



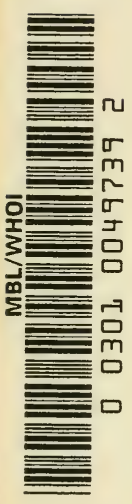





\title{
THE BIOCHEMISTRY OF B VITAMINS
}

\author{
Roger J.' Williams \\ Robert E. Eakin \\ Ernest Beerstecher, Jr. \\ William Shive
}

University of Texas, Austin, Texas

BOOK DIVISION

REINHOLD PUBLISHING CORPORATION

330 West Forty-second St., New York 18, U. S. A. 
Copyright, 1950, BY

REINHOLD PUBLISHING CORPORATION

All rights reserved

Printed in the United States of America by

THE HADDON CRAFTSMEN, INC., SCRANTON, PA. 


\section{GENERAL INTRODUCTION}

\section{American Chemical Society's Series of}

\section{Chemical Monographs}

By arrangement with the Interallied Conference of Pure and Applied Chemistry, which met in London and Brussels in July, 1919, the American Chemical Society was to undertake the production and publication of Scientific and Technologic Monographs on chemical subjects. At the same time it was agreed that the National Research Council, in cooperation with the American Chemical Society and the American Physical Society, should undertake the production and publication of Critical Tables of Chemical and Physical Constants. The American Chemical Society and the National Research Council mutually agreed to care for these two fields of chemical progress. The American Chemical Society named as Trustees, to make the necessary arrangements for the publication of the Monographs, Charles L. Parsons, secretary of the Society, Washington, D. C.; the late John E. Teeple, then treasurer of the Society, New York; and the late Professor Gellert Alleman of Swarthmore College. The Trustees arranged for the publication of the ACS Series of (a) Scientific and (b) Technological Monographs by the Chemical Catalog Company, Inc. (Reinhold Publishing Corporation, successor) of New York.

The Council of the American Chemical Society, acting through its Committee on National Policy, appointed editors (the present list of whom appears at the close of this sketch) to select authors of competent authority in their respective fields and to consider critically the manuscripts submitted.

The first Monograph of the Series appeared in 1921. After twenty-three years of experience certain modifications of general policy were indicated. In the beginning there still remained from the preceding five decades a distinct though arbitrary differentiation between so-called "pure science" publications and technologic or applied science literature. By 1944 this differentiation was fast becoming nebulous. Research in private enterprise had grown apace and not a little of it was pursued on the frontiers of knowledge. Furthermore, most workers in the sciences were coming to see the artificiality of the separation. The methods of both groups of workers are the same. They employ the same instrumentalities, and frankly recognize that their objectives are common, namely, the search for new knowledge for the service of man. The officers of the Society therefore combined the two editorial Boards in a single Board of twelve representative members.

Also in the beginning of the Series, it seemed expedient to construe rather broadly the definition of a Monograph. Needs of workers had to be 
recognized. Consequently among the first hundred Monographs appeared works in the form of treatises covering in some instances rather broad areas. Because such necessary works do not now want for publishers, it is considered advisable to hew more strictly to the line of the Monograph character, which means more complete and critical treatment of relatively restricted areas, and, where a broader field needs coverage, to subdivide it into logical sub-areas. The prodigious expansion of new knowledge makes such a change desirable.

These Monographs are intended to serve two principal purposes: first, to make available to chemists a thorough treatment of a selected area in form usable by persons working in more or less unrelated fields to the end that they may correlate their own work with a larger area of physical science discipline; second, to stimulate further research in the specific field treated. To implement this purpose the authors of Monographs are expected to give extended references to the literature. Where the literature is of such volume that a complete bibliography is impracticable, the authors are expected to append a list of references critically selected on the basis of their relative importance and significance.

\section{AMERICAN CHEMICAL SOCIETY}

BOARD OF EDITORS

William A. Hamor, Editor of Monographs

Associates
L. W. BASS
T. H. Chilton
Barnett Cohen
FarRington Daniels
J. BenNetT Hili
E. H. Huntress

S. C. Lind

C. H. Mathewson

W. T. READ

Walter A. Schmidt

E. R. WEIDLEIN

W. G. Whitman 
This volume is dedicated to

The Clayton Foundation For Research

which has generously and continuously supported research dealing with the $\mathrm{B}$ vitamins. 


\section{ACKNOWLEDGEMENTS}

We wish to express our thanks to Dr. R. R. Williams and to our former colleague Professor E. E. Snell for critically reading substantial portions of the manuscript. We also acknowledge gratefully the help and encouragement received from all of the members of the Biochemical Institute staff. Individual members have read portions of the manuscript, helped in its final preparation and aided in numerous ways to make the book possible. To all of these we express our sincere gratitude and thanks.

Permission to use the following material is acknowledged with thanks: Table 2, pp. 250-251, Table 3, p. 253 and Table 4, p. 253, from Williams and Spies: Vitamin $B_{1}$ and Its Use In Medicine, copyright 1938, by the Macmillan Company. Table 6, p. 257, Journal of Nutrition, L. J. Roberts, Figure 3, p. 313, Indian Journal of Medical Research, P. S. Sarma. Table 20, pp. 324-325, Food and Nutrition Board, National Research Council. Tables 29 and 30, pp. 366-367, Academic Press, C. W. Denko. Table 34, p. 389, Proceedings of the Society for Experimental Biology and Medicine, F. G. Brazda. Figure 17, p. 405, Journal of Nutrition, J. Salcedo, Jr. and L. E. Holt, Jr. Figures 18-20, pp. 416-418, Lancet, T. D. Spies. 


\section{PREFACE}

We have sought in this volume to fulfill, with respect to the chosen field, the two fundamental purposes set forth at the time the American Chemical Society Monograph Series was instituted.

First, we have attempted to present the material "in readable form, intelligible to those whose activities may be along a wholly different line" to the end that they may gain perspective and interest and appreciation of the fundamental unity existing in the broad area involving physical as well as biological science.

Second, we have sought to promote research in the field of the B vitamins "by furnishing a well digested survey of the progress already made in that field and by pointing out directions in which investigation needs to be extended." In connection with this latter purpose we have had in mind two groups: the oncoming advanced students who from year to year must have a means of becoming acquainted with the field, and the ever increasing number of chemists whose fundamental training has been in some other branch but who are turning to biochemistry as an attractive field of investigation.

In keeping with the title of the volume and the purposes set forth, we have not included full discussions of the organic chemistry or technology of the substances involved, nor have we given a historical treatment of their discovery and identification. To trace all the misconceptions and inevitable blunderings which have entered into the development of our present knowledge and at the same time to do justice to the numerous investigators would be an impossible task, and from the standpoint of our purposes an attempt would not be worthwhile.

We agree with the late G. N. Lewis' statement, "a monograph of this sort belongs to the ephemeral literature of science. The studied care which is warranted in the treatment of the more slowly moving branches of science would be out of place here. Rather with the pen of a journalist we must attempt to record a momentary phase of current thought, which may at any instant change with kaleidoscopic abruptness."

Although the four authors have found themselves to be in substantial agreement on most points, in the presentation of the material in the four sections they have used their respective judgments, expressed their own 
individual opinions and organized the material in accordance with their own thinking on the subject. One of our primary aims has been to present constructive and suggestive viewpoints, and in so doing we have had to run the risk of being in error. This risk could have been avoided by compiling an uncritical review with none of the reviewers' opinions expressed, but to have done this would have effectively defeated the purpose of the book.

We wish to beg the indulgence of our readers for mistakes and imperfections that may be found, and if they are such as can be remedied in later printings or editions we will appreciate having them called to our attention. There has been some unavoidable duplication in different areas of the discussion, but this has been retained whenever doing so would contribute materially to the unity and understandability of the particular section. In view of the magnitude of the task, which turned out to be even greater than we had anticipated, and because the preparation of the manuscripts had to be superimposed upon active research and/or teaching programs, we hope that our readers will be charitable in judging the product of our labors.

It has not been feasible to synchronize our efforts completely and in the respective sections the literature has not been completely reviewed up to exactly the same date. Even within sections the up-to-dateness may vary slightly from topic to topic. For example, the citation of individual crucial papers up through April or May, 1950, does not mean that all of the literature has been covered up to that point. We cannot hope that the material which we have presented will remain up-to-date for long. Indeed, a sincere desire to promote research is equivalent to hoping that this volume will rapidly become out-of-date. But, like Professor Lewis, we can hope that necessary changes will involve "matters of detail rather than of essence."

Roger J. Williams

Robert E. Eakin

Ernest Beerstecher, Jr.

William Shive

Austin, Texas

June 15, 1950 


\section{CONTENTS}

General Introduction

iii

Preface

Section A: Characterization, Distribution, Assay and Biogenesis of B Vitamins

Chapter IA: B Vitamins: IVhat They Are 3

Chapter IIA: Distribution of B Vitamins 18

Chapter IIIA: Combined Forms-Extraction 30

Chapter IVA: Assay Methods 45

Chapter VA: Biogenesis of the B Vitamins 78

Section B: The Catalytic Functions of the B Vitamins

Chapter IB: Biochemical Reactions and Their Catalysts 95

Chapter IIB: Coenzymes Derived from B Vitamins 123

Chapter IIIB: The Functions of the B Vitamins in Metabolic Processes 216

Section C: The Role of the B Vitamins in Animal and Plant Organisms

Chapter IC: Methods of Assessing B Vitamin RequireMENTS

Chapter IIC: Factors Influencing B Vitamin RequireMENTS

Chapter IIIC: The B Vitamin Requirements of Animals and Plants

Chapter IVC: Metabolism of the B Vitamins

Chapter VC: Physiological, Pharmacological, and ToxICOLOGICAL EFFECTS 377

Chapter ViC: B Vitamin Deficiency States

Section D: The Comparative Biological Activities of the B Vitamins and Related Compounds

Chapter ID: Introduction and Theoretical ConsideraTIONS 
Chapter IID: Utilization of Competitive AnalogueMetabolite Inhibition in the Elucidation of Biochemical Processes Involving Vitamins

Chapter IIID: $p$-Aminobenzolc Acid 481

Chapter IVD: Biotin

Chapter VD: The Folic Acid Group

Chapter VID: The Nicotinic Acid Group 604

Chapter VIID: Pantothenic Acid 620

Chapter Vilid: The Vitamin $B_{6}$ Group $\quad 652$

Chapter IXD: Riboflavin $\quad 669$

Chapter XD: Thiamine 684

Chapter XID: Biological Activities of Other Nutritional Factors of Doubtful Status 
Section A

CHARACTERIZATION, DISTRIBUTION, ASSAY AND BIOGENESIS OF B VITAMINS

Roger J. Williams 



\section{Chapter IA}

\section{B VITAMINS: WHAT THEY ARE}

Historically the term "vitamin B". was applied to the water-soluble organic material present in yeast, wheat germ, protein-free milk, etc., which was found to be necessary in small amounts for the nutrition of young animals. At the time this designation came into general use the dietary importance of minerals, proteins (amino acids), carbohydrates, fats, "vitamin A," and vitamin C was recognized, and "vitamin B" meant something distinct from these other recognized food materials.

When it became evident that vitamin B was not a single substance but several, the designations $\mathrm{B}_{1}, \mathrm{~B}_{2}, \mathrm{~B}_{3}$, etc., were introduced. These have more recently given way in most cases to names for the specific chemical substances involved: thiamine, riboflavin, etc. We shall discuss later specific cases of substances which according to one's point of view may or may not be included among the "B vitamins."

The time may well arrive when the term "B vitamin" will be abandoned, and each specific chemical substance will be considered entirely as a separate entity. At the present time, however, there is good reason for retaining the term, because $\mathrm{B}$ vitamins appear to have common attributes which set them apart from all other vitamins.

Microbiological assay methods have made it possible to learn that the specific compounds commonly considered as members of the B family are universally distributed in all living cells, whether of plant, animal or bacterial origin. Since this appears not to be true of any of the other vitamins, it was suggested elsewhere that $B$ vitamins may be tentatively defined as those which occur as indispensable constituents of all living matter. ${ }^{1}$ If this suggestion is valid, their fundamental importance is selfevident.

Studies relating to the functioning of individual members of the B family of vitamins have demonstrated that they are integral parts of biological catalytic systems and that they constitute essential factors in the metabolic machinery of widely diverse forms. It seems probable that this is true of all of the $B$ vitamins, and an amended definition may include this idea. In this case, we may say that B vitamins are those which enter into the catalytic systems of all living cells.

Since the water solubility or fat solubility of a compound is not absolute 
but rather a matter of degree, we have not included the loose term "water solubility" as an essential characteristic of a B vitamin. Indeed, if the idea of the existence of a distinctive group of vitamins which function catalytically in all living cells is a valid one, it is conceivable that we may eventually come to include some of the "fat-soluble" compounds among the B vitamins. Of such compounds already recognized, possibly the most likely candidates for inclusion are the vitamins E, which appear to be widely distributed in diverse organisms and tissues. ${ }^{2}$ If these vitamins should be found to be part of the catalytic machinery of all cells, there would seem to be no very valid reason for excluding them from the $\mathrm{B}$ vitamins. The fact that a separate letter designation has been used for them would not preclude this possibility. Biotin, which was early called "vitamin H," is now recognized as a member of the B family. The question of the universal occurrence of $\mathrm{B}$ vitamins in relation to other vitamins is discussed further in Chapter IIA.

One of the interesting observations which in a measure appears to differentiate the $\mathrm{B}$ vitamins from the members of the so-called fat-soluble group is the fact that in the case of the fat-soluble vitamins there are in every instance several naturally occurring and distinct chemical structures which possess the specific vitamin activity, whereas among the $\mathrm{B}$ vitamins the physiological activity is more specifically associated with a single chemical structure. It is true that in several instances among the $B$ vitamins, modified structures possess specific vitamin activity, but in general each B vitamin is represented by a single substance or at least by a few very closely related structures. As an instance of the latter, in case a specific vitamin (e.g., nicotinamide, riboflavin) is involved as a catalytic hydrogen carrier, it is reasonable that both the oxidized and reduced forms should have physiological activity; likewise, if a vitamin is a catalyst for ammonia transfer (e.g., pyridoxal), it is not surprising that an aminated form (pyridoxamine) should possess physiological activity.

We may now profitably consider in a critical manner the meaning and significance of the word "vitamin"-a term the meaning of which we have so far in this discussion taken for granted. It involves historically a nutritional concept and has come to be applied almost exclusively to certain organic substances which function in the nutrition of higher animals. While a considerable number of the B vitamins were discovered and isolated using yeasts and bacteria as test organisms, they have not been admitted to the family of vitamins unless they have been found to be nutritionally effective for higher animals.

A substance which counteracts a vitamin deficiency is not, however, necessarily designated a vitamin. If this were so, thiamine pyrophosphate, 
coenzymes I and II, riboflavin nucleotides, and even flavoproteins, etc., would be considered as vitamins because they are capable of counteracting respectively thiamine, niacin and riboflavin deficiencies. Actually, they are not designated as vitamins. The easiest way out of the difficulty in a specific case is to consider as a vitamin only the simplest compound capable of performing the specific nutritional function. In cases where two or more compounds of about the same complexity function alike nutritionally, each may conveniently be called a vitamin. Nicotinic acid and nicotinamide on the one hand, and pyridoxal, pyridoxamine and pyridoxine on the other, are examples.

The importance of some of the compounds commonly designated as vitamins does not rest, moreover, solely upon their functioning in nutrition. Nicotinamide from the nutritional standpoint may not be essential for animals if tryptophan is abundantly supplied, yet it is a nutritional substance and is important in that it constitutes a part of the metabolic machinery in every cell. Even though mammals generally, including human beings, are probably capable of synthesizing nicotinamide in their bodies from tryptophan, it is nonetheless a compound of great biochemical interest and importance. Likewise, the importance of thiamine, riboflavin, pantothenic acid and other members of the B family of vitamins does not depend only upon the fact that they cannot be synthesized by higher animals. As essential parts of the metabolic machinery, they are most fundamental, regardless of their nutritional importance. Their nutritional functioning may even be considered of secondary significance.

Looking at the matter with these facts in mind we may suspect that the B vitamins actually bclong to a larger group of organic catalytic units which are indispensable to all cells, but which may or may not be vitamins in the nutritional sense. Some of these indispensable units may be uniformly synthesized by higher animals. We have no name for this inclusive group of catalytic substances, ${ }^{*}$ if such exists, and it appears premature to discuss them at present. Until the time arrives when we fully recognize the existence of such a group, it will be well to retain the term "vitamin" and the nutritional concept which underlies it. A substance therefore cannot be classed as a vitamin unless it functions nutritionally for higher animals.

Following this line of reasoning we may expand our definition of a B vitamin to include those organic substances which act catalytically in all living cells and which function nutritionally for at least some of the higher animals. We cannot guarantee, of course, that this delineation of $B$ vitamins will remain valid indefinitely. If it should be found that some of the typical B vitamins lack a catalytic function or that some of them

* The name "catalins" has, however, occurred to the author as an appropriate one. 
are absent from certain types of cells, then our definition might have to be modified immediately. At present such modification does not appear likely.

\section{Chemically Recognized B Vitamins}

There are a number of specific chemical substances of known structure which are universally recognized as B vitamins. We may, therefore, gain a more specific idea of what is meant by the term " $\mathrm{B}$ vitamin" by discussing briefly these individual compounds.

Thiamine (aneurin) earlier received the designation " $\mathrm{B}_{1}$ " in keeping with the fact that it was the first B vitamin to be discovered and isolated. There is substantially no confusion resulting from identifying vitamin<smiles></smiles>

Thiamine chloride hydrochloride

$B_{1}$ as thiamine, because thiamine is the only naturally occurring structure which is capable of performing the vitamin functions (p. 684). More complex structures containing the thiamine unit as a part may function nutritionally, as may also products formed by reversible oxidation and reduction, if such exist. The chemistry of thiamine has been reviewed by R. R. Williams. ${ }^{3}$

Riboflavin, earlier called vitamin $\mathrm{B}_{2}$, has the structure indicated below and is the only naturally occurring structure possessing the characteristic vitamin activity. Several synthetic flavins have lesser biological activity;<smiles>Cc1cc2cc3c(=O)[nH]c(=O)nc-3n(CC(O)C(O)C(O)CO)c2cc1C</smiles> 
esters and other simple derivatives of riboflavin may possess full activity, and conjugated forms such as the nucleotides or flavoproteins may be nutritionally effective in proportion to their content of combined riboflavin. The (reversibly) reduced form of riboflavin and its conjugates are also physiologically active. Since the establishment of its constitution and its synthesis in 1935 by Kuhn and Karrer and their co-workers, there have been few advances in the organic chemistry of riboflavin. A complete review of this topic may be found in the literature. ${ }^{4}$

Nicotinic Acid, Nicotinamide. These two compounds interchangeably possess vitamin activity and the only other naturally occurring compounds which can function nutritionally in the same manner are more complicated derivatives which may act because they contain the essen-

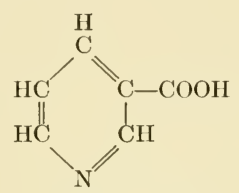

Nicotinic acid

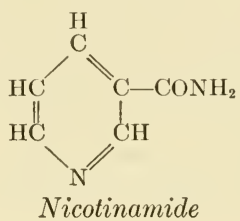

Nicotinamide

tial structure in combined form. Combined forms, even naturally occurring ones, are not necessarily wholly effective. The chemistry of nicotinic acid is too old a topic in the field of organic chemistry to require comment here.

Pantothenic Acid. Probably this compound was mainly responsible for what was first designated "vitamin $\mathrm{B}_{3}$ "; it is the only naturally occurring one capable of performing the nutritional function. Conjugated forms<smiles>CC(C)(CO)C(O)C(=O)NCCC(=O)O</smiles>

\section{Pantothenic acid}

may possess vitamin activity because they contain the fundamental structure in combination. The chemistry of pantothenic acid has been reviewed ${ }^{5}$ and detailed material on the subject will be found in the literature cited in the review. Other material clealing with the chemistry of pantothenic acid will be found elsewhere in this volume (p. 464).

Pyridoxal, Pyridoxamine, Pyridoxine. For animals these three forms are nutritionally interchangeable and are often thought to be in equilibrium in vivo. Lactic acid bacteria often show incomparably greater response to pyridoxal or pyridoxamine or their phosphates ${ }^{6}$ than to pyridoxine, so these forms of the vitamin which were discovered later 
appear to be fundamentally more important biocatalytically than pyridoxine. The three members of the group possess for animals what has been called "vitamin $\mathrm{B}_{6}$ " activity. The chemistry of these compounds<smiles>Cc1ncc(CO)c(C=O)c1O</smiles>

Pyridoxal<smiles>Cc1ncc(CO)c(CN)c1O</smiles>

Pyridoxamine<smiles>Cc1ncc(CO)c(CO)c1O</smiles>

Pyridoxine

is discussed in a series of articles by Heyl et al. and in earlier contributions from the same laboratory. ${ }^{7,8,9,10}$

Biotin. This substance is the only naturally occurring one (except its conjugates and possibly oxybiotin discussed below) known to be capable of counteracting the deficiency induced by feeding raw egg white or avidin, its active constituent, to animals. It is an example of a B vitamin which appears to be of little or no practical importance nutritionally

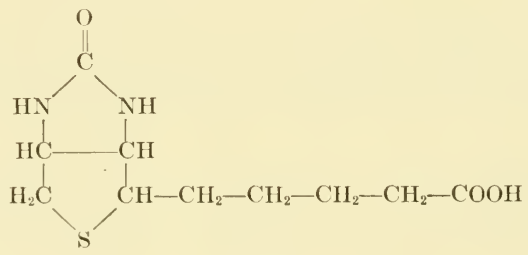

Biotin

(because it is so readily produced by intestinal organisms) but is nonetheless most interesting as a universal cellular constituent which probably acts catalytically. It is effective in unusually low concentrations.

Oxybiotin, the compound corresponding to biotin with oxygen replacing the sulfur, is especially interesting as a substance which almost falls within our definition of a $\mathrm{B}$ vitamin. Certain microorganisms, at least, can use it in place of biotin and do not convert it into biotin. ${ }^{11}$ Oxybiotin in these cells can act as a part of the metabolic machinery, but since it appears ordinarily not to be a constituent of cells, and certainly there is no evidence that it is present in all cells, its inclusion in the group of $B$ vitamins is not justified.

The organic chemistry of biotin and its derivatives has been reviewed by Hofmann ${ }^{12}$ and by Melville, ${ }^{13}$ and further discussion of its chemistry will be found in Section D of this volume. 
Folic Acid. The vitamin activity which characterizes this substance most clearly is its ability to prevent a specific type of anemia in chicks. No other naturally occurring structure (except its conjugates) is known to be capable of performing this function.

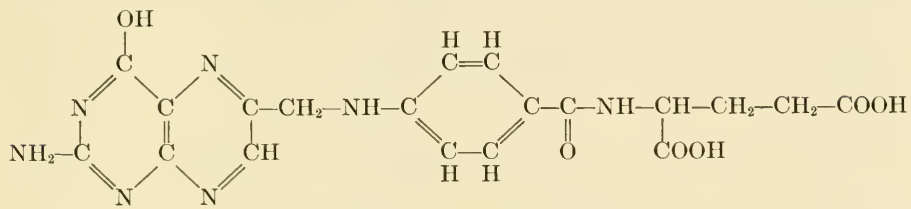

\section{Folic acid (Pteroylglutamic acid)}

More complicated structures occur naturally and their physiological significance will be discussed in later chapters, but the simplest compound capable of performing the vitamin function is the one pictured above, and in accordance with our previous discussion it is the only one which will be considered as a B vitamin. There is no serious doubt, on the basis of microbiological evidence, but that it functions universally in living matter. The fact that it is effective in very minute amounts strongly suggests a catalytic role.

A series of articles dealing with the chemistry of folic acid and its derivatives treat this subject rather fully. ${ }^{14,15,16,17,18,19}$

\section{Structurally Known Compounds of Somewhat Doubtful Status}

In addition to the seven chemically characterized vitamins with distinct nutritional functions listed above, there are three other well recognized compounds of nutritional signficance: inositol, choline, and $p$-aminobenzoic acid, which merit consideration because they possess at least some of the characteristics of B vitamins.

We have used three criteria in our previous discussions. To belong in the B group a compound must: (1) function nutritionally for higher animals, (2) be universally present in living cells, and (3) act or be presumed to act catalytically. These criteria are listed, in our opinion, in the approximate order of their importance. The status of each of the three substances mentioned above can be questioned on the basis of at least one of these criteria.

Inositol appears to function nutritionally for various experimental animals (though contrary evidence has been obtained ${ }^{20}$ ), and evidence for its universal occurrence is perhaps as good as for other members of the group, but its relative abundance in tissues and its occurrence as a structural unit in recently discovered lipides, makes one question its inclusion. It is not uncommon for inositol to be present in tissues in 
amounts 100 or even 1000 times that of the more typical B vitamins. Favoring the inclusion of inositol as a member of the group is the finding that it constitutes a functioning portion of pancreatic amylase. ${ }^{21 *}$ This indicates a catalytic role. It may be that inositol does indeed act cat-

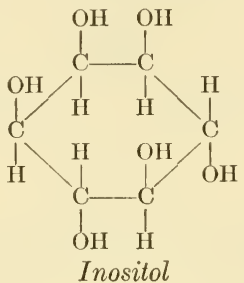

alytically like the other $\mathrm{B}$ vitamins and that its relative abundance is explained by the fact that it also is a constituent of certain widely occurring lipides.

The relatively recent determination of the configuration of inositol is alluded to in a later chapter (p. 18).

Choline is without question of great nutritional importance and has some of the earmarks of a B vitamin. If any one of a number of mammals and fowls is fed a diet deliberately made up so as to be low in choline content, a serious deficiency results which can be counteracted by the addition of pure choline salts to the diet. However, methionine and<smiles>C[N+](C)(C)CCO</smiles>

\section{Choline chloride}

betaine (as well as other substances which do not occur naturally) are also effective under these conditions, and it is clear that they may perform at least part of the function of choline. It appears likely that they may replace choline entirely, especially in the presence of some other unidentified substance. ${ }^{22}$ Ethanolamine can evidently be methylated by Type III pneumococcus ${ }^{23}$ to produce choline which in combined form (phospholipides) is probably always present in living cells. Furthermore, evidence involving mutant strains of Neurospora shows clearly that the synthesis of choline takes place through the intermediate formation of ethanolamine. ${ }^{24}$

It appears clear that the naturally occurring substances, choline, betaine and methionine alike, are able to furnish animals with transferable methyl groups which are essential to life, and that in addition choline

* Contradictory evidence has been reported. ${ }^{21 a}$ 
itself is an essential cell constituent and must be furnished in the food or built up by animals. Just what is required for this building up process is not known. If ethanolamine is the simplest substance which in conjunction with methionine can overcome the lack of choline in the diet, it instead of choline should be designated as the vitamin, according to our previous discussion. From the practical nutritional standpoint, however, choline is more important than ethanolamine, but its status as a vitamin is complicated by the existence of other natural food substances which at least partially replace it.

From the standpoint of universality in cells, choline appears to be like the typical B vitamins. Its catalytic functions have not been demonstrated and its relative abundance and the high "requirements" of animals may be cited against this possibility. On the other hand, it may (like inositol?) be both a catalytic unit and a constituent of certain phospholipides. There is some resistance to designating choline as a vitamin because it was known to be a common constituent of natural foods (combined in phospholipides) long before the typical vitamins were discovered.

$p$-Aminobenzoic acid is unique among the vitamins or vitamin-like substances so far considered in that it makes up an integral part of one of the typical B vitamins, namely folic acid. Its nutritional functioning

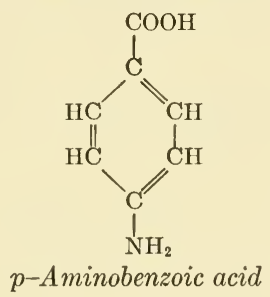

has been demonstrated, but not in connection with a diet already containing an adequate supply of folic acid. Furthermore, relatively large quantities have been used. It is possible, since $p$-aminobenzoic acid makes up a part of the folic acid molecule (p. 9), that its nutritional value is dependent upon its use as a building unit out of which folic acid may be made by intestinal bacteria or otherwise. Even before the presence of the $p$-aminobenzoic acid residue in folic acid was established, there was evidence that feeding $p$-aminobenzoic acid to chicks promoted intestinal production of folic acid..$^{25}$

The crucial questions which have not been conclusively answered at the present writing are whether $p$-aminobenzoic acid is required nutritionally in addition to folic acid, and whether it has catalytic functions independent of folic acid. There are strong indications of a positive 
answer to the second question, and these will be discussed in later chapters. $p$-Aminobenzoic acid occurs universally in living matter in its combination in folic acid and without much doubt in other forms also (p. 41). It is a highly important catalytic unit and appears to constitute a unique example of a "vitamin within a vitamin."

\section{B Vitamins and Related Principles Not Completely Characterized Chemically}

In the infancy of vitamin investigations vitamin $B$ was thought to be a single entity. Since that time, one after another well defined chemical substance has been found to contribute to the physiological activity which resides in crude extracts of yeast, liver, etc. How much further this discovery of new organic components possessing vitamin activity will go, no one can say.

When the time arrives that the list is complete, it will be possible to feed baby chicks and young rats completely synthetic diets, raise them to maturity in a healthy condition, have them reproduce normally generation after generation, and raise young as they do when fed natural diets. Furthermore, it will be possible to accomplish this feat when the animals are kept under sterile conditions, free from the symbiotic aid of microorganisms. Until this latter is possible, one cannot be sure but that some essential vitamins of bacterial origin are as yet unrecognized. Biotin is an example of a vitamin which might have been overlooked in non-sterile feeding experiments were it not for the experimental use of raw egg white in diets and the presence in it of avidin, which combines quantitatively with biotin, rendering it inactive.

Many of the $B$ vitamins have been discovered and concentrated by the use of microorganisms, but historically they were not admitted to the family of $\mathrm{B}$ vitamins until their functioning in animal nutrition had been demonstrated. The study of microbial nutrition therefore constitutes an important means whereby hitherto unrecognized nutrilites may be discovered. Their acceptance as vitamins may depend upon the demonstration of their functioning in the nutrition of higher animals.

There is the point of view among some active vitamin investigators, particularly those who have approached the subject from the standpoint of enzyme chemistry or microbial nutrition, that catalytic substances of relatively small molecular dimensions may properly be regarded as vitamins whether or not they are nutritionally required by higher animals. This point of view has merit and may eventually be adopted. An alternative suggestion has already been made, namely, that the term "vitamin" retain its historical nutritional connotation and that perhaps a new, more inclusive term (e.g., catalins, p. 5) will be needed to designate all 
biocatalysts of low molecular weight, regardless of their nutritional significance or insignificance.

The search for substances which are potentially $B$ vitamins is an active one at the present time. Advance in this field is so rapid that this monograph cannot hope to carry up-to-the-minute information. It is not surprising that the author of this section should be in possession of a certain amount of unpublished information which is pertinent to this discussion. The only safe procedure in view of the activity in this field, however, seems to be to treat all unpublished information which may be in the writer's possession as though it did not exist. The discussions which follow will, therefore, be based almost entirely upon results which have received general publication.

If we retain the nutritional point of view with respect to the meaning of the term "vitamin," then before any new substance can be accepted as a member of the family, evidence must be available with respect to its nutritional need by animals. We shall therefore discuss first those substances for which such need has been demonstrated.

Second, before any growth factor can be discussed intelligently, its unitary nature and its existence as a chemical entity must be reasonably well established. In order to meet this criterion, the substance in question must have been characterized chemically or concentrated to a relatively high degree.

Before we inquire what is meant by "concentrating a vitamin to a high degree," it will be well to look at some historical data with respect to the vitamins which are already well recognized.

Rice polish was the starting material used for the first isolation of thiamine. It contains in round figures $33 \mu \mathrm{g}$ of thiamine per gram. Thiamine is therefore about 30,000 times as active physiologically as rice polish, and this figure represents the extent to which concentration had to be carried to yield the active principle. Using the same type of calculation, we arrive at the values for the typical B vitamins listed below.

\begin{tabular}{ll}
\multicolumn{1}{c}{ Vitamin } & \multicolumn{1}{c}{ Source } \\
Thiamine & Rice polish \\
Riboflavin & Whey solids \\
& Liver \\
Nicotinic acid & Liver \\
Pantothenic acid & Liver \\
Folic acid & Spinach \\
& Liver \\
Biotin & Egg yolk \\
& Liver
\end{tabular}

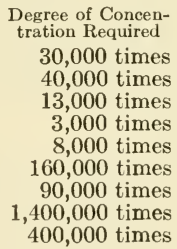

From these data we may infer that before a new vitamin is obtained in pure form, it is probable that it will have to be concentrated several thousand times at least, starting with any rich natural source as a refer- 
ence point. Concentration may have to be carried up to a millionfold or more before the substance is pure. In view of these facts it apears undesirable to refer to a "vitamin concentrate" or to a vitamin having been obtained "in concentrated form" unless its concentration has bcen carried to a point at least 100 times that of a rich natural source. In the literature in the past numerous "concentrates" have been so designated when they were not substantially more active than crude extracts of liver or yeast. In the writer's own experience, the difference between having a "concentrate" which is a crude extract and one which is several thousand times as active as the starting material may involve the expenditure of many thousands of man-hours of effort. To designate all such products "concentrates" regardless of how far the process has been carried shows a lack of proper discrimination.

Anti-Pernicious Anemia Principle (Vitamin $B_{12}$ ). Of the vitamins which have not as yet been completely characterized chemically, by far the most interesting one at the present writing is the anti-pernicious anemia principle which has been isolated approximately simultaneously in crystalline form both in England ${ }^{26}$ and in the United States. ${ }^{27}$

It is a red crystalline substance which does not melt before decomposition; it contains cobalt, phosphorus and nitrogen, and is reported to have a molecular weight of about 1500 . It is active in clinical pernicious anemia in doses of about $1 \mu \mathrm{g}$ per day, and is thought to be identical with the "animal protein factor" ${ }^{28}$ as well as the "cow manure factor" ${ }^{29}$ which have been reported to be required by chickens.

The physiological activity of the substance as judged by its effect on human subjects is much greater, weight for weight, than that of any of the vitamins discussed. Its purification from liver represents several millionfold concentration.

The effectiveness of this principle in extremely small doses suggests its catalytic functioning, which has been studied by inhibition analysis (p. 475). Its relationship to normal nutrition is not entirely clear, but the requirement of chickens and its relationship to macrocytic anemias of nutritional origin make its status as a vitamin relatively secure. While its universal presence in living cells has not been explored, it appears to be widely distributed in nature and to be produced by various bacteria and molds. ${ }^{30}$

It is interesting and significant to note that, although numerous laboratories have engaged in studying and attempting to concentrate the antipernicious anemia principle, in terms of chronology no outstanding progress was reported until after the development of a microbiological test. Actually, workers in England, however, appear to have used the red color obtained chromatographically as a basis for clinical testing. This vitamin 
extends further the list of $\mathrm{B}$ vitamins for the elucidation of which microbiological investigations have proved most valuable. It is also worthy of note that every vitamin isolated or concentrated by using microbiological tests has fallen into the category of " $B$ vitamins" by common consent and on the basis of the criteria which we have outlined (p. 5).

Thymidine is worthy of note in this connection because of its functional relation to the anti-pernicious anemia vitamin ${ }^{31}$ and the fact that in relatively large doses it is able to replace the vitamin in microbiological tests. ${ }^{32}$ Other desoxyribosides also function in a similar manner.

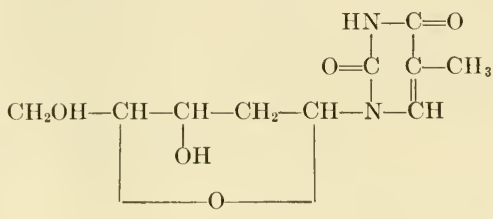

Thymidine

The interrelationship between thymidine and the pernicious anemia vitamin will be discussed in a later section (p. 474).

Strepogenin. This growth principle was originally found in liver and was effective for certain hemolytic streptococci. ${ }^{33}$ Subsequently it was found to be more abundant in certain purified proteins, notably insulin and trypsinogen, and to be released most effectively by tryptic digestion. ${ }^{34}$ A convenient test organism which is in current use is $L$. casei. ${ }^{35}$ What appears to be the same substance is also effective in promoting the growth of mice. ${ }^{36,37}$ The available evidence indicates that the substance is of peptide nature and predominantly acidic, and one synthetic peptide, seryl glycyl glutamic acid, has been found to have appreciable strepogenin activity.

If strepogenin is a peptide of known amino acids without any novel feature in its structure, it could hardly be classified as a B vitamin. The growth principle as tested for in the usual way has not been obtained in a form such that a minute amount is effective, so a catalytic function for strepogenin cannot be taken for granted. It is an important nutritional principle, however, and it is discussed briefly here because, pending the complete elucidation of its chemical nature, it is a potential member of the B vitamin family. Recently, Chattaway et al., using diphtheria organisms for testing, obtained a highly potent preparation from yeast which is suggestive of strepogenin. ${ }^{38}$ It is reported to possess activity by virtue of the presence of two peptides and a fraction which is stable to acid hydrolysis, all three of which entitics are required for maximum growth. 
"Vitamin $B_{13}$ " represents a growth factor for rats which has been obtained in highly concentrated form by Novak and Hauge. ${ }^{39}$ The concentrate prepared was effective for rats at a level of $2 \mu \mathrm{g}$ per day and gave a maximum response when $10 \mu \mathrm{g}$ per day were administered. Its ultraviolet absorption curve showed a maximum at $2820 \AA$, and it exhibited fluorescence. The material was readily soluble not only in water but also in acetone, ethanol, ether, chloroform and benzene. If this substance becomes established as a member of the B family of vitamins, it will be unique with respect to its solubilities. This fact emphasizes the undesirability of using solubility as a criterion for classifying vitamins.

"Vitamin $B_{14}$," a crystalline substance having high growth-promoting activity on reticulocytes and tumor cells in vitro and on the anemia in rats induced by sulfathiazole administration, has been reported by Norris and Majnarich. ${ }^{40}$ It was isolated from urine and contains 19.6 per cent nitrogen, 4 per cent phosphorus and no cobalt, and is thought to be functionally related to folic acid. ${ }^{41}$

The literature contains numerous references to additional growth substances for microorganisms and animals which are potentially members of the B vitamin family. However, neither their concentration nor characterization has proceeded far enough to justify individual discussion.* The history of the discovery and identification of individual B vitamins is replete with nutritional factors, often designated by letter names and numbers, which are more or less composite in nature and which have remained poorly defined indefinitely. When a new growth substance is identified and obtained in crystalline form, usually by the help of microbiological tests, confusion with respect to various nutritional factors previously discovered in animal work tends to be dispelled.

\section{Bibliography}

1. Williams, R. J., "A.A.A.S. Research Conference on Cancer," Science Press Printing Co., Lancaster, Pa., 1945, pp. 253-66.

2. Williams, R. J., "Vitamins and Hormones," Vol. I, Academic Press, Ine., New York, N. Y., 1943, p. 231.

3. Williams, R. R., Ergeb. Vitamin-Hormonforsch., 1, 213-62 (1938).

4. Rosenberg. H. R., "Chemistry and Physiology of the Vitamins," Interscience Publishers, New York, N. Y., 1942, pp. 155-70.

* Since the above was written the concentration of three additional potential members of the B vitamin family has been accomplished: Folinic acid (citrovorium factor), Bond, T. J., Bardos, T. J., Sibley, M. and Shive, W., J. Am. Chem. Soc. 71, 3852 (1949) and Bardos, T. J, Bond, T. J., Humphreys, J. and Shive, W., ibid.; the Lactobacillus bulgaricus factor, Williams, W. L., Hoff-J $\varnothing$ rgensen, E. and Snell, E. E., J. Biol. Chem. 177, 933-40 (1949); an acetate factor (pyruvate oxidation factor, "protogen"), Snell, E. E. and Broquist, H. P., Arch. Biochem. 23, 326 (1949). Other bacterial factors are discussed by Snell, E. E., Ann. Rev. Microbiol. III, 97 (1949). 
5. Williams, R. J., "Advances in Enzymology," Vol. III, Interscience Publishers, New York, N. Y., 1943, pp. 253-87.

6. McNutt, W. S., and Snell, E. E., J. Biol. Chem., 173, 801-2 (1948).

7. Harris, S. H., Heyl, D., and Folkers, K., J. Am. Chem. Soc., 66, 2088-92 (1944).

8. Heyl, D., J. Am. Chem. Soc., 70, 3434-6 (1948).

9. Heyl, D., Harris, S. A., and Folkers, K., J. Am. Chem. Soc., 70, 3429-31 (1948).

10. Heyl, D., et al., J. Am. Chem. Soc., 70, 3669-71 (1948).

11. Hofmann, K., and Winnick, T., J. Biol. Chem., 160, 449-53 (1945).

12. Hofmann, K., "Advances in Enzymology," Vol. III, Interscience Publishers, New York, N. Y., 1943, pp. 289-311.

13. Melville, D. B., "Vitamins and Hormones," Vol. II, Academic Press, Inc., New York, N. Y., 1944, pp. 29-66.

14. Stokstad, E. L. R., et al., Ann. N. Y. Acad. Sci., 48, 269-72 (1946).

15. Hutchings, B. C., et. al., Ann. N. Y. Acad. Sci., 48, 273-8 (1946)

16. Mowat, J. H., et. al., Ann. N. Y. Acad. Sci., 48, 279-82 (1946).

17. Waller, C. W., et al., Ann. N. Y. Acad. Sci., 48, 283-8 (1946).

18. Pfiffner, J. J., et al., J. Am. Chem. Soc., 69, 1476-87 (1947).

19. Gates, M., Chem. Rev., 41, 63-96 (1947).

20. Woolley, D. W., J. Nutrition, 28, 305-14 (1944).

21. Lane, R. L., and Williams, R. J., Arch. Biochem., 19, 329-35 (1948).

21a. Fischer, E. H., and Bernfeld, P., Helv. Chim. Acta., 32, 1146-50 (1949).

22. McGinnis, J., Norris, L. C., and Heuser, G. F., Proc. Soc. Exptl. Biol. Med., 56, 197 (1944).

23. Badger, E., J. Biol. Chem., 153, 183-91 (1944).

24. Horowitz, N. H., J. Biol. Chem., 162, 413 (1946).

25. Briggs, G. M., et al., Proc. Soc. Exptl. Biol. Med., 52, 7-10 (1943).

26. Smith, E. L., Nature, 161, 638 (1948).

27. Rickes, E. L., et al., Science, 107, 396 (1948).

28. Ott, W. H., Rickes, E. L., and Wood, T. R., J. Biol. Chem., 174, 1047 (1948).

29. Lillie, J. L., Denton, C. A., and Bird, H. R., J. Biol. Chem., 176, 1477-8 (1948).

30. Rickes, E. L., et al., Science, 108, 634-5 (1948).

31. Shive, W., Ravel, J. M., and Eakin, R. E., J. Am. Chem. Soc., 70, 2614 (1948).

32. Wright, L. D., Skeggs, H. R., and Huff, J. W., J. Biol. Chem., 175, 475-6 (1948).

33. Woolley, D. W., J. Exptl. Med., 73, 487-92 (1941).

34. Wright, L. D., and Skeggs, H. R., J. Bact., 48, 117 (1946).

35. Sprince, H., and Woolley, D. W., J. Exptl. Med., 80, 213-7 (1944).

36. Woolley, D. W., J. Biol. Chem., 159, 753 (1945).

37. Woolley, D. W., J. Biol. Chem., 162, 383-8 (1946).

38. Chattaway, F. W., et al., Biochem. J., 43, lix (1948).

39. Novak, A. F., and Hauge, S. M., J. Biol. Chem., 174, 647 (1948).

40. Norris, E. R., and Majnarich, J. J., Science, 109, 32-3 (1949).

41. Norris, E. R., and Majnarich, J. J., Science, 109, 33-5 (1949).

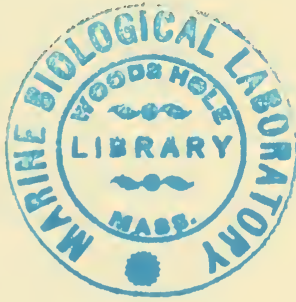




\section{Chapter II A}

\section{DISTRIBUTION OF B VITAMINS}

Of the ten members or possible members of the B vitamin family discussed in Chapter IA, four (inositol, nicotinic acid, choline, and $p$-aminobenzoic acid) were well recognized chemicals long before vitamins were discovered. The earlier information regarding these is therefore presented briefly first.

Inositol was known during the last century as a compound of wide distribution having been found in the sprouts, leaves, fruits, seeds and rhizomes of a considerable number of plants and in the blood, urine and various organs and tissues of animals such as cattle, guinea pigs and dogs. It was found also in fowls and cephalopods and in human urine. Early materials used in the preparation of inositol from natural sources included walnut leaves, mistletoe berries and beef lung or beef heart. The complete stereochemical structure of the naturally occurring substance, however, was not elucidated until $1942 .^{1,2}$

Nicotinic acid has been known chemically for about 80 years as a product formed by strong oxidation of nicotine. It was first isolated from natural sources (rice polishings) by Suzuki, Shamimura and Odake ${ }^{3}$ and soon after $\mathrm{Funk}^{4}$ isolated it from both yeast and rice polishings in his attempt to concentrate the anti-beriberi vitamin. Its natural occurrence was not, however, mentioned in the 4 th edition of Beilstein (1935). It was only after its coenzymic and vitamin functions were known that its widespread occurrence was recognized.

Choline, first isolated as a hydrolytic product of a phosphatide fraction in 1865 , was originally called "neurin." It was found later to be widespread in ergot, in mushrooms, in the germs of seeds and in other plant tissues: leaves, fruits, flowers, rhizomes. In these plant sources it was said to be partly free and partly combined in what are now called phospholipides. It was also found in animal tissues: glandular tissues, brain, blood, sperm, etc. If, as is commonly thought, lecithins and related phospholipides are always present in living cells, the universal distribution of choline in combined form is evident.

$p$-Aminobenzoic acid has been known chemically since the infancy of synthetic organic chemistry, but knowledge of its natural occurrence dates from its isolation from yeast in $1940-41 .^{5,6}$ It was administered to dogs 
as long ago as $1889^{7}$ and was found to be without physiological effect. It was found to be excreted unchanged in later experiments. ${ }^{8}$ Only after its physiological functioning was suspected ${ }^{9}$ has its widespread natural occurrence been established.

\section{Evidence for Universal Biological Occurrence of B Vitamins}

The universal presence of each B vitamin in living cells is indicated by microbiological evidence which will be discussed in later paragraphs. Before this method of study became so generally applicable, it was realized, for example, that thiamine occurs widely. Williams and Spies* list in tabular form about 90 different types of products which contain thiamine. These include animal tissues, fish, dairy products, legumes, cereals, vegetables, fruits, nuts and yeast. The quantitative information given by them is based entirely upon animal experiments.

In early investigations involving riboflavin, it was found in yeast, kidney, liver, suprarenals, corpus luteum, egg yolk, egg white, milk, urine, blood serum and the retinas of fish eyes. ${ }^{10}$ Advantage was taken in this case of the fact that riboflavin exhibits characteristic fluorescence. Its fluorimetric determination will be discussed later. For most of the evidence bearing on the universal distribution of the $\mathrm{B}$ vitamins in general, however, we must depend on microbiological evidence.

The first evidence as to the universal occurrence in living matter of what is now a recognized B vitamin was obtained in connection with pantothenic acid. ${ }^{11} \mathrm{~A}$ systematic study based upon a microbiological test was presented in 1933, which indicated that the same acid growth determinant was present in all types of living organisms. The materials examined represented eleven different biological phyla: Chordata, Arthropoda, Echinodermata, Mollusca, Annulata, Plathylminthes, Mxyomycetes, Bacteria, Fungi, Algae and Spermatophytes, and in the case of the more common phyla several examples were tested. Because of the evidence for its diverse distribution, this substance was named pantothenic acid (from the Greek, meaning from everywhere). It should be noted by contrast that the seemingly diverse sources of inositol and choline mentioned above on page 18 represent in each case only three phyla. Likewise, the evidence for the widespread occurrence of thiamine based upon animal tests above and the requirements of some insects involved not more than four phyla: Chordata, Arthropoda, Fungi, and Spermatophytes. It may be mentioned at this point, though the question will be discussed later, that ascorbic acid, which is not considered a B vitamin, is widely present in the tissues of about seven phyla, yet it is not present, to the

* Williams, R. R. and Spies, T. D., "Vitamin B (Thiamin) and Its Use in Medicine," The Macmillan Co., New York, N. Y., 1939, 411 pp. 
best of our knowledge, in representative members of the groups represented by Protozoa, Bacteria and Yeasts. Other examples to be cited later indicate that widespread occurrence in several phyla is not equivalent to universal biological occurrence.

It was not apparent at the time, but the root name, pantothen-, could have been given appropriately to any member of the B vitamin family because microbiological evidence now reveals that each one is universally present in the same sense that pantothenic acid is.

The most extensive and systematic study of the distribution of the B vitamins was undertaken in the writer's laboratories. This series of explorations was carried with the full realization that data would be subject to later revision because of improvements in microbiological methods, but they served to demonstrate, as had not been done before, that thiamine, riboflavin, nicotinic acid, pantothenic acid, pyridoxine (pyridoxal, see p. 8), biotin, folic acid and inositol are to be found in any type of biological material that is examined.

In one study ${ }^{12}$ the autolyzates of 50 animal tissues (rat, mouse, beef and swine) were found to contain substantial amounts of every member of the group. Embryonic, immature and mature livers, hearts and brains of rats and chickens were also assayed. ${ }^{13}$ In other studies enzymatic digests of seven representative rat tissues, ${ }^{14}$ seventeen representative human tissues (three individuals), ${ }^{15}$ twenty-three human cancers, 18 mouse and rat cancers, ${ }^{16}$ cell nuclei from heart and cancer tissues ${ }^{17}$ were assayed, always with the same result: substantial amounts of all the substances in question were found. More comprehensive exploration, ${ }^{18}$ including thirty-four representative materials from eight different biological phyla (Chordata, Arthropoda, Mollusca, Annelida, Protozoa, Bacteria, Fungi, and Spermatophytes) showed again the universal presence of ali eight substances, as had earlier bcen shown for pantothenic acid. In another study, ${ }^{19}$ milk from six species (human, mare, cow, goat, dog, mouse) was assayed and all eight substances found in every sample.

Numerous scattered studies have contributed information and have tended to corroborate the universal occurrence of the B vitamins. The most comprehensive recent compilations of data with respect to their quantitative distribution in foods are cited in the bibliography. ${ }^{20,21,22,23}$ Due allowances must be made in every case for the shortcomings of the methods used.

\section{Contrast Between the Distribution of B Vitamins and that of Other Vitamins}

In view of the possible importance of distribution as a criterion for determining whether or not a substance should be classified as a $B$ vita- 
min, it will be well to examine critically the available information regarding the distribution of those vitamins which are not considered within this group.

Ascorbic Acid. Of the vitamins not considered in the B group probably ascorbic acid is most widespread. Certainly, there is no question but that it is generally, and probably universally, present in the tissues of mature higher animals. ${ }^{24}$ Extremely interesting in this connection, however, is the fact that it is absent from unincubated hen eggs and only appears on incubation, when the amount increases during the first two weeks. ${ }^{25}$ During embryonic development, as judged by the acid silver nitrate staining technique, it does not appear in all of the chick tissues. It disappears from the liver after the tenth day and does not appear in the adrenals until the twelfth day. ${ }^{26}$ These results are in striking contrast to those observed in connection with the B vitamins. All of these are present in eggs from the start, even when they are synthesized (e.g., nicotinic acid) by the chick tissues during incubation. ${ }^{27}$ Certainly, the B vitamins do not appear to be absent from any actively growing tissue.

Ascorbic acid likewise is widespread in higher plants. Unlike B vitamins, however, it is practically absent from typical seeds but is produced during embryonic development.

It also seems clear that ascorbic acid is present in the tissues of many lower organisms: earthworms, six molluses, sea urchins, crustaceans, ${ }^{28}$ crabs, ${ }^{29}$ thirteen marine invertebrates, seven marine plants, ${ }^{30}$ cockroaches, ${ }^{31}$ and mushrooms. ${ }^{32,}{ }^{33}$ It is also produced by Aspergillus niger. ${ }^{34}$ In many of these organisms its distribution in the various organs strongly suggests that it is of functional importance. The fact that cockroaches synthesize it and maintain it at about the same level whether or not it is present in the diet, as do rats, points to the same conclusion.

When we consider the monocellular organisms, bacteria, yeasts and protozoa, however, we find that the preponderance of evidence indicates that in these organisms ascorbic acid is generally absent and nonfunctional. Although ascorbic acid has been reported repeatedly to promote the growth of specific bacteria, ${ }^{35,36}$ this effect has always been interpretable as due to the change in the oxidation-reduction potential of the medium rather than to its action as a specific nutrilite. Though the formation of vitamin $\mathrm{C}$ by bacteria has been reported, ${ }^{36,37}$ there is no clear-cut proof that it is actually ascorbic acid and not some other highly reducing substance that is produced. There is no doubt that intestinal bacteria utilize ascorbic acid, ${ }^{38,} 39$ but in these cases it is probably serving simply as an energy source. The fact that no one has ever identified ascorbic acid either chemically or biologically as a constituent of yeast places it in striking contrast to the $\mathrm{B}$ vitamins, all of which (for which adequate 
evidence is available) are present in significant amounts in this organism. The requirement of vitamin $\mathrm{C}$ for the growth of certain protozoa (Trichomonads) has been reported, ${ }^{40}$ but not enough information is given to rule out the effect on the oxidation-reduction potential as an important factor. The definite absence of ascorbic acid from Paramecium caudatum, $P$. bursaria, Stentor coeruleus, Opalina and Nictoterus has also been reported, ${ }^{41}$ and in the same study it was found that intestinal trypanosomes in the guinea pig may or may not contain ascorbic acid, depending upon whether or not the guinea pig diet is deficient.

So far as vitamin $\mathrm{C}$ is concerned, it appears to belong definitely in a different category from the $\mathrm{B}$ vitamins in that it is not universally distributed, being absent from eggs and seeds, from certain embryonic organs and in general from bacteria, yeast and protozoa.

Vitamin $A$ in its various forms occurs widely in nature, but shows a marked contrast to the $\mathrm{B}$ vitamins inasmuch as it does not appear to be present in all mammalian tissues, ${ }^{42}$ and has been found to be absent during the entire life cycle of cockroaches. ${ }^{43}$ Its distribution in numerous lower forms has apparently not been ascertained, but the two facts cited above are sufficient to show that its distribution shows a marked contrast to that of the B vitamins. It is interesting that in plants there is some relationship between the distribution of the carotenoids and ascorbic acid. ${ }^{44}$

Vitamins $D$. Information regarding the distribution of the $\mathrm{D}$ vitamins, except for relatively rich sources, is scanty. ${ }^{45}$ The fact that they are practically absent from plant foods and that they appear to be unimportant for microorganisms indicates that they are not universal. Yeasts, fungi, and other lower forms contain sterols which may be converted to $\mathrm{D}$ vitamins by ultraviolet light, but since many of these organisms can live entirely in the dark, there is no reason to think that $D$ vitamins function in their physiology. How the D vitamins are distributed in animal organs and tissues is unknown.

Vitamins $E$. Comparatively little quantitative information is available regarding the distribution of tocopherols ( $\mathrm{E}$ vitamins) in mammalian tissues, ${ }^{46,47}$ though it appears to be generally present. Still less is known about its presence in lower animals. Its principal known sources are seed germ oils and certain plants (lettuce, alfalfa). The mold Phycomyces appears to contain none at all. ${ }^{45}$ Yeast evidently contains none because it is included in vitamin E-deficient diets which are fed to rats. "Royal jelly," which is fed to the bee larvae that are to become fertile queens, is devoid of any significant amount of vitamin E. ${ }^{49}$ In contrast, royal jelly is a relatively rich source of most of the B vitamins and is even richer in pantothenic acid than beef liver. ${ }^{50}$ Sea-urchin eggs are a rich source of 
vitamin $\mathrm{E}^{51}$; this is in contrast to hen's eggs which are a poor source of $\mathrm{E}$ vitamins. The occurrence of $\mathrm{E}$ vitamins in the germs of seeds (and in some eggs) makes their distribution resemble that of the $B$ vitamins more than that of ascorbic acid, but the available evidence indicates that they are absent and nonfunctional in many lower forms of life.

Vitamins $K$. The distribution of the $\mathrm{K}$ vitamins is particularly interesting in that they are produced by bacteria, are widespread in the chloroplasts of green plants ${ }^{52}$ and are known to function in higher animals. However, one bacterium out of ten produced no demonstrable amount of vitamin $\mathrm{K}$, and yeast contains little or none. ${ }^{53}$ Substances with vitamin $\mathrm{K}$ activity have no effect on the growth, respiration or fermentation of yeast. ${ }^{54}$ The studies of Dam and co-workers ${ }^{54}$ have shown that vitamin $\mathrm{K}$ is present in all chlorophyll-bearing plant organs and that it is absent or present in low amounts in plant organs which normally do not carry chlorophyll throughout development. Chloroplast preparations were found to be about 60 times as rich as cytoplasm preparations. Seeds contain very little. Photosynthesizing algae and bacteria were found to contain vitamin $K^{54,55,56}$ Mushrooms were found to contain roughly $1 / 40$ of the amount in green leaves.

The absence or near absence of $\mathrm{K}$ vitamins from seeds, yeast, certain bacteria, nonchlorophyll-bearing higher plant organs and most animal tissues makes it appear that their occurrence is not universal. Certainly their distribution offers a strong contrast to that of the B vitamins.

\section{Quantitative Relationships Pertaining to the Distribution of B Vitamins}

Some of the quantitative relationships with respect to the distribution of the $B$ vitamins are worthy of note, in spite of the fact that available data are not all dependable. Our present discussion of these relationships will be limited to six substances: thiamine, riboflavin, nicotinic acid, pantothenic acid, biotin and inositol. The available data with respect to pyridoxal, etc., are not sufficiently reliable because of the difficulties involved in assay and in releasing these forms quantitatively from tissues without destruction. The available folic acid values are too low by a variable and unknown amount due to the fact that when most of the comparable assays were made, enzymes capable of freeing it completely from tissues were not known. ${ }^{57}$ Data regarding $p$-aminobenzoic acid and choline which might be used for comparative purposes are not available.

From the data compiled in Table 1 it may be noted first that in terms of the absolute amounts, inositol is always the most abundant and biotin the least abundant. In 17 out of 24 cases, the following order is maintained: (1) inositol, (2) nicotinic acid, (3) pantothenic acid, (4) ribo- 
flavin, (5) thiamine, (6) biotin. Only the following exceptions may be noted: In five eases (including the 3 seeds) the thiamine content exceeds that of the riboflavin; in two cases, riboflavin exceeds pantothenic acid; and in one ease pantothenic acid exceeds nicotinic acid. Otherwise the order of their occurrences falls into the same pattern, and the regularity observed in the diverse forms is remarkable.

Among the sources given, the values vary least in the case of inositolprotozoa are 12 times richer than brewers' yeast-and most in the case

TABLE 1. Relative Abundance of B Vitamins in Whole Organisms (dry wt.).*

$\begin{array}{lcccccr} & \begin{array}{c}\text { Thiamine } \\ (\gamma / \mathrm{g})\end{array} & \begin{array}{c}\text { Riboflavin } \\ (\gamma / \mathrm{g})\end{array} & \begin{array}{c}\text { Nicotinic } \\ \text { Acid } \\ (\gamma / \mathrm{g})\end{array} & \begin{array}{c}\text { Panto- } \\ \text { thenate } \\ (\gamma / \mathrm{g})\end{array} & \begin{array}{c}\text { Biotin } \\ (\gamma / \mathrm{g})\end{array} & \begin{array}{r}\text { Inositol } \\ (\gamma / \mathrm{g})\end{array} \\ \text { Rat } & 5.0 & 10.5 & 180 & 38 & 0.33 & 560 \\ \text { Fish } & 9.5 & 5.2 & 78 & 24 & 0.31 & 880 \\ \text { Frog } & 6.4 & 11.4 & 53 & 17 & 0.57 & 1230 \\ \text { Horned toad } & 11 . & 21 . & 170 & 36 & 0.7 & 2100 \\ \text { Snake } & 5.1 & 45 . & 142 & 25 & 0.25 & 1070 \\ \text { Chick embryo } & 8.3 & 13.4 & 405 & 370 & 1.75 & 1180 \\ \text { Red ant } & 7.3 & 14 . & 47 & 29 & 0.37 & 2200 \\ \text { Cockroach } & 16.2 & 26 . & 120 & 65 & 0.48 & 1340 \\ \text { Termite } & 12.8 & 26.5 & 175 & 88 & 0.66 & 2150 \\ \text { Dros. larvae } & 24 . & 47 . & 210 & 116 & 2.05 & 930 \\ \text { Dros. larvae } & 23 . & 43 . & 195 & 108 & 1.95 & 1320 \\ \text { Oyster } & 11 . & 13 . & 73 & 30 & 0.53 & 2700 \\ \text { Earthworm } & 7.8 & 25 . & 48 & 10 & 0.25 & 520 \\ \text { Protozoa } & 38 . & 17 . & 90 & 105 & 0.75 & 3300 \\ \text { A. aerogenes } & 10.6 & 43 . & 240 & 145 & 3.9 & 1360 \\ \text { S. marcescens } & 27 . & 35 . & 235 & 124 & 4.1 & 1160 \\ \text { P. fluorescens } & 26 . & 68 . & 210 & 90 & 7.1 & 1700 \\ \text { C. butylicum } & 9.3 & 55 . & 250 & 92 & 1.7 & 860 \\ \text { Mushrooms } & 8.8 & 26 . & 540 & 138 & 1.4 & 1350 \\ \text { Brewers' yeast } & 8.5 & 15.2 & 126 & 42 & 0.07 & 280 \\ \text { Mold } & 0.44 & 4.7 & 60 & 15 & 0.10 & 1280 \\ \text { Wheat seed } & 5.5 & 1.8 & 45 & 13 & 0.06 & 1900 \\ \text { Lima bean } & 5.7 & 1.4 & 12 & 9 & 0.12 & 1800 \\ \text { Blackeyed peas } & 8.5 & 1.5 & 14 & 11 & 0.22 & 2500 \\ \text { * Too much reliance should not be placed upon the exact numerical values, since incomplete extractions } \\ \text { and other limitations are involved in connection with the methods used. } \text { The material given represents } \\ \text { the only data on the subject that are available. } & & & & & \\ & & & & & & \end{array}$

of biotin-P. fluorescens is 120 times as rich as wheat seed. Sixty per cent of the values given in Table 1 , however, do not differ from the mean value for that vitamin by more than a factor of two.

Since microbiological methods are extremely sensitive and can be used to determine infinitesimally minute amounts, the question may be raised whether the mere finding of measurable amounts of the various B vitamins in all organisms is really significant. If we select the lowest values for each of the vitamins in Table 1 we find that they correspond to the following sources: (1) for thiamine the mold is poorest and the whole rat a poor second; (2) for riboflavin the three seeds are lowest; (3) for nicotinie acid again the seeds are lowest; $(4)$ the same is true for pantothenic acid; (5) for biotin, wheat and brewers' yeast are the poorest 
sources; (6) for inositol, brewers' yeast and rat carcass are the poorest sources. If the B vitamins are present in any organisms in insignificant amounts, the ones cited above are likely examples; therefore let us consider these sources individually.

In view of Schopfer's extensive work dealing with the importance of thiamine for molds, its indispensable role in these organisms can hardly be questioned. Certainly no one would say that thiamine is insignificant for rats. The fact that seeds are the poorest sources of riboflavin, nicotinic acid and pantothenic acid does not indicate a lack of importance of these vitamins in seed plants, because each increases during germination (at least in blackeyed peas and lima beans), ${ }^{58}$ and there is abundant indirect evidence of a diverse nature that they function in the enzyme systems of seed plants. In view of the importance of biotin as a nutrilite for yeasts, no one could question that it exists in significant amount in brewers' yeast. Wheat has about the same amount, and its significance is undoubted, especially since biotin is relatively abundant in many seed plants. In view of the importance of inositol as a nutrilite for yeasts, the relatively low amount in brewers' yeast cannot be taken as an indication of lack of importance. There is no reason to think that inositol is present in rat tissues in insignificantly low amounts, especially in view of the fact that it is unevenly distributed in the various tissues in accordance with a definite pattern, and the total amount present in the carcass of a 200 gram rat is about $40 \mathrm{mg}$.

On the basis of these facts we can safely conclude that the B vitamins are present in all organisms in significant amounts.

The quantitative distribution of the $B$ vitamins in different tissues of the same species is of interest because of the relative uniformity of the amounts present and apparent presence of significant amounts in every tissue and at every stage of development.

The most complete data available are those obtained by the assay of 17 human tissues. In Table 2 is given a summary of the values obtained from the tissues of three persons, two males and one female. An examination of these results shows that in 14 out of the 17 tissues, the absolute amounts of the substances present are in the same order as for the whole biological kingdom, namely inositol (1), nicotinic acid (2), pantothenic acid (3), riboflavin (4), thiamine (5), biotin (6). In the other three tissues the same order is maintained except that riboflavin slightly exceeds pantothenic acid in amount (by from 3-10 per cent).

The variation in content from tissue to tissue is greatest in the case of biotin (see also Table 1) ; liver is about 50 times as rich as seminal duct. The variation is least in the case of nicotinic acid; liver is about seven times as rich as skin. Curiously, when the results in Table 1 are compared with those in Table 2, a striking resemblance is observed. In the latter 
case, 58 per cent of the values differ by less than a factor of two from the average value for the vitamin in question, whereas for Table 1, 60 per cent is the corresponding figure. These data seem to indicate that insofar as the content of the various B vitamins can be taken as an index of metabolic characteristics, the diversity of these characteristics is just about as great for the various tissues of a mammal as it is for the various organisms in the whole biological kingdom.

Unfortunately, data comparable to that given in Tables 1 and 2 are lacking for each of the vitamins other than those belonging to the $\mathrm{B}$

TABLE 2. B Vitamins in Human Tissues. (wet wt. $\gamma / g$.)*

$\begin{array}{lcccccr} & \text { Thiamine } & \text { Riboflavin } & \begin{array}{c}\text { Nicotinic } \\ \text { Acid }\end{array} & \begin{array}{c}\text { Pantothenic } \\ \text { Acid }\end{array} & \text { Biotin } & \text { Inositol } \\ \text { Heart } & 3.6 & 8.3 & 41 & 16 & 0.17 & 500 \\ \text { Liver } & 2.2 & 16 & 58 & 43 & 0.74 & 660 \\ \text { Brain } & 1.6 & 2.5 & 20 & 15 & 0.58 & 1510 \\ \text { Lung } & 1.5 & 1.9 & 18 & 5 & 0.19 & 400 \\ \text { Kidney } & 2.8 & 20 & 37 & 19 & 0.67 & 1240 \\ \text { Spleen } & 1.1 & 3.6 & 23 & 5.4 & 0.06 & 1030 \\ \text { Skel. muscle } & 1.2 & 2.0 & 47 & 12 & 0.035 & 450 \\ \text { Sm. muscle } & 1.2 & 2.3 & 31 & 6.2 & 0.06 & 580 \\ \text { Adrenal } & 1.6 & 8.2 & 24 & 8 & 0.35 & 690 \\ \text { Stomach } & 0.56 & 5.2 & 19 & 6.1 & 0.19 & 760 \\ \text { Ileum } & 0.55 & 4.2 & 19 & 5.3 & 0.06 & 750 \\ \text { Colon } & 1.0 & 2.1 & 13 & 5 & 0.09 & 780 \\ \text { Mammary gland } & 0.43 & 2.4 & 10 & 3.9 & 0.04 & 270 \\ \text { Ovary } & 0.61 & 4.3 & 18 & 3.9 & 0.025 & 580 \\ \text { Testes } & 0.8 & 2.0 & 16 & 5 & 0.09 & 1600 \\ \text { Seminal ducts } & 0.69 & 1.0 & 9.2 & 2.0 & 0.015 & <100 \\ \text { Skin } & 0.52 & 1.2 & 8.6 & 3.1 & 0.022 & 200\end{array}$

* Too much reliance should not be placed upon the exact numerical values, since incomplete extractions and other limitations are involved in connection with the methods used. The material given represents the only data on the subject that is available.

family. From the fragmentary evidence available we can be reasonably sure that both vitamin $\mathrm{A}$ and vitamin $\mathrm{C}$ would be found to be absent from a number of the sources listed in Table 1. Insofar as data are available, it appears that ascorbic acid is present in all adult human tissues; but the presence of vitamin A has not been demonstrated in the epithelial layers of the skin, in ovaries after menopause, in testicles before puberty or after involution, in the normal duodenal mucosa, etc. ${ }^{42}$ The complete absence of vitamin A from the livers of some animals has been reported, ${ }^{59}$ and tremendous variation in tissue content is common. In this respect, the quantitative distribution of vitamin A shows a strong contrast to that of the $\mathrm{B}$ vitamins.

\section{Distribution of B Vitamins in Tumors}

The quantitative distribution of the $\mathrm{B}$ vitamins in cancerous tissues is interesting both from the standpoint of the B vitamins and because of the light that it sheds on the cancer problem. In a series of 23 human 
tumors of various types almost without exception the same order of occurrence was observed as in other studies, with inositol the most abundant and biotin the least abundant of the six. Some of the specimens assayed were estimated to be 70-80 per cent cancer tissue, while a few contained as little as 20 per cent. ${ }^{16,60,61,62,63}$

For comparing a group of tissues with each other, on the basis of their content of B vitamins, a simple mathematical scheme was used by which to calculate from the assay values the coefficient of uniformity (100 per cent minus the coefficient of variation) of the group of tissues compared. On this basis eight diverse normal human tissues showed a uniformity of only 27 per cent, whereas eight diverse cancers showed a much higher uniformity, namely, 66 per cent. Three normal human tissues (kidney, ovary and mammary gland) showed a uniformity of only 11 per cent, whereas three cancers derived from these same three tissues showed a 60 per cent uniformity. These observations led to the idea that cancer tissue, regardless of its origin, may represent, from the standpoint of its inherent metabolic machinery, a specific tissue type.

Further evidence supporting this idea was obtained by comparing various groups of tissues as indicated in Table 3.

TABLE 3. "Vitamin Uniformity" in Human, Rat, and Mouse Normal and Cancer Tissues.

8 diverse normal rat tissues

5 rat cancers of diverse origin

9 heart tissues from mice

12 diverse mouse cancers

9 heart tissues, 3 each from human, rat and mouse

22 cancers ( 8 human, 5 rat, 9 mouse)
Coefficient of Uniformity

29.7

62.8

76.0

58.0

61.2

53.3

In all cases when groups of cancers were compared, regardless of whether they originated in humans, in rats or mice, or in what type of tissue they originated or whether they were induced or spontaneous, the coefficient of uniformity was above 50 per cent; however, when diverse tissues, for example, those in which the cancers originated, were compared, the uniformity was far lower than this.

The conclusion derived from these findings is important because it has been corroborated, largely on the basis of enzyme studies. In this connection, Greenstein says, "It is possible to speak of cancer tissue in much the same way as one speaks of hepatic tissue or renal tissue, namely as a tissue with limited and ascertainable properties." 64

In general, cancer tissue (on a moist basis) tends to have a relatively low content of $\mathrm{B}$ vitamins, perhaps 50 per cent as much as an average of other mammalian tissues. Part of this difference is due to the relatively high water content of cancer tissue. However, some of the B vitamins, 
viz., riboflavin, biotin and pyridoxine, ${ }^{*}$ were found to be proportionately much lower than in other tissues, and inositol and folic acid* were relatively abundant. These findings are interesting in that both inositol and folic acid have been found to be effective in causing regression of eancer when injected intravenously into mice. ${ }^{65}$

Unfortunately neither the studies of the content of B vitamins nor extensive enzyme studies have been extended to cover fowl tumors which are known to be virus-induced. If these tumors should be found to follow the same pattern, this will constitute further circumstantial evidence that mammalian tumors are also induced by viruses.

\section{Bibliography}

1. Posternak, T., Helv. Chim. Acta, 25, 746-52 (1942).

2. Dangschat, G., and Fischer, H. O. L., Naturwiss., 30, 146-7 (1942).

3. Suzuki, U., Shamimura, T., and Odake, S., Biochem. Z., 43, 89-153 (1912).

4. Funk, C., J. Physiol., 46, 173-9 (1913).

5. Rubbo, S. D., and Gillespie, J. M., Nature, 146, 838-9 (1940).

6. Blanchard, K. C., J. Biol. Chem., 140, 919-26 (1941).

7. Gibbs, W., and Hare, H. A., Am. Chem. J., 11, 435-48 (1889).

8. Hildebrand, H., Beitr. Z. Chem. Physiol. Pathol., 3, 365 (1903).

9. Woods, D. D., Brit. J. Exptl. Path., 21, 74-90 (1940).

10. Euler, H. v., and Alder, E., Z. Physiol. Chem., 228, 1-12 (1934).

11. Williams, Roger J., et. al., J. Am. Chem. Soc., 55, 2912-27 (1933).

12. Wright, L. D., et. al., Univ. Texas Pub., 4137, 38-9 (1941).

13. Williams, R. J., Taylor, A., and Cheldelin, V. H., Univ. Texas Pub., 4137, 61-6 (1941).

14. Mitchell, H. K., and Isbell, E. R., Univ. Texas Pub., 4237, 37-40 (1942).

15. Taylor, A., Pollack, M. A., and Williams, R. J., ibid., 41-55.

16. Pollack, M. A., Taylor, A., and Williams, R. J., ibid., 56-71.

17. Isbell, E. R., et al., ibid., 81-3.

18. Woods, A. M., et. al., ibid., 84-6.

19. Williams, R. J., Cheldelin, V. H., and Mitchell, H. K., ibid., 97-104.

20. Adams, G., and Smith, S. L., U. S. Dep. Agr. Misc. Pub., 536, 88 pp. (1944).

21. Cheldelin, V. H., and Williams, R. J., Univ. Texas Pub., 4237, 105-24 (1942).

22. U. S. Dep. Agr. Misc. Pub., 572, (1945).

23. Com. on Food Comp., Natl. Research Council (U. S.) 1944.

24. Giroud, A., Ergeb. Vitamin-Hormonforsch., 1, 68-113 (1938).

25. Suomalainen, P., Ann. Acad. Sci. Fennicae, Ser. A53, No. 8, 13 pp. (1939).

26. Barnett, S. A., and Bourne, G., Quart. J. Microscop. Sci., 83, 299-316 (1942).

27. Snell, E. E., and Quarles, E., J. Nutrition, 22, 483-9 (1941).

28. Giroud, A., and Ratsimamanga, R., Compt. rend. soc. biol., 120, 763-5 (1935).

29. Ludány, G., Biochem. Z., 284, 108-10 (1936).

30. Eekelen, M. van, Acta. Brevia Neerland. Physiol. Pharmacol. Microbiol., 3, 119-20 (1933).

31. Wollman, E., Giroud, A., and Ratsimamanga, R., Compt. rend. soc. biol., 124, 434-5 (1937).

32. Anderson, E. E., and Fellers, C. R., Proc. Am. Soc. Hort. Sci., 41, 301-4 (1942).

* The values for pyridoxine and folic acid in the publication under discussion ${ }^{16}$ are of comparative value only, since the release of these vitamins could not be accomplished in a quantitative manner at the time the investigation was carried out. 
33. Kawakami, K., and Miyayosi, H., Rept. Inst. Sci. Research Manchoukuo, 4, 399-403 (1940).

34. Bernhauer, K., Görlich, B., and Kocher, E., Biochem. Z., 286, 60-5 (1936).

35. Illényi, A., Zentr. Bakt. Parasitenk. Abt. I, Orig., 114, (7/8), 502 (1937).

36. Illényi, A., and Kenessey, S., Zentr. Bakt. Parasitenk. Abt. I, Orig., 146, 204-7 (1940).

37. Berencsi, G., and Illényi, A., Biochem. Z., 298, 298-300 (1938).

38. Young, R. M., and James, L. H., J. Bact., 44, 75-84 (1942).

39. Kendall, A. I., and Chinn, H., Quart. Bull. Northwestern Univ. Med. School, 15, 205-10 (1941).

40. Cailleau, R., Rept. Proc. 3rd Intern. Congr. Microbiol., 1939, 493 (1940).

41. Roskin, G., and Nastyukova, O., Compt. rend. acad. sci. U.R.S.S., 32, 566-8 (1941).

42. Popper, H., Physiol. Revs., 24, 205-24 (1944).

43. Bowers, R. E., and McCay, C. M., Science, 92, 291 (1940).

44. Giroud, A., et al., Bull. soc. chim. biol., 18, 573-89 (1936).

45. Williams, R. J., "Vitamins and Hormones," Academic Press Inc., New York, N. Y., 1943, Vol. IV, p. 231.

46. Mason, K. E., J. Nutrition, 23, 71-81 (1942).

47. Hines, L. R., and Mattill, H. A., J. Biol. Chem., 149, 549-54 (1943).

48. Schopfer, W. H., and Blumer, S., Z. Vitaminforsch., 9, 344-9 (1939).

49. Mason, K. E., and Melampy, R. M., Proc. Soc. Exptl. Biol. Med., 35, 459-63 (1936).

50. Pearson, P. B., and Burgin, C. J., Proc. Soc. Exptl. Biol. Med., 48, 415-7 (1941).

51. Lieck, H., and Willstaedt, H., Svensk. Kem. Tid., 57, 134-9 (1945).

52. Gaffron, H., J. Gen. Physiol., 28, 259-68 (1945).

53. Almquist, H. J., Pentler, C. F., and Mecchi, E., Proc. Soc. Exptl. Biol. Med., 38, 336-8 (1938).

54. Dam, H., Glavind, J., and Nielsen, N., Z. physiol. Chem., 265, 80-7 (1940).

55. Dam, H., Am. J. Botany, 31, 492-3 (1944).

56. Dam, H., Glavind, J., and Svendsen, I., Biochem. J., 32, I, 485-7 (1938).

57. Cheldelin, V. H., et al., Univ. Texas Pub., 4237, 15-36 (1942).

58. Cheldelin, V. H., and Lane, R. L., Proc. Soc. Exptl. Biol. Med., 54, 53-5 (1943).

59. Karrer, P., Euler, H. v. and Schöpp, K., Helv. Chim. Acta, 15, 493-5 (1932).

60. Pollack, M. A., et al., Cancer Research, 2, 739-43 (1932).

61. Taylor, A., et al., Cancer Research, 2, 744-7 (1942).

62. Pollack, M. A., et al., Cancer Research, 2, 748-51 (1942).

63. Taylor, A., et al., Cancer Research, 2, 752-4 (1942).

64. Greenstein, J. P., "Biochemistry of Cancer," Academic Press Inc., New York, N. Y., 1947, p. 370.

65. Lewisohn, R., et al., Proc. Soc. Expll. Biol. Med., 52, 269-72 (1943). 


\section{Chapter IIIA}

\section{COMBINED FORMS-EXTRACTION}

It is an experimental fact that in spite of their relatively high water solubility in the free state, the B vitamins often resist aqueous extraction from plant and animal materials.

In the case of at least one of the B vitamins (biotin), its distribution in various tissues was originally determined quantitatively on the assumption that it would be extracted by water, since it exhibited a hydrophilic character in the free state. Subsequent investigations showed that a small and uneven fraction of the vitamin in the various tissues had been extracted. ${ }^{1}$ Since the extraction of each of the $\mathrm{B}$ vitamins presents special problems, we must consider them separately.

\section{Thiamine}

Historically, yeast and rice polish have been the most important materials used in attempts to concentrate and purify this vitamin. These two sources offer considerable contrast, because yeast requires autolysis before it becomes a favorable source while rice polish can be effectively extracted directly with acidulated water. ${ }^{2}$

Since the work of Auhagen ${ }^{3}$ and Lohmann and Schuster, ${ }^{4}$ it has been recognized that a considerable part of the thiamine in yeast and in animal tissues is combined in the form of diphosphothiamine (cocarboxylase), and this may undergo cleavage to produce free thiamine. Actually the diphosphothiamine is itself in combined form in the holoenzyme, carboxylase. Carboxylase has been isolated in pure form and contains 0.46 per cent diphosphothiamine and 0.13 per cent magnesium. ${ }^{5}$ In the presence of high salt concentrations the cocarboxylase is firmly bound, but in dilute salt solutions or in alkaline or acid ammonium sulfate solution it is almost completely dissociated. ${ }^{6}$

Various commercial enzyme preparations (taka-diastase, malt diastase, clarase, polidase, high phosphatase mylase, etc.) catalyze the hydrolysis of diphosphothiamine, usually at $\mathrm{pH} 4.5^{7,8}$ and hence can be used to free thiamine more or less completely from its natural combination in various tissues.

Westenbrink ${ }^{9}$ postulated the existence of more than one type of combination in undissociated carboxylases, and Sarett and Cheldelin, ${ }^{10}$ by 
inhibition studies, found evidence that thiamine itself may form a relatively undissociable linkage with protein, which possesses enzymatic activity. Tatum and Bell, ${ }^{11}$ using the same inhibiting agent (pyrithiamine) on mutant strains of Neurospora, interpret their somewhat similar findings to indicate that endogenous thiamine is more effectively utilized than exogenous thiamine. In a subsequent study of the dissociation of carboxylases in different yeasts and yeast preparations, Parvé and Westenbrink ${ }^{12}$ concluded that the various phenomena could be explained on the supposition that cocarboxylase combines with proteins other than the unaltered apoenzyme.

There are a number of other suggestive findings with respect to the extraction of thiamine from various sources; the need for further research is indicated. Of great importance in this connection is the fact that plants often contain very little of their thiamine in the form of cocarboxylase, and many unexplained irregularities occur. ${ }^{13}, 14$ Rice polishings yield free thiamine without enzymatic treatment. Wheat does not contain cocarboxylase, although an enzyme is present which is capable of catalyzing its hydrolysis..$^{15}$ Jackbeans and soybeans both contain relatively large and comparable amounts of thiamine; jackbeans are rich in carboxylase, ${ }^{16}$ but soybeans are said to have none. Under strongly acid conditions commercial phosphatase preparations were found effective in releasing thiamine from wheat embryos, but were wholly ineffective in releasing the vitamin from brewers' yeast. ${ }^{17}$ In a wholly different experiment in a different laboratory, one out of three samples of wheat germ yielded a slightly increased amount of thiamine upon enzymatic treatment. ${ }^{14}$ The availability of the thiamine present in different yeasts varies widely, suggesting differences in the method of combination. In the case of three brewers' yeast, $93-100$ per cent utilization by rats was reported, ${ }^{18}$ whereas in the case of some bakers' type yeasts the utilization by human subjects is said to be as low as 17 per cent (see p. 291). ${ }^{19}$ Utilization by rats in these cases was also low. ${ }^{20}$

Unexplained irregularities have also been observed in connection with animal tissues. In liver, brain and kidney a very large percentage of the total thiamine is present as cocarboxylase, while in muscle the concentration of free thiamine may nearly equal to (or in some cases even exceed) that of cocarboxylase. ${ }^{21,}{ }^{22}$ In milk, only 50-60 per cent of the thiamine is in the free state, and the rest is combined in nondialyzable form, which is not released by phosphatases but requires a proteolytic agent such as papain. ${ }^{23,24}$ Melnick and co-workers found that thiamine is apparently destroyed 50-90 per cent when it is incubated with bile, but that subsequent treatment with a special enzyme from yeast caused a recovery of a substantial part of the lost activity. The precursor of this recovered 
activity was not cocarboxylase or its combined form. ${ }^{25}$ Goodhart and Sinclair ${ }^{26}$ have presented evidence for the existence in serum of thiamine not in the form of diphosphothiamine bound to protein.

The presence of thiamine-destroying principles in the tissues of fishes, clams and ferns ${ }^{27}$ complicates the extraction of the vitamin from these sources, as well as the more fundamental problem of whether thiamine has diverse modes of linkage in the tissues of various organisms. The inconclusive investigations of Myrback ${ }^{28}$ and Shönberg ${ }^{29}$ and their respective associates with respect to the predominant presence or absence of an oxidized form of thiamine (presumably thiamine disulfide, in which the thiazole ring is cleaved and the sulfur oxidized) in bakers' yeast, have a bearing upon the problem of thiamine utilizability mentioned above. According to Myrback and his associates, bakers' yeast, when highly aerated, contains no substantial amount of cocarboxylase or thiamine as such, and this explains the lack of fermentation under these conditions (Pasteur reaction). Shönberg and his associates deny the existence of the oxidized form in substantial amounts.

It should be evident that complete information regarding the various ways in which thiamine is bound in tissues would constitute an important contribution to understanding the catalytic functions which thiamine performs.

\section{Riboflavin}

The history of riboflavin is closely associated with the flavoproteins of which it is a part, and from which it may be split by extraction with $0.1 \mathrm{~N}$ HCl. Warburg and Christian's "old yellow enzyme," ${ }^{30}$ which antedated exact knowledge regarding riboflavin, was the original flavoprotein discovered, and from it riboflavin was subsequently obtained.

Since that time a dozen or more specific flavoproteins have been isolated in pure form from natural sources, or at least have been concentrated or identified enzymatically. ${ }^{31-44}$ Many of these have been obtained from yeast, others from various animal tissues and from milk, Neurospora and other molds. In addition, there is the L-amino acid oxidase of snake venoms on which information is not available as to whether or not it is a flavoprotein. ${ }^{45}$ In the great majority of these flavoproteins, the prosthetic group is flavin adenine dinucleotide, made up as follows: isoalloxazine-D-ribose-phosphate-phosphate-D-ribose-adenine. However, in three of the flavoproteins, the "old yellow enzyme," the L-amino acid oxidase isolated from rat kidney, and cytochrome-c reductase, ${ }^{40}$ the prosthetic group is a mononucleotide: isoalloxazine-D-ribose-phosphate. The flavoproteins are dissociable into the protein and prosthetic parts which readily recombine to form the flavoprotein with its original enzymatic activity. 
By combination of the protein from one flavoprotein with the prosthetic group from another, new flavoprotein enzymes can be formed. ${ }^{34}$ Some of the flavoproteins are thought to have prosthetic groups in addition to the flavin nucleotides. ${ }^{44}$

In view of the fact that riboflavin appears always to be attached to proteins through a phosphate radical, it is not surprising that its extraction from tissues has not generally been a serious problem. Snell and Strong ${ }^{46}$ recommend for quantitative extraction autoclaving in the presence of a large amount of water. Extraction with $0.1 \mathrm{~N}$ acid is also mentioned by these authors and has been widely used. Neither procedure effects a quantitative extraction from all materials, even though the extraction may often be complete or nearly so. ${ }^{8}$ Recently the use of $0.25 \mathrm{~N} \mathrm{HCl}$ in 25 per cent acetone and 75 per cent water has been recommended. ${ }^{47}$ The U.S. Pharmacopoeia method involves autoclaving at 15 lbs. pressure for 30 minutes with $0.04 N \mathrm{HCl}$.

Digestion with a phosphatase preparation to obtain free riboflavin has theoretical justifications because of the existence of riboflavin bound to phosphoric acid, and the lack of definite information with respect to the hydrolysis of this combination under acid conditions. Alkaline hydrolysis cannot be used because riboflavin is unstable under alkaline conditions.

Recent experimental studies have shown that the cooking (and storage) of various foods causes a considerable increase in their assayable riboflavin content. This extra amount of riboflavin appears not to be released by phosphatase preparations, and to be determinable both by fluorometric and microbiological methods. ${ }^{48,} 49$

\section{Nicotinic Acid, Nicotinamide}

Nicotinic acid occurs principally in the form of its amide, and in tissues this is for the most part linked directly through the pyridine nitrogen to a ribose residue which makes up a part of a dinucleotide, such as coenzyme I: nicotinamide-ribose-phosphate-phosphate-ribose-adenine. This coenzyme is in turn linked to various proteins (apoenzymes). Coenzyme II has a structure similar to coenzyme I except that there is another phosphate radical the position of which is uncertain. Nicotinamide is linked in the same manner in both coenzyme I and coenzyme II, either of which in turn may be combined with a number of proteins. Sumner and Somers describe about 15 dehydrogenases of which about twice as many contain coenzyme I as contain coenzyme II..$^{50}$ The isolation of dinicotinyl ornithine from the excreta of chicks ${ }^{51}$ shows that in natural materials nicotinic acid may be combined through an amide linkage to other structures. 
The extraction of nicotinamide or nicotinic acid may be accomplished in various ways, depending upon the purpose. If one wishes, as is most often the case, to determine the total amount of the vitamin forms present and is not concerned with which form is present, he may extract a large part simply by autoclaving the material with water, as suggested by Snell and Wright. ${ }^{52}$ This does not effect quantitative removal in all cases, and the more common procedure involves autoclaving with $N$ acid or some similar treatment. ${ }^{53,54}$ When applied to animal tissues and milk, this treatment gives results comparable with those obtained by alkaline digestion or enzyme digestion, but with cereals the values are substantially higher, due it is thought, to the conversion of some unknown substance into nicotinic acid. ${ }^{55,56,57}$

Alkaline extraction in the case of cereals, especially if relatively concentrated alkali is used, gives values very much higher than by watery extraction. ${ }^{58}$ This may be due in part to the conversion of trigonelline which does not function as a vitamin into nicotinic acid which does.

Enzymatic digestion seems to give satisfactory release so far as the microbiological determination is concerned, ${ }^{8,55}$ because nicotinamide and the corresponding coenzymes are active in this test; but this method of extraction has not been generally adopted.

Considerable more research will be required before the extraction of nicotinic acid and nicotinamide can be completely controlled. This phase of study has not received adequate study, partly because of the lack of a satisfactory chemical method which is specific for a single chemical species.

\section{Pantothenic Acid}

Long before the vitamin properties of pantothenic acid were demonstrated, it was known to exist in a bound form particularly in liver, the then richest known source. ${ }^{59}$ From this combination it was freed by autolysis for purposes of concentration. ${ }^{60}$

The problem of extraction of pantothenic acid from tissues is different from that involved in the case of the other vitamins so far discussed because this vitamin is easily destroyed by hydrolytic cleavage under acid or alkaline conditions. Autolysis and the use of added enzymes are the available methods. Using uncooked brain and heart tissues as starting materials, Cheldelin et al. found that proteolytic enzymes, pepsin, trypsin, pancreatin, papain were relatively ineffective in the release of pantothenic acid, and that with hog kidney and spinach, papain was ineffective. "Takadiastase" in most cases gave the highest yields. Waisman et al. ${ }^{61}$ found more effective release to be accomplished by pancreatin digestion. The increased yield due to the use of pancreatin was 2 or 3 -fold in the 
case of some cooked meats. The differences in comparable results from different laboratories are probably due in part to the fact that crude enzyme preparations are not uniform. Willerton and Cromwell ${ }^{62}$ found that clarase digestion of yeast and liver preparations caused a severalfold increase in the available pantothenic acid in some cases, and brought the assay values for these materials up to the point where they agreed substantially with chick assay values. In all laboratories phosphatase preparations are effective in releasing pantothenic acid, and except in the case of cooked meats, release by this method is at least near the maximum. Recently Neilands and Strong ${ }^{63}$ have made combined use of liver enzyme and alkaline phosphatase to release pantothenic acid from foodstuffs. They emphasize the incomplete release of this vitamin by previously used procedures.

The form (or forms) in which pantothenic acid is bound in natural materials is largely unknown. On the basis of the fact that pantothenic acid itself is readily hydrolyzed by acid or alkaline hydrolysis but that $\beta$-alanine is not readily released from tissues by this means, Williams postulated that combination, presumably with proteins, takes place through the $\beta$-alanine portion of the molecule. ${ }^{64}$ Since esters of pantothenic acid are readily hydrolyzed (this can be accomplished without cleaving the pantothenic acid) and since pantothenic acid has not been removed from tissues by this means, the amide linkage suggests itself as most probable. The question of whether pantoic acid is readily split from the naturally combined forms of pantothenic acid has apparently never been determined. ${ }^{65}$

The fact that the coenzyme of Lipmann was found to contain about 10 per cent pantothenic acid has a tremendous bearing upon the problem of the combined form or forms of pantothenic acid. ${ }^{66}$ Since the functioning of pantothenic acid supposedly centers in this coenzyme, it may be presumed that pantothenic acid occurs naturally combined in this form, which constitutes the prosthetic group of one or more enzymes. It is interesting that pantothenic acid was freed only very slowly from this coenzyme by clarase-papain digestion, according to Cheldelin et al. ${ }^{8}$ After $\beta$-alanine had been found as a significant hydrolytic product, a combination of a liver enzyme and an alkaline phosphatase which together had previously been found to inactivate the coenzyme was found to release the pantothenic acid quantitatively.

\section{$B_{6}$ Group: Pyridoxal, Pyridoxine, and Pyridoxamine}

Although the chemistry of vitamin $\mathrm{B}_{6}$ appeared to be cleared up with the isolation and synthesis of pyridoxine, a biologically active vitamin, in 1938 and 1939, it was shown conclusively vears later 67, 68 that "vitamin 
$\mathrm{B}_{6}$ " and pyridoxine are by no means synonymous, and that pyridoxamine is fundamentally just as important as pyridoxine, and pyridoxal is even more so.

The question of the combined forms of "vitamin $B_{6}$ " was not answerable until these findings were made, and all conclusions based upon the supposition that vitamin $B_{6}$ and pyridoxine are one and the same were made obsolete by these discoveries.

Pyridoxal phosphate, presumably pyridoxal esterified with phosphoric acid in the 5-position, is now known to be an important coenzyme involved in decarboxylation of amino acids ${ }^{69}$ and in transamination. ${ }^{70,71,72}$ This constitutes one of the most important combined forms of vitamin $\mathrm{B}_{6}$ and is itself the prosthetic group of a number of enzymes, some of which have been purified. ${ }^{71}$ Pyridoxal readily forms combinations with amino acids in vitro, and such combinations may be important in nature. ${ }^{73}$

Pyridoxamine phosphate is another important bound form of vitamin $\mathrm{B}_{6}$, and appears to be the principal form occurring in yeast, where it is at least partly in the free state. ${ }^{74} \mathrm{It}$ is not hydrolyzed by alkaline extraction and may be extracted by this means from liver and grass preparations. In these latter materials it exists in combined forms presumably with proteins, and appears to constitute the predominant form of the vitamin. Pyridoxamine phosphate is also reported to be present in certain transaminase preparations. ${ }^{75}$

Pyridoxine, rather than pyridoxal or pyridoxamine, appears to be the principal form of the vitamin in certain seeds (rice and wheat), but little is known regarding its mode of combination. ${ }^{70}$ These cereals, it will be noted, are not metabolically active materials, as are those containing predominantly pyridoxal and pyridoxamine.

The complete extraction of all forms of vitamin $B_{6}$ in the free condition from tissues in general must take into account each of the forms. Pyridoxamine phosphate is stable toward alkaline hydrolysis and is hydrolyzed less readily than pyridoxal phosphate in acid medium. ${ }^{74}$ Stronger acid $(2 N)$ is far less effective in the release of free vitamin from yeast than is acid of lower concentration $(0.055 N) .77,78$ Rabinowitz and Snell ${ }^{78}$ found that for many materials autoclaving for 5 hours in $0.055 \mathrm{~N}$ $\mathrm{HCl}$ at $20 \mathrm{lbs}$. pressure gave maximum yields. A rice bran concentrate, however, required hydrolysis with $2 N$ acid, and dried green peas and oats yielded less vitamin $\mathrm{B}_{6}$ with the regular procedure than animal tests indicated to be present. This discrepancy may be due to intestinal synthesis.

The problem of extraction of all forms of vitamin $B_{6}$ in the free form is made less acute by the fact that there appears to be no destruction by 
acid treatment. ${ }^{78}$ This makes possible the utilization of one treatment after another, if necessary.$^{79}$ The present evidence is against the idea that other acid labile forms of vitamin $B_{6}$ exist, as once appeared to be the case $^{80}$

\section{Biotin}

Though biotin itself is readily water-soluble, only a minute portion of that present in tissues, sometimes as little as 0.1 per cent, is extracted by hot water. ${ }^{81}$ Unquestionably biotin exists naturally primarily in combined forms, but little information is as yet available regarding these forms.

Avidin, ${ }^{82,83,84}$ a protein constituent of egg white, appears to combine stoichiometrically with biotin to form a heat-labile complex. Avidinbiotin constitutes one of the combined forms of biotin, but the distribution of avidin is limited, so far as is known, to eggs and oviduct tissues, ${ }^{85}$ and so from the standpoint of general occurrence this form can be of only minor importance. The more abundant forms of combined biotin differ from avidin-biotin in that they are not dissociated in hot water.

Biotin unquestionably is associated with protein enzymes as a coenzyme or portion thereof, as will be discussed in a later section, but knowledge regarding these biotin-containing enzymes is almost nonexistent.

Extraction of biotin from various tissues indicates that it may not always be combined in the same way. For example, exhaustive dialysis of egg yolk (the original source of biotin used by Kögl and co-workers) followed by cold-water extraction yields a nondialyzable form which is active for yeast. Dialysis of liver tissue, on the other hand, does not render the biotin extractable by cold water, though it could be released by enzymes. ${ }^{81}$ Probably the most widely used extraction method is to autoclave the material with $6 N$ sulfuric acid for 2 hours at $15 \mathrm{lbs}$. pressure $81,86,87$; in most cases this yields the maximum amount of biotin. Prolonged autoclaving under these conditions causes appreciable destruction. Autoclaving with $18 \mathrm{~N}$ sulfuric acid for 2 hours caused 20-40 per cent destruction.

This method of extraction cannot be accepted as universally the best since in some cases, particularly plant materials, there is considerable destruction. ${ }^{8,} 88$ This difference in behavior may not be due to differences in combination but to the presence of substances in certain extracts which may, under the conditions used, interact with biotin. Incidentally, it may be remarked that the term "heat lability" as applied to vitamins is a very uncertain and indefinite one, inasmuch as the substances with which a vitamin is heated may be fully as important in determining rate of destruction as is the temperature of heating. 


\section{Inositol}

The most widely recognized bound form of inositol is "phytic acid," which has been obtained from plant sources in various states of crudity many times, most often in the form of mixed salts. Posternak ${ }^{89}$ synthesized the hexaphosphoric acid ester of inositol and gave good evidence as to its identity with a natural material, but perusal of the literature shows that often the term "phytin" (supposedly an acid calcium magnesium salt of phytic acid) has often been used uncritically for material of indefinite composition. Other compounds, including lower phosphoric esters and not agreeing in analysis with the classical formula, have often been obtained. $.^{90}, 91$ The enzyme phytase, ${ }^{92}$ which of course is no more definite than its substrate, is present in plant materials and catalyzes the hydrolysis of the inositol phosphoric esters.

Inositol is also found in bound form in the phospholipide material. Anderson ${ }^{93}$ first found it in a phosphatide fraction from tubercle bacilli, and Klenk and Sakai ${ }^{94}$ obtained from the cephalin fraction of soybean phosphaticle a material which on hydrolysis yielded inositol and an inositol monophosphoric ester. Folch and Woolley ${ }^{95}$ obtained brain phosphatide fractions containing from 6.8 per cent up to 10 per cent inositol, and Woolley ${ }^{96}$ obtained a preparation from soybean, "lipositol," with a content of 16 per cent inositol. Partial hydrolysis yielded inositol monophosphate. An inositol galactoside linkage was thought to be present. Complete hydrolysis yielded besides inositol, 15.5 per cent galactose, 8.3 per cent tartaric acid, 23.6 per cent oleic acid, cerebronic, palmitic and stearic acids totalling about 21 per cent, phosphoric acid 9.8 per cent, and ethanolamine. The content of the various fat acids and the analysis for phosphoric acid indicate that the material is not a pure compound, though the same conclusion holds for many refined phospholipide preparations.

A third, and from the functional standpoint, highly significant form of bound inositol is pancreatic amylase. ${ }^{97}$ The purified enzyme not only contains over 0.4 per cent inositol, but inhibition studies show that without inositol the amylase cannot function.98* The implications of this finding are far-reaching, especially if it should be found that starch- and glycogen-splitting enzymes containing inositol are widespread. This might account for the universal presence of inositol in living cells.

The extraction of the total inositol from tissues in uncombined condition requires refluxing with 18 per cent $\mathrm{HCl}$ for 6 hours. ${ }^{99} \mathrm{~A}$ considerable but variable portion can be extracted by milder procedures. Autolysis 100 usually frees considerably less than does enzymatic treatment. ${ }^{8}$ No critical study has been found recorded of the way inositol may be released

* See footnote p. 10. 
from its various combined forms. Platt and Glock ${ }^{101}$ have shown that when fresh rat tissues are carefully dried in the frozen and finely divided condition the water extract of the powder contains partly free and partly combined (requiring acid hydrolysis) inositol.

\section{Choline}

The principal bound forms in which choline occurs are the lecithins and sphingomyelins. Since the chemistry of naturally occurring phospholipides and related compounds is not satisfactory, it being extremely difficult or impossible to obtain such compounds in the pure state, one would not dare be dogmatic as to the existence of other lipides in which choline also is bound.

From the standpoint of physiology, an extremely important bound form of choline is acetylcholine. From the quantitative standpoint, however, the amount of natural choline which is bound in this manner is extremely small.

There are certain close relatives of choline (dimethylaminoethanol, monomethylaminoethanol, and ethanolamine) all of which are possible components of lipides. These should be considered in connection with choline studies.

The extraction of total choline, both combined and uncombined, from tissues is accomplished by the use of exhaustive absolute methanol extraction. ${ }^{102}$ Mixtures of ethanol and ether and other solvents have been used, but the yield in every case is smaller. ${ }^{102,}{ }^{103}$

Lecithins which contain most of the bound choline can be precipitated from an aqueous medium with acetone, leaving free choline in solution.

Acid digestion of tissues has been used to free choline from its combinations. ${ }^{104}$ Autoclaving the entire tissue with $3 \mathrm{~N} \mathrm{HCl}$ for 2 hours yields a solution which contains choline in the free form. ${ }^{105}$

\section{Folic Acid (Pteroylglutamic Acid, P.G.A.)}

This vitamin has not been known long enough for its more complex combined forms to be recognized. There is every reason to suppose that it acts catalytically as a coenzyme and is therefore bound (perhaps loosely) to proteins. These protein combinations are unknown.

Of the three combined forms, probably the most revealing is the simplest, i.e., formylfolic acid. ${ }^{106}$ This has been made synthetically and evidence for its natural occurrence and biological functioning has accumulated. The next combined form, listed in order of simplicity, has two extra (three total) glutamic acid residues joined to the glutamic acid portion by peptide linkage. This was designated "fermentation $L$. casei factor," since it was obtained from a fermentation residue. ${ }^{107}$ A third 
form has a total of seven glutamic acid residues and has been called "vitamin $\mathrm{B}_{\mathrm{c}}$ conjugate." 108

For the liberation of free folic acid (which is active alike in S. fecaelis, L. casei and chick tests) from tissues, a special enzyme (or enzymes) is necessary ${ }^{109,110}$; drastic acid or alkaline treatment, of course, causes destruction of the vitamin. Even autoclaving certain bacteria in the presence of water destroys most of it.111 Treatment with commercial phosphatase-containing and proteolytic enzymes or autolysis frees only a fraction of the vitamin present.

Specific enzymatic treatments yield sufficiently divergent results, when applied to different tissues, to suggest the probability that folic acid exists in different types of combination. ${ }^{112}$ The fact that folic acid is particularly associated with green leaves, ${ }^{113}, 114$ where it probably functions in a special way, suggests that special combined forms exist in leaves. The observation of Bird and co-workers ${ }^{115}$ that no enzymatic treatment of plant extracts was found that would cause the microbiological assay values to equal those obtained by chick assays, is in line with this suggestion.

\section{p-Aminobenzoic Acid}

Three well defined naturally occurring combined forms of $p$-aminobenzoic acid are known: folic acid (including conjugates), rhizopterin ${ }^{116}$ and $p$-aminobenzoylpolyglutamic acid. ${ }^{117}$ In addition, the acetyl derivative may occur in blood and urine. ${ }^{118,} 119$ Other information regarding combined forms is based upon indirect evidence. Most of the $p$-aminobenzoic acid of yeast (about 90 per cent) is in the free state; that is, it is extractable and utilizable by microorganisms. ${ }^{120,}{ }^{121}$ In different tissues tested, the amounts of "bound" versus total $p$-aminobenzoic acid varied from 6 per cent in potatoes to 93 per cent in rat kidney. Eight animal tissues averaged about 80 per cent bound, whereas miscellaneous materials mostly of plant origin averaged 44 per cent bound.

The quantitative extraction of $p$-aminobenzoic acid from tissues offers difficulties that have not been fully overcome.

Landy and Dicken ${ }^{122}$ autoclaved the material to be assayed with water and obtained maximum yields. No data were given as to what other procedures were used for comparison. Lewis ${ }^{123}$ found greater destruction with acid than with alkali, and autoclaved samples with $N \mathrm{NaOH}$ for 30 minutes to obtain assay values. Thompson et al. ${ }^{120}$ obtained yields about 3 times as high when the material (beef liver and kidney) was autoclaved for 1 hour with $6 \mathrm{~N} \mathrm{H}_{2} \mathrm{SO}_{4}$, than when alkaline hydrolysis (mild compared to that used by Lampen and Peterson below) was used. Under these acid conditions they found about a 15 per cent destruction. 
Lampen and Peterson ${ }^{119}$ made a careful study of hydrolytic conditions using as the principal material to be assayed a "dry powdered liver sample." They found liberation by acid hydrolysis to be rapid, but that it never appeared to be complete. Subsequent digestion of the same soluble material with alkali caused an increase in the total $p$-aminobenzoic acid, indicating that the acid treatment had extracted the compound from the liver but had not rendered it available to the test organism $(\mathrm{Cl}$. acetobutylicum). Freeing of the available form took place more slowly under alkaline conditions, but proceeded considerably further. The curve for autoclaving (15 lbs.) with $2 \mathrm{~N} \mathrm{NaOH}$ had not reached a plateau even at 20 hours. Some destruction was observed as a result of long alkaline treatments. The question of whether the $p$-aminobenzoic acid being formed during drastic alkaline treatment might be an artifact was investigated, with negative indications. Preliminary treatment with acid seemed to cause the subsequent liberation to be more rapid under alkaline conditions.

These four studies cited involved the use of four different assay organisms, and this may be partially responsible for the differences observed. It is possible that the "aminobenzoicless" Neurospora used in the study of Thompson et al. ${ }^{120}$ responds to a conjugate produced by acid treatment, and that the Clostridium acetobutylicum does not. This would help to explain the difference between the last two studies cited.

The numerous observations, as well as those relating to folic acid, suggest that $p$-aminobenzoic acid may be combined in nature in a number of ways and that some of these combinations are extraordinarily stable. Further study is required to clarify the picture.

A comparison of available $p$-aminobenzoic acid assay values for beef liver, spinach and egg with corresponding folic acid assay values indicates that there is 3-10 times as much $p$-aminobenzoic acid present as could be derived from the folic acid present. Lampen and Peterson ${ }^{119}$ found a maximum of $8 \mu \mathrm{g}$ per gram of $p$-aminobenzoic acid in liver powder. This would require $25.6 \mu \mathrm{g} / \mathrm{gm}$ of folic acid if it were all in this form. The folic acid content of beef liver (no conjugase used) on a dry basis was $2.8 \mu \mathrm{g} / \mathrm{gm}$ (calculated on the basis of 160,000 potency). Even if this value were to be doubled or tripled by conjugase action, there would still be more than half of the total $p$-aminobenzoic acid present in liver in some conjugated form other than folic acid and its conjugates.

\section{Bibliography}

1. Kögl, F., and Hasselt, W. van, Z. physiol. Chem., 243, 189-94 (1936).

2. Williams, R. R., Waterman, R. E., and Keresztesy, J. C., J. Am. Chem. Soc., 56, 1187-91 (1934). 
3. Auhagen, E., Z. physiol. Chem., 204, 149-67 (1932).

4. Lohmann, K., and Schuster, P., Biochem. Z., 294, 188-214 (1937).

5. Green, D. E., Herbert, D., and Subrahmanyan, V., J. Biol. Chem., 135, 795-6 (1940).

6. Weil-Malherbe, N., Biochem. J., 33, 1997-2007 (1939).

7. Conner, R. T., and Straub, G. J., Ind. Eng. Chem., Anal. Ed., 13, 380-4 (1941).

8. Cheldelin, V. H., et al., Univ. Texas Pub., 4237, 15-36 (1942).

9. Westenbrink, H. G. K., Willebrands, A. F., and Kamminga, C. E., Enzymologia, 9, 228-35 (1941).

10. Sarett, H. P., and Cheldelin, V. H., J. Biol. Chem., 156, 91-100 (1944).

11. Tatum, E. L., and Bell, T. T., Am. J. Bot., 33, 15-20 (1946).

12. Parve, E. P. S., and Westenbrink, H. G. K., Z. Vitaminforsch, 15, 1-52 (1944).

13. Tauber, H., Proc. Soc. Exptl. Biol. Med., 37, 541-43 (1937).

14. Hennessy, D. J., and Cerecedo, L. R., J. Am. Chem. Soc., 61, 179-83 (1939).

15. Obermeyer, H. G., Fulmer, W. C., and Young, J. M., J. Biol Chem., 154, 557-9 (1944).

16. Cohen, P. P., J. Biol. Chem., 164, 685-89 (1946).

17. Sure, B., and Easterling, L., J. Biol. Chem., 162, 139-47 (1946).

18. Sure, B., and Easterling, L., Arch. Biochem., 4, 413-8 (1944).

19. Parsons, H. T., Foeste, A., and Gilberg, H., J. Nutrition, 29, 383-9 (1945).

20. Hochberg, M., Melnick, D., and Oser, B. L., J. Nutrition, 30, 201-8 (1945).

21. Westenbrink, H. G. K., and Goudsmit, J., Nature, 142, 150-1 (1938).

22. Ochoa, S., and Peters, R. A., Biochem. J., 32, 1501-15 (1938).

23. Houston, J., and Kon, S. K., Nature, 143, 558 (1939).

24. Halliday, N., and Deuel, H. J., J. Biol. Chem., 140, 555-61 (1941).

25. Melnick, D., Robinson, W. D., and Field, H., J. Biol. Chem., 138, 49-61 (1941).

26. Goodhart, R. S., and Sinclair, H. M., Biochem. J., 33, 1099-1108 (1939).

27. Weswig, D. H., Freed, A. M., and Haag, J. R., J. Biol. Chem., 165, 737-8 (1946).

28. Myrback, K., and Vallin, I., Svensk. Kem. Tid., 57, 72-4 (1945). (in German)

29. Schönberg, K., and Sperber, E., Svensk. Kem. Tid., 57, 117-23 (1945). (in English)

30. Warburg, O., and Christian, W., Biochem. Z., 254, 438-58 (1932).

31. Corran, H. S., and Green, D. E., Biochem. J., 32, 2231-40 (1938).

32. Haas, E., Biochem. Z., 298, 378-90 (1938).

33. Warburg, O., and Christian, W., Biochem. Z., 298, 150-68 (1938).

34. Warburg, O., and Christian, W., Biochem. Z., 298, 368-77 (1938).

35. Straub, F. B., Nature, 143, 76-7 (1939).

36. Adler, E., Euler, H. v., and Günther, G., Nature, 143, 641-2 (1939).

37. Fischer, F. G., Roedig, A., and Rauch, K., Naturwiss., 27, 197 (1939).

38. Subrahmanyan, V., Green, D. E., and Gordon, A. H., Nature, 144, 1016 (1939).

39. Green, D. E., et. al., J. Biol. Chem., 138, 775-82 (1941).

40. Haas, E., Harrer, C. J., and Hogness, T. R., J. Biol. Chem., 143, 341-9 (1942).

41. Ratner, S., Nocito, V., and Green, D. E., J. Biol. Chem., 152, 119-133 (1944).

42. Horowitz, N. H., J. Biol. Chem., 154, 141-9 (1944).

43. Blanchard, M., et al., J. Biol. Chem., 161, 583-97 (1945).

44. Ball, E. G., J. Gen. Physiol., 29, 413-8 (1946).

45. Zeller, E. A., and Maritz, A., Helv. Chim. Acta, 27, 1888-1902 (1944).

46. Snell, E. E., and Strong, F. M., Ind. Eng. Chem., Anal. Ed., 11, 346-50 (1939).

47. Scott, M. L., et al., J. Biol. Chem., 165, 65-71 (1946).

48. Hinman, W. F., et al., Ind. Eng. Chem., Anal. Ed., 18, 296-301 (1946).

49. Feaster, J. F., et al., Ind. Eng. Chem., 38, 87-90 (1946).

50. Sumner, J. B., and Somers, G. F., "Chemistry and Method of Enzymes," Academic Press, New York, N. Y., 1947, pp. 243-70.

51. Dann, W. J., and Huff, J. W., J. Biol. Chem., 168, 121-7 (1947).

52. Snell, E. E., and Wright, L. D., J. Biol. Chem., 139, 675-85 (1941). 
53. "Pharmacopoeia of the United States of America," 13th Revision, Mack Printing Co., Easton, Pa., 1947, p. 669.

54. Steele, H. K., Cereal Chem., 22, 448-54 (1945).

55. Cheldelin, V. H., and Williams, R. R., Ind. Eng. Chem., Anal. Ed., 14, 671-5 (1942).

56. Krehl, W. A., and Strong, F. M., J. Biol. Chem., 156, 1-12 (1944).

57. Krehl, W. A., Elvehjem, C. A., and Strong, F. M., J. Biol. Chem., 156, 13-19 (1944).

58. Kodicek, E., Biochem. J., 34, 712-23 (1940).

59. Rohrman, E., Burget, G. E., and Williams, R. J., Proc. Soc. Exptl. Biol. Med., 32, 473-4 (1934).

60. Williams, R. J., et al., J. Am. Chem. Soc., 60, 2719-23 (1938).

61. Waisman, H. A., et al., J. Nutrition, 23, 239-48 (1942).

62. Willerton, E., and Cromwell, H. W., Ind. Eng. Chem., Anal. Ed., 14, 603-4 (1942).

63. Neilands, J. B., and Strong, F. M., Arch. Biochem., 19, 287-91 (1948).

64. Williams, R. J., "The Biological Action of the Vitamins," Univ. Chicago Press, Chicago, Ill., 1942, p. 122.

65. Sarett, H. P., and Cheldelin, V. H., J. Biol. Chem., 159, 311-9 (1945).

66. Lipmann, F., et al., J. Biol. Chem., 167, 869-70 (1947).

67. Snell, E. E., Guirard, B. M., and Williams, R. J., J. Biol. Chem., 143, 519-30 (1942).

68. Snell, E. E., "Annual Review of Biochemistry," Vol. XV, Stanford Univ. Press, Stanford University, California, 1946, pp. 383-6.

69. Gunsalus, I. C., Bellamy, W. D., and Umbreit, W. W., J. Biol. Chem., 155, 685-6 (1944).

70. Schlenk, F., and Snell, E. E., J. Biol. Chem., 157, 425-6 (1945).

71. Green, D. E., et al., J. Biol. Chem., 161, 559-82 (1945).

72. Lichstein, H. C., Gunsalus, I. C., and Umbreit, W. W., J. Biol. Chem., 161, 311-20 (1945).

73. Oser, B. L., "Annual Review of Biochemistry," Vol. XVII, Stanford Univ. Press, Stanford University, Calif., 1948, p. 419.

74. Rabinowitz, J. C., and Snell, E. E., J. Biol. Chem., 169, 643-50 (1947).

75. Schlenk, F., and Fisher, A., Arch. Biochem., 12, 69-78 (1947).

76. Rabinowitz, J. C., and Snell, E. E., J. Biol. Chem., 169, 631-42 (1947).

77. Rubin, S. H., Scheiner, J., and Hirschberg, E., J. Biol. Chem., 167, 599-611 (1947).

78. Rabinowitz, J. C., and Snell, E. E., Anal. Chem., 19, 277-80 (1947).

79. Rabinowitz, J. C., and Snell, E. E., J. Biol. Chem., 176, 1157-67 (1948).

80. Melnick, D., et al., J. Biol. Chem., 160, 1-14 (1945).

81. Thompson, R. C., Eakin, R. E., and Williams, R. J., Science, 94, 589-90 (1941).

82. Eakin, R. E., Snell, E. E., and Williams, R. J., J. Biol. Chem., 136, 801-2 (1940).

83. Eakin, R. E., Snell, E. E., and Williams, R. J., J. Biol. Chem., 140, 535-43 (1941).

84. Pennington, D. E., Snell, E. E., and Eakin, R. E., J. Am. Chem. Soc., 64, 469 (1942).

85. Hertz, R., and Sebrell, W. H., Science, 96, 257 (1942).

86. Schweigert, B. S., et al., J. Nutrition, 26, 65-71 (1943).

87. Wright, L. D., and Skeggs, H. R., Proc. Soc. Exptl. Biol. Med., 56, 95-8 (1944).

88. Lampen, J. O. Bahler, G. D., and Peterson, W. H., J. Nutrition, 23, 11-21 (1942).

89. Posternak, S., Compt. rend., 169, 138-40 (1919).

90. Anderson, R. J., J. Biol. Chem., 18, 441-6 (1914).

91. Boutwell, P. W., J. Am. Chem. Soc., 39, 491-503 (1917).

92. Anderson, R. J., J. Biol. Chem., 20, 483-91 (1915).

93. Anderson, R. J., J. Am. Chem. Soc., 52, 1607-8 (1930). 
94. Klenk, E., and Sakai, R., Z. physiol. Chem., 258, 33-8 (1939).

95. Folch, J., and Woolley, D. W., J. Biol. Chem., 142, 963-4 (1942).

96. Woolley, G. W., and White, A. G. C., J. Biol. Chem., 147, 581-91 (1943).

97. Williams, R. J., Schlenk, F., and Eppright, M. A., J. Am. Chem. Soc., 66, 896-8 (1944).

98. Lane, R., and Wiliams, R. J., Arch. Biochem., 19, 329-35 (1948).

99. Woolley, D. W., J. Biol. Chem., 140, 453-9 (1941).

100. Williams, R. J., et al., Univ. Texas Pub., 4137, 27-30 (1941).

101. Platt, B. S., and Glock, G. E., Biochem. J., 37, 709-12 (1943).

102. Engel, R. W., J. Biol. Chem., 144, 701-10 (1942).

103. Rhian, M., Evans, R. J., and St. John, J. L., J. Nutrition, 25, 1-5 (1943).

104. Fletcher, J. D., Best, C. H., and Solandt, O. M., Biochem. J., 29, 2278-84 (1935).

105. Luecke, R. W., and Pearson, P. B., J. Biol. Chem., 155, 507-12 (1944).

106. Gordon, M., et al., J. Am. Chem. Soc., 70, 878 (1948).

107. Angier, R. B., et al., Science, 103, 667-9 (1946).

108. Pfiffner, J. J., et al., J. Am. Chem. Soc., 68, 1392 (1946).

109. Mims, V., Totter, J. R., and Day, P. L., J. Biol. Chem., 155, 401-5 (1944).

110. Bird, O. D., et al., J. Biol. Chem., 163, 619-59 (1946).

111. Stokes, J. L., and Larsen, A., J. Bact., 50, 219-27 (1945).

112. Loo, Y. H., and Williams, R. J., Univ. Texas Pub., 4507, 123-34 (1945).

113. Mitchell, H. K., Snell, E. E., and Williams, R. J., J. Am. Chem. Soc., 63, 2281 (1941).

114. Olson, O. E., Burris, R. H., and Elvehjem, C. A., J. Am. Dictetic Assoc., 23, 200-3 (1947).

115. Bird, O. D., et al., J. Biol. Chem., 159, 631-6 (1945).

116. Rickes, E. L., Chaiet, L., and Keresztesy, J. C., J. Am. Chem. Soc., 69, 2749-51 (1947).

117. Ratner, S., Blanchard, M., and Green, D. E., J. Biol. Chem., 164, 691-701 (1946).

118. Harrow, B., Mazur, A., and Sherwin, C. P., J. Biol. Chem., 102, 35-8 (1933).

119. Lampen, J. O. and Peterson, W. H., J. Biol. Chem., 153, 193-202 (1944).

120. Thompson, R. C., Isbell, E. R., and Mitchell, H. K., J. Biol. Chem., 148, 281-7 (1943).

121. Lampen, J. O., et al., Arch. Biochem., 7, 277-86 (1945).

122. Landy, M., and Dicken, D. M., J. Biol. Chem., 146, 109-14 (1942).

123. Lewis, J. C., J. Biol. Chem., 146, 441-50 (1942). 


\section{Chapter IVA}

\section{ASSAY METHODS}

There are three important types of assay methods which may, theoretically at least, be developed for each of the B vitamins: (1) chemical (or physical-chemical) methods; (2) microbiological methods using bacteria, yeasts and molds; and (3) biological (animal assay) methods. In addition, it might be desirable, of course, to use combinations of these methods. Other organisms, such as higher plants or lower animals that have potentialities for vitamin assay work, may be considered in connection with gaining the broadest picture. Animal or plant tissues may be used in some cases. Even human beings can be used for vitamin assay and the newest (probable) member of the $\mathrm{B}$ vitamin family, the antipernicious anemia principle, was assayed for exclusively in this manner until a useful microbiological test was finally developed.

There are three general purposes which underlie the performance of vitamin assays: (1) they may be performed for purposes of exploration with respect to distribution in nature, in foods and in food products; (2) they may be used as a guide in isolating, purifying and determining the functions of a principle; (3) they may be used for the assay and control of commercial concentrates and of synthetic products. The method to be used must be selected on the basis of its availability and applicability to the intended purpose. A chemical or physical-chemical method, for example, might be extremely valuable in testing nearly pure commercial concentrates or synthetic mixtures, and yet might be of no value whatever in connection with the assay of materials where the principle exists only in traces.

Other things being equal, the chemical and physical-chemical methods, when applicable, are most advantageous because of their accuracy, speed and definition. In general, however, such methods become progressively less applicable as we pass from preparations which are relatively pure to those in which the substance tested for is present, for example, to the extent of only a few parts per billion. In the latter cases, chemical or physical-chemical tests are likely to be far too insensitive. When such tests are sensitive enough for the assay of a natural mixture, they are likely to be interfered with by extraneous substances, which, however, it may be feasible to remove. 
Microbiological tests, in general, rate next to the chemical or physicalchemical tests with regard to speed and accuracy, and have the tremendous advantage with respect to natural extracts of very great sensitivity and often high specificity. Interference by extraneous substances is always a possibility in every type of assay, and microbiological tests, like all others, are more readily applied to concentrates than to tracecontaining mixtures. However, in many cases there is no substantial difficulty in this regard, and extremely small amounts can be determined microbiologically.

The importance of microbiological tests in vitamin research may be gauged by the fact that by their use pantothenic acid, biotin, pyridoxal, pyridoxamine and folic acid were discovered. They also formed the basis for the discovery of the vitamin properties of niacin, inositol and $p$-aminobenzoic acid, and for the isolation of the anti-pernicious anemia principle.

Biological assays using mammals or fowls constituted the first recognized vitamin tests, and because of the pre-eminent nutritional function of vitamins, these tests will always remain fundamentally important. Chemical tests and microbiological tests demand that the vitamin to be tested shall be in solution. Animal assays do not have this limitation; and in the sense that vitamins are concerned, by definition, with animal nutrition, the animal tests are the most direct vitamin tests.

Actually, in animal assays the important factor of availability, as well as presence, comes in. ${ }^{1}$ For example, if one were to determine by animal assay the amount of thiamine in raw bakers' yeast, the relatively low result would reflect the unavailability of the vitamin present. From the standpoint of the practical nutrition of animals or human beings the amounts of the various vitamins present in different foodstuffs are of no consequence if the vitamins are unavailable, and only a direct test with animals will give the fundamental information as to how much effective vitamin is present.

Unfortunately, it is not always safe to carry results obtained using one species of animals over to other species, because the physiological availability may not be the same. If one's interest is in human nutrition, it is necessary to test the materials in question upon human subjects to gain completely trustworthy information. All nutritional tests on animals and humans are complicated by the problem of intestinal synthesis of vitamins, as will be discussed in a later section.

Biological assays for vitamins using experimental animals are costly, time-consuming and lack accuracy. Nevertheless, for some purposes they are most necessary because vitamins are fundamentally concerned with animal and human nutrition. 


\section{Thiamine}

For physiological research involving thiamine and its functioning, many different organisms and procedures may profitably be used which fall outside the scope of our present discussion. We shall be concerned primarily with methods which are designed to determine thiamine content, and which neglect all other questions involving availability to any particular organism.

Thiochrome Method. Since the original publication by Jansen ${ }^{2}$ which forms the basis of this highly important method, dozens of articles dealing with applications, modifications and refinements have been published. Among the more important of these are those of Hennessy and Cerecedo ${ }^{3}$ and Conner and Straub, ${ }^{4}$ who respectively introduced the use of adequate base exchange procedures for eliminating interfering substances and enzyme digestion using commercially available enzyme preparations to free thiamine from its combination in cocarboxylase.

The experimental essentials of the method as it is now applied include seven steps:

(1) Quantitative extraction from the material under examination of all of the free thiamine and cocarboxylase present. This is accomplished by the use of acidulated water.

(2) Enzymatic digestion of the cocarboxylase present by the use of clarase or some other suitable commercial preparation rich in phosphatase.

(3) Selective quantitative adsorption of the free thiamine by "Decalso" or other suitable agent.

(4) Quantitative recovery by elution, yielding a thiamine solution from which many interfering substances have been discarded.

(5) Oxidation of the thiamine to thiochrome by the use of alkaline potassium ferricyanide solution.

(6) Extraction of thiochrome by isobutyl alcohol.

(7) Measurement of the fluorescence produced by irradiating the thiochrome solution with ultraviolet light (fluorophotometer) and evaluation in terms of thiochrome.

The key reaction on which the method is based is the quantitative production of the fluorescent pigment from thiamine by oxidation.

In the application of this method to specific cases, some of the steps may become superfluous. For example, in the assay of wheat and wheat products, Step 2 can be omitted because cocarboxylase is absent, and the results are the same whether or not this step is performed. If one is dealing with a vitamin concentrate containing free thiamine and not too much interfering material, steps 1-4 may be dispensed with. If one wishes to determine free thiamine in the presence of cocarboxylase, Step 2 is 
omitted and advantage is taken of the fact that the pigment produced by the oxidation of cocarboxylase is not soluble in isobutyl alcohol, whereas thiochrome is. Experimental details of procedure and a partial bibliography will be found in the reference cited. ${ }^{5}$

As ordinarily performed, the thiochrome solution examined (Step 7) corresponds to the order of $1.0 \mu \mathrm{g}$ of thiamine. A micromethod using a Spekker Fluorometer has been devised ${ }^{6}$ which is accurate to \pm 20 per cent when $0.001 \mu \mathrm{g}$ of thiamine is determined, and to about \pm 3 per cent when the amount of thiamine is $0.05 \mu \mathrm{g}$.

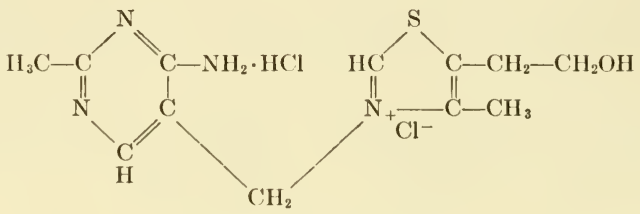

Thiamine hydrochloride

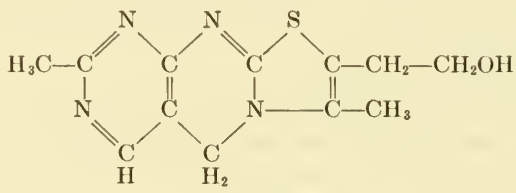

Thiochrome

The thiochrome procedure has been successfully applicd to the analysis of cereals and cereal products, ${ }^{7,8}$ to miscellaneous foods and tissues, ${ }^{9,10}$ to urine, ${ }^{11,12}$ to blood, ${ }^{13,14,14 a}$ and to pharmaceuticals. ${ }^{15,16}$

Colorimetric Method. Another chemical method of some value involves the formation of a colored pigment (usually red) when thiamine is allowed to react in alkaline medium with a diazotized aromatic amine. The most important amine for this purpose, $p$-aminoacetophenone, which yields a red dye insoluble in water but soluble in xylene, was first used by Prebluda and McCollum. ${ }^{17}$ The development of quantitative methods, suitable for laboratory use, involving this reagent is the work of Melnick and Field. ${ }^{18}$

The Melnick and Field method, including modifications thereof, has not been used as extensively as the thiochrome method mainly because of its lack of sensitivity, though in some laboratories, at least, it has been found more reliable. ${ }^{15}$ It requires, in order to be applied, a concentration of 2-3 $\mu \mathrm{g}$ of thiamine per $\mathrm{ml}$, whereas the thiochrome method is applicable to solutions containing as little as $0.05 \mu \mathrm{g}$ per $\mathrm{ml}$. For the analysis of 
relatively potent concentrates or even for clinical examination of urine, ${ }^{19}$ the colorimetric method is, however, probably superior to any other.

Yeast Fermentation Method. This method, which is probably second to the thiochrome method with respect to wideness of use, was developed by Schultz, Atkin and Frey ${ }^{20,21}$; it utilizes the fact, discovered by them, that fermentation by suitable yeast is enhanced by the presence of free thiamine. In its modified form the method takes advantage of the fact that thiamine is cleaved quantitatively by sulfite, and the cleavage products have no effect upon the fermentation. The difference between the enhancement produced by an untreated solution and a sulfite-treated one is used as a measure of the thiamine content.

The application of this method requires the use of a special apparatus, a Fermentometer, which is constructed so that 12 fermentations can be run simultaneously. One-half gram of yeast is used in each bottle. The carbon dioxide evolution after 3 hours of fermentation is measured and the enhancement induced by $1 \mu \mathrm{g}$ and $2 \mu \mathrm{g}$ of thiamine are taken as standards for comparison. Errors in assay under favorable conditions are not more than a very few per cent.

By the use of a Warburg apparatus thiamine can be assayed on an ultramicro scale $(0.005 \mu \mathrm{g}$ to $0.025 \mu \mathrm{g})$ with errors no greater than \pm 5 per cent. ${ }^{22}$

Comparative studies have shown that the yeast fermentation method gives results generally comparable in accuracy with those obtained by the thiochrome method. ${ }^{716,23,24}$ Details of procedure are described by the originators. ${ }^{21}$

Microbiological Growth Methods. The yeast-growth method has not been used extensively except in the laboratory where it originated, but for certain types of investigation involving minute amounts of material it had a distinct advantage. The growth of certain strains of yeast ("Old Process") in an otherwise complete medium is greatly stimulated by minute amounts of thiamine. ${ }^{25,26,27}$ Thiamine degradation products are likely to be effective, however, and these must be ruled out. ${ }^{24}$ The effect of thiamine is stimulatory rather than essential for growth, and differences in growth rates are therefore the basis of the response. By this means it is possible to determine with satisfactory accuracy in unprocessed materials, amounts of thiamine as little as $0.0001 \mu \mathrm{g}$, and by reducing the volume of the cultures, a lower limit can be reached.

The use of the mold Phycomyces Blakesleeanus as a test organism has been advocated by Schopfer ${ }^{28}$ and was used, for example, by Meiklejohn ${ }^{29}$ for the determination of thiamine in the blood. Sinclair, however, has emphasized that the response is nonspecific. ${ }^{30}$ Later this investigator introduced a correction which was thought to take care of 
extraneous effects. ${ }^{31}$ Even so, the growth period in the test is 10 days, and in view of later developments the use of this organism has little to recommend it.

Niven and Smiley ${ }^{32}$ have suggested the use of Streptococcus salivarius as a test organism. It is extremely sensitive to minute amounts of thiamine ( 0.1 to $2 \mu \mathrm{g}$ per tube) and yields satisfactory assay values. Thiamine fragments are inactive; cocarboxylase is 40 per cent more active than thiamine. The method has the disadvantage that the medium has a $\mathrm{pH}$ value of 7.4 and thiamine must be added to it aseptically after autoclaving.

Sarett and Cheldelin ${ }^{33}$ have developed an assay method using Lactobacillus fermenti, which responds in their test to from 0.005 to $0.05 \mu \mathrm{g}$ of thiamine. The recommended growth period is 16 to 18 hours and under these conditions, cocarboxylase is 30 per cent more active than thiamine. The method has been applied by the authors to a considerable number of foods, to animal tissues, and to urine with excellent results. ${ }^{34}$

Biological Assays. Probably the most widely used biological assay methods for thiamine involve curative tests on rats. In the U.S.P. method, ${ }^{35}$ rats are kept upon a thiamine free diet until they show signs of acute polyneuritis, whereupon at least eight such rats are given a standard dose of pure thiamine which "cures" the polyneuritic condition for from 5 to 15 days, depending upon the dose given and the condition of the individual rats. As soon as the rats have regressed and reached the same stage of polyneuritis as before dosing, a single dose of the unknown material to be tested is administered. If the curative effect of this preparation lasts as long or longer than that of the standard (based upon the sum of the cured days for each animal), it is determined to contain as much or more thiamine than the standard.

It is obvious that the assay of unknown mixtures by this method involves the use of a large number of animals and a large amount of time, since the depletion period may last as long as 8 or 10 weeks and the assay period for a single test is from 10 to 30 days longer.

A less time-consuming curative test is one devised by Smith ${ }^{36}$ and modified by Birch and Harris. ${ }^{37}$ In this modified test animals are placed upon a thiamine-deficient diet containing, however, a small amount of thiamine in the form of $0.4-0.5$ per cent brewers' yeast. On this diet they develop polyneuritic symptoms more regularly and do not die of extreme thiamine deficiency before they become useful test animals. After depletion, which may require 50 days or more, they are injected intravenously with the material to be tested and the minimal amount required to produce a three-day cure is determined. If a larger than minimal dose is given, the excess can be judged by the longer duration 
of the cure, the duration being roughly proportional to the amount of thiamine administered. The same animal can be used over and over, 5 to 10 times or more, and if laboratory animals are continually kept in condition for testing, assays can be run with relative speed, and the results may be as accurate as those obtained by the U.S.P. method. ${ }^{15}$

Biological tests for thiamine involving rat growth, ${ }^{38}$ pigeon weight maintenance, ${ }^{39}$ prevention of bradycardia in rats involving the use of an electrocardiograph, ${ }^{37}$ chick growth, ${ }^{40}$ and postponement of death from polyneuritis in chicks ${ }^{41}$ have all been successfully used; but except for those interested in the particular species involved, these tests are largely of historical interest only, since they are not used at present as thiamine assay methods per se. The "catatorulin" test of Peters and co-workers 42 should also be mentioned in this connection. Oxygen consumption by brain tissue from avitaminotic pigeons is low, and is increased by minute amounts of thiamine. The test is sensitive to about $0.2 \mu \mathrm{g}$ of thiamine.

\section{Riboflavin}

Aside from the rat growth and chick growth methods which are basic, but belong in a class by themselves because of cumbersomeness and the time and expense involved, there are two methods that have been widely used and are recommended for riboflavin assay, namely, the fluorometric and the microbiological.

Fluorometric Method. This method takes advantage of the fact that riboflavin fluoresces strongly when exposed to light of wave length 440 to $500 \mathrm{~m} \mu$, and the intensity of the fluorescence is proportional to the concentration of riboflavin in the solution examined. It must be performed on solutions or extracts containing riboflavin, and the extraction procedures involve simultaneous hydrolysis of the bound forms (p. 33). If one were dealing with solutions containing riboflavin as the only fluorescing substance, the application of the method would be relatively simple. However, in practice, particularly with some types of products, it is necessary to take elaborate precautions to eliminate the effects of other substances.

One expedient that is always used to eliminate the effect of interfering substances involves the use of a sodium hydrosulfite (dithionite), $\mathrm{Na}_{2} \mathrm{~S}_{2} \mathrm{O}_{4}$, which reduces riboflavin quantitatively to its nonfluorescing leuco-form, and leaves unaltered some of the other colored fluorescent substances which may be present. After the fluorometric reading is taken on the final solution, regardless of previous treatments, the riboflavin is destroyed with hydrosulfite and a blank fluorometric reading taken. This reading is subtracted from the riboflavin reading. 
Another means of eliminating the effect of interfering substances is to reduce with stannous chloride and sodium hydrosulfite all the fluorescent pigments including riboflavin, and then reoxidize by contact with air; this procedure brings back riboflavin to its original fluorescent form, but leaves in reduced form some of the substances which would otherwise interfere.

Another procedure involves the use of dilute permanganate under controlled conditions to oxidize interfering pigments without affecting the riboflavin. The excess permanganate is removed with hydrogen peroxide.

Another procedure, regarded as necessary only when the materials tested are of relatively low potency or highly pigmented, involves adsorbing the riboflavin selectively upon a column of "Florosil" and eluting it with 20 per cent pyridine in 2 per cent acetic acid. This, of course, leaves behind many fluorescing substances, but introduces an extra step into the procedure; hence it is likely to introduce errors as well as expend time.

Scott and co-workers ${ }^{43}$ have recently published a complete procedure which has been applied with excellent results to many types of materials, including milk and milk products, ${ }^{44}$ dried leguminous seeds, ${ }^{45}$ miscellaneous cereal products, fermentation residues, leaf meals, meat and fish scrap, and yeasts. ${ }^{43}$ In this method, only two of the expedients discussed above are used: permanganate oxidation and hydrosulfite reduction at the end to obtain a blank reading. Details of procedure are to be found in the original article. ${ }^{43}$ It is said to require less time than the microbiological method..$^{45}$

A fuller discussion of the various alternate procedures involved in the fluorometric method will be found elsewhere. ${ }^{46}$

Microbiological Method. This method, which is essentially that of Snell and Strong, ${ }^{47}$ has been widely used and in spite of the natural handicap inherent in the use of an unfamiliar type of technique, it has been adopted by the U. S. Pharmacopoeia. ${ }^{48}$

The method is based upon the fact that the growth of Lactobacillus casei and its ability to produce lactic acid requires riboflavin in the medium. The riboflavin can be in the free form, but its combined forms, e.g., flavoprotein, flavin adenine dinucleotide, and presumably riboflavin phosphate, are equally effective under the experimental conditions prescribed for the assay. ${ }^{49}$

Theoretically, for this or any other comparable assay, one should have a basal medium perfect in every other respect except for the lack of the one item to be assayed for. Actually, it is only necessary in this case that the basal medium be such that under laboratory conditions other 
substances present in the extracts, aside from riboflavin, will have a negligible effect.

Subsequent investigations dealing with the nutrition of the test organism have shown that the basal medium recommended by Snell and Strong is far from ideal in the sense of the previous paragraph. Roberts and Snell ${ }^{50}$ have developed a vastly improved medium in which the organism responds to riboflavin to a much higher degree. Nevertheless, the original medium is such that it has given very satisfactory results comparable to those obtained using the improved medium, and it serves for practical purposes. If one were assaying materials never assayed before, the results could be accepted with greater certainty using the more complete medium of Roberts and Snell.

The most serious extraneous factors that can complicate riboflavin assay by this method are the fatty substances, ${ }^{51,52,53}$ the effects of which are sometimes stimulatory and sometimes inhibitory depending upon the agents, their concentration and upon the presence or absence of other agents in the extract. These disturbing factors can be eliminated by preliminary solvent extraction, or more simply by careful filtration of the extract at $\mathrm{pH} 4.5$ to obtain a clear solution. The latter procedure is the one used in the U. S. Pharmacopoeia method.

In the application of the method to urine, urea can be present in sufficient amount to introduce an error due to its inhibitory effect. This effect can be corrected for if necessary. ${ }^{54}$

There are two valid methods for evaluating the response of the organism to riboflavin: turbidimetric measurement of growth after 24 hours or less ( 16 hours in case the Roberts-Snell medium is used) and titration of the acidity developed after 72 hours. Either method gives wholly satisfactory results; the titration method possibly is a little less exacting, requires no special apparatus and is specified in the U. S. Pharmacopoeia. The turbidity method, however, gives results overnight which for some purposes may be a tremendous advantage. It requires a turbidity measuring device; a suitable photoelectric colorimeter will serve, but even better, however, is a thermoelectric turbidimeter such as has been in use in the author's laboratory for twenty years. ${ }^{55}$

Biological Methods. In view of the satisfactory assay of riboflavin by the two methods discussed, the animal assay methods are mostly of historical interest. This does not mean, of course, that animal experiments involving riboflavin are outmoded.

The rat growth method of Bourquin and Sherman ${ }^{56}$ has been used with minor modifications for a number of years and was the standard with which the newer methods were initially compared. These investigators were fortunate in preparing an alcoholic extract ( 80 per cent) of 
whole wheat which contained very little riboflavin but a relatively good supply of other B vitamins required by rats. Hence, when a riboflavincontaining material was supplied, its effect was largely due to the riboflavin. The method, of course, was particularly useful in connection with assaying relatively rich sources. Rats were first depleted for about two weeks and then fed supplements containing control and unknown amounts of "vitamin G," and the comparisons evaluated on the basis of the growth rates over a period of several weeks. Diets of substantially different character have been used more recently for the assay of riboflavin using rat growth, but we shall not go into detail here. ${ }^{57,} 58,59$

Extensive use has not been made of chicks in the assay of riboflavin, though methods were early proposed. ${ }^{60,61}$ An improved method was devised by Jukes ${ }^{62}$ in which the growth response at lower levels was found to be approximately proportional to the amount of riboflavin fed. The basal medium contained in addition to yellow corn, wheat middlings, casein, and supplements, 7 per cent of a "rice bran filtrate," which served to supply the chicks with the "filtrate factor" (pantothenic acid), and other unknowns. This was designed to make the test more specific and was apparently successful.

\section{Nicotinic Acid, Nicotinamide}

Two types of methods have been used almost exclusively for the assay of nicotinic acid or its amide: colorimetric methods and microbiological methods. Biological methods are relatively unimportant.

Colorimetric Methods. The colorimetric methods which have been applied widely to nicotinic acid assays all involve the interaction between it, cyanogen bromide and an aromatic amine. The chemistry of the reactions is not well known, but rupture of the pyridine ring is thought to be involved. ${ }^{63}$ Among the aromatic amines used for this purpose are $p$-aminoacetophenone, ${ }^{64}$ metol $^{65}$ ( $p$-methylaminophenol), aniline, ${ }^{66}$ $\beta$-naphthylamine, ${ }^{67}$ and $p$-phenylenediamine. ${ }^{68}$

One of the more extensively used amines has been $p$-aminoacetophenone. This reagent has been investigated by Kodicek, who agrees with the originators that it is 3 to 5 times as sensitive as aniline or metol; he has applied it with success to a variety of plant and animal products. ${ }^{69}$ It has been applied principally to cereal products by Bina and co-workers. ${ }^{70}$

Metol has been used by a number of workers, including Perlzweig and co-workers, ${ }^{71}$ Dann and Handler, ${ }^{72}$ and Steel and collaborators. ${ }^{73}$ The color produced is said to be more stable than that obtained with $p$-aminoacetophenone, ${ }^{73}$ and the reaction is reported to be more specific for nicotinic acid. ${ }^{72}$ Aniline has been used by Melnick and Field ${ }^{74,75}$ and 
Pearson, ${ }^{76}$ who indicates that it is four times as sensitive as $\beta$-napthylamine.

There have been at least two obstacles in the way of coming to an agreement upon a chemical method which will be generally recognized as acceptable. One is the fact that the amount of nicotinic acid appearing in the extract depends upon the method of extraction, and there has not been common agreement on this point. It is evident that some unknown substance is converted to nicotinic acid by certain extraction procedures. ${ }^{69,77}$

Another more serious obstacle is the fact that the fundamental reactions involved in producing the dye or dyes are not understood. The reaction is not specific for nicotinic acid, ${ }^{75}$ but with different aromatic reagents other pyridine derivatives such as nicotinamide, nicotinuric acid and nicotine as well as pyridine itself react to produce more or less color.

In spite of these obstacles, it is probable that several aromatic amines can be used successfully for particular types of products with good success. The colorimetric methods in general are not as sensitive, however, as the microbiological method which has now become pretty well standardized and accepted.

Microbiological Method. The principle of this method is the same as for the riboflavin method, but the microbiological procedure for nicotinic acid is in an especially favorable position because of the lack of any very satisfactory competing chemical method.

As with the microbiological methods generally, the substance to be assayed must first be extracted and brought into solution. In the Snell and Wright method, ${ }^{78}$ which has received wide acceptance, ${ }^{79},{ }^{80}$ nicotinic acid, nicotinamide, nicotinuric acid, and cozymase all have the same biological activity when tested in equimolecular amounts. ${ }^{78}$ This makes it unnecessary to bring about complete hydrolysis of the combined forms. The formation of nicotinic acid by acid and alkali treatment from precursors which may or may not have vitamin activity, ${ }^{77}$ presents an uncertainty which has not been overcome.

The organism used in this assay is Lactobacillus arabinosis 17-5 and the evaluation of the response involves titration, usually at the end of 72 hours. When this method is used, the turbidity of the extracts does not interfere. The method is 20 to 100 times as sensitive as the chemical methods, ${ }^{78}$ and once the nicotinic acid or its derivative is in solution, there is no obstacle to its satisfactory determination in amounts down to $0.05 \mu \mathrm{g}$ or less if drop cultures are used. ${ }^{81}$ The organism used for this assay is not as sensitive to fat acids as is $L$. casei. Krehl and co-workers found that with the possible exception of linoleic acid, they did not interfere. 
The basal medium as originally proposed has been modified by the addition of more glucose and sodium acetate, the elimination of half of the biotin, and the introduction of $p$-aminobenzoic acid. ${ }^{82}$ In the study referred to earlier in connection with the riboflavin assay, Roberts and Snell ${ }^{50}$ found that the new medium for Lactobacillus casei also supports heavy growth of Lactobacillus arabinosus in $\mathbf{1 6}$ hours, and suggest that this improved medium may be made the basis of a nicotinic assay. In spite of improvements which may be made in the basal medium, no serious errors are introduced by using the medium as originally proposed.

Recently two new microbiological assay methods for nicotinic acid have been developed, one using a yeast, Torula cremoris, ${ }^{83}$ the other a nonpathogenic bacteria, Proteus $H X 19 .{ }^{84}$ Both methods are more sensitive and more rapid than the Snell-IVright method, and it remains to be seen how widely they are used. One sure advantage, from the standpoint of research, of having several available methods is the fact that the different organisms respond characteristically to different natural derivatives.

Biological Tests. Although assays for nicotinic acid have been made to a limited extent using $\operatorname{dogs}^{85,86,78}$ and chicks ${ }^{86}$ as experimental animals, the methods have not been standardized. As a result of recently accumulated knowledge regarding the common transformation of tryptophan to nicotinic acid (p. 353) in mammals, it is obvious that no biological test which leaves out of consideration this transformation can be specific for nicotinic acid.

\section{Pantothenic Acid}

Although attempts have been made to determine pantothenic acid chemically by lead tetraacetate oxidation of the lactone derived from it, no serviceable method was developed, ${ }^{87}$ and the only available assay methods are microbiological and biological.

Microbiological Methods. Many microorganisms require pantothenic acid for growth or are stimulated by it, and all of these are potential test organisms.

The yeast test served adequately in the discovery and isolation of this vitamin 88,89 but later was not used, partly because of the effectiveness of $\beta$-alanine, a cleavage product, as a yeast growth stimulant, and also because the pantothenic acid concentration governed the rate of growth rather than the total growth, as in the case of the lactic acid bacteria. ${ }^{90,91}$ Because of this latter fact, the multiplicity of yeast nutrilites, ${ }^{92}$ and the complicated effects of amino acids, ${ }^{93}$ it was feared that the yeast method would not be as specific as would be required in dealing with all sorts of materials.

Recently the yeast test has been revived: a different strain of yeast 
is used, and the $\beta$-alanine effect has been cancelled out by using more asparagin in the medium; the results appear to be satisfactory. ${ }^{93}$ It has the advantage of speed (16 hours) over the titration procedure using lactic acid bacteria, but has the disadvantage that, as described, the cultures have to be continuously shaken.

Lactic acid bacteria ${ }^{94}$ have been used most extensively for pantothenic acid assay following the similar methods of Pennington et al. ${ }^{90}$ and Strong et al. ${ }^{95}$ The organism $L$. case $i$ has been used successfully in the assay of a great many types of materials. ${ }^{96-106}$ Fatty substances interfere, as in the case of riboflavin assay, ${ }^{52}$ but this difficulty can often be cared for by careful filtration at $\mathrm{pH} 4.5$ or preliminary fat extraction, as in the case of the riboflavin assay. Inhibiting substances produced by the clarase digestion of yeasts and other materials are reported to interfere with the determination by causing a downward drift of values with increasing test dosage. ${ }^{103}$ Such substances would be most disturbing if present in low-potency material; but yeast, which was the worst offender, is, of course, a relatively rich source. Thompson, Cunningham and Snell ${ }^{106}$ found in the assay of canned foods that there was often a downward drift in the assay values with increasing dosage which, however, tended to reach a definite level. Presumably in such cases the effect is that of extraneous stimulating substances which are effective at low dosage levels. Neal and Strong, ${ }^{10 .}$ by suitable supplements to the basal medium, eliminated such drifts which, however, appear not to prevent obtaining satisfactory assay values, provided the higher dosage values (lower assay values) are accepted as correct. ${ }^{106}$

While $L$. casei has been used extensively as a test organism for pantothenic acid assay, it has the disadvantage that its growth is greatly affected by fatty substances and by unknowns which must be introduced into the basal medium in the form of crude extracts. Hoag, Sarett and Cheldelin ${ }^{107}$ and Skeggs and Wright ${ }^{108}$ have developed assay methods using $L$. arabinosus 17-5. In one laboratory, the Pennington et al. ${ }^{90}$ medium was modified, principally by using norite-treated autolyzed yeast and rice bran concentrate in the basal medium in place of the alkalitreated yeast extract. In the other, the medium of Snell and Wright ${ }^{78}$ for nicotinic acid assay was modified by altering the amounts of some of the constituents, by introducing xanthine and $p$-aminobenzoic acid, and by substituting nicotinic acid for pantothenic acid. For this organism the effects of fatty substances are less marked, but are enough to introduce substantial errors. Hoag et al. advocate a preliminary ether extraction for samples of high fat content. Skeggs and Wright introduced oleic acid, which they found to be stimulative, into the basal medium. If this is not done, they advocate a preliminary ether extraction. In both 
laboratories it was found that turbidimetric measurement of the response in 18 hours or less could be used, and in one laboratory ${ }^{107}$ titration after 24 to 40 hours was found to be satisfactory.

On the basis of general experience with the two organisms, it seems likely that $L$. arabinosus should be a more satisfactory one for pantothenic acid assays than $L$. casei. Whether the $L$. casei methods are too well entrenched or whether the $L$. arabinosus methods offer enough advantage to merit their general adoption remains to be seen.

Among the other microorganisms used for pantothenic acid assay are Streptococcus lactis, ${ }^{109}$ Streptobacterium plantarum, ${ }^{110}$ Proteus morganii, ${ }^{111}, 112,113$ Streptococcus faecalis, ${ }^{114}$ and Acetobacter suboxidans, which responds to pantoic lactone and pantoic acid. ${ }^{115,116}$

Chick Assay. While it would be feasible to develop an assay method for pantothenic acid using the growth of any one of a number of animals as a basis, only chicks have been used with any consistency.

The assay method involves the use of a basal diet the predominant ingredients of which (yellow corn meal, wheat middlings and commercial casein) have been heated in the dry state to $120^{\circ} \mathrm{C}$ for 36 hours. This heat treatment effectively destroys pantothenic acid. This mixture is supplemented with minerals, sources of vitamins $A, D$ and $K$ and riboflavin (whey adsorbate). ${ }^{117,}{ }^{118}$ Chicks are kept on a normal diet for four days and then placed upon the heated diet for 5 days of depletion. After this they are divided into groups and fed the heated diet supplemented by standard and "unknown" materials containing pantothenic acid for comparison. The growth of chicks on the heated diet is very slight, but in the presence of graded doses of pantothenic acid it is increased in accordance with the dosage used.

The basal diet as described is not ideal for the purpose of pantothenic acid assay, but by supplementing it in accordance with newer developments in the field of chick nutrition it can be made the basis of a test which grows more and more specific. ${ }^{119}$

Because of their relative ease and speed, it is probably safe to say that microbiological assays for pantothenic acid have been run at least ten times as often as chick assays. The microbiological tests have thus been more thoroughly explored than has the chick test. In many cases the microbiological and chick methods give concordant results when direct comparisons have been made. Yeast and liver preparations have very often given values by the chick method which are much higher than those by microbiological methods. According to Willerton and Cromwell ${ }^{103}$ this difference largely disappears when the material is properly digested before the microbiological assay is applied. Other workers have not been successful in closing this gap. ${ }^{104,107}$ In view of the recent findings of 
Lipmann et al. ${ }^{120}$ with respect to the release of pantothenic acid from coenzyme A, the subject needs to be reinvestigated. Certainly there is some hazard involved in using crude, non-uniform enzyme preparations, just as there is in the use of chemicals of low purity.

More recently a rat growth method for the estimation of pantothenic acid has been proposed in which wheat germ, in addition to thiamine, riboflavin, pyridoxine, inositol, nicotinic acid and choline, is included in the basal diet. ${ }^{121}$ This material is relatively deficient in pantothenic acid but contains other vitamins essential to rapid growth, and hence is said to serve excellently as a constituent of the basal diet.

\section{Vitamin $B_{6}$-Pyridoxal, Pyridoxamine, Pyridoxine}

The available methods for determining these forms of the vitamin either individually or together are chemical, microbiological and biological. Much material which is primarily of historical interest, that antedating the discovery of pyridoxal and pyridoxamine, ${ }^{122}$ will be omitted from the present discussion.

Chemical Methods. Since each of the three forms of vitamin $\mathrm{B}_{6}$ possesses a phenolic group in the 3 position and the 6 position is unsubstituted, it is not surprising that they can be condensed with various reagents to yield dyes. Among the reagents which have been used in attempts to develop colorimetric methods for vitamin $B_{6}$ are diazotized sulfanilic acid, ${ }^{123}$ 2,6-dichloroquinone chlorimide ${ }^{124,}{ }^{125,} 126$ and diazotized $p$-amino acetophenone. ${ }^{127}$ The earlier attempts were, of course, based upon the assumption that vitamin $\mathrm{B}_{6}$ and pyridoxine are identical.

In natural materials there are numerous phenolic and other reactive substances, however, and the application of such color reactions to natural products is a quite different matter from their application to the pure substances. In developing colorimetric methods it has been necessary to eliminate or rule out other reacting substances, and in some cases this has required an elaborate procedure. ${ }^{123}$ The results obtained using such precautions have often agreed substantially with animal assays of similar materials, though actually the number of available animal assay values on materials which are in any sense duplicable is exceedingly small. The question of the reliability of the animal assays will be discussed in a later paragraph.

One of the interesting facts which has a bearing upon the applicability of this colorimetric procedure is that all three forms of the vitamin give colors when treated with diazotized sulfanilic acid. Pyridoxal gives a bright yellow, pyridoxamine yields orange to pink, and pyridoxine gives an orange color. ${ }^{128}$ It is easy to see that a natural mixture might give a a color for which all three components might be partially responsible. 
In view of these facts and because of the existence in nature of other reacting substances, it seems unlikely that any simple colorimetric procedure can be worked out for the detcrmination of all forms of vitamin $\mathrm{B}_{6}$. Spectrophotometric analysis of the colors produced will probably have to be employed. By the use of borate which under proper conditions forms an inactive complex with pyridoxine only, it is possible, by difference, to obtain a color value due to pyridoxine alone. ${ }^{129}$ This has been applied using the quinone chloroimide reagent. In the absence of borate under prescribed conditions both pyridoxal and pyridoxamine are about one-half as chromogenic as pyridoxine.

Microbiological Methods. Pyridoxine was first shown to be a growth substance for microorganisms by Möller, ${ }^{130}$ who found it to be stimulatory toward lactic acid bacteria and a bottom yeast from sauerkraut. About this time Schultz, Atkin and Frey ${ }^{131}$ and Eakin and Williams ${ }^{132}$ independently and simultaneously announced its stimulative effect on bakers' yeast. A yeast growth method (Saccharomyces carlsbergensis) for its determination as worked out in the Fleischmann Laboratories ${ }^{133}$ is at present the best method for the simultaneous determination of all three forms of the vitamin. A yeast growth method (Saccharomyces cerevisiae) was earlier developed and applied by Williams and co-workers, ${ }^{134}, 135$ which contributed because of contrast with results obtained with Streptococcus lactis (faecalis) to the discovery of "pseudo pyridoxine," later identified as pyridoxal and pyridoxamine. ${ }^{122,136}$

According to Snell and Rannefeld ${ }^{137}$ and Melnick and co-workers, ${ }^{129}$ pyridoxal, pyridoxamine and pyridoxine are approximately equivalent in their effects on Saccharomyces carlsbergensis; but for Saccharomyces cerevisiae the newer members of the group are materially less active under the prescribed conditions.

The effects of pyridoxal, pyridoxamine and pyridoxine on seventeen test organisms which had been suggested or used, were studied thoroughly by Snell and Rannefeld, ${ }^{137}$ and the use of Strep. faecalis as a test organism for the assay of pyridoxal and pyridoxamine was developed by Rabinowitz and Snell. ${ }^{114}$ Pyridoxal has been determined by Rabinowitz et al. ${ }^{137 a}$

It seems feasible by a combination of colorimetric and biological methods to develop means of determining each of the three members of the group. Rabinowitz and Snell have determined each of the three components by microbiological means. ${ }^{137 \mathrm{~b}}$ The yeast growth method ( $S$. carlsbergensis) ${ }^{133}$ is at present reasonably satisfactory for determining all three forms together, provided extraction is adequate (p. 36).

Stokes ${ }^{138}$ has recommended the 5-day mold test using the Neurospora mutant discovered by Beadle and co-workers. For some studies the 5-day incubation period would be a serious disadvantage. 
Biological Assay. The only biological assay method that has been used extensively and which has been applied to pyridoxal and pyridoxamine is the method of Conger and Elvehjem ${ }^{139}$ which has recently been improved by Sarma, et al. ${ }^{139 a}$ This involves the growth response of rats which had been depleted 4 to 6 weeks on a basal diet containing $\mathrm{B}$ vitamins in the form of thiamine, riboflavin, nicotinic acid, choline and pantothenic acid in pure form and 1:20 liver concentrate powder. The growth response is linear with respect to the amount of vitamin $\mathrm{B}_{6}$ added. As originally designed, this test, like the microbiological tests, was for the assay of pyridoxine, on the assumption that pyridoxine and vitamin $\mathrm{B}_{6}$ were identical. Pyridoxamine and pyridoxal are about equally active in the test, however, so it constitutes a method for determining all three forms of the vitamin simultaneously. Assay values obtained by this method for very few duplicable materials are available, and a wider application of the test, particularly to low-potency materials might easily show it to possess serious flaws. The biological method of Dimick and Schreffer, ${ }^{140}$ which appears to be less specific, has also been employed in some recent studies. ${ }^{129}$ Elvehjem has recently suggested an improved basal diet for the rat growth test. ${ }^{141}$ This has been investigated in only a preliminary way.

\section{Biotin}

The only available methods for the assay of biotin involve the use of microorganisms or higher animals. The outstanding obstacle to the development of a chemical method which would be applicable to natural material is the fact that biotin occurs naturally in exceedingly low concentrations which are beyond the reach of most kinds of chemical tests.

\section{Microbiological Tests:}

1. Yeast Growth Method. Yeast was the test organism used initially in the discovery and isolation of biotin by Kögl and co-workers. ${ }^{\mathbf{1 4 2}}$ However, the assay method utilized fresh yeast (heavy seeding) obtained directly from a brewery and hence was not applicable to laboratories in general. The most widely used assay method was that developed in the Texas laboratories, which utilizes a pure culture of bakers' yeast. ${ }^{143}$

The basal medium for this yeast test contained only known substances, including inositol, $\beta$-alanine (synthetic calcium pantothenate was not yet available), thiamine and pyridoxine; 16-hour growth responses were obtained from the addition to $12-\mathrm{ml}$ cultures of biotin in amounts of 25 to 250 micromicrograms $\left(10^{-12}\right.$ gram $)$. This method has the advantage of speed and is also specific to a high degree, since biotin is an extremely potent nutrilite and the amounts of material (in the form of a tissue 
extract, for example) which need to be added to the cultures are small enough so that extraneous growth stimulants are not likely to interfere. This is particularly true if the lower portion of the "growth curve" is utilized.144 Turbidity and color of extracts for the same reason usually offer no problem whatever. This method has been used extensively in the Cornell ${ }^{145}$ and Texas laboratories and elsewhere. Hofmann ${ }^{146}$ characterized it as "simple, accurate and fast," and as "the most satisfactory technique." In view of the striking effect which amino acids were later found to have on yeast growth, when the necessary nutrilites are supplied, ${ }^{147}$ Hertz improved the basal medium by the addition of a casein digest and by the obvious substitution of calcium pantothenate for $\beta$-alanine. ${ }^{148}$

The yeast test is not applicable for the determination of biotin in the presence of desthiobiotin, biotin diaminocarboxylic acid, desthiobiotin diaminocarboxylic acid, ${ }^{149}$ oxybiotin, ${ }^{150}$ biotin sulfoxide methyl ester, ${ }^{151}$ and a few less active compounds because they too possess biotin activity for yeast. However, as these are synthetic substances and are not encountered in many types of investigations, the limitation on the method is often not serious. Other microbiological procedures are limited to some extent in the same way, but yeast possesses great synthetic powers and can utilize some compounds in place of biotin which lactic acid bacteria, for example, cannot. ${ }^{152}$

In applying the yeast test to metabolism studies, Oppel ${ }^{153}$ found that only a portion of the total biotin (as measured by the yeast test) of dog, rabbit, rat and human urines was avidin-combinable, but that "only minute amounts of the non-avidin-combining biotin were found in diets and stool specimens." In the case of one subject who consumed $35-55 \mu \mathrm{g}$ of biotin on alternate days but no raw egg white, the "non-combinable biotin" of the urine was about 35-40 per cent of the total, whereas when large amounts of egg white and biotin were both consumed, the "noncombinable" constituted 75-85 per cent of the total urinary excretion.

Burk and Winzler, ${ }^{154}$ however, reported that uncombinable biotin is abundant and widespread constituting 90 to 100 per cent of that in the urine of rats and mice fed avidin and in Squibb urease, 30 to 50 per cent in "Vitab" (rice bran concentrate) and unhydrolyzed beer, and 1 to 10 per cent in rat lung, spleen, testes, adrenals, lymph nodes, skin, intestinal tract contents, feces, polished rice, dried yeast and food mixtures. Various active substances possessing biotin activity were designated by these investigators as "miotin," "tiotin" and "rhiotin."

Chu and Williams ${ }^{144}$ corroborated the findings of Oppel in that they found substantial amounts of "uncombinable biotin" in urine but nowhere else. They were unable to find evidence for the existence of "uncombinable 
biotin" in "Vitab," rat brain, beer or Squibb urease, and warned against the use of high dosage levels in applying the yeast test. As larger and larger amounts of extracts are introduced into the yeast medium, the test becomes less specific, because of the existence of other yeast nutrilites which may not be in any way related to biotin..$^{155}$

The existence and significance of the "uncombinable biotin" in urine has never been clarified. Probably the most reasonable explanation at the present time is that in urine there exist biotin degradation products which do not combine with avidin and yet can be utilized by yeast in lieu of biotin. The existence of such substances has never been completely clarified and their interference with other asasy methods has not been adequately studied.

Hofmann and Winnick ${ }^{\mathbf{1 5 6}}$ have utilized the fact that oxybiotin is active for yeast in the determination of oxybiotin in the presence of biotin. Biotin is destroyed by dilute permanganate under conditions that oxybiotin is unaffected, so that the response after oxidation is a measure of the oxybiotin present.

2. Methods Utilizing Lactic Acid Bacteria. Several papers have been published describing biotin assay methods or modifications thereof, which utilize $L$. casei as the test organism..$^{157,158,159,160}$ Difficulties are encountered in obtaining a biotin-free basal medium, and the same interferences, particularly of fatty substances, occur as with other tests involving this organism. If the titration method is used for evaluating responses, more time is required to obtain results than in the yeast test.

More recently Wright and Skeggs ${ }^{161}$ have developed the use of $L$. arabinosus for biotin assay. Because the basal medium needs to contain only known substances, and for other reasons, this organism appears to have distinct advantages. A recent survey (1945) is reported to indicate that seven out of nine prominent laboratories use L. arabinosus for biotin assays. ${ }^{152}$ However, the amount of published analytical data accumulated using this method is small, and any shortcomings that the method may possess can be discovered only by extensive use. Because of the nature of the medium and high sensitivity of the organism to biotin, one would not anticipate any serious difficulties in the general application of the method. In the test as originally described, ${ }^{161}$ titration after 72 hours was used to evaluate responses. Subsequently, IVright ${ }^{152}$ has indicated that satisfactory results may be obtained turbidimetrically after about 24 hours of growth, or by titration after 48 or even 24 hours. Speeding up the response in this test could doubtless be accomplished by suitable modifications (if necessary) of the improved medium for $L$. casei developed by Roberts and Snell. ${ }^{50}$

Other biotin assays have utilized as test organisms Rhizobia, ${ }^{154}$ Clos- 
tridium butylicum, ${ }^{162}$ and Neurospora crassa,${ }^{163}$ among others, but these have not been used extensively.

Biological Assay. Biological assays of biotin are relatively of little importance. By introducing raw egg white into the diet of rats they become deficient in biotin (vitamin $\mathrm{H}$ ) and develop characteristic lesions. The daily dose of biotin required to cure this condition was used as a "unit." 164 Other animals, chicks, guinea pigs, rabbits, monkeys and dogs are capable of developing egg white injury and may therefore be used for assay purposes. Ansbacher and Landy ${ }^{165}$ produced biotin deficiency in chicks by feeding a heated diet low in biotin and suggested the use of this procedure as the basis of an assay method.

Trager ${ }^{\mathbf{1 6 6}}$ has reported the presence in the plasma of various animals, of fat-soluble material-not oleic acid-which replaces biotin in the tests involving lactic acid organisms and which is active for biotin-deficient chicks. Contrary to these findings, however, Axelrod, Mitz and Hofmann, ${ }^{167}$ as a result of a thorough study, conclude that the biotin-like activity present in plasma is explicable on the basis of the content of known fat acids.

\section{Inositol}

For the assay of inositol, chemical and microbiological methods are available. In addition, some use of experimental animals has been made.

Chemical Method. The only serviceable chemical method for inositol is that of Platt and Glock, ${ }^{168}$ and it is subject to limitations both as to specificity and to convenience and speed. Earlier chemical methods were investigated in the author's laboratory ${ }^{169}$ and were judged to be almost valueless. Actual isolation and weighing of the inositol was one of the expedients used, but this obviously has very serious drawbacks. ${ }^{170}$

The method of Platt and Glock involves very briefly: (1) extraction with water, (2) precipitation of extraneous material with acetone, (3) removal of glucose by fermentation, (4) removal of other extraneous substances with base exchange resins, (5) differential oxidation of the residual glycerol and inositol with periodic acid. The last step takes advantage of the fact that glycerol can be oxidized quantitatively without more than a very small fraction of the inositol being attacked. As Woolley ${ }^{171}$ has indicated, however, there are in certain tissues several other substances closely related to inositol including isomers, and these presumably would not be eliminated by any of the treatments. On this basis there is some reason to question the specificity of the method. Furthermore, its application is relatively exacting and time-consuming.

Microbiological Methods. The microbiological determination of inositol stems from the finding of Eastcott ${ }^{172}$ that inositol serves as a growth 
substance for yeast. Woolley ${ }^{173}$ and Williams and co-workers ${ }^{174}$ independently developed assay methods based upon this observation. The work of the latter group was in part an outgrowth of earlier work ${ }^{\mathbf{1 6 9}}$ in the author's laboratory.

The two methods are essentially alike, the main difference being in some of the constituents of the basal medium. In order to take care of unknown yeast nutrilites, ${ }^{155}$ Williams and co-workers introduced into the basal medium a liver autolyzate which was rich in unknown nutrilites but relatively poor in inositol. This small amount of inositol caused the growth in the basal medium to be heavier than would otherwise be the case; but adequate responses were obtained by the addition of $0.1 \mu \mathrm{g}$ to $0.8 \mu \mathrm{g}$ inositol, and the method was applied extensively ${ }^{96-101}$ with success. Woolley used a dialyzed rice bran extract (which was lacking in free inositol) as an ingredient of his basal medium because it contained unknown yeast-stimulating material; he obtained satisfactory results.

It appears that, provided the problem of extraction is cared for (p. 38), either of the yeast methods yields satisfactory results. It is also probable that either method could be improved if it were reinvestigated in the light of present knowledge. The extensive application of one of the yeast methods to the study of the contents of tissues yielded results which in one case apply to the amount freed by autolysis and in a later study to the amount freed by enzymatic digestion. ${ }^{96-101}$ The total inositol values were generally materially higher than those obtained by autolysis or enzymatic digestion. ${ }^{85}$ From the nutritional standpoint the total inositol values may well be the most important, because bound forms of inositol are utilizable by mice. On the other hand, the general nutritional importance of inositol is problematical and the availability of different forms of inositol to different animals is largely unknown. The fact that phytic acid interferes with the assimilation of calcium in diets ${ }^{\mathbf{1 7 5}}$ suggests that inositol is not always freed by digestion and hence is not uniformly available in this form.

Woolley ${ }^{176}$ has made a careful study of the specificity of the yeast test for inositol, and has found that only meso-inositol is effective. Other naturally occurring polyalcohols including isomers are inactive, as are also inositol esters, including phytic acid and soybean lipositol. The bound inositol in amylase is freed by enzymatic action, since about the same values were obtained whether enzymatic digestion or acid digestion was used. ${ }^{177}$

Another microbiological method for the determination of inositol involves the use of a mutant strain of Neurospora. ${ }^{178}$ This appears to yield satisfactory results but has not been extensively applied. The dosage 
levels at which inositol is effective in the test is from 10 to $30 \mu \mathrm{g}$, as compared with 0.1 to $0.8 \mu \mathrm{g}$ in the yeast tests.

Biological Test Using Mice. The ability to cure alopecia in mice which have been fed inositol-free diets was used as an assay method by Woolley, ${ }^{179}$ but the results obtained were only roughly quantitative, partly because relatively few levels were tested. Phytin and mesoinositol, for example, were found to be "active" when tested at the same level ${ }^{177}(0.1$ per cent of the diet). If the active ingredient is inositol, phytin should be about $1 / 5$ as active. The relative activities of phytin and inositol for mice would throw some light on the assimilation of phytin and its effects on calcium assimilation.

\section{Choline}

Chemical, microbiological, physiological and animal assay methods for choline have been described. Each has its usefulness, though the methods in general are not as satisfactory as many that have been described in previous sections.

Chemical Assay. A large number of chemical tests have been applied to the problem of the estimation of choline. ${ }^{180}$ Among these precipitation with reineckate is without question the most valuable. The use of this method has a long history which we shall not take time to trace. Among more recent papers those by Beattie, ${ }^{181}$ Jacobi and co-workers, ${ }^{182}$ Engel, ${ }^{183}$ Marenzi and Cardini, ${ }^{184}$ Enteman et al. ${ }^{185}$ Glick, ${ }^{186}$ and Winzler and Meserve, ${ }^{187}$ appear to be most important. The subject has been reviewed recently by Handler. ${ }^{188}$

In most cases choline is freed by alkaline hydrolysis, and choline reineckate precipitated under prescribed conditions is determined colorimetrically in acetone solution. Marenzi and Cardini, ${ }^{184}$ however, determined chromium colorimetrically in the insoluble reineckate, and Winzler and Meserve ${ }^{185}$ used ultraviolet light instead of visible light.

This general method has been applied to tissues and feeds most extensively by Engel ${ }^{189}$ and Rhian and co-workers, ${ }^{190}$ with results which appear satisfactory. None of the methods is extremely sensitive. For the direct colorimetric determination of the reineckate in acetone about 200 $\mu \mathrm{g}$ of choline must be in the sample; when ultraviolet light is used ${ }^{185}$ the minimum determinable amount is about $50 \mu \mathrm{g}$. Colorimetric analysis for chromium ${ }^{184}$ makes possible a determination of $15 \mu \mathrm{g}$. As long as one is concerned with the total choline content of tissues, which is relatively high, the methods are serviceable from the standpoint of sensitivity.

Means have been devised whereby most of the interfering substances commonly found in food materials, including betaine, are eliminated. Dimethyl aminoethanol may be carried down to some extent along with 
choline reineckate, however. ${ }^{191}$ It is doubtful whether the method could be used directly, unless by the Winzler and Meserve modification, for the determination of choline in plants that contain substantial amounts of alkaloids.

Microbiological Assay. The microbiological method for choline which has been applied to tissues, blood and urine is that originated by Horowitz and Beadle, ${ }^{192}$ and involves the use of a cholineless mutant of Neurospora. This organism has lost by ultraviolet radiation its ability to synthesize choline and gives corresponding growth responses when amounts of choline, $1 \mu \mathrm{g}$ to $50 \mu \mathrm{g}$, are added to $25 \mathrm{ml}$ of culture medium. The responses are evaluated after 72 hours' growth by weighing the dried mycelia (ca. 5.0-50 mg). An alternate simplified procedure involves measuring the diameter of the mold cultures as grown in 16 hours on agar in Petri dishes. ${ }^{193,} 194$

The response of this cholineless mutant as well as other cholineless strains ${ }^{194}$ is not quite specific. Betaine and ethanolamine are inactive, but about 50 per cent of the choline of lecithins ${ }^{192}$ is effective in the 72 -hour test; methionine is 0.2 per cent as active as choline; and dimethylaminoethanol and monoethylaminoethanol, acetylcholine, phosphorylcholine and arsenocholine are highly active. Certain other synthetic homologues, dimethylethylhydroxyethylamine, and diethylmethylhydroxyethylamine are also highly active. ${ }^{194}$

One cannot help feeling that these nonspecificities are less important than they appear. The authors are to be commended for thoroughness in investigating the question of specificity, and the method should not be damned because its specificity has been thoroughly investigated. Lecithins and methionine can be eliminated from test solutions, and the other active compounds for the most part are not known to occur naturally in quantities likely to interfere. Dimethylaminoethanol occurs in an alkaloid found in certain leguminous plants ${ }^{195}$; but its general distribution, while possible, and perhaps even probable ${ }^{196}$ has not been demonstrated. Leucke and Pearson have applied the mold method to free choline in plasma and urine, ${ }^{197}$ and the free and bound choline of a few animal tissues, ${ }^{198}$ with results which appear to be satisfactory and in agreement with those obtained by the reineckate method. Hodson has applied the method to milk products. ${ }^{163} \mathrm{~A}$ combination of the reineckate and microbiological methods suggests itself as desirable in crucial cases.

The basis for another microbiological test of limited interest is the requirement of Type III Pneumococcus for choline. ${ }^{199}$ A large number of compounds, mostly synthetic, showed activity, including dimethylethanolamine (100 per cent) and ethanolamine (10 per cent). The nonspecificity 
and the pathogenic nature of the organism used are deterrents to its use as an assay method.

Physiological Assay. An interesting and important method for choline determination involves acetylating it quantitatively and determining the acetylcholine by its stimulation of the contraction of isolated muscle from rabbit intestine. The amount of acetylcholine necessary to elicit a contraction about 75 per cent of the maximum may be from 0.01 to $0.03 \mu \mathrm{g}$ per ml, depending upon the muscle preparation and the exact conditions; so the method has high sensitivity. ${ }^{200}$

There are interfering substances and the method possesses many dangerous pitfalls for the chemist, ${ }^{201}$ but inherently it has great advantages and it seems likely that cooperative studies by biochemists (who are more expert in preparing suitable extracts and eliminating by adsorption and otherwise interfering substances) and physiologists (who are more expert with respect to dealing with muscle preparations) might evolve an assay method more sensitive, more specific, and more satisfactory generally than any yet devised. In its present form it has been applied to many materials. ${ }^{200}$ Duplicate assays occasionally differed by almost 30 per cent.

Animal Assays. The development of an animal assay procedure for choline is complicated by the fact that choline is not an absolute requirement; methionine and other naturally occurring substances possess choline-like activity when fed to animals. ${ }^{202}$ Engel, ${ }^{183}$ nevertheless, has developed an assay method for choline and choline-like substances which depends upon its ability to prevent kidney hemorrhages in rats receiving a choline-deficient diet. The rats had to be carefully matched and used in considerable numbers (more often 11 or 12 for each test), but the results were such that a 10 per cent increase or decrease of the material tested could be detected. Liver was found to be about 25 per cent more effective than could be accounted for on the basis of its choline content as determined chemically by the reineckate method, and this was interpreted to mean the presence in it of other substances which are physiologically like choline. Otherwise, the bioassays agreed with the chemical method rather closely.

\section{Folic Acid (Pteroyl Glutamic Acid, P.G.A.)}

Determination of folic acid may be performed (under delimiting conditions) chemically, microbiologically or by animal assays. None of the methods has been fully standardized, particularly so as to be readily applied to tissues of all types.

Chemical Determination. Hutchings, et al. ${ }^{203}$ have developed a chemical method for the determination of folic acid and related compounds, taking advantage of the fact that by reduction in acid solution with zinc 
dust, folic acid yields characteristically a pteridine and an aromatic amine. The latter can be determined colorimetrically after the method of Bratton and Marshall. ${ }^{204}$ The presence of aromatic amines in the original solution before reduction is taken care of by the use of a blank, and the amount before reduction subtracted from that obtained after reduction is used as a measure of the folic acid present.

The specificity of the test has not been explored (in spite of the ten investigators involved) and its sensitivity is not high. It may be used only for concentrates which contain 5 per cent or more of folic acid.

Microbiological Tests. The two organisms which are most often used for folic acid assays are $L$. casei, the organism used initially in observations dealing with the "norite eluate factor" 205 and Streptococcus faecalis $\mathrm{R}$ (earlier called Strep. lactis R) which was the principal organism used in connection with first obtaining folic acid in highly concentrated form ${ }^{206}$ and in determining its distribution in tissue autolyzates and in enzymedigested tissues and foods.

The principal differences between the behaviors of the two organisms in these tests are: (1) L. casei assays are materially affected by the introduction of additional alanine into the medium; S. faecalis assays are not. ${ }^{207}$ The same general statement appears to apply also to other amino acids (leucine, isoleucine, threonine ${ }^{208,209}$ ), though direct comparisons appear not to have been made. (2) $L$. casei requires for maximum growth unknown substances such as occur in enzymatically hydrolyzed casein ${ }^{50}$ and norite-treated peptone. ${ }^{210}$ Such addenda have not been required when S. faecalis is used. ${ }^{210}$ (3) Thymine in sufficient concentration has stimulating effect on both organisms as well as certain other lactic acid bacteria ${ }^{211}, 212,213$ however, $L$. case $i$ is considerably more sensitive to small concentrations. ${ }^{209}$ On the other hand, thymine in sufficient amounts has an ultimate effect practically equivalent to folic acid for $S$. faecalis, but incomplete fermentation only results when excess thymine is furnished L. casei. ${ }^{213}$ This latter observed difference may be eliminated when it is possible to include all the stimulatory substances affecting $L$. casei, except folic acid, in the basal medium. (4) S. faecalis responds to rhizopterin 214 (S.L.R. factor) as it does to folic acid, but this glutamic-acid-free relative of folic acid is substantially inactive for $L$. casei. (5) $L$. casei responds readily to pteroyl triglutamic acid while for $S$. faecalis this combined form is only about 6 per cent as active. ${ }^{215}$ (Both organisms respond equally to folic acid and fail to respond to pteroyl heptaglutamic acid.) (6) $L$. casei tests have usually involved relatively long growth periods and titrametric evaluation of the responses. S. faecalis tests, on the other hand, have usually involved short growth periods and turbidimetric measurement of the response. 
A number of the apparent defects mentioned above are relatively easily remedied. The basal media can be adjusted to take care of the effects of alanine and other amino acids, and with less satisfaction the unknown stimulatory substances.

A drawback so far as $L$. case $i$ is concerned is the relatively high activity of thymine-as little as $1 \mu \mathrm{g}$ in a sample would be enough to introduce serious error. ${ }^{209}$ For $S$. faecalis, however, folic acid is at least 5000 times as active as thymine. ${ }^{213}$ This discrepancy is not as important as might be supposed, however, since $L$. casei responds to smaller concentrations of folic acid than does S. faecalis. Stokstad and Hutchings ${ }^{216}$ suggest that with $S$. faecalis the total response minus the residual response after selectively destroying the folic acid could be used as a measure of the true folic acid content. Whether such a procedure is necessary or practical has not been demonstrated.

The most serious defect of the $S$. faecalis assay is the response to a simpler compound, rhizopterin. The degree of importance of this observation depends upon the distribution of rhizopterin in nature. If it is widespread and relatively abundant, the defect is quite serious. Whether or not this is so can be determined only by comparative assays on completely digested samples, using both $L$. casei and S. faecalis. Because the two organisms do not respond equally to combined forms of folic acid, ${ }^{215}$ one would have to be sure that digestion had been complete before valid conclusions could be drawn.

From the foregoing discussion, it is clear that no well standardized and completely satisfactory microbiological method for folic acid assay has been devised. Stokstad and Hutchings wisely suggest the use of both $L$. casei and S. faecalis. ${ }^{216}$ Complete digestion of the combined forms without destruction constitutes one crucial problem (p. 40).

\section{Animal Assay Methods.}

Folic acid assays may be performed using chicks, rats or monkeys. The use of rats involves the feeding of sulfa drugs to prevent intestinal synthesis ${ }^{217}$ and involves so many complications and uncertainties that it does not appear to be a useful assay method at the present time. Use can be made of rhesus monkeys ${ }^{218}$ but the expense and inconvenience could hardly be justified on the basis of an assay method.

Chick Assay. While O'Dell and Hogan ${ }^{219}$ and their co-workers used the cure of anemia in chicks in connection with concentrating "vitamin $\mathrm{B}_{\mathrm{c}}$ " this method of assay has apparently not been applied to the determination of the vitamin in foods and tissues.

The preferred chick assay method involves the use of a 4-week prophylactic test period. It was applied successfully by Bird et al. ${ }^{220}$ to natural 
materials, but has been only briefly described. ${ }^{221} \mathrm{~A}$ number of changes result from lack of the vitamin, ${ }^{221}, 222$ including failure to grow, but it is not apparent how Bird and co-workers ${ }^{220}$ calculated their results. In the assay technic recommended by Day and Totter, ${ }^{223}$ weights of the chicks at the end of four weeks are the basis for calculating the results.

The chick assay method as applied by Bird and co-workers yielded results which in general agreed substantially with microbiological assays when the materials for the latter were treated enzymatically with preparations from hog kidney and almonds. ${ }^{20}$ Plant extracts, however, gave higher results with chick assays than were obtainable by microbiological assay. It may be that plants contain combined forms of folic acid which are not hydrolyzed by any of the treatments used. Chicks respond equally to folic acid, the tri- and heptaglutamate forms, and presumably to other combined forms which may occur naturally (p. 31).

\section{p-Aminobenzoic Acid}

While $p$-aminobenzoic acid can be determined colorimetrically by diazotizing and coupling with dimethyl- $\alpha$-napthylamine ${ }^{224}$ and by the use of thiamine as a reagent, ${ }^{225}$ such methods are neither highly specific nor are they sensitive in comparison with microbiological methods and have not been used as general assay procedures.

Animal experiments have demonstrated the physiological effect of feeding $p$-aminobenzoic acid under prescribed conditions to chicks, ${ }^{226,227}$ mice, ${ }^{228,}{ }^{229}$ and rats, ${ }^{226,} 230$ but the effects may be indirect and not always reproducible, ${ }^{231}$ and no assay method has resulted from these observations.

Microbiological Assays. Four microbiological assays for $p$-aminobenzoic acid have been developed. They utilize respectively, Acetobacter suboxydans, ${ }^{232}$ Lactobacillus arabinosus $17-5,{ }^{233}$ a mutant strain (aminobenzoicless) of Neurospora, ${ }^{234}$ and $\mathrm{Cl}$. acetobutylicum..$^{235}$ In the table below is given certain crucial information regarding these tests.

\section{Microbiological Assays for $p$-Aminobenzoic Acid}

\begin{tabular}{lcll}
$\quad$ Organism & $\begin{array}{c}\text { Growth } \\
\text { period } \\
\text { (hrs.) }\end{array}$ & $\begin{array}{c}\text { Assay range } \\
(\mu \mathrm{g})\end{array}$ & \multicolumn{1}{c}{$\begin{array}{c}\text { Method of evaluating } \\
\text { response }\end{array}$} \\
(1) Acetobacter suboxydans & 48 & $5.0-50$ & Turbidity \\
(2) L. arabinosus & 72 & $0.15-0.5$ & Titration \\
(3) Neurospora & 20 & $4.0-40$ & Measurement of culture size \\
(4) Cl. acetobutylicum & $20-24$ & $0.3-1.5$ & Turbidity
\end{tabular}

It is probable that each of the four methods is capable of yielding satisfactory results and for the most part they embody methods which are now common in microbiological assays. In method 3, the size of the mold cultures is measured with calipers which is something of an innova- 
tion, and in method 4 , the organism has to be grown under anerobic conditions.

Assays for $p$-aminobenzoic acid are in general exacting partly because laboratory equipment, glassware, etc., becomes so readily contaminated with $p$-aminobenzoic acid that consistent assays are impossible. This is particularly true when the more sensitive methods are used. Segregation of the glassware used for this purpose and the use of a separate room are desirable precautions, in addition to scrupulous care in the cleaning of glassware.

The problem of extraction of $p$-aminobenzoic acid, which has already been discussed (p. 40), is a crucial one and the four methods above have never been compared under conditions where the extraction procedures were the same. The values obtainable for different materials assayed are therefore usually not comparable. The evidence indicates that the response in every case is quite specific for $p$-aminobenzoic acid, in the sense that no simple known chemicals have more than a slight effect. Information is lacking as to how the unknown combined forms, which probably exist naturally, affect the different organisms, and no tests involving pure folic acid and its conjugates or rhizopterin have been found.

\section{Bibliography}

1. Kline, O. L., "Biological Symposia," Vol. 12, Jaques Cattell Press, Lancaster, Pa., 1947, pp. 524-31.

2. Jansen, B. C. P., Rec. trav. chim., 55, 1046-52 (1936).

3. Hennessy, D. J., and Cerecedo, L. R., J. Am. Chem. Soc., 61, 179-83 (1939).

4. Conner, R. T., and Straub, G. J., Ind. Eng. Chem., Anal. Ed., 13, 380-4 (1941).

5. The Association of Vitamin Chemists, Inc., "Methods of Vitamin Assay," Interscience Publishers, New York, N. Y., 1947, p. 73.

6. Hinton, J. J. C., Biochem. J., 37, 585-9 (1943).

7. Vitamin $B_{1}$ Subcommittee of the Accessory Food Factors Committee of the Medical Research Council and The Lister Institute, Biochem. J., 37, 433-9 (1943).

8. Andrews, J. S., et al., Cereal Chem., 21, 388-97 (1944).

9. Harris, L. J., and Wang, Y. L., Biochem. J., 35, 1050-67 (1941).

10. Lane, R. L., Johnson, E., and Williams, R. R., J. Nutrition, 23, 613-24 (1942).

11. Egaña, E., and Meiklejohn, A. P., J. Biol. Chem., 141, 859-70 (1941).

12. Mickelsen, O., Condiff, H., and Keys, A., J. Biol. Chem., 160, 361-70 (1945).

13. Friedemann, T. E., and Kmieciak, T. C., J. Lab. Clin. Med., 28, 1262-8 (1943).

14. Greenberg, L. D., and Rinehart, J. F., Proc. Soc. Exptl. Biol. Med., 59, 9-13 (1945).

14a. Lowry, O. H., J. Biol. Chem., 173, 677-82 (1948).

15. Brown, R. A., et al., Ind. Eng. Chem., Anal. Ed., 15, 494-5 (1943).

16. Hennessy, D. J., Wapner, S. and Truhlar, J. Eng. Chem., Anal. Ed., 16, 476-8 (1944).

17. Prebluda, H. J. and McCollum, E. V., J. Biol. Chem., 127, 495-503 (1939).

18. Melnick, D., and Field, H., J. Biol. Chem., 127, 505-14, 515-30, 531-40 (1939).

19. Hochberg, M., and Melnick, D., J. Biol. Chem., 156, 53-9 (1944).

20. Schultz, A. S., Atkin, L., and Frey, C. N., J. Am. Chem. Soc., 59, 948-9 (1937). 
21. Schultz, A. S., Atkin, I., and Frey, C. N., Ind. Eng. Chem., Anal. Ed., 14, 35-9 (1942).

22. Atkin, L., Schultz, A. S., and Frey, C. N., J. Biol. Chem., 129, 471 (1939).

23. Frey, C. N., and Hennessy, D. J., Cereal Chemists Bull., 2, 233 (1942).

24. Eppright, M. A., and Williams, R. J., Ind. Eng. Chem., Anal. Ed., 16, 576-9 (1944).

25. Williams, R. J., and Roehm, R. R., J. Biol. Chem., 87, 581-90 (1930).

26. Williams, R. J., MeMahan, J. R., and Eakin, R. E., Univ. Texas Pub., 4137, 31-5 (1941).

27. Cheldelin, V. H., et al., Univ. Texas Pub., 4237, 15-36 (1942).

28. Schopfer, W. H., Z. Vitaminforschung, 4, 67, 187 (1935).

29. Meiklejohn, A. P., Biochem. J., 31, 1441-51 (1937).

30. Sinclair, H. M., Biochem. J., 32, 2185-99 (1938).

31. Sinclair, H. M., Biochem. J., 33, 2027-36 (1939).

32. Niven, C. F., and Smiley, K. L., J. Biol. Chem., 150, 1-9 (1943).

33. Sarett, H. P., and Cheldelin, V. H., J. Biol. Chem., 155, 153-60 (1944).

34. Sarett, H. P., and Cheldelin, V. H., J. Nutrition, 30, 25-30 (1945).

35. "Pharmacopoeia of the United States of America," 13th Revision, Mack Printing Co., Easton, Pa., 1947, p. 703.

36. Smith, M. I., U. S. Pub. Health Repts., 45, 116-29 (1930).

37. Birch, T. W., and Harris, L. J., Biochem. J., 28, 602-21 (1934).

38. Chase, E. F., and Sherman, H. C., J. Am. Chem. Soe., 53, 3506-10 (1931).

39. Cowgill, G. R., "The Vitamin B Requirements of Man," Yale Univ. Press, New Haven, Conn., 1934, p. 33.

40. Arnold, A., and Elvehjem, C. A., J. Nutrition, 15, 403-10 (1938).

41. Jukes, T. H., and Heitman, H., J. Nutrition, 19, 21-30 (1940).

42. Passmore, R., Peters, R. A., and Sinclair, H. M., Biochem. J., 27, 842-50 (1933).

43. Scott, M. L., et al., J. Biol. Chem., 165, 65-71 (1946).

44. Daniel, L., and Norris, L. C., Food Research, 9, 312-8 (1944).

45. Daniel, L., and Norris, L. C., J. Nutrition, 30, 31-6 (1945).

46. The Association of Vitamin Chemists, Inc., "Methods of Vitamin Assay," Interscience Publishers, New York, N. Y., 1947, pp. 102-110.

47. Snell, E. E., and Strong, F. M., Ind. Eng. Chem., Anal. Ed., 11, 346-50 (1939.

48. "Pharmacopoeia of the United States of America," 13th Revision, Mack Printing Co., Easton, Pa., 1947, p. 685.

49. Feeney, R. E., and Strong, F. M., J. Biol. Chem., 133, xxxi (1940).

50. Roberts, E. C., and Snell, E. E., J. Biol. Chem., 163, 499-509 (1946).

51. Bauernfeind, J. C., Sotier, A. L., and Boruff, C. S., Ind. Eng. Chem., Anal. Ed., 14, 666-71 (1942).

52. Strong, F. M., and Carpenter, L. E., Ind. Eng. Chem., Anal. Ed., 14, 909-13 (1942).

53. Kodicek, E., and Worden, A. N., Biochem. J., 39, 78-85 (1945).

54. Isbell, H., Wooley, J. G., and Fraser, H. F., U.S. Pub. Health Repts., 56, 282-5 (1941).

55. Williams, R. J., McAlister, E. D., and Roehm, R. R., J. Biol. Chem., 83, 315-20 (1929).

56. Bourquin, A., and Sherman, H. C., J. Am. Chem. Soc., 53, 3501-5 (1931).

57. El Sadr, M. M., Macrae, T. F., and Work, C. E., Biochem. J., 34, 601-7 (1940).

58. Wagner, J. R., et al., J. Biol. Chem., 136, 357-64 (1940).

59. Emmett, A. D., et ai., Ind. Eng. Chem., Anal. Ed., 13, 219-21 (1941).

60. Wilgus, H. S., Norris, L. C., and Heuser, G. F., J. Agri. Research, 51, 383-99 (1935)

61. Heiman, V., and Carver, J. S., Poultry Sci., 16, 434-7 (1937).

62. Jukes, T. H., J. Nutrition, 14, 223-33 (1937).

63. Konig, J., Prakt. Chem., 70, 19 (1904).

64. Harris, L. J., and Raymond, W. D., Biochem. J., 33, 2037-51 (1939). 
65. Bandier, E., and Hald, J., Biochem. J., 33, 264-71 (1939).

66. Swaminathan, M., Nature, 141, 830 (1938).

67. Euler, H. v., et al., Z. physiol. Chem., 256, 208-28 (1938).

68. Teeri, A. E., and Shimer, S. R., J. Biol. Chem., 153, 307-11 (1944).

69. Kodicek, E., Biochem. J., 34, 712-23, 724-35 (1940).

70. Bina, A. F., Thomas, J., and Brown, E. B., Cereal Chem., 18, 661-6 (1941).

71. Perlzweig, W. A., Levy, E. D., and Sarett, H. P., J. Biol. Chem., 136, 729-45 (1940).

72. Dann, W. J., and Handler, P., J. Biol. Chem., 140, 201-13 (1941).

73. Steel, H. K., Cereal Chem., 22, 448-54 (1945).

74. Melnick, D., and Field, H., J. Biol. Chem., 134, 1-16 (1940); 135, 33 (1940).

75. Melnick, D., Robinson, W. D., and Field, H., J. Biol. Chem., 136, 131-44 (1940).

76. Pearson, P. B., J. Biol. Chem., 129, 491-4 (1939).

77. Cheldelin, V. H., and Williams, R. R., Ind. Eng. Chem., Anal. Ed., 14, 671-5 (1942).

78. Snell, E. E., and Wright, L., J. Biol. Chem., 139, 675-85 (1941).

79. "Pharmacopoeia of the United States of America," 13th Revision, Mack Printing Co., Easton, Pa., 1947, p. 669.

80. The Association of Vitamin Chemists, Inc., "Methods of Vitamin Assay," Interseience Publishers, New York, N. Y., 1947, p. 128.

81. Williams, R. J., Univ. Texas Pub., 4237, 7-13 (1942).

82. Krehl, W. A., Strong, F. M., and Elvehjem, C. A., Ind. Eng. Chem., Anal. Ed., 15, 471-5 (1943).

83. Williams, W. L., J. Biol. Chem., 166, 397-406 (1946).

84. Grossowicz, N., and Sherstinsky, E., J. Biol. Chem., 167, 101-5 (1947).

85. Elvehjem, C. A., et al., J. Nutrition, 17, Suppl. 11 (1939).

86. Krehl, W. A., Elvehjem, C. A., and Strong, F. M., J. Biol. Chem., 156, 13-9 (1944).

87. Williams, R. J., "Advances in Enzymology and Related Subjects," Vol. 3, Interseience Publishers, Inc., New York, N. Y., 1943, p. 265.

88. Williams, R. J., et al., J. Am. Chem. Soc., 55, 2912 (1933).

89. Williams, R. J., et al., J. Am. Chem. Soc., 60, 2719-23 (1938).

90. Pennington, D., Snell, E. E., and Williams, R. J., J. Biol. Chem., 135, 213-22 (1940).

91. Williams, R. J., Biol. Rev., 16, 49-80 (1941).

92. Mitchell, H. K., and Williams, R. J., Biochem. J., 34, 1532-6 (1940).

93. Atkin, L., et al., Ind. Eng. Chem., Anal. Ed., 16, 67-71 (1944).

94. Cheldelin, V. H., Hoag, E. H., and Sarett, H. P., J. Bact., 49, 41-5 (1945).

95. Strong, F. M., Feeney, R. E., and Earle, A., Ind. Eng. Chem., Anal. Ed., 13, 566-70 (1941).

96. Mitchell, H. K., and Isbell, E. R., Univ. Texas Pub., 4237, 37-40 (1942).

97. Taylor, A., Pollack, M. A., and Williams, R. J., Univ. Texas Pub., 4237, 41-55 (1942).

98. Pollack, M. A., Taylor, A., and Williams, R. J., Univ. Texas Pub., 4237, 56-71 (1942).

99. Woods, A. M., et al., Univ. Texas Pub., 4237, 84-6 (1942).

100. Williams, R. J., Cheldelin, V. H., and Mitchell, H. K., Univ. Texas Pub., 4237, 97-104 (1942).

101. Cheldelin, V. H., and Williams, R. J., Univ. Texas Pub., 4237, 105-24 (1942).

102. Waisman, H. A., et al., J. Nutrition, 23, 239-48 (1942).

103. Willerton, E., and Cromwell, H. W., Ind. Eng. Chem., Anal. Ed., 14, 603-6 (1942).

104. Neal, A. L., and Strong, F. M., Ind. Eng. Chem., Anal. Ed., 15, 654-7 (1943).

105. Bauernfeind, J. C., Norris, L. C., and Heuser, G. F., Poultry Sci., 21, 136-41 (1942). 
106. Thompson, M. L., Cunningham, E., and Snell, E. E., J. Nutrition, 28, 123-9 (1944).

107. Hoag, E. H., Sarett, H. P., and Cheldelin, V. H., Ind. Eng. Chem., Anal. Ed., $17,60-2$ (1945).

108. Skeggs, H. R., and Wright, L. D., J. Biol. Chem., 156, 21-6 (1944).

109. Mitchell, H. K., et al., J. Am. Chem. Soc., 62, 1776-9 (1940).

110. Kuhn, R., and Wreland, T., Ber., 73, 962 (1940).

111. Pelczar, M. J., and Porter, J. R., Proc. Soc. Exptl. Biol. Med., 43, 151-4 (1940).

112. Pelczar, M. J., and Porter, J. R., J. Biol. Chem., 139, 111-9 (1941).

113. Pelczar, M. J., and Porter, J. R., Proc. Soc. Exptl. Biol. Med., 47, 3-7 (1941).

114. Rabinowitz, J. C., and Snell, E. E., J. Biol. Chem., 169, 631-42 (1947).

115. Underkofler, L. A., Bantz, A. C., and Peterson, W. H., J. Bact., 45, 183-90 (1943).

116. Sarett, H. P., and Cheldelin, V. H., J. Biol. Chem., 159, 311-9 (1945).

117. Jukes, T. H., J. Biol. Chem., 117, 11-20 (1937).

118. Jukes, T. H., J. Biol. Chem., 129, 225-31 (1939).

119. Jukes, T. H., and McElroy, L. W., Poultry Sci., 22, 438-41 (1943).

120. Lipmann, F., et al., J. Biol. Chem., 167, 869-70 (1947).

121. Bacon, J. S. D., Jenkins, G. N., and Irwin, J. O., Biochem. J., 37, 492-7 (1943).

122. Snell, E. E., J. Biol. Chem., 154, 313-4 (1944).

123. Swaminathan, M., Nature, 145, 780 (1940).

124. Scudi, J. V., J. Biol. Chem., 139, 707-20 (1941).

125. Bina, A. F., Thomas, J. M., and Brown, E. B., J. Biol. Chem., 148, 111 (1942).

126. Hochberg, M., Melnick, D., and Oser, B. L., J. Biol. Chem., 155, 109-14 (1944).

127. Brown, E. B., Bina, A. F., and Thomas, J. M., J. Biol. Chem., 158, 455-61 (1945).

128. Ormsby, A. A., Fisher, A., and Schlenk, F., Arch. Biochem., 12, 79-81 (1947).

129. Melnick, D., el al., J. Biol. Chem., 160, 1-14 (1945).

130. Möller, E. F., Z. physiol. Chem., 254, 285-6 (1938).

131. Schultz, A. S., Atkin, L., and Frey, C. N., J. Am. Chem. Soc., 61, 1931 (1939).

132. Eakin, R. E., and Williams, R. J., J. Am. Chem. Soc., 61, 1932 (1939).

133. Atkin, L., et al., Ind. Eng. Chem., Anal. Ed., 15, 141-4 (1943).

134. Williams, R. J., Eakin, R. E., and McMahan, J. R., Univ. Texas Pub., 4137, 24-6 (1941).

135. Williams, R. J., Univ. Texas Pub., 4237, 9-10 (1942).

136. Snell, E. E., Guirard, B. M., and Williams, R. J., J. Biol. Chem., 143, 519-30 (1942).

137. Snell, E. E., and Rannefeld, A. N., J. Biol. Chem., 157, 475-89 (1945).

137a. Rabinowitz, J. C., Mondy, N. I., and Snell, E. E., J. Biol. Chem., 175, 147-53 (1948).

137b. Rabinowitz, J. C., and Snell, E. E., J. Biol. Chem., 176, 1157-67 (1948).

138. Stokes, J. L., "Biological Symposia," Vol. 12, Jaques Cattell Press, Lancaster, Pa., 1947, p. 231.

139. Conger, T. W., and Elvehjem, C. A., J. Biol. Chem., 138, 555-61 (1941).

139a. Sarma, P. S., Snell, E. E., and Elvehjem, C. A., J. Nutrition, 33, 121-8 (1917).

140. Dimick, M. K., and Schreffler, C. B., J. Nutrition, 17, 23-9 (1939).

141. Elvehjem, C. A., "Biological Symposia," Vol. 12, Jaques Cattell Press, Lancaster, Pa., 1947, pp. 213-26.

142. Kögl, F., and Tönnis, B., Z. physiol. Chem., 242, 43-73 (1936).

143. Snell, E. E., Eakin, R. E., and Williams, R. J., J. Am. Chem. Soc., 62, 175-8 (1940).

144. Chu, E. J., and Williams, R. J., J. Am. Chem. Soc., 66, 1678-80 (1944).

145. du Vigneaud, V., et al., J. Biol. Chem., 140, 643-51 (1941).

146. Hofmann, K., "Advances in Enzymology and Related Subjects," Vol. 3, Interscience Publishers, Inc., New York, N. Y., 1943, pp. 292, 306.

147. Mitchell, H. K., and Williams, R. J., Biochem. J., 34, 1532-6 (1940).

148. Hertz, R., Proc. Soc. Exptl. Biol. Med., 52, 15-7 (1943).

149. Dittmer, K., and du Vigneaud, V., Science, 100, 129-31 (1944). 
150. Pilgrim, F. J., et al., Science, 102, 35-6 (1945).

151. du Vigneaud, V., Chem. Eng. News, 23, 623-5 (1945).

152. Wright, L. D., "Biological Symposia," Vol. 12, Jaques Cattell Press, Lancaster, Pa., 1947, p. 305.

153. Oppel, T. W., Am. J. Med. Sci., 204, 856-75 (1942).

154. Burk, D., and Winzler, R. J., Science, 97, 57-60 (1943).

155. Williams, R. J., Eakin, R. E., and Snell, E. E., J. Am. Chem. Soc., 62, 1204-7 (1940).

156. Hofmann, K., and Winnick, T., J. Biol. Chem., 160, 449-53 (1945).

157. Shull, G. M., Hutchings, B. L., and Peterson, W. H., J. Biol. Chem., 142, 913-20 (1942).

158. Shull, G. M., and Peterson, W. H., J. Biol. Chem., 151, 201-2 (1943).

159. Landy, M., and Dicken, D. M., J. Lab. Clin. Med., 27, 1086-92 (1942).

160. Tomlinson, F. F., and Peterson, W. H., Arch. Biochem., 5, 221-31 (1944).

161. Wright, L. D., and Skeggs, H. R., Proc. Soc. Exptl. Biol. Med., 56, 95-8 (1944).

162. Lampen, J. O., Bahler, G. P., and Peterson, W. H., J. Nutrition, 23, 11-21 (1942).

163. Hodson, A. Z., J. Biol. Chem., 157, 383-5 (1945).

164. György, P., J. Biol. Chem., 131, 733-44 (1939).

165. Ansbacher, S., and Landy, M., Proc. Soc. Exptl. Biol. Med., 48, 3-5 (1941).

166. Trager, W., Proc. Soc. Exptl. Biol. Med., 64, 129-34 (1947).

167. Axelrod, A. E., Mitz, M., and Hofmann, K., J. Biol. Chem., 175, 265-74 (1948).

168. Platt, B. S., and Glock, G. E., Biochem. J., 37, 709-12 (1943).

169. King, Anne, Master's Thesis, Oregon State College, 1938.

170. Winter, L. B., Biochem. J., 34, 249-50 (1940).

171. Woolley, D. W., "Biological Symposia," Vol. 12, Jaques Cattell Press, Lancaster, Pa., 1947, pp. 279-89.

172. Eastcott, E. V., J. Phys. Chem., 32, 1094-11 (1928).

173. Woolley, D. W., J. Biol. Chem., 140, 453-9 (1941).

174. Williams, R. J., et al., Univ. Texas Pub. 4137, 27-30 (1941); see also references 89-94.

175. MeCance, R. A., and Widdowson, E. M., Nature, 153, 650 (1944).

176. Woolley, D. W., J. Biol. Chem., 140, 461-6 (1941).

177. Williams, R. J., Schlenk, F., and Eppright, M. A., J. Am. Chem. Soc., 66, 896-8 (1944).

178. Beadle, G. W., J. Biol. Chem., 156, 683-9 (1944).

179. Woolley, D. W., J. Biol. Chem., 139, 29-34 (1941).

180. Best, C. H., and Lucas, C. C., "Vitamins and Hormones," Vol. I, Academic Press, Inc., New York, N. Y., 1943, pp. 9-16.

181. Beattie, F. J. R., Biochem. J., 30, 1554-9 (1936).

182. Jacobi, H. P., Baumann, C. A., and Meek, W. J., J. Biol. Chem., 138, 571-82 (1941).

183. Engel, R. W., J. Biol. Chem., 144, 701-10 (1942).

184. Marenzi, A. D., and Cardini, C. E., J. Biol. Chem., 147, 363-70 (1943).

185. Entenman, C., Taurog, A., and Chaikoff, I. L., J. Biol. Chem., 155, 13-8 (1944).

186. Glick, D., J. Biol. Chem., 156, 643-51 (1945).

187. Winzler, R. J., and Meserve, E. R., J. Biol. Chem., 159, 395-7 (1945).

188. Handler, P., "Biological Symposia," Vol. 12, Jaques Cattell Press, Lancaster, Pa., 1947, pp. 361-72.

189. Engel, R. W., J. Nutrition, 25, 441-6 (1943).

190. Rhian, M., Evans, R. J., and St. John, J. L., J. Nutrition, 25, 1-5 (1943).

191. Jukes, T. H., and Dornbush, A. C., Proc. Soc. Exptl. Biol. Med., 58, 142-3 (1945).

192. Horowitz, N. H., and Beadle, G. W., J. Biol. Chem., 150, 325-33 (1943).

193. Thompson, R. C., Isbell, E. R., and Mitchell, H. K., J. Biol. Chem., 148, 281-7 (1943).

194. Horowitz, N. H., Bonner, D., and Houlahan, M. B., J. Biol. Chem., 159, 145-51 (1945). 
195. Faltis, F., and Holzinger, L., Ber., 72B, 1443-50 (1939).

196. Jukes, T. H., and Oleson, J. J., J. Biol. Chem., 157, 419-20 (1945).

197. Luecke, R. W., and Pearson, P. B., J. Biol. Chem., 153, 259-63 (1944).

198. Luecke, R. W., and Pearson, P. B., J. Biol. Chem., 155, 507-12 (1944).

199. Badger, E., J. Biol. Chem., 153, 183-91 (1944).

200. Fleteher, J. D., Best, C. H., and Solandt, O. M., Biochem. J., 29, 2278-84 (1935).

201. Best, C. H., and Lucas, C. C., "Vitamins and Hormones," Vol. I, Academic Press, Inc., New York, N. Y., 1943, p. 16.

202. Jukes, T. H., "Annual Review of Biochemistry," Vol. 16, Stanford Univ. Press, Stanford University, Calif., 1947, pp. 193-222.

203. Hutchings, B. L., et al., J. Biol. Chem., 168, 705-10 (1947).

204. Bratton, A. C., and Marshall, E. K., Jr., J. Biol. Chem., 128, 537 (1939).

205. Snell, E. E., and Peterson, W. H., J. Bact., 39, 273-85 (1940).

206. Mitchell, H. K., Snell, E. E., and Williams, R. J., J. Am. Chem. Soc., 63, 2284 (1941).

207. Snell, E. E., Proc. Soc. Exptl. Biol. Med., 55, 36-9 (1944).

208. Dolby, D. E., and Waters, J. W., Nature, 153, 139-40 (1944).

209. Krueger, K., and Peterson, W. H., J. Biol. Chem., 158, 145-56 (1945).

210. Tepley, L. J., and Elvehjem, C. A., J. Biol. Chem., 157, 303-9 (1945).

211. Snell, E. E., and Mitchell, H. K., Proc. Natl. Acad. Sci. U.S., 27, 1-7 (1941).

212. Stokstad, E. L. R., J. Biol. Chem., 139, 475-6 (1941).

213. Stokes, J. L., J. Bact., 48, 201-9 (1944).

214. Rickers, E. L., Chaiet, L., and Keresztesy, J. C., J. Am. Chem. Soc., 69, 2749-51 (1947).

215. Hutchings, B. L., et al., Science, 99, 371 (1944).

216. Stokstad, E. L. R., and Hutchings, B. L., "Biological Symposia," Vol. 12. Jaques Cattell Press, Lancaster, Pa., 1947, pp. 339-60.

217. Day, P. L., and Totter, J. R., ibid., pp. 329-34.

218. Day, P. L., and Totter, J. R., ibid., pp. 316-24.

219. O'Dell, B. L., and Hogan, A. G., J. Biol. Chem., 149, 323-37 (1943).

220. Bird, O. D., et al., J. Biol. Chem., 159, 631-6 (1945).

221. Campbell, C. J., Brown, R. A., and Emmett, A. D., J. Biol. Chem., 152, 483-4 (1944).

222. Campbell, C. J., et al., Am. J. Physiol., 144, 348-54 (1945).

223. Day, P. L., and Totter, J. R., "Biological Symposia," Vol. 12, Jaques Cattell Press, Lancaster, Pa., 1947, pp. 327-8.

224. Eckert, H. W., J. Biol. Chem., 148, 197-204 (1943).

225. Kirch, E. R., and Bergeim, O., J. Biol. Chem., 148, 445-50 (1943).

226. Ansbacher, S., Science, 93, 164-5 (1941).

227. Briggs, G. M., et al., Proc. Soc. Exptl. Biol. Med., 52, 7-10 (1943).

228. Martin, G. J., and Ansbacher, S., J. Biol. Chem., 138, 441 (1941).

229. Martin, G. J., and Ansbacher, S., Proc. Soc. Exptl. Biol. Med., 48, 118-20 (1941).

230. Martin, G. J., Am. J. Physiol., 136, 124-7 (1942).

231. Henderson, L. M., et al., J. Nutrition, 23, 47-58 (1942).

232. Landy, M., and Dicken, D. M., J. Biol. Chem., 146, 109-114 (1942).

233. Lewis, J. C., J. Biol. Chem., 146, 441-50 (1942).

234. Thompson, R. C., Isbell, E. R., and Mitchell, H. K., J. Biol. Chem., 148, 281-7 (1943).

235. Lampen, J. O., and Peterson, W. H., J. Biol. Chem., 153, 193-202 (1944). 


\section{Chapter VA}

\section{BIOGENESIS OF THE B VITAMINS}

Some discussion of the biogenesis of the $\mathrm{B}$ vitamins in general will be included below in the section on thiamine. Otherwise we shall treat each member of the group separately and endeavor to present in the respective sections the present status of knowledge with regard to where they originate in nature and in some cases the probable raw materials. Certain details regarding the biosynthesis of the $\mathrm{B}$ vitamins which hinge on plant and animal metabolism, intestinal bacterial synthesis in relation to supplying the requirements of higher organisms, and upon inhibition studies will be presented in later chapters.

\section{Thiamine}

Several positive answers can be given to the question as to where thiamine is produced in nature. First, we may say that it is produced by numerous bacteria, presumably all those which can grow on simple media, and by many which require other vitamins. Definitive information on this matter is fragmentary. Peterson and Peterson, ${ }^{1}$ in a list of 136 organisms which have "growth factor" requirements, indicated 8 which have been found to synthesize thiamine, 12 others which do not require thiamine (and presumably synthesize it), 36 which require or at least are stimulated by it, and 80 for which no information is available. In another table, 23 organisms reported to synthesize thiamine were listed (p. 299). Of the bacteria which are stimulated by thiamine, probably a good many actually synthesize it, as has been demonstrated with certain yeasts. ${ }^{2}$ Knight ${ }^{3}$ has listed 10 bacteria which are capable of synthesizing thiamine, and Najjar and Barrett ${ }^{4}$ list 18 such bacteria. The latter list, however, includes organisms for which synthetic ability was indicated before thiamine and other members of the B family of vitamins were clearly differentiated. Thompson ${ }^{5}$ studied 5 bacteria chosen because of their diverse characteristics, all of which, however, were capable of being grown on relatively simple media, and found that they all produced substantial amounts of thiamine.

The extent to which thiamine synthesis by bacteria in the soil and in the digestive tracts of animals, particularly in the rumen of cattle, etc., figures in the economy of nature is difficult to assess (see Chapter II C), 
but the available evidence indicates that the contribution is substantial. To think of green plants as the sole substantial producers of thiamine and other B vitamins is certainly unwarranted.

Second, we can be sure that thiamine is produced in nature by yeasts and molds. Brewers' yeast was one of the earliest discovered rich sources of "vitamin B," and that the source of thiamine in yeasts is not entirely in the culture medium has been amply demonstrated., ${ }^{2,6}$ While some strains of the yeast Saccharomyces cerevisiae respond by enhanced growth to thiamine, ${ }^{7}$ many do not, and at least some of those which do respond are capable of thiamine synthesis. ${ }^{2,8}$ It is interesting that yeasts, when furnished with thiazole and pyrimidine intermediates used in the chemical manufacture of thiamine, may produce relatively large amounts of thiamine. This fact has found commercial application. ${ }^{9}$

It is presumed that thiamine synthesis takes place in many molds because culture media for them have since Raulin's time been made up from constituents of known composition excluding all vitamins. More definite evidence of synthesis has been found, for example, in the case of Aspergillus oryzae ${ }^{10}$ and Aspergillus niger. ${ }^{11}$ Schopfer and co-workers have made extensive studies which have revealed that while some fungi can synthesize thiamine using simple starting materials, others require either the thiazole or pyrimidine portions of the molecule, or both, before synthesis and growth are accomplished. ${ }^{12}$ The vitamin requirements of fungi have also been investigated to some extent by Williams and Honn ${ }^{13}$ and by Robbins and Kavanagh. ${ }^{\mathbf{1 4}}$

The importance of fungi as producers of thiamine and of other B vitamins in nature is indicated. While the number of mold organisms in the soil is small compared with bacteria, the total mold substance present is said to be larger. Furthermore, the close association of fungi with the roots of many green plants is suggestive.

The synthesis of thiamine in green plants during the course of their natural growth is undoubtedly of prime importance in connection with producing the thiamine supply of nature. This does not mean, however, that one can glibly affirm that green plants are the responsible agents in this synthesis. To demonstrate conclusively the synthetic production of thiamine by a green plant, one would have to grow the plant aseptically from seeds rendered aseptic, and show an increase in the thiamine content of the system. While this is probably possible, it has rarely, if ever, been done.

In this connection it is necessary to call attention to the most unsatisfactory state of our knowledge with respect to biochemical facts underlying the symbiosis which commonly exists between green plants and mycorhizal fungi and the bacteria of the soil. Because of this lack of 
knowledge it is impossible to interpret with any certainty the action on plant growth of animal manures and decaying organic matter-materials which always contain substantial amounts of $B$ vitamins.

Many experiments have been conducted to ascertain whether or not the $B$ vitamin content of a crop can be increased by using natural fertilizers. In general, the effect, if any, has been small. ${ }^{15}, 16,17$ In some experiments, at least, larger crop yields (which means larger total vitamin yields) have been obtained from plots treated with manure. Of course, it is unsafe to ascribe the effect of a natural manure to its vitamin content without further investigation. More often than not, investigations in this general field have been directed toward practical ends and have not been designed to answer fundamental biochemical questions.

It should be pointed out in this connection that, in higher organisms generally, amounts of thiamine beyond an organism's needs are not produced or stored in quantity, nor are they physiologically valuable or active. Without an ample supply, however, an organism cannot live. Hence, the finding that plants grown under different cultural conditions always contain about the same amount of thiamine throws more light on the question of how thiamine functions than upon how it originates.

There are several well authenticated facts which have a bearing on the problem of how thiamine is produced during the growth of green plants and the extent to which microorganisms participate. (1) Some plant roots (tomatoes) are tremendously stimulated in growth by very low concentrations of thiamine $(0.0001 \mu \mathrm{g} \text { per } \mathrm{ml} \text { or less })^{18}$ such as may occur in the soil and originate in the bacteria and molds present. (2) Mycorhizal fungi, which may be either "endotrophic" or "exotrophic," not only play an indispensable role in the life of such plants as orchids, but are likewise always associated with many common flowering plants and with many forest trees (conifers, oaks, beeches, alders, willows, poplars, etc.). In many of these latter the mycorhiza are essential to continued life, and the production of specific B vitamins such as thiamine by the mycorhizal fungi is not ruled out as an important factor in the symbiotic relationship. (3) During the early stages of growth of a seedling, especially if the seed is small and therefore contains little storage food, the seedling is likely to respond to tissue extracts and vitamin supplements by increased growth. (4) Pea seedlings grown 8 days in the dark contain one-half to one-third as much thiamine as those grown in the light, as determined by the Phycomyces test. ${ }^{19}$ (5) Certain plant roots (tomato) are capable of synthesizing the pyrimidine portion of thiamine and supplying their thiamine needs if the thiazole portion alone is supplied..$^{20}(6)$ The bacteria found in the "rhizosphere" of plants are reported to have more growth factor requirements than those more distant from the plant roots. ${ }^{21}$ 
It is elear that some of the above facts have a bearing on the problem of biosynthesis of $\mathrm{B}$ vitamins other than thiamine.

An interpretation of the various findings is in line with the idea that microorganisms-bacteria and molds-often play an important role in furnishing green plants with thiamine (and probably various other B vitamins) especially during the initial stages of growth of seedlings. Synthesis of thiamine by green plants themselves probably can take place, but in nature a complex symbiosis is the rule rather than the exception. The nutritional interrelationships between the symbiotic organisms are mostly obscure, and it would be unscientific to ascribe the effects of the symbiosis to one organism (the green plant) merely on the basis of its being more prominent visually.

Little is known about the precise mode of thiamine biosynthesis, beyond the fact that the last step involves the coupling of the pyrimidine and thiazole fragments. It has been suggested, ${ }^{22,23,24}$ however, that the thiazole fraction may be synthesized by the condensation of methionine, acetaldehyde and ammonia, since similar condensations are well known:

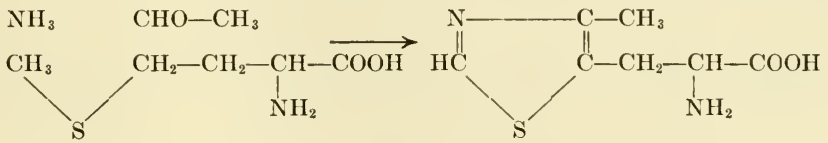

The $\alpha$-amino- $\beta$-(4-methylthiazole-5)-propionic acid so formed may then , be converted to the thiazole fraction, and it has been demonstrated that yeast cells are able to perform this latter step in a manner analogous to that in which they convert most $\alpha$-amino acids to alcohols:<smiles>Cc1ncsc1CCc1sc([C@@H](N)C(=O)O)nc1C</smiles>

Another mode of biosynthesis involving thioformamide and acetopropyl alcohol has been suggested by the work cited above.

\section{Riboflavin}

The bacterial synthesis of riboflavin has been widely observed. Among the 136 bacteria with "growth factor" requirements listed by Peterson and Peterson, ${ }^{1} 18$ synthesize riboflavin, 16 do not require it (and presumably synthesize it), while 45 require it for growth, and no information is available for 57. In another table the authors list 75 bacteria which have been found to synthesize riboflavin. Molds and fungi are also able to produce riboflavin; one fungus Eremothecium ashbyii produces enough so that it or a derivative crystallizes in the vacuoles. ${ }^{25}$ The synthesis of 
riboflavin by Aspergillus niger is more effective when the culture medium is relatively deficient in magnesium. ${ }^{26}$

The importance of bacteria and fungi as producers of riboflavin in nature cannot seriously be doubted. The demonstration is particularly clear in connection with the action of intestinal bacteria and of the organisms inhabiting the rumen of cattle and sheep. ${ }^{27}$ Milk is a highly important nutritional source of riboflavin, and it is clear that the feed which the cow consumes is not the only source of the vitamin, since the output of riboflavin in the milk may be ten times as great as the intake in the food. The riboflavin output in the milk of cows and goats is independent of the content of the feed, and the rumen content of riboflavin may be 100 times that of the feed. Intestinal synthesis of riboflavin has been demonstrated in rats, ${ }^{28}$ fowls ${ }^{29}$ and in man, ${ }^{30}$ and presumably takes place in animals generally. In ruminants the production takes place higher in the intestinal tract and the utilization is therefore expedited. Intestinal synthesis in animals and in man is not sufficient, of course, to insure against riboflavin deficiency.

The production of riboflavin during the growth of green plants is indicated by the fact that leafy vegetables are generally good food sources, but little definite information is available as to the locus of the synthesis or whether symbiotic microorganisms are important. The discussion of the general topic of $\mathrm{B}$ vitamin synthesis in the previous section on thiamine is applicable also at this point.

\section{Nicotinic Acid}

The synthesis of nicotinic acid by bacteria has been observed to be far less widespread than, for example, the synthesis of riboflavin. In the list of organisms having growth factor requirements previously mentioned, ${ }^{1}$ 48 bacteria are stimulated by or require nicotinic acid, 9 do not require it (and presumably carry out its synthesis), for 3 bacteria its synthesis has been demonstrated, and no information was available concerning 76 others. In the same review, 14 organisms which synthesize nicotinic acid are listed in another table. Here, as in other cases, some strains of a given species may have a requirement while other strains do not. Thompson's study ${ }^{5}$ showed that five diverse organisms which are capable of growing on a relatively simple medium all carry out nicotinic acid synthesis. Very little attention has been paid to the question of the production of nicotinic acid by yeasts and fungi, though unquestionably those which can grow on simple media carry out its synthesis.

While some observations have been made with respect to the stimulatory action of nicotinic acid or nicotinamide on plant roots ${ }^{31,32}$ and 
pea embryos, ${ }^{33}$ and while it is presumed that green plants synthesize nicotinic acid, little attention has been paid to the problem.

The relatively recent demonstrations that nicotinic acid is nutritionally replaced by tryptophan in higher animals (p. 279) has, of course, an important bearing on the problem of its biogenesis. While intestinal synthesis of nicotinic acid in rats has been demonstrated, ${ }^{34}$ it is not known how important such synthesis is because nicotinic acid is also reported to be formed in rat tissues. ${ }^{35}$ Human beings also excrete more nicotinic acid degradation products when tryptophan is fed ${ }^{36}$ It appears certain that the production of nicotinic acid from tryptophan in animals is important in the economy of nature, whether or not bacteria play an important role symbiotically in the transformation.

The precise mode in which nicotinic acid is synthesized is at present under intensive investigation, and our insight into this problem is hampered more by the vast amount of conflicting information than by lack of data. Present trends tend to emphasize the biosynthetic sequence starting from tryptophan and proceeding as follows:<smiles></smiles>

Tryptophan<smiles>Nc1c(O)cccc1C(=O)O</smiles>

6-Hydroxy-anthranilic acid<smiles>Nc1ccccc1C(=O)CC(N)C(=O)O</smiles>

Kynurenin<smiles>CCCCCCCCCCC(=O)O</smiles>

Nicotinic acid

This plan has much to support it; it is discussed in greater detail in the sections that follow (pp. 279, 353). It is, however, well known that both ornithine and proline are involved in niacin biosynthesis, and these facts are completely neglected in the above scheme. Proline, ornithine and glutamic acid have all been shown to be effective in increasing the production of trigonellin by rice plants.<smiles>C[n+]1cccc(C(=O)Oc2ccccc2)c1</smiles>

Trigonellin 
Indeed, Bovarinck ${ }^{37}$ has shown that if glutamic acid and asparagin are boiled in aerated water containing a trace of manganous sulfate, the solution will manifest niacin activity. Niacin synthesis by rats is stimulated by a mixture of glycine and $d l-\delta$-amino- $n$-valeric acid. It is also known that guvacine will substitute for nicotinic acid in the nutrition of Staphylococcus aureus and Proteus vulgaris. A consideration of these facts has led to the following proposed biosynthetic sequence:

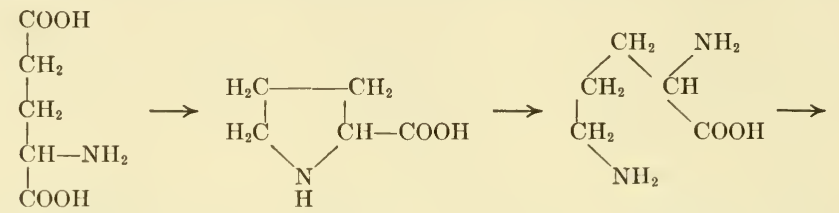

Glutamic acid -Proline Ornithine<smiles>NCCCCC(=O)O</smiles>

$\delta$-Amino-n-valeric acid

Guvacine

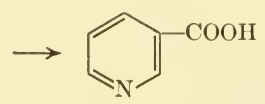

Nicotinic acid

\section{Pantothenic Acid}

Pantothenic acid is required by, or is at least stimulative toward, a relatively large proportion of the bacteria which have been tested. Thus, Peterson and Peterson ${ }^{1}$ list 52 bacteria having growth factor requirements which respond to pantothenic acid specifically, 2 which synthesize it and 3 additional ones which do not require it. In another table they list a total of nine bacteria which are reported to synthesize it. These include the 5 organisms tested by Thompson, ${ }^{5}$ all of which grow on relatively simple media and produce other B vitamins, and Rhizobium melilote ${ }^{38}$ which grows in the root nodules of legumes.

Despite the relatively small number of bacteria known to synthesize pantothenic acid, there is good evidence on which to base the opinion that bacterial synthesis is an important natural source. Its production has been demonstrated to take place, for example, in the rumen of sheep and 
cattle. ${ }^{27,}{ }^{39}$ Milk is reported to contain twice as much pantothenic acid as the total dietary intake of the cow, but the assays on which such reports are based are not entirely reliable due to incomplete extraction (p. 34). Synthesis also takes place in rats, particularly in the cecum. It has been estimated on the basis of balance studies that 10 to 60 per cent of the requirement of the rat may be furnished in this way. ${ }^{35}$ The amount produced is dependent upon the type of diet and the intestinal flora which is favored. Certain strains of $C$. diphtheriae, like yeasts, are able to produce pantothenic acid when $\beta$-alanine alone is supplied, indicating that they possess the metabolic machinery for synthesizing pantoic acid but not for producing $\beta$-alanine. ${ }^{40}$ Acetobacter suboxidans, on the other hand, is stimulated by the pantoic acid portion of the pantothenic acid molecule and evidently possesses the ability to synthesize $\beta$-alanine but not pantoic acid. ${ }^{41}$ Present evidence seems to indicate that the biosynthesis of pantothenic acid occurs through the direct coupling of $\beta$-alanine and pantoic acid. A considerable amount of evidence (p. 465) indicates that the $\beta$-alanine is formed by the decarboxylation of aspartic acid, while the pantoic acid has been suggested as arising from an amino acid, pantonine (p. 289), thus:<smiles>NC(C(=O)O)C(=O)O</smiles>

Aspartic Acid<smiles>CC(C)(CO)C(N)C(=O)O</smiles>

"Pantonine"

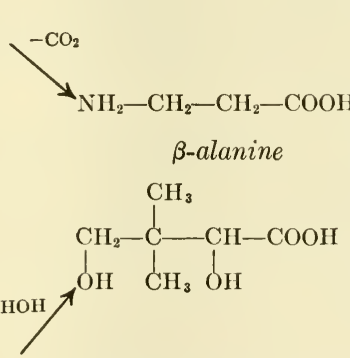

Pantoic acid

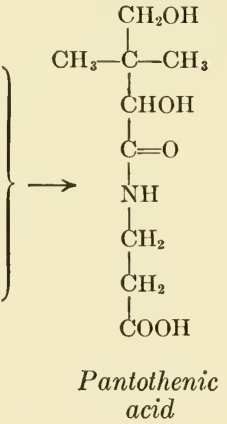

Further discussion bearing on the mechanism of the biosynthesis of pantothenic acid will be given in a later section (p. 464).

The production of pantothenic acid by common strains of bakers' yeast, in the absence of $\beta$-alanine, is certainly not important, but other yeasts and fungi which are able to grow on simple media undoubtedly produce the whole molecule. No worthwhile information regarding the importance of fungi as producers of pantothenic acid in the soil or elsewhere appears to be available. 
The relation of pantothenic acid to the growth of green plants has been studied.38 Aseptically grown alfalfa seedlings were found to respond to pantothenic acid by increased growth, but when the plants had developed for several days, they were assayed and found to contain much more pantothenic acid than could be accounted for other than by synthesis. These experiments, carried out in the author's laboratory, were performed at a time when minute amounts of nearly pure pantothenic acid were available, and there is no reason to doubt the substantial biological purity of the preparations used. The only criticism of the demonstration of pantothenic acid synthesis by green plants was the failure to show at the end of the experiment that molds and other bacteria were entirely absent from the sand cultures. While there was no macroscopic evidence of mold or bacterial growth, the resistance of some spores to autoclaving may introduce a small uncertainty.

\section{Pyridoxal, Pyridoxamine, Pyridoxine}

Especially because of relatively recent clarification with respect to the chemical nature of the " $\mathrm{B}_{6}$ group" of vitamins, the available information regarding their biosynthesis is relatively unsatisfactory. Studies involving "vitamin $\mathrm{B}_{6}$ " have revealed that of the approximately 40 bacteria about which information is available, about one-half are stimulated by or require it, and the other half either are known to synthesize it or have been shown at least not to require it for growth. The five organisms studied by Thompson ${ }^{5}$ all produced it.

Just how the results of such studies would appear if all three forms of the vitamin were taken into account is not entirely clear. It is certain, however, that the three forms are not always interchangeable in nature, since some organisms are unable to convert pyridoxine into the active pyridoxal form, ${ }^{42}$ and the form which predominantly exists differs from source to source. Pyridoxine appears to be a relatively inactive storage form which occurs predominantly in metabolically inactive seeds (p. 36).

Yeasts, molds and fungi which are able to grow on simple media produce at least one form of the vitamin because assays by a yeast method, which responds to all forms, indicates the universal presence of these, as well as the other B vitamins (p. 60). By x-ray induction a mutant strain of Neurospora crassa was produced early in the investigations of Beadle and Tatum ${ }^{43}$ which is unable to grow unless pyridoxine, pyridoxamine or pyridoxal is added to the medium. For this organism the three forms are interchangeable, and the x-ray has destroyed a gene which is essential for the building of any of the forms.

The same uncertainty exists with respect to the mode of production of the " $\mathrm{B}_{6}$ group" during the growth of green plants as exists in connec- 
tion with the other $\mathrm{B}$ vitamins. Tomato roots, for example, are stimulated in growth by pyridoxine, ${ }^{44}$ and in this respect pyridoxine shows a resemblance to some of the other $B$ vitamins.

From recent work it appears that alanine is not, as formerly supposed, ${ }^{45}$ a direct precursor of vitamin $B_{6}$ and that $D$-alanine, which is reported to be a consistent cell eonstituent for several organisms, can replaee $\mathrm{B}_{6}$ in culture media without giving rise to additional $\mathrm{B}_{6}{ }^{45 a}$

\section{Biotin}

The bacterial synthesis of biotin undoubtedly constitutes an important natural source. While biotin defieiency has often been indueed in animals it is usually by introdueing egg white or avidin into the diet, or administering sulfa drugs, or eliminating bacteria from the intestinal tract ${ }^{44}$ that it is accomplished (see p. 428). Usually feeding a biotindefieient diet is itself ineffeetive, though not necessarily so with baby chicks. Peterson and Peterson ${ }^{1}$ list 21 bacteria which produce biotin, among them several common intestinal bacteria. The synthesis of biotin is not limited to the bacteria which fail to respond to it; thus Rhizobia which are stimulated by biotin (eonzyme $R$ ) ean be cultured under conditions in which preformed biotin is excluded. ${ }^{46}$ As Knight ${ }^{47}$ has aptly pointed out, very limited growth of a microorganism may oceur in a biotin-deficient medium even though the organism is able to synthesize biotin. The rate of growth may under these eonditions be limited by the rate of synthesis, in which case the addition of biotin to the culture medium may greatly accelerate growth.

The synthesis of biotin by bacteria is attended often by a large amount of release into the culture medium. In four organisms studied by Thompson ${ }^{5}$ the amount of biotin found in the medium averaged about 8 times that in the bacterial cells. This release into the medium is not due to the autolysis of dead cells as it oceurs progressively in a rapidly growing culture.

The bacterial production of biotin in the rumen of cattle has been demonstrated, ${ }^{48,49}$ and rats on a biotin-deficient ration excrete much more biotin than they take in, ${ }^{50}$ as do also human beings. ${ }^{51}$ The presence of relatively large amounts of biotin in bacterial eulture media which have aceidentally been contaminated by organisms from the air bespeaks the ubiquity of organisms capable of producing this vitamin.

Yeasts and lower fungi as well as bacteria produce biotin. While many yeasts are stimulated by biotin they often are capable of its synthesis. ${ }^{2,} 8$

Knight ${ }^{52}$ lists 12 lower fungi, ineluding Aspergilli and Penicillia, which do not require biotin, and it is relatively safe to conclude that they synthesize it. One notable genus of molds that requires biotin, however, 
is the Neurospora, which requires this single vitamin and no other. The wild strains require biotin; they lack the enzyme(s) necessary for its synthesis. The mutant strains in addition lack other specific enzymes due to the destruction of specific individual genes.

Of the work relating biotin to the activities of green plants, one of the most significant bits is the demonstration that it acts as a growth substance in stimulating root production on etiolated cuttings of pea roots. ${ }^{53}$ Stimulative effects of this sort suggest that biotin may not be produced by green plants, or at least that the synthesis takes place in leaves rather than roots, and that the roots may depend for at least part of their supply on soil microorganisms. The stimulative effect of biotin on Rhizobia suggests that the green plants may furnish biotin (synthesis taking place probably in leaves) to the microorganism as an important factor in the symbiotic relationship. The ability of some of these organisms to synthesize biotin at a slow rate has already been noted. No specific information appears to be available regarding the relationship of biotin to mycorhizal growth. In general, we may say that there appears to be no other B vitamin for which production by microorganisms is as important as it is in the case of biotin.

Eakin and Eakin ${ }^{54}$ have shown that pimelic acid stimulates the synthesis of biotin by Aspergillus niger, and that this stimulation is further enhanced by the presence of cysteine or cystine. Pimelic acid and biotin have been found to be interchangeable as growth stimulants for some organisms, so that it appears likely that pimelic acid is a precursor of biotin. There is some evidence to suggest that the synthesis proceeds through desthiobiotin as a precursor (p. 468).

\section{Folic Acid, Inositol, Choline, p-Aminobenzoic Acid, "Vitamin $\mathbf{B}_{12}$ "}

With respect to the biogenesis of other members of the $B$ vitamin family, hardly enough information is available to warrant more than a very brief discussion.

Folic acid is required by a considerable number of microorganisms, but on the basis of available information it is produced by all those bacteria which can grow on simple chemically defined media. ${ }^{5}$ Its production in the intestinal tracts of rats has been studied, ${ }^{34}$ and numerous demonstrations of deficiency have involved the prevention of intestinal synthesis by sulfa drugs. Quantitative information in this field is relatively unsatisfactory because of the difficulties of complete release of the vitamin from its combined forms for assay (p. 40). The fact that deep green leaves are an unusually rich source of folic acid, ${ }^{55}$ as well as other facts regarding its distribution, strongly suggest that this vitamin is formed in green plants. 
Few studies have dealt with bacterial growth in relation to inositol synthesis. In the previously mentioned study of Thompson, ${ }^{5}$ its production by five diverse bacteria was demonstrated. One study indicated that it is not produced to a substantial degree by intestinal organisms in rats. ${ }^{34}$ The relative abundance of inositol in plant materials ${ }^{56,57}$ suggests that it is probably synthesized by plants. It is stimulatory to certain fungi, but in view of the ability of many of these organisms to grow on simple media, it is presumably synthesized by them.

Choline is probably widely synthesized in nature. Few bacteria have been found to require it, ${ }^{58}$ and wild strains of Neurospora evidently synthesize it. ${ }^{59}$ In animals, as in Type III Pneumococci, ${ }^{58}$ methionine and other compounds containing available methyl groups serve as precursors. Ethanolamine is probably important as a precursor in the biosynthesis of choline (p. 353). Choline biosynthesis has been extensively studied and it is now felt that the exact sequence involved is well known. In brief, it may be indicated thus:

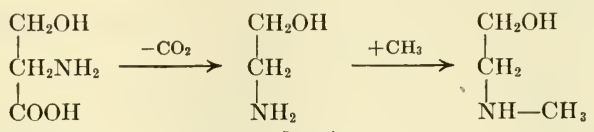

Serine Ethanolamine

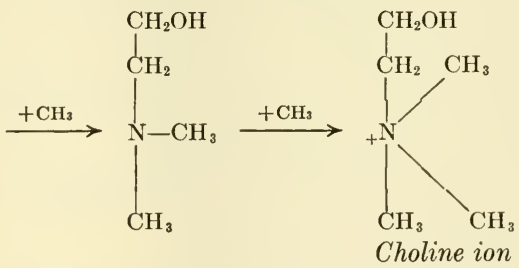

Certain aspects of this sequence of reactions are considered in greater detail in a later section (p. 353).

$p$-Aminobenzoic acid, like choline, is probably produced widely in nature. Although a considerable number of bacteria are stimulated by its presence in the culture medium, Peterson and Peterson ${ }^{1}$ list 13 bacteria which have been found to synthesize it. Wild strains of Neurospora synthesize this substance as do yeasts. Presumably green plants do as it enters into the make-up of folic acid, but no direct evidence on this point has been found. 


\section{Bibliography}

1. Peterson, W. H., and Peterson, M. S., Bact. Rev., 9, 49-109 (1945).

2. Williams, R. J., Eakin, R. E., and Snell, E. E., J. Am. Chem. Soc., 62, 1204-7 (1940).

3. Knight, B. C. J. G., "Vitamins and Hormones," Vol. III, Academic Press, Inc., New York, N. Y., 1945, pp. 105-228b.

4. Najjar, V. A., and Barrett, R., "Vitamins and Hormones," Vol. III, Academic Press, Inc., New York, N. Y., 1945, pp. 27-8.

5. Thompson, R. C., Univ. Texas Pub., 4237, 87-96 (1942).

6. Livshits, M. I., Proc. Sci. Inst. Vitamin Research, U.S.S.R., 3, No. 1, 84-8 (1941); C.A., 36, 3219 (1942).

7. Williams, R. J., and Roehm, R. R., J. Biol. Chem., 87, 581-90 (1930).

8. Leonian, L. H., and Lilly, V. G., Science, 95, 658 (1942).

9. Standard Brands, Inc., Brit. Patent 532,013, Jan. 15, 1941.

10. Scheunert, A., and Schieblich, M., Biochem. Z., 286, 66-71 (1936).

11. Fawns, H. T., and Jung, A., Biochem. J., 27, 918-33 (1933).

12. Schopfer, W. H., "Plants and Vitamins," Chronica Botanica Co., Waltham, Mass., 1943, pp. 101-29.

13. Williams, R. J., and Honn, J. M., Plant Physiol., 4, 629-41 (1932).

14. Robbins, W. J., and Kavanagh, V., Botan. Rev., 8, 411-71 (1942).

15. Scheunert, A., Ernährung, 3, 67-9 (1938).

16. Rowlands, M. J., and Wilkerson, B., Biochem. J., 24, 199-204 (1930).

17. Harris, L. J., J. Agri. Sci., 24, 410-5 (1934).

18. Robbins, W. J., and Bartley, M. A., Science, 85, 246 (1937).

19. Bonner, J., and Green, J., Botan. Gaz., 100, 226 (1934).

20. Robbins, W. J., and Bartley, M. A., Proc. Natl. Acad. Sci. U.S., 23, 358-8 (1937).

21. West, P. M., and Lochhead, A. G., Can. J. Research, 18, C, 129-35 (1940).

22. Harington, C. R., and Moggridge, R. C. G., Biochem. J., 34, 685-9 (1940).

23. Harington, C. R., and Moggridge, R. C. G., J. Chem. Soc., (1939) 443-6.

24. Bonner, J., and Buchman, E. R., Proc. Natl. Acad. Sci. U.S., 24, 431-8 (1938).

25. Guillermond, A., Rev. de Mycol., 1, 115 (1937).

26. Lavollay, J., and Laborey, F., Compt. rend., 206, 1055-6 (1938).

27. MeElroy, L. W., and Goss, H., J. Nutrition, 20, 527-40 (1940).

28. Griffith, W. H., J. Nutrition, 10, 667-74 (1935).

29. Lamoureux, W. F., and Schumacher, R. S., Poultry Sci., 19, 418 (1940).

30. Najjar, V. A., et al., J. Am. Med. Assoc., 126, 357 (1944).

31. Bonner, J., Am. J. Botany, 27, 692 (1940).

32. White, P. R., Am. J. Botany, 27, 811 (1940).

33. Bonner, J., Plant Physiol., 13, 865 (1938).

34. Mitchell, H. K., and Isbell, E. R., Univ. Texas Pub., 423,7, 125-34 (1942).

35. Dann, WV. J., J. Biol. Chem., 141, 803 (1941).

36. Sarett, H. P., and Goldsmith, G. A., J. Biol. Chem., 167, 293 (1947).

37. Bovarnick, M. R., J. Biol. Chem., 151, 467-75 (1943).

38. McBurney, C. H., Bollen, W. B., and Williams, R. J., Proc. Natl. Acad. Sci. U.S., 21, 301-4 (1935); also unpublished observations by the same authors.

39. McElroy, L. W., and Goss, H., J. Biol. Chem., 130, 437-8 (1939).

40. Mueller, J. H., and Kilotz, A. WV., J. Am. Chem. Soc., 60, 3086-7 (1938).

41. Underkofler, L. A., Bantz, A. C., and Peterson, WV. H., J. Bact., 45, 183-90 (1943).

42. Snell, E. E., and Rannefeld, A. N., J. Biol. Chem., 157, 475-89 (1945).

43. Beadle, G. W., and Tatum, E. L., Proc. Natl. Acad. Sci. U.S., 27, 499-506 (1941).

44. Robbins, W. J., and Schmidt, M. B., Proc. Natl. Acad. Sci. U.S., 25, 1-3 (1939).

45. Snell, E. E., and Guirard, B. M., Proc. Natl. Acad. Sci. U.S., 29, 66-73 (1943).

45a. Holden, J. T., and Snell, E. E., J. Biol. Chem., 178, 799-809 (1949).

46. Wilson, J. B., and Wilson, P. W., J. Bact., 43, 329 (1942). 
47. Knight, B. C. J. G., "Vitamins and Hormones," Vol. III, Academic Press, Inc., New York, N. Y., 1945, p. 162.

48. McElroy, L. W., and Jukes, T. H., Proc. Soc. Exptl. Biol. Med., 45, 296-7 (1940).

49. Wegner, M. I., el al., Proc. Soc. Expll. Biol. Med., 45, 769-71 (1940)

50. Nielsen, E., et al., J. Nutrition, 24, 523-33 (1942).

51. Oppel, T. W., J. Clin. Invest., 21, 630 (1942).

52. Knight, B. C. J. G., "Vitamins and Hormones," Vol. III, Academic Press, Inc., New York, N. Y., 1945, p. 167.

53. Went, F. W., and Thimann, K. V., "Phytohormones," Macmillan Co., New York, N. Y., 1937, 294 pp.

54. Eakin, R. E., and Eakin, E. A., Science, 96, 187-8 (1942).

55. Olson, O. E., Burris, R. H., and Elvehjem, C. A., J. Am. Dielet. Assoc., 23, 200-3 (1947).

56. Woods, A. M., et al., Univ. Texas Pub., 4237, 84-6 (1942).

57. Cheldelin, V. H., and Williams, R. J., Univ. Texas Pub., 4237, 105-25 (1942).

58. Badger, E., J. Biol. Chem., 153, 183-91 (1944).

59. Horowitz, N. H., and Beadle, G. W., J. Biol. Chem., 150, 325-33 (1943).

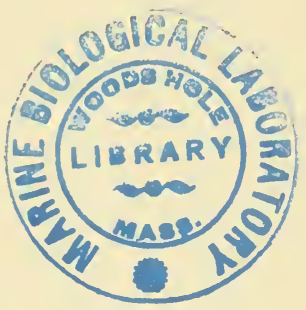



Section B

\title{
THE CATALYTIC FUNCTIONS OF THE
}

B VITAMINS

\author{
Robert E. Eakin
}





\section{Chapter IB}

\section{BIOCHEMICAL REACTIONS AND THEIR CATALYSTS}

When a mammal or some lower form of life is deprived of an adequate amount of one of the $B$ vitamins it requires, serious changes in the metabolism of the organism occur.* The deviations from the normal processes induced by the deficiency are reflected in a variety of physiological effects, many of which can be readily detected. In general, however, the different changes which are observed in even a simple deficiency of a single vitamin are so diverse in character that it is impossible to relate the symptoms physiologically or to establish any connection between the chemical structure of the vitamin and its physiological effects. An excellent illustration of the complex nature of vitamin function is the variation in the ways by which a deficiency manifests itself, not only in different types of life but even among members of the same species (see Chapter VI C). Before any plausible explanations for these diverse physiological and clinical effects of the vitamins can be deduced, it is necessary to explore much deeper and uncover the specific chemical or physical reactions in which they are participating.

The elucidation of the specific manner in which a vitamin performs its duties in a biological system usually does not arouse the popular interest that attends the discovery of a vitamin or the accomplishment of its synthesis. Nevertheless, for the medical sciences, understanding exactly how these compounds work, and why, is just as important as knowing what they are. Until the specific chemical functions of a vitamin are determined, knowledge about the vitamin is indeed incomplete.

The purpose of this section, then, will be to review those investigations which have been reported which we feel to be most pertinent in answering the question, "Into what specific reactions does each B vitamin enter?" and to correlate these chemical reactions with the observed effects of the vitamins upon the metabolism of cells and tissues.

During the early investigations on essential dietary factors, it was only natural that relatively little experimental work was done on the bio-

* A number of topics which will be referred to from a general viewpoint in this chapter are discussed in more detail in other parts of the book where they are documented with references to the original publications. The citation of references in this chapter will be limited for the most part to material not elaborated upon more thoroughly elsewhere. 
chemical mechanisms in which they functioned. The goal of the pioneers in the field of vitamin research was to determine the chemical structure of the vitamins so that their syntheses could be effected and preparations made available for the treatment of nutritional deficiency diseases. Also, it was impossible to attack successfully the problem of the mechanisms of vitamin action prior to the time that the multiple nature of vitamin $B$ had been clarified, and before potent concentrates of the individual vitamins free from other substances having biological activity became generally available.

Many early investigators of vitamins, particularly those having biochemical training and viewpoints, however, recognized that the vitamins must act as "catalysts," since only minute amounts were needed to produce such profound changes in biological systems; but the nature of the "catalytic activity," of course, at that time could not be explained.

Fortunately, the study of enzymes and enzyme activity was proceeding simultaneously with the development of the nutritional sciences. It is interesting to note that 1926 was an epochal year in both fields, for it marked the isolation for the first time of a vitamin in crystalline form (thiamine from rice bran) ${ }^{1}$ and the first isolation of an enzyme (crystalline urease from jack bean meal). ${ }^{2}$ These two accomplishments offered irrefutable evidence that the activity of both vitamins and enzymes could, at least in some cases, be attributed to specific chemical compounds. Previous to this date some scientists had speculated on the relationship between enzymes and ritamins, and had pointed out that one of the most likely means by which the vitamins could exert their "catalytic activity" was to participate in some way in enzymatic reactions. It is probably difficult for the younger scientists of today, who have always thought of the vitamins and enzymes as specific chemical entities and who in their training have always associated vitamin activity with enzyme action, to realize that twenty-five years ago explaining a vitamin function in terms of enzymes was only a conjecture, and that even fifteen years ago the hypothesis that a $\mathrm{B}$ vitamin functioned directly in a biochemical reaction had yet to be demonstrated.

The actual proof that a B vitamin is an integral part of an enzymatic reaction was not made until 1935, when a component of the "yellow enzyme" was identified as riboflavin (a substance whose status as a vitamin at that time was debatable). ${ }^{3}$ Two years later an essential part of the enzyme system required for the biological decarboxylation of pyruvic acid was shown to be a derivative of thiamine. ${ }^{4}$ By 1935 nicotinamide had been identified as a part of molecules essential for reactions taking place when glucose is utilized by erythrocytes and yeast, ${ }^{5}$ 
but two years elapsed before the vitamin activity of nicotinamide (or the corresponding acid) was established. ${ }^{6}$ It is interesting to note that, whereas in 1937 a two-year interval had separated the identification of an enzyme component and its trial in the treatment of the most prevalent nutritional disease of this country, today the time that would elapse between discovery of a new organic molecule essential for some important enzymatic reaction and its trial in many diseases would be at most a matter of weeks - an excellent illustration of the change that has taken place in the last decade in the general acceptance of the enzymevitamin relationship.

A related concept which was early appreciated by some scientists working with lower types of life, but which was long neglected by most workers in the field of mammalian nutrition and physiology, was that of the universal occurrence of the $B$ vitamins and their importance to all forms of life. The significance of this fact and its effect upon vitamin research was pointed out in the previous section. Brilliant investigations early in this century demonstrated that in their metabolism of carbohydrates both mammalian tissues and unicellular organisms (yeast) utilize almost identical series of enzymatic reactions. Had the intimate relationship of nutritional factors and enzyme systems been more fully appreciated, there would have been earlier recognition by all biochemists of the similarity of the nutritional requirements of lower forms of life and those of mammals.

It was in the period immediately following the establishment of the vitamin-enzyme relationship of riboflavin, thiamine, and nicotinic acid that the complex tangle of the other $B$ vitamins was unsnarled; the biological activities of countless factors which had been reported during the previous twenty years were resolved into pantothenic acid, pyridoxine, biotin, $p$-aminobenzoic acid, inositol, and folic acid, or combinations and derivatives of these substances. Since the three members of the complex originally recognized were known to function as parts of enzyme systems, considerable effort in a number of laboratories was directed toward demonstrating that substances more recently established as B vitamins were also involved in some type of enzyme reactions as yet uncharacterized, or that these newer vitamins were present in significant amounts in the purified preparations of known enzymes. Although a number of suggestive leads were obtained, it was impossible as late as 1943 to ascribe any definite enzymatic functions to any of these "newer" B vitamins. The failure to pin these substances down to specific enzymatic functions led to some speculation that it might be found that not every B vitamin would exhibit its "catalytic activity" by being an integral part 
of an enzyme system. ${ }^{7}$ Some weight was given to such interpretations, since at that time it appeared that there might be almost no end to the list of growth-promoting substances that would eventually be found; also, none of the numerous plant and animal hormones had been implicated in specific enzyme reactions.

However, in the five-year period 1943 to 1948, pantothenic acid, pyridoxal and inositol were found to be parts of definite enzyme systems involved in fat, protein, and carbohydrate metabolism; and folic acid, $p$-aminobenzoic acid, biotin, and a new member of the group which was isolated during this period, vitamin $\mathrm{B}_{12}$, were each shown to function in one or more fundamental enzymatic reactions which, at the time of this writing, are in the process of being more fully characterized. During this same interval it also became increasingly apparent that the number of unknown B vitamins might not be as great as previously assumed, and that many of the remaining uncharacterized factors of general biological significance which had been reported would be found to be mixtures or derivatives of compounds already known.

Later in this chapter (p. 104) an outline will be given of the fundamental types of enzymatic reactions which have been found to constitute the chemical routes over which the basic processes in carbohydrate, lipide, and nitrogen metabolism must proceed. A number of these enzyme systems contain as an indispensable part one of the B vitamins. Of greater interest is the fact that, except for inositol, each one of the B vitamins is always required in at least one group of these essential reactions, thus establishing a definite association between every typical B vitamin and the processes which form the foundation upon which life is built. If any one of these types of reactions is lacking, a series of gaps results which cannot be effectively bridged or by-passed, and the procession of reactions necessary for the maintenance of cell activity must cease. ${ }^{*}$ It is for this reason that these vitamins occur universally and are a prerequisite for life.

From the standpoint of organic evolution the thesis that there exist throughout the entire plant and animal kingdoms a certain number of essential and fundamental types of enzymatic processes common to all organisms seems not only reasonable but almost necessary. An appreciation of this situation has been of invaluable help to those investigators interested in studying the details of metabolism, for it means that they can choose as their biological tool any organism or species with which

* See pp. 174, 187, and 202 for a discussion concerning possible exceptions-the interesting cases where the reactions catalyzed by biotin, pyridoxal, and folic acid may be by-passed in certain bacteria. 
they can conveniently work with reasonable assurance that the information so obtained will be of general biochemical interest and will be applicable, with limitations, to other forms of life. As a matter of fact, the outline of the basic reactions mentioned above was assembled from information obtained from studying organisms at the extremes on the scale of biological development.

Now that it is definite that each of the B vitamins can be associated with an enzymatic reaction, a pertinent question that still must be considered is: What clear-cut evidence is there that any of the B-vitamins have fundamental roles which cannot be associated with the enzyme systems in which they are known to participate? Most of the clinical symptoms of deficiencies, as well as a number of miscellaneous observations that have been reported, have not as yet been correlated with the enzymatic reactions in which the vitamins function; but there is no clearly defined positive evidence that any of the typical B vitamins has any indispensable roles other than those associated with the specific type reactions for which it is required. In view of this it seems reasonable to offer as a general hypothesis that the only function of the B vitamins per se is to participate directly in certain specific enzymatic reactions.

In addition to these basic reactions essential for every cell, there are numerous "specialized" biochemical reactions which are not observed in all forms of life, but which are characteristic of particular types of organisms or tissues. These reactions are not necessarily needed by the individual cells themselves, but they are essential if the complex organization of life is to be maintained. Examples of such reactions are photosynthesis in green plants, nitrogen fixation in certain types of bacteria, hormone production by glandular tissue, the reactions involved in transmission of impulses in nerve tissue, etc. Do the $\mathrm{B}$ vitamins function in such reactions? Information that has been obtained on this point seems scant, especially when one considers the importance of many of these phenomena and the amount of investigation they have received. It would seem only natural that in the evolutionary development of the "specialized functions" the organism would utilize, wherever possible, the catalysts already present and functioning in the basic metabolism of the cell. The little information available on these functions of the vitamins is summarized in a later chapter.

The following sections of this chapter will be devoted to a general discussion of the fundamental enzyme reactions.

\section{Enzymatic Reactions}

Any discussion attempting to explain in detail the mechanisms by which B vitamins catalyze chemical reactions would necessarily become a 
treatise on enzyme chemistry and hence cannot be undertaken here. However, before going into the detailed account of the specific reactions in which the vitamins are involved, it seems advantageous to discuss a few topics about the general processes and mechanisms of enzyme reactions which have particular bearing upon points to be treated individually in the chapters which follow.

A biochemical reaction, like any chemical reaction, involves changes in the chemical constitution of one or more molecules with an accompanying transformation of energy. These two changes - the material and the energy - take place simultaneously and cannot be divorced from each other. There has been an unwarranted tendency on the part of some workers in the biological sciences to separate biochemical reactions (including those mediated by derivatives of $\mathrm{B}$ vitamins) into energyproducing reactions and reactions utilized for the synthesis of cellular components. Such an idea is conveyed in the categorical statements often made to the effect that "carbohydrates and fats are used for the storage and production of energy while proteins are used for building cell structure." In the chemical reactions by which energy is obtained from carbohydrates and lipides, cells are at the same time forming, in addition to "waste" or excretory products, a large number of compounds which are either incorporated directly into the structure of cells or are converted into other compounds which are essential units of cellular constituents. On the other hand, the energy liberated by the chemical changes occurring during protein metabolism is utilized in a manner identical to that energy made available during carbohydrate and lipide metabolism.

Before an enzyme reaction can be considered as well characterized, at least three things must be known:

(a) the net energy transformations taking place,

(b) the exact chemical changes occurring (i.e., the specific reactants and products), and

(c) the components of the catalyst mediating the reaction. These will be considered in turn.

Energy Transformations in Biological Systems. The role of the biological catalysts involved in producing energy transformations can be better appreciated after comparing the conditions involved in cellular reactions with those employed to effect comparable changes without the aid of enzymes. In nonbiological systems, the production of utilizable energy by the oxidation or degradation of organic compounds (wood, petroleum, alcohol, etc.) almost always involves some kind of combustive process wherein the chemical energy of the organic compounds is first converted into heat with an attending production of high temperatures. 
This thermal energy is then used for work or chemical syntheses by transforming it into mechanical, electrical, or chemical energy. This transformation of heat, a "degraded" form of energy, is always inefficient from the standpoint of thermodynamics, and involves other wasteful losses due to practical difficulties. In cells, where only small changes in temperature can be tolerated, heat cannot generally be used to transfer the energy derived from one metabolic reaction to another process in which it will be utilized for locomotion, establishment of electrical potentials, absorption against osmotic pressure, or chemical synthesis. Energy-producing processes must be carried out in a carefully controlled, stepwise manner to prevent appreciable rises in temperature and to permit the liberated energy to be stored and utilized as needed.

It has been found that a relatively simple device, though somewhat unique from the standpoint of classical physics and chemistry, is employed in most if not all living systems-that of conserving the chemical energy arising from the degradation and oxidation of organic compounds (and perhaps from other sources available to some organisms) by converting it into another type of chemical energy, popularly termed "highenergy phosphate bonds." This conversion may be more accurately described by saying that the energy-producing metabolic reactions result in the formation of acid anhydrides of phosphoric acid, compounds which are most versatile in their reactions and from which the chemical energy inherent in the acid anhydride linkages can be readily utilized by biological systems.

Although the B vitamins themselves are not the substances which act as transporting agents for these high-energy phosphate units, they are usually involved in the reactions by which these agents are formed and often in subsequent processes wherein they are utilized. Quantitative values for the amount of free energy (the energy available for useful work) liberated or absorbed during a reaction $(\Delta F)$ enable one to predict which metabolic reactions can be used to create the high-energy units and which processes will necessarily require expenditure of some of the cell's reserve of these energy units. This same thermodynamic information also enables one to calculate the relative concentrations of the reactants and products of a reaction at equilibrium. From this it is possible to determine the direction in which an enzymatic reaction will proceed under any given set of conditions.

The problem of the "reversibility of a reaction" involves the question whether a reaction is theoretically capable of proceeding in either direction. Reversibility implies that a reaction and the reverse transformation are taking place simultaneously, although the conditions may be such as 
to favor a net change, which is the forward reaction. As a corollary, there should be some critical set of conditions for a reaction in which there is no net change, in which case the system will be at equilibrium.

In the past the reversibility of many enzymatic reactions has been frequently questioned, since attempts to demonstrate enzymatic synthesis, as opposed to hydrolysis and other forms of degradation, in a direct manner have in some cases proved wholly unsuccessful. Even if a catalyst is present in excess it may be impossible to achieve the concentrations of products required to produce a measurable resynthesis of the original substances. Today, with isotopes available, it is easy to demonstrate that in uncomplicated enzymatic reactions the products are in dynamic equilibrium with the reactants, even though it is sometimes impossible to achieve the conditions necessary to completely effect the reverse reaction. This can best be shown by labelling one of the products with an isotopic atom, adding it to an enzyme system during the course of a reaction, and stopping the reaction while there is still some of the reactant left. If the reactant contains some of the isotopic element added in the product, then it is obvious that the "backward reaction" has been taking place.

Consequently, today the question should not be, "Is this enzyme reaction reversible?" but, "Do the conditions required for reversing this reaction occur or can they be achieved?" To answer the latter question it is necessary to know the relative concentration of reactants and products when they are at equilibrium. At equilibrium a reaction can be forced to go in either direction by making only slight changes in the concentration of one of the participating substances. In cells a number of reactions mediated by enzyme systems, including many of these containing $\mathrm{B}$ vitamins, are at equilibrium most of the time. The direction in which these reactions proceed may be constantly alternating because of the slight changes produced by other reactions in cells. In the reaction, glycogen $+\mathrm{H}_{3} \mathrm{PO}_{4} \rightleftarrows$ glucose-1-phosphate, only slight changes in the intracellular concentration of inorganic phosphate are needed to stop the process in which glycogen is utilized and to initiate its synthesis. This is an example of one of the important methods by which metabolic processes are regulated. It is also possible to find reactions at the other extremes-hydrolytic reactions and some decarboxylations are good examples. Here, even though the catalyst is present, the ratio of the concentrations of products to reactants at equilibrium is so large that it is impossible to achieve the concentration of the products needed to reverse the reaction effectively.

A knowledge of relative concentrations at equilibrium is not only of value in understanding or predicting the mechanisms and chemical routes 
of metabolic processes, but it also enables the investigator who is studying an isolated system to have some idea of the concentration conditions that he must use to demonstrate the activity of an enzyme preparation.

Exact statements of the energy relationships and equilibrium conditions involve the use of thermodynamic concepts and equations. However, without these it can be stated as an approximation that when the amount of free energy liberated by a reaction is comparatively small, then the equilibrium concentrations of the reactants and products can usually be obtained, such that it is possible for an organism to make use of either the forward or the reverse reaction. On the other hand, if an enzymatic reaction liberates a large amount of energy, it would probably be difficult to establish the concentrations needed to reverse the reaction. If this reverse process is needed for synthetic purposes, some indirect route in which high-energy units are utilized will have to be employed (pp. 218 and 235).

Types of Biochemical Reactions. Next to be considered are the fundamental chemical reactions required for the synthesis and interconversion of constituents essential for cell structure and function. These processes involve such diverse reactions as the formation and hydrolysis of peptide, ester, and glucosidic linkages; the oxidation of alcohols, aldehydes, and amines; the reduction of acids and aldehydes; the hydrogenation and dehydrogenation of hydrocarbon chains; the formation and cleavage of carbon-to-carbon bonds; and the synthesis and degradation of heterocyclic compounds. The demands presented by the variety and complexity of these reactions upon the synthetic abilities of the cell are equalled by the drastic limitations put upon the conditions under which the reactions must be carried out. All transformations must take place within a very narrow temperature range, with extremely low concentrations of reactants, at an approximately neutral $\mathrm{pH}$ and a temperate redox potential. The powerful but caustic agents which are indispensable to the synthetic organic chemist must be wholly avoided. Even the simplest types of biochemical reactions would be impossible under such conditions, if it were not for the remarkable catalytic abilities of the enzymes.

It is interesting to speculate on the number of enzyme systems required to account adequately for all these reactions which must be taking place within cells. The number must indeed be large. If, however, one tabulates according to type the numerous enzymatic reactions which have been demonstrated and postulated for the normal metabolism of carbohydrates and fats, he will find that the reactions can be classified chemically into a surprisingly small number of groups. By suitable combinations of these relatively few types of reactions it is possible to carry 
a carbohydrate or fat through the series of steps by which it is believed to be normally utilized. These same types of reactions will also adequately account for the synthesis of polysaccharides and fats from the intermediary compounds arising during metabolism (Chapter IIIB). Unfortunately, it is not possible to make this statement general for all carbohydrates and lipides, since at present one can do nothing more than speculate about the biosynthesis of sterols.

There still remain to be identified a number of the chemical pathways by which the individual amino acids, purines and pyrimidines are connected with each other and with the intermediates which they have in common with carbohydrates and lipides. At the present time this problem is receiving a great deal of attention and interest, and pertinent findings appear in almost every current issue of biochemical journals. The mechanism by which these component units are elaborated into protein molecules is still an enigma and represents the biggest gap in our knowledge of fundamental biochemical processes.

Below is presented an outline of the general types of enzymatic reactions necessary to account for the basic processes by which carbohydrates are known to be utilized and by which fats are presumably synthesized and degraded. Also included are the fundamental reactions associated with nitrogen metabolism which appear to be reasonably well characterized and of general biological importance. A discussion of the specific reactions and their role in general metabolic processes can be found in the following chapters.

In this outline, the vitamin or group of vitamins associated with each particular type of reactions has been indicated. On the basis of present knowledge, it can be assumed that the specified vitamin or a member of the specified group will generally be required for the particular type of enzymatic action irrespective of the specific substrate used or the biological source furnishing the corresponding enzyme.

\section{Types of Enzymatic Reactions Utilized in Essential Metabolic Processes}

\section{Types of Reactions}

I. Reactions in which acetal, ester, amide, and acid anhydride linkages are formed or cleaved by the addition or removal of the elements of water or phosphoric acid.

II. Intramolecular hydrations and dehydrations.

III. Intramolecular isomerizations.
Vitamins required none

none

none 
IV. Simple oxidations and reductions in Nicotinic acid which the following types of double Riboflavin bonds are created or reduced by the Porphyrins* removal or addition of hydrogen atoms (or the addition or loss of electrons).

A.

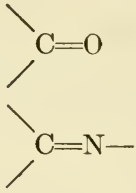

C.

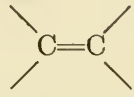

D. $\mathrm{O}=\mathrm{O}$

V. Decarboxylations of keto acids and the reverse reactions.
A. $\alpha$-Keto acid decarboxylations
Thiamine
B. $\beta$-Keto acid decarboxylations
(Biotin?)
VI. Reactions of amino acids requiring Pyridoxal activation of the $\alpha$-position.
A. Transaminations
B. $\alpha$-Amino acid decarboxylations

VII. Condensations and cleavages creating or rupturing carbon-to-carbon bonds, (other than carboxylations).

A. Aldol type

none

B. Reactions in which acetyl derivaPantothenic acid tives are condensed or formed

C. Reactions in which "activated single carbon unit participates $\dagger$ cleaved $\dagger$

VIII. Other reactions in which the single carbon unit participates $\dagger$

$p$-Aminobenzoic acid Folic acid

$p$-Aminobenzoic acid Folic acid

To place vitamin $B_{12}$ in the classification now would be premature, since concentrates known to be free from other biological agents have been available for only a few months. It is possible, however, to indicate that vitamin $B_{12}$ functions (perhaps indirectly) in the group of reactions for which $p$-aminobenzoic acid or folic acid are essential.

* The reasons for including porphyrins with the $\mathrm{B}$ vitamins in this discussion will be taken up later (p. 151).

$\uparrow$ This group of reactions is not yet as completely characterized as are the other types. There is some question concerning the exact compounds which enter into the reactions and the information on the component parts of the enzymes is still incomplete. However, these types of reactions have been included because of the abundance of circumstantial evidence concerning them (see p. 198). 
In the preparation of this outline several types of reactions, which so far have been demonstrated in only certain forms of life, have been omitted since their inclusion would necessitate cumbersome qualifying phrases in the description of the general types of reactions. For example, the detoxification of amines by amide formation in the liver and the formation of an acetate ester, acetylcholine, in nerve tissue represent cases where the reactive acetyl derivative, which usually reacts to form carbon-to-carbon bonds, has been utilized for specialized functions. From the standpoint of function, the syntheses of such acetyl amides and esters (requiring pantothenic acid, p. 195) can be considered entirely distinct from the amide and ester syntheses necessary to produce fats and proteins (no vitamin requirement).

It may be that another general type of reaction in which carbon-tocarbon bonds are formed will eventually be added to this group. Some microorganisms can effect the condensation of the amino acid serine with indole to form tryptophan (p. 183). Here it is the hydroxymethyl group adjacent to the $\alpha$-carbon atom (rather than the carboxy or amino group) which reacts after the amino acid is activated by a pyridoxal-containing enzyme. To date, this reaction is the only instance known in which pyridoxal catalyzes the formation of a carbon-to-carbon bond.

Inositol has recently been implicated as a necessary component of $\alpha$-amylase, a hydrolytic enzyme. Attempts to associate it with other hydrolytic enzymes have not yet been reported. The vitamin analyses of several other purified enzymes catalyzing hydrolysis indicate that inositol does not function generally in hydrolytic reactions. When polysaccharides are synthesized and broken down intracellularly, it is by a phosphorolytic process rather than a hydrolytic one, i.e., the elements of phosphoric acid instead of water take part in the formation and cleavage of glycosidic linkages. It would be interesting to know if inositol is needed for this type of reaction. If so, then inositol could take its place with the other members of the B group as a compound which is essential for a reaction required in the metabolism of practically all cells. The earlier discovery that inositol is a component of cell lipides (which could account for its nutritional importance) is probably partly responsible for so little work being directed toward establishing other possible functions. The association of inositol with enzymatic activity warrants a thorough investigation of its catalytic role in metabolism.

In view of the large number of separate reactions involved in the metabolism of carbohydrates, fats, and proteins and the variety of chemical compounds produced during these processes, it seems quite significant that the types of enzymatic reactions are limited; also, that each vitamin is specific for a given type of reaction, and that all the 
typical B vitamins appear on this essential list. Cognizance of the relationship between each vitamin and a highly specific type reaction enables an investigator to predict which $\mathrm{B}$ vitamin, if any, will be involved in a newly discovered enzyme system and which ones would be unlikely to function. Before it was shown that thiamine functioned only in the decarboxylation of $\alpha$-keto acids, efforts were often made to demonstrate that thiamine was a component of enzymes which were effective in decarboxylating other types of acids.

The list of fundamental types of reactions will be incomplete until the biosyntheses of sterols, certain protein constituents, and some other essential compounds have been elucidated. On the basis of the information now available about the formation and degradation of these compounds it seems reasonable to predict that not too many more types of reactions will be found necessary to account for their metabolism.

Just as it is possible to produce an almost infinite number of proteins from combinations of nineteen or so amino acids, so it should be possible, if the catalysts are available, to produce almost any type of chemical change which a cell requires by the proper combinations of a limited number of relatively simple types of reactions. It may be pointed out here that a limited number of enzymes in the digestive tract are capable of hydrolyzing an inestimable number of different proteins. Could a similar number of enzymes accomplish the reverse synthetic processes? An answer must wait until more knowledge is available concerning the manner in which the amino acids are placed in order during the synthesis of proteins. If the amino acids and intermediate peptides are oriented in some nonenzymatic fashion (by a gene or "organizer"), a small number of specific enzymes could accomplish the syntheses of most simple proteins.

Such speculation concerning the number of types of enzyme reactions offers an independent approach on which to base estimates as to the number of B ritamins yet to be discovered. Undoubtedly several new types of enzymatic reactions occurring generally throughout the biological realms will be found. Some of these will probably require as cocatalysts specific organic compounds which will be chemically unrelated to any of the known vitamins or other growth-promoting substances. When such substances are found, there will be good reasons for grouping them with the known $B$ vitamins in any classification of biochemical substances based upon functions.

At one time it seemed necessary to postulate the existence of a large number of types of enzymatic reactions unrelated to those then known in order to account for the catalytic activity of the numerous growthpromoting factors and vitamins which had not yet been associated with 
definite enzymes. Is such an assumption still necessary? This question, as well as the related question concerning whether or not there are many undiscovered $B$ vitamins, is certainly debatable. The thesis that there are only a small number of $\mathrm{B}$ vitamins yet to be found may have substantial foundation.

Components of Enzyme Systems. Once the relationship of a vitamin to a particular type of chemical reaction has been established, there still remain many questions to be considered regarding the relationship of the vitamin to the enzyme system itself.

Although thiamine, riboflavin, and nicotinic acid are as effective agents as can be found for the treatment of the corresponding nutritional deficiencies, none of the three is effective as such in the biochemical reactions for which they are required; each has to be incorporated into a more complex molecule before it can take part in its reactions. All the other typical vitamins whose functions have been completely elucidated have been shown to behave in an analogous manner-they act as catalysts in enzymatic reactions only after they have been built up into coenzymes of higher molecular weight than the vitamin itself.

Enzyme systems vary considerably in their complexity. The component parts of the system acting upon a substrate include:

A. Substance required to produce a suitable environment in which the reaction can be carried out. This group includes water, and the compounds necessary to establish the proper $\mathrm{pH}$, a suitable redox potential, and appropriate ionic concentrations.

B. A protein.

C. Co-factors.

(1) Specific inorganic ions.

(2) Specific organic compounds-the coenzymes.

Some of the simpler systems do not require any cofactors; others require only an activating inorganic ion, or a coenzyme; many enzymes, however, require both.

There is little specificity about the substances needed to establish a favorable environment for a reaction, and one has considerable leeway in his choice of buffering agents, poising agents, and salts which can be used. These factors should not be confused with or classified as coenzymes. The concentration of the nonspecific components can usually be varied over a considerable range. It is impossible to set down a single optimum value for the $\mathrm{pH}$, redox potential, and salt concentrations, since the most effective levels will depend upon the other conditions imposed upon the system. In contrast, the substances listed as cofactors are much more specific, and during a reaction are combined with the protein component in stoichiometric amounts. The divalent ions-magnesium, calcium, man- 
ganese, cobalt, and zinc-are the ionic substances most often encountered. Sometimes there exists a certain amount of interchangeability among these metallic ions, but usually it will be found that one particular metal will be much more effective than any of the others, and probably is the one associated with the enzyme system in its natural environment.

The organic molecules required as cofactors are highly specific, and in almost all cases only one specific compound will function; without it the protein is completely inactive.

Enzyme chemists are always particularly interested in determining whether a coenzyme is required for a biochemical reaction under investigation. During the purification of an enzyme, attempts are made to see if the enzyme molecule can be separated into inactive components which, upon recombination, will possess the original activity of the enzyme. If such a dissociation can be achieved, the complete enzyme is called the holoenzyme; the inactive protein component, the apoenzyme; and the smaller entities which are split off, the coenzymes or prosthetic groups. The ease with which the coenzymes may be separated from their apoenzymes varies considerably. Dialysis has been the method usually employed for separating these components, since this procedure is less likely to alter irrevocably the protein molecule than any other means of separation now known. Extraction of insoluble cellular material with alkaline or acidic buffers often can effect a resolution of the holoenzyme. If one is interested in obtaining only the coenzyme, heat will often liberate the coenzyme but will leave a denatured apoenzyme.

A large number of enzymes exist which cannot be dissociated by dialysis or any other method yet tried. It may be that this group of enzymes includes some proteins that contain tightly bound prosthetic groups which, except for their nondissociability, are analogous to the B vitamin coenzymes. Indeed, some of the enzymes in which the $\mathrm{B}$ vitamins are implicated have not yet been successfully resolved into their component parts. This offers some basis for postulating the existence of unidentified "factors" which must be incorporated into enzymes catalyzing certain fundamental processes occurring in most cells. From the standpoint of the nutritional requirements of animals such factors could be either essential or nonessential.

The classification of organic substances which have been definitely shown to be prosthetic groups for important enzyme systems is very simple. They will be found to be one of three types:

(1) adenylic acid or a phosphorylated derivative,

(2) a derivative of one of the $B$ vitamins, or

(3) a metallic complex of a porphyrin.

Very few substances that have been definitely characterized as true 
coenzymes will not fit into this classification. The number of substances known to function coenzymatically is small, and the $\mathrm{B}$ vitamin derivatives constitute a major portion of the list.

Several naturally occurring oxidizing and reducing agents, including ascorbic acid and glutathione, have been classified as "enzyme activators." The requirement for these substances, however, is not specific since other compounds can generally be substituted. It would seem, then, that these compounds should be considered as poising agents needed to adjust the redox potential to a level at which the protein will be active. An exception to this may be the requirement for glutathione, or closely related compounds, necessary for the functioning of glyoxalase. ${ }^{8}$ Here glutathione appears to participate chemically in the reaction.

There are a number of important compounds which are essential for certain cyclic enzymatic processes. Ornithine in the urea cycle and oxalacetic acid in the tricarboxylic acid cycle (p. 224) are familiar examples. These substances go through a series of enzymatic transformations, but are regenerated at the completion of the cycle. For this reason such a substance is sometimes referred to as a "carrier" for the compound which is produced or consumed during the process. Although it is possible to show a great deal of similarity between the manner in which such "carriers" function and the way in which some of the coenzymes function (p. 137), these "carriers" are commonly thought of as a type of catalyst distinct from the typical coenzymes.

There are a number of questions concerning the relation of the $\mathrm{B}$ vitamins to their respective coenzymes and the relation of the coenzymes to the proteins that will be considered in the following section of this chapter. The individual coenzymes will be discussed in detail in Chapter IIB.

\section{The Coenzymes}

Intensive research on the structures and functions of the coenzymes derived from the $\mathrm{B}$ vitamins is currently being conducted in a number of laboratories. It is probable that many important contributions directly related to the topics to be discussed are about to be reported in the journal literature. The information available as this book goes to press will be summarized in the following paragraph.

The coenzymes derived from thiamine, riboflavin, nicotinic acid, and pyridoxine have all been isolated and their chemical structures determined. A crystalline preparation of a pantothenic acid-containing coenzyme has not yet been announced, but the properties and hydrolytic products of highly active preparations have been reported. Absolute proof that biotin is a part of a specific coenzyme has not yet been pub- 
lished. Biotin activity is associated with several molecules more complex than the simple vitamin itself and it would indeed be unusual if one or more of these complexes were not a coenzyme for some type of reaction. Although a great many facts have been learned about the metabolic processes in which $p$-aminobenzoic acid, folic acid, and vitamin $\mathrm{B}_{12}$ participate, no work has been reported which justifies drawing any conclusions concerning the chemical relationship of these vitamins to their respective coenzymes. Inositol has recently been shown to be an active constituent of $\alpha$-amylase. It has not been definitely demonstrated that this simple molecule is identical with the coenzyme, but it would appear that this could be so (p. 125).

Coenzymatic Activity of the Simple Vitamins. Can the B vitamins, in their simplest chemical forms, ever serve as coenzymes? With the exception of inositol, just mentioned, there is no evidence that any B vitamin participates in vivo in a catalytic function until it has been transformed into its corresponding coenzyme (s).

It is possible in some cases to demonstrate, in vitro, chemical transformations in which a particular vitamin behaves in a manner analogous to the behavior of its coenzyme, in vivo. An example is the conversion (transamination) of pyridoxal to pyridoxamine by heating the former with amino acids. The conditions required for a reasonable yield make it appear most unlikely that within the cell such nonenzymatic reactions of the free vitamin could be important. It is also possible to demonstrate that free riboflavin can take part directly in oxidation and reduction processes. These reactions are so sluggish in an environment comparable to that found intracellularly that they would undoubtedly be useless.

Some of the free vitamins produce very distinctive pharmacological responses (Chapter $\mathrm{VC}$ ) ; however, these are entirely independent of the vitamin function of the compound and have no necessary connection with processes in cellular metabolism for which the vitamin is required.

There are two possible ways in which a free vitamin can conceivably influence the metabolism of a cell. It is possible for a vitamin to act as an inhibitor of its own coenzyme (Section D). Because of the structural similarity between a vitamin and its coenzyme, it is possible to produce effects which can best be explained by assuming that the vitamin competes with its coenzyme for the apoenzyme. In in vitro studies with bacteria and yeast, however, the concentrations of a vitamin required to produce inhibition are entirely outside the limit which would be found in natural circumstances. The ability of an organism to detoxify the inhibitor (the vitamin) by converting it to the coenzyme would also reduce the probability of achieving concentrations producing inhibition within the cell. 
High levels of a vitamin might, however, exert an effect which is just the opposite, i.e., the high concentration might increase the apparent activity of a coenzyme by slowing down its destruction. Thus, nicotinamide has been observed to inhibit the enzymatic destruction of its coenzyme in minced tissues. ${ }^{9}$ The investigators making this observation believed that the extent of inactivation of the coenzyme was decreased by the increased concentration of one of the products (nicotinamide) of the inactivating reaction. Thiamine has been shown to be a specific inhibitor for certain enzymes which catalyze the hydrolytic destruction of its coenzyme (p. 156).

Biosynthesis of the Coenzymes. In vivo, the coenzymes are formed by enzymatic reactions in which the vitamins themselves serve as substrates. The coenzymes vary in their chemical complexity, and presumably some vitamin-to-coenzyme transformations require more than one reaction. The simplest coenzymes are those in which the vitamin is converted to a phosphoric acid ester. Thiamine, riboflavin, and pyridoxal fall in this group. Riboflavin, nicotinic acid, pantothenic acid, and perhaps biotin form more elaborate molecules which contain the nucleotide, adenylic acid, as a component. The simpler coenzymes have all been synthesized chemically by direct phosphorylation of the vitamin. Every attempt to synthesize adenylic acid-containing coenzymes by chemical means has been unsuccessful.

It is interesting to consider the possible existence of unrecognized vitamins required for the reactions converting $B$ vitamins to their coenzymes. Thus, if some cofactor of a system essential for a reaction which produces one of the recognized coenzymes could not be synthesized by an animal, this cofactor could be an essential dietary factor or vitamin. However, the amount of a catalyst needed to catalyze the formation of a catalyst would be extremely small; hence the nutritional requirements for such factors may be extremely minute, and demonstrating their existence would be difficult. Such a coenzymatic role has been suggested for vitamin $B_{12}$ (in the biosynthesis of the coenzymes of $p$-aminobenzoic and folic acids, p. 203). This would account for the clinical activity of vitamin $B_{12}$ in one-thousandth the amounts required for other vitamins.

It may often happen that appreciable amounts of a vitamin may be supplied to an organism in a chemical form which cannot be directly utilized as the substrate for the synthesis of the necessary coenzyme. Other reactions must then precede the final transformation. If an organism is deficient or lacking in enzymes capable of catalyzing the needed change, then that form of the vitamin will show less activity or be totally inactive. It appears, for example, that pyridoxine must be oxidized to the aldehyde before it can be converted into a coenzyme; hence the vitamin 
$\mathrm{B}_{6}$ activity of pyridoxine for an organism is a measure of that organism's enzymatic capacity for carrying out the preliminary oxidation (p. 179).

For some cells the coenzyme, or compounds intermediate between the coenzyme and vitamin in their chemical complexity, must be enzymatically cleaved before absorption can take place, thus necessitating the complete resynthesis within the cell. An interesting example is cited in a report in which it was shown that if the monophosphate of thiamine is supplied as the substrate for the formation of the coenzyme (which is a di(pyro)-phosphate), the monophosphate must first be hydrolyzed to the unphosphorylated thiamine. ${ }^{10}$ In such cases the coenzyme or intermediate will be useless nutritionally to an organism if it cannot elaborate the extracellular enzymes needed to degrade the more complex forms of the vitamin. It is for this reason that the coenzyme of thiamine cannot be used to supply the nutritional requirements of some thiamine-requiring yeasts. ${ }^{11}$

The chemical mechanisms involved in the biosynthesis of a coenzyme may not always include a reaction involving the vitamin itself. Thus some bacteria can convert $\beta$-alanine into a pantothenic acid complex more rapidly than they can produce the complex if pantothenic acid is the initial substrate. ${ }^{12}$

Rate of Coenzyme Synthesis. How rapidly is a coenzyme synthesized from a vitamin? Such conversions can be carried out very quickly by most cells. This is demonstrated by the rapidity with which one can get a response when a cell deficient in some coenzyme is supplied the essential vitamin. There is, to be sure, some lag in time between the addition of the vitamin to the culture medium and a detectable response due to the coenzyme formed. It must be remembered that a deficient cell is essentially a dormant cell, since the retardation of vital metabolic processes forces most of the cell's activities to come to a standstill. It is only natural that some time should elapse before the cell can reach an active state again. In cells in which the deficiency is not acute the conversion of a vitamin to a coenzyme may take place almost immediately. This is demonstrated in the fermentation assay method for thiamine. ${ }^{13}$ The yeast used contains sufficient thiamine coenzyme to carry on some fermentation, and the cells are in a moderately active phase. The addition of thiamine to a medium containing such cells results in an immediate increase in the rate of fermentation.

Extent of Coenzyme Synthesis. There is insufficient information on which one could base a general statement concerning the relative concentrations of all the vitamins and their coenzymes within cells. It appears, however, that intracellularly the vitamins are predominantly in the form of their active coenzymes. In blood, for example, it has been shown 
that, whereas the thiamine, nicotinic acid, riboflavin, and pantothenic acid occur as such in the serum, they are almost entirely in the form of their respective coenzymes inside the erythrocytes. ${ }^{14,}{ }^{15}, 16,17$ It may be that part or all of the conversion of a vitamin to a coenzyme takes place during its absorption into the cell. Many absorptive processes are known to involve phosphorylation. Intestinal mucosa, a tissue in which absorptive mechanisms are extremely highly developed, has been shown to be a good source for obtaining preparations which will carry out the enzymatic syntheses (phosphorylation) of thiamine and riboflavin coenzymes. ${ }^{18,19}$

Do all cells possess the enzymes necessary to carry out the syntheses of their coenzymes from the vitamins? The known cases in which a vitamin must be furnished in the form of the intact coenzyme are rare. Two related species of bacteria cannot utilize nicotinic acid or nicotinamide (p. 136). Their nutritional requirements are usually supplied in the form of the intact coenzyme, but actually only a portion of the whole coenzyme is necessary. Some bacteria do not respond to pantothenic acid, but must have either the coenzyme or some substance of intermediate complexity that has been elaborated from pantothenic acid. ${ }^{12}$ In neither of these cases can we say that the organisms have a specific requirement for the coenzyme. Recently some organisms have been found which cannot utilize unphosphorylated derivatives of pyridoxal. ${ }^{20}$ The rarity of cases to date in which the intact coenzymes have been shown to be essential nutritional factors might lead one to expect that they will seldom be found to be irreplaceable nutrilites. To draw such a conclusion now is unwise. The coenzymes of only half of the known vitamins are as yet chemically identified, and suitable preparations of several of the identified compounds have not been generally available. At present it is possible to obtain from commercial sources only two coenzymes-cocarboxylase (thiamine) and coenzyme I (nicotinic acid). Furthermore, a large number of organisms have never been successfully grown on media whose chemical composition is known. Many of the chemically undefined nutritive requirements which can now be furnished only by natural extracts may be found to be unidentified coenzymes (or their intermediates) of the known $B$ vitamins.

Number of Coenzymes. Is more than one coenzyme synthesized from each vitamin? Those coenzymes which function as carriers of specific groups or atoms naturally will exist in two states. Hydrogen carriers will exist in the oxidized and reduced forms. A coenzyme which transports an active group (the active acetyl radical, for example) probably exists part of the time in chemical combination with the active substance. The situation in which pyridoxamine phosphate may be formed from pyridoxal 
phosphate during transaminations is of the same category. A phenomenon entirely different from this duality in forms of a single coenzyme occurs when there are synthesized from a single vitamin two specific coenzymes which cannot be substituted for one another in an enzyme system. Nicotinic acid and riboflavin are the only vitamins whose coenzymes are known at this time to fall definitely in this category.

All the reactions catalyzed by thiamine, pyridoxal, and pantothenic acid can be explained on the basis of a single coenzyme for each vitamin.

Most of the known enzymatic reactions involving riboflavin require the more complex coenzyme (called the dinucleotide). A coenzyme less complex in structure, riboflavin phosphate (designated as the mononucleotide), however, activates at least three apoenzymes (p. 146). One of these apoenzymes, that of the old yellow enzyme, can be reactivated equally well by either the mononucleotide or the dinucleotide. The oxidation mediated by this yellow enzyme proceeds at such a sluggish rate that it could not be of use in a metabolically active cell; hence it is believed to be a "derived" enzyme formed during its isolation from a more reactive flavoprotein. Two other recognized mononucleotide enzymes, cytochrome $\mathrm{c}$ reductase and L-amino acid oxidase, are capable of catalyzing the transfer of hydrogen atoms at a rate rapid enough to meet adequately the demands imposed upon them by natural systems. No report has been made concerning the question of whether or not equally active systems could be formed by using the dinucleotide as the coenzyme.

Most of the reactions catalyzed by nicotinic acid, however, fall into two distinct classes, each of which utilizes a specific coenzyme. The only difference in chemical structure between the two nicotinic acid coenzymes is one phosphate group. There are a few systems which are known to be activated by either coenzyme. Upon reinvestigation of some systems for which such claims had originally been made, it was shown that the coenzyme containing the extra phosphate group was degraded by traces of phosphatases present as impurities in the apoenzyme preparation. ${ }^{21}$ These phosphatases convert the triphospho-coenzyme (coenzyme II), which might be inactive itself, into the active diphospho-compound (coenzyme I), and it would appear that either coenzyme could function.

It may be well, before leaving the topic of vitamin-coenzyme conversion, to speculate as to why a vitamin must be transformed into a coenzyme before it is active. In the case of no coenzyme now known does it appear from the standpoint of theory of how they act that the nonvitamin portion should be useful or necessary for the catalytic activity observed. Thus in oxidation and reduction reactions, the addition or donation of hydrogen atoms during the transfer between substrates takes place in the 
vitamin component of the coenzyme.* In vitro, pyridoxal can participate in transamination reactions with amino acids without being phosphorylated; although the mechanism by which thiamine performs its catalytic function is not understood, there is no reason to expect that the phosphate part of the coenzyme molecule is altered during decarboxylations. It seems in most instances that the chemical changes catalyzed by a vitamin-containing enzyme are directly mediated by the vitamin component of the coenzyme, but the vitamin itself does not readily associate itself with the apoenzyme; hence, the function of the nonvitamin portion must in some way be concerned with the mechanism by which the coenzyme associates itself with the apoenzyme. Until some better explanation can be offered, it can be postulated that an important reason for the conversion of a vitamin to its coenzyme is to enable the molecule to contain the chemical groups which are essential for the formation of the coenzyme-protein bond.

Formation of Holoenzymes. One of the most intriguing properties exhibited by many proteins is their remarkable ability for combining with specific compounds of smaller molecular weight. It is impossible to give any adequate explanation of this phenomenon in terms of the classical concepts of chemical bonds. The inadequacy of our knowledge is frequently hidden behind the veil of such ambiguous terms as "protein complexes" and "enzyme-substrate union."

The binding of an apoenzyme with its coenzyme should be considered as a special case of the general phenomenon of combination between enzymes and substrates. In fact, in a number of reactions the participating coenzyme can more properly be termed a substrate than a catalyst. These are the reactions in which coenzymes are chemically altered during the course of the reaction and must be reconverted to their original form by a second reaction entirely separate from the first (p. 137). In such cases the coenzyme is in a strict sense the catalyst for a process, but not for the individual reactions.

Any attempt to develop a general explanation for the association between coenzymes and proteins is further complicated by the extreme variation in the stability of the union. Some systems exist in which the dissociated apoenzyme and coenzyme are present in greater concentrations than the holoenzyme; at the other extreme are the enzymes whose prosthetic groups are so firmly bound that hydrolysis of the protein is necessary to release the vitamin component. Also, a particular coenzyme does

* In the case of nicotinic acid, the conversion of the tertiary amine (the vitamin) to the quaternary ammonium base (the coenzyme) is probably essential for establishing the redox potential appropriate for the reactions catalyzed by the nicotinic acid-enzyme systems. 
not necessarily have the same affinity for the different apoenzymes which it activates.

The most stable type of combination might result from the formation of an ester or amide from the two components; more labile unions could resemble those found in compounds which dissociate readily in aqueous solution-hemiacetals, ammonia-aldehyde types, and hydrates; the bonding in some systems whose degree of dissociation is so sensitive to electrolyte concentration and $\mathrm{pH}$ changes might be explained by ionic attraction and salt formation.

Until recently no one had ever questioned the assumption that the union of a substrate and its enzyme was one involving actual physical contact of the two molecules. However, it has been reported that enzymes whose surfaces were believed to be completely coated with a polymer film could still activate their substrates and effectively catalyze reactions. ${ }^{22}$ That this procedure prevented the physical union between enzyme and substrate has been challenged; ${ }^{23}$ but if this finding can be substantiated and shown to be of general application to enzyme systems, many current concepts concerning enzyme mechanisms, including the functioning of coenzymes, will have to be radically altered.

Any theory concerning the chemical and physical forces which bind the components during a reaction should account for the extreme specificity which the protein may exhibit in its choice of substrates. Often, the slightest alterations in the structure of a substrate or coenzyme will affect its capacity for uniting with the enzyme, even though no change has been made in any of its reactive groups or the isoelectric point. This is indicative that the spatial configuration is critical.

The similarity in the chemical composition of the nonvitamin moiety of all the coenzymes is indicative of a general requirement for phosphate ester and adenylic acid components in the establishment of the coenzymeapoenzyme bond. The additional acid and amino groups introduced when the coenzyme is created increase the number of points where the coenzyme molecule can become attached to the protein by the formation of a salt. Perhaps adenylic acid possesses a chemical structure which is particularly adapted for combining with some configuration common to a number of proteins. Thus adenylic acid itself serves as a coenzyme for some reactions p. 134) ; its phosphorylated derivatives are essential dissociable parts of most enzyme systems in which high-energy phosphate bonds are created or utilized; and nucleic acids which contain adenylic acid constitute the prosthetic groups of a number of important types of proteins.

There is a difference in the affinity of apoenzymes for the oxidized and reduced states of the coenzymes of nicotinic acid. This can be attributed at least partly to the acid-base changes which accompany the oxidation 
and reduction of the coenzymes. When the coenzyme is reduced, the quaternary ammonium ion in the pyridine ring is converted to a tertiary amine. This elimination of a strongly basic group increases the acid properties of the coenzyme. Since the reduced acidic compound may dissociate more readily, it leaves the apoenzyme and is free to transport the hydrogen atoms to another enzyme system where these atoms will be passed on to another substrate. Other processes which involve shuttling atoms or reactive groups from one enzyme system to another may likewise function because of differences in the degree of dissociation of the two forms of a coenzyme.

The association of a substrate with its enzyme probably is always accompanied with a redistribution of intramolecular energies within both the substrate molecule and the protein. These changes are responsible for the "activation" of a certain atom or group of the substrate molecule. When a coenzyme unites with a protein the same thing occurs. The effect is more than just the tying together of two substances, and even though the union may be a "loose" one it results in profound changes in some of the chemical properties and reactivity of both the coenzyme and the protein.

An interesting example in which a quantitative expression can be derived for the change that takes place within a coenzyme when it combines with a protein is riboflavin phosphate. The standard redox potential of the riboflavin coenzyme is considerably different from the potential of a riboflavin enzyme system. ${ }^{24}$ This means that in combining with the apoenzyme some changes take place within the riboflavin moiety which greatly increase its tendency to accept hydrogen atoms (actually electrons, p. 128). The magnitude of the changes is such that the system can catalyze an entirely different group of biological oxidations and reductions than it could if it retained the potential of the unassociated coenzyme (p. 146).

The changes in the stability of apoenzymes when dissociated from their coenzymes can be used to substantiate the fact that the separation of a coenzyme from its protein may induce critical changes within the protein molecule. For example, when the coenzyme is removed from $\alpha$-amylase by dialysis, the protein rapidly deteriorates and cannot be reactivated again, even by the addition of the coenzyme. However, it can be shown that the denatured protein still possesses its original capacity for combining with the coenzyme (p. 125), although the complex formed is enzymatically inactive. This indicates that in the dissociation of the holoenzyme an alteration in the molecular structure of the protein occurs at some position other than the exact site where the coenzyme attaches itself. 
The association of a coenzyme with its apoenzyme to form the holoenzyme is sometimes an essential step in the biosynthesis of a coenzyme. Thus, the enzymatic synthesis of cocarboxylase from thiamine requires the presence of the apoenzyme, and as soon as there has been sufficient synthesis to saturate the protein the synthesis of the coenzyme ceases. ${ }^{10}$ A reasonable explanation for this is that, when there is no longer any apoenzyme present to combine with the coenzyme as it is formed, the uncombined coenzyme cannot protect itself from the action of phosphatases which hydrolyze the coenzyme as rapidly as it is formed.

Specificity of Coenzymes. The inactivity of most hydrolytic and other degradation products of coenzyme molecules indicates that the structural specificity required by apoenzymes of their coenzymes usually applies to the whole coenzyme molecule rather than to one particular type group or linkage within the molecule. The specificity of some apoenzymes for their coenzyme cannot be said to be absolute, however. The well substantiated cases in which an analogue of a vitamin has vitamin-like activity (Chapter VID) necessitate assuming that coenzymes containing those vitamin analogues are synthesized enzymatically, and that the resulting analogues of the natural coenzymes are able to combine with the apoenzymes and catalyze certain reactions. A few analogues of coenzymes have been prepared synthetically and tested in vitro and found to have some coenzymatic activity.

Analytical Methods for the Coenzymes. It would be gratifying in a general discussion of the analytical methods for determining the coenzymes (and the enzyme systems in which they participate) to be able to describe general methods which could be used for all the B vitamin coenzymes. Unfortunately, this is not possible at the present time. Studies of the distribution of the B vitamins in cells and tissues have yielded some very interesting results (Chapter IIA). Such data would have considerably more significance if they indicated how much of the vitamin was in an active form and how the coenzyme was distributed among the various systems for which it is required.

It may be that microbiological methods, as general in their applicability as the vitamin assays, can eventually be developed for the coenzymes. This will depend upon success in finding organisms which require specifically the intact coenzymes. There are no organisms known which satisfy this requirement in the strictest sense, the closest case being an organism whose vitamin $\mathrm{B}_{6}$ requirement can be met only by pyridoxal phosphate or pyridoxamine phosphate. ${ }^{20}$ If suitable organisms cannot be found, inhibitors perhaps will offer the solution to the problem of obtaining simple general methods for the analyses of the coenzymes. The ideal inhibitors for these analyses would effectively block the last reaction in 
the conversion of a vitamin to its coenzyme. If such compounds were available, they could be used to adapt the simple microbiological procedures used in vitamin determinations to specific assay methods for the coenzymes.

Today the only specific methods of assay for most coenzymes involve a direct determination of the effect of the addition of a substance upon an enzymatic reaction in which the particular coenzyme is the limiting factor. This entails the preparation of the appropriate apoenzyme, either from cells or tissues deficient in the coenzyme or from protein preparations in which the coenzyme has been destroyed or removed. Such enzymatic assay methods for coenzymes have a number of disadvantages not encountered in the microbiological methods. They require equipment, chemicals, and biological preparations which may not be readily available; technically, more training and skill is usually needed than for a microbiological assay. In addition, a general standardized procedure for all B vitamin coenzymes cannot be used, since the analysis for each coenzyme constitutes a special method.

Occurrence of Coenzymes. Since one of the reasons for grouping the $B$ vitamins together is the similarity of their natural distribution, it would be of interest to know if the enzymes containing them are associated together physically within cells. Some of the fundamental processes which involve a number of steps may require at most a single $B$ vitamin coenzyme, e.g., the elaborate mechanism by which glycogen is converted to lactic acid in muscle requires fourteen separate enzymes, but only one $B$ vitamin coenzyme is needed (p. 219). On the other hand, there are processes every step of which must be mediated by a different B vitamin. The conversion of carbohydrate to fat involves a series of reactions in which each pyruvic acid molecule eventually lengthens a fatty acid by two carbon atoms. This conversion requires enzymes containing thiamine, pantothenic acid, nicotinic acid, and probably riboflavin (p. 226). This series of reactions cannot be demonstrated if the structure of the cell has been destroyed. It is not known if this process is carried out by a number of separate enzymes which are physically separated from one another in the cell. It may be that the transformation requires an enzyme complex in which the component proteins and coenzymes are actually combined, and that this system would be inactive if its organization were disturbed.

Recently a process has been described for preparing a protein complex from mammalian tissue containing all the enzymes necessary for the aerobic oxidation of pyruvic acid and certain fatty acids through the tricarboxylic acid cycle. In its isolation, the protein complex containing all the essential component enzymes separates out as a gel. ${ }^{25}$ When the gel loses its ability to carry through the series of reactions there is an 
alteration in the appearance of the gel. The associated complex of enzymes is believed to be localized in certain morphological structures occurring in cytoplasm, the mitochondria. ${ }^{26,27}$

Most of the individual reactions utilized in the synthesis of the units for cell structure and in the production of energy are only component parts of some process involving a large number of reactions. Usually the intermediate compounds formed by the reactions are utilized immediately. In many instances the concentration of these intermediates under normal conditions is so low that it is difficult to demonstrate their existence; also some of the processes in which such intermediates occur are known to proceed very rapidly. The simplest explanation to account for the rapidity of a long process, when the concentration of the reactants for a number of the individual reactions is so low, is that the enzymes are in some way associated together in the cell.

There probably are also special means by which labile intermediates and by-products arising in one part of the cell can be transported to another. If enzyme systems containing cofactors that are easily dissociable are used, it is then possible that a reaction can be carried on in one part of the cell to furnish chemical intermediates or energy for utilization elsewhere within the cell. For example, adenosine diphosphate, the coenzyme for most reactions in which a high-energy phosphate bond is generated, is converted in these reactions to adenosine triphosphate. This form of the coenzyme can dissociate and is free to transport its energy "unit" to the other parts of the cell where it can be utilized. Hydrogen atoms arising from the numerous dehydrogenations of cellular reactions can be transported to other parts of the cell by dissociable coenzymes. It may be found that several of the highly reactive labile substances like the activated acetate derivative and the single carbon units are transported from one part of the cell to another by the coenzymes of pantothenic acid and $p$-aminobenzoic acid. Thus the complete series of enzymes needed for a particular cell function need not all be located exactly at the site of the function.

One of the present goals of enzyme chemists is the establishment of the exact location of the enzyme systems within cells. Some information has already been obtained by carrying out vitamin and enzyme analyses on the fractions obtained by separating cells into their gross components. Any procedure, however, that disrupts cell organization even slightly will not give all the information needed to explain what actually takes place within normal cells.

Hence, now that the relationship between most of the vitamins and their chemical functions has been established, many investigators are seriously engaged in an equally challenging study - that of associating 
the vitamins and their enzymes with the biological organization of the cells in which they function.

\section{Bibliography}

1. Jansen, B. C. P., and Donath, W. F., Chem. Weekblad, 23, 201 (1926).

2. Sumner, J. B., J. Biol. Chem., 69, 435 (1926).

3. Theorell, H., Biochem. Z., 278, 263 (1935).

4. Lohmann, K., and Schuster, P., Biochem. Z., 294, 188 (1937).

5. Warburg, O., and Christian, W., Biochem. Z., 275, 464 (1935).

6. Elvehjem, C. A., Madden, R. J., Strong, F. M., and Woolley, D. W., J. Am. Chem. Soc., 59, 1767 (1937).

7. Williams, R. J., Vitamins and Hormones, 1, 229 (1942).

8. Jowett, M., and Quastel, J. H., Biochem. J., 27, 486 (1933).

9. Mann, P. J. G., and Quastel, J. H., Biochem. J., 35, 502 (1941).

10. Weil-Malherbe, H., Biochcm. J., 33, 1997 (1939).

11. Williams, R. J., McMahan, J. R., and Eakin, R. E., University of Texas Publication, 4137, 34 (1941).

12. King, T. E., Fels, I. G., and Cheldelin, V. H., J. Am. Chem. Soc., 71, 131 (1949).

13. Schultz, A. S., Atkin, L., and Frey, C. N., J. Am. Chcm. Soc., 59, 2457 (1937).

14. Goodhart, R. S., and Sinclair, H. M., Biochem. J., 33, 1099 (1939).

15. Kohn, H. I., and Klein, J. R., J. Biol. Chem., 130, 1 (1939).

16. Klein, J. R., and Kohn, H. I., J. Biol. Chem., 136, 177 (1940).

17. Kaplan, N. O., and Lipmann, F., J. Biol. Chem., 174, 37 (1948).

18. Tauber, H., Enzymologia, 2, 171 (1937).

19. Hübner, H., and Verzar, F., Helv. Chim. Acta, 21, 1006 (1938).

20. MeNutt, W. S., and Snell, E. E., J. Biol. Chem., 173, 801 (1948).

21. Schlenk, F., Adv. in Enzymology, 5, 212 (1945).

22. Rothen, A., J. Am. Chem. Soc., 70, 2732 (1948).

23. Karush, F., and Siegel, B. M., Science, 108, 107 (1948).

24. Kuhn, R., and Boulanger, P., Ber. Itsch. chem. Ges., 69, 1557 (1936).

25. Green, D. E., Loomis, W. F., Auerbach, V. H., J. Biol. Chem., 172, 389 (1948).

26. Schneider, W., J. Biol. Chem., 176, 259 (1948).

27. Kennedy, E. P., and Lehninger, Albert, J. Biol. Chem., 172, 847 (1948). 


\section{Chapter IIB}

\section{COENZYMES DERIVED FROM B VITAMINS}

The problem of determining the specific chemical reactions catalyzed by a coenzyme derived from a $\mathrm{B}$ vitamin is not one which can be attacked in a straightforward manner. There is no standardized procedure the utilization of which would assure an investigator of success in determining the function of a newly discovered vitamin. The relationships between $B$ vitamins and their enzyme systems have been established through the correlation of information obtained from many types of investigation of metabolic processes.

In a critical examination of biochemical processes, there are logical reasons for studying the enzymatic reactions in their natural environment, i.e., within intact cells and as an integral part of a series of reactions. On the other hand, it is impossible to characterize a reaction completely - to determine the specific compounds reacting and the component parts of the catalyst-unless an isolated system free from all interfering phenomena is studied. Obviously, it is not possible to achieve both of these goals with the same techniques. This difference in purpose has led to some argument concerning the relative merits of establishing the existence of biological processes in cells by the use of isotopes, inhibitors, mutants, etc., and of studying specific isolated enzymatic reactions in detail. An adequate explanation of metabolism can ultimately be achieved only by approaching the individual problems from both directions.

The coenzymatic functions of some of the B vitamins were discovered by investigators whose primary interests were in the enzyme systems they were isolating; in such cases, specific vitamins have fortuitously been found to be component parts of particular enzymes-that is, the investigators up to time of finding the presence of a given vitamin, may have had no particular interest in that or any other vitamin.

In other cases the known functions of B vitamins have been ascertained as a result of observing the effect of vitamins upon metabolism. In such direct attempts to study the chemical changes catalyzed by the vitamins, normal biological systems are compared with those in which the vitamin has been prevented from functioning. The most direct method of limiting the reactions catalyzed by a vitamin is to produce a deficiency of that 
vitamin in the cells or tissues of an organism requiring the vitamin. Although this method is naturally limited to organisms which cannot synthesize adequate amounts of a given vitamin, its applicability has been greatly extended by inducing mutations in the genes controlling vitamin syntheses, thus artificially producing organisms with the desired nutritional requirements.

Reactions requiring a specific vitamin can be blocked even in organisms which do not normally require the vitamin. This is accomplished by subjecting the cells to some treatment which inactivates the coenzyme. Coenzymes essential for some reactions can be destroyed by placing the organism in an unfavorable environment. A method of general application for inactivating specific enzymes within the cell involves the use of inhibitors. The methods by which inhibitors can be used in investigating biological reactions is discussed in more detail in the last section of this monograph.

If the metabolic processes mediated by a coenzyme derived from a vitamin are blocked by the use of any of the procedures just described, by-products will accumulate. Chemical identification of the substances accumulating indicates the nature of the substrate of the transformation which has been blocked. Finding biochemical substances (chemically unrelated to the vitamin) which will partially or wholly counteract the changes produced in the deficient organism serves as a means of identification of some of the products which would normally be formed from the blocked reactions.

The chemical changes resulting from inactivation of a vitamin-containing system can be demonstrated by use of classical chemical procedures only when the substances involved are present in measurable concentrations. In many important instances, however, the intracellular concentrations of the reactants and products participating in a series of reactions are so low that changes from the normal metabolic pattern cannot be observed by ordinary methods. This obstacle was very effectively surmounted when the isotopes of carbon, hydrogen, nitrogen, sulfur and phosphorus became available for biological tracer studies. The use of compounds containing labelled atoms has been responsible for a number of recent contributions which have established coenzymatic functions of the vitamins: (1) the unexpected discovery of two extremely important biochemical intermediates (activated derivatives of formic and acetic acids) ; (2) the disclosure of a number of fundamental metabolic routes; and (3) the verification of the existence of postulated reactions previously undemonstrable.

The purpose of this chapter is to present a discussion of the individual coenzymes and their enzymes. These discussions will not include those 
contributions which have no direct bearing on the functions of the coenzymes nor will enzymatic reactions be cited whose existence is not well substantiated.

\section{Coenzymes Required for the Synthesis and Cleavage of Ester, Acetal, and Amide Linkages}

Most of the high molecular weight compounds-fats, polysaccharides, proteins, nucleic acids, etc.-must be cleaved into simpler substances before they can be absorbed and incorporated into essential structures of cells. This extracellular digestion is catalyzed by a group of enzymes which directly hydrolyze the ester, acetal, and amide linkages of the macromolecules. In intracellular syntheses, their hydrolytic productssimple sugars, fatty acids, amino acids, etc.-are recombined by reactions which recreate the acetal, ester, and amide bonds. These synthetic reactions within cells cannot be mediated by the same enzymes which catalyzed the hydrolysis, since the intracellular concentrations of amino acids, free fatty acids, and monosaccharides are probably never high enough to reverse the direction of the corresponding hydrolytic reactions. The formation of these larger molecules must proceed by indirect routes by which are incorporated reactions which introduce the energy necessary for the coupling of the component units (usually by the formation of phosphorylated intermediates).

With one exception, there is no evidence that the $\mathrm{B}$ vitamins function in either the direct hydrolytic reactions or in the synthetic mechanisms utilizing phosphorylated intermediates. However, each of the B vitamins is required for the production of some of the ultimate units from which the fats, carbohydrates, and proteins are formed, and most of the vitamins are essential for the energy-producing processes which supply the energy needed for the synthetic reactions.

Inositol as a Coenzyme. One reaction which is probably an exception to the statement in the previous paragraph is the hydrolysis of amylose by a pancreatic enzyme. Inositol appears to function as an essential component of this enzyme, $\alpha$-amylase. The coenzymatic activity of inositol was first suggested when a highly purified preparation of $\alpha$-amylase was shown to contain $4.1 \mathrm{mg}$ of inositol per gram. ${ }^{1}$ Subsequently this enzyme was shown to dissociate upon dialysis into a dialyzable thermostable component (a coenzyme) and a protein having no enzymatic activity. ${ }^{2}$ The dissociation of the enzyme during dialysis produces a change in the protein component, so that it is no longer enzymatically active even when recombined with the dialyzable fraction. This change, however, does not affect the capacity of the protein for combining with the coenzyme. This was demonstrated by showing that the dialyzed 
protein can inactivate an active undialyzed preparation (presumably by competing with the active enzyme for the coenzyme) ; but a combination of the dialyzed protein and the dialyzable coenzyme does not exhibit the inhibitory effect upon an undialyzed preparation. Furthermore, no inactivation of the protein component occurs if the enzyme is dialyzed against a solution containing the thermostable coenzyme.

In view of these findings a study using inositol inhibitors was carried out to demonstrate a possible relationship of the coenzyme to inositol. The $\gamma$-isomer of $1,2,3,4,5,6$-hexachlorohexane, a compound whose inhibitory action on the growth of yeast and molds can be prevented by mesoinositol, was found to inactivate purified $\alpha$-amylase preparations. ${ }^{3}$ The presence of inositol, however, counteracted this inhibition. To demonstrate the inactivation of the enzyme, it was necessary that the inhibitor be incubated with the enzyme for at least fifteen hours. The amount of inactivation produced by the inhibitor depended on the ratio of inhibitor to inositol. When the molar ratio of hexachlorohexane to inositol was 10 , only a 10 per cent inactivation occurred. Increasing this ratio to $50 \mathrm{com}$ pletely inactivated the enzyme. Thus, the authors concluded that inositol is an active constituent of $\alpha$-amylase.

Although the results obtained with the inositol inhibitor are highly indicative, they do not conclusively answer a pertinent question: Is "free" inositol the coenzyme of $\alpha$-amylase? The only logical explanation for the inhibition by hexachlorohexane and its reversal by inositol in the highly purified system used is that inositol itself is a dissociable component of the system, or that it can effectively replace some dissociable component. It would be interesting to know whether inositol by itself would behave in a manner identical to the thermostable dialyzable component obtained by dialysis of the enzyme. If the coenzyme is simply inositol, $\alpha$-amylase could be dialyzed against a solution of inositol with no loss in activity; also, the inactivation of undialyzed preparations by dialyzed protein fractions could be prevented by the addition of inositol along with the dialyzed protein.

In considering possible mechanisms by which inositol, which contains no salt-forming groups, could combine with the apoenzyme, the relationship of the chemical structure of inositol to that of the glucosidic units in the amylase substrate should not be overlooked.

A crystalline preparation from hog pancreas was used for the dialysis experiments which demonstrated the existence of a coenzyme for $\alpha$-amylase. Since that time, $\alpha$-amylases have been isolated in a crystalline state from human pancreas, ${ }^{4}$ human saliva, ${ }^{5}$ and Bacillus subtilis. ${ }^{6}$ The hog pancreatic amylase is not the same protein as the enzyme isolated from human pancreas, but apparently the human salivary amylase is 
identical with human pancreatic amylase. Inositol analyses on these preparations have not been reported, nor is information on the inositol content of saliva available.

Inositol, according to indications, has two possible biological functions independent of the one just described. It is a component of certain phospholipides (Section D), and may be an important factor in the regulation of fat metabolism in mammals. It is impossible to give clear presentation of the latter function because of disagreements which have not yet been resolved concerning the manner in which dietary factors influence the production of fatty livers. The confusion one encounters in attempting to follow the literature is due to several facts: different investigators have used different means of producing fatty livers; the types of lipides deposited have not always been well characterized; and it has been necessary to use crude liver extracts rather than pure substances to supply certain vitamins. ${ }^{7,}, 8,9$

"Lipocaic" was the name given to an uncharacterized heat-stable substance $(\mathrm{s})$ present in pancreas which was effective in preventing the fatty livers induced by diets containing liver extracts of high biotin content. Inositol, at one time, was claimed to be an active component of lipocaic, but its activity apparently is not as great as once believed and inositol per se is not as effective as choline..$^{10}$

Another lipotropic factor, protein-like in nature, obtained from the pancreas will prevent the fatty liver which results from pancreatectomy of dogs. This type of fatty liver can also be treated by adding choline to the diet. It is believed that the protein-like lipotropic factor functions by catalyzing during digestion certain hydrolytic reactions which are necessary to liberate methionine and choline from food, and that these compounds are the actual agents which prevent the abnormal deposition of fat in the liver. ${ }^{11}$ Apparently no effort has been made, however, to determine if the lipocaic action of inositol may be due to its presence in the lipotropic protein of the pancreas. If appreciable amounts of inositol were found in the more active preparations, it would be indicative that inositol is a component of another hydrolytic enzyme produced by the pancreas.

\section{Hydrases and Isomerases}

A class of reactions distinct from the synthetic and hydrolytic processes just discussed is the intramolecular hydrations and dehydrations-reactions in which the elements of water are added to or removed from a single molecule. A number of essential steps in the metabolism of carbohydrates and fats are of this type: fumaric acid $\rightleftarrows$ malic acid; phosphoglyceric acid $\rightleftarrows$ phosphopyruvic acid; $\beta$-hydroxybutyric acid $\leftrightarrows$ crotonic acid (p. 
$222)$; isocitric acid $\rightleftarrows$ cis-aconitic acid; etc. Each of these reactions involves the dehydration of a $\beta$-hydroxy acid, a type of reaction often encountered in organic chemistry. When catalyzed enzymatically, no vitamin-containing coenzyme has been shown to be required.

The isomerases which act upon phosphohexoses and phosphotrioses constitute a class of enzymes essential for most, if not all, living organisms that metabolize carbohydrates. These two enzymes catalyze structural rearrangements by establishing an equilibrium between:

(1) glucose-6-phosphate and fructose-6-phosphate;

(2) glyceraldehyde-3-phosphate and dihydroxyacetone phosphate. The conversion of an aldose phosphate to the corresponding ketose phosphate may take place as a result of the intermediate formation of an enol form common to both. A coenzyme requirement for such systems has not been demonstrated. These reactions produce isomers having the molecular configuration required for the subsequent aldol condensation or cleavage by which trioses and hexoses are interconverted (p. 219).

\section{Coenzymes Mediating Biological Oxidations and Reductions}

Biological oxidations and reductions constitute a class of enzymatic processes which has been intensively and thoroughly investigated during the past half century. Probably no other biochemical phenomenon has received the amount of attention that has been devoted to the study of the mechanisms by which organic substances are oxidized and reduced under the conditions imposed by an environment compatible with life.

It was early recognized that most of the reactions classified as oxidations do not involve molecular oxygen or any of the oxygen-donating types of compounds commonly employed as oxidizing agents in laboratory syntheses. The reactions in which metabolic substrates are "oxidized" can more properly be designated dehydrogenations, for the mechanism is in most instances one involving the transfer of hydrogen atoms; indeed, only rarely are oxygen atoms exchanged during the transformation. Even in aerobic processes, which require molecular oxygen, the particular component reaction in which the oxygen is utilized is usually one in which the oxygen molecule acts as an acceptor for hydrogen atoms which have been transported from the initial substrate by a series of enzymatic reactions. The oxygen molecule is, in fact, hydrogenated, forming hydrogen peroxide, and the oxygen atoms of the oxygen molecule are not incorporated into any of the organic molecules undergoing oxidation.

A more exact interpretation of the mechanism of hydrogenationdehydrogenation reactions can be set forth by explaining the phenomenon in terms of the donation and acceptance of electrons rather than hydrogen atoms. Excellent discussions of the current theories regarding the mecha- 
nisms of oxidations, particularly those types occurring in biological systems, have been published recently. ${ }^{12,}{ }^{13}$ Since the changes in the structure of organic molecules ean be much more easily indicated in terms of hydrogen atoms, this convention of depicting "oxidations" will be used in preference to the more exact one in which the electronic changes are described.

The enzymatic oxidation of many organic compounds involves no changes other than the removal of two hydrogen atoms. The catalysts for this type of reaction are found to contain, as cofactors, derivatives of either nicotinic acid, riboflavin, or a porphyrin. There are several types of oxidative processes which are not simple dehydrogenations. Oxidative decarboxylations and transaminations are illustrative of the more complex types of oxidative reactions. In these cases it will be found that vitamins other than the ones just mentioned function in the catalytic processes; these reactions will be considered elsewhere. The dehydrogenations considered below (catalyzed by enzymes containing nicotinic acid, riboflavin, and porphyrins) are those in which a double bond of one of the following types is ereated by the removal of two hydrogen atoms and an existing double bond in another molecule is reduced by the addition of these two atoms:
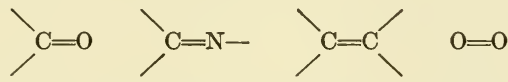

An interpretation of the oxidation and reduction of inorganic substances can often be most easily made by considering the process in terms of a galvanic cell. Likewise, the presentation of certain facts pertaining to biological processes in which there are oxidations and reductions occurring can be most easily made by drawing an analogy between the biological systems and a galvanic cell.

The galvanic cell is composed of two half-cells; each half-cell contains both an oxidized and a reduced form of some substance capable of existing in the two states (for example, a metal and its ion); these oxidized and reduced components of a half-cell must be in a state of dynamic equilibrium; and the two half-cells must be connected by suitable conductors before chemical changes and energy production take place. When the circuit between the two half-cells is closed, a reduction will take place at one electrode, and a compensating oxidation will occur at the other. Which part (oxidation or reduction) of the overall reaction will occur at a specific electrode and how much energy will be released by the reaction are determined by the relative potentials of the two half-cells composing the cell. At a given temperature and $\mathrm{pH}$ this electrode potential of a half cell is a function of the components of the half-cell and their relative 
concentrations, and this value is a quantitative expression of the capabilities of the component pair as an oxidizing or a reducing agent.

In each of the individual reactions of a biological oxidation there are two metabolite systems: each system consists of a substance capable of existing in an oxidized and reduced state; the oxidized and reduced forms of a particular system must be in a state of dynamic equilibrium; and the two systems must be connected by suitable means before chemical changes and energy production take place. When this connection between the two systems is established a reduction will take place in one system and an equivalent oxidation in the other. Which system will be reduced and which oxidized and how much energy will be liberated by the reaction are determined by the relative potentials of the two metabolite systems composing the reaction. In this case, the potential is called the redox potential; it is a function of the components of the system, their relative concentrations, the temperature, and, usually, the $\mathrm{pH}$ at which the reaction is carried out. This potential is a quantitative expression of the tendency of the metabolite pair to undergo oxidation or reduction. The lower the potential (based on the positive and negative notation found in all biochemical literature), the greater is the tendency of a system to accept hydrogen atoms and to exist predominantly in the reduced form.

In many common galvanic cells no catalyst other than water is needed to establish the equilibrium between the oxidized and reduced states of the material composing the half-cell. There are instances, however, in which a catalyst must be introduced to establish this equilibrium. For example, a platinum electrode must be used to establish the equilibrium between molecular hydrogen and its oxidized state, the hydrogen ion. Several systems which often form component parts of biological oxidations (for example, the sulfide-disulfide system, the ascorbic-dehydroascorbic acid system, or certain ferrous-ferric complex systems) require no catalyst (at least, no enzyme) to establish the equilibrium between their oxidized and reduced states. When two such systems are coupled, a nonenzymatic oxidation-reduction reaction occurs. Usually, however, the situation is more analogous to the hydrogen-hydrogen ion half-cell, and a catalyst (enzyme) must be introduced to establish the equilibrium between the oxidized and reduced form of the organic metabolite. The classical example is the succinic-fumaric acid system. These two acids cannot be reversibly interconverted by any chemical means yet known, and they cannot function by themselves as a hydrogen donor or an acceptor. Yet, in the presence of an appropriate enzyme, these two acids rapidly reach a state of equilibrium, and this system can act as either an oxidizing or reducing agent depending upon the potential of the system with which it becomes linked. 
The specific purposes of the enzymes which mediate biological oxidations and reductions are (1) to establish the equilibrium between the two states of a metabolite system and (2) to link this system with another of appropriate potential. When these criteria are met a reaction will then automatically occur.

The relative electrode potential of a half-cell or the relative redox potential of a metabolite system can be calculated from the standard potential of the half-cell or the metabolite system. The standard potential is that potential, referred to an arbitrary standard (the potential of a $\frac{1}{2} \mathrm{H}_{2}-\mathrm{H}^{+}$system), of a half-cell or metabolite system when the oxidized and reduced states are present in equal concentrations (or, are at equal activities). The actual potential of a system having other concentration ratios differs from the standard potential by a factor which includes the ratio of the oxidized and reduced forms. Hence the actual potential of a system and its tendency to act as an oxidizing or reducing agent can be varied by changing the relative concentration of the two components.

In a galvanic cell it is possible to reverse the direction of the current and the direction in which the chemical reaction is proceeding by changing the ratio of the oxidized and reduced forms of the components of the half-cells, provided the standard potentials of the two half-cells are not too far apart. If the standard potentials for two metabolite systems composing a reaction are approximately the same value, it is possible to cause the reaction to proceed in either direction by establishing appropriate ratios between the oxidized and reduced forms of the two component systems. Such reactions are those in which the net chemical changes have been observed to be reversible. If, however, there is appreciable difference between the standard potentials of the two systems composing the reaction it is impossible to achieve concentration gradients sufficient to reverse the usual course of the reaction. In this case the system having the lower potential will always be observed to be oxidized while the one having the higher potential will be reduced.

For convenience in studying redox systems, reactions are often set up in which one of the component systems is an artificial one, i.e., is not encountered under natural circumstances. The redox dyes, such as methylene blue, are commonly used for this purpose. Their use often simplifies the process to be studied, enables the investigator to by-pass technical difficulties, and through the change in the color of the dyes at a critical redox potential serves as a convenient indicator in following the progress of the reaction.

Coenzymes derived from nicotinic acid, riboflavin, and the porphyrins exist in both oxidized and reduced states and, with their appropriate apoenzymes, form redox systems. It is not surprising that the enzyme 
systems derived from each of these three cofactors have characteristic, but widely differing, potentials. Because of this difference, one type of cofactor is usually much more suitable for a particular reaction than are the other two. The enzyme systems containing nicotinic acid have the lowest potential, those containing riboflavin intermediate, and those related to porphyrins the highest. These vitamin-containing systems either become linked with one another or with a specific metabolite system, thus establishing an oxidation-reduction reaction. Such reactions are responsible for most of the biological oxidations which take place. A more detailed account of individual reactions will be discussed in connection with their specific coenzymes.

\section{Coenzymes Containing Nicotinic Acid}

The first organic coenzyme to be recognized (1904) was a heat-stable factor which activated preparations of zymase, the complex of enzymes in yeast which catalyzes the alcoholic fermentation of carbohydrates. ${ }^{14}$ This cofactor was designated "cozymase." Its chemical constitution, however, was not established until much later, after a second coenzyme, coenzyme II (the codehydrogenase of Zwischenferment), had been discovered, isolated, and shown to be a derivative of nicotinamide (1934).$^{15}$ (Zwischenferment, an enzyme of historical interest, dehydrogenates glucose-6-phosphate.) A year later nicotinamide was also isolated from cozymase and the chemical relationship of the two coenzymes was thus established. ${ }^{16}$

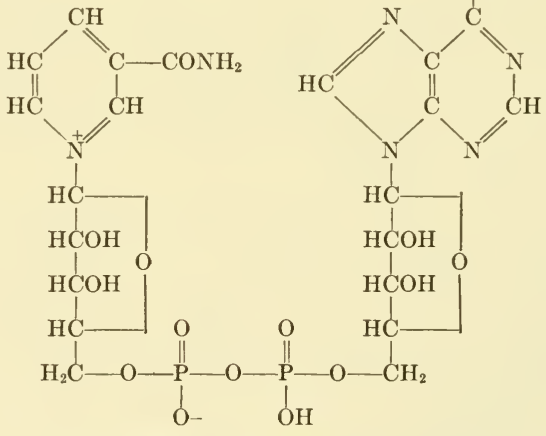

Diphosphopyridine nucleotide (Cozymase)

Upon hydrolysis the two coenzymes yield identical products; however, only two mols of phosphoric acid are present in cozymase, while three 
mols are liberated from coenzyme II. The results of degradation of the coenzymes have excluded all but one possible structure for cozymase, ${ }^{17}$ but have not given sufficient data to establish the exact formula for coenzyme II. They are both inner salts of ribose dinucleotides in which the organic bases are nicotinamide and adenine. The problems encountered in attempting to synthesize such dinucleotides have not yet been solved, and it has been impossible to verify the proposed structures by synthesis.

It was originally postulated that the third phosphate group of coenzyme II formed a linear pyrophosphate "chain" with the two other phosphate residues. ${ }^{18}$ However, it is now believed that the additional phosphate is attached in some position as a "side chain," presumably by forming an ester with one of the hydroxyl groups of the adenosine component. This structure would account for the ease with which the triphospho- coenzyme can be degraded to cozymase, since the reaction would then not entail a cleavage and resynthesis of the dinucleotide bond during the conversion.

A number of trivial synonyms have been coined for the two coenzymes; for most of them there are obvious objections. The thiamine coenzyme is also a cofactor essential for zymase activity; hence, it too is a "cozymase" and a "coferment." "Coreductase" and "codehydrogenase" can logically be applied equally well to coenzymes which contain no nicotinamide. Both coenzymes of nicotinamide plus several degradation products can serve as "Factor V," the growth factor required by Hemophilus influenzae. Designation of compounds by numerals or by the names of investigators can be very confusing even to scientists well acquainted with the historical aspects of biochemistry. Names based upon chemical structure are the desirable choice. For this reason, diphosphopyridine nucleotide (DPN) will be used in preference to cozymase, coenzyme I, codehydrogenase I, coferment of alcoholic fermentation, Harden's coferment, coreductase, or Factor V; triphosphopyridine nucleotide (TPN) will be used to designate the compound which was originally called coenzyme II (codehydrogenase II) or Warburg's coferment.

The formula and names given above represent the oxidized states of the coenzymes. The reducd forms are referred to as the dihydro compounds. In the oxidized state, the nucleus of the nicotinamide exists as a quaternary pyridinium ion which forms an inner salt with one of the ionizable acid groups in the pyrophosphate bridge between the two nucleosides. Upon reduction, the heterocyclic nitrogen atom is converted to the weakly basic tertiary amine; hence the reduced disphosphopyridine nucleotide behaves as a dibasic acid. The reduction of either coenzyme is thus always accompanied by an increase in the number of titratable hydrogen ions. Which one of the two ortho positions ( 2 or 6 ) is hydro- 
genated when the pyridine nucleus is reduced has not yet been established.

The reduction of the coenzymes not only changes the acidic and basic properties of the compounds, but also causes characteristic alterations in their absorption spectra, in their stability toward acidic and alkaline treatment, and in their affinity for their apoenzymes. The reduced coenzymes both show in their absorption spectra a very distinct band at $320-360 \mathrm{~m} \mu$ which is totally absent from the spectra of the oxidized molecules. The production of this band during reduction of the coenzymes is so characteristic of these compounds that it can be used as an analytical method for determining the di- and triphospho nucleotides in purified preparations. ${ }^{19}$ Such spectrographic analysis also is an excellent method for following the progress of enzymatic reductions or other enzymatic reactions which can be coupled to a reaction involving a nicotinic acid coenzyme. ${ }^{20}$ When irradiated with ultraviolet light, only the reduced coenzymes exhibit a strong whitish fluorescence. ${ }^{21}$

The coenzymes in their oxidized state are rapidly inactivated by standing at room temperature in dilute alkaline solution $(0.1 N)$, but the rate of destruction is much slower in acidic solutions $(0.1 N)$; the reduced molecules, on the other hand, are inactivated immediately by weakly acidic conditions, but are unaffected by the alkaline treatment. ${ }^{18}$

The immediate destruction of the reduced molecules by acid has been attributed to the formation of an addition compound in which a molecule of acid adds to one of the double bonds of the dihydropyridine nucleus. ${ }^{22}$ The stability of the dihydro coenzymes (trivalent nitrogen atom) in alkaline solution is comparable to the stability of the glucosidic-like linkage through the nitrogen atom of purine nucleosides (likewise trivalent). Hence, the unusual alkaline lability of the oxidized nicotinamide nucleoside can probably be attributed to the difference in properties of a glucosidic bond linking a quaternary ammonium nitrogen ion to a pentose. The initial cleavage occurring when diphosphopyridine nucleotide is subjected to either acidic or basic hydrolysis takes place at the bond linking the pyridine base to the ribose.

Adenosine diphosphate and adenylic acid can be obtained from the alkaline hydrolyzates of diphosphopyridine nucleotide, ${ }^{23}$ the latter possessing the coenzymatic activity necessary for the activation of certain apophosphorylases. Attempts to demonstrate (by this enzymatic analysis) the formation of adenylic acid by a comparable treatment of the triphosphopyridine nucleotide have been unsuccessful. This is the reason for believing that the third phosphate group of the latter coenzyme is attached in the form of a phosphate ester of the adenosine portion of the molecule rather than as a portion of the pyrimidine nucleoside or the connecting pyrophosphate bridge. Alkaline cleavage of the former type 
compound would yield an esterified derivative of adenylic acid which would be inactive when tested for "cophosphorylase" activity. ${ }^{24}$

An enzyme occurring in rabbit tissue has been shown to cleave the pyrophosphate linkage in diphosphopyridine nucleotide to produce the two mononucleotides, ${ }^{24 a}$ and an enzyme preparation has been obtained from almonds which cleaves this coenzyme in a fashion such that the products are the labile nicotinamide nucleoside, adenosine, and phosphoric acid. ${ }^{25}$

Since the coenzymes cannot be prepared synthetically, they must be isolated from natural sources. Yeast is a rich source from which the diphospho derivative is usually isolated, although it has been suggested that better preparations can be obtained if muscle is used as the source. Several investigators have reported alterations in procedure for improving the older methods of concentration and purification. ${ }^{26,27}$ Erythrocytes were the source originally used for preparing the triphospho compound $;^{15}$ it has also been isolated from liver ${ }^{28}$ and yeast. ${ }^{29}$ The diphospho nucleotide can now be purchased from commercial sources, and preparations possessing triphospho nucleotide activity can be prepared chemically from the former coenzyme.$^{30}$ Although in their chemical properties the di- and triphospho nucleotides are very similar, there are sufficient differences in their tendencies to be absorbed ${ }^{29}$ and in the solubilities of their salts that their separation from one another is not difficult. ${ }^{29,31,32}$

The dihydro derivatives can be readily prepared by chemical reduction of the oxidized forms of the coenzymes. Sodium hydrosulfite is a convenient reducing agent for this transformation. ${ }^{33}$

Assay Methods. The quantitative determination of the coenzymes of nicotinic acid has been carried out by spectographic measurements, enzymatic analyses, microbiological assays, and chemical methods.

Both the characteristic absorption band at $340 \mathrm{~m} \mu^{27}$ and the fluorescence of the dihydrocompounds ${ }^{21}$ have been employed for estimating the coenzyme content of concentrates. These methods do not distinguish between the di- and triphospho derivatives of nicotinic acid, but they do serve as a means of establishing the combined amounts of the reduced forms of the two coenzymes.

The only specific method for determining the concentration of the individual coenzymes is by use of enzymatic systems. With an appropriate apoenzyme an assay for either the diphospho or the triphospho nucleotide can be accomplished. In this case both the oxidized and reduced states of the particular coenzyme are equally active and are not distinguished by the method. In the determination of diphosphopyridine nucleotide care must be taken to have an apoenzyme preparation free from phosphatases which would degrade any triphospho coenzyme present into the compound being measured. 
The activation of a "zymase" system has been the classical method employed in determining the concentration of diphosphopyridine nucleotide, since the preparation of the apozymase can be readily accomplished. ${ }^{34}$ The repeated extraction of dried brewers' yeast gives a residue which contains all the components of the alcohol fermentation system except diphosphopyridine nucleotide. In the presence of the apozymase preparation the rate of fermentation (measured by following the evolution of carbon dioxide) gives a direct measure of the amount of coenzyme introduced into the system. In this process the coenzyme is needed to accept hydrogen atoms from glyceraldehyde-phosphate and subsequently to donate them to acetaldehyde. Aerobic processes (such as oxidation of lactic acid ${ }^{35}$ and malic acid) ${ }^{27}$ have also been used for the enzymatic estimation of diphosphopyridine nucleotide. In addition to the appropriate apoenzyme and substrate the system must contain the other enzymes needed for accepting the hydrogen atoms from the reduced coenzyme and transporting them to the final hydrogen acceptor. If molecular oxygen is the acceptor, the reaction can be followed manometrically; if methylene blue is used, the rate of decolorization is measured.

The oxidation of glucose-6-phosphate (Robinson ester) to 6-phosphogluconic acid is catalyzed by a dehydrogenase (Zwischenferment), the coenzyme of which is triphosphopyridine nucleotide. ${ }^{36}$ This reaction can be used as a specific method for the determination of this coenzyme, since the diphospho nucleotide cannot act here as the hydrogen acceptor. The reaction can be followed in several ways depending upon the hydrogen transporting systems to which it is coupled. The most sensitive and accurate method is one in which cytochrome-c is the component finally reduced..$^{37}$ The rate of reduction of cytochrome-c can be easily followed spectrometrically. By this procedure quantities as small as 0.02 microgram of the coenzyme can be measured.

Hemophilus influenzae and Hemophilus para-influenzae cannot synthesize the nicotinamide nucleoside from nicotinic acid or nicotinamide $;^{38}$ consequently, these organisms do not respond to nicotinic acid or nicotinamide but must have an exogenous supply of either of the coenzymes or certain degradation products in which the pyridine-ribose bond is intact. ${ }^{39}$ These organisms have been used to assay for the coenzymes by conventional microbiological procedures. Although this method lacks specificity because some degradation products are active, the interference by such substances in most instances probably is negligible.

The chemical reagents employed in the chemical determination of the free vitamin also react with the coenzymes, and cannot be directly used to distinguish between the simple vitamin and its more complex derivatives. However, chemical methods can be of use when it is known that 
all the nicotinic acid activity of a preparation exists in the form of the coenzymes or if the various nicotinic acid-containing compounds are separated by means of paper chromatography, for example.

The use of any of these methods for the analysis of natural substances depends upon obtaining extracts suitable for analysis. Considerable variation can often be noted in the analytical results of different investigators who have tested crude materials. This would indicate that the accuracy of these methods may be limited by the factors involved in the preparation of the extracts. The instability of the coenzymes and the enzymatic destruction which occurs when cells are macerated must always be taken into account. In making differential assays for the two coenzymes the possibility of enzymatic interconversion must be considered. The rapidity with which the original equilibrium of the oxidized and reduced forms of the two coenzymes can be disturbed can lead to erroneous conclusions when values for the two states of each of the coenzyme are sought. The reduced coenzymes, for example, are oxidized by air during hot-water extraction. ${ }^{18}$

Occurrence. In view of the number of essential reactions for which the coenzymes of nicotinic acid are required, it is not surprising that they have been found in all cells which have been examined for their presence. It would be most unexpected if some type of cell were found which did not contain these coenzymes. The quantities of the two individual enzymes as well as the ratio between the di- and triphospho nucleotides vary over a wide range for different types of cells. ${ }^{18}$ Yeast, the richest source of the diphospho nucleotide yet encountered, contains as high as $0.5 \mathrm{mg}$ per gram of moist cells. The triphospho nucleotide is always present in smaller quantities than the simpler coenzyme, which would be expected inasmuch as it functions in fewer reactions.

The degree of association of these coenzymes with their apoenzymes is less than that encountered in any of the other $\mathrm{B}$ vitamin coenzymes. ${ }^{40}$ It has been pointed out that the coenzymes really function as substrates of two independent reactions and that they are, in effect, catalysts for a complex oxidation-reduction process rather than for simple individual reactions. The coenzymes must alternate between two separate apoenzymes; the one on which the coenzyme is reduced and the one on which the reoxidized form is regenerated. Since comparable rates of reaction are found in both intact cells and in solutions in which there is no organization of the enzymes, it is believed that these two apoenzymes between which the coenzyme shuttles need not be coupled together sterically within the cell structure. ${ }^{41}$ These coenzymes have consequently been described as "mobile coenzymes" in order to contrast their behavior with that of the fixed coenzymes which remain attached to one apoenzyme 
Because of this mobile function these two coenzymes have been found to occur in quantities which on an equivalent basis are considerably in excess of the amounts of apoenzymes. Since the oxidized and reduced forms of the coenzymes are kept in equilibrium by these apoenzymes, the ratio of reduced and oxidized molecules will be determined by the redox potential of the cellular environment. It is not surprising, therefore, that of the total diphosphopyridine nucleotide present in a number of animal tissues, a fairly uniform percentage (35-45 per cent) is in the reduced state. ${ }^{42}$ In certain malignant tissues the ratio of reduced form to oxidized form is increased, ${ }^{43}$ a change which presumably results from the lowered redox potential maintained by such cells.

Biosynthesis. The synthesis of the coenzymes from their component units (those formed upon hydrolysis) can apparently be carried out by most organisms. The only known instances in which this total synthesis cannot be accomplished are encountered in the two strains of influenza bacteria previously mentioned ( $p .136)$. In higher organisms practically all the intracellular nicotinic acid is in the form of its coenzymes, but the extracellular fluids (such as plasma, milk, and urine) contain little, if any, coenzyme. The enzymatic synthesis of both coenzymes is known to occur in vitro in most intact cells, and an enzymatic synthesis of triphosphopyridine nucleotide from nicotinamide, ribose, and adenosine triphosphate has been reported in which only a cell-free extract was used. ${ }^{44}$

Investigations of the specificity of the pyridine component used for the biosynthesis of the coenzyme have disclosed several interesting variations among different types of cells in regard to the utilization of nicotinic acid and nicotinamide. It appears that the route of synthesis may differ in different types of cells, and that nicotinamide sometimes cannot be directly used for the biosynthesis of the coenzymes. ${ }^{45}$

The synthesis of the triphospho nucleotide from the diphospho compound can be accomplished enzymatically. ${ }^{46}$ Chemically the conversion can be made by use of phosphorus oxychloride. ${ }^{30}$ The phosphorylated substances formed by the enzymatic and chemical syntheses are assumed to be identical with the naturally occurring compound, but chemical proof of their identity has not been established.

When the organization of cells is disrupted the coenzymes of nicotinic acid are rapidly inactivated by enzymatic hydrolyses. The coenzymes are vulnerable to attack by hydrolytic enzymes at a number of points. In animal tissue, most of the enzymes responsible for the inactivation are believed to hydrolytically cleave the nicotinamide-ribose bond, liberating free nicotinamide. ${ }^{47}$ 
An interesting phenomenon observed in yeast is one in which the presence of a substrate appears to protect a coenzyme from destruction. The crude apozymase preparations rapidly inactivate diphosphopyridine nucleotide when the latter is added alone, ${ }^{48}$ but if hexose diphosphate is added simultaneously the destruction does not take place. Likewise, if the yeast fermentation is inhibited by the presence of the fluoride ion a rapid decrease in coenzyme content results. Apparently, active fermentation is necessary to produce the conditions required either for decreasing the rate of hydrolysis of the coenzyme or for increasing the rate of its resynthesis from its hydrolytic products. A demonstration of coenzyme synthesis by these apoenzyme preparations, however, has not as yet beer. achieved.

Reactions Catalyzed by Nicotinic Acid-containing Coenzymes. Although the reduction of the nicotinic acid coenzymes is often pictured as the simple addition of two hydrogen atoms to one of the $-\mathrm{N}=\mathrm{C}<$ bonds of the pyridine nucleus, the reduction of the coenzyme is probably not so direct. There is evidence that the reduction is a stepwise procedure in which the intermediate formation of a stabilized semiquinoid radical (monohydronucleotides) allows the addition to the coenzyme of a single hydrogen atom at a time, ${ }^{49}$ and facilitates the establishment of an equilibrium between the oxidized and reduced states. ${ }^{13}$

A wide degree of variation has been noted in the specificity of various nicotinic acid dehydrogenases in their requirements for the two coenzymes. $^{50}$ In most of the reactions that have been investigated there is a distinct preference, if not an absolute requirement, for one or the other of the two compounds. One enzyme (glutamic acid dehydrogenase from animal tissues), however, can use either the di- or triphospho nucleotide equally well.

Approximately forty different enzyme reactions have been reported to be catalyzed by one or the other of these coenzymes. Although many of these reactions are probably of limited importance in the normal functioning of cells, a number of indispensable reactions taking place during basic metabolic processes are found in the list.

The redox potentials for most of the important systems with which pyridine coenzymes are coupled fall in the same range as those of the coenzymes themselves. Consequently, the direction in which these reactions proceed can be reversed (1) when changes in metabolism alter the concentration ratio of the metabolite-pair within the cell or (2) when the coenzyme-reduced coenzyme ratio changes because of variations in the intracellular redox potential brought about by other processes taking place. For example, in many instances the direction in which the lactic acid-pyruvic acid conversion proceeds is constantly alternating because 
of slight changes in the local concentrations of these two substances at the site of each enzyme molecule or because of variations in the redox potential during the so-called aerobic-anaerobic phases of cellular activity.

One group of reactions in which these coenzymes participate include those in which energy-rich phosphate compounds are formed by the "oxidation" of an aldehyde; the reverse reactions, ones in which energyrich bonds are utilized for synthetic purposes, are the "reduction" of acids. The "oxidation" of phosphoglyceraldehyde is a good example. The aldehyde forms a loosely bound addition product with phosphoric acid (a compound analogous to an aldehyde hydrate). On dehydrogenation this inorganic phosphate is converted into a reactive acyl phosphate group which can be transferred to adenosine diphosphate (ADP), forming adenosine triphosphate (ATP).

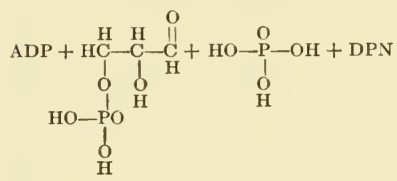<smiles>C1=CC=C1</smiles><smiles>O=P(O)(O)OCC(O)C(O)O[PbH]</smiles>

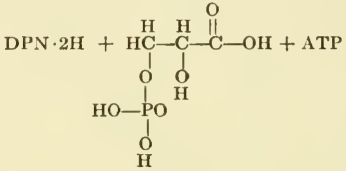

11

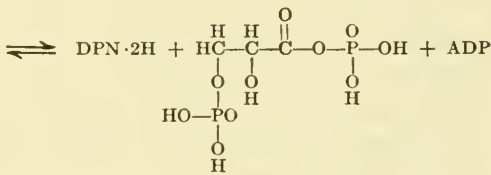

During catabolic phases of activity when glycogen and other organic substrates are being oxidized for the purpose of supplying energy to cells, this reaction (and other comparable ones) proceeds in the direction causing the dehydrogenation of the substrate and the creation of the reactive phosphate bond. During anabolic phases, when part of the glycogen is resynthesized by the organism, the reaction proceeds in the reverse direction and an energy-rich bond of adenosine triphosphate is utilized to reduce, in effect, an acid to an aldehyde. This reaction represents a general biological mechanism in which the phosphopyridine nucleotides mediate the conversion of the latent chemical energy of reduced organic substrates into the readily utilizable energy of the reactive phosphoric anhydride compounds, or the reverse process in which available energy is conserved by the formation of more highly reduced compounds.

The numerous redox systems which have been shown to be enzymatically coupled with the phosphopyridine coenzymes can be classified 
according to the following outline (in which some of the more important specific systems have been included as examples) : ${ }^{50,51}$

Aldehyde-primary alcohol

Ketone-secondary alcohol

Acyl phosphate-aldehyde-l-phosphate

Acid-aldehyde (hydrate) (acetaldehyde-ethanol)

(pyruvic acid-lactic acid)

(oxalacetic acid-malic acid)

(oxalsuccinic acid-isocitric acid)

$(\beta$-hydroxybutyric acid-acetoacetic acid)

(1,3-diphosphoglyceric acid-1,3-diphosphoglyceraldehyde)

(gluconic acid-glucose)

(phosphogluconic acid-glucose-6-phosphate)

(phosphoglyceric acid-phosphoglyceraldehyde)

Imine-amine

Both the glucose-6-phosphate and isocitric acid systems, as well as the glutamic acid dehydrogenase of yeast and bacteria, ${ }^{50}$ must be coupled with the triphospho nucleotide; the glutamic dehydrogenase system of animal tissue requirement is nonspecific; and all the other well characterized enzyme systems react most readily with the diphospho coenzyme.

Several other reactions have been reported to be activated when these coenzymes are added to specific substrates in the presence of unrefined preparations of the apoenzymes. These include several interesting chemical transformations; the reduction of nitrate to nitrite, ${ }^{52}$ the oxidation of luciferin (the substrate that on oxidation produces bioluminescence), ${ }^{53}$ and the dehydrogenation of formic acid. ${ }^{54}$ When crude enzyme preparations are used, there is always a chance of the observed reaction being a secondary effect and further characterization should be undertaken before the reaction is definitely catalogued as to its type.

\section{The Riboflavin-Containing Coenzymes}

The role of riboflavin-containing enzymes as hydrogen carriers was first definitely established in 1932 when a yellow enzyme isolated from yeast was found to serve as a connecting link in a complex system in which hexose monophosphate is oxidized and molecular oxygen reduced. ${ }^{55}$ The specific purpose of the flavoprotein in this system is to catalyze the reaction in which the hydrogen atoms accepted by triphosphopyridine nucleotide from the substrate are passed on to oxygen. Later, other flavoproteins were found to function in a comparable fashion with diphosphopyridine nucleotide, and it became evident that one of the fundamental purposes of the riboflavin enzymes is to serve as "bridges" over which the hydrogen atoms accumulating on the nicotinic acid coenzymes can be passed to systems which will carry out the reduction of oxygen. An 
equally important function for the riboflavin coenzymes was also early appreciated-that of being the initial acceptor for hydrogen atoms in a number of metabolically important dehydrogenations of aldehydes, amino acids, and purines.

When the yellow enzyme was discovered, it was realized that the yellow pigment possessed the characteristics of a "flavin," a class of biological pigments observed as early as $1879 .{ }^{56}$ In 1933 , riboflavin was isolated in crystalline form and shown to possess vitamin activity for the rat. ${ }^{57}$ At first, it was assumed that riboflavin itself was the chromophoric com-<smiles>Cc1cc2nc3c(=O)[nH]c(=O)nc-3n(CC(O)c3ccccc3)c2cc1COP(=O)(O)O</smiles>

Riboflavin phosphate

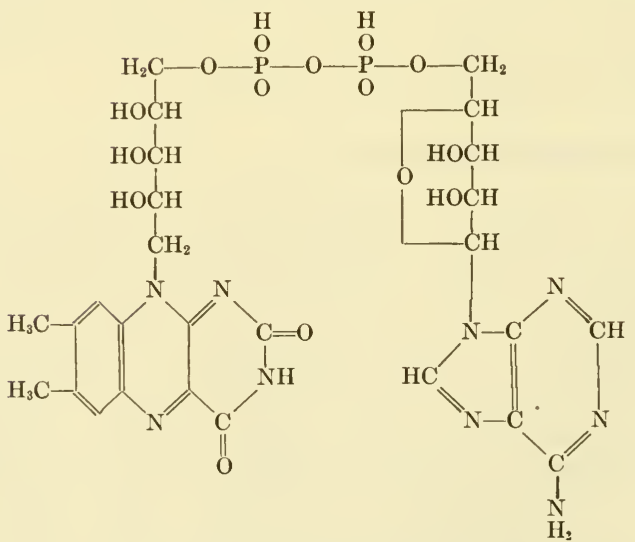

Flavin adenine dinucleotide 
ponent of the yellow enzyme; but the addition of pure riboflavin to the protein component did not result in reconstitution of the flavoprotein. Isolation of the pure coenzyme from the enzyme showed the coenzyme to be a phosphoric acid ester of riboflavin. ${ }^{58}$

Riboflavin forms two coenzymes. The simpler one is the phosphoric acid ester just mentioned. This ester is often referred to as a mononucleotide, although in a strict sense this is incorrect since the compound is derived not from the sugar, D-ribose, but from the corresponding alcohol, D-ribitol. The more complex coenzyme, called the dinucleotide, can be described as a molecule in which adenylic acid (muscle) and riboflavin phosphate are united by the formation of a pyrophosphate bond.

The location of the phosphoric acid at the 5 position on the ribityl unit has been definitely established. Oxidation of the mononucleotide does not yield formaldehyde, a product that would be formed were the primary hydroxyl group unesterified. ${ }^{59} \mathrm{~A}$ synthesis of riboflavin-5'-phosphate by a method establishing the location of the phosphate confirmed the structure of the mononucleotide, since the synthetic product had the same coenzymatic activity as the naturally occurring compound. ${ }^{58}$

The structure of the dinucleotide has not been proved by synthesis. However, the identity of the two mononucleotides obtained upon hydrolysis is certain. ${ }^{60}$ The structure indicated in the formula above has been accepted as the most likely one for the dinucleotide.

Enzymes containing either of the two coenzymes are often referred to as yellow enzymes or flavoproteins. The coenzymes are sometimes designated by the method of classification in which the name of the aromatic nucleus, alloxazine (or more properly, isoalloxazine), is specified. The simpler expression, flavin adenine dinucleotide (FAD), is now more popular than the cumbersome expression isoalloxazine adenine dinucleotide.

The coenzymes of riboflavin resemble the parent vitamin in many of their physical and chemical characteristics: they exhibit the same characteristic color and fluorescence; they are decomposed when irradiated; they can be reduced by chemical reagents to leuco derivatives; they are autooxidizable (i.e., the reduced leuco compounds can be oxidized by air back to the original pigment); during reduction in strongly acidic solution there is formed a red intermediate having the properties of a semiquinoid radical; ${ }^{61}$ and the redox potential of the riboflavin phosphate system is comparable to that of a simple riboflavin system. ${ }^{62}$

The introduction of the acidic phosphate groups, however, makes the coenzymes acidic and profoundly affects the capacity of the molecule for combining with the apoenzyme (p. 145). 
Assay Methods. A D-amino acid oxidase system is the one commonly employed for the qualitative and quantitative determination of flavin adenine dinucleotide. ${ }^{63}$ No other naturally occurring flavin derivative is known to exhibit appreciable activity in this assay; hence, it is used as a specific test for the dinucleotide coenzyme. The apoenzyme concentrate used in the assay is obtained by resolving the enzyme present in crude extracts prepared from hog kidney. As in most flavoproteins, the pigment dissociates from the protein when a solution of the holoenzyme is acidified, and the protein component can usually be separated by ammonium sulfate precipitation. D-alanine is the substrate most often used with this sytsem, and oxygen is used as the hydrogen acceptor, since methylene blue is reduced very slowly. The rate of the reaction is followed by measuring the oxygen uptake or by determining quantitatively either the amount of ammonia or of the keto acid formed by the oxidation of the amino acid.

No enzymatic method has been developed to determine specifically riboflavin phosphate. The use of the "old yellow enzyme"- the system in which riboflavin phosphate was first recognized-will not suffice, since the system is quite nonspecific in regard to its coenzyme requirements. The dinucleotide, as well as certain riboflavin derivatives prepared synthetically, can reactivate the apoenzyme (p. 150). Cytochrome reductase or L-amino acid oxidase, the only other enzyme systems whose coenzymes are definitely known to be riboflavin phosphate, may be used. Whether these systems are specific or not, however, is uncertain since no report has been made concerning the activity of the dinucleotide when tested with the apoenzymes of these protcins.

Occurrence. Qualitatively, it is known that the coenzymes of riboflavin have a widespread distribution in cells and tissues. The presence of flavoenzymes (especially the dinucleotide type) has been demonstrated in a variety of material, and the coenzymes themselves have been concentrated and isolated from microorganisms and several different animal tissues. Much of the intracellular riboflavin is in a bound form from which it can be liberated by phosphatases. If riboflavin resembles the other $\mathrm{B}$ vitamins in respect to the distribution of the free vitamin and its coenzymes, one would expect the intracellular vitamin content to reflect predominantly the concentration of the coenzymes. However, practically no quantitative data, based on actual determination of the coenzymes, are yet available, nor is it possible to estimate the relative concentrations of the two coenzymes.

Biosynthesis. The biosynthesis of riboflavin phosphate from the vitamin has been carried out in vitro by using phosphorylating enzymes of the intestinal mucosa of mammals. ${ }^{64}$ The biosynthesis of the dinucleotide has never been observed except in intact cells. Since this coenzyme cannot 
be prepared synthetically, it can be obtained only by direct isolation from tissues or yeast, or by separating it from preparations of its flavoproteins. The ability to synthesize the two coenzymes must be common to most living organisms, since no instances are known in which either of the intact coenzymes must be supplied to an organism.

Properties of the Flavoproteins. When the riboflavin coenzymes combine with their apoenzymes to form the flavoproteins, certain changes can be detected in the properties of the pigment. The flavin no longer exhibits its characteristic fluorescence, although its yellow color is imparted to the enzyme. ${ }^{62}$

The redox potential of the flavoprotein system is appreciably higher than for the uncombined flavins, i.e., the flavoprotein has a greater tendency to accept hydrogen atoms and become reduced. ${ }^{62}$

Several different lines of evidence indicate that riboflavin phosphate is united to its apoenzyme through both the acid group of the phosphate ester and the imide nitrogen of the isoalloxazine nucleus, presumably by the formation of salts with acidic and basic groups of the protein: the affinity of unphosphorylated riboflavin for the protein is small; the substitution of groups upon the imide nitrogen destroys all vitamin and coenzyme activity; the formation of salts of the imide is known to destroy the fluorescence of isoalloxazines; and the flavin loses its fluorescence when it combines with apoenzymes to form the flavoproteins.

Unlike the nicotinic acid coenzymes, the flavin coenzymes do not have to alternate from one apoenzyme to another during the progress of a reaction. Hence, (in neutral solutions) the riboflavin enzymes are only slightly dissociated. For this reason, when the "old yellow enzyme" is resynthesized from its resolved parts, the apoenzyme takes up the added riboflavin phosphate in stoichiometric fashion and is completely saturated by the time an equimolecular amount of coenzyme is added. Flavoproteins are not readily resolved by dialysis in neutral solution, although they are easily dissociated in acidic solutions in which the pigment dialyzes away from the protein. ${ }^{65} \mathrm{~A}$ simpler procedure is to salt out the protein from the acid solution leaving the prosthetic group in the supernatant liquid. ${ }^{66}$ This difference in the stability of the intact enzyme under neutral and acidic conditions is probably due to ionic and tautomeric changes in the groups of the coenzyme and the protein which form the salt linkages.

In addition to the differences in motility of their respective coenzymes, there are a number of other dissimilarities in the enzymatic reactions catalyzed by flavoproteins and those catalyzed by the nicotinic acid systems: (1) a considerably smaller number of reactions have been characterized for the flavoproteins; (2) there is much more specificity in the coupling of the two systems which oxidize and reduce the flavoproteins 
than there is for the nicotinic acid enzyme systems, e.g., a flavoprotein which is reduced by a particular substrate can be reoxidized only by certain specific substances, whereas a pyridine nucleotide reduced by one metabolic system can be reoxidized by any of a number of other metabolic systems; (3) many of the reactions catalyzed by the phosphopyridine coenzymes are those in which the potentials of the reacting systems approximate those of the coenzymes, and hence the reactions may proceed in either direction. The redox potential of riboflavin, however, is intermediate between the low values of most organic metabolite systems (and the nicotinic acid coenzymes) and the high values of the cytochrome systems or oxygen itself. The differences are of sufficient magnitude that most reactions catalyzed by flavoproteins may proceed effectively in one direction only. Consequently, reversal of the direction in which a reaction catalyzed by a flavoprotein proceeds is seldom encountered, and it has become customary to specify one system as the hydrogen donor and the other as the hydrogen acceptor, rather than to treat them in general terms of coupled systems.

When the coenzymes of riboflavin are alternately reduced and oxidized it is believed to be by a process in which the hydrogen atoms are accepted or donated one at a time. This mechanism is possible since the flavin nucleus can exist in an intermediate state of reduction in which a stabilized semiquinoid radical is formed-a process which can be observed experimentally. ${ }^{62}$ The predominant structures among the various resonance forms of the coenzymes are indicated as:
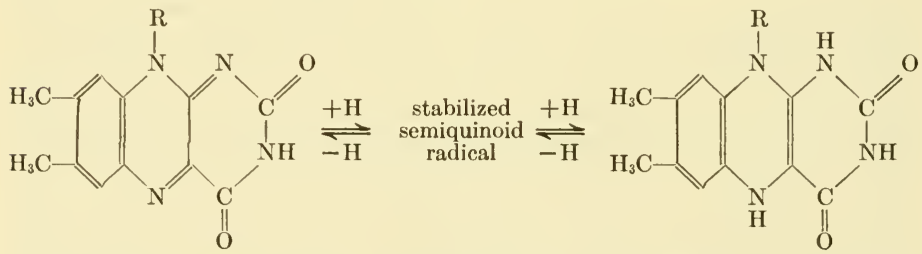

Reactions Catalyzed by Flavoproteins. For discussion, the reactions catalyzed by flavoproteins may be classified in two groups: (1) those in which the enzyme reacts directly with the primary substrate being metabolized, and (2) those in which the pigment is a secondary acceptor of the hydrogen atoms. The substrates with which the flavoproteins have been shown to react directly are D-amino acids, L-amino acids, glycine, L-hydroxy acids, aldehydes, purines, and substances not ordinarily encountered under natural conditions, such as quinine and reduced dyes. In the other group of reactions, the flavoprotein reacts with an "intermediate carrier," which is either one of the two dihydropyridine nucleo- 
tides or a reduced thiamine system which has catalyzed an oxidative decarboxylation (p. 165).

The reactions of flavoproteins could equally well be classified on the basis of the substances which have been found to be hydrogen acceptors: (1) the cytochromes, (2) molecular oxygen, (3) fumaric acid, and (4) artificial acceptors such as methylene blue.

An interpretation of the primary intracellular functions of flavoproteins on the basis of results obtained from isolated proteins is hazardous. A number of instances have been observed in which it appears that the enzyme after isolation has lost its ability to react with certain substrates. The rate at which some of the isolated enzymes catalyze reactions in vitro is too slow to enable them to be of importance in vivo. With some flavoproteins which have been isolated it has been impossible to reconstruct systems using substrates known to occur in living cells. It is still impossible to characterize completely the most important (from a quantitative standpoint) reaction which flavoproteins mediate in aerobic organisms, the dehydrogenation of reduced dihydro-diphosphopyridine nucleotide.

The changes which may occur in the enzymatic capacities of the flavoproteins during their concentration make it difficult to establish the identity of enzymes studied by different investigators. On several occasions enzymes have been obtained from the same source material in separate laboratories and not all their properties have. checked. Consequently, the reactions of the flavoproteins will be classified on a general basis according to their substrates and no attempt will be made to characterize the individual enzymes as reported.

Amino acid oxidases. Oxidation of amino acids is a two-step process in which (1) the $\alpha$-amino group is dehydrogenated to form an $\alpha$-imino acid, and (2) the imino group hydrolyzes spontaneously to yield ammonia and the corresponding keto acid.

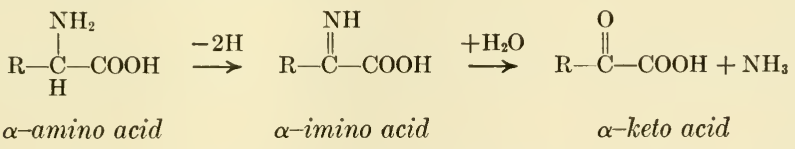

Three types of amino acid oxidases which are flavoproteins have been recognized: (1) D-amino, (2) L-amino, and (3) glycine. The potential of these systems is such that the reverse process could not be effectively utilized directly for amino acid synthesis.

D-amino acid oxidase catalyzes the oxidation of all the common D-amino acids except D-lysine, although there are extreme differences in the rate of reaction of the individual amino acids. ${ }^{67}$ The enzyme occurs in most animal tissues, but its purpose is not understood since its sub- 
strates are not the naturally occurring isomers. Only the dinucleotide can function as the prosthetic group of this flavoprotein.

L-amino oxidase ${ }^{68}$ (L-hydroxy acid oxidase) is an interesting and unusual enzyme in that it will not only catalyze the oxidation of at least thirteen amino acids but will also bring about comparable dehydrogenation of $\mathrm{L}-\alpha$-hydroxy acids having structures related to the $\alpha$-amino acids. ${ }^{69}$ Its coenzyme is riboflavin monophosphate. ${ }^{70}$ The enzyme was isolated from rat kidney. It has been shown that certain bacteria possess a similar enzyme, some properties of which differ from the animal preparations. ${ }^{71}$ Molds are the source for a third preparation, the activity of which differs in minor respects from both the mammalian and bacterial enzymes. ${ }^{72}$ One of the active principles in snake venom is an L-amino acid oxidase, and although its relation to the other oxidases just mentioned has not been ascertained, it is believed to be a flavoprotein. ${ }^{73}$

Glycine is oxidized by neither of these types of enzymes, but is attacked by a specific enzyme, glycine oxidase. ${ }^{74}$ The cofactor is flavin adenine dinucleotide.

Aldehyde oxidases. It has been shown that there are three flavoproteins capable of catalyzing the oxidation of aldehydes. It is believed that each enzyme of this group contains adenine dinucleotide as its prosthetic group. Milk was the first substance to be used as a source of an "aldehyde oxidizing" flavoprotein, an enzyme which catalyzes the dehydrogenation of formaldehyde hydrate and other aliphatic and aromatic aldehydes. ${ }^{75}$ Strangely enough, this enzyme, or another flavoprotein which cannot be separated from it, was later found to catalyze the oxidation of hypoxanthine and xanthine. ${ }^{76} \mathrm{~A}$ second aldehyde oxidase was isolated from plant tissues, ${ }^{77}$ but this enzyme was incapable of oxidizing purines. That it is likewise a flavoprotein has never been demonstrated. Liver has been found to contain an aldehyde oxidase which differs from liver xanthine oxidase. ${ }^{78}$ Penicillium notatum produces a flavoprotein which was prematurely classified as an antibiotic, notatin. ${ }^{79}$ Further investigation showed this flavoprotein to be a glucose oxidase. The bactericidal effect of this enzyme is observed only in the presence of glucose and oxygen in which case hydrogen peroxide accumulates, killing bacteria which do not contain peroxidases. ${ }^{80}$ It has been shown that xanthine oxidase can be made to demonstrate similar action against certain bacteria. ${ }^{81}$

Xanthine oxidase. Xanthine oxidase catalyzes the oxidation of hypoxanthine and xanthine to uric acid by removing two hydrogen atoms from the hydrated purine base.

Milk serves as an especially good source of this enzyme, although it is found widely distributed in animal tissues. Highly purified preparations 
of this enzyme show it to be a flavoprotein containing the riboflavin adenine dinucleotide prosthetic group ${ }^{66,82}$ and perhaps an additional nonflavin cofactor. ${ }^{83}$ No naturally occurring purine other than hypoxanthine and xanthine is acted upon by this enzyme. ${ }^{84}$ This high specificity is interesting when compared with the much lower substrate specificity exhibited by the aldehyde and amino acid oxidases.

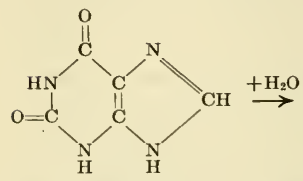

xanthine

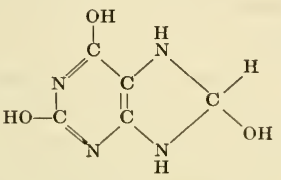

xanthine hydrate

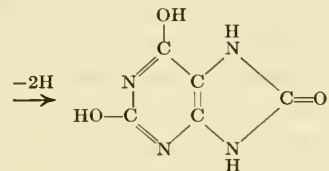

uric acid

Oxygen is the common hydrogen acceptor for the amino acid, aldehyde and purine oxidases. Methylene blue can be substituted in most of the reactions if the Thunberg technique (rate of decolorization) is used. In the case of the D-amino acid oxidase, however, the dye is reduced much more slowly than is oxygen. In no case is there any evidence that a cytochrome system is linked with these flavoproteins which catalyze the direct oxidation of metabolites, i.e., when riboflavin coenzymes directly accept the hydrogen atoms of organic substrates (rather than through the intermediation of the pyridine nucleotides), they transport these atoms directly to oxygen instead of reducing a cytochrome.

The second group of flavoproteins differs from those just discussed in at least two important respects: (1) they do not directly catalyze the dehydrogenation of a metabolite, but have for substrates the reduced forms of intermediate hydrogen carriers; (2) although they react with oxygen slowly, cytochrome systems are believed to be the hydrogen acceptors in vivo.

Flavoproteins can accept hydrogen atoms from three different reduced coenzyme-enzyme systems: (1) diphosphopyridine nucleotide, (2) triphosphopyridine nucleotide, and (3) probably a reduced thiamine enzyme system.

The oxidation of reduced diphosphopyridine nucleotide is the specific function of flavoproteins (designated diaphorases) isolated from yeast, ${ }^{61}$ heart muscle, ${ }^{85}$ and milk. ${ }^{86}$ They possess the following common properties: (1) their coenzymes are dinucleotides; (2) they can be oxidized by methylene blue or oxygen, although the latter process is slow; and (3) there has been no definite demonstration of a system linking these particular flavoproteins with any specific cytochrome system. Although it is still believed that there is some means by which these flavin mediators can pass the hydrogen atoms from reduced diphosphopyridine nucleotide 
to a cytochrome system, and although extensive study has been devoted to this problem, as yet the proof of such a mechanism is lacking.

The oxidation of the reduced triphosphopyridine nucleotide is carried out by cytochrome c reductase. This enzyme has been isolated from yeast and differs from the diaphorases mentioned above in that (1) its coenzyme is the riboflavin mononucleotide, (2) its substrate is the triphosphopyridine coenzyme, and (3) that the cytochrome system to which it is linked has been identified. ${ }^{s 7}$

Two flavin enzymes: (1) the "old yellow enzyme" 55 which was isolated from yeast and contains riboflavin phosphate as the prosthetic group, and (2) the synthetic yellow enzyme ${ }^{66}$ in which the dinucleotide is substituted for riboflavin phosphate upon the same apoenzyme, may act upon either the di- or triphosphopyridine nucleotides. No cytochrome system has been shown to function with either of these enzymes; the flavoproteins in this case pass the hydrogen atoms directly to oxygen at a sluggish rate and the protein is believed to be a "derived" enzyme rather than a "native" one.

The other secondary hydrogen donor that is coupled with flavoproteins is the reduced thiamine system. When thiamine functions in oxidative decarboxylation reactions involving the removal from $\alpha$-keto acids of carbon dioxide and two hydrogen atoms (see p. 165), either the thiamine coenzyme or some group of the apoenzyme must be temporarily reduced. It has been shown, using a crude protein extract from bacteria, that a flavin dinucleotide must be present when this type of reaction is carried out aerobically. ${ }^{88}$ On this basis it has been postulated that a yellow enzyme is essential for the reoxidation of the reduced thiamine systems.

The mechanism by which energy is conserved in aerobic respiration is still not clear. Energy balances indicate that there are approximately three high-energy phosphate bonds formed for every two hydrogen atoms passed from a substrate via the phosphopyridine and riboflavin nucleotides to oxygen. ${ }^{89}$

The flavin-containing enzymes which have been enumerated function primarily as carriers of hydrogen atoms in processes which are believed to be unidirectional in their natural environment, i.e., the net process is always one in which organic substrates are the hydrogen donors, and molecular oxygen (in vivo) is the final acceptor of these atoms. Does riboflavin function in anaerobic systems? Undoubtedly flavoproteins catalyze reactions carried out by anaerobic organisms, and in these instances the enzymes must have some organic substrate as a hydrogen acceptor. To date only one example of this type of reaction has been disclosed i.e., fumaric dehydrogenase, a flavin adenine dinucleotide enzyme.$^{90}$ Fumaric acid appears to be the specific hydrogen acceptor, but 
as yet no naturally occurring hydrogen donor has been found and the catalytic role of the flavoprotein can be demonstrated only when an artificial donor (a leuco dye) is used.

It is of interest to note that a flavoprotein can catalyze the conversion of fumaric to succinic acid because it may be indicative of a general function of flavoproteins which has not yet been established-that of being a general catalyst for the production or saturation of ethylenic bonds. A difficultly soluble protein, succinoxidase, catalyzes a similar reaction (reverse direction) in which succinic acid is dehydrogenated and a cytochrome is reduced.91 Whether this enzyme is a flavoprotein is questionable, but it has been shown that the concentration of succinoxidase is less in the tissues of animals depleted of riboflavin than in the tissues of animals on adequate diets. ${ }^{92}$

\section{The Cytochromes}

Numerous compounds which are derivatives of porphyrins are essential for extremely diverse types of biological function ranging from the oxygen-transporting duties of hemoglobin to the energy transformations catalyzed by chlorophyll. The porphyrins of particular interest in a discussion of the functioning of the B vitamins are the ones which act in the same oxidation systems as do riboflavin and nicotinic acid. These compounds are the cytochromes. It is believed that in the cells of all aerobic organisms the cytochromes act as the final mediators in most, but not all, processes in which the utilization of oxygen takes place.

The porphyrins have many properties in common with the B vitamins: they are found in all forms of life which make use of aerobic processes; their distribution parallels other B vitamins; and they constitute a nutritional requirement for some bacteria and protozoa. ${ }^{93}$ Except for one class of insects, ${ }^{93 a}$ no higher types of life, however, have been encountered which cannot synthesize their own requirements for the porphyrins. Since all the known reactions of the porphyrin-containing enzymes are those in which molecular oxygen is involved, it is not surprising that organisms which are true anaerobes do not contain detectable amounts of porphyrin derivatives.

There is still considerable confusion regarding the identity and chemical function of the cytochromes that have been recognized and characterized almost entirely on the basis of their absorption spectra-cytochromes a, $a_{1}, a_{2}, a_{3}, b$ and $b_{2} .{ }^{94}$ Two cytochrome components, cytochrome $c$ and cytochrome c oxidase, however, have been well characterized on the basis of their enzymatic activity, and their function in biological oxidations has been well established. 
Cytochrome $\mathrm{c}$ is a protein (M.W. about 13,000 ) containing as a prosthetic group the same iron-porphyrin unit, hematin, as occurs in hemoglobin. ${ }^{95}$ Unlike hemoglobin, though, it functions by changing its valency state, alternately existing as a ferro and ferric complex. It is not autooxidizable but can react with molecular oxygen only in the presence of a specific enzyme, cytochrome c oxidase, which is itself a porphyrin derivative.

The reduction of cytochrome $\mathrm{c}$ in vitro at a rate compatible with the requirements of an actively metabolizing cell can be demonstrated either with (1) certain dehydrogenases of organic metabolites for which no other coenzyme requirement is known to exist, or with (2) the reduced triphosphopyridine nucleotide, provided the neecssary flavin catalyst, cytochrome $\mathrm{c}$ reductase, is present. $^{87}$ It seems logical to expect that a third type system, involving the enzymes by which the majority of dehydrogenations are initiated, the diphosphopyridine dehydrogenases, would likewise be associated with the cytochrome c mechanism. In spite of an intensive search, the enzyme (flavoprotein?) capable of linking the two systems has yet to be well characterized, although crude preparations which link the diphosphopyridine nucleotide-cytochrome c systems have been reported..$^{96,97}$

The dehydrogenases having no recognized coenzymes which presumably pass their hydrogen atoms directly to cytochrome $c$ are: succinic acid dehydrogenase, ${ }^{91}$ lactic acid dehydrogenase, ${ }^{98,99} \alpha$-glycerophosphate dehydrogenase II, ${ }^{100}$ a formic acid dehydrogenase, ${ }^{101}$ a fatty acid dehydrogenase, ${ }^{102}$ and sarcosine dehydrogenase. ${ }^{103}$ Most of these enzymes are intimately associated with the cell structure and cannot be brought into solution. Since tissues from riboflavin deficient animals have a subnormal amount of succinic acid dehydrogenase, ${ }^{92}$ a question can be raised concerning the possibility that not only this enzyme but also other insoluble cytochrome-linked enzymes may be flavoproteins.

In addition to these natural enzymatic systems, a number of phenols and amines can directly (nonenzymatically) serve as artificial agents for reducing cytochrome c. $^{95}$

As is the case with the other dehydrogenase coenzymes, the reduction of cytochrome $\mathrm{c}$ is brought about by a univalent change in which the cytochrome $\mathrm{c}$ accepts the hydrogen atoms one at a time from the organic substrates or reduced coenzymes. Each atom so transferred in effect reduces a ferric-porphyrin group to the ferro complex with the accompanying creation of a hydrogen ion:

$$
[\mathrm{H}]+\mathrm{Fe}^{+++} \text {complex } \rightleftarrows \mathrm{H}^{+}+\mathrm{Fe}^{++} \text {complex }
$$

The reduction causes a very distinctive alteration in the absorption spectra of the porphyrin complex which can be followed spectrophoto- 
metrically. ${ }^{104}$ An even more sensitive method is a polarigraphic one. ${ }^{105}$

The reoxidation of reduced cytochrome $\mathrm{c}$ is brought about by molecular oxygen in the presence of cytochrome oxidase, a porphyrin-containing protein so intimately bound within the structure of cells that it was once assumed that it could not be extracted and obtained in solution. A soluble preparation, however, has been recently reported. ${ }^{106}$ The exact mechanism of the reaction catalyzed by this enzyme is obscure. Since no hydrogen peroxide results from the reaction, the oxygen molecule either is reduced by a process which does not proceed through the reduction state corresponding to hydrogen peroxide, or else this state constitutes an unstable intermediate which is instantaneously decomposed. In view of the general peroxidase activity of the iron-porphyrin enzymes, the latter postulate seems the more reasonable.

When the cytochrome-cytochrome c oxidase systems are blocked by the heavy metal poisons, $\mathrm{CN}-, \mathrm{H}_{2} \mathrm{~S}, \mathrm{NaN}_{3}$, etc., their function in reconstructed systems or in intact cells can be partially taken over by appropriate autooxidizable dyes. When these artificial hydrogen carriers are used, oxygen is always reduced to hydrogen peroxide.

One more type of iron-porphyrin enzyme, catalase, ${ }^{107}$ should be mentioned, to complete the presentation of the catalysts involved in dehydrogenations. In all reactions in which the utilization of molecular oxygen is accomplished by enzymes or catalysts not containing iron or copper, hydrogen peroxide is formed. If the peroxide were not decomposed at once it not only would destroy the enzyme system catalyzing its formation but would inactivate most adjacent proteins as well. Consequently, catalase is an essential component of all cells having aerobic metabolic processes, and hence is found widely distributed in nature.

\section{Coenzymes Essential for the Carboxylation and Decarboxylation of Keto Acids}

In the metabolic processes essential for life, a number of keto acids are continually being formed and utilized. Probably no other type of compound is capable of participating in such a variety of enzymatic reactions. Because of this, they occupy key positions in most metabolic processes and are the essential links which interconnect the metabolism of carbohydrates, proteins, and fats. From the standpoint of molecular turnover, one of the most important mechanisms by which these compounds are metabolized is decarboxylation, resulting in the cleavage of a carbon-tocarbon bond and formation of carbon dioxide (or sometimes its reductive product, formic acid). The reactions can most conveniently be grouped together as decarboxylative processes. On the basis of the coenzymes needed as catalysts, this group can be broken down into two definite classes: those which require thiamine pyrophosphate and those which do 
not. These two classes are also chemically distinct with respect to whether they involve $\alpha$ - or $\beta$-keto acids.

$\alpha$-Decarboxylation. Thiamine is essential only when the substrate is an $\alpha$-keto acid and only when the reaction results in the rupture of the bond between the keto carbon atom and the adjacent carboxyl group.

$\beta$-Decarboxylation. The other reactions in which carbon dioxide is formed from a keto acid are those which have been termed $\beta$-decarboxylations because they result in the direct decarboxylation of acids in which the keto group is $\beta$ to the reacting carboxyl group. Biotin has been associated with this type of reaction, although it is questionable if its role in these reactions is a direct one.

\section{The Coenzyme Derived from Thiamine}

Carboxylase, an enzyme which converts pyruvic acid to carbon dioxide and acetaldehyde, was one of the first components to be recognized in zymase, the complex of enzymes used by yeast in fermenting sugars. ${ }^{108}$ Some twenty years after its discovery it was shown (1932) that an essential thermostable organic component could be removed from the holoenzyme by washing with weakly alkaline solution. ${ }^{109}$ This coenzyme was designated as cocarboxylase. The isolation of the coenzyme in crystalline form was achieved by using an enzymatic assay method (reactivation of a carboxylase apoenzyme) to follow the concentration of the active principle. The chemical structure was established by both degradation and synthesis and found to correspond to the pyrophosphoric acid ester of thiamine. ${ }^{110}$

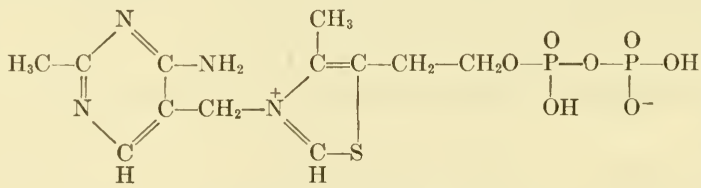

Thiamine pyrophosphate

The compound has been most often referred to as cocarboxylase, but other synonyms and abbreviations are frequently encountered-thiamine pyrophosphate (TPP), diphosphothiamine (DPT), and aneurin pyrophosphate (APP). This coenzyme should not be confused with codecarboxylase, the name often used to denote pyridoxal phosphate. The synonym, thiamine pyrophosphate, has been chosen as the one most suitable for use in the discussions which follow, since it indicates the vitamin component, describes the chemical nature of the coenzyme, and eliminates confusion with other types of coenzymes catalyzing decar- 
boxylations. Also, there are instances in which the reactions requiring "cocarboxylase" do not produce or utilize carbon dioxide.

Thiamine pyrophosphate can be prepared synthetically by chemical phosphorylation of the vitamin ${ }^{111}$ and can be purchased from commercial firms which stock biochemicals.

The conversion of thiamine to the phosphoric acid ester profoundly affects its biological reactivity even though, chemically, the change is not a drastic one. Upon oxidation the coenzyme forms a fluorescent compound, thiochrome pyrophosphate, by a reaction analogous to that which produces thiochrome from the vitamin; ${ }^{*}$ the coenzyme can also be cleaved by sulfurous acid into a pyrimidine and a phosphorylated thiazole; ${ }^{110}$ the phosphorylation of the vitamin does not alter the susceptibility of the molecule to cleavage by the "anti-thiamine" enzyme present in raw fish for the coenzyme is split just as rapidly by this means as is the vitamin.

The most obvious chemical change in forming the coenzyme is the creation of a strongly acidic compound from the organic base. This is accompanied by an increase in the resistance of the thiazole nucleus to reduction and reoxidation by chemical agents, ${ }^{112}$ a fact of some importance when considering possible mechanisms of the functioning of thiamine (p. 168).

Assay Methods. The determination of the thiamine pyrophosphate content of natural materials is usually carried out by a manometric procedure in which the rate of pyruvic acid decarboxylation is followed. The crude preparations of the apoenzyme needed for the procedure are obtained by washing dried yeast cells with an alkaline phosphate buffer. ${ }^{110}$ The extent of reactivation of the washed cells by extracts (in the presence of $\mathrm{Mg}^{++}$) is a direct measure of their coenzyme content.

Thiamine itself often shows some activity when it is added to crude apoenzyme preparations, presumably because of the presence of phosphorylating enzymes which convert the vitamin to the coenzyme during the course of the determination. When brewers' yeast is used as a source of the apoenzyme, free thiamine is found to exert an "activating" effect upon the carboxylase enzyme. ${ }^{113}$ Thiamine itself is completely inactive when added to the apoenzyme, yet when added to the apoenzyme along with the coenzyme, it increases the activity of the reconstructed system. This effect is observed even when the coenzyme is present in excess; hence the thiamine effect cannot be attributed to a direct synthesis of additional coenzyme. This "activation" effect is believed to be due to the ability of

* The acidic pyrophosphoric acid ester of thiochrome (obtained from the coenzyme) is not extracted from alkaline solutions by butyl alcohol; hence, the coenzyme does not interfere in the thiochrome assay for the free vitamin when the usual procedures are used (p. 46). 
thiamine to inhibit certain phosphatases present in the brewers' yeast which cause the hydrolysis and inactivation of the intact coenzyme. ${ }^{114}$ Thus thiamine can appear to be active in the system merely because it is sparing the destruction of its active derivative. The monophosphoric ester and the pyrimidine moiety of the vitamin behave similarly in "activating" the carboxylase system from brewers' yeast.

These interfering activities of the vitamin in the manometric assay for the coenzyme have been eliminated by using bakers' yeast instead of brewers', ${ }^{115}$ by adding sodium iodoacetate to inhibit the phosphorylation of thiamine, ${ }^{116}$ or by assaying all preparations in the presence of an excess of thiamine. ${ }^{117}$ Concise directions for carrying out the manometric determination of the coenzyme have been published in a standard reference text. ${ }^{118}$ An analyst using this procedure on crude materials should be aware of the many complicating effects which the extraneous matter can produce.

No organisms are known in which the thiamine requirements can be met only by its pyrophosphoric ester. Certain atypical strains of gonococcus respond much better to "a cocarboxylase-like substance" than to thiamine, but their requirement for the intact coenzyme is not absolute. ${ }^{119}$

Microbiological and chemical methods can be adapted to coenzyme analysis of tissues and biological products if it is known that all the "bound" thiamine is the pyrophosphate and if an assay method is used in which only the free vitamin is active (yeast growth and thiochrome methods). If such is the case, the difference in thiamine content of extracts before and after treatment with phosphatases will represent the amount of coenzyme. Due to the differences in solubility of the thiochromes resulting from the oxidation of thiamine and its phosphate esters, this chemical method can be adapted for the determination of both the vitamin and its phosphorylated derivatives. ${ }^{120}$

The presence of the coenzyme has been directly demonstrated in a number of different plant and animal tissues and in microorganisms. From the results obtained, it would appear that intracellularly most of the thiamine present is in the form of its coenzyme, whereas in plasma and other extracellular fluids (including urine and cerebrospinal fluid) the vitamin occurs predominantly in the free state. ${ }^{121}$ Practically all the microorganisms so far tested which cannot synthesize their thiamine requirements can utilize the free vitamin at least as effectively as the phosphorylated derivatives, whereas many organisms cannot use the coenzyme as a nutrient in place of the free vitamin. It is apparent, then, that the biosynthesis of the coenzyme must take place within the cells of most organisms and tissues.

Biosynthesis. The presence of enzymes capable of phosphorylating thiamine has been directly demonstrated in a number of different types 
of cells. The results of studies of the biological synthesis indicate that adenosine triphosphate is probably the usual phosphorylating agent. ${ }^{122,}{ }^{123}$ It is needed in only catalytic amounts, however, if other acyl phosphates are supplied or if the biosynthetic reaction is coupled with enzymatic processes in which reactive phosphate derivatives are created. A cell free extract of rat kidney which converts thiamine to its coenzyme has been reported. ${ }^{124}$

The pyrophosphate linkage in the coenzyme is readily hydrolyzed by the phosphatases (distinct from the phosphorylases catalyzing its synthesis) and usually any excess coenzyme present in a cell will be rapidly hydrolyzed. ${ }^{114}$ If, however, the coenzyme is combined with apocarboxylase it is quite resistant to attack by any hydrolytic enzymes present. This may be one explanation for the fact that the synthesis of the coenzyme from thiamine in a number of organisms is observed only as long as uncombined apoenzyme is present.

Mention has already been made of the inhibition of thiamine pyrophosphatases by thiamine and its derivatives. This type of inhibition has been observed only in certain yeast, however. It probably accounts for the exceptional case in which considerable unbound coenzyme can be found in yeast cells. If these particular yeasts are cultured in media containing appreciable amounts of thiamine, they absorb the vitamin almost quantitatively and convert most of it to the pyrophosphate (in amounts much greater than could be bound to carboxylase apoenzymes). ${ }^{125}$ The coenzyme synthesized by these cells is not hydrolyzed, since their specific phosphatases present have been inactivated by the high thiamine concentrations.

The pyrophosphate linkage between the two phosphate residues is also readily hydrolyzed by dilute acid. The monophosphoric acid ester formed by either the acid or enzymatic hydrolysis of the coenzyme is much more slowly attacked by phosphatases. The presence of appreciable quantities of the monophosphoric acid ester of thiamine in natural extracts probably is the result of the breakdown of the coenzyme during the preparation of the sample.

The mechanism for the formation of holoenzymes from thiamine pyrophosphate and its apoenzymes has been the subject of several studies. The easily prepared apocarboxylase from yeast has been used as the protein source for the most detailed investigation. ${ }^{126}$ The presence of the $\mathrm{Mg}^{++}$ or $\mathrm{Mn}^{++}$ion is essential for the union. Other divalent ions are much less effective. In earlier reports it had been assumed that the artificially reconstituted holoenzyme was different from the original native system, since the addition of a given amount of thiamine pyrophosphate to washed yeast cells did not elicit as great a response as was obtained when unwashed cells containing the equivalent amount of the coenzyme were 
used. ${ }^{127}$ It is now believed that the difference is due to the presence of inactivated apocarboxylase and possibly other apoenzymes which combine with some of the added coenzyme and render it unavailable to the active apocarboxylase. ${ }^{126}$

That the pyrophosphoric acid group is at least partially responsible for the association of the apoenzyme with the coenzyme is indicated by the inhibition of carboxylase activity by other molecules containing pyrophosphate groups-adenosine triphosphate ${ }^{123}$ and thiazole pyrophosphate ${ }^{128} \mathrm{Ca}^{++}$ions interfere with the formation of the holoenzyme, presumably by competing with the $\mathrm{Mg}^{++}$or $\mathrm{Mn}^{++}$ions. ${ }^{129}$ Consequently, it is desirable to use water free from calcium in the preparation of apoenzymes. This interference may account for some of the discrepancies found in the earlier studies on the recombination of the carboxylase holoenzymes.

Phosphorylated thiamine does not pass through cell membranes easily. ${ }^{130}$ This accounts for its relative inactivity as a thiamine source for certain microorganisms, and explains why it is not as effective as thiamine in stimulating decarboxylations by tissues from deficient animals. ${ }^{131}$ The impermeability of cell membranes to the intact coenzyme may account for the effects observed when thiamine and its pyrophosphate were tested for their relative activities in reversing the inhibition of bacterial growth induced by pyrithiamine. The vitamin analogue more effectively inhibits the coenzyme than it does the vitamin-a phenomenon not ordinarily encountered. ${ }^{132}$ To account for this, it was postulated that thiamine is attached to the apoenzyme before it is phosphorylated, and that the coenzyme so formed is more firmly bound than is preformed coenzyme. An equally logical explanation is that the coenzyme added to the medium must be hydrolyzed before absorption can take place, thus necessitating subsequent resynthesis. The thiamine added in the free form would be more rapidly absorbed than would the thiamine which had to be first liberated from the coenzyme; hence the former would produce a higher intracellular ratio of thiamine to pyrithiamine and would be the more effective agent for reversing the inhibition.

Reactions Catalyzed by the Thiamine Coenzyme. The enzymatic reactions in which thiamine has been demonstrated to function in vivo are limited to only two substrates, pyruvic acid and $\alpha$-ketoglutaric acid, or their degradation products. Some of these enzyme preparations have been found to decarboxylate other $\alpha$-keto acids-for example, $\alpha$-ketobutyric and $\alpha$-ketovaleric acids. The latter compounds, though, have never been shown to be a part of metabolic processes, and there seems little reason to believe that these substances normally occur in vivo. Two other $\alpha$-keto acids which are important intermediates often formed during metabolism, 
oxalacetic acid and oxalsuccinic acid, may be assumed to undergo decarboxylative reactions analogous to those found for pyruvic and ketoglutaric acids. However, these two acids not only are $\alpha$-keto, but also $\beta$-keto acids, and they normally undergo $\beta$-decarboxylation rather than cleaving at the alpha bond.

The reactions of pyruvic acid which are catalyzed by the thiamine derivative were shown in 1936 not to be limited to simple decarboxylations. It was found that the coenzyme for carboxylase also is an essential component of the enzymatic systems by which pyruvic acid is oxidized in animal tissue, and that the initial reaction is one in which a dehydrogenation takes place simultaneously with the decarboxylation. ${ }^{133} \mathrm{~A}$ number of different processes are now known which require the thiamine coenzyme, but all can be explained on the basis of the catalysis of one of these two types of reactions: (1) simple decarboxylation of either pyruvic or $\alpha$-ketoglutaric acid, or (2) oxidative decarboxylation of one of these two metabolites.

The experimental work which led to the identification of most of the reactions in which thiamine pyrophosphate participates has been presented in detail in several reviews. ${ }^{88,} 134,135$

The coenzyme of thiamine has been shown to be essential for reactions in which the following substances are produced from pyruvic acid: acetaldehyde, acetic acid, molecular hydrogen, lactic acid, a reactive phosphorylated derivative of acetic acid, formic acid, acetoin (acetylmethylcarbinol), acetylethylcarbinol, dicarboxylic acids containing four carbon atoms, citric acid, $\alpha$-ketoglutaric acid, acetoacetic acid, and carbon dioxide and water. On the basis of the diverse chemical nature of the substances listed it might be inferred that a number of functions would necessarily have to be ascribed to the single catalyst. However, two related hypotheses, independently advanced, suggest mechanisms by which it is possible to explain in terms of a general type reaction most, if not all, of the reactions, both aerobic and anaerobic, catalyzed by thiamine pyrophosphate. ${ }^{136,137}$ Although the hypothetical intermediates postulated for these reactions have not been experimentally demonstrated (and it may be impossible to do so) it is believed that the extension of these hypotheses offers the most convenient method for presenting the reactions catalyzed by the thiamine coenzyme and enables one to better appreciate the chemical relationships between the various products of these reactions. The individual reactions will therefore be discussed from the standpoint of the postulated mechanisms.

The diverse reactions of pyruvic acid catalyzed by thiamine-containing enzymes can be logically explained if it is assumed that the holoenzyme activates pyruvic acid in a manner such that three fragments are made 
available for recombination in any one of a number of ways to produce the recognized products of pyruvic acid metabolism.

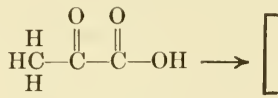

pyruvic acid<smiles>CC(Cl)(Cl)OC(=O)CCCCC(=O)C(=O)O</smiles>

ketenyl

radical

The specific products formed depend upon the manner in which the enzymes utilized or disposed of the $\mathrm{C}_{2}$ fragment (designated hereafter as a ketenyl radical) and the atoms of available hydrogen. The ketenyl radical can react (1) with water to form acetic acid, (2) with phosphoric acid to yield a phosphorylated derivative of acetic acid, (3) with acetaldehyde (or other aldehydes) to form acetoin (or its homologs), (4) with another ketenyl radical and hydrogen to form the dimer, diacetyl, or (5) with the available hydrogen atoms to form acetaldehyde.

The available hydrogen atoms also must be utilized in some fashion if they are not accepted by the hypothetical ketenyl radical (forming acetaldehyde) or the dimer, diacetyl (yielding acetoin). They can be accepted by another molecule of pyruvic acid, forming lactic acid; they can be disposed of in the form of molecular hydrogen; they can associate themselves with the elements of carbon dioxide to produce formic acid; or under aerobic conditions, the hydrogen atoms can be taken by riboflavin-containing enzymes to be passed on and eventually accepted by molecular oxygen. The three reactions of $\alpha$-ketoglutaric acid known to be catalyzed by thiamine pyrophosphate are analogous to three of the reactions in which pyruvic acid is the substrate.

Reactions of Pyruvic Acid Catalyzed by Thiamine Pyrophosphate. Thiamine pyrophosphate and a divalent cation $\left(\mathrm{Mg}^{++}\right.$or $\left.\mathrm{Mn}^{++}\right)$and the appropriate apoenzyme have been shown to be the coenzymatic factors for the following eight reactions:

(1) Simple Decarboxylation. The simplest reaction which thiamine pyrophosphate catalyzes is the direct decarboxylation of pyruvic acid. When the keto acid molecule is cleaved, the hydrogen atoms become attached to the $\mathrm{C}_{2}$ fragment and the end products are acetaldehyde and carbon dioxide:

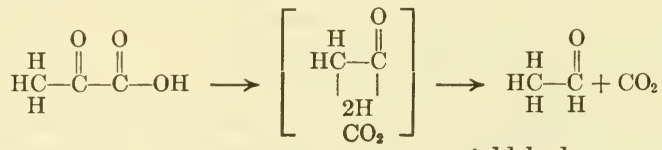


This reaction is a necessary step in the production of ethanol from sugar and constitutes the primary method of pyruvate metabolism in yeast when cultured anaerobically. Carboxylase, the enzyme catalyzing this reaction, has been shown to occur in yeast, bacteria, fungi, and higher plants, but it never has been found to constitute a part of the enzymatic systems by which carbohydrates are utilized in animal metabolism. Free phosphoric acid is not required for this particular reaction since no utilizable energy units are produced.

(2) Acetoin Formation. When acetaldehyde is added to preparations from animal tissues capable of metabolizing pyruvic acid, it is found that acetoin is formed. ${ }^{138}$ The reaction is presumed to involve a condensation of a reactive ketenyl radical (arising from pyruvic acid) and a molecule of acetaldehyde forming diacetyl which then acts as the acceptor for the two hydrogen atoms.<smiles>O=CC(=O)O</smiles>

pyruvic acid<smiles>C=O</smiles>

acetaldehyde

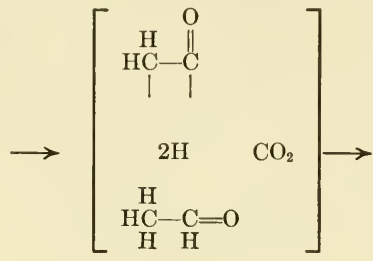

$\mathrm{H} \quad \mathrm{H}$

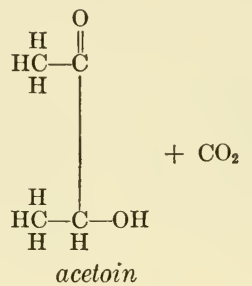

The requirement for inorganic phosphate ion in connection with this reaction has not been settled. If an energy-containing phosphate intermediate were formed, it would be decomposed and its energy utilized in the condensation creating the carbon-to-carbon bond (p. 189). When propionaldehyde was used as a substrate with pyruvate instead of acetaldehyde, the homologue of acetoin, acetylethylcarbinol, was the product of the reaction.

In the absence of acetaldehyde muscle tissues still produce acetoin from pyruvate but only at one-fourth the rate, and the yield from a given amount of pyruvate is only half that which would be obtained in the presence of acetaldehyde. Although the investigators could not detect free acetaldehyde as an intermediate under these conditions, it is presumed that the reaction is the result of a two-step process in which acetaldehyde is a transitory intermediate. The requirement of phosphate for this reaction has not been determined.

In certain bacteria acetoin is the primary product from the anaerobic decarboxylation of pyruvic acid. ${ }^{139}$ The mechanism of acetoin formation in Aerobacter aerogenes apparently is somewhat different from that in 
animal tissues, since acetaldehyde has not been shown to increase the yield, i.e., both of the $\mathrm{C}_{2}$ radicals which condense must be formed from pyruvic acid. It is possible that added acetaldehyde does not unite with the enzyme system to form the necessary enzyme-substrate complex, whereas the "acetaldehyde," or its equivalent, produced in situ condenses instantaneously. Inorganic phosphate was found to be essential for this reaction, but no phosphorylated intermediates have been directly demonstrated.

The reverse reaction, in which carbon dioxide is assimilated, has been reported. ${ }^{140,1+1}$ The enzyme preparation did not require a thiamine coenzyme for activation, but no evidence was presented to prove that it was not a bound component of the material used.

(3) Formic Acid Production. Escherichia coli and certain other bacterial species have been shown to cleave pyruvic acid in the presence of inorganic phosphates. Until recently it was assumed that formic acid and acetyl phosphate were the primary products of this phosphoroclastic reaction. ${ }^{142}$ It has recently been shown, however, that the product first formed from the $\mathrm{C}_{2}$ radical is not acetyl phosphate but is a related compound whose structure is as yet unknown. ${ }^{248}$ This compound, which in this chapter is designated as the "phosphoryl-acetyl intermediate" to distinguish it from acetyl phosphate, is a very reactive acetylating agent as well as an efficient phosphorylating agent. It is a participant in all the reactions known to be mediated by the pantothenic acid coenzyme. (See p. 191 for a detailed discussion of its recognized properties.) The reaction by which it is formed may be considered to be one in which (1) the reactive $\mathrm{C}_{2}$ radical is combined in some fashion with phosphoric acid, perhaps through a common carrier (designated in the formula as $\mathrm{X}$ ), and (2) the hydrogen atoms associate themselves with the carbon and oxygen atoms which usually form carbon dioxide in the other types of reactions:
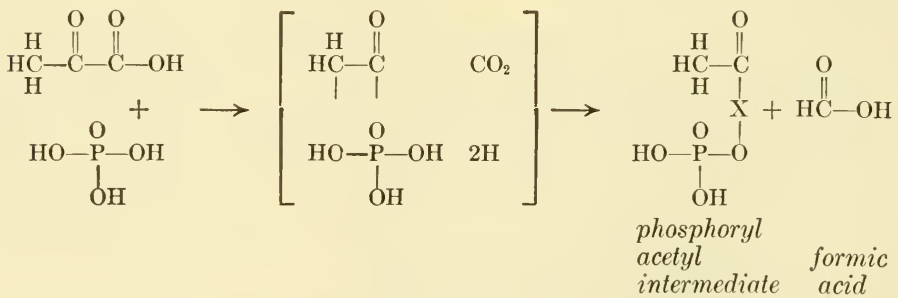

An alternate mechanism, advanced when the reaction product was presumed to be acetyl phosphate, was that phosphoric acid first formed an 
acid-carbonyl addition product with the keto acid before the cleavage of the carbon-to-carbon bond, ${ }^{143}$ as indicated below:

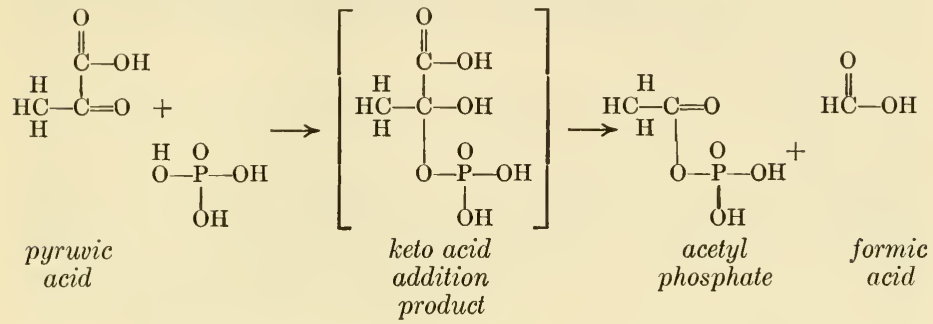

The acid-keto addition product of this earlier hypothesis could very well be an intermediate in the mechanisms recently postulated.

The "phosphoryl-acetyl intermediate," if not used immediately for acetylation, probably reacts with adenosine diphosphate, producing adenosine triphosphate and acetic acid. In this way most of the energy resulting from the degradation of pyruvic acid to acetic acid is conserved in the formation of a high-energy pyrophosphate bond which can be used by the cell for subsequent energy-requiring processes. The reactions for the overall process can be summed up in this equation:

\section{Pyruvic acid $+\mathrm{H}_{3} \mathrm{PO}_{4}$ +adenosine diphosphate $\longrightarrow$ \\ acetic acid +formic acid +adenosine triphosphate}

Attempts to demonstrate the reversibility of this process led to the experiments which clarified the nature of the acetyl derivative. Synthetic acetyl phosphate, when added to formic acid in the presence of the enzyme system, did not yield measurable amounts of pyruvic acid, but biological preparations of the phosphoryl-acetyl intermediate (prepared by an enzymatic synthesis from acetic acid and adenosine triphosphate) were found to be almost quantitatively converted to pyruvic acid when an excess of formic acid was used.142a

(4) Production of Molecular Hydrogen by a Phosphoroclastic Splitting of Pyruvic Acid. Clostridium butylicum possesses an enzyme which carries out a reaction similar to the one just discussed, except that the available hydrogen atoms are disposed of as molecular hydrogen instead of combining with the elements of carbon dioxide to form formic acid. Acetic acid was first thought to be a primary product of the reaction, ${ }^{144}$ but when phosphate was found to be an essential part of the system it was postulated, on the basis of substantial evidence, that acetyl phosphate rather than acetic acid was first formed, and that the acetic acid was a 
product of the decomposition of acetyl phosphate. ${ }^{144}$ On the basis of the recent finding discussed above, it is anticipated that the phosphorylacetyl intermediate will be found to be the initial product of the reaction rather than acetyl phosphate itself, and that the equation representing the reaction should be:

$$
\text { pyruvic acid }+\mathrm{H}_{3} \mathrm{PO}_{4} \longrightarrow \text { phosphoryl acetyl intermediate }+\mathrm{CO}_{2}+\mathrm{H}_{2}
$$

The reaction as indicated might be the result of the summation of two individual enzymatic reactions - a phosphoroclastic cleavage producing formic acid, immediately followed by the decomposition by a hydrogenlyase of the formic acid into carbon dioxide and molecular hydrogen. This mechanism is excluded, however, since the enzyme preparations do not decompose formic acid.

(5) The Acetic Acid-Lactic Acid Dismutation of Pyruvic Acid. One additional means of hydrogen disposal under aerobic conditions has been observed in bacterial cultures - the case in which the available hydrogen atoms are accepted by a second molecule of the pyruvic acid substrate. The products are acetic acid (phosphorylated derivatives?), carbon dioxide, and lactic acid.
(a) pyruvic acid $+\mathrm{H}_{2} \mathrm{O} \longrightarrow(2 \mathrm{H})+$ acetic acid $+\mathrm{CO}_{2}$ (or $\mathrm{H}_{3} \mathrm{PO}_{4}$ ?) (phosphorylated?)

(b) pyruvic acid $+(2 \mathrm{H}) \longrightarrow$ lactic acid

Net: 2 pyruvic acid $+\mathrm{H}_{2} \mathrm{O} \longrightarrow$ lactic acid + acetic acid $+\mathrm{CO}_{2}$

This dismutative anaerobic utilization of pyruvic acid has been observed in a number of animal tissues, ${ }^{145}, 146,147$ and in several species of bacteria. $88,147,148$

There is a question which has not yet been conclusively answered: Is the reaction in which the second molecule of pyruvic acid is reduced an independent reaction requiring a separate enzyme? If so, is a hydrogen carrier necessary to transfer the available hydrogen atoms from the thiamine enzyme to the pyruvic acid reductase? In one instance in which this reaction was studied in a cell-free system, the evidence favored the concept of coupled reactions and indicated that hydrogen carriers are needed to link the two distinct reactions of this dismutation. ${ }^{88}$

The use of radioactive isotopes has made it possible demonstrate the assimilation of carbon dioxide by the reverse process. ${ }^{149}$

(6) Aerobic Production of Acetic Acid. If a hydrogen acceptor (other than the intermediates or substrate) is available, pyruvic acid can be metabolized in the fashion indicated below. Under natural conditions, a riboflavin-containing protein is believed to accept initially the available 
hydrogen from the thiamine system, and these atoms are then aerobically metabolized via a hydrogen transport system.

$$
\begin{aligned}
& \text { pyruvic acid }+\mathrm{H}_{3} \mathrm{PO}_{4}+\text { riboflavin-containing enzyme } \longrightarrow \\
& \text { phosphoryl-acetyl intermediate }+\mathrm{CO}_{2}+\text { reduced flavoprotein }
\end{aligned}
$$

This type of reaction has been thoroughly studied using preparations from Lactobacillus debrückii as the enzyme source. ${ }^{133}$ Phosphoric acid is an essential component of this system and undoubtedly is utilized in the same manner as in the reactions previously described. If, however, the phosphoryl-acetyl intermediate is not required for synthetic purposes, it is degraded, its available energy dissipated as heat, and acetic acid becomes the end product of the process:

$$
\text { phosphoryl-acetyl intermediate } \longrightarrow
$$

$$
\text { acetyl phosphate } \stackrel{\mathrm{H}_{2} \mathrm{O}}{\longrightarrow} \text { acetic acid }+\mathrm{H}_{3} \mathrm{PO}_{4}
$$

(7) Aerobic Utilization of Pyruvic Acid. Many of the diverse "reactions" in pyruvic acid metabolism formerly postulated are now believed to consist of a series of two or more enzymatic steps. All the processes have a common initial reaction catalyzed by thiamine pyrophosphate. This reaction is analogous to the one just described occurring in $L$. debruckii, except that in this case a pantothenic acid enzyme picks up the phosphoryl-acetyl intermediate from the thiamine system:

pyruvic acid $+\mathrm{H}_{3} \mathrm{PO}_{4}+$ riboflavin-containing enzyme + pantothenic acid coenzyme $\longrightarrow \mathrm{CO}_{2}$ +reduced flavoprotein + phosphoryl acetyl intermediate associated with pantothenic acid coenzyme

The many different ways in which the phosphoryl-acetyl intermediate can be utilized are taken up in the discussion of pantothenic acid function, but some of the important end products which have been associated with pyruvic acid and thiamine metabolism will be enumerated here. The phosphoryl-acetyl intermediate produced from pyruvic acid by the thiamine-catalyzed reaction is, in the presence of a suitable pantothenic acid system, used for: acetylating choline; acetylating aromatic amines; forming acetoacetic acid and its homologues, which are intermediates in fatty acid synthesis; condensing with oxalacetic acid to form cis-aconitic acid, which is a precursor of citric acid, $\alpha$-ketoglutaric acid (and glutamic acid), the $\mathrm{C}_{4}$ dicarboxylic acids, etc. For this reason thiamine or its coenzyme has been reported at one time or another as a necessary catalyst for each of these processes.

The condensation of the reactive intermediate with oxalacetic acid initiates the tricarboxylic acid cycle by which pyruvic acid is completely "oxidized" to carbon dioxide and water (p. 224). Consequently, normal pyruvic acid metabolism in animal tissues can proceed only in the 
presence of pantothenic acid and catalytic amounts of some $\mathrm{C}_{4}$ dicarboxylic acid (precursor of oxalacetic acid) in addition to a divalent ion, inorganic phosphate, thiamine pyrophosphate, a hydrogen transport system, and oxygen.

(8) Aerobic Oxidation of Pyruvic Acid in the Absence of Phosphate. Cell-free preparations have been prepared from animal tissue and bacteria which are capable of oxidizing pyruvic acid in the absence of inorganic phosphate. ${ }^{150}$ The reaction observed is:

$$
\text { pyruvic acid }+1 / 2 \mathrm{O}_{2} \longrightarrow \text { acetic acid }+\mathrm{CO}_{2}
$$

Thiamine pyrophosphate is an essential component of this system. Since a requirement for, or presence of any other cofactors, could not be demonstrated, the disposition of the hydrogen atoms in this system must be by a mechanism which has not been previously encountered. When cells metabolize pyruvic acid in this fashion they presumably cannot conserve in a chemical form the energy of the oxidation.

In the presence of thiamine pyrophosphate, the enzyme preparation referred to above can carry out another reaction, not wholly unrelatedthe dismutation of diacetyl. ${ }^{150}$ It may appear that this dismutation represents a new type of thiamine function, since it involves neither a keto acid nor a decarboxylation. However, if only one enzyme in the preparation is responsible for both pyruvic acid oxidation and diacetyl dismutation, the latter reaction can be considered as one in which the enzyme establishes an equilibrium between two different sets of end products of pyruvic acid metabolism through formation of their common intermediates, namely, ketenyl radicals and available hydrogen atoms. The equilibrium is such that the fragments, upon recombination, form primarily acetic acid rather than diacetyl; but the mechanism for the formation of acetic acid is the same as if the fragments had been formed by the decomposition of pyruvic acid. The overall reaction can be represented in this fashion:

(a)
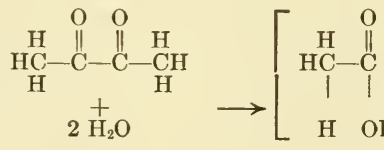<smiles>O=C(O)CC(=O)O</smiles>

$$
\begin{array}{cccc}
\mathrm{H} & \stackrel{\mathrm{O}}{\mathrm{O}} & \mathrm{OH} \\
\mathrm{HC}- & \stackrel{\mathrm{C}}{\mathrm{C}}-\underset{\mathrm{C}}{\mathrm{C}}-\mathrm{CH} \\
\mathrm{H} & & \mathrm{H} & \mathrm{H}
\end{array}
$$

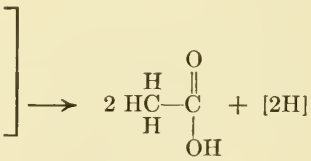

(b)

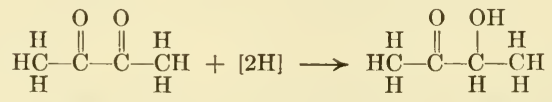

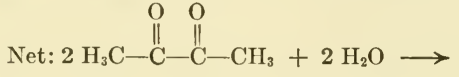

diacetyl<smiles>CC(=O)[18CH]CC(=O)C(C)O</smiles>

acetic acid

acetoin 
Reactions of $\alpha$-Ketoglutarate Catalyzed by Thiamine Pyrophosphate. $\alpha$-Ketoglutaric acid has been shown to be enzymatically decarboxylated by three different mechanisms.

(1) Simple decarboxylation ${ }^{150}$

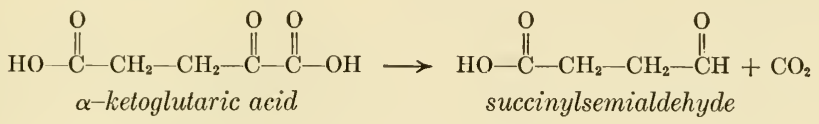

(2) Aerobic oxidation requiring phosphate ${ }^{151}$
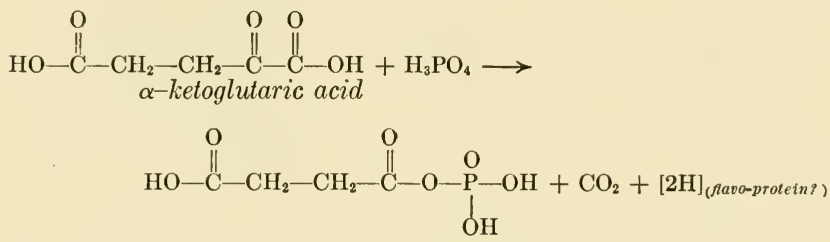

succinyl phosphate

(3) Aerobic oxidation independent of phosphate ${ }^{150}$

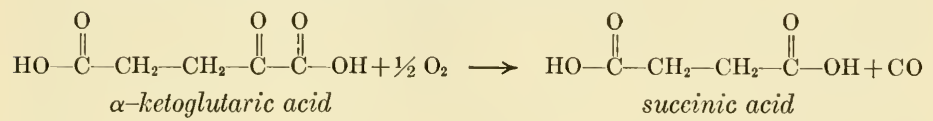

Each of these reactions resembles a comparable one in which pyruvic acid is the substrate; hence they need not be discussed in detail. The apoenzymes for the two substrates have similar physical properties, but they are not identical and cannot substitute for one another. The same ions $\left(\mathrm{Mg}^{++}\right.$or $\left.\mathrm{Mn}^{++}\right)$are required as cofactors. No demonstration has been made of the existence of a phosphorylated succinyl compound analogous to the phosphoryl-acetyl intermediate; hence succinyl phosphate is shown as the initial product of the phosphorylative oxidation.

A phosphoroclastic cleavage, forming succinyl phosphate and formic acid, has never been shown to occur in any organism. However, in muscles perfused with pyruvic acid considerable amounts of succinic and formic acids accumulate (p. 197). This suggests that there may be enzymes present to handle the $\alpha$-ketoglutaric acid (formed from pyruvic acid) by such an anaerobic cleavage if the oxidative decarboxylation system is overtaxed or not functioning.

Relationship of the Structure of Thiamine to its Function. Attempts have naturally been made to correlate the structure of thiamine with the mechanism by which its coenzyme functions. The initial formation of a 
Schiff's base by the elimination of the elements of water from the amino group of thiamine and the carbonyl group of the substrate has been postulated. ${ }^{137}$ The essentiality of the amino group on the pyrimidine ring of the vitamin suggests the formation of such an intermediate, but its existence has not been demonstrated.

Whenever the reaction catalyzed is of an oxidative type the enzyme system is momentarily in possession of the equivalent of two available hydrogen atoms. The enzyme system must, therefore, exist in both an oxidized and reduced state. It has been postulated that the coenzyme would be the most likely component of the enzyme system to undergo a reversible oxidation and reduction, since such is the case in other enzymes transporting hydrogen atoms. The possibility that some group of the apoenzyme component (rather than the coenzyme) may be the actual hydrogen carrier, possibly by constituting a thioldisulfide system, should not be overlooked. Attempts were first made to demonstrate a reversible reduction and reoxidation of the thiazole nucleus in a manner analogous to the pyridine-dihydropyridine interconversions of the nicotinic acid coenzymes. This possibility is no longer seriously considered. However, it has been recently pointed out that the dihydrothiamine pyrophosphate has never actually been prepared, since all the attempts to reduce the thiazole nucleus chemically resulted in a cleavage of the molecule at the methylene bridge connecting the two aromatic nuclei of the vitamin..$^{\mathbf{1 5 2}}$

A second mechanism, wherein an oxidized and reduced state of thiamine would also exist, has been postulated on the basis of the observed thiamine activity of "thiamine disulfide," a dimer in which the thiazole nucleus opens. ${ }^{153,154}$ If the suggested equilibrium occurs, the structure always ascribed to the vitamin represents the reduced, rather than the oxidized form. Although the disulfide analogues of either thiamine or its coenzyme are active when tested with intact cells or organisms, these compounds do not reactivate cell free preparations of apocarboxylase. ${ }^{\mathbf{1 5 5}}$ This would indicate that the disulfides are not active oxidized forms of the vitamin or coenzyme but are instead compounds which, although inactive per se, can be reduced by cells to form the vitamin or coenzyme having an intact thiazole nucleus. Since the decarboxylation in which thiamine disulfide was tested is a nonoxidative one, it can justifiably be argued that the reaction should not be used for testing the validity of any hypothesis concerned with oxidized and reduced states of the coenzyme.

The Function of Thiamine. In making a statement concerning a general mode of action for the thiamine coenzyme in the decarboxylation of $\alpha$-keto acids, one should consider three questions: 

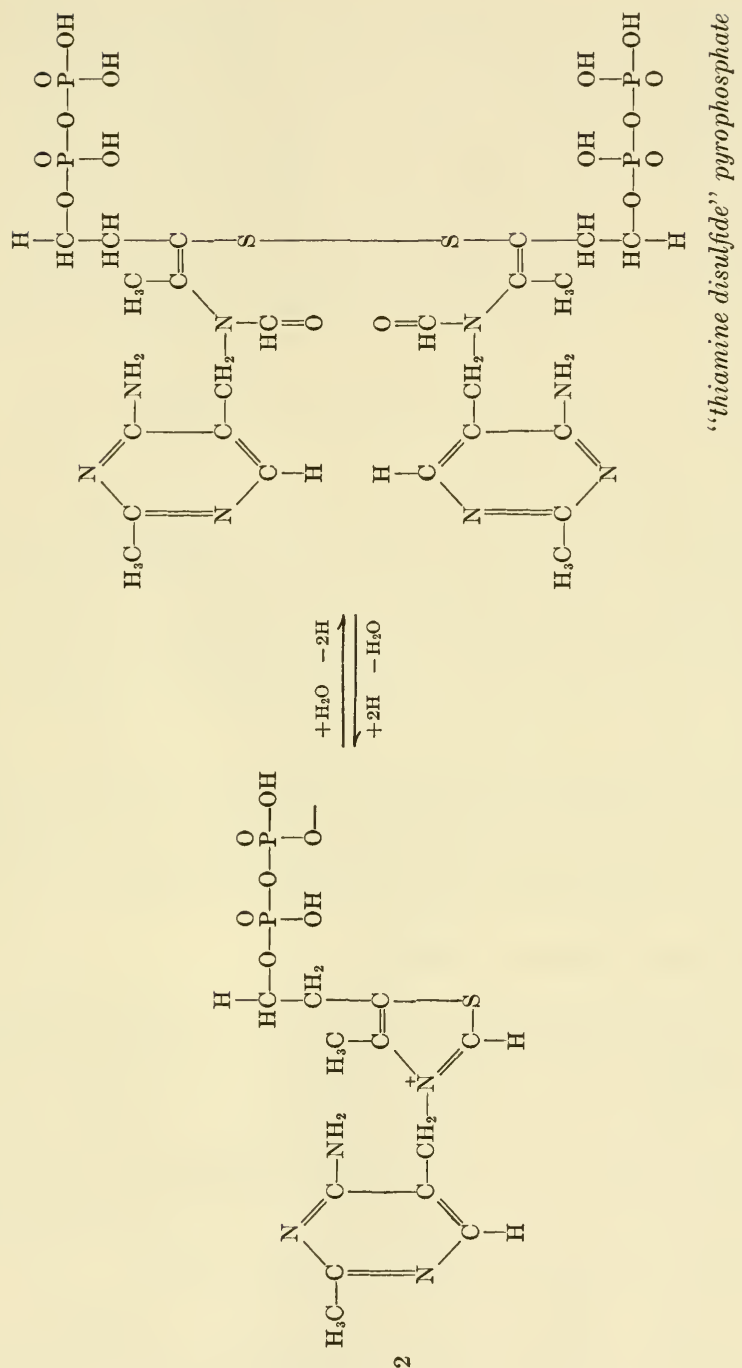
(1) Are there other cofactors which can catalyze the decarboxylation reactions of pyruvic and ketoglutaric acids? A cofactor which is essential for the oxidative decarboxylation of pyruvic acid by certain bacteria has been demonstrated recently. ${ }^{156}$ Its structure is not yet known, but on the basis of stability studies it cannot be related chemically to the usual coenzyme. However, no report has been made which would justify the conclusion that the system does not also contain thiamine pyrophosphate.

(2) Is thiamine pyrophosphate necessary for the biological decarboxylation of other $\alpha$-keto acids? One enzyme system in which oxalacetate is cleaved by an $\alpha$-decarboxylation (oxidative) rather than a $\beta$-cleavage has been reported. ${ }^{157}$ This is an oxidative decarboxylation and produces malonic acid. The reaction is analogous to the oxidative decarboxylation of $\alpha$-ketoglutaric acid in which succinic acid is produced. However, in this instance, the enzyme has been shown to be a porphyrin-containing protein and contains no thiamine. ${ }^{158}$

$\alpha$-Keto acids are produced by the oxidative deamination of amino acids. Some of these at least are known to be metabolized by oxidative decarboxylations (phenylpyruvic acid, for example, is converted to phenylacetic acid). Thiamine has never been shown to be necessary for these reactions; but since they have not been studied in well resolved systems, one cannot make any statement concerning its function in these reactions.

(3) Is thiamine pyrophosphate a coenzyme for any type of reaction other than the decarboxylation of $\alpha$-keto acids? There is no apparent necessity for postulating any additional type of function for the thiamine coenzyme, if the reactions of the diacetyl mutase type are regarded as special cases involving intermediates of $\alpha$-decarboxylations.

\section{The Coenzymatic Functions of Biotin}

The search for the specific enzymatic reactions mediated by biotin has, at the time of this writing, not been wholly successful. Although considerable information is now available concerning metabolic products whose syntheses depend upon the presence of biotin, the exact reactions in which the biotin coenzyme participates still cannot be stated with certainty. The evidence based on all the information reported to date necessitates the assumption that biotin functions in several processes which seem to have nothing in common-a situation which, if unexplained, leaves this one member of the typical B group in a unique category.

Four metabolic processes have been shown to be influenced by the biotin available to cells or tissues: (1) the $\beta$-decarboxylation of polybasic keto acids and the reverse carboxylation; (2) the biosynthesis of 
aspartic acid; (3) the deamination of certain amino acids; and (4) the biosynthesis of oleic acid. In each instance, however, more than one mechanism for biotin activity can be justifiably postulated, and in no case has a specific catalytic function been proved. The questions posed by the many seemingly unrelated phenomena in biotin metabolism have stimulated considerable interest, and they are under intensive investigation. The answer to the basic question-does biotin have more than one type of function?-should be fortheoming soon.

The Role of Biotin in $\beta$-Decarboxylations. The specific enzymatic systems to which biotin was first tentatively assigned were $\beta$-decarboxylases, the enzymes catalyzing the reactions:

$$
\begin{aligned}
\text { oxalacetic acid } & \rightleftharpoons \text { pyruvic acid }+\mathrm{CO}_{2} \\
\text { oxalsuccinic acid } & \rightleftharpoons \alpha \text {-ketoglutaric }+\mathrm{CO}_{2}
\end{aligned}
$$

The existence of these two reactions had been previously established, and their importance in metabolism (tricarboxylic acid cycle, p. 224; carbon dioxide fixation, p. 221) clearly recognized. Both enzymes had been concentrated and shown to be specific for their respective substrates. They did have comparable equilibrium constants and a requirement for the same cofactor, $\mathrm{Mn}^{++},{ }^{159,} 160$ which is indicative of a common mechanism. Although no essential organic cofactor can be directly demonstrated, biotin has been postulated as a component of such systems because of nutritional relationships between biotin and the metabolites which participate in those two reactions: (1) oxalacetic acid (or its amino acid analogue, aspartic acid) effectively replaced the biotin requirement of microorganisms under certain conditions ${ }^{161,162}$; oxalacetic acid prevented the inhibition of growth effected by a biotin analogue ${ }^{161}$; (3) pyruvic acid and biotin in the absence of bicarbonate were ineffective in meeting the aspartic acid requirements of an organism, but when the cultures were grown in a bicarbonate-containing medium, this vitamin could replace the amino acid ${ }^{161}$; (4) the uptake of carbon dioxide by a lactobacillus, followed by use of isotopically labelled bicarhonate, was not observed until sufficient biotin was added to substitute for the aspartic acid requirement ${ }^{164}$; the capacity of biotin-deficient bacteria to decarboxylate oxalacetate is much less than that of normal cells, ${ }^{163}$ and $\alpha$-ketoglutaric acid prevented the inhibition of a biotin analogue. ${ }^{161}$ The most logical explanation for these observations is that the oxalacetic acid (and aspartic acid) and $\alpha$-ketoglutaric acid requirements of the organisms either can be furnished in the form of the metabolites themselves, or can be supplied by the synthetic reactions under discussion, provided the catalyst-a biotin enzyme-is supplied and is 
not inhibited by biotin analogues. The inability of biotin-deficient tissues to metabolize pyruvic acid ${ }^{165,166}$ or of biotin-deficient yeast cells to utilize glucose aerobically ${ }^{167}$ can be attributed to the deficit of oxalacetic acid needed to catalyze the tricarboxylic acid cycle by which these substrates are "oxidized."

However, the following observations which are not in line with this hypothesis suggest that the function of biotin in $\beta$-decarboxylations may not be a direct one: (1) aspartic acid, but neither oxalacetic acid nor any other dicarboxylic acids which can be converted to oxalacetic acid, alters the biotin requirement of yeast ${ }^{168} ;(2)$ the biotin content of oxalacetic acid decarboxylase preparations from a bacterium decreased during purification, ${ }^{169}$ and no biotin at all was found in a purified preparation of animal origin. However, the biotin might have been in a form which was inactive in the microbiological assays. ${ }^{170}$

Biotin Function in Aspartic Acid Synthesis. The sparing effect of aspartic acid on the biotin requirements of yeast ${ }^{171,172}$ and bacteria, ${ }^{161}, 162,173,174$ can best be interpreted on the basis of biotin functioning either directly or indirectly in the synthesis of this amino acid. Since aspartic acid is effective when no other $\mathrm{C}_{4}$ dicarboxylic acids are, ${ }^{168}$ it is possible that the reaction in aspartic acid synthesis which is catalyzed by biotin is not one in which oxalacetic acid is directly formed from pyruvic acid by carboxylation. This would also explain why aspartic acid is always more effective than oxalacetic acid in substituting for biotin, and why other $\mathrm{C}_{4}$ dicarboxylic acids, which should be easily converted to oxalacetic acid, are inactive both as substitutes for biotin and as agents for reversing biotin inhibitors.

The diminished rate of respiration of biotin-deficient yeast ${ }^{167,175}$ is increased by the addition of either aspartic acid or biotin plus ammonium salts. Biotin alone is ineffective. This observation suggests that the reaction catalyzed by biotin in the synthesis of aspartic acid is one involving an amination.

Biotin as a Catalyst for Deaminations. The type of reaction in which biotin has been most directly implicated is the deamination of certain amino acids. When bacterial cells are suspended in acid buffers they rapidly lose their ability to decarboxylate aspartic, malic, and oxalacetic acids ${ }^{176}$ and to deaminate aspartic acid, threonine, and serine. ${ }^{177}$ Extracts of dried cells can be reactivated by the addition of yeast extract, or by biotin plus adenylic acid (muscle), but not by biotin alone. ${ }^{178}$ Although the biotin-adenylic acid mixture is as effective as yeast extract initially, the combination becomes ineffective after the cell preparations have been stored; the yeast extract, on the other hand, maintains its ability to reactivate the stored preparations of the deaminase systems. These 
results have been interpreted as showing that a biotin coenzyme is destroyed by subjecting the cells to an acid environment; that the coenzyme can be resynthesized from adenylic acid and biotin by fresh preparations, but that on standing the enzymes bringing about the synthesis of the coenzymes deteriorate; and that yeast extract contains the intact coenzyme and, hence, can reactivate the older preparations.

The Role of Biotin in the Synthesis of Oleic Acid. Oleic acid and related lipides, in the presence of aspartic acid, can effectively replace biotin in the medium of certain lactobacilli that would otherwise require this vitamin $179,180,181,182,183$ and can satisfy the biotin requirement of mosquito larvae. ${ }^{184}$ This fatty acid can also effectively reverse biotin inhibitors. The results of such investigations indicate that biotin functions in the biosynthesis of oleic acid. Efforts to prove that the reverse is true, i.e., that oleic acid is a precursor of biotin (presumably the aliphatic side chain attached to the biotin nucleus), have not been successful. ${ }^{179}$ Equally unsuccessful have been the attempts to ascribe the activity of the acid solely to some physical action (such as alteration of cell permeability). The function of biotin in the synthesis of oleic acid is not one in which carbon dioxide is fixed, for no carbon dioxide (isotopically labelled) is taken up when biotin is used to satisfy the oleic acid requirement. ${ }^{164}$

Biotin Coenzymes. The question concerning the number of biotin coenzymes cannot be satisfactorily answered until the number of "biotins" is known. There seems to be irrefutable evidence for the existence of two chemically distinct isomers (Section D), $\alpha$-biotin and $\beta$-biotin, having identical biological properties in all systems in which they have been compared. There is still some question concerning the exact structure of the $\alpha$-isomer, but on the basis of the configuration proposed it is difficult to see how the two structures could exist in equilibrium or be readily interconverted.

In addition to these two isomers a number of uncharacterized substances of diverse complexities have been shown to possess varying degrees of biotin activity. The existence of these biotin isotels * has been established by comparing the biotin activities of naturally occurring substances with respect to their ability to stimulate growth in different organisms, ${ }^{185}$ their avidin combinability, ${ }^{185}$ their rate of migration during chromatographic separations, ${ }^{186}$ and their effectiveness in counteracting the growth inhibitions produced by biotin analogues. ${ }^{186}$ If the composition and distribution of the biotin coenzyme(s) resembles that of other $\mathrm{B}$ vitamin coenzymes, then it can be assumed that one or more of these naturally

* Chemically distinct compounds which perform the same physiological function. Williams, R. J., Science, 98, 386 (1943). 
occurring substances, more complex in structure than biotin, will be found to be a coenzyme.

Biotin and certain of its isotels form very stable complexes with avidin and other proteins, a combination that cannot be effectively dissociated by any means yet tried. The release of the biotin component can be accomplished only by destroying the protein. ${ }^{187} \mathrm{~A}$ similar nondissociation of coenzyme-apoenzyme may exist in biotin-containing enzymes.

An insight into the chemical nature of one biotin coenzyme is given by the investigations on the reactivation of amino acid deaminases (p. 173). The system which these investigators have developed should serve as a method which could be adapted for the quantitative determination of this biotin coenzyme provided the preparations were aged a sufficient length of time so that the component parts of the cleaved (?) coenzyme in the apoenzyme preparation were no longer active.

Is biotin always an essential cell constituent? In certain "biotin-requiring" bacteria, biotin seems to be essential for only two processes, one related to the synthesis of aspartic acid and one to oleic acid production. Any other metabolites produced by biotin-catalyzed reactions are apparently dispensable or else can be obtained by alternate mechanisms. Consequently, if these particular organisms are furnished an exogenous supply of aspartic and oleic acids, they no longer require detectable amounts of biotin for growth and reproduction. In this instance the synthesis of demonstrable quantities of biotin by the organism does not occur. Hence, this may be one instance in which a $\mathrm{B}$ vitamin becomes nonessential for life. Possibilities which have not been completely eliminated are that extremely minute quantities of the vitamin are present in the culture medium as impurities, or else that small amounts are being synthesized intracellularly. Such undetectable amounts could still be performing certain duties essential for the survival of the organism. Since the addition of avidin to the medium does not alter the situation, the presence of an exogenous source seems unlikely unless the impurity is one of the avidinuncombinable isotels. ${ }^{185}$ Intracellular synthesis, however, cannot yet be conclusively ruled out, since current assay procedures may not determine all forms of bound biotin.

The intracellular concentration of both vitamin $B_{6}$ and folic acid may likewise be reduced to negligible quantities under similar circumstances wherein products of their functioning are supplied preformed to bacterial cells (pp. 187 and 202).

\section{The Coenzyme Activating $\alpha$-Amino Acids}

Most organisms are capable of synthesizing from other metabolic intermediates at least part of their amino acid requirements. Processes of a 
reverse type in which amino acids are degraded and metabolized are also of common biological occurrence. A general method of synthesis or degradation, reductive amination or oxidative deamination, has already been pointed out in the discussion of reactions catalyzed by the coenzymes of nicotinic acid and riboflavin (pp. 141 and 147). Several other types of reactions, however, constitute alternate pathways by which amino acids may be elaborated or utilized. At least three of these types are reactions catalyzed by enzymes having a common coenzyme-one derived from vitamin $B_{6}$. In several instances, at least, reactions catalyzed by this vitamin are the only methods by which an organism can adequately produce particular amino acids and essential metabolites derived from them.

\section{The Vitamin $\mathbf{B}_{6}$ Coenzyme}

A period of almost six years separated the time when pyridoxine was first synthesized (1939) and the date when the biocatalytic functions of vitamin $B_{6}$ were discovered. Then, within a period of a few months, two distinct metabolic processes involving entirely separate types of chemical reactions were shown to be dependent upon the presence of vitamin $B_{6}$ derivatives. Two years later several reactions constituting a third type of chemical reaction were shown to require the identical pyridoxal coenzyme as did the two processes earlier recognized.

The decarboxylation of tyrosine and several other amino acids by bacterial cells had been observed to be catalyzed by an enzyme containing a dissociable codecarboxylase. ${ }^{18 s}$ None of the coenzymes then known, however, were active in reconstituting purified preparations from which the coenzyme had been separated, but a concentrate having 15,000 times the coenzymatic activity of the richest natural source (yeast extract) had been prepared. ${ }^{189}$ Concurrently, it was observed that the decarboxylation of tyrosine by resting cells of a lactic acid bacterium was influenced by the medium in which the organism had been cultured. ${ }^{190}$ For the prodiction of cells possessing optimum decarboxylase activity the medium in which the organism was grown had to contain three times the amount of pyridoxine needed to promote maximum growth. Since the enzymatic activity of the cells varied according to the amount of pyridoxine furnished them during growth, pyridoxine and its analogues, pyridoxamine and pyridoxal (which had just become available), were tested with cell suspensions of bacteria collected from cultures grown on a vitamin $B_{6}$-free medium. (The vitamin can be omitted from the medium if high levels of alanine are used-see p. 187.) The addition of pyridoxal to the "vitamin-deficient" cells increased the rate of decarboxylation twentyfold; pyridoxamine and pyridoxine were inactive. ${ }^{191}$ It was subsequently shown 
that the bacterial cells first phosphorylate the added pyridoxal before it becomes active, ${ }^{192}$ and that this phosphorylated pyridoxal is also the codecarboxylase for at least six of the known amino acid decarboxylases.

The vitamin $\mathrm{B}_{6}$ activity of pyridoxine-supplied bacteria had been observed in many instances to depend upon an activation of this compound occurring when it was autoclaved with the other components (particularly the amino acids) of the medium. ${ }^{193}$ An investigation into the nature of the chemical changes resulting from the autoclaving led to the discovery of pyridoxal and pyridoxamine (p. 186), and to the recognition of a nonbiological reaction (a transamination) by which these two forms of the vitamin are interconverted. ${ }^{194}$ Glutamic acid (and most other common $\alpha$-amino acids) can serve as an amino donor for the formation of pyridoxamine from pyridoxal, and $\alpha$-ketoglutaric acid is an efficient acceptor for the amino group in the reverse reaction.<smiles>C=Cc1ncc(CO)c(C=O)c1O</smiles>

glutamic acid

pyridoxal<smiles>Cc1ncc(CO)c(O)c1C(N)N</smiles>

$\alpha$-ketoglutaric acid

pyridoxamine

A similar intermolecular exchange of amino and carbonyl groups, catalyzed by enzymes, had been previously recognized as occurring in the tissues of animals. ${ }^{195}$ These enzymes, classified as transaminases, catalyze the interconversion of certain $\alpha$-keto and $\alpha$-amino acids; consequently, it was suggested that a possible function for vitamin $\mathrm{B}_{6}$ was to serve as an intermediate in transamination reactions by alternating between the aldehyde and amine states. ${ }^{194}$ To test this hypothesis, tissues of vitamin $\mathrm{B}_{6}$-deficient rats were compared to controls from normal animals. ${ }^{196}$ The deficient tissues were found to be definitely inferior in their ability to catalyze a transamination reaction (the glutamic acid-aspartic acid system). The apoenzymes from resolved bacterial decarboxylases were shown to be reactivated by boiled extracts of transaminase concentrates (prepared from animal tissue), indicating the existence of a 
common coenzyme for these two systems. ${ }^{197}$ Subsequently, the reconstruction of a transaminase system from an inactive apoenzyme and pyridoxal phosphate was accomplished, thus proving conclusively the identity of the vitamin $B_{6}$ coenzyme and cotransaminase. ${ }^{198}$

Reactions in which tryptophan either is synthesized from indole or is cleaved to produce it have been demonstrated in bacteria and molds. The enzymes required can be resolved into inactive components. Synthetic pyridoxal phosphate can reactivate the apoenzymes ${ }^{199,200}$ and is presumably identical with naturally occurring "cotryptophanase."

Structure of the Coenzyme. On.the basis of its chemical composition, pyridoxal phosphate is probably the simplest of the $B$ vitamin coenzymes. Yet four years have elapsed since the first synthetic preparation of the coenzyme was made, and its structure still cannot be stated to have been proved. When pyridoxal is treated with phosphorylating agents, a phosphorylated derivative (obtained as a crude barium salt) is formed. ${ }^{192}$ This "synthetic coenzyme" has the biological properties of the natural codecarboxylase, cotransaminase, and cotryptophanase. 192, 198, 201

The active compound was first prepared synthetically by American scientists who have now conclusively shown it to be not the 3-phosphate (the phenolic ester), ${ }^{202}$ although in their original report properties were reported for the ester which suggested it was pyridoxal-3-phosphate. ${ }^{203}$ Meanwhile, Swiss chemists had prepared the acetal of pyridoxal-3phosphate by methods which leave no doubt as to the structure of their product. ${ }^{204}$ They had claimed codecarboxylase activity for their synthetic product, but were unable to activate an apotransaminase system with it. ${ }^{205,} 206$ The dispute concerning the activity of the $3 \triangleleft$ phosphate ester has been resolved by simultaneously testing the products prepared in different laboratories on the same biological system. ${ }^{202}$ The activity of the 3-phosphate is so low compared to that of the active "synthetic coenzyme" of the American group that the slight response elicited by the former might be attribued to traces of an active isomer formed by an intramolecular transesterification.

The active synthetic derivative when cleaved yields equimolecular amounts of pyridoxal and inorganic phosphate. ${ }^{203}$ Since it is not the phenolic ester, its structure is presumably that of the phosphoric ester of the hydroxymethyl group (position 5 on the pyridine nucleus). Pyridoxamine can be readily produced in quantitative yields by heating pyridoxal phosphate with an excess of glutamic acid in a neutral solution, ${ }^{207}$ and it possesses the specific growth-promoting properties of the compound prepared by a direct phosphorylation of pyridoxamine. ${ }^{208}$ The esters are quite stable in alkaline solution (no destruction after heating for five hours in $1 \mathrm{~N} \mathrm{NaOH}$ at $120^{\circ} \mathrm{C}$ ), but they are rapidly 
hydrolyzed when heated with dilute acid $(0.05 N) .{ }^{207}$ Stronger concentrations of acid are less effective. The ester of the amine is hydrolyzed by acid more rapidly than is pyridoxal phosphate. Decomposition of the latter ester was noted when neutral solutions were stored in the refrigerator.<smiles>Cc1ncc(COP(=O)(O)O)c(C=O)c1O</smiles>

pyridoxal phosphate<smiles>Cc1ncc(COP(=O)(O)O)c(CN)c1O</smiles>

pyridoxamine phosphate

Assay Methods. The method generally favored for the enzymatic determination of pyridoxal phosphate is the system in which tyrosine apodecarboxylase is reactivated. The source of apoenzyme is a dried cell powder prepared from Streptococcus faecalis $R$., which has been grown on an alanine-rich, vitamin $\mathrm{B}_{6}$-deficient medium. A detailed description of the assay procedure has been published. ${ }^{192}$ The powder is easily prepared and is stable over long periods of time. When preparations are assayed with the powder and a substrate, the rate of carbon dioxide evolution is a measure of the amount of pyridoxal phosphate in the preparations. More elaborate procedures are needed to prepare the apoenzymes of decarboxylases, transaminases, and tryptophanases from normal cells or tissues; this makes their use as testing agents less convenient than the procedure employing deficient cells.

A microorganism which responds only to the phosphorylated derivatives of either pyridoxal or pyridoxamine has been encountered. ${ }^{209}$ The amine phosphate is three to five times as active as the aldehyde ester. The individual determination of each of the unphosphorylated components of the $\mathrm{B}_{6}$ group-pyridoxine, pyridoxal, and pyridoxaminecan be accomplished by a differential method in which samples are analyzed by use of three organisms which respond differently to the three compounds. ${ }^{210}$ If materials are tested by such a procedure both before and after dilute acid hydrolysis (which cleaves the phosphate ester linkage), it is possible to get a reasonably accurate estimate of both the pyridoxal phosphate and the pyridoxamine phosphate contents of crude extracts. ${ }^{207}$ The ease with which the amine and aldehyde forms can be interconverted, by nonenzymatic as well as enzymatic reactions, should always be considered when interpreting results obtained by this method.

The formation of the amine phosphate from the aldehyde phosphate has been followed spectrometrically at an alkaline $\mathrm{pH}$. By this procedure 
the two phosphates can be distinguished from each other and from their nonphosphorylated derivatives. ${ }^{208}$ This method, of course, is limited to solutions of the pure substances and cannot be used with extracts of crude material.

Occurrence. Before the relationship of pyridoxal and codecarboxylase was recognized, the coenzyme had been shown by enzymatic analyses to occur in a variety of biological substances. ${ }^{189}$ Since then, vitamin $B_{6}$ has been shown by microbiological assays to occur primarily in bound forms, presumably the phosphates of pyridoxal and pyridoxamine. On the basis of distribution studies, using the differential analysis technique previously mentioned, it appears that pyridoxal phosphate is the predominant form of vitamin $B_{6}$ in most animal tissues. Liver appears to be an exception, for in most samples of this tissue the phosphate of the amine accounts for the greater part of the vitamin $\mathrm{B}_{6}$ content. Yeast extract is also a rich source of pyridoxamine phosphate. ${ }^{207}$

Biosynthesis. It has been observed that the synthesis of pyridoxal phosphate is rapidly carried out by yeast, molds, and bacteria, but only from the particular components of the vitamin $\mathrm{B}_{6}$ group that serve to satisfy the nutritional requirements of each particular organism. ${ }^{212}$ It has also been shown that the coenzyme content of rat tissues is directly related to the dietary intake of pyridoxine. If the bacterial cells are resting, i.e., suspended in solutions lacking nutrients needed for growth, the rate of phosphorylation of pyridoxal is decreased to one tenth that observed in the metabolically active cells; the conversion of pyridoxamine to the coenzyme under resting conditions cannot be detected unless a keto acid (pyruvic acid) is added during the incubation. In this organism the route of biosynthesis would appear to be limited to the one in which only pyridoxal can be phosphorylated, i.e., the amine must first undergo a transamination (enzymatic?) with a keto acid to form the aldehyde. Studies of this nature have been so limited in number that no general statement can yet be made concerning the possible utilization by other organisms of alternate routes of synthesis (via pyridoxamine phosphate, for example).

One of the earliest methods of obtaining a "synthetic" coenzyme for use with preparations of apoenzymes from treated cells was to add pyridoxal and adenosine triphosphate to the protein preparation before the substrate for the decarboxylation was introduced. ${ }^{192}$ Whereas either the vitamin or phosphorylating agent is inactive when used alone, together they can effectively replace the missing coenzyme. It is assumed that the phosphorylation is an enzymatic process catalyzed by a phosphorylating enzyme present in the crude decarboxylase preparations, although this 
enzymatic biosynthesis of the coenzyme has not been critically studied and is therefore still poorly characterized.

The phosphorylated esters of the vitamin components are quite stable and are not as readily inactivated by enzymatic hydrolysis as are the coenzymes of other vitamins in which pyrophosphoric linkages occur. A high degree of association between the coenzyme and its recognized apoenzymes has been demonstrated. This affinity of the apoenzymes for their coenzymes accounts for the difficulties encountered in trying to resolve some of the holoenzymes by simple procedures; it may also be an important factor in preventing the hydrolysis of the coenzyme by intracellular phosphatases.

The dissociation constant has been measured for a representative enzyme of each of the three recognized types of pyridoxal-catalyzed reactions. ${ }^{201,213}$ The three values have similar orders of magnitude and are so small that the amount of uncombined pyridoxal phosphate is negligible as long as there is even a small excess of an apoenzyme. The affinity can also be demonstrated by the use of an inhibitor, an analogue of the coenzyme. The effectiveness of the analogue in inactivating a decarboxylase system depends upon the order in which the analogue and the coenzyme are added to the apoenzyme. ${ }^{214}$ If the coenzyme is added first the analogue is quite ineffective as an antagonist, whereas if the order of addition is reversed the analogue is an effective inhibitor. The association is thus great enough so that the equilibrium between the inhibitor-apoenzyme and coenzyme-apoenzyme systems is not readily achieved, and in such an instance considerable time would have to elapse before the ratio of inhibitor to coenzyme would be the factor determining the degree of inhibition.

Dialysis of fresh preparations of the holoenzyme does not accomplish its resolution. The most effective means of securing the apoenzyme from the cellular material of normal organisms, i.e., those supplied adequate amounts of vitamin $\mathrm{B}_{6}$, is a process of aging. Storing the intact tissue or extracts for a period of time results in a gradual inactivation of the preparation and the release of the apoenzymes. ${ }^{198,}$ 199, 215 The process, however, is not one of simple dissociation but rather one in which destruction of the coenzyme precedes the dissociation. Dissociation of the complete enzyme is also achieved whenever the source of the enzyme is carried through the number of fractionating procedures needed to effect a substantial concentration of the enzyme.216 The extent of destruction of the coenzyme during such separations has not been indicated.

Reactions Catalyzed by Pyridoxal Phosphate. The chemical changes catalyzed by pyridoxal phosphate may, on first inspection, appear to have little in common since they represent extremely divergent types of 
reactions: (1) simple decarboxylations, (2) oxidative transfer of amino groups, and (3) condensations establishing carbon-to-carbon bonds or the reverse process in which these bonds are cleaved. However, in every reaction known to be catalyzed by this coenzyme there is a common type of substrate, an $\alpha$-amino acid, and in every instance the reaction involves a group or atom attached to the $\alpha$-carbon atom.<smiles>[R]CC(N)C(=O)O</smiles>

decarboxylation<smiles>[R]CC(N)C(=O)O</smiles>

transamination<smiles>[R]C([2H])C(N)C(=O)O</smiles>

tryptophan synthesis and cleavage

I. Amino Acid Decarboxylation. Amino acid decarboxylation is undoubtedly an important means by which bacteria can metabolize some amino acids. This type of reaction also provides these organisms a means of producing alkaline substances with which they can alter an undesirable acidic environment. ${ }^{217}$ In addition, the decarboxylation of amino acids seems to be the most likely source for certain polyamines which have been shown to be bacterial metabolites.

Decarboxylation of $\alpha$-amino acids by mammalian tissues can be demonstrated, although they never possess activity comparable to that observed in many bacteria. This type of reaction is not believed to be an important general mechanism for the catabolism of amino acids, but it may be the process by which are formed histamine (from histidine), taurine (from cysteic acid), ethanolamine (from serine), $\beta$-alanine (from aspartic acid), adrenalin (from 3,4-dihydroxyphenylalanine-"dopa") and putrescine (from ornithine). Detailed reviews of mammalian and bacterial decarboxylases were published in 1945 and $1946 .{ }^{218,217}$

Pyridoxal phosphate has been conclusively shown to be the catalyst for these six reactions:<smiles>[R]C(N)C(=O)OCC(=O)O</smiles>

1. tyrosine

$\longrightarrow \mathrm{CO}_{2}+$ tyramine. ${ }^{219}$

2. dopa

$\longrightarrow \mathrm{CO}_{2}+3,4$-dihydroxyphenylethylamine. ${ }^{219,}{ }^{197}$ (precursor of adrenaline.)

3. lysine

$\longrightarrow \mathrm{CO}_{2}+$ cadaverine. ${ }^{220}$

4. ornithine

$\longrightarrow \mathrm{CO}_{2}+$ putrescine. ${ }^{221}$

5. arginine

$\longrightarrow \mathrm{CO}_{2}+$ argamine.221, 215

6. glutamic acid

$\mathrm{CO}_{2}+\gamma$-aminobutyric acid. ${ }^{215}$ 
In every case the apoenzyme used for reconstructing the system was of bacterial origin, with the exception of dopa decarboxylase. ${ }^{197}$ No direct answer has been obtained to the question of whether pyridoxal phosphate functions in the decarboxylation of other amino acids by mammalian tissue. Until it has, the lack of a positive demonstration should be attributed to the weakness of such systems in mammalian tissues and the difficulty of resolving the holoenzyme.

II. Transamination. Pyridoxal phosphate is known to be the coenzyme for two transaminase reactions, the so-called glutamic-aspartic system and the glutamic-alanine system.
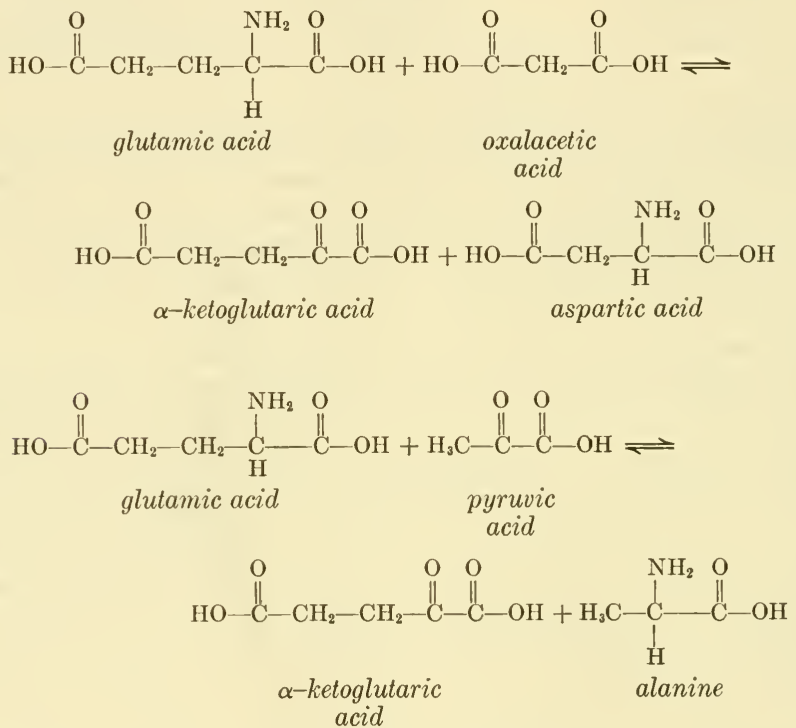

A combination of these two systems gives what amounts to an asparticalanine system:

aspartic acid+pyruvic acid $\rightleftharpoons$ oxalacetic acid+alanine.

This reaction was originally believed to be catalyzed by a single distinct enzyme, ${ }^{222}$ having another "coenzyme" in addition to pyridoxal phosphate. It is now recognized, however, that this reaction is brought about by a combination of these two enzyme systems and catalytic amounts of glutamic acid (or $\alpha$-ketoglutaric acid). ${ }^{223}$ These latter substances are the "coenzyme" of the combined system; they shuttle back and forth between 
the two enzymes and thus make possible the coupling of the two reactions. The individual systems have been found to occur in animal tissue, in plant material, ${ }^{224}$ and in bacterial cells, but as yet only those of animal and bacterial origin have been resolved and tested for their coenzyme requirements. The existence of a third distinct transaminase is known. It catalyzes a glutamic-cysteic acid system, but it has not been studied from the standpoint of its component parts. ${ }^{225}$ Although the occurrence of additional transaminases in which other amino acids form part of the system has been indicated, absolute proof of their existence is still lacking.

III. Reactions in which the Methylene Groups Attached to the $\alpha$-Carbon Atom React. Two instances are known in which pyridoxal phosphate catalyzes a process in which it is the methylene group bonded to the $\alpha$-carbon atom (rather than the amino or carboxyl group) that reacts. In one tryptophan is the product; in the other, it is the substrate.

A. Synthesis of Tryptophan from Indole and Serine. A mutant of Neurospora crassa can utilize indole in place of tryptophan.226 An analysis of the mechanism, using extracts from such cells, demonstrated that the biosynthesis of tryptophan can be achieved by this reaction. ${ }^{199}$<smiles>NC(C(=O)O)c1c[nH]c2ccc(OC(=O)C(N)C(N)C(=O)O)cc12</smiles>
indole

serine

tryptophan

The equilibrium for this reaction must decidedly favor the synthetic process, rather than its reverse, since the degradation of tryptophan into serine and indole cannot be demonstrated with the enzymatic preparation.

B. Non-Oxidative Degradation of Tryptophan. An extract prepared from Escherichia coli has been found to catalyze the breakdown of tryptophan in the following manner ${ }^{201}$ :

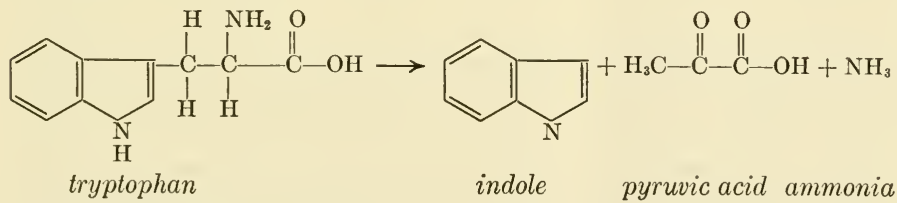

After inactivation of the enzyme system by aging and dialysis the protein can be completely reactivated by pyridoxal phosphate. The possibility of a two-step reaction in which one enzyme would hydrolytically 
cleave tryptophan to form serine and a second would catalyze the deamination of serine, yielding the observed products, ammonia and pyruvic acid, was ruled out when it was shown that the preparation cannot catalyze the deamination of serine.

An independent investigation has been carried out upon the enzymatic degradation of tryptophan by cellular extracts from this same organism. ${ }^{200}$ In this study, the biological preparations used had apparently not been adequately resolved into individual enzyme systems, since the production of indole and tryptophan was accelerated by each of four substances: a nicotinic acid coenzyme, free riboflavin, pyridoxal phosphate, and a porphyrin-containing enzyme (verdoperoxidase). A maximum rate of reaction was achieved only when all four of the stimulating factors were added. By chromatographic analysis it was shown that alanine was formed during the "reaction." It could, however, have been formed secondarily from pyruvic acid following the initial cleavage. Were alanine a primary product of the reaction, it would mean that the cleavage is a reductive one, and the reaction would of necessity have to be coupled with a second reaction in which hydrogen atoms are made available. The effect of the hydrogen-transporting agents upon the rate of indole formation probably is the result of the oxidative removal of the other products of the primary reaction.

Relationship of Vitamin $B_{6}$ and Amino Acid Requirements in Bacterial Metabolism. The determination of the amino acid requirements of several bacteria has indicated processes in amino acid syntheses, other than those completely characterized, for which the vitamin $\mathrm{B}_{6}$ coenzyme is undoubtedly essential. In most instances, the variations in amino acid requirements resulting from the addition of pyridoxal or pyridoxamine can be explained by assuming catalysis of reactions of the types which have been demonstrated in cell-free systems.

It has been observed that the presence of three amino acids in particular -lysine, threonine and alanine-radically affects the pyridoxal or pyridoxamine requirement of certain bacteria. ${ }^{227,228,} 229$ In the absence of these amino acids the vitamin $\mathrm{B}_{6}$ requirement of these organisms may be more than ten times that when they are present. It was observed that these amino acids were no longer essential when pyridoxine was replaced by pyridoxal or pyridoxamine in the media of certain bacteria. The effect presumably did not occur as the result of an independent function of pyridoxine, but rather reflected an inadequate chemical conversion of the inactive pyridoxine during autoclaving; hence, the organisms were not supplied sufficient amounts of active precursors of the vitamin $\mathrm{B}_{6}$ coenzyme to carry out the synthesis of these three amino acids. Formation of these acids was independent of the carbon dioxide tension, and it is 
presumed that their synthesis probably involves transamination reactions, either directly or indirectly. Imidazole pyruvic acid has been shown to replace the histidine requirements of a bacterium provided adequate vitamin $\mathrm{B}_{6}$ is supplied. ${ }^{230}$ The amino acid could be formed directly from the former compound by transamination.

The ability of an organism to use $\mathrm{O}$-amino acids in place of the natural isomers has been shown to be dependent upon the availability of pyridoxal or pyridoxamine. Transamination reactions in which optically inactive keto acids are formed would be a convenient method for making such compounds utilizable.

The availability of pyridoxal and pyridoxamine also exerts a sparing action upon the requirements for three additional amino acids-arginine, phenylalanine and tyrosine. ${ }^{228}$ In these instances, however, adequate carbon dioxide tension must be maintained over the cultures. In a carbonate free system the vitamin is incapable of altering the requirements for these particular amino acids. It was at first postulated that a carboxylation of amines can be effectively used for the biosynthesis of these amino acids. If such were the case, however, one would have to account for the origin of these amines by some process other than decarboxylation of amino acids. In addition, the equilibrium is such that relatively high concentrations of the rather toxic amines would have to exist for the reaction to be directed toward amino acid synthesis. When phenylethylamine was supplied the microorganism, only a slight conversion to phenylalanine was observed, even in the presence of high concentrations of carbon dioxide. It seems probable, therefore, that the carbon dioxide effect is an indirect one, and that vitamin $\mathrm{B}_{6}$ catalyzes the syntheses of these three amino acids by some process other than a direct amine carboxylation.

The third type of $\mathrm{B}_{6}$ reaction-tryptophan synthesis-has also been indicated by similar studies of nutritional requirements. Either pyridoxal or pyridoxamine must be supplied in greater amounts if a bacterium is to use indole or anthranilic acid (an indole precursor) in place of tryptophan. ${ }^{231}$

In animals, the utilization of tryptophan for nicotinic acid synthesis is dependent upon an adequate vitamin $B_{6}$ intake (p. 279), and low intakes of vitamin $\mathrm{B}_{6}$ result in abnormal tryptophan catabolism and the production of urinary products not normally detected (p. 427).

Vitamin $\mathbf{B}_{6}$ and Fat Metabolism. Several of the earliest observations upon the physiological results of vitamin $B_{6}$ deficiencies involved this vitamin in fat metabolism, particularly the metabolism of the unsaturated fatty acids. ${ }^{232}, 233,234,235$ No explanation for these observations can be 
made on the basis of any of the reactions now recognized as being catalyzed by pyridoxal phosphate.

Mechanism of Pyridoxal Phosphate Action. The nonenzymatic transamination reactions in which pyridoxal and pyridoxamine participate can most logically be explained on the basis of an intermediate formation of a Schiff's base which can tautomerize and be hydrolytically cleaved in a manner such as this:<smiles>O=Cc1ncc(CO)c(C=O)c1O</smiles>

pyridoxal<smiles>[R]C(N)C(=O)O</smiles>

$\alpha-\operatorname{amino}$

acid<smiles>[R]C(N=Cc1c(C(O)C=C)cnc(CO)c1O)C(=O)O</smiles><smiles>[R]C(=NC=Cc1c(C(O)O)cnc([CH])c1O)C(=O)O</smiles>

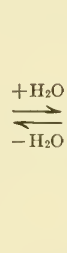<smiles>[R]C(=O)C(=O)O</smiles>

pyridoxamine $\alpha-$ keto
acid

Compounds of the Schiff's base type formed from pyridoxal and amino acids are believed to exist in biological materials. It has been assumed that the formation of such an intermediate is the mechanism by which pyridoxal phosphate activates the amino acid molecule. The energies in the postulated Schiff's base are localized in such a way that any one of the groups attached to the $\alpha$-carbon atom may undergo a reaction if the pyridoxal phosphate is attached to the appropriate apoenzyme.

When the substrate for a reaction is an amino acid, the aldehyde phosphate would be the appropriate state of the coenzyme for the formation of the postulated intermediate, and pyridoxamine phosphate would not be expected to be active per se as the coenzyme for decarboxylases or tryptophanases; and it is not. However, in transamination reactions (wherein both amino and keto acids must be present as substrates) it is postulated that the coenzyme alternates between two states, the aldehyde 
and amine, and it would seem logical to expect either the aldehyde or amine form to be active. Until recently this was thought to be the case. ${ }^{236}$ Reports of recent experiments in which a highly purified preparation of glutamic-aspartic transaminase was resolved indicate that in this instance, at least, only pyridoxal phosphate is active in reconstituting the enzyme. $^{208}$ If this is found to be generally true when refined preparations are used, the original hypothesis (which led to the discovery of cotransaminase) may have to be modified. It may be that only the aldehyde phosphate can establish the initial apoenzyme-coenzyme bond.

The phosphorylation of the vitamin is essential for its incorporation into the enzyme complexes. It has also been demonstrated that an analogue of the vitamin does not associate to any degree with a decarboxylase, and hence is inactive, whereas the phosphorylated analogue is an effective inhibitor.

The Essentiality of Vitamin $\mathbf{B}_{6}$. The sparing action of alanine, ${ }^{237}$ and in particular D-alanine, ${ }^{238}$ upon the vitamin $B_{6}$ requirements of some organisms had been presumed to be due to its utilization in the synthesis of pyridoxal. Although the decarboxylase and transaminase activities of cells cultured upon $\mathrm{D}$-alanine in the absence of a vitamin $\mathrm{B}_{6}$ source are very slight, ${ }^{192,} 198$ it has been commonly assumed that the use of $\mathrm{D}$-alanine results in the synthesis of only the minimum amounts of vitamin $B_{6}$ needed for growth. It has now been established that D-alanine is not a precursor of vitamin $\mathrm{B}_{6}$, but is a direct product of its catalytic activity, or else can indirectly function by sparing the requirement for some metabolite produced directly when vitamin $\mathrm{B}_{6}$ is available..$^{239,240}$

The phenomenon of the nonessentiality of a $\mathrm{B}$ vitamin was discussed when biotin functions were considered (p. 174). Pyridoxal is another B vitamin which may be nonessential for the growth and metabolism of certain organisms. Since its primary function is the synthesis of amino acids and amines, it may be possible to dispense with its reactions entirely if the organism is supplied with all the essential products preformed (L-amino acids, D-alanine, amines, and unidentified products in casein hydrolyzates). ${ }^{238}$

In higher forms of life, where amino acids and hormones like histamine and adrenalin must be synthesized in situ, and where extensive degradation of amino acids must precede excretion, the vitamin $\mathrm{B}_{6}$ requirement could never be completely abolished.

\section{The Coenzymes Involved in Condensations Forming Carbon-to-Carbon Bonds}

In the elaboration of more complex organic compounds from simpler ones there must be condensation reactions in which carbon-to-carbon 
bonds are established. The condensations carried out by biological systems resemble the reactions which are commonly employed by organic chemists, and many of the limitations concerning the types of reactive compounds and the manner in which they unite are common both to laboratory syntheses and to those occurring intracellularly.

The earliest enzymatic condensation to be recognized was the one in which hexoses are formed from trioses by a typical aldol condensation:
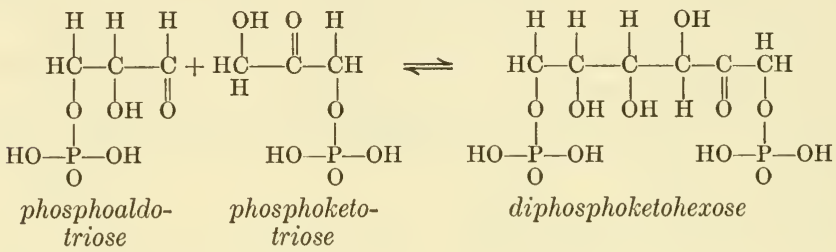

diphosphoketohexose

In this reaction, the condensation takes place between a molecule containing a carbonyl group and one containing an active hydrogen atom, i.e., one bonded to a carbon atom alpha to a carbonyl group. This type of reaction (aldol condensation) occurs readily in vitro with alkali as a catalyst; the enzyme (aldolase) which mediates the reaction pictured above has never been resolved into dissociable components, and is believed to contain no recognized vitamin. The reaction is one of the essential steps in the general process by which most organisms metabolize hexoses or synthesize them from metabolic products. The isomerization of the phosphate ester of the aldotriose to produce the corresponding ketose is an essential reaction that must precede the condensation depicted, for the condensation of two aldotrioses would give a branched chain hexose. Likewise, the isomerization of the glucose diphosphate to the corresponding fructose ester must occur prior to the cleavage, since an aldose cleavage of glucose could only occur between the $\alpha$ - and $\beta$-carbon atoms, producing a biose and tetrose.

A second type of condensation often used by organic chemists is the so-called Claisen type, a condensation which involves (1) the carbonyl group of an acid anhydride or ester and (2) a carbon and a hydrogen atom alpha to a carbonyl group. The enzymatic condensations by which fatty acids, sterols, amino acid precursors, and probably several other important products are elaborated are of this type.

In Claisen type condensations in biological systems one of the reacting molecules is usually (if not always) an acyl phosphate, the mixed acid anhydride of phosphoric acid and an organic acid. In most reactions, it is 
an acetic acid derivative. The reaction has been pictured as occurring in this fashion:<smiles>CC[14CH]OP(=O)(O)OC(=O)C=CC=O</smiles><smiles>O=CC=CC(=O)OP(=O)(O)OCCO</smiles>

Recent investigations have definitely disclosed that the phosphoric acid derivative of acetic acid is not the simple acetyl phosphate pictured above (p. 190), but it probably reacts in a comparable manner. The biologically active phosphoryl derivative of acetic acid is associated with a pantothenic acid coenzyme, and the numerous reactions in which it can be utilized constitute essential steps in many fundamental processes.

A third type of condensation utilized by living organisms is one which involves a reactive single carbon unit related to formic acid but not to carbon dioxide (or the carbonate ion). The only synthetic reaction of this type now recognized occurs in the conversion of glycine to serine (p. 201) and is dependent upon a $p$-aminobenzoic acid-containing enzyme and possibly vitamin $\mathrm{B}_{12}$. The exact mechanism of this type of condensation is not known, nor is it yet possible to state how important reactions of this type will ultimately prove to be in the synthetic activities of cells.

A fourth type of condensation which has been observed is one catalyzed by a thiamine system - the formation of acetoin from the intermediates formed during the metabolism of pyruvic acid. This reaction has never been shown to be of value to any cell from the standpoint of synthesis of cellular components, and it is believed to be a mechanism the only purpose of which is to dispose of end products of carbohydrate metabolism.

Two reactions, catalyzed by pyridoxal phosphate, constitute a fifth type of condensation: (1) the formation of tryptophan from indole and serine, and (2) the cleavage of tryptophan into indole, pyruvic acid and ammonia (p. 183). It should be noted that the $\alpha$-amino group in the presence of pyridoxal-containing enzymes is a "potential keto" group (p. 186); hence these reactions of $\alpha$-amino acids are comparable to those involving the reactive methylene carbon and hydrogen atoms alpha to carbonyl groups.

Because the equilibria of the decarboxylation reactions discussed in the 
preceding divisions of this chapter are so decidedly in favor of the cleavage of carbon-to-carbon bonds rather than the reverse carboxylation, these reactions have sometimes been ignored when effective enzymatic mechanisms for increasing the number of carbon atoms in a molecule are considered. However, carboxylations, both of the $\alpha$ and $\beta$ types, catalyzed by the coenzymes of thiamine and biotin (?), may be utilized to a greater extent in biological syntheses than was once realized..$^{241}$

The type of condensation for which the greatest number of reactions are recognized resembles the Claisen type (second in the listing above). The many processes in which it is employed were not understood until the recent discovery of an essential metabolite-a derivative of acetic acid. Acetic acid had long been recognized as the principal end product in certain types of fermentations, but its importance as an intermediate compound in metabolism was not fully realized until it was shown that acetic acid (labelled with isotopic atoms), when introduced into organisms, was converted into some activated derivative which participated in a number of metabolic processes. ${ }^{242}$ Although the concentration of acetic acid in normal animal tissues is too low to be measured by conventional methods, it was shown by the use of isotopes that in a 24-hour period an adult rat produces a quantity of acetic acid (a phosphorylated derivative) equal to 1 per cent of its body weight. ${ }^{243}$ In the meantime, other investigators found that acetic acid was a substrate for (1) the reaction in which acetylcholine is formed, and (2) the enzymatic acetylation of aromatic amines. Previously it had been recognized that the acetate ion which is used for buffering media in which bacteria are grown has functions independent of its buffering capacity, ${ }^{244}$ and later this metabolic utilization of acetic acid by bacteria was shown to be related to lipide synthesis. ${ }^{245}, 246$

To discuss the reactions in which acetic acid can participate it is necessary to consider the chemical nature of the activated acetyl molecule which is first formed and with which pantothenic acid is associated. This reactive compound, which can be formed enzymatically from acetic acid, is believed actually to be the substance participating in most, if not all of the acetate reactions. It is a molecule which can act both as a phosphorylating and as an acetylating agent ${ }^{247}$ but it is not identical with acetyl phosphate. $248,249,250$ Although there were a number of reasons for assuming the active intermediate to be acetyl phosphate, this compound, when prepared synthetically, was inactive and could not be substituted as the substrate replacing the so-called "active acetate" arising from the oxidative decarboxylation of pyruvate. ${ }^{249}$ Moreover, the "active acetate" is not decomposed readily by the specific enzyme, acetyl phosphatase, whereas the synthetic acetyl phosphate is. ${ }^{248}$ Partial purification of the 
active phosphorylated acetate (prepared enzymatically from acetic acid and adenosine triphosphate by dried bacterial cells) has been reported.251 It contains equimolecular amounts of reactive acetyl and phosphoryl groups; hence, it is not acetyl pyrophosphate. In an acidic environment (pH 1.5) the "active acetate" spontaneously undergoes a transformation producing a compound which is indistinguishable from synthetic acetyl phosphate. ${ }^{251}$ To distinguish the biologically active compound from acetyl phosphate the former will be referred to as the reactive phosphoryl-acetyl intermediate.

Origin of the Reactive Phosphoryl-Acetyl Intermediate. The reactive acetyl compound is formed during carbohydrate metabolism by the oxidative phosphorylative decarboxylation of pyruvic acid which is catalyzed by thiamine pyrophosphate (p. 162). It seems logical that this is also the compound formed during fatty acid (and sterol?) degradation when the $\mathrm{C}_{2}$ fragments are successively cleaved from the molecule. It probably is the intermediate formed from ketogenic amino acids when they are catabolized. Substances metabolically related to fatty acids-acetoacetic acid and ethanol-undoubtedly are metabolized via metabolic pathway in which the reactive acetyl intermediate occurs. It has been shown that the tricarboxylic acids can be cleaved to yield oxalacetic acid and the reactive acetyl molecule by a reversal of the reactions in which these acids are formed. ${ }^{241}$ When an exogenous supply of acetic acid is available, cells can use a pantothenic acid enzyme, "acetylphosphorylase," to "activate" the acetic acid molecule; adenosine triphosphate is the phosphorylating agent.

\section{The Coenzyme Derived from Pantothenic Acid}

Ten years elapsed between the time (1936) that pantothenic acid was first found to participate in carbohydrate metabolism ${ }^{253}$ and the time that the mechanism of its function was established.

Physiologists interested in nerve metabolism discovered (in 1942) an enzyme system in brain tissue which was responsible for the synthesis of acetylcholine from acetic acid, choline, and adenosine triphosphate. ${ }^{254}$ At about the same time another group of investigators demonstrated the presence in liver of an enzyme which converts sulfonamides and other aromatic amines into the less toxic amides by acetylation. ${ }^{249}$ They showed that the preparations contained a heat-stable, dissociable component which would reactivate enzyme systems which had been inactivated by dialysis. This coenzyme was subsequently shown to be a necessary component of the system which acetylated choline. ${ }^{255}$

Initial attempts to demonstrate the presence of a $\mathrm{B}$ vitamin in this coenzyme were unsuccessful, because pantothenic acid, the essential vita- 
min component, is bound in such a way that it is not released by the ordinary methods currently used for preparing samples for microbiological assay. Acid hydrolysis of the coenzyme did, however, yield appreciable amounts of $\beta$-alanine. ${ }^{256}$ The coenzymatic activity of preparations of various degrees of purity paralleled the $\beta$-alanine content. It was also shown that pantothenic acid could be liberated in its uncombined form by use of a combination of a phosphatase and an enzyme obtained from liver. ${ }^{256,} 257$

The presence of pantothenic acid in this coenzyme established at least one function of this vitamin, and indicated its involvement in reactions in which acetic acid is utilized. It was realized, however, that neither of the rather specialized reactions studied could account for the general importance of the vitamin. This led to the subsequent demonstration of the essentiality of the pantothenic acid-containing coenzyme for a number of reactions of general importance in carbohydrate and fat metabolism where tracer studies and investigations of acetate metabolism in bacteria had indicated involvement of acetic acid. This coenzyme is now usually referred to as coenzyme A (A for acetylation), and will be so designated in this discussion.

The Chemical Structure of Coenzyme A. The structure of coenzyme A has not yet been announced. The rate at which it diffuses through sintered glass membranes indicates that its molecular weight probably lies between 750 and $850 .^{258}$ A preparation of the coenzyme containing 11 per cent pantothenic acid, contained 9 per cent phosphorus, 18 per cent adenine, 22 per cent pentose and some cysteine. On the basis of its pantothenic acid content and apparent molecular weight, this preparation was only 50 per cent pure, but its analysis does indicate the presence of adenylic acid. Glutamic acid is probably an additional constituent, since it is essential for the biosynthesis of a pantothenic acid complex, ${ }^{259}$ which is believed to be identical with a product obtained during the enzymatic degradation of the coenzyme. ${ }^{258}$ Studies of the enzymatic degradation of the compound indicate that there are at least two linkages which must be cleaved before pantothenic acid is liberated. Both an intestinal phosphatase and an enzyme present in liver extract must be allowed to act upon the molecule before the coenzyme will be active in the microbiological assays usually employed. Either enzyme alone renders the coenzyme inactive (each producing a different product), but neither enzyme by itself liberates panthothenic acid. ${ }^{257}$

Assay Methods. The microorganisms commonly employed in B vitamin assays cannot utilize coenzyme A. ${ }^{256}$ Acetobacter suboxydans, which responds very slowly to pantothenic acid, has been shown to grow rapidly in the presence of a pantothenic acid conjugate (PAC) concentrated from 
liver or heart muscle.259 Intact coenzyme A produces a comparable response with this organism..$^{258}$ The cleavage in the coenzyme catalyzed by the liver enzyme produces a derivative, still phosphorylated, which is equally active for Acetobacter suboxydans. Incubation of either the intact coenzyme or its phosphorylated intermediate with phosphatases, however, destroys the activity for this organism.

The pantothenic acid conjugate (PAC) isolated from heart muscle was inactive when tested in enzymatic acetylation systems. ${ }^{259}$ It seems likely, then, that it is identical with the phosphorylated intermediate derived from coenzyme A by treatment with the liver enzyme and probably is formed from coenzyme A by an autolytic process during its concentration from tissues. A. suboxydans consequently cannot be used for the specific microbiological assay for the intact coenzyme.

At present the only method of differentiating coenzyme A from some of its degradation products is by the use of enzyme analyses. By a combination of assays, using Lactobacillus arabinosis, A. suboxydans, and enzymatic acetylation, it should be possible to work out a differential assay for coenzyme A, free pantothenic acid, and the two compounds of intermediate complexity. The activity under various testing conditions is summarized:

Free pantothenic acid

Coenzyme A

Phosphorylated intermediate (PAC?)

Phosphatase-treated coenzyme

Growth of

L. arabinosis

$+$

$-$

$\overline{-}$

\pm long incubation or high concentration required.

An enzymatic determination of coenzyme A can be made either by (1) following the acetylation of choline (using as an indicator a biological response-muscle contraction) ${ }^{260}$ or (2) determining the rate of acetylation of aromatic amines. ${ }^{249}$ As a routine method of analysis, the latter is preferred because of its greater simplicity and accuracy. A detailed description of this assay procedure has been published.261 This method can be conveniently adapted to laboratories equipped for microbiological analyses. The preparation of a suitable apoenzyme is not a problem, since crude liver extracts can be used. The coenzyme originally present in these extracts is completely inactivated if the extract is allowed to stand for four hours at room temperature.

Occurrence. By use of the rate of acetylation method just described, the coenzyme A content of a number of animal tissues and various plant materials has been ascertained. ${ }^{261}$ The pantothenic acid content of these sources was simultaneously measured by the conventional microbiological procedure. A comparison indicates that within cells pantothenic acid exists 
almost exclusively in the form of its coenzyme. As is the case with several of the other B vitamins, the plasma pantothenic acid is in the form of the free vitamin, whereas the vitamin within the red blood cells has been converted quantitatively to the coenzyme.

Biosynthesis. The chemical routes by which pantothenic acid is converted to coenzyme $\mathrm{A}$ are not known. Before coenzyme $\mathrm{A}$ was discovered, it had been shown that the addition of pantothenic acid quickly activated the pyruvate metabolism of bacterial cells which were deficient in pantothenic acid. ${ }^{262}$ Later it was shown that incubation of pantothenic aciddeficient yeast and bacteria with pantothenic acid resulted in a rapid synthesis of coenzyme A. ${ }^{263,264}$ This indicates that these cells possess adequate mechanisms for the rapid synthesis of the coenzyme. Since the tissues of animals receive their pantothenic acid in an uncombined form from the blood stream, they too must be able to carry out this conversion. Strangely enough, however, the addition of pantothenic acid to surviving deficient tissues of ducks and rats in vitro does not result in any demonstrable synthesis of the coenzyme. ${ }^{265}$

Incubating resting yeast cells with glutamic acid and pantothenic acid (or $\beta$-alanine) results in the production of a conjugate which occasionally shows a thousand times the activity of an unincubated control containing these same substances. ${ }^{259}$ That the formation of the conjugate may not be direct from the vitamin itself was indicated by the fact that the incubation product of $\beta$-alanine was consistently more active than that obtained from pantothenic acid.

Reactions Catalyzed by Coenzyme A. Although all the reactions catalyzed by coenzyme A may involve a common substrate, they result in the formation of a variety of chemical compounds: amides, esters, acid anhydrides, and compounds produced by the condensation of an acetate radical with keto acids or acid phosphates.

The equations for enzymatic reactions in which coenzyme A is a catalyst are tabulated on the following page. Following this list is a summary of the enzyme reactions in which pantothenic acid has been definitely implicated, but which have not been sufficiently well characterized to enable one to say with certainty that coenzyme $\mathrm{A}$ is the coenzyme.

It should be noted that the reaction in which the acetyl derivative condenses with oxalacetic acid to form a tricarboxylic acid is the one which initiates the cycles by which both carbohydrates and fatty acids are metabolized aerobically, and by which they are converted to the dicarboxylic acids, $\alpha$-ketoglutaric, glutamic, fumaric, tartaric, malic, oxalacetic, and aspartic acids (p. 223). When carbohydrate metabolism provides the acetyl molecule, thiamine and pantothenic acid are required; when fatty acids or ethanol are the source of the reactive phosphoryl- 
acetyl intermediate, only pantothenic acid is needed to mediate the initial condensation.

I. Reactions demonstrated using cell-free preparations in which coenzyme $\mathrm{A}$ is the cofactor.

A. Formation of acid phosphates acetic acid + ATP $\longrightarrow$ phosphoryl-acetyl intermediate +ADP

B. Formation of esters choline +acetic acid $+\mathrm{ATP} \longrightarrow$ acetylcholine $+\mathrm{ADP}+\mathrm{H}_{3} \mathrm{PO}_{4}{ }^{260}$

C. Formation of amides $p$-aminobenzoic acid (or sulfonamides) +acetic acid +ATP $\longrightarrow$ acetylated amine $+\mathrm{ADP}+\mathrm{H}_{3} \mathrm{PO}_{4}^{266}$

D. Condensation reactions*

2 acetic acid $+\mathrm{ATP} \longrightarrow$ acetoacetic acid $+\mathrm{ADP}+\mathrm{H}_{3} \mathrm{PO}_{4}^{200}$

II. Conversions requiring pantothenic acid which can be demonstrated in vivo by the use of pantothenic acid antagonists, deficient cells, isotopes.

acetic acid $\longrightarrow$ fatty acids (bacteria) ${ }^{267}, 268$

acetic acid $\longrightarrow$ phloroglucinol-like compound (bacteria) ${ }^{269}$

acetic acid $\longrightarrow$ sterols (bacteria) ${ }^{267,268}$

acetic acid $\longrightarrow$ aromatic amino acids (bacteria) ${ }^{269}$

acetic acid $\longrightarrow$ cis-aconitate $\longrightarrow \alpha$-ketoglutarate (bacteria) ${ }^{268 *}$

acetic acid $\longrightarrow \mathrm{CO}_{2}+\mathrm{H}_{2} \mathrm{O}$ (yeast) ${ }^{264}$

glucose, or pyruvic acid, or lactic acid $\longrightarrow \mathrm{CO}_{2}+\mathrm{H}_{2} \mathrm{O}$ (rat liver) ${ }^{270,}{ }^{266}$ (duck tissues) ${ }^{266}$ (bacteria) ${ }^{262,} 271$

proteins or carbohydrates $\longrightarrow$ fats (rat) ${ }^{272}$

III. Conversions involving acetic acid for which pantothenic acid has not yet been shown essential.

acetic acid $\longrightarrow$ formic acid (pigeon) ${ }^{273}$

acetic acid $\longrightarrow$ porphyrins $(\mathrm{dog})^{274}$

ketogenic substances $\longrightarrow$ acetone bodies (mammals)

acetic acid +acetic acid $\longrightarrow$ succinic acid (molds) ${ }^{275}$

The synthesis of fats from either carbohydrate or protein as far as is now known must go on through processes in which reactions mediated by pantothenic acid cause the condensation of reactive acetyl molecules. The pantothenic acid-requiring reactions in which carbohydrates and fats are oxidized and in which fatty acids are synthesized are undoubtedly essential in many forms of life, while the reactions in which amines and alcohols are acetylated may be of importance only in specific phyla having special functions in which an acetylating agent is needed for the production of acetic acid esters or amides.

No detailed studies of the possible dissociation of coenzyme A from the apoenzyme while the coenzyme is still associated with the phosphorylated acetic acid have yet been undertaken; hence, it is not known whether (1) both the creation and utilization of the "active acetate" must take place simultaneously in a coupled system, or (2) coenzyme A accepts the active molecule from one enzymatic reaction, dissociates itself from this

* The reaction in which citric acid is formed by the condensation of an activated acetate with oxalacetate should be added to the well characterized reactions since coenzyme $\mathrm{A}$ has now been shown to be the coenzyme which reactivates an aged cell-free preparation capable of accomplishing this synthesis (see footnote p. 223). 
enzyme, and transports the acetyl derivative to another system where it is utilized.

It is impossible to do more than speculate upon possible linkages that may exist between coenzyme A and the phosphorylated acetate molecule. Studies of the specificity of chemical structure for compounds that will replace or inhibit pantothenic acid have been undertaken (p.620), but the information obtained is not sufficient to justify any conclusions concerning the question posed in the previous sentence, or to decide what groups of the coenzyme are essential for the formation of the enzymecoenzyme bond.

Is there more than one pantothenic acid coenzyme? All enzymatic phenomena in which pantothenic acid has been implicated can be explained on the basis of a single coenzyme. A fact offering corroborative evidence for this statement is that all the intracellular pantothenic acid is in a form which can serve as the active coenzyme for the aromatic amine acetylation system. If the coenzyme requirement for this enzyme is specific, then within cells there can be only one pantothenic acid coenzyme present in measurable quantities.

Does the pantothenic acid coenzyme mediate reactions other than those involving acetic acid derivatives? On the basis of information now available, there seems to be no justification for postulating other functions for pantothenic acid.

\section{Coenzymes Involved in the Utilization of the Single Carbon Unit}

Formic acid is a common by-product of bacterial fermentation. Like acetic acid, it had long been regarded as a waste product of inefficient catabolic processes, but its role in essential synthetic reactions had gone unrecognized. The first indication of its participation in biologically important synthetic reactions resulted from exploratory tracer studies designed to establish the precursors of purines when they are synthesized de novo by animal tissues. It was found that neither carbon dioxide nor the carboxyl group of pyruvic acid could serve as a source of the carbon atoms in the ureide portion of the purine molecule (positions 2- and 8-), although it had been anticipated that they would be found to be precursors of the single carbon units. ${ }^{276}$ When labelled formic acid was used, however, the isotopic carbon was incorporated into the purine nucleus in the 2 and 8 positions. ${ }^{273}$ This discovery created considerable interest in possible metabolic roles of formic acid and stimulated further investigations, which have now disclosed other important reactions involving the single carbon unit derived from formic acid.

No statement can be made at this time as to the exact chemical nature of this single carbon unit which is normally produced and utilized in 
biological systems. It may simply be formic acid; it may be a reactive derivative similar to the phosphorylated derivatives of acetic acid. Another possibility is that the "formyl group" is chemically combined with a "formate-carrying" coenzyme from the time of its formation until it is utilized, so that the formic acid or the formate ion would not necessarily be present as such. Until this question has been settled, it seems unwise to designate the reactive intermediate as formic acid or formate; hence, the expression "single carbon unit" has been used.

Origin of the Single Carbon Unit. Exogenous sources of formic acid itself can be utilized for syntheses requiring the single carbon units (at least, by bacteria, fowl, and mammals). ${ }^{277}, 273,278$ Consequently, biological reactions producing formic acid may serve as sources of the single carbon unit. Before isotopically labelled compounds were available, free formic acid had been shown to be formed by:

(1) many species of bacteria, in most cases by a phosphoroclastic cleavage of pyruvic acid (p. 162);

(2) muscle perfused with pyruvic acid (perhaps by a phosphoroclastic cleavage of $\alpha$-ketoglutaric acid) (p. 167);

(3) the enzymatic degradation of the imidazole nucleus of histidine (by histidinase)..$^{279}$

(4) insects (mechanism unexplored).

Other potential sources of the single carbon unit recently disclosed by the use of tracers are: either carbon atom of glycine, ${ }^{280,} 281$ the $\beta$-carbon atom of serine, ${ }^{282}$ the carboxyl carbon atom of acetic acid, ${ }^{276}$ and the carbon atoms of the $\mathrm{N}$ - and $\mathrm{S}$-methyl groups of choline and methionine. ${ }^{282}$

Although the carboxyl group of pyruvic acid is the precursor of the formic acid produced by bacterial fermentation, it has been shown that this group can not be the primary source of the single carbon unit in the metabolism of animals. ${ }^{276}$ The simultaneous accumulation of succinic acid and formic acid when muscles are perfused with pyruvic acid indicates that a phosphoroclastic splitting of $\alpha$-ketoglutaric acid may be an important source of the single carbon unit in organisms in which pyruvic acid is metabolized via the tricarboxylic acid cycle (p. 223). Such a reaction could account for the ultimate incorporation of the carboxyl carbon atom of acetic acid and the carbonyl carbon atom of pyruvic acid into compounds in the same positions where labelled carbon atoms of formic acid have been shown to appear. (In aerobic metabolism, these particular carbon atoms in the acetic and pyruvic acid molecules eventually form the $\alpha$-carboxyl group of $\alpha$-ketoglutaric acid). Histidine was for a long time believed to be the precursor of purines in animals since ingestion of histidine increased the excretion of purines. ${ }^{283}$ It was naturally assumed that the histidine was the precursor of the purine's imidazole nucleus. 
Recent studies with isotopic nitrogen have indicated that this concept is invalid, since the nitrogen atoms of the imidazole ring of histidine are not used in the formation of purines. ${ }^{284}$ However, the formic acid or a derivative arising from the hydrolysis of the $\mathrm{N}=\mathrm{C}-\mathrm{N}$ bonds of histidine by histidinase could serve as the source for the single carbon units needed in purine synthesis, and the histidine effect could be entirely attributed to its activity as a formic-donor. It has not been shown whether or not the ureide carbon atoms of purines and pyrimidines can be effectively used as sources for the single carbon intermediate (in reactions which reverse the processes of their syntheses). Neither serine, glycine, methionine, choline, nor histidine can be considered as primary sources of the single carbon unit in organisms which do not require an exogenous supply of these substances.

Vitamins Associated with the Metabolism of the Single Carbon Unit. Both $p$-aminobenzoic acid-containing coenzymes and vitamin $\mathrm{B}_{12}$ (derivatives?) are catalysts in some stages of the processes by which formic acid or its derivatives are utilized for synthetic purposes.

\section{Coenzymes Derived from p-Aminobenzoic Acid and Folic Acid}

The specific enzyme systems in which folic acid and other $p$-aminobenzoic acid derivatives participate are not as yet known. In no instance can we be certain of the exact structure of the compounds entering into or produced by the individual reactions, nor do we know the chemical components of the coenzymes derived from these vitamins. However, a general hypothesis which explains most of the biochemical reactions in which these vitamins are implicated can be arrived at from information acquired during the course of a number of investigations whose primary objectives were entirely unrelated.

Exploration of the mechanism of sulfonamide inhibition led not only to the recognition of the vitamin activity of $p$-aminobenzoic acid, but also to the establishment of a metabolic relationship between $p$-aminobenzoic acid and purines, pyrimidines, and certain amino acids; all these substances can at least partially overcome the toxic effect of sulfonamides upon bacterial growth (Chapter II D). However, the manner in which the amino acids and nitrogen bases acted as "reversing agents" and their relationship to $p$-aminobenzoic acid were at first obscure. It was suggested that they might be products of reactions involving $p$-aminobenzoic acid and that the presence of sulfonamides suppressed their synthesis. ${ }^{285}$

Subsequent studies on the nutritional requirements of organisms and mutants requiring an exogenous supply of $p$-aminobenzoic acid or folic acid demonstrated that here also the amounts of these vitamins needed 
to produce growth responses were definitely determined by the presence or absence of purines, thymine, and the amino acid, methionine. ${ }^{286,287}$

The hypothesis that these essential metabolites were products of processes, one or more reactions of which involve $p$-aminobenzoic acid, was greatly strengthened when quantitative concepts developed for isolated enzyme systems were shown to be applicable also to the inhibition of growth in bacteria. ${ }^{288}$ It was deduced that a simple mathematical analysis of the manner in which a compound counteracts inhibition of bacterial growth indicates the role of this substance in the inhibited system. That is, it is possible to determine whether a substance is (1) the substrate (or precursor of the substrate) of the reaction, (2) a product of the reaction (or derived from it), or (3) a part of the catalytic mechanism of the reaction (Chapter ID).

Whereas the antagonism between $p$-aminobenzoic acid and the sulfonamides is competitive, the concentrations of the pyrimidines, purines, and amino acids required to initiate growth in cultures which had been completely inhibited by sulfonamides were found to be entirely independent of the level of the inhibitor, thus substantiating the belief that these compounds are "products" of $p$-aminobenzoic acid functioning, ${ }^{289}$ and that this vitamin is required for their synthesis. What these synthetic processes have in common, though, was not recognized until (1) tracer studies had demonstrated the existence of the single carbon intermediate, and (2) inhibition studies had established the type of reactions catalyzed by $p$-aminobenzoic acid.

The link connecting $p$-aminobenzoic acid with the metabolism of the single carbon unit was disclosed when the chemical nature of a reactant in a $p$-aminobenzoic acid catalyzed process was established. ${ }^{290} \mathrm{~A}$ substance of unknown structure accumulated in the culture medium of Escherichia coli whenever the growth of the organism was partially inhibited by sulfonamides. ${ }^{291}$ The compound presumably piled up because, after its formation, sulfonamides blocked the reaction by which it normally was utilized. Hence it represented a compound related to the substrate of a $p$-aminobenzoic acid-catalyzed reaction. The compound which accumulated was shown to possess a molecular structure which would make it a logical precursor of purines. It is an imidazole so substituted that if direct condensation with a single carbon unit were effected, a naturally occurring purine hypoxanthine would be formed. It was therefore postulated that one of the functions of $p$-aminobenzoic acid was to catalyze a reaction in which the single carbon unit is introduced into the 2 position of the purine nucleus and that one of the direct effects of sulfonamide inhibition is the blocking of reactions in which the single carbon unit participates. ${ }^{290}$ 


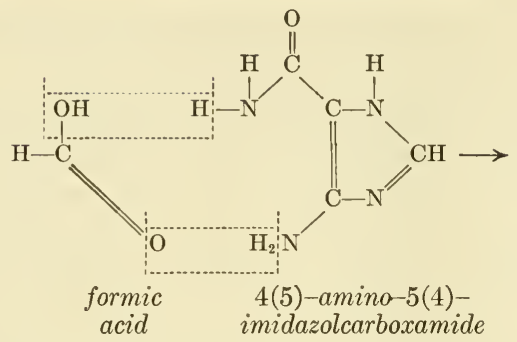

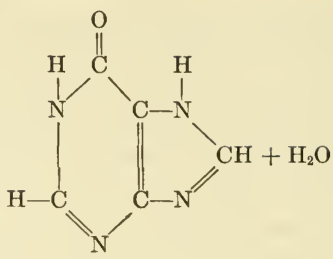

hypoxanthine

The possible involvement of folic acid in the metabolism of formic acid derivatives was indicated a few months later when the structure of the factor which could be substituted for folic acid in culturing Streptococcus faecalis $R$. (SLR factor) was shown to be formylpteroic acid (I) ${ }^{292}$ The glutamic acid peptide of this substance, formylfolic acid (II), was synthesized and shown to be more active than folic acid itself in reversing inhibitors structurally related to folic acid..$^{293}$<smiles>Nc1nc(O)c2nc(CN(C=O)c3ccc(C(=O)O)cc3)cnc2n1</smiles>

formylpteroic acid (I)<smiles>C=CN(Cc1cnc2nc(N)nc(O)c2n1)c1ccc(ONC(CCC(=O)O)C(=O)O)cc1</smiles>

formyl folic acid (II)

Since $p$-aminobenzoic acid derivatives occur in both a formylated and unformylated state, and since both states are biologically active, it is tempting to postulate that the coenzymes of this vitamin catalyze the reactions of single carbon units by serving as their carriers in the same way that the nicotinic acid coenzymes are considered to be hydrogen carriers. As yet, however, no direct evidence has been offered to prove or disprove the hypothesis that the coenzymes mediating the single carbon 
condensations are alternately formylated in one reaction and then regenerated by acting as formylating agents in a second.

Reactions Catalyzed by Coenzymes Derived from $p$-Aminobenzoic Acid and Folic Acid. Since a direct demonstration of the participation of these vitamins in any defined enzyme system has not been achieved, no equations will be written to depict the specific reactions for which they or their derivatives are required. The following isolated observations, however, can be explained and integrated if one accepts a general hypothesis in which it is assumed that: derivatives of $p$-aminobenzoic acid function as coenzymes for metabolic reactions in which the single carbon units (related to formic acid) are utilized for synthetic purposes.

\section{Amino Acid Synthesis}

A. Serine and Glycine. A p-aminobenzoic acid coenzyme is required for the reductive condensation by which serine is formed from glycine and formic derivatives, or for the reverse oxidative cleavage by which glycine is produced from serine, because: serine increases the sulfonamide $p$-aminobenzoic acid ratio needed to inhibit bacteria 294,295 ; serine is a precursor of glycine, ${ }^{296}$ but in the presence of sulfonamides this conversion cannot take place ${ }^{297}$; a synthesis of serine from formic acid and glycine has been demonstrated 280, 281; and folic acid has been shown to be involved in the interconversion of glycine and serine. ${ }^{293}$

B. Methionine. A $p$-aminobenzoic acid coenzyme is believed to be an essential catalyst for some reaction in the reductive process by which the single carbon unit is converted to an S-methyl group needed for the synthesis of methionine from homocysteine, because: methionine increases the sulfonamide $p$-aminobenzoic acid ratio needed to inhibit bacteria, ${ }^{285,} 289$ and decreases the requirements of organisms (and mutants) unable to synthesize their own $p$-aminobenzoic acid ${ }^{287}$; but homocysteine is ineffective in either of these situations ${ }^{299}$; the S-methyl carbon atom of methionine, isotopically labelled, is found to be incorporated into the $\beta$-position when serine is formed biosynthetically from glycine.282

\section{Purines and Pyrimidines.}

Derivatives of folic acid and $p$-aminobenzoic acid are presumed to be coenzymes for the reactions in which the single carbon unit is incorporated into purine and pyrimidine nuclei, because: in the presence of amino acids the folic acid requirements of some microorganisms can be replaced by thymine and purines ${ }^{300}$; in the presence of amino acids, the $p$-aminobenzoic acid requirements of other microorganisms can be replaced by thymine and purines ${ }^{287}$; in the presence of amino acids the inhibition produced by folic acid analogues can be prevented by thymine and 
purines (section I) ; in the presence of amino acids the inhibition produced by $p$-aminobenzoic acid inhibitors can be prevented by thymine and purines (section D); for some bacteria, 4(5)-amino-5(4)-imidazolcarboxamide is partially effective as a purine substitute, and the presence of formic acid enhances its activity ${ }^{277}$; this amine accumulates when sulfonamides block the synthesis of purines. ${ }^{290}$ Folic acid can cause a hematopoiesis in certain macrocytic anemias; thymine and nucleotides of purines and pyrimidines also have produced a similar response (p. 414).

Mention has been previously made of instances in which the nutritional requirements of certain bacteria for a particular vitamin (biotin, p. 173 or pyridoxal, p. 184) can be completely satisfied by supplying only the products of the reactions for which the coenzyme of the vitamin is required, and none of the vitamin itself. This phenomenon was first demonstrated with folic acid. In a medium containing purines and amino acids, thymine can substitute for the folic acid required for growth of a lactobacillus. No synthesis of folic acid could be detected in the cells grown upon this medium. ${ }^{301}$

Composition of the Coenzymes. Of a number of questions still unanswered concerning these vitamins, one of the most perplexing is: Are $p$-aminobenzoic acid and folic acid used to form the same coenzymes? An independent requirement for both $p$-aminobenzoic acid and folic acid has never been demonstrated; consequently one cannot, on the basis of nutritional requirements, imply the existence of separate coenzymes for these two vitamins. Nothing is known concerning the chemical nature of their coenzymes; hence, it is impossible to use chemical composition as a criterion for deciding the question. No coenzymatic activity has yet been associated with the various combined forms of folic acid and $p$-aminobenzoic acid; as a result, a quantitative comparison of the relative distribution of "combined folic acid" and "combined $p$-aminobenzoic acid" cannot provide a solution based upon the distribution of their actual coenzymes. Nor can a decision be based upon information concerning the biological activities of the two vitamins under various conditions; the relative responses produced by a vitamin, its coenzymes, and compounds of intermediate complexity depend entirely upon the type of biological system used; and, except in the case of coenzymatic activity in cell-free systems, the responses of the various derivatives have no predictable relationship to their chemical complexity.

Consequently, a definite statement as to the structure of the coenzymes derived from these two vitamins and their identity or relationship to each other must be delayed until isolated systems can be employed to establish the identity of the specific cofactors. 
If only coenzymes common to both vitamins exist, there must be two alternative routes for their syntheses: (1) one in which folic acid is first synthesized from $p$-aminobenzoic acid or else must be supplied preformed to the organism (in which case $p$-aminobenzoic acid might be ineffective as a substitute for folic acid); and (2) one in which the order of the assembling of the components is such that folic acid does not constitute an intermediate (in which case folic acid might be ineffective as a substitute for $p$-aminobenzoic acid).*

Biosynthesis of the Coenzymes. Although the chemical nature of the biosynthetic processes by which coenzymes are formed from folic acid and $p$-aminobenzoic acid is unknown, mention should be made of four observations which may have some bearing on mechanisms of the conversion of the vitamins to their active forms. (1) The occurrence and activities of the formyl derivatives of folic and pteroic acids have been previously pointed out; the formylation of the amino group of a $p$-aminobenzoyl moiety may be a reaction occurring at some stage in the formation of the coenzymes. (2) An enzyme, designated as vitamin $B_{c}$ conjugase by its discoverers, catalyzes the hydrolysis of the polyglutamyl derivatives of folic acid which have been found to occur in yeast, plant, and animal tissues ${ }^{302}$; this enzyme may catalyze reactions necessary for converting the pteroyl polypeptides into a form which can be used for coenzyme synthesis. (3) Vitamin $\mathrm{B}_{12}$ has been postulated as functioning in the utilization of $p$-aminobenzoic acid, probably by promoting some reaction which is a necessary step in producing the active cofactors of $p$-aminobenzoic and folic acids (p. 207). (4) The amount of folic acid necessary to cause a remission in pernicious anemia is markedly decreased if the vitamin is first incubated with a liver preparation of "xanthopterin oxidase." ${ }^{303}$ Folic acid, when incubated either with concentrates possessing xanthine oxidase activity or with crude extracts of gastric mucosa is reported to be "activated" in a fashion such that it has vitamin $B_{14}$ activity (p. 16), i.e., it can (a) stimulate erythropoiesis in bone marrow tissue cultures and (b) inhibit proliferation of tumor cells cultured in vitro. Untreated folic acid is inactive. No further information on the chemical or functional relationship of vitamin $\mathrm{B}_{14}$ and folic acid has been disclosed.

* An announcement of the natural occurrence of a group of substances structurally and functionally related to folic acid which are 100 times as active as folic acid in preventing the inhibition of a folic acid inhibitor was made in November, 1949 (Bond, T. J., Bardos, T. J., Sibley, M., and Shive, W., J. Am. Chem. Soc., 71, 3852 (1949)). These forms of the folic acid vitamin, designated the folinic acid group, replace the thymidine requirement of an organism which does not utilize folic acid itself. Hence, this group may constitute folic acid coenzymes or be compounds more complex than folic acid itself which are elaborated during the synthesis of the coenzyme (s). 
Other Catalytic Functions of Folic Acid. The catabolism of tyrosine is altered in folic acid deficiencies produced in animals, ${ }^{304}$ in scorbutic guinea pigs, ${ }^{305}$ and in humans afflicted with pernicious anemia. ${ }^{306}$ This metabolic derangement in the clinical anemias is so consistently observed that it has been suggested as a test having diagnostic value (p. 416). In these three instances the normal oxidation of tyrosine is blocked, causing the accumulation of phenolic keto acids (probably mono- and dihydroxyphenylpyruvic acids). The oral administration of either ascorbic acid, folic acid, or liver corrects the defective tyrosine metabolism in the scorbutic guinea pigs ${ }^{307}$; treating the pernicious anemia patients with liver extracts (containing vitamin $\mathrm{B}_{12}$, but no folic acid) caused a drop in the concentration of phenolic substances in the blood and urine ${ }^{308}$; and addition of folic acid in vitro to liver slices from folic acid-deficient rats markedly increased their ability to oxidize tyrosine. ${ }^{309}$ These results are of considerable interest, inasmuch as both folic acid and ascorbic acid have been demonstrated to have some metabolic relationship to vitamins $B_{12}$, both in the nutrition of microorganisms (p. 206) and in the treatment of pernicious anemia in humans (p. 416); however, the interrelationship in terms of cellular reactions is obscure. It may be that folic acid is a component of the tyrosine oxidase system and has functions entirely independent of its role in the metabolism of formic acid derivatives; or, since only intact tissues have been used, the function demonstrated for this vitamin in tyrosine oxidation may be an indirect one. The amounts of folic acid required for correcting the faulty tyrosine metabolism either in vivo or in vitro are of higher order of magnitude than are those required for alleviating other symptoms of folic acid deficiencies.

Folic acid has been reported to increase the choline esterase content of blood. ${ }^{310}$ The concentration of this enzyme, which catalyzes the hydrolysis of acetylcholine following its release by neural activity, apparently rises when folic acid is administered to experimental animals or even when folic acid is added to samples of blood serum in vitro. In an independent investigation, no evidence was obtained which would support the original claims of folic acid activity. ${ }^{311}$ How direct an action folic acid may have upon choline esterase is not known, but in view of its other functions it seems unlikely that folic acid would be directly involved as a coenzyme in this hydrolytic enzyme.

Dopa decarboxylase (extract of rat kidney) has been postulated to have a coenzyme related to folic acid since pterin analogues inhibit the activity of the enzyme, and the inhibition can be prevented by folic acid. ${ }^{312}$ A pyridoxal analogue, which inhibits tyrosine decarboxylase, was inactive. The pyridoxal-like activity of folic acid in this particular 
instance cannot be easily correlated with any other known function of folic acid. It may have some relationship to the role of folic acid in the oxidation of tyrosine. ${ }^{304}$

\section{Coenzymes Derived From Vitamin $\mathbf{B}_{12}$}

Vitamin $B_{12}$ and chemically related substances have only recently become available to investigators interested in studying the chemical functions of these compounds. As a result, their enzymatic role has not yet been clearly defined. On the basis of preliminary and incomplete reports, however, it would appear that the cobalt-containing compounds effective in the treatment of pernicious anemia should be added to the list of factors necessary for important reactions of general biological occurrence. The inclusion of the $\mathrm{B}_{12}$ group in the list of $\mathrm{B}$ vitamins thus seems warranted, not only from the standpoint of its distribution and nutritional importance, but also on the basis of its function.

Until the end of 1948, all available preparations used clinically and for research were concentrated by procedures which had beeen developed using clinical responses as the method of assay. Consequently, it is not surprising that, in addition to the cobalt-containing factors, these crude concentrates contain appreciable amounts of thymidine and other desoxyribosides which possess "vitamin $\mathrm{B}_{12}$ " activity under some conditions (p. 206). The presence of these nucleosides may be desirable from the standpoint of the effectiveness of the product, but it makes it impossible to interpret accurately the results of experiments in which "injectable liver concentrates," "purified antipernicious anemia preparations," "refined liver extracts," etc. were used as sources of vitamin $\mathrm{B}_{12}$.

It is impossible as yet to make any statement concerning the chemical relationship of the substances possessing vitamin $B_{12}$ activity to the coenzyme(s) derived from them. Two cobalt-containing compounds which are therapeutically active in the treatment of pernicious anemia have been reported by English investigators. ${ }^{313}$ Both these compounds satisfy the nutritional requirements of a microorganism when tested under conditions where "vitamin $\mathrm{B}_{12}$ " is essential. ${ }^{314}$ They can be most easily characterized by the rate at which they travel during chromatographic separation. The slower-moving compound, presumably the more complex of the two, predominates in fresh liver tissue. During autolysis, however, this form of the vitamin is apparently converted enzymatically to the faster-moving compound. ${ }^{315}$ Crystalline substances independently isolated in the United States to which the names vitamin $\mathrm{B}_{12}{ }^{316}$ and erythrotin ${ }^{317}$ have been given are believed to be identical with the faster-moving component. 
The ultraviolet absorption spectrum of a crystalline preparation isolated by a fourth laboratory indicates that the vitamin is "porphyrin-like." 318 One of the cobalt compounds already identified may be an intact coenzyme if the constitution of the prosthetic group of the $B_{12}$ enzymes is no more complex than the porphyrin groups of the iron and copper porphyrin enzymes.

The reactions taking place during the biosynthesis, digestion, absorption, and utilization of this vitamin group are still obscure, and its relationship to the "intrinsic" and "extrinsic" factors has yet to be explained. A vitamin $B_{12}$ preparation was found to be inactive (when administered orally to pernicious anemia patients) unless it had been previously incubated with normal gastric juice. ${ }^{319}$ Another interesting fact which further clouds the picture is that the anemias in cattle resulting from cobalt deficiency can be corrected only by the oral administration of cobalt; parenteral injection of the ion is ineffective. ${ }^{320}$

The type of reactions directly catalyzed by the vitamin $\mathrm{B}_{12}$ coenzyme cannot be stated with certainty, but they definitely are involved in many of the processes which utilize single carbon units. The vitamin appears to be intimately associated functionally with folic acid and $p$-aminobenzoic acid.

To date, vitamin $\mathrm{B}_{12}$ has been implicated in the following processes:

(1) the syntheses of purines and pyrimidines and their derivatives,

(2) the syntheses of methionine and serine, and

(3) the utilization of $p$-aminobenzoic acid and folic acid.

The announcement of the isolation of thymidine ${ }^{321}$ from antipernicious anemia preparations resulted in a number of studies designed to determine what relationship may exist between vitamin $\mathrm{B}_{12}$ and nucleic acid derivatives. When tested on the bacteria which had been found to respond to vitamin $\mathrm{B}_{12}$ (Lactobacillus lactis Dorner and Lactobacillus leishmanii), it was found that either thymidine or the desoxyribosides of other pyrimidines and purines could be substituted for this vitamin.322, 323, 324, 325 Also it was shown that, in the presence of vitamin $\mathrm{B}_{12}$ (and folic acid), purines could be omitted from the medium used to culture L. Lactis. ${ }^{326}$

In considering possible relationship of vitamin $B_{12}$ to folic acid, it is important to note that in the cases just mentioned vitamin $B_{12}$ and folic acid cannot be effectively substituted for each other. An interesting demonstration of their independence of function is contained in a report in which it is implied that the vitamin $\mathrm{B}_{12}$ required by $L$. leishmanii can be replaced by thymidine but not by thymine, whereas folic acid can be replaced (somewhat inadequately) by either thymine or thymidine. ${ }^{326}$ On the basis of the nutritional needs of these two bacteria there has been some speculation as to the possibility of vitamin $\mathrm{B}_{12}$ functioning in the 
formation of desoxyribosides, either by promoting the synthesis of a reactive derivative of desoxyribose ${ }^{322}$ or by catalyzing the union of the sugar with the nitrogen bases. ${ }^{323}$

Vitamin $B_{12}$ has since been shown to participate in other types of biosynthetic processes - those by which serine and methionine are formed. This was first demonstrated by the use of sulfonamide inhibition of Escherichia coli. ${ }^{295,317}$ Two other findings offer collaborative evidence for the methionine functions: vitamin $\mathrm{B}_{12}$ can completely replace the methionine necessary to promote the growth of a $p$-aminobenzoic acid requiring mutant of $E$. coli, when it is cultured in a medium containing suboptimal amounts of $p$-aminobenzoic acid ${ }^{327}$; and the incidence of renal damage in rats resulting from a diet deficient in choline and methionine can be appreciably reduced by the administration of vitamin $\mathrm{B}_{12} \cdot{ }^{328}$

A very plausible explanation for the manner in which vitamin $B_{12}$ functions has been obtained through the use of inhibitors. ${ }^{295}, 317$ In $E$. coli the biosynthesis of methionine, purines, serine and folic acid (or thymine) by enzyme systems containing $p$-aminobenzoic acid can be effectively blocked by sulfonamide inhibition. But in each of these four distinct processes the presence of vitamin $\mathrm{B}_{12}(0.00005 \mu \mathrm{gm} / \mathrm{ml})$ reduces by twothirds the amount of $p$-aminobenzoic acid required to counteract the specific inhibition and restores adequate synthesis of the respective compounds (section D). The investigators felt that their results could be best explained by assuming that vitamin $\mathrm{B}_{12}$ is a catalytic factor necessary for the utilization of $p$-aminobenzoic acid (and folic acid?). This explanation could be interpreted to mean that vitamin $\mathrm{B}_{12}$ functions as a catalyst for the formation of the coenzymes necessary in the reactions involving single carbon units. Such a hypothesis seems reasonable and would explain why vitamin $\mathrm{B}_{12}$ is involved in the biosynthesis of a variety of types of chemical compounds. It should be noted that these compounds include all those in which $p$-aminobenzoic acid and folic acid are definitely known to function (p. 201). It would also imply an independent requirement of both vitamin $\mathrm{B}_{12}$ (catalyst for coenzyme formation) and $p$-aminobenzoic acid or folic acid (substrate for coenzyme formation). Hence folic acid could not be expected to substitute completely for vitamin $\mathrm{B}_{12}$ in the treatment of macrocytic anemias, nor would vitamin $\mathrm{B}_{12}$ be expected to exhibit appreciable activity in the treatment of blood dyscrasias corrected by folic acid therapy (Chapter VI C).

It has been suggested on the basis of clinical evidence that vitamin $B_{12}$ is necessary for the conversion of folic acid conjugates to other derivatives which are more readily utilized. ${ }^{329,} 330,331$ Although the investigators later retracted their conclusions, ${ }^{332}$ a direct demonstration of such a 
function for the vitamin appeared in a recent preliminary report. ${ }^{333}$ The livers from day-old chicks hatched from eggs laid by hens whose diet contained no "animal protein" were used as a source of tissue deficient in vitamin $\mathrm{B}_{12}$. These livers were incapable of liberating folic acid from pteroylheptaglutamic acid. However, the addition of crystalline vitamin $\mathrm{B}_{12}$ initiated conjugase activity. This would indicate that vitamin $\mathrm{B}_{12}$, or a derivative of it formed by the liver cells, is a cofactor for some reaction in the process by which the folic acid conjugate is degraded. The same reaction, or one related to it chemically, may very well be one of the essential steps involved in the conversion of folic acid and $p$-aminobenzoic acid to their active coenzymes.

The particular reaction(s) catalyzed by $\mathrm{B}_{12}$ may be of an oxidative type if the molecular structure of the vitamin is indicative of its mode of functioning. All other metallo-porphyrin enzymes mediate oxidative reactions (p. 151). A similar mechanism for vitamin $B_{12}$ action may be anticipated if further investigation establishes its structure to be that of a typical porphyrin.

There is some metabolic relationship, either direct or indirect, between this vitamin and ascorbic acid (or other reducing compounds). These agents alter the nutritional requirements of lactobacilli to such an extent that in their presence the organism can no longer be demonstrated to require an exogenous supply of vitamin $B_{12}$ or the desoxyribosides. ${ }^{325,334}$ The ascorbic acid-vitamin $\mathrm{B}_{12}$ effect in microorganisms may be related to the synergistic effects of liver extracts and vitamin $\mathrm{C}$ in the treatment of pernicious anemia. ${ }^{335}$

\section{Bibliography}

1. Williams, R. J., Schlenk, F., and Eppright, M. A., J. Am. Chem. Soc., 66, 896 (1944).

2. Meyer, K. H., Fischer, E. H., and Bernfeld, P., Helv. Chim. Acta, 30, 64 (1947)

3. Lane, R. L., and Williams, R. J., Arch. Biochem., 19, 329 (1948).

4. Meyer, K. H., Fischer, E. H., Bernfeld, P., and Duckert, F., Arch. Biochem, 18, 205 (1948).

5. Meyer, K. H., Fischer, E. H., Bernfeld, P., and Staub, A., Experientia, 3, 455 (1947).

6. Di Carlo, F. J., and Redfern, S., Arch. Biochem., 15, 333 (1947).

7. McHenry, E. W., Biological Symposia, 5, 177 (1941).

8. Lucas, C. C., and Best, C. H., Vitamins and Hormones, 1,1 (1943).

9. Chaikoff, I. L., and Entenman, C., $A d v$. in Enzymology, 8, 171 (1948).

10. Best, C. H., Lucas, C. C., Patterson, J. M., and Rideout, J. H., Science, 103, 12 (1946).

11. Chaikoff, I. L., Entenman, C., and Montgomery, M. L., J. Biol. Chem., 160, 489 (1945).

12. Michaelis, L., "Currents in Biochemical Research," Interscience Publishers, Inc., (New York), 1946, p. 207.

13. Michaelis, L., American Scientist, 54, 573 (1946). 
14. Harden, A., and Young, W. J., J. Physiol., 32, Proc. of Nov. 1904 (1905).

15. Warburg, O., and Christian, W., Biochem. Z., 274, 112 (1934).

16. Euler, H. v., Albers, H., and Schlenk, F., Z. physiol. Chem., 237, I (1935).

17. Schlenk, F., and Euler, H. v., Naturwiss., 24, 794 (1936).

18. Schlenk, F., "A Symposium on Respiratory Enzymes," Univ. of Wisconsin Press (Madison, Wisconsin), 1942, p. 104.

19. Warburg, O., Ergeb. Enzymforschung, 7, 210 (1938).

20. Warburg, O., and Christian, W., Biochem. Z., 311, 209 (1942).

21. Warburg, O., and Christian, W., ibid., 286, 81 (1936).

22. Haas, E., ibid., 288, 123 (1936).

23. Euler, H. v., Schlenk, F., and Vestin, R., Naturwis., 25, 318 (1937).

24. Schlenk, F., Högberg, B., and Tingstam, S., Svenska Akad. Arkiv. f. Kemi, 13A, 11 (1939).

24a. Kornberg, A., and Lindberg, O., J. Biol. Chem., 176, 665 (1948).

25. Bredereck, H., Beuchelt, H., and Richter, G., Z. physiol. Chem., 244, 102 (1936).

26. Schlenk, F., and Schlenk, T., Arch. Biochem., 14, 131 (1947).

27. LePage, G. A., J. Biol. Chem., 168, 623 (1947).

28. Warburg, O., Christian, W., and Griese, A., Biochem. Z., 282, 157 (1935).

29. Euler, H. v., and Adler, E., Z. physiol. Chem., 238, 233 (1936).

30. Schlenk, F., Naturwiss., 25, 668 (1937).

31. Euler, H. v., Z. physiol. Chem., 240, 113 (1936).

32. Warburg, O., and Christian, W., Biochem. Z., 287, 291 (1936).

33. Green, D. E., and Dewan, J. G., Biochem. J., 31, 1069 (1937).

34. Myrbäck, K., Z. physiol. Chem., 177, 158 (1928).

35. Krishman, P. S., Science, 95, 295 (1947).

36. Warburg, O., Christian, W., and Griese, A., Biochem. Z., 279, 143 (1935).

37. Haas, E., Harper, C. J., and Hogness, T. R., J. Biol. Chem., 142, 835 (1942).

38. Lwoff, A., and Lwoff, M., Proc. Roy. Soc. (London), B122, 253, 360 (1937).

39. Gingrich, W. D., and Schlenk, F., J. Bact., 47, 535 (1944).

40. Meyerhof, O., Ergeb. Physiol. exptl. Pharmakol., 39, 10 (1936).

41. Straub, F. B., Z. physiol. Chem., 275, 63 (1942).

42. Ochoa, S., and Ochoa, C. G., Nature, 140, 1097 (1937).

43. Euler, H. v., Schlenk, F., Heiwinkel, H., and Högberg, B., Z. physiol. Chem., 256, 208 (1938).

44. Altman, K. I., and Evans, E. A., Jr., J. Biol. Chem., 169, 463 (1947).

45. Hoagland, C. L., Ward, S. M., and Shank, R. E., J. Biol. Chem., 151, 369 (1943).

46. Vestin, R., Naturwiss., 25, 667 (1937).

47. Handler, P., and Klein, J. R., J. Biol. Chem., 143, 49 (1942).

48. Lennerstrand, A., Svenska Vet. Akad. Arkiv. Kemi., B14, 1 (1940).

49. Adler, E., Hellstrom, H., and Euler, H. v., Z. physiol. Chem., 242, 225 (1936).

50. Mehler, A. H., Kornberg, A., Grisolia, S., and Ochoa, S., Abstract in Federation Proceedings, 6, 278 (1947).

51. Schlenk, F., Advances in Enzymology, 5, 207 (1945).

52. Adler, R., and Sreenwasaya, M., Z. physiol. Chem., 249, 24 (1937).

53. Johnson, F. H., and Eyring, H., J. Am. Chem. Soc., 66, 848 (1944).

54. Wirth, J. C., and Nord, F. F., Arch. Biochem., 1, 143 (1942).

55. Warburg, O., and Christian, W., Biochem. J., 254, 438 (1932).

56. Blyth, A. W., J. Chem. Soc., 35, 530 (1879).

57. Kuhn, R., György, P., Wagner-Jauregg, Th., Ber. Deutsch. Chem. Ges., 66, 1034 (1933).

58. Kuhn, R., Rudy, H., and Weygand, F., Ber., 69, 2034 (1936).

59. Karrer, P., Frei, P., and Meerwein, H., Helv. Chim. Acta, 20, 79 (1937).

60. Warburg, O., and Christian, W., Biochem. Z., 296, 150 (1938).

61. Haas, E. Biochem. Z., 290, 291 (1937).

62. Kuhn, R., Boulanger, P., Ber. Deutsch. Chem. Ges., 69, 1557 (1936).

63. Krebs, H. A., Enzymologia, 7, 53 (1939). 
64. Rudy, H., Naturwiss., 23, 286 (1935).

65. Theorell, H., Biochem. Z., 278, 263 (1935).

66. Warburg, O., and Christian, W., Biochem. Z., 298, 368 (1938).

67. Klein, J. R., and Handler, P., J. Biol. Chcm., 139, 103 (1941).

68. Green, D. E., Moore, D. H., Nocito, V., and Ratner, S., J. Biol. Chem., 156, 383 (1944).

69. Blanchard, M. Green, D. E., Nocito, V., and Ratner, S., J. Biol. Chem., 163, 137 (1946).

70. Blanchard, M., Green, D. E., Nocito, V., and Ratner, S., J. Biol. Chem., 161, 583 (1945).

71. Roulet, R., Wydler, H., and Zeller, E. A., Helv. Chim. Acta, 29, 1973 (1946).

72. Knight, S. G., J. Bact., 55, 401 (1948).

73. Singer, T. P., and Kearney, E. B., Fed. Proc., 8, 251 (1949).

74. Ratner, S., Nocito, V., Green, D. E., J. Biol. Chem., 152, 119 (1944).

75. Schardinger, F., Z. Untersuch. Nahr. Genussm., 5, 22 (1902).

76. Morgan, E. J., Stewart, C. P., and Hopkins, F. G., Proc. Roy. Soc. (London), B94, 109 (1922-1923).

77. Franke, W., and Schumann, R., Ann., 552, 243 (1942).

78. Lemberg, R., Wyndham, R. A., and Henry, N. P., Australian J. Exptl. Biol. Med. Sci., 14, 259 (1936).

79. Coulthard, C. E., Michaelis, R., Short, IV. R., Sykes, G., Skrimshire, G. E. H., Standfast, A. F. B., Birkinshaw, J. H., and Raistrick, H., Biochem. J., 39, 24 (1945).

80. Schales, O., Arch. Biochem., 2, 487 (1943).

81. Lipmann, F., and Owen, C. R., Science, 98, 246 (1943).

82. Ball, E. G., Science, 88, 131 (1938).

83. Corran, N. S., Dewan, J. G., Gordon, A. H., and Green, D. E., Biochem. J., 33, 1694 (1939).

84. Booth, V. H., Biochem. J., 32, 494 (1938).

85. Straub, F. B., Biochem. J., 33, 787 (1939).

86. Ball. E. G., J. Biol. Chem., 128, 51 (1939).

87. Haas, E. Horecker, B. L., and Hogness, T. R., J. Biol. Chem., 136, 747 (1940).

88. Lipmann, F., Cold Spring Harbor Symp. Quant. Biol., 7, 248 (1939).

89. Lipmann, F., "Currents in Biochemical Research," Interscience Publishers, Inc. (New York) 1946, p. 137.

90. Fischer, F. G., Roedig, A., and Rauch, K., Naturwiss., 27, 197 (1939).

91. Stotz, E., and Hastings, A. B., J. Biol. Chem., 118, 479 (1937).

92. Axelrod, A. E., Potter, V. R., and Elvehjem, C. A., J. Biol. Chem., 142, 85 (1942).

93. Lwoff, A., and Lwoff, M., Ann. Inst. Pasteur, 59, 129 (1937).

93a. Lwoff, M., and Nicolle, P., Compt. rend. soc. biol., 138, 164 (1941).

94. Stotz, E., "A Symposium on Respiratory Enzymes," The University of Wisconsin Press (Madison, Wisconsin) 1942, p. 149.

95. Sumner, J. B., and Somers, G. F., "Chemistry and Methods of Enzymes," Academic Press, Inc. (New York), 1943, p. 169.

96. Potter, V. R., J. Biol. Chem., 165, 311 (1946).

97. Heppel, L. A., Fed. Proc., 8, 205 (1949).

98. Stephenson, M., Biochem. J., 22, 605 (1928).

99. Bernheim, F., Biochem. J., 22, 1178 (1928).

100. Horecker, B. L., Stotz, E., and Hogness, T. R., J. Biol. Chem., 128, 251 (1939).

101. Gale, E. F., Biochem. J., 33, 1012 (1939).

102. Munoz, J. M., and Leloir, L. F., J. Biol. Chem., 147, 355 (1943).

103. Bernheim, F., and Bernheim, M. L. C., J. Biol. Chem., 143, 391 (1942).

104. Rosenthal, O., and Drabkin, D. L., J. Biol. Chem., 149, 437 (1943).

105. Carruthers, C., J. Biol. Chem., 171, 64 (1947). 
106. Wainis, W. W., Cooperstein, S. J., Kollen, S., and Eichel, B., J. Biol. Chem., 173, 145 (1948).

107. Lipton, M. A., Arnold, A., and Berger, J., "Respiratory Enzymes," Burgess Pub. Co. (Minneapolis, Minn.), 1939, p. 55.

108. Neuberg, C., and Karczag, L., Biochem. Z., 36, 68 (1911).

109. Auhagen, E., Z. physiol. Chem., 204, 149 (1932).

110. Lohmann, K., and Schuster, P., Biochem. Z., 294, 188 (1937).

111. Weijlard, J., and Tauber, H., J. Am. Chem. Soc., 60, 2263 (1938).

112. Barron, E. S. G., and Lyman, C. M., J. Biol. Chem., 141, 951 (1941).

113. Ochoa, S., and Peters, R. A., Biochem. J., 32, 1501 (1938).

114. Westenbrink, H. G. K., Van Dorp, D. A., Gruder, M., and Veldman, H., Enzymologia, 9, 73 (1940).

115. Lipton, M. A., and Elvehjem, C. A., J. Biol. Chem., 136, 637 (1940).

116. Silverman, M., and Werkman, C. H., Enzymologia, 5, 385 (1938-39).

117. Goodhart, R., J. Biol. Chem., 135, 77 (1910).

118. Sumner, James B., and Somers, G. Fred, "Chemistry and Methods of Enzymes," Academic Press, Inc., (New York), 1943, p. 287.

119. Lankford, C. E., and Skaggs, P. K., Arch. Biochem., 9, 265 (1946).

120. Westenbrink, H. G. K., and Goudsmit, J., Enzymologia, 5, 307 (1938).

121. Ochoa, S., "The Biological Action of the Vitamins," The University of Chicago Press, (Chicago, Ill.), 1942, p. 20.

122. Lipton, M. A., and Elvehjem, C. A., Cold Spring Harbor Symp. Quant. Biol., 7, 184 (1939).

123. Weil-Malherbe, H., Biochem. J., 33, 1997 (1939).

124. Cedrangolo, F., and Villano, F., Boll. soc. ital. biol. sper., 17, 558 (1942).

125. Westenbrink, H. G. K., and Veldman, H., Enzymologia, 10, 255 (1942).

126. Parvi, E. P. S., and Westenbrink, H. G. K., Z. Vitaminforschung, 15, 152 (1944).

127. Parvi, E. P. S., Chem. Zentr., I, 2000 (1943).

128. Buchman, E. R., Heegaard, E., and Bonner, J., Proc. Nat. Acad. Sci., 26, 561 (1940).

129. Grob, E. C., Z. Vitaminforschung, 17, 98 (1946).

130. Banga, I., Ochoa, S., and Peters, R. A., Biochem. J., 33, 1109 (1939).

131. Peters, R. A., Biochem. J., 31, 2240 (1937).

132. Saratt, H. P., and Cheldelin, V. H., J. Biol. Chem., 156, 91 (1944).

133. Lipmann, F., Enzymologia, 4, 65 (1937).

134. Ochoa, S., "The Biological Action of the Vitamins," The University of Chicago Press (Chicago, Ill.), 1942, pp. 23-38.

135. Stotz, E., Advances in Enzymology, 5, 145 (1945).

136. Martius, C., Z. physiol. Chem., 279, 96 (1943).

137. Weil-Malherbe, H., Nature, 145, 106 (1940).

138. Green, D. E., Westerfeld, W. W., Vennesland, B., and Knox, W. E., J. Biol. Chem., 140, 683 (1941).

139. Silverman, M., and Werkman, C. H., J. Biol. Chem., 138, 35 (1941).

140. Watt, D., and Krampitz, L. O., Federation Proc., 6, 301 (1947).

141. Gross, N. H., and Werkman, C. H., Arch. Biochem., 15, 125 (1947).

142. Utter, M. R., Werkman, C. H., and Lipmann, F, J. Biol Chem., 154, 723 (1944).

143. Lipmann, F., Advances in Enzymology, 6, 231 (1946).

144. Koepsell, H. J., and Johnson, M. J., J. Biol. Chem., 145, 379 (1942).

145. Krebs, H. A., and Johnson, W. A., Biochem. J., 31, 645 (1937).

146. Weil-Malherbe, H., Biochem. J., 31, 2202 (1937).

147. Barron, E. S. G., and Lyman, C. M., J. Biol. Chem., 127, 143 (1939).

148. Krebs, H. A., Biochem. J., 31, 661 (1937).

149. Wiken, T., Watt, D., White, A. G. C., and Werkman, C. H., Arch. Biochem., 14, 478 (1947).

150. Green, D. E., Stumpf, P. K., and Zarudnoya, K., J. Biol. Chem., 167, 811 (1947). 
151. Ochoa, S., J. Biol. Chem., 155, 87 (1944).

152. Karrar, P., Graf, W., and Schukri, J., Helv. Chim. Acta, 29, 711 (1946).

153. Zima, O., and Williams, R. R., Ber. deutsch. chem. Gesellsch., 73, 941 (1940).

154. Zima, O., Ritsert, K., and Moll, Th., Ztschr. physiol. Chem., 267, 210 (1941).

155. Karrar, P., and Viscontini, M., Helv. Chim. Acta, 29, 711 (1946).

156. O'Kane, D. J., and Gunsalus, I. C., J. Bact., 56, 499 (1948).

157. Vennesland, B., and Evans, E. A., Jr., J. Biol. Chem., 156, 783 (1944).

158. Vennesland, B., Evans, E. A. Jr., and Francis, A. M., J. Biol. Chem., 163, 573 (1946).

159. Evans, E. A., Jr., Vennesland, B., and Slotin, L., J. Biol. Chem., 147, 771 (1943).

160. Ochoa, S. and Weisz-Tabori, E., J. Biol. Chem., 159, 245 (1947).

161. Shive, W., and Rogers, L. L., J. Biol. Chem., 169, 453 (1947).

162. Lardy, H. A., Potter, R. L., and Elvehjem, C. A., J. Biol. Chem., 169, 451 (1947).

163. Korkes, S. and Ochoa, S., J. Biol. Chem., 176, 463 (1948).

164. Lardy, H. A., Abstract 112th Meeting Am. Chem. Soc., Sept., 1947.

165. Pilgrim, F. J., Axelrod, A. E., and Elvehjem, C. A., J. Biol. Chem., 145, 237 (1942).

166. Summerson, W. H., Lee, J. M., and Partridge, C. W. H., Science, 100, 250 (1944).

167. Winzler, R. J., Burk, D., and du Vigneaud, V., Arch. Biochem., 5, 25 (1944).

168. Benz, L. G., and Eakin, R. E., Unpublished data.

169. Plaut, G. W. E., and Lardy, H. A., Fed. Proc., 8, 237 (1949).

170. Ochoa, S., et al., J. Biol. Chem., 170, 413 (1949).

171. Koser, S. A., Wright, M. H., and Dorfman, A., Proc. Soc. Exptl. Biol. Med., 51, 204 (1942).

172. Benz, L. G., Master Thesis, University of Texas, August, 1947.

173. Stokes, J. L., Larsen, A., and Gunness, M., J. Bact., 54, 219 (1947).

174. Potter, R. L., and Elvehjem, C. A., J. Biol. Chem., 172, 531 (1948).

175. Axelrod, A. E., Purvis, S. E., and Hofmann, K., J. Biol. Chem., 176, 695 (1948).

176. Lichstein, H. C., and Umbreit, W. W., J. Biol. Chem., 170, 329 (1947).

177. Lichstein, H. C., and Christman, J. R., J. Biol. Chem., 175, 649 (1948).

178. Lichstein, H. C., J. Biol. Chem., 177, 125 (1949).

179. Williams, V. R., and Fieger, E. A., J. Biol. Chem., 166, 335 (1946).

180. Williams, W. L., Broquist, H. P., and Snell, E. E., J. Biol. Chem., 170, 619 (1947).

181. Axelrod, A. E., Hofmann, K., and Daubert, B. F., J. Biol. Chem., 169, 761 (1947).

182. Axelrod, A. E., Mitz, M., and Hofmann, K., J. Biol. Chem., 175, 265 (1948).

183. Trager, W., J. Biol. Chem., 176, 133 (1948).

184. Trager, W., J. Biol. Chem., 176, 1211 (1948).

185. Burk, D., and Winzler, R. J., Science, 97, 57 (1943).

186. Jones, M., and Eakin, R. E., unpublished data.

187. Eakin, R. E., Snell, E. E., and Williams, R. J., J. Biol. Chem., 140, 535 (1941).

188. Gale, E. F., Biochem. J., 34, 392 (1940).

189. Gale, E. F., and Epps, H. M. R., Biochem. J., 38, 250 (1944).

190. Bellamy, W. D., and Gunsalus, I. C., J. Bact., 46, 573 (1943).

191. Gunsalus, I. C., and Bellamy, W. D., J. Biol. Chem., 155, 357 (1944).

192. Umbreit, W. W., Bellamy, W. D., and Gunsalus, I. C., Arch. Biochem., 7, 185 (1945).

193. Snell, E. E., Guirard, B. M., and Williams, R. J., J. Biol. Chem., 143, 519 (1942).

194. Snell, E. E., J. Am. Chem. Soc., 67, 194 (1945).

195. Braunstein, A. E., and Kritzmann, M. G., Enzymologia, 2, 129 (1937).

196. Schlenk, F., and Snell, E. E., J. Biol. Chem., 157, 425 (1945).

197. Green, D. E., Leloir, Luis, F., and Nocito, V., J. Biol. Chem., 161, 559 (1945). 
198. Lichstein, H. C., Gunsalus, I. C., and Umbreit, W. W., J. Biol. Chem., 161, 311 (1945).

199. Umbreit, W. W., Wood, W. A., and Gunsalus, I. C., J. Biol. Chem., 165, 731 (1946).

200. Dawes, E. A., Dawson, J., and Happold, F. C., Nature, 159, 645 (1947).

201. Wood, W. A., Gunsalus, I. C., and Umbreit, W. W., J. Biol. Chem., 170, 313 (1947).

202. Umbreit, W. W., and Gunsalus, I. C., J. Biol. Chem., 179, 279 (1949).

203. Gunsalus, I. C., Umbreit, W. W., Bellamy, W. D., and Foust, C. E., J. Biol. Chem., 161, 743 (1945).

204. Karrer, P., and Viscontini, M., Helv. Chim. Acta, 30, 52 (1947).

205. Ibid., 30, 524 (1947).

206. Ibid., 30, 528 (1947).

207. Rabinowitz, J. C., and Snell, E. E., J. Biol. Chem., 169, 643 (1947).

208. Umbreit, W. W., O'Kane, D. J., and Gunsalus, I. C., J. Biol. Chem., 176, 629 (1948).

209. McNutt, W. S., and Snell, E. E., J. Biol. Chem., 173, 801 (1948).

210. Rabinowitz, J. C., and Snell, E. E., J. Biol. Chem., 176, 1157 (1948).

211. Herbst, E. J., and Snell, E. E., J. Biol. Chem., 176, 989 (1948).

212. Bellamy, W. D., Umbreit, W. W., and Gunsalus, I. C., J. Biol. Chem., 160, 461 (1945).

213. O'Kane, D. E., and Gunsalus, I. C., J. Biol. Chem., 170, 425 (1947).

214. Umbreit, W. W., and Waddell, J. G., Proc. Soc. Exptl. Biol. Med., 70, 293 (1949).

215. Umbreit, W. W., and Gunsalus, I. C., J. Biol. Chem., 159, 333 (1945).

216. Gale, E. F., and Epps, H. M. R., Biochem. J., 38, 238 (1944).

217. Gale, E. F., Advances in Enzymol., 6, 1 (1946).

218. Blaschko, H., Advances in Enzymol., 5, 67 (1945).

219. Epps, H. M. R., Biochem. J., 38, 242 (1944).

220. Gale, E. F., and Epps, H. M. R., Biochem. J., 38, 232 (1944).

221. Taylor, E. S., and Gale, E. F., Biochem. J., 39, 52 (1945).

222. Braunstein, A. E., and Kritzmann, M. G., Biochimia, 8, 1 (1943).

223. O'Kane, D. E., and Gunsalus, I. C., J. Biol. Chem., 170, 433 (1947).

224. Albaum, H. G., and Cohen, P. P., J. Biol. Chem., 149, 9 (1943).

225. Cohen, P. P., J. Biol. Chem., 136, 565 (1940).

226. Tatum, E. L., and Bonner, D., Proc. Nat. Acad. Sci., 30, 30 (1944).

227. Stokes, J. L., and Gunness, M., Science, 101, 43 (1945).

228. Lyman, C. M., et al., J. Biol. Chem., 167, 177 (1947).

229. Lyman, C. M., and Kiuken, K. A., Federation Proc., 7, 770 (1948).

230. Broquist, H. P., and Snell, E. E., Federation Proc., 8, 188 (1949).

231. Schweigert, B. S., J. Biol. Chem., 168, 283 (1947).

232. Birch, T. W., J. Biol. Chem., 124, 775 (1938).

233. Halliday, N., J. Nutrition, 16, 285 (1938).

234. Quackenbush, F. W., and Steenbock, H., Proc. XVI Intern. Physiol. Congr. Zurich, 1938, p. 108.

235. Salmon, W. D., Proc. Am. Soc. Biol. Chem., 34, LXXXII (1940). .

236. Schlenk, F., and Fisher, A., Arch. Biochem., 12, 69 (1947).

237. Snell, E. E., and Guirard, B. M., Proc. Nat. Acad. Sci., 29, 66 (1943).

238. Snell, E. E., J. Biol. Chem., 158, 497 (1945).

239. Holden, J. T., Furman, C., and Snell, E. E., J. Biol. Chem., 178, 789 (1949).

240. Holden, J. T., and Snell, E. E., J. Biol. Chem., 178, 799 (1949).

241. Ochoa, S., "Currents in Biochemical Research," Interscience Publishers (New York), 1946, p. 165.

242. Rittenberg, D., and Block, K., J. Biol. Chem., 154, 311 (1944).

243. Block, K., "Currents in Biochemical Research," Interscience Publishers (New York), 1946, p. 200. 
244. Snell, E. E., Strong, F. M., and Peterson, W. H., Biochem. J., 31, 1789 (1937).

245. Guirard, B. M., Snell, E. E., and Williams, R. J., Arch. Biochem., 9, 361 (1946).

246. Ibid., 9, 381 (1946).

247. Lipmann, F., Advances in Enzymol., 6, 231 (1946).

248. Kaplan, N. O., and Lipmann, F., Federation Proc., 7, 163 (1948).

249. Lipmann, F., J. Biol. Chem., 160, 173 (1945).

250. Strecker, H., Krampitz, L. O., and Wood, H. G., Federation Proc., 7, 194 (1948).

251. Kaplan, N. O., and Lipmann, F., J. Biol. Chem., 176, 459 (1948).

252. Kaplan, N. O., and Soodak, M., Federation Proc., 8, 211 (1949).

253. Williams, R. J., Mosher, W. A., and Rohrman, E., Biochem. J., 30, 2036 (1936).

254. Nachmansohn, D., and Machado, A. L., J. Neurophysiol., 6, 397 (1943).

255. Lipmann, F., and Kaplan, N. O., J. Biol. Chem., 162, 743 (1946).

256. Lipmann, F., et al., J. Biol. Chem., 167, 869 (1947).

257. Novelli, G. D., Kaplan, N. O., and Lipmann, F., J. Biol. Chem., 177, 97 (1949).

258. Novelli, G. D., Flynn, R. M., and Lipmann, F., J. Biol. Chem., 177, 493 (1949).

259. King, T. E., Fels, I. G., and Cheldelin, V. H., J. Am. Chem. Soc., 71, 131 (1949).

260. Nachmansohn, D., and Berman, M., J. Biol. Chem., 165, 55 (1946).

261. Kaplan, N. O., and Lipmann, F., J. Biol. Chem., 174, 37 (1948).

262. Dorfman, A., Berkman, S., and Koser, S. A., J. Biol. Chem., 144, 393 (1942).

263. Novelli, G. D., and Lipmann, F., Arch. Biochem., 14, 23 (1947).

264. Novelli, G. D., and Lipmann, F., J. Biol. Chem., 171, 833 (1947).

265. Olson, R. E., and Kaplan, N. O., J. Biol. Chem., 175, 515 (1948).

266. Soodak, M., and Lipmann, F., J. Biol. Chem., 175, 999 (1948).

267. Guirard, B. M., unpublished observations.

268. Shive, W., Ackermann, W. W., Ravel, J. M., and Sutherland, J. E., J. Am. Chem. Soc., 69, 2567 (1947).

269. Gordon, M., Ph. D. Dissertation, University of Texas, June, 1948.

270. Pilgrim, F. J., Axelrod, A. E., and Elvehjem, C. A., J. Biol. Chem., 145, 237 (1942).

271. Hills, G. M., Biochem. J., 37, 418 (1943).

272. McHenry, E. W., and Gavin, G., J. Biol. Chem., 138, 471 (1941).

273. Buchanan, J. M., and Sonne, J. C., J. Biol. Chem., 166, 781 (1946).

274. Block, K., and Rittenberg, D., J. Biol. Chem., 159, 45 (1945).

275. Slade, H. D., and Werkman, C. H., Arch. Biochem., 2, 97 (1943).

276. Buchanan, J. M., Sonne, J. C., and Delluva, A. M., J. Biol. Chem., 166, 395 (1946).

277. Shive, W., Lane, A. E., and Eakin, R. E., unpublished observations.

278. Sakami, W., J. Biol. Chem., 176, 995 (1948).

279. Edelbacher, S., Z. physiol. Chem., 157, 106 (1926).

280. Sakami, W., J. Biol. Chem., 178, 519 (1949).

281. Winnick, T., Moring-Claesson, I., and Greenberg, D. M., J. Biol. Chem., 175, 127 (1948).

282. Sakami, W., Federation Proc., 8, 246 (1949).

283. Rose, W. C., and Cook, K. G., J. Biol. Chem., 64, 325 (1925).

284. Shemin, D., and Rittenberg, D., J. Biol. Chem., 167, 875 (1947).

285. Kohn, H. I., Ann. N. Y. Acad. Sci., 44, 503 (1943).

286. Housewright, R. D., and Koser, S. A., J. Infect. Dis., 75, 113 (1944).

287. Lampen, J. O., Roepke, R. R., and Jones, M. J., J. Biol. Chem., 164, 789 (1946).

288. Shive, W., and Macow, J., J. Biol. Chem., 162, 451 (1946).

289. Shive, W., and Roberts, E. C., J. Biol. Chem., 162, 463 (1946).

290. Shive, W., et al., J. Am. Chem. Soc., 69, 725 (1947).

291. Stetten, M. R., and Fox, C. L., Jr., J. Biol. Chem., 161, 333 (1945).

292. Wolf, D. E., et al., J. Am. Chem. Soc., 69, 2753 (1947).

293. Gordon, M., Ravel, J. M., Eakin, R. E., and Shive, W., J. Am. Chem. Soc., 70, 878 (1948).

294. Winkler, K. C., and de Haan, P. G., Arch. Biochem., 18, 97 (1948). 
295. Alexander, E. R., Master Thesis, University of Texas, June, 1949.

296. Shemin, D., J. Biol. Chem., 162, 297 (1946).

297. Ravel, J. M., Eakin, R. E., and Shive, W., J. Biol. Chem., 172, 67 (1948).

298. Holland, B. R., and Meinke, W. W., J. Biol. Chem., 178, 7 (1949).

299. Shive, W., and Ravel, J. M., unpublished observations.

300. Stokstad, E. L. R., J. Biol. Chem., 139, 476 (1941).

301. Stokes, J. L., J. Bact, 48, 201 (1944).

302. Bird, O. D., et al., J. Biol. Chem., 157, 413 (1945).

303. Jacobson, W., and Good, P. M., Int. Physiol. Cong. Abstracts, Oxford, XVII, 157 (1947).

304. Rodney, G., Swendseid, M. E., and Swanson, A. L., J. Biol. Chem., 168, 395 (1947).

305. Sealock, R. R., and Silberstein, H. E., J. Biol. Chem., 135, 251 (1940).

306. Swendseid, M. E., Burton, I. F., and Bethel, F. H., Proc. Soc. Exptl. Biol. and Med., 52, 202 (1943).

307. Woodruff, W. C., and Darby, W. J., J. Biol. Chcm., 172, 851 (1948).

308. Swendseid, M. E., Wandruff, B., and Bethel, F. H., J. Lab. Clin. Med., 32, 1242 (1947).

309. Rodney, G., Sw'endseid, M. E., and Swanson, A. L., J. Biol. Chem., 179, 19 (1949).

310. Davis, J. E., Proc. Soc. Exptl. Biol. and Med., 63, 287 (1946).

311. Hawkins, R. D., Arch. Biochem., 17, 97 (1948).

312. Martin, G. J., and Beiler, J. M., Arch. Biochem., 15, 195 (1947).

313. Smith, E. L., Nature, 161, 638 (1948).

314. Cuthbertson, W. F. J., and Smith, L. E., Biochem. J., 44, v (1949).

315. Smith, E. L., and Parker, L. F., Biochem. J., 43, viii (1948).

316. Rickes, E. L., et al., Science, 107, 396 (1948).

317. Shive, W., Arn. N. Y. Acad. Sci., in press.

318. Ellis, B., Petrow, V., and Snook, G., J. Pharm. Pharmacol., 1, 287 (1949).

319. Berk, et al., New England J. Med., 239, 911 (1948).

320. McCance, R. A., Rev. Clin. Espãn., 8, 371 (1943).

321. Shive, W. Eakin, R. E., Harding, W. M., Ravel, J. M., and Sutherland, J. E., J. Am. Chem. Soc., 70, 2299 (1948).

322. Kitay, E., MeNutt, W. S., and Snell, E. E., J. Biol. Chem., 177, 993 (1949).

323. Wright, L. D., Skeggs, H. R., and Huff, J. W., J. Biol. Chem., 175, 475 (1948).

324. Kocher, Von v. and Schindler, O., Sonderdruck aus Internationale Zeitschrift für Vitaminforschung, 20, 441 (1949).

325. Shive, W., Ravel, J. M., and Eakin, R. E., J. Am. Chem. Soc., 70, 2614 (1948).

326. Shive, W., Ravel, J. M., and Harding, W. M., J. Biol. Chem., 176, 991 (1948).

327. Sibley, M., and Shive, W., unpublished observations.

328. Schaefer, A. E., Salmon, W. D., and Strength, D. R., Federation Proc., 8, 395 (1949).

329. Heinle, R. W., and Welch, A. C., Ann. N. Y. Acad. Sci., 48, 343 (1936).

330. Welch, A. D., Heinle, R. W., Nelson, E. M., and Nelson, H. V., J. Biol. Chem., 164, 787 (1946).

331. Bethell, F. H., et al., Univ. Hosp. Bull., Univ. of Mich., 12, 42 (1946).

332. Welch, A. D., Federation Proc., 6, 471 (1947).

333. Scott, M. L., Hill, C. H., and Norris, L. C., Federation Proc., 8, 249 (1949).

334. Kocher, Von v., Sonderdruck aus Internationale Zeitschrift für Vitaminforschung, 20, 369 (1949).

335. Dyke, S. C., Della Vida, B. L., and Kelikat, E., Lancet, 2, 278 (1942). 


\section{Chapter III B}

\section{THE FUNCTIONS OF THE B VITAMINS IN METABOLIC PROCESSES}

In Chapter I B there was presented a brief discussion of the fundamental reactions common to most organisms. The purpose of the present chapter is to examine in greater detail those processes the chemical steps of which have been fairly well established and which are believed to be generally utilized by many different types of life. There are undoubtedly some reactions included in this discussion which cannot be demonstrated to occur in all organisms, and likewise other reactions omitted which may be universal. The purpose of this chapter would be defeated and our perspective lost if an attempt was made to be encyclopedic with respect to all the metabolic reactions which have been demonstrated or postulated, and to discuss in detail exceptions to the general schemes. The material presented here is intended (1) to indicate the basic patterns which are usually followed by most cells, (2) to elucidate the nature of the chemical reactions involved, and (3) to establish the positions where the $B$ vitamins are essential.

Organisms show extreme variation in their ability to carry out many reactions. The numerous end products of glucose metabolism which are produced by different organisms illustrate this point well. However, the differences are often one of degree rather than absolute. Although we often think of one or two specific compounds as being the end products of a particular fermentation process, actually many substances are produced. In no fermentation involving intact cells are the principal products formed exclusively.

Variations in the chemical processes taking place in cells occur (1) when different organisms are compared, (2) when cells from one tissue are compared with those of another tissue in the same organism, (3) when cells at one age are compared with similar cells at a different age, and (4) when cells and tissues from one individual organism are compared with those of another individual of the same species. These variations, taken as a group, are probably more often of a quantitative rather than a qualitative nature. Many organisms and cells probably have the ability to carry out fundamental reactions, such as we are considering, even though such reactions take place slowly and in many cases have not 
been specifically demonstrated. The production of partial genetic blocks which result, for example, in impaired synthetic abilities on the part of the cells may cause cells to require for rapid metabolism a substance which otherwise would never be a limiting factor. In such cases, however, the cells have not lost their synthetic ability completely, and the difference between these cells and the unaltered ones is quantitative, not qualitative. The capacity of cells to adapt themselves to the utilization of completely new substances indicates that they possess latent potentialities with respect to enzymatic reactions which are ordinarily not observed.

Variations in the fundamental processes in different cells, in different species, and in different individuals of the same species are of extreme importance and interest, but their discussion does not belong in a summary of the chemical processes which appear to be common to cells in general.

There would be certain advantages in preparing an elaborate diagrammatic scheme showing all the known and postulated relationships between the fundamental biochemical compounds. Such a chart could be used to indicate in a concise manner where the individual reactions discussed in the previous chapter fit into the general processes of cell metabolism. It is technically impossible, however, to prepare a diagram that would not be more confusing than enlightening, since some of the intermediate compounds, like pyruvic acid, are involved in a multitude of reactions. The metabolic processes, therefore, will be treated in turn according to the classical divisions of biochemical substances: carbohydrates, lipides, and amino acids and proteins. Following this will be a discussion of the role of the $B$ vitamins in the fundamental physiological processes involving energy transformations.

\section{The Utilization of Carbohydrates}

Most of the chemical steps involved in the utilization of carbohydrates have been well established, and a number of excellent reviews on this aspect of metabolism have appeared during the last ten years. ${ }^{1-7}$ The general metabolic pathways by which the carbohydrates are stored, degraded, or converted to intermediates that can be used for the synthesis of compounds of other types involve a large number of reactions. These can be most conveniently considered as components of four different phases: (1) the synthesis and cleavage of the polysaccharides; (2) the glycolytic process-glycogen (or starch) $\leftrightarrows$ pyruvate; (3) the anaerobic utilization of pyruvate; and (4) the aerobic utilization of pyruvate.

The Synthesis and Cleavage of Polysaccharides. The initial phase of carbohydrate utilization includes the reactions by which complex sugars 
are enzymatically hydrolyzed extracellularly to yield simpler sugars which can be absorbed, and the subsequent intracellular processes by which these absorbed compounds are converted into polysaccharides in which form they are stored until utilized. A number of enzymes hydrolyzing carbohydrates have been well characterized. Of these, only a single specific enzyme, pancreatic amylase, has been shown to contain a coenzyme (inositol) (p. 125).

The intracellular synthesis of glycogen and starch from simple hexoses cannot be carried out directly since an input of energy is required for the formation of the acetal bonds. The energy for the synthesis is introduced by a reaction in which glucose is initially converted to a phosphate ester ${ }^{8}$ by adenosine triphosphate, a transformation that uses up an energy-rich phosphate bond generated previously in some metabolic reaction. The phosphate ester initially formed, glucose-6-phosphate, is in equilibrium with its isomer, glucose-1-phosphate, due to the presence of an enzyme which catalyzes this intramolecular transesterification. The glucose-1-phosphate molecules polymerize to form the polysaccharide by a reaction in which the phosphate ester linkage is cleaved (liberating inorganic phosphate), but an acetal bond is created. The energy transformations involved in this reaction are small and the hexose-phosphate and polysaccharide are usually in equilibrium.

$x$ glucose-1-phosphate $\rightleftharpoons$ polysaccharide $+x \mathrm{H}_{3} \mathrm{PO}_{4}$

When an organism expends energy, the phosphoric acid anhydrides, in which energy has been stored, are hydrolytically cleaved and the inorganic phosphate concentration increases. This increase in inorganic phosphate upsets the equilibrium between the hexose phosphate and glycogen and causes the breakdown of glycogen to glucose-1-phosphate. This, in turn, initiates the glycolytic process in which the glycogen is metabolized. The energy liberated during this process is utilized through the resynthesis of the phosphoric acid anhydrides from the inorganic phosphate. The formation of energy-containing phosphate bonds continues until the cell reaches a state wherein the inorganic phosphate concentration will have been reduced to such a level that the equilibrium shifts to favor the formation of glycogen instead of its breakdown. As far as is now known, the reactions involved in the intracellular formation of polysaccharides require only adenylic acid and its phosphorylated derivatives as coenzymes. The $\mathrm{B}$ vitamins are involved only indirectly; they are needed for producing the energy units used in the synthetic process-the phosphoric acid anhydrides.

Glycolysis. When the second phase of carbohydrate utilization, the glycolytic process, is initiated by the phosphorolysis of glycogen (or 
starch), a series of reactions occur which eventually produce pyruvic acid. Although other ways in which hexoses can be degraded are known, ${ }^{3}$ the mechanism employed by almost all organisms is the one represented by the classical scheme of fermentation. ${ }^{1}$ The individual reactions involve the formation and cleavage of phosphate esters, isomerizations, the cleavage of the hexose diphosphate into two triose phosphates (a reaction which is the reverse of an aldol condensation), the dehydration of a $\beta$-hydroxy acid, and one dehydrogenation:

\section{Transesterification:}

glucose-1-phosphate $\rightleftharpoons$ glucose- 6 -phosphate

2. Phosphorylation: glucose-6-phosphate $+\mathrm{ATP} \longrightarrow$ glucose-1,6-diphosphate + ADP

3. Isomerization: glucose-1,6-diphosphate $\rightleftharpoons$ fructose-1,6-diphosphate

4. Aldol cleavage or formation: fructose-1,6-diphosphate $\rightleftharpoons$ glyceraldehyde -3 -phosphate + dihydroxyacetone phosphate

5. Isomerizalion:

dihydroxyacetone phosphate $\rightleftharpoons$ glyceraldehyde-3-phosphate

6. Nonenzymatic formation and disintegration of a carbonyl-phosphoric acid addition product (see p. 140):

2 (glyceraldehyde- 3 -phosphate) $+2 \mathrm{H}_{3} \mathrm{PO}_{4} \rightleftharpoons$

2 (glyceraldehyde-3-phosphate) - phosphoric acid addition product

7. Hydrogenation-dehydrogenation:

2 (glyceraldehyde -3 -phosphate) phosphoric acid addition product +

$2 \mathrm{DPN} \rightleftharpoons 2(3-$ phosphoglyceroyl phosphate $)+2(\mathrm{DPN} \cdot 2 \mathrm{H})$

8. Phosphorylation:

$2(3$-phosphoglyceroyl phosphate $)+2 \mathrm{ADP} \underset{2(3-\text { phosphoglyceric acid })+2 \mathrm{ATP}}{\rightleftharpoons}$

9. Transesterification:

$2(3$-phosphoglyceric acid $) \rightleftharpoons 2(2$-phosphoglyceric acid)

10. Dehydration or hydration (involving $\beta$-hydroxy acid):

$2\left(2\right.$-phosphoglyceric acid $\rightleftharpoons 2$ (phospho(enol)pyruvic acid) $+2 \mathrm{H}_{2} \mathrm{O}$

11. Phosphorylation:

2 (phospho(enol)pyruvic acid) $+2 \mathrm{ADP} \rightleftharpoons 2$ (pyruvic acid) $+2 \mathrm{ATP}$

Of the eleven reactions, only one, the dehydrogenation of the diphosphate derivative of glyceraldehyde, is of the type which requires a $B$ vitamin coenzyme. The hydrogen atoms from this reaction are accepted by the diphosphopyridine nucleotide. The net transformation of the organic molecules brought about by the process and the B vitamin coenzymes involved may be summed up thus:

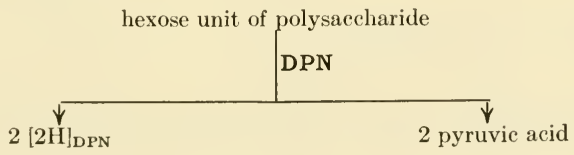


When this stage of the process is reached, the organism is confronted with the problem of the disposition of the pyruvic acid and of the hydrogen atoms which have been temporarily taken up by the diphosphopyridine nucleotide coenzyme. This coenzyme must be reconverted to the oxidized form for re-use, for, if no suitable hydrogen acceptor can be found, the glycolytic process will stop when all the coenzyme is tied up in its reduced state.

Under anaerobic conditions pyruvic acid itself or other products derived from it acts as the acceptor for the hydrogen atoms. When oxygen is available and can be utilized, it can serve as the final acceptor for the hydrogen atoms and will in addition permit the pyruvic acid to be converted, by reactions involving further dehydrogenations, into compounds which are in a sense oxidation products; or pyruvic acid may be completely degraded to carbon dioxide and water.

The reactions into which pyruvic acid can enter are numerous, and we find a great deal of variation among the various forms of life in the manner in which they carry forward the carbohydrate metabolism from this point. ${ }^{5}$ Probably most often several of the reactions are utilized simultaneously, although one reaction may predominate to such an extent that the others are completely overlooked.

Anaerobic Utilization of Pyruvic Acid. When a cell has only a limited supply of molecular oxygen or when it lacks the porphyrin enzymes (cytochromes) which catalyze the utilization of molecular oxygen as the final hydrogen acceptor, it must dispose of the hydrogen atoms temporarily associated with the reduced nicotinic acid and of the pyruvic acid by an anaerobic process. The anaerobic processes can be classified in three groups.

The simplest and most direct process is that in which the pyruvic acid itself accepts the hydrogen atoms from the coenzyme forming lactic acid. This type of reaction is the predominant method in a number of bacteria and in the tissues of vertebrates. The only vitamin involved is the nicotinic acid which is present in the reduced coenzyme.

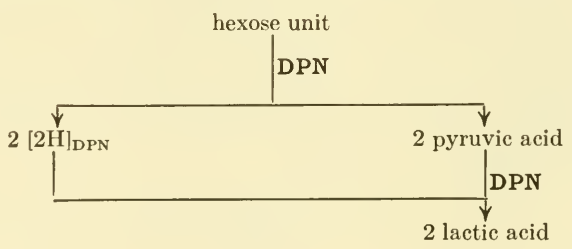

A second important type process is one in which the reduction of pyruvic acid takes place after it has undergone carboxylation.$^{9}$ Oxalacetic 
acid is first formed by $\beta$-carboxylation of pyruvic acid and on subsequent reduction yields malic acid. In biological systems, malic acid is usually in equilibrium with its dehydration product, fumaric acid. Whether or not biotin is generally required to mediate the $\beta$-carboxylation step is still an open question (p. 171), and it may be that this method of carbohydrate utilization is another instance in which the nicotinic acid coenzyme is the only vitamin coenzyme participating. (Further reduction of the fumaric acid, utilizing hydrogen atoms from other metabolic reactions, can occur and results in the production of succinic acid. The enzyme needed for this reaction, a fumaric reductase, might be expected to be one of the riboflavin enzymes) (p. 150).

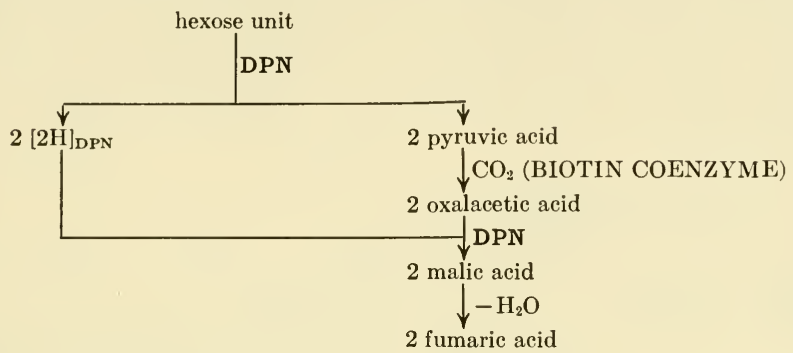

The third type anaerobic process is one in which pyruvic acid is first decarboxylated, and the resulting $\mathrm{C}_{2}$ compounds or their condensation products are then reduced by the hydrogen atoms of the nicotinic acid coenzyme. The thiamine coenzyme is believed to be absolutely essential for the production of every one of these fermentation products which are formed from pyruvic acid by decarboxylation. ${ }^{3}$ However, they may be divided into two subgroups depending upon whether or not there is a pantothenic acid requirement. This requirement appears to be directly determined by the type of decarboxylation. Those processes in which thiamine catalyzes a simple decarboxylation to produce acetaldehyde or its dimer, acetylmethylcarbinol, do not require pantothenic acid. Con-

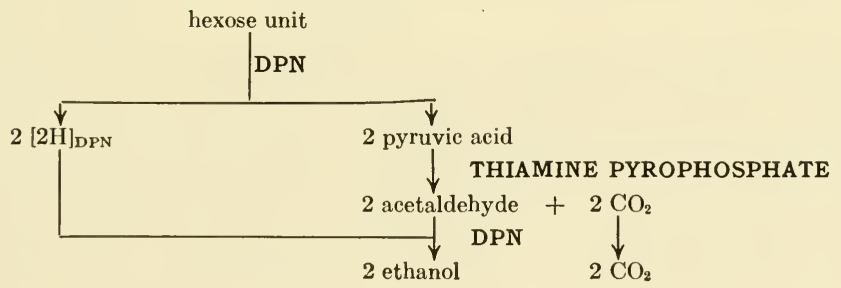


sequently, pantothenate is not required for the synthesis of ethanol or 2,3-butylene glycol (formed by the reduction of acetylmethylcarbinol). Many of the thiamine-promoted processes, however, involve an "oxidative" decarboxylation of pyruvic acid which produces the reactive acetylating intermediate, and hence the subsequent steps in these processes all require the presence of a pantothenic acid coenzyme (p. 191). The mechanism of transporting the two additional hydrogen atoms released by each pyruvic acid molecule during the oxidative decarboxylation is not known. In aerobic processes they are probably accepted by a riboflavin-containing enzyme. ${ }^{10}$ In anaerobic processes these hydrogen atoms are used to reduce the products formed from the acetyl derivatives, possibly through the intermediation of flavoproteins or some other hydrogen carrier. A possible mechanism for the formation of butyric acid and butyl alcohol (based upon demonstrated reactions catalyzed by thiamine, nicotinic acid, and pantothenic acid) is shown as an example of this type of process:

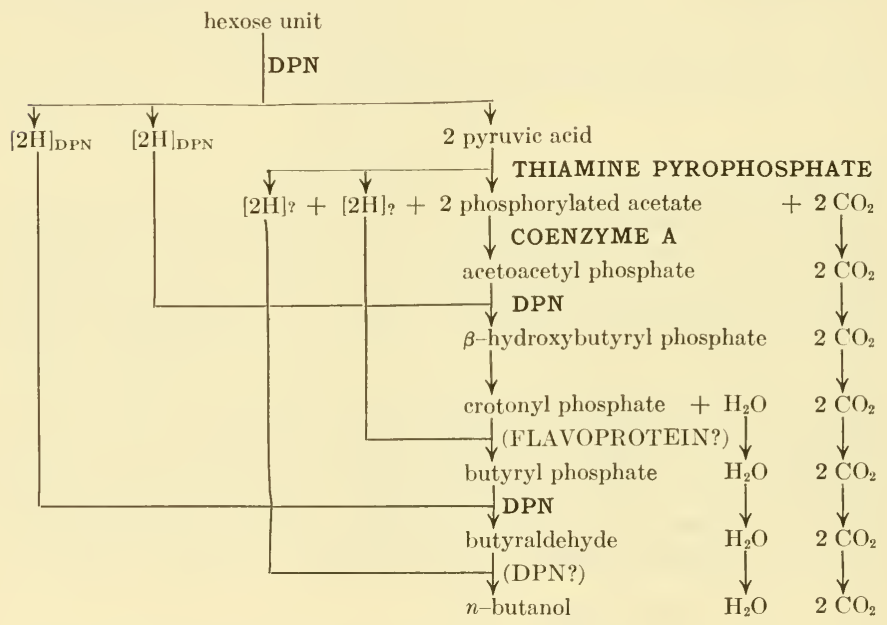

The synthesis of fatty acids from carbohydrates probably is carried out by an analogous procedure. Hence, any conversion of carbohydrates to fats or fat-like substances requires the coenzymes of thiamine, nicotinic acid, pantothenic acid, and probably riboflavin. In the synthesis of fat from proteins, the same vitamins will be essential for the utilization of those "anti-ketogenic" amino acids which on deamination are metabolized via processes that involve carbohydrate intermediates.

The Aerobic Utilization of Pyruvate. The first step in the aerobic 
metabolism of pyruvic acid presumably always involves the thiaminerequiring oxidative decarboxylation. The hydrogen atoms donated to riboflavin ${ }^{10}$ in this reaction, as well as those donated to the diphosphopyridine nucleotide previously during the glycolytic process, are "transported" by the elaborate dehydrogenase-cytochrome systems described in Chapter IIB and are finally oxidized in reactions which reduce molecular oxygen. The active phosphorylated acetyl molecule which is formed by the oxidative decarboxylation of pyruvate can be directly used for energy by employing it in reactions in which it acts as a phosphorylating agent. ${ }^{11}$ Acetic acid is then the end product and accumulates. A more common aerobic mechanism, however, is one in which the activated acetate is completely oxidized to carbon dioxide and water by a series of reactions designated as the tricarboxylic acid cycle.2, 4,12 In this cyclic process,

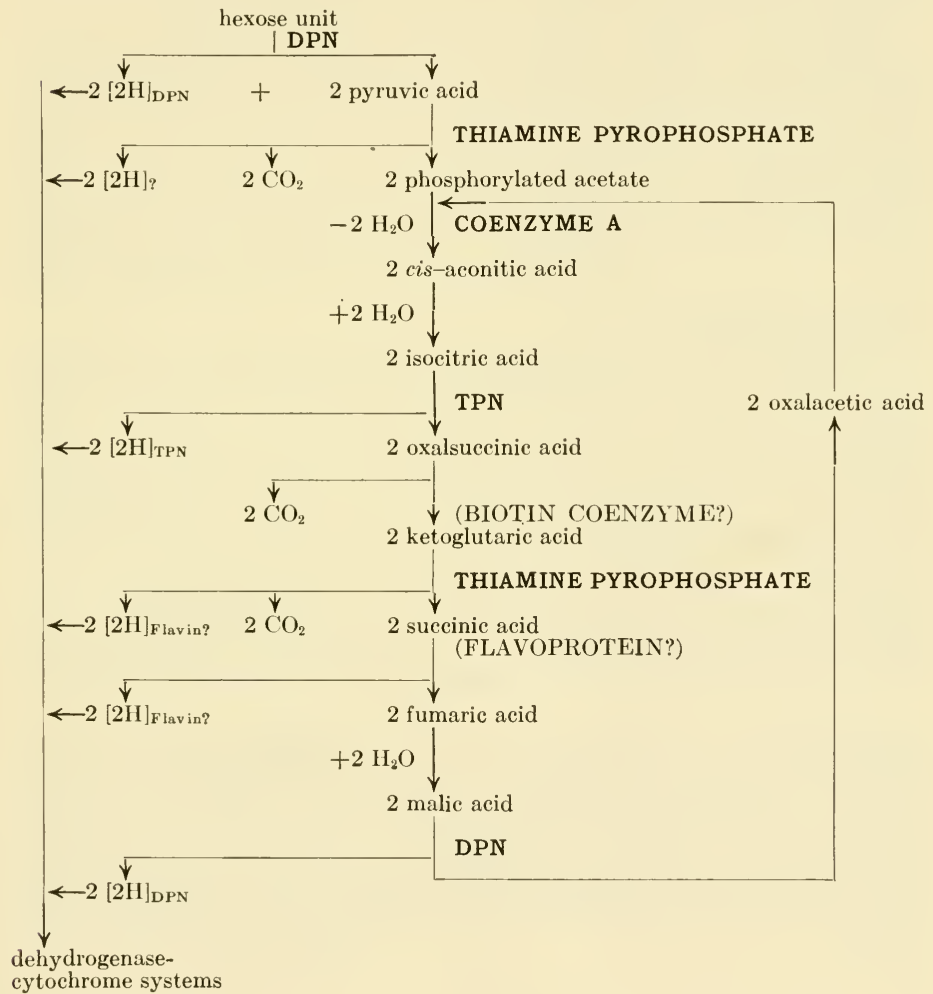


the activated acetate is believed to undergo first a condensation with oxalacetic acid to form cis-aconitic acid; then, by a series of dehydrogenations, hydrations and dehydrations, and decarboxylations the cisaconitic acid is degraded to oxalacetic acid, which can then react with another activated acetate and "carry" it through the same cycle."

Pantothenic acid is necessary for the initial condensation of the activated acetate (p. 195); thiamine is required for one step in the cycle, the oxidative decarboxylation of $\alpha$-ketoglutaric acid; nicotinic acid is necessary for the dehydrogenation of the hydroxy acids, isocitric and malic; a riboflavin enzyme may be the hydrogen acceptor in the conversion of succinic acid to fumaric acid (p. 151). It is interesting to note that thiamine, riboflavin, nicotinic acid, and pantothenic acid are the vitamins required for both the aerobic oxidation of carbohydrates and for the anaerobic processes in which the carbohydrates are converted to fats.

There are a number of compounds of general biological occurrence and importance which are related chemically to the carbohydrates and which are undoubtedly produced from them. These include the pentoses, desoxypentoses, amino sugars, ascorbic acid, inositol, and the hexonic and hexuronic acids. Nicotinic acid-containing coenzymes have been shown to catalyze the dehydrogenation of glucose ${ }^{13}$ or its phosphate ester ${ }^{14}$ to the corresponding acid, but the involvement of vitamin-containing coenzymes in the biosynthesis of other sugar derivatives has not yet been established.

The mechanism by which the pentoses and desoxypentoses are generally formed has not yet been definitely established. This subject is under active investigation due to the present interest in nucleic acid metabolism. When the mechanisms for the formation of these compounds, as well as the other carbohydrate-like substances, are worked out, the vitamin requirements will probably be obvious; or, conversely, if certain vitamins are shown to be directly involved in the biosynthesis of any of these substances, it will give some insight into the mechanisms and the intermediates in their biosyntheses. If, for example, pantothenic acid were shown to be essential for the synthesis of desoxyribose, a reasonable

* In the past, on the basis of certain tracer experiments, citric acid has been assigned the role of a metabolic by-product of the tricarboxylic acid cycle rather than that of a necessary intermediate. In a more critical analysis of these tracer studies it was pointed out that such an interpretation need not be made, and subsequent to the preparation of this diagram it was shown experimentally that citrate, rather than cis-aconitate, is the initial compound formed by the condensation of the reactive acetyl unit with oxalacetate. (See Stern, J. R., and Ochoa, S., J. Biol. Chem., 179, 491 (1949) and Potter, V. R., and Heidelberger, C., Nature, 164, 180 (1949)). 
postulation would be that the precursor of this compound was formed by a condensation of a triose and an activated acetate unit.

\section{The Metabolism of Lipides}

A general scheme of lipide metabolism must account for fatty acid synthesis and degradation, glycerol formation and utilization, and for the reactions by which fat molecules are synthesized from their component parts, and should also explain the origin of sterols.

Fatty acid metabolism. The mechanism now postulated for the synthesis and degradation of fatty acids ${ }^{15}$ is very similar to that shown in the scheme for the production of butyl alcohol, and the same combination of vitamins-thiamine, pantothenic acid, nicotinic acid, and riboflavinis needed. When proteins are converted to fats a fifth vitamin, pyridoxal is also undoubtedly required to catalyze the deamination of amino acids. ${ }^{\mathbf{1 6}}$ A route of synthesis by which butyryl phosphate can be formed from a hexose unit has been diagrammed (p. 222). The synthesis of higher fatty acids presumably is carried out similarly by a series of reactions which lengthen the carbon chain of a fatty acyl phosphate in some fashion corresponding to the diagram on the following page. The net result of this process is an anaerobic utilization of each hexose unit for increasing the chain of a fatty acid by four carbon atoms.

The discovery of the reactive phosphoryl acetyl compound cleared up a number of points previously obscure concerning the synthesis and degradation of fatty acids. This fundamental acetylating agent which can be formed by a number of metabolic processes furnishes the units from which fatty acids are constructed, and in turn these same acetyl units are regenerated when a fat is metabolized. The mechanism of fatty acid catabolism ${ }^{15}$ is believed to be just the reverse of the synthetic process; fatty acyl phosphates are degraded by dehydrogenations, hydrations, and phosphoroclastic cleavage of the $\beta$-keto acids to yield the reactive phosphorylated acetyl units (associated with coenzyme A?) and the hydrogenated coenzymes of nicotinic acid and riboflavin. If the aerobic mechanisms for oxidation of the acetyl units (tricarboxylic acid cycle) are inhibited from functioning or are overloaded by the intermediates of carbohydrate or protein metabolism, then the accumulation of the acetyl units will result in their condensation, and fats will be formed. On the other hand, if there is insufficient carbohydrate or protein degradation to furnish sufficient substrate for the energy-producing tricarboxylic acid cycle the fats will be degraded to supply the acetyl units for the oxidative cycle. 


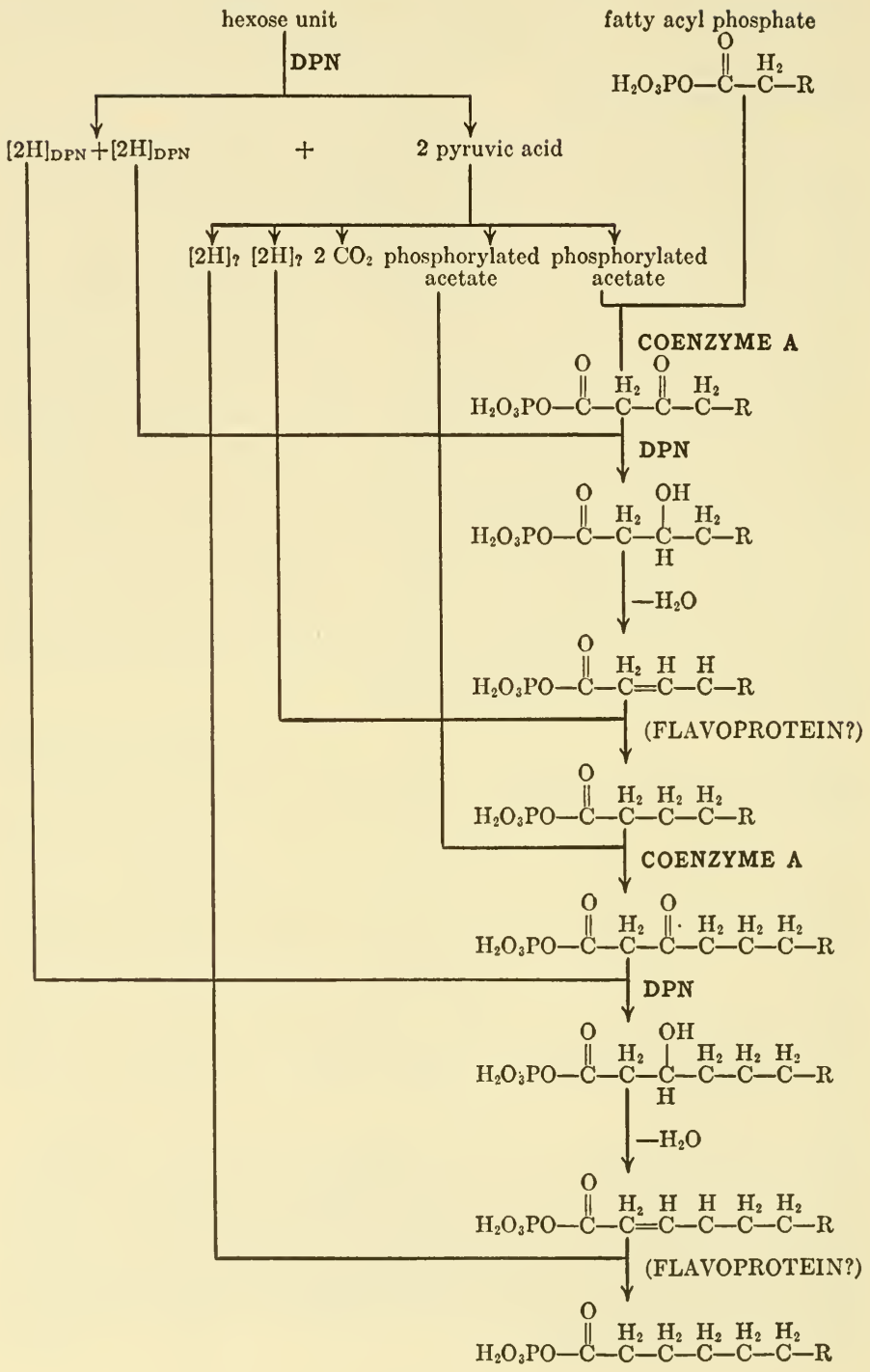


It is interesting to note that fatty acids have an appreciable sparing effect upon the pantothenic acid ${ }^{17}$ and riboflavin ${ }^{18}$ requirements of lactic acid bacteria, indicating that a considerable fraction of each of these vitamins is being used by these organisms in the fat synthesis systems, but that lipides have only a slight effect upon the nicotinic acid response. ${ }^{19}$ (A reduction in utilization of an enzyme system is reflected in the lowered demands of the organism for the cofactors.) The sparing effect of fats upon the thiamine requirement of mammals (p. 000) can be attributed to the fact that the body is oxidizing acetyl units primarily derived from fatty acids rather than those arising from the decarboxylation of pyruvic acid.

The reverse process, in which a deficiency of an enzyme system alters the dietary requirements, can also be of physiological importance. For example, in lactic acid bacteria where the thiamine system is inherently deficient, the organisms need an exogenous supply of acetate or fatty acids as a source of acetyl units to supplement the sub-optimal quantities furnished by the thiamine enzymes. ${ }^{20}$

An additional problem encountered in the synthesis of fats is that of the formation of unsaturated fatty acids. The requirement for specific unsaturated fatty acids in both bacteria and mammals would indicate that these organisms are deficient in enzymes designed for this purpose. Einzymes that dehydrogenate fatty acids are known, ${ }^{21}$ but have never been shown to be dependent upon any B vitamin coenzymes for activation. However, the biotin-sparing activity of oleic acid and other unsaturated fatty acids for certain microorganisms (p. 173) is suggestive of a function of biotin related in some way to the formation of a double bond by dehydrogenation of saturated fatty acids.

Glycerol Formation and Utilization. The glycerol needed for lipide synthesis is a by-product of carbohydrate metabolism. The phosphorylated triose, dihydroxyacetone phosphate (formed by the hexose diphosphate cleavage), is reduced by the corresponding dehydrogenasi, the specific hydrogen donor required is the hydrogenated diphosphopyridine nucleotide. The phosphorylated glycerol is then available for reactions producing phospholipides-compounds which are assumed to be necessary intermediates in fat synthesis.

Nicotinic acid, in the form of its coenzyme, is also essential for the utilization of the glycerol liberated from fat, since the glycerol (after phosphorylation) must be dehydrogenated by DPN to a triosephosphate before it can be metabolized in the carbohydrate system..$^{22}$ After the initial dehydrogenation, the substrate can be utilized during anabolic phases of cell activity for synthesis of glycogen or starch; during periods in which the catabolic activity of the cells predominate, it will be utilized 
in one of the previously described processes in which pyruvic acid is an intermediate.

Synthesis and Hydrolysis of Fats. A number of lipases from both plant and animal sources have been thoroughly investigated. ${ }^{23}$ These esterases catalyze the hydrolysis of fats, liberating free fatty acids, glycerol, and the other components found in complex lipides. In no case has a coenzyme requirement been demonstrated for this hydrolysis.

The synthesis of fats by the reverse reaction can be accomplished in vitro by these same enzymes if the molar ratio of fatty acids to fat is greater than two to one. ${ }^{24}$ Since the intracellular concentration of fatty acids is very low, the synthesis of fats here must be by a different process. . The mechanism probably is similar to that involved in the formation of polysaccharides from simple sugars and involves phosphorylated intermediates. In the condensations and reductions by which the phosphorylated acetyl units are elaborated into the fatty acids, it was indicated that the fatty acid derivatives actually produced are undoubtedly fatty acyl phosphates. (This supposition is in line with evidence concerning the reverse process, wherein it has been demonstrated that before a fatty acid can be degraded enzymatically it must be first converted to the corresponding acyl phosphate. ${ }^{25}$ ) The fatty acyl phosphates react directly with the hydroxyl groups of glycerol phosphate or other alcohols to yicld the esters which constitute the saponifiable lipides. These reactions arc analogous to those employed for the nonenzymatic synthesis of esters wherein acyl halides are used. The equilibrium reached in this reaction is so greatly in favor of ester formation that the reaction can be assumed for practical purposes to have "gone to completion." Both in the synthesis of fats and in the utilization of the fatty acids from fats the individual fatty acids of different carbon chain length appear to be used in a nonspecific and random fashion. ${ }^{26}$

The vitamin requirements for fat metabolism are summarized in the diagram on the following page.

Sterol Metabolism. Very little is known about the reactions by which sterols are formed. Tracer studies using isotopes of carbon first showed the importance of acetate in the synthesis of sterols. ${ }^{27}$ An independent demonstration was provided by the study of the acetate requirements of organisms which produce lactic acid. ${ }^{20}$ These bacteria are very limited in their ability to convert pyruvic acid to the reactive two-carbon unit. Consequently, they require an exogenous supply of acetate in order to function normally. Sterols as well as fatty acids were found to have a significant sparing action upon this acetate requirement of these organisms. 


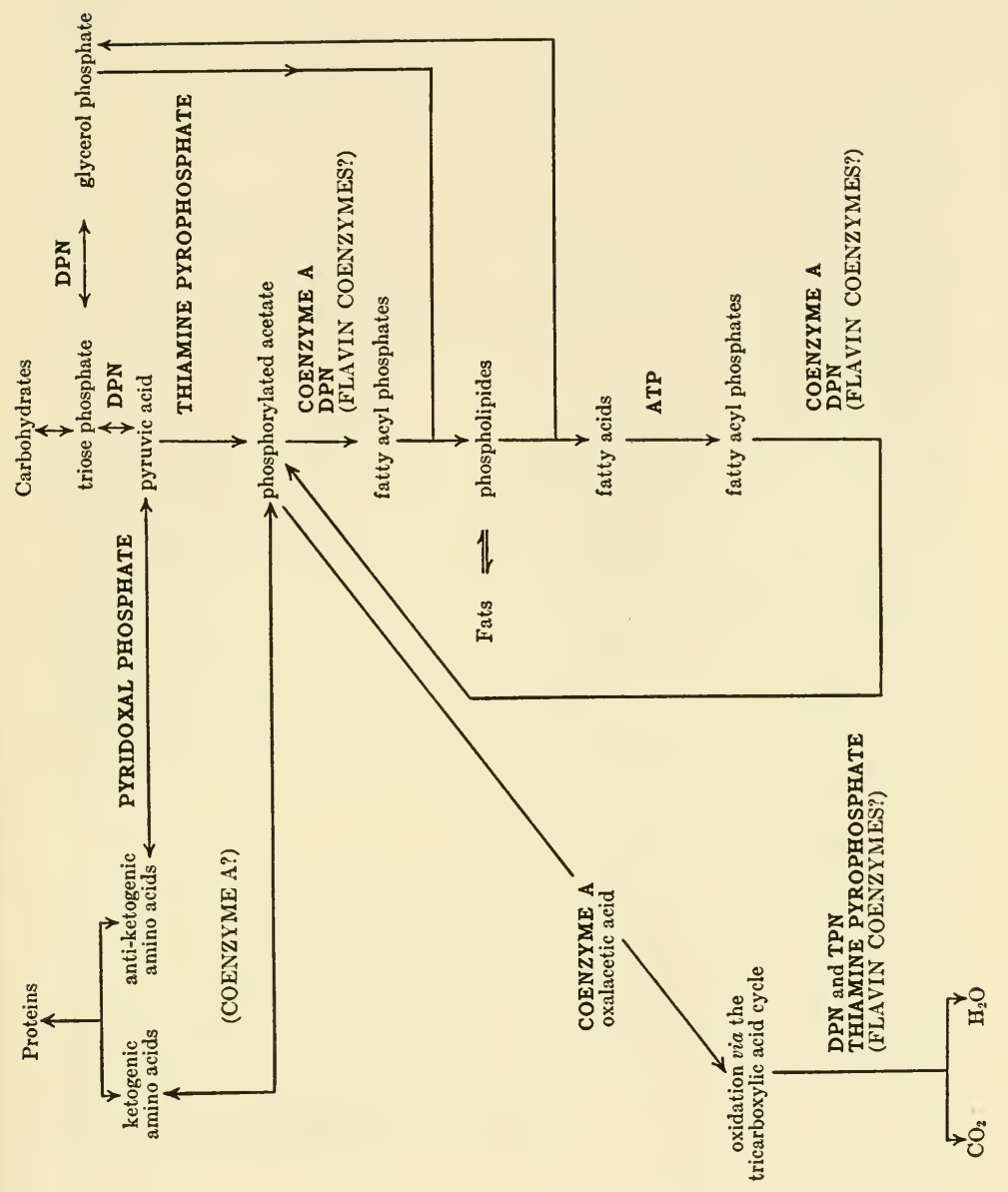


When the function of pantothenic acid was shown to be that of a mediator of reactions involving active forms of acetate, this vitamin was naturally implicated in sterol synthesis. It was shown that sterols had a sparing action upon the pantothenic acid requirement of Lactobacilli, ${ }^{17}$ and that a sterol could partially reverse the toxicity of pantothenic acid inhibitors. ${ }^{28}$ The reversal was noncompetitive, indicating that sterols are products of a series of reactions, part of which are catalyzed by pantothenic acid enzymes.

The mechanism for the formation of both the polynuclear cholane nucleus and the hydrocarbon side chains is completely unknown, but it appears impossible to postulate any mechanism involving acetate condensations which does not include the hydrogenation of keto groups and of ethylenic bonds. For these reactions nicotinic acid and riboflavin are undoubtedly required.

Pantothenic acid, riboflavin, and nicotinic acid may be the only vitamins required for sterol synthesis if a cell has a potential source of acetate in the form of fatty acids or acetate itself. If carbohydrates must be used as the initial source of carbon and hydrogen, then thiamine, of course, will be required to form the active acetyl units from pyruvic acid.

Biotin administration at one time was believed to cause the formation of excessive amounts of cholesterol in the liver. ${ }^{29}$ Critical reexamination of this phenomenon has cast doubts upon this role of biotin. ${ }^{30}$ Biotin. however, could function in some fashion in the process responsible for the formation of the unsaturated linkages in the sterol molecules in a manner comparable to its possible function in the formation of the ethylenic linkages in oleic acid (p. 227).

\section{The Metabolism of Nitrogen Compounds}

From a chemical standpoint, the metabolism of proteins is more complicated than that of carbohydrates and fats. The presentation of the basic reactions is likewise more difficult. Unlike the simple sugars or the fatty acids, the amino acids-the component units of proteins-vary considerably in their chemical structure, and many reactions are necessary to account for the synthesis of the individual amino acids. Polysaccharides are usually polymers of a single hexose, and in fats the arrangement of fatty acids is a random one; but in the formation of the proteins, polymerization of the different amino acids must take place according to a highly specific pattern.

Synthesis and Hydrolysis of Proteins. The proteolytic enzymes catalyzing the direct hydrolysis of the amide bonds require no coenzymes. Almost nothing is known of the enzymes responsible for the intracellular synthesis and hydrolysis of proteins. Several enzymatic processes in which 
simple amide bonds are created have been partially characterized and shown to require adenosine triphosphate or else a coupling with an aerobic system in which this phosphorylating agent is presumably generated:

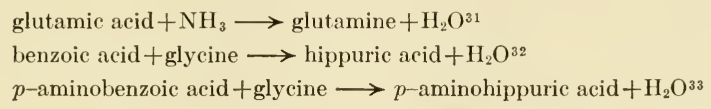

The individual steps of these processes and the phosphorylated intermediates which may be formed have not yet been established. The occurrence of acyl phosphates derived from the amino acids has never been demonstrated. Hence, the formation of the peptide bond cannot be explained on the basis of the utilization of acid anhydrides as was the case in the formation of the ester and acetal linkages of fats and carbohydrates. No $B$ vitamin has as yet been directly implicated in the synthetic processes.

Synthesis of Amino Acids. Many of the enzymatic reactions which are utilized by organisms for the synthesis of their "nonessential" amino acid requirements have yet to be clearly defined. The disclosure of certain types of enzymatic reactions of general occurrence has indicated certain steps which probably take place during most of these syntheses; but the gaps which still exist in any outline of the total processes indicate how much remains to be learned before a scheme for amino acid biosynthesis can be drawn which will in any measure deserve the designation "complete." An effective synthesis of amino acids usually takes place in one of two ways: (1) by the direct amination of the corresponding keto acid, or (2) by a reaction in which one $\alpha$-amino acid is transformed into another amino acid by a chemical alteration of the molecule which leaves the original amino and carboxyl groups intact.

The synthesis by amination of a keto acid either (1) utilizes an amino (or amide) group of some other organic compound, a transamination, or (2) introduces a molecule of inorganic ammonia into the organic structure by a reductive amination. In the former instance pyridoxal has been shown to be required in all cases adequately characterized; in the latter instance, nicotinic acid. Riboflavin enzymes catalyze most of the recognized oxidative deaminations of amino acids (p. 147) and it may be assumed that these function in amino acid synthesis by the reversal of such reactions. The equilibrium established by the flavoprotein enzyme, however, is so much in favor of deamination that this system has not yet been shown to be a method by which amino acid synthesis can effectively occur. However, the glutamic acid dehydrogenases, which are activated by nicotinic acid coenzymes, catalyze a reaction in which concentrations can exist which will favor the reverse reaction, amino acid formation. ${ }^{34}$ It is significant that, of all the amino acids, only glutamic acid has been 
found to participate as a substrate in the reversible nicotinic acid systems. For this reason, ketoglutaric acid may be an extremely important intermediate for the fixation of ammonia into organic molecules. Equally significant is the fact that of the known transamination reactions, by which the $\alpha$-amino group of one acid can be passed on to other keto acids, all have as one amino acid component glutamic acid. ${ }^{35}$ Although only a few transaminases are known, it seems not unlikely that others exist. If so, a general scheme for the formation of these amino acids would be the reductive amination of ketoglutaric acid, followed by a transfer of the amino group to other keto acids. In this way the ketoglutaric acidglutamic acid system could function as a type of ammonia carrier and the system would be one requiring nicotinic acid and pyridoxal. Since the amination of $\alpha$-ketoglutaric acid is reductive, it entails having the reaction coupled with the dehydrogenation of some organic substrate in order to supply the hydrogen atoms which will keep regenerating the reduced form of the nicotinic acid coenzyme. Thus the energy for the conversion of ammonia to an organic amine is derived indirectly from the "oxidation" of another substrate. In addition to pyridoxal and nicotinic acid, biotin may also be another B vitamin directly involved in the biosynthesis of at least one amino acid, aspartic acid (p. 172).

For many microorganisms the amides of aspartic and glutamic acids, asparagine and glutamine, are better sources of nitrogen than are inorganic ammonium salts. It has bcen shown that glutamine is formed by a reaction in which inorganic ammonia is fixed at the expense of a high energy phosphate bond in adenosine triphosphate, ${ }^{31}$ and this conversion of inorganic nitrogen into an intermediate amide nitrogen may often constitute an essential step in the formation of amino compounds from ammonia.

A number of different observations offer evidence indicating that the requirements for specific amino acids by organisms reflects a deficiency in their ability to form the carbon skeleton of the essential molecules rather than an incapacity to form the specific amino acid itself. These observations include (1) the ability of most organisms to utilize the corresponding keto acids in place of essential amino acids; (2) the demonstrated equilibrium existing between inorganic ammonia and the $\alpha$-amino nitrogen of all amino acids except lysine $;^{36}$ and (3) the utilization of ammonia by mammals in place of the nitrogen usually supplied by the nonessential amino acid. ${ }^{37}$

Identified reactions in which one amino acid is formed from another by changes leaving the original $\alpha$-amino and carboxyl groups intact include several transformations which are known to depend upon the presence of a $\mathrm{B}$ vitamin coenzyme: 
glycine $\rightleftharpoons$ serine $\left(p \text {-aminobenzoic acid, folic acid, vitamin } \mathrm{B}_{12}\right)^{38}$ homocysteine $\rightleftharpoons$ methionine $\left(p \text {-aminobenzoic acid, vitamin } \mathrm{B}_{12}\right)^{39}$ serine $\longrightarrow$ tryptophan (pyridoxal) ${ }^{40}$

Degradation of Amino Acids. The degradation of amino acids can be conveniently divided into three groups: (1) those in which the amino acid is first deaminated; (2) those in which the amino acid is converted into another amino acid; and (3) those in which the amino acid is decarboxylated.

The following types of reactions (discussed in detail in the preceding chapter) which cause the deamination of amino acids have been shown to be dependent upon the presence of coenzymes of the vitamins indicated:

(1) Oxidative deamination of glycine and most D- and L-amino acids by riboflavin-containing enzymes.

(2) Oxidative deamination of L-glutamic acid by enzymes whose coenzymes contain nicotinic acid.

(3) Transaminations which result in the deamination of glutamic, aspartic and cysteic acids, alanine, and probably other amino acids by pyridoxal-containing transaminases.

(4) The simultaneous deamination and degradation of tryptophan by a system requiring pyridoxal phosphate.

(5) Deamination of aspartic acid, threonine, and serine by biotinreactivated systems.

The B-vitamin-catalyzed reactions transforming one amino acid into another which were previously listed above undoubtedly are important to the organisms, not only from the standpoint of a means of synthesis, but also as a means of catabolic utilization of certain amino acids.

The decarboxylation of amino acids results in the formation of monoamines, diamines, $\gamma$-aminobutyric acid, and $\beta$-alanine, and it can be anticipated that pyridoxal phosphate will be an essential part of most, if not all, the enzymes carrying out this type of degradation.

A check of the reactions just discussed directly implicates all of the typical B vitamins, except thiamine and pantothenic acid, in the catalysis of one or more reactions in which amino acids participate.

Pyrimidines and Purines. The demonstration of a direct involvement of a $\mathrm{B}$ vitamin in reactions utilized for the biosynthesis of purines and pyrimidines has yet to be accomplished. However, by the use of inhibitors and isotopically labelled substrates the general routes of synthesis have been indicated, the important role of the single carbon unit established, and the essentiality of $p$-aminobenzoic acid (or folic acid) and vitamin $\mathrm{B}_{12}$ demonstrated (Chapter IIB). In purine syntheses these two vitamins may in many instances be required not only for the introduction of the single carbon unit into the purine nucleus, but also for the biosynthesis 
of glycine (from serine, p. 201) which is needed as the source of the metabolic unit from which carbon atoms 4 and 5 (and probably nitrogen atom 7) of the purine nucleus originate. ${ }^{41}$ In the case of fowls and reptiles which excrete most of their metabolic nitrogen in the form of uric acid, the extensive purine synthesis which must be accomplished is reflected in the unusually high glycine requirement. ${ }^{42}$

The initial step in the catabolism of purines is the deamination of adenine and guanine (no vitamin requirement) producing hypoxanthine and xanthine, which are then oxidized by the flavoprotein, xanthine oxidase, to yield uric acid. No B vitamin has been implicated in the further degradations which uric acid has been found to undergo.

Biosynthesis of the B Vitamins. The biological origin of the individual vitamins has been taken up elsewhere (Chapter VA). However, it is logical at this point to indicate that the synthesis of one vitamin probably often depends upon the presence of another B vitamin. In the intestinal tract the biosynthesis of one $\mathrm{B}$ vitamin by bacteria may be influenced by the dietary level of other vitamins which are essential for the growth of the intestinal flora accomplishing the synthesis. A more direct interrelation exists when an enzymatic reaction necessary for the synthesis of one B vitamin requires another B vitamin as a coenzyme. No such case has been unequivocally demonstrated, but when the individual steps in the biosyntheses of vitamins have been better defined, it will undoubtedly be found that many of the reactions involved are of the types which require B vitamin coenzymes. For example, pyridoxal phosphate probably catalyzes the decarboxylation of aspartic acid to form the $\beta$-alanine required for the synthesis of pantothenic acid; and it can be anticipated that the pyrimidine portion of the pterin (folic acid) and isoalloxanine (riboflavin) molecules will be formed by processes utilizing single carbon units in a manner analogous to that observed in purine synthesis, and will be mediated by a $p$-aminobenzoic acid coenzyme.

Choline. The methylation of ethanolamine, forming choline, is dependent upon an adequate dietary source of substances containing available methyl groups ${ }^{43}$ (methionine being the most important) or the capacity of the organism for producing them from other metabolic processes.

In microorganisms it has now been established that the coenzymes involved in the metabolism of the single carbon unit ( $p$-aminobenzoic acid, folic acid, and vitamin $\mathrm{B}_{12}$ ) likewise function in the conversion of homocysteine to methionine. ${ }^{39}$ Also, on the basis of studies using isotopically labelled compounds, it is known that the methyl groups of methionine and choline can serve as sources of "formate" (p. 197). Hence, it is indicated that the single carbon unit will be found to be one of the precursors of the available methyl groups. That mammals may possess to a limited 
extent the enzyme systems capable of the conversion of the single carbon unit to utilizable methyl groups is indicated in an abstract reporting the choline-sparing action of vitamin $\mathrm{B}_{12}$ in the nutrition of rats and chicks. ${ }^{44}$

Porphyrins. Lack of either folic acid ${ }^{45}$ or vitamin $\mathrm{B}_{12}{ }^{46}$ (in rats) results in a decrease in porphyrin synthesis, indicating a possible role of the single carbon unit in porphyrin metabolism. A recent report indicates that glycine may be a complete substitute for the folic acid requirement needed for normal porphyrin synthesis. ${ }^{45}$ Thus the involvement of the catalysts of the single carbon unit may be partially or wholly due to their role in the production of glycine from serine. Glycine had previously been shown to be one of the metabolic units needed for the biosynthesis of the pyrrol rings in the porphyrin nucleus. ${ }^{47}$ Isotopically labelled acetate has also been shown to be incorporated into the porphyrin structures, implicating a pantothenic acid requirement for porphyrin synthesis. ${ }^{48}$

\section{Fundamental Physiological Processes Requiring Energy}

Knowledge concerning the mechanisms by which the chemical energy inherent in the organic substrates metabolized by organisms is converted into other forms of energy must serve as the basis for understanding the fundamentals of physiological processes. By the degradation of organic substrates, and in some cases oxidation of inorganic substances, living organisms transform chemical energy into mechanical energy (including work against osmotic pressure), thermal energy, electrical energy and radiant energy. What is known concerning the role that the $\mathrm{B}$ vitamins play in these energy transformations?

Chemical Energy. The general mechanisms by which the energy released during the oxidation or degradation of organic compounds is made available to organisms for other purposes which require an energy supply has been previously indicated. In almost all cases it appears that the energy is conserved, transported, and eventually utilized through the intermediate formation of compounds that are acid anhydrides of phosphoric acid, compounds containing the so-called "high energy phosphate bonds." 49,50

Three types of reactions for which there is some evidence indicating the mode of formation of the energy-carrying phosphate bonds have been discussed in connection with the reactions catalyzed by the coenzymes of nicotinic acid, thiamine, and pantothenic acid:

(1) The dehydrogenation of an aldehyde-inorganic phosphate addition product by the coenzymes of nicotinic acid in effect utilizes the energy derived from the oxidation of an aldehyde to an acid to convert a molecule of inorganic phosphate to an energy-rich acyl phosphate. (See p. 140 for the mechanism.) 
(2) The energy liberated in the coupled dehydrogenation and decarboxylation of the inorganic phosphate-carbonyl addition products of pyruvic acid and $\alpha$-ketoglutaric acid by thiamine-containing enzymes produces the energy-rich phosphorylated intermediate (p. 163 and 167).

(3) The degradation of the inorganic phosphate-carbonyl addition product of $\beta$-ketoacyl phosphates (for example, acetoacetyl phosphate) results in the cleavage of a carbon-to-carbon bond and the formation of an additional acyl phosphate (p. 189).

In addition to these reactions it has been shown that in aerobic processes additional inorganic phosphate is converted into energy-laden pyrophosphates by the reactions in which the hydrogen atoms are transported to oxygen via the riboflavin and porphyrin-containing enzymes. ${ }^{49}$ In these instances the mechanism by which the phosphate transformation is coupled to the transfer of hydrogen atoms is unknown, but it has been postulated to take place through the addition of phosphoric acid to ethylenic bonds. ${ }^{49}$

Conversely, the energy of the phosphate bonds may be utilized for synthetic purposes by serving as the sources of energy for the formation of glucosidic, ester, and probably peptide bonds (reactions requiring no $B$ vitamins) and for the reductions and condensations catalyzed by nicotinic acid, thiamine, and pantothenic acid (reactions which are the reverse of those tabulated for the formation of the energy-laden bond).

Mechanical and Thermal Energy. On the basis of the current state of knowledge it appears that the B vitamins, having catalyzed the processes by which the high-energy phosphate bonds are formed, have no further function in transforming this energy into either mechanical work or thermal energy. Thus, in none of the following instances has a B vitamin requirement been shown: the contractions of muscle are the result of the transformation into kinetic energy of the energy liberated during the hydrolysis of adenosine triphosphate by an enzyme, adenosine triphosphatase (a component of muscle myosin) ${ }^{50}$; the chemical mechanisms involved in protoplasmic movement (which are responsible for the contractility and mobility of living cells) are not yet understood; the work against osmotic pressure, performed during absorption, is a process often involving phosphorylation of the absorbed molecules by adenosine triphosphate; extra thermal energy (over and above that normally resulting from metabolic processes) can be produced by the hydrolytic action of pyrophosphatases ${ }^{23}$ upon the energy-rich bonds, causing the dissipation in heat of all the energy of the bonds.

Electrical Energy. Little can be said concerning the manner in which the energy derived from metabolic reactions is utilized to establish the electrostatic membrane potentials maintained by viable cells or concern- 
ing the way the electric currents resulting from localized changes in these potentials are controlled. Studies on the enzymatic capacities of tissues in which the electrical potentials are pronounced (nerves and the electric organs of electric eels and fish) established a correlation between the acetylcholine metabolism of these tissues and the electrical potentials which they could develop. ${ }^{51}$ On the basis of considerable evidence it was postulated that the electric current responsible for the conduction of impulses in nerve ${ }^{51}$ and muscle fibers ${ }^{52}$ was due to the release and hydrolysis of acetylcholine, a process which was believed to cause a local change in the permeability of the cell membrane and a resultant flow of the "action current," and that the energy released during the hydrolysis of acetylcholine originally was derived from the high-energy phosphate bonds utilized in the synthesis of acetylcholine. Since this postulate was advanced, convincing arguments against such a direct involvement of acetylcholine in conduction have been offered $;^{53}$ its exact function in nervous and electric tissue is still open to question.

One of the first functions demonstrated for pantothenic acid was the requirement for its coenzyme (coenzyme A) in choline acetylase, ${ }^{54}$ the enzyme catalyzing the reaction in which choline is acetylated by the phosphoryl-acetyl intermediate derived either (1) from the oxidative decarboxylation of pyruvic acid (thiamine pyrophosphate essential) or (2) from the direct phosphorylation of acetic acid (coenzyme A required). Hence, these two coenzymes are of specific importance for the functioning of cells where conduction takes place.

Radiant Energy. The biological conversion of radiant energy to chemical energy by the reduction of carbon dioxide (photosynthesis) and the reverse process, the emission of radiant energy during biological oxidations of organic substrates (bioluminescence), are the result of two processes whose mechanisms are entirely unrelated. The chemical reactions responsible for the latter phenomena have been well established, but the former process, which is indispensable from the standpoint of the economy of the biological world, cannot yet be described in terms of specific chemical reactions.

It is surprising, in view of the vast amount of study which has been devoted to the photosynthetic phenomenon, that nothing has been learned concerning what roles the $\mathrm{B}$ vitamins may play in this process. It can be anticipated, however, that this question will soon be answered, at least in part, as a result of current investigations which have already yielded much information not previously obtained by the classical methods that have been used in attacking the problem. Two of these recent approachs which are proving to be especially valuable are the study of photosynthesis from the standpoint of comparative biochemistry ${ }^{55}$ and the cor- 
relation of the chemistry of the photosynthetic process with that of certain chemosynthetic mechanisms which have been well established. ${ }^{56}$ Chemosynthesis, the assimilation of carbon dioxide by reactions which utilize energy derived from other metabolic reactions rather than radiant energy, is the result of the reversal of the processes in which there are decarboxylation reactions; hence the accomplishment of carbon dioxide fixation by chemosynthetic means is dependent upon exactly the same vitamins and enzymes that carry out these carboxylation reactions. It seems reasonable to expect that many, if not most, of the photosynthetic "dark reactions" which take place after the initial "light reaction" and lead to the ultimate formation of carbohydrates will be reactions that are the reverse of those used for carbohydrate catabolism, and hence will be catalyzed by the same enzymes and cofactors. When the mechanisms which the photosynthetic and chemosynthetic processes have in common are eventually established, then at least some of the functions of the B vitamins in photosynthesis will have been determined.

Bioluminescence is the result of the action of an enzyme (luciferase) upon a reduced substrate, dihydroluciferin, in the presence of oxygen. ${ }^{57}$

$$
\text { luciferin } \cdot 2 \mathrm{H}+\mathrm{O}_{2} \stackrel{\text { luciferase }}{\longrightarrow} \text { luciferin }+\mathrm{H}_{2} \mathrm{O}_{2}
$$

The release of energy in the form of light is a specific characteristic of the enzyme, since the oxidation of dihydroluciferin by other agents is not accompanied by the emission of light. The reduced coenzymes of nicotinic acid and riboflavin as well as sodium dithionite and hydrogen (Pt catalyst) can be used in place of dihydroluciferin as hydrogen donors for the luminescent reaction. Luciferin, though once postulated to be a flavin-like compound, is now known to be chemically related to vitamin K. ${ }^{58}$

\section{Bibliography}

1. Meyerhof, O., Biol. Symposia, 5, 141 (1941).

2. Evans, E. A., Jr., Biol. Symposia, 5, 157 (1941).

3. Barron, E. S. G., Advances in Enzymol., 3, 149 (1943).

4. Krebs, H. A., ibid., 3, 247 (1943).

5. Stotz, E., ibid., 5, 129 (1945).

6. Sumner, J. B., and Somers, E. F., "Chemistry and Methods of Enzymes," Academic Press, Inc. (New York), 1943, pp. 302-331.

7. Baldwin, E., "Dynamic Aspects of Biochemistry," Macmillan Company (New York), 1947, pp. 305-383.

8. Cori, C. F., Biol. Symposia, 5, 131 (1941).

9. Woods, H. G., and Werkman, C. H., Biochem. J., 32, 1262 (1938).

10. Lipmann, F., Cold Spring Harbor Symposia Quant. Biol., 7, 248 (1939).

11. Lipmann, F., Advances in Enzymol., 6, 231, (1946).

12. Lardy, H. A., and Elvehjem, C. A., Ann. Rev. Biochem., 14, 1 (1945).

13. Lynen, F., and Franke, W., Z. physiol. Chem., 270, 271 (1941).

14. Negelein, E., and Gerischer, W., Biochem. Z., 284, 289 (1936). 
15. Baldwin, E., op. cit., pp. 384-415.

16. MeHenry, E. W., and Gavin, G., J. Biol. Chem., 138, 471 (1941).

17. Guirard, B. M., unpublished observations.

18. Krehl, W. A., Strong, F. M., and Elvehjem, C. A., Ind. Eng. Chem. Anal. Ed., 15, 471 (1943).

19. Bauernfeind, J. C., Sotier, A. L., and Boruff, C. S., Ind. Eng. Chem., Anal. Ed., 14, 666 (1942).

20. Guirard, B. M., Snell, E. E., and Williams, R. J., Arch. Biochem., 9, 361 (1946).

21. Lang, K., Z. physiol. Chem., 261, 240 (1939).

22. Baer, E., and Fischer, H. O. L., J. Biol. Chem., 128, 463 (1939).

23. Sumner, J. B., and Somers, E. F., op. cil., pp. 45-67.

24. Baldwin, E., op. cit., p. 83.

25. Lehninger, A. L., J. Biol. Chem., 157, 368 (1941).

26. Longnecker, H. E., Biol. Symposia, 5, 99 (1941).

27. Bloch, K., and Rittenberg, D., J. Biol. Chem., 145, 625 (1942).

28. Shive, W., Ackermann, W. W., Ravel, J. M., and Sutherland, J. E., J. Am. Chem. Soc., 69, 2567 (1947).

29. Gavin, G., and McHenry, E. W., J. Biol. Chem., 141, 619 (1941).

30. Handler, P., J. Biol. Chem., 162, 77 (1946).

31. Speck, J. F. S., J. Biol. Chem., 168, 403 (1947).

32. Borsook, H., and Dubnoff, J. W., J. Biol. Chem., 132, 307 (1940).

33. Cohen, P. P., and McGilvrey, R. W., J. Biol. Chem., 166, 261 (1946).

34. v. Euler, H., Adler, E., Günther, G., and Das, N. B., Z. physiol. Chem., 254, 61 (1938).

35. Cohen, P. P., J. Biol. Chem., 136, 565 (1940).

36. Schoenheimer, R., Ratner, S., and Rittenberg, D., J. Biol. Chem., 127, 333 (1939).

37. Lardy, H. A., and Feldott, G., J. Biol. Chem., 179, 509 (1949).

38. Shemin, D., J. Biol. Chcm., 162, 297 (1946).

39. Shive, W., Ann. N. Y. Acad. Sci., in press.

40. Tatum, E. L., and Bonner, D., Proc. Nat. Acad. Sci., 30, 30 (1944).

41. Buchanan, J. M., Sonne, J. C., and Delluva, A. M., J. Biol. Chem., 166, 395 (1946).

42. Almquist, H. J., and Mecchi, E., J. Biol. Chem., 135, 356 (1940).

43. du Vigneaud, V., et al., J. Biol. Chem., 134, 787 (1940).

44. Shaefer, A. E., Salmon, W. D., and Strength, D. R., Federation Proc., 8, 395 (1949).

45. Totter, J. R., Sims, E., and Day, P. L., Proc. Soc. Exptl. Biol. Med., 66, 7 (1947).

46. Dunning, J. S., Keith, C. K., Totter, J. R., and Day, P., Fedcration Proc., 8, 381 (1949).

47. Totter, J. R., Amos, E. S., and Keith, C. K., J. Biol. Chem., 178, 847 (1949).

48. Bloch, K., and Rittenberg, D., J. Biol. Chem., 159, 45 (1945).

49. Lipmann, F., "Currents in Biochemical Research," Interscience Publishers, Inc. (New York), 1946, pp. 137-148.

50. Engelhardt, IV. A., and Lynbimowa, M. N., Nature, 144, 668 (1939).

51. Nachmansohn, D., "Currents in Biochemical Research," Interscience Publishers, Inc. (New York), 1946, pp. 335-356.

52. Bullock, T. H., Grundfest, H., Nachmansohn, D., and Rothenberg, M. A., J. Neurophysiol., 10, 11 (1947).

53. Grundfest, H., Ann. Rev. Physiol., 9, 477 (1947).

54. Lipmann, F., and Kaplan, N. O., J. Biol. Chem., 162, 743 (1946).

55. Van Niel, C. B., "Photosynthesis in Plants," Iowa State College Press (Ames, Iowa), 1949 , pp. 437-495.

56. Ochoa, S., "Currents in Biochemical Research," Interseience Publishers, Inc. (New York), 1946, pp. 165-186.

57. Sumner, J. B., and Somers, E. F., op. cit., p. 275.

58. Kluyver, A. J., van der Kirk, G. J. M., and van der Burg, A., Proc. Nederl. Akad. van Wetenschappen, 45, 962 (1942). 

Section C

THE ROLE OF THE B VITAMINS IN ANIMAL AND PLANT ORGANISMS

Ernest Beerstecher, Jr. 


\section{PROLOGUE}

A logical consideration of the role of the $\mathrm{B}$ vitamins in living organisms might well follow an outline centering around the answers to these four major questions:

What are the $\mathrm{B}$ vitamin requirements of living organisms?

What happens to the $\mathrm{B}$ vitamins in living organisms?

How do the $\mathrm{B}$ vitamins affect living organisms?

How does $B$ vitamin deprivation affect living organisms?

The first three chapters of this section are an attempt to answer the first of these questions, while the ensuing chapters are devoted respectively to the last three questions. The answers, however, are by no means as explicit as are the questions. 


\section{Chapter IC}

\section{METHODS OF ASSESSING B VITAMIN REQUIREMENTS}

\section{General Considerations}

After the foregoing consideration of the general nature of the B vitamins and their role in the chemistry of cells and aggregates of cells, it would be desirable to proceed by a logical sequence of study to the part played by the $B$ vitamins in entire organisms. Unfortunately, however, the present understanding of the facts does not permit so smooth a transition. In the study of vitamin deficiencies in particular, wherein lie man's most urgent interests, there is seldom more than a vague similarity between the biochemical functions of the vitamins as we have considered them and the clinical characteristics of the avitaminoses. This section of the monograph is developed largely along a new pathway; it therefore draws on the more basic biochemical factors previously considered only on those rare occasions when the circumstances will permit.

Implicit in the study of the B vitamins as a group is the fact that in nature they always occur together and are essential in the economy of all living cells. Since the problems involving the requirement of any one $B$ vitamin are common to all members of the $B$ group, an attempt is made to present the discussion of these requirements in a general and integrated way, rather than to stress the consideration of each vitamin individually.

When the supply, whether intracellular or extracellular, of any one of the $\mathrm{B}$ vitamins is cut off, the entire metabolic process rapidly comes to a standstill. From the standpoint of a single cell, the time required for this to occur is largely dependent upon how rapidly the various chemical events progressing within the cell bring about attrition of vitamincontaining catalyst molecules. In the absence of a renewed supply of coenzyme, this generally ensues quite rapidly, and the cell becomes functionless in a normal sense when the critical vitamin reaches an inoperably low level. The quantitative requirement of that cell for any B vitamin is that amount which it must supply, or which must be supplied to it, to continue in normal operation. This concept is basically true for cell aggregates, whether tissues or entire organisms. Cell aggregates for architectural reasons, however, are able to buffer themselves against deficiency, and therefore do not respond as rapidly to vitamin privation. 
The present discussion will not be primarily concerned with that portion of the requirement with which living matter is able to supply itself. This fraction of the requirement has been assessed from a practical standpoint in the consideration of the sources of vitamins for nutritional purposes, and from an academic standpoint in the discussion of biosynthetic processes. Far more expedient is the evaluation of that portion of the requirement which must be supplied to the cell, tissue, and complete organism; and this qualitative and quantitative fraction of the total will hereafter be referred to as the "requirement," as is the custom. The term "nutritional requirement" differs critically from this, in that it is that part of the requirement which must be supplied in the diet, and does not include that portion which may be supplied by symbiants such as intestinal bacteria.

The natures of the $\mathrm{B}$ vitamin requirements of living organisms are as diverse as are the forms of life themselves. To a considerable extent the members of the plant kingdom are able to meet their own $\mathrm{B}$ vitamin requirements, demonstrating thereby a higher degree of synthetic ability than is found generally in the animal kingdom. This fact is most fundamental to the overall economy of life. Green plants have, therefore, been considered in this discussion primarily as a food source; and, though they will be referred to again when considerations arise that seem to warrant their separate discussion, a more extended consideration of the B vitamins in green plants does not at present seem practical. Similarly, the role of the $\mathrm{B}$ vitamins in the lower plant forms is of importance from a number of diverse aspects, which seems to dictate the advisability of their discussion as the occasion arises rather than by separate treatment. The $B$ vitamins are therefore presented here in their relationship to the animal as a whole, while the plant kingdom is considered only as the pattern of the treatment and as the availability of data permit.

From a purely qualitative standpoint, organisms exist which require none of the $\mathrm{B}$ vitamins, and others exist which require all those now known and probably still other substances of chemical natures which are at present unknown. Although many bacteria are able to synthesize all the $B$ vitamins in sufficient amounts to meet their needs, this cannot be said at present of any member of the animal kingdom. Indeed, no higher animal has as yet been found which is able to survive even when all the presently known $\mathrm{B}$ vitamins are supplied; and it must be concluded that as yet unidentified factors, whether they turn out to be $B$ vitamins or not, are necessary in animal nutrition. A summary of some of these factors is given on pp. 12-16.

Qualitative requirements may not, however, be dismissed as a matter of "required" or "not required," since a variety of factors influence the 
decision. While nutritional requirements of ruminants for B vitamins are virtually nonexistent, this is only by virtue of an extensive system of bacterial symbiosis; and ruminants do actually require a supply of these substances, even though it may be derived from within the confines of the gastrointestinal tract. Moreover, certain B vitamins may substitute for others. Thus, as will be shown later, substitutions similar to that of alanine for vitamin $\mathrm{B}_{6}$ in the nutrition of $S$. fecaelis $\mathrm{R}^{1}$ may occur in the animal kingdom.

The nature of quantitative $\mathrm{B}$ vitamin requirements is by comparison far more complex and will require extended discussion. It should be pointed out here, however, that the conditions under which any given quantitative $\mathrm{B}$ vitamin requirement exists are so limited that the fixing of a practical value for any particular species is possible only within very broad limits.

Finally, fundamental to the nature of any requirement is the problem of what criterion shall be taken in judging whether a substance is required and how much is required. ${ }^{2}$ Thus, the amount of thiamine necessary to protect a rat from convulsive seizures might be defined as the requirement, even though the animal suffered from other pathological manifestations. The requirement might also be defined as the amount necessary to maintain the animal in a state of health, or as the amount required to promote growth, or optimum growth, or as the amount necessary to promote longevity. Each of these criteria has found use, and a variety of similar cases makes it necessary to reach an agreement on, or an understanding of, this issue. Prejudicial interpretation of this factor in prison camps during the recent war has resulted in extensive discussion of deficiency criteria. A more extended discussion of this factor, as it refers to the prevention of specific pathology, occurs in the following section. Criteria based on benefits exceeding growth and maintenance are discussed more fully in later chapters.

Associated with this problem is the one of poor usage and ill-defined terminology. Expressions such as "health," "physical fitness," "optimal requirements," and "maximal requirements," are common to the field and portray clearly the uncertainty and the lack of development of satisfactory scientific criteria and nomenclature. ${ }^{3}$ One definition of good nutrition, for instance, is "that condition which permits the development and maintenance of the highest state of fitness." "Fitness," however, is a term of little advantage, since, as Keys points out, "everyone knows what it means but not how to measure it."

In addition to the problem of precise requirements, there is the further question of recommended intake, i.e., the safety margin believed to be advisable above the absolute requirement. This consideration is largely 
significant in view of wide individual variations in nutritional requirements (p. 273). Unquestionably the most generally accepted recommendations or "allowances" are those of the Food and Nutrition Board of the National Research Council (p. 324). Based on broad considerations and a variety of studies, these are nevertheless frequently challenged, sometimes because of their interpretation as "requirements" rather than recommended intakes, but more often on a seemingly valid basis. Indeed, it is quite apparent that no single recommended level will suffice for all purposes, ${ }^{6-10}$ since recommendations for the allowances necessary to ward off deficiency diseases will obviously differ from those used in planning a dietary regimen for therapeutic purposes, or those used in planning broad agricultural or public health programs. These factors will be considered in greater detail somewhat later.

\section{Methods of Assessing B Vitamin Requirements}

Man's first interest has always been with man; but because of the limitations which have been placed on experimentation involving humans, great ingenuity has been required in assessing human vitamin requirements. A variety of approaches has been employed, all more or less indirect, and therefore subject to interpretation. It has generally been possible to determine the nutritional requirements of other species with far greater precision as a result of the experimental freedom which it is possible to achieve. This fact led to one of the earlier approaches to the assessment of human requirements, Cowgill's study of comparative requirements which is discussed first.

Clear-cut experimental data regarding requirements are rare, and this is due among other reasons to the difficulty in obtaining animals free from symbiotic organisms. Some conclusions can be drawn with considerable certainty in fowls, inasmuch as the interiors of eggs are generally germfree. Thus, since the riboflavin content of hens' eggs does not increase during incubation, we know with certainty that the foetal chicken, at least, does not synthesize riboflavin. ${ }^{11}$ Similarly, when hens' eggs are injected with tryptophan, and then incubated and analyzed, they show a higher niacin content than uninjected eggs;11a this is among the more convincing facts that indicate that animal tissues do convert tryptophan to niacin without the aid of bacterial symbiants. A more adequately controlled repetition of this work would seem to be desirable. Unfortunately, however, extensive data of this nature are lacking.

Comparative Studies of Requirements. It has long been known that the food consumption per unit of body weight and the basal metabolic rate per unit of body weight are much greater for small animals than for larger ones. It is thus not strange that it was readily apparent from 
the earliest studies that the thiamine requirement per unit body weight varied from species to species, being greater for smaller animals. Cowgill ${ }^{12}$ has made an extended study of this relationship and its application to the determination of human requirements. While the actual data obtained are not generally accepted today, and the relationships obtained reflect to some extent the impure nature of the crude extracts used at the time the work was done, the general concept behind the conclusions is of considerable interest and importance. In studies using the mouse, the rat, the pigeon, and the $d o g$, and using the maintenance of appetite as a criterion of satisfaction of the requirement, he found that for any one species, the thiamine requirement was proportional to the five-thirds power of the weight of an individual animal, and that the proportionality constant was characteristic for the species.

$$
\text { Thiamine requirement }=K_{S} W_{i}^{3 / 3}
$$

When the logarithm of the maxium recorded normal weights ever attained by an individual of the species is plotted against the logarithms of the species constants, a straight line is obtained, which may be expressed by the equation

$$
\text { Thiamine requirement }(\mu \mathrm{g} / \mathrm{day})=\frac{0.98 \times W_{i}^{0.66} \times W_{i}}{W_{\max }}
$$

A study of several species indicated that

$$
W_{i}=\frac{\mathrm{Cal}_{i}}{1.5}
$$

and the final equation is thus obtained,

$$
\text { Thiamine requirement }(\mu \mathrm{g} / \mathrm{day})=\frac{0.654 \times W_{i} \times \mathrm{Cal}_{i}}{\bar{W}_{\max }}
$$

where $W_{i}$ is the weight of an individual, $W_{\max }$ is the maximum weight obtained by the species, and $\mathrm{Cal}_{i}$ is the daily food intake in Calories. Employing the last equation, one concludes that for a $70-\mathrm{kg}$ man $\left(W_{\max }\right.$ is $115 \mathrm{~kg}$ ) with an intake of 2500 Calories, the daily nutritional thiamine requirement is about $1000 \mu \mathrm{g}(1.0 \mathrm{mg})$.

This general approach has been subjected to considerable criticism, and is obviously subject to numerous errors. ${ }^{13}, 14$ Nevertheless, the estimate so obtained is not greatly different from that obtained by numerous other means. In view of this fact, it is indeed surprising that this general approach has not been employed with other members of the B group of vitamins, where the general principles involved should apply with equal validity (pp. 319-323).

Diets controlled to produce a given symptom in a given species. Perhaps the most direct approach to the assessment of requirements con- 
sists of depleting the nutrition of a factor and then observing the exact amount of the vitamin required to prevent active clinical manifestations of deficiency. While this was possibly the earliest technique, only in recent years has it been directly applied to humans. Even so, such a direct approach to the problem of vitamin requirements is not entirely satisfactory for a number of reasons. Primarily, the results obtained will depend completely upon the symptoms to be prevented. Careful perusal of the tabulated requirements in Chapter IIIC will demonstrate this forcibly as it applies to many species, since requirements have been variously adjudged over wide ranges based on this fact alone. This may perhaps best be illustrated in the case of the folic acid requirements of the chick which have been extensively investigated in recent years. ${ }^{15}$ The symptoms which are prevented by different levels of folic acid in the chick diet are indicated in Table 1.

\section{TABLE 1. Functions Supported in the Chick by Various Levels of} Folic Acid

Level of folic acid (per $100 \mathrm{gm}$ diet) $(\mu \mathrm{g})$

25
30
35
45
55
$50-110$

Criterion

survival

prevention of perosis

normal hemoglobin formation normal growth normal feathering

optimal growth
Reference

(15)

Thus for any vitamin and species a number of criteria may be taken (and frequently have been taken) as indicating a deficiency or lack of it. Obviously, the criterion suited to the purpose is that which insures the "well-being" and "general normal character" of the animal in question, such terms being about as indefinite as the requirement itself. Since the full consequences of avitaminoses are seldom realized simultaneously with the first recognition of the etiology of the disease, the optimal type of criteria mentioned is not always definable, and realization of this has done much to foster the study of subclinical deficiencies.

Another difficulty inherent in this approach is that concerned with the association of specific symptoms with a deficiency. Thus when the list of vitamins consisted largely of "A," "B," "C," and "D," certain symptoms were associated with a deficiency of vitamin "B," and only in more recent times has it been possible to associate certain of these with the precise chemical factor or factors whose absence was responsible for these symptoms. In many cases the exact correlation is still unclear. Green and Brunschwig, ${ }^{18}$ for instance, in assessing the physiological activity of choline, have only recently come to the conclusion that the factors responsible for hepatic fatty infiltration and for parenchymal necrosis may not be identi- 
cal. Whether or not the finding that thiomalic and thiolactic acids afford no protection against the latter but seem to promote the fatty infiltration actually supports this belief remains to be seen.

It is seldom as simple as it might appear to those unfamiliar with the field to exclude one factor from the diet without simultaneously omitting another. ${ }^{19}$ In recent studies using dogs, a diet apparently complete in all necessary factors but biotin produced paralysis and death. Biotin, moreover, appeared to prevent these effects. ${ }^{20,21}$ Later work ${ }^{22,23}$ showed, however, that the symptoms were primarily due to a potassium deficiency. While biotin produced some temporary responses for several hours, the protective effect of a single adequate dose of potassium lasted for six to ten weeks. Similar cases are well known throughout the history of the assessment of $\mathrm{B}$ vitamin requirements by depletion methods.

It is only in recent years that it has been possible to apply this type of study directly to humans. This is due to a considerable extent to the difficulty in controlling a multitude of variables which must be controlled in order to obtain significant data. Realizing this, the National Research Council's Committee on Diagnosis and Pathology of Nutritional Deficiencies has outlined certain of these factors which they feel to be of the greatest importance. ${ }^{24}$ While primarily designed for assessing conditions of deficiency, these factors apply in every sense to the assessment of requirements in animals as well as humans. Briefly they are: (1) the adequacy of the criterion for determining the nutritional status; $(2)$ the nutritional status previous to the experimental period; (3) the diet prior to and during the experimental period; $(4)$ the conditions which influence the relationship between the supply and requirements; $(5)$ the indices set by the observer to measure the criteria; (6) the initial status of the subjects with regard to growth, physical performance, resistance to disease, etc.; (7) the method of selection of subjects; (8) the number of subjects; (9) the nature and potency of supplements; and (10) the length of the experiment.

In addition to these factors, adequate controls are essential. This is particularly true in human experimentation when psychic factors may be magnified..$^{25}$ The supplement must be matched in such control groups with placebos, indistinguishable from the supplement in taste and appearance. Not only the subjects, but equally important, the observers, must be ignorant of which individual receives a placebo and which a supplement. Even in experimentation designed to determine the essentiality of a nutritional source of some $B$ vitamin, it is necessary to observe the factors outlined above. In a recent study of the effects of a vitamin $B_{6}$ deficient diet in humans, ${ }^{26}$ almost no definite conclusions could be reached because 
TABLE 2. Analysis of Beriberi-Producing and Beriberi-Preventing Diets. (From Williams and Spies)

Designation of Diet

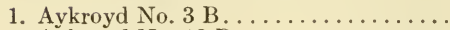

2. Aykroyd No. 13 B...............

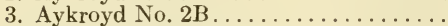

4. Strong and Crowell IV ..............

5. Selangor Jail $1892 \ldots \ldots \ldots \ldots \ldots$.

6. East Indian Navy Natives (fish) ......

7. Fraser and Stanton I . . . . . . . . .

8. Indian Troops Trincomalee (1900) .....

9. Singapore Prison $1869-75 \ldots \ldots \ldots \ldots$

10. Strong and Crowell II ............

11. Selangor 1901 Ordinary ............

12. Kuala Lumpur I . . . . . . . . . . . .

13. East Indian Native Sailors $1874 \ldots \ldots$.

14. Selangor Jail, Penal, 1902 . . . . . . . .

15. Bilibid Prison 1901-02 . . . . . . . . .

16. Selangor Jail, Penal, $1900 \ldots \ldots \ldots \ldots$

17. Negro Laborers Congo I . . . . . . . . .

18. Singapore Prison $1876 \ldots \ldots \ldots \ldots \ldots$

19. Philippine Scouts $1908 \ldots \ldots \ldots \ldots \ldots$

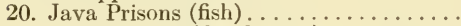

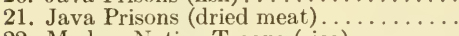

22. Madras Native Troops (rice) .........

23. Dutch E. Ind. Navy, Native 1874 (fish).

24. Mediterranean Troops A1 ..........

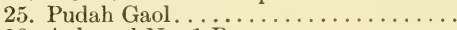

26. Aykroyd No. 1 B . . . . . . . . . . . .

27. Kut-el-Amara British Jan. $22 \ldots \ldots \ldots$.

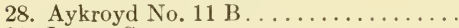

29. Lascar Seamen ...................

30. Dutch E. Ind. Navy Natives $1878 \ldots .$.

31. Java Prisons (beef and pork) .........

32. Aykroyd No. 9 B . . . . . . . . . . . .

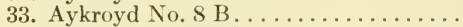

34. Selangor 1902 Ordinary . . . . . . . .

35. Japanese Ryujo Marines. . . . . . . . .

36. Bilibid $1902 \ldots \ldots \ldots \ldots \ldots \ldots \ldots \ldots$

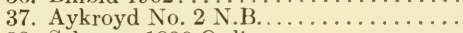

38. Selangor 1899 Ordinary ............

39. Aykroyd No. 4 B ...............

40. Selangor 1900 Ordinary .............

41. Singapore Prison July $1880 \ldots \ldots \ldots \ldots$

42. Akroyd No. 5 B . . . . . . . . . . . .

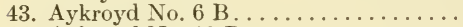

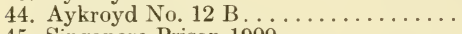

45. Singapore Prison $1900 \ldots \ldots \ldots \ldots \ldots$

46. Mediterranean Troops A2 .........

47. Singapore Prison $1897 \ldots \ldots \ldots \ldots \ldots$

48. Dutch E. Ind. Navy, 1874 Europeans .

49. Aykroyd No. 10 B ................

50. Selangor 1895 Ordinary ...........

\begin{abstract}
Thiamine
per day
$(\mu \mathrm{g})$
\end{abstract}

225

300

308

268

256

420

346

446

336

268

402

443

565

284

368

306

475

342

578

420

440

573

600

672

487

442

469

934

670

948

550

818

676

482

521

478

629

561

495

468

535

727

576

557

645

918

624

1091

632

618
Calories
per day

1974

2480

2520

2310

2581

3810

3060

3922

2952

2310

3150

3379

4208

2084

2661

2193

3295

2315

3908

2831

2927

3804

3960

4400

2970

2700

2839

5530

3956

5534

3211

4720

3870

2690

2875

2622

3430

3032

2660

2454

2720

3665

2860

2740

3128

4400

3005

5189

3020

2942
$\frac{T(\mu \mathrm{g})}{\text { Cal. }}$

0.074

0.078

0.079

0.099

0.099

0.110

0.113

0.114

0.114

0.115

0.128

0.131

0.134

0.136

0.138

0.140

0.144

0.148

0.148

0.148

0.150

0.151

0.151

0.153

0.164

0.164

0.165

0.169

0.170

0.171

0.171

0.173

0.175

0.175

0.181

0.182

0.183

0.185

0.186

0.190

0.197

0.198

0.201

0.203

0.206

0.208

0.208

0.209

0.209

0.210
Extent of beriberi

B

B

B

XXXX

XXX

XXXX

XXX

XXX

XXX

XXY

XXX

XXX

XXX

XXXX

XXXX

IXXX

XXXX

XXX

XXX

XX

XX

XXX

XX

XX

B

$\mathrm{XXX}$

B

X

$\mathrm{X}$

XX

B

B

X

XXXX

$\mathrm{X}$

No

XXX

B

XXX

XX

B

B

B

XXX

XX

X

B X 
TABle 2. Analysis of Beriberi-Producing and Beriberi-Preventing Diets-(Continued). (From Williams and Spies)

Designation of Diet

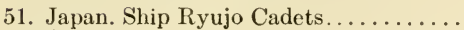

52. Aykroyd No. 7 B.

53. Megaw and Bhattacharjee Parsibazar.

54. Megaw and Bhattacharjee (Campbell).

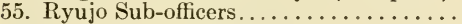

56. Strong and Crowell III.............

57. Jap. Navy Food Act. $1884 \ldots \ldots \ldots \ldots$.

58. Ryujo Officers.................

59. Kala Bagan . . . . . . . . . . .

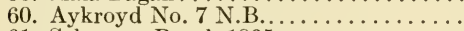

61. Selangor, Penal, $1895 \ldots \ldots \ldots \ldots \ldots$

62. Selangor, Penal, $1893 \ldots \ldots \ldots \ldots \ldots$

63. Singapore Prison $1898-99 \ldots \ldots \ldots \ldots$

64. Bengali Girls . ................

65. Singapore Prison Sept.-Dec. $1881 \ldots .$. .

66. Singapore Prison 1882-85 . . . . . . .

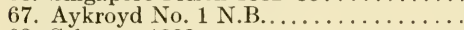

68. Selangor 1892 .

69. Mediterranean Troops.............

70. Megaw and Bhattacharjee Hindus.....

71. Aykroyd No. 3 N.B..................

72. Mediterranean Troops B..........

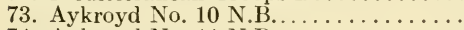

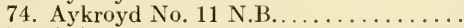

75. Aykroyd No. 4 N.B..............

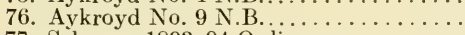

77. Selangor 1893-94 Ordinary ..........

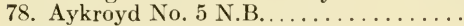

79. Megaw and Bhatt. Anglo-Ind........

80. Aykroyd No. 12 N.B.

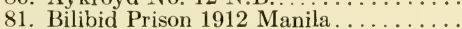

82. Kut-el-Amara Indians March 11 .......

83. Richmond Asylum-Dublin 1897 ......

84. Kut-el-Amara British March $11 \ldots \ldots$.

85. Garrison, San Juan Jan.-Apr. . . . . . . .

86. Megaw and Bhatt. Mohammedans....

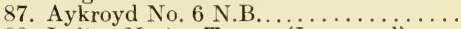

88. Indian Native Troops (Improved) .....

89. Kut-el-Amara British Feb. $10 \ldots \ldots \ldots$

90. Singapore Prison 1885-1896 . . . . . .

91. Kut-el-Amara Indians Mar. $4 \ldots \ldots \ldots$.

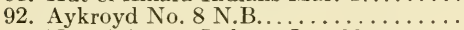

93. Kut-el-Amara Indians Jan. $22 \ldots \ldots \ldots$

94. U. S. Garrison San Juan June.......

95. Kut-el-Amara British Mar. 4 . . . . . .

96. Philippine Scouts after $1911 \ldots \ldots \ldots \ldots$

97. Trincomalee Troops $1901 \ldots \ldots \ldots \ldots \ldots$

98. Frazer and Stanton $1907-08 \ldots \ldots \ldots$.

99. Kuala Lumpur Asylum . . . . . . . . . .

100. Ind. Native Troops Attah ...............

$\underset{\substack{\text { Thiamine } \\ \text { per day } \\(\mu \mathrm{g})}}{\cos }$

651

510

464

506

660

442

995

819

432

751

646

710

581

532

904

970

703

1034

1013

616

764

1033

1035

875

820

1497

965

1664

932

1597

941

726

982

771

600

778

954

1500

1063

585

983

2070

1217

866

1168

1770

2194

1695

2170

3925
Calories
per day

$\frac{T(\mu \mathrm{g})}{\text { Cal. }}$

Extent of

3072

0.212

0.212

0.220

0.230

2100

2200

2867

1916

4295

3508

1822

3160

2720

2982

2424

2200

3475

3652

2590

3766

3620

2295

2710

3590

3690

2945

2710

4980

3142

5510

3000

4980

2911

2208

2994

2304

1718

2180

2580

4025

2835

2514

2149

5020

2843

1882

2420

3672

4200

3054

3381

3994
0.230

0.231

0.232

0.233

0.237

0.238

0.238

0.238

0.240

0.242

0.260

0.266

0.271

0.274

0.280

0.281

0282

0.288

0.288

0.297

0.300

0.300

0.308

0.302

0.310

0.321

0.324

0.328

0.328

0.334

0.350

0.357

0.370

0.373

0.375

0.385

0.457

0.413

0.428

0.460

0.481

0.522

0.555

0.624

0.985
0.482
XXX

B

B

B

$\mathrm{XX}$

$\mathrm{XX}$

X

$\mathrm{X}$

No

XXX

$\mathrm{X}$

XXX

No

$\mathrm{XXX}$

$\mathrm{X}$

No

No

No

No

No

No

No

No

No

No

No

No

No

No

No

XXX

No

$\mathrm{X}$

No

No

No

No

No

No

No

No

No

No

No

No

No

No
No

Prevalence of beriberi is indicated by number of $X^{\prime} s$, except when the number of cases is not recorded or the group was too small for statistical consideration, in which case the existence of beriberi is indicated by $B$.

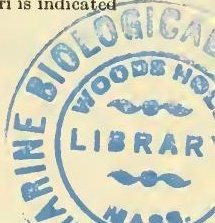


a number of these factors mentioned had not been considered in the experiment.

With rats, mice, chickens, and most other smaller animals, growth has frequently been taken as a suitable criterion for assessing the fulfillment of the B vitamin requirement. In humans this is not the case, supposedly because of the difficulties in the interpretation of such data. Indeed, studies involving vitamin supplementation of adequate diets have been frequently subjected to criticism for adopting "growth" as a criterion without any further consideration of other factors involved. Moreover, from a physiological point of view, "increased" and "improved" growth may be very different things, there being no guarantee that the most rapid growth rate is the most desirable. ${ }^{27}$

Melnick et al. ${ }^{28}$ have developed $\mathrm{B}$ vitamins bioassay methods which employ human subjects. These methods (p. 283) depend upon the study of the urinary excretion of the B vitamins, which is presumably a function of the intake. While not bearing directly upon the problem at hand, the work of these investigators deserves mention at this time in that it is subject to the variables previously mentioned, yet illustrates the value that is attached to carefully controlled experimentation with human subjects.

Dietary surveys. Somewhat akin to controlled diet studies are those on healthy and avitaminotic populations. By a careful consideration cf numerous dietary surveys, it is sometimes possible to estimate the level of nutrition which will bring about a deficiency of one or more of the B vitamins. Excellent examples of this approach have resulted from studies of prison camps and of circumscribed populations during the recent war, since the diet was frequently rigidly controlled and permitted unusually accurate assessment of the vitamin intake. One of the classical studies of this nature, however, is an earlier one dealing with thiaminedeficient diets in the Orient.

Cowgill ${ }^{12}$ studied some 180 human diets in regard to their thiamine and calorific content and association with beriberi. Williams and Spies ${ }^{14}$ later reassessed these data and arrived at a more accurate estimate of the minimal human requirement necessary to prevent beriberi. A modified list of the diets studied in increasing order of the ratio of thiamine to Calories in the diet is given in Table 2. A summary of the results is given in Table 3.

The validity of these data and the conclusions derived from them are borne out adequately by a study of a large number of American dietaries (Table 4).$^{29}$ The thiamine-to-calorie ratio seems generally to be above that associated with clinical beriberi, other dietary factors (i.e., fat; page 
TaBle 3. Classification of Diets in Table 2 as to Thiamine Deficiency.

Thiamine ( $\mu \mathrm{g} /$ day)

$\begin{array}{lc}\text { Calories per day } & \begin{array}{c}\text { Diets producing } \\ \text { Beriberi }\end{array} \\ 0.074-0.229 & 52 \\ 0.230-0.249 & 9 \\ 0.250-0.279 & 2 \\ 0.280- & 3\end{array}$

Non-Beriberi Diets

1
2
2

29
Total Diets

53

11

4

Total $\frac{32}{100}$

TABLE 4. Vitamin $B_{1}$ Content of Diets of Families of Wage Earners and Low-Salaried Clerical Workers.

Region, number of families

North Atlantic, 1394 white families

Pacific: 688 white families

East South Central: 426 white families

South: 284 Negro families

Sherman-Average American Diet (Cowgill p. 186)

American Family on Food Relief (Cowgill p. 194)

Assumed boderline for clinical beriberi

$\begin{array}{cccc}\begin{array}{c}\text { Weekly } \\ \text { expenditure per } \\ \text { food constituent }\end{array} & \begin{array}{c}\text { Thiamine } \\ \text { per day } \\ (\mu \mathrm{g})\end{array} & \begin{array}{c}\text { Calories } \\ \text { per } \\ \text { day }\end{array} & \begin{array}{c}\mathrm{T}(\mu \mathrm{g}) \\ \text { per } \\ \mathrm{Cal} .\end{array} \\ \$ 1.33-\$ 1.99 & 600 & 2550 & 0.235 \\ 2.00-2.66 & 735 & 2960 & .248 \\ 2.67-3.32 & 885 & 3310 & .276 \\ 3.33-3.99 & 945 & 3840 & .246 \\ 4.00-4.66 & 1200 & 4140 & .290 \\ 1.33-1.99 & 780 & 2570 & .303 \\ 2.00-2.66 & 840 & 3100 & .271 \\ 2.67-3.32 & 960 & 3660 & .262 \\ 3.33-3.99 & 1065 & 4140 & .257 \\ 4.00-4.66 & 1125 & 4340 & .259 \\ .67-1.32 & 495 & 2620 & .189 \\ 1.33-1.99 & 735 & 3050 & .241 \\ 2.00-2.66 & 855 & 3470 & .246 \\ 2.67-3.32 & 1095 & 3980 & .275 \\ .67-1.32 & 570 & 2450 & .232 \\ 1.33-1.99 & 840 & 3460 & .243 \\ 2.00-2.66 & 1180 & 4470 & .242 \\ 2.67-3.32 & 1260 & 4880 & .259 \\ & & & \\ & 900 & 2500 & .360 \\ & & & \\ & 600 & 2500 & .400 \\ & & & .250\end{array}$

276) doubtless contributing a somewhat greater element of safety than is apparent in these lower values.

Goldberger's classical studies on the nicotinic acid requirement of man are likewise of a survey nature. Frazier and Friedemann have recently re-evaluated these studies, including the dietary records of some 1863 human subjects ${ }^{30}$ and concluded on this basis that the human requirement for nicotinic acid, when other factors are present in good supply, is about $4 \mathrm{mg} /$ day, but may be as high as $7.5 \mathrm{mg} /$ day on a marginal diet high in corn products. Similarly, Williams ${ }^{31}$ studied the B vitamin content of mixed human diets known to be adequate for human nutrition, of a highly satisfactory animal ration, and of a rat carcass, and found them highly similar on an isocalorific basis, as is shown in Table 5 . He concluded, therefore, that these values probably indicate "safe" levels for a daily human intake. 
These values for a recommended intake obviously represent a much higher level than is arrived at for the requirement by other means. They are, however, not extravagant as an assessment for practical use in view of the great variation which may exist in individual needs (p. 273).

Table 5. Vitamin B Content of Various Materials (per 2500 Calories).

$\begin{array}{lllllllll} & \begin{array}{c}\text { Thi- } \\ \text { amine } \\ (\mathrm{mg})\end{array} & \begin{array}{c}\text { Nico- } \\ \text { tinic } \\ \text { Acid } \\ (\mathrm{mg})\end{array} & \begin{array}{c}\text { Ribo- } \\ \text { flavin } \\ (\mathrm{mg})\end{array} & \begin{array}{c}\text { Panto- } \\ \text { thenic } \\ \text { Acid } \\ (\mathrm{mg})\end{array} & \begin{array}{c}\text { Biotin } \\ (\mathrm{mg})\end{array} & \begin{array}{c}\text { Inosi- } \\ \text { tol } \\ (\mathrm{mg})\end{array} & \begin{array}{c}\text { Pyrid- } \\ \text { oxine } \\ (\mathrm{mg})\end{array} & \begin{array}{c}\text { Folic } \\ \text { Acid } \\ (\mathrm{mg})\end{array} \\ \begin{array}{l}\text { Mixed diet } \\ \text { Dog food }\end{array} & 3.6 & 40.1 & 3.67 & 11.2 & 0.25 & 987 & 1.77 & 1.39 \\ & 2.8- & 24- & 3.7- & 10- & 0.114- & 1170- & 1.13- & 0.66- \\ \begin{array}{l}\text { Rat carcass } \\ \text { Recommended } \\ \text { daily intake }\end{array} & 1.46 & 40 & 6.2 & 10.9 & 1.14 & 2909 & 1.86 & 0.94 \\ & 3.2 & 40 & 3.7 & 11 & 0.14 & 1000 & 1.5 & 1.0\end{array}$

Studies of Vitamin Excretion. A fourth approach to the assessment of requirements for $\mathrm{B}$ vitamins is the evaluation of vitamin excretion levels in terms of the $\mathrm{B}$ vitamin intake. Actually this represents a variation of a previous approach wherein the excretion is taken as the criterion of deficiency or sufficiency. Economizing processes in living tissues presumably work to retain and utilize essential nutrients when the requirement is not being met by the nutrition or when a so-called "tissue hunger" exists for some factor. When, however, the tissues have absorbed as much of a vitamin as they require, an organism will frequently excrete amounts of essential food constituents proportional to the intake. Melnick ana co-workers ${ }^{28}$ have utilized this latter principle extensively in biological assay work employing human subjects, and in the study of the biological availability and inactivation of certain B vitamins. More generally, however, test doses of a given vitamin are administered to a subject and, depending upon the portion excreted as compared with known values from individuals on an adequate diet, an estimate is made of whether a depletion exists and therefore whether a diet of known vitamin content meets the requirement. Still simpler but infinitely less satisfactory, one may attempt to determine whether the level of urinary excretion of a $B$ vitamin is within the range of values considered to be normal.

This general approach, like the others, has its drawbacks. Unquestionably the greatest errors have been due to the individual variability in the proportion of an arministered test dose which is excreted. Another source of question has been concerned with the validity of the general principle that the absorption capacity of the body is a true function of the requirement. In general, results obtained by this method agree well with other evaluations of requirements, although they tend to be somewhat higher. A third source of error, particularly in earlier studies, is in the measurement of inappropriate excretion products. Increased understanding of 
$B$ vitamin metabolism and of the chemical nature of excretion products has done much to remedy this situation (p. 365).

Johnson et $a l .^{32}$ have critically analyzed many of the technical factors involved in this type of study, devoting special attention to the thiamine, riboflavin and $\mathrm{N}^{\prime}$-methylnicotinamide content of urine. A comparison of fasting specimens, random specimens and samples after an oral loading test seemed to indicate that fasting urinary excretion studies may be far more accurate than studies using a loading test. While there is little doubt that random urine samples are valueless as compared with the fasting samples, it is apparent (p. 351) that the many factors involved in the storage and excretion of the $B$ vitamins have caused many workers to express grave doubt concerning the value of fasting urinary levels of $B$ vitamins in assessing dietary requirements. This doubt, aggravated by extensive individual variability, seems well founded, as judged by most recent studies. ${ }^{33,34}$ Dietary interrelationships may operate to make such errors even greater. Illustrative of this is the demonstration that very high nicotinamide intakes have been shown to increase thiamine excretion by as much as 70 per cent. ${ }^{35}$

In spite of these and other difficulties, much instructive information is obtainable in this manner. Studies on individuals whose vitamin intakes have been cut by 28 to 66 per cent from the normal levels show that the change was generally reflected in the urinary levels within a single week. In these studies, however, physical changes were not observable throughout the test period of five weeks. ${ }^{36}$ There can be little doubt therefore that when cautiously interpreted, results from urinary excretion studies may be of extreme value. It is possible to discuss in the limited space available only a few of the more recent applications of this method, principally as illustrative examples of the possibilities of this approach.

In an extended series of studies, Oldham and co-workers, on the basis of urinary excretion data obtained from urine samples from young women, ${ }^{37}$ have decided that the thiamine requirement is somewhat less than $1.0 \mathrm{mg} /$ day or $20 \mu \mathrm{g} / \mathrm{kg}$ body weight.

Michelson et al..$^{38}$ have studied in detail the problems inherent in assessing the level of thiamine nutrition by this means, and have pointed out several important considerations. The attainment of excretion equilibrium at a given intake level requires considerable time, and this was not realized in much earlier work. They found that a change in daily intake of thiamine is on an average only half reflected in the excretion in ten days. These authors studied both thiamine and pyramin * excretion over prolonged periods, and found large (threefold) variations between individuals in thiamine excretion at high levels of thiamine intake. They

* 2-methyl-4-amino-5-hydroxymethylpyrimidine. 
report that pyramin excretion increases exponentially with increases in thiamine intake, approaching linearity at normal levels, as contrasted with linear increases in thiamine excretion throughout the range studied. Moreover, pyramin excretion remains measurable at low intakes of thiamine, when thiamine excretion falls to essentially zero. For these reasons, pyramin would seem to be a better end product to study than thiamine.

Comparing the excretion technique with others, Berryman et al..$^{39}$ find that urinary excretion of $\mathrm{B}$ vitamins falls off rapidly following the change of men to a deficiency diet, but that fecal levels remain unchanged. Decline in physical and mental states are more gradual, and these states improve more slowly when the vitamin supply is increased than do the urinary excretion levels. The great individual variability in response was again noted by these workers. Still other studies ${ }^{40,41}$ indicate quite clearly that a decreased dietary intake is rather rapidly followed by a decrease in urinary output, followed only gradually by decreases in tissue content, and only much later by the manifestations of a clinical deficiency.

The most advantageous use of excretion studies in the assessment of vitamin requirements comes about in those cases in which, unlike thiamine, other methods of approach to the requirement are less feasible. The riboflavin requirement is an example of this situation. Hagedorn, ${ }^{42}$ in a study of prison inmates, found great variability in riboflavin excretion $(0.05-2.4 \mathrm{mg} / 24 \mathrm{hrs})$ and in the retention of test doses without apparent cause, and experienced further difficulty in obtaining useful results from fasting subjects due to the large errors in measuring such low riboflavin concentrations as were obtained. Despite the apparent advantages of dietary control, prison inmates have seldom been a source of entirely satisfactory data, and other workers have obtained better results with more satisfactory subject material. Oldham et al. concluded from a study of the riboflavin excretion of institutionalized children ${ }^{43}$ that $1.15-1.6 \mathrm{mg} /$ day of riboflavin met their nutritional requirements. Other workers, by studying excretion at different levels of intake and the per cent of a 3-mg test dose of riboflavin excreted in 24 hours at each level of intake, decided that $1.3-1.5 \mathrm{mg} /$ day is adequate for an intake of 2100-2300 Calories. ${ }^{44}$ Oldham et al., in their earlier studies, estimated that the riboflavin nutritional requirement was about $1 \mathrm{mg}$ /day or 0.50 mg per 1000 Calories. A summary of their results is given in Table 6 .

All these results on riboflavin agree well with the earlier observations of $\mathrm{R}$. D. Williams et al., ${ }^{46}$ but the requirements arrived at are less than those proposed by Sebrell et al. . $^{47}$ It is noteworthy that nearly all workers are in agreement that fecal riboflavin does not vary with the intake.

Briggs ${ }^{48}$ studied two subjects who had been pellagrins, and who were placed on a "corn-poor" diet containing about $2.4 \mathrm{mg}$ of nicotinic acid 
per day. Although urinary excretion tests suggested a niacin deficiency, only the mildest of clinical symptoms were observed. Najjar and coworkers ${ }^{49}$ in similar experiments maintained subjects on diets containing 1.5-2.0 mg of nicotinic acid per day and observed neither signs of nicotinic acid deficiency nor a reduction in $\mathrm{N}^{\prime}$-methylnicotinamide excretion, which would suggest depletion of body tissues. These authors attribute such survival on obviously low levels of nicotinic acid to intestinal synthesis. Cossandi, ${ }^{50}$ on the basis of loading tests with infants in which only 1 per cent recovery was obtained as compared with 5-10 per cent in adults, concluded that up to one year of age there exists a relative niacin deficiency, more particularly in breast-fed as compared with bottlefed infants.

TABLE 6. Percentage of the Average Total Riboflavin Intake and of the Increase in Average Intake Excreted at Each Dietary Level. ${ }^{45}$

$\begin{array}{rcccccc}\text { Period } & \begin{array}{c}\text { Total } \\ \text { Intake }\end{array} & \begin{array}{c}\text { Increase in } \\ \text { Intake }\end{array} & \begin{array}{c}\text { Total } \\ \text { Excretion }\end{array} & \begin{array}{c}\text { Increase in } \\ \text { Excretion }\end{array} & \begin{array}{c}\text { Total Intake } \\ \text { Excreted }\end{array} & \begin{array}{c}\text { Increase in } \\ \text { Intake } \\ \text { Excreted } \\ (\%)\end{array} \\ \text { I } & (\mu \mathrm{g}) & (\mu \mathrm{g}) & (\mu \mathrm{g}) & (\mu \mathrm{g}) & (\%) & \\ \text { II } & 1017 & & 113 & & 19 & 9 \\ \text { III } & 1234 & 417 & 150 & 37 & 15 & 52 \\ \text { IV } & 7171 & 517 & 263 & 113 & 21 & 69 \\ \text { V } & 1206 & & 4344 & 4081 & 61 & \end{array}$

Normal persons excrete about $2.3 \mu \mathrm{g}$ of folic acid per day in urine, and after oral doses of 5-16 mg, excrete about 28 per cent of the dose according to a recent study. In patients hospitalized for various causes, recoveries were much lower, however. There is therefore some reason to believe that an estimate of human folic acid requirements may be derived from such studies. ${ }^{51}$ In the case of some other factors, such a possibility seemingly does not exist. Thus, since biotin excretion nearly always exceeds the intake $^{52}$ (due to intestinal synthesis) accurate results seem improbable. In other cases where intestinal synthesis is of a relatively low order, the error so introduced is not as serious an objection to the method. A more detailed consideration of many of the factors involved in the validity of these studies occurs in a later section on B vitamin excretion (p. 364).

Studies of Levels in Various Biological Materials. Closely related to those studies in which the $\mathrm{B}$ vitamin requirement has been assessed on the basis of excretory levels are those studies of vitamin tissue concentrations which contribute to an understanding of $\mathrm{B}$ vitamin requirements. This latter type of study, however, has been very little pursued, to some extent because of the uncertainty regarding the variable nature of tissue storage, but in most cases because of the difficulty in obtaining suitable material.

Studies of fecal vitamin content have proved to be almost totally valueless, because of both the influence of bacteria directly and the influence 
of other dietary constituents on bacteria. Thus, fecal elimination of most of the $\mathrm{B}$ vitamins in rats is more of a criterion of the dietary protein level than of any other factor, ${ }^{53}$ this being particularly true for biotin, pantothenic acid, and nicotinic acid. Protein levels likewise influence hepatic storage of $\mathrm{B}$ vitamins, making the liver an uncertain tissue for study. On diets in which factors other than the $\mathrm{B}$ vitamins are held constant, it has been found ${ }^{54}$ that the thiamine content of feces is quite constant and independent of the intake. Similarly, the biotin in the combined urine and feces of humans on low, moderate, and high biotin intakes, respectively, is about nine, three, and one to five times the dietary level, or approximately constant. ${ }^{55}$

Milk which is generally quite available for study is unfortunately also influenced in its B vitamin content by a variety of factors other than dietary vitamin levels ${ }^{55}$ (p. 347), and has not as yet proved of great value in this regard. It has been shown, however, that there is some correlation between the thiamine levels in human blood and in milk, other factors being constant, ${ }^{57}$ and that a daily intake of $1.5 \mathrm{mg}$ of thiamine produces a level of about $20 \mu \mathrm{g}$ per cent in the milk. Moreover, both the thiamine and riboflavin levels in milk seem to vary with urinary excretion, indicating some possibility of studies of the requirement during lactation. ${ }^{58}$ This type of study has not, however, been extensive as yet.

A variety of other materials has been found somewhat more satisfactory for assessing tissue vitamin sufficiency. It seems well established that pork generally reflects the dietary thiamine level of the hog, but studies have not as yet been reported on the assessment of porcine thiamine requirements by such a method. ${ }^{59}$ This technique of assessing vitamin requirements, like the urinary excretion method, lends itself best to those cases where more direct means are not practical.

A typical example of this approach is the work of Czaczkes and Guggenheim ${ }^{60}$ on the riboflavin requirements of the rat. On diets containing no riboflavin or $5 \mu \mathrm{g}$ of riboflavin per day, the riboflavin content of liver, kidney, muscle, and urine steadily decreases. On a level of $7.5 \mu \mathrm{g}$ per day, however, balance is maintained, while on $10 \mu \mathrm{g}$, the tissue content increases. Thus, it seems logical to conclude that for these 50 -gm rats, the riboflavin requirement is about $7.5 \mu \mathrm{g}$ per day. These workers point out that the blood content remains constant, while the kidney content does not show an excess, but readily reflects a deficit. Both liver and muscle, however, were good indicators of the nutritional status.

Not only must the validity of the particular tissue as an indicator be established as above, but while employing any tissue, all other dietary constituents must be kept constant. Thus, it has been found that vitamin $\mathrm{C}$ controls riboflavin storage to a marked degree, ${ }^{61}$ as does protein. More- 
over, the riboflavin level of the liver is a function not only of dietary protein, but also of methionine and cystine. ${ }^{62}$

Interesting results concerning the riboflavin requirements of fowls have been obtained from studies employing eggs. Jackson and coworkers ${ }^{63}$ have found that the highest level of dietary riboflavin that would affect the riboflavin concentration in hens' eggs was from 1400 to $1600 \mu \mathrm{g}$ per pound of feed. This is about $330 \mu \mathrm{g}$ per cent and very close to the requirement of hens as assessed by other means (p. 327).

In order to maintain a normal blood level of niacin in humans $(0.6-0.65$ mg per cent), it has been found that a daily intake of from 12 to $16 \mathrm{mg}$ is required. Since the blood level of nicotinic acid varies with the dietary intake, this value may be taken as an estimate (albeit a high one) of the average human requirement. ${ }^{64}$ In white rats the liver and spinal fluid levels of nicotinic acid have also been shown to be a function of niacin intake, ${ }^{65,66}$ but also to be inversely related to the thiamine intake. Oldham ${ }^{39}$ has concluded that urinary and fecal niacin excretion are independent of the intake, each being about $1 \mathrm{mg}$ per day in the young women studied.

Urinary and fecal pantothenic acid, however, have been found by Oldham et al. ${ }^{37}$ to vary with the dietary intake. Pearson et al. found that in the chick, both the blood and muscle tissue reflected the dietary supply of pantothenic acid, although the liver did not. ${ }^{67}$ Dietary levels of pantothenic acid greater than those required for "adequate nutrition," however, do not further increase the muscle pantothenic acid level in the chick. Silber ${ }^{68}$ similarly finds that in dogs the blood pantothenic acid level reflects the nutritional supply.

With regard to vitamin $\mathrm{B}_{6}$, studies have shown that the level in rat liver is independent of the level of intake, for dietary levels greater than $25 \mu \mathrm{g}$ per day, thereby suggesting this as the dietary requirement for the rat. The value so obtained does not vary greatly from other assessments of this requirement.

Unquestionably, studies of the kind mentioned will be more numerous in the future. Techniques such as that recently developed for the determination of riboflavin in very small amounts of serum ${ }^{68 a}$ may eventually make this approach widespread in nutritional survey work. Moreover, by similar processes it will be possible eventually to study the nutritional requirements of individual animal tissues grown in vitro and thus arrive at a more fundamental understanding of B vitamin requirements. Such studies, already under way, ${ }^{69}, 70$ may well be one of the great advances in this field within the immediate future.

Natural Selection Studies. It has been reported from time to time that among lower animals at least there exists an instinctive tendency to 
select diets rich in some factor which may be deficient in their nutrition, and this would seem to provide still another approach to the assessment of the B vitamin requirements of certain species. However, because of the imperfect understanding of the phenomena involved and due to the relatively recent inception of this type of study ${ }^{71}$ little progress has been made with regard to this particular aspect of self-selection diets. Scott et al. ${ }^{72}, 73$ have shown clearly, however, that in rats fed appropriately deficient diets (but not in normal controls) appetites are developed for thiamine, riboflavin, and the vitamins $\mathrm{B}_{6}$, but not for pantothenic acid. Much progress in this field will undoubtedly be made in the years immediately ahead as our understanding of the physiological nature of specific hungers is further developed. (See p. 433.)

The Use of "Anti-Vitamins." Finally, it is worthy of mention that the new and rapidly developing study of anti-vitamins has contributed in some degree to our knowledge of $\mathrm{B}$ vitamin requirements and seems to provide an unique approach to their study. Later sections of this monograph will consider in detail how avidin has made possible the elucidation of the biotin requirement of various species (p. 428), how live yeast has been used to produce thiamine depletion (p. 291), and how the sulfa drugs have been similarly employed (p. 298). It seems possible that the requirements for as yet unidentified members of the $B$ vitamin group may await studies of this kind. Challenging in its implications for future possibilities along these lines is the recent work involving lyco-marasmine. Plattner and Clausen-Kaas ${ }^{74}$ isolated from Fusarium lycospersici Sacc. a substance, "lyco-marasmine," which is responsible for the wilting of

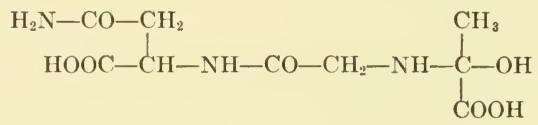

Lycomarasmine (after Woolley)

plants on which the Fusarium is parasitic. Analysis of this substance has indicated the probability of a tripeptide nature involving serine, glycine and aspartic acid. Strepogenin (p. 15), a possible new member of the B vitamin group, is considered to be similar in structure, and because of this it was thought that an anti-vitamin relationship might exist. This is apparently so, since strepogenin reverses the toxic action of lycomarasmine, and it therefore seems likely that strepogenin is important in the higher plants (as it is in bacteria). This conclusion is based on reasoning by methods analogous to those used in explaining the reversal of sulfa drug bacterial inhibition with $p$-aminobenzoic acid (Chapter IIID).$^{75,76}$ 
Thus, we find that extensive methodology has accrued about the assessment of B vitamin requirements. No single method as yet developed is perfect or free from just criticism. The extent to which more accurate methods of assessment are required is in itself questionable in view of the many factors influencing the requirements. Since the very nature of the nutritional $\mathrm{B}$ vitamin requirement is largely a mosaic of these other factors, it seems quite essential to consider them in some detail in the following chapter.

\section{Bibliography}

1. Snell, E. E., and Guirard, B. M., Proc. Natl. Acad. Sci. U. S., 29, 66-73 (1943).

2. Nutrition Revs., 3, 293-5 (1945).

3. Keys, A., Nutrition Revs., 3, 95 (1945).

4. Keys, A., Henschel, A. F., Michelsen, O., and Brozek, J. M., J. Nutrition, 26, 399-415 (1943).

5. Keys, A., Nutrition Revs., 5, 129-31 (1947).

6. Dann, W. J., and Darby, W. J., Physiol. Revs., 25, 326-46 (1945).

7. Leitch, I., Nutrition Abstracts \& Revs., 11, 509-21 (1942).

8. Nutrition Revs., 4, 8-11, 39-41 (1946).

9. Sinclair, H. M., Vitamins and Hormones, 6, 102-62 (1948).

10. Pett, L. B., Morrell, C. A., and Hanley, F. W., Can. J. Pub. Health, 36, 232-9 (1945).

11. Clandinin, D. R., Poultry Sci., 25, 223-31 (1946).

11a. Schweigert, B. S., German, H. L., and Garber, M. J., J. Biol. Chem., 174, 383-4 (1948).

12. Cowgill, G. R., "The Vitamin B Requirement of Man," Yale University Press, New Haven, Conn., 1934, p. 183.

13. Cowgill, G. R., "The Vitamins, A Symposium," American Medical Association, Chicago, Ill., 1939, p. 232.

14. Williams, R. R., and Spies, T. D., "Vitamin $B_{1}$ and Its Use in Medicine," The Macmillan Co., New York, N. Y., 1939, p. 95.

15. Robertson, E. I., Daniel, L. J., Farmer, F. A., Norris, L. C., and Heuser, G. F., Proc. Soc. Exptl. Biol. Med., 62, 97-101 (1946).

16. Daniel, L. J., Farmer, F. A., and Norris, L. C., J. Biol. Chem., 163, 349-50 (1946).

17. Hutchings, B. L., Oleson, J. J., and Stokstad, E. L. R., J. Biol. Chem., 163, 447 (1946).

18. Green, J., and Brunschwig, A., Proc. Soc. Exptl. Biol. Med., 61, 348-50 (1946).

19. Nutrition Revs., 4, 159-60 (1946).

20. Smith, S. G., Science, 100, 389-90 (1944).

21. Smith, S. G., and Lester, T. E., Am. J. Physiol., 144, 175-88 (1945)

22. Ruegamer, W. R., Elvehjem, C. A., and Hart, E. B., Proc. Soc. Exptl. Biol. Med., 61, 234-8 (1946).

23. Smith, S. G., Proc. Soc. Exptl. Biol. Med., 63, 339-41 (1946).

24. Kruse, H. D., Bessey, O. A., Jolliffe, N., McLester, J. S., Tisdall, F. F., Wilder, R. M., and Sydenstricker, V. P. W., Arch. Internal Mcd., 74, 258-79 (1944).

25. Muench, H., "Proceedings of a Research Conference on the Relationship of Nutrition to Public Health," The Nutrition Foundation, New York, N. Y., 1943 , p. 45.

26. Hawkins, W. W., and Barsky, J., Science, 108, 284-6 (1948).

27. Nutrition Revs., 3, 99-100 (1945).

28. Melnick, D., Hochberg, M., and Oser, B. L., J. Nutrition, 30, 67-88 (1945). 
29. Baker, A. Z., Wright, M. D., and Drummond, J. C., J. Soc. Chem. Ind. London, 56, 191-4T (1937).

30. Frazier, E. I., and Friedemann, T. E., Quart. Bull. Northwestern Univ. Med. School, 20, 24-48 (1946).

31. Williams, R. J., J. Am. Med. Assoc., 119, 1-3 (1942).

32. Johnson, R. E., Henderson, C., Robinson, P. F., and Consolazio, F. C., J. Nutrition, 30, 89-98 (1945).

33. Berryman, G. H., Henderson, C. R., French, C. E., Goorley, J. T., Harper, H. A., Pollock, H., and Harkness, D. M., Am. J. Physiol., 145, 625-31 (1946).

34. D'Agostino, L., Boll. soc. ital. biol. sper., 20, 628-9 (1945).

35. Malaguzzi-Valeri, C., and Conese, G., Boll. soc. ital. biol. sper., 20, 613-14 (1945).

36. Cogswell, R. C., Berryman, G. H., Henderson, C. R., Denko, C. W., Spinella, J. R., Friedemann, T. E., Ivy, A. C., and Youmans, J. B., Am. J. Physiol., 147, 39-48 (1946).

37. Oldham, H. G., Davis, M. V., and Roberts, L. J., J. Nutrition, 32, 163-80 (1946).

38. Michelsen, O., Caster, W. O., and Keys, A., J. Biol. Chem., 168, 415-31 (1947).

39. Berryman, G. H., Henderson, C. R., Wheeler, N. C., Cogswell, R. C., Jr., Spinella, J. R., Grundy, W. E., Johnson, H. C., Wood, M. E., Denko, C. W., Friedemann, T. E., Harris, S. C., Ivy, A. C., and Youmans, J. B., Am. J. Physiol., 148, 618-47 (1947).

40. Berryman, G. H., and Henderson, C. R., Am. J. Physiol., 149, 142-8 (1947).

41. Berryman, G. H., French, C. E., Baldwin, H. R., Bell, S. L., and Henderson, C. R., Am. J. Physiol., 149, 254-63 (1947).

42. Hagedorn, D. R., Kyhos, E. D., Germek, O. A., and Sevringhaus, E. L., J. Nutrition, 29, 179-89 (1945).

43. Oldham, H., Roberts, L. J., and Young, M., J. Pcdiat., 27, 418-27 (1945).

44. Brewer, W., Porter, T., Ingalls, R., and Ohlson, M. A., J. Nutrition, 32, 583-96 (1946).

45. Davis, M. V., Oldham, H. G., and Roberts, L. J., J. Nutrition, 32, 143-61 (1946).

46. Williams, R. D., Mason, H. L., Cusik, P. L., and Wilder, R. M., J. Nutrition, 25, 361-77 (1943).

47. Sebrell, W. H., Butler, R. E., Wooley, J. G., and Isbell, H., Pub. Health Repts., 56, 510-19 (1941).

48. Briggs, A. P., Singal, S. A., and Sydenstricker, V. P., J. Nutrition, 29, 331-9 (1945).

49. Najjar, V. A., Holt, L. E., Jr., Johns, G. A., Medairy, G. C., and Fleischmann, G., Proc. Soc. Exptl. Biol. Med., 61, 371-4 (1946).

50. Cossandi, E., Boll. soc. ital. biol. sper., 16, 703-6 (1941).

51. Steinkamp, R., Shukers, C. F., Totter, J. R., and Day, P. L., Proc. Soc. Exptl. Biol. Med., 63, 556-8 (1946).

52. Gardner, J. Parsons, H. T., and Peterson, W. H., Am. J. Med. Sci., 211, 198-204 (1946).

53. Wright, L. D., and Skeggs, H. R., Proc. Soc. Exptl. Biol. Med., 63, 327-33 (1946).

54. Alexander, B., and Landwehr, G., J. Clin. Invest., 25, 287-93 (1946).

55. Gardner, J., Parsons, H. T., and Peterson, W. H., Arch. Biochem., 8, 339-48 (1945).

56. Nutrition Revs., 4, 134-7 (1946).

57. Schultz, F. W., Semana méd. Buenos Aires, 50, 689-91 (1946).

58. Roderuck, C. E., Williams, H. H., and Macy, I. G., J. Nutrition, 32, 249-65 (1946).

59. Heinemann, W. W., Ensminger, M. E., Cunha, T. J., and McCulloch, E. C., J. Nutrition, 31, 107-25 (1946).

60. Czaczkes, J. W., and Guggenheim, K., J. Biol. Chem., 162, 267-74 (1946).

61. Cimino, S., Boll. soc. ital. biol. sper., 22, 291-3 (1946).

62. Riesen, H. W., Schweigert, B. S., and Elvehjem, C. A., Arch. Biochem., 10, 387-95 (1946). 
63. Jackson, S. H., Drake, T. G. H., Slinger, S. J., Evans, E. V., and Pocock, R., J. Nutrition, 32, 567-81 (1946).

64. Gounelle, H., Vallette, A., and Raoul, Y., Compt. rend. soc. biol., 139, 16-17 (1945).

65. Malaguzzi-Valeri, C., and Neri, F., Boll. soc. ital. biol. sper., 17, 644-5 (1942).

66. Malaguzzi-Valeri, C., and Neri, F., Boll. soc. ital. biol. sper., 17, 645-6 (1942).

67. Pearson, P. B., Melass, V. H., and Sherwood, R. M., J. Nutrition, 32, 187-93 (1946).

68. Silber, R. H., J. Nutrition, 27, 425-33 (1944).

68a. Burch, H. B., Bessey, O. A., and Lowry, H., J. Biol. Chem., 175, 457-70 (1948).

69. Nutrition Revs., 5, 189-90 (1947).

70. Hetherington, D. C., Proc. Soc. Exptl. Biol. Med., 62, 312-15 (1946).

71. Scott, E. M., J. Nutrition, 31, 397-406 (1946).

72. Scott, E. M., and Quint, E., J. Nutrition, 32, 285-91 (1946).

73. Scott, E. M., Smith, S. J., and Verney, E. L., J. Nutrition, 35, 281-6 (1948).

74. Plattner, P. A., and Clauson-Kaas, N., Helv. Chim. Acta, 28, 188-95 (1945).

75. Plattner, P. A., and Clauson-Kaas, N., Experientia, 1, 195-6 (1945).

76. Woolley, D. W., J. Biol. Chem., 176, 1291-1308 (1948). 


\section{Chapter IIC}

\section{FACTORS INFLUENCING B VITAMIN REQUIREMENTS}

There are few aspects of an individual, his nutrition, or his environment that do not to an appreciable extent influence his B vitamin requirements. These effects may be exerted by (a) varying the actual physiological requirement, (b) varying the proportion of the total vitamin intake which may be utilized, or (c) varying the amount of vitamin supplied by intestinal flora. The ensuing discussion of these effects proceeds in that order. To consider even a major portion of the factors involved would be neither possible nor practical in this volume. Rather, it seems expedient to consider briefly those major factors which have proved to be the most important in influencing $\mathrm{B}$ vitamin dietary requirements. It is impractical to do more than briefly delineate these relationships at this time, although a more fundamental explanation of their basis in many cases is provided in the succeeding chapters.

\section{Factors Influencing the Physiological Requirement for the B Vitamins}

Species and Strain Variability. Thiamine was the first of the B vitamins to be discovered and thoroughly studied, and consequently knowledge of the variability of its requirement among different species is perhaps the most complete of all. Since many of the more recently discovered members of the B vitamin family follow the same trends as does thiamine in this regard, it is advantageous to consider vitamin $\mathrm{B}_{1}$ in greater detail from this standpoint than would otherwise be the case.

TABLE 7. Relationship of the Thiamine Requirement to the Size of a Species.

\begin{tabular}{|c|c|c|c|c|c|c|}
\hline \multirow[b]{2}{*}{ Species } & \multirow{2}{*}{$\begin{array}{l}\text { Average } \\
\text { weight } \\
\text { (gms) }\end{array}$} & \multirow{2}{*}{$\begin{array}{c}\text { Basal } \\
\text { Metabolic } \\
\text { rate } \\
\text { (Cal./kg/day) }\end{array}$} & \multirow{2}{*}{$\begin{array}{l}\text { Days } \\
\text { required for } \\
\text { polyneuritis } \\
\text { to develop }\end{array}$} & \multicolumn{3}{|c|}{$\begin{array}{l}\text { Thiamine required } \\
\text { to prevent polyneuritis }\end{array}$} \\
\hline & & & & $\mu \mathrm{g} /$ day/animal & $\begin{array}{l}\mu \mathrm{g} / \mathrm{day} / \mathrm{kg} \\
\text { body wt. }\end{array}$ & $\begin{array}{c}\mu \mathrm{g} / \mathrm{gm} \\
\text { food }\end{array}$ \\
\hline $\begin{array}{l}\text { Tirbolium } \\
\text { confusum }\end{array}$ & & & & & & \\
\hline $\begin{array}{l}\text { confusum } \\
\text { Rice bird }\end{array}$ & 0.5 & $\overline{250}$ & $\overline{9-12}$ & $\overline{2}$ & 66 & 4 \\
\hline Mouse & 20 & 160 & $15-20$ & 1 & 50 & 0.5 \\
\hline Rat & 125 & 80 & $35-60$ & 2 & 16 & 0.4 \\
\hline Pigeon & 400 & 100 & $20-30$ & 9 & 22.5 & 0.7 \\
\hline Chick & 300 & 50 & 30 & - & $\longrightarrow$ & 0.7 \\
\hline Dog & 8000 & 25 & & 64 & 8 & 0.35 \\
\hline Man & 60,000 & 25 & - & 600 & 10 & 0.60 \\
\hline
\end{tabular}

We have previously mentioned (p. 246) Cowgill's extensive study of species requirements and his conclusion that smaller species require a greater amount of thiamine per unit of body weight than do larger ones. 
To a large extent this is probably due to the increased relative food intake of smaller animals, which is in turn related to the greater body surface and basal metabolic rate of small animals per unit of body weight. The data in Table $7^{1}$ are presented only to illustrate these relationships, and are not proposed as absolute values in any case.

Beyond doubt, similar tabulations might be made for the other B vitamins (p. 319).

Strain differences may be as marked as the differences between species and a large number have been reported. For instance, Light and Cracas ${ }^{2,3}$ compared the thiamine requirements of three different strains of rats and found indications of considerable variation in the requirement. Their data are summarized in Table 8 .

TABLE 8. Thiamine Requirements of Three Strains of Rats. Average growth in grams per rat for a five-week test period

$\begin{array}{cccc}\text { Strain } & 2 \mu \mathrm{g} \text { Thiamine/rat/day } & 4 \mu \mathrm{g} \text { Thiamine/rat/day } & \begin{array}{c}\text { On level of } \\ 8 \mu \mathrm{g} \text { Thiamine/rat/day }\end{array} \\ 1 & 33.3 \pm 1.9 & 52.8 \pm 2.3 & \\ 2 & 29.8 \pm 3.38 & 27.0 \pm 1.04 & 52.9 \pm 2.8\end{array}$

Lamoureux and Hutt ${ }^{4}$ developed strains of white leghorn chickens which gained 50 per cent more in weight than other strains on a given thiamine intake. Engel ${ }^{5}$ similarly obtained strains of rats varying broadly in their choline requirements. On a given dietary intake one strain suffered 1.5 per cent deaths as contrasted with 42.4 per cent for the other group. The females of the former strain had an incidence of kidney hemorrhages of 19.7 per cent as compared with 93.9 per cent for the latter group. Lucas, Heuser, and Norris, ${ }^{6}$ in their studies of chick nutrition, found that Red Rock cross chicks require as much as 20 times the vitamin $\mathrm{B}_{6}$ levels in their diet as is required by other strains such as White Leghorns in order to prevent a severe vitamin $B_{6}$ deficiency. Similarly, Ershoff ${ }^{7}$ has found that while most rats do not require nicotinic acid, one strain did not nurse their young while on a niacin deficient diet, although they appeared otherwise normal. The report of Rhoads et al. ${ }^{8}$ that there is no difference in the response of colored and white children to normal and vitamin supplemented diets should not be interpreted as meaning that differences in vitamin requirements do not exist between human races, although there is little information available bearing upon this nutritionally important question.

Variations with Age and Weight. Mention has previously been made (p. 247) of Cowgill's conclusion that the thiamine requirement varies within a species according to the five-thirds power of the body weight. This has been disputed, ${ }^{1}$ and it is at present felt that there is no general relationship between the thiamine requirement and the age or weight of 
an individual, except as these factors influence the calorific intake. Thus, for a given species, the requirement expressed as the vitamin-to-calorie ratio is independent of the weight of the individual. General considerations would indicate that this is similarly true for the other B vitamins, and it is possible that this constitutes a distinguishing characteristic of the B group.

Unfortunately, little work has as yet been done on the effects of age, as distinguished from weight, on B vitamin requirements. Rafsky and Newman ${ }^{9,10}$ have studied the niacin requirement of the aged, and more recently their thiamine excretion. ${ }^{11} \mathrm{~A}$ study was made of 31 persons, 14 men and 17 women, ranging from 65 to 81 years of age and on adequate thiamine intakes (0.51-1.11 mg/day). Forty-five per cent of the subjects were found to excrete less than $50 \gamma$ per day of thiamine, which is considerably below the generally accepted normal value. Despite the fact that a similar percentage of the group had a low gastric hydrochloric acid secretion and six had total achlorhydria, this was not correlatable with the low thiamine excretion. Mills et al. ${ }^{12}$ have recently shown that the thiamine requirement of adult rats per gram of food increases greatly with old age and increasing weight. Since in the aged the caloric requirements may be much less than in younger individuals, these would seem to be findings of great significance. The presumption that in old age the efficiency of vitamin utilization is greatly curtailed would seem to be of considerable theoretical interest to the growing field of geriatrics. ${ }^{12 a}$

In the extremely young, the situation is somewhat more lucid. In children from one to ten years there is a gradual increase in caloric requirement with age, and the $\mathrm{B}$ vitamin requirements parallel this. ${ }^{13}$ This is not to say that extraneous factors may not influence the requirement in young individuals and then become insignificant later. Thus, Schweigert et al. ${ }^{14}$ found that young mice were much more sensitive to the influence of protein levels on the niacin requirement than were older ones. A variety of similar observations will be discussed at later points in our studies. Suffice it to say that much of our knowledge of B vitamin requirements has been derived from experiments upon adults, and much remains to be learned before this can safely be extrapolated to the very young and the very old.

Influence of Sex. The influence of sex on the B vitamin requirements, so far as is now known, is exerted by virtue of the known differences in basal metabolism and caloric intake between the sexes, and the increased requirement during reproduction. Up to five years, the average caloric intakes of boys and girls are the same, and this appears true of the B vitamin requirements also. Subsequently, however, boys have a higher caloric intake and therefore a higher requirement for B vitamins. ${ }^{13}$ Here 
again, however, the eomplete picture is not available. For example, it is not entirely clear why the administration of higher levels of choline produces a considerable growth increase in female turkeys at 24 weeks, but not in females at 10 weeks, nor in male turkeys at all. ${ }^{55}$ Morris, Palmer, and Kennedy, in studying the relationship of efficiency of food utilization to inheritance in rats, ${ }^{16}$ found that there were appreeiable sex differences, the average female effieiency being 70 per cent greater than that for the male during the six-week study period. It seems likely, however, that present advanees in our understanding of the effect of the sex hormones on metabolism in general, and in particular upon protein metabolism, may ultimately serve to clarify certain of these relationships.

Influence of Occupation. While it is commonly stated that persons engaged in hard physical labor require an increased amount of vitamins in their nutrition, there is little to support so sweeping a statement. ${ }^{17}$ Nevertheless, the effect of work in increasing the caloric requirement does undoubtedly bring about inereased $\mathrm{B}$ vitamin requirements (but probably not increased requirements for other vitamins), although this increase may not be as great as for the ealorie requirement. As contrasted with such hard physical labor, Forbes ${ }^{18}$ has pointed out that emotionally tiring work performed by the average industrial worker is completely unstudied in regard to its effect on $\mathrm{B}$ vitamin requirements. During recent years, as our understanding of nutritional values has improved, there has been in the United States a tendeney for the calorific value of diets to rise. ${ }^{13}$ Concurrently there has therefore been an increase in $\mathrm{B}$ vitamin requirements, but there is little to indicate to what extent the increased pace of life in these same years has influenced our nutritional vitamin requirements.

In occupations involving such extreme physieal exertion that perspiration is excessive, there is some possibility that increased vitamin exeretion affects the $B$ vitamin requirement. This possibility is considered at greater length in the paragraphs that follow.

Effects of Climate. Throughout the war years there appeared a variety of conflicting reports with regard to the effects of elimate on vitamin requirements. Many of these were particularly concerned with the thiamine requirement, which probably serves as an excellent criterion for the problem in question. Since in tropical elimates there is a decreased caloric requirement, ${ }^{19}$ it might be anticipated that there would be deereased $\mathrm{B}$ vitamin requirements. Despite this, Mills et al. ${ }^{20,21}$ found that rats have increased thiamine requirements at higher temperatures, and Sarett and Perlzweig ${ }^{22}$ later extended these studies by measuring the tissue thiamine in rats at different temperatures. Edison, however, found that rats at $90^{\circ} \mathrm{F}$ and 70 per cent relative humidity required no more 
thiamine than those at $72^{\circ} \mathrm{F}$ and 50 per cent relative humidity (and perhaps less) for normal growth. ${ }^{23}$

A solution to this dilemma came with the study of Kline, Friedman and Nelson, ${ }^{24}$ wherein the basal diets were thiamine-free and the thiamine dosage was administered separately. Therefore, thiamine intake was not a function of food intake, as it had been in the previous studies. The rats were allowed to develop polyneuritis and then a dose of thiamine hydrochloride was given and an observation made of the length of time that protection was afforded. It was found by this method that $6 \mu \mathrm{g}$ of thiamine hydrochloride protected a rat for an average of 8.7 days of $78^{\circ} \mathrm{F}$ and 12.4 days at $85^{\circ} \mathrm{F}$. In a second experiment a series of ten rats underwent four successive depletion and treatment periods. The results are summarized in abbreviated form in Table 9.

TABLE 9. Effect of Temperature on the Thiamine Requirement of Rats.

$\begin{array}{ccccc}\text { Experiment } & \text { Period } & \begin{array}{c}\text { Dose of thiamine } \\ \text { hydrochloride }(\gamma)\end{array} & \begin{array}{c}\text { Temperature } \\ \left({ }^{\circ} \mathrm{F}\right)\end{array} & \begin{array}{c}\text { Days of } \\ \text { protection }\end{array} \\ 1 & - & 6 & 78 & 8.7 \\ 2 & 1 & 6 & 85 & 12.4 \\ & 2 & 3 & 78 & 9.1 \\ & 3 & 6 & 90 & 11.6 \\ & 4 & 3 & 78 & 9.9\end{array}$

It was found, moreover, that with a given daily dietary intake of thiamine, there was more rapid growth of rats at $90^{\circ} \mathrm{F}$ than at $78^{\circ} \mathrm{F}$. Earlier workers were unable to arrive at this conclusion because the rats used were receiving optimal amounts of thiamine, under which conditions the food intake, and therefore the growth response, were controlled by the environmental temperature and could not therefore be related to the thiamine intake or requirement.

Mills et $a l .{ }^{25}$ point out nevertheless that since vitamin intake is normally a function of the amount of food eaten, their conclusions are still significant. Extending their data to chicks, they have found the polyneuritis threshold level is $1 \mathrm{mg} / \mathrm{kg}$ diet at $70^{\circ} \mathrm{F}$ and $3 \mathrm{mg} / \mathrm{kg}$ at $90^{\circ} \mathrm{F}$. At these temperatures no differences were found in the chick requirements for folic acid, niacin, pyridoxine, or choline. (The choline requirement apparently varies with the temperature for rats.) It is thus apparent that the previously conflicting evidence regarding the temperature effect is in reality in no conflict at all, and that the question depends upon whether the thiamine requirement or the amount actually consumed is under consideration.

Despite the obvious and acknowledged importance of these findings, it has been pointed out that man differs from the rat to a considerable degree in his mechanism for temperature regulation, and that data per- 
fectly valid for fur-bearing animals are not always as significant when applied to man for this reason. Extensive studies have therefore been made upon the increased excretion of $\mathrm{B}$ vitamins in sweat.

Spector, Hamilton, and Mitchell ${ }^{26}$ have found that an increase in relative humidity from 65 to 92 per cent at $32.2^{\circ} \mathrm{C}$ produces an increase in the average net loss of body weight of 176 per cent, but an increase of 221 per cent in the average dermal excretion of pantothenic acid by humans. When the temperature was increased from 28.9 to $38.3^{\circ} \mathrm{C}$, the hourly dermal excretion similarly increased from 5.1 to $27.7 \mu \mathrm{g}$, and the total urinary and dermal excretion was increased 11.6 per cent. Johnson, Mitchell and Hamilton ${ }^{27}$ made similar studies on inositol excretion in sweat, and found that it increased from $27 \mu \mathrm{g} /$ hour under comfortable conditions to $118 \mu \mathrm{g} /$ hour under hot, moist conditions. The general opinion at present resulting from these studies is that $\mathrm{B}$ vitamin losses in sweat under tropical conditions are quite small as compared to the normal urinary output, and that there is thus considerable support for the belief that tropical climates do not increase $B$ vitamin requirements in humans.

With regard to the other extreme of climate, there has been less actual study, perhaps because no one has as yet seriously questioned the obviously increased requirement necessary in cold climates to support the increased caloric requirement. ${ }^{27 a}$ Johnson and Kark ${ }^{28}$ have presented data showing a linear relation between voluntary caloric intake in men (American soldiers), and the environmental temperature, the range being from $92^{\circ} \mathrm{F}$ and 3100 Calories to $-30^{\circ} \mathrm{F}$ and 4900 Calories! Studies have shown ${ }^{29}$ that a nutritional vitamin intake greater than that normally required is without effect in enhancing the ability of men to withstand the harmful effects of repeated exposure to cold climates.

Effects of Pregnancy and Lactation. Extensive studies have been made of the effects of pregnancy and lactation on vitamin requirements, and there is no doubt that under these circumstances there is a considerable increase in the required dietary level of $\mathrm{B}$ vitamins. Thus, Williams ${ }^{30}$ points out that in Manila the majority of women of the poorer classes show signs of deficiency during the child-bearing age. In many of these same regions, moreover, infantile beriberi is widespread. To a large extent at least this is due to the increased metabolic rate in pregnancy, and to lactation. Since the total metabolism has been shown to be equal to the metabolism of the mother plus the metabolism of the fetus, it might be anticipated that the thiamine requirement under such conditions could be calculated. There is not at present sufficient evidence to assess the validity of such a process, however.

Siddell and Mull ${ }^{31}$ have made a study of urinary excretion in a group of 42 pregnant women. Their results are shown in Table 10. 
All the patients in this study seemed quite normal and gave birth to normal and well-nourished children. Since normal adults on well-balanced diets containing about $0.86 \mathrm{mg}$. of thiamine per day excrete more than $200 \mu \mathrm{g}$ of thiamine per day in the urine, ${ }^{32}$ it would seem from this study that there is little justification for the widespread vitamin supplementation of the diets of pregnant women. Beyond this, however, there is little information on the problem at hand, since the women were all on ample diets and it is not possible to say how much their food intake varied during the course of pregnancy.

\begin{tabular}{|c|c|c|c|}
\hline Trimester & $\begin{array}{c}\text { Group I } \\
\text { Well-balanced } \\
\text { diet only }\end{array}$ & $\begin{array}{c}\text { Group II } \\
\text { Same diet }+0.75 \mathrm{mg} \\
\text { thiamine } / \text { day }\end{array}$ & $\begin{array}{c}\text { Group III } \\
\text { Same diet }+1.50 \mathrm{mg} \\
\text { thiamine } / \text { day }\end{array}$ \\
\hline 1 & 286 & 428 & $\overline{039}$ \\
\hline 2 & 263 & 620 & 932 \\
\hline 3 & 249 & 483 & 1131 \\
\hline
\end{tabular}

Kennedy and Palmer, ${ }^{33}$ from studies on sows and on rats on egg-white diets, have concluded that biotin is needed early in the life of the fetus, and also later for normal lactation. The existence of a high biotin requirement in rapidly growing tissue is not entirely unexpected in view of its biochemical function (p. 170).

During lactation the $\mathrm{B}$ vitamin requirement remains high, since it still represents the requirement of two or more individuals. When lactation is possible, but for some reason does not occur, any increased vitamin requirement is imperceptible. Apparently, the extent of the increased requirement during pregnancy and lactation is not the same for each vitamin. Thus, rats normally require about three times as much pantothenic acid as pyridoxine, but during lactation the ratio increases to six times or higher. ${ }^{34}$

A number of factors work to raise the requirements during lactation, chief among these being the fact that the physiological requirement of the mother is now logically an amount such as will provide a sufficient level in the milk to nourish the infant. It is quite clear that the thiamine level in the milk is largely dependent upon the amount in the diet (p. 347). Therefore, a large increase in dietary intake might conceivably be necessary to provide a sufficiently high level in milk. Rats are stated to require five times the maintenance amount of thiamine to nurse a litter successfully. ${ }^{35,}{ }^{36}$ For these reasons the study of increased B vitamin requirements during lactation received quite early attention, ${ }^{37}$ although little scientific elucidation occurred until recent years.

Roderuck et $a l{ }^{38}$ have recently made excellent studies of the thiamine and riboflavin requirements of humans during lactation. Fourteen women were studied for an extended period during which analyses were made 
of duplicate meals, of 24-hour collections of urine, and of fasting onehour samples of urine. During the period of lactation the thiamine intake varied from 0.73 to $1.59 \mathrm{mg} /$ day and the riboflavin intake from 2.2 to $3.6 \mathrm{mg} /$ day. Sample data from one individual in which the average thiamine intake was $1.11 \mathrm{mg} /$ day and the average daily riboflavin intake was $2.95 \mathrm{mg}$ are given in Table 11 .

\begin{tabular}{|c|c|c|c|c|}
\hline $\begin{array}{l}\text { Days } \\
\text { ost partum }\end{array}$ & $\begin{array}{l}\text { Thiamine } \\
\text { in milk } \\
(\mathrm{mg} \%)\end{array}$ & $\begin{array}{l}\text { Total thiamine } \\
\text { secretion in milk } \\
\text { (mg) }\end{array}$ & $\begin{array}{c}\text { Riboflavin } \\
\text { in milk } \\
(\mathrm{mg} \%)\end{array}$ & $\begin{array}{l}\text { Total riboflavin } \\
\text { secretion in } \\
\text { milk (mg) }\end{array}$ \\
\hline $\begin{array}{l}1 \\
6\end{array}$ & $\begin{array}{l}0.002 \\
0.005\end{array}$ & $\begin{array}{l}0.014 \\
0.086\end{array}$ & $\begin{array}{l}0.033 \\
0.040\end{array}$ & $\begin{array}{l}0.26 \\
0.72\end{array}$ \\
\hline 78 & 0.015 & 0.126 & 0.048 & 0.41 \\
\hline 162 & 0.012 & 0.112 & 0.042 & 0.38 \\
\hline 239 & 0.013 & 0.089 & 0.043 & 0.29 \\
\hline 302 & 0.016 & 0.062 & 0.046 & 0.18 \\
\hline
\end{tabular}

Total thiamine secretion in milk was from 2 to 12 per cent of the intake, and total riboflavin, 6 to 32 per cent. For all subjects, the maximum daily thiamine secreted in milk never exceeded 15 per cent of the intake and varied in urine from 2 to 57 per cent of the intake. Assuming that there is no waste of thiamine in the production of milk by mammary tissue, and since there was no indication of an avitaminosis in the subjects, the authors conclude that a 15 per cent increase in the dietary supply of the requirements of normal women supplied any increased requirements due to lactation. On this basis the National Research Council's recommendations of $3 \mathrm{mg}$ of riboflavin per day and $2 \mathrm{mg}$ of thiamine per day on a 3000-Calorie basis seem adequate, and the absolute requirement is obviously much lower. The National Research Council also suggests $20 \mathrm{mg}$ per day as a suitable nicotinic acid intake under similar conditions. Their recommendations for lactation all involve an increase of from one-half to one-third of the level (on a 3000-Calorie basis) for a very active normal woman.

Pathological States of the Body. It is readily apparent that a large variety of pathological situations might arise which could hamper the efficient utilization by the organism of an otherwise adequate B vitamin supply. Some of these which occur with sufficient frequency to permit their recognition as distinct clinical entities are discussed in later chapters as deficiency conditions (Chap. VI C). Many other clinical and acute pathological conditions, however, appear only from time to time, and yet effectively cause a distinct rise in B vitamin requirements. While such conditions are of major importance in hospital dietetics, there is not at present sufficient information to permit rational treatment in these cases. Largely because of this, "shot-gun vitamin therapy" has become a rather standardized procedure. 
Perhaps of the most striking interest in recent years has been the study of B vitamin requirements in severe injury. Most of such studies have shown, however, only that increased vitamin intakes produce some type of beneficial result. Thus, Govier and Greer ${ }^{39}$ found that in dogs with hemorrhage-induced shock, although all eventually succumbed, the average survival time of thiamine-treated controls was 2.4 times that of an untreated group. They found that the elevated pyruvic acid (4.5 mg per cent), ${ }^{*}$ blood sugar and blood lactic acid levels occurring in shock were all returned to normal by thiamine administration. Moreover, a high plasma thiamine level seems to add to the resistance to the onset of shock. ${ }^{40}$ Finally, cocarboxylase is dephosphorylated in shock, but is apparently resynthesized as a result of thiamine administration. ${ }^{41,42} \mathrm{In}$ these cases, as Govier points out, a thiamine deficiency in a sense may exist even though there is an ample thiamine supply, since the thiamine is converted to a metabolically useless form. Greig ${ }^{43}$ has shown that this same situation prevails in anoxia, and that similar breakdowns of coenzyme I and flavin adenine dinucleotide may occur in these cases. Thus, the requirements under such acute pathological conditions are obviously considerably elevated at least for thiamine, riboflavin and niocin.

These observations have been confirmed to some extent in humans. Andreae, Schenker and Browne ${ }^{43}$ studied riboflavin excretion in 23 cases of burns and injuries. Among healthy controls about one-half of a 5-mg oral dose of riboflavin was retained, whereas patients with acute injuries showed a much higher retention for 3 to 5 days immediately following the injury. Subsequently there was abnormally high riboflavin excretion, followed by a return to normal at about ten days following the injury. One might suppose that the vitamin retained during the first period (or that formed from the coenzyme decomposition) was not further used, but stored in some unusual manner for a period and then excreted. Levenson et al. ${ }^{45}$ studied six patients with severe, acute surgical conditions and found similar abnormalities in thiamine, riboflavin and nicotinic acid metabolism. All this work seems to accord well with the known facts regarding coenzyme breakdown ${ }^{46}$ (p. 352).

There has been a variety of reports regarding the effects of gastrointestinal disturbances and surgery upon B vitamin requirements. In a typical case, signs of pellagra were observed some four weeks after stomach resection. These disappeared on thiamine and nicotinic acid therapy. In another case, polyneuritis attendant upon adhesions of the omentum was cured by relaparotomy followed by thiamine therapy. ${ }^{47}$ Little definite information is available regarding the basis for such effects. The effects of endemic disease on the $\mathrm{B}$ vitamin requirements are vir-

* Normal, 1-2 mg per cent. 
tually unstudied and present a field of real interest for obvious reasons. Other aspects of disease and its interrelationships with $\mathrm{B}$ vitamins are more fully presented in Chapter VI C. At present it is possible to do little more than recognize the existence of a vast and unstudied group of socalled "conditioned nutritional deficiencies," wherein pathological conditions have raised individual $\mathrm{B}$ vitamin requirements.

It has previously been mentioned (p. 247) that the thiamine requirement varies with the metabolic rate of an individual. Under conditions in which there is an augmented metabolism (hyperthyroidism, prolonged fever) the requirement is therefore notably increased. Details as to the extent of this increase are lacking, however.

Inherent Individual Variations in B Vitamin Requirements. When finally we consider the existence of the many factors which go to delineate the vitamin requirements of a species and a strain under highly defined conditions, we are still faced with the indisputable fact that individual animals, and indeed litter mates, differ from each other in many ways, among which are their B vitamin requirements. R. J. Williams, in a more extended discussion of the aspects of individual variability in metabolic patterns, has pointed out the extreme importance of such variation. $\mathrm{He}$ states: ${ }^{48}$

"It would be presumed on the basis of what we know about the inheritance of enzyme catalysts and the heritability of vitamin requirements in animals that the requirements for each vitamin would be inherited as a separate unit. A requirement for one vitamin might be very high, for another it might be low, and for a third it might be about average, and so on. The inheritance of individual vitamin requirements, which is closely akin to the inheritance of enzyme catalysts, does not rule out the fact that environmental conditions such as infectious disease may alter requirements and make for variation, though information on this point is largely lacking. ..."

"On the basis of what we know about the requirements of animals it is safe to assume that individual human beings differ widely from one another in the amounts of different vitamins that they require. It is not at all improbable that specific individuals may have requirements for certain vitamins which are several times those of their associates. These differences may be due to relative failure to digest or assimilate, increased tendency to excrete, a failure in the ability to build the vitamins into the tissues, or to other reasons."

"As I have said, information on variation in vitamin requirements is largely lacking; those who have been investigating vitamins in nutrition have not been interested in possible individual differences but have been pleased if they could get information about the average man, and have 
been content to neglect the exceptional individual whose performances are out of line. Even information regarding the average man has been difficult to obtain."

"Casual information suggesting individual variability in vitamin requirements is readily available. Probably every doctor who deals in his practice with vitamin requirements could cite cases of unusual benefits from vitamins, or cases in which administration of a vitamin was effective in one case and wholly ineffective in another."

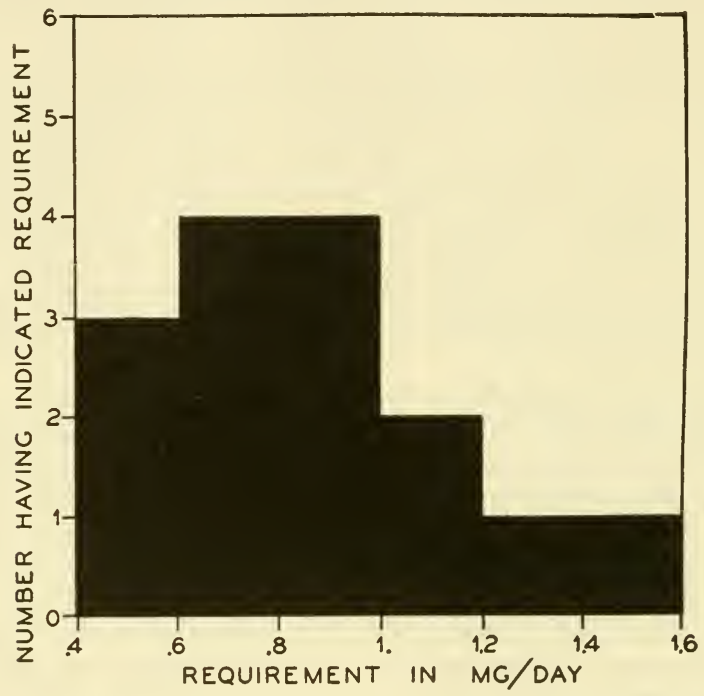

Figure 1. Distribution of the daily thiamine requirement among fifteen individuals.

Actual cases of individual variations might be enumerated in great length. In some, the variability may be extreme. For instance, even when all presently recognized vitamins are added to the diets of chicks it is known that a certain small percentage of them (about 2 per cent) still develop perosis. ${ }^{49}$ Bloomfield ${ }^{50}$ found great variation in the weight losses among rats on deficiency diets, and found that after recovery, the same individuals again lose the most weight on a second test on the deficiency diet.

Berryman et $a l .^{51}$ have come across the same problem in B vitamin excretion studies, finding that different human individuals, even under the best controlled conditions, may still excrete widely varying amounts 
of vitamins. In the study of Denko et al. ${ }^{52}$ on the B vitamin excretion of a number of individuals, individual differences are readily apparent, and the similarities in excretion levels between a pair of brothers are most noteworthy in suggesting the genetic basis which must exist to explain individual variations (p. 369).

Pett has considered this aspect of the vitamin requirements at great length and has emphasized the great danger of taking the average, mean, or modal value for a dietary standard. ${ }^{53}$ He points out that when such a standard is taken, a distribution curve for this figure should be available. In line with this suggestion he has presented such a curve for thiamine prepared from data in the literature. ${ }^{54}$ These data are indicated in Figure 1. Unfortunately, however, no one has apparently as yet endeavored to prepare more accurate data of this kind for thiamine or for the other members of the B vitamin group.

\section{Factors Involving the Nature of the B Vitamin Nutritional Supply}

For a given individual the amount of a $\mathrm{B}$ vitamin which may be required nutritionally varies considerably according to the efficiency with which the vitamin actually present in the food may be utilized. Thus, other components of the diet or circumstances attendant upon dietary habit may influence the requirement. It has also been found that when more than one form of a given vitamin exists in nature, the relative potencies of these forms for various species may not be the same, and some forms may be completely unavailable due to their occurrence in "bound" states which cannot be utilized by the animal in question. Further specific inhibitions and inactivations exist which in some cases prevent utilization of the vitamin. All these things effectively influence the amount of the vitamin which must be present in the diet to meet the needs of the organism, and they must therefore be considered in some detail. Still other factors conditioning the requirement, such as the effect of the state of nutrition on the proportion of vitamin assimilated, are at present too poorly understood to provide more than passing recognition of their existence.

Effect of Other Nutritional Components. The ways in which other components of the diet may influence the $B$ vitamin requirement are varied and only a few interrelationships are well understood. In some cases it is quite clear that a given B vitamin is involved in the metabolism of some particular nutritional component and that the amount of that component consumed will directly influence the vitamin requirement. In other cases, certain species are able to utilize biosynthetic precursors of the vitamin so as to increase their supply. In many instances it is not clear whether the animal itself or symbiotic organisms bring about the 
particular effect. Conflicting reports exist in many cases, making a thorough understanding of many relationships impossible, at least for the present. The discussion which follows then is of necessity a brief one, intended merely to point out the major associations of this type which are recognized at present.

Effect of Carbohydrates and the Calorific Intake. Thiamine functions in the form of thiamine pyrophosphate (cocarboxylase) in the metabolism of carbohydrates (the decarboxylation of pyruvate and ketoglutarate) (p. 158). For this reason the thiamine requirement is determined almost completely by the carbohydrate intake. It is apparently immaterial to the requirement whether the carbohydrate is used immediately for energy or converted to fat. ${ }^{55}$ Because of this, the thiamine requirement is frequently expressed in terms of thiamine per Calorie, or even better per "non-fat Calorie." Fat, and to a lesser extent protein, by displacing carbohydrate from the diet, exert a "sparing action" on the thiamine requirement.

Dann ${ }^{56}$ has maintained rats for a period of a year on a substantially thiamine-free diet containing 80 per cent protein and no carbohydrate. At the end of this period, the animals appeared to be in excellent condition although they had not grown rapidly. In this case, it would appear that intestinal synthesis by symbionts was ample to meet most requirements on the carbohydrate-free diet.

Thiamine is not required in the immediate metabolism of ethyl alcohol, although it was long believed otherwise; and thiamine deficiencies in alcoholics are believed to be due to low intakes and possibly poor assimilation. Lowry et al. ${ }^{57}$ found in fact that alcohol delayed the symptoms of an acute thiamine deficiency in rats on a deficiency diet, when it either supplemented or replaced part of the carbohydrate of the diet. Westerfeld and Doisy, ${ }^{58}$ in similar studies with pigeons, found that either alcohol or fat had a thiamine-sparing action, 16 grams of fat being equivalent in this regard to 9 grams of alcohol. It therefore seems well established that the isocaloric substitution of alcohol or fat for carbohydrate decreases the thiamine requirement. Recent studies have also shown that replacement of the dietary carbohydrate on an isocaloric basis with ethyl alcohol results in an increased excretion of thiamine, $\mathrm{N}^{\prime}$-methylnicotinamide, and pyridoxic acid, but not pantothenic or folic acid. ${ }^{59}$

High caloric diets also apparently create an increased folic acid requirement, and this adds to the many other difficulties inherent in assessing the folic acid requirements of the chick (p. 248). Luckey and co-workers further found the nature of the diet to be a major factor. ${ }^{60}$ Diets high in fat or in which the only carbohydrate was glucose, sucrose or starch necessitated a much higher level of folic acid than did cornmeal and 
dextrin diets or high protein-low fat diets. In experiments with rats it has also been found that diets containing dextrin as the carbohydrate require less pyridoxine than diets in which sucrose is the carbohydrate component. ${ }^{61}$

Effect of Proteins and Amino Acids. Much attention has been drawn to the effects of protein levels on the riboflavin requirement. Reference has previously been made to the studies of Czaczkes and Guggenheim ${ }^{62}$ on the correlation of the dietary requirements of rats for riboflavin with tissue and urinary riboflavin levels. In this study it was found that the fat or protein content of the diet profoundly affected the requirement. These results are summarized in Table 12. It is apparent that at least part of this variation is due to variation in bacterial synthesis in the gut, and it seems doubtful whether increased protein increases the riboflavin requirement beyond this. Trufanov ${ }^{63}$ has found that in rats on a low protein diet the riboflavin content of the urine increases, while that of liver and muscle decreases. He claims moreover that there is no further synthesis of flavin-adenine-dinucleotide in the liver and tissues after fifty days on this diet. There is little apparent relationship between these data and the observation that thiamine and riboflavin tend to counteract the degenerative changes wrought upon the liver and spleen of experimental animals by protein-free diets. ${ }^{64}$

TABLE 12. The Effect of Protein on the Riboflavin Requirement of the Rat.

Diet

Standard

High-fat

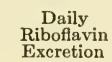

Fat Protein $\begin{aligned} & \text { Riboflavin } \\ & \text { Excretion }\end{aligned}$

$20 \% \quad 20 \%$

$\begin{array}{ll}20 \% & 20 \% \\ 40 \% & 20 \%\end{array}$

\section{Riboflavin Riboflavin \\ $\begin{array}{ll}\text { Level } & \text { Level } \\ \text { in Organs } & \text { in Feces }\end{array}$}

Remarks

diminished diminished diminished Requires two times amount of riboflavin required to maintain normal level in organs.

Low-fat $\quad 2 \% \quad 20 \%$ increased increased increased Requires half of

High-protein $20 \% \quad 34 \%$ diminished diminished diminished Requires twice norLow-protein $20 \% \quad 11 \%$ no change no change no change mal amount.

Lack ability to store riboflavin.

Our present understanding of the function of vitamin $B_{6}$ in amino acid metabolism * would lead one to predict that an increased dietary protein level would increase the vitamin $B_{6}$ requirement and this is found to be the case. Thus, in mice on a vitamin $\mathrm{B}_{6}$-deficient 50 per cent casein diet, the liver vitamin $B_{6}$ after 3 weeks was $0.97 \mu \mathrm{g} / \mathrm{gm}$, whereas in a similar series on 10 per cent casein, the level was $3.7 \mu \mathrm{g} / \mathrm{gm}$ and reached the low

* Lyman's studies of bacteria are particularly interesting in this regard. ${ }^{65}$ For instance, with pyridoxine present, L. arabinosus loses its nutritional requirements for threonine, lysine, and alanine. 
level previously mentioned only after 12 weeks. ${ }^{14}$ Still more direct evidence was produced by Morgan et al., ${ }^{66}$ who were able to produce a vitamin $\mathrm{B}_{6}$ deficiency in dogs on a 45.8 per cent casein diet in 79 to 123 days, but no deficiency on an 18 per cent casein diet for 169 to 190 days.

Pantothenic acid is believed to function in the metabolism of carbohydrates and more particularly in acetylation processes, and one might expect that it might be less required in diets in which the main energy component was protein. This expectation may be further strengthened by the fact that amino acids function as precursors for both the $\beta$-alanine and pantoic acid moieties of pantothenic acid, and may thus encourage synthesis in the intestine. Nelson and Evans ${ }^{67}$ have recently shown that rats raised on a pantothenic acid-deficient diet fare much better on a high-protein diet (64 per cent casein) than on a lower one (24 per cent casein).

The nutritional requirement for choline is greatly affected by the protein intake of the diet, largely by virtue of the interrelationship between choline and the amino acids serine and methionine. The precursor function of serine for choline is discussed elsewhere (p. 89) in relationship to the general cellular metabolism. The reciprocal relationships of choline and methionine for growth and lipotropism ${ }^{68}$ are most frequently encountered in animal nutrition, however. Choline functions in the transport of fats, and in the absence of an adequate supply a variety of symptoms, including a neutral fat type fatty liver, may develop. Methionine may replace choline in the diet inasmuch as it serves as a source of methyl groups for choline synthesis in vivo. Thus, in the presence of ample serine, rats may grow at a normal rate (41.8 gms/21 days) and have normal liver lipides (8.9 mg per cent) if the diet contains $1200 \mathrm{mg}$ per cent of methionine. ${ }^{68}$ In the presence of $500 \mathrm{mg}$ per cent of methionine, however, the growth rate is only $23.8 \mathrm{gms} / 21$ days and liver lipides are $24.7 \mathrm{mg}$ per cent. Addition to this diet of $100 \mathrm{mg}$ per cent of choline restores liver lipides to normal but does not improve the growth rate. These data taken from Treadwell's study are summarized in Table 13, and are interpreted as meaning that on a choline-free diet, the methionine requirement is $1200 \mathrm{mg}$ per cent, of which about half is required for lipotropism (i.e., choline synthesis) and half for growth.

However, it should also be noted that starch, or some impurity therein other than choline, has an appreciable effect in preventing the development of the hemorrhagic kidneys found in young white rats on a cholinedeficient diet. ${ }^{69,70}$

For chick growth, a very similar relationship holds, as it does in a variety of other animals. McKittrick ${ }^{71}$ has found that the essential choline (the limit beyond which reduction of choline cannot proceed with- 
out reducing growth below optimum levels, regardless of the methionine present) was about 0.1 per cent of the diet (containing 0.55 per cent L-cystine) and the essential level of methionine was 0.5 per cent of the diet.

Thus, it seems generally true that the choline requirement is quite dependent upon methionine and serine. A further discussion of these interrelationships is found in a later chapter.

TABLE 13. Effect of Cystine and Methionine on the Choline Requirement.

$\begin{array}{ccccc}\begin{array}{c}\text { Choline } \\ \mathrm{mg} / 100 \mathrm{gm} \\ \text { diet }\end{array} & \begin{array}{c}\text { Methionine } \\ \mathrm{mg} / 100 \mathrm{gm} \\ \text { diet }\end{array} & \begin{array}{c}\text { Cystine } \\ \mathrm{mg} / 100 \mathrm{gm} \\ \text { diet }\end{array} & \begin{array}{c}\text { \% Gain in } \\ \text { weight in } \\ 21 \text { days }\end{array} & \begin{array}{c}\text { Liver Lipides } \\ \mathrm{gm} / 100 \mathrm{gm} \\ \text { moist tissue }\end{array} \\ 0 & 500 & 100 & 23.8 & 24.7 \\ 0 & 600 & 100 & 37.0 & 20.5 \\ 0 & 700 & 100 & 38.3 & 18.8 \\ 0 & 1000 & 100 & 43.3 & 14.9 \\ 0 & 1200 & 100 & 41.8 & 8.9 \\ 0 & 500 & 100 & & \\ 0 & 500 & 200 & 23.8 & 24.7 \\ 0 & 500 & 300 & 26.4 & 24.3 \\ 0 & 500 & 400 & 29.3 & 26.1 \\ 0 & 500 & 600 & 30.6 & 21.9 \\ 0 & & & 26.5 & 16.1 \\ 0 & 500 & 100 & & \\ 100 & 500 & 100 & 23.8 & 24.7 \\ 200 & 500 & 100 & 20.2 & 7.0 \\ & & & 27.2 & 6.6\end{array}$

Effect of Tryptophan on the Nicotinic Acid Requirement. While in reality a subject belonging to the discussion of the previous section, the effect of tryptophan on the nicotinic acid requirement is of such farreaching importance that it merits entirely separate treatment. For the sake of clarity of discussion, however, many of the aspects of the closely associated effect of corn on the nicotinic acid requirement will be treated in greater detail in a later section dealing specifically with inhibitory effects.

Despite the fact that by 1938 the identity of nicotinic acid as the pellagra-preventive vitamin was well established, it was still apparent that other factors were involved in the etiology of this condition. Aykroyd and Swaminathan ${ }^{72}$ observed that in Moldavia the staple corn diet supplied $15 \mathrm{mg}$ of nicotinic acid daily and that there was endemic pellagra, whereas in southern India the $5 \mathrm{mg} /$ day of nicotinic acid derived from rice diets produced only rare cases. A variety of similar observations led to the conclusion that corn in some manner antagonized the utilization of nicotinic acid. Krehl et al. ${ }^{73}$ showed, moreover, that in the rat, which normally is able to synthesize its own supply of nicotinic acid, corn grits almost completely prevented growth, but that this effect was entirely reversed by the addition of $1 \mathrm{mg}$ per cent of nicotinic acid to the diet. Moreover, the nicotinic acid requirement of dogs on purified diets con- 
taining corn grits was tripled..$^{74}$ Wintrobe $^{75}$ found a similar relationship in young pigs.

In Krehl's study it was found that raising the casein in the basal diet from a level of 15 per cent to a level of 20 per cent also prevented the antagonism due to corn. Both lysine and tryptophan occur in low concentrations in corn but in higher levels in casein; and Krehl et al. ${ }^{76}$ found that whereas lysine was not effective, 0.05 per cent of L-tryptophan was as effective in reversing the effect of corn as was nicotinic acid itself. At the time, this was believed to be due to intestinal synthesis of nicotinic acid by bacteria, which were believed unable to grow on corn diets due to inadequate tryptophan. It was also found 77 that in diets in which wheat gluten or gelatin (which are lower in tryptophan) were used as the protein supplement, poor growth ensued which was cured by either tryptophan or nicotinic acid. When egg albumin, fibrin or soybean globulin were used as the protein components ${ }^{7 \mathrm{~s}}$ it was found that no inhibition occurred when corn grits were added to the diet, and that this could be explained on the basis of the high tryptophan content of these protein materials. Thus, while an antagonist effect of corn was not ruled out, its low tryptophan content was at least in part concerned with the nicotinic aciddeficiencies observed.

Singal and co-workers ${ }^{79}$ found that there was an increased urinary excretion of niacin and its metabolic products in rats when tryptophan was added to the corn grit diet. Rosen, Huff, and Perlzweig ${ }^{80}$ similarly observed that $50 \mathrm{mg}$ of DL- or L-tryptophan either given orally or subcutaneously produced a five- to tenfold increase in nicotinic acid excretion over the level excreted on 15 per cent casein diets. Moreover, there was a relative constancy of fecal nicotinic acid whether or not tryptophan was administered. From these observations it became readily apparent that the tryptophan effect was not due to intestinal synthesis, and that tryptophan must therefore be involved in nicotinic acid metabolism, probably as a precursor. This, moreover, seemed more in line with the observation that, when $4 \mathrm{mg}$ /day of 3-pyridylmethylketone, a structural analogue of nicotinic acid, was fed to mice on niacin-free diets, 11 out of 12 died within 10 days, but that this toxic action was prevented by both nicotinic acid and tryptophan. ${ }^{81}$ This work was based on the well established theory of the interference by structural analogues with the operation of a metabolite through saturation of enzymes involved in the metabolism of the latter substance, and reversal of such toxic action by precursors or products of the metabolite. It led naturally to the reemphasis of a "pellagragenic" agent in corn, which acts in a manner similar to that of 3-pyridylmethylketone (Chap. VI D). More recently, work with Neurospora mutants ${ }^{\$ 2}$ makes it seem certain that the effect 
of tryptophan is as a metabolic precursor of nicotinic acid. The mode of conversion is by no means known at this time, however, nor is there unequivocal reason to select the indole-nitrogen over the $\alpha$-amino-nitrogen as the precursor of that nitrogen atom which occurs in niacin (see p. 356).

Recent studies have indicated that the tryptophan-nicotinic acid relationship holds with a variety of other species. The lack of growth of guinea pigs on a corn-soybean oil meal-alfalfa ration is reversed by nicotinic acid or tryptophan. ${ }^{83}$ Chicks on a 10 per cent gelatin diet exhibit depressed growth rates which are corrected by $5 \mathrm{mg}$ of nicotinic acid or $200 \mathrm{mg}$ of DL-tryptophan per $100 \mathrm{gm}$ of diet. ${ }^{84}$ Krehl has found ${ }^{77}$ that for rats 1 to $1.5 \mathrm{mg}$ of nicotinic acid are equivalent to $50 \mathrm{mg}$ of tryptophan per $100 \mathrm{gm}$ of diet. Monkeys, however, develop a deficiency on such diets, which is corrected by neither nicotinic acid nor tryptophan, nor both, but by liver powder or by lyophilized liver. ${ }^{85}$ Various other workers have also recently produced a variety of evidence to show that man does actually convert tryptophan into nicotinic acid ${ }^{86,87}$ (see p. 354). Present indications are that neither indole, anthranilic acid, nor indoleacetic acid may serve in lieu of tryptophan as a nicotinic acid precursor. ${ }^{88}$ Additional data bearing on the problem of the conversion of tryptophan to nicotinic acid will be found in the discussion of nicotinic acid metabolism in Chapters V A and IV C. Without further consideration it seems fair to assume that, for most higher animals at least, the nicotinic acid requirement can be stated only in the light of some estimate of the tryptophan intake. Frazier and Friedemann ${ }^{89}$ have shown that on corn-free diets the human requirement may be as low as $4 \mathrm{mg} /$ day of nicotinic acid, but that this is increased to about $5 \mathrm{mg}$ /day by corn when there is a high vitamin and protein level, and to $7.5 \mathrm{mg}$ /day when vitamin and protein levels are lower.

Of considerable interest is the recent report ${ }^{90}$ that bacteriologically sterile Drosophila melanogaster require both tryptophan and niacin, and that the niacin requirement is increased by higher tryptophan levels in the diet. This indicates that there may be wide variation in the animal kingdom with regard to this interrelationship, and to some extent tends to suggest the theory that there is at least contributory intestinal synthesis of nicotinic acid by bacteria. Other recent work showing that sulfonamides decrease the excretion of nicotinic acid metabolites ${ }^{91}$ has further brought this earlier hypothesis into prominence again. Nutritional studies with "germ-free animals" (p. 300) may do much to resolve this dilemma, and it seems probable that both factors may eventually be shown to be involved.

Vitamin Interrelationships. Strangely enough, little is as yet known about the effects of vitamins on the requirements of other vitamins, As 
concerns the fat-soluble group, it has been reported that the thiamine requirement of the rat is considerably increased (as indicated by loading tests) in vitamin A deficiency, and that large doses of thiamine delay the appearance of symptoms of avitaminosis A. ${ }^{92}$

While rats are normally not susceptible to nicotinic acid deficiency, it has been reported that a multiple deficiency of nicotinic acid, pantothenic acid, and $p$-aminobenzoic acid produces a syndrome similar to that of pellagra. While all these factors are required for its cure, ${ }^{93}$ nicotinic acid alone can delay the symptoms. There is at present a rapidly increasing body of evidence which indicates that nicotinic acid and folic acid are intimately associated in some manner in the cure of the symptoms of nicotinic acid deficiency. ${ }^{94}$ This problem is more appropriately discussed in later chapters (pp. 408 and 412).

There is at present little explanation for the observation that thiamine and cholic acid substitute for pantothenic acid in the Hall strain of Clostridium botulinum Type A..$^{95}$

Nutritional Customs, Habits, and Taboos. Of great practical concern to the nutritionist is the problem of dietary habits and their effect on nutritional requirements. Generally the effect of such habits is exerted through obvious means (excessive carbohydrate intake, high protein diets, high raw egg intake, etc.), but subtle factors may also be at work which may influence dietary calculations to a considerable degree. Thus even drinking water may contain appreciable quantities of $\mathrm{B}$ vitamins, and the consumption of excessive quantities of coffee with its relatively high trigonellin content might certainly influence the nicotinic acid requirement (p. 288). The habit of the Mexican natives of consuming beans with their tortillas undoubtedly prevents widespread pellagra in Mexico. Such factors should then influence the calculation of adequate dietary requirements as much as they influence the adequacy of the diet itself. While it is necessary to abbreviate what might well be an extended discussion on this point, it is none the less important to emphasize the fundamental nature of sociological aspects in assessing nutritional requirements.

The Processing of Foods. Whereas the vitamin requirements of an individual might well be met on the basis of the native vitamin content of the diet, the situation may well be otherwise by the time the dietary components have been marketed, stored, canned, cooked, baked, or otherwise modified by circumstances of time, temperature, and chemical treatment. Losses of B vitamins in food preparation vary widely with the food and the precise methods involved; there have been a large number of studies and publications on this subject. The topic, however, does not fall sufficiently within the realm of this monograph to merit a detailed discussion. Moreover, the ready availability of extensive data on this 
topic further lessens the expediency of a consideration of it here. It should be emphasized in passing, however, that the topic is of extreme importance in estimating the $\mathrm{B}$ vitamin requirements of any population, and must be weighed carefully in evaluating the adequacy of diets and of new processes in food technology.

Biological Potency and Availability. It frequently occurs among the $B$ vitamins that one vitamin may have a number of structural forms, the biological activities of which differ for a given species, and between different species. The requirement for a $\mathrm{B}$ vitamin in a given animal cannot be met by a form that is without activity for that animal, regardless of how high its activity may be for another. It is necessary to insure that in the diet there is an adequate amount of B vitamin analogues which are active for the species in question, if the requirement is to be met. For this reason, the nutritional requirement cannot be stated accurately in terms of, say, vitamin $B_{6}$, since vitamin $B_{6}$ has a number of active forms with different biological potencies. It is therefore necessary to consider, as one aspect of the problem of assessing the nutritional requirements, what the various naturally occurring forms of the $B$ vitamins are, and how they compare with each other as regards their biological activity.

Beyond this, it has been found in recent years that certain forms of some $B$ vitamins are not available to animals for use; indeed the problem of the availability of vitamins, even when they are present in otherwise adequate amounts, is one worthy of considerable attention.

Human Bioassay Techniques. While it is generally possible to determine the vitamin requirements of lower animals and the potencies of various diets or vitamin derivatives for any given species by a suitable direct approach, it is seldom possible to employ such direct methods with man himself. Laboratory depletion studies upon members of the human race are relatively rare, ${ }^{96}$ and indirect methods must generally be used. These have largely employed studies of vitamin excretion.

Melnick et $a l .{ }^{97}$ have applied such studies to a number of problems. Generally the assay involves suitably sized groups of about five individuals on diets containing ample vitamins for their requirements. Twentyfour-hour samples of urine are collected and the basal level of vitamins determined. Immediately thereafter a known dose of vitamin is administered, and the percentage of this dose recovered in the urine is observed. When the test dose is then administered, it is assumed that the percentage recovery in the urine is similar, and the potency of the test material can presumably thus be calculated. Despite the fact that there are large individual variations in basal levels, control experiments are reported to indicate an accuracy generally as good as that obtained in other animal assays. In view of the various factors discussed previously in regard to 
excretion studies, it is apparent that such an approach must involve several assumptions (e.g., that the excretion is a function of the intake) and techniques (e.g., eventual testing of the excreted products by nonhuman means) which leave much to be desired. Nevertheless, when the conditions are adequately controlled, considerable valuable information may be so obtained, and extended further studies of this nature seem to be urgently required. The application of such human bioassay techniques to the study of the availability of thiamine in yeast ${ }^{98,99}$ (p. 291) and its enzymatic destruction ${ }^{100}$ (p. 292), have indeed opened a valuable new approach in the science of nutrition.

The Relative Potencies of the Various Naturally Occurring Forms of the $B$ Vitamins. It seems most probable that much remains to be discovered concerning the variety of naturally occurring substances possessing $\mathrm{B}$ vitamin activity. This is largely due to the great difficulties inherent in the separation of minute quantities of structurally similar compounds. Recently techniques employing a combination of paper partition chromatography ${ }^{101}$ and plate growth of assay organisms ${ }^{102}$ have been developed, and these show promise of adding to our knowledge of nutrilite derivatives in general. For the present, however, the discussion which follows must be considered in terms of the probability of the existence in nature of a far greater number of $\mathrm{B}$ vitamin isotels. ${ }^{103}$

The discussion of these various vitamin forms might well be undertaken in a pedagogic fashion from the standpoint of their relationship to the generally accepted form (s) of the vitamin, but it is certainly worthwhile to point out that other rather obvious categories of isotels do exist. Thus we have the immediate vitamin structures such as the $\mathrm{B}_{6}$ "triad" and the folic acid "triad." Secondly, bound forms, available and unavailable, are known for most of the B vitamins. Vitamin precursors frequently show vitamin activity as in the case of the precursors of choline and nicotinic acid, and the functional forms, frequently coenzymes, are generally active. Catabolic products of vitamin metabolism also show activity for some species. Finally, there is a large group of biologically active substances which seem to have little apparent structural relationship to the B vitamins themselves, although one might theorize extensively on their activity. For practical purposes, however, it has been deemed advisable to consider the various known analogues in relation to each of the individual vitamins, and thus in a more systematic, though less erudite fashion.

\section{(1) Thiamine}

Thiamine may occur in free and combined forms (p. 30), and little is known of the nature of bound thiamine. It is apparent however that various animal species may utilize some, but not all, of these bound 
forms. Thiamine also occurs in the form of the pyrophosphate (cocarboxylase), but the relative potency of this form for living things other than bacteria is apparently unknown. (Certain strains of Neisseria gonorrheae even require this form for growth, and thiamine itself is not only inactive, but inhibits growth, being competitively reversed in its action by the pyrophosphate.) ${ }^{104}$ Certain protozoa at least, and perhaps a few higher animals, are able to use either the thiazole or the pyrimidine fraction of thiamine for growth, ${ }^{105}$ so that in a general sense, the presence of these substances and their activity must be considered in the nutrition of members of phyla below the chordates.

A thiol form of thiamine, and its oxidized disulfide form (and their pyrophosphates), are believed to exist, ${ }^{106}$ and both thiamine and cocarboxylase disulfide have been shown to be active in the catatorulin test with deficient pigeon brain (p. 51). The presence of these forms must be considered, if they are found to have biological activity for any species under consideration.

Finally, Polonovski et al. ${ }^{107}$ recently reported an interesting series of studies in which a number of natural and synthetic pterins were found to substitute for thiamine (or riboflavin) in pigeons and rats. Fluorescyanine (a fluorescent pigment from the scales of certain fish) was able to eliminate the symptoms of deficiency in thiamine-deficient rats and pigeons, and to increase the oxygen uptake and carbon dioxide evolution from thiamine deficient rat brain. Injection of 50 to $100 \mu \mathrm{g} /$ day of a number of synthetic pterins produced similar effects, and the fact that oral dosage was similar in effect showed that the result was not due to intestinal synthesis by microorganisms. Indeed the pterins were found to be incapable of replacing thiamine for microorganisms. It was also reported that in cecectomized rats ${ }^{108}$ xanthopterin, isoxanthopterin, folic acid, and lumazine could be substituted for thiamine, isoxanthopterin being the most active. Such results, if verified, suggest the possibility that the biological and structural specificity of many of the B vitamins (and other nutritionally active substances) may not be as great as has been generally presumed heretofore, and that the specificity may rest rather in the chemical structure and groupings involved in the precise functions of the vitamin.

\section{(2) Riboflavin}

Riboflavin is known to exist in free and combined forms and as the 5 -phosphate, in the form of the flavin-adenine-dinucleotide, and in both oxidized and reduced states (p. 32). The relative potencies and availability of these forms, however, for various species, are not known, though it is generally assumed that riboflavin is seldom present in forms in which 
it cannot be used. In the previously mentioned work of Polonovski et al. ${ }^{107}$ it was found that certain pterins could substitute for riboflavin in pigeons and rats. Ten $\mu \mathrm{g}$ per day of fluorescyanine produced a growth rate in riboflavin deficient rats of $2 \mathrm{gm} / \mathrm{day}$, similar to that produced by riboflavin. A large variety of synthetic riboflavin derivatives have been prepared, and much has been learned in this manner regarding the structural specificity associated with the riboflavin molecule, but these substances per se are of little interest in the present discussion.

\section{(3) Nicotinic Acid}

There are probably more known nutritionally active substances isotelic with nicotinic acid than with any of the other B vitamins. Many of these analogues are known to function in nature either as precursors or products in nicotinic acid metabolism, and there seems to be considerable variation in the abilities of various species to utilize these analogues. In general the activity of nicotinic acid is believed due to its conversion to nicotinic acid amide and thence to Coenzymes I and II, and consequently substances that may be readily converted to nicotinic acid or nicotinic acid amide may be expected to show nicotinic acid activity. Mueller's finding that nicotinic acid is more potent than nicotinic acid amide for diphtheria organisms, ${ }^{109}$ and the discovery by Dorfman et al. ${ }^{110}$ that nicotinic acid amide is more potent than Coenzymes I or II for dysentery organisms, while in discord with this view, do not prove unequivocally that it is untenable, but rather indicate the fastidious nature of certain cells with regard to their nutritional source of building blocks for intracellular coenzymes.

In order to obviate an unduly lengthy discussion regarding the activity of these derivatives for various species, the available information is summarized in Table 14. The effects of a large number of synthetic pyridine derivatives which do not occur in nature have been reviewed adequately in the literature ${ }^{111}$ and in Chapter VI D of this monograph. Much of the tryptophan-nicotinic acid interrelationship has been worked out with Neurospora mutants, and for that reason data are included to show the metabolites which have been found active for some Neurospora mutants.

Few quantitative interrelationships are known. It is generally true that nicotinic acid and nicotinic acid amide are about equally active, though exceptions exist, particularly among bacteria. The drug coramine is not nearly as active as nicotinic acid, but seems to be sufficiently active to be an effective therapeutic agent in man. $\beta$-Picoline has a low activity, but the $\beta$-methyl group can apparently be oxidized to niacin. For rats on a corn diet, Krehl ${ }^{7 \tau}$ found $1.0-1.5 \mathrm{mg}$ nicotinic acid to be approximately 
equivalent to $50 \mathrm{mg}$ of tryptophan. For chicks, $5 \mathrm{mg}$ of nicotinic acid are equivalent to about $200 \mathrm{mg}$ of DL-tryptophan, ${ }^{84}$ and for dogs, $1 \mathrm{gm}$ of L-tryptophan is equivalent to $5.6-10 \mathrm{mg}$ of nicotinic acid. ${ }^{111 \mathrm{~b}}$ There are considerable individual variations in this regard, but in general the overall efficiency of the conversion seems to be of the order of 1 or 2 per cent. Only L-tryptophan is effective. Fifty to $100 \mathrm{gm}$ of protein per day would be required to meet the niacin requirement of man solely through synthesis from tryptophan on this basis. Since many animals excrete $\mathrm{N}^{\prime}$-methylnicotinamide $\left(\mathrm{F}_{2}\right)$, it was thought that some might be able to demethylate this product to nicotinamide, but to date only divergent and generally questionable results have been obtained (p. 359).

Table 14. Nicotinic Acid Activity of Some Metabolically Related Compounds.

\begin{tabular}{|c|c|c|c|c|}
\hline Compound & Man & Dog & Chick & Neurospora \\
\hline Nicotinic acid & + & + & + & + \\
\hline Nicotinamide & + & + & + & + \\
\hline Coenzyme I & + & + & + & \\
\hline Coenzyme II & + & + & + & \\
\hline Nicotinuric acid & - & $-(?)$ & & \\
\hline Trigonellin & - & - & & \\
\hline $\mathrm{N}^{\prime}$-methylnicotinamide $\left(\mathrm{F}_{2}\right)$ & $\pm(?)$ & $\pm(?)$ & & \\
\hline$\beta$-Picoline & + & + & & \\
\hline$\beta$-Aminopyridine & - & - & & \\
\hline Tryptophan & + & + & + & + \\
\hline Kynurenine & & & & + \\
\hline Anthranilic acid & - & & & - \\
\hline 3-Hydroxyanthranilic acid & $*$ & & & + \\
\hline $\begin{array}{l}\text { Coramine (synthetic drug) } \\
(\mathrm{N}, \mathrm{N} \text {-diethylnicotinamide) }\end{array}$ & + & & & \\
\hline $\mathrm{N}$-methylnicotinamide & & + & & \\
\hline Quinolinic acid ${ }^{111} \mathrm{c}^{*}$ & & & & + \\
\hline
\end{tabular}

* Active for the rat in increasing $\mathrm{F}_{2}$ exeretion and growth.ma

It has been stated as a generalization that animal tissues contain a preponderance of nicotinamide, and that plant tissues contain a smaller and more variable amount in this form. ${ }^{112}$ There is also evidence indicating that cereals contain a nicotinic acid precursor that is liberated only upon alkaline extraction. This precursor is apparently unavailable to dogs or chicks unless liberated by hydrolysis prior to feeding. ${ }^{113-115}$

The recent isolation of dinicotinylornithine from natural sources pro- 
vides still another niacin derivative which may figure in meeting the nicotinic acid requirements of man. ${ }^{116}$

It has been previously mentioned that cooking may modify the nutritional value of a vitamin source. An interesting example of improved nutrition resulting in this manner is in the case of coffee. Raw coffee contains a preponderance of trigonellin over nicotinic acid, but in the roasting process the trigonellin is largely converted to nicotinic acid, so that a cup of coffee generally contains from 1 to $2 \mathrm{mg}$ of nicotinic acid. ${ }^{117}$

\section{(4) The Vitamins $B_{6}$}

The three major forms of vitamin $B_{6}$ are pyridoxine, pyridoxal, and pyridoxamine, to which may be added the functional vitamin form, codecarboxylase, or pyridoxal phosphate, and pyridoxamine phosphate, which is now known to be a nutritional requirement for certain lactic acid bacteria. In addition, "bound" forms exist, and there is increasing evidence to suggest the possibility of a variety of vitamin $\mathrm{B}_{6}$-amino acid complexes (Schiff bases) with biological activity. Finally the ability of some lactic acid bacteria to employ certain amino acids interchangeably with vitamin $B_{6}$ may eventually be found to have broader biological significance. ${ }^{118}$

In vitamin $\mathrm{B}_{6}$-deficient mice, ${ }^{119}$ using xanthurenic acid excretion as a criterion of response, Miller and Bowman found that pyridoxine hydrochloride returned the excretion to normal within four days, while pyridoxal and pyridoxamine required seventeen and twenty-four days respectively. Growth rates on the three analogues and survival times on lower levels similarly indicated the same order of activities.

For the rat, it has been shown that the three forms are about equally active ${ }^{120}$ in promoting growth and in restoring the ability to convert tryptophan to niacin. ${ }^{120 a}$ It has also been reported that for the rat and chick, all three forms and pyridoxal phosphate are equally active when fed by eye dropper or injected intraperitoneally, ${ }^{121}$ although when added to the ration, pyridoxine appears somewhat more active. The three forms are also known to be equally active in promoting growth and blood regeneration in vitamin $\mathrm{B}_{6}$-deficient dogs. ${ }^{122}$

Although certain studies would suggest the existence of further analogues of vitamin $B_{6}$ in nature, ${ }^{125}$ the work of Rabinowitz and Snell ${ }^{123,124}$ indicates strongly that the $\mathrm{B}_{6}$ trilogy, their phosphates, and their proteinbound forms account for the vitamin $B_{6}$ content of tissues. Little is as yet known of the biological activity of the various products of vitamin $B_{6}$ metabolism in mammals, although 4-pyridoxic acid is known to be inactive for rats, chicks, and all the bacteria so far tested. 


\section{(5) Pantothenic Acid}

Pantothenic acid is known to occur in the form of the free acid, as coenzyme $\mathrm{A}$, in one or more bound forms, and possibly in the form of conjugates with one or more amino acids (glutamic acid particularly has been suggested). In addition, certain lower organisms at least can utilize either the $\beta$-alanine or the pantoyl fraction of the molecule in lieu of pantothenic acid ${ }^{126,127}$; and an amino acid which lias recently been indicated as occurring naturally has been suggested as a precursor of pantoic acid, and may have pantothenic acid activity. ${ }^{128}$ Coenzyme $\mathrm{A}$ is apparently available to animals, but $\beta$-alanine is inactive for at least rats and chicks, and probably all higher animals. Pantothenyl alcohol, a synthetic compound presumably not occurring in nature, is reported to be utilized by humans as readily as pantothenic acid, and may in some cases be even more effective, perhaps due to its apparently greater stability to acid. ${ }^{129-132}$ It cannot be oxidized by bacteria to the acid, however, and so is inactive in supporting bacterial growth.

\section{(6) Biotin}

Whereas biotin may have a large number of stereoisomers, there is little evidence to indicate that they occur naturally. There are reputed to be, however, as yet unelucidated avidin uncombinable forms, and bound forms. The bound forms which occur naturally seem to be readily utilized by most animals. There is also some evidence for the existence of a biotin coenzyme which is more active than biotin. ${ }^{132 a} \mathrm{O}-$ Heterobiotin (oxybiotin) which is a synthetic analogue, and desthiobiotin, which may possibly occur naturally, have however been studied quite extensively and compared to biotin earefully as regards their potency. Other substances with structures as yet unknown, and some with structures unlike that of biotin (oleic acid) substitute for biotin in some lower forms of life. ${ }^{133}$

McCoy et al. ${ }^{134}$ have shown that DL-oxybiotin is about 17 per cent as effective as biotin for the chick. It is $\mathbf{2 5}$ per cent as active as D-biotin for Saccharomyces cerevisiae and Lactobacillus casei, and 50 per cent for $L$. arabinosus. ${ }^{135}$ It is inactivated by avidin, and its effect for $L$. casei is inhibited by desthiobiotin. DL-Oxybiotin prevents chick dermatitis at levels of about $20 \mu \mathrm{g}$ per $100 \mathrm{~g}$ diet. ${ }^{136}$ Using growth and the disappearance of skin lesions as criteria, DL-oxybiotin is found to be only 4 per cent as active as D-biotin in curing egg-white injury in the rat. ${ }^{137}$

Tatum has suggester that desthiobiotin functions in the biosynthesis of biotin. ${ }^{138}$ Desthiobiotin is one-twentieth as active as D-biotin in curing egg-white injury in rats. ${ }^{135}$ Wright et al. have recently isolated a crystalline biotin-protein complex. ${ }^{191}$ 


\section{(7) Folic Acid}

The elucidation of the structure and functions of the folic acid group of compounds has come in recent years, so that comparatively little is known as to the relative merits of its various analogues. Folic acid is known to occur in free and bound forms, as the tri- and heptaglutamate, and also in formylated derivatives. Methods for assessing responses generally depend upon hematopoietic response, and a variety of substances have been found to influence such responses (e.g., pyridoxic acids, p. 421). Even 5-methyl uracil (thymine) in doses of $4.5 \mathrm{gm}$ or more produced hematological responses in six patients in relapse stages of Addisonian pernicious anemia, ${ }^{139}$ which is in line with the suspected function of folic acid in purine and pyrimidine formation. Indeed, for bacteria at least, the replacement of the pteridin moiety of folic acid by a quinazoline or a benzimidazole ring gives compounds with some biological activity. ${ }^{140,141}$ Petering et al. ${ }^{142}$ found that a combination of 4-pyridoxic acid and folic acid was better than folic acid alone in stimulating growth and hemoglobin formation in deficient chicks, although other workers have been unable to confirm this. Folic acid and the tri- and heptaglutamate are all active in curing sprue, ${ }^{1+3}$ and are active for monkeys. The monoand triglutamate at least are active in nutritional macrocytic anemia, and folic acid at least is active in treating pernicious anemia. (The heptaglutamate has up till now given highly divergent results. ${ }^{144}{ }^{145}$ The recently isolated vitamin $B_{12}$ should also be mentioned at this point as a substance which in minute quantities is capable of alleviating pernicious anemia. Both pteroic acid and formylpteroic acid are inactive for $L$. casei or humans, ${ }^{146-14 s}$ while pteroylheptaglutamate is inactive for bacteria, but is twice as active as the monoglutamate for Tetrahymena gelii. ${ }^{149}$ Various other possibilities exist for the presence of folic acid activity in natural sources ${ }^{149 \mathrm{a}}$ which are more conveniently discussed in a later chapter (pp. 413 to 422 ).

\section{(8) Choline}

Because choline is a structural part of such a wide varicty of naturally occurring compounds, its analogues in a sense may be considered to be numberless. In the phospholipides, however, when digestive hydrolysis occurs, free choline is doubtless liberated, so that the efficiency of lipide choline is quite high. This variety of choline derivatives, however, makes the assessment of choline requirements difficult, since it involves an estimate of the ability of an organism to liberate the choline. ${ }^{150}$ In addition to its esters, the various substances involved in choline synthesis are also active in many cases. Mono- and dimethylethanolamine are active in 
preventing perosis in chicks, for instance, but the mono- derivative will not promote growth, and the dimethyl compound only does so to the extent that methionine is present. ${ }^{151}$ These relationships are discussed at greater length elsewhere (p. 353).

\section{(9) p-Aminobenzoic Acid}

$p$-Aminobenzoic acid occurs in both free and combined forms in nature and as part of the folic acid group of compounds. Little is known beyond this concerning the variety of its occurrence, or the relative potency of its forms (p. III D).

\section{(10) Inositol}

Inositol occurs in nature in the free form, as the hexaphosphoric ester and its salts, and in certain "cephalins." ${ }^{152}$ In addition it occurs in bound forms that have been as yet unelucidated. ${ }^{153}$ The majority of these forms are apparently available to most higher animals, except those cases where the insoluble calcium or magnesium salts of the phosphate make solution and liberation of the inositol impossible.

Availability of Thiamine from Yeast. Mention has been made of the existence of bound and/or unavailable forms of a number of the B vitamins. In no case, however, has this fact been so vitally important and so vividly demonstrated as in the case of the thiamine of yeast. Particularly is this so because of the extensive use of yeast as a thiamine source.

It has been known for some time that dried yeast is superior to live yeast as a thiamine source for rats, ${ }^{154}$ but it was not until recent years that this was shown to be true for man. ${ }^{99}$ In some cases indeed, as little as 17 per cent of the total thiamine was found to be available for nutritional purposes in humans. ${ }^{97,98}$ Recently, it has been shown that live yeast, when added to a diet containing adequate thiamine, ${ }^{155}$ decreases the available thiamine in the diet, and the technique has even been employed to produce thiamine deficiencies in man. In one experiment, for instance, five women on a diet containing $1.6 \mathrm{mg}$ per day of thiamine were fed $15 \mathrm{gm}$ of live baker's yeast, which decreased the average urinary thiamine level from 374 to $101 \mu \mathrm{g}$ per day. As little as $150 \mathrm{gm}$ of yeast was found to depress the excretion to $40 \mu \mathrm{g}$ per day.

A variety of studies on this phenomenon have resulted in the conclusion that the effect is due to the inability of the intestine to absorb the thiamine, for mechanical reasons. An inverse relationship exists in such cases between the fecal and urinary thiamine, and it appears that a large proportion of the living yeast cells ingested pass through the intestinal tract without rupturing. These cells apparently have an active thiamine uptake, and the yeast thiamine, whether free or phosphorylated, does not 
readily diffuse out of the intact cell. In this manner a mechanical barrier materially influences the availability of the thiamine. Boiling, or other measures which fracture the yeast cells effectively, destroy the antithiamine effect. In general, the other $B$ vitamins of live yeast appear to be similarly unavailable for nutritional purposes by virtue of the same effect. Effects such as these are by no means limited to yeast, and emphasize the desirability of obtaining information regarding the availability of the B vitamins in any particular food source for any species dependent upon that source. ${ }^{155 a}$

\section{Natural Inhibitors and Inactivators}

It has been discovered in recent years that there exists in nature a group of substances which are able to exercise a pronounced effect upon the nutritional value of $\mathrm{B}$ vitamins, either by destroying them, by irreversibly binding them, or by competing with them for some enzyme system involved in the function of the vitamin. Typical of these effects are the thiaminases, which enzymatically destroy thiamine; avidin, which tightly binds biotin; and a possible pellagragenic factor in corn, which competes with nicotinic acid. In addition to these rather clear-cut cases, a large number of less thoroughly studied examples are known where the nature of the effect is not as yet clear. The consideration of these various effects is here undertaken in the order mentioned, to emphasize the type of mechanism involved, rather than the details of any specific case.

The effects of a large group of synthetic inhibitors are considered in a later section; the application of the use of inhibitors and inactivators to the assessment of $\mathrm{B}$ ritamin requirements has already received passing mention (p. 260).

Enzymatic Inactivation of B Vitamins. It was in 1932 on the farm of J. S. Chastek that a fatal disease of domestic foxes was first observed, although it was not until some years later, when the affliction had reached major economic proportions, that it was realized that the fatal symptoms followed closely upon the incorporation of raw fish into the animal stock diet. The careful studies of Green et al. ${ }^{156}$ indicated clearly that the affliction, which generally terminated fatally in from two to three months, was probably due to a thiamine-splitting enzyme in the raw fish. This was soon shown to be so, ${ }^{157}$ and the thiaminase was found in most uncooked fresh water fish and molluses, and in a few salt-water forms. It was also found that the enzyme produced similar effects in the diet of cats ${ }^{158}$ and could potentially act in this manner in man. It was also active in suppressing the growth-promoting effects of thiamine on yeast ( $E n$ domyces vernalis). ${ }^{159}$ The enzyme consists of a heat-labile, nondialyzable 
and a heat-stable, dialyzable fraction (possibly manganese), and catalyzes the overall reaction:<smiles>Cc1ncc(Cn2sc(CO)[n+]2Cl)c(N)n1</smiles><smiles>Cc1ncc(CO)nc1CCO</smiles>

that is,

$$
\mathrm{R}^{\prime} \mathrm{CH}_{2} \mathrm{~N}^{+} \mathrm{R}_{3}+\mathrm{H}_{2} \mathrm{O} \rightleftharpoons \mathrm{R}^{\prime} \mathrm{CH}_{2} \mathrm{OH}+\mathrm{NR}_{3}+\mathrm{H}^{+}
$$

The reaction is somewhat unique in that a hydrogen ion is formed in the process. Studies of the reaction employing enzyme extracts yield unidentified pyrimidine derivatives, while the pyrimidylmethyl alcohol is only obtained by using whole tissue suspensions. Whereas a number of $o$-amino derivatives of aromatic compounds are able to inhibit this reaction, $m$ substituted compounds generally activate it. This activation by substances such as $m$-nitroaniline and $m$-aminobenzoic acid is believed to occur by virtue of a combination between the $m$-amino group of the accelerator and the 5-methylene group of the pyrimidine moiety as it is split off. When $m$-nitroaniline is used as the accelerator (or acceptor) for instance, it is possible to isolate from the reaction mixture $\mathrm{N}$-(2-methyl-6-aminopyrimidyl-5-methyl)-m-nitroaniline. Presumably the enzyme itself catalyzes only the first reaction, a sort of transmethylation in which the methyl group is substituted with a pyrimidine derivative, and in which the substituted methyl group is transferred from a quaternary amine to a primary amine. Subsequent reactions in vivo then hydrolyze the secondary amine formed to the pyrimidylmethyl alcohol. ${ }^{159 a}$ The enzyme is of additional interest in that it rapidly destroys the thiamine in dead carp, unless the enzyme is inactivated by immediate heating after death. Employing known bioassay techniques (p. 283), it has been found that 42 per cent of a test dose of $7.5 \mathrm{mg}$ of thiamine (and 50 per cent of the thiamine in the basal diet) is destroyed by the consumption of $100 \mathrm{gm}$ of raw clams. ${ }^{100}$ The anti-thiamine activity of a large variety of plant materials has been shown to be due to nonenzymatic factors, and the "Chastek factor" remains the principal example of enzymatic B vitamin destruction. Inactivation of B Vitamins by Binding Agents. In $1916^{160}$ Bateman 
made the observation that raw egg white has a toxic effect when incorporated into the diet, and in 1927 Boas ${ }^{161}$ observed the presence in certain foods of an organic substance that would protect against this effect. As the result of extended subsequent researches Eakin et al. found that the symptoms of egg-white toxicity were due to the presence in egg white of a protein, avidin, ${ }^{162-164}$ which is able to bind biotin in a firm complex which is not readily broken by the usual digestive processes. An enzyme is present in the blood, however, which can break the combination. Subsequently, the feeding of raw egg white has become a standard procedure in producing the symptoms of biotin deficiency in a wide variety of animals. ${ }^{165}$

It has recently been suggested that the retardation of sexual development in chickens, which occurs on diets containing otherwise optimal amounts of vitamins and minerals, but large amounts of whole milk powder, may be due to a similar avidin-like effect. ${ }^{166}$

Inhibition of B Vitamin Activity by Competitive Action. For three centuries preceding the discovery that nicotinic acid deficiency was involved in the etiology of pellagra, it was realized that the affliction was associated with the use of corn as a major portion of the diet, and it was suggested from time to time that pellagra was due to some toxic agent in the corn. This theory completely disappeared for a time when it was shown that pellagra primarily indicates a nicotinic acid deficiency. It was soon realized, however, that in consideration of the amounts of nicotinic acid and tryptophan present in various corn diets, there remained far too high an incidence of pellagra. Thus on the rice diets of India, which provided about $5 \mathrm{mg}$ of nicotinic acid per day, pellagra was rare, while it was endemic in Moldavia, where the staple corn dicts provided $15 \mathrm{mg}$ per day. ${ }^{72}$ Similar paradoxes were encountered broadly in the United States. ${ }^{167}$ It thus appears that corn does, in fact, contain a pellagragenic factor.

Woolley ${ }^{168}$ has suggested that the toxic action may be due to some structural analogue of nicotinic acid, or its associated metabolites, which competes with the nutrilite for some enzyme (Chap. VI D), thus preventing the full nicotinic acid activity. A one hundred thousandfold concentration of the factor was achieved, using mice as assay animals, and the toxic effect was found to be reversed by nicotinamide. Since it was reported that 3 -indoleacetic acid produced a corn-like pellagragenic action in rats, and this substance is known to be present in corn in considerable amounts, heteroauxin was believed to be the toxic agent. ${ }^{169}$ Subsequent experiments, however, have indicated this to be untrue, and the chemical nature of the pellagragenic agent in corn, if it exists, remains as yet unknown. ${ }^{170-172}$ Subsequent to the study of thiaminase, it was found that a wide variety 
of plant materials have anti-thiamine activity, and it was at first thought that a similar enzymatic principle was involved in these plants. Later, however, it was found that these antithiamine effects were due to thermostable principles, so that it now appears, but not unequivocally, that an antimetabolite principle is involved.

Bhagvat and Devi ${ }^{173}$ found that chloroform-water extracts of rice polishings, ragi (Eleusine coracana), green grain (Phaseolus radiatus), mustard seed (Brassica juncea), yellow cotton seed (Gossipum sp.), and linseed (Linum usitatissimum) have an inactivating effect on both free and combined thiamine. The active factor contains a dialyzable and a nondialyzable portion. Incubation for short periods of the crude material or organic extract with thiamine or thiamine-containing products indicated a loss of thiamine activity, as shown by thiochrome tests and rat and pigeon assays. The product was, however, active for mosquito larvae, suggesting the possibility of a chemical cleavage analogous to the bisulfite cleavage, in which the fission products have activity for some species. This would not involve inhibitory effects in the sense that we are considering them, and chemical nonenzymatic inactivation would be more analogous to enzymatic destruction if this mode of action is proved to exist. Unfortunately, further information upon this point is lacking. "Fern poisoning," which afflicts horses and cattle that consume considerable amounts of the fern Pteris aquilina, has also been shown to exert its toxic effect by producing a thiamine deficiency, ${ }^{174}$ and has indeed been proposed as another way of bringing about such deficiencies experimentally. In this ease the highly thermostable material seems to differ from the active substance in Bhagvat and Devi's work in some characteristics, and although the mode of action is unknown, an antimetabolite effect seems unlikely.

A naturally occurring vitamin $\mathrm{B}_{6}$ inhibitor has been shown to exist in linseed oil meal, ${ }^{174 a}$ and vitamin $B_{6}$ deficiency is rapidly induced in chicks when their diet contains 30 per cent of this meal. Usual dietary levels of vitamin $B_{6}$ do not affect the inhibitor, but synthetic pyridoxine added in higher levels completely prevents the anti-pyridoxine activity. Water pretreatment of the meal also abolishes the activity, but it does not change the vitamin $B_{6}$ content.

Rat growth on a low casein diet is inhibited by threonine, and this inhibition is prevented by either tryptophan or niacin. ${ }^{174 \mathrm{~b}}$ Both threonine and phenylalanine are said to intensify the symptoms of niacin-tryptophan deficiency in the rat. ${ }^{174}$

The possible presence in synthetic vitamin preparations of impurities - which have inhibitory effects should be given some consideration. A recent example of this was the report that folic acid inhibited the activity 
of milk and liver xanthopterin oxidase and xanthine oxidase. ${ }^{174 d, \mathrm{e}} \mathrm{A}$ later report ${ }^{17+f}$ indicated that the effect was due to an impurity, probably a pteridyl aldehyde photofission product, which also had the ability to inhibit rabbit liver quinine oxidase. It is at present uncertain whether or not this impurity could account for the undesirable neurological effects observed in the treatment of macrocytic anemias with synthetic folic acid. (p. 416)

It has recently been reported that the feeding of 300 to $500 \mathrm{mg}$ of adenine per day to dogs produces a pellagra-like condition, and it seems possible that the large excess of this substance might well interfere by competition with the function or synthesis of some other metabolite. ${ }^{175}$

Mention has previously been made (p. 260) of the toxic effect of lycomarasmine upon certain species, and the reversal of this effect by strepogenin. In species that require nutritional strepogenin, it seems apparent that the ingestion of quantities of the tomato wilt might readily influence nutritional requirements for strepogenin.

It is also worthy of passing mention that such synthetic products as araboflavin and dulcitoflavin inhibit riboflavin activity in rats; ${ }^{176}$ and naturally occurring analogues of a similar nature, should they exist, might be expected to be similarly effective in causing increased requirements for riboflavin in the diet. These and other synthetic inhibitors are considered in a later section.

Miscellaneous Antivitamin Effects. Various other instances of antivitamin effects have been reported from time to time in the literature, but have not appeared to be of sufficient importance to merit further study. A review of these reports would be tedious and uninformative. Mention should be made, however, of several recent reports of this general nature.

With the advent of rapid transportation and refrigeration, a variety of new and strange food plants may appear in the markets of the civilized world. It should be anticipated that from some of these, unique dietary problems may arise. The fact that a plant has been utilized as a food by some remote native population is not in itself a guarantee of the desirability of its incorporation into a diet. Indeed, B vitamin deficiencies are common among primitive tribes (contrary to the general opinion), and have probably existed from prehistoric times. While this is due to a wide variety of factors, antivitamin effects play at least some part. An interesting example of these points is the case of manioc. ${ }^{177}$ A preparation of this plant, called "gari," is one of the staple foods in Nigeria, although its incorporation into the diet produces a syndrome (in native school children), which is improved considerably by riboflavin (but not niacin) and cured by the administration of marmite. 
It is unnecessary, however, to consider strange food sources to find such effects. Gross dietary distortions in a normal dietary regime may bring such quantities of an apparently innocuous substance into the diet that effects which are usually too slight to measure become important. Thus the addition of various quantities of roasted coffee to the diet of rats and dogs produced in the dogs diuresis, catharsis, weight loss, flaccid paralysis, greying of the hair, an eye condition, and convulsions; and produced alopecia, weight loss, edema, and death in the rats. ${ }^{178}$ In dogs, inositol appeared to cure the paralysis, and biotin cured the "weepy eye" symptoms. The curative properties of inositol and whole liver were not, however, complete in the rat. The alopecia in the rat appears to be due to the caffeine. The similarity of caffeine to various other purines involved in metabolism (and the known presence in coffee of a wide variety of other analogues of $B$ vitamins and other metabolites) might suggest the existence of such toxic effects.

Finally, new processing methods in food technology may result in antivitamin effects by the production of synthetic antivitamins. Thus the agenizing of flour (bleaching with $\mathrm{NCl}_{3}$ ) forms products which have been shown to be responsible for canine distemper, and it has been suggested ${ }^{179}$ that this is due to chlorination of the aromatic groups in the gluten. Such chloro-amino acids are known to be inhibitors of amino acid . metabolism, and similarly modified $\mathrm{B}$ vitamins would be capable of a similar effect. To date, fortunately, no such case has been reported.

\section{Influence of the Intestinal Flora upon the B Vitamin Requirement}

It has long been known that the microorganisms of the digestive tract are in many cases able to synthesize many of the B vitamins, and that these may well have a significant role in supplementing the nutritional supply in order to meet the B vitamin requirement. Studies of this aspect of vitamin nutrition have been numerous and extensive, but because of the equivocal nature of the techniques available for such studies, the results obtained have been at best qualitative, and frequently difficult to interpret. Because of the uncertain nature of the results, the vast amount of data bearing on the subject, and the presence in the literature of excellent reviews on this topic, ${ }^{180-181}$ it does not seem practical or expeditious in this volume to consider the problem in detail. For this reason, only a brief summary of the major points of interest is here presented.

Numerous studies have dealt at great length with the analysis of intestinal and rumenal flora, and the abilities of microorganisms to synthesize the various B vitamins. Some organisms which are known to synthesize specific B vitamins are listed in Table 15. Analyses have shown 
a varied and extensive list of organisms which inhabit the intestinal tract, frequently predominated by the coliform group, lactobacilli, enterococci, and yeasts. ${ }^{182}$ Intestinal bacteria which require nutritional sources of B vitamins also exist, e.g., lactobacilli (p. 307), most frequently in relatively small numbers although this fortuitous fact is seldom considered and has been little investigated. The nature of the factors influencing the intestinal flora, and its variation among species has been studied at length, but the valid information derived from such studies is limited for the most part to the influence of diet on the nature of the organisms present. ${ }^{182 \mathrm{a}}$

The nature of the carbohydrate, protein, fat, and vitamin content of the diet has a considerable effect upon the relative numbers of the various types of organisms to be found in the intestinal lumen, depending upon the selective effects of these dietary constituents in promoting or depressing the growth of the various organisms. The phenomenon of "refection," 183 in which high starch diets protect experimental rats on a B-deficient diet, has long been known, and is generally believed to be due to the stimulation of thiamine-producing organisms in the rat intestine. Various drugs also have pronounced effects, and antibiotic substances may depress intestinal vitamin synthesis to a very low level.

It is generally recognized that the entire $\mathrm{B}$ vitamin nutritional requirement of ruminants may be met by the microflora of the rumen, so that $\mathrm{B}$-avitaminoses are rare in this group of animals. In young ruminants, before the microflora is well established, some deficiencies may occur, however. ${ }^{184}$ In most other higher animals, a lesser and more variable part of the requirement is so met. Limited evidence suggests that the diet of the pig is more subject to fluctuations in intestinal synthesis than most other species, ${ }^{185}$ although the reasons for this are unknown. Relatively little is known at all concerning the effects or nature of intestinal flora in the nonmammalian vertebrates, or in the invertebrates, although there is ample evidence to suggest that bacterial vitamin synthesis may be a critical factor in fulfilling nutritional requirements in the entire plant and animal kingdoms.

The study of the extent to which intestinal synthesis may affect the nutritional requirement in any given case has been approached in several ways. Most frequently sulfonamides and antibiotics have been fed, and their effect on the nutritional requirement as judged by the various techniques previously discussed (Chap. I C) has been observed. ${ }^{186}$ Such studies applied to man and domestic and laboratory animals have given us most of the existing data regarding the intestinal synthesis of the B vitamins in general, and biotin and folic acid in particular. Other special diets have also been used from time to time with some success, when the 
TABLE 15. Some Common Bacteria which Synthesize B Vitamins*

\begin{tabular}{|c|c|c|c|c|c|c|c|c|}
\hline Organism & $\begin{array}{l}\text { Thia- } \\
\text { mine }\end{array}$ & $\begin{array}{l}\text { Ribo- } \\
\text { flavin }\end{array}$ & Niacin & $\underset{\mathrm{B}_{6}}{\operatorname{Vitamin}}$ & $\begin{array}{l}\text { Panto- } \\
\text { thenic } \\
\text { acid }\end{array}$ & Biotin & $\begin{array}{l}\text { Folic } \\
\text { acid }\end{array}$ & Inosito \\
\hline Pfeiffer's bacillus & + & & & & & & & \\
\hline B. vulgatus & + & + & + & & & & & \\
\hline B. proteus & + & & & & & & & \\
\hline B. subtilis & + & & & & & + & & \\
\hline B. adhoerans & + & & & & & & & \\
\hline B. lactis aerogenes & + & & & & & & & \\
\hline $\begin{array}{l}\text { B. alcaligenes } \\
\text { fecalis }\end{array}$ & + & + & + & & & + & & \\
\hline $\begin{array}{l}\text { Dysentery bac. } \\
\text { strain }\end{array}$ & + & + & & & & & & \\
\hline $\begin{array}{l}\text { Corynebacterium } \\
\text { diphtheriae }\end{array}$ & + & + & & & & & & \\
\hline B. aerogenes & + & + & + & & & + & & \\
\hline B. mesentericus & + & + & + & & & & & \\
\hline E. coli & + & + & + & & & + & & \\
\hline Ps. fluorescens & + & + & + & + & + & + & + & + \\
\hline Prot. vulgaris & + & + & + & + & + & + & + & + \\
\hline Cl. butylicum & + & + & + & + & + & + & + & + \\
\hline B. bifidus & + & & & & & & & \\
\hline $\begin{array}{l}\text { Lactic acid bact. } \\
\text { strain }\end{array}$ & + & + & & & & & & \\
\hline B. vulgaris & + & + & & & & + & & \\
\hline $\begin{array}{c}\text { Aerobacter } \\
\text { aerogenes }\end{array}$ & & & + & & & + & + & + \\
\hline Staph. flavis & + & & & & & & & \\
\hline Prop. bact. strain & + & & $\cdot$ & & & & & \\
\hline Serratia mares & + & & & & & + & & \\
\hline Ps. aeruginosa & + & & & & & + & & \\
\hline E. typhosa & + & & & & & + & & \\
\hline
\end{tabular}

* Neither are the data in this table complete, nor is the table a complete list. Rather is it meant to indicate representative data, and includes only those cases where study has definitely shown synthesis by the indicated organism. $+=$ synthesize the indicated B vitamin.

diet could be suitably balanced so as to affect adversely the growth of the bacteria without influencing the dietary requirement of the host. ${ }^{187}$ Cecectomy has given some valuable data, but is technically difficult, and therefore has been rarely used. ${ }^{188}$ In addition, this technique creates a different animal from the normal one-a factor worth weighing in both 
this technique and in those methods where drugs are involved, which may likewise modify other factors influencing the requirement. In many cases, moreover, cecectomy merely serves to shift the area of bacterial synthesis to another portion of the intestinal tract.

Some idea of the extent of bacterial synthesis in humans may be gained from the excellent study of Denko et al., ${ }^{52}$ in which the urinary and fecal excretion of eight $B$ vitamins was measured over a period of time in seven individuals on controlled and analyzed diets. They found that the urinary and fecal folic acid averaged 5.5 times the intake, $p$-aminobenzoic acid 2.3 times, biotin 3.8 times, and pantothenic acid 1.1 times. Riboflavin was slightly less than the intake, and thiamine, niacin, and vitamin $\mathrm{B}_{6}$ considerably less. In this latter regard, however, certain known metabolic products of vitamin $B_{6}$, niacin, thiamine and riboflavin were not measured; hence it is likely that the excretion of these vitamins, too, may exceed the intake.

It was also found in this same study that the fecal excretion of riboflavin and $p$-aminobenzoic acid was 1.5 times the urinary excretion; fecal thiamine and niacin were double the urinary excretion; fecal biotin was four times as much; and fecal folic acid 75 times as much. Only about 60 per cent of the pantothenic acid and pyridoxine appeared in the urine, however. It would thus appear that in these well nourished individuals, bacterial synthesis of the $B$ vitamins may well exceed the normal intake.

Most nearly approaching the ideal from the scientific standpoint would be the study of the $\mathrm{B}$ vitamin requirements of bacteriologically sterile animals. While it has been possible to obtain such animals for over fifty years by aseptic delivery on Cesarean section, it has been only in recent years that the techniques have been so improved as to be practical by the efforts of Glimmer ${ }^{189}$ and Reyniers. ${ }^{190}$ Reyniers et al. have been able to obtain and raise to maturity a variety of "germ-free" animals in this fashion; and the study of the nutritional requirements of these animals promises to be one of the great advances in the science of nutrition in the years ahead. Unfortunately even in this case, however, the animals must be considered as very different from their symbiotic "cousins," and results so obtained will be viewed with caution because of this fact. Life as it exists is a highly symbiotic process, and the knowledge that is so obtained with germ-free animals will not apply with certainty to animals living in natural surroundings. No one of the approaches mentioned can be expected to supply the desired information; but over an extended period, the synthesis of data obtained by the various techniques will result in an increase in knowledge regarding the functioning of intestinal symbionts. 


\section{Bibliography}

1. Williams, R. R., and Spies, T. D., "Vitamin $B_{1}$ and Its Use in Medicine," The Macmillan Company, New York, N. Y., 1939, pp. 309-15.

2. Light, R. F., and Cracas, L. J., Science, 87, 90 (1938).

3. Nutrition Revs., 5, 237-41 (1947).

4. Lamoreux, IV. F., and Hutt, F. B., Genetics, 28, 79-80 (1943).

5. Engel, R. W., Proc. Soc. Exptl. Biol. Med., 52, 281-2 (1943).

6. Lucas, H. L., Heuser, G. F., and Norris, L. C., Poultry Sci., 25, 137-42 (1946).

7. Ershoff, B. H., Arch. Biochem., 9, 81-4 (1946).

8. Rhoads, T. F., Rapaport, M., Kennedy, R., and Stokes, J., Jr., J. Pediat., 26, 415-53 (1945).

9. Rafsky, H. A., and Newman, B., Am. J. Med. Sci., 201, 749-56 (1941).

10. Rafsky, H. A., and Newman, B., Am. J. Med. Sci., 205, 209-13 (1943).

11. Rafsky, H. A., and Newman, B., Gastroenterology, 1, 737-42 (1943).

12. Mills, C. A., Cottingham, E., and Taylor, E., Arch. Biochem., 9, 221-7 (1946).

12a. Horwitt, M. K., Liebert, E., Kreisler, O., and Wittman, P., Bull. Nat. Research Council U. S., 116 (1948).

13. Beal, V. A., Burke, B. S., and Stuart, H. C., Am. J. Diseases Children, 70, 214-19 (1945).

14. Schweigert, B. S., Sauberlich, H. E., Elvehjem, C. A., and Baumann, C. A., J. Biol. Chem., 165, 187-96 (1946).

15. Slinger, S. J., Mac Ilraith, J. J., and Evans, E. V., Poultry Sci., 25, 628-41 (1946).

16. Morris, H. P., Palmer, L. S., and Kennedy, C., Minn. Agr. Exp. Sta. Tech. Bull., 92 (1933).

17. Nutrition Revs., 3, 201-2 (1945).

18. Forbes, W. H., Milbank Mem. Fund Quart., 23, 89-96 (1945).

19. Herrington, L. P., Am. J. Physiol., 129, 123-39 (1940).

20. Mills, C. A., Am. J. Physiol., 133, 525-31 (1941).

21. Mills, C. A., Cottingham, E., and Taylor, E., Arch. Biochem., 9, 221-7 (1946).

22. Sarett, H. P., and Perlzweig, W. A., J. Nutrition, 26, 611-20 (1943).

23. Edison, A. O., Silber, R. H., and Tennent, D. M., Am. J. Physiol., 144, 643-51, (1945).

24. Kline, D. L., Friedman, L., and Nelson, E. M., J. Nutrition, 29, 35-42 (1945).

25. Mills, C. A., Cottingham, E., and Taylor, E., Am. J. Physiol., 149, 376-82 (1947).

26. Spector, H., Hamilton, T. S., and Mitchell, H. H., J. Biol. Chem., 161, 145-52 (1945).

27. Johnson, B. C., Mitchell, H. H., and Hamilton, T. S., J. Biol. Chem., 161, 357-60 (1945).

27a. Smith, E. D., Ershoff, B. H., Winzler, R. J., and Deuel, H. J., Jr., J. Nutrition, 35, 39-4S (1948).

28. Johnson, R. E., and Kark, R. M., Science, 105, 378-9 (1947).

29. Glickman, N., Keeton, R. W., Mitchell, H. H., and Fahnestock, M. K., Am. J. Physiol., 146, 538-58 (1946).

30. Williams, R. R., and Spies, T. D., "Vitamin $\mathrm{B}_{1}$ and Its Use in Medicine," The Macmillan Company, New York, N. Y., 1939, p. 293.

31. Siddall, A. C., and Mull, J. W., Am. J. Obstet. Gynecol., 49, 672-6 (1945).

32. Melnick, D., J. Nutrition, 24, 139-51 (1942).

33. Kennedy, C., and Palmer, L. S., Arch. Biochem., 7, 9-13 (1945).

34. Sure, B., Proc. Soc. Exptl. Biol. Med., 60, 250-3 (1945).

35. Evans, H. M., and Burr, G. O., J. Biol. Chem., 76, 263-72 (1928).

36. Sure, B., J. Biol. Chem., 76, 685-700 (1928).

37. League of Nations Health Committee, "Technical Committee Report on the Physiological Basis of Nutrition, London, 1935.

38. Roderuck, C., Williams, H. H., and Macy, I. G., J. Nutrition, 32, 240-65 (1946). 
39. Govier, W. M., and Greer, C. M., J. Pharmacol. Exptl. Therap., 72, 317-20 (1941).

40. Govier, W. M., J. Pharmacol. Exptl. Therap., 77, 40-9 (1943).

41. Greig, M. E., and Govier, W. M., J. Pharmacol. Exptl. Therap., 79, 169-75 (1943).

42. Alexander, B., J. Clin. Invest., 23, 259-62 (1944).

43. Greig, M. E., J. Pharmacol. Exptl. Therap., 81, 164-73 (1944).

44. Andreae, W. A., Schenker, V., and Browne, J. S. L., Federation Proc., 5, 3 (1946).

45. Levenson, S. M., Green, R. W., Taylor, F. H. L., Robinson, P., Page, R. C., Johnson, R. E., and Lund, C. C., Ann. Surg., 124, 840-56 (1946).

46. Ochoa, S., Biochem. J., 33, 1262-70 (1939).

47. Fretheim, B., Nord. Med., 27, 1574-7 (1945).

48. Williams, R. J., "The Human Frontier," Harcourt, Brace and Company, New York, N. Y., 1946, pp. 38-40.

49. Richardson, L. R., Hogan, A. G., and Kempster, H. L., Missouri Agr. Expt. Sta., Rescarch Bull. 390, 12 pp. (1945).

50. Bloomfield, A. L., J. Nutrition, 14, 111-16 (1937).

51. Berryman, G. H., Henderson, C. R., French, C. E., Goorley, J. T., Harper, H. A., Pollock, H., and Harkness, D. M., Am. J. Physiol., 145, 625-31 (1946).

52. Denko, C. W., Grundy, W. E., Porter, J. W., Berryman, G. W., Friedemann, T. E., and Youmans, J. B., Arch. Biochem., 10, 33-40 (1946).

53. Pett, L. B., "Proceedings of a Research Conference on the Relationship of Nutrition to Public Health," The Nutrition Foundation, New York, N. Y., 1943, p. 22.

54. Pett, L. B., Can. J. Pub. Health, 36, 69-73 (1945).

55. Baxter, G. E., and Stetten, D., Jr., J. Biol. Chem., 153, 607-16 (1944).

56. Dann, W. J., Federation Proc., 4, 153 (1945).

57. Lowry, J. V., Sebrell, W. H., Daft, F. S., and Ashburn, L. L., J. Nutrition, 24, 73-83 (1942).

58. Westerfeld, W. W., and Doisy, E. A., Jr., J. Nutrition, 30, 127-36 (1945).

59. Butler, R. E., and Sarett, H. P., J. Nutrition, 35, 539-48 (1948).

60. Luckey, T. D., Moore, P. R., Elvehjem, C. A., and Hart, E. B., Proc. Soc. Exptl. Biol. Med., 62, 307-12 (1946).

61. Sarma, P. S., Snell, E. E., and Elvehjem, C. A., J. Biol. Chem., 165, 55-63 (1946).

62. Czaczkes, J. W., and Guggenheim, K., J. Biol. Chem., 162, 267-74 (1946).

63. Trufanor, A. V., Biokhimiya, 11, 33-43 (1946).

64. Aschkenasy, A., and Mignot, J., Compt. rend. soc. biol., 140, 206-8 (1946).

65. Lyman, C. M., Moseley, O., Wood, S., Butler, B., and Hale, F., J. Biol. Chem., $167,177-87$ (1947).

66. Morgan, A. F., Groody, M., and Axelrod, H. E., Am. J. Physiol., 146, 723-38 (1946).

67. Nelson, M. M., and Evans, H. M., Proc. Soc. Exptl. Biol. Med., 60, 319-20 (1945).

68. Treadwell, C. R., J. Biol. Chem., 160, 601-7 (1945).

69. Griffith, IV. H., J. Nutrition, 22, 239-53 (1941).

70. Baxter, J. H., J. Nutrition, 34, 333-49 (1947).

71. McKittrick, D. S., Arch. Biochem., 15, 133-55 (1947).

72. Aykroyd, W. R., and Swaminathan, M., Indian J. Med. Research, 27, 667-77 (1940).

73. Krehl, W. A., Teply, L. J., and Elvehjem, C. A., Science, 101, 283 (1945).

74. Krehl, W. A., Teply, L. J., and Elvehjem, C. A., Proc. Soc. Exptl. Biol. Med., 58, 334-7 (1945).

75. Wintrobe, M. M., Stein, H. J., Follis, R. H., Jr., and Humphreys, S., J. Nutrition, 30, 395-412 (1945).

76. Krehl, W. A., Teply, L. J., Sarma, P. S., and Elvehjem, C. A., Science, 101, 489-90 (1945). 
77. Krehl, WV. A., Sarma, P. S., Teply, L. J., and Elvehjem, C. A., J. Nutrition, 31, 85-106 (1946).

78. Krehl, W. A., Sarma, P. S., and Elvehjem, C. A., J. Biol. Chem., 162, 403-11 (1946).

79. Singal, S. A., Briggs, A. P., Sydenstricker, V. P., and Littlejohn, J., Federation Proc., 5, 154 (1946).

80. Rosen, F., Huff, J. W., and Perlzweig, W. A., J. Biol. Chem., 163, 343-4 (1946).

81. Woolley, D. W., J. Biol. Chem., 157, 455-9 (1945).

82. Mitchell, H. K., and Nyc, J. F., Proc. Natl. Acad. Sci. U. S., 34, 1-5 (1948).

83. Cannon, M. D., Mannering, G. J., Elvehjem, C. A., and Hart, E. B., Proc. Soc. Exptl. Biol. Med., 63, 414-17 (1946).

84. Briggs, G. M., J. Biol. Chem., 161, 749-50 (1945).

85. Cooperman, J. M., MiCall, K. B., Ruegamer, W. R., and Elvehjem, C. A., J. Nutrition, 32, 37-45 (1946).

86. Sarett, H. P., and Goldsmith, G. A., J. Biol. Chem., 167, 293-4 (1947).

87. Perlzweig, W. A., Rosen, F., Levitas, N., and Robinson, J., J. Biol. Chem., 167, 511-14 (1947).

88. Krehl, W. A., Henderson, L. M., de la Huerga, J., and Elvehjem, C. A., J. Biol. Chem., 166, 531-40 (1946).

89. Frazier, E. I., and Friedemann, T. E., Quart. Bull. Northwestern Univ. Med. School, 20, 24-8 (1946).

90. Schultz, J., and Rudkin, G. T., Fcderation Proc., 7, 185 (1948).

91. Ellinger, P., and Kiader, M. M. A., Nature, 160, 675-6 (1947).

92. Cimino, S., Boll. soc. ital. biol. sper., 19, 142-4 (1944).

93. Lecoq, R., Compt. rend. soc. biol., 139, 861-3 (1945).

94. Nutrition Revs., 5, 205-6, (1947).

95. Lamanna, C., and Lewis, C., J. Bact., 51, 398-9 (1946).

95a. Kratzer, F. H., and Williams, D. E., J. Nutrition, 36, 297-305 (1948).

95b. Singal, S. A., Sydenstricker, V. P., and Littlejohn, J. M., J. Biol. Chem., 176, 1063-8 (1948).

95c. Hankes, L. V., Henderson, L. M., Brickson, IV. L., and Elvehjem, C. A., J. Biol. Chem., 174, 873-81 (1948).

95d. Kalckar, H. M., and Klenow, H., J. Biol. Chem., 172, 349-50 (1948).

95e. Kalckar, H. M., and Klenow, H., J. Biol. Chem., 172, 351-2 (1948).

95f. Kalcker, H. M., Kjeldgaard, N. O., and Klenow, H., J. Biol. Chem., 174, 771-2 (1948).

96. Hawkins, W. W., and Barsky, J., Science, 108, 284-6 (1948).

97. Melnick, D., Hochberg, M., and Oser, B. L., J. Nutrition, 30, 67-79 (1945).

98. Hochberg, M., Melnick, D., and Oser, B. L., J. Nutrition, 30, 201-8 (1945).

99. Parsons, H. T., and Collord, J., J. Am. Dietet. Assoc., 18, 805-10 (1942).

100. Melnick, D., Hochberg, M., and Oser, B. L., J. Nutrition, 30, 81-8 (1945).

101. Williams, R. J., and Kirby, H., Science, 107, 481-3 (1948).

102. Winsten, W. A., and Eigen, E., Proc. Soc. Exptl. Biol. Med., 67, 513-17 (1948).

103. Leonian, L. H., and Lilly, V. G., West Virginia Agr. Expt. Sta. Bull., 319, 35 pp. (1945).

104. Lankford, C. E., and Skaggs, P. K., Arch. Biochem., 9, 255-83 (1946).

105. Knight, B. C. J. G., Vitamins and Hormones, 3, 121-3 (1945).

106. Peters, R. A., Nature, 158, 707 (1946).

107. Polonovski, M., Busnel, R. G., and Pesson, M., Helv. Chim. Acta, 29, 1328-33 (1946).

108. Benard, H., Busnel, R. G., Chauchard, P., Mazoue, H., and Polonovski, M., Compt. rend., 223, 826-8 (1946).

109. Mueller, J. H., J. Bact., 34, 429-41 (1937).

110. Dorfman, A., Koser, S. A., Horwitt, M. K., Berkman, S., and Saunders, F., Proc. Soc. Exptl. Biol. Med., 43, 434-8 (1940).

111. Elvehjem, C. A., and Teply, L. J., Chem. Revs., 33, 185-208 (1943). 
111a. Mitchell, H. K., Nyc, J. F., and Owen, R. D., J. Biol. Chem., 175, 433-8 (1948).

111b. Singal, S. A., Sydenstricker, V. P., and Littlejohn, J. M., J. Biol. Chem., 176, 1051-62 (1948).

111c. Henderson, L. M., J. Biol. Chem., 181, 677-85 (1949).

112. Krehl, W. A., de la Huerga, J., Elvehjem, C. A., and Hart, E. B., J. Biol. Chem., 166, 53-7 (1946).

113. Andrews, J. S., Boyd, H. M., and Gortner, W. A., Ind. Eng. Chem., Anal. Ed., 14, 663-6 (1942).

114. Krehl, W. A., and Strong, F. M., J. Biol. Chem., 156, 1-12 (1944).

115. Krehl, W. A., Elvehjem, C. A., and Strong, F. M., J. Biol. Chem., 156, 13-19 (1944).

116. Dann, W. J., and Huff, J. W., J. Biol. Chem., 168, 121-7 (1947).

117. Hughes, E. B., and Smith. R. F., J. Soc. Chem. Ind. London, 65, 284-6 (1946).

118. Snell, E. E., and Guirard, B. M., Proc. Natl. Acad. Sci. U. S., 29, 66-73 (1943).

119. Miller, E. C., and Bauman, C. A., J. Biol. Chem., 159, 173-83 (1945).

120. Snell, E. E., and Rannefeld, A. N., J. Biol. Chem., 157, 475-89 (1945).

120a. Junqueira, P. B., and Schweigert, B. S., J. Biol. Chem., 174, 605-10 (1948).

121. Sarma, P. S., Snell, E. E., and Elvehjem, C. A., J. Biol. Chem., 165, 55-63 (1946).

122. Sarma, P. S., Snell, E. E., and Elvehjem, C. A., Proc. Soc. Exptl. Biol. Med., 63, 284-6 (1946).

123. Rubin, S. H., and Scheiner, J., J. Biol. Chem., 162, 389-90 (1946).

124. Melnick, D., Hochberg, M., Himes, H. W., and Oser, B. L., J. Biol. Chem., 160, 1-14 (1945).

125. Nutrition Revs., 4, 111-12 (1946).

126. Woolley, D. W., J. Biol. Chem., 130, 417-19 (1939).

127. Knight, B. C. J. G., Vitamins and Hormones, 3, 155-6 (1945).

128. Ackermann, W. W., and Kirby, H., J. Biol. Chem., 175, 483-4, 867-70 (1948).

129. Pfaltz, H., Z. Vitaminforsch., 13, 236-49 (1943).

130. Burlet, E., Z. Vitaminforsch., 14, 318-31 (1944).

131. Rubin, S. H., Cooperman, J. H., Moore, M. E., and Scheiner, J., J. Nutrition, 35, 499-509 (1948).

132. Silber, R. H., Arch. Biochem., 7, 329-36 (1945).

132a. Lichstein, H. C., J. Biol. Chem., 177, 125-7 (1949).

133. Williams, V. R., and Fieger, E. A., J. Biol. Chem., 166, 335-43 (1946).

134. McCoy, R. H., Felton, J. R., and Hofmann, K., Arch. Biochem., 9, 141-7 (1946).

135. Rubin, S. H., Flower, D., Rosen, F., and Drekter, L., Arch. Biochem., 8, 79-90 (1945).

136. Moore, P. R., Luckey, T. D., Elvehjem, C. A., and Hart, E. B., Proc. Soc. Exptl. Biol. Med., 61, 185-7 (1946).

137. Axelrod, A. E., Pilgrim, F. J., and Hofmann, K., J. Biol. Chem., 163, 191-4 (1946).

138. Tatum, E. L., J. Biol. Chem., 160, 455-9 (1945).

139. Frommeyer, W. B., Jr., Spies, T. D., Vilter, C. F., and English, A., J. Lab. Clin. Med., 31, 643-9 (1946).

140. Martin, G. J., Moss, J., and Avakian, S., J. Biol. Chem., 167, 737 (1947).

141. Edwards, P. C., Starling, D., Mattocks, A. M., and Skipper, H. E., Science, 107, 119-20 (1948).

142. Petering, H. G., Marvel, J. P., Glausier, C. E., Jr., and Waddell, J., J. Biol. Chem., 162, 477-89 (1946).

143. Suarez, R. M., Welch, A. D., Heinle, R. W., Suarez, R. M., Jr., and Nelson, E. M., J. Lab. Clin. Med., 31, 1294-1304 (1946).

144. Bethell, F. H., Meyers, M. C., Andrews, G. A., Swendseid, M. E., Bird, O. D., and Brown, R A., J. Lab. Clin. Med., 32, 3-22 (1947).

145. Scharp, E. A., and VanderHeide, E. C., Am. J. Clin. Path., 17, 761-9 (1947).

146. Spies, T. D., and Stone, R. E., Southern Med. J., 40, 46-55 (1947).

147. Jones, E., Warden, H. F., and Darby, W. J., Am. J. Med., 3, 506 (1947). 
148. Spies, T. D., Lopez, G. G., Stone, R. E., Milanes, F., Brandenberg, R. O., and Aramburu, T., Blood, 3, 121-6 (1948).

149. Kidder, G. W., and Dewey, V. C., Proc. Natl. Acad. Sci. U. S., 33, 95-102 (1947).

149a. Olson, O. E., Fager, E. E. C., Burris, R. H., and Elvehjem, C. A., J. Biol. Chem., 174, 319-26 (1948).

150. Lucas, H. L., Norris, L. C., and Heuser, G. F., Poultry Sci., 25, 373-5 (1946).

151. Jukes, T. H., Oleson, J. J., and Dornbush, A. C., J. Nutrition, 30, 219-23 (1945).

152. Woolley, D. W., J. Biol. Chem., 147, 581-92 (1943).

153. Woolley, D. W., J. Biol. Chem., 139, 29-34 (1941).

154. Walker, R., and Nelson, E. M., Am. J. Physiol., 103, $25-9$ (1933).

155. Ness, H. T., Price, E. L., and Parsons, H. T., Science, 103, 98-9 (1946).

155a. Everson, G., Wheeler, E., Walker, H., and Caulfield, W. J., J. Nutrition, 35, 209-23 (1948).

156. Green, R. G., Carlson, W. E., and Evans, C. A., J. Nutrition, 23, 165-74 (1942).

157. Sealock, R. R., Livermore, A. H., and Evans, C. A., J. Am. Chem. Soc., 65, 93540 (1943).

158. Smith, D. C., and Proutt, L. M., Proc. Soc. Exptl. Biol. Med., 56, 1-3 (1944).

159. Krampitz, L. O., and Woolley, D. W., J. Biol Chem., 152, 9-17 (1944).

159a. Sealock, R. R., and Davis, N. C., J. Biol. Chem., 177, 987-8 (1949).

160. Bateman, W. G., J. Biol. Chem., 26, 263-91 (1916).

161. Boas, M. A., Biochem. J., 21, 712-24 (1927).

162. Eakin, R. E., Snell, E. E., and Williams, R. J., J. Biol. Chem., 140, 535-43 (1941).

163. Eakin, R. E., MeKinley, W. A., and Williams, R. J., Science, 92, 224-5 (1940).

164. Eakin, R. E., Snell, E. E., and Williams, R. J., J. Biol. Chem., 136, 801-2 (1940)

165. Hertz, R., Physiol. Revs., 26, 479-94 (1946).

166. Alibrandi, A., Arch. fisiol., 44, 163-79 (1945).

167. Dann, W. J., Federation Proc., 3, 159-61 (1944).

168. Woolley, D. W., J. Biol. Chem., 162, 179-80 (1946).

169. Kodicek, E., Carpenter, K. J., and Harris, L. J., Lancet, 251, 491-2 (1946).

170. Rosen, F., and Perlzweig, W. A., Arch. Biochem., 15, 111-14 (1947).

171. Krehl, W. A., Carvalho, A., and Cowgill, G. R., Federation Proc., 6, 413 (1947).

172. Sarett, H. P., and Goldsmith, G. A., Federation Proc., 6, 288 (1947).

173. Bhagvat, K., and Devi, P., Indian J. Med. Research, 32, 131-7 (1944).

174. Weswig, P. H., Freed, A. M., and Haag, J. R., J. Biol. Chem., 165, 737-8 (1946).

175. Raska, S. B., Science, 105, 126-7 (1947).

176. Emerson, G. A., Wurtz, E., and Johnson, O. H., J. Biol. Chem., 160, 165-7 (1945).

177. Moore, D. F., J. Trop. Med. Hyg., 45, 129-31 (1942).

178. Briggs, G. M., Jr., Mills, R. C., Elvehjem, C. A., and Hart, E. B., Proc. Soc. Exptl. Biol. Med., 51, 59-61 (1942).

179. Mellanby, E., Brit. Med. J., 2, 885 (1946).

180. Najjar, V. A., and Barrett, R., Vitamins and Hormones, 3, 23-48 (1945).

181. Nutrition Revs., 4, 76-8 (1946).

182. Evenson, A., MeCoy, E., Geyer, B. R., and Elvehjem, C. A., J. Bact., 51, 513-21 (1945).

182a. Gall, L. S., Illingworth, B. A., Cowgill, G. R., and Fenton, P. F., J. Nutrition, 35, 27-38 (1948).

183. Roscoe, M. H., Hyg., 27, 103-7 (1927).

184. Johnson, B. C., Wiese, A. C., Mitchell, H. H., and Nevens, W. B., J. Biol. Chem., 167, 729-36 (1947).

185. Nutrition Revs., 3, 81-2 (1945).

186. Daft, F. S., and Sebrell, W. H., Vitamins and Hormones, 3, 49-72 (1945).

187. Friedman, H., J. Nutrition, 12, 165-72 (1936).

188. Taylor, A., Pennington, D., and Thacker, J., Univ. Texas Pub., 4237, pp. 135-44 (1942).

189. Glimstedt, G., Skand. Arch. Physiol., 73, 48-62 (1936).

190. Reyniers, J. A., Trexler, P. C., and Ervin, R. F., Lobund Rept., 1, 1-120 (1946).

191. Wright, L. D., Cresson, E. L., Skeggs, H. R., Wood, T. R., Peck, R. L., Wolf, D. E., and Folkers, K., J. Am. Chem. Soc., 72, 1048 (1950). 


\section{Chapter IIIC}

\section{THE B VITAMIN REQUIREMENTS OF ANIMALS AND PLANTS}

\section{Requirements of Invertebrates}

Biochemistry in the broadest sense is concerned with the chemistry of all living species. Generally, however, biochemical investigations have been most intense in those fields that seem the most likely to bring the accomplishment of immediate results. Coupled with this, the ever urgent need for deeper insight into the factors involved in human nutrition, and to a lesser extent in the nutrition of domestic animals, has resulted in a neglect in the study of the nutritional requirements of invertebrates.

In very recent years, however, a number of factors have brought about some change for the better in this regard. In the first place, commercial interests, increasingly mindful of the economic benefits of research, have diverted investigations in several cases into this field of inquiry. The manufacturers of insecticides and those in the sea-food industries have thus taken an active interest in what quite recently was purely an academic subject. Secondarily, the problems of the recent war and life in tropical places have brought about a renaissance in parasitology, which has resulted in considerable new knowledge of the vitamin requirements of a number of parasitic and vector invertebrates.

Aside from the purely academic, commercial, and medical reasons for inquiry into invertebrate nutrition, an increasing awareness of the benefits to be derived from a study of comparative biochemistry seems likely to bring about even greater interest in this field. We have already considered one such topic-Cowgill's application of comparative requirements in assessing human vitamin requirements (p. 246). The challenge for investigation in comparative biochemistry is very great. In many common phyla (porifera, coelenterata, echinodermata, annelida) literally nothing is known about vitamin requirements (or indeed nutritional requirements). Still greater is the challenge, because up to the time of this writing, no animal had as yet been reared and bred on a chemically defined diet. In view of these considerations there are many limitations to anything resembling a thorough review of invertebrate $\mathrm{B}$ vitamin requirements, and as to these limitations we are helpless. 
One might for comparative purposes wish to start a review with a consideration of the B vitamin requirements of green plants. Most existing evidence indicates that green plants are able to meet their own vitamin requirements by synthesis. Such plants, however, are seldom studied under sterile conditions, and plant embryos and roots are known to require in many cases a nutritional source of $B$ vitamins; hence this field of investigation offers limitless opportunities for study. A brief consideration of green plant requirements, therefore, occurs later in this chapter.

Passing briefly to the bacteria whose $B$ vitamin requirements have been studied extensively, we find a broad spectrum of requirements. While many bacteria are able to meet all their nutritional requirements by synthesis, others require one or many of the known $\mathrm{B}$ vitamins, and some are even more fastidious. ${ }^{1,2}$ The $B$ vitamin requirements of bacteria have been extensively reviewed several times in recent years, ${ }^{3}$ and various aspects of bacterial and plant nutrition are considered in greater detail elsewhere (p. 336). As concerns the most "simple" living forms, the viruses, we know so little of their metabolism and nutrition that almost nothing can be said. ${ }^{4}$

Like the bacteria, the protozoa constitute a heterogeneous group nutritionally as well as taxonomically. Many of the more plant-like forms grow well in inorganic salts media, and have the ability to synthesize all the B vitamins, while the so-called "higher" protozoa require most if not all of them. Still others require as yet unidentified growth factors. The nutrition of the protozoa has been intensively studied in recent years, and excellent reviews are available. ${ }^{5,6}$

Thus in passing from the higher plants to the higher animals, we pass, in general, from cells requiring none to cells requiring most of the B vitamins. At the juncture of the two kingdoms, we find single-celled organisms that run the gamut of requirements, in general resembling nutritionally the kingdom that they most resemble on the basis of other conventional taxonomical considerations. If the line of demarcation between the plant and animal kingdoms is thus sharply drawn nutritionally within the single-celled forms, we may expect that as we ascend the evolutionary scale in the animal kingdom, no perceptible trend in B vitamin requirements will occur, i.e., the lowest metazoan forms would require many if not most of the $B$ vitamins. As shown in the discussion which follows, the limited information available suggests that this is essentially so. The recent development of methods for the study of the biosynthetic abilities of developing embryos should even further assist in examining this generalization in the years to come. ${ }^{7}$

The Lower Invertebrates. In considering the B vitamin requirements of the lower invertebrates the absolute lack of evidence concerning the 
nutrition of the Porifera and Coelenterata creates a great gap. The economic importance of these phyla may soon, however, induce sufficient effort into the study of this group of animals to overcome the experimental difficulties inherent in the investigation of their nutrition.

Regarding the Plathelminthes, studies on the nutrition of Planaria maculata have shown that a heat-labile, ether-soluble fraction of liver is required for growth, ${ }^{8}$ and that a similar and possibly identical factor is inactivated by egg white. Despite the conflicting nature of the descriptions, there is some reason for believing the factor to be biotin. It has been shown ${ }^{9}$ that the number of tapeworms (Hymenolepis diminutia) in rats on various vitamin-deficient diets varies broadly. Thiaminedeficient rats have worm populations similar to those of normal rats, whereas rats deficient in the "B complex" vitamins have reduced numbers of worms. We shall see shortly that, by contrast, studies of parasitic round worm infections in humans and rats show increased populations of worms in malnutrition.

The fish tapeworm, Diphyllobothrium latum, infects humans and causes a macrocytic anemia differing from Addisonian pernicious anemia in that spinal cord involvement is rare, gastric acid is seldom decreased, the anemia is cured by removal of the worm, etc. Extracts or suspensions of the worm are inactive, and considerable evidence suggests that the effect produced may possibly be due to absorption by the worm of the antipernicious anemia factor (vitamin $\mathrm{B}_{12}$ ) in the host, in a manner similar to that in which live yeast may deplete the intestinal tract of thiamine (p. 291),${ }^{10}$ and produce the corresponding avitaminosis. One is caused to wonder in passing whether a metabolic factor is involved in the production of rat liver sarcoma by the flat worm Cysticercus. ${ }^{11}$

The nutrition of a number of round worms (Anguilla oxophilla, ${ }^{12}$ Neoplectana glaseri, ${ }^{13}$ and Ancylostoma caninum ${ }^{14}$ ) has been studied, but never in such a manner as to indicate much with regard to their $B$ vitamin requirements. All require complex media (yeast autolysatepeptone, dextrose-veal infusion-live yeast, live bacteria) and in many cases, the diet is still inadequate for reproduction without supplementation with other materials of a complex nature.

It is generally believed that hookworm infection in man is commonest in areas where malnutrition is prevalent, and that the improvement of the general dietary status of an individual may frequently purify the body of the parasite. Moreover, in experiments using vitamin-deficient rats and the round worm Nippostrongylus muris, ${ }^{15}$ it was found that thiamine- and riboflavin-deficient rats contained appreciably more parasites than did normal controls. Mention of this work is made to emphasize the unreliability of data obtained by deducing the nutritional require- 
ments of the parasite from the nutritional status of the host, since it is extremely improbable that the diminished amounts of thiamine and riboflavin per se improved the nutrition of the parasite. Unfortunately, much of our knowledge of the nutrition of parasitic organisms stems from data of this nature.

If the information so far presented seems sparse, it is even more striking that data are virtually nonexistent regarding the $\mathrm{B}$ vitamin requirements of the Echinodermata and Annelida. This is particularly strange in view of the relative ease in the handling of such animals as the earthworms and leeches. Similarly, but more obviously because of experimental difficulties, nothing at all is known of the $\mathrm{B}$ vitamin requirements of Mollusca, although some indirect data ${ }^{16}$ suggest that the requirement is a complex one.

The Arthropoda. We have seen that the requirement for no single $B$ vitamin has been definitely established for any metazoan invertebrate below the Arthropoda, and a similar lack of information exists with regard to the economically important Crustacea. The Insecta, then, represent the only class of metazoan invertebrates concerning which we have reliable information as to $\mathrm{B}$ vitamin requirements, and for some insects relatively complete data are available. For this reason alone, the discussion of this class is more extensive than that of the other phyla.

Aside from the need for information regarding the nutritional requirements of insects, there are many factors to encourage their study. In general, they are readily bred in a small space and with little time and effort, and require a minimum of food and equipment for their study. Various species are herbivorous, carnivorous, and omnivorous, and hence vary widely in their natural nutrition. Factors such as weight, food and liquid intake, and environment may be readily controlled, and for these reasons insects are generally ideal experimental animals. Insects have the further advantage in nutritional experimentation in that they possess symbiotic microorganisms which may function to meet their requirements, in a manner similar to that of the vertebrates. Thus of five beetles studied by Fraenkel and Blewett, ${ }^{17}$ Tribolium confusum and Ptinus Tectus required thiamine, riboflavin, nicotinic acid, pyridoxine, pantothenic acid, biotin and choline, while Lasioderma serricorne, Sitodrepa panicea, and Silvanus surinamesis did not require all of these because of the presence of intraocellular symbionts. It is possible, on the other hand, to raise many insects in a germ-free condition, a feat involving great difficulties with higher animals. Under such sterile conditions the nutritional requirements are generally found to be more fastidious, reflecting extra needs for metamorphosis, etc. 
है

옹

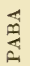

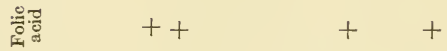
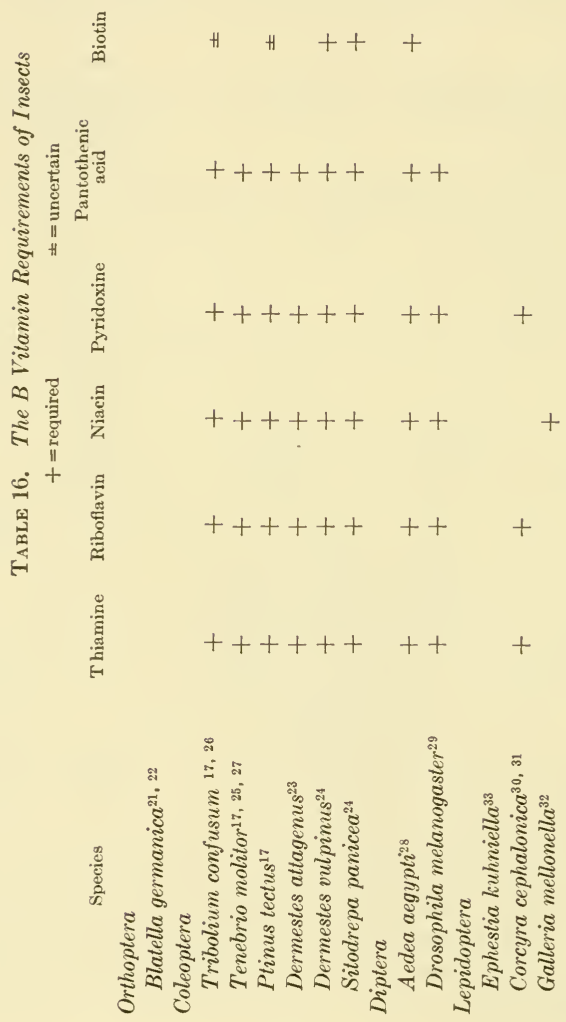
To an extent exceeding that in the vertebrates, insect B vitamin requirements vary with the phases of the animal's life. Larval requirements are much higher (and B vitamin content apparently lower) than in the adult, and the requirements for successful pupation, exuviation, and reproduction may be even more extreme. Thus Aedes aegypti larvae from bacteriologically sterile eggs complete metamorphosis on a sterile diet

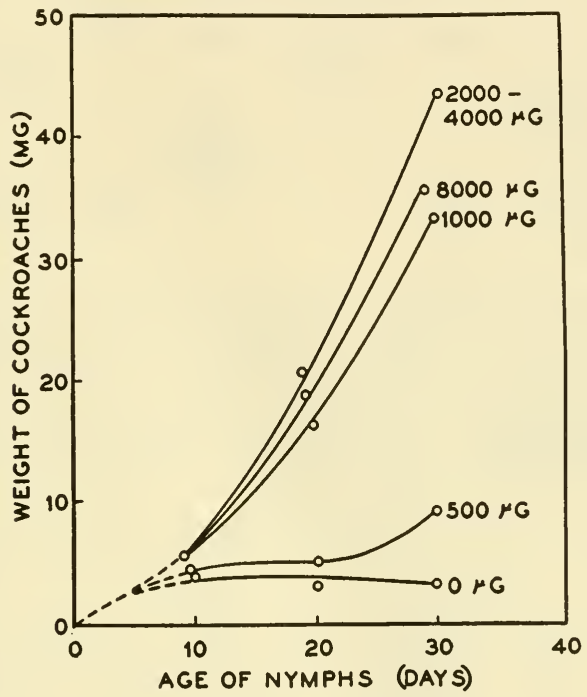

Figure 2. Growth of cockroach nymphs on various levels of choline.

only when it contains adequate amounts of biotin, ${ }^{18}$ and the creation of abnormally high $\mathrm{B}$ vitamin levels in serum causes premature shedding of the old skin of Tristoma infestans larvae. ${ }^{19}$ The high level of pantothenic acid in the royal jelly of the bee is also suggestive in this regard. ${ }^{20}$

While a relatively large number of insects has been studied, the number is actually small in view of the great variety of known species. This variety provides the broadest possible latitude for experimental work, as previously stated, and with relatively few difficulties. Illustrative of this fact are the studies of Sarma et al. with the rice moth larva (Corcyra cephalonica St.), which have demonstrated in a variety of ways to be discussed later the similarity that exists between vertebrate and arthropod vitamin metabolism. In a manner similar to that in which higher experi- 
mental animals are kept, the larvae are reared in a $30^{\circ} \mathrm{C}$ incubator on a whole wheat stock diet. Ten day-old larvae are then placed upon appropriate experimental diets, and growth measured by weekly weighing. In this manner, the experimental results obtained accord beautifully with those obtained in nutritional research with higher animals.

TABLE 17. Quantitative B Vitamin Requirements of Several Insect Species ( $\mu \mathrm{g} /$ unit of diet)

Species

Thiamine

$\begin{array}{cc}\text { Riboflavin } & \text { Niacin } \\ 0.4 & \\ \mu \mathrm{g} / \mathrm{ml} & \\ & 1.0 \\ & \mu \mathrm{g} / \mathrm{ml} \\ & 0.05-0.10 \\ & \mu \mathrm{g} / \mathrm{gm}\end{array}$

Tribolium confusum

4.0

Blatella germanica $\mu \mathrm{g} / \mathrm{gm}$

$\begin{array}{ccc}\text { Folic } & & \\ \text { acid } & \text { Biotin } & \text { Choline } \\ & 0.05 & \\ & \mu \mathrm{g} / \mathrm{ml} & \end{array}$

$0.2 \quad 0.10$

$\mu \mathrm{g} / \mathrm{gm} \quad \mu \mathrm{g} / \mathrm{gm}$

2000. -4000 . $\mu \mathrm{g} / \mathrm{gm}$

Qualitatively, the B vitamin requirements of insects appear to resemble closely those of the vertebrates. A summary of much of the existing data in this regard is given in Table 16. The limited amount of quantitative data as yet available is insufficient to make valid comparisons, but is summarized in Table 17. Consideration of these data in terms of their relationship to insect vitamin composition ${ }^{34,} 35$ shows that, as in higher

TaBle 18. Results of B Vitamin Deprivation for Several Insect Species

\begin{tabular}{|c|c|c|c|c|c|c|c|c|c|}
\hline \multirow{2}{*}{$\begin{array}{l}\text { Vitamin } \\
\text { missing from } \\
\text { Diet }\end{array}$} & \multicolumn{3}{|c|}{ Tribolium } & \multicolumn{3}{|c|}{ Ptinus } & \multicolumn{3}{|c|}{ Silvanus } \\
\hline & $\begin{array}{l}\text { Growth } \\
\text { rate* }\end{array}$ & $\begin{array}{c}\text { Number } \\
\text { sur- } \\
\text { vivingt }\end{array}$ & Index $\ddagger$ & $\begin{array}{c}\text { Growth } \\
\text { rate }\end{array}$ & $\begin{array}{l}\text { Number } \\
\text { sur- } \\
\text { viving }\end{array}$ & Index & $\begin{array}{l}\text { Growth } \\
\text { rate }\end{array}$ & $\begin{array}{c}\text { Number } \\
\text { sur- } \\
\text { viving }\end{array}$ & Index \\
\hline Thiamine & 42 & 17 & 714 & 70 & 2 & 140 & 97 & 16 & 1552 \\
\hline Riboflavin & 31 & 0 & 93 & 0 & 0 & 0 & 94 & 9 & 846 \\
\hline Niacin & 27 & 1 & 27 & 66 & 1 & 66 & 78 & 1 & 78 \\
\hline Vitamin $\mathrm{B}_{6}$ & 70 & 16 & 1120 & 63 & 13 & 349 & 91 & 16 & 1456 \\
\hline $\begin{array}{l}\text { Pantothenic } \\
\text { Acid }\end{array}$ & 0 & 0 & 0 & 70 & 2 & 140 & 103 & 1 & 103 \\
\hline Choline & 88 & 19 & 1672 & 74 & 14 & 1036 & 89 & 16 & 1424 \\
\hline Inositol & 97 & 17 & 1649 & 98 & 17 & 1666 & 91 & 15 & 1365 \\
\hline PABA & 86 & 18 & 1548 & 98 & 18 & 1764 & 94 & 14 & 1316 \\
\hline $\begin{array}{l}\text { Yeast-fed } \\
\text { controls }\end{array}$ & 100 & 18 & 1800 & 100 & 18 & 1800 & 100 & 16 & 1600 \\
\hline
\end{tabular}

* Growth Rate: Reciprocal of the period in days in which $50 \%$ of the insects were completely developed, expressed as the percentage of the period required by a yeast fed control group.

$\dagger$ Number surviving: The number of the original group of twenty insects that completed development.

Index: Product of the growth rate and number surviving.

animals, there is a rough correlation between the amounts of the various vitamins required and the amounts found in the animal. The nicety of response obtained in determining requirements is indicated by Figure 2, which shows the growth of cockroach nymphs on various levels of 
choline. ${ }^{21}$ Such responses, however, must be assessed in terms not only of weight, but also of the time required to reach the adult stage, and the number of nymphs surviving. Indeed some workers use the product of the number of insects reaching a certain stage of development and the reciprocal of the average time required to reach that stage as a criterion

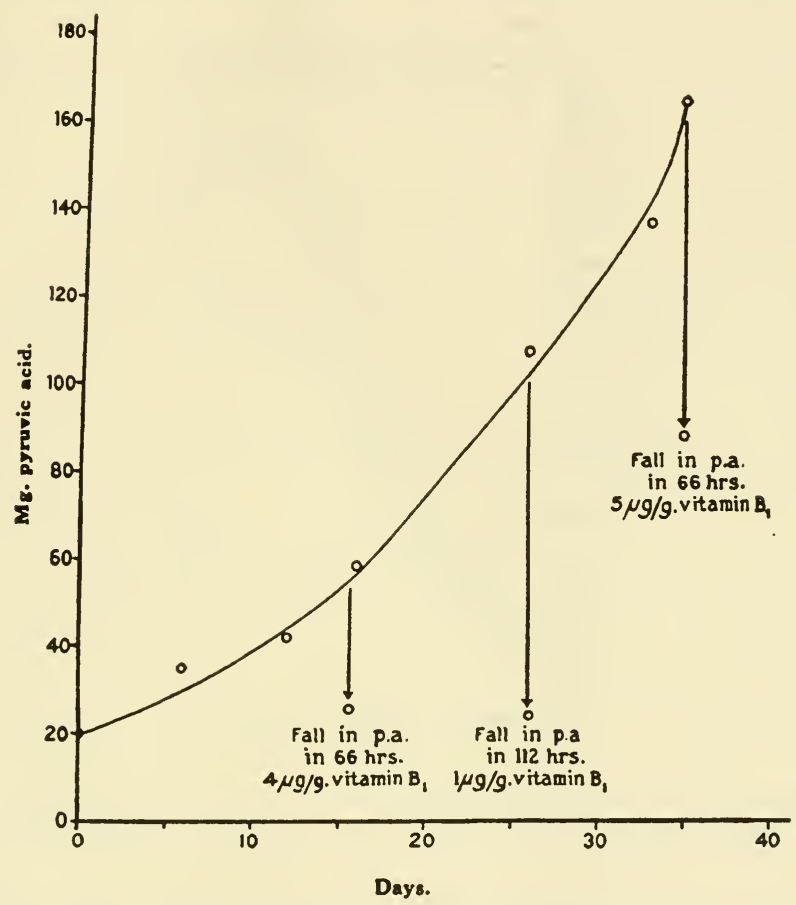

FIGURe 3. Increase in pyruvic acid in Corcyra cephalonica St. larvae on a thiamine deficient diet. Ten larvae were used in each test group. Pyruvic Acid is in $\mathrm{mg}$ per $100 \mathrm{gm}$ of larvae.

of response. ${ }^{18}$ Certain interesting conclusions are apparent when such a criterion is applied to some of Fraenkel and Blewett's data, ${ }^{17}$ as shown in Table 18. Species differences are readily apparent, as witness the critical nature of riboflavin for Ptinus. Certainly riboflavin, niacin, and pantothenic acid stand out for these three species as the B vitamins most critically required in their nutrition. 
Because of the nicety of quantitative response obtainable, Sarma and co-workers have employed Corcyra as an assay animal for thiamine, riboflavin, and pyridoxine, and obtained results which agree well with those obtained by other methods. Strain differences, however, are sufficiently great that "pure" strains would be desirable for routine insect assay work. ${ }^{30,31,36}$

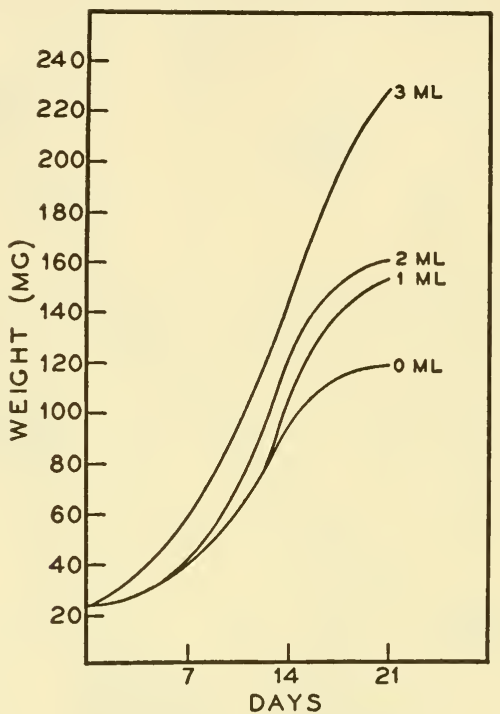

Figure 4. Growth of Corcyra Cephalonica St. larvae with various amounts of a biotin concentrate added to an egg-white diet. Ten larvae per test group.

Of even greater interest, however, is the work done by this group on vitamin metabolism in insects. ${ }^{37}$ In a manner entirely analogous to that of higher animals, thiamine-deficient Corcyra larvae show fatty tissue and nerve degeneration, and the pyruvic acid content of the deficient larvae increases to eight times that of the normal animals (p. 403), and decreases again on a high thiamine diet. Again, vitamin $B_{6}$ deficient larvae on high tryptophan diets excrete a yellow pigment which disappears on supplementation of the diet with pyridoxine, in a manner remarkably suggestive of the abnormal xanthurenic acid (yellow) excretion in vitamin $B_{6}$ deficient mammals (p. 428). As in mammals, tryptophan alone 
is able to inerease excretion of the pigment. Finally, raw egg white or avidin eoncentrate, when added to the diet, causes death of the larvae in about four weeks, while pretreatment of the eoneentrate or egg white by means which destroy avidin permits normal larval growth. When larvae are changed from egg white diets to diets containing various levels of biotin, the growth is renewed at rates in accordance with the biotin present. $^{37}$ The nicety of these responses is illustrated in Figures 3 and 4. Thus in many ways at least the rice moth larva resembles the vertebrates in its metabolism of thiamine, pyridoxine, and biotin. ${ }^{171-80}$ The extension of these studies of the Coonoor Nutrition Researeh Laboratories will be anticipated with great interest.

The insects mentioned above represent a number of the more important orders of the class Inseeta. Many others have not been investigated. The order Thysanura (silverfish), beeause of its primitive nature, ubiquitous distribution, and economic importance, certainly challenges the investigator. Indeed the entire class Arachnida, for many reasons important, remains virtually unstudied with regard to its $B$ vitamin requirements. Perhaps the single exception to this statement is the work of de Meillon and eo-workers on the blood-sucking tick, Ornithodorus moubata. These workers found that this tick (as well as the common bedbug, Cimex lactularis), when feeding on thiamine-deficient rats, requires an average of 79.7 days to develop as compared with 41.1 days on normal rats. ${ }^{38}$ They further found that definite toxic effects were observable in bloodsucking arthropods that fed on rabbits injected with $\gamma$-hexachlorocyclohexane, suggesting an interference with inositol metabolism. ${ }^{39}$ It is truly remarkable that the vast effort put into the study of tick-borne diseases has not produced similar more extended studies of the nutrition of the insect vectors. Indeed future studies of insect-borne disease may well start with a study of the nutrition of the host, as an approach to insectieides that might be organized on logical lines of reasoning, based upon present knowledge of the inhibition of vitamin metabolism.

In summary, our knowledge of invertebrate nutrition is practically nonexistent, and, at best, is based upon presumptive evidence, in the phyla below Arthropoda. This scant evidence, however, suggests that these lower phyla have extensive $\mathrm{B}$ vitamin requirements. ${ }^{40}$ Among the Arthropoda, information approaching adequacy exists only in the class Insecta, where the available data indicate that $\mathrm{B}$ vitamin requirements are as extensive as those of the vertebrates. The problems involved in invertebrate nutrition are such as to offer great indueement to the investigator, and are further significant for physiologieal, medical, economic, and taxonomic reasons. 


\section{The B Vitamin Requirements of Green Plants}

Because the major source of the B vitamins for the animal kingdom ultimately comes from green plants, and because green plants are so frequently referred to as autotrophic, and even further because of the widespread culture of plants in mineral salts-water solutions, it is customary to think of them as having no $\mathrm{B}$ vitamin requirements. It is true that the green plant as a whole will grow without an apparent exogenous supply of B vitamins or other organic nutrients. It is also true, however, that in many cases the plant may be deriving considerable nourishment from symbiotic microorganisms. It also seems reasonably well established that many green plants may not be able to synthesize sufficient $B$ vitamins for optimum growth. It has been frequently observed (and also denied) that thiamine, at least, frequently causes more luxuriant growth of some plants, when added to the nutriment. Added riboflavin has been reported to be of benefit to eggplant cultured in a synthetic medium, ${ }^{41}$ and it seems likely that other specific cases may arise involving others of the B vitamins.

Green plant embryos, young roots, cuttings, and pollen grains are, however, heterotrophic, and all are dependent upon supplies of at least some of the B vitamins from stored foods or other portions of the plant. While only a limited amount of information is available, it seems that thiamine and riboflavin ${ }^{42,} 43$ have pronounced effects on the germination of pollen grains. Biotin is highly active in stimulating the growth of roots on cuttings, ${ }^{44,182}$ but in this case its activity has been likened to that of a hormone. Indeed biotin functions in an auxin-like fashion in roots, in which it is concentrated in the tips (and also in the tips of coleoptiles) ${ }^{45}$ Biotin is synthesized by all roots, and even excreted in considerable amounts. ${ }^{46}$ Its effect on root formation in peas, for instance, is quite marked, inducing a 100 per cent increase in root formation on pea cuttings. ${ }^{4+}$ The pea embryo, however, cannot synthesize biotin, and in this case the function of biotin is more likely associated with the metabolic activities of the embryo than with its differentiation. Biotin shares with auxin $a$ and $b$ the structural feature of a five-membered ring joined to a valeric acid derivative, and may well owe its auxin-like activity to this resemblance.

The roots and embryos of green plants have been more extensively studied, but plant tissue culture techniques are generally so recent in origin as to have supplied little information. Excised roots have been grown in apparently sterile media, and show a wide variety of organic nutritional requirements. White has found some nine amino acids to be required by excised tomato roots-histidine, phenylalanine, lysine, leucine, 
isoleucine, valine, glutamic acid, proline, and serine. ${ }^{47,48}$ Thiamine is apparently not synthesized by any roots, ${ }^{49}$ but is necessary for their growth, and is active in dilutions as low as $1: 4 \times 10^{13} .{ }^{50}$ There is also some evidence to indicate that it is a stimulating factor for root formation. ${ }^{51}$ Roots are apparently able to utilize the thiazole and pyrimidine fractions of thiamine about as well as thiamine itself. ${ }^{52}$ Some, such as the tomato root, require only the thiazole portion, being able to synthesize the pyrimidine part; but others, such as the pea root, can synthesize neither part, requiring a nutritional source of both.

Work with plant embryos requires that the embryo be removed from the seed and the cotyledons from the embryo as early as possible. When these precautions are taken, the resulting embryo is found to be a heterotrophic organism, requiring a nutritional source of at least some of the B vitamins. Thiamine and nicotinie acid have been found to be required by most roots and embryos, while pyridoxine is required by a lesser number. The requirement for riboflavin and pantothenic acid is less clearly known, due to less thorough study. The requirements for the more recently discovered members of the B group of vitamins have not been reported to date. A summary of much of the known data is shown in Table 19.

\section{TABLE 19. Known B Vitamin Requirements of the Roots and Embryos of Some Green Plants}

\section{Species}

Tomato roots

Pea roots

Pea embryos

Cosmos roots

Radish roots ${ }^{1}$

Alfalfa roots

Flax roots

Clover roots

Cotton roots

Carrot roots

Datura roots

Sunflower roots

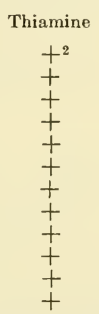

\section{Riboflavin}

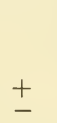

$+=$ require (for thiami

$\mathrm{S}=$ stimulates growth

- = not required.

$1=$ thiamine and niacin alone as effective as yeast extract.

$2=$ requires thiazole portion of thiamine only.

In summary, we find that the ability of green plants to synthesize B vitamins is very unevenly distributed, and is concentrated generally in those parts of the plant reserved specifically for general synthetic activity. To what extent the activities of the various tissues of animals vary in respect to B vitamin synthesis is largely unknown. While green plants as a whole survive readily without an exogenous source of $B$ vitamins, it seems apparent that parts of the plant (and phases in its life) exist that 
need some, and may in the future be shown to need most, of the B vitamins required by other organisms.

\section{The B Vitamin Requirement of the Vertebrates}

Since we have considered earlier the many factors that may so profoundly influence $B$ vitamin requirements, it would indeed be strange to set down absolute specifications for the vitamin intake of man or any other species. A casual survey of the literature reveals data pertaining to the requirements of many species, and in some cases, excellent reviews of the requirements of a particular species are available ( $\operatorname{man},{ }^{53}$ mouse,${ }^{54}$ birds, ${ }^{56}$ pigs ${ }^{57}$ ruminants, ${ }^{58}$ other animals $\left.{ }^{59,181}\right)$. For the sake of convenience alone some of the useful data are reproduced here in tabular form. Any discussion of their validity, however, would be redundant, and is deliberately avoided. In addition, the current National Research Council table of recommended daily dietary allowances for humans is here reproduced for the convenience of those who are constrained to settle upon some figure for dietary calculations. ${ }^{60}$ The amounts cited in this table, as in any other, might well be subjected to extended discussion, but a critical appraisal of human $B$ vitamin requirements would require a volume in itself. ${ }^{61}$ It may be said, however, that it would be difficult for practical reasons to construct a diet meeting the stated allowances that was still inadequate in B vitamin content. It is thus hoped that the salient fact emerging from our discussion of $B$ vitamin requirements is that even in a monograph devoted to the subject of the $\mathrm{B}$ vitamins, the authors cannot conscientiously make a statement as to the precise requirements of any species for any vitamin.

Reference was made earlier to Cowgill's comparison of the thiamine requirements of different species (p. 246). It would be expected that a similar general relationship would hold for the other B vitamins, and this is apparently so, judging from the limited data available. Despite the many errors, qualifications, and interpretations inherent in any presentation of such data, Figures 5 to 14 are presented, to emphasize the general trend that exists for smaller species to have a higher relative $\mathrm{B}$ vitamin requirement than larger ones. ${ }^{183}$

When one considers at length the conflicting evidence regarding whether or not various animal species require certain $\mathrm{B}$ vitamins, the conclusion is inevitably reached on the basis of the existing evidence that all the species studied require an exogenous source of all the $B$ vitamins, with the probable exception of nicotinic acid (and choline, although this has "nonvitamin" functions and the requirement should be considered in a different light because of this). In those cases where there is no nutritional requirement, intestinal flora account adequately for the discrepancy. In 


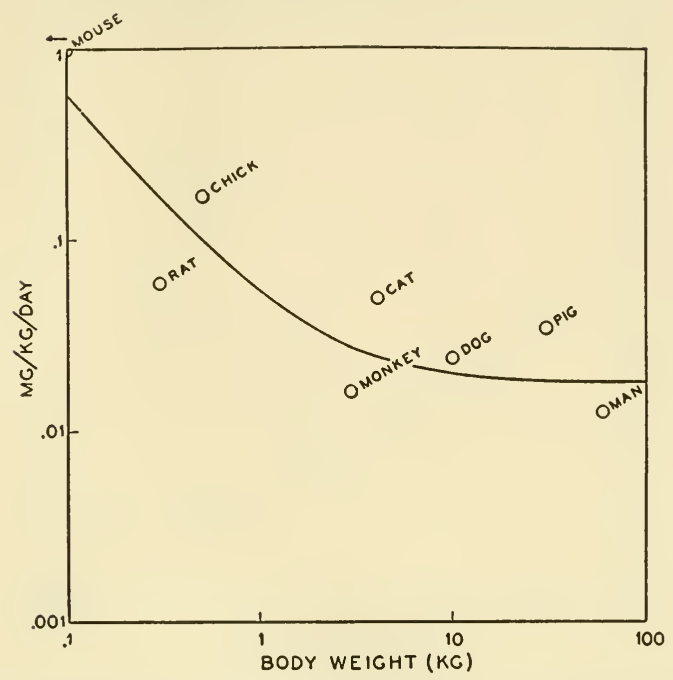

Figure 5. Relationship of the size of a species to its thiamine requirement.

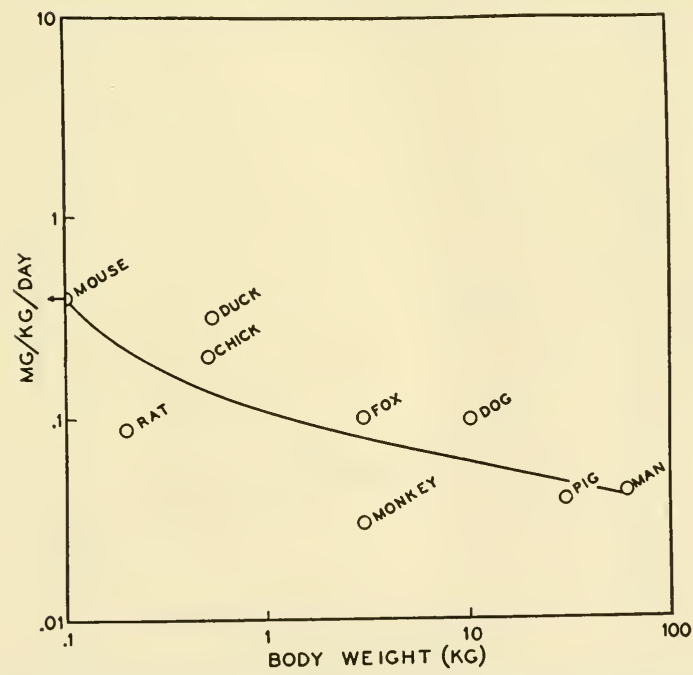

Figure 6. Relationship of the size of a species to its riboflavin requirement. 


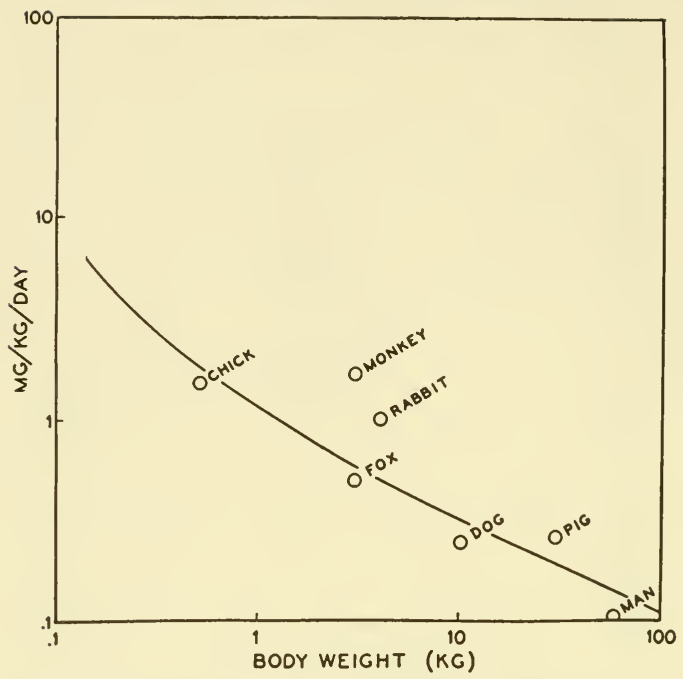

Figure 7. Relationship of the size of a species to its nicotinic acid requirement.

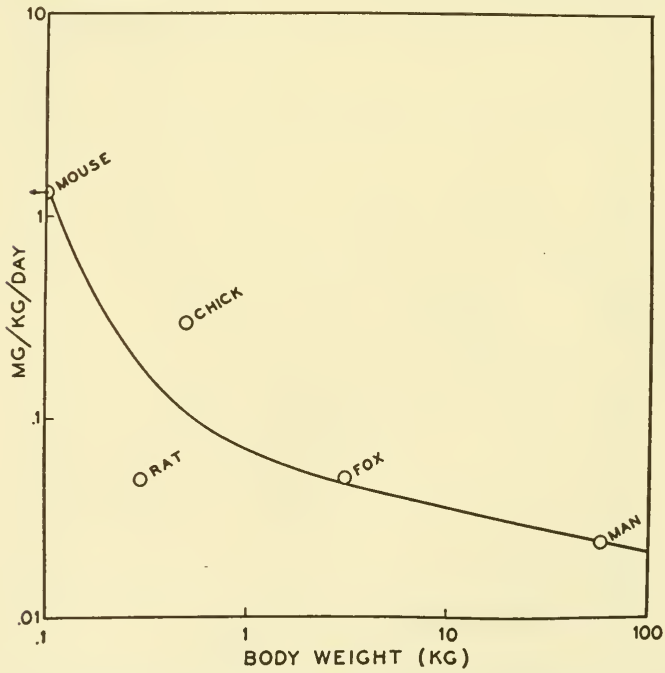

Figure 8. Relationship of the size of a species to its pyridoxine requirement. 


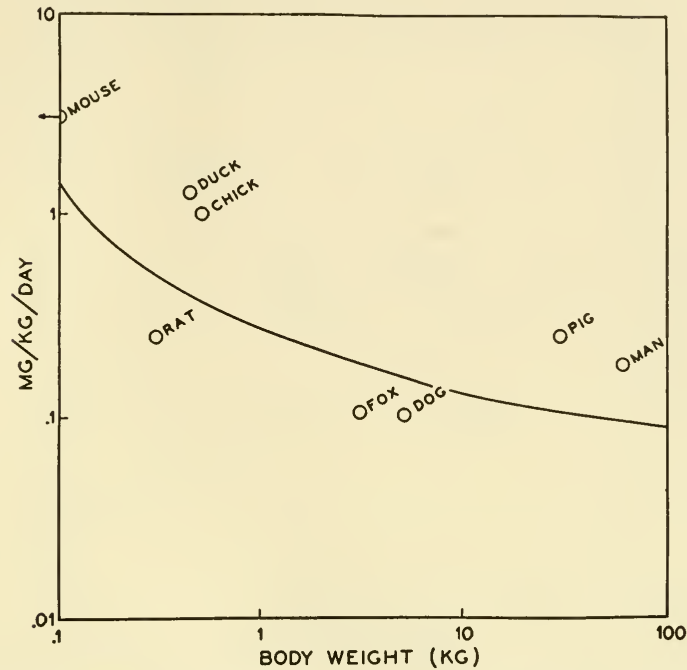

Figure 9. Relationship of the size of a species to its pantothenic acid requirement.

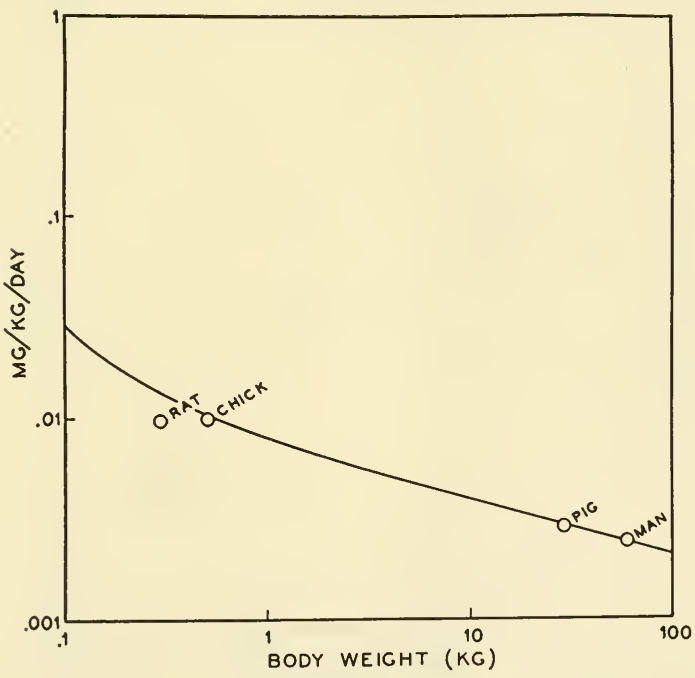

FigURE 10. Relationship of the size of a species to its biotin requirement. 


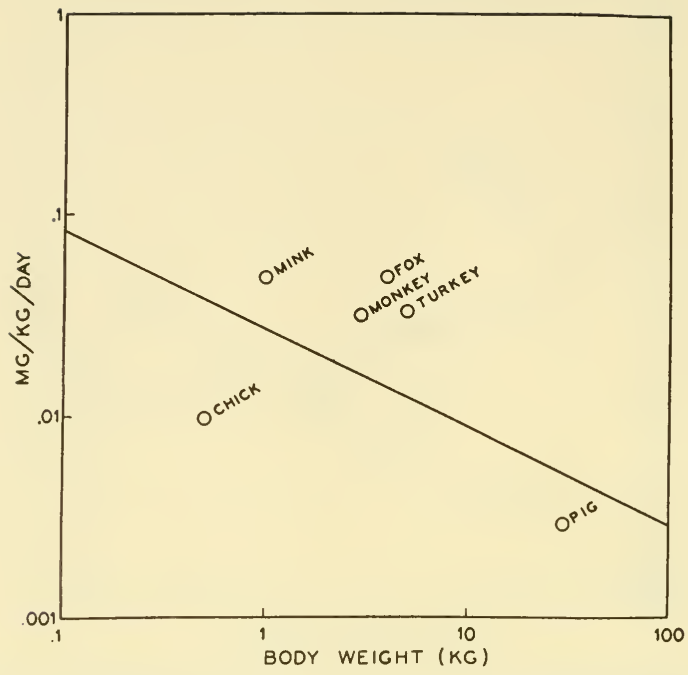

Figure 11. Relationship of the size of a species to its Folic Acid requirement.

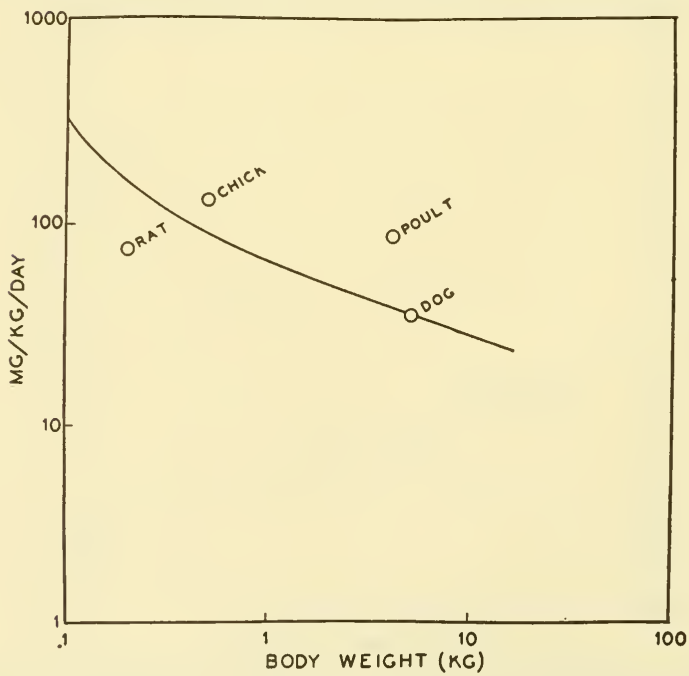

Figure 12. Relationship of the size of a species to its Choline requirement. 


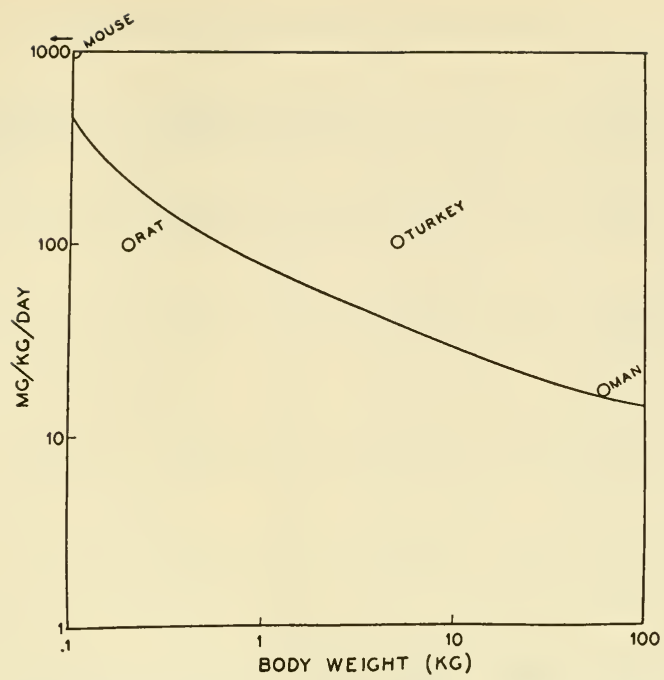

Figure 13. Relationship of the size of a species to its inositol requirement.

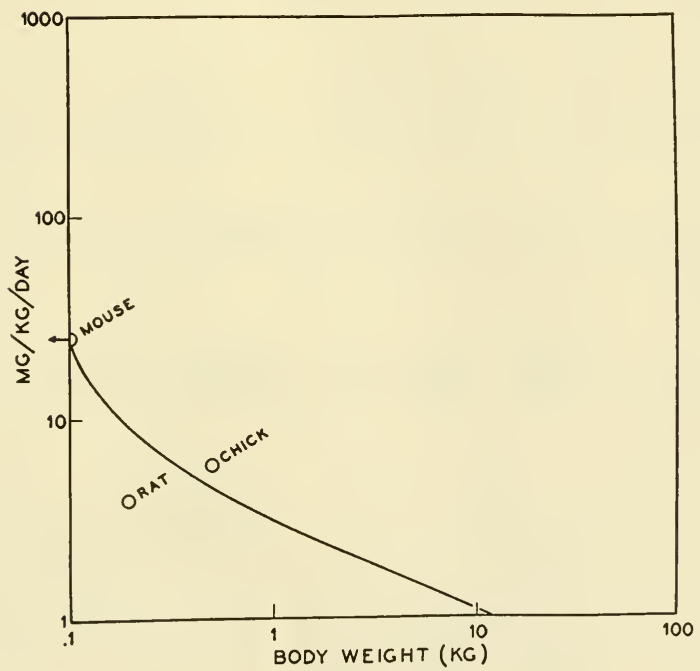

Figure 14. Relationship of the size of a species to its $p$-aminobenzoic acid requirement. 


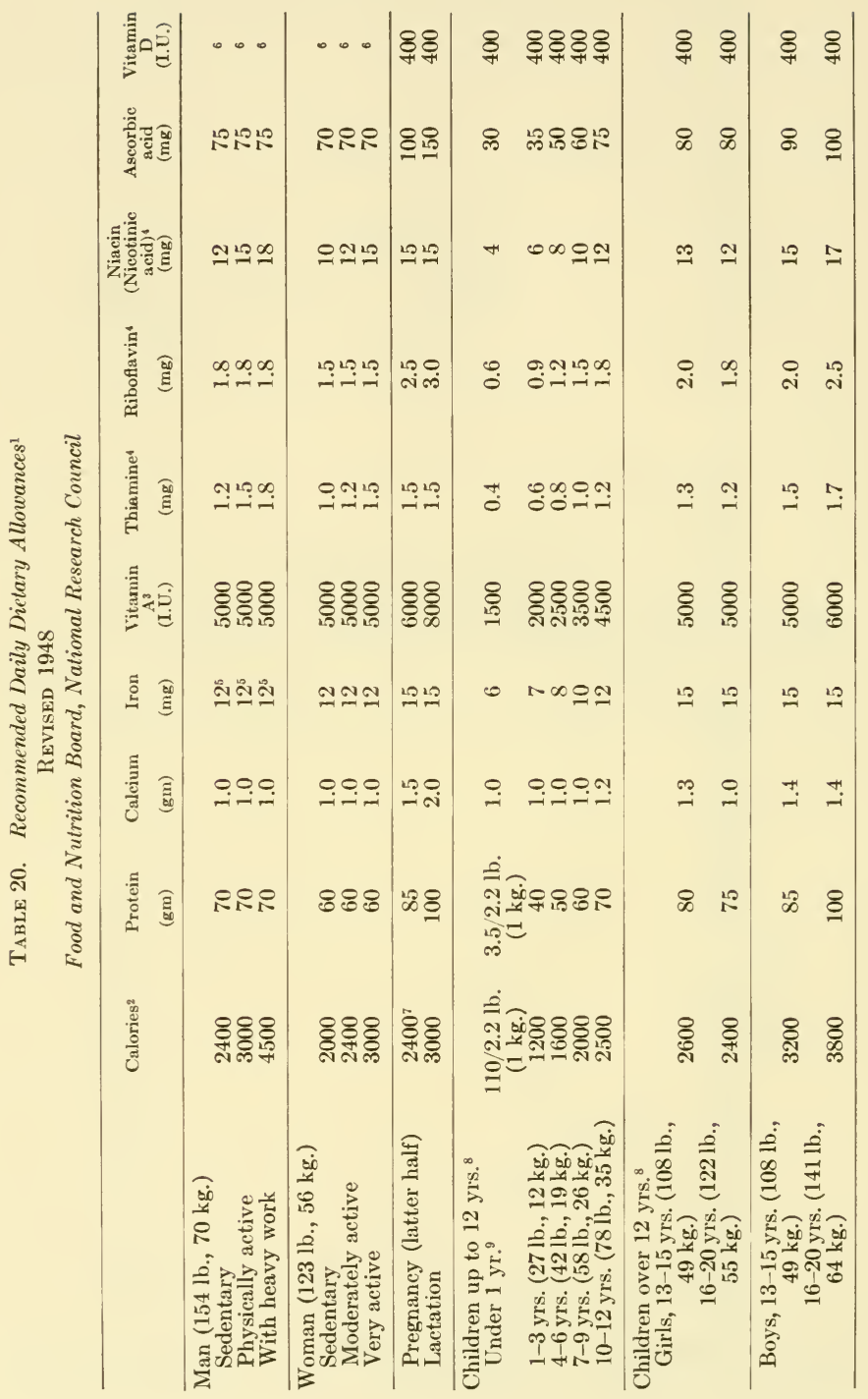




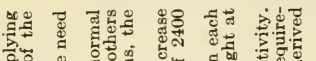

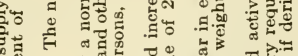

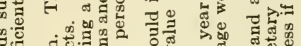

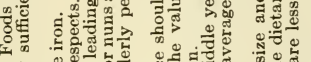

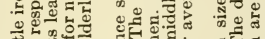

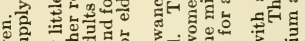

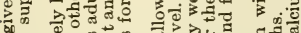

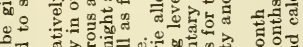

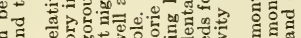

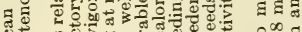

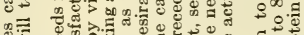

至

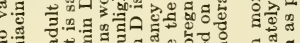

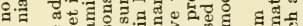

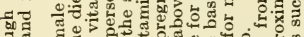

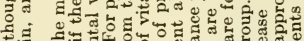

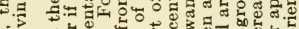

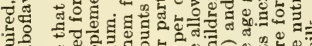

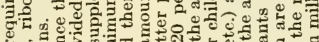
.

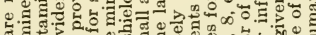
4

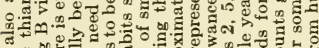

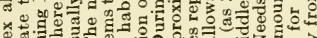

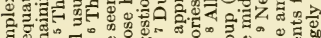

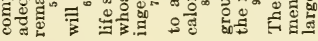

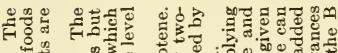

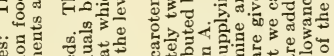

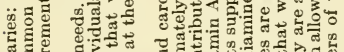

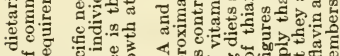
ᄀ내

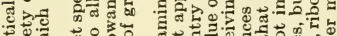
它.

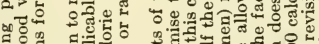

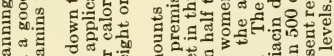
돈듀

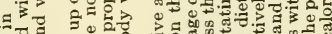

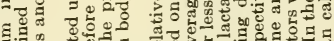

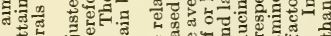

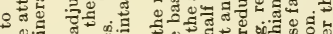

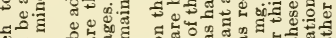
롱

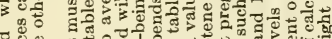

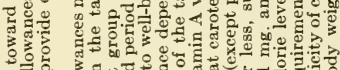

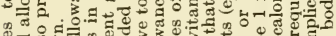

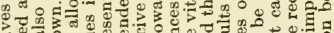
巳ั

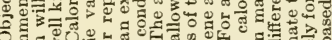

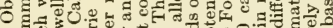

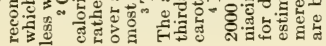

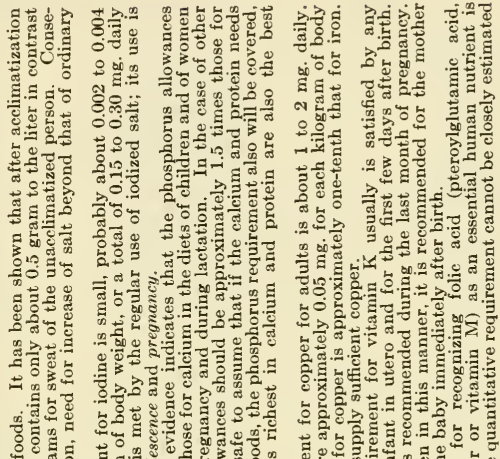

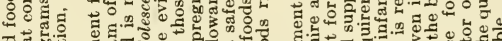
ơ

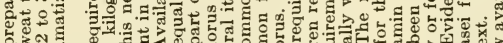

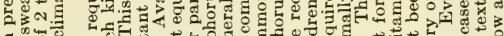
$\Xi$ क. क

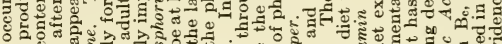
$\rightarrow \infty$

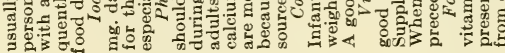

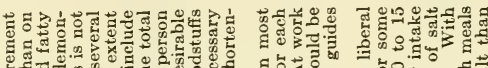

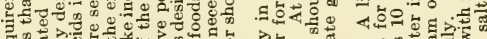

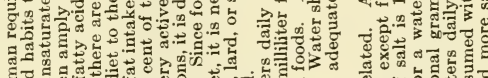

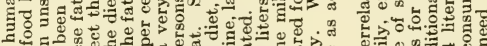
-

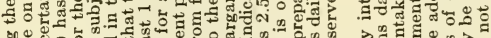
o

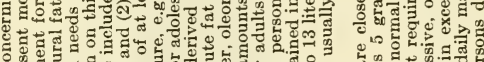

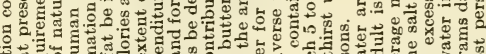

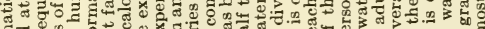
舟啳 国 a.

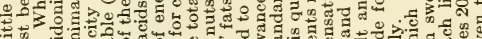

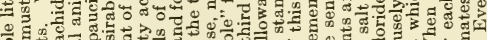

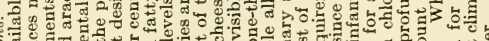
贾 용.

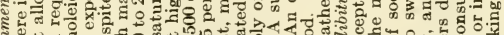
政

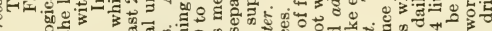

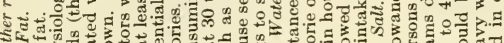
5心 
TABLE 21. Some Reported Requirements of Humans for B Vitamins Vitamin

Amount

Remarks

Reference

Thiamine

$1.0-1.8 \mathrm{mg}$ per day

$3.2 \mathrm{mg}$ per day

$1.06 \mathrm{mg}$ per day

$0.44 \mathrm{mg}$ per $1000 \mathrm{Cal}$.

Recommended intake and not requirement

Based on good diets

Minimum

Minimum for "normal"

male

Riboflavin

1. 5- $1.8 \mathrm{mg}$ per day

$3.7 \mathrm{mg}$ per day

Recommended intake

Recommended intake

Based on excretion studies

1.3 - 1.4 mg per day

Recommended intake

$\begin{array}{lrr}\text { Nicotinic acid } \quad 10.0-18.0 & \mathrm{mg} \text { per day } \\ 40.0 & \mathrm{mg} \text { per day }\end{array}$

Recommended intake

$15.0-20.0 \quad \mathrm{mg}$ per day

Based on blood level studies

$1.5-2.0 \mathrm{mg}$ per day

$4.0-7.5 \mathrm{mg}$ per day

Based on excretion studies

Minimum requirement

Pantothenic acid

$9.0-12.0 \quad \mathrm{mg}$ per day or per $2500 \mathrm{Cal}$.

Vitamin $\mathrm{B}_{6}$ $1.5 \mathrm{mg}$ per day

Biotin

\section{$0.15-0.3 \mathrm{mg}$ per day} $0.14 \mathrm{mg}$ per day

Folic acid

$0.20 \mathrm{mg}$ per day

Inositol

$1.0 \mathrm{gm}$ per day

Recommended

Recommended

$(62,68)$

Recommended

Recommended

Recommended

Recommended

Under remarks, "recommended" means that the authors cited in the reference infer that this level is suitable for the normal diet of individuals in order to maintain a state of good health. It will be noted that in some cases where there may be no nutritional requirement under normal circumstances, it is nevertheless suggested that a level of the vitamin be provided in the diet as a precaution in view of our lack of knowledge regarding the subject.

TABLE 22. Some Reported Requirements of Rats for B Vitamins

Vitamin

Thiamine

Riboflavin

Pantothenic acid

Vitamin $\mathrm{B}_{6}$

Biotin

Inositol

$p$-Aminobenzoic acid

Choline
Amount

$0.0125 \mathrm{mg}$ per day $0.02 \%$ solution ad lib

$0.0075 \mathrm{mg}$ per day

$0.010 \mathrm{mg}$ per day

$0.018 \mathrm{mg}$ per day

$0.036-0.090 \mathrm{mg}$ per day

$0.01 \mathrm{mg}$ per day

$0.10 \mathrm{mg}$ per day

$0.15 \mathrm{mg}$ per $100 \mathrm{gm}$ diet

$0.01 \mathrm{mg}$ per day

0.001-0.003 mg per day

$20.0 \mathrm{mg}$ per day

$0.75 \mathrm{mg}$ per day

$1.5-5.0 \mathrm{mg}$ per day $120-200 \mathrm{mg} / \mathrm{kg}$ per day
Remarks

Reference

Minimum

For reproduction

Maximum growth. Growth is linear to $0.075 \mathrm{mg}$

Minimum

Recommended. Avidin required to deplete.

Cures deficiency symptoms.

Required in diet only by cotton rats.

Cures nutritional achromotrichia

To maintain lipid tissues

Prevents kidney degeneration and liver lipotropism 
TABLE 23. Some Reported Requirements of Mice for B Vitamins

Vitamin

Thiamine

$0.005 \mathrm{mg}$ per day

0.008

0.010

0.005

Riboflavin

$0.0015 \mathrm{mg}$ per $\mathrm{gm}$ food

$0.004 \mathrm{mg}$ per day

Pantothenic acid

Vitamin $\mathrm{B}_{6}$

Biotin

Inositol

$p$-Aminobenzoic acid
$0.030 \mathrm{mg}$ per day

$0.0005 \mathrm{mg}$ per $\mathrm{gm}$ food

0.001

$0.008 \mathrm{mg}$ per $100 \mathrm{gm}$ diet $10.0 \mathrm{mg}$ per $100 \mathrm{gm}$ diet

$0.25 \mathrm{mg}$ per day
Remarks

Reference

Maintenance of body weight of weanlings

Suboptimal growth

Normal growth

Maintenance in adults

Minimum requirement for adults

Minimum requirement for adults

Minimum requirement

Half normal growth of weanlings

Good growth of weanlings

Cures deficiency symptoms

Prevents depigmentation of fur

TABLE 24. Some Reported Requirements of Chicks for B Vitamins

Vitamin

Thiamine

Riboflavin

Nicotinic acid

Pantothenic acid

Vitamin $\mathrm{B}_{6}$

Biotin

Folic acid

Vitamin $\mathrm{B}_{12}$

$0.01 \mathrm{mg}$ per $100 \mathrm{gm}$ diet $0.0025-.010 \mathrm{mg}$ per day

$0.020 \mathrm{mg}$ per $100 \mathrm{gm}$ diet

$0.030 \mathrm{mg}$ per day

$4.0 \mathrm{mg}$ per $100 \mathrm{gm}$ diet

$0.2 \mathrm{mg}$ per $100 \mathrm{gm}$ diet

$0.050 \mathrm{mg}$ per $100 \mathrm{gm}$ diet

$6.0 \mathrm{mg}$ per $1000 \mathrm{gm}$ diet

$30.0 \mathrm{mg}$ per $1000 \mathrm{gm}$ diet

$p$-Aminobenzoic acid

Choline

Strepogenin
$7.5 \mathrm{mg}$ per $100 \mathrm{gm}$ diet $100-200 \mathrm{mg}$ per $100 \mathrm{gm}$ diet

$12,000 \mathrm{mg}$ units per $100 \mathrm{gm}$ diet
Remarks

Reference

Prevents deficiency and suppressed growth

Optimal growth

Recommended level

Recommended

To maintain hatchability of eggs in adults

Recommended

On $10 \%$ gelatin diet

To prevent dermatitis

For optimal growth

Optimal growth

Increases rapidity of growth

Minimum

Minimum. Done with depleted chicks

Minimum

Minimum

Minimum to prevent deficiency

To prevent anorexia, weight loss, and decreased hatchability

Minimum

Minimum

DL-O-Heterobiotin minimum

(104)

$(109,110)$

Recommended (see Table p. 248)

Stimulates chick growth on animal protein factor deficient diet

Above optimum requirement

Recommended

Recommended to prevent perosis

Optimum growth 
view of this fact, the nutritional requirement is a rather flexible quantity, depending as it does upon the difference between the physiological requirement and the bacterial synthesis; and it is easy to understand the large number of conflicting reports in the literature on this topic.

In an extended discussion of human nutrition, it would be appropriate to consider at this point what the actual average consumption of $\mathrm{B}$ vitamins was for the many population groups in the world that have been studied in this regard. This formidable task is not here undertaken because the quantity of information is so great, the quality so poor, and the space available so limited. Suffice it to say that for the research statistician a wealth of material lies buried in the literature awaiting analyses from which may come a better understanding of the B vitamins in nutrition. Since the actual consumption of the B vitamins varies among individuals, groups, and geographical areas even more than does the requirement, no simple analysis of this subject is now possible.

TABlE 25. Some Reported Requirements of Other Domestic Birds for B Vitamins ( $m g$ per $100 \mathrm{gm}$ diet)

\begin{tabular}{|c|c|c|c|}
\hline Vitamin and species & Amount & Remarks & Reference \\
\hline $\begin{array}{c}\text { Thiamine } \\
\text { Pigeon }\end{array}$ & 0.125 & Minimum requirement & $(128)$ \\
\hline Riboflavin & & & \\
\hline $\begin{array}{l}\text { Poult } \\
\text { Duck }\end{array}$ & $\begin{array}{l}0.3-0.4 \\
0.3\end{array}$ & $\begin{array}{l}\text { Minimum } \\
\text { Required for growth }\end{array}$ & $\begin{array}{c}(94,112,129) \\
(130,131)\end{array}$ \\
\hline $\begin{array}{l}\text { Nicotinic acid } \\
\text { Poult }\end{array}$ & $\begin{array}{l}5.0 \\
2-5 \mathrm{mg}\end{array}$ & $\begin{array}{l}\text { On } 10 \% \text { gelatin diet } \\
\text { For good growth, etc. }\end{array}$ & $\begin{array}{c}(132) \\
(129,133)\end{array}$ \\
\hline $\begin{array}{l}\text { Pantothenic acid } \\
\text { Ducklings }\end{array}$ & 1.1 & Minimum & $(131)$ \\
\hline$\underset{\text { Duck }}{\text { Vitamin }} \mathrm{B}_{6}$ & 0.25 & Minimum & $(134)$ \\
\hline $\begin{array}{l}\text { Folic acid } \\
\text { Poult } \\
\text { Choline }\end{array}$ & 0.08 & Required to prevent deficiency & $(129,135,136)$ \\
\hline $\begin{array}{l}\text { Choline } \\
\text { Poult }\end{array}$ & 170.0 & Required to prevent perosis & (94) \\
\hline $\begin{array}{r}\text { Inositol } \\
\text { Poult }\end{array}$ & 1000.0 & Maximum growth & $(129)$ \\
\hline
\end{tabular}

\section{Philosophic Considerations}

In retrospect, the member's of the plant kingdom seem able to synthesize the $B$ vitamins while the members of the animal kingdom, as far as we know, have completely lost this ability, except for nicotinic acid. Generalizations are always dangerous, but it is remarkable how consistently this one holds true. While parts of plants may be heterotrophic, there is no certainty that small vital cell groups in animal tissue do not have the ability to synthesize B vitamins. Further, whereas certain Thallophytes are heterotrophic and certain protozoa autotrophic, the general nutritional 
trend in even these intermediate species follows closely trends in their morphological classification. If one were compelled to state a single distinctive difference between the two kingdoms, it would be difficult to find a better one than that of the ability to synthesize the B vitamins.

TABLE 26. Some Reported Requirements of Miscellaneous Mammals for B Vitamins

Vitamin and species

Thiamine

Monkey

Dog
Swine
Cat
Riboflavin
Dog

Swine

Fox

Monkey (young)

Nicotinic acid

Monkey

Dog

Pig

Rabbit

Fox

Pantothenic acid

Dog (young)

Pig (growing)

\section{Fox}

Biotin

$$
\text { Pig }
$$

Folic acid

Monkey

\section{Fox}

Mink

Choline

Dog
Amount

$0.04 \mathrm{mg}$ per day

$0.05 \mathrm{mg}$ per day

$0.075 \mathrm{mg}$ per day

$0.0275-0.075 \mathrm{mg}$ per 100 gm diet

$0.037 \mathrm{mg} / \mathrm{kg} /$ day

less than $0.05 \mathrm{mg}$ per day

$0.01-0.02 \mathrm{mg} / 100 \mathrm{gm}$

body weight

$0.002-0.006 \mathrm{mg} / 100 \mathrm{gm}$ body weight

$0.12-.40 \mathrm{mg}$ per $100 \mathrm{gm}$ diet

$0.025-0.030 \mathrm{mg} / \mathrm{kg} /$ body weight

$5.0 \mathrm{mg}$ per day

$0.5-1.5 \mathrm{mg} / \mathrm{kg} /$ day

$6.5-10 \mathrm{mg}$ per day

$0.2 \mathrm{mg}$ per $\mathrm{kg}$

$0.5-1 \mathrm{mg}$ per $\mathrm{kg}$

5. $-10 \mathrm{mg}$ per $\mathrm{kg}$

0.39-2 mg per $\mathrm{kg}$ body weight

$0.1 \mathrm{mg} / \mathrm{kg}$ body wt/day

$7.8-11.8 \mathrm{mg} / 100 \mathrm{lbs}$ animal/day

$0.25-1.5 \mathrm{mg} / 100 \mathrm{gm}$ food

$0.1 \mathrm{mg}$ per day

$0.1 \mathrm{mg}$ per day

$0.2-0.3 \mathrm{mg}$ per day $B_{c}$

$0.5 \mathrm{mg}$ per day

less than $0.05 \mathrm{mg} / \mathrm{day}$

$50 \mathrm{mg} / \mathrm{kg}$ body weight
Remarks

Reference

To maintain body weight

For growth response

Optimum growth

Requirement

Requirement

Requirement

(137-139)

Requirement

Requirement

Requirement

Requirement

Requirement

Requirement

Normal growth

Minimum for maintenance

Slight growth

Maximum growth

Requirement

Requirement (adults less)

Requirement

To prevent alopoecia, etc.

Requirement

Requirement

Adequate diet

Requirement

Why this nutritional dichotomy developed in the evolutionary process is a matter for considerable speculation. It is apparent that both plants and animals may survive upon the earth in a biological balance when only the one group has the higher synthetic ability, and a philosophy might readily be worked out in terms of a conserving economy in the distribution of metabolic abilities. More practically, since on a global scale the plant and animal kingdoms are interdependent, this difference 
may provide one means whereby the animal kingdom cannot exceed the balance that exists, just as the plant kingdom is held in check by the carbon dioxide content of the air, the supply of which is influenced by the animal population. It may be surmised that the dichotomy may have developed by a stepwise genetic process, leaving in the primitive forms of both kingdoms (Thallophytes and protozoa) members having the ability to synthesize intermediate numbers of $\mathrm{B}$ vitamins; and on this basis the fact that the protozoa and fungi vary in their abilities would be expected. Arriving thus at a common primitive progenitor with fully developed synthetic abilities does not seem as improbable as arriving at one with none, according to present concepts ${ }^{168}$ of evolution.

Despite the unverifiable nature of these suggestions, one is tempted to wonder why nicotinic acid stands out as an exception to the fact that animals cannot synthesize B vitamins. (There is some evidence that Tetrohymena gelii and Drosphila melanogaster may be exceptions to this). If one were forced into the ludicrous discussion as to which one of the $B$ vitamins is the most vital, he would probably select niacin. Ultimately, life is characterized by activities; these require energy, and energy is generally derived in animals from high-energy phosphate bonds, which are most frequently created by a reduction involving Cozymase and thus nicotinic acid. An extremely primitive system might conceivably subsist with this B vitamin, whereas the other B vitamins could not serve this prime purpose of energy production. (In plants, the utilization of radiant energy presumably involves a reduction of fixed carbon dioxide, and here again it may well be that nicotinic acid is the first vitamin involved in the linear process.) It may be considered providential, at least, that more flexibility is permitted animals in niacin synthesis, allowing the conversion from tryptophan. It may also be because of the particularly crucial place assigned to niacin in metabolism, that animals, which are still limited by the tryptophan nutrition and the efficiency of the process, tend to store nicotinic acid in as nearly finished a product as possible, nicotinamide, ${ }^{169}$ whereas the less limited plants store it primarily as nicotinic acid. Indeed, our existing scanty knowledge suggests that the routes of synthesis of niacin may differ in the animals and higher plants, since asparagin, glutamic acid, proline and ornithine seem to be intimately involved in plant synthesis, ${ }^{170}$ but not in animals, while tryptophan generally seems to be the major precursor in animals, but not in plants. This point in the discussion at least should receive elucidation in the near future. 


\section{Bibliography}

1. Knight, B. C. J. G., Vitamins and Hormones, 3, 108-228 (1945).

2. Snell, E. E., Ann. Rev. Biochem., 15, 375-96 (1946).

3. Peterson, W. H., and Peterson, M. S., Bact. Revs., 9, 49-109 (1945).

4. Bauer, J. D., Nature, 159, 438-9 (1947).

5. Hall, R. P., Vitamins and Hormones, 1, 249-68 (1943).

6. Sebrell, W. H., Ann. Rev. Biochem., 13, 441-66 (1944).

7. Ackermann, W. W., and Taylor, A., Proc. Soc. Exptl. Biol. Med., 67, 449-52 (1948).

8. Wulzen, R., and Bahrs, A. M., Physiol. Zool., 4, 204-13 (1931).

9. Addis, C. J., and Chandler, A. C., J. Parasitol., 30, 229-36 (1944).

10. Bonsdorff, B. v., Acta med. Scand., 129, 59 (1947).

11. Curtis, M. R., Dunning, W. F., and Bullock, F. D., Am. J. Cancer, 17, 894-923 (1933).

12. Zimmerman, A., Rev. suisse zool., 28, 357-80 (1921).

13. Glaser, R. W., J. Exptl. Zool., 84, 1-12 (1940).

14. McCoy, O. R., Am. J. Hyg., 10, 140-56 (1929).

15. Watt, J. Y. C., Am. J. Hyg., 39, 145-51 (1944).

16. Howes, N. H., and Whellock, R. B., Biochem. J., 31, 1489-98 (1937).

17. Fraenkel, G., and Blewett, M., Biochem. J., 37, 686-92 (1944).

18. Trager, W., J. Biol. Chem., 176, 1211-23 (1948).

19. Lwoff, M., and Nicolle, P., Compt. rend., 219, 246-8 (1944).

20. Pearson, P. B., and Burgin, C. J., Proc, Soc. Exptl. Biol. Mcd., 48, 415-17 (1941).

21. Noland, J. L., and Baumann, C. A., Proc. Soc. Exptl. Biol. Med., 70, 198-201 (1949).

22. Noland, J. L., Lilly, J. H., and Baumann, C. A., Ann. Entomol. Soc. Am., (in press).

23. Moore, W., Ann. Entomol. Soc. Am., 36, 483-5 (1943).

24. Fraenkel, G., Sci. J. Roy. Coll. Sci., 13, 59-69 (1943).

25. Martin, H. E., and Hare, L., Biol. Bull., 83, 428-37 (1942).

26. Grob, C. A., and Brunner, Th., Experientia, 2, 449-50 (1946).

27. Fraenkel, G., and Blewett, M., Nature, 157, 697 (1946).

28. de Meillon, B., Goldberg, L., and Lavoipierre, M., J. Exptl. Biol., 21, 84-9 (1945).

29. Tatum, E. L., Proc. Natl. Acad. Sci. U. S., 27, 193-7 (1941).

30. Swamy, B. G. L., and Sreenevasaya, M., Current Sci., 8, 365-7 (1939).

31. Sarma, P. S., Swamy, B. G. L., and Sreenevasaya, M., Current Sci., 11, 332-3 (1942).

32. Haydak, M. H., Ann. Entomol. Soc. Am., 29, 581-8 (1936).

33. Fraenkel, G., and Blewett, M., Biochem. J., 41, xviii (1947).

34. Woods, A. M., Taylor, J., Hofer, M. J., Johnson, C. A., Lane, R. L., and MeMahan, C. R.. Univ. Texas Pub. 4237, 84-6 (1942).

35. Kozloff, L., and Pijoan, M., Naval Med. Research Inst., Natl. Naval Med. Center, Bethesda, Md., Research Project X-334, Rept. 4, 1-3 (1946).

36. Sarma, P. S., Indian J. Med. Research, 32, 117-22 (1944).

37. Sarma, P. S., Indian J. Med. Research, 32, 149-53 (1944).

38. de Meillon, B., and Goldberg, L., Nature, 159, 171 (1947).

39. de Meillon, B., Nature, 158, 839 (1946).

40. Trager, W., Physiol. Revs., 21, 1-35 (1941).

41. Dennison, R., Science, 92, 17 (1940).

42. Dandliker, W. B., Cooper, W. C., and Traub, H. P., Science, 88, 622 (1938).

43. Cooper, W. C., Botan. Gaz., 100, 844-52 (1939).

44. Went, F. W., and Thimann, K. V., "Phytohormones," Macmillan and Co., New York, 1937. 
45. Kogl, F., and Haagen-Smith, A. J., Z. physiol. Chem., 243, 209-26 (1936).

46. West, P. M., Nature, 144, 1050-1 (1939).

47. White, P. R., Plant Physiol., 12, 777-90 (1937).

48. White, P. R., Plant Physiol., 12, 793-802 (1937).

49. Robbins, W. J., and Bartley, M. A., Proc. Natl. Acad. Sci. U. S., 23, 385-8 (1937).

50. Robbins, W. J., and Schmidt, M. B., Proc. Natl. Acad. Sci. U. S., 25, 1-3 (1938).

51. Bonner, J., and Addicott, F., Botan. Gaz., 99, 144-70 (1937).

52. Bonner, J., and Axtman, G., Proc. Natl. Acad. Sci. U. S., 23, 453-7 (1937).

53. National Research Council, Reprint and Circ. Ser., No. 122, 1945, pp. 3-18.

54. Morris, H. P., Vitamins and Hormones, 5, 175-95 (1947).

55. Day, P. L., Vitamins and Hormones, 2, 71-106 (1944).

56. Bird, H. R., Vitamins and Hormones, 5, 163-73 (1947).

57. Ellis, N. R., Nutrition Abstracts and Revs., 16, 1-10 (1946).

58. National Research Council, "Recommended Nutrient Allowances for Domestic Animals. III. Recommended Nutrient Allowances for Dairy Cattle" (1945).

59. Huffman, C. F., and Duncan, C. W., Ann. Rev. Biochem., 13, 477-80 (1944).

60. National Research Council, Reprint and Circ. Ser., No. 129, 1948, pp. 16-17.

61. Hegsted, D. M., J. Nutrition, 35, 399-409 (1948).

62. Williams, R. J., J. Am. Med. Assoc., 119, 1-3 (1942).

63. Alexander, B., and Landwehr, G., J. Clin. Invest., 25, 287-93 (1946).

64. Brewer, W., Porter, T., Ingalls, R., and Ohlson, M. A., J. Nutrition, 32, 583-96 (1946).

65. Gounelle, H., Vallette, A., and Raoul, Y., Compt. rend. soc. biol., 139, 16-17 (1945).

66. Najjar, V. A., Holt, L. E., Jr., Johns, G. A., Medairy, G. C., and Fleischmann, G., Proc. Soc. Exptl. Biol. Med., 61, 371-4 (1946).

67. Frazier, E. I., and Friedemann, T. E., Quart. Bull. Northwestern Univ. Med. School, 20, 24-48 (1946).

68. Gordon, E. S., "Biological Action of the Vitamins," E. A. Evans, Editor, University of Chicago Press. Chicago, (1942), p. 136.

69. Gyorgy, P., and Rose, C. S., Proc. Soc. Exptl. Biol. Med., 43, 73-4, (1940).

70. Supplee, G. C., Bender, R. C., and Kahlenberg, O. J., J. Nutrition, 20, 109-23 (1940).

71. Richter, C. P., Am. J. Physiol., 145, 107-14 (1945).

72. Czaczkes, J. W., and Guggenheim, K., J. Biol. Chem., 162, 267-74 (1946).

73. Mannering, G. F., Lipton, M. A., and Elvehjem, C. A., Proc. Soc. Exptl. Biol. Med., 46, 100-4 (1941).

74. Everson, G., Williams, E., Wheeler, E., Swanson, P., Spivey, M., and Eppright, M., J. Nutrition, 36, 463-78 (1948).

75. Grussner, A., Gatzi-Fichter, M., and Reichstein, T., Helv. Chim. Acta, 23, 1276-86 (1940).

76. Unna, K., Am. J. Med. Sci., 200, 848 (1940).

77. Sarma, P. S., Snell, E. E., and Elvehjem, C. A., J. Biol. Chem., 165, 55-63 (1946).

78. Dimick, M. K., and Schreffler, C. B., J. Nutrition, 17, 23-9 (1939).

79. Ringrose, A. T., Norris, L. C., and Heuser, G. F., Poultry Sci., 10, 166-77 (1931).

80. Pavcek, P. L., and Baum, H. M., Science, 93, 502 (1941).

81. Martin, G. J., and Ansbacher, S., J. Biol. Chem., 138, 441 (1941).

82. Borglin, N. E., Acta Pharmacol. Toxicol., 3, Supplement 1, 123 pp. (1947).

83. Nutrition Revs., 4, 116-8 (1947).

84. Martin, G. J., and Ansbacher, S., Proc. Soc. Exptl. Biol. Med., 48, 118-20 (1941).

85. Hauschildt, J. D., Proc. Soc. Exptl. Biol. Med., 49, 145-7 (1942).

86. Morris, H. P.. and Dubnik, C. S., Proceedings of the American Chemical Society, New York (1944).

87. Morris, H. P., and Robertson, W. v.B., J. Natl. Cancer Inst., 3, 479-89 (1943).

88. Lippincott, S. W., and Morris, H. P., J. Natl. Cancer Inst., 2, 601-10 (1942).

89. Morris, H. P., and Lippincott, S. W., J. Natl. Cancer Inst., 2, 29-38 (1941). 
90. Miller, E. C., and Baumann, C. A., J. Biol. Chem., 157, 551-62 (1945).

91. Nielsen, E., and Block, A., J. Nutrition, 28, 203-8 (1944).

92. Arnold, A., and Elvehjem, C. A., J. Nutrition, 15, 403-10 (1938).

93. Jukes, T. H., and Heitman, H., Jr., J. Nutrition, 19, 21-30 (1940).

94. Cravens, W. W., Almquist, H. J., Bethke, R. M., Norris, L. C. and Titus, H. W., "Recommended Nutritional Allowances for Poultry," National Research Council (1946).

95. Bird, F. H., Asmundson, U. S., Kratzer, F. H., and Lepkovsky, S., Poultry Sci., 25, 47-51 (1946).

96. Bethke, R. M., and Record, P. R., Poultry Sci., 21, 147-54 (1942).

97. Bolton, W., J. Agr. Sci., 34, 198-206 (1944).

98. Norris, L. C., Wilgus, H. S., Jr., Ringrose, A. T., Heiman, V., and Heuser, G. F., Cornell Univ. Agr. Exp. Sta. Mem. 660, (1936).

99. Davis, H. J., Norris, L. C., and Heuser, G. F., Poultry Sci., 17, 81-6 (1938).

100. Heuser, G. F., Wilgus, H. S., and Norris, L. C., Poultry Sci., 17, 105-8 (1938).

101. Bird, J. H., Asmundson, U. S., Kratyer, J. H., and Lepkovsky, S., Poultry Sci., 25, 47-51 (1946).

102. Clandinin, D. R., Poultry Sci., 25, 223-31 (1946).

103. Briggs, G. M., Jr., Mills, R. C., Elvehjem, C. A., and Hart, E. B., Proc. Soc. Exptl. Biol. Mcd., 51, 59-61 (1942).

104. Jukes, T. H., J. Biol. Chem., 129, 225-31 (1939).

105. Bauernfeind, J. C., and Norris, L. C., Science, 89, 416-17 (1939).

106. Jukes, T. H., J. Biol. Chem., 117, 11-20 (1937).

107. Bird, H. R., and Rubin, M., Poultry Sci., 25, 87-9 (1946).

108. Richardson, L. R., Hogan, A. G., and Kempster, H. L., Missouri Agr. Exp. Sta., Research Bull., 390, 12 pp. (1945).

109. Briggs, G. M., Jr., Mills, R. C., Hegsted, D. M., Elvehjem, C. A., and Hart, E. B., Poultry Sci., 21, 379-83 (1942).

110. Hogan, A. G., Richardson, L. R., Patrick, H., O'Dell, B. L., and Kempster, H. L., Poultry Sci., 20, 180-3 (1941).

111. Hegsted, D. M., Oleson, J. J., Elvehjem, C. A., and Hart, E. B., J. Biol. Chem., 130, 423-4 (1939).

112. Lucas, H. L., Heuser, G. F., and Norris, L. C., Poultry Sci., 25, 137-42 (1946).

113. Cravens, W. W., Sebesta, E. E., Halpin, J. G., and Hart, E. B., Poultry Sci, 25, 80-2 (1946).

114. Hegsted, D. M., Niells, R. C., Briggs, G. M. Jr., Elvehjem, C. A., and Hart, E. B., J. Nutrition, 23, 175-9 (1942).

115. MeCoy, R. H., Felton, J. R., and Hofmann, K., Arch. Biochem., 9, 141-7 (1946).

116. Robertson, E. I., Daniel, L. J., Farmer, F. A., Norris, L. C., and Heuser, G. F., Proc. Soc. Exptl. Biol. Med., 62, 97-101 (1946).

117. Oleson, J. J., Hutchings, B. L., and Sloane, N. H., J. Biol. Chem., 165, 371-5 (1946).

118. Daniel, L. J., Farmer, F. A., and Norris, L. C., J. Biol. Chem., 163, 349-50 (1946).

119. Hutchings, B. L., Oleson, J. J., and Stoksted, E. L. R., J. Biol. Chem., 163, 447-53 (1946).

120. Luckey, T. D., Moore, P. R., Elvehjem, C. A., and Hart, E. B., Science, 103, 682-4 (1946).

121. Petering, H. G., Marvel, J. P., Glausier, C. E., Jr., and Waddell, J., J. Biol. Chem., 162, 477-89 (1946).

122. Briggs, G. M., Jr., Luckey, T. D., Mills, R. C., Elvehjem, C. A., and Hart, E. B., Proc. Soc. Exptl. Biol. Med., 52, 7-10 (1943).

123. Ott, W. H., Rickes, E. L., and Wood, T. R., J. Biol. Chem., 174, 1047-8 (1948).

124. Jukes. T. H., Oleson, J. J., and Dornbush, A. C., J. Nutrition, 30, 219-23 (1945).

125. Record, P. R., and Bethke, R. M., Poultry Sci., 21, 271-6 (1942).

126. Abbott, O. D., and DeMasters, C. U., J. Nutrition, 19, 47-55 (1940). 
127. Scott, M. L., Norris, L. C., and Heuser, G. F., J. Biol. Chem., 167, 261-72 (1947).

128. Swank, R. L., and Bessey, O. A., J. Nutrition, 22, 77-89 (1941).

129. Jukes, T. H., Stokstad, E. L. R., and Belt, M., J. Nutrition, 33, 1-12 (1947).

130. Fritz, J. C., Archer, W., and Barker, D., Poultry Sci., 18, 449-54 (1939).

131. Hegsted, D. M., and Perry, R. L., J. Nutrition, 35, 411-17 (1948).

132. Briggs, G. M., Jr., J. Nutrition, 31, 79-84 (1946).

133. Briggs, G. M., Jr., J. Biol. Chem., 161, 749-50 (1945).

134. Hegsted, D. M., and Rao, M. N., J. Nutrition, 30, 367-74 (1945).

135. Richardson, L. R., Hogan, A. G., and Kempster, H. L., J. Nutrition 30, 151-7 (1945).

136. Lance, B. G., and Hogan, A. G., J. Nutrition, 36, 369-79, (1948).

137. Waisman, H. A., and MeCall, K. B., Arch. Biochem., 4, 265-79 (1944).

138. Leblond, C. P., and Chaulin-Serviniere, J., Am. J. Med. Sci., 203, 100-9 (1942).

139. McCarrison, R., Brit. Med. J., I, 249-53 (1920).

140. Arnold, A., and Elvehjem, C. A., Am. J. Physiol., 126, 289-98 (1939).

141. Van Etten, C., Ellis, N. R., and Madsen, L. L., J. Nutrition, 20, 607-25 (1940).

142. Everett, G. M., Am. J. Physiol., 141, 439-48 (1944).

143. Street, H. R., and Cowgill, G. R., Am. J. Physiol., 125, 323-34 (1939).

144. Hughes, E. H., J. Nutrition, 20, 233-8 (1940).

145. Schaefer, A. E., Whitehair, C. K., and Elvehjem, C. A., J. Nutrition, 34, 131-9 (1947).

146. Cooperman, J. M., Waisman, H. A., McCall, K. B., and Elvehjem, C. A., J. Nutrition, 30, 45-57 (1945).

147. Harris, L. J., Biochem. J., 32, 1479-81 (1938).

148. Margolis, G.. Margolis, L. H., and Smith, S. G., J. Nutrition, 16, 541-8 (1938).

149. Sebrell, W. H., Onstott, R. H., Fraser, H. F., and Daft, F. S., J. Nutrition, 16, 355-62 (1938).

150. Elvehjem, C. A., Madden, R. J., Strong, F. M., and Woolley, D. W., J. Biol. Chem., 123, 137-49 (1938).

151. Birch, T. W., J. Nutrition, 17, 281-92 (1939).

152. Hughes, E. H., Hilgardia, 11, 595-612 (1939).

153. Wintrobe, M. M., Am. J. Physiol., 126, 375-87 (1939).

154. Braude, R., Kon, S. K., and White, E. G., Biochem. J., 40, 843-55 (1947).

155. Hogan, A. G., and Hamilton, J. W., J. Nutrition, 23, 533-43 (1942).

156. Wooley, J. G., and Sebrell, W. H., J. Nutrition, 29, 191-9 (1944).

157. Olcese, O., Pearson, P. B., and Schweigert, B. S., J. Nutrition, 35, 577-90 (1948).

158. Schaefer, A. E., McKibbin, J. M., and Elvehjem, C. A., J. Biol. Chem., 143, 321-30 (1942).

159. Hughes, E. H., and Ittner, N. R., J. Animal Sci., 1, 116-19 (1942).

160. Cunha, T. J., Lindley, D. C., and Ensminger, M. E., J. Animal Sci., 5, 219-25 (1946).

161. Cooperman, J. M., Elvehjem, C. A., MeCall, K. B., and Ruegamer, W. R., Proc. Soc. Exptl. Biol. Med., 61, 92-7 (1946).

162. Cooperman, J. M., McCall, K. B., Ruegamer, W. R., and Elvehjem, C. A., J. Nutrition, 32, 37-45 (1945).

163. Schaefer, A. E., Whitehair, C. K., and Elvehjem, C. A., Arch. Biochem., 12, 349-57 (1947).

164. Schaefer, A. E., Whitehair C. K., and Elvehjem, C. A., Proc. Soc. Exptl. Biol. Med., 62, 169-74 (1946).

165. MeKibbin, J. M., Thayer, S., and Stare, F. J., J. Lab. Clin. Med., 29, 1109-22 (1944).

166. Ruegamer, W. R., Michaud, L., Elvehjem, C. A., and Hart, E. B., Am. J. Physiol., 145, 23-7 (1945).

167. Enterman, C., and Chaikoff, I. L., J. Biol. Chem., 138, 477-85 (1941).

168. Horowitz, N. H., Proc. Natl. Acad. Sci. U. S., 31, 153-7 (1945). 
169. Krehl, W. A., de la Huerga, J., Elvehjem, C. A., and Hart, E. B., J. Biol. Chem., 166, 53-7 (1946).

170. Ellinger, P., and Kader, M. M. A., Biochem. J., 42, ix (1948).

171. Sarma, P. S., and Sreenevasaya, M., Current Sci., 8, 551 (1939).

172. Sarma, P. S., and Sreenevasaya, M., Current Sci., 10, 525-6 (1941).

173. Ramaswami, S., Sarma, P. S., and Sreenevasaya, M., Current Sci., 11, 53-4 (1942).

174. Swamy, B. G. L., and Sreenevasaya, M., Current Sci., 11, 147-8 (1942).

175. Sarma, P. S., and Bhaguat, K., Current Sci., 11, 331-2 (1942).

176. Swamy, B. G. L., and Sreenevasaya, M., Current Sci., 9, 493 (1940).

177. Sarma, P. S., and Bhaguat, K., Current Sci., 11, 394 (1942).

178. Sarma, P. S., Indian J. Med. Research, 31, 161-3 (1943).

179. Sarma, P. S., Indian J. Med. Research, 31, 165-71 (1943).

180. Bhaguat, K., and Sarma, P. S., Indian J. Med. Research, 31, 173-81 (1943).

181. Schweigert, B. S., Vitamins and Hormones, 6, 55-67, (1948).

182. Bonner, J., and Bonner, H., Vitamins and Hormones, 6, 225-275, (1948).

183. Beerstecher, E., Jr., Science, 111, 300-312, (1950). 


\section{Chapter IVC}

\section{METABOLISM OF THE B VITAMINS}

It is proposed to outline in this chapter the essential facts concerning the processes which the $\mathrm{B}$ vitamins undergo from the time they are synthesized or ingested by the organism until they are excreted. As in other chapters in this section, the information in most cases is empirical; a true understanding of the subject does not exist. In both plants and animals, however, there are certain broad categories which may be referred to as "stages" in the metabolism. For convenience, these may be referred to as digestion, absorption, distribution, anabolism and catabolism, and excretion, and the following discussion proceeds in this order. Since plant metabolism involves many differences in detail, and its consideration is limited by the meager amount of data bearing upon the subject, it is considered independently.

\section{Metabolism in Plants}

Origin in the Plant. The precise pathways involved in B vitamin biosynthesis have been considered briefly in an earlier section. The cytological location of this synthesis in the single-celled plants is not known, but it seems relatively certain that the synthesis occurs in the leaves of the higher green plants, which are generally considered to be the major focus of synthetic activity in the plant. Since many alkaloids are also synthesized in this highly active metabolic area, ${ }^{224}$ it is no wonder that some B vitamin moieties may be recognized in certain alkaloids (arecoline, guvacine, nicotine, ergot alkaloids). B Vitamins are also supplied to the higher plants from the soil and from symbiotic microorganisms, although the extent to which this source is important to the higher plants is unknown. The $\mathrm{B}$ vitamins reach the heterotrophic portions of the plant largely through the translocation stream by which other leaf synthetic products are transported, but also by diffusion from neighboring cells and by absorption from the exterior environment.

Digestion and Cellular Absorption in Plants. Since the B vitamins are frequently found in plants in bound forms, it is apparent that the plants must first release the stored vitamins in order to utilize them for their own purposes, and annual cyclic variations in the relative amounts of free and bound vitamins are known to occur in some cases. Since plants 
are capable of binding the $\mathrm{B}$ vitamins, either to create structural enzymecoenzyme forms or insoluble storage forms, our knowledge of the mechanism of catalysis would suggest that they also would be able to liberate these forms. If, as suggested (p. 316), biotin functions as a hormone in plants, flowing downward from the tip, then its solubilizing release from the bound form in an auxin-like fashion (p. 37) is of major importance in plant differentiation., 225 In the case of many Thallophytes, digestion as we know it may frequently occur outside the plant. Although the bacteria particularly are well known for their ability to liberate vitamins from combination, in some cases, as in the avidin-biotin complex, even bacteria may be incapable of digesting the bound form. There is a more detailed discussion of enzymatic liberation of $\mathrm{B}$ vitamins earlier in this volume (Chap. III A).

It is generally assumed that water-soluble forms of the $B$ vitamins may diffuse readily in and out of plant cells, but that bound forms, particularly protein-bound forms, do not. These assumptions are frequently unjustified, and are based largely on analogy with questionable data in the animal kingdom. Since there is little evidence bearing directly on the subject in plant cells and tissues, it seems pertinent at least to point out that the diffusion across a cell membrane or tissue barrier may involve intermediate formation, and is most frequently a selective process not involving simple diffusion in the strictly physical sense. The active absorption of thiamine by live yeast in the animal intestine is particularly significant in this regard. Further, some bound forms, even proteinaceous ones, may be quite capable of diffusion across "semipermeable" membranes. Further extended studies will therefore be required to determine the nature of this process in plants.

Distribution, Catabolism, and Excretion in Plants. The salient facts with regard to the distribution of the $B$ vitamins in plants have been considered in an earlier section (Chap. II A). Thiamine, vitamin $\mathrm{B}_{3}$, niacin, pantothenic acid, and biotin seem largely concentrated in the seeds, while riboflavin, inositol, and folic acid are most concentrated in the leaves. Without dwelling unduly upon this latter fact, and without questioning the importance of all $\mathrm{B}$ vitamins to the photosynthetic process, it seems of sufficient interest to note that inositol occurs in leaves largely as the hexaphosphate, ${ }^{2}$ and may function in this case as a phosphate storage form; that riboflavin has been implicated in phototropism in plants, ${ }^{3}$ a phenomenon that is closely integrated with photosynthesis; and that folic acid may well have a very special role in the photosynthetic process.

Strangely, little is known concerning the role of the $B$ vitamins in photosynthesis, and in one recent monograph on photosynthesis ${ }^{4}$ no $B$ vitamin is even so much as mentioned in the index. It is known that 
pantothenic aeid is not synthesized in the leaves of green plants until the photosynthetic process commences, but whether this is a cause or an effect is uncertain. Beyond this, little is known of annual cyclic variations in those plants that maintain their photosynthetic ability throughout the year.

In the Thallophyta, and within single cells in general, very little is as yet known with regard to the possible localization of $\mathrm{B}$ vitamins. It is not possible to report any reliable information as to distribution between the nucleus, eytoplasm, and cell wall, or as to distribution in different parts of the cytoplasm, or as to distinet changes during mitosis. There will undoubtedly be much intensive study of this subject during the next few years as a result of the intensive rescarch efforts now in effect in the field of cytochemistry, and of the rapidly increasing number of techniques now being reported which should facilitate such study. ${ }^{226}$

Practically nothing is known with regard to the breakdown products of $\mathrm{B}$ vitamins in green plants. A fertile and relatively simple field for exploration awaits the investigator of this point, with the information found in animal catabolism well developed to serve as a guide. In the case of the Thallophyta, furthermore, the breakdown products of B vitamins have been but little more studied, despite the vast amount of information available on the bacterial catabolism of other metabolites.

Plants excrete $B$ vitamins into the surrounding medium, and vitamin production by certain bacteria has already been noted (p. 299). Indeed in certain molds (Eremothecium ashbyae, Ashbya gossypii) riboflavin excretion is so pronounced as to form riboflavin crystals about the mycelium. ${ }^{5}$ Higher plants seerete vitamins into the soil from their roots, and in several instances (i.e., thiamine, inositol) extended studies have been made of the subject; these have indicated that the high localized vitamin content of the soil is a factor in increasing the bacterial population in these areas. ${ }^{6}$ The exact process involved in excretion, as in absorption, however, is unknown, and such factors as threshold values are as yet unavailable.

\section{Digestion in the Animal Organism}

The many complex activities that proceed in the animal gastrointestinal tract may produce three major changes in the nutritional vitamin forms: liberation, activation, and destruetion. Most frequently, the bound unabsorbable vitamin complexes may be broken down and the vitamin liberated and made available for absorption. Associated with this process, the cellular structure of the food may (or may not) be destroyed. Less often, the vitamin may be so modified in the intestinal tract as to provide a more active form than that ingested, for example, a form more closely related to the functional form. Finally, destruction or inactivation of 
$B$ vitamins may occur. These effects may be produced as the result of enzymes indigenous to the animal, or as the result of intestinal microflora; but in most cases it is not now possible to distinguish whether one or both factors are involved. The establishment of whether the digestive effect is due to animal or microfloral action is important, since the assessment of factors which may affect the digestive process must be made in terms of which member of this digestive partnership is involved. When large amounts of a nutritional component are processed in the digestive tract, bacterial effects upon the component may be negligible; but in the case of small amounts of catalytic materials, bacteria may move the digestive process into a radically different channel.

The part of the B vitamin nutritional intake which is supplied by bacteria is (most logically) largely present in the intestine in free form, and is not further materially affected by the digestion. A large fraction of the exogenous B vitamin nutrition is in bound form, however. In some cases cooking of the food may suffice to break the complex. This is reported to be markedly true in the case of riboflavin, and to a variable and lesser extent in the case of the other vitamins; it is, of course, dependent upon the conditions of temperature, $\mathrm{pH}$, and concentrations of other ingredients. Cooking does not produce sufficient liberation of available forms to be considered as a major factor in the process. While some liberation may occur in the stored uncooked food as the result of ripening processes or autolysis, this factor seems not to be of any considerable importance in the overall liberation. Thus, water-soluble choline compounds (choline glycerophosphoric esters) appear quite rapidly when rat intestine and stomach are allowed to autolyze, but there is only very slow liberation in lung and kidney autolyzates, and scarcely any in brain, liver, and heart. ${ }^{7}$

The $\mathrm{B}$ vitamin-protein complexes of the food are to a great extent broken down in the gastrointestinal tract, and a limited amount of evidence suggests that this process occurs largely in the duodenum. This process may not be as efficient in some cases as in others, and in the case of pantothenic acid complexes particularly, there is some evidence to suggest that the liberation is not as complete as in the case of the other $B$ vitamins. ${ }^{8}$ Since it seems quite likely that the functional form of pantothenic acid, unlike most of the other $B$ vitamins, involves a union of the vitamin with an amino acid (glutamic acid), it seems pertinent to suggest that the binding of pantothenic acid may be stronger for this reason. Similarly the apparent unavailability of some of the more complex forms of folic acid to some bacteria and to pernicious anemia patients suggests that a vitamin connected in its functional form (coenzyme) to 
an amino acid (e.g., glutamic acid) may not be as readily released as vitamins not normally so linked in the functional form.

A brief consideration of the structures of the B vitamins shows that a diversity of active groups is present which may participate in binding to proteins. Carboxylic acid groups are present in five of the B vitamins, hydroxyl groups in six, aliphatic or aromatic primary amino groups in four, and phenolic groups in three, with a scattering of other active structural groupings. Generally phenolic groups and aromatic amino groups remain intact in metabolism so as to function in redox reactions; and hydroxyl groups are frequently phosphorylated in functional forms. It would, therefore, seem most likely that the B vitamins are generally bound via their acid or aliphatic amino groups or via the acid groups of their phosphates to suitable active groups in proteins, and that the amido and salt linkages so formed should be quite readily hydrolyzed by $\mathrm{pH}$ extremes or certain phosphatases and digestive enzymes. Thus thiamine, riboflavin, choline, and inositol are the $\mathrm{B}$ vitamins which have well known and widely occurring phosphates, but few other good groups for protein binding, and so may logically be bound via phosphate molecules to protein; whereas nicotinic acid, pantothenic acid, $p$-aminobenzoic acid, biotin, and folic acid do not have widely distributed (or well known) phosphates, but do have carboxylic acid groups capable of combining with free protein amino groupings. Nicotinamide may possibly be bound through its amide grouping; indeed either it or nicotinic acid might be produced upon liberation, depending upon the course of the action. The $\mathrm{B}_{6}$ vitamins most logically would be bound via their 5 -hydroxymethyl group (phosphorylated?) since other active groupings are involved in the vitamin function and must necessarily remain intact. Such reasoning supposes that each vitamin contains separate groupings for performing its primary function and for attaching it to its protein enzyme-an hypothesis which fits well the existing information on this subject. In vitro studies with pure enzyme preparations should do much to elucidate the nature of the binding involved in each case, and the enzymes capable of vitamin liberation. The fact that the avidin-biotin complex is not broken by the intestinal processing indicates that the nature of the binding may not be deduced with complete accuracy on the basis of structural considerations alone, although the $-\mathrm{CO}-\mathrm{NH}-$ grouping common to both biotin and protein linkages may be suggestive of secondary binding effects.

Individual differences in digestive ability, whether within physiological or pathological limits, are doubtless among the important factors which influence the broad individual differences in $\mathrm{B}$ vitamin requirements. This fact is extremely evident in the case of the folic acid conjugases, 
which may be well utilized by normal persons, but are apparently unavailable to patients with pernicious anemia, although folic acid itself is effective. In this instance there is some reason to believe that "vitamin $B_{12}$ " functions in the enzyme which hydrolyzes vitamin $B_{c}$ conjugate to folic acid. ${ }^{9}$ A variety of other disturbances which involve the gastrointestinal tract have similarly been shown to produce B vitamin deficiencies, and may involve drastically reduced abilities to liberate bound forms of the B vitamins. Among healthy persons, the differences may not be so manifest, but they undoubtedly do exist and may markedly predispose certain individuals and groups to avitaminoses. The factors influencing intestinal liberation of the B vitamins are at least as manifold as those affecting the digestive process in the broader sense. They lack experimental elucidation at present, but may be surmised generally on the basis of our overall knowledge of gastrointestinal digestion. Because of the lack of data, a more extended consideration of these factors is not now possible.

Mention has previously been made of the unavailability of the B vitamins in live yeast (p. 291), and this must be considered in the broader sense as a digestive limitation. It seems equally certain that other cellular forms that are not disrupted during digestion may similarly withhold their vitamins, so that cellular disintegration is a critical factor in the digestive process. While the cells of most food material are apparently not as resistant to fracture as are yeast cells, further investigation of this point is merited. The possibility of irreversible adsorption of the B vitamins upon other nutritional components in the intestine seems also worthy of consideration in this regard. Fuller's earth adsorbates of rice polishing extract were early used as a thiamine standard, but it has been subsequently shown that only about half of the thiamine present could be eluted in the animal digestive tract. ${ }^{10}$ Similar adsorbents are now broadly used as medicants, and undoubtedly they similarly limit the available thiamine in some cases. Cellulose may exert a similar effect, although the evidence now available seems to disprove this belief. ${ }^{11}$

The free vitamin may in some instances be converted by the digestive process to an even more active form, although this is not apparently a general process. Phosphorylation and bacterial conversion to functional forms probably account for the cases in which this is so, although it is by no means certain that other vital changes in vitamin structure do not occur in the intestinal tract of some species. The conversion of the higher homologues of folic acid to folic acid in the animal digestive tract must, moreover, be considered as one example of an increase in vitamin activity in the light of our present understanding of this group of substances. In addlition, it is probable that many other such apparent effects are actually 
due to the favorable influence of the exogenous vitamin on endogenous bacterial synthesis in general, rather than to actual interconversion of an exogenous molecule by a bacterium to a more active derivative of the same molecule.

Finally, the chemical processes encountered in the digestive tract undoubtedly result in the destruction of some percentage of ingested $\mathrm{B}$ vitamins; this factor is a major one in certain of the cases where parenteral vitamin administration is markedly more effective than feeding per os. Biotin, for instance, is said to be five times more active parenterally, ${ }^{12}$ and it might be expected that the $-\mathrm{CH}-\mathrm{NH}-\mathrm{CO}-\mathrm{NH}-\mathrm{CH}-$ structure of its ring would receive some destruction by enzymes active upon peptide linkages. For the same reason, folic and pantothenic acids may be hydrolyzed to some extent in the intestine, and there may be some cleavage of carbon-nitrogen bonds, such as those on the ribitol in riboflavin and on the thiazole moiety in thiamine. Pyridoxal and pyridoxamine are also less active and may be partially decomposed when fed orally, as might be anticipated from their general chemical reactivity in vitro. Vitamin $B_{12}$ is readily destroyed by the digestive processes, and it is essential that this vitamin be protected by conversion into a bound form. Ternberg and Eakin 12a have recently shown that "intrinsic factor" (p. 415), a protein material present in the gastric juices, has this ability of combining with vitamin $\mathrm{B}_{12}$ and protecting it from digestive destruction. In pernicious anemia, intrinsic factor is absent from the gastric juice, and a vitamin $B_{12}$ deficiency results due to the digestive destruction of the unprotected vitamin.

Since our nutrition is oral, and the $\mathrm{B}$ vitamin nutritional requirement is a summation of all these effects, digestive destruction is not generally a matter for extreme concern. In experimentation or medication where parenteral administration is used, it is important, however, to take cognizance of the fact that a much larger amount of vitamin may reach the animal via this route than by supplying a similar amount orally, from the standpoint both of the physiological effects that may result, and of exceeding the limits below which the vitamin is not toxic. As will be shown later in some detail, parenteral administration is most commonly practiced with the two vitamins that appear to be most toxic, thiamine and nicotinic acid. In other cases, as in these, extrapolation of the oral therapeutic dose to the parenteral one is dangerous, largely because of our limited knowledge of the degree of destruction of the oral dose in the intestine.

Finally, it should be pointed out that despite the fact that the $\mathrm{B}$ vitamins are markedly soluble in water and generally just as insoluble in organic solvents, they may in some cases nevertheless have to undergo 
transformations, like the fat-soluble vitamins, to render them absorbable. This is most certainly so in the case of some of the absorbed forms of choline, and may be true of other vitamins, such as inositol. For some of the lower forms of life, for which the nutrition must reach the absorptive membrane in particulate form, and liquid media will not suffice, a similar consideration may well be involved. In any case, the water solubility of a substance cannot be considered a priori to be the final end to be achieved in the digestive process.

\section{The Absorption of B Vitamins}

The state of knowledge of the processes involved in the absorption of the individual $\mathrm{B}$ vitamins is to a large extent a function of the time that the $\mathrm{B}$ vitamin in question has been well recognized. The more explicit information which is available concerning thiamine and riboflavin absorption indicates clearly that the absorption of the $\mathrm{B}$ vitamins cannot be regarded as a simple process, even though it is frequently assumed to be for the more recently discovered vitamins. Passage of a metabolite across a living membrane seldom is a matter of passive transfer or simple diffusion. This is certainly a good generalization for the B vitamins, even in view of the lack of much experimental data to verify the assertion. It should also be reemphasized at this point that vitamin-protein complexes may be absorbed in some cases, just as undigested proteins in general may be absorbed to a limited degree.

In the case of both thiamine and riboflavin, it is believed that phosphorylation occurs in the intestinal mucosa prior to absorption. ${ }^{13}$ Throughout the gamut of physiological processes, phosphorylation is a frequent adjunct to the passage of metabolites of many kinds across membranes, so that in the cases of thiamine and riboflavin the process is by no means unique. Both thiamine and its phosphate are readily absorbed in the small gut; ${ }^{1+}$ and although thiamine occurs in the blood plasma in the free form ${ }^{15}$ (part of which may be again secreted in the gastric juice), duodenal phosphorylase readily phosphorylates thiamine in vitro, ${ }^{16,17}$ so that phosphorylation and dephosphorylation apparently are involved in passage across the intestinal wall into the circulation, as in the case of transfer of many other metabolites. Riboflavin, its 5'-phosphate, and its adenine dinucleotide are all available to the higher animals, but it is possible that the coenzymes are broken down prior to absorption. Free riboflavin is phosphorylated prior to absorption, ${ }^{13,18}$ and the phosphorylation may be done in vitro with mucosal extracts. Interference with the process by iodoacetate or by adrenalectomy in rats causes a prompt lack of free riboflavin absorption and ensuing cessation of growth, which may 
however be prevented by nutritional riboflavin-5'-phosphate or the dinucleotide coenzyme form. ${ }^{19}$

Only scant knowledge exists in other cases. Free B vitamins are generally believed to be readily absorbed in the intestine, but probably not as such. It is not certain whether cozymase and inositol phosphate are absorbed directly or first split to simpler products, but both nicotinic acid and its amide are apparently absorbed. Recent evidence indicates that coenzyme A is probably hydrolyzed prior to pantothenate absorption. ${ }^{20}$ By analogy with thiamine and riboflavin, it seems possible that many of these vitamins undergo phosphorylation prior to absorption; this point merits further investigation. Since absorption processes and "thresholds" are apparently major factors in influencing the individual differences in efficiency of $\mathrm{B}$ vitamin utilization and requirements, it is indeed surprising that more extended study of this subject has not been undertaken to date.

It is readily apparent that a large number of factors may influence the ability to absorb the B vitamins. Diets high in fats may mechanically prevent ready access of the vitamin to the absorptive membrane. Pharmaceutical derivatives of riboflavin have been prepared with such low solubility as to be poorly absorbable. In the case of renal resorption of amino acids, competition may exist between amino acids that are resorbed by a similar mechanism for the limited metabolic activity of the membrane, ${ }^{21}$ and it seems quite logical that an excess of metabolites that are transported across the intestinal wall by a mechanism (e.g., phosphorylation) similar to that involved with a vitamin may diminish the absorbability of that vitamin. This factor may explain the observation that the presence of food in the gastrointestinal tract diminishes the absorption of calcium pantothenate in dogs..$^{22}$ Certainly any factor that influenced the phosphorylation activity of the intestine would have this effect, and this consideration may well be advanced as one other that favors a balanced diet containing a variety of nutrients. At present, however, it is entirely impossible to estimate what portion of vitamin malnutrition is due to absorption difficulties. It is known that wide variations do exist in the ability to use dietary components, and further extended studies are necessary to show what effect the state and nature of the nutrition have on the ability of an individual to utilize B vitamins. We have already noted (p. 300) that large amounts of B vitamins occur in the feces, and it is most uncertain what portion of this material is of exogenous origin and whether its presence is due to its inavailability to the animal or to its not being required. Over 60 per cent of the B vitamins of feces are said to be water-soluble, and should be available. ${ }^{23}$ Perhaps by a better understanding of the absorptive process, we may some day 
hope to understand this, as well as the innumerable clinical reports dealing with the marked beneficial effects of large doses of some one vitamin in an individual where the pathology bears no apparent relation to the medication.

\section{Distribution of the B Vitamins}

Much specific information has been included in an earlier chapter on the occurrence and distribution of the $\mathrm{B}$ vitamins that might be reconsidered at this point. It would seem more appropriate, however, to consider in more general terms and from a somewhat dynamic standpoint the various general relationships that exist in the distribution process, leaving the interpretation of the voluminous data found in the literature on $\mathrm{B}$ vitamin occurrence for those whose needs justify the labor that must necessarily be involved in so arduous a task. The allocation by the circulation of the various $B$ vitamins to the tissues is a complex process, and we can at best do little more than guess at the principles involved.

State and Levels in the Circulation. Once the B vitamins are introduced into the circulation, they are rapidly distributed between the cellular elements and the plasma, and almost as rapidly between the blood and the tissues, so that an equilibrium is usually maintained. It is generally true that the plasma does not in itself modify the B vitamins, although in some cases "binding" may occur. The blood is generally incapable of any vitamin liberation, being unable to break, for instance, the avidin-biotin complex. ${ }^{24}$ Parenteral administration of the complex, however, results in its destruction by tissue oxidative processes.

The thiamine in blood plasma is largely in the free state, but not entirely so, whereas that in the cellular elements of the blood is mostly, if not entirely in the form of cocarboxylase. ${ }^{25}$ Apparently all nucleated cells are capable of performing the phosphorylation, and adult red blood cells are believed to have acquired their cocarboxylase content prior to loss of the nucleus while in the bone marrow. ${ }^{26}$ The total blood thiamine in normal humans generally ranges between 8 and $9 \mu \mathrm{g}$ per cent, although some workers have reported values as high as $14.5 \mu \mathrm{g}$ per cent. ${ }^{27}$ Of this, apparently 70-90 per cent is esterified.28-30 Pig blood apparently contains about $20 \mu \mathrm{g}$ per cent; ${ }^{31}$ oxblood, $5.7 \mu \mathrm{g}$ per cent;25 pigeon blood 20.2 $\mu \mathrm{g}$ per cent; $;^{25}$ rat blood $7 \mu \mathrm{g}$ per cent; $;^{32,33}$ and rabbit blood about 28.3 $\mu \mathrm{g}$ per cent of total thiamine. ${ }^{34}$ Various reported figures relating to the distribution of free and esterified thiamine between plasma and cells, and in pregnancy, and placental and amniotic fluid, ${ }^{36}$ and infant blood ${ }^{37}$ may be found in the literature, but are not at present sufficiently substantiated to merit general acceptance. 
Riboflavin is converted by the red blood cells (probably only nucleate erythrocytes) and most other cells of the body into flavin adeninedinucleotide, but this reaction cannot be performed by the plasma. ${ }^{35}, 38$ There is apparently a rather constant equilibrium between the coenzyme levels in cells and plasma; and the level in the whole blood remains quite steady under most conditions. Human blood is said to contain the equivalent of about $21.2 \mu \mathrm{g}$ per cent, varying somewhat annually in places where there are marked seasonal dietary changes. ${ }^{39}$ The blood plasmas of several Brazilian snakes contain levels ranging from 180 to $300 \mu \mathrm{g}$ per cent. ${ }^{40}$

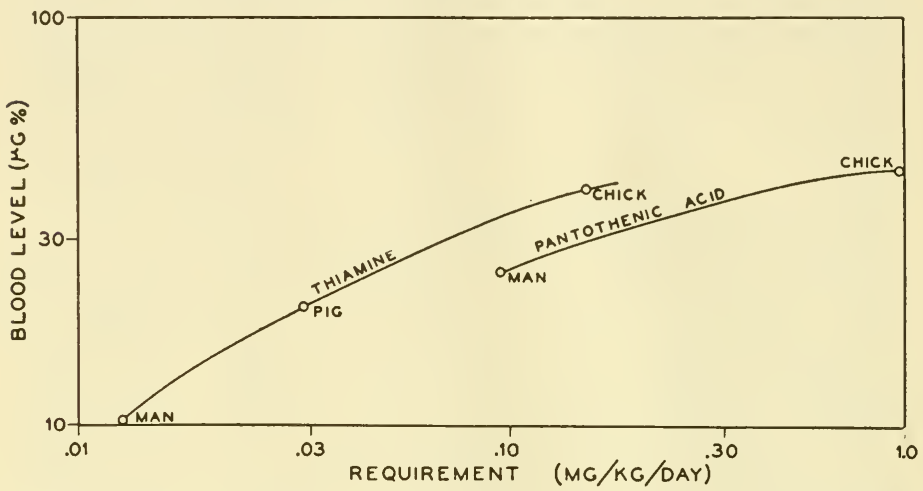

Figure 15. Relationship of the requirements by various species for thiamine and pantothenic acid to the blood levels of these vitamins.

Nicotinic acid and nicotinamide enter the blood, as far as is now known, as such, although it is possible that small amounts of cozymase may escape digestion and be directly absorbed. Blood plasma contains both the acid and the amide, and these are converted to cozymase ${ }^{41}$ in nucleate blood cells (for the most part white cells, which contain very little of the free vitamin). In humans, the blood level normally ranges from 400 to $700 \mu \mathrm{g}$ per cent, of which about one-third is free and the balance is combined as coenzyme. ${ }^{42-45}$ The blood level of nicotinic acid is not generally lowered appreciably in pellagra. ${ }^{46}$ Horse blood contains about $180 \mu \mathrm{g}$ per cent free and $140 \mu \mathrm{g}$ per cent combined nicotinic acid, while in the cow the corresponding levels are about 268 and $332 \mu \mathrm{g}$ per cent, respectively.

Pantothenic acid is found in the blood of man in concentrations of about $30 \mu \mathrm{g}$ per cent, ${ }^{47}$ and in chicks in levels of about $40 \mu \mathrm{g}$ per cent $(50 \mu \mathrm{g} \text { per cent in the plasma) })^{48}$ but little is known of its state or dis- 
tribution, and even less of the states of the remainder of the B vitamins in the circulation. On the basis of thiamine, riboflavin, and nicotinic acid, it would appear that free B vitamins entering the plasma are unchanged there, but largely enter the nucleate blood and tissue cells where they are converted to coenzymes, leaving only a low residual free vitamin content in the blood. How true this is of the other vitamins remains to be determined.

With regard to the relationship of the blood levels of $\mathrm{B}$ vitamins to the nutritional requirement for these vitamins, three general relationships are apparent. Except those vitamins that may be supplied to a large extent by endogenous sources such as niacin and biotin, the blood levels of any given vitamin for a number of species tend to increase as the nutritional requirements on a unit body weight basis increase, i.e., as the sizes tend to decrease (p. 246).$^{227}$ This is in line with Williams' observation that there is a "tendency for the vitamin content to be lower in the tissues of larger animals." 49 With the same exceptions, for any given species, the blood levels of various $B$ vitamins tend to vary with the vitamin requirements. These relationships are shown in Fig. 15. Finally, as indicated in an earlier section, at levels of vitamin intake below the nutritional requirement, the blood level tends to reflect the intake, whereas at higher dietary levels it does not. The urinary levels, conversely, reflect only the higher intake levels, for obvious reasons. With regard to this last generalization, so many qualifications and apparent exceptions exist, and, as previously mentioned, so many other dietary factors influence the balance, that it must be taken only as a rather self-evident and frequently demonstrable trend, and not relied upon quantitatively.

Levels in Milk. For a number of readily apparent reasons, there has been extensive study of the levels of the various B vitamins in the milk of a number of species. Much of the existing knowledge as to actual levels is summarized in Table 27. Present limited information suggests that thiamine, biotin, and inositol occur in milk in bound forms (or in more firmly bound forms than the other B vitamins); but little is actually known about the precise vitamin forms in milk. Within certain limits, the vitamin content of the milk reflects that of the diet. Among a number of species, it is known that the $\mathrm{B}$ vitamin content of the milk generally increases as the requirement increases, and in any one species, the relative amounts of $B$ vitamin in milk tend to vary with the requirements, as shown in Fig. 16.

The colostrum in ruminants seems to be higher in vitamins $\left(B_{1}\right.$ and $\mathrm{B}_{2}$ ) than is the adult milk, ${ }^{50}$ whereas the inverse seems to be true in the human. Macy et al. ${ }^{51,52}$ have shown that the following variations occur in human milk: 


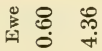

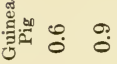

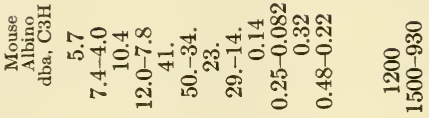

हี

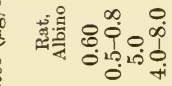

每

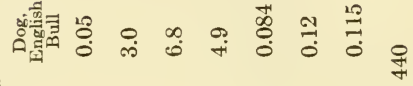

亏ั้

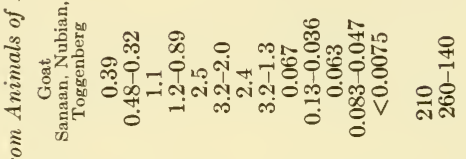

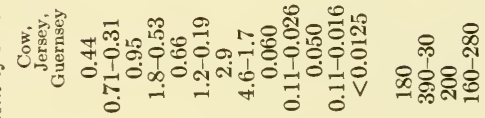

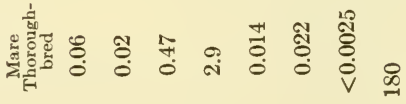

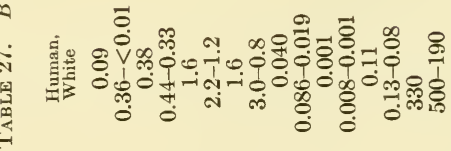

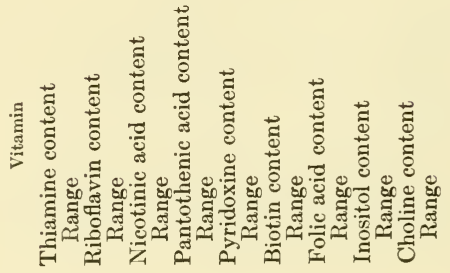


(a) Thiamine: 0.9-2.4 $\mu \mathrm{g}$ per cent during first few days after parturition, rising to $8.1 \mu \mathrm{g}$ per cent on tenth day and about $148 \mu \mathrm{g}$ per cent in mature milk. Free thiamine starts at $1 \mu \mathrm{g}$ per cent, rising to 5-7 $\mu \mathrm{g}$ per cent, and averaging 37 per cent of the total.

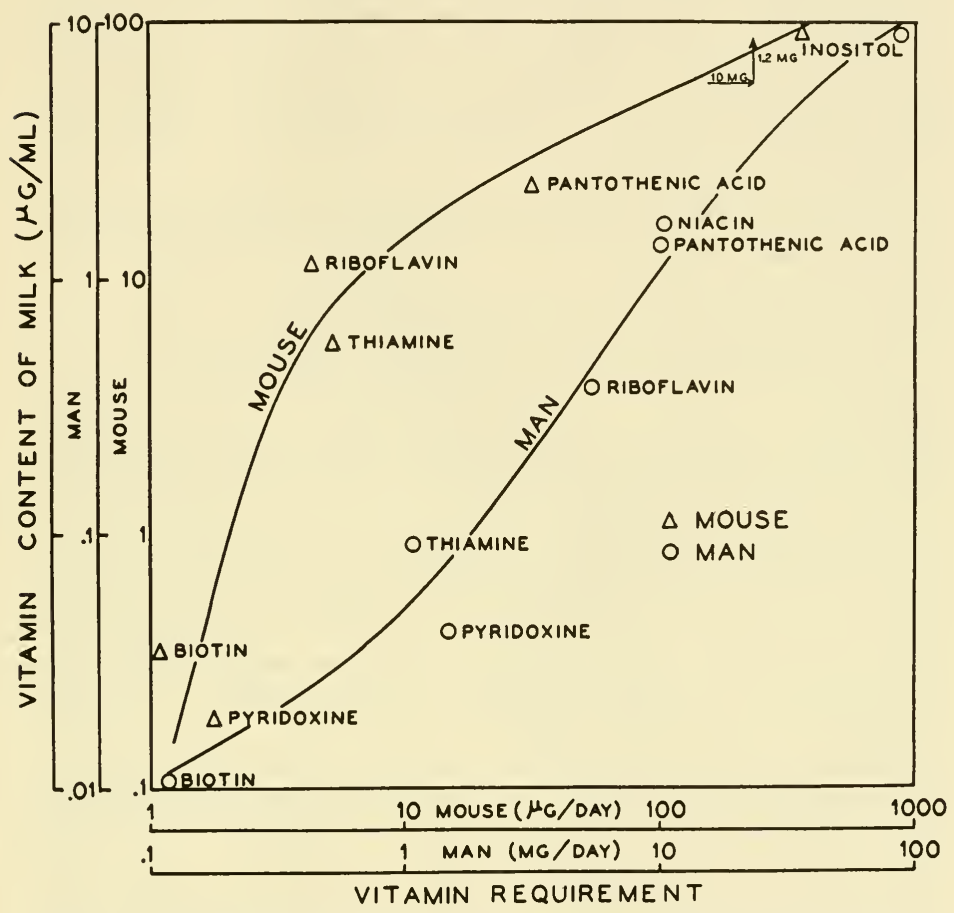

Figure 16. Relationship of the $B$ vitamin requirements of man and the mouse to the levels of $B$ vitamins found in the milk of these species.

(b) Riboflavin: $19.6 \mu \mathrm{g}$ per cent on first day of lactation, rising to $39.2 \mu \mathrm{g}$ per cent on the tenth day, with free riboflavin rising more slowly. Mature milk contained a total of about $35-49 \mu \mathrm{g}$ per cent, with free riboflavin composing 43-86 per cent of the total.

(c) Nicotinic acid: $100 \mu \mathrm{g}$ per cent on the first day, decreasing slightly from second to fourth day and then rising rapidly to about $250 \mu \mathrm{g}$ per cent on tenth day. Mature milk contained 175-200 $\mu \mathrm{g}$ per cent. 
(d) Pantothenic acid: $48 \mu \mathrm{g}$ per cent on first day to $245 \mu \mathrm{g}$ per cent on the fourth day and rising to $304 \mu \mathrm{g}$ per cent on the tenth day, with mature milk containing about $250 \mu \mathrm{g}$ per cent.

(e) Biotin: Very low first five days, rising to about $0.38 \mu \mathrm{g}$ per cent on ninth day and $0.80 \mu \mathrm{g}$ per cent in mature milk.

Pearson's data on cows and ewes show, however, that these trends are not necessarily general. Thus: ${ }^{50}$

(a) Cow colostrum contains $62 \mu \mathrm{g}$ per cent thiamine and $610 \mu \mathrm{g}$ per cent riboflavin, while cow milk contains $38 \mu \mathrm{g}$ per cent thiamine and $177 \mu \mathrm{g}$ per cent riboflavin.

(b) Ewe colostrum contains $180 \mu \mathrm{g}$ per cent thiamine and $2008 \mu \mathrm{g}$ per cent riboflavin, while ewe milk contains $60 \mu \mathrm{g}$ per cent thiamine and $436 \mu \mathrm{g}$ per cent riboflavin.

(c) Nicotinic acid content of cow colostrum and milk are about the same, but ewe milk is twice as rich as ewe colostrum.

(d) Pantothenic acid is higher in the milk of both species.

All workers in this field seem generally agreed that variations between individuals are great in regard to milk vitamin content. Since the level in the milks of individuals receiving similar diets must involve the intestinal-circulatory absorption threshold, the excretory thresholds, and the circulatory-acinus threshold, there is ample reason to anticipate such marked individual differences. In consideration of the low content of human and cow's milk in relationship to infant requirements, particularly in regard to thiamine and niacin, at a time when there is only limited intestinal synthesis in the young, and of the broad individual differences in human milk, the concept of milk as a perfect nutrient for the young should not be accepted uncritically.

Distribution and Storage in Tissues and Body Fluids. The B vitamins are circulated and pass readily from the blood into the various tissues and fluids of the body, in each case establishing an equilibrium between the content of the tissue and the environment. A discussion of tissue profiles and various interrelationships that exist in this regard are to be found elsewhere in this volume. Ultimately, studies of distribution constants may make possible the calculation of tissue contents from vitamin intakes, but this is not now generally possible. In the tissues, the vitamins are frequently fixed into firmly bound coenzyme-enzyme complexes or vitamin-protein storage forms. It has been shown that practically all the pantothenic acid of the body tissues is in coenzyme form, ${ }^{53,54}$ and it seems apparent that in the cases of most other B vitamins it will eventually be shown that the coenzyme form accounts for the majority of the vitamin present. It has previously been mentioned that nicotinamide is the predominating form of that vitamin in animal tissues (p. 330), while 
nicotinic acid predominates in plant tissues. In animal tissues and yeast pyridoxal and pyridoxamine predominate, while pyridoxine is present in larger amounts in plants. ${ }^{55}$ Of theoretical interest in this regard is the fact that oxybiotin functions per se in animal tissues and is not converted to biotin. ${ }^{56}$ Its storage in tissues parallels closely that of biotin. ${ }^{57}$ Vitamin storage in animals is a limited process, being influenced by many factors, most frequently by the protein intake, as previously mentioned (p. 277). Whereas storage doubtless occurs throughout the body, the liver seems to be particularly effective as a storage depot in many cases. As previously noted, rats on a low-protein diet are not able to store riboflavin, regardless of the intake, but excrete unused excesses promptly, whereas high riboflavin intakes on a high-protein diet bring about prompt liver storage of riboflavin. ${ }^{58}$ Similar relationships have been observed for other vitamins, ${ }^{59}$ although storage abilities vary among species and for each vitamin. Thus the depletion of some vitamin in one species (e.g., thiamine in the rat) may cover a long period of progressive pathology, while in others (e.g., thiamine in the mouse), the depletion is so rapid that death is one of the first observable symptoms. ${ }^{60}$ For this reason, the general nature of and trends in B vitamin storage are so erratic as to make generalization impossible for practical purposes. At best, the possibilities of building up extensive B vitamin reserves are limited, unlike the fatsoluble vitamins, and current trends in nutritional thought along these lines should be considered critically in regard to both humans and farm animals. ${ }^{61}$ The desirability of increasing the vitamin level in meats in certain areas is great, and it has been shown that it is possible to increase the thiamine level in hog tissues by feeding higher levels of thiamine in the diet. ${ }^{2}$ It should be noted that the hearts and livers of hogs on lower thiamine levels, however, had more riboflavin than did those tissues from animals receiving adequate thiamine. In the chicken, increases in muscle pantothenic acid cannot be achieved by dietary supplementation with pantothenic acid levels above those required for adequate nutrition, however. A considerable amount of data has recently shown that within limits the riboflavin content of hens' eggs may be increased by increasing the dietary riboflavin, and in such cases when the efficiency and limits of effectiveness are known, higher feeding levels are obviously desirable in fowls. ${ }^{63-66}$

\section{Synthesis and Catabolism in Animals}

The biogenesis of the $\mathrm{B}$ vitamins has been discussed at some length in an earlier chapter, but certain considerations make it seem appropriate to review some aspects of this subject as it applies to the higher animals. Whereas most animals have the ability to convert the commonly recog- 
nized forms of the $\mathrm{B}$ vitamins to their respective functional forms, only in the cases of nicotinic acid and choline are the animals apparently able to synthesize the vitamin from some precursors. Special consideration must be given to the synthesis of these vitamins, therefore, and also to the breakdown of nicotinic acid, concerning which we have considerable knowledge. The transformation of the other B vitamins to their excretory products has been little studied and few data are available in their regard.

Coenzyme Synthesis. Thiamine, riboflavin, pyridoxal, nicotinamide, and pantothenic acid are known to function in the specific coenzymes cocarboxylase, flavin mononucleotide and dinucleotide, codecarboxylase, coenzymes I and II, and coenzyme A, respectively, all of which contain phosphate. In the case of cocarboxylase, flavin mononucleotide, and codecarboxylase, the vitamin phosphate apparently constitutes the entire coenzyme, and the synthesis of the coenzymes by phosphorylases is apparently performed within the various cells of the animal in a rather direct manner. This is certainly so in the case of thiamine, since preparations from liver and kidney, and to a lesser extent muscle and brain actually convert it to cocarboxylase. ${ }^{67}$ Rat kidney extracts in phosphate buffer at $\mathrm{pH} 8.4$ and containing a trace of arsenite are particularly effective in this respect, ${ }^{68}$ and it has been shown that some energyyielding system is necessary to perform the phosphorylation, ${ }^{69}$ as would be expected. It is unclear, however, just how the vitamin $B_{6}$ triad functions in maintaining the codecarboxylase concentration, although it seems, likely that pyridoxine is first oxidized and then phosphorylated to pyridoxal phosphate; this then functions in a reversible transamination system with pyridoxamine phosphate in those processes which activate the $\alpha$ carbon atom of $\alpha$-amino acids, such as transamination, decarboxylation, and trytophan decomposition. Present evidence indicates at least that pyridoxamine requires the presence of a keto acid for its conversion to pyridoxal ${ }^{69}$ (p. 176). It further seems likely, but is not proved, that riboflavin is first phosphorylated and then coupled with adenylic acid, since adenylic acid and riboflavin-5'-phosphoric acid are formed on the enzymatic hydrolysis of the "flavin-adenine-dinucleotide." 70 In this regard, riboflavin must be considered to be a step more evolved toward the coenzyme than nicotinic acid, animals apparently not being able to couple ribitol to the 6,7-dimethyl-isoalloxazine nucleus, as they are able to couple ribose to nicotinamide. The steps involved in the conversion of nicotinamide to coenzymes I and II are not known, but by analogy with riboflavin one might expect the process to involve combinations with ribose, phosphorylation, and coupling with adenylic acid successively. It has been shown that rabbit brain contains a DPN 
nucleosidase which splits only nicotinamide from DPN, whereas rabbit kidney contains a pyrophosphatase which forms adenylic acid and nicotinamide mononucleotide from DPN; ${ }^{71}$ and it may be that the coenzyme synthesis similarly has more than one route. The synthesis of the coenzyme does not directly involve pyridoxine or its derivatives. ${ }^{72}$ The DPN of chick embryos increases with the niacin content, suggesting that the ability to synthesize coenzymes is present in the animal from the earliest stages of its development. ${ }^{73}$ Apparently only leucocytes and other nucleated cells are able to perform the synthesis. ${ }^{41,74}$ The tissues of pantothenic acid-deficient rats and ducks exhibit a deficiency of coenzyme A in a manner analogous to many of the other B vitamins. Nitrogen, arsenite, and glucose are reported to interfere with coenzyme A synthesis in vitro. ${ }^{75}$ With regard to biotin, there is some evidence to indicate that adenylic acid is involved in the synthesis of its coenzyme, ${ }^{228}$ which is reported to be more active biologically than biotin itself. ${ }^{76}$ Steps involved in the formation of active forms of the other $\mathrm{B}$ vitamins are unknown and must await further elucidation of the structure of the functional forms.

Choline Synthesis. Any discussion of choline synthesis must be considered in the light of the fact that in many ways choline is not a typical $B$ vitamin, and appears to occur and function in relatively large amounts in the living organism. In an earlier chapter it has been shown that in Neurospora and other organisms, choline is synthesized via the process: $\mathbf{7 7}, \mathbf{7 8}$

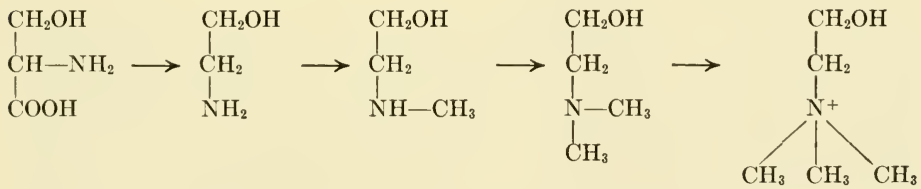

and that the necessary methyl groups may be derived from a source such as methionine or betaine. Apparently the same series of reactions occurs in all the higher animals if sufficient serine and methionine are available, ${ }^{79}$ and in practice it is necessary to limit the methionine content of the diet in order to produce choline deficiencies. Isotopic studies in the rat have done much to show that the pathway indicated above does in fact exist in animals. ${ }^{80}$

Nicotinic Acid Synthesis. It has previously been mentioned that animals have the ability to convert tryptophan to nicotinic acid (p. 83), and that in Neurospora the pathway presumably involves kynurenine, 3-hydroxy-kynurenine, and 3-hydroxy-anthranilic acid (p. 280), but that 
even in Neurospora the exact sequence is not as yet well understood. While there is much reason to believe that the process is similar in the higher animals, there is not at present sufficient evidence to indicate that this is unequivocably so.

The primary indications that tryptophan is converted to nicotinic acid in animals are the facts that the administration of tryptophan to the rat, ${ }^{81}$ horse, ${ }^{83}$ pig, ${ }^{84}$ dog, ${ }^{85}$ calf, ${ }^{86}$ and humans, ${ }^{87}$ results in the excretion of increased amounts of nicotinic acid metabolites in the urine, ${ }^{88}$ and that tryptophan supplants niacin in preventing a niacin deficiency in the rat ${ }^{89}$ chick, ${ }^{90}$ mouse,${ }^{91}$ dog,${ }^{85}$ pig,${ }^{84}$ guinea pig, ${ }^{82}$ rabbit ${ }^{92}$ and humans. ${ }^{93}$ While many authorities have attempted to explain these facts on the basis of intestinal bacterial synthesis, a number of ingenious experiments have largely eliminated this possibility. Thus the injection of chick eggs with tryptophan causes an increase in niacin content. ${ }^{95}$ In addition, a variety of measures designed to lower or minimize the effects of intestinal symbiants have been without effect upon the conversion. ${ }^{96}$ Perhaps the only known exceptions to this fact in the animal kingdom are the cases of germ-free Drosophila - which require both tryptophan and niacin, and in which tryptophan, when increased, causes a higher niacin requirement ${ }^{\mathbf{9 7}}$ and Tetrahymena. ${ }^{98}$ It is also known that 3 -pyridylmethylketone, a structural analogue of niacin, is toxic to mice. ${ }^{99}$ Since nicotinic acid prevents its toxicity, the analogue presumably interferes with niacin formation, and since tryptophan similarly reverses the toxicity, it presumably functions as a niacin precursor.

The balance of the data bearing on the problem center around the fact that in vitamin $B_{6}$ deficiency there is a decrease in urinary kynurenic acid and an increase in urinary xanthurenic acid, and that simultaneously there is a decrease in nicotinic acid metabolites in the urine. ${ }^{100}$ Since kynurenic acid is known to be derived from tryptophan, ${ }^{101}$ and kynurenine is a known intermediate in niacin synthesis from tryptophan in Neurospora, it seems logical to suppose that vitamin $\mathrm{B}_{6}$ is involved somewhere in the intermediate process. Actually, however, kynurenine does not lead to increased nicotinic acid synthesis in the rat, as indicated by the $\mathrm{N}^{\prime}$-methylnicotinamide excretion studies, and kynurenine does not produce growth in the rat in the absence of tryptophan and niacin. ${ }^{102-104}$ 3-Hydroxy-anthranilic acid does bring about increased growth and increased niacin and $\mathrm{F}_{2}$ excretion in rats, however. ${ }^{105}$ Since, however, the experimental basis for these reports was limited, and the work is not as yet confirmed, it seems best at present to leave the subject of the involvement of kynurenine in animal metabolism open while considering the known facts regarding this general metabolic pathway. 
In tracer studies with DL-tryptophan- $\beta-\mathrm{C}^{14}$, the labelled atom may be found in kynurenic acid, but not in niacin.

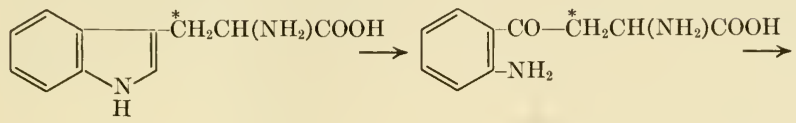<smiles>O=C(O)c1cccnc1</smiles>

This indicates clearly that the conversion does not involve the pyridine ring of kynurenic acid as a ring precursor of niacin. ${ }^{106}$ When, however, DL-tryptophan-3-C ${ }^{14}$ is employed, the tracer atom is found in the carboxylic acid group of niacin, ${ }^{107}$ strongly suggesting that it is the benzene ring of tryptophan which eventually becomes the pyridine ring of nicotinic acid.

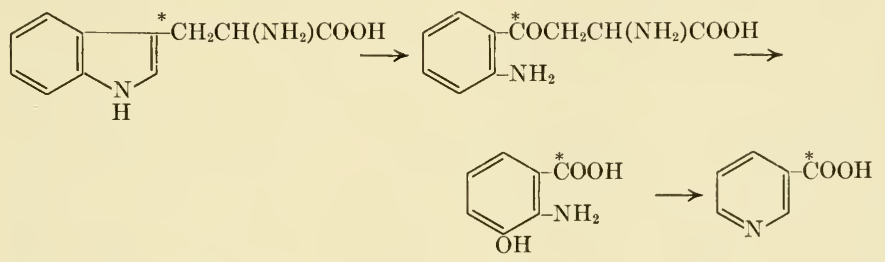

The ring rearrangement presumably involved in the final step of the process is of a type not entirely unknown to organic chemistry. Very recently Henderson and co-workers ${ }^{107 a-c}$ have shown that a probable intermediate in this step is quinolinic acid. Rats on a $9 \%$ casein diet containing little niacin but $2.5 \%$ added DL-tryptophan excrete 6-11. $\mathrm{mg}$ of this acid per day. The injection of 1 . mM of tryptophan or 3hydroxyanthranilic acid into such rats increases their niacin excretion tenfold, their $\mathrm{F}_{2}$ excretion twenty-five to one hundredfold, and their quinolinic acid excretion one hundred to three hundredfold. Quinolinic acid is slightly active for rats in replacing dietary niacin, is active in some Neurospora strains, and accumulates in the culture medium of other strains. Rat liver slices or homogenates are able to convert 3hydroxyanthranilic acid to quinolinic acid, and quinolinic acid is converted by weak acid to a substance having niacin activity for Lactobacillus arabinosus. 
Kynurenic acid (4-hydroxyquinaldic acid) was the earliest known product of tryptophan metabolism in the higher animals, ${ }^{108}$ and it is known that the liver is one of the principal sites of its formation. Only the $\mathrm{L}$-form of tryptophan is active in its formation, and 3-indole-pyruvic acid and kynurenine are equally as active as tryptophan in kynurenic acid formation. ${ }^{109}$ In the rabbit, which normally excretes only kynurenic acid, thiamine deficiency produces a simultaneous excretion of kynurenine, so that thiamine presumably catalyzes the conversion of kynurenine to kynurenic acid. ${ }^{110}$ Kynurenic acid will not substitute for tryptophan in the diet. Work with methylkynurenic acid and methyltryptophan derivatives in the rabbit further indicates that it is the $\alpha$-amino nitrogen atom of the tryptophan that ends up in the kynurenic acid structure, rather than the pyrrole nitrogen. ${ }^{111}$ The presence in urine of two other products, xanthurenine (3-hydroxykynurenine) and xanthurenic acid (4,8-dihydroxyquinaldic acid $)^{112,113}$ has been recognized more recently. As previously stated, the occurrence of the yellow xanthurenic acid in the urine of various species (rice moth larvae, ${ }^{114}$ mice, ${ }^{115}$ rats, ${ }^{116,117}$ rabbits, ${ }^{118}$ $\operatorname{dogs},{ }^{118}$ swine, ${ }^{119}$ ) made vitamin $B_{6}$ deficient by depletion diets or by an inhibitor, 4-desoxypyridoxine, ${ }^{120}$ is closely associated in some manner with the pathway of niacin synthesis, and is the only certain intermediate point so far found that can be studied in animals. The general interpretation of this fact is that vitamin $B_{6}$ normally mediates tryptophan metabolism so as to prevent (or limit) ${ }^{121}$ its oxidation to xanthurenic acid. A quinine oxidase, recently isolated, has the property of oxidizing the carbon atom next to the nitrogen atom in a large variety of heterocyclic compounds. ${ }^{122}$ Although this enzyme is involved in niacin breakdown, as we shall see shortly, it does not affect tryptophan, xanthurenic acid, or kynurenic acid, and is thus probably not involved in niacin synthesis. It does oxidize indole, but neither indole, 3-indoleacetic acid, nor anthranilic acid is effective in niacin synthesis. ${ }^{123}$ Thus, in summary, whereas there is considerable direct and indirect evidence concerning the general pathway of niacin synthesis in animals, there is little certainty as to the actual steps involved, and the problem remains as one of the outstanding ones to be worked out in the future.

Products of Nicotinic Acid. There is probably more evidence bearing upon the nature of the products formed from nicotinic acid in animals than upon the metabolism of all of the other B vitamins combined. This is largely because these products are diverse in nature and occur in relatively large amounts. Further, because of their diversity, they have complicated the study of nicotinic acid requirements by means of the urinary excretion methods, which have been so successful in other cases. It is 
therefore now possible to account for a fair portion of the nicotinic acid metabolized in the animal body (see p. 365 ).

There are at present some eight known derivates of nicotinic acid which are excreted by various animal species. These are:<smiles>O=C(O)c1cccnc1</smiles>

Nicotinic acid<smiles>C[n+]1cccc(C(=O)O)c1</smiles>

Trigonellin<smiles>NC(=O)c1cccnc1</smiles>

Nicotinamide<smiles>C[n+]1cccc(C(N)=O)c1</smiles>

$N^{\prime}-$ Methylnicotinamide $\left(\mathrm{F}_{2}\right)$
Nicotinuric acid<smiles>O=C(O)c1cccnc1C(=O)O</smiles>

Quinolinic acid<smiles>C[n+]1cccc(C(N)=O)c1</smiles>
$N^{\prime}$-Methyl-6-pyridone-3-carboxylamide<smiles>O=C(NCCCCC(NC(=O)c1cccnc1)C(=O)O)c1cccnc1</smiles>

Dinicotinylornithine

In addition to these compounds, evidence has been presented to show that many other nicotinic acid metabolites are formed in the body. ${ }^{124-126}$ It seems well established that coenzymes I or II are not excreted, and there is little evidence to suggest that riboside derivatives of niacin are found in the excreta, so that these coenzymes are apparently hydrolyzed at the nicotinamide bond as a general procedure in their metabolism (see p. 352).

Our knowledge of niacin metabolism in various species must be interpreted carefully, in view of the fact that much earlier work was done with analytical procedures that were not specific or sensitive, and that certain compounds closely related to those measured were not known until recent years. Most species probably excrete at least some nicotinic acid and nicotinamide as such, although there is some conflicting evidence 
on this point. Thus, in the dog earlier reports stated that there was no free nicotinic acid or nicotinamide in the urine, ${ }^{127}$ whereas later workers report its presence. ${ }^{128}$ It has similarly been stated that birds excrete only nicotinic acid, ${ }^{129}$ although this seems unlikely, and indeed they have been shown to excrete dinicotinylornithine in their droppings (chicks). ${ }^{130}$

It has long been known that when pyridine is administered to animals, it is excreted in the form of $\mathrm{N}^{\prime}$-methylpyridinium ions, ${ }^{131}$ and heterocyclic nitrogen compounds in general seem to be largely methylated prior to excretion by both plants and animals. Even before the role of nicotinic acid as a vitamin was known, it had been shown that this substance, when administered to dogs, was excreted as trigonellin (and nicotinic acid), ${ }^{128}$ and it might be anticipated that trigonellin would be a major metabolic product of niacin. Despite the fact that the dog excretes nearly all of a 100-mg dose of nicotinic acid as trigonellin and nicotinic acid, rabbits do not excrete trigonellin, ${ }^{128}$ and apparently cannot methylate niacin. While humans excrete some trigonellin, it is now believed that this is all exogenous, and the result only of the trigonellin ingested. Oral administration of trigonellin to humans does not result in significantly increased excretion of other nicotinic acid metabolites, but does result in almost complete excretion of the entire dose as trigonellin. ${ }^{132-136}$ Although trigonellin is apparently ineffective in curing niacin deficiency in at least some species, generalizations are dangerous since, as in the case of other metabolites to be considered, results may vary depending upon the magnitude of the dosage and the nutritional state of the animal. At present it would seem best to believe that whereas in some species (e.g., the dog) trigonellin may be a major end product of niacin metabolism, it is probably found in only very small amounts in many others. While there is apparently little tendency to amidate trigonellin in animals, since ingested trigonellin is largely excreted as such, trigonellin in the dog may result from deamidation of $\mathrm{N}^{\prime}$-methylnicotinamide. This may well be the case in view of the ability of all animals studied to methylate nicotinamide, and the relatively lower levels of free nicotinic acid available to the dog for conversion directly to trigonellin. IVe may well assume for the present, therefore, that trigonellin arises as a product of methylated nicotinamide, and that animals that do not excrete measurable trigonellin lack the tendency to deamidate $\mathrm{F}_{2}$.

$\mathrm{N}^{\prime}$-methylnicotinamide $\left(\mathrm{F}_{2}\right)$ is apparently one of the major metabolic products of nicotinic acid in all the animals so far studied in this regard (man, ${ }^{87}$ rat $^{81}$ horse, ${ }^{83}$ calf, ${ }^{86}$ pig ${ }^{84}$ ). It is said to account for 94 per cent of the total nicotinic acid and its metabolites found in the urine. ${ }^{23}$ When rats are fed large amounts of niacin in which the acid group is labelled 
with $\mathrm{C}^{13}, 95.7$ per cent of the original concentration of $\mathrm{C}^{13}$ is recovered as $\mathrm{F}_{2} \cdot{ }^{143}$ Tracer work with $\mathrm{C}^{14}$ indicates, however, that both nicotinic acid and its amide are to some extent decarboxylated, ${ }^{144}$ although the products are as yet unknown. $\mathrm{F}_{2}$ is distinguished in urine by its formation of a bluish white fluorescent compound when examined under ultraviolet light in the presence of alkali and butanol, ${ }^{137},{ }^{138}$ and its part in nicotinic acid metabolism has been realized only during the past few years. It does not promote growth in Lactobacillus arabinosus (which can utilize both nicotinamide and nicotinuric acid) nor in Leuconostoc mesenteroides (which cannot utilize low levels of nicotinamide or any levels of nicotinuric acid, but requires free niacin). ${ }^{139}$ Liver slices in vitro, but not kidney or muscle tissue, convert nicotinamide to $\mathrm{F}_{2}$, and in vivo studies have shown that the intact liver is capable of performing this methylation at a rapid rate, whereas the intact kidney is not. ${ }^{140}$ The liver is thus believed to be the sole site of $\mathrm{F}_{2}$ formation; and $\mathrm{F}_{2}$ formation from nicotinamide has been shown to be a valuable test of hepatic function, employing as the test dose a physiological compound within physiological levels, as contrasted with the usual tests employing substances foreign to the animal body. ${ }^{141,1+2}$ The reaction has been of some value in the study of transmethylation in healthy persons, and it appears that in studies with rat liver slices, the methylation is frequently enhanced by methionine. ${ }^{140}$ Bearing upon the nature of the methylation process, as well as the utilization of $\mathrm{F}_{2}$ as a nicotinamide source, is the demonstration that the incorporation of 2 per cent of $\mathrm{F}_{2}$ into the Griffith and Wade fatty liver diet of four rats for three months resulted in liver fats of 24.5, 8.2, 10.6 and 23.5 per cent, as compared to rats on the unsupplemented diet with liver fats of $36.1,39.1,39.7$ and 34.7 per cent. It would thus appear that the methyl group of $\mathrm{F}_{2}$ is available for choline formation (p. 353). ${ }^{145}$

As a corollary of this latter study, ${ }^{146}$ it was found that 1 per cent $\mathrm{F}_{2,}$ in the diets of four rats on this diet produced after three months an average daily excretion of $11.8 \mu \mathrm{g}$ per day of nicotinamide, as compared with $4.5 \mu \mathrm{g}$ per day in four controls on this diet. While these data have been criticized on the basis of the smallness of the groups and the possibility of nicotinamide contamination in the large amounts of $\mathrm{F}_{2}$ employed, general metabolic considerations would make it seem likely that $\mathrm{F}_{2}$ was at least to some extent available as a nicotinamide source. Tests have to date strongly indicated that $\mathrm{F}_{2}$ is ineffective in the treatment of pellagra ${ }^{146}$ and blacktongue, ${ }^{147}$ although in the latter case seriously conflicting reports do exist. ${ }^{148,} 149$

That $\mathrm{F}_{2}$ is derived rather directly from nicotinamide is shown by the facts (1) that nicotinamide is more effective than nicotinic acid in producing urinary $\mathrm{F}_{2},{ }^{150}(2)$ that oral doses of equivalent amounts of nico- 
tinamide and $\mathrm{F}_{2}$ produce similar amounts of urinary $\mathrm{F}_{2},{ }^{151}$ and (3) that, as previously mentioned, rats fed nicotinic acid in which the carbon atom of the carboxylic acid group was labelled with $\mathrm{C}^{13}$ excrete amounts of labelled $\mathrm{F}_{2}$ in the urine which indicate that all of the $\mathrm{F}_{2}$ is derived from nicotinic acid. $\mathrm{F}_{2}$ is absent from the urine of pellagrins and its excretion is in general a function of the nicotinic acid and tryptophan intake of animals. ${ }^{152}$ Wide individual variations exist, however, and some individuals with an apparently adequate niacin intake do not excrete any $\mathrm{F}_{2} \cdot{ }^{153}$ There are, moreover, apparently significant variations among species in their ability to form $\mathrm{F}_{2}$. Thus in the horse on a low-niacin diet tryptophan stimulates only slightly higher nicotinic acid excretion, and no increase in $\mathrm{F}_{2}$ excretion, ${ }^{154}$ whereas in the cotton rat tryptophan stimulates an increase in both, ${ }^{155}$ but principally in the $\mathrm{F}_{2} \cdot{ }^{156}$ In humans, tryptophan apparently does not affect niacin excretion, but does increase the excretion of $\mathrm{F}_{2}$ markedly. ${ }^{157}$ About half the niacin metabolites excreted by calves are active for Lactobacillus arabinosus, the remainder being largely $\mathrm{F}_{2}{ }^{158} \mathrm{In}$ vitamin $\mathrm{B}_{6}$ deficient rats, it has previously been mentioned that there is no $\mathrm{F}_{2}$ excretion. When pyridoxine is administered to these animals, there is a prompt disappearance of xanthurenic acid from the urine, but only a very gradual reappearance of $\mathrm{F}_{2} .{ }^{159}$ Therefore, although $\mathrm{F}_{2}$ may be formed rather directly from nicotinamide, it would seem that such a wide variety of factors influence its formation that extended study will yet be required to assess its exact place in niacin metabolism.

It has been realized for some time that the level of the excretion of $\mathrm{F}_{2}$ is the result of two major factors: its synthesis from nicotinamide and its conversion to still other metabolic products. ${ }^{160}$ It has recently been discovered that liver contains an enzyme capable of oxidizing quinine and many other nitrogen heterocycles, the enzymatic oxidation characteristically involving the conversion of the carbon atom adjacent to the nitrogen into a keto group. ${ }^{122,123}$ This oxidase readily converts $\mathrm{F}_{2}$ into $\mathrm{N}^{\prime}$-methyl-6-pyridone-3-carboxylamide in vitro, and it has been found that the administration of $600-900 \mathrm{mg}$ of nicotinamide to humans results in the urinary excretion of about $100 \mathrm{mg}$ of this same compound. It thus appears that this substance is a major product of niacin metabolism in at least some species. To date, however, it is uncertain as to what extent its presence may have influenced earlier determination of other niacin metabolites. In any case it seems likely that further analogous pyridones may eventually be discovered among the metabolic products of niacin.

Nicotinuric acid (nicotinylglycine) was found to be a major product of niacin metabolism in the dog long before niacin was implicated as a 
vitamin. ${ }^{161}$ Rabbits similarly excrete nicotinuric acid, but humans apparently do not. ${ }^{162}$ In vitro studies with hog intestinal hippurase have shown that it cleaves nicotinuric and hippuric acids at roughly equal rates, and it has been suggested therefore that nicotinuric acid is probably formed by hippurase in vivo. ${ }^{163}$ Although this point lacks further verification, it seems likely that the liver and kidneys may well perform this function along with their more extensive hippuric acid-synthesizing functions.

Dinicotinylornithine has been isolated from dried chicken droppings, ${ }^{130}$ but nothing is known as yet concerning its metabolism or significance. The structure is of particular interest, however, because of the relationship of ornithine to nicotinic acid synthesis in the higher plants (p. 84). The recent isolation of quinolinic acid from rat urine is mentioned on page 355 , but little more is yet known concerning the significance of this substance in the urine. ${ }^{107 a-c}$

A considerable portion of ingested nicotinic acid remains still to be accounted for, and several probable, but as yet undetected, products have been suggested in the preceding discussion. In the studies previously mentioned employing nicotinic acid in which the acid group was labelled with $\mathrm{C}^{14}$, it was shown that 15 per cent of the $\mathrm{C}^{14}$ of the acid group disappears from mice as exhaled carbon dioxide, so that considerable decarboxylation, possibly preceded by ring rupture, occurs. These studies further showed that the gross metabolism of niacin and its amide is identical in the mouse. ${ }^{144}$ It thus appears likely that in addition to the 3 -carboxylic acid derivatives of pyridine now known, other pyridine derivatives may also occur and eventually be detected. In view of this likelihood, a reinvestigation of the numerous pyridine derivatives already discovered in nature with regard to their urinary occurrence would seem to be in order. ${ }^{124}, 125,164$

Metabolic Products of the Other B Vitamins. As previously stated, little is actually known of the metabolic products of the other B vitamins, although one might hazard extensive guesses as to the probable hydrolytic, oxidative, and conjugative changes that they may logically undergo by analogy with the metabolism of other structurally similar compounds. Rather than venture so far, therefore, it seems better to outline what little is known in this regard, leaving the speculation for a time when it might be better strengthened by the discovery of new and suggestive data in this largely unstudied realm of knowledge.

On normal levels of dietary intake, only a small portion of the $\mathrm{B}$ vitamins consumed can be accounted for on the basis of their excretion in urine. This may be due to their conversion to as yet unidentified but closely related decomposition products, ${ }^{165,166}$ or to their conversion to 
very dissimilar products. In this connection it should be pointed out that they may be completely metabolized to carbon dioxide, water, and ammonia. Tracer studies have indicated that the nutritionally active isomer of inositol is at least partially converted to glucose in the rat, ${ }^{167}$ and the probable existence of similar conversions of some other vitamins may make it impossible ever to obtain completely balanced data on intake and excretion. This seems even more likely since the urinary and fecal excretion so frequently exceeds the intake (pp. 300 and 368), and it is uncertain what portion of the $\mathrm{B}$ vitamins present in both urine and feces originated in the diet.

A variety of types of metabolic conversions break down the B vitamins to their known excretory products. Urine apparently contains no cocarboxylase, so that the thiamine present in urine must largely result from the action of phosphatases on the pyrophosphate. ${ }^{168}$ Thiamine is split in the human body in apparently much the same way as by the thiaminase of aquatic animals (p. 292), and the resulting pyrimidine moiety, called pyramin (2-methyl-4-amino-5-hydroxymethylpyrimidine) is found in the urine in considerable quantities. ${ }^{169}$ When an individual is saturated with thiamine, practically all excess thiamine given can be recovered either as thiamine or pyramin, ${ }^{170}$ so that it would appear that there are not normally other major end products of thiamine metabolism. However, although the amount of thiamine excreted is highly characteristic for the individual (p. 255), pyramin excretion seems to be a relatively constant process. ${ }^{171}$ Since on the same diets one individual may consistently excrete three times as much thiamine as another, while excreting an essentially identical level of pyramin, it is apparent that still another pathway of thiamine metabolism must exist.

Riboflavin is principally excreted as such, but varying amounts up to half of the total may be excreted as the phosphate. ${ }^{172}$ Aquaflavin, or uroflavin, a degradation product of riboflavin, is also found in most urine samples. ${ }^{173}$ In cow, goat, and sheep milk and urine there is a considerable discrepancy between riboflavin determinations done fluorometrically and microbiologically, and it appears that an as yet structurally unidentified degradation product of riboflavin is present which exhibits marked fluorescence but no microbiological activity. ${ }^{17 t} \mathrm{As}$ this metabolic product is not present in the urine of humans or rats, it is possible that the product is formed in the rumen of the animals mentioned. Certain microorganisms are well known for their ability to oxidize riboflavin to lumichrome, ${ }^{175}$ and such a reaction may be responsible for the presence of this fluorescent pigment in the urine and milk of ruminants.

Pantothenic acid is partially excreted as such, but a large part of that ingested has an unknown fate. ${ }^{176,177}$ It is well established that there is 
no pantoyl lactone in the urine, and that the lactone administered intravenously or orally is recovered in the urine quantitatively and unchanged; hence hydrolysis of the vitamin molecule probably does not normally occur to any appreciable extent. ${ }^{178}$ Since the tissue vitamin is almost solely in the form of the coenzyme, it would seem that the search for traces of coenzyme fragments in the urine may well lead to clues as to the exact structure of this coenzyme. Synthetic pantothenyl alcohol is converted by the body to pantothenic acid, ${ }^{179}$ but since this former compound apparently does not appear in nature, the reaction has no great significance.<smiles>Cc1ccc2nc3[nH]c(=O)[nH]c(=O)c3nc2c1</smiles>

Lumichrome

Little is known of the breakdown of folic acid in the body, but it is known that "conjugase" of the liver, kidney and pancreas converts the triglutamate and heptaglutamate to folic acid. ${ }^{180}$ Strangely enough, in vitro studies with chicken pancreas conjugase have shown that only the terminal glutamic acid molecule is removed from the triglutamate, so that there is a considerable lack of understanding of how folic acid is ultimately produced in vivo. ${ }^{181}$ Even in sprue, administered pteroyl triglutamate is converted to the monoglutamate, as indicated by urinary excretion of the latter. ${ }^{182}$ The fact that folic acid-free liver extracts further increase the excretion of folic acid in this case suggests that the metabolism of the conjugates is in some manner mediated by the erythrocyte maturation factor.

Although little is known of the normal metabolism of $p$-aminobenzoic acid, larger amounts when administered are excreted as the acetylated product. ${ }^{183}$ Normal rats acetylate about 70 per cent of the excreted portion of a 1 to $2.5-\mathrm{mg}$ dose of $p$-aminobenzoic acid, and this ability is decreased in pantothenic acid-deficient rats. ${ }^{184}$ Sulfanilamide is similarly acetylated in the animal body, ${ }^{185}$ and if, as seems likely, the mechanism is the same, the process involves adenosine triphosphate, acetate, and coenzyme A. ${ }^{186}$ The activity of liver preparations in this regard suggests that this organ is at least one major site of the reaction. Since however all these considerations refer to levels of $p$-aminobenzoic acid that are somewhat above the physiological range, there can be no absolute cer- 
tainty that a similar mechanism is involved normally in PABA metabolism.

Regarding vitamin $B_{6}$, apparently small amounts of all three forms may be excreted in the urine. The major metabolic product of pyridoxine, however, is 4-pyridoxic acid, which accounts for about five-sixths of the known vitamin $B_{6}$ metabolites in the urine.

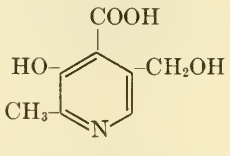

\section{4-Pyridoxic acid}

Urinary choline appears to be entirely in the free state, although there does not seem as yet to be ample evidence to substantiate the absence of other derivatives. Practically nothing is known of the breakdown products of biotin, or "vitamin $\mathrm{B}_{12}$," although the latter is said to be stored by both normal and pernicious anemia patients, even when it is administered in high dosage. ${ }^{187}$ Whereas a variety of biotin metabolic products have been suggested, the recent demonstration of the biotinlike activity for microorganisms of a number of structurally unrelated compounds (p. 173) seems to cast serious doubts on the existing data concerning the presence of biotin degradation products in urine.

Thus at present there are many gaps in our knowledge of the breakdown products of the $\mathrm{B}$ vitamins. These gaps may reasonably be expected to be filled in the years immediately ahead, largely by the use of isotopic tracer techniques, which have already been so preeminently successful in problems of this kind, and also by means of the rapidly developing technique of paper chromatography, which is so well adapted to the separation and identification of small amounts of structurally similar compounds. As in many other cases discussed in this monograph, this field is one in which true progress has barely commenced.

\section{Excretion}

A great many of the aspects of $B$ vitamin excretion have been discussed previously in regard to the assessment of $B$ vitamin requirements ( $p$. 254 ), the effects of climate and other factors upon the requirements (p. 269), the concentration in milk (p. 347) which may be regarded as an excretory product, and with regard to the breakdown products of the $B$ vitamins (p. 361). Still other aspects are more appropriately discussed in the consideration of deciency states. Consequently our present discus- 
sion will be in the nature of a summary of the general picture at present available of the levels of excretion themselves.

Levels in Urine. Table 28 summarizes the major known end products of vitamin metabolism found in the urine. In cases where a variety of products exist, variations occur in the relative amounts of the products, and in some cases at least these variations have been extensively studied. For the most part, however, little is known of the significance of the various forms and their relative amounts.

TABLE 28. Known Urinary Products of Vitamin Metabolism

B Vitamin

Thiamine

Riboflavin

Nicotinic acid

Vitamin $B_{6}$

Pantothenic acid

Biotin

Folic acid (group)

$p$-Aminobenzoic acid

Choline

Inositol

\section{Urinary form}

Thiamine (but not pyrophosphate)

Pyramin

Riboflavin (and phosphate)

Uroflavin

Nicotinic acid (and amide)

$\mathrm{N}^{\prime}$-methylnicotinamide

N'methyl-6-pyridone-3-carboxylamide

Nicotinuric acid

Quinolinic acid

Vitamin $\mathrm{B}_{6}$

4-Pyridoxic acid

Pantothenic acid

Biotin

Folic acid

Acetyl PABA

Choline

Inositol

The excretion of the $\mathrm{B}$ vitamins and their derivatives via the kidney or skin involves processes considerably more complex than passive diffusion. While few data are available on the topic, it appears that the renal and dermal thresholds are maintained, in some cases at least, by intermediate formation similar to that which occurs upon the passage of a vast number of other metabolites across biological membranes. Whereas there have been some studies of the renal clearance and glomerular filtration of the B vitamins, ${ }^{186,187}$ this field of endeavor is relatively unexploited at present.

In considering the levels of $\mathrm{B}$ vitamin excretion in humans, and their relation to the dietary intake, one could do little better than to consider the data shown in Tables 29 and 30, which are taken from the excellent study of Denko et al..$^{23}$ The subjects were seven healthy men from 23 to 28 years of age, who were maintained on a balanced diet for a twelveweek period. Various aspects of these data have been previously discussed (pp. 275 and 300), and they are sufficiently complete and lucid that an extended discussion of their various aspects at this time would seem unwarranted. The data are in excellent agreement with the great mass of published material on this topic, and have the advantage of being far 


\begin{tabular}{|c|c|c|c|c|c|}
\hline 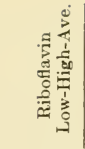 & $r$ & 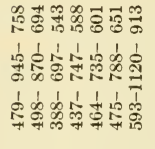 & $\mid \begin{array}{l}\infty \\
0 \\
0 \\
1 \\
0 \\
0 \\
6 \\
1 \\
07 \\
20\end{array}$ & 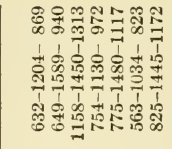 & 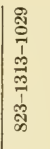 \\
\hline 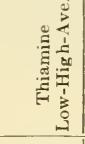 & $\lambda$ & 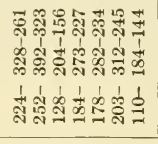 & 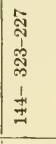 & 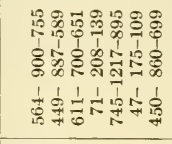 & $\begin{array}{l}\infty \\
10 \\
10 \\
10 \\
0 \\
1 \\
1 \\
8\end{array}$ \\
\hline 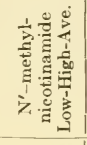 & $\stackrel{\dot{\theta}}{\Xi}$ & 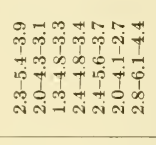 & $\begin{array}{l}0 \\
0 \\
i \\
i \\
i \\
i \\
i \\
i\end{array}$ & $\stackrel{*}{*}$ & \\
\hline 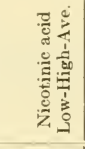 & ही & 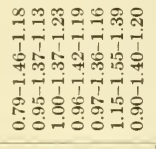 & 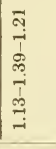 & 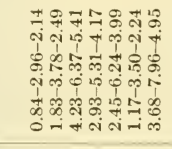 & 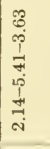 \\
\hline 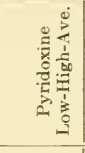 & छी & 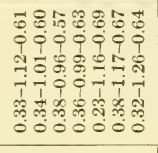 & $\begin{array}{l}3 \\
0 \\
0 \\
0 \\
0 \\
0 \\
0 \\
1 \\
10 \\
0 \\
0\end{array}$ & 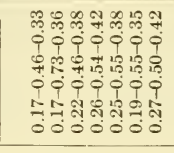 & 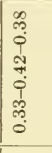 \\
\hline 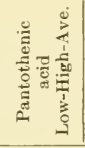 & $\stackrel{\infty}{\Xi}$ & 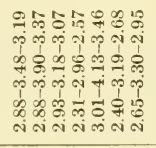 & $\begin{array}{l}+1 \\
0 \\
o p \\
0 \\
0 \\
01 \\
01 \\
0 \\
0 \\
0 \\
ن\end{array}$ & 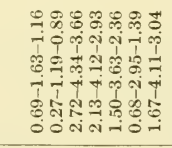 & $\begin{array}{l}\text { d̂ } \\
\text { 1 } \\
1 \\
0 \\
0 \\
0 \\
0 \\
0 \\
0 \\
0\end{array}$ \\
\hline 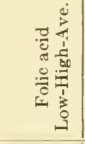 & $r$ & 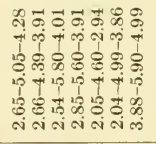 & 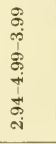 & 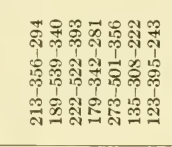 & 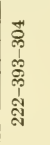 \\
\hline 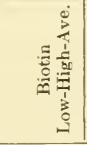 & $\succ$ & 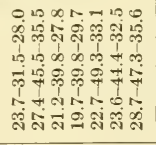 & 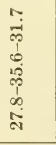 & 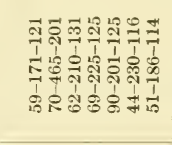 & 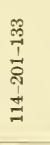 \\
\hline 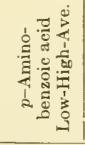 & c & 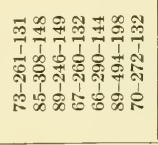 & 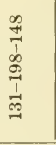 & 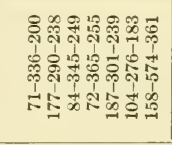 & $\begin{array}{l}0 \\
\text { N } \\
\frac{1}{0} \\
0 \\
0 \\
\infty \\
0\end{array}$ \\
\hline & \multicolumn{2}{|c|}{ 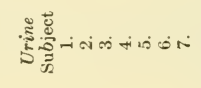 } & 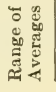 & ङूँ & 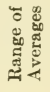 \\
\hline
\end{tabular}




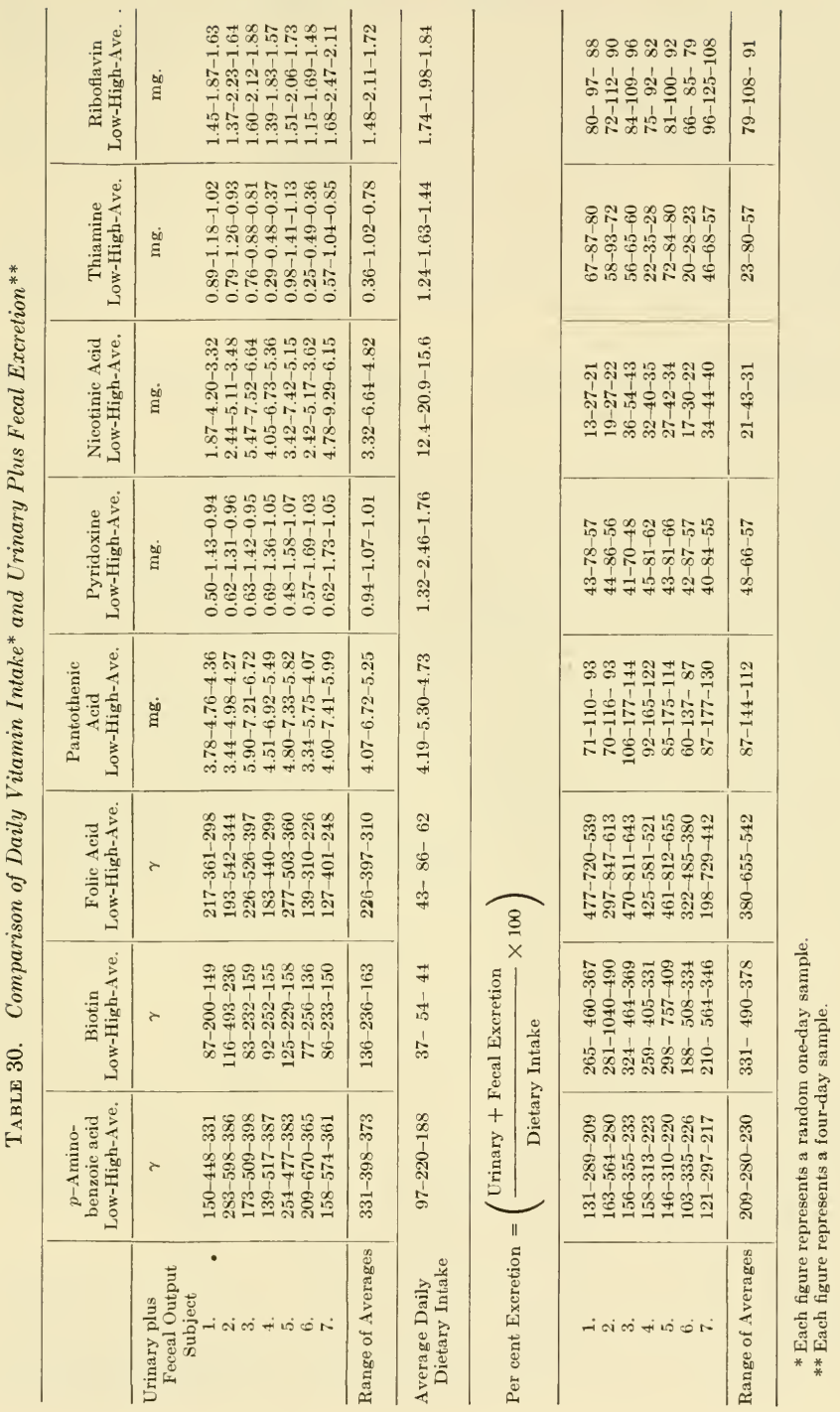


more complete than most similar studies. Those interested in specific aspects of the excretion problem may readily find a vast current literature dealing with the individual vitamins: thiamine, ${ }^{188-196}$ riboflavin, ${ }^{197-204}$ niacin, ${ }^{205-211}$ pantothenic acid, ${ }^{212-213}$ biotin, ${ }^{214,} 215$ folic acid, ${ }^{216-218}$ vita$\min \mathrm{B}_{6},{ }^{219} p$-aminobenzoic acid and inositol, ${ }^{220}$ and the antipernicious anemia factor. ${ }^{221}$ Problems dealing with obstetrics and pediatrics are similarly well covered in the current literature, $188,199,200,207,210,222$ and ready access to the vast earlier literature on the topic may be gained through these references. Unfortunately, there is not at present sufficient information to draw any conclusion as to the variations in levels among various species, however.

Fecal and Dermal Excretion. As shown in Tables 29 and 30, except for pantothenic acid and pyridoxine, fecal excretion of the B vitamins far exceeds urinary excretion, but there is little information to indicate what part of the fecal excretion represents dietary intake and what part intestinal synthesis. Undoubtedly the greater part of the fecal vitamin content is of bacterial origin, since the combined fecal and urinary excretion of biotin, pantothenic acid, PABA, and folic acid exceeds the intake, and since the remainder of the $B$ vitamins have other major products of metabolism which were not considered in this study. Moreover, the consideration of dermal excretion was not made in assessing these data. Furthermore, there is no apparent correlation between the combined urinary and fecal excretion and the dietary intake.

It seems quite certain that fecal thiamine is almost entirely in the form of cocarboxylase, in contrast with urinary thiamine. ${ }^{194}$ Little is known of the precise form of the other vitamins in the feces, although from 60 to 100 per cent of the fecal B vitamin content is said to be "water-soluble." ${ }^{27}$ Similarly, little is known as to the precise forms of the B vitamins excreted in sweat. Vitamin concentrations in sweat appear to be quite similar to those in urine, and it is consequently generally felt that under normal environmental conditions the dermal excretion is negligible by comparison with urinary excretion. Pantothenic acid normally occurs in sweat in concentrations of about $3.8 \mu \mathrm{g}$ per cent, ${ }^{212}$ and the fact that this level cannot be increased by higher dosage with pantothenic acid suggests that the excretory ability of the skin is more limited than that of the kidney. Inositol and PABA occur in sweat in concentrations of about 21. $\mu \mathrm{g}$ per cent and $0.24 \mu \mathrm{g}$ per cent, respectively. ${ }^{220}$

Recent studies on urinary and dermal excretion of-pyridoxine and its derivatives are of interest in that they strongly suggest that sweat contains metabolites of $\mathrm{B}$ vitamins quite similar to those in urine. Table 31, taken from Johnson, Hamilton and Mitchell's paper, ${ }^{223}$ indicates that sweat normally contains pyridoxal, pyridoxamine and 4-pyridoxic acid. 
When higher levels of pyridoxine are administered, dermal excretion of pyridoxine also occurs.

TABLE 31. Average Dermal and Urinary Excretion of Pyridoxine and its Metabolites

\begin{tabular}{|c|c|c|c|c|c|c|}
\hline \multirow[b]{2}{*}{ Subject } & \multicolumn{3}{|c|}{ Urine (mg/24 hours) } & \multicolumn{3}{|c|}{ Sweat (mg/8 hours) —_ } \\
\hline & pyridoxine $\dagger$ & $\begin{array}{c}\text { pyridoxal }+ \\
\text { pyridoxamine* }\end{array}$ & $\begin{array}{c}\text { 4-pyridoxic } \\
\text { acid }\end{array}$ & pyridoxine & $\begin{array}{c}\text { pyridoxal }+ \\
\text { pyridoxamine* }\end{array}$ & $\begin{array}{c}4 \text {-pyridoxic } \\
\text { acid }\end{array}$ \\
\hline 1 & 0.114 & 0.192 & 2.83 & 0 & 0.030 & 0.159 \\
\hline 2 & 0.187 & 0.375 & 3.02 & 0 & 0.030 & 0.193 \\
\hline 3 & 0.118 & 0.236 & 3.29 & 0 & 0.032 & 0.257 \\
\hline 4 & 0.131 & 0.181 & 3.36 & 0 & 0.035 & 0.189 \\
\hline Average & 0.138 & 0.246 & 3.13 & 0 & 0.031 & 0.198 \\
\hline$\%$ of total & 3.9 & 6.8 & 89.3 & 0 & 13.6 & 86.4 \\
\hline
\end{tabular}

* Measured in terms of pyridoxal.

† The data cannot be accepted in a quantitative sense, because the assay methods used are now known to give results that do not represent the specific analogues indicated. The data do illustrate the points cited in the text, however.

Individual Variations. Despite the fact that there may be large daily variations in the excretion of most of the $B$ vitamins, marked individual differences do exist of such a nature that for some of the B vitamins, two individuals on the same dietary intake may excrete widely divergent amounts. This is to be expected in consideration of the many genetically controlled enzyme systems that mediate vitamin metabolism. Fecal B vitamin excretion varies more than does the urinary excretion, since the bulk of fecal vitamins is controlled by the bacteria present rather than by the host; but even in this case individuals do differ by virtue of these differences in intestinal tracts, etc. This may be noted, for instance, by considering the values in Table 29 for subjects 4 and 6 . These subjects were brothers, and a distinct resemblance occurs in the urinary and fecal excretion of both thiamine and riboflavin.

An excellent example of urinary individual differences is that of thia-

TABLE 32. Urinary and Fecal Excretion of Thiamine and Pyramin by Six Men on Three Days*

\begin{tabular}{|c|c|c|c|c|c|c|c|c|c|c|}
\hline \multirow{3}{*}{ Subject } & \multicolumn{5}{|c|}{ Thiamine ( $\mu \mathrm{g} /$ day) } & \multicolumn{5}{|c|}{ Pyramin $(\mu \mathrm{g} /$ day $) \dagger$} \\
\hline & \multicolumn{4}{|c|}{ Urine } & \multirow{2}{*}{ Feces } & \multicolumn{4}{|c|}{ Urine } & \multirow{2}{*}{ Feces } \\
\hline & I & II & III & mean & & I & I & III & mean & \\
\hline 1 & 118 & 75 & 79 & 91 & 540 & 187 & 169 & 157 & 171 & 383 \\
\hline 2 & 36 & 21 & 39 & 32 & 510 & 162 & 170 & 142 & 158 & 208 \\
\hline 3 & 60 & 48 & 53 & 54 & 800 & 148 & 178 & 178 & 168 & 37 \\
\hline 4 & 85 & 83 & 86 & 85 & 550 & 178 & 198 & 179 & 185 & 532 \\
\hline 5 & 55 & 46 & 49 & 50 & 720 & 188 & 173 & 146 & 169 & 61 \\
\hline 6 & 62 & 52 & 60 & 58 & 666 & 159 & 169 & 150 & 159 & 94 \\
\hline Mean & 69 & 54 & 61 & 62 & 631 & 170 & 176 & 159 & 168 & 219 \\
\hline
\end{tabular}

\footnotetext{
* In the above table, the measurements of thiamine were not made on the same three days as were the measurements of pyramin.

† Expressed as 2-methyl-4-amino-5-ethoxymethylpyrimidine.
} 
mine excretion, as shown in Table 32. The subjects in this study of Mickelson et al. ${ }^{171}$ were on a thiamine intake of approximately $1.5 \mathrm{mg}$ per day, and under these conditions distinct differences are shown in thiamine excretion among the subjects. Pyramin excretion, however, was most remarkably constant from day to day and individual to individual, and has been suggested for this reason as a more accurate indication of the status of thiamine nutrition as judged by excretion studies (p. 256). It will be noted that fecal thiamine does not vary with either the thiamine or pyramin excretion in the urine. Certainly the investigation of the source and high variability of fecal pyramin would seem to be a worthwhile undertaking, together with the extension of this study to a larger number of individuals.

Relationship to Dietary and Body Levels. The levels of the various B vitamins found in the urine do not correlate with the requirements for these vitamins because of the variety of ways in which the various members of the group are metabolized. Above a certain minimal level, the amount of a vitamin in the diet is generally correlated with its content in the urine. In a general manner, the levels in the urine resemble those in milk and in sweat. As yet there are insufficient data to tell whether this is also true of saliva. The addition of some one vitamin in a single high dose to the diet of an individual "saturated" with a B vitamin causes an increase in the blood level rather rapidly, but an almost equally rapid fall to normal and corresponding increase in the urine. A full understanding of these relationships, however, must await the elucidation of the variations which occur in the various $B$ vitamin derivatives, the fate of the unaccounted for fraction of the intake, and the source and qualitative nature of the fecal fraction of the vitamins eliminated. These problems at present are all under intensive investigation, and may well be to a large extent resolved in the near future.

\section{Bibliography}

1. Schopfer, W. H., "Plants and Vitamins," Chronica Botanica Co., Waltham, Mass., 1943, p. 75.

2. Rosenberg, H. R., "Chemistry and Physiology of the Vitamins," Interscience Publishers, Inc., New York City, N. Y., 1945, p. 276.

3. Heiman, M., Wien. klin. Wochschr., 49, 398-9 (1936).

4. Rabinowitch, E. I., "Photosynthesis and Related Processes," Interscience Publishers, Ine., New York City, N. Y., 1945.

5. Raffy, A. Compt. rend. soc. biol., 126, 875-7 (1937).

6. West, P. M., Nature, 144, 1050-51 (1939).

7. Kahane, E., and Levy, J., Boll. soc. chim. biol., 27, 544-58 (1945).

8. Cheldelin, V. H., Eppright, M. A., Snell, E. E., and Guirard, B. M., Univ. Texas Pub. 4237, 15-36 (1942).

9. Nutrition Revs., 7, 19-20 (1949). 
10. Keresztesy, J. C., and Sampson, W. L., Proc. Soc. Exptl. Biol. Med., 36, 686-7 (1937).

11. Williamson, A., and Parsons, H. T., J. Nutrition, 29, 51-9 (1945).

12. Gyorgy, P., and Rose, C. S., Proc. Soc. Exptl. Biol. Med., 43, 73-4 (1940).

12a. Ternberg, J. L., and Eakin, R. E., J. Am. Chem. Soc., 71, 3858 (1949).

13. Verzar, F., and Laszt, L., Enzymologia, 3, 16-20 (1937).

14. Stockholm, M., Althausen, T. L., and Boison, T. J., Proc. Soc. Exptl. Biol. Med., 46, 387-90 (1941).

15. Sinclair, H. M., Biochem. J., 33, 1816-21 (1939).

16. Tauber, H., Science, 86, 180 (1937).

17. Tauber, H., J. Biol. Chem., 123, 499-506 (1938).

18. Verzar, F., and Laszt, L., Arch. ges. Physiol. (Pfïgers), 236, 693 (1935).

19. Verzar, F., and Laszt, L., Arch. ges. Physiol. (Pflügers), 237, 476-72 (1936).

20. Hegsted, D. M., and Lipmann, F., J. Biol. Chem., 174, 89-92 (1948).

21. Wright, L. D., Trans. N. Y. Acad. Sci., 10, 271-7 (1948).

22. Silber, R. H., Arch. Biochem., 7, 329-36 (1945).

23. Denko, C. W., Grundy, W. E., Wheeler, N. C., Henderson, C. R., Berryman, G. H., Friedemann, T. E., and Youmans, J. B., Arch. Biochem., 11, 109-17 (1946).

24. Gyorgy, P., and Rose, C. S., Proc. Soc. Exptl. Biol. Med., 53, 55-7 (1943).

25. Ochoa, S., and Peters, R. A., Biochem. J., 32, 1501-15 (1938).

26. Goodhart, R. S., and Sinclair, H. M., Biochem. J., 33, 1099-1108 (1939).

27. DeCaro, L., and Fornaroli, P., Boll. soc. ital. biol. sper., 15, 1068-70 (1940).

28. Bang, H. O., Acta Med. Scand., 122, 38-59 (1945).

29. Lodi, M., Z. Vitaminforsch., 17, 36-52 (1946).

30. Carleen, M. H., Weissman, N., and Ferrebee, J. W., J. Clin. Invest., 23, 297302 (1944).

31. Pence, J. W., Miller, R. C., Dutcher, R. A., and Thorp, W. T. S., J. Biol. Chem., 158, 647-51 (1945).

32. Schultz, A. S., Light, R. F., Cracas, L. J., and Atkin, L., J. Nutrition, 17, 143-9 (1939).

33. Florijn, E., and Smits, G., Nature, 162, 220-1 (1948).

34. Tangari, C., Med. sper. arch. ital., 6, 169-78 (1940).

35. Klein, J. R., and Kohn, H. I., J. Biol. Chem., 136, 177-89 (1940).

36. Paladino, T., and Vasta, S., Boll. soc. ital. biol. sper., 20, 479-81 (1945).

37. Schultz, F. W., Semana med., 50, 689-91 (1946).

38. Strong, F. M., Feeney, R. E., Moore, B., and Parsons, H. T., J. Biol. Chem., 137, 363-72 (1941).

39. Bradford, E. A. M., and Coke, H., Biochem. J., 39, 379-85 (1945).

40. Villela, G. G., and Hargreaves, A. B., Mem. inst. Oswaldo Cruz, 42, 167-76 (1945).

41. Vilter, S. P., Vilter, R. W., and Spies, T. D., Nature, 144, 943 (1939).

42. Chu, H. I., Kuo, P. T., and Chang, K. P., Chinese Med. J., 61, 181-91 (1942).

43. Gounelle, H., Vallette, A., and Raoul, Y., Compt. rend. soc. biol., 139, 16-17 (1945).

44. Gounelle, H., Raoul, Y., and Marche, J., Compt. rend. soc. biol., 139, 144-5 (1945).

45. Niemeijer, J. A., Rec. trav. chim., 65, 84-8 (1946).

46. Kochhar, B. D., Ann. Biochem. Exptl. Mcd. (India), 3, 85-94 (1943).

47. Wright, L. D., J. Biol. Chem., 147, 261-2 (1943).

48. Pearson, P. B., Melass, V. H., and Sherwood, R. M., J. Nutrition, 32, 187-93 (1946).

49. Williams, R. J., Vitamins and Hormones, 1, 236 (1943).

50. Pearson, P. B., Darnell, A. L., and Weir, J., J. Nutrition, 31, 51-7 (1946).

51. Coryell, M. N., Harris, M. E., Miller, S., Williams, H. H., and Macy, I. G., Am. J. Diseases Children, 70, 150-61 (1945). 
52. Roderuck, C. E., William, H. H., and Macy, I. G., Am. J. Diseases Children, 70, 162-70 (1945).

53. Lipmann, F., Kaplan, N. O., and Novelli, G. D., Federation Proc., 6, 272 (1947).

54. Kaplan, N. O., and Lipmann, F., J. Biol. Chem., 174, 37-44 (1948).

55. Rabinowitz, J. C., and Snell, E. E., J. Biol. Chem., 176, 1157-67 (1948).

56. McCoy, R. H., McKibben, J. N., Axelrod, A. E., and Hofmann, K., J. Biol. Chem., 176, 1327-31 (1948).

57. McCoy, R. H., McKibben, J. N., Axelrod, A. E., and Hofmann, K., J. Biol. Chem., 176, 1319-26 (1948).

58. Czaczkes, J. IV., and Guggenheim, K., J. Biol. Chem., 162, 267-74 (1946).

59. Wright, L. D., and Skeggs, H. R., Proc. Soc. Exptl. Biol. Med., 63, 327-33 (1946).

60. Morris, H. P., Vitamins and Hormones, 5, 176 (1947).

61. Nutrition Revs., 5, 41-2 (1947).

62. Heinemann, W. W., Ensminger, M. E., Cunha, T. J., and McCulloch, E. C., J. Nutrition, 31, 107-25 (1946).

63. Stamberg, O. E., Petersen, C. F., and Lampman, C. E., Poultry Sci., 25, 320-6 (1946).

64. Clandinin, D. R., Poultry Sci., 25, 223-31 (1946).

65. Ringrose, R. C., and Davis, H. A., Poultry Sci., 25, 646-7 (1946).

66. Jackson, S. H., Drake, T. G. H., Slinger, S. J., Evans, E. V., and Pocock, R., J. Nutrition, 32, 567-8 (1946).

67. Westenbrink, H. G. K., and Goudsmit, J., Enzymologia, 5, 307-20 (1938).

68. Cedrangolo, F., and Villano, F., Boll. soc. ital. biol. sper., 17, 558-9 (1942).

69. Bellamy, W. D., Umbreit, W. W., and Gunsalus, I. C., J. Biol. Chem., 160, 46172 (1945).

70. Warburg, O., and Christian, W., Biochem. Z., 298, 150-68, 368-77 (1938).

71. Kornberg, A., and Lindberg, O., J. Biol. Chem., 176, 665-77 (1948).

72. Ling, C. T., Hegsted, D. M., and Stare, F. J., J. Biol. Chem., 174, 803-12 (1948).

73. Levy, M., and Young, N. F., J. Biol. Chem., 176, 185-91 (1948).

74. Kohn, H. J., and Klein, J. R., J. Biol. Chem., 130, 1-11 (1939).

75. Olson, R. E., and Kaplan, N. O., J. Biol. Chem., 175, 515-29 (1948).

76. Lichstein, H. C., J. Biol. Chem., 177, 125-7 (1949).

77. Horowitz, N. H., J. Biol. Chem., 162, 413-19 (1946).

78. Horowitz, N. H., Bonner, D., and Houlahan, M. B., J. Biol. Chem., 159, 14551 (1945).

79. duVigneaud, V., Chandler, J. P., Simmonds, S., Moyer, A. W., and Cohn, M., J. Biol. Chem., 164, 603-13 (1946).

80. Stetten, DeW., J. Biol. Chem., 142, 629-33 (1942).

81. Rosen, F., Huff, J. W., and Perlzweig, W. A., J. Biol. Chem., 163, 343-4 (1946).

82. Cannon, M. D., Mannering, G. J., Elvehjem, C. A., and Hart, E. B., Proc. Soc. Exptl. Biol. Med., 63, 414-17 (1946).

83. Schweigert, B. S., Pearson, P. B., and Wilkening, M. C., Arch. Biochem., 12, 139-45 (1947).

84. Luecke, R. W., McMillen, W. N., Thorp, F., Jr., and Tull, C., J. Nutrition, 33, 251-61 (1947).

85. Singal, S. A., Sydenstricker, V. P., and Littlejohn, J., Federation Proc., 6, 422 (1947).

86. Johnson, B. C., Mitchell, H. H., Hamilton, T. S., and Nevens, W. B., Federation Proc., 6, 410-11 (1947).

87. Perlzweig, W. A., Rosen, F., Levitas, N., and Robinson, J., J. Biol. Chem., 167, 511-14 (1947).

88. Sarett, H. P., and Goldsmith, G. A., J. Biol. Chem., 177, 461-75 (1949).

89. Krehl, W. A., Teply, L. J., Sarma, P. S., and Elvehjem, C. A., Science, 101, 489-90 (1945).

90. Briggs, G. M., J. Biol. Chem., 161, 749-50 (1945).

91. Woolley, D. W., J. Biol. Chem., 162, 179-80 (1946). 
92. Rosen, F., Huff, J. W., and Perlzweig, W. A., J. Biol. Chem., 163, 343-4 (1946).

93. Vilter, R. W., Mueller, J. F., and Bean, W. B., J. Lab. Clin. Med., 34, 409-13 (1949).

94. Ellinger, P., and Kader, M. M. A., Nature, 160, 675-6, (1947).

95. Schweigert, B. S., German, H. L., and Garber, M. J., J. Biol. Chem., 174, 383-4 (1948).

96. Snyderman, S. E., Ketron, K. C., Carretero, R., and Holt, L. E., Jr., Proc. Soc. Exptl. Biol. Med., 70, 569-71 (1949).

97. Schultz, J., and Rudkin, G. T., Federation Proc., 7, 185 (1948).

98. Kidder, G. W., Dewey, V. C., Andrews, M. B., and Kidder, R. R., J. Nutrition, 37, 521-9 (1949).

99. Woolley, D. W., J. Biol. Chem., 157, 455-9 (1945).

100. Rosen, F., Huff, J. W., and Perlzweig, W. A., J. Nutrition, 33, 561-7 (1947).

101. Homer, A., J. Physiol. (London), 48, ii (1914).

102. Mitchell, H. K., and Nyc, J. F., Proc. Natl. Acad. Sci. U. S., 34, 1-5 (1948).

103. Nyc, J. F., and Mitchell, H. K., J. Am. Chem. Soc., 70, 1847-8 (1948).

104. Bonner, D., Proc. Natl. Acad. Sci. U. S., 34, 5-9 (1948).

105. Albert, P. W., Scheer, B. T., and Deuel, H. J., Jr., J. Biol. Chem., 175, 479-80 (1948).

106. Heidelberger, C., Gullberg, M. E., Morgan, A. F., and Lepkovsky, S., J. Biol. Chem., 175, 471-2 (1948).

107. Heidelberger, C., Abraham, E. P., and Lepkovsky, S., J. Biol. Chem., 176, 1461-2 (1948).

107a. Henderson, L. M., and Hirsch, H. M., J. Biol. Chem., 181, 667-75 (1949).

107b. Henderson, L. M., J. Biol. Chem., 181, 677-85 (1949).

107c. Henderson, L. M., and Ramasarme, G. B., J. Biol. Chem., 181, 687-92 (1949).

108. Homer, A., J. Physiol. (London), 46, 18 (1912).

109. Matsuoka, Z., Takemura, S., and Yashimatsu, N., Z. physiol. Chem., 143, 199205 (1925).

110. Kotake, Y., and Iwao, J., Z. physiol. Chem., 195, 139-47 (1931).

111. Robson, W., Biochem. J., 22, 1157-68 (1928).

112. Lepkovsky, S., and Nielsen, E., J. Biol. Chem., 144, 135-8 (1942).

113. Kotake, Y., Jr., and Ito, N., J. Biochem. (Japan), 25, 71-7 (1937).

114. Sarma, P. S., Proc. Soc. Exptl. Biol. Med., 58, 140-1 (1945).

115. Miller, E. C., and Bauman, C. A., J. Biol. Chem., 159, 173-83 (1945).

116. Lepkovsky, S., Roboz, E., and Haagen-Smit, A. J., J. Biol. Chem., 149, 195-201 (1943).

117. Reid, D. F., Lepkovsky, S., Bonner, D., and Tatum, E. L., J. Biol. Chem., 155, 299-303 (1944).

118. Axelrod, H. E., Morgan, A. F., and Lepkovsky, S., J. Biol. Chem., 160, 155-64 (1945).

119. Cartwright, G. E., Wintrobe, M. M., Jones, P., Lauristen, M., and Humphreys, S., Bull. Johns Hopkins Hosp., 75, 35-47 (1944).

120. Porter, C. C., Clark, I., and Silber, R. H., J. Biol. Chem., 167, 573-9 (1947).

121. Chiancone, F. M., Boll. soc. ital. biol. sper., 22, 470-4 (1946).

122. Knox, W. E., J. Biol. Chem., 163, 699-711 (1946).

123. Knox, W. E., and Grossman, W. I., J. Biol. Chem., 166, 391-2 (1946).

124. Roggen, J. C., Rec. trav. chim., 64, 229-38 (1945).

125. Roggen, J. C., Rec. trav. chim., 65, 89-90 (1946).

126. Field, H., Jr., Foa, P. P., and Foa, N., Arch. Biochem., 9, 45-9 (1946).

127. Ackermann, D., Z. Biol., 59, 17-22 (1912).

128. Sarett, H. P., J. Nutrition, 23, 35-45 (1942).

129. Komori, Y., and Sendju, Y., J. Biochem. (Japan), 6, 163-70 (1926).

130. Dann, W. J., and Huff, J. W., J. Biol. Chem., 168, 121-7 (1947).

131. His, W., Arch. exptl. Path. Pharmakol., 22, 253 (1887).

132. Nutrition Revs., 3, 263-6 (1945). 
133. Nutrition Revs., 5, 234-5 (1947).

134. Field, H., Foa, P. P., and Foa, N., Arch. Biochem., 9, 45-9 (1946).

135. Foa, P. P., and Glicksman, A. S., Arch. Biochem., 11, 477-9 (1946).

136. Sarett, H. P., Huff, J. W., and Perlzweig, W. A., J. Nutrition, 23, 23-34 (1942).

137. Najjar, V. A., and Wood, R. W., Proc. Soc. Exptl. Biol. Med., 44, 386-90 (1940).

138. Najjar, V. A., and Holt, L. E., Jr., Science, 93, 20-1 (1941).

139. Johnson, B. C., J. Biol. Chem., 159, 227-30 (1945).

140. Perlzweig, W. A., Bernheim, M. L. C., and Bernheim, F., J. Biol. Chem., 150, 401-6 (1943).

141. Najjar, V. A., Hall, R. S., and Deal, C. C., Bull. Johns Hopkins Hosp., 76, 83-91 (1945).

142. Erdei, A., Lancet, 256, 503 (1949).

143. Handley, J. M., and Bond, H. W., J. Biol. Chem., 173, 513-17 (1948).

144. Roth, L. J., Leifer, E., Hogness, J. R., and Langham, W. H., J. Biol. Chem., 176, 249-57 (1948).

145. Najjar, V. A., and Deal, C. C., J. Biol. Chem., 162, 741-2 (1946).

146. Vance, G. A., Bull. Johns Hopkins Hosp., 77, 393 (1945).

147. Najjar, V. A., Hammond, M. M., English, M. A., Wooden, M. B., and Deal, C. C., Bull. Johns Hopkins Hosp., 74, 406-14 (1944).

148. Woolley, D. W., Strong, F. M., Madden, R. J., and Elvehjem, C. A., J. Biol. Chem., 124, 715-23 (1938).

149. Tepley, L. J., Krehl, W. A., and Elvehjem, C. A., Proc. Soc. Exptl. Biol. Med., 58, 169-71 (1945).

150. Nutrition Revs., 3, 233-4 (1945).

151. Huff, J. W., Thesis, Duke University (1945).

152. Mickelsen, O., and Erickson, L. L., Proc. Soc. Exptl. Biol. Med., 58, 33-6 (1945).

153. Sargent, F., Robinson, P., Johnson, R. E., and Castiglione, M., J. Clin. Invest., 23, 714-19 (1944).

154. Huff, J. W., Pearson, P. B., and Pearlzweig, W. A., Arch. Biochem., 9, 99-104 (1946).

155. Schweigert, B. S., Pearson, P. B., and Wilkening, M. C., Arch. Biochem., 12, $139-45$ (1947).

156. Rosen, F., Huff, J. W., and Pearlzweig, W. A., J. Biol. Chem., 163, 343-4 (1946).

157. Sarett, H. P., and Goldsmith, G. A., J. Biol. Chem., 167, 293-4 (1947).

158. Johnson, B. C., Wiese, A. C., Mitchell, H. H., and Nevens, W. B., J. Biol. Chem., 167, 729-36 (1947).

159. Rosen, F., Huff, J. W., and Perlzweig, W. A., J. Nutrition, 33, 561-7 (1947).

160. Perlzweig, W. A., and Huff, J. W., J. Biol. Chem., 161, 417-18 (1945).

161. Ackermann, D., Z. Biol., 59, 17-22 (1912).

162. Johnson, B. C., Hamilton, T. S., and Mitchell, H. H., J. Biol. Chem., 159, 231-6 (1945).

163. Lanfranchi, F., Atti accad. Italia, Rend. (7), 3, 103-12 (1941).

164. Elvehjem, C. A., and Teply, L. J., Chcm. Revs., 33, 185-208 (1943).

165. Nutrition Revs., 4, 310-13 (1946).

166. Nutrition Revs., 4, 58-60 (1946).

167. Stetten, M. R., and Stetten, DeW., Jr., J. Biol. Chem., 164, 85-91 (1946).

168. Goudsmit, J., and deJong, S., Nederland. Tijdschr. Geneesk., 90, 617-20 (1946).

169. Schultz, A. S., Atkin, L., and Frey, C. N., Ind. Eng. Chem., Anal. Ed., 14, 35-9 (1942).

170. Alexander, B., Landwehr, G., and Mitchell, F., J. Clin. Invest,, 25, 294-303 (1946).

171. Mickelsen, O., Caster, W. O., and Keys, A., Proc. Soc. Exptl. Biol. Med., 62, 254-8 (1946).

172. Klein, J. R., and Kohn, H. I., J. Biol. Chem., 136, 177-89 (1940).

173. Koschara, W., Z. physiol. Chem., 232, 101-16 (1935).

174. Pearson, P. B., and Schweigert, B. S., J. Nutrition, 34, 443-53 (1947). 
175. Foster, J. W., J. Bact., 47, 27-41 (1944).

176. Pennington, D., Snell, E. E., and Williams, R. J., J. Biol. Chem., 135, 213-22 (1940).

177. MeLean, R. A., Smith, D. T., and Smith, S. G., Abstracts, Am. Chem. Soc., 108th Meeting, New York City, New York, 1944.

178. Sarett, H. P., J. Biol. Chem., 159, 321-5 (1945).

179. Rubin, S. H., Cooperman, J. M., Moore, M. E., and Scheiner, J., J. Nutrition, 35, 499-509 (1948).

180. Daniel, L. J., Scott, M. L., Norris, L. C., and Hauser, G. F., J. Biol. Chem., 160, 265-71 (1945).

181. Kazenko, A., and Laskowski, M., J. Biol. Chem., 173, 217-21 (1948).

182. Suarez, R. M., Welch, A. D., Heinle, R. W., Suarez, R. M., Jr., and Nelson, E. M., J. Lab. Clin. Med., 31, 1294-1304 (1946).

183. Bernhard, K., Z. physiol. Chem., 267, 91-8 (1940).

184. Riggs, T. R., and Hegsted, D. M., J. Biol. Chem., 172, 539-45 (1948).

185. Lipmann, F., Federation Proc., 4, 97 (1945).

186. Nachmansohn, D., and John, H. M., J. Biol. Chem., 158, 157-71 (1945).

187. Blanchaer, M. C., and Cameron, E., J. Nutrition, 35, 391-7 (1948).

188. Roderuck, C. E., Williams, H. H., and Macy, I. G., J. Nutrition, 32, 249-65 (1946).

189. Oldham, H. G., Davis, M. V., and Roberts, L. J., J. Nutrition, 32, 163-80 (1946).

190. Fabriani, G., and Spadoni, M. A., Boll. soc. ital. biol. sper., 20, 730-1 (1945).

191. Geill, T., and Lindholm, H., Acta Med. Scand., 124, 522-34 (1946).

192. Malaguzzi-Valeri, C., and Conese, G., Boll. soc. ital. biol. sper., 20, 613-14 (1945).

193. Hathaway, M. L., and Strom, J. E., J. Nutrition, 32, 1-8 (1946).

194. Alexander, B., and Landwehr, G., J. Clin. Invest., 25, 287-93 (1946).

195. Gifft, H. H., and Hauck, H. M., J. Nutrition, 31, 635-45 (1946).

196. Alexander, B., Landwehr, G., and Mitchell, F., J. Clin. Invest., 25, 294-303 (1946).

197. Slater, E. C., and Morell, D. B., Australian J. Exptl. Biol. Med. Sci., 24, 121-2 (1946).

198. Brewer, W., Porter, T., Ingalls, R., and Ohlson, M. A., J. Nutrition, 32, 583-96 (1946).

199. Blazso, S., and Bubrauszky, Z., Z. Vitaminforsch., 14, 13-24 (1943).

200. Dubrauzky, V., and Blazso, S., Z. Vitaminforsch., 14, 2-13 (1943).

201. Hathaway, M. L., and Lobb, D. E., J. Nutrition, 32, 9-18 (1946).

202. Davis, M. V., Oldham, H. G., and Roberts, L. J., J. Nutrition, 32, 143-61 (1946).

203. Hou, H. C., and Dju, M. Y., Chinese Med. J., 61, 121-30 (1942).

204. Truvanov, A. V., Biokhimiya, 11, 33-43 (1946).

205. Sarett, H. P., and Goldsmith, G. A., J. Biol. Chem., 167, 293-4 (1917).

206. Cayer, D., and Cody, S., Am. J. Med. Sci., 215, 273-7 (1948).

207. Cossandi, E., Boll. soc. ital. biol. sper., 16, 703-6 (1941).

208. Huff, J. W., Pearson, P. B., and Perlzweig, W. A., Arch. Biochem., 9, 99-104 (1946).

209. Butler, R. E., and Sarett, H. P., J. Nutrition, 35, 539-48 (1948).

210. Coulson, R. A., and Stewart, C. A., Proc. Soc. Exptl. Biol. Med., 61, 364-9 (1946).

211. Hou, H. C., and Dju, M. Y., Chinese Med. J., 61, 192-8 (1942).

212. Spector, H., Hamilton, T. S., and Mitchell, H. H., J. Biol. Chem., 161, 145-52 (1945).

213. Wright, L. D., Beyer, K. H., Skeggs, H. R., Russo, H. F., Patch, E. A., Am. J. Physiol., 145, 633-7 (1946).

214. Gardner, J., Parsons, H. T., and Peterson, W. H., Arch. Biochem., 8, 339-48 (1945). 
215. Gardner, J., Parsons, H. T., and Peterson, W. H., Am. J. Med. Sci., 211, 198204 (1946).

216. Steinkamp, R., Shukers, C. F., Totter, J. R., and Day, P. L., Proc. Soc. Exptl. Biol. Med., 63, 556-8 (1946).

217. Suarez, R. M., Welch, A. D., Heinle, R. W., Suarez, R. M., Jr., and Nelson, E. M., J. Lab. Clin. Med., 31, 1294-1304 (1946).

218. Jukes, T. H., Franklin, A. L., Stokstad, E. L. R., Boehne, J. W., J. Lab. Clin. Med., 32, 1350-5 (1947).

219. Rabinowitz, J. C., and Snell, E. E., Proc. Soc. Exptl. Biol. Med., 70, 235-40 (1949).

220. Johnson, B. C., Mitchell, H. H., and Hamilton, T. S., J. Biol. Chem., 161, 357-60 (1945).

221. Lange, R. D., Ramsey, R. H., and Moore, C. V., J. Lab. Clin. Med., 30, 1048-55 (1945).

222. Hamil, B. M., Coryell, M. M., Roderuck, C., Kaucher, M., Moyer, E. Z., Harris, M. E., and Williams, H. H., Am. J. Diseases Children, 74, 434-6 (1947).

223. Johnson, B. C., Hamilton, T. S., and Mitchell, H. H., J. Biol. Chem., 158, 619-23 (1935).

224. Dawson, R. F., Advances in Enzymol., 8, 203-51 (1948).

225. Bonner, J., and Bonner, H., Vitamins and Hormones, 6, 225-75, (1948).

226. Van Lanen, J. M., and Tanner, F. W., Jr., Vitamins and Hormones, 6, 163224, (1948).

227. Beerstecher, E., Jr., Science, 111, 300-302, (1950).

228. Wright, L. D., Cresson, E. L., Skeggs, Wood, T. R., Peck, R. L., Wolf, D. E., and Folkers, K., J. Am. Chem. Soc., 72, 1048 (1950). 


\section{Chapter VC}

\section{PHYSIOLOGICAL, PHARMACOLOGICAL, AND TOXICOLOGICAL EFFECTS}

The four preceding chapters have presented the available evidence bearing upon the problems of what the $\mathrm{B}$ vitamin requirements of living things are, and the metabolic fate of the vitamins themselves, and the next chapter is to deal with the effects of B vitamin deprivation. In accordance with the outline proposed for this section, it is necessary to consider here certain overall effects of the $\mathrm{B}$ vitamins on living organisms.

It is immediately apparent that the major effect of the B vitamins is to maintain the animal and all its parts in an efficient functional state, and regarding this aspect there would seem to be little cause for extended discussion. The exact manner in which the $B$ vitamins function in the maintenance of cellular and tissue metabolism has been considered at length in earlier sections. Moreover, the possible role of the B vitamins in preventing and curing various pathological conditions, primarily avitaminotic and otherwise, is more conveniently discussed in the next chapter, dealing with deficiency states. There are however certain special relationships which exist between the various $\mathrm{B}$ vitamins and specific physiological systems and functions, and these require some brief consideration at this point.

In considering the biological effects of any substance it is important to realize that different concentrations of a substance frequently manifest markedly different activities. What may be an innocuous and even required substance at one level of administration may become a dangerous drug at another. There is frequently a distinct intermediate level at which the substance becomes effective in producing in the activity of the organism certain changes which are neither particularly hazardous in the usual sense nor of a nature similar to its physiological function. These three levels of activity-the physiological, the pharmacological, and the toxicological - are quite distinct for a number of the $\mathrm{B}$ vitamins. The cause of the varying activities exhibited by different concentrations of these and other substances is obscure, but it may be surmised that $B$ vitamins in increased concentrations may have two major effects: they may increase the normal physiological reaction to a point where it be- 
comes so disproportionate as to upset the usual functions and become manifest, or they may at higher concentrations react in enzyme systems in which they are normally alien, in the manner of analogue inhibitors (Sect. D). These considerations have not as yet been intensively investigated, and there is little real knowledge in this regard.

The study of the pharmacology and toxicology of the B vitamins is of interest largely in that it throws light on two major problems: what the effects of large doses or prolonged treatment with a given vitamin will be, and to what extent the special physiological relationships to be considered here (and their derangement, considered in the next chapter) may be related to the pharmacological activity of the vitamin. Most authorities now feel that there is little real relationship between the often apparent similarity in physiological and pharmacological activities of the $B$ vitamins, being so decided largely by virtue of the thousandfold difference that generally exists between the two levels. Whether or not such an opinion is justified, it is certainly true that the three levels of activity of the $\mathrm{B}$ vitamins can be most adequately expressed in terms of micrograms, milligrams, and grams, respectively. In this regard, one of the most distinctive and remarkable things about the B vitamin group is certainly the high physiological activity per unit weight, coupled with the low toxicity exhibited by these substances which, on the basis of structural considerations alone, might be predicted to be highly toxic. A further reason for the consideration of certain pharmacological aspects of the B vitamins lies in the aspect that the precise mode of action of most drugs is as yet unknown. Present knowledge indicates, however, that it will eventually be shown that most drugs, at least, exert their action through specific enzyme systems. In view of the fact that so many of these systems involve B vitamins as coenzymes, it may be surmised that a growing field of interest will develop in B vitamin-drug interrelationships.

\section{Physiological Relationships}

Many of the special physiological interrelationships of the B vitamins are only discernible in the light of their derangement, and are therefore almost of necessity discussed in the next chapter. Most of the others seem to be associated with certain categories that have been established by tradition in the field of physiology-metabolism, endocrine function and reproduction, nervous function, and mental activity. It is apparent that these terms have little real meaning from the standpoint of being distinct functional entities: they are so interdependent as to be in practice inseparable. Nevertheless they do convey sufficient indications of various aspects of physiology for the present purpose, and are therefore 
used here as reference points rather than to designate functional boundaries.

Metabolic Interrelationships. Many of the relationships between vitamins and metabolism primarily involve endocrine function and are discussed in a following section, while other relationships that involve biochemically specific metabolic reactions are discussed at other appropriate places. Drawing the many facets of metabolism together results in the concept of "total metabolism" or the overall metabolic rate. The metabolic rate is a function of all the factors that go into a myriad of metabolic processes, and may be limited by any factor that is deficient, so that when any $B$ vitamin is present in inadequate amounts, the metabolism may be slowed down, and addition of that B vitamin may increase the metabolic rate to the point where some other factor becomes limiting. Beyond this, the $\mathrm{B}$ vitamins have little effect upon the metabolic rate in the sense that many other physiologically active substances do. When for some reason the metabolic rate is increased, the heightened cellular activity of the body results in an increased vitamin attrition and therefore an increased requirement for the B vitamins, as it does other nutritional elements.

As far as is now known, these same considerations apply to the interrelationships between the B vitamins and growth. Supplementation of the diet with increased amounts of $\mathrm{B}$ vitamins will undoubtedly increase the growth rate in many cases, ${ }^{117}$ but this effect is primarily due to the fact that in these cases there are originally present insufficient supplies of vitamin to meet the requirements for the maximum rate of growth permitted by other metabolic factors. Whereas there is some evidence to indicate that certain B vitamins may function specifically to increase the mitotic rate of certain plant tissues, it seems unlikely that such an effect occurs in the higher animals. When $\mathrm{B}$ vitamins are added to the diet to a point that they no longer stimulate growth, it is apparent that some other factor then becomes limiting in the process. This factor may well be one whose limiting nature is unimportant, but it may also be one that will result in serious consequences. Thus in an overly rapid rate of growth various other nutritional substances associated with protein metabolism or bone formation may become limiting; and as previously stated, there is no assurance that the fastest rate of growth is the best. There would seem to be some justification, therefore, in permitting growth to proceed at what experience has taught is a reasonable rate, allowing the limiting catalytic activity of the $\mathrm{B}$ vitamin, when it exists, to remain the controlling factor. Because of the high rate of turnover of raw materials in the growing animal, and the consequent high metabolic rate, there is an increased B vitamin requirement on a weight basis. In well 
nourished populations, however, this factor seems adequately compensated for by the increased appetite and consumption of other nutritional materials. Except when specific factors make it seem expedient, there consequently seems little justification for the B vitamin supplementation of the diets of growing children. When however the practice is instituted, supplementation of the diet with a variety of other important nutritional factors would also seem to be highly desirable.

Endocrine Function and Reproduction.* The known interrelationships between the $\mathrm{B}$ vitamins and endocrine function are numerous; ${ }^{1 \mathrm{a}}$ at our present state of knowledge many are nebulous, but a few cases at least have received considerable attention. The endocrine glands represent areas of high metabolic activity, and it is logical to assume that they would demonstrate unusual sensitivity to changes in vitamin balance for that reason. They function, moreover, in a catalytic role frequently associated with the control of specific metabolic processes, and the effects produced by the superimposition of two catalytic factors would for kinetic reasons be extremely pronounced.

As mentioned earlier, B vitamin deficiency results in a pathological condition of all the body tissues, and depleted glandular tissue will generally manifest such a condition by cytological changes and deranged function. While such a condition is not always observed in all the endocrines, there seems ample evidence to indicate that it does occur in those cases where other factors do not produce death before the effect is observable. It is remarkable that relatively little is known about the hypophysis in this regard, and this fact may be a reflection of special preservative powers possessed by this key gland. In other cases, little is known regarding vitamin interrelationships aside from the natural degenerative changes occurring in deficiency. Certain special relationships are known, however, that seem to involve more than this, and it is with these that our concern here rests. They involve primarily the thyroids, the adrenal cortex, and the gonads. Were our knowledge adequate, it would of course be apparent that these relationships extend through these endocrines to the others.

Thyroid relationships. The thyroid gland exerts its effects by virtue of its relationship to the metabolic rate, which in turn has a profound influence on $B$ vitamin requirements. There is seldom any pronounced thyroid change in B vitamin deficiency states. Experimental hyperthyroidism in animals produces a number of characteristic changes--loss of weight, interrupted sexual cycles, hepatic damage, cardiovascular disorders, and a drop in liver glycogen. These symptoms can for the most

* The function of the $B$ vitamins as plant hormones has been discussed earlier (p. 316), and has recently been thoroughly reviewed in the literature (1). 
part be prevented or cured by B vitamin, liver or yeast administration. $^{2,3}, 115$ The thiamine content of rat tissues decreases when the animal is rendered hyperthyroid, and there is increased thiamine excretion. ${ }^{4,5}$ Thiamine administration in some cases of Graves' disease has been shown to be beneficial, and it seems apparent that many of the symptoms of hyperthyroidism are due to the conditioned malnutrition caused by increased vitamin requirements. There is known to be a real possibility that the thyroid and other glands have a marked influence on vitamin metabolism, particularly at points involving absorption and excretion, but the nature of such effects is at present too obscure to permit any detailed consideration. The involvement of the so-called "digestive hormones" in this regard would seem to be of special interest, but has been little studied.

Adrenal Cortical Relationships. Pantothenic acid-deficient animals develop a characteristic lesion of the adrenal cortex, ${ }^{6}$ and adrenalectomy in such animals prevents the typical gray hair syndrome, ${ }^{7}$ although desoxycorticosterone administration permits hair graying to occur. ${ }^{8}$ This relationship has been extensively studied, and is discussed in greater detail in the next chapter. Despite the fact that there is still considerable disagreement as to whether apantothenosis produces adrenal hypofunction or hyperfunction, it is at least clear that there is involved here an intimate relationship between pantothenic acid, desoxycorticosterone, and melanin formation, and that this bids fair to be the first direct vitaminhormone interrelationship to be understood in any detail. Many of the other symptoms of pantothenic acid deficiency, such as the deranged water and salt balance, are undoubtedly manifest through adrenal malfunction..$^{9-12}$

Interrelationships with the Gonads and Reproduction. Whereas certain of the fat-soluble vitamins have been linked with sexual and reproductive functions by tradition, there is no fundamental reason to attribute such a role to them and not the B vitamins. B Vitamin deficiency most certainly results in sterility, and this point will be mentioned again later. There are rather specific manners, however, in which the androgenic and estrogenic substances are related to the $\mathrm{B}$ vitamins, and these are considered here.

Certain steroid hormones, largely androgenic in nature, have now been found to have an important role in nitrogen, inorganic phosphorus, and potassium retention, in castrate animals, eunuchs, and normal animals including man. ${ }^{13}$ The administration of testosterone, for instance, produces an abrupt drop in urea excretion but also a hypoproteinemia, and it is felt that nitrogen retention is involved with the laying down of tissue protein. Whether nitrogen retention is a cause or result of this 
is unclear, but testosterone simultaneously produces a marked rise in renal arginase and $d$-amino acid oxidase, so that it is felt that the kidney is in some manner involved in these overall retention effects. It has previously been pointed out that the role of the B vitamins is closely associated with nitrogen metabolism in a variety of ways including absorption, storage, and metabolic function. It is therefore apparent that the $\mathrm{B}$ vitamins must be considered from a number of standpoints in interpreting the overall metabolic effects of the androgens. ${ }^{14}$

Considerably more concise is the relationship between the $\mathrm{B}$ vitamins and estrogen inactivation. ${ }^{15-19} \mathrm{~A}$ great variety of ingenious experimental work has shown that the liver inactivates estrogen and androgens both in vivo and in vitro. In the case of estrogens, but not androgens, this inactivation does not occur when the experimental animals are on thiamine or riboflavin deficient diets, and it has been suggested that the liver inactivation normally involves oxidative steps which are impaired in deficiency. Indeed, it seems well established that cozymase is critical in this conversion. ${ }^{20}, 21$ Chick liver converts testosterone to 17-ketosteroids, while rat liver carries the process beyond this, further metabolizing the latter substances. ${ }^{22}$ It has been pointed out moreover, that the differences in action on estrogens and androgens during deficiency works to produce a severe imbalance, and working more or less from this viewpoint, Biskind et al..$^{23}$ have reported the successful use of $\mathrm{B}$ vitamin therapy in the treatment of cases of menorrhagia, metrorrhagia, cystic mastitis, and premenstrual tension. Administration of large doses of thiamine or riboflavin rapidly restores the ability of livers in deficient animals to detoxify estrone or alpha-estradiol, but the restoration of diethylstilbestrol inactivation is less rapid. ${ }^{24}$ There is no apparent relationship between liver damage and function in this regard. Inanition and malnutrition, particularly protein deficiency, in general exert a similar effect, and it has been claimed that the effects of thiamine and riboflavin in this regard are not specific, the effect being due to the inanition produced..$^{25}$ While this may be true, it seems clear that livers from animals made deficient in pyridoxine, pantothenic acid, biotin and vitamin $\mathrm{A}$ do not lose their inactivating ability. ${ }^{26}$ It has been suggested, moreover, that the pronounced gynecomastia seen in many male prisoners-of-war in the Orient during the last war may have been due to this effect brought about by the accompanying malnutrition-quite frequently accompanied by ariboflavinosis and mild beriberi. ${ }^{27}$ Excess estrogen, moreover, is known to cause adrenal hypertrophy, and it seems likely that the adrenal hypertrophy associated with certain avitaminoses in rats may be a result of this same impaired liver function. ${ }^{28}$

A second relationship of considerable interest involves the fact that the 
marked (fortyfold) hypertrophy induced in the chick oviduct by stilbestrol does not occur in folic acid-deficient chicks and to only a moderate degree in pantothenic acid-deficient chicks. ${ }^{29}$ Thus in experiments in which chicks were maintained on appropriate deficient diets for fifteen to nineteen days following which they received daily subcutaneous injections for six days of $0.5 \mathrm{mg}$ of stilbestrol, autopsy revealed folic acid-deficient chicks to have an average oviduct weight of $62 \mathrm{mg}$; pantothenic-deficient chicks had average oviduct weights of $281 \mathrm{mg}$, with weights for controls receiving $20 \mu \mathrm{g}$ of folic acid daily from birth of 450 mg. It has been shown that there is a direct quantitative relationship between the oviduct response to stilbestrol and the dietary level of folic acid. Riboflavin and pyridoxine at least do not behave in a similar manner. ${ }^{30}$ Later work has indicated strongly that a similar relationship is true in the folic acid-deficient monkey on estradiol treatment, and that it is probably a general phenomenon. ${ }^{31}$ It has been suggested ${ }^{32}$ that all rapid cell proliferation requires special amounts of folic acid for the necessarily rapid synthesis of nuclear thymine and purine bases. This suggestion is of some particular interest in view of the role of folic acid in erythropoiesis and the observed effects of folic acid analogues on tumor growth. ${ }^{33}$

Reference to this response of the chick oviduct calls for at least passing mention of the fact that it is in the oviduct that biotin-binding avidin is laid down in the hen's egg. ${ }^{34}$ An extended discussion of the genesis and significance of this naturally occurring antivitamin is not expedient at this time, although it should be noted that there is as yet no acceptable explanation for the functional occurrence of this substance. Despite earlier hopes, little of significance from the standpoint of an understanding of its fundamental role in metabolism has been discovered.

A consideration of various aspects of the reproduction process-gametogenesis, mating behavior, the estrus cycle, embryonic development, lactation, and maternal instinct-shows at once that adequate $\mathrm{B}$ vitamin nutrition is essential to the process for many reasons that may be considered in terms of general health. ${ }^{35}$ Thus, the production of viable sperm and ova, development of the embryo, and the maintenance of satisfactory lactation are linked to B vitamin activity, largely because normally functioning cellular and tissue elements are essential to these processes. No specific B vitamin relationship is known to be directly involved beyond this, although special "lactation factors" have been reported in experimental work from time to time. ${ }^{36-39}$ Indirectly, B vitamin-endocrine relationships may influence these processes as well as mating and maternal behavior. The estrus cycle, and all the phases of reproduction directly influenced by estrogens, are directly concerned with the $\mathrm{B}$ vitamins in 
the manner previously mentioned, however. It would thus appear that the role of the $\mathrm{B}$ vitamins in the reproductive process per se is no more than a composite of the various facets previously discussed: the increased requircments (p. 269), the general metabolism, and the endocrine relationships. The clinical synthesis of these items is a problem beyond the realm of this monograph.

Nerve Function. The "special" functions of the B vitamins considered in this section may be considered as "special" largely because they occur in the higher animals and may not be considered in terms of the fundamental metabolism of a single cell. Thus endocrine relationships and the metabolism of large aggregates of cells are considerations peculiar to higher animals as contrasted to single cells. Nervous function is similarly a specialized activity, and one in which several vitamins are particularly involved in addition to their fundamental role in the life of the neuron. From the standpoint of maintaining the lipoid myelin sheath and the transfer of the nerve impulse along the nerve cell, special relationships may well exist which can as yet only be surmised. For nerve impulse transmission, in which acetylcholine is critically involved, thiamine and pantothenic acid at least must play particularly important roles. Coenzyme A is known to function in the acetylation of choline, and the vital nature of this reaction would dictate a critical role in the metabolic interchange in the neurone.

Thiamine, by virtue of its role in the conversion of pyruvate to acetate, is obviously important in the supplying of a constant source of acetyl groups for the pantothenate-mediated coupling, thus:

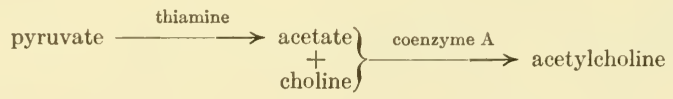

It has been observed that diffusible thiamine is present in stimulated nerves in four to eight times the concentration that it exists in resting nerves, as measured by bradycardia tests and Phycomyces tests, but that equal amounts are indicated by yeast fermentation tests. ${ }^{40,}{ }^{41}$ This excess of diffusible thiamine disappears rapidly after stimulation, however. In nerve poisoned with iodoacetate, by contrast, there is an apparent preponderance of diffusible thiamine in resting nerve. ${ }^{42}$ Thiamine (but not cocarboxylase) is said to block acetylcholine action upon the heart, moreover. ${ }^{43}$ The significance of these observations on the basis of the role of thiamine proposed above is not too clear, and indeed Muralt has proposed that thiamine must be considered not only as a catalyst, but also as a metabolic substance in nerve biochemistry. ${ }^{44}$ The justification for such 
a proposal, however, does not at present seem sufficient to warrant its general acceptance.

Mental Activity. A further field of interest characteristic of animals involves reflex and mental activity, and because of the pronounced defects that occur in these functions in vitamin deprivation, various groups have sought to establish some special relationship between them and the B vitamins. To a large extent such attempts have been unsuccessful. There is no doubt that mental activity as measured by sensory acuity and performance tests of various kinds, reflex activity, and general personality are all influenced greatly in B vitamin deficiency of even a mild nature. Thus dogs that have developed conditioned reflexes to auditory stimuli such that they showed a 100 per cent differentiation between two tones for some months, lose their ability to differentiate in from four to fifteen days on $\mathrm{B}$ vitamin-deficient diets. ${ }^{45}$ While this loss became worse over a two-month deficiency period, other deficiency signs were lacking in the experiment. Vitamin supplementation rapidly restored the ability to differentiate, and similar results were obtainable by repeating the sequence on the same animals.

A number of studies have been made to determine whether thiamine supplementation improves the mental response of children, ${ }^{46,47}$ and the results have indicated that such is not the case when the individuals concerned have an adequate thiamine intake. Similar results from adults on adequate and restricted diets ${ }^{48-50}$ seem to indicate clearly that inadequate $\mathrm{B}$ vitamin nutrition results in a frequently severe and general breakdown in mental, personality and reflex processes, but that supplementation with $\mathrm{B}$ vitamins above the level required for adequate nutrition as assessed by other means has little or no beneficial effect.

\section{Pharmacological and Toxicological Effects ${ }^{50 a}$}

Previous mention has been made of the several important reasons for a brief consideration of the pharmacodynamic action of the B vitamins. Such a discussion might well be undertaken either from the standpoint of the pharmacological effects observed in particular physiological systems, or in terms of the known effects of structurally similar compounds. The latter alternative, i.e., the consideration of each B vitamin separately, is here adopted because of the desirability of considering the picture so obtained in the light of deficiency symptoms. The impetus of current research in this field is such that what is at present a sparsely studied topic may be expected to expand in the immediate future to a major field of endeavor. Certainly such an event will be essential before a thorough understanding of the biochemical processes involved in drug action can be elucidated and drug design can become a science. 
Thiamine. The pharmacodynamic action of thiamine may best be described as "curare-like." ${ }_{51}$ In frogs, rats, and dogs, except for the dosage, dextro-tubocurarine and thiamine have been demonstrated to have almost identical effects. Rapid intravenous injection of $50 \mathrm{mg} / \mathrm{kg}$ of thiamine hydrochloride into dogs causes respiratory paralysis, hypotension, bradycardia, and vasodilation, but if respiration is maintained these effects are transient. Toe contractions resulting from stimulation of the isolated sciatic nerve are prevented by both thiamine and dextro-tubocurarine, although the muscle still responds to direct stimulation. Similar effects may be demonstrated on the exposed sciatic nerve and gastrocnemius muscle of frogs. These results seem closely related to the symptoms following administration of lethal doses to experimental animals-weakness, tetany, labored breathing, and death from respiratory failure. Thiamine has found some use in the treatment of labor pains ${ }^{52}$ in intramuscular doses of $60 \mathrm{mg}$.

The lethal doses of thiamine by intravenous administration are 125 , 250,300 and $350 \mathrm{mg} / \mathrm{kg}$ for the mouse, rat, rabbit and dog respectively, and the toxic subcutaneous and oral doses are about six and forty times as high respectively. There is no apparent cumulative effect. The therapeutic index (ratio of therapeutic dose to minimum lethal dose) is therefore about 600 for mice, 5000 for rats, and 70,000 for dogs. Haley ${ }^{54}$ has carefully determined thiamine toxicity for the mouse and rabbit and finds that for the mouse the intravenous LD 50 * is $84.24 \mathrm{mg} / \mathrm{kg}$, with a standard error of \pm 1.14 for the mononitrate salt. Intraperitoneally it is 387.3 $\mathrm{mg} / \mathrm{kg} \pm 1.65$; and $329.8 \pm 3.93$ for the hydrochloride. For the rabbit the average intravenous lethal dose of the mononitrate is $112.58 \mathrm{mg} / \mathrm{kg}$ and for the hydrochloric $117.45 \mathrm{mg} / \mathrm{kg}$. He quotes Molitor as having found the intravenous hydrochloride toxicity for the mouse to be $85 \mathrm{mg} / \mathrm{kg}$.

The rapidity of the onset of thiamine pharmacological activity is such as to largely preclude any possibility of action other than interference in some enzyme system. Death seems to result generally from paralysis of the respiratory center of the medulla, ${ }^{55,56}$ and the administration of thiamine by cisternal puncture ${ }^{57}$ or direct application to the cerebral cortex ${ }^{58}$ produces particularly marked effects.

Thiamine toxicity in man is well known and presents a number of special problems. ${ }^{59}$ It appears reasonably certain that the untoward effects reported as resulting from thiamine administration in man result. either from sensitization and the resulting allergic type of response or in a very few cases from some inherent susceptibility to this compound. All the cases reported in this regard, including two deaths ${ }^{60,61}$ and several near deaths ${ }^{62}$ had histories of extensive previous thiamine therapy, and

* The dose that produces death in $\mathbf{5 0}$ per cent of the test animals given that dose. 
the doses involved have never exceeded $100 \mathrm{mg}$ or so, and were generally less $(5-10 \mathrm{mg})$. They were generally intravenous. These levels are far below the lethal dose that might be anticipated from toxicity data in lower animals. Unfortunately, however, little accurate toxicity data exist for lower animals, and much of the data mentioned earlier is subject to extreme question as regards its accuracy. The symptoms described in acute cases involve those classically associated with anaphylaxis, ${ }^{63-67}$ and in some cases at least hypersensitivity has been demonstrated by passive transfer. ${ }^{68}$ In sensitive persons, subcutaneous injection of small amounts of thiamine generally causes large wheals, ${ }^{68}$ although the validity of such a skin test has been challenged. ${ }^{69}$ Other symptoms often associated with thiamine toxicity include herpes zoster ${ }^{70},{ }^{71}$ headache, trembling, and a rapid pulse. The symptoms have been likened to those occurring from an overdose of thyroid extract,,$^{72}$ and in general bear little resemblance to the curare-like action seen in lower animals. ${ }^{73-82}$ Attempts to sensitize rabbits, dogs, and guinea pigs to thiamine have been unsuccessful. $^{83}$

Thiamine toxicity is a major problem from the practical standpoint because large doses of thiamine are so frequently administered. It is for this reason as much as any that a large number of toxic effects have been reported for this B vitamin and not for the others. It seems quite clear that the effects observed in man have little or no relationship to those seen in lower animals-that in man the result is an allergic one in probably every case reported. ${ }^{84,85}$ That similar results occur from a large variety of other popular pharmaceuticals-the sulfa drugs are a classic example-would indicate that such an effect might well be anticipated. Some workers have strongly advocated the complete abandonment of high thiamine therapy, particularly by intravenous administration, for this reason. In any case, it is readily apparent that two things are badly needed at present: a thorough evaluation of individual responses to thiamine, and accurate data on the toxicity levels of thiamine for lower animals other than the mouse. Such information should certainly precede any acceleration in the present trends toward massive vitamin therapy.

Nicotinic Acid. Nicotinic acid and its amide like many pyridine derivatives, have marked pharmacological activity. One of the most important manifestations of this in man is the nitroid reaction, which is similar to the effects of histamine and caused by nicotinic acid but not the amide. ${ }^{86-92}$ Shortly after a suitable dose of nicotinic acid is administered, there is a marked flushing of the face, neck, and arms. The reaction is due to a transient vasodilation which lasts for an hour or so and may be accompanied by itching or burning. This flushing action is said to be prevented by a previous oral dose of $30-60 \mathrm{gm}$ of glycine. ${ }^{93}$ There is an 
increased peripheral blood flow ${ }^{94}$ and a rise in cutaneous temperature. The reaction is not considered to be a "frank toxic manifestation" 95 in view of the low dosage that evokes it and its absence on nicotinamide administration, the latter being about twice as toxic as the free acid. Approximately $30 \mathrm{mg}$ of niacin per day orally may be sufficient to arouse the reaction, while $10 \mathrm{mg}$ intravenously or $60 \mathrm{mg}$ intramuscularly are frequently effective. At present, $16-18 \mathrm{mg}$ of nicotinic acid are generally added to each pound of flour in this country, and there has been at least one report of a flushing reaction from this source. In general it seems that there is a great individual variation in response, that all individuals react to some dosage, and that the manifestation is a harmless one. It does however make the amide the vitamin isotel of choice, since oral doses as high as $500 \mathrm{mg}$ are without a nitroid effect.

The pharmacological effects of niacin and many other closely related pyridine derivatives have been intensively studied, and it should be mentioned that the diethylamide of nicotinic acid, coramine or nikethamide, is a widely used respiratory stimulant. ${ }^{96,97}$ Nicotinic acid, as regards its flushing action, has been likened to histamine, acting in a manner antagonistic to epinephrine. Nicotinic acid has little or no effect on the blood pressure or pulse rate however, its effects on peripheral blood flow being apparently local ones. It is without effect on the autonomic ganglia, isolated rabbit intestine, or isolated guinea pig uterus. Nicotinic acid has been compared with its amide, the mono- and diethyl amides, and pyridine as regards their effects on curare, prostigmine, and acetylcholine action on the quadriceps and soleus muscles of cats anaesthetized with nembutal. ${ }^{98}$ These results are indicated in Table 33 .

\section{TABLE 33. Some Pharmacological Effects of Niacin and Similar Compounds}

Derivative

Pyridine

Nicotinic acid

Nicotinamide

Ethylnicotinamide

Diethylnicotinamide

$\begin{array}{lc}\text { Curare Effect } & \text { Prostigmine Effect } \\ \text { antagonistic } & \text { reinforcing } \\ \text { antagonistic } & \text { antagonistic } \\ \text { antagonistic } & \text { reinforcing } \\ \text { reinforcing } & \text { reinforcing } \\ \text { reinforcing } & \text { reinforcing }\end{array}$

Acetylcholine Effect

enhancing enhancing enhancing enhancing

It has similarly been convenient to compare the toxicity of niacin with that of many structurally similar compounds. As regards chronic toxicity, it seems that doses as high as $2 \mathrm{gm} / \mathrm{kg}$ daily for several months do not affect dogs, rats, or chicks. ${ }^{99}$ Brazda and Coulson have determined the subcutaneous LD 50's of a number of pyridine derivatives for rats, ${ }^{100}$ and their results are summarized in Table 34 . Unna ${ }^{101}$ has similarly found the LD 50 of niacin for mice and rats to be from $4-5 \mathrm{gm} / \mathrm{kg}$ subcutaneously and $5-7 \mathrm{gm} / \mathrm{kg}$ orally, and the toxicity of nicotinamide 
TABLE 34. LD 50 of Several Pyridine Derivatives for $50-100 \mathrm{gm}$ Rats by Subcutaneous Injection

\begin{tabular}{lcc}
\multicolumn{1}{c}{ Derivative } & $\begin{array}{c}\mathrm{LD} \mathrm{50} \\
(\mathrm{gm} / \mathrm{kg})\end{array}$ & Order of Toxicity \\
Pyridine & 1.0 & 5.00 \\
Pyridine methochloride & 0.28 & 17.9 \\
Niacin & 5.0 & 1.0 \\
Niacin methochloride (Trigonellin) & 5.0 & 1.0 \\
Nicotinamide & 1.68 & 3.0 \\
Nicotinamide methochloride $\left(\mathrm{F}_{2}\right)$ & 2.40 & 2.08 \\
Coramine & 0.24 & 20.8 \\
Coramine methochloride & 1.90 & 2.63
\end{tabular}

to be about double this. By contrast the closely related alkaloid, arecoline, has a subcutaneous lethal dose for mice of $0.065 \mathrm{gm} / \mathrm{kg} .{ }^{102}$<smiles>COC(=O)C1=CCCN(C)C1</smiles>

Arecoline<smiles>CN1CCCC1c1cccnc1</smiles>

Nicotine

Minimum lethal doses have also been determined for niacin intravenously for a number of species and nicotine was similarly assessed in parallel experiments, ${ }^{103}$ with the results shown in Table 35 .

$\begin{array}{lcc}\text { TABLE 35. Intravenous Toxicity of Sodium Nicotinate and Nicotine } & \begin{array}{c}\text { Sicth } \\ \text { Species }\end{array} & \begin{array}{c}\text { Nose }(\mathrm{mg} / \mathrm{kg})- \\ \text { Sodium nicotinate }\end{array} \\ \text { White mice } & 0.8 & 4500 \\ \text { Rats } & 1.0 & 3500 \\ \text { Guinea pigs } & 4.5 & 3500\end{array}$

Death from niacin, as from nicotine, follows severe clonic convulsions. Other symptoms associated with niacin toxicity include generalized paralysis, depressed respiration, and cyanosis, death generally occurring in from 12 to 36 hours.

Attention should also be called to the fact that large but sublethal doses of pyridine derivatives may have a growth depressing effect by virtue of the fact that they are generally excreted as $\mathrm{N}^{\prime}$-methyl derivatives, and they may thus draw critically on the available methyl group supply of the body.

Choline. The pharmacological effects of choline have been well known for some time ${ }^{104}$ and choline chloride has found some use as a therapeutic agent. It exerts a muscarine effect peripherally, stimulating parasympathetic endings, and also a nicotine effect, stimulating and paralyzing 
autonomic ganglia. By vagal stimulation it slows and weakens the heart and causes a drop in blood pressure. Choline increases salivary, lacrimal, and other secretions and both gastroc and intestinal peristalsis. Doses of $600 \mathrm{mg}$ in $240 \mathrm{ml}$ physiological saline may be slowly administered intravenously (15-20 minutes) without great danger.

Levels of 1,2, and 4 per cent choline in chick diets produce retarded growth but no other apparent ill effects. In rat diets, $2.7,5$, and 10 per cent choline decreases rat growth by 20,45 , and 100 per cent, respectively. When 1 per cent choline, corresponding to $500 \mathrm{mg} / \mathrm{kg} /$ day is used, it is not effective. The 2.7 per cent level corresponds to 1350 $\mathrm{mg} / \mathrm{kg} /$ day. Drinking water containing 1 per cent choline decreases growth, the dose being $750 \mathrm{mg} / \mathrm{kg} /$ day. ${ }^{105}$ The LD 50 of choline given intraperitoneally to mice is $320 \mathrm{mg} / \mathrm{kg}$, and the oral LD 50 for rats is 6.7 $\mathrm{gm} / \mathrm{kg}$. Other studies have reported the oral LD 50 for rats to vary from 3.4 to $6.1 \mathrm{gm} / \mathrm{kg}$ depending upon the solution concentration used. ${ }^{106,109}$ It has been pointed out that the same toxicity applied to man would mean that minimum effects would occur at $15-70 \mathrm{gm} /$ day and the LD 50 dose would be about 200-400 gm. ${ }^{107}$

Pyridoxine. The lethal dose (LD 50) of pyridoxine for rats by subcutaneous injection is about $3.1 \mathrm{gm} / \mathrm{kg} .{ }^{108}$ The oral LD 50 is $4 . \mathrm{gm} / \mathrm{kg}$. Toxic symptoms involve tonic convulsions some 24 hours after dosing, the hind limbs being stretched away from the body. These convulsive attacks continue for from several days to three weeks unless death intervenes. At lethal doses death occurs in from 36 to 72 hours. The closeness of the subcutaneous and oral toxic doses is of course a reflection of the delayed nature of the symptoms. Autopsy of these animals reveals adrenal enlargement with occasional cortical hemorrhage. In man pyridoxine is said to have a sedative effect. Daily feeding of $10 \mathrm{mg} / \mathrm{kg}$ to rats, dogs, and monkeys for a period of three months produces no significant change, and $20 \mathrm{mg} / \mathrm{kg}$ given to cats intravenously is ineffective, as are single doses up to one gram. ${ }^{110}$

Riboflavin. There is little or no information available regarding the toxicology and pharmacology of riboflavin. Five thousand times the therapeutic dose for mice (i.e.: $340 \mathrm{mg} / \mathrm{kg}$ ) is not effective in producing any observable action and it has been estimated that on this basis $20 \mathrm{gm}$ per day for a $70-\mathrm{kg}$ man should be harmless. ${ }^{111-113}$

Folic Acid. Folic acid is relatively nontoxic, judging by the limited available data. The intravenous LD $50 \mathrm{in} \mathrm{mg} / \mathrm{kg}$ is 600 for the mouse, 500 for the rat, 410 for the rabbit, and 120 for the guinea pig. Death is apparently caused by the obstruction of the renal tubules as the result of precipitation of folic acid. Taylor and Carmichael ${ }^{116}$ have recently found a high sex differential in the toxicity of folic acid for dba mice, 
the males being much more resistant. Thus, whereas $600 . \mathrm{mg} / \mathrm{kg}$. produced a 100 per cent mortality in females, $1600 \mathrm{mg} / \mathrm{kg}$. did not kill any male mice. Five $\mathrm{mg} / \mathrm{kg}$ intraperitoneally does not affect rats or rabbits when administered daily over long periods, but doses of 50 to $75 \mathrm{mg} / \mathrm{kg}$ do have some slight effects. All efforts to demonstrate any pharmacological activity have been unsuccessful. ${ }^{114}$

Pantothenic Acid, Biotin, p-Aminobenzoic Acid, and Inositol. Little is known concerning the pharmacology of pantothenic acid. Intravenous doses as high as $100 \mathrm{mg}$ have been given to man without apparent effects $^{99}$ (p. 422). Monkeys have been fed as high as one gram per day and rats have also been given one gram per rat per day without apparent harmful effects. Biotin, $p$-aminobenzoic acid and inositol are also known to be relatively nontoxic but have been little studied in this regard. A number of esters of $p$-aminobenzoic acid (the ethyl ester, "Benzocaine," and the butyl ester, "Butesin") have however been widely used as local anaesthetics.

\section{Summary of Relationships}

It is apparent that the B vitamins play certain physiological roles in the higher animals that differ from their maintenance functions in cellular metabolism, and these roles occur by virtue of the existence of differentiated physiological functions in the higher animals. In these cases the vitamins undoubtedly act in the same type of reaction, but the process in which the reaction occurs is not one characteristic of all living cells. The fact that only a few such relationships can be demonstrated lucidly at present is probably the result of the state of our learning rather than an indication of a limitation to the number of "special cases." It seems entirely reasonable to expect that in the evolutionary scale, increased morphological complexity would demand an increased complexity in catalyst functions, and this view should receive considerable elucidation in the years ahead.

The symptoms of vitamin deprivation, and physiological and pharmacological levels of $\mathrm{B}$ vitamin administration, apparently bear little relationship to one another. To some extent, this may be a reflection upon the present status of knowledge. It is also apparent however that closely similar structural analogues may have vastly different pharmacodynamic properties. Of considerable theoretical and practical interest in this regard is the allergic reaction to thiamine, since the symptoms in hypersensitivity bear little or no relationship to the structure of the causative reagent. The implications of this situation in regard to future plans for high vitamin therapy are of the greatest significance, and it may be anticipated that similar sensitivities will appear for other B vitamins. One cannot but 
wonder whether modern man will be forced by a trick of his genius to desensitize himself against these substances so critical to his existence.

\section{Bibliography}

1. Bonner, J., and Bonner, H., Vitamins and Hormones, 6, 225-75 (1948).

1a. Biskind, M. S., Vitamins and Hormones, 4, 147-85 (1946).

2. Ershoff, B. H., and Hershberg, D., Am. J. Physiol., 145, 16-22 (1945).

3. Drill, V. A., Overman, R., and Leathem, J. H., Endocrinology, 32, 327-33 (1943).

4. Sure, B., and Ford, Z. W., Jr., Endocrinology, 32, 433-6 (1943).

5. Betheil, J. J., Wiebelhaus, V. D., and Lardy, H. A., J. Nutrition, 34, 431-41 (1947).

6. Daft, F. S., and Sebrell, W. H., Pub. Health Repts., 54, 2247-50 (1939).

7. Ralli, E. P., and Graef, I., Endocrinology, 32, 1-12 (1943).

8. Ralli, E. P., and Graef, I., Endocrinology, 37, 252-61 (1945).

9. Supplee, G. C., Bender, R. C., Kahlenberg, O. J., and Babcock, L. C., Endocrinology, 30, 355-64 (1942).

10. Gaunt, R., Liling, M., and Mushett, C. W., Endocrinology, 38, 127-32 (1946).

11. Ralli, E. P., Endocrinology, 39, 225-33 (1946).

12. Spoor, H. J., and Ralli, E. P., Endocrinology, 35, 325-37 (1944).

13. Kochakian, C. D., Vitamins and Hormones, 4, 255-310 (1946).

14. Strom, J. E., and Hathaway, M. C., J. Nutrition, 32, 327-43 (1946).

15. Biskind, M. S., and Shelesnyak, M. C., Endocrinology, 30, 819-20 (1942).

16. Biskind, M. S., and Biskind, G. R., Endocrinology, 31, 109-14 (1942).

17. Sengher, H. O., Taylor, H. C., Jr., Rhoads, C. P., and Unna, K., Endocrinology, 35, 226 (1944).

18. Shipley, R. A., and Gyorgy, P., Proc. Soc. Exptl. Biol. Med., 57, 52-5 (1944).

19. Sengher, H. O., Kensler, C. J., Taylor, H. C., Jr., Rhoads, C. P., and Unna, K., J. Biol. Chem., 154, 79-86 (1944).

20. Cappedge, R. L., Segaloff, A., Sarett, H. P., and Altschul, A. M., J. Biol. Chem., $173,431-2$ (1948).

21. Sweat, M. L., and Samuels, L. T., J. Biol. Chem., 175, 1-5 (1948).

22. Sweat, M. L., and Samuels, L. T., J. Biol. Chcm., 173, 433-1 (1948).

23. Biskind, M. S., Biskind, G. R., and Biskind, L. H., Surg. Gynecol. Obstet., 78, $49-60$ (1944).

24. Segaloff, A., and Segaloff, A., Endocrinology, 34, 346-50 (1944).

25. Drill, V. A., and Pfeiffer, C. A., Endocrinology, 38, 300-7 (1946).

26. Unna, K., Sengher, H. O., Kensler, C. J., Taylor, H. C., Jr., and Rhoads, C. P., Proc. Soc. Exptl. Biol. Med., 254-6 (1944).

27. Jacobs, E. C., Ann. Internal Mled., 28, $792-7$ (1948).

28. Plaut, A., Z. Ges. Expll. Med., 32, 300 (1923).

29. Hertz, R., and Sebrell, WV. H., Science, 100, 293-4 (1944).

30. Hertz, R., Endocrinology, 37, 1-6 (1945).

31. Hertz, R., Proc. Soc. Exptl. Biol. Med., 67, 113-15 (1948).

32. Nutrition Revs, 6, 281-5 (1948).

33. Moore, A. E., Stock, C. C., Sugiura, K., and Rhoads, C. P., Proc. Soc. Exptl. Biol. Med., 70, 396-8 (1949).

34. Hertz, R., Physiol. Revs., 26, 479-94 (1946).

35. Hertz, R., Vitamins and Hormones, 4, 135-46 (1936).

36. Nelson, M. M., von Nouhuys, F., and Evans, H. M., Proc. Soc. Exptl. Biol. Med., 61, 74-5 (1946).

37. Spitzer, R. R., and Phillips, P. H., J. Nutrition, 32, 631-9 (1946).

38. Vinson, L. J., and Cerecedo, L. R., Arch. Biochem., 3, 389-97 (1944). 
39. Richardson, L. R., and Hogan, A. G., Federation Proc., 4, 161 (1945).

40. Muralt, A. v., and Zemp, J., Arch. Ges. Physiol. (Pflugers), 246, 746-7 (1943).

41. Wyss, A., and Wyss, F., Helv. Physiol. et Pharmacol. Acta, 3, C30 (1945).

42. Wyss, A., and Wyss, F., Expericntia, 1, 160-1 (1945).

43. Kaiser, P., Arch. ges. Physiol. (Pflugers), 242, 504-7 (1939).

44. Muralt, A. v., Vitamines and Hormones, 5, 93-118 (1947).

45. Gantt, W. H., and Wintrobe, M., Federation Proc., 4, 22 (1945).

46. Harrell, R. F., J. Nutrition, 31, 283-98 (1946).

47. Robertson, E. C., Tatham, C. M., Walker, N. F., and Weaver, M. R., J. Nutrition, 34, 691-700 (1947).

48. Guetzkow, H., and Brozek, J., Am. J. Psychol., 59, 538 (1946).

49. Henderson, C. R., Wheeler, N. C., Johnson, H. C., Cogswell, R. C., Jr., Berryman, G. H., Ivy, A. C., Friedemann, T. E., and Youmans, J. B., Am. J. Med. Sci., 213, 488-93 (1947).

50. Graves, P. R., Brit. Med. J., 1, 253 (1947).

50a. Molitor, H., and Emerson, G., Vitamins and Hormones, 6, 69-100 (1948).

51. Smith, J. A., Foa, P. P., and Weinstein, H. R., Science, 108, 412 (1948).

52. Shub, P., Am. Rev. Soviet Med., 4, 476 (1947).

53. Molitor, H., Sampson, W. L., E. Merck's Jahresber., 50, 51-64 (1936).

54. Haley, T. J., and Flesher, A. M., Science, 104, 567-8 (1946).

55. Smith, J. A., Foa, P. P., Weinstein, H. R., Ludwig, A. S., and Wertheim, J. M., J. Pharmacol. Exptl. Therap., 93, 294-304 (1948).

56. Hecht, G., and Weese, H., Klin. Wochschr., 16, 414-5 (1936).

57. Stern, E. L., Am. J. Surg., 39, 495 (1938).

58. Dias, M. V., Science, 105, 211-3 (1947).

59. Lancet, 252, 223-4 (1947).

60. Reingold, I. M., and Webb, F. R., J. Am. Med. Assoc., 130, 491-2 (1946).

61. Mills, C. A., J. Am. Med. Assoc., 117, 1500-1 (1941).

62. Schiff, L., J. Am. Med. Assoc., 117, 609 (1941).

63. Stiles, M. H., J. Allergy, 12, 507 (1941).

64. Stiles, M. H., J. Am. Med. Assoc., 117, 954 (1941).

65. Eisenstadt, W. S., Minnesota Med., 25, 861 (1942).

66. Leitner, Z. A., Lancet, $245,474-5$ (1943).

67. Stein, W., and Morgenstein, M., Ann. Internal Med., 20, 826 (1944).

68. Laws, C. L., J. Am. Med. Assoc., 117, 176 (1941).

69. Kulz, F. J., Investigative Dermatol., 5, 135 (1942).

70. Sternberg, C. L., Am. J. Digestive Diseases, 5, 680-1 (1938).

71. Sternberg, C. L., J. Am. Med. Assoc., 116, 2713 (1941).

72. Mills, C. A., J. Am. Med. Assoc., 116, 2101 (1941).

73. Demole, V., Kongressber., XVI Int. Physiol. Kongr., Zurich, 1938, p. 19.

74. Pick, E. P., and Unna, K., J. Pharmacol. Exptl. Therap., 83, 59-70 (1945).

75. Smith, J. A., Science, 106, 622 (1947).

76. Smith, J. A., Foa, P. P., and Weinstein, H. R., Federation Proc., 6, 204 (1948).

77. Smith, J. A., Foa, P. P., Weinstein, H. R., Ludwig, A. S., and Wertheim, J. M., Federation Proc., 7, 116-7 (1948).

78. Smith, J. A., Foa, P. P., Weinstein, H. R., Ludwig, A. S., and Wertheim, J. M., J. Pharmacol. Exptl. Therap., 93, 294-304 (1948).

79. Haley, T. J., Proc. Soc. Exptl. Biol. Med., 68, 153-7 (1948).

S0. Perla, D., Proc. Soc. Exptl. Biol. Med., 37, 169-72 (1937).

81. Zaidi, S. H., Indian Med. Gaz., 82, 181 (1947).

82. Hamovici, H., and Pick, E. P., Proc. Soc. Exptl. Biol. Med., 62, 234-7 (1946).

83. Molitor, H., Federation Proc., 1, 309-15 (1942).

84. Leitner, Z. A., Lancet, 252, 345-6 (1947).

85. Haley, T. J., Lancet, 252, 500 (1947).

86. Smith, D. T., Ruffen, J. M., and Smith, S. G., J. Am. Med. Assoc., 109, 2054-5 (1937). 
87. Sebrell, W. H., and Butler, R. E., J. Am. Med. Assoc., 111, 2286-7 (1938).

88. Spies, T. D., Bean, W. B., and Stone, R. E., J. Am. Med Assoc., 111, 584-92 (1938).

89. Popkin, R. J., Am. Heart J., 18, 697 (1939).

90. Field, H., Jr., and Robinson, W. D., Am. J. Med. Sci., 199, 275-6 (1940).

91. Alport, A. C., and Ghalioungui, P., Arch. Schiffs- u. Tropen-Hyg., 43, 34 (1939).

92. Robertson, D. S., Brit. Med. J., 1, 339 (1941).

93. Bean, W. B., and Spies, T. D., J. Am. Med. Assoc., 114, 439 (1940).

94. Abramson, D. I., and Katzenstein, K. H., Am. J. Med. Sci., 200, 96-102 (1940).

95. Nutrition Revs., 4, 65-7 (1946).

96. Goodman, L., and Gilman, A., "The Pharmacological Basis of Therapeutics," Macmillan, New York, 1941, p. 270.

97. Huidobro, F., and Jordon, J., J. Pharmacol. Exptl. Therap., 86, 49-59 (1946).

98. Valenzuela, F., and Huidobro, F., J. Pharmacol. Exptl. Therap., 92, 1-14 (1948)

99. Spies, T. D., Hightower, P. P., and Hubbard, L. H., J. Am. Med. Assoc., 115, 292-7 (1940).

100. Brazda, F. G., and Coulson, R. A., Proc. Soc. Exptl. Biol. Med., 62, 19-21 (1946).

101. Unna, K., J. Pharmacol. Exptl. Therap., 65, 95-103 (1939).

102. Hunt, R., and Renshaw, R. R., J. Pharmacol. Exptl. Therap., 35, 75-98 (1929).

103. Chen, K. K., Rose, C. L., and Robbins, E. B., Proc. Soc. Exptl. Biol. Med., 38, 241-5 (1938).

104. "The Merck Index," 5th Ed., Merck and Co., Inc., Rahway, N. J., 1940, p. 142.

105. Melass, V. H., Pearson, P. B., and Sherwood, R. M., Proc. Soc. Exptl. Biol. Med., 62, 174-6 (1946).

106. Neuman, M. W., and Hodge, H. C., Proc. Soc. Exptl. Biol. Med., 58, 87-8 (1945).

107. Nutrition Revs., 5, 116-18 (1947).

108. Unna, K., and Antopol, W., Proc. Soc. Exptl. Biol. Med., 43, 116-18 (1940).

109. Hodge, H. C., Proc. Soc. Exptl. Biol. Med., 58, 212-15 (1945).

110. Unna, K., and Molitor, H., Am. J. Physiol., 129, 483-4 (1940).

110a. Weigand, C. G., Eckler, C. R., and Chen, K. K., Proc. Soc. Exptl. Biol. Med., 44, 147-51 (1940).

111. Kuhn, R., and Boulanger, P., Z. physiol. Chem., 241, 233-8 (1936).

112. Kuhn, R., Klin. Wochschr., 17, 222-3 (1938).

113. Demole, V., Z. Vitaminforsch., 7, 138-43 (1938).

114. Harned, B. K., Cunningham, R. W., Smith, H. D., and Clark, M. C., Ann. N. Y. Acad. Sci., 48, 289-98 (1946).

115. Ershoff, B. H., Proc. Soc. Exptl. Biol. Med., 70, 398-401 (1949).

116. Taylor, A., and Carmichael, N., Proc. Soc. Exptl. Biol. Med., 71, 544-5 (1949).

117. Wetzel, N. C., Fargo, W. C., Smith, I. H., and Helikson, J., Science, 110, 651-3 (1949). 


\section{Chapter VIC}

\section{B VITAMIN DEFICIENCY STATES}

\section{General Considerations}

When for some reason the supply of one of the B vitamins to an animal organism is diminished to a level below the nutritional requirement, the $B$ vitamin content of the organism slowly diminishes, as a result of both molecular attrition and excretion. The time required for the shortage to become manifest varies with both of these factors and with the rate at which the nutritional deficit is incurred. Eventually, however, the shortage of vitamin will be felt in the individual metabolizing cells of the body and the cellular function becomes retarded or deranged in some other manner. The various organs and tissues of the body vary in their ability to fend for themselves under such adverse conditions, as evidenced by the fact that deficiencies do not primarily affect all portions of the animal with equal severity, and that death from B vitamin deficiency generally results from the loss of function of some one most susceptible organ or tissue, and seldom from general inanition.

Since all the B vitamins are required by every living cell, and except for nicotinic acid and choline, animal tissues are apparently incapable of B vitamin synthesis, it would appear that all B vitamin deficiencies might result in largely the same symptoms in any given species of animal. This is generally true, except for those instances where a particular tissue has an unusually high requirement for some one vitamin $;^{1}$ in this case the cells of that tissue may suffer disproportionately from the deficit, and the tissue will develop a specific pathology in response. It is therefore believed that the symptoms of the avitaminoses are generally not due to any particular biochemical effect but rather to a diminished function of the cells of the various body tissues, resulting in a pathological condition of the tissue concerned.

From the overall standpoint, the progress of an avitaminosis generally follows a rather set pattern, in which the course of the disease is first manifest in general feelings of poor health, accompanied by a decreased fasting urinary vitamin excretion level. As the symptoms progress there is a gradual decrease in tissue vitamin levels until the point is reached where clinical deficiency signs and symptoms occur and macroscopically and microscopically demonstrable pathology results ${ }^{2}$ (see Figure 17). 
The symptoms associated with B vitamin deficiencies are consequently of a broad and general nature, ${ }^{3}$ affecting all the organs and systems of the animal. Neurological symptoms are manifest psychiatrically and by both central and peripheral neurological malfunction and degeneration. ${ }^{4}$ Cardiovascular and gastrointestinal symptoms are almost invariably found. Epithelial degeneration is characteristic, and hematological and endocrinological involvements are pronounced in deficiencies of at least some of the B vitamins. A more detailed consideration of specific deficiencies follows in a later section, but it is important to emphasize at this point the general nature of the symptomatology, which frequently makes clinical diagnoses of avitaminoses extremely difficult. A consequence of this fact is that much of our knowledge concerning deficiencies has come from "epidemiological" studies, where the disease was deduced from the nature of the diet. Largely as a result of this development, a diagnosis of an avitaminosis in an individual living in a generally well nourished population area is seldom made, and our understanding of individual differences in requirements and of subclinical vitamin deficiencies is a recent but only slowly progressing effort to remedy this. ${ }^{2,5,6}$

B Vitamin deficiencies generally occur as the result of an unbalanced diet, and when a dietary intake is so poor as to bring the level of one $B$ vitamin below a critical level, it rather frequently happens that more than one vitamin is lacking in the diet in adequate amounts. Consequently, many of the classical pictures of deficiency are actually compound deficiencies, and clear-cut clinical cases involving a deficiency of one and only one nutritional factor are seldom seen. ${ }^{7-12}$ This fact is exemplified by the many difficulties experienced by most earlier workers, and in some cases by workers even today, in producing diets for animal experimentation which are lacking in only the factor under study. ${ }^{13}$ As a result of the compound nature of most clinical avitaminoses, there is a great wealth of practical information available on the pathology of naturally occurring deficiency diseases, but much less information on the picture involved in any particular B vitamin deficiency. In considering clinical data bearing upon the medical aspects of deficiency, it is therefore most important to keep in mind the probable complex nature of the deficiency. Moreover, many case reports from nonendemic populations involve deficiencies secondary to some other affliction, in which case the symptomatology is similarly a complex one in which the distribution of symptoms between a number of causative factors is difficult if not impossible to analyze. ${ }^{14,15}$

Finally, there is a growing realization at present that the incipient early stages of deficiency may manifest themselves over extended periods in individuals upon a slightly submarginal B vitamin intake. ${ }^{16}$ The 
extent to which such subclinical deficiencies occur is at present unknown, but a growing body of evidence seems to suggest that even in populations having a high standard of living there may be a high incidence. ${ }^{17}$ The early rapid commercial overexploitation of vitamins which resulted in a universal "vitamin consciousness" has now been followed by a reactionary era of public resistance to the general topic of vitamins, with the result that progress in the study and treatment of subclinical deficiencies may well be extremely difficult for some years.

An extended discussion of $\mathrm{B}$ vitamin deficiencies has not been undertaken in this volume for a number of reasons. Primarily this monograph is concerned with the more biochemical aspects of B vitamins, and a very extensive discussion of clinical material would be out of place. The clinical picture, moreover, is more appropriately one for clinicians and pathologists, and is not within the scope of interest of either the authors or most of the readers of this book. The references found in this section, therefore, are generally the recent publications which add something to the long known overall picture of deficiency. Finally, a great number of excellent clinical treatises exist, which may be turned to by those whose interests in this aspect go beyond the coverage given here. In keeping with the purposes set forth in the preface, therefore, this chapter presents a broad general survey of the essential facts concerning the B avitaminoses, omitting a voluminous mass of supplementary data of interest only to those who would pursue the clinical problems in an extended manner. Only by this course may the vast majority of those for whom this treatise is chiefly intended hope to obtain some picture of the general field of B vitamin deficiencies.

\section{B Vitamin Deficiencies in the Lower Forms of Life}

Very little is known regarding the natural occurrence of $\mathrm{B}$ vitamin deficiencies in organisms other than the vertebrates. In single-celled organisms, the lack of some required B vitamin results in a cessation of growth, although some portions of the metabolic machinery may function for short periods under these conditions. For this reason there is little to be gained from a consideration of the known facts regarding this condition in bacteria and protozoa, aside from certain metabolic derangements that might be discussed, as they shed light upon similar situations in the higher animals. Perhaps the nearest approach to such a state among the green plants occurs when some foreign toxic substance inhibits the activity of a growth factor, as in the case of the lycomarasmine inhibition of a strepogenin activity in the tomato (p. 260). Laboratory-induced deficiencies have been produced in at least one lower animal, the rice moth as previously mentioned (p. 314), it having been rendered 
deficient in each of several B vitamins by means of techniques similar to those used with higher animals. Certain insecticides such as "Gammexane" (hexachlorocyclohexane) may function to produce conditioned deficiencies in some other insects by antagonizing vitamin activity, ${ }^{26}$ and it is interesting to reflect on the possible action of certain "weed killers" by a similar mechanism. In general there seems to be little present interest in this general field of endeavor, however, despite the many intriguing possibilities that exist for improving the growth of both plants and economically important lower animals, and the destruction of undesirable members of both kingdoms by vitamin analogues.

\section{B Vitamin Deficiencies in the Higher Animals}

Introduction. While the roles of thiamine and nicotinic acid in the etiology of beriberi and pellagra have been recognized for only a relatively few years, these two important $B$ avitaminoses have been recognized as human afflictions for centuries. Beriberi was well known and is clearly described in the literature of the 7th century in China, although its spread to Japan was not apparent until a thousand years later, possibly as a result of the introduction of polished rice into the Japanese diet at that time. Descriptions of pellagra apparently do not occur before the early part of the 18th century, although the affliction probably occurred before this time. It is difficult to estimate the full effect of these two diseases during the course of history. In the Philippine Islands as late as 1947 the mortality rate due to beriberi was 132 per 100,000 population, being second only to pulmonary tuberculosis. ${ }^{18}$ In the Japanese navy prior to Takaki's work the incidence was 23 per cent. ${ }^{19}$ Just as beriberi is endemic in the Orient, so pellagra has been in many other areas of the world, including the southern United States. In 1941 in this country there were 1868 deaths from this disease reported. ${ }^{20}$ All these figures constitute only that small part of the total cases that terminated fatally, and reflect only those deaths reported to the authorities and known to be due to this cause. Moreover, the indirect effects of this malnutrition undoubtedly resulted in a far higher mortality than the avitaminosis itself. At present, with the technical ability available to eradicate these and other avitaminoses, the overall picture is changing only slowly, because of the enormous problems involved in practical implementation of the prophylaxis and therapy in most of the populations where these diseases occur. ${ }^{21}$

The discussion of the avitaminoses which follows must of necessity be from the standpoint of a consideration of undesirable afflictions that have no place in a well regulated society. It is indeed difficult to picture disease as serving any good purpose, and yet in some instances we find reports of 
increased resistance to some infectious agents in deficiency states. ${ }^{22-24}$ Of even greater interest, however, is a consideration of the increased muscular activity which results in some cases of nutritional deprivation. It has been shown repeatedly in rats deprived of thiamine or riboflavin (but not carotenol or mineral salts) that in the period before the usual signs of deficiency occur, the running activity of these animals is markedly increased. Wald and Jackson ${ }^{25}$ have pointed out that in a free environment this activity would increase the probability of the animal encountering what it lacks. "In effect it represents a gamble in which the animals' metabolic reserves are staked against the chance of finding its necessities. The possibility of a successful outcome for the individual, however, is not the only point of the reaction. It probably represents also the behavioral basis of mammalian emigration." These authors feel that emigration serves more to relieve nutritional pressures on the home population than to rescue the individual emigré from starvation.

Thiamine Deficiency. There are three known major causes of thiamine deficiency in the higher animals. Of primary importance, the lack of an adequate nutritional source of thiamine results in a number of conditions typified in man by beriberi. Vying with this for importance, the second cause is an increased or inherently high requirement for thiamine in specific individuals, due (1) to physiological conditions previously mentioned in the discussion of vitamin metabolism, (2) to the action of toxic agents or pathological conditions, or (3) to circumstances involving an abnormal dietary. Finally, mention has previously been made of the production of deficiencies of thiamine in nature by the action of thiaminases and other anti-thiamine compounds. Since the last two etiological factors have already been discussed in some detail, and their result is much the same as in the case of primary nutritional deficiency, the discussion will be principally concerned with this latter situation.

Beriberi is endemic in regions where polished rice is a major item in the nutrition, polished rice containing about 6 per cent $(0.02 \mathrm{mg}$. per cent) of the thiamine of brown rice (0.3-0.4 mg. per cent). White wheat flour and other high carbohydrates staples in the diet have also been responsible for beriberi from time to time. ${ }^{27,28}$ Certain other factors, essentially those which increase the thiamine requirement, ${ }^{29}$ tend to predispose toward the condition. The incidence is higher in warm months and under condition of high humidity, much commoner in men than women, most frequent between the ages of 15 and 30 years, and greater in individuals having other forms of disease.

Infant beriberi is also common in many children breast-fed by mothers with beriberi, and is most common in the second month of life. A recently reported acute case in the United States was found in an infant at birth 
that was delivered by a mother with only mild symptoms, however. ${ }^{30}$ In the Philippines in 1947 about sixty-six per cent of the deaths from beriberi were infants, ${ }^{18}$ and it may be that infantile beriberi is commoner in the United States than is generally appreciated..$^{31,32}$

Thiamine deficiency in the Western world is generally a more subtle affliction, for although outbreaks of beriberi have been known here, it is not common. Generally the result of inadequate thiamine in this case is manifest in one of two ways: by cardiac manifestations ${ }^{28}$ or by neurological symptoms. ${ }^{33-35}$ The latter type is commonly referred to as nutritional polyneuritis, and is a frequent result of chronic alcoholism. Similar to this form, but frequently of less severity, is an almost general incidence of very mild beriberi in the Orient, and below this in severity is the widespread existence of so-called "subclinical" thiamine deficiency, ${ }^{6}$ which, as medical experience progresses, becomes more and more clearly defined and less and less "sub"-clinical. The epidemic dropsy seen in India and Africa may well be an atypical form of beriberi. ${ }^{36}$ Another frequently referred to but relatively rare form of thiamine deficiency is the encephalopathy of Wernicke, or Wernicke's disease. ${ }^{37}$ Naturally occurring thiamine deficiency in vertebrates other than man is apparently rare, the "Chastek" paralysis of foxes (p. 292) being perhaps the best known example. In general, the symptoms of thiamine deficiency in most animals follow closely those in human beriberi.

Beriberi is generally referred to as acute or chronic, and "wet" or "dry," the latter terminology being dependent upon the presence or absence of severe edema. The symptoms of the disease are conveniently considered in three categories: cardiovascular, neurological, and edematous. It appears that palpitation of the heart and dyspnea (difficult or labored breathing) are among the earliest symptoms; there is hypertrophy of the heart, and its action is increased. Ellis found that in 125 cases of beriberi that came to autopsy the average heart weight was $379 \mathrm{gm}$ as compared with $255 \mathrm{gm}$ for 204 patients dying from other causes. ${ }^{38}$ The pulse becomes rapid (120-130), and subject to change upon the slightest exertion. Diastolic pressure alone is low. Despite these pronounced changes, there is often little change in the electrocardiogram. Nervous symptoms are both motor and sensory but there is seldom any sensory disturbance apparent that is not located peripherally. The sensory symptoms involve hyperaesthesia (increased sensitivity) commencing in the lower extremities and frequently the finger tips, followed by pain and frequently cramps. In severe cases other areas of the body may be involved, but the motor disturbances are generally limited to the lower extremities.

It is of interest that the sensory disturbances do not reflect the distribution of particular nerves, and that both motor and sensory symptoms 
in many cases are more pronounced on one side of the body. Motor disturbances may vary from a general sense of weakness to absolute paralysis. In severe cases there is extensive edema which is present only to an incipient degree in the milder ones. Fever and vomiting also occur in the advanced stages; there is no respiratory pulsation of the diaphragm, and severe pulmonary edema aggravates the generally terminal cardiac failure. While the disease may run a protracted course throughout most of which dietotherapy provides rapid recovery, a much feared variation which occasionally develops - Shoshin - brings about rapid aggravation of the symptoms and death within a few days. Findings on autopsy are generally in line with those to be expected from the symptoms: edematous effusions, typical cardiac changes, and frequent peripheral nervous degeneration. Histologically demonstrable nerve degeneration has never, however, been proved to be the result of thiamine deficiency. ${ }^{39}$

In the less severe deficiencies more frequently encountered in the Western world, loss of appetite is one of the first symptoms. ${ }^{40}$ In many cases there is an accompanying disturbance in gastric motility and in experimental animals, at least, a more severe gastric disturbance frequently occurs. It has been shown that in thiamine-deficient rats there is a much greater volume of gastric secretion, but there is no change in acidity, peptic power, or total chloride concentration. ${ }^{41}$ There is a high incidence of gastric ulcer in protein-deficient rats on a restricted calorific intake which is independent of thiamine intake; but thiamine-deficient rats have a greater incidence, number, and severity of lesions than rats having adequate thiamine. ${ }^{41,42}$

In the type of deficiency manifest largely by cardiac symptoms, the most frequent disturbances are dyspnea, tachycardia (increased heart rate), and palpitation, and the general picture with its less frequently occurring auxiliary symptoms of edema, pulmonary congestion, and systolic and diastolic murmurs is such as to show little difference from the similar phase in beriberi itself. Weiss and Wilkins state that thiamine deficiency in their experience is a more frequent cause of heart disease than either subacute bacterial endocarditis or congenital heart disease. ${ }^{43}$ In the neuritic type of disturbance, mild cases frequently manifest only absence of knee jerks, plantar dysesthesia (impaired sensitivity of the sole of the foot), and a tenderness of the calf muscles; prompt recovery generally results from thiamine administration. In more severe cases these symptoms become progressively worse, resembling those of beriberi, although in this case there may be a more frequent involvement of mental difficulties (Korsakoff's syndrome?). The course of the disease may be as short as a few weeks, but is frequently much longer, and when acute, seldom responds completely to thiamine therapy. 
Wernicke's disease is marked by rather different symptoms, largely of a cerebral nature. ${ }^{37} \mathrm{~A}$ typical hemorrhagic lesion of the third and fourth ventricles of the brain frequently associated with polyneuritis and alcoholism (and found in thiamine-deficient pigeons) is the principal anatomical sign, while symptoms involve lethargy and excitability, coma, nystagmus (a rapid involuntary oscillation of the eyeball), vomiting, and cardiac and respiratory irregularities. The reasons for the manifestation of thiamine deficiency in different individuals in such a variety of ways are unknown. It has been variously proposed that the critical factor is a matter of degree of deficiency, of compound deficiencies or pathologies, or of varying sensitivities of the several affected systems in different individuals. A large number of other animal species have been rendered thiamine-deficient and their symptomatology studied; to a large extent, the principal symptoms are those found in beriberi.

In the complete absence of thiamine mice die so rapidly that typical symptoms do not develop; but in a more gradual depletion they cease to grow, lose weight, undergo convulsions (especially when spun by the tail), and exhibit brain and muscular lesions and testicular degeneration. ${ }^{44}$

Rats follow a similar course, exhibiting a failure to grow and a loss of weight, followed by typical convulsions and polyneuritis. Assay methods exist for thiamine which depend on either growth, ${ }^{45}$ or the prevention or cure of polyneuritis in rats. ${ }^{46}$ The normal heart rate of rats is from 500 to 530 beats per minute, and on a thiamine-free diet this drops to 250 to 300 per minute after about two weeks. The remission of this bradycardia has also found considerable use as a thiamine assay technique, because of its rapidity, economy of animals, and relative ease and accuracy. ${ }^{47}$

Thiamine deficiency in the fox, ferret, and mink is generally referred to as Chastek paralysis (p. 292). In young foxes, death occurs before other typical symptoms occur; but in older ones, a period of anorexia is followed by general weakness, ataxia (lack of ability to coordinate muscular movements), and spastic paralysis. The paralysis is characterized by extreme board-like stiffness with the heads drawn back, but by no signs of mental effects. Death may occur before the paralysis is complete, or may not ensue until the complete paralysis has been developed for some time. Autopsy reveals cardiac edema and degeneration, and hepatic congestion, hemorrhage, and necrosis. Typical Wernicke lesions are found in the brain of foxes after about 40 days' depletion, whereas they occur in dogs only after prolonged chronic deficiency.

Thiamine deficiency has been studied in a wide variety of other animals, important among which are cats ${ }^{48}$ monkeys ${ }^{49}$ eattle, ${ }^{50}$ and birds. Eijkman and Grijn's original observations on thiamine deficiency and the curative effects of rice polishings were made on chickens. Pigeons were extensively 
used as assay animals in earlier work, both the cure of polyneuritis and the maintenance of weight having been used as the measured criteria in the assays. ${ }^{51}$ Thiamine has also been measured by its effect on the oxygen uptake of avitaminotic pigeon brain tissue, once referred to as the "catatorulin effect." 52 It is of interest in this regard that differences apparently occur in the ability of tissues from different species to take up oxygen. ${ }^{53}$ Deficient pigeons first exhibit a general lack of activity and cease to eat normally. One of the most characteristic symptoms of thiamine deficiency in the pigeon is head retraction, and this symptom has been utilized as a criterion in assay work. The rapid cure by thiamine administration of this grotesque, ruffled-feather, drawn back neck with upside down head appearance (opisthotonus) is one of the most sensational experimental pictures conceivable, particularly in view of the convulsions and death which normally follow rapidly upon this stage. As in rats, pigeons that are thiamine-deficient exhibit a marked bradycardia.

The principal biochemical features involved in thiamine deficiency are an increased blood and urinary pyruvate and lactate and decreased thiamine and cocarboxylase. As previously discussed (p. 255), thiamine retention in loading tests is increased. The pyruvism is obviously due to the lack of ability to convert pyruvate to acetate, a reaction involving thiamine. Since the oxidative metabolism of pyruvate is further blocked in the cyclophorase system at the thiamine-mediated conversion of ketoglutarate to succinate, the decreased oxygen uptake of thiaminedeficient pigeon brain would certainly be expected. Studies on the pyruvate exchange in the heart of thiamine-deficient dogs indicate that the heart normally oxidizes completely the products regularly formed within it from carbohydrate metabolism, and in addition some of the products derived from other organs via the blood, and that in thiamine deficiency this function is so impaired that the increased blood pyruvate may to a large extent originate from the heart. ${ }^{54}$ Since many factors influence blood pyruvate and lactate levels, it seems that pyruvate and lactate determinations are not more sensitive and accurate as diagnostic tests for thiamine deficiency than thiamine levels themselves. ${ }^{55}$

There is some evidence to indicate that episthotonos in the pigeon and some other symptoms in other species are a consequence of lactate accumulation, which may be demonstrated in the liver, heart, muscles, and brain. There is similarly an accumulation of tissue pyruvate but the actual amounts of either acid are so small as to make doubtful their contribution to the toxic effect. Other evidences of a deranged carbohydrate metabolism follow as a sequence to the blocked pyruvate oxidation. Pigeons exhibit a hyperglycemia and depletion of liver glycogen, and rats show abnormally high glucose tolerance curves (reduced glucose 
tolerance). In human beriberi, too, some degree of hyperglycemia is common. It is noteworthy in this regard that both the islets of Langerhans and the adrenal medulla are frequently hypertrophied in beriberi. It has also been observed that there is a decrease in gastric acidity in beriberi, frequently progressing to achlorhydria, and Goodhart and Sinclair ${ }^{56}$ have demonstrated a definite correlation between gastric acidity and blood cocarboxylase. Mention should be made of the evidence that milk from women suffering from beriberi contains a toxic factor, as do the blood, urine and tissues of thiamine-deficient experimental animals, and that such toxic factors may be in part responsible for some of the deficiency symptoms. ${ }^{57}$ Methylglyoxal has been suggested in this regard, although there is insufficient evidence available to evaluate properly the numerous papers dealing with the presence of this substance in thiamine-deficient animals.

Horwitt ${ }^{58}$ has recently given considerable attention to the study of blood lactate and pyruvate in mild thiamine deficiency and has concluded that the basal levels are of little diagnostic value, since at this stage the organism can still retain a blood equilibrium. Only after pronounced clinical signs of deficiency become apparent do the blood levels change, and even then exceptions occur. During mild deficiency, lactate and pyruvate determination following glucose administration are significant if correlated with the blood glucose. It was further found in this study that mild exereise after glucose administration made it readily possible to detect distinct characteristies in mild thiamine deficiency at an early stage in its development. A formula termed the "Index of Carbohydrate Metabolism" or "(CI)" was developed to relate the amounts of blood lactate $(L)$, pyruvate $(P)$, and glucose $(G)$ in milligrams per cent, and a change in this index seemed to be highly indicative of thiamine restriction.

$$
(\mathrm{CI})=\frac{\left(L-\frac{G}{10}\right)+\left(15 P-\frac{G}{10}\right)}{2}
$$

Patients receiving $200 \mu \mathrm{g}$ of thiamine per day showed increases of this index to a pathological level within 10 weeks or less, and clinical signs of deficiency followed within one to four months after the first significant rise. This study merits particular attention from the student of thiamine nutrition because of its extended nature (three years), its coordinated approach involving biochemical, clinical, neurological, and psychological investigations, its carefully controlled nature, and the tremendous emphasis placed on the study and reporting of individual data rather than average data. While it was primarily, concerned with the 
problems of thiamine and riboflavin nutrition in gerontology and mental disease, it is one of the major contributions to the literature on the subject of vitamin deficiency, and may well serve as a model for further studies of this nature, much needed with regard to nonclinical individuals and cases involving the remainder of the $\mathrm{B}$ vitamin group.
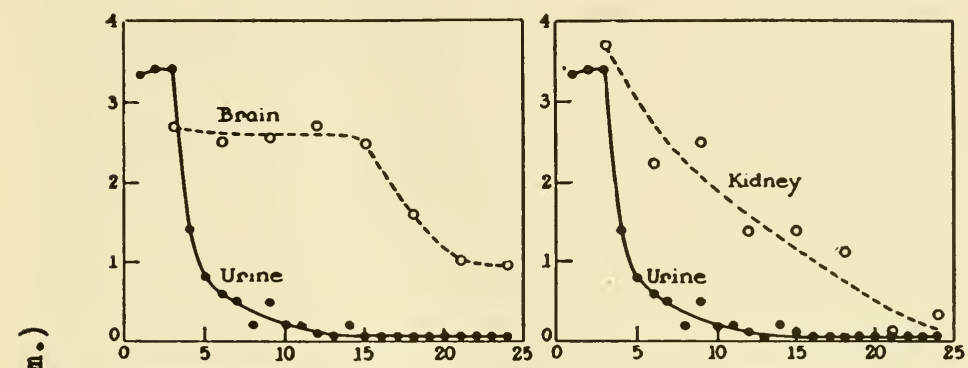

造
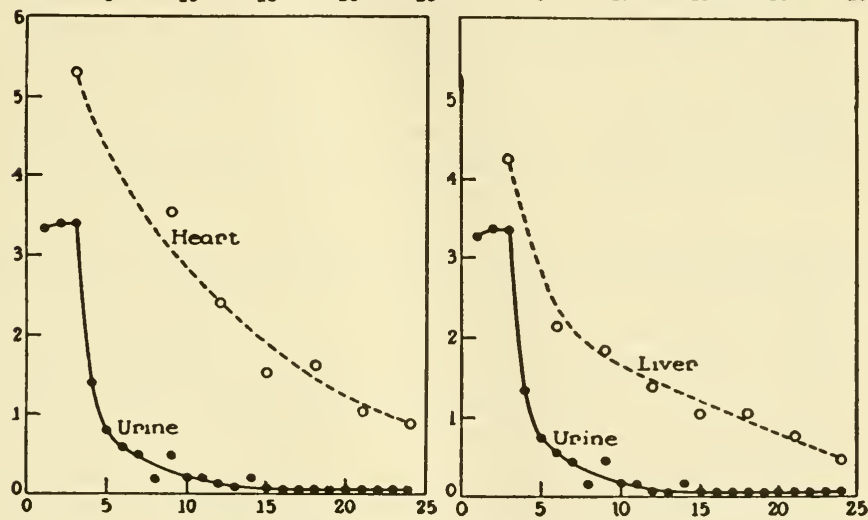

Days on Thiamine Deficient Diet

FIGURE 17. Decline in urinary and tissue levels of thiamine during the course of thiamine depletion in the rat.

Previous mention has been made of the fact that in thiamine restriction, the rapid drop in urinary thiamine is followed by a much more gradual decline in tissue levels. Salsedo et al. ${ }^{59}$ have studied these changes in thiamine-deficient rats, and their results are summarized in Figure 17. These workers have suggested that the drop in tissue levels coinciding 
with the time at which urinary thiamine disappears may provide a means of assessing the thiamine requirement from urinary studies. It is further apparent that brain tissue seems particularly able to preserve its thiamine content in periods of thiamine deficiency.

Riboflavin Deficiency. Riboflavin deficiency was first recognized in man about 1935, although experimental deficiencies in a number of animals had been produced considerably before this time. The vast majority of cases of ariboflavinosis seem to be associated with other deficiencies, characteristically pellagra, but a sufficient number of cases of noncomplicated deficiency have now been observed to indicate that the affliction is a rather common and distinct clinical entity. ${ }^{60}$ Whereas there is little to indicate its extent, it seems apparent that ariboflavinosis is relatively common in the southeastern United States, in the Orient, and among native populations in the West Indies. The major cause of riboflavin deficiency so far as is known is an inadequate intake, and corn and polished rice are low enough in riboflavin to insure a deficiency if some other high riboflavin source is not incorporated into the diet.

The symptoms of ariboflavinosis in man principally involve the mouth, tongue, nose, and eyes, although general body weakness and dermatitis may also occur. ${ }^{61}$ Primarily there is an inflammation of the tip and margin of the tongue (glossitis), and lesions at the muco-cutaneous juncture of the mouth with the development of painful fissures. ${ }^{62}$ There is an increased redness of the lips, but a pallor to the mucosa, aind a scaly, greasy desquamation about the nose and ears. A nasolabial seborrhea and seborrhoic and follicular keratosis of the face is common. Undoubtedly, however, the glossitis is the most prominent symptom, the papillae being large and flattened, and the tongue itself being a reddish purple color, appearing clean, but frequently manifesting fissures. Of equal diagnostic importance, however, is the marked corneal vascularization and attendant ocular symptoms involving circum-corneal injection, photophobia, burning of the eyes, corneal opacity, and pigmentation of the iris. ${ }^{63,64}$ There is little doubt that the eye is one of the most sensitive organs of the body to riboflavin deficiency. Out of 47 cases of ariboflavinosis studied by Sydenstricker et al. ${ }^{65}$ six had cataract.

Ariboflavinosis is identical with an affliction of children long known in the southern United States as "perleche." The symptoms of ariboflavinosis generally respond promptly to riboflavin administration, but only in patients with teeth does the cheilosis disappear. Ariboflavinosis in edentulous patients presents certain unique problems, therefore, with regard to successful therapy. ${ }^{66}$ Ariboflavinosis is common in many parts of Africa, and in Nigeria it is said to be present in 16 per cent of the adult population. A syndrome also seen in Africa in which about 50 per 
cent of the cases are under two years of age is known as Kwashiorkor, or "infantile pellagra," and is thought to be a compound deficiency in which ariboflavinosis is predominant. ${ }^{67,68} \mathrm{~A}$ recent case of ariboflavinosis in India was characterized by vulval pathology, a symptom not generally seen in cases reported from other areas, ${ }^{69}$ and still other atypical forms may arise among different population groups.

Riboflavin deficiency was first studied in the rat (in 1926) and termed "rat pellagra," and the curative agent was termed the "pellagra-preventive factor," although it is now known that the symptoms studied were those of ariboflavinosis, and that the factor involved was riboflavin and not the main curative agent for human pellagra. The first symptoms in the rat are a cessation of growth, and this fact has long been used as the basis for a riboflavin assay technique. Shortly thereafter there is a general loss of fur and a characteristic symmetrical dermatitis of the ears, upper chest, and extremities. The tail becomes dry and scabby, the eyeballs sunken and lids swollen; corneal vascularization occurs as in man, and is among the most striking characteristics. Cataract has been reported by some workers, but there is much disagreement as to its general occurrence. ${ }^{70}$ Granulocytopenia and anemia also occur in severe cases ${ }^{71}$ of ariboflavinosis in rats, dogs, swine, and monkeys, although in some cases folic acid deficiency seems to be involved in an unclear manner with this symptom. Swine are said to develop cataracts when suffering from ariboflavinosis, but there is apparently no corneal vascularization.

In young chicks on a prolonged mild deficiency there is a highly characteristic "curled toe paralysis." On a completely deficient diet chicks exhibit an acute paralysis and dystonia, rapidly followed by death. Dermatitis is rare but does occur in turkeys. Eggs from hens on a low riboflavin diet fail to hatch, although injection of riboflavin into the egg on the first day of incubation remedies this defect. ${ }^{2}$

Riboflavin deficiency has been extensively studied in dogs. These animals rapidly become weak, reluctant to walk, and exhibit an ataxia. Vomiting and occasional convulsion also occur. After some time in this unhealthy stage the animals develop a diarrhea, followed suddenly by collapse, coma, and death within a few hours. This rather dramatic rapid terminal stage is not seen in rats, and only in chicks on a completely deficient diet..$^{73}$

The biochemical changes occurring in ariboflavinosis have not been well studied, although a number of pertinent observations have been made. There is of course a diminished level of riboflavin and its adenine dinucleotide in the body and urine under deficiency conditions. The xanthine ovidase (p. 148) activity of deficient rat liver, as measured by oxygen consumption rates, is very low. ${ }^{74}$ Liver slices from deficient rats 
have a decreased D-amino acid oxidase activity, which is increaed by the addition of riboflavin dinucleotide, indicating that the apoenzyme is present and only the coenzyme deficient. ${ }^{75}$ In ariboflavinosis there are, moreover, some signs of a water metabolism derangement similar to those that have been observed in pantothenic acid deficiency (p. 424). ${ }^{76}$ In hens and dogs that have died of ariboflavinosis there is an increased liver fat content, and a high fat diet seems to aggravate the symptoms of riboflavin deficiency in rats.

It has been suggested that the oxidative role played by riboflavin in the normally avascular cornea is counteracted in deficiency by supply of the needed oxygenation by vascularization. It has been reported that in riboflavin deficiency there is an increased magnesium requirement. ${ }^{77}$ Since riboflavin and thiamine have been reported to have limited sparing actions on each other and since magnesium is involved as a cofactor with thiamine, it is possible that the magnesium effect is due to increased thiamine activity. B vitamins have been reported to reverse atabrine toxicity for $\mathrm{rats}^{78}$ and riboflavin may be active in this regard, as it is in reversing atabrine inhibition in the tryptophanase reaction. ${ }^{79}$ Generally, however, it is apparent that there is little true understanding of the biochemical picture in ariboflavinosis.

Nicotinic Acid. Nicotinic acid deficiency occurs most frequently in the form of pellagra, although classical pellagra is not purely a nicotinic acid deficiency. This disease is common throughout the world, frequently compounded with beriberi and other avitaminoses, and at one time or another has been either endemic or epidemic in nearly every area excepting northern Europe. It is endemic in the southeastern United States, ${ }^{80}$ in South Africa, ${ }^{9,81}$ and in some other tropical and semitropical areas of the world. It is considered in many respects to be more dangerous than beriberi, and is certainly the major clinical avitaminosis encountered in the United States. It is considered to be an economic disease, as Drummond says, a matter of pounds, shillings, and pence. ${ }^{82}$

Most generally pellagra is associated with populations in which corn is a staple in the diet, just as polished rice is in beriberi. As previously mentioned (p. 279), this appears to be due to the low nicotinic acid and tryptophan content of the corn, coupled with the possible presence of a pellagragenic factor in the corn. Pellagra and other somewhat atypical forms of niacin deficiency also occur, however, under other circumstances when the dietary levels of niacin and tryptophan are low. ${ }^{83}$

As with thiamine, nicotinic acid deficiency is a frequent result of chronic alcoholism and has been observed secondary to drug addiction. ${ }^{84}$ It is readily apparent, however, that there are many factors other than nutritional ones which are secondarily involved in the etiology of pel- 
lagra. Sunlight in some manner and to an unknown extent plays an important role in the disease. ${ }^{85}$ With the exception of the characteristic vaginal and scrotal lesions, the early skin lesions generally follow exposed parts of the body, and it has been suggested that some photodynamic action at the site of the chromatophores may promote this pathological characteristic. Strong sunlight is also known to cause relapse in patients in remission. It has further been suggested that the characteristic outbreak of the disease in the spring may involve sunlight, although it seems more likely that this is due to the accumulated effects of the nutritional deficiency acquired during the winter months.

A number of factors combine to make pellagra particularly pernicious. Pellagragenic diets are generally deficient in thiamine, riboflavin, and pantothenic acid, and perhaps still other factors, and the multiple nature of the avitaminosis has a weakening effect on the body which leaves it poorly able to withstand the most pronounced deficiency of niacin. Disturbed gastrointestinal function also plays a major role in the etiology of niacin deficiency. Dysentery, colitis, intestinal parasitism, and surgery are all known to be strong predisposing factors toward pellagra, as they are toward beriberi. It would seem that in these deficiencies there is a strong probability of the creation of a vicious cycle, the deficiency leading to further gastrointestinal disturbances which in turn encourage the avitaminosis. The exact manner in which such organic afflictions promote deficiency is unknown, although faulty digestion and absorption seem likely. It is of considerable interest that gastric preparations have been successfully used for many years in the treatment of pellagra, and there is at least some evidence to indicate that their efficacy may not be due to the niacin content. ${ }^{86}$ (Certain liver fractions which have a low niacin content are similarly quite active).

In its essence, as Goldberger pointed out, the major causes of pellagra seem to involve first dietary factors, and secondly factors that prevent the normal utilization of the diet by the individual. ${ }^{87}$ The disease is common in children and persons of all ages and racial stocks, being most pronounced in adult married women. Finally, when all the known etiological factors are taken into consideration, it is still difficult to explain the fact that in both man and experimental animals and without any dietary change, there is a frequent spontaneous remission of niacin deficiency which, however, is seldom long-lasting.

As previously stated, pellagra characteristically develops in the early months of the year. In some acute cases the symptoms become progressively worse and death ensues. In the majority, however, the symptoms develop for only a few months and then disappear for the most part and in some cases never return. Generally there is an annual recurrence 
which continues until the progressively weakened condition of the pellagrin results in death, most often in about five years. Nicotinic acid therapy is highly and rapidly effective for all the symptoms that are clearly due to the niacin deficiency, but since pellagra is a multiple deficiency, complete cure is obtained only by multiple vitamin therapy. In advanced cases where irreversible pathological changes have occurred, of course, vitamin therapy has only limited efficacy.

The cardinal symptoms of pellagra are often referred to as the three "D's," dermatitis, diarrhea, and dementia. Gastric disturbances, anorexia, headache, and loss of weight and strength are among the earliest symptoms. Diarrhea may occur quite early, and frequently becomes one of the severest symptoms in later stages. Para-sprue, a condition that is widespread in India and similar in many respects to sprue (p. 417), is undoubtedly a compound deficiency, principally involving niacin deficiency, and distinguishable from true sprue by the nonfatty nature of the stools. ${ }^{88}$ In pellagra, redness of the tip and margin of the tongue ${ }^{89}$ and lining of the mouth, and a characteristic gingivitis and gastric irritation are almost invariable; at the height of the disease the tongue becomes swollen and cracked, and peels and appears cyanotic in many cases. $^{90}$ Nausea is frequent, and along with this and the diarrhea, 40 per cent of the cases develop an achlorhydria. The dermatitis is bilaterally symmetrical in most but not all cases, ${ }^{85}$ clearly defined, and generally restricted to a necklace or gauntlet pattern about the neck, and to the dorsal surfaces of the hands and forearms, although it may occur on other areas exposed to sunlight (the face and lower legs) and on areas subject to chafing. Frequent characteristic scrotal lesions are also believed to be due to niacin deficiency, although this point seems uncertain..$^{91,92}$ The dermatitis generally originates in the form of deep red areas that gradually become brown and coalesce and later become thickened and scaly.

Unlike the symptoms in beriberi or ariboflavinosis, there is a high incidence of mental symptoms in pellagra, and these may range from mild psychoneurosis and insomnia to stupor or mania. The existence of acute mental disorders in this case, and their rapid cure by niacin therapy merits special consideration from the standpoint of its bearing upon the nature of mental disease in general. The longstanding and widespread concept of a purely psychological etiology in the insanities has resulted in little general advance in the cure of these diseases. Very recently a few workers have attempted to discover physiological bases for mental disturbances, but in the face of widespread opposition from groups that hold the "psychic" hypothesis. A thorough consideration of the dramatic cure of the mania of pellagra by niacin should do much to promote further in- 
vestigations of mental disease from the nutritional and metabolic standpoints, and to remove the laissez faire policy now applying to the natural sciences in the study of the mental processes.

A variety of nervous symptoms also occur in pellagra, but it is uncertain to what extent these are due to an accompanying thiamine deficiency. ${ }^{93,}{ }^{94}$ While there are apparently no marked cardiovascular changes in pellagra, a macrocytic hyperchromic anemia is frequent. ${ }^{95}$ Atypical forms of pellagra are frequently encountered as a result of compound deficiencies. In addition, most workers feel that subclinical pellagra is common in the United States, and to some considerable degree even in the North. Finally, recent years have brought forth an increasing number of reports of cases of nervous and mental disease that have responded markedly to niacin therapy. To a lesser extent there have been obtained similar remissions of other of the characteristic symptoms of pellagra that occasionally appear singly in individuals not exhibiting the general pattern of the avitaminosis.

Post-mortem examination of pellagrins shows little to indicate the cause of the disease other than the skin lesions. There is seldom any change in the stomach, but the colon is thickened, red and typically stippled with cystic lesions. Nervous lesions are common, but generally believed to be due to deficiencies of other factors. The heart appears quite normal, but the liver, while normal in size, is yellowish gray and mottled, and characterized by fatty degeneration and fibrosis. From the overall standpoint, it is apparent that the marked differences between the symptomatology of niacin deficiency and that of thiamine and riboflavin deficiency do not in any way reflect obvious relationships of these vitamins to their coenzyme functions. Indeed, the mental symptoms associated with pellagra might far more logically result from beriberi, since thiamine occupies a special place in nerve function (p. 384); and since niacin and riboflavin function metabolically in an intricate fashion, it is strange indeed that there is not a greater similarity between the symptoms of their deficiencies.

Until recent years, the mortality from pellagra varied, but was generally above 66 per cent of the cases. Recently, however, due to improved dietotherapy and hospital methods, and the specific use of niacin, this has dropped to below 5 per cent. Niacin, in doses of from 50 to $1,000 \mathrm{mg}$ daily when administered orally (or intravenously in acute cases), generally achieves a rapid remission of the symptoms of niacin deficiency, the redness of the tongue disappearing in a day and the lingual ulcers in several days, nausea and vomiting ceasing immediately, the mental symptoms vanishing within a week, and the dermatitis regardless of its severity eventually disappearing completely. 
In areas where pellagra is endemic, dogs frequently develop a canine counterpart of the disease known as "blacktongue." In general the symptoms follow closely those found in humans. The mouth is typically dark red due to necrosis, which in turn causes a drooling appearance and a fetid odor. There is a generalized gastrointestinal disturbance, and the scrotal lesions seen in human pellagra are generally found in blacktongue in dogs. A macrocytic anemia is frequently found, ${ }^{96}$ and almost invariably nervous degeneration occurs. The same disease has been produced in dogs in experimental studies by the use of niacin-deficient diets. Because rats do not readily develop a niacin deficiency, dogs have been of great value in the experimental study of niacin deficiency. Recent work has indicated, however, that the frequent anemia seen in dogs with blacktongue may primarily be a simultaneous folic acid deficiency. ${ }^{97}, 98$ That the etiology of pellagra and blacktongue is not fully understood is readily apparent from the fact that the injection of saline alone is frequently curative for blacktongue. ${ }^{99}$ In any case it is apparent that many of the symptoms of blacktongue induced in dogs on certain diets are those of folic acid deficiency, and that this condition in the dog is frequently more similar to sprue than pellagra, the balance of the symptoms in blacktongue depending largely on the diet by which the deficiency is induced. ${ }^{100}$

Pig pellagra also occurs naturally, although with much less frequency, and pigs have been used somewhat in the study of niacin deficiency 101 because of the possibility of producing this condition experimentally. In this case there is no glossitis or stomatitis; the symptoms involve anorexia, slowed growth, a scurfy skin, colitis, and a diarrhea which is generally followed within a month by death. The condition responds promptly to $50 \mathrm{mg}$ /day of niacin. Monkeys fed on a niacin-deficient diet similarly become ill and develop anorexia, diarrhea, and dermatitis, and are cured by doses of 5 to $25 \mathrm{mg}$ of niacin. As previously mentioned, rats do not normally develop a niacin deficiency on niacin deficient diets, nor do lambs.

Biochemical changes in niacin deficiency are not well studied. A change in the urine that frequently occurs in pellagra and has been used in laboratory diagnosis involves an increase in pigments which have been variously identified as coproporphyrins I and III or urorosein and indirubin. ${ }^{102}$ It is generally presumed that these pigments are formed as a result of impaired liver function, and may be responsible when deposited in the skin for the characteristic photosensitive effects in pellagra. It seems well established now, however, that this diagnostic criterion is of little or no value, since a similar result occurs in a number of unrelated conditions, and since many pellagrins do not give a positive reaction. ${ }^{\mathbf{1 0 3}}$ 
Significantly, however, the original work upon which this test was based was done upon a group of pellagrins most of whom suffered the disease as a consequence of alcoholism, and pellagra secondary to alcoholism may differ in some respects from other forms. As a result of the extreme nausea and diarrhea, a hyperproteinemia may develop in advanced cases and a disturbance of acid-base equilibrium may also occur. The decrease in urinary levels (and blood and tissue levels in extreme cases) of niacin and its metabolites has already been mentioned in earlier chapters; this constitutes one of the most marked biochemical changes occurring during early or mild deficiency. ${ }^{104}$ It has also been reported that pellagrins are extremely sensitive to insulin and are refractory to adrenalin, but these factors have not as yet received adequate study. ${ }^{105}, 106$

Folic Acid and Vitamin $B_{12}$ Deficiency. ${ }^{107-111}$ A large number of different clinical conditions result in the production of anemias, and in recent years it has become readily apparent that many of these have a definite nutritional deficiency involved in their etiology. Of these a rather significant number of anemias have been found to respond to a greater or lesser degree to the administration of the two most recently identified members of the B group of vitamins, folic acid and vitamin $B_{12}$. While it is by no means certain that these anemias are caused by a nutritional deficiency, it is apparent that for one reason or another the bodies of patients so afflicted do not receive an adequate supply of these factors. It is undoubtedly true that a deficiency of any of the B vitamins would ultimately result in anemia, ${ }^{112,113}$ and anemias are associated with deficiencies of a number of them; but the acute macrocytic anemias that respond to the two vitamins here discussed are so marked and well recognized as to leave little doubt that these factors have a particularly important function in normal erythrocyte physiology. The conditions that respond to these factors are almost without exception marked by severe anemia, but this is generally only one of a group of symptoms associated with each clinical entity to be considered here. Thus there is no doubt that deficiencies of these vitamins are manifest throughout the body, and the weight given the hematological aspect should not distract attention from the other symptoms of these avitaminoses.

A unique situation exists with regard to these avitaminoses: a number of factors other than low dietary levels contribute to the majority of the recognized cases, and these factors bring about a number of different, prevalent, clinically identifiable forms of the deficiency. The situation is even more unusual in that two different members of the B group of vitamins are generally effective in curing most of the deficiency conditions, and that a naturally occurring pterin and pyrimidine are also known which are effective, though to a lesser degree. This multiplicity of 
curative factors is undoubtedly due to the fact that the substances involved are closely related to one another metabolically, and various considerations may eventually cause the folic acid group and vitamin $\mathrm{B}_{12}$ to be considered as a functional unity.

It is important to realize that the afflictions considered here have been recognized for a great many years; they are widespread, and in terms of their overall effects they compete with beriberi and pellagra as avitaminoses: it is for that reason that their discussion occurs at this point. As a consequence, the consideration of these conditions upon which numerous volumes have been written must be extremely abbreviated. Further, the discoveries of the folic acid group of substances and vitamin $\mathrm{B}_{12}$ and their remarkable clinical efficacy have been so recent that there is at the time of writing a considerable degree of uncertainty and even confusion regarding the true picture of their function. The metabolic function of these vitamins, discussed elsewhere (p. 198), is at present only poorly understood. ${ }^{113 a}$ For these reasons it seems best to present in barest outline form the facts as they now stand, realizing full well that in these paragraphs more than any other, the overall concept may change drastically within a few months.

The following clinical conditions have been found to respond in some degree to one or both of the vitamins here discussed:

(a) Addisonian pernicious anemia.

(b) sprue

(c) nutritional macrocytic anemia

(d) macrocytic anemias of pregnancy

(e) macrocytic anemias (megaloblastic) of infancy

(f) macrocytic anemias following surgery, alcoholism, cancer, and infectious agents.

In addition, thymine (5-methyluracil) in massive doses has been found to be effective in a number of these conditions.<smiles>Cc1c[nH]c(=O)[nH]c1=O</smiles>

thymine

It seems most convenient to discuss the effects of these vitamins and thymine in relation to each of the conditions mentioned above, and in the approximate order given. 
Addisonian pernicious anemia is a spontaneously occurring macrocytic anemia further characterized by a permanent, histamine-refractory achlorhydria, hyperplastic megaloblastic bone marrow, glossitis, and frequent neurological manifestations ranging from peripheral neuritis to degenerative lesions of the spinal cord. It is frequently accompanied by depigmentation of the hair, increased urinary urobilin, and increased plasma iron and bilirubin. There are generally high blood and urinary levels of phenolic compounds. ${ }^{114}$ Tyrosine seems to increase the efficacy of some liver extracts, and it is readily apparent that there is a close interrelationship between folic acid, ascorbic acid, and tyrosine metabolism;115, 116 but the precise manner in which folic acid influences the ascorbic acid-controlled oxidation of tyrosine is at present obscure. Impaired absorption of tyrosine has been made the basis of a "tolerance test" in which, after a 4 -gm oral dose, the blood levels reach a peak only after three hours, as contrasted with one hour in healthy persons. Pernicious anemia patients differ from those with cirrhosis in that the blood tyrosive level is markedly elevated. ${ }^{117}, 118$ During remissions there is occasionally a transitory edema of unexplained etiology, ${ }^{119}$ and a few pernicious anemia patients exhibit cardiac symptoms. ${ }^{120}$

The cause of pernicious anemia is unknown, but it has generally been considered to be an acquired metabolic defect that results in an impairment of erythrocyte maturation. Liver has long been known to sustain patients with the disease, but its continued consumption is necessary, and highly potent concentrates of the antipernicious anemia factor have been used parenterally for some time. As previously mentioned, achlorhydria is an invariable accompaniment of this disease, and it has been found that the gastric juice from normal persons when incubated with beef steak produces a curative substance, but the gastric juice from patients does not. The active principle is produced by the mucosa of the pyloric and cardiac regions of the stomach, and the commencement of the duodenum, and desiccated defatted hog stomach may be used therapeutically as an alternative to liver. Ternberg and Eakin have recently shown ${ }^{120 a}$ that the active principle in gastric juice, long designated as the "intrinsic factor," is in reality a protein which combines with vitamin $\mathrm{B}_{12}$ (extrinsic factor) to form a complex which is resistant to the destructive changes wrought upon vitamin $\mathrm{B}_{12}$ itself by the digestive processes (p. 342). In pernicious anemia, the intrinsic factor is absent, and consequently a "conditioned" vitamin $\mathrm{B}_{12}$ deficiency results even in the presence of an adequate nutritional supply, since the vitamin consumed is destroyed before it can be absorbed. ${ }^{121-123}$ It has been suggested that these interrelationships favor the substitution of the term erythrotin for vitamin $\mathrm{B}_{12}$, apoerythein for intrinsic factor, and erythein for the complex. 
Thymine, folic acid, and vitamin $B_{12}$ are all effective in bringing about a remission of the symptoms of pernicious anemia, but the relative effective doses of these substances varies a millionfold. From 5 to 10 grams of thymine daily, or approximately $10-\mathrm{mg}$ daily of folic acid are required, while an initial intramuscular injection of $15 \mu \mathrm{g}$ of vitamin $\mathrm{B}_{12}$ is effective for a number of days. Spies et al. ${ }^{124}$ have compared the effects of these three substances in a case of pernicious anemia that was admitted to the hospital for treatment three successive times. Their graphs are reproduced

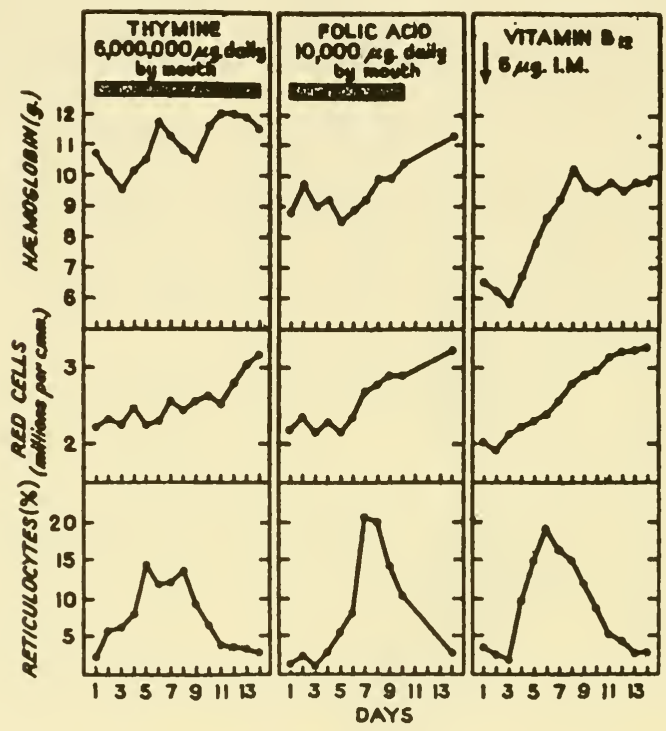

Figure 18. Hematological response in a case of pernicious anemia.

in Figure 18 and are of particular value because of the analogous data obtained from cases of tropical sprue and nutritional macrocytic anemia shown in Figures 19 and 20.

The evidence now available suggests that pteroyl heptaglutamate is not appreciably active in pernicious anemia, ${ }^{125}, 126$ and that none of the folic acid vitamins are effective in curing all the symptoms. Indeed, neither folic acid nor thymine prevents the development of subacute combined degeneration of the spinal cord, nor do they retard it once it is initiated. ${ }^{127}$ Folic acid in some eases seems to aggravate the neurological symptoms, although this effect may possibly be due to impurities in 
the preparations used ${ }^{128}$ (p. 295). In this regard, however, there is good evidence to indicate that folic acid increases cholinesterase formation in the body, and folic acid is apparently quite effective in counteracting the hyperchromic anemia produced by choline injection. It therefore seems well to reserve judgment in this regard until the functions of folic acid in neural physiology are more clearly elucidated. ${ }^{129,130}$ Recent indications are that vitamin $B_{12}$ does improve the neurological status of at least most patients with subacute combined degeneration of the spinal cord. ${ }^{131,132}$

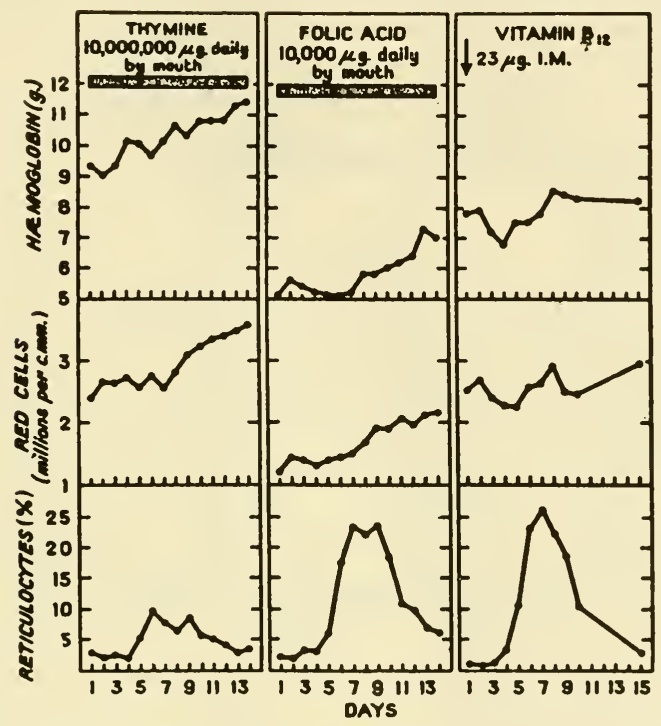

Figure 19. Hematological response in a case of tropical sprue.

The achlorhydria of pernicious anemia does not respond to treatment by any known means.

Sprue is a clinical condition that has been well recognized for some two centuries and is characterized by a macrocytic anemia with moderate leucopenia and bone marrow hyperplasia, steatorrhea ${ }^{133}$ (high fat in the feces), glossitis, diarrhea, skin pigmentation, loss of body weight, and impaired absorption from the gastrointestinal tract. It differs from parasprue, which is more basically a niacin deficiency and in which the diarrhea is of the nonfatty type. ${ }^{88}$ It is generally felt to be a deficiency disease resulting from impaired absorption of the antipernicious anemia 
factor. There is generally an accompanying deficiency of the fat-soluble vitamins due to faulty absorption, ${ }^{134,135}$ together with nightblindness, hypocalcemia and hypoprothrombinemia. ${ }^{136}$ It differs from pernicious anemia in the skin pigmentation, frequent presence of the intrinsic factor, frequent occurrence before puberty, general presence of gastric acid, absorption defects, and frequent association with poor diets. There is a typically flat oral glucose curve, but a normal intravenous one, and no rise in blood phosphate after oral glucose. Impaired glucose absorption in this case has been interpreted as due to an impaired phosphorylation mechanism in the intestinal wall, fructose absorption being apparently normal. ${ }^{137}$

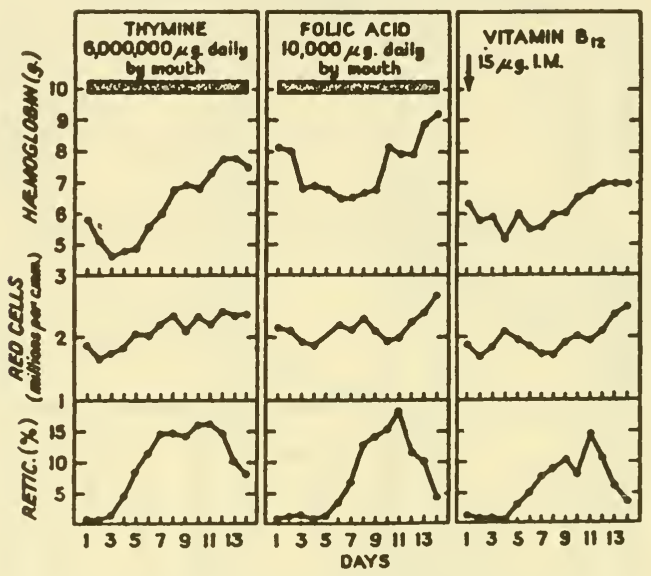

Figure 20. Hematological response in a case of nutritional macrocytic anemia.

Frequent classification into tropical and nontropical varieties seems justified only on the basis of its more frequent occurrence and severer forms in the tropics. Sprue must be regarded as a multiple deficiency, initiated perhaps by infectious conditions or general malnutrition, and propelled by the diarrhea and other factors that make absorption inefficient and create a vicious cycle. The symptoms generally respond well to liver therapy and improved diet. The response of sprue to thymine, folic acid, and vitamin $\mathrm{B}_{12}$, is almost identical with that of pernicious anemia, and similar data from the Spies et al. study previously mentioned ${ }^{124}$ is shown for a case of tropical sprue in Figure 19. In separate studies, ${ }^{138}$ it has been shown that massive doses of thymine are followed by at least a year of freedom from sprue and its symptoms. Pteroyldiglutamic and 
triglutamic acids are also both effective in the treatment of sprue. ${ }^{139,140}$ Some cases of sprue, like those of pernicious anemia, are apparently refractory to folic acid however. ${ }^{141}$

Nutritional macrocytic anemia is an ill-defined condition that resembles both sprue and pernicious anemia, and in many cases may actually represent stages of one or the other of these diseases. It frequently differs from sprue in that there is a normal oral glucose curve, and from pernicious anemia in the frequent presence of gastric acid and intrinsic factor. It is most generally a compound deficiency and is often associated with more marked manifestations of other avitaminoses, such as pellagra. Spies et al. ${ }^{124}$ have presented evidence to show a similar efficacy in this case of thymine, folic acid, and vitamin $\mathrm{B}_{12}$ to that in sprue and pernicious anemia, as shown in Figure 20.

The macrocytic anemia of pregnancy is also an ill-defined condition similar to those already discussed, but characterized by its temporary nature. It is generally thought to be a manifestation of the heavy demands upon the mother made by the foetus, since recovery generally follows delivery, and continuous therapy is seldom necessary. This condition responds to folic acid, ${ }^{142}$ thymine, ${ }^{127}$ or liver therapy, vitamin $\mathrm{B}_{12}$ being as yet unreported upon. It has recently been reported, however, that this condition, which is cured by crude liver preparations, does not respond to some highly purified preparations that are very active against pernicious anemia, and there is therefore considerable reason to suspect a basic difference between the two types of anemia. ${ }^{143,144}$

The megaloblastic macrocytic anemias of infancy and childhood constitute a number of clinical entities: celiac disease or steatorrhea, generally characterized as infantile sprue; true pernicious anemia of childhood which is extremely rare; a condition characterized as "temporary pernicious anemia"; and "goat's milk" anemia. The first three of these conditions are known to respond to folic acid therapy, the last being as yet unreported upon. The effects of thymine and vitamin $B_{12}$ in these conditions are also unknown. ${ }^{145}$

Finally, a variety of miscellaneous conditions, such as diarrhea, gastric cancer, gastrectomy, and alcoholism, have resulted in conditions of macrocytic anemia which resemble more or less the syndrome noted above, and have as their basis the absence of extrinsic factor or an inadequate supply of folic acid or vitamin $B_{12}$. Some of these, such as gastrectomy and the so-called "chronic diarrheas," have already been shown to respond well to folic acid, but further studies are as yet lacking. A macrocytic anemia caused by infestation with the fish tapeworm, Diphyllobothrium latum, resembles pernicious anemia in many respects and is noteworthy because of the extreme nature of the causative agent in this case. This 
latter condition responds to removal of the parasite, to anti-pernicious anemia liver extract, and stomach preparations, no report of vitamin therapy being as yet available. ${ }^{146}$

It is thus apparent that a variety of common long recognized severe afflictions must now be considered as avitaminoses, and that the symptoms of these diseases generally respond well to both folic acid and vitamin $B_{12}$. It is equally apparent, however, that much work remains to be done in elucidating the relationships of these $B$ vitamins to the full measure of the antipernicious effect of liver, and to erythrocyte maturation. There are present indications that vitamin $\mathrm{B}_{12}$ may function in the synthesis of folic acid, and that folic acid functions in purine synthesis. ${ }^{147}$ The relationship of these observations to the etiology of the macrocytic anemias remains for the present obscure, as do the relations of the other $\mathrm{B}$ vitamins to these hematological symptoms. ${ }^{148}$

Deficiencies in Vertebrates other than Man. Folic acid deficiencies have been produced in a number of lower animals, most notably chicks, ${ }^{149,}{ }^{164}$ and monkeys. ${ }^{150}$ In chicks the symptoms involve slow growth and deficient feathering and a macrocytic anemia, and in monkeys, diarrhea, gingivitis, and a macrocytic anemia. Folic acid is effective in causing a remission in both cases. Folic acid deficiencies have been produced in mice by the use of the inhibitors, 1-methylfolic acid and 4-aminofolic acid (Chap. V D). ${ }^{151}$ Typical deficiency has also been produced in rats. Both humans and rats develop severe blood dyscrasias when treated with certain chemotherapeutic agents. In rats the agranulocytosis and bone marrow hypoplasia produced in this manner (sulfaguanidine, sulfasuxidine) respond rapidly to folic acid administration. Folic acid deficiency also apparently occurs in rats made deficient in pantothenic acid (p. 423), ${ }^{152}$ and in some cases of blacktongue in $\log _{9}{ }^{96,98}$ (p. 412), in which case the syndrome is more like canine sprue than pellagra. Similar unclear relationships exist between folic acid and the anemias resulting from riboflavin deficiency in rats, dogs, swine, and monkeys. ${ }^{153,154}$ Rats also have impaired lactation when folic acid-deficient. ${ }^{155}$ Folic acid is only partially effective in treating the blood changes induced in rats by gastrectomy, ${ }^{156}$ and thymine is ineffective in replacing folic acid in the rat for either hematopoiesis or lactation, or in the chick at all. ${ }^{157}$<smiles>N=c1nc(O)c2nccnc2[nH]1</smiles> 
Salmon and trout both develop anemias that are cured or prevented by xanthopterin ${ }^{158}$ and by folic acid; and xanthopterin apparently ${ }^{159}$ has at least some activity in the monkey and possibly the goat's milk anemia of rats, but not in the chick. ${ }^{160}$ The incubation of xanthopterin with rat liver gives results that indicate, though not unequivocally, that this substance is converted to folic acid or enhances the liberation of folic acid from bound forms. Studies with regard to $\mathrm{B}_{12}$ deficiency in lower animals are as yet fragmentary, although the apparent identity of this vitamin with the "animal protein factor" 161 and the "cow manure factor" 162 provides some evidence of the necessity of this factor in the diets of rats and chicks and of the symptoms resulting from some degree of nutritional deprivation. Anemia has been produced in a pig, however, by using vitamin-free casein and 2 per cent sulfasuxidine in the diet, and a remission obtained by the use of purified liver extract; thus vitamin $\mathrm{B}_{12}$ deficiency may be attainable in a number of species when the conditions are properly selected. Prior to the isolation of vitamin $B_{12}$, however, a satisfactory biological response to the antipernicious anemia factor in animals other than man had been long and ardently sought for in vain as an assay device. The relationship which may exist between vitamin $\mathrm{B}_{12}$ and cobalt metabolism should now be reviewed in the light of the cobalt content of vitamin $B_{12}$ and the known lowering of vitamin $\mathrm{B}_{6}$ blood levels in cobalt-deficient animals.

Mention should be made of the apparent stimulatory effect of $\alpha$ - and $\beta$-pyracins upon the pteroyltriglutamate activity on hemoglobin formation and growth in anemic chicks. Either of the pyracins alone is ineffective. However, either pyracin with the triglutamate stimulates its effectiveness ( $\beta$-pyracin being somewhat more active) in improving growth and

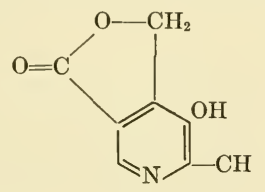

$\alpha$-pyracin

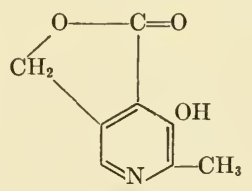

$\beta$-pyracin

preventing anemia. $\beta$-pyracin does not further augment the activity of the monoglutamate in the anemic chick. All three forms of folic acid are apparently active to some degree for both chicks and monkeys, and the pyracins seem to influence favorably the conversion of the triglutamate to the monoglutamate in the chick. Whether this implies an involvement of pyridoxine metabolism in pteroylmonoglutamate formation from its higher conjugates remains unknown. Indeed, recent reports have chal- 
lenged the fact that the pyracins have any biological activity whatsoever in this regard.

Formylpteroic acid (rhizopterin, SLR factor) is inactive in curing rat leucopenia, and its magnesium salt and the magnesium salt of formylfolic acid, presumably a functional form of folic acid, are both apparently inactive in hematopoiesis in man, ${ }^{163}$ as is pteroic acid itself. Indeed, formylpteroic acid is inactive in curing folic acid deficiency in rats or chicks in concentrations 50 times the curative dose of folic acid. The present limited evidence regarding the inefficacy of formylfolic acid in man should be viewed with caution, however. These observations are of interest in view of the inactivity of pteroylpolyglutamates for bacteria, unlike man, and the high activity of formylpteroic acid in some bacteria.

Pantothenic Acid Deficiency. While there is no acute widespread disease of man now known to be associated with a lack of pantothenic acid, there is great danger in assuming that wiclespread and dangerous deficiencies of this vitamin do not exist. The function of riboflavin as a vitamin had been well established for a considerable period before it was realized that its lack was responsible for any special difficulty in man-far less the serious consequences that we now associate with ariboflavinosis. The efficacy of pantothenic acid in curing an increasing number of individual cases of various acute symptoms would seem to suggest, therefore, that as diagnostic experience is acquired, pantothenic acid deficiency may be discovered to be a relatively common affliction in man. There is little doubt that pantothenic acid deficiency is commonly present in many conditions of malnutrition, and is a frequent complication in beriberi, ariboflavinosis, and pellagra; and a low blood pantothenic acid level is frequently observed in these three conditions. A number of cases of peripheral neuritis and delirium tremens and one of Korsakoff's syndrome that did not respond to other vitamins have apparently responded promptly to pantothenic acid.

Clear-cut pantothenic acid deficiencies have been produced in a variety of other animals. Indeed before its isolation and identification, this substance unknowingly had been studied by various groups as a factor for weight maintenance in pigeons (vitamin $\mathrm{B}_{3}$ ), the chick "anti-dermatitis" factor, and a liver filtrate factor required by rats. ${ }^{165}$

Pantothenic acid deficiency in the chick results in extensive spinal cord lesions, thymus involution, a fatty liver, keratitis, dermatitis, and retarded feathering. In black chicks there is a feather depigmentation, and force-feeding chicks on the deficiency diet results rapidly in death. Hens are apparently more resistant to deficiency, but do eventually develop a mild dermatitis of the lower shanks and feet when on a pantothenic acidfree diet. A deficiency of any proportions, however, drastically curtails 
reproduction and hatchability of eggs, although its effect on egg production is only slight.

In rats, deficiency of this vitamin results in a wide variety of symptoms, including graying of the hair in dark rats, "blood-caked whiskers" due to porphyrin deposition from the Harderian gland, dermatitis, sore mouth and nose, subcuticular hemorrhage, kidney and heart damage, marked and highly characteristic adrenal damage, and sudden death. In highly deficient rats there is also a severe anemia, granulocytopenia, and bone marrow hypoplasia. The anemia responds, but only slowly, to pantothenic acid, whereas the granulocytopenia is apparently a folic acid deficiency. It seems, therefore, that the one deficiency is able to create a deficiency of another factor (or factors) in this case. In this regard it has been shown that feeding rats a purified diet containing sulfasuxidine causes a reduction in hepatic stores of folic acid, pantothenic acid, and biotin, but not of other B vitamins. Under these circumstances a typical pantothenic acid deficiency develops on a diet which normally would contain an adequate level of the vitamin, and administration of folic acid and biotin (but not pantothenic acid) causes recovery from the avitaminosis. ${ }^{166}$

The course of pantothenic acid deficiency in dogs is quite different from that in the foregoing cases, being marked by the rapidity that the symptoms progress. Sudden prostration or coma, convulsions, violent gastrointestinal disturbances, and an accelerated respiration and heart rate all are prominent and terminate rapidly in death. The suddenness of the onset of these symptoms makes treatment quite difficult and frequently ineffectual. Autopsy reveals a generally severe gastroenteritis, hemorrhagic kidneys, a mottled fatty liver, and a mottled thymus, but little adrenal damage.

Whereas pantothenic acid deficiency has beeen studied a number of times in mice, the results seem generally to be complicated by the uncertain role played by other nutritional factors in the symptoms observed. Although graying of hair is caused in mice on a pantothenic aciddeficient diet and can be cured by administration of the vitamin, biotin administration seems necessary for the indefinite maintenance of a normal pelt. Alopecia is also caused in mice by abiotinosis; but it has been claimed that the curative effect of pantothenic acid in this case is by stimulation of intestinal inositol synthesis, inositol deficiency being responsible for the alopecia. Adult mice lose weight on a pantothenic acid-deficient diet; and in deficient mice, while the adrenals remain normal, there are desquamative dermatosis, myelin degeneration in the sciatic nerve and spinal cord with accompanying paralysis of the hind quarters, spinal curvature, and a serous exudate from the eyes. 
Deficient pigs show poor growth and become emaciated, develop a rough, dry coat, and later lose their hair, have a prominent gastroenteritis, and an uncoordinated gait, described as goose-stepping with the hind legs. ${ }^{167,168}$

Pantothenic acid is now known to function as part of coenzyme $\mathrm{A}$ in acetylation processes. These processes are at present believed to include the acetylation of choline and aromatic amines, and the condensation of oxalacetate to form cis-aconitate, although they may be much more extensive (p. 195). While a large number of facts of biochemical interest are known concerning pantothenic acid deficiency, these do not as yet fit neatly together to provide a clear-cut explanation of the "biochemical lesions" that occur in this condition. An almost certain factor, however, lies in the adrenal atrophy that is so pronounced in rats, since many of the symptoms of the avitaminosis are characteristic of adrenal pathology. While the biogenesis of the adrenocortical hormones is unknown, it is at least tempting to suggest that it proceeds through an acetylation at the 17 position which may be pantothenate-mediated, followed by oxidation of the 21-methyl group, in somewhat the following manner:
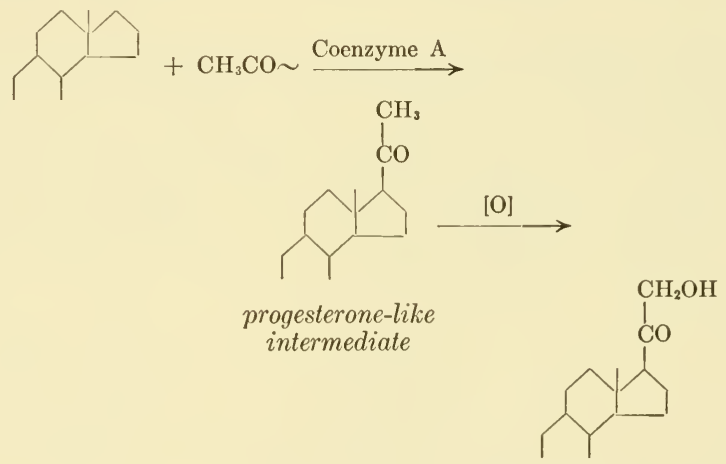

desoxycorticosterone, etc.

In this manner failure of the acetylation process might well result in adrenal insufficiency. Desoxycorticosterone itself is not effective in treating the hair graying in deficient animals, but other closely related ketosteroids are well known for their efficacy in restoring hair pigmentation in man under many other conditions. It has been shown, morêover, that deficient animals do actually have an adrenal cortical insufficiency, which produces such sequelae as the loss of abdominal fat, decreased testicular function, deranged water metabolism and reduced growth. ${ }^{169-171}$ 
Studies on adrenalectomized rats have done much to elucidate this relationship. ${ }^{172}$ Adrenalectomy has been shown to accelerate the growth of new hair in both normal and pantothenic acid-deficient rats, and in the avitaminotic animals it restores the color of the hair and the skin. Finally, it accelerates recovery when pantothenic acid is administered. A bluish pigmentation of the skin develops in the adrenalectomized rat; it reaches a maximum after about two weeks and then fades. This coloration is due to widespread accumulation of melanin in hair bulbs and follicles, and hair graying in pantothenate deficiency results from atrophy of the hair apparatus and a cessation of melanin deposition. The skin pigmentation occurs in all the operated animals, but lasts for a shorter time in the deficient animals. Finally, in adrenalectomized deficient rats, desoxycorticosterone acetate prevents the effects of the operation on hair growth and color. It is thus clear that any adrenal insufficiency in pantothenic acid deficiency is not primarily concerned with the absence of desoxycorticosterone, since the latter substance is necessary to produce the gray hair symptom. ${ }^{173}$ This vitamin-hormone interrelationship is one that merits further study in the immediate future.

The bronzing of the skin in Addison's disease is in some ways suggestive of the fundamental role of the adrenals in pigmentation. The structural relationships between the medullary adrenaline and the known precursors of melanin, and between adrenaline and tyramine, which is an endocrine substance controlling pigmentation in certain molluscs, further suggest that both portions of the suprarenal gland function in pigmentation. The avitaminosis-induced hair graying and porphyrin deposition might conceivably both be linked to the adrenal atrophy. It is well known that the adrenals are intimately associated with the mineral balance, and a number of interesting factors in apantothenosis are undoubtedly associated with this fact. Low salt diets seem to favor the hair-graying process, and deficient animals have an increased salt appetite. The involvement of the adrenals in water balance recalls the blood-soaked whiskers appearance of rats due to water deprivation-a condition characteristic of this avitaminosis but in this case not cured by pantothenic acid.

While nervous lesions are common to most $\mathrm{B}$ vitamin deficiencies, the role of pantothenic acid in acetylcholine synthesis would cause one to predict particularly severe symptoms in this case. If pantothenate functions in the multiple acetate condensation that occurs in fat synthesis and oxidation, then the fatty liver symptom found in the avitaminosis might be explained.

Finally, other symptoms of pantothenate deficiency are doubtless associated with the role of pantothenate in carbohydrate metabolism. 
It is believed that the hypoglycemia (and presumed low liver glycogen) seen in dogs in advanced stages of depletion is responsible for the coma that occurs in these animals. The fact that thyroid administration increases the pantothenate requirement is apparently associated with the overall metabolic stimulation, since the pyridoxine and thiamine requirements are similarly increased.

Pantothenic acid is apparently associated with the mobilization of liver riboflavin, but the mechanism is obscure. ${ }^{174}$ Vitamin $\mathrm{B}_{6}$ deficient animals are said to be more resistant to hair graying in pantothenate deficiency, and cystine is believed to speed the remission of this symptom on pantothenate treatment; but the reasons for these relationships are unknown. ${ }^{175}$ Finally, and perhaps most peculiar, is the evidence that chronic zinc chloride poisoning causes a syndrome extremely similar to pantothenate deficiency, that is said to respond to administration of the vitamin. ${ }^{176}$

Vitamin $\mathbf{B}_{6}$ Deficiency. As in the case of pantothenic acid, no well recognized syndrome in man is known to be due to vitamin $B_{6}$ deficiency, although a variety of conditions have been reported to respond to vitamin $\mathrm{B}_{6}$ administration. There seems little doubt that vitamin $\mathrm{B}_{6}$ deficiency is a factor in some compound deficiencies, such as beriberi, pellagra, and sprue, but attempts to deplete humans of this vitamin specifically have been generally quite unsuccessful (p. 249).

It has been reported that many of the nervous symptoms that remain in pellagrins after thiamine, riboflavin, and niacin therapy respond rapidly to pyridoxine administration. ${ }^{177}$ There is also some evidence that the characteristic cheilosis of ariboflavinosis may also frequently be due to vitamin $\mathrm{B}_{6}$ deficiency, this vitamin having been effective in the cure of this symptom in a number of cases. ${ }^{178}$ Other reports suggesting the efficacy of pyridoxine in epilepsy, Parkinson's disease, pseudohypertrophic muscular dystrophy, and macrocytic anemia are scattered and lack general confirmation, although it is entirely certain that some benefit would result in any one of these conditions should the nature of the affliction in some manner interfere with the normal degree of efficiency in vitamin $B_{6}$ metabolism. Generally, therefore, it seems that vitamin $B_{6}$ plays an important role in human nutrition, but the circumstances attending vitamin $B_{6}$ deficiency in man are as yet obscure. ${ }^{179}$

Vitamin $\mathrm{B}_{6}$ deficiency has been induced in a number of animals by the use of depletion diets, and the rat has been much studied in this regard. Most characteristic of the vitamin $\mathrm{B}_{6}$-depleted rat is the dermatitis or acrodynia, which is manifest on the peripheral portions of the animal such as the tail, ears, nose, mouth and paws. There is an accompanying scaliness and edema, an ulcerated tongue, and cessation of growth with 
accompanying reduction of the accessory organs of reproduction and decreased sexual behavior. In adult mice, vitamin $\mathrm{B}_{6}$ deficiency results in failure to maintain body weight, and death inside of two months. In young mice there is a paralysis of the hind legs, but no dermatitis which is frequently seen in the rat. In adult mice, however, the acute stages of deficiency do show pathological skin manifestations and frequently necrotic tails.

There is no dermatitis in the apyridoxic chick, but growth is slow and accompanied by anorexia and general signs of debility. Pigs and dogs have also been studied with regard to vitamin $B_{6}$ deficiency and both develop anemias. ${ }^{180,181}$ In the pig the anemia is microcytic, the bone marrow hyperplastic, and both it and the spleen and liver are siderotic (contain excessive iron deposits). One of the most significant changes seen in dogs, rats, chicks, and pigs during vitamin $\mathrm{B}_{6}$ deficiency is the typical epileptiform seizures. ${ }^{182}$ The attack appears suddenly, the animal running about wildly in great excitement, then falling and undergoing both clonic and tonic convulsions, followed by coma and collapse. Slow, confused recovery follows upon the rather brief period during which the attack occurs. Nervous degeneration occurs in acute vitamin $B_{6}$ deficiency, as in the case of the other B vitamins, and in the dog at least there is pronounced cardiac hypertrophy.

The epileptiform seizures seen in lower animals have been responsible for the study of pyridoxine efficacy in human epilepsy. The results in this regard, however, have not been promising. Davenport and Davenport ${ }^{183}$ have shown that pyridoxine increases the electroshock threshold of mildly apyridoxic rats, but not of normal animals. Glutamic acid similarly increases the threshold, and tryptophan, which intensifies vitamin $\mathrm{B}_{6}$ deficiency, lowers it. In severe deficiency, pyridoxine causes only a slow rise in the electroshock threshold unless there has been previous loading with glutamic acid. These facts all tend to suggest that maintenance of transaminase activity is critical for a high electroshock threshold-a suggestion with many implications in the field of brain metabolism and mental disease.

Dairy cattle apparently develop a natural vitamin $B_{6}$ deficiency-a fact that is remarkable both because of the resistance of ruminants to avitaminoses, and the apparently rare occurrence of natural apyridoxosis. In cattle the affliction is manifest by anorexia, thinness, a poor hair coat, retarded growth, and most characteristic of all by a poikilocytosis. This condition has been found to respond rapidly to pyridoxine administration, and it will be of interest to see whether the corresponding poikilocytosis in man (sickle cell anemia) bears any relationship to vitamin $B_{6}$ deficiency. ${ }^{184}$ In cobalt deficiency in sheep there is apparently a lowering of 
blood vitamin $\mathrm{B}_{6}$ levels - $\mathrm{a}$ fact further involving vitamin $\mathrm{B}_{6}$ with vitamin $\mathrm{B}_{12}{ }^{185}$ in the overall picture of normal hematopoiesis. The relationship of the pdracins to folic acid has already been discussed (p. 421).

Previous mention has been made of many of the biochemical aspects of vitamin $\mathrm{B}_{6}$ deficiency. There is a lowering of blood, tissue and body levels of this vitamin in most cases, and loading tests have been employed in the diagnosis of apyridoxosis. Studies of the anemia produced in swine have resulted in the conclusion that hemoglobin synthesis is decreased in this deficiency. Thus during vitamin $\mathrm{B}_{6}$ deficiency in swine, erythrocyte protoporphyrin is reduced by over 50 per cent, plasma iron is more than doubled and plasma copper is decreased by 20 per cent, while urinary coproporphyrin remains constant. It is thus felt that the fundamental disturbance involves impaired protoporphyrin synthesis. ${ }^{186}$

One of the most striking changes that occurs in apyridoxosis is the interference in tryptophan metabolism which results in increased urinary excretion of xanthurenic acid and decreased excretion of kynurenic acid by deficient animals. Various aspects of this derangement have been discussed in relation to the earlier study of vitamin metabolism (p. 354). Transaminase activity is distinctly decreased in vitamin $\mathrm{B}_{6}$-deficient rat tissue, ${ }^{187-190}$ and can be increased by the addition of pyridoxal and adenosine triphosphate. Pyridoxine is said to alleviate the toxic symptoms of DL-serine in rats. ${ }^{191}$

Biotin Deficiency. So far as is now known, biotin deficiencies are not naturally occurring, probably largely because of the considerable intestinal synthesis of this vitamin. Deficiencies induced in a number of species by the feeding of avidin-containing raw egg white are well known, however. Sydenstricker et al. fed a group of human volunteers a diet containing 30 per cent of the total calories in the form of desiccated egg white and observed a fine scaly dermatitis; a variety of mental symptoms, including depression, lassitude, hallucinations and panic; pallor; and a drop in urinary excretion of biotin from 29-62 $\mu \mathrm{g}$ per day to 3.5-7.5 $\mu \mathrm{g}$ per day after ten weeks on the diet. All these symptoms disappeared after administration of biotin. ${ }^{192}$ There have been a number of reports of successful biotin therapy of various skin disturbances (acne vulgaris, rosacea, furunculosis, baldness due to seborrhea), but there is at present insufficient evidence to support such claims.

Egg-white injury has been extensively studied in the rat, the symptoms being highly typical. There is a cessation of growth, a spectacle-eyed appearance, a desquamous dermatitis of the neck and groin which becomes generalized, and stiffened joints, which result in an awkward movement of the animal. Progressive emaciation terminates in death. Such rats are said to be unusually susceptible to infection with various organisms, 
Trypanosoma lewisi infection and pneumonia having been particularly studied. ${ }^{193} \mathrm{~A}$ deep brown pigmentation has also been reported on the back of deficient rats, particularly males (and deficient human infants). Male rats are said to be more sensitive than females.

Biotin-deficient chicks also develop a characteristic dermatitis: the feet become calloused and cracked, the corners of the mouth and the area about the beak develop severe lesions, and the eyelids become swollen and stick together. Similar symptoms of no distinctive interest occur as the result of egg-white feeding to a wide variety of other animalsrabbits, monkeys, ${ }^{194,195}$ mice, guinea pigs, and swine. Little is known of the biochemical changes that occur in abiotinosis. Studies with liver slices from biotin-deficient rats have shown that added biotin increases the efficacy of this material in utilizing lactate, and it may be that the conversion of pyruvate to oxalacetate will be shown to be seriously impaired in this deficiency (p. 171). ${ }^{196}$ Impaired ovalacetate formation might cause a collateral impairment of pyruvate oxidation, which may explain the observed depression in oxygen consumption of biotin-deficient duck heart. ${ }^{197}$

p-Aminobenzoic Acid Deficiency. Relatively little is known about $p$-aminobenzoic acid (PABA) deficiency and its incidence in man, or about the relationship of this vitamin to another in which it is contained, folic acid. There is good evidence to indicate, however, that it is effective in the treatment of certain types of hair graying in man. Folic and pantothenic acids have been reported to have similar effects, and it has been suggested that $p$-aminobenzoic acid may act through folic acid synthesis. There is some reason to believe that pantothenic acid functions with $\mathrm{PABA}$ in hair pigmentation. The effect of PABA ultimately is involved in melanin formation however, and the oxidation of tyrosine to melanin may in some manner be related to the apparent relationship between tyrosine and folic acid (p. 415).

PABA stimulates the growth of rats and chicks on a deficiency diet, and is a growth requirement for certain microorganisms. In the black or piebald rat, deficiency results in graying of the hair (nutritional achromotrichia) - a condition which, like hydroquinone-induced achromotrichia, can be cured by PABA administration. Female albino rats have been reported to have lactation disturbances when fed a PABA-deficient diet. The high efficacy of the sulfonamides in inhibiting bacterial growth indicates that PABA plays an unusually critical role in cellular metabolism, particularly since analogues of many other metabolites have not proved effective therapeutic agents. It would seem, therefore, that PABA occupies some key metabolic position, which when blocked causes the breakdown of a number of metabolic sequences. The reversal of sulfanilamide 
by folic acid and vitamin $\mathrm{B}_{12}$ indicates indeed that PABA is intimately related to these substances (see Chapter III D). In view of these considerations it is remarkable that the physiological effects of PABA and its deficiency in the higher animals are not more pronounced.

Inositol Deficiency. Inositol deficiency is little understood, probably because of the paucity of evidence regarding this avitaminosis in man. Possibly the only effect known in man is its prevention of the fatty liver induced by cholesterol feeding. Mice and rats apparently require inositol for normal growth, and mice on deficiency diets become hairless and develop a severe dermatitis. Rats develop a spectacle-eyed appearance and a high-cholesterol fatty liver. The alopoecia and other symptoms undergo remission on inositol administration. The fatty livers induced by biotin or liver extract are similarly cured by inositol, but not choline. ${ }^{198}$ Inositol does not affect "fat type" fatty livers as does choline, however. Along with the association of inositol as one of the lipocaic factors of the pancreas, other relationships appear. Inositol has recently been reported to be highly efficacious in the treatment of diabetes mellitus, and despite the caution that must be used in viewing such reports, it seems probable that as yet unknown dietary considerations will eventually be shown to play an integral role in the etiology of this disease. The relationship is more interesting in view of the possible role of inositol as a coenzyme in amylase (p. 125). ${ }^{199,200}$

One other aspect of inositol deserves mention at this point. Beyond its wide occurrence in the free state and as its esters and their salts, inositol is found in certain cephalins called "lipositols," which occur in brain tissue, soybean oil, etc. Regardless of what other roles they play, these cephalins undoubtedly function in the mobilization and transport of fat, and the lipotropic activity of inositol may be due to its occurrence in lipositols. Beyond this, the antagonistic activities between streptomycin and lipositol in bacteria suggest that lipositol may play an integral role in cellular function. ${ }^{201}$

Choline Deficiency. Choline deficiency occurs naturally among domestic fowls and it is thought to occur on rare occasion in other animals, but it may be readily induced in most species. An important function of choline is as a supply of methyl groups for transmethylation, ${ }^{202}$ and although this function is vital, it is not a vitamin function. For this reason, and because the topic is a large and important one in its own right, ${ }^{203}$ the function of choline as a raw material for methionine synthesis cannot be considered at length in this monograph. It is recognized, however, that choline may function as a vitamin by acting catalytically as a carrier of methyl groups, or as a donor in the methylation of some other catalyst. It is difficult to attribute a vitamin function to choline by virtue 
of its fat transport functions which involve large amounts of the substance that are not compatible with our present concept of B vitamin activity. One might associate a vitamin function with choline for its role in acetylcholine synthesis, were it not so difficult to extend this to microorganisms, some of which require a nutritional choline source. Because of the multiplicity of symptoms associated with its deficiency and despite the several possible reasons for these, it is difficult to discuss choline deficiency in the light of an avitaminosis, although it may well be true that choline functions as a vitamin, and that some of the manifestations of its deficiency are due to the breakdown of this function.

The usual symptoms of choline deficiency are retarded growth, fatty livers with interlobular cirrhosis, renal tubular degeneration, enlarged spleen, and a generalized hemorrhagic condition. ${ }^{204,205}$ In chicks and turkeys, perosis is also characteristic and egg production subsides. In albino rats normal lactation ceases, and young rats raised from mothers approaching such a state develop a flaccid paralysis of the hind quarters. There is a high nonprotein nitrogen excretion in choline deficiency, and the general indications of impaired renal and hepatic function. Choline is said to depress the polycythemia induced by cobalt ${ }^{206}$ and to prevent the necrosis, cirrhosis, and cancer of liver induced by "butter yellow" (dimethylaminoazobenzene). ${ }^{207}$ Neoplasms are frequently formed, moreover, in the livers of choline-deficient rats. ${ }^{208}$ There is little in this overall picture to suggest the consequences that result from an impaired function of choline in cellular metabolism, and further studies of choline function in cellular and enzyme systems where fat mobilization and transmethylation effects can be eliminated are needed to elucidate the vitamin role of choline. Choline deficiency has been studied in a variety of other animals, dogs being particularly suitable for such studies. ${ }^{209}$

\section{The Relationship of the B Vitamins to Various "Nondeficient" States.}

A number of pathological conditions are known which, while they bear no apparent relationship to any of the avitaminoses studied, yet respond to $\mathrm{B}$ vitamin, liver, or yeast therapy. Whether this is due to insufficient study of the therapeutic effects of the various members of the B group in each case, or the presence of as yet unidentified factors is at present uncertain. Also worthy of consideration are a wide variety of conditions of presumably known etiology which bear no apparent relationship to avitaminoses, but respond favorably to B vitamin therapy. At the present time, when the impetus for study of most of the classical nutritional deficiencies has subsided, the possibilities for spectacular advances in this new field seem unlimited. Only a few suggestions are as yet available, however, to indicate the directions in which such advance may proceed. 
A list of topics that have been suggested in this regard is provided in Table 36. Only two are of sufficient promise to merit any greater discussion at present.

$p$-Aminobenzoic acid has recently been shown to be an highly effective and specific chemotherapeutic agent against various rickettsial diseases.

TABle 36. Various Pathological Conditions Associated with or Reported as Responding to B Vitamin Therapy Condition

Reference

Infectious Diseases

Bacterial

Salmonella infection

Tuberculosis

210,211

Tabes dorsalis

212

Pneumonia

213

General

214

Protozoa

Trypanosomes

215-218

Malaria

193

Rickettsiae and Viruses

Rocky Mountain Spotted Fever and Typhus

Equine encephalomyelitis

Poliomyelitis

219,220

Immunity

Antibody production

Invasion

Phagocytosis

Resistance

221

222

223

224,225

226

227

228,229

Organic Diseases

Eye, ear, nose, and throat disorders

230-232

Mental and neurological disorders

233-238

Genetotrophic diseases, alcoholism

239

Diabetes

Inanition and anestrous

Arthritis

40-242

Cancer

243

244

245

Disorders of pregnancy

246

Anorexia nervosa

247

Intestinal obstruction, etc.

Seborrhea

248,249

250

Injuries

Burns

Shock

Anoxia

Wounds

General

In mice infected with murine typhus, incorporation of 3 per cent of PABA into the diet results in a 100 per cent survival, compared with zero survival for untreated controls. Similar results have been obtained with Rocky Mountain spotted fever in guinea pigs, ${ }^{221}$ and the use of PABA in humans infected with these diseases has been shown to be highly effective. The precise reasons for these effects are obscure, however. The 
results so obtained are of particular interest in that analogues of this vitamin have met with such spectacular success in combatting bacterial infections.

Of interest from both theoretical and practical standpoints is the work of Williams et al. on genetotrophic diseases. ${ }^{239}$ Whereas the realization that there may exist genetically induced high vitamin requirements is not entirely new, the recent coupling of such high requirements with nutritional states to produce a hitherto unconsidered type of deficiency disease merits attention. Experimental evidence has as yet been produced bearing on only one-compulsive drinking. Thus, whereas rats from an ordinary colony normally show a wide range in individual appetites for alcohol as measured by self-selection of water or 10 per cent alcohol, animals on deficient diets tend eventually to drink large quantities of alcohol. The alcohol consumption so induced may be cured by nutritional means by correcting the nutritional deficiency to which the particular animal is subject. The extension of these studies to clinical trials with humans, and to other diseases, ${ }^{259}$ should certainly be one of the most fascinating directions in which results are to be expected in the near future in this field.

\section{Bibliography}

1. Nutrition Revs., 5, 319 (1947).

2. Berryman, G. H., Frank, C. E., Baldwin, H. R., Bell, S. L., and Henderson, C. R., Am. J. Physiol., 149, 254-263 (1947).

3. Nutrition Revs., 3, 195-7 (1945).

4. Denny-Brown, D., Medicine, 26, 41 (1947).

5. Proceedings, Research Council on the Relation of Nutrition to Public Health, Nutrition Foundation, New York, 1943, p. 16.

6. Hulse, M. C., Weissman, N., Stotz, E., Clinton, M., and Ferrebee, J. W., Ann. Internal Med., 21, 440-5 (1944).

7. Aykroyd, W. R., and Gopalan, C., Indian Med. Gaz., 80, 68 (1945).

8. Cartwright, G. E., and Wintrobe, M. M., J. Lab. Clin. Med., 31, 886-99 (1946).

9. Gillman, J., and Gillman, T., Nutrition Revs., 5, 353-5 (1947).

10. Graves, P. R., Brit. Med. J., 1, 253 (1947).

11. Passmore, R., Trans. Roy. Soc. Trop. Med. Hyg., 41, 189 (1947).

12. Davies, J. N. P., Lancet, 254, 317-20 (1948).

13. Nutrition Revs., 5, 253-4 (1947).

14. Metcoff, J., and McQueeney, A. J., New Engl. J. Med., 235, 451-60 (1946).

15. Bloom, S. M., Merz, E. H., and Taylor, W. W., Am. J. Ophthalmology, 29, 1248 (1946).

16. Keys, A., Nutrition Revs., 5, 129-31 (1947).

17. Bessey, O. A., and Lowry, O. H., Am. J. Pub. Health, 35, 941-6 (1945).

18. Salcedo, J., Jr., Carrosco, E. O., Jose, F. R., and Valenzuelo, R. C., J. Nutrition, 36, 561-77 (1948).

19. Eddy, W. H., and Dalldorf, G., "The Avitaminoses," Wilkins \& Wilkins Co., Baltimore, 1944, p. 175.

20. Eddy, W. H., and Dalldorf, G., Ibid., p. 222.

21. Nutrition Revs., 4, 304-6 (1946). 
22. Foster, C., Jones, J. H., Henle, W., and Dorfman, F., J. Exptl. Med., 79, 221-34 (1944).

23. Seeler, A. O., and Ott, W. H., J. Infectious Diseases, 75, 175-8 (1944).

24. Rasmussen, A. F., Jr., Waisman, H. A., Elvehjem, C. A., and Clark, P. F., $J$. Infectious Diseases, 74, 41-7 (1944).

25. Wald, G., and Jackson, B., Proc. Natl. Acad. Sci. U.S., 30, 255-63 (1944).

26. Schopfer, W. H., Pasternak, T., and Boss, M. C., Schweiz. Z. Path. Bakt., 10, 443-54 (1947).

27. Dansey-Browning, G. C., and Rich, W. M., Brit. Med. J., 1, 20 (1946).

28. Hibbs, R. E., Ann. Internal Med., 25, 270-82 (1946).

29. Eddy, W. H., and Dalldorf, G., op. cit., p. 200.

30. Van Gelder, D. W., and Darby, F. V., J. Pediat., 25, 226-35 (1944).

31. Krishnan, B. G., Ramachandran, S., and Sadhu, K., Indian Med. Gaz., 80, 521 (1945).

32. Rascoff, H., J. Am. Med. Assoc., 120, 1292-3 (1942).

33. Spillane, J. D., Lancet, 249, 317-18 (1945).

34. Clarke, C. A., and Sneddon, I. B., Lancet, 250, 734-7 (1946).

35. Rudy, A., and Epstein, S. H., J. Clin. Endocrinol., 5, 92-8 (1945).

36. Cullinan, E. R., Kerwick, A., Watts, A. S., and Titman, W. L., Quart. J. Med., 15, 91-105 (1946).

37. Riggs, H. E., and Boles, R. S., Quart. J. Studies Alc., 5, 361-70 (1944).

38. Ellis, W. G., Lancet, II, 985 (1898).

39. Nutrition Revs., 3, 21-4 (1945).

40. Berkman, J. M., Ann. Internal Med., 22, 679-91 (1945).

41. Shay, H., Komarov, S. A., Gruenstein, M., and Fels, S. S., Gastroenterology, 6, 199-212 (1946).

42. Rafsky, H. A., Newman, B., and Jolliffe, N., J. Lab. Clin. Med., 32, 118-23 (1947).

43. Weiss, S., and Wilkins, R. WV., Ann. Internal Med., 11, 104-48 (1937).

44. Morris, H. P., Vitamins and Hormones, 5, 175-95 (1947).

45. Sherman, H. C., and Spohn, A., J. Am. Chem. Soc., 45, 2719-2S (1923).

46. Cook, E. F., J. Am. Pharm. Assoc., 28, 267-8 (1939).

47. Birch, T. W., and Harris, L. J., Biochem. J., 28, 602-21 (1934).

48. Everett, G. M., Am. J. Physiol., 141, 439-48 (1944).

49. Waisman, H. A., and McCall, K. B., Arch. Biochem., 4, 265-79 (1944).

50. Johnson, B. C., Hamilton, T. S., Nevens, W. B., and Boley, L. E., J. Nutrition, 35, 137-45 (1948).

51. Kinnersley, H. W., Peters, R. A., and Reader, V., Biochem. J., 19, 820-6 (1928).

52. Peters, R. A., Biochem. J., 32, 2031-6 (1938).

53. Olson, R. E., Pearson, O. H., Miller, O. N., and Stare, F. J., J. Biol. Chem., 175, 489-501 (1948).

54. Randles, F. S., Himwich, W. A., Homburger, E., and Himwich, H. E., Am. Heart J., 33, 341-5 (1947).

55. Nutrition Revs., 6, 259-61 (1948).

56. Goodhart, R., and Sinclair, H. M., J. Biol. Chem., 132, 11-21 (1940).

57. Williams, R. R., in "The Vitamins, A Symposium," American Medical Association, Chicago, 1939, p. 154.

58. Horwitt, M. K., Bull. Natl. Research Council, 116, 12-46 (1948).

59. Salcedo, J., Jr., Najjar, V. A., Holt, L. E., Jr., and Hutzler, E. W., J. Nutrition, 36, 307-13 (1948).

60. Darby, W. J., and Milam, D. F., Am. J. Pub. Health, 35, 1014-21 (1945).

61. Hagedorn, D. R., Kyhos, E. D., Germek, O. A., and Sevringhaus, E. L., J. $N u$ trition, 29, 179-89 (1945).

62. Cayer, D., Ruffin, J. M., and Perlzweig, W. A., Southern Med. J., 38, 111-17 (1945).

63. Mann, T., Am. J. Ophthalmology, 28, 243-7 (1945). 
64. Spies, T. D., Perry, D. J., Cogswell, R. C., and Frommeyer, W. B., J. Lab. Clin. Med., 30, 751-65 (1945).

65. Sydenstricker, V. P., Sebrell, W. H., Cleckley, H. M., and Kruse, H. D., J. Am. Med. Assoc., 114, 2437-45 (1940).

66. Mann, A. W., Mann, J. M., and Spies, T. D., J. Am. Dental Assoc., 32, 1357-67 (1945).

67. Davies, J. N. P., Lancet, 254, 317-20 (1948).

68. Williams, C. D., Lancet, 256, 711-12 (1949).

69. Vakil, R. J., Indian Med. Gaz., 80, 148 (1945).

70. Hall, W. K., Bowles, L. L., Sydenstricker, V. P., and Schmidt, H. C., Jr., J. Nutrition, 36, 277-82 (1948).

71. Endicott, K. M., Kornberg, A., and Ott, M., Blood, 2, 164-74 (1947).

72. Engel, R. W., Phillips, P. H., and Halpin, J. G., Poultry Sci., 19, 135-42 (1940).

73. Sebrell, IV. H., and Onstott, R. H., Pub. Health Repts., 53, 83-94 (1938).

74. Axelrod, A. E., and Elvehjem, C. A., J. Biol. Chem., 140, 725-38 (1941).

75. Rossiter, R. J., J. Biol. Chem., 135, 431-6 (1940).

76. Gaunt, R., Liling, M., and Mushett, C. W., Endocrinology, 38, 127-32 (1946).

77. Tufs, E. V., and Greenberg, D. M., J. Biol. Chem., 122, 715-26 (1938).

78. Ershoff, B. H., J. Nutrition, 35, 269-80 (1945).

79. Dawson, J., Biochem. J., 40, xli (1946).

80. De Kleine, W., Southern Med. J., 35, 992-6 (1942).

81. Cluver, E. H., Brit. Med. J., 2, 751-4 (1929).

82. Drumond, J. C., "Science and Nutrition," Bacharach, Watts, \& Co., London, 1938.

83. Musselman, M. M., War Med., 8, 325-32 (1945).

84. Comerford, C., and Kirman, B. H., Brit. Med. J., 2, 44-5 (1945).

85. Bean, W. B., Spies, T. D., and Vilter, R. W., Arch. Dermatol. Syphilol., 49, 335-45 (1944).

86. Sydenstricker, V. P., Armstrong, E. S., Derrick, C. J., and Kemp, P. S., Am. J. Med. Sci., 192, 1-9 (1936).

87. Eddy, W. H., and Dalldorf, G., op. cit., p. 225.

88. Cook, A. B., Indian Med. Gaz., 79, 429-37 (1944).

89. Bakwin, H., et al., Am. J. Discases Childien, 74, 657-68 (1947).

90. Sevringhaus, E. L., and Kyhos, E. D., Arch. Internal Med., 76, 31-3 (1945).

91. Landor, J. V., and Pallister, R. A., Trans. Roy. Soc. Trop. Med. Hyg., 29, 121 (1935).

92. Spies, T. D., J. Am. Med. Assoc., 105, 1028-30 (1935).

93. Spillane, J. D., and Scott, G. I., Lancet, 249, 261-4 (1945).

94. Stannus, H. S., Brit. Med. J., 2, 103, 140 (1944).

95. Handler, P., and Featherston, W. P., J. Biol. Chem., 151, 395-404 (1943).

96. Krehl, W. A., Torbet, N., de la Huerga, J., and Elvehjem, C. A., Arch. Biochem., 11, 363-9 (1946).

97. Ruegamer, W. R., Brickson, W. L., Torbet, N. J., and Elvehjem, C. A., J. Nutrition, 36, 425-35 (1948).

98. Krehl, W. A., and Elvehjem, C. A., J. Biol. Chem., 158, 173-9 (1945).

99. Handler, P., and Dann, W. J., J. Biol. Chem., 145, 145-53 (1942).

100. Darby, W. J., Jones, E., and Johnson, H. C., Science, 103, 108 (1946).

101. Luecke, R. W., McMillen, W. N., Thorp, F., and Tull, C., J. Nutrition, 33, 251-61 (1947).

102. Watson, C. J., and Layne, J. A., Ann. Internal Med., 19, 183-99 (1943).

103. Rimington, C., and Leitner, Z. A., Lancet, 249, 494-6 (1945).

104. Singal, S. A., Sydenstricker, V. P., and Littlejohn, J. M., J. Biol. Chem., 176, 1069-73 (1948).

105. Mainzer, F., Acta Med. Scand., 100, 208-30 (1939).

106. Tscherkes, L. A., Litvack, J. I., and Korovitzky, L. K., Acta Med. Scand., 87, 459-69 (1936). 
107. Minot, G. R., and Strauss, M. B., Vitamins and Hormones, 1, 269-91 (1943).

108. Pfiffner, J. J., and Hogan, A. G., Vitamins and Hormones, 4, 1-34 (1946).

109. SubbaRow, Y., Hastings, A. B., and Elkin, M., Vitamins and Hormones, 3, 237-96 (1945).

110. Berry, L. J., and Spies, T. D., Blood, 1, 271-306 (1946).

111. SubbaRow, Y., et al., Ann. N. Y. Acad. Sci., 48, 255-350 (1946).

112. Kornberg, A., Daft, F. S., and Sebrell, W. H., Arch. Biochem., 8, 431-7 (1945).

113. Kornberg, A., J. Biol. Chem., 164, 203-12 (1946).

113a. Hutchings, B. L., and Mowat, J. H., Vitamins and Hormones, 6, 1-25 (1948).

114. Swendseid, M. E., Wandruff, B., and Bethell, F. H., J. Lab. Clin. Med., 32, 1242-7 (1947).

115. Woodruff, C. W., and Darby, W. J., J. Biol. Chem., 172, 851-2 (1948).

116. Sealock, R. R., and Lepow, J. P., J. Biol. Chem., 174, 763-4 (1948).

117. Bernhart, F. W., and Schneider, R. W., Am. J. Med. Sci, 205, 636-43 (1943).

118. Swenseid, M. E., and Bethell, F. H., Proc. Central Soc. Clin. Research, 17, 40 (1944).

119. Holmes, E. G., Brit. Med. J., 2, 561-4 (1945).

120. Hunter, A., Quart. J. Med., 15, 107-21 (1946).

120a. Ternberg, J. L., and Eakin, R. E., J. Am. Chem. Soc., 71, 3858 (1949).

121. Castle, W. B., and Townsend, W. C., Am. J. Med. Sci., 178, 764-77 (1929).

122. Stokstad, E. L. R., and Jukes, T. H., Proc. Soc. Exptl. Biol. Med., 62, 112-13 (1946).

123. Welch, A. D., Heinle, R. W., Nelson, E. M., and Nelson, H. V., J. Biol. Chem., 164, 787-8 (1946).

124. Spies, T. D., Stone, R. E., Lopez, G. G., Toca, R. L., and Aramburu, T., Lancet, 255, 519-22 (1948).

125. Sharp, E. A., and Vonder Heide, E. C., Am. J. Clin. Path., 17, 761-9 (1947).

126. Bethell, F. H., Meyers, M. C., Andrews, G. A., Swendseid, M. E., Bird, O. D., and Brown, R. A., J. Lab. Clin. Med., 32, 3-22 (1947).

127. Vilter, C. F., Vilter, R. W., and Spies, T. D., J. Lab. Clin. Med., 32, 262-73 (1917).

128. Heinle, R. W., and Welch, A. D., J. Am. Med. Assoc., 133, 739-41 (1947).

129. Davis, J. E., Science, 104, 37-8 (1946).

130. Meyer, L. M., Sawitsky, A., Ritz, N. D., and Fitch, H. M., J. Lab. Clin. Med., 33, 189-202 (1948).

131. Spies, T. D., Stone, R. E., Hartus, S., and Aramburu, T., Southern Med. J., 41, 1030-1 (1948).

132. Berk, L., Denny-Brown, D., Finland, M., and Castle, W. B., New Engl. J. Med., 239, 328 (1948).

133. Nutrition Revs., 6, 182-5 (1948).

134. Cayer, D., Ruffin, J. M., and Perlzweig, W. A., Am. J. Med. Sci., 210, 200-7 (1945).

135. Darby, W. J., Cherrington, M. E., and Ruffin, J. M., Proc. Soc. Exptl. Biol. Med., 63, 310-2 (1946).

136. Cayer, D., Ruffin, J. M., and Perlzweig, W. A., Am. J. Med. Sci., 212, 179-84 (1946).

137. Maegraith, B. G., Adams, A. R. D., Havard, R. E., King, J. D., and Millet, R. F., Lancet, 249, 635 (1945).

138. Lopez, G. G., Milones, F., Toca, R. L., Aramburu, T., and Spies, T. D., Am. J. Med. Sci., 216, 270-4 (1948).

139. Spies, T. D., and Suarez, R. M., Blood, 3, 1213-20 (1948).

140. Suarez, R. M., Welch, A. D., Heinle, R. W., Suarez, R. M., Jr., and Nelson, E. M., J. Lab. Clin. Med., 31, 1294-1304 (1946).

141. Weir, J. F., and Comfort, M. W., J. Lab. Clin. Med., 32, 1231-41 (1947).

142. Darby, W. J., Vitamins and Hormones, 5, 119-61 (1947).

143. Watson, J., and Castle, W. B., Proc. Soc., Exptl. Biol. Med., 58, 84-6 (1945). 
144. Heinle, R. W., Dingle, J. T., and Weisberger, A. S., J. Lab. Clin. Med., 32, 970-81 (1947).

145. Peterson, J. C., and Dunn, S. C., Am. J. Diseases Children, 71, 252-68 (1946).

146. Bonsdorff, B. v., Acta Med. Scand., 129, 59 (1947).

147. Shive, W., Ravel, J. M., and Harding, W. M., J. Biol. Chem., 176, 991-2 (1948).

148. Davis, L. J., and Brown, A., Blood, 2, 407-25 (1947).

149. Campbell, C. J., MeCabe, M. M., Brown, R. A., and Emmett, A. D., Am. J. Physiol., 144, 348-54 (1945).

150. Day, P. L., Vitamins and Hormones, 2, 71-105 (1944).

151. Franklin, A. L., Stokstad, E. L. R., and Jukes, T. H., Proc. Soc. Exptl. Biol. Med., 67, 398-400 (1948).

152. Daft, F. S., Kornberg, A., Ashburn, L. L., and Sebrell, W. H., Pub. Health Repts., 60, 1201-15 (1945).

153. Endicott, K. M., Kornberg, A., and Ott, M., Blood, 2, 164-74 (1947).

154. Waisman, H. A., Proc. Soc. Exptl. Biol. Med., 55, 69-71 (1944).

155. Nelson, M. M., and Evans, H. M., Arch. Biochem., 13, 265-75 (1947).

156. Higgins, G. M., Ingle, D. J., and Joneson, O. R., J. Lab. Clin. Med., 32, 635-43 (1947).

157. Daniel, L. J., Scott, M. L., Norris, L. C., and Heuser, G. F., J. Biol. Chem., 173, 123-35 (1948).

158. Norris, E. R., and Simmons, R. W., J. Biol. Chem., 158, 449-53 (1945).

159. Totter, J. R., Mims, V., and Day, P. L., Science, 100, 223-5 (1944).

160. O'Dell, B. L., and Hogan, A. G., J. Biol. Chem., 149, 323-37 (1943).

161. Zucker, L. M., and Zucker, T. F., Arch. Biochem., 16, 115-29 (1948).

162. Rubin, M., and Bird, H. R., J. Nutrition, 34, 233-45 (1947).

163. Spies, T. D., Lopez, G. G., Stone, R. E., Milanes, F., Brandenberg, R. O., and Aramburu, T., Blood, 3, 121-6 (1948).

164. Keith, C. K., Brooch, W. J., Warren, D., Day, P. L., and Totter, J. R., J. Biol. Chem., 176, 1095-1101 (1948).

165. Williams, R. J., Advances in Enzymology, 3, 253-87 (1943).

166. Wright, L. D., and Welch, A. D., J. Nutrition, 27, 55-66 (1944).

167. Follis, R. H., Jr., and Wintrobe, M. M., J. Exptl. Med., 81, 539-52 (1945).

168. Luecke, R. W., MeMillen, W. N., Thorp, F., and Tull, C., J. Nutrition, 33, 251-61 (1947).

169. Gaunt, R., Liling, M., and Mushett, C. W., Endocrinology, 38, 127-32 (1946).

170. Supplee, G. C., Bender, R. C., Kahlenberg, O. J., and Babcock, L. C., Endocrinology, 30, 355-64 (1942).

171. Wintrobe, M. M., Follis, R. H., Jr., Alcayaga, R., Paulson, M., and Humphreys, S., Bull. Johns Hopkins Hosp., 73, 313-41 (1943).

172. Ralli, E. P., and Graef, I., Endocrinology, 32, 1-12 (1943).

173. Ralli, E. P., and Graef, I., Endocrinology, 37, 252-61 (1945).

174. Supplee, G. C., Jensen, C. G., Bender, R. C., and Kahlenberg, O. J., J. Biol. Chem., 144, 79-85 (1942).

175. Lunde, G., and Kringstad, H., J. Nutrition, 19, 321-32 (1940).

176. Gross, P., Harvalek, Z., and Runne, E., J. Investigative Dermatol., 4, 385-98 (1941).

177. Rosenblum, L. A., and Jolliffe, N., J. Am. Med. Assoc., 117, 2245-8 (1941).

178. Machella, T. E., Am. J. Med. Sci. 203, 114-20 (1942).

179. Stoerk, H. C., Proc. Soc. Exptl. Biol. Med., 62, 90-6 (1946).

180. Cartwright, G. E., Wintrobe, M. M., and Humphreys, S., J. Biol. Chem., 153, 171-82 (1944).

181. Mushett, C. W., Stebbins, R. B., and Barton, M. N., Trans. N. Y. Acad. Sci., 9, 291-6 (1947).

182. Nutrition Revs., 3, 269-71 (1945).

183. Davenport, V. D., and Davenport, H. W., J. Nutrition, 36, 263-75 (1948).

184. Reid, J. T., Huffman, C. F., and Duncan, C. W., J. Nutrition, 30, 413-23 (1945). 
185. Ray, S. N., Weir, W. C., Pope, A. L., and Phillips, P. H., J. Nutrition, 34, 595-9 (1947).

186. Cartwright, G. E., and Wintrobe, M. M., J. Biol. Chem., 172, 557-65 (1948).

187. Miller, E. C., and Baumann, C. A., J. Biol. Chem., 157, 551-62 (1945).

188. Schlenck, F., and Snell, E. E., J. Biol. Chem., 157, 425-6 (1945).

189. Ames, S. R., Sarma, P. S., and Elvehjem, C. A., J. Biol. Chem., 167, 135-41 (1947).

190. Bellamy, W. D., Umbreit, W. W., and Gunsalus, I. C., J. Biol. Chem., 160, 461-72 (1945).

191. Fishman, W. H., and Artom, C., Proc. Soc. Exptl. Biol. Med., 57, 241-3 (1944).

192. Sydenstricker, V. P., Singal, S. A., Briggs, A. P., De Vaughn, N. M., and Isbell, H., J. Am. Med. Assoc., 118, 1199-1200 (1942).

193. Caldwell, F. E., and Gyorgy, P., J. Infectious Diseases, 81, 197 (1947).

194. Lease, J. G., Parsons, H. T., and Kelly, E., Biochem. J., 31, 433-7 (1937).

195. Waisman, H. A., McCall, K. B., and Elvehjem, C. A., J. Nutrition, 29, 1-11, (1945).

196. Summerson, W. H., Lee, J. M., and Partridge, C. W. H., Science, 100, 250-1 (1944).

197. Olson, R. E., Miller, O. N., Topper, Y. J., and Stone, F. J., J. Biol. Chem., 175, 503-14 (1948).

198. Best, C. H., Lucas, C. C., Patterson, J. M., and Ridout, J. H., Biochem. J., 40, 368-73 (1946).

199. Williams, R. J., Schlenk, F., and Eppright, M. A., J. Am. Chem. Soc., 66, 896-8 (1944).

200. Lane, R. L., and Williams, R. J., Arch. Biochem., 19, 329-35 (1948).

201. Rhymer, I., Wallace, G. I., Byers, I. W., and Carter, H. E., J. Biol. Chem., 169, 457-8 (1947).

202. Nutrition Revs., 4, 37-9 (1946).

203. Best, C. H., and Lucas, C. C., Vitamins and Hormones, 1, 1-58 (1943).

204. Nutrition Revs., 4, 345-7 (1946).

205. Handler, P., J. Nutrition, 31, 621-33 (1946).

206. Davis, J. E., Am. J. Physiol., 127, 322-7 (1939).

207. Jacobi, H. P., and Bauman, C. A., Cancer Research, 2, 175-80 (1942).

208. Copeland, D. H., and Salmon, IV. D., Am. J. Path., 22, 1059-79 (1946).

209. Schaefer, A. E., McKiblen, J. M., and Elvehjem, C. A., Proc. Soc. Exptl. Biol. Med., 47, 365-8 (1941).

210. Kligler, I. J., Guggenheim, K., and Hernheiser, H., J. Infectious Diseases, 78, 60-2 (1946).

211. Guggenheim, K., Nutrition Revs., 5, 63 (1947).

212. Getz, H. R., Nutrition Revs., 5, 97-8 (1947).

213. Kesert, B. H., and Grossman, M. O., J. Nervous Mental Disease, 101, 372-7 (1945).

214. Hitchings, G. H., and Falco, E. A., Proc. Soc. Exptl. Biol. Med., 61, 54-7 (1946).

215. Schneider, H. A., and Webster, L. T., J. Exptl. Med., 81, 359-84 (1945).

216. Schneider, H. A., J. Exptl. Med., 84, 305-22 (1946).

217. Peraita, M., Brit. Med. J., 2, 784 (1946).

218. Metcoff, J., Darling, D. B., Scanlon, M. H., and Stare, F. J., J. Lab. Clin. Med., 33, 47-66 (1948).

219. Trager, W., J. Exptl. Med., 77, 557-82 (1943).

220. Seeler, A. O., and Ott, W. H., J. Infectious Diseases, 77, 82-4 (1945).

221. Anigstein, L., and Bader, M. N., Science, 101, 591-2 (1945).

222. Kearney, E. B., Pond, W. L., Plass, B. A., Maddy, K. H., Elvehjem, C. A., and Clark, P. F., J. Infectious Diseases, 82, 177-86 (1948).

223. Nutrition Revs., 3, 69-72 (1945).

224. Stoerk, H. C., Proc. Soc. Exptl. Biol. Med., 62, 90-6 (1946). 
225. Axelrod, A. E., Carter, B. B., McCoy, R. H., and Geisinger, R., Proc. Soc. Exptl. Biol. Med., 66, 137-40 (1947).

226. Guest, M. M., Ware, A. G., and Seegers, W. H., Am. J. Physiol., 150, 661-9 (1947).

227. Cottingham, E., and Mills, C. A., J. Lab. Clin. Med., 30, 498-502 (1945).

228. Riddle, J. W., Spies, T. D., and Hudson, N. P., Proc. Soc. Exptl. Biol. Med., 45, 361-4 (1940).

229. Berry, L. J., Davis, J., and Spies, T. D., J. Lab. Clin. Med., 30, 684-94 (1945).

230. Nutrition Revs., 3, 14-15 (1945).

231. Carroll, F. D., Am. J. Ophthalmol., 30, 172-6 (1947).

232. Kinsey, V. E., Nutrition Revs., 6, 65-6 (1948).

233. Nutrition Revs., 4, 56-8 (1946).

234. Harrell, R. F., J. Nutrition, 31, 283-98 (1946).

235. Guetzkow, H., and Brozek, J., Am. J. Psychol., 59, 358-81 (1946).

236. Williams, R. D., Mason, H. L., Smith, B. F., and Wilder, R. M., Arch. Internal Med., 69, 721-38 (1942).

237. Glusman, M., Am. J. Med., 3, 211-23 (1947).

238. Zimmerman, F. T., Burgemeister, B. B., and Putnam, T. J., Am. J. Psychiat., 104, 593 (1948).

239. Williams, R. J., Berry, L. J., and Beerstecher, E., Jr., Arch. Biochem., 23, 275-90 (1949).

240. Lowry, P. T., and Hegsted, D. M., J. Lab. Clin. Med., 30, 839-43 (1945).

241. Banerjee, S., Science, 106, 128-30 (1947).

242. Himsworth, H. P., Lancet, 256, 465-73 (1949).

243. Drill, V. A., and Burrill, M. W., Endocrinology, 35, 187-92 (1944).

244. Stephens, C. A. L., Borden, A. L., Holbrook, W. P., and Hill, D. F., Ann. Internal Med., 27, 420-32 (1947).

245. Rusch, H. P., and Baumann, C. A., Nutrition Revs., 4, 353-5 (1946).

246. Hesseltine, H. C., Am. J. Obstet. Gynecol., 51, 82-6 (1946).

247. Berkman, J. M., Ann. Internal Med., 22, 679-91 (1945).

248. Rinehart, J. F., and Greenberg, L. D., Am. J. Path., 24, 710 (1948).

249. Leithauser, D. J., Surg. Gynecol. Obstet., 86, 543 (1948).

250. Oppel, T. W., Am. J. Med. Sci., 215, 76-83 (1948).

251. Lund, C. C., Levenson, S. M., Green, R. W., Paige, R. W., Robinson, P. E., Adams, M. A., McDonald, A. H., Taylor, F. H. L., and Johnson, R. E., Arch. Surg., 55, 557-83 (1947).

252. Nutrition Revs., 3, 74-6 (1945).

253. Govier, W. M., J. Am. Med. Assoc., 126, 749-50 (1944).

254. Katzin, L. I., and Warren, S. L., J. Clin. Invest., 24, 152-3 (1945).

255. Greig, M. E., and Govier, W. M., J. Pharmacol. Exptl. Therap., 79, 169-75 (1943).

256. Williams, R. H., and Bissell, G. W., Arch. Surg., 49, 225-7 (1944).

257. Levenson, S. M., Green, R. W., Taylor, F. H. L., Robinson, P., Page, R. C., Johnson, R. E., and Lund, C. C., Ann. Surg., 124, 840-56 (1946).

258. Wald, G., Science, 109, 482-3 (1949).

259. Williams, R. J., Beerstecher, E., Jr., and Berry, L. J., Lancet, 258, 287-9 (1950). 

Section D

THE COMPARATIVE BIOLOGICAL ACTIVITIES OF THE B VITAMINS AND RELATED COMPOUNDS

By William Shive 



\section{Chapter ID}

\section{INTRODUCTION AND THEORETICAL CONSIDERATIONS}

The preparation and testing of analogues of biologically active compounds has long been an accepted approach in the search for more active principles. This approach was very successful in the field of chemotherapy and pharmacology but has yielded meager results with compounds analogous to the $\mathrm{B}$ vitamins as far as nutrition is concerned.

A few compounds have been found to be partially, but almost never fully, active in replacing their analogous B vitamins in the nutrition of various organisms. Other analogues have been observed to replace the corresponding vitamin in carrying out some, but not all, of its biological functions. In most instances, analogues have been found to be essentially inert biologically; however, in some cases, certain analogues of the B vitamins and of related metabolites have been found to be toxic for some organisms. The action of substances which are toxic for an organism has been presumed to involve a combination of the substance with an essential cell constituent. This type of action is usually represented by a combination of the toxic agent with an enzyme in such a fashion that the enzyme can no longer effectively serve as a catalyst.

Much of our knowledge concerning enzymes has been obtained by the use of toxic substances capable of reducing the velocity of enzymatic reactions. According to the modes of action of these inhibitory substances, the inhibitions can usually be classified into one of two general types. One type is termed competitive inhibition, in which case the effectiveness of the inhibitor in preventing an enzymatic reaction depends upon the concentration of the substrate, and a direct relationship exists between the rate of the reaction and the relative concentrations of inhibitor and substrate. The inhibitor appears to compete with the substrate for the same reactive groups of the enzyme. In the other type, noncompetitive inhibition, the effective enzyme concentration is diminished by the inhibitor irrespective of the concentration of the substrate.

Noncompetitive inhibitors such as fluoride, ${ }^{1,2,3}$ which prevents the functioning of enolase and allows the accumulation of 2-phosphoglycerate, have been utilized successfully as specific enzyme "poisons" permitting the substrate to accumulate in a biological system. In the fluoride inhibition of enolase, which converts 2-phosphoglycerate to 2-phosphopyruvic 
acid, the fluoride forms a fluorophosphate with the magnesium of enolase. ${ }^{4}$ Iodoacetate appears to inhibit noncompetitively enzymes which contain sulfhydryl groups essential for their activity. It has been postulated that hydrocyanic acid, mercuric salts, sodium azicle, hydroxylamine and many other noncompetitive inhibitors likewise react with specific groups or ions essential for the activity of the enzymes. These inactivations are not prevented by excess substrate, as is the case in the inactivation of an enzyme by a competitive type of inhibitor.

The earliest reports of inhibitions which appear to be of a competitive nature concerned inhibitions of enzymatic reactions by products of the reactions. ${ }^{5}$ Maltose and glucose inhibit maltose production from starch by malt amylase. ${ }^{6}$ In this and many other instances, such inhibitions induced by the products of a reaction cannot be ascribed totally to a reversal of the reaction by a mass action effect. Thus, fructose or $\beta$-glucose prevents the hydrolysis of sucrose by yeast saccharase, and $\alpha$-glucose competitively prevents the action of Aspergillus saccharase ${ }^{7}$ glycine as well as alanine prevents the hydrolysis of glycylglycine by intestinal peptidases; $;$ pyruvic acid inhibits competitively the dehydrogenation of lactic acid by lactic dehydrogenase $;^{9}$ and ornithine prevents the hydrolysis of arginine by arginase. ${ }^{10}$ In all these cases the products of the enzymatic reactions appear to be able to combine with the enzyme in competition with the substrate. In a number of instances compounds somewhat similar to the substrate were known to prevent an enzymatic reaction. Thus, 3-methylxanthine as well as guanine, uric acid or 1- or 7 -methylguanine prevents the oxidation of xanthine by xanthine oxidase. ${ }^{11}$ Some of the inhibitions were shown to be competitive; e.g., physostigmine competitively prevents the hydrolysis of acetylcholine by choline esterase. $^{12}$

The realization that substances structurally related to the substrate may inhibit the action of an enzyme came with the report of Quastel and Wooldridge ${ }^{9}$ that the dehydrogenation of succinic acid by succinic dehydrogenase was competitively prevented by malonic acid and a number of structurally related compounds.

Although in this and in many other cases the competitive inhibitors structurally resembled the substrate, and although the fundamental theories for both competitive and noncompetitive inhibitions had been previously developed, especially for isolated enzyme systems, the importance of inhibition of enzymatic action by an analogue of the substrate as related to chemotherapy and growth inhibition was not fully realized until the report of Woods and Fildes in $1940^{13,14}$ that the bacteriostatic action of sulfanilamide was competitively prevented by $p$-aminobenzoic acid, a compound previously not known to have a biological function. 
This report prompted a widespread search for chemotherapeutic agents among compounds which were structurally related to catalytic metabolites, but which inhibited the biological functioning of the metabolite.

Many investigators directed their efforts toward the discovery of new and effective chemotherapeutic agents. Others prepared and utilized analogues of metabolites in the study of biochemical transformations. Out of these efforts a new field and new tools for the study of biochemistry have been developed.

\section{Inhibition of Enzymatic Action}

Inhibition of the functioning of an enzyme by an inhibitor results in a decrease in the rate of the enzymatic reaction and of the biological process in which the enzyme is involved. The mode of action of substances inhibiting biological processes has in general been determined either by a study of the relative concentrations of the inhibitor and substrate (metabolite) which are necessary to obtain a defined degree of inhibition after the lapse of a specified time, or by a study of the effect of the substrate on the rate of the process at a specified concentration of inhibitor. For a competitive inhibition, the ratio of the concentration of inhibitor to the concentration of substrate necessary for a defined degree of inhibition of a biological process in a specified time is constant over a range of concentrations and is termed the inhibition index. The effect of the substrate on the rate of the biological process inhibited by a competitive analogue is such that at high concentrations of substrate the rate approaches that of the normal process in the absence of the inhibitor. Such data are usually presented graphically, as subsequently indicated.

The Inhibition Index. Competition of an inhibitor $I$ (analogue) with a substrate $S$ (metabolite) for an enzyme is frequently represented by the following equations analogous to those first developed by Michaelis and Menten, ${ }^{15}$ where $P$ represents the product and ES and EI represent the enzyme-substrate complex and enzyme-inhibitor complex, respectively:

$$
\begin{gathered}
E+S \rightleftharpoons E S \longrightarrow E+P \\
E+I \rightleftharpoons E I
\end{gathered}
$$

By the Law of Mass Action:

$$
\frac{[E][S]}{[E S]}=K_{S}
$$

where $K_{S}$ is the dissociation constant of the enzyme-substrate complex, and

$$
\frac{[E][I]}{[E I]}=K_{I}
$$


where $K_{I}$ is the dissociation constant of the enzyme-inhibitor complex. By dividing equation 2 by equation 1 , one obtains

$$
\frac{[I]}{[S]}=\frac{K_{I}[E I]}{K_{S}[E S]}
$$

If $\left[E_{t}\right]$ represents the total enzyme concentration, both free and combined, by definition

$$
\left[E_{t}\right]=[E]+[E I]+[E S]
$$

In the application of the above equations to biological systems, certain experimental conditions designed to limit some of the variables greatly simplify the problem. In order to study the effect of an inhibitor on an entire biological system, conditions must be such that an observable effect on the rate of a biological process will result from the interaction of the inhibitor with an enzyme; this specific enzymatic reaction then becomes the limiting reaction of the biological process. In an isolated enzyme system, the observable effect may be a decrease in the rate of formation of a product. The observable effect on bacterial cells or any isolated culture of cells such as tissue cultures may be a decreased growth rate or complete inhibition of growth; or the effect observed in an animal or embryo may be on the rate of growth, time of survival or time necessary for the development of certain deficiency symptoms. Hence, any observable effect resulting from a decreased rate of reaction of an inhibited enzyme system can be used to determine the effect of an inhibitor on a specific enzyme system. One method of studying the mode of action of an inhibitor is to determine the relationship between the concentration of inhibitor and the concentration of substrate necessary to obtain a defined observable effect within a constant period of time. Other experimental conditions of the biological system are not allowed to vary. For example, in bacterial studies, the concentrations of inhibitor just necessary to attain a defined inhibition of growth, e.g., maximum or halfmaximum inhibition, are determined with variable concentrations of substrate under conditions of a defined medium, constant size of inoculum, and a defined time and temperature of incubation.

The quantitative response of biological systems under such conditions is dependent upon the rate, $r$, of the limiting reaction, which can be expressed as follows:

$$
r=k[E S]
$$

where $k$ is the rate constant for the reaction. Under the defined experimental conditions, the variables which may affect $[E S]$ and in turn the rate of the reaction are the concentrations of the inhibitor and substrate as well as the total enzyme concentration, $\left[E_{t}\right]$. The inhibitor and sub- 
strate are usually employed at concentrations sufficiently high that their utilization by the biological system does not appreciably alter their concentrations during the experimental period. If the total enzyme concentration of the biological system does not vary during the course of the experiment, the variables of equation 3 and 4 are not a function of time. Even in a system in which cells are multiplying, the mother and daughter cells have similar composition, and the concentrations of their intracellular enzymes would not be expected to vary appreciably. Consequently, for both static and growing systems, the enzyme concentration in all its various forms can be assumed to be constant during the course of the experiment and not a function of time.

Varied concentrations of inhibitor and substrate are established at the outset of the experiment in such a manner that one can determine the critical ratio which will reduce the response of the biological system to a defined quiantity after the lapse of a constant period of time. The total response of the organism, then, is a function of the rate of the limiting inhibited enzymatic reaction and of time. In order to obtain the defined response after a constant period of time, the rate of the limiting reaction, $r$, must be reduced to a value which is specific for this particular selected response. The rate of the inhibited reaction is proportional to the concentration of the enzyme-substrate complex; hence, to achieve the critical rate which will produce the chosen biological response in a specified time, the concentration of the active complex [ES] must be reduced to a specific value, $C_{E S}$, at the onset of the process.

For increasing concentrations of substrate and inhibitor, particularly those approaching enzyme saturation, the value of $[E]$ approaches zero and becomes negligible in comparison with $[E I]$. If the total enzyme concentration, $\left[E_{t}\right]$, is assumed to be constant for the reasons previously indicated, it is apparent from equation 4 that the concentration of the enzyme-inhibitor complex, $[E I]$, must be essentially a constant value, $C_{E I}$, since $\left[E_{t}\right]$ and $[E S]$ are constant, and $[E]$ can be neglected.

By substitution of these constant values, $C_{E I}$ and $C_{E S}$ for $[E I]$ and $[E S]$, respectively, in the general equation 3 , one obtains an equation for the molar ratio of analogue to metabolite which must be established to produce the necessary inhibition that will result in the defined response of the biological system under the conditions outlined above. This ratio is a constant, $K$, the inhibition index.

$$
\frac{[I]}{[S]}=\frac{K_{I} C_{E I}}{K_{S} C_{E S}}=K
$$

The assumption that the intracellular concentration of all the forms of a particular enzyme in a biological system does not vary with changes in the substrate and inhibitor concentrations may not always be valid. The 
decrease in concentration of enzyme-substrate complex induced by the inhibitor may possibly affect the biosynthesis of the enzyme by a system of multiplying cells. Any such change in concentration of enzyme under these conditions would be expected to be a function of $[E S]$ and of time. It has been shown that even if such changes in the intracellular concentration of the enzyme do occur during the course of an experiment, there still is a specific unique value of $[I] /[S]$ which must be initially established in order to obtain the specified response of the system at the end of a constant experimental period. ${ }^{16}$

It can likewise be demonstrated that even when a growing system develops a resistance to an inhibitor during the course of an experiment, there is still a definite initial ratio of inhibitor to substrate concentration for the particular system which will produce the specific response for the constant experimental period.

For experimental conditions in which the concentrations of inhibitor and substrate must be regulated in a medium outside the biological system wherein the reaction takes place, i.e., bacterial growth experiments, etc., the ratio of the concentrations of the inhibitor to the substrate within the cell is a function of this ratio of the concentrations existing outside the cell. Consequently, this latter ratio is also constant for a defined inhibition after a specific period of time and is termed the inhibition index for such systems, even though it does not represent the actual intracellular ratio.

For biological systems in which high concentrations of inhibitor and substrate cannot be employed, e.g., embryonated eggs, the amount of the substrate synthesized by or present in the system must be considered. The inhibition index would be $[I] /\left(\left[S_{1}\right]+\left[S_{2}\right]\right)$, where $\left[S_{1}\right]$ represents the contribution of the biological system and $\left[S_{2}\right]$ represents the contribution of the exogenous supply to the total concentration of substrate.

If an inhibitor reacts with the enzyme-substrate complex, a constant inhibitor-substrate ratio for a defined inhibition is not obtained, and the inhibition is of the noncompetitive type.

Even though a vitamin is initially utilized as substrate in the formation of a coenzyme, an analogue may prevent the combination of this coenzyme with an apoenzyme. This can be illustrated by the following:

$$
\begin{aligned}
& E_{a}+\mathrm{Co} \rightleftharpoons E_{a} \mathrm{Co} \\
& E_{a}+I \rightleftharpoons E_{a} I \\
& E_{a} \mathrm{Co}+S \rightleftharpoons E_{a} \mathrm{Co} S \longrightarrow P+E_{a} \mathrm{Co}
\end{aligned}
$$

where $E_{a}$, Co and $I$ represent the apoenzyme, coenzyme and inhibitor, respectively. $E_{a} \mathrm{Co}$, and $E_{a} I$ represent the complete enzyme and the apoenzyme inhibitor complex, respectively. $S, E_{a} C o S$ and $P$ represent the 
substrate, enzyme-substrate complex and the product of the enzyme system. The following equation can be derived in a manner analogous to equation (3):

$$
\frac{[I]}{[\mathrm{Co}]}=\frac{K_{I}\left[E_{a} I\right]}{K_{C}\left[E_{a} \mathrm{Co}\right]}
$$

where $K_{I}$ and $K_{C}$ are the dissociation constants for the apoenzyme-inhibitor complex and for the complete enzyme, respectively. By the Law of Mass Action:

$$
\frac{[S]\left[E_{a} \mathrm{Co}\right]}{\left[E_{a} \mathrm{Cos} S\right]}=K_{S}
$$

where $K_{S}$ is the dissociation constant of the enzyme-substrate complex.

The total apoenzyme concentration, $\left[E_{a_{t}}\right]$ becomes

$$
\left[E_{a_{\ell}}\right]=\left[E_{a}\right]+\left[E_{a} I\right]+\left[E_{a} \mathrm{Co}\right]+\left[E_{a} \mathrm{Co} S\right]
$$

In the application of these equations to the determination of the effect of the concentrations of inhibitor and coenzyme on the degree of inhibition of a biological system, the rate of the reaction in which the final product, $P$, is formed would be expected to govern the rate of the biological process. The effect of any change in substrate concentrations, resulting from the lack of the complete enzyme in optimal concentration, would be a function of time under the experimental conditions. For a defined degree of inhibition after a constant experimental period, the rate of the reaction is limited in the manner previously indicated for substrate inhibition. Consequently, the concentration of the enzyme-substrate complex, $\left[E_{a} \mathrm{CoS}\right]$, must be reduced to a defined amount at the outset of the biological process in order to attain a defined inhibition. Since $[S]$ is not a variable at the outset of the experiment, it is apparent from equation (8) that the concentration of $E_{a} \mathrm{Co}$ must be reduced initially to a defined amount. Since the concentration of $\left[E_{a}\right]$ becomes negligible, in comparison with $\left[E_{a} I\right]$, with increasing concentrations of inhibitor and coenzyme, and since the total enzyme concentration is considered to be constant, it follows from equation (9) that $E_{a} I$ must then become a defined concentration initially in order for a defined inhibition to be obtained.

Since both $E_{a} C_{o}$ and $E_{a} I$ must initially be defined quantities for a defined inhibition, it follows from equation (7) that the ratio of inhibitor to coenzyme necessary for a defined inhibition is constant. Consequently, the inhibition index may be applied to such a system.

The problem of the reverse rate of reaction being so slow that equilibrium conditions are not attained becomes a reality for reactions of many apoenzymes with their coenzymes or analogues of the coenzymes. In such cases, it is essential that both the inhibitor and coenzyme be added 
simultaneously in order to study the enzyme system; otherwise, one of the factors may combine with the enzyme completely before the other has a chance to combine with the apoenzyme. This problem exists particularly with isolated and nongrowing systems.

Effect of Substrate on Velocity of Inhibited Enzymatic Reactions. ${ }^{17}, 18$ In some instances, determination of the rate of a biological process involving an inhibited enzyme system has advantages over the inhibition index method both in ease of obtaining experimental data and in its interpretation. This is true particularly for biological systems in which concentrations of inhibitor and substrate sufficiently high to approach enzyme saturation cannot be employed. Such rate studies are frequently employed in the elucidation of the mechanisms of inhibition of isolated enzyme systems. The equations which may be applied to a general system of this type are analogous to those previously indicated in the derivation of the inhibition index. Under these conditions of suboptimal substrate concentrations the possibility of the combination of the inhibitor with the enzyme-substrate complex as well as with the free enzyme must be considered. Thus,

$$
\begin{aligned}
& E+S \rightleftarrows E S \longrightarrow P+E \\
& E+I \rightleftarrows E I \\
& E S+I \rightleftarrows E S I
\end{aligned}
$$

where the symbols represent the quantities previously indicated in the inhibition index method, and ESI represents the enzyme-substrateinhibitor complex. By the Law of Mass Action, equations (1) and (2) apply to the dissociation of the enzyme-substrate complex and to the dissociation of the enzyme-inhibitor complex. Similarly, the dissociation constant, $K_{S I}$, of the enzyme-substrate-inhibitor complex can be obtained.

$$
\frac{[E S][I]}{[E S I]}=K_{S I}
$$

The total enzyme concentration, $\left[E_{t}\right]$, may be represented as follows:

$$
\left[E_{t}\right]=[E]+[E I]+[E S]+[E S I]
$$

Solving equation (1) for $[E]$, equation (2) for $[E I]$, and equation (10) for $[E S I]$ and substituting these values in equation (11), one obtains:

$$
E_{\imath}=\frac{K_{S}[E S]}{[S]}+\frac{K_{S}[E S][I]}{K_{I}[S]}+[E S]+\frac{[E S][I]}{K_{S I}}
$$

The velocity of the enzymatic reaction is proportional to [ES], so that $r=k[E S]$, where $r$ is the rate and $k$ is the rate constant of the reaction. The maximum rate, $R$, of the enzymatic reaction is similarly proportional to the total enzyme concentration since $[E S]$ becomes equal to $\left[E_{t}\right]$ 
under such conditions. Thus, the ratio, $R / r$ is equal $\left[E_{t}\right] /[E S]$ for which an expression can be obtained from equation (12) by dividing by $[E S]$ :

$$
\frac{\left[E_{t}\right]}{[\overline{E S}]}=\frac{R}{r}=\frac{K_{S}}{[S]}+\frac{K_{S}[I]}{K_{r}[S]}+1+\frac{[I]}{K_{S I}}
$$

These equations can be applied with slight modification to (a) enzymatic reaction in the absence of an inhibitor, (b) competitive inhibition in which the inhibitor combines only with the free enzyme and not with the enzyme-substrate complex and (c) noncompetitive inhibition in which the inhibitor combines with the enzyme-substrate complex and may or may not combine with the free enzyme.

In the absence of an inhibitor, the $[E I]$ and $[E S I]$ terms of equation (11) are zero, and the corresponding terms of equation (13) can be omitted to obtain an expression for the velocity of an enzymatic reaction in terms of the concentration of the substrate.

$$
\frac{1}{r}=\frac{K_{S}}{R[S]}+\frac{1}{R}
$$

In the case of competitive inhibition, the inhibitor does not combine with the enzyme-substrate complex, so that the $[E S I]$ term of equation (11) and the corresponding term of equation (13) can be omitted.

$$
\frac{1}{r}=\frac{1}{[S]}\left[\frac{K_{S}}{R}+\frac{K_{S}[I]}{K_{I} R}\right]+\frac{1}{R}
$$

For strictly noncompetitive inhibition, the inhibitor combines with both the enzyme and the enzyme-substrate complex; so equation (13) rearranged applies:

$$
\frac{1}{r}=\frac{1}{[S]}\left[\frac{K_{S}}{R}+\frac{K_{S}[I]}{K_{I} R}\right]+\frac{1}{R}+\frac{[I]}{R K_{S I}}
$$

Since the dissociation constants, $K_{I}$ and $K_{S I}$ may not be identical, the extreme can be represented by a competitive inhibition in which the inhibitor does not combine with the enzyme-substrate complex and by a noncompetitive enzyme-substrate inhibition in which inhibitor does not combine with the free enzyme but combines only with the enzymesubstrate complex. In the latter case, which has been termed "uncompetitive" inhibition, ${ }^{18}$ the $[E I]$ term of equation (11) and the corresponding term of equation (13) can be omitted:

$$
\frac{1}{r}=\frac{K_{S}}{R[S]}+\frac{1}{R}+\frac{[I]}{R K_{S I}}
$$

In order to determine the type of inhibition, the rate of an enzymatic reaction is determined over a wide range of substrate concentrations in the absence of an inhibitor and at two or more concentrations of inhibitor. 
By plotting the reciprocal of the rate, $1 / r$, against the reciprocal of the concentration of the substrate, $1 /[S]$, a linear relationship should result at a constant concentration of inhibitor. The characteristics of slope and extrapolated intercept at $1 /[S]=0$ are as follows for various types of inhibitions.

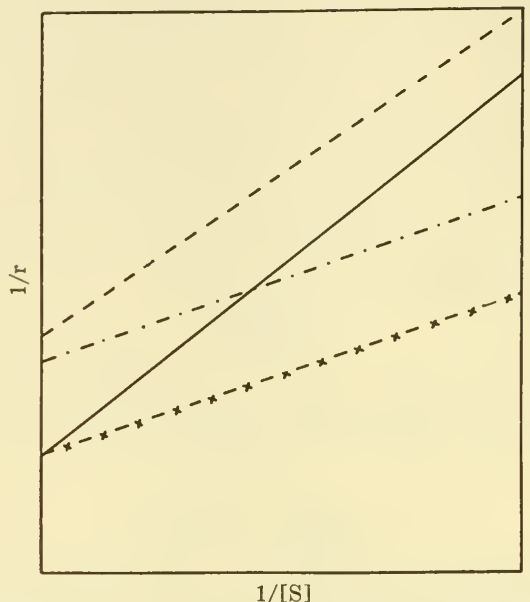

Figure 1. Relationship of reciprocal of the rate of an enzymatic reaction to the reciprocal of substrate concentration as a method of determining type of inhibition.

Competitive Inhibition

-... Noncompetitive Inhibition

$-\mathrm{x}-\mathrm{x}-\mathrm{x}-\mathrm{x}$ In absence of Inhibitor

For the uninhibited enzyme reaction, the extrapolated intercept should represent $1 / R$, where $R$ is the maximum rate of the enzymatic reaction at enzyme saturation, and the slope should be $K_{S} / R$. The ratio of slope to intercept is the dissociation constant of the enzyme-substrate complex.

For competitive inhibition, the intercept for various concentrations of the inhibitor is constant, $1 / R$. However, the slope of the linear relationship is $K_{S} / R+K_{S}[I] / K_{I} R$, and is dependent upon the concentration of the inhibitor.

For noncompetitive inhibition, both the slope and intercepts are changed. The usual case is such that the combination of the inhibitor with either enzyme or enzyme-substrate complex occurs with approximately equal affinity, so that $K_{I}$ and $K_{S I}$ are approximately equal. Under 
such circumstances the slope and the intercept are increased by a factor of $1+[I] / K_{I}$, as compared to the linear relationship in the absence of the inhibitor.

For the specific case of noncompetitive inhibition ("uncompetitive in-

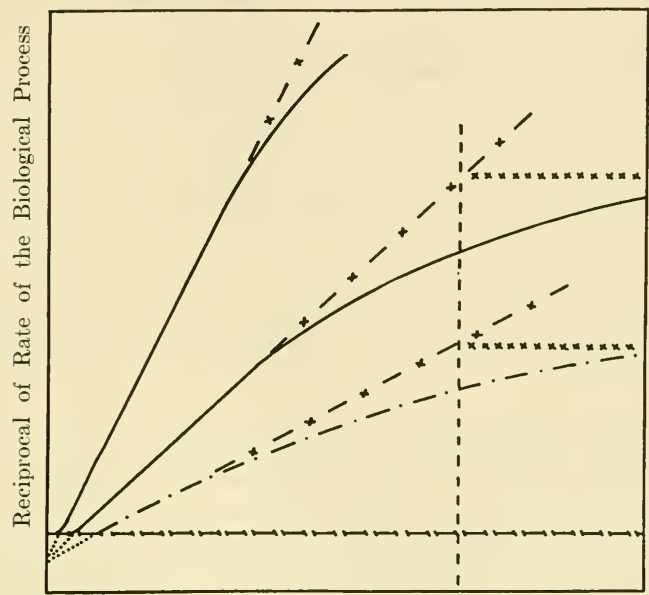

$1 /[\mathrm{S}]$

Figure 2. Relationship of the reciprocal of the rate of a biological process to the reciprocal of the substrate concentration.

-.... Relationship in the absence of an inhibitor.

Relationship in the presence of a competitive inhibitor at a defined concentration.

ーーーールー Maximum rate of the biological process at optimal substrate concentrations.

Extrapolated theoretical values for rate exceeding the maximum rate of the biological process which is then limited by other enzymatic processes.

_. . . - Maximum value of $1 /[\mathrm{S}]$ for biological systems either synthesizing or containing unknown amounts of substrate.

$\mathrm{x} \times \mathrm{x} \times \mathrm{x} \times \mathrm{x}$ Maximum value of the reciprocal of rate of the biological process for systems synthesizing or containing unknown amounts of substrate under experimental conditions.

$-\mathrm{x}-\mathrm{x}-\mathrm{x}-\mathrm{x}$ Extension of theoretical relationships for biological systems incapable of synthesis of substrate or not containing unknown amounts of substrate.

hibition") in which the inhibitor combines only with the enzyme-substrate complex, addition of the inhibitor does not change the slope as compared to the linear relationship in the absence of inhibitor; but the intercept increases by a factor of $1+[I] / K_{S I}$.

These changes are indicated in Figure 1, which illustrates the varia- 
tions of slope and intercept which distinguish competitive inhibition from noncompetitive inhibitions.

A more specialized type of inhibition, "quadratic" inhibition, has been described, in which the plots for different concentrations of inhibitor have different intercepts and begin with near zero slopes which change to a common slope identical with that in the absence of the inhibitor. ${ }^{18}$ This is easily differentiated from competitive inhibition.

In a consideration of the rates of biological processes rather than of isolated enzymes, the application of the methods of differentiation of competitive inhibition from other types by rate studies is somewhat complicated by the interrelationship of the rate of the biological process and the rate of the specific inhibited enzymatic reaction. In the presence of the inhibitor at effective concentrations, the rate of the specific enzymatic reaction is the limiting process in eliciting an observable response from the biological system. Under such conditions, the reciprocal of the rate of the biological process plotted against the reciprocal of the substrate concentration would give a linear function over a defined region in the plot. The extrapolated intercept would not represent the maximum rate of the biological process, but rather the theoretical maximum rate of the biological process when the inhibited enzyme is saturated with substrate and is still the limiting reaction. This is a purely hypothetical condition, since many enzyme systems other than the specific one concerned would most likely be somewhat less efficient under these conditions. As indicated in Figure 2, the rate can increase only to the maximum rate of the complete biological process and not to the maximum theoretical rate of the specific enzyme.

Another complication is the fact that many biological systems have the ability to synthesize the substrate. Hence, while the concentration of the substrate supplied to the system can be diminished to zero, the concentration synthesized by the biological system limits the lowest concentration of substrate attainable. Thus, the value of $1 /[S]$ can be increased only to a definite value, and further decreases in exogenous supply of the substrate do not alter the rate of the biological process. This is indicated in Figure 2. With increasing $1 /[S]$ values the transition from the normal linear relationship of $1 /[S]$ versus $1 / r$ to the constant minimum rate of the biological process for a defined concentration of inhibitor may be either an abrupt change or a gradual one depending upon the system. For example, there may be a very abrupt change in systems in which the concentration of substrate outside the biological system is not appreciably altered by the biological system, and the concentration of substrate within and without the system attain equilibrium. Concentrations of exogenous substrate below that synthesized by the system would not 
alter the internal concentration of the factor, while concentrations of the externally supplemented substrate higher than that synthesized by the system would attain an equilibrium with the concentration within the system. Since the amount of substrate synthesized would merely be additive to total external and internal concentrations, the total external and resulting internal concentration would not be materially affected by such synthesis in many systems, e.g., inhibition of growth of a small inoculum in bacterial growth medium.

On the other hand, the transition may be gradual in some systems, e.g., embryonated eggs, in which the amount of substrate synthesized or initially present is appreciable in comparison with the total amount of substrate supplied. The transition indicated in Figure 2 is minimized to indicate the intermediate effect between two possible extremes.

These effects have not seriously limited the application of this method in determining the type of inhibition in certain biological systems, but may have altered some of the quantitative aspects of data derived by the method.

This method was applied by Wyss ${ }^{19}$ to the effect of $p$-aminobenzoic acid on the growth inhibition of Escherichia coli resulting from the action of sulfanilamide. The results indicate that sulfanilamide competitively inhibits the functioning of $p$-aminobenzoic acid. The rate of growth of the organism inhibited by sulfanilamide was considered to be proportional to the rate of the inhibited enzymatic reaction, and the total enzyme concentration of the bacterial cell was assumed to be constant, since mother and daughter cells have the same enzyme concentration. Essentially, it is assumed that no resistance to sulfonamide develops under the testing conditions. Although some resistance may develop during the determination of growth rate, it does not seriously affect the qualitative results, but may affect conclusions which are based on quantitative calculation. This method has also been applied to the reversal by $p$-aminobenzoic acid of the toxicity of other sulfonamides for Escherichia coli. ${ }^{20}$

By a method similar to that used in the derivation of the above rate equations, an expression can be derived for the competitive inhibition of the combination of a coenzyme with an apoenzyme. Thus

$$
\frac{1}{r}=\frac{K_{S} K_{C}}{R[S][C o]}\left[1+\frac{[I]}{K_{I}}\right]+\frac{1}{R} \frac{K_{S}}{[S]}+1
$$

where the symbols are identical with those previously indicated. In the graphical presentation of the data with $1 / r$ plotted against $1 /[\mathrm{Co}]$, the intercept of $1 / r$ at $1 /[\mathrm{Co}]=0$ does not change with changes in inhibitor concentration, but the slope of the linear relationship increases with increasing concentrations of inhibitor. 
Biological Action of Analogues of B Vitamins. Although some vitamin analogues are converted in vivo to the corresponding vitamin, such is not always the case. An analogue may combine with the enzyme involved in the conversion of the vitamin to its coenzyme. The enzyme-analogue complex either may be nonfunctional, resulting in competitive inhibition, or it may function in a manner identical with that of the vitamin. In the latter case an analogue of the normal coenzyme will be formed. If the modified coenzyme cannot be utilized by the proper apoenzyme, the result is still a competitive inhibition of coenzyme formation. However, if modified coenzymes resulting from the analogue can be utilized by the apoenzymes, the analogue is capable of replacing the vitamin in the biological system. Some modified coenzymes apparently can be utilized by only some of the apoenzymes which normally combine with the natural coenzyme. In such cases, supplements of the products of the enzyme systems which cannot utilize the modified form of the coenzyme are essential for the vitamin-like activity of an analogue which can replace all other coenzyme functions of the vitamin. Similar considerations apply in case more than one coenzyme is derived from the vitamin. Only one of the coenzymes might be replaced by a similar product derived biologically from the analogue.

The analogue of a vitamin may prevent the utilization of a vitamin at any stage in the biosynthesis and functioning of the coenzyme; however, if two successive stages in the utilization of a vitamin are inhibited by a single analogue, the vitamin would be capable of preventing the inhibition only over a small range of concentrations.

Structural Modifications Producing Vitamin Analogues witl Biological Activity. In order for a compound to inhibit the functioning of or replace a natural metabolite in a biological system, the substance must possess the ability to combine with a particular enzyme utilizing the metabolite. The ability to combine with a particular enzyme at the specific point at which the metabolite associates with the enzyme is determined not only by the presence of certain functional groups which are instrumental in effecting such a combination, but also upon the shape, size and configuration of the molecule. Consequently, most of the compounds which either replace or inhibit a vitamin in its functioning in a biological system are related structurally to the vitamin.

The types of modifications which produce compounds with such biological activities include replacement of groups occurring in the metabolite as follows: $-\mathrm{S}-$ by $-\mathrm{CH}=\mathrm{CH}-,-\mathrm{CH}_{2}-\mathrm{CH}_{2}-$ or $-\mathrm{SO}_{2}-$; benzene nucleus by aromatic heterocyclic nuclei such as thiophene, pyrimidine, pyridine, etc. nuclei; $-\mathrm{COOH}$ by $-\mathrm{SO}_{2}-\mathrm{NH}_{2},-\mathrm{SO}_{3} \mathrm{H},-\mathrm{SO}_{2}-\mathrm{R},-\mathrm{SO}-\mathrm{R}$, $-\mathrm{PO}_{3} \mathrm{H}_{2},-\mathrm{PO}_{2} \mathrm{H},-\mathrm{AsO}_{3} \mathrm{H}_{2},-\mathrm{CH}_{2} \mathrm{OH},-\mathrm{COR}$, etc.; $-\mathrm{CH}_{3}$ by $-\mathrm{Cl}$, 
$-\mathrm{C}_{2} \mathrm{H}_{5}$, etc. $-\mathrm{NH}_{2}$ by $-\mathrm{OH},-\mathrm{NO}_{2}$, etc.; $-\mathrm{H}$ by $-\mathrm{Cl},-\mathrm{CH}_{3},-\mathrm{OH}$, etc., and these and other groups by such similar groups.

A number of excellent reviews by Woolley, ${ }^{21-2+}$ by Welch, ${ }^{25}$ and by Roblin ${ }^{26}$ have indicated the developments in the general field of competitive inhibitors of the biochemical functioning of essential metabolites and have indicated more extensively the changes in structure most likely to produce metabolic antagonists.

The biological activities of the $\mathrm{B}$ vitamins and related compounds including modifications such as these indicated above are listed in subsequent chapters. The biological activities in replacing the vitamin are indicated in terms of per cent activity on a molar basis relative to the particular $B$ vitamin. The inhibitory activities are indicated in terms of the inhibition index which, unless otherwise indicated, is the lowest ratio of the concentration of the inhibitor to the concentration of the corresponding $B$ vitamin at which maximum inhibition of the biological system occurs.

\section{Bibliography}

1. Embden, G., and Haymann, C., Z. physiol. Chem., 137, 154 (1924).

2. Lohmann, K., Biochem. Z., 222, 324 (1930).

3. Embden, G., and Deuticke, H. J., Z. physiol. Chem., 230, 50 (1934).

4. Warburg, O., and Christian, W., Naturwiss., 29, 589 (1941); Biochem. Z., 310, 384 (1941).

5. Tammann, G., Z. physiol. Chem., 16, 271 (1892); Armstrong, E. F., Proc. Soc. Roy. Soc. (London), 73, 516 (1904).

6. Wohl, A., and Glimm, E., Biochem. Z., 27, 349 (1910).

7. Kuhn, R., Z. physiol. Chem., 129, 57 (1923).

8. Euler, H. v., and Josephson, K., Z. physiol. Chem., 157, 122 (1926).

9. Quastel, J. H., and Woolridge, W. R., Biochem. J., 22, 689 (1928).

10. Gross, R. E., Z. physiol. Chem., 112, 236 (1920).

11. Coombs, H. I., Biochem. J., 21, 1259 (1927).

12. Loewi, O., and Navratil, E., Arch. ges. Physiol. (Pfluger's), 214, 678 (1926).

13. Woods, D. D., and Fildes, P., Chem. Ind., 59, 133 (1940).

14. Woods, D. D., Brit. J. Exptl. Path., 21, 74 (1940).

15. Michaelis, L., and Menten, M. L., Biochem. Z., 49, 333 (1913).

16. Shive, W., "Conference on Development and Uses of Antimetabolites," New York Acad. Sci., Feb., 1949 (in press).

17. Lineweaver, H., and Burk, D., J. Am. Chem. Soc., 56, 658 (1934).

18. Ebersole, E. R., Guttentag, C., and Wilson, P. W., Arch. Biochem., 3, 399 (1944).

19. Wyss, O., Proc. Soc. Exptl. Biol. Med., 48, 122 (1941).

20. Klotz, I. M., and Gutmann, H. R., J. Am. Chem. Soc., 67, 558 (1945).

21. Woolley, D. W., Science, 100, 579 (1944).

22. Woolley, D. W., Harvey Lectures, Ser. 41, 189 (1946).

23. Woolley, D. W., Advances in Enzymol., 6, 129 (1946).

24. Woolley, D. W., Physiol. Revs., 27, 308 (1947).

25. Welch, A. D., Physiol. Revs., 25, 687 (1945).

26. Roblin, R. O., Chem. Revs., 38, 255 (1946). 


\section{UTILIZATION OF COMPETITIVE ANALOGUE-METABOLITE INHIBITION IN THE ELUCIDATION OF BIOCHEMICAL PROCESSES INVOLVING VITAMINS}

Rapid methods of developing relatively specific assays for metabolites of biochemical systems and of elucidating the biochemical processes involving these substances are desirable in the study of biochemistry. One of the recently developed methods whereby this can be accomplished involves a study of the effects of known metabolites and of biological extracts on a specific competitive analogue-metabolite inhibition of a biological system. These effects can be studied by a variety of testing techniques to determine the interrelationship of the metabolite to the substances affecting the inhibition. The ability of unknown substances in natural extracts to exert an influence on the system can be used as a basis for assay of unknown naturally occurring substances directly related to the metabolite. Consequently, this method, which has been termed inhibition analysis, offers a direct approach to specific problems of biochemistry which may otherwise be difficult to solve.

\section{Theoretical Considerations}

From a theoretical standpoint, ${ }^{1-5}$ exogenous substances other than the metabolite which are capable of preventing the inhibitory effect of the analogue in competitive analogue-metabolite inhibitions of biological systems include (1) substances which increase the effective concentration of the metabolite, e.g., precursors of the metabolite; $(2)$ the product, or its equivalent, of the inhibited enzymatic reaction; (3) substances which decrease the quantity of the product necessary for normal functioning of the biological system, i.e., substances exerting a "sparing action" on the product of the inhibited enzyme system; $(4)$ agents increasing the effective concentration of the inhibited enzyme; and (5) substances which assist in the destruction of the inhibitory analogue.

(1) Precursor Effect. The effective concentration of the metabolite may be increased in a biological system by supplementation of the system with several types of substances other than the metabolite. The most common is a limiting precursor, an additional amount of which allows the organism to synthesize an increased concentration of the metabolite. 
However, the addition of a catalytic factor normally limiting the biosynthesis of the metabolite may also cause an increased synthesis, resulting in a higher concentration of the metabolite in the biological system. Further, if the metabolite is utilized in reactions which are not essential for the response of the biological system, substances which prevent the loss of the metabolite by such pathways may also increase the effective concentration of the metabolite; but the magnitude of such an effect would usually be relatively small in comparison with other precursor effects.

A relatively simple testing technique has been developed which characterizes the precursor type of effect on a competitive analogue-metabolite inhibition of a biological system. The increased metabolite concentration resulting from enhanced synthesis by the biological system results in a corresponding increase in the amount of analogue (inhibitor) necessary for a defined inhibition. However, if the metabolite is supplied to the system in concentrations in excess of that from the enhanced synthesis by the biological system, substances which exert such a precursor effect do not appreciably influence the amount of inhibitor necessary for the defined inhibition in the presence of the metabolite at such a concentration. Thus, the minimum inhibitory concentration of the analogue is increased by such substances, but the inhibition index determined over the remainder of a range of concentrations of the metabolite is not altered.

In many instances increasing concentrations of a limiting precursor of a metabolite may prevent the inhibition caused by the analogue over a rather wide range of concentrations. However, the conversion of the precursor to the metabolite usually becomes less efficient at higher concentrations, and the concentration of precursor relative to the inhibitory analogue of the metabolite for a defined inhibition increases markedly with increasing concentrations.

(2) Product Effect. If the product of the inhibited enzymatic reaction is of such a nature that it can be supplied to the biological system from an external source, the inhibited enzyme system becomes nonessential for the biological process as a whole when this product is made available to the system in adequate quantities. If the analogue does not prevent the functioning of any other enzymes utilizing the metabolite, it is no longer inhibitory to the biological system.

However, the metabolite (substrate) may be utilized by several different enzymes involved in the synthesis of several products, $P_{1}, P_{2}, P_{3}$, etc., and the specific analogue, $I$, may prevent the conversion of the metabolite, $S$, to one or more of these products. If more than one system is inhibited by the analogue, one of these enzyme systems, e.g., $E_{1}$, would be expected to become the limiting reaction of the biological process 
before the others. The equations relating the inhibition index to equilibrium constants and other constants (p. 447) would apply to the particular enzyme system, $E_{1}$, and the inhibition index, $K_{1}$, would be related to this particular enzyme system.

Although the analogue may not prevent the formation of $P_{2}$, it may at higher concentrations prevent the conversion of $S$ to $P_{3}$ sufficiently to inhibit the biological system. Consequently, an exogenous supply of $P_{1}$ would not completely prevent the toxicity of the analogue, since another enzyme system, $E_{3}$, becomes the limiting process of the biological system, and the particular equilibrium, rate, and other constants of this enzyme determine the inhibition index, $K_{3}$. Thus, in the presence of adequate amounts of $P_{1}$, the inhibition index is increased from the $K_{1}$ value corresponding to the inhibition of the biosynthesis of $P_{1}$, to the value $K_{3}$, corresponding to the inhibition of the biosynthesis of $P_{3}$.

Since $P_{1}$ becomes the first limiting product, an external supply of $P_{3}$ would not be expected to exert any effect on the inhibition in the absence of $P_{1}$. However, if both $P_{1}$ and $P_{3}$ are supplied to the biological system, the analogue either becomes ineffective as an inhibitor of the system, or at a still higher inhibition index it prevents an additional function of the metabolite.

Hence, regardless of the metabolite concentration, the addition of the product of an inhibited enzyme system will result either in a complete reversal of the toxicity of the analogue, or in a higher analogue-metabolite ratio corresponding to the inhibition index of another enzyme system utilizing the metabolite. If an analogue prevents the formation of a series of products from the metabolite, there is a definite order in which the products must be added if the effect of each substance is to be demonstrated. In the absence of a product the biosynthesis of which is inhibited at a lower inhibition index, all other products the biosyntheses of which are inhibited at higher inhibition indices do not affect the inhibition index.

The general shape of the graph obtained by plotting the growth response of the biological system against increasing concentration of an inhibitor at a constant concentration of substrate is related to a specific enzyme system and is dependent upon the dissociation constants, etc., of the particular system (p. 447). Since two separate enzyme systems involving the same metabolite and inhibitory analogue would not be expected to have similar dissociation constants, etc., the general shape of such a graph or any type of data which depends upon such constants can be employed to show that different enzyme systems are involved. The ratio of the index for maximum inhibition to that for half-maximum inhibition can be useful in such cases. 
(3) "Sparing Effect" on the Product. The following equation can be obtained by solving equation (4) (p. 446) for $[E I]$ and substituting the value in equation (3) (p. 446).

$$
\frac{[I]}{[S]}=\frac{K_{I}\left(\left[E_{t}\right]-[E S]-[E]\right)}{K_{S}[E S]}
$$

If exogenous substances act in such a manner as to decrease the amount of product, $P$, necessary for a constant response of the biological system, the concentration of the enzyme-substrate complex must be decreased accordingly, to maintain a defined response of the system in the presence of such substances. This can be accomplished by increasing the ratio of inhibitor to substrate. Since $[E]$ is relatively small and can be neglected, and since $\left[E_{t}\right]$ is relatively constant and is large with respect to $[E S]$ under the testing conditions, it is apparent from equation (19) that the inhibition index varies approximately inversely with $[E S]$, which is defined by the amount of product necessary for the defined response of the biological system. Consequently, the decrease in the amount of product required by the biological system for a constant response as a result of the addition of substances exerting a "sparing action" is reflected by a proportional decrease in $[E S]$, and a practically proportional increase in the inhibition index.

If the product, $P$, of the inhibited enzyme system is involved in the biosynthesis of secondary products, $P_{a}, P_{b}, P_{c}$, etc., these secondary products can exert an effect on the inhibited biological system. If a secondary product is formed by a reversible reaction and if the reverse reaction can take place under the testing conditions, the secondary product will affect the inhibition in a manner analogous to the primary product. However, in many instances the secondary products cannot form the primary product by a reversible reaction. In such cases, one particular secondary product would be expected to become the substance limiting the biological response, and would be capable of exerting a "sparing action" on the primary product. A definite order in which the secondary products exert their effects would be expected.

While analogues of vitamins may in some cases inhibit at the coenzyme stage, analogues often prevent coenzyme formation from the vitamin, as illustrated in Figure 3. The coenzyme in turn is required by several apoenzymes to form a series of enzymes, $E_{a}, E_{b}, E_{c}$, etc. These apoenzymes vary in their relative concentrations and in their affinity for the coenzyme, and the enzymes are required by the biological system in different concentrations. Consequently, one particular enzyme system, e.g., $E_{a}$, requiring the coenzyme would be expected to become the limiting factor of the biological system. If the product, $P_{a}$, of such an enzyme is supplied 
to the biological system, a decreased rate of coenzyme formation would be essential to maintain the defined response. To decrease the rate of coenzyme formation, an increase in the inhibition index is required such that another enzyme system, e.g., $E_{c}$, requiring the coenzyme becomes the limiting system of the biological process. There is a definite order in which the secondary products exert their effects. If a biological system is somewhat deficient in a secondary substrate, e.g., $S_{a}$, when the conversion of this substrate to product, $P_{a}$, is the limiting reaction of the system, supplements of this substrate will allow the reaction to proceed more efficiently. To maintain the defined inhibition of the system, the concentration of the enzyme, $E_{a}$, must be decreased by decreasing the rate of coenzyme formation. This necessitates an increase in the ratio of analogue to vitamin. The increase in the inhibition index resulting from such additions will seldom approach that obtained with the product, $P_{a}$.

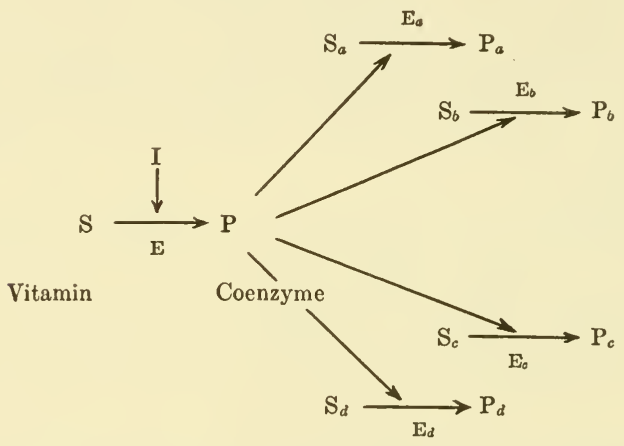

Frgore 3. Relationship of Enzymatic Reactions to Inhibition of Coenzyme Formation by Analogues of Vitamins

Substances which prevent the product of the inhibited enzyme reaction from being utilized in a manner not essential for the biological response would be expected to exert a "sparing action" on the product. Undoubtedly still other types of substances exerting such an action will be discovered.

(4) Changes in Total Effective Enzyme Concentration. From equation (19) it is apparent that exogenous substances which increase the total effective enzyme concentration, $E_{t}$, will affect an inhibition in a manner such that an increased ratio of analogue to metabolite is required in order to obtain the same degree of inhibition as in the absence of such substances. Since $[E S]$ is small in comparison with $\left[E_{t}\right]$ and is maintained at a defined value under these conditions where only $\left[E_{t}\right],[I]$ and 
$[S]$ are varied, the inhibition index varies approximately directly with the total effective enzyme concentration.

The substances which can produce such an increase in the effective enzyme concentration include (a) a limiting coenzyme or a precursor of a limiting coenzyme when excess apoenzyme is synthesized by the biological system; and (b) a limiting second substrate which is involved in the utilization of the inhibited substrate in the enzymatic reaction. Since the biological system normally synthesizes these substances in near optimal amounts, these effects usually are relatively moderate in comparison with the other types.

(5) Destruction of the Inhibitor. Substances which allow rapid destruction of an inhibitory analogue by the biological system will render the analogue ineffective as an inhibitor; however, if destruction occurs more slowly, other effects, e.g., one similar to a precursor effect, may be observed.

Other Theoretical Considerations. Reversing agents of type 1 are easily distinguished from those of types 2,3 , and 4 by determination of the inhibition index over a wide range of concentrations. Since substances of the latter three types exert their action by increasing the inhibition index, methods whereby these groups can be further differentiated are desirable. A number of substances exerting such an effect on competitive inhibition can usually be classified into the various groups. Since reversing agents of type 2 but not of types 3 and 4 involve more than one enzyme, data which involve the dissociation and other constants will permit classification of substances of type 2 . Hence, the ratio of the inhibition index for maximum to that for half-maximum inhibition would be expected to change with the addition of an agent of type 2, but not of types 3 and 4 . Reversing agents of type 3 and type 4 exert their effects independently of each other and are synergistic; but neither exerts an effect on the inhibition in the presence of the product of type 2 .

When two analogues prevent enzymatic reactions at two successive stages in a biosynthetic sequence, a synergistic action of the inhibitions is usually obtained. When the first biosynthetic transformation is inhibited to such an extent that the biosynthesis of the immediate product is reduced to half the normal rate, the amount of an analogue of the product necessary to inhibit completely the response of the biological system is reduced to half. However, the amount of the first analogue necessary to reduce the rate of the reaction to half the normal rate is often only a small fraction of that necessary to inhibit completely the response of the biological system. Consequently, the effects of the inhibitors are not additive but synergistic. Demonstration of such synergistic effects can often be useful in indicating such a biosynthetic sequence. 


\section{Application of Inhibition Analysis to Elucidation of Biochemical Transformations}

Biosynthesis of Pantothenic Acid. The specificity of the yeast assay for pantothenic acid which resulted in the discovery of the vitamin is dependent upon the presence of asparagine in the medium. ${ }^{6}$ Asparagine prevents the response of the organism to $\beta$-alanine, which in the absence of asparagine replaces pantothenic acid in stimulating the growth of yeast. Similar results have been obtained with a number of $\alpha$ - and $\beta$-amino acids, ${ }^{7}$ and with propionic acid ${ }^{8}$ (p. 645).

The involvement of aspartic acid in the biosynthesis of $\beta$-alanine and of pantothenic acid in Escherichia coli has been demonstrated with two analogues of aspartic acid. Cysteic acid ${ }^{9}$ and hydroxyaspartic acid ${ }^{1}$ competitively prevent the functioning of aspartic acid in Escherichia coli cultured in a salts-glucose medium. The inhibition indices are 30-100 and $3-16$, respectively. If a supplement of either $\beta$-alanine or pantothenic acid is added to the growth medium, cysteic acid does not affect the growth of the organism even at relatively high concentrations; but hydroxyaspartic acid at high concentrations still prevents it. The inhibition index in the latter case is increased to 20-30. Thus, cysteic acid apparently prevents only the conversion of aspartic acid to $\beta$-alanine, whereas hydroxyaspartic acid prevents that reaction and at least one additional transformation involving aspartic acid.

Inhibitions of the biosynthesis of pantothenic acid by several analogues of pantoic acid have been reported. Salicylic acid has been considered in this eategory ${ }^{10}$ (p. 646). Either $\alpha$-hydroxy- $\beta, \beta$-dimethylbutyric acid or $\beta, \gamma$-dihydroxy- $\beta$-methylbutyric acid prevents growth of Saccharomyces cerevisiae G. M. stimulated by $\beta$-alanine, but does not affect growth stimulated by pantothenic acid ${ }^{11}$ (p. 646); hence, these compounds apparently prevent the biosynthesis of pantothenic acid by preventing the combination of pantoic acid with $\beta$-alanine.

Biochemical Functions of Pantothenic Acid. A study of the effect of known metabolites on the inhibition of pantothenic acid synthesis by cysteic acid in Escherichia coli has been utilized in an effort to determine the metabolic function of pantothenic acid. ${ }^{12}$ As the organism becomes deficient in synthesis of the vitamin and the corresponding coenzyme, one particular secondary enzyme system would be expected to become the first limiting reaction for growth, since the various apoenzymes requiring the coenzyme would be expected to differ in their affinity for the coenzyme and in the quantity of the coenzyme required for normal enzymatic activity. Addition of the product of this secondary enzyme system would allow growth until the rate of synthesis of pantothenic acid was decreased 
to an extent such that another secondary enzyme system requiring the factor became the limiting reaction of the biological system. Since the rate of synthesis of pantothenic acid under the testing conditions is determined by the ratio of cysteic acid to aspartic acid, the addition of such a product of a secondary enzyme system would be expected to result in an increase in the inhibition index-a type 3 effect.

Such an effect is obtained with eitric acid, cis-aconitic acid, or $\alpha$-ketoglutaric acid. The inhibition index determined over a range of aspartic acid concentrations is increased approximately tenfold when one of these substances is added to the medium of Escherichia coli. Although inactive alone, supplements of oxalacetic and pyruvic acids together increased the inhibition index slightly. Acetate had a similar slight effect. These effects were not comparable to those of the tricarboxylic acids or of $\alpha$-ketoglutaric acid. Pantoic acid was inactive. Since the sparing action of citric acid, cis-aconitic acid, or $\alpha$-ketoglutaric acid cannot be duplicated by the precursors of the tricarboxylic acids, it appears that in Escherichia coli pantothenic acid functions in the biosynthesis of the tricarboxylic acids, citric and cis-aconitic acids, from oxalacetic acid and pyruvate or acetate. It has since been shown that coenzyme $\mathrm{A}$, which was found to contain pantothenic acid while the work just mentioned was being completed, ${ }^{13}$ functions in the oxidation of acetate in yeast ${ }^{14}$ and in the formation of citric acid by pigeon liver from oxalacetic acid and acetate. ${ }^{15}$ Phenylpantothenone has been reported to prevent the conversion of pantothenic acid to coenzyme A in yeast. ${ }^{16}$

The "sparing action" of $\alpha$-ketoglutaric acid on pantothenic acid and the precursor effect of glutamic acid in the biosynthesis of aspartic acid result in an unusual effect of glutamic acid on the toxicity of cysteic acid for Escherichia coli. Apparently a very rapid transamination with oxalacetic acid results in the formation of essentially equivalent amounts of aspartic acid from supplementary glutamic acid. The presence of $\alpha$-ketoglutaric acid results in a "sparing action" on the product of the system such that glutamic acid is 3 to 10 times as effective as aspartic acid in preventing the toxicity of cysteic acid. ${ }^{9}$ This puzzling situation of an apparent precursor of a metabolite being more effective than the metabolite itself led to the elucidation of the complete cycle in which glutamic acid simultaneously acts as a limiting precursor and as the product of a secondary enzyme system utilizing the product of the blocked enzymatic reaction as indicated in Figure 4.

At an inhibition index of approximately 3,000, N-pantoyl- $n$-butylamine inhibits the utilization of pantothenic acid by Lactobacillus arabinosus 17-5. A supplement of either oleic acid or "Tween 80 " increases the inhibition index approximately tenfold to a value of 30,000 . Since either acetate 


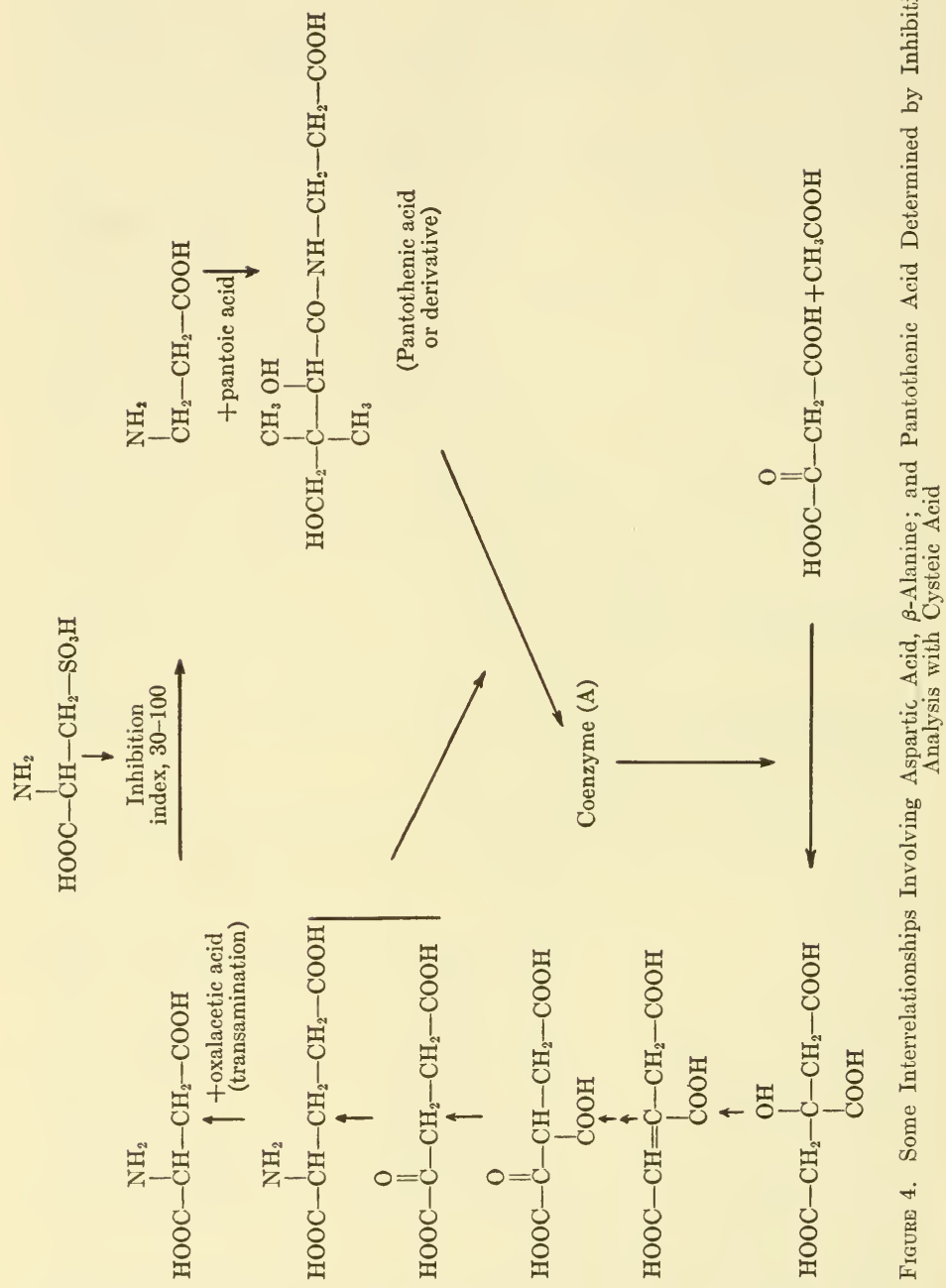


or oleic acid is required by Lactobacillus arabinosus for growth, it appears that pantothenic acid functions in the conversion of acetate to oleic acid or its equivalent. ${ }^{12}$

A strain of Leuconostoc mensenteroides which requires either acetate or an aromatic amino acid (phenylalanine, tryptophan or tyrosine) for growth is inhibited by $\mathrm{N}$-pantoyl- $n$-butylamine. ${ }^{5}$ The inhibition is prevented competitively by pantothenic acid, resulting in an inhibition index of 300 in the presence of either aromatic amino acids or acetate; but in the presence of both an aromatic amino acid and acetate the inhibition index is increased to 3,000 . Phloroglucinol, particularly in the presence of increased phosphate, was just as effective as the aromatic amino acids, which are interchangeable in exerting this effect. Although ineffective in replacing the aromatic compounds, sterols such as cholesterol and coprosterone exert a sparing effect on the amount of phloroglucinol or aromatic amino acids necessary to prevent the toxicity of the analogue of pantothenic acid. The data suggest that pantothenic acid functions in the biosynthesis of an intermediate common to the biosynthesis of all these aromatic amino acids, and possibly to the biosynthesis of sterols which are not readily reconverted to the intermediate. Phloroglucinol, which can be considered a condensation product of three acetate radicals, either is such an intermediate or is converted to it by the organism. ${ }^{5}$

In studies with Proteus morganii, the relative effects of glutamic acid and cis-aconitic acid suggested that glutamic acid might be involved in pantothenic acid metabolism in still an additional manner. Accordingly, a number of synthetic conjugates of pantothenic acid, including those with all the naturally occurring amino acids and with certain peptides, have been prepared. ${ }^{5}$ The conjugates containing glutamic acid were somewhat more active than pantothenic acid in preventing the toxicity of analogues of pantothenic acid for certain lactic acid bacteria. ${ }^{5}$ Concentrates of coenzyme A contain glutamic acid and glycine, ${ }^{17}$ as well as cysteine or cystine. ${ }^{18}$ A naturally occurring conjugate of pantothenic acid is also reported to contain glutamic acid. ${ }^{19}$

Biochemical Interrelationships Involving Biotin. The inhibitory effect of $\gamma$-(3,4-ureylenecyclohexyl) butyric acid on the growth of Lactobacillus arabinosus is prevented competitively by biotin, resulting in an inhibition index of approximately 30,000 in a medium from which aspartic acid is omitted. ${ }^{20}$ Either aspartic acid or oxalacetic acid affects the inhibition in such a manner that the inhibition index is increased to approximately 300,000 . Although sodium bicarbonate, particularly in the presence of pyruvate, exerts an effect on the inhibition index, the two substances together are not as effective as either aspartic acid or oxalacetic acid. Oleic acid or "Tween 80 " has no effect on the inhibitory action of the 
analogue in the absence of an exogenous aspartic acid supplement; but the inhibition index determined in the presence of both aspartic and oleic acids varies from $1,000,000$ to $10,000,000$. Often when the inhibition index is relatively low $(1,000,000)$, cis-aconitic acid and related tricarboxylic acids increase the inhibition index several fold. However, the effect of the tricarboxylic acids is not always obtained; consequently, even if the tricarboxylic acids are essential products, presumably derived from $\alpha$-ketoglutaric acid, additional enzymatic reactions involving biotin are essential for growth of Lactobacillus arabinosus. The implications of these data are indicated in Figure 5, which shows that the biotin analogue presumably prevents the formation of a coenzyme which is either directly or indirectly concerned with the carboxylation of pyruvic acid to form aspartic acid, the biosynthesis of oleic acid or an analogous product, and at least one additional product. ${ }^{5,20}$

The carboxylation of pyruvic acid as a function of biotin was independently and almost simultaneously discovered in three different laboratories. ${ }^{21}, 20,22,23$ An interrelationship of aspartic acid and biotin had been established for yeast much earlier,24 and was extended to bacteria. ${ }^{25}$ The ability of oleic acid to replace the nutritional requirement of some microorganisms for biotin had been previously reported. ${ }^{26}$

Homobiotin, which inhibits the utilization of biotin by Lactobacillus casei at a relatively low inhibition index, does not inhibit the growth of that organism in a medium containing oleic acid, ${ }^{27}$ even at relativeiy high concentrations. Since analogues of biotin do not usually inhibit the utilization of biotin synthesized by an organism, it is possible that oleic acid as a limiting product reduces the biotin requirement of Lactobacillus casei to such a point that the organism is capable of synthesis of the small amount of the vitamin needed for other enzyme systems. The possibility of complete replacement of a vitamin by all the products of the enzyme systems in which it functions must be considered in this case. In view of the results with Lactobacillus arabinosus, it appears that synthesis of the small biotin requirement by the organism may be the most logical explanation.

Desthiobiotin competitively prevents the toxicity of 2-oxo-4-imidazolidinecaproic acid for Escherichia coli. ${ }^{28}$ The inhibition index is approximately 100 . Since biotin, at slightly greater concentration than the lowest giving any response, prevents the toxicity of even relatively high concentrations of the desthiobiotin analogue, it appears that the analogue prevents the formation of biotin from a metabolite identical with or similar to desthiobiotin. A supplement of either glutamic acid or $\alpha$-ketoglutaric acid prevents the toxicity of the inhibitor in such a manner as to increase the inhibition index to 300 . Either glutamic acid or $\alpha$-keto- 
glutaric acid exerts a "sparing effect" on biotin which results in an increase in the ratio of analogue to desthiobiotin necessary for the defined inhibition of growth of Escherichia coli. ${ }^{20}$ Since neither cis-aconitic acid nor citric acid exerted such an effect, one would suppose by analogy with the carboxylation of pyruvic acid that biotin functions in the decarboxylation of oxalsuccinic acid. ${ }^{20}$

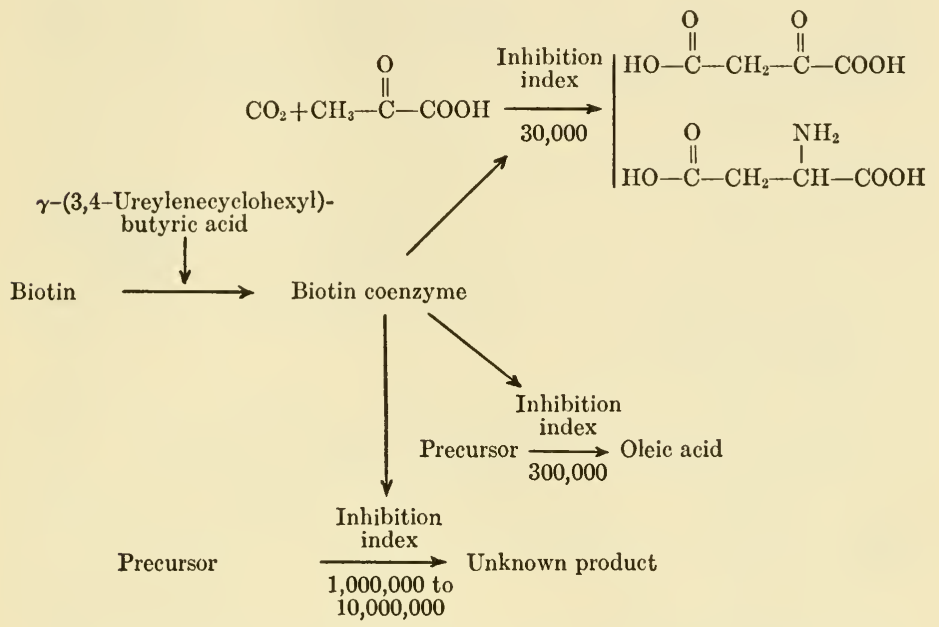

Figure 5. Interrelationships of Biotin Indicated by Inhibition Analysis with $\boldsymbol{\gamma}$-(3,4-Ureylenecyclohexyl)butyric acid

Biochemical Functions of $p$-Aminobenzoic Acid and Folic Acid. Substances other than $p$-aminobenzoic acid which prevent the toxicity of sulfonamides have been known to occur in natural extracts for some time. ${ }^{29}, 30$ Methionine ${ }^{30-33}$ has some ability-which is enhanced by purines ${ }^{32,33}$ - to prevent the toxicity of sulfanilamide for Escherichia coli. Adenine or hypoxanthine is reported to be as active on a weight basis as $p$-aminobenzoic acid in preventing the protective action of sulfanilamide against infections of Streptococcus hemolyticus in mice. ${ }^{34}$ Purines under specific conditions also prevent the toxicity of sulfonamides for lactic acid bacteria ${ }^{35}$ and for Eremothecium ashbyii. ${ }^{36}$

Such effects of structurally unrelated compounds were early considered to be evidence against the competitive analogue-metabolite theory of sulfonamide action. However, adequate explanations for such action are 
offered by inhibition analysis, which allows some insight into the metabolic functions of this and related vitamins.

Sulfanilamide, in preventing the functioning of $p$-aminobenzoic acid in Escherichia coli, inhibits a series of biochemical transformations, as indicated in Figure 6. 57,38 The inhibition index determined in a salts-<smiles>NC(CCS)C(=O)O</smiles>

Homocysteine (or related precursor)<smiles>CSCCC(N)C(=O)O</smiles>

Methionine<smiles>Cc1nccnc1/N=C\C=O</smiles>

5(4)-Amino-4(5)-imidazolecarbox-

amide (or derivative)<smiles>NCC(=O)O</smiles>

Glycine<smiles>CNC(C(=O)O)C(C)O</smiles>

Threonine

Precursor<smiles>Cc1c[nH]c(=O)[nH]c(=O)c1=O</smiles>

Hypoxanthine Xanthine Adenine Guanine or derivatives

Figure 6. Interrelationships Involving $p$-Aminobenzoic Acid Determined by Inhibition Analysis with Sulfanilamide

glucose medium is approximately 3000 ; but in the presence of supplements of methionine a higher ratio, approximately 10,000 , is required for the same degree of inhibition of growth of the organism. ${ }^{37}$ The inhibition is further affected by certain purines, which are ineffective in the absence of supplementary methionine. ${ }^{37}$ The inhibition index is approximately 30,000 when determined in a medium containing both methionine and purines. The purines and derivatives effective in replacing the purine 
requirement under these conditions include adenosine, xanthine, guanine and inosine. Adenine not only is ineffective, but is usually synergistic with sulfonamides in preventing growth of Escherichia coli under these conditions. ${ }^{37}$ Thus, the first limiting enzymatic reaction involves the biosynthesis of methionine, probably from homocysteine, since the latter, a precursor, does not affect the inhibition. ${ }^{5,38}$ The next limiting transformation, which becomes apparent when exogenous methionine is supplied to the organism, is the biosynthesis of purines, presumably from 5(4)amino-4(5)-imidazolecarboxamide or a derivative of this amine, since the amine accumulates in the medium under these conditions of sulfonamide inhibition. ${ }^{39,40}$ Although the amine stimulates growth of Lactobacillus arabinosus in a manner similar to purines and disappears slowly from the medium during the process, ${ }^{5}$ many organisms including Escherichia coli cannot utilize the amine, and it remains unchanged in the medium. It appears likely that the free amine is derived from a conjugated form, e.g., riboside or desoxyriboside, which is the normal precursor of purines.

Glycine, which increases the production of the aminoimidazolecarboxamide in Escherichia coli, is apparently a precursor of the amine. ${ }^{41} \mathrm{Al}-$ though less effective than glycine, threonine similarly increases the production of the amine and is apparently converted into glycine by the organism. The inability of serine to replace glycine, particularly in view of reported mutants of Escherichia coli requiring either serine or glycine for growth, indicates that the conversion of serine to glycine is blocked by sulfanilamide. ${ }^{41}$

The involvement of $p$-aminobenzoic acid in the biosynthesis of serine is further indicated by the effect of serine on the inhibition of growth of Escherichia coli by sulfanilamide in a medium containing both methionine and purines. ${ }^{38}$ The inhibition index is increased from 30,000 to 50,000 100,000 by supplements of serine under these conditions. ${ }^{5,38}$

Thymine and folic acid are somewhat interchangeable in affecting the inhibition index determined in a medium containing methionine, purines and serine. ${ }^{38}$ The inhibition index is usually increased two- or threefold to approximately 200,000 to $300,000 .^{5,38}$ High concentrations of sulfanilamide which are necessary for determination of these high inhibition indices often do not affect solely enzymes utilizing $p$-aminobenzoic acid; consequently, the results are sometimes variable. ${ }^{5,38}$

For each of these products to exert its effect on the inhibition of growth of Escherichia coli by sulfanilamide, the products must be added in a definite sequence as indicated in Figure 6 . All products whose biosyntheses are related to lower inhibition indices must be available to the organism from an exogenous supply in order that a product whose biosynthesis is 
related to a higher inhibition index may exert its effect on the inhibition. However, the order in which the products exert their effect may vary to some extent under different testing conditions and with inocula treated differently. For example, it is occasionally possible in Escherichia coli to obtain an effect with folic acid in the absence of serine.

The requirement of certain mutant strains of Escherichia coli for $p$-aminobenzoic acid can be replaced by a mixture of amino acids, purines and thymine, indicating further the involvement of $p$-aminobenzoic acid in the biosynthesis of these substances. ${ }^{42}$

The index for inhibition of growth of Lactobacillus arabinosus by sulfanilamide is increased from 100 to 1000 by purines. ${ }^{37}$ For this organism and Streptobacterium plantarum 10S, thymine at high concentrations in the presence of purines is reported to prevent the toxicity of sulfonamides over a wide range of concentrations. ${ }^{43}$ Both purines and thymine are necessary to replace the requirement of $p$-aminobenzoic acid for growth of Lactobacillus arabinosus. ${ }^{43}$ Thymine exerts a similar effect on the toxicity of sulfanilamides for certain strains of Streptococcus faecalis and Streptococcus zymogenes. ${ }^{44}$

One common feature in the metabolic reactions in which $p$-aminobenzoic acid participates is the involvement of a single carbon unit in the final product. Hence, it appears that $p$-aminobenzoic acid functions directly or indirectly in the introduction of single carbon units into purines, pyrimidines, serine (from glycine), and methionine (from homocysteine or a related precursor). A close association of all these single carbon units with the possibility of a common precursor is suggested by these results. The incorporation of isotopically labelled formate in the 2 and 8 positions of uric acid previously indicated a single carbon unit precursor for the purines. ${ }^{45}$ The involvement of a common precursor was further indicated recently by results indicating the incorporation of isotopically labelled formate into the $\beta$-carbon atom of serine, ${ }^{46}$ and the incorporation of isotopically labelled methyl groups of choline into the $\beta$-carbon atom of serine. $^{47}$

On the basis of published research the interrelationship of $p$-aminobenzoic acid and folic acid cannot be completely described. Folic acid has little or no activity in replacing the nutritional requirement of a number of organisms for $p$-aminobenzoic acid. However, sulfonamides prevent the biosynthesis of a microbiologically active form(s) of folic acid in a wide variety of organisms which do not require folic acid for growth (p. 490). Furthermore, the biological functions of folic acid closely parallel those of $p$-aminobenzoic acid. Thus, thymine and purines replace or partially replace the folic acid requirement of certain organisms, ${ }^{48-50}$ and with Lactobacillus casei, inhibition analysis indicates that folic acid 
functions in the biosynthesis of these substances. ${ }^{51}$ The inhibition index obtained with methylfolic acid, a competitive analogue of folic acid, is 30 in the absence of purines, 100 in their presence, and 1000 in the presence of both purines and thymine. ${ }^{51}$ Thymine is inactive alone. For Streptococcus faecalis $\mathrm{R}$, the toxicity of methylfolic acid is completely prevented by both purines and thymine. ${ }^{52}$ Purines alone exert a very slight effect on the inhibition index, and thymine alone exerts no effect. An involvement of folic acid in the biosynthesis of serine in Streptococcus faecalis $\mathrm{R}^{53}$ appears to be similar to the involvement of $p$-aminobenzoic acid in the same biosynthesis in Escherichia coli.

The involvement of $p$-aminobenzoic acid in biosyntheses involving a single carbon unit led to a search for folic acid derivatives capable of serving as a formate carrier. Pteroylhistidine which was prepared synthetically did not exert any pronounced activity. However, the structure of rhizopterin, $\mathrm{N}^{10}$-formylpteroic acid, gave a clue as to how formate could be carried by a functional derivative of folic acid. Accordingly, formylfolic acid was prepared and found to be approximately 30 times as active as folic acid in preventing the toxicity of methylfolic acid for Streptococcus faecalis $\mathrm{R}$. The derivative was just as active as folic acid in promoting growth of this and a number of other organisms requiring folic acid. ${ }^{54}$

While it is not possible as yet on the basis of published results to conclude whether $p$-aminobenzoic acid and folic acid are converted to an identical coenzyme, or whether two or more different coenzymes are involved, inhibition analysis applied to natural extracts has been useful in the discovery of a form of folic acid which is more widely active, and of other related factors from which a solution to the perplexing problem appears possible in the near future.

\section{Utilization of Inhibition Analysis in the Development of Assays for Naturally Occurring Unknown Factors}

The techniques of inhibition analysis can be used to develop microbiological assays for unknown factors which may be difficult to detect by other means. This offers a specific, direct approach to the discovery and isolation of factors related to a specific metabolite. Such a problem presented itself recently when folic acid was found to be effective in the treatment of pernicious anemia, but was not the anti-pernicious anemia principle (s) of refined liver extracts. It was highly desirable at that time to develop specific assays for unknown substances related to folic acid or $p$-aminobenzoic acid and occurring in refined liver extracts. It has been reported that through the use of inhibition analysis and related approaches more than twenty different assays have been developed for 
unidentified substances related to $p$-aminobenzoic acid, folic acid or related factors. ${ }^{5}$

Thymidine. One particular factor was especially interesting since it prevented the toxicity of methylfolic acid for Leuconostoc mesenteroides 8293 and the toxicity of either sulfanilamide or 6,7-diphenyl-2,4-diaminopteridine for Lactobacillus arabinosus $17-5 .^{5,55}$ Since an inhibition analysis indicated that the factor was a product of the biological functioning of folic acid and $p$-aminobenzoic acid, the high activity of refined liver extracts suggested the possibility that the factor might be a conjugated form of folic acid. However, on isolation of the factor from hog liver, a colorless crystalline compound was obtained and identified as thymidine which was present in some refined liver extracts to the extent of 1 per cent of the solids. ${ }^{55}$

The inhibition index for Leuconostoc mesenteroides inhibited by methylfolic acid is increased from 3,000 to 30,000 by the addition of thymidine to the medium. Although thymine and thymidine are interchangeable for many organisms, thymine is inactive in the above tests. Consequently, the biosynthesis of thymidine does not appear to take place through the intermediate formation of thymine. ${ }^{55}$

The Vitamin $B_{12}$ Group. Of the many tests developed for factors occurring in refined liver extracts, five were found to involve a functionally related group of factors involved in the biochemical functioning, of $p$-aminobenzoic acid in Escherichia coli. ${ }^{5}$ One testing method utilized sulfanilamide in a concentration sufficient to prevent the biosynthesis of methionine in Escherichia coli grown in a salts-glucose medium supplemented with known vitamins, xanthine, thymine, serine and glutamic acid. Under these conditions, the organism responded to very small amounts of refined liver extract; and by use of this assay technique, a crystalline red compound was isolated from refined liver extracts after a 20,000-fold concentration. ${ }^{5}$ Because of its distinctive color and biological properties, the factor was termed "erythrotin." This factor is apparently identical with or closely related to vitamin $\mathrm{B}_{12}{ }^{5,56}$ Still another factor, which moves more slowly in organic solvents used as eluants during various chromatographic separations, is also active in the Escherichia coli assay; this has been tentatively named "erythrotide." 5 The root prefix erythro- has been suggested as a basis for naming the individual members of this group of factors which have the same biological function but slightly different chemical structures.

Although methionine replaces erythrotin for Escherichia coli under the testing conditions, the latter compound is from 100,000 to 300,000 times as active. Consequently, no interference by methionine was encountered 
in the isolation of the factors from liver or in the assay of relatively good sources of this group of factors. ${ }^{5}$

Whether the biosynthesis of methionine, purines, serine or thymine (or folic acid) is the limiting reaction in the growth of Escherichia coli inhibited by sulfanilamide, the inhibition index is increased about threefold by the addition of erythrotin at a concentration of $0.0005 \gamma$ per $10 \mathrm{cc}$ as indicated in Table 1.5 This effect is not enhanced by increasing the concentrations of erythrotin, even to $0.1 \gamma$ per 10 cc. From these results it is apparent that erythrotin plays a catalytic role in the biosynthesis of methionine, purines, serine, thymine and additional products. The catalytic role of erythrotin involves either the conversion of $p$-aminobenzoic acid to the coenzyme form involved in these syntheses or a separate catalytic role in combining the single carbon unit into these metabolites. Thus, the close association of the functioning of the vitamin $\mathrm{B}_{12}$ group and $p$-aminobenzoic acid is further indicated. The biological action of other unknown factors which are currently being isolated by similar techniques will further clarify the exact role of this new vitamin.

TABLE 1. Effect of Erythrotin on Sulfanilamide Inhibition of E. coli

\begin{tabular}{|c|c|c|}
\hline \multirow[b]{2}{*}{ Supplement } & \multicolumn{2}{|c|}{ Inhibition Index } \\
\hline & $\begin{array}{l}\text { Without added } \\
\text { Erythrotin }\end{array}$ & $\begin{array}{l}\text { With added } \\
\text { Erythrotin } \\
0.0005 \gamma \text { per } 10 \mathrm{cc}\end{array}$ \\
\hline None & 3,000 & 10,000 \\
\hline Methionine, $100 \gamma$ per $10 \mathrm{ec}$ & 10,000 & 30,000 \\
\hline $\begin{array}{l}\text { Methionine, } 100 \gamma \text { per } 10 \mathrm{cc} \\
\text { Xanthine, } 100 \gamma \text { per } 10 \mathrm{cc}\end{array}$ & 30,000 & 100,000 \\
\hline $\begin{array}{l}\text { Methionine, } 100 \gamma \text { per } 10 \mathrm{cc} \\
\text { Xanthine, } 100 \gamma \text { per } 10 \mathrm{cc} \\
\text { Serine, } 100 \gamma \text { per } 10 \mathrm{cc}\end{array}$ & $50,000-100,000$ & $200,000-300,000$ \\
\hline $\begin{array}{l}\text { Methionine, } 100 \gamma \text { per } 10 \mathrm{cc} \\
\text { Xanthine, } 100 \gamma \text { per } 10 \mathrm{cc} \\
\text { Serine, } 100 \gamma \text { per } 10 \mathrm{cc} \\
\text { Folic acid, } 0.03 \gamma \text { per } 10 \mathrm{cc}\end{array}$ & $100,000-200,000$ & $300,000-500,000$ \\
\hline
\end{tabular}

It is interesting that neither thymidine nor other desoxyribosides which replace the vitamin $\mathrm{B}_{12}$ group for both Lactobacillus lactis and Lactobacillus leichmanni is active in replacing erythrotin for Escherichia coli. ${ }^{5}$ Similarly ascorbic acid, glutathione and related compounds at concentrations which can replace vitamin $\mathrm{B}_{12}$ under certain conditions for these lactic acid bacteria do not affect appreciably the response of Escherichia coli in the sulfonamide assay. ${ }^{5}$

\section{Mechanisms of Resistance to Competitive Inhibitors}

If an organism is allowed to grow in the presence of sub-inhibitory concentrations of a vitamin analogue or drug, the concentration of the in- 
hibitor can usually be increased gradually until the organism becomes resistant to relatively high concentrations of the inhibitory compound. The resistance acquired by the organism in the early stages of the development of resistant strains is usually lost after the organism is cultured in the absence of the inhibitor. The initial resistance may be highly specific for the inlibitory analogue, or the organism may be sensitive to analogues of similar structure. However, the resistance gained after prolonged culturing in the presence of the inhibitor usually is relatively permanent, and may be specific not for the individual inhibitor but for the majority, if not all, of the inhibitory analogues of the vitamin, the functioning of which is prevented by the inhibitor against which the resistance is developed.

The development of resistant strains of an organism would be expected to result either from a selection of a naturally occurring mutant strain, or from an induced mutation, or a combination of both. Obviously, in the presence of the drug, the environment favors the selection of natural resistant strains, and no convineing data indicating that an inhibitor specifically induces the mutation to a strain which is resistant have been presented. While the process of development of resistance is gradual in most instances, suggesting that the final mutant strain which possesses the resistance is not present in the initial population, the possibility exists that continual selection of progeny more and more resistant to the inhibitor allows the isolation of a mutant strain which occurs normally, but only so infrequently that an ordinary culture would have little chance initially of containing a single such resistant cell.

Many types of biochemical differences between normal strains of an organism sensitive to an inhibitory analogue of a metabolite and strains resistant to the inhibitor could account for the development of resistant strains. Biochemical differences between resistant and parent strains include: (1) increase in the biosynthesis and concentration of the metabolite in the cells; (2) increase in the effective concentration of the inhibited enzyme; (3) increase in the synthesis of other factors limiting the utilization of the product of the inhibited enzyme system, i.e., factors which exert a "sparing action" on the product; (4) more extensive development of other mechanisms by which the product of the inhibited enzyme system is synthesized; (5) presence of an enzyme of slightly different structure with normal affinity for the metabolite, but with less or no affinity for the analogue, i.e., different cells of the same organism may not necessarily produce structurally identical molecules of an enzyme catalyzing the same reaction in all the cells, and such variations could conceivably occur within a single cell; (6) presence of an enzyme system which destroys the inhibitor; (7) decrease in cell permeability specifically to the inhibitor. 
Many organisms resistant to an inhibitory analogue do produce inereased amounts of the metabolite. This can be demonstrated by assay of the cultures for the metabolite. Although in such a case considerably more of the analogne is required for inhibition of growth in the absence of an exogenous supply of the metabolite, the inhibition index appearing with increased supplemental concentrations of the metabolite does not differ from that of the normal strain.

For resistant strains of organisms showing increased inhibition indices, classification in the above groups is difficult, since resistant organisms of all the types 2-5 would be expected to have increased inhibition indices. If the resistant organisms of type 4 are able to dispense completely with the normal route of biosynthesis, such organisms would be expected to be completely resistant to the analogue.

Destruction of the inhibitor by the organism is known to account for the resistance in some strains which tolerate larger amounts of the inhibitor. If the rate of destruction of an inhibitory analogue is not too rapid, the inhibition index determined at high concentrations of metabolite and inhibitor would not be expected to be appreciably altered.

Specific phases of acquired resistance to the inhibitory action of analogues are discussed in subsequent sections.

\section{Competitive Metabolite Antagonists and Biochemical Genetics}

When Escherichia coli is sub-cultured in an inorganic salts-glucose medium containing sulfanilamide and methionine as well as glycine, serine and xanthine, a strain develops which requires methionine for growth ${ }^{58}$ and cannot utilize homocystine. ${ }^{59}$ The mutant strain does not develop in the absence of either sulfanilamide or methionine. Though evidence has been presented which indicates that the mutant strains of this type have a greater growth rate than the parent strain, ${ }^{60}$ the mechanism by which the mutant strain is obtained appears to be more complex than that of spontaneous mutation and selection. This mutant strain is particularly interesting, since sulfanilamide prevents the biosynthesis of methionine at the stage corresponding to the deficiency of the mutant strain. ${ }^{59}$ Similarly, a strain of Escherichia coli requiring both methionine and purines has been obtained from serial sub-cultures in the presence of sulfanilamide in a medium containing both methionine and purines. ${ }^{61}$ The purine requirements of the mutant strain are analogous to the requirements of the parent strain cultured in the presence of sulfonamides. ${ }^{61}$ From these results, it appears possible that competitive inhibitors of metabolites may play a role in the elucidation of the biochemical relationship of enzyme to gene.

From crosses of a sulfanilamide-resistant strain to wild type, a strain of Neurospora which appeared to require sulfanilamide for growth was 
obtained ${ }^{62} p$-Aminobenzoic acid inhibited the growth-promoting action of sulfanilamide in a competitive manner. The ratio of $p$-aminobenzoic acid to sulfanilamide giving growth inhibition was approximately 0.001 . Sulfanilamide was not specific, since sulfapyridine and sulfathiazole also promoted growth. The latter was the most effective. A double mutant obtained from crosses of the sulfonamide-requiring strain and a $p$-aminobenzoic acid-requiring strain required both $p$-aminobenzoic acid and sulfanilamide for growth, a maximum being obtained at a ratio of sulfanilamide to $p$-aminobenzoic acid of approximately $1000{ }^{63}$ Investigation of this double mutant revealed that low concentrations of $p$-aminobenzoic acid stimulated the growth of the organism in the absence of sulfanilamide, but that higher concentrations of $p$-aminobenzoic acid were toxic to the organism. ${ }^{64}$ The toxicity of $p$-aminobenzoic acid at these higher concentrations could be prevented by sulfanilamide. Thus, the sulfonamide-requiring mutant produced more than the tolerated amount of $p$-aminobenzoic acid. ${ }^{64}$ The detrimental enzymatic transformations involved in the utilization of $p$-aminobenzoic acid are inhibited by sulfanilamide, which thereby prevents the toxicity of excess $p$-aminobenzoic acid. A similar situation exists with yeasts for which thiamine is toxic, and the toxicity is prevented by pyrithiamine. ${ }^{65}$

More and more it is being realized that the basis for many of the genetic blocks of enzymatic reactions involves inhibitions by normal metabolic products of the organism. These inhibitions are not unrelated in character to those obtained with synthetic analogues of metabolites.

\section{Bibliography}

1. Shive, W., and Macow, J., J. Biol. Chem., 162, 451 (1946).

2. Shive, W., and Roberts, E. C., J. Biol. Chem., 62, 463 (1946).

3. Beerstecher, E., Jr., and Shive, W., J. Biol. Chem., 164, 53 (1946).

4. Harding, W. M., and Shive, W., J. Biol. Chem., 174, 743 (1948).

5. Shive, W., Trans. New York Acad. Science, in press; presented before the New York Acad. of Science, Feb., 1949

6. Williams, R. J., and Rohrmann, E., J. Am. Chem. Soc., 58, 695 (1936); Weinstock, H. H., Jr., Mitchell, H. K., Pratt, E. F., and Williams, R. J., J. Am. Chem. Soc. 61, 1421 (1939).

7. Nielsen, N., Naturwiss., 31, 146 (1943) ; Nielsen, N., and Johansen, G., Naturwiss., 31, 235 (1943) ; Hartelius, V., Naturwiss., 31, 440 (1943); Nielsen, N., Hartelius, V., and Johansen, G., Naturwiss., 32, 294 (1944); Chem. Abstr., 40, 3154 (1946); Compt. rend. trav. lab. Carlsberg, Ser. physiol., 24, 39 (1944); Chem. Abstr., 40, 5796 (1946); Sarett, H. P., and Cheldelin, V. H., J. Bact., 49, 31 (1945); Kjerulf-Jensen, K., and Schmidt, V., Acta Pharmacol. Toxicol. (Copenhagen), 1, 346 (1945); Chem. Abstr., 40, 6541 (1946).

8. Wright, L. D., and Skeggs, H. R., Arch. Biochem., 10, 383 (1946); King, T. E., and Cheldelin, V. H., J. Biol. Chem., 174, 273 (1948).

9. Ravel, J. M., and Shive, W., J. Biol. Chem., 166, 407 (1946).

10. Ivanovies, G., Naturwiss., 30, 104 (1942); Z. physiol. Chem., 276, 33 (1942). 
11. Cheldelin, V. H., and Schink, C. A., J. Am. Chem. Soc., 69, 2625 (1947).

12. Shive, W., Ackermann, W. W., Ravel, J. M., and Sutherland, J. E., J Am. Chem. Soc., 69, 2567 (1947).

13. Lipmann, F., Kaplan, N. O., Novelli, G. D., Tuttle, L. C., and Guirard, B. M., J. Biol. Chem., 167, 869 (1947).

14. Novelli, G. D., and Lipmann, F., J. Biol. Chem., 171, 833 (1947).

15. Stern, J. R., and Ochoa, S., J. Biol. Chem., 179, 491 (1949).

16. Novelli, G. D., and Lipmann, F., Federation Proc., 7, 177 (1948).

17. Gordon, M., and Shive, W., unpublished work.

18. Lipmann, F., Kaplan, N. O., and Novelli, G. D., Federation Proc., 6, 272 (1947).

19. King, T. E., Locher, L. M., and Cheldelin, V. H., Arch. Biochem., 17, 483 (1948); King, T. E., Fels, I. G., and Cheldelin, V. H., J. Am. Chem. Soc., 71, 131 (1949).

20. Shive, W., and Rogers, L. L., J. Biol. Chem., 169, 453 (1947).

21. Eakin, R. E., and Benz, L. G., unpublished work; Benz, L. G., M. A. Thesis, University of Texas, August, 1947.

22. Lardy, H. A., Potter, R. L., and Elvehjem, C. A., J. Biol. Chem., 169, 451 (1947).

23. Lichstein, H. C., and Umbreit, W. W., J. Biol. Chem., 170, 329 (1947).

24. Koser, S. A., Wright, M. H., and Dorfman, H., Proc. Soc. Exptl. Biol. Med., 51, 204 (1942).

25. Stokes, J. L., Larsen, H., and Gunness, M., J. Biol. Chem., 167, 613 (1947).

26. Williams, V. R., and Fieger, E. A., Ind. Eng. Chem., Anal. Ed., 17, 127 (1945); J. Biol. Chem., 170, 619 (1947).

27. Shive, W., unpublished observations.

28. Rogers, L. L., and Shive, W., J. Biol. Chem., 169, 57 (1947).

29. Loomis, T. A., Hubbard, R. S., and Neter, E., Proc. Soc. Exptl. Biol. Med., 47, 159 (1941).

30. Kohn, H. I., and Harris, J. S., J. Pharmacol. Exptl. Therap., 73, 383 (1941).

31. Bliss, E. A., and Long, P. H., Bull. Johns Hopkins Hosp., 69, 14 (1941).

32. Harris, J. S., and Kohn, H. I., J. Biol. Chem., 141, 989 (1941).

33. Kohn, H. I., and Harris, J. S., J. Pharmacol. Exptl. Therap., 73, 343 (1941); 77, 1 (1943).

34. Martin, G. J., and Fisher, C. V., J. Biol. Chem., 144, 289 (1942).

35. Snell, E. E., and Mitchell, H. K., Arch. Biochem., 1, 93 (1943).

36. Schopfer, W. H., and Guilloud, M., Helv. Physiol. Pharmacol. Acta, 4, C24 (1946).

37. Shive, W., and Roberts, E. C., J. Biol. Chem., 62, 463 (1946).

38. Winkler, K. C., and de Haan, P. G., Arch. Biochem., 18, 97 (1948).

39. Stetten, M. R., and Fox, C. L., Jr., J. Biol. Chem., 161, 333 (1945).

40. Shive, W., Ackermann, W. W., Gordon, M., Getzendaner, M. E., and Eakin, R. E., J. Am. Chem. Soc., 69, 725 (1947).

41. Ravel, J. M., Eakin, R. E., and Shive, W., J. Biol. Chem., 172, 67 (1948).

42. Lampen, J. O., Roepke, R. R., and Jones, M. J., J. Biol. Chem., 164, 789 (1946).

43. Lampen, J. O., and Jones, M. J., J. Biol. Chem., 170, 133 (1947).

44. Lampen, J. O., and Jones, M. J., J. Biol. Chem., 166, 435 (1946).

45. Sonne, J. C., Buchanan, J. M., and Delluva, A. M., J. Biol. Chem., 173, 69 (1948);

Buchanan, J. M., and Sonne, J. C., J. Biol. Chem., 166, 781 (1946).

46. Sakami, W., J. Biol. Chem., 176, 995 (1948).

47. Sakami, W., J. Biol. Chem., 179, 495 (1948).

48. Snell, E. E., and Mitchell, H. K., Proc. Nat. Acad. Sci., 27, 1 (1941).

49. Stokstad, E. L. R., J. Biol. Chem., 139, 475 (1941).

50. Stokes, J., J. Bact., 48, 201 (1944).

51. Rogers, L. L., and Shive, W., J. Biol. Chem., 172, 751 (1948).

52. Stokstad, E. L. R., Regan, M., Franklin, A. L., and Jukes, T. H., Federation Proc., 7, 193 (1948).

53. Holland, B. R., and Meinke, W. W., J. Biol. Chem., 178, 7 (1949). 
54. Gordon, M., Ravel, J. M., Eakin, R. E., and Shive, W., J. Am. Chem. Soc., 70, 878 (1948).

55. Shive, W., Eakin, R. E., Harding, W. M., Ravel, J. M., and Sutherland, J. E., J. Am. Chem. Soc., 70, 2299 (1948).

56. Rickes, E. L., Brink, N. G., Koniuszy, F. R., Wood, T. R., and Folkers, K., Science, 107, 396 (1948).

57. Smith, E. L., and Parker, L. F. J., Biochem. J., 43, viii (1948).

58. Kohn, H. I., and Harris, J. S., J. Bact., 44, 717 (1942).

59. Lampen, J. O., Roepke, R. R., and Jones, M. J., Arch. Biochem., 13, 55 (1947).

60. Roepke, R. R., Libby, R. L., and Small, M. H., J Bact., 48, 401 (1944).

61. Alexander, E. A. R., and Shive, W., unpublished work.

62. Emerson, S., and Cushing, J. E., Federation Proc., 5, 379 (1946).

63. Emerson, S., J. Bact., 54, 195 (1947).

64. Zalokar, N., Proc. Natl. Acad. Sci., U.S., 34, 32 (1948).

65. Rabinowitz, J. C., and Snell, E. E., Federation Proc., 8, 240 (1949). 


\section{Chapter IIID}

\section{p-AMINOBENZOIC ACID}

The discovery by Woods ${ }^{1}$ in 1940 that $p$-aminobenzoic acid, first synthesized by Fischer ${ }^{2}$ in 1863, is an essential metabolite was not the result of nutritional studies, but was made on the basis of the ability of the factor to prevent the bacteriostasis of sulfanilamide and related compounds. Even the evolvement of the sulfonamides and related compounds as chemotherapeutic agents was almost as novel, particularly since sulfanilamide had been synthesized by ${ }^{~ G e l m o}{ }^{3}$ in 1908, approximately a quarter of a century before the chemotherapeutic action of this type of compound was discovered.

The inhibitory activity of many dyes against bacteria in vitro led to the preparation of a number of azo compounds including those in which diazotized sulfanilamide was coupled with a number of aromatic amines and related compounds. ${ }^{4.5}$ The activity of one of these compounds, prontosil, ${ }^{6}$ attracted widespread attention because of its effectiveness in vivo,

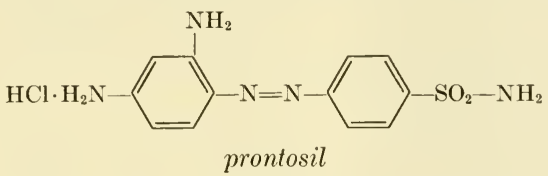

particularly against staphylococcal and $\beta$-hemolytic streptococcal septicemia. ${ }^{7}$ However, the compound was inactive in preventing the growth of these bacteria in vitro. Also, it was found that a number of active compounds could be prepared by coupling diazotized sulfanilamide with a variety of aromatic amines and phenols; but the sulfanilamide portion of the molecule was highly specific, since a wide variety of other diazotized amines did not form active compounds on coupling with these same aromatic amines and phenols. ${ }^{8}$ The specificity of sulfanilamide in the synthesis of active azo derivatives and the fact that the azo compounds were not effective in vitro led to the discovery that sulfanilamide was fully active in replacing prontosil. ${ }^{8}$ Reduction of the azo group in vivo apparently allows the formation of the active principle, which is also active in vitro.

Studies of the mechanism of the bacteriostasis produced by sulfanila- 
mide led to indirect evidence suggesting that inactivation of essential enzymes was involved. ${ }^{9,10}$ Extracts of streptococci, ${ }^{11}$ Brucella abortus ${ }^{12}$ and yeast ${ }^{1}$ were effective in preventing the toxicity of sulfanilamide for streptococci; and the active substance from yeast extract reversed inhibition in a manner reminiscent of the competitive inhibition of enzyme reactions by substances structurally related to the substrate. ${ }^{1}$ By testing substances structurally related to sulfanilamide, Woods ${ }^{1}$ discovered that $p$-aminobenzoic acid was extremely effective in preventing competitively the toxicity of sulfanilamide, and that the compound exhibited a high degree of specificity.<smiles>Nc1ccc(C(=O)O)cc1</smiles>

$p$-aminobenzoic acid

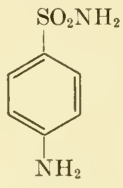

sulfanilamide

It was proposed that $p$-aminobenzoic acid is an essential metabolite synthesized in adequate amounts by many organisms but that it might be an essential growth factor for some organisms. ${ }^{1}$

Within a short time the nutritional importance of the compound was demonstrated by Rubbo and Gillespie, ${ }^{13}$ who isolated $p$-aminobenzoic acid as the N-benzoyl derivative from yeast, and demonstrated that $p$-aminobenzoic acid is an essential growth factor for Clostridium acetobutylicum. $p$-Aminobenzoic acid was subsequently isolated from yeast in the free form and as the $\mathrm{N}$-acetyl derivative by Blanchard, ${ }^{14}$ and as the methyl ester by Kuhn and Schwartz. ${ }^{15}$ Many other organisms were subsequently shown to require this factor.

\section{Specificity}

The specificity of the nutritional requirements of various organisms for $p$-aminobenzoic acid, as well as the specificity of this substance in preventing the toxicity of sulfonamides for various organisms, is indicated in Table 2, in which the activities of various compounds related to $p$-aminobenzoic acid are listed.

A majority of the compounds which possess activity in replacing $p$-aminobenzoic acid are substances which presumably can be easily converted to $p$-aminobenzoic acid by the various organisms. These include 4 -aminocyclohexanecarboxylic acid, $p$-nitrobenzoic acid, $p$-hydroxylaminobenzoic acid, N-glycosido- $p$-aminobenzoic acids, esters and certain amide derivatives of $p$-aminobenzoic acid-all of which can be converted 


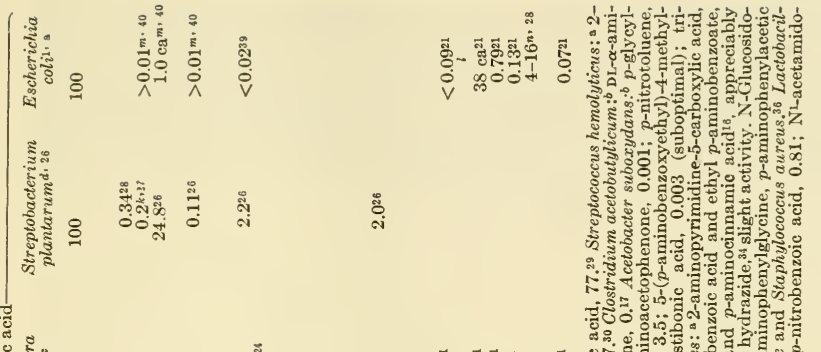

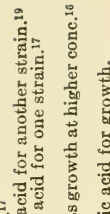
ב.

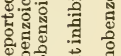

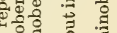
t.e. ฮ है है है

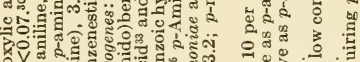

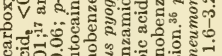
क० 궁.

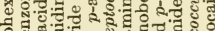
웅.

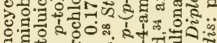

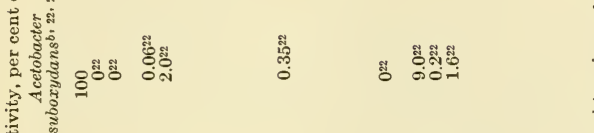

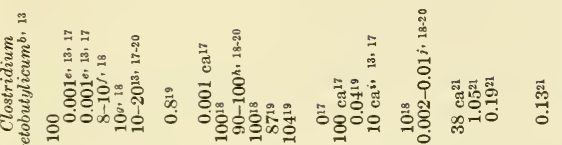
มี हैं

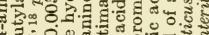

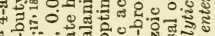

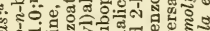
看行 o 3.

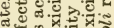

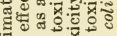
중 \&

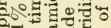
뎌의

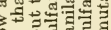

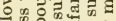

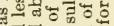

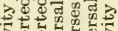

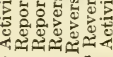
I 0 . स्त्व

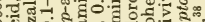
竞

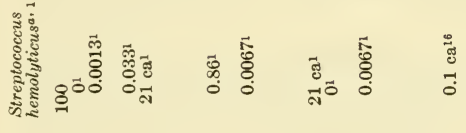

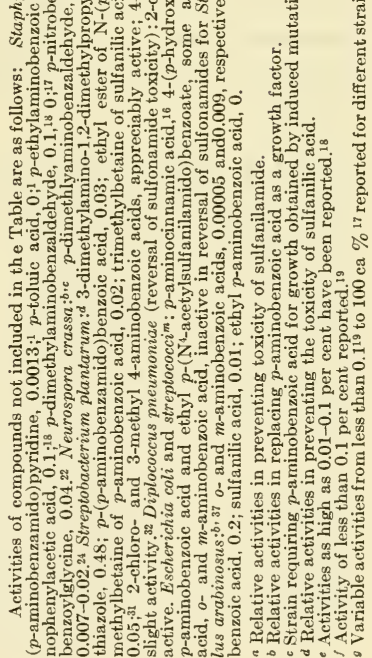


easily to $p$-aminobenzoic acid by oxidation, reduction or hydrolytic reactions. However, there is no such explanation for the activity of several compounds which apparently may be utilized as such in carrying out the biochemical functions of the growth factor. Among these are 2-fluoro-4aminobenzoic acid ${ }^{21}$ and the corresponding 2 -bromocompound, ${ }^{21}$ as well as 2-aminopyrimidine-5-carboxylic acid. ${ }^{31}$<smiles>Nc1ccc(C(=O)O)c(F)c1</smiles>

2-fluoro-4-aminobenzoic acid

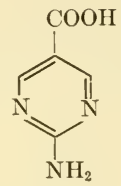

2-aminopyrimidine-5-carboxylic acid

2-Fluoro-4-aminobenzoic acid is reported by Wyss, Rubin and Strandskov 21 to be approximately 38 per cent as effective as $p$-aminobenzoic acid in promoting growth of either Clostridium acetobutylicum or a mutant strain of Neurospora crassa requiring $p$-aminobenzoic acid for growth, and has approximately this same relative activity (38 per cent) in preventing the toxicity of sulfanilamide for Escherichia coli. Since the ratio of sulfanilamide to 2 -fluoro-4-aminobenzoic acid is indicated to be constant over a range of concentrations, the utilization of the analogue as such in building the appropriate coenzyme is indicated. If conversion to $p$-aminobenzoic acid were the mechanism of action, the per cent conversion would be expected to decrease with increased concentration of the analogue.

2-Hydroxy-4-aminobenzoic acid (4-aminosalicylic acid) is particularly interesting. Though this compound appears to inhibit effectively the utilization of $p$-aminobenzoic acid in Mycobacterium tuberculosis, ${ }^{\mathbf{4 1}, 42}$ it does not have such an action in a wide variety of other organisms (p. 525). On the contrary, for two mutant strains of Escherichia coli which require $p$-aminobenzoic acid, the hydroxy derivative is 4 to 16 per cent as effective as $p$-aminobenzoic acid in promoting growth. ${ }^{28}$ Thus, the analogue replaces $p$-aminobenzoic acid in promoting growth of one organism and inhibits the functioning of this factor in another organism.

The activity of $p$-nitrobenzoic acid varies considerably. It is almost inactive in preventing the toxicity of sulfanilamide for Streptococcus hemolyticus, but is almost as effective as $p$-aminobenzoic acid in promoting growth of some strains of Clostridium acetobutylicum. The nitro compound is only slightly active for a " $p$-aminobenzoicless" strain of Neurospora crassa, ${ }^{24}$ and inhibits the growth of Streptococcus viridans ${ }^{43}$ (p. 522). The growth inhibition is prevented by $p$-aminobenzoic acid or 
by long incubation, particularly with higher concentrations of nitrobenzoic acid, indicating that the organism actually converts the $p$-nitrobenzoic acid slowly to $p$-aminobenzoic acid. With growth, the nitro compound is converted into $p$-aminobenzoic acid in sufficient quantity that the conversion can be demonstrated by quantitative determination of diazotizable amine and by the ability of the product to reverse the toxicity of sulfathiazole for the organism. ${ }^{44}$

The activity of $p$-hydroxylaminobenzoic acid in preventing the toxicity of sulfanilamide for Streptococcus hemolyticus approaches that of $p$-aminobenzoic acid. ${ }^{1}$ Although it is possible that samples of the compound contain traces of $p$-aminobenzoic acid, such highly active substances would not be expected to contain sufficient $p$-aminobenzoic acid as a contaminant to account for the high activity. ${ }^{1}$ The difference in activity of $p$-nitro- and $p$-hydroxylaminobenzoic acids for Streptococcus hemolyticus is interesting.

For Staphylococcus aureus, 4-aminocyclohexanecarboxylic acid is approximately 77 per cent as effective as $p$-aminobenzoic acid in preventing the toxicity of sulfanilic acid. This type of dehydrogenation in vivo also occurs with nicotinic acid derivatives (p. 607). $p$-Aminobenzaldehyde is apparently as active as $p$-aminobenzoic acid for Clostridium acetobutylicum.

While the $\mathrm{N}$-alkyl derivatives of $p$-aminobenzoic acid are almost devoid of activity, ${ }^{30} \mathrm{~N}$-glycosido- $p$-aminobenzoic acids retain the activity of $p$-aminobenzoic acid. N-D-Ribosido- and N-L-arabinosido- $p$-aminobenzoic acid are as active as $p$-aminobenzoic acid for Clostridium acetobutylicum. ${ }^{19}$

Since $p$-aminobenzoylglycine as well as $p$-nitrobenzoylglycine is just as active as $p$-aminobenzoic acid for Clostridium acetobutylicum, ${ }^{18}$ the cleavage of peptide groups appears to occur. However, $p$-aminobenzamide is utilized inefficiently, if at all, by most of the organisms and inhibits the functioning of $p$-aminobenzoic acid in some strains of Escherichia $\operatorname{coli}^{45,46}$ (p. 522). p-Acetamidobenzoic acid is utilized only with difficulty by many organisms. Clostridium acetobutylicum appears to be able to utilize $p$-benzamidobenzoic acid about one-tenth as effectively as $p$-aminobenzoic acid, ${ }^{17}$ but the activity for what is presumably another strain of this organism is reported to be considerably less. ${ }^{19}$

$p$-Aminophenylacetic acid has been reported to be ten times as effective as $p$-aminobenzoic acid for Clostridium acetobutylicum, ${ }^{17}$ but other reports indicate that the compound is only 0.002 to 0.01 per cent as effective as $p$-aminobenzoic acid as a growth factor, ${ }^{18-20}$ and it is ineffective in preventing the toxicity of sulfanilamide for this organism. ${ }^{47}$ For other 
organisms the analogue is not particularly effective in replacing $p$-aminobenzoic acid (Table 2).

Alkyl esters of $p$-aminobenzoic acid are not readily hydrolyzed by many organisms. Also, considerable variation in activities has been reported in some cases. Thus, ethyl $p$-aminobenzoate is reported to be from less than 0.1 per cent ${ }^{19}$ to almost 100 per cent as effective as $p$-aminobenzoic acid for Clostridium acetobutylicum. ${ }^{17}$

An ester such as diethylaminoethyl $p$-aminobenzoate (procaine) appears to be more readily hydrolyzed by various organisms than either the methyl or ethyl esters. Since this and related compounds are widely used as local anesthetics, the ability of these compounds to prevent sulfonamide-induced bacteriostasis has received widespread attention. ${ }^{30,40,48-64}$ As indicated in Table 2, procaine is almost one-fourth as active as $p$-aminobenzoic acid for Streptobacterium plantarum ${ }^{26}$ and Streptococcus hemolyticus. It is also utilized by Acetobacter suboxydans, ${ }^{22}$ Clostridium acetobutylicum, ${ }^{13}, 17-20$ Escherichia coli, ${ }^{40}$ Staphylococcus aureus, ${ }^{40}$ Eberthella typhosa, ${ }^{64}$ and pneumococci. ${ }^{48,}{ }^{63}$ The inhibitory effect of sulfanilamide on sprouting wheat is decreased by a number of local anesthetics derived from $p$-aminobenzoic acid. ${ }^{59}$

Different sulfonamides are similarly affected by procaine. The latter compound has been reported to prevent the bacteriostasis resulting from sulfanilamide, ${ }^{50,53,61,63}$ sulfapyridine, ${ }^{40,48}$ sulfathiazole, ${ }^{40,49,60}$ bis ( $p$ aminophenyl) sulfoxide, ${ }^{49}$ bis ( $p$-aminophenyl) sulfone, ${ }^{49} \quad p$-aminophenyl $p$-hydroxyphenyl sulfone, ${ }^{49} p$-aminophenyl $p$-nitrophenyl sulfoxide, ${ }^{49}$ and prontosil. ${ }^{62}$

Other anesthetics containing $p$-aminobenzoic acid are also active. $\gamma$-Dimethylamino- $\alpha, \beta$-dimethylpropyl, $\quad \gamma$-diethylamino- $\beta, \beta$-dimethylpropyl, $\beta$-diethylaminoisohexyl and $\gamma$-di- $n$-butylpropyl $p$-aminobenzoates are 1-10, 0.1-1.0, 1.0 ca. and 0.1 ca. per cent, respectively, as effective as $p$-aminobenzoic acid in preventing the toxicity of sulfapyridine for Escherichia coli. ${ }^{40}$ For Staphylococcus aureus, the first two compounds are somewhat more than 1 per cent, and procaine approximately 10 per cent, as effective as $p$-aminobenzoic acid in preventing the toxicity of sulfathiazole. ${ }^{40} \gamma$-Di- $n$-butylpropyl $p$-aminobenzoate is considerably less active than procaine in preventing sulfanilamide bacteriostasis. ${ }^{61}$

Procaine is reported to be hydrolyzed by an esterase in human and mouse blood. ${ }^{50}$ The mortality of mice infected with streptococci and treated with sulfanilamide is slightly greater when procaine is injected with the sulfanilamide. ${ }^{50,53}$ The effect is significant only when maximum nontoxic doses of procaine are administered. ${ }^{53}$ The pneumococcal therapeutic action of sulfapyridine in mice is decreased by administration of local anesthetics derived from $p$-aminobenzoic acid. ${ }^{51}$ Procaine counter- 
acts the therapeutic effect of locally applied sulfathiazole against gasgangrene, ${ }^{60}$ and antagonizes the action of sulfanilamide on pneumococci in vivo as well as in vitro. ${ }^{63}$

From the in vitro activity of procaine for Streptococcus hemolyticus ${ }^{1}$ (Table 2) it has been calculated that the average amount of procaine used in minor surgery should be sufficient to inhibit all the sulfanilamide in the human body, even during intensive treatment. ${ }^{53,58}$ The concentration in the pleural fluid of patients under procaine anesthesia has been reported to be 0.0002 per cent, which is sufficient to prevent completely the bacteriostasis resulting from 0.05 per cent sulfapyridine for Pneumococcus III (T $3-1$ ) in vitro. ${ }^{48}$

However, procaine and its metabolic products are rapidly excreted within 10 to 12 hours by the rabbit, ${ }^{52}$ and presumably by other organisms. This tends to minimize the deleterious effect of such anesthetics on the chemotherapeutic action of sulfonamides. Three clinical cases, one streptococcal infection treated with sulfanilamide and one streptococcal and one pneumococcal infection on sulfapyridine therapy, have been presented in which procaine was used as a local anesthetic without more than transient ill effect on therapy. ${ }^{\overline{3} 3}$ However, the use of local anesthetics derived from $p$-aminobenzoic acid is contraindicated during sulfonamide therapy.

Since $\beta$-dimethylaminoethyl $p$ - $n$-butylaminobenzoate (Table 2 ) is relatively inactive in preventing sulfonamide bacteriostasis, a group of esters and amides of $p$-alkylaminobenzoic acids have been prepared as possible local anesthetics with diminished ability to prevent sulfonamide bacteriostasis. These compounds in general were ineffective in preventing sulfonamide bacteriostasis for Escherichia coli and Streptococcus hemolyticus. ${ }^{30,56,57}$

$\mathbf{N}$-(p-Aminobenzoyl)-L-glutamic Acid. The first indication of the involvement of glutamic acid in the metabolism of $p$-aminobenzoic acid was the report of Auhagen, ${ }^{65}$ who found that $\mathrm{N}$-( $p$-aminobenzoyl $)-\mathrm{L}-$ glutamic acid is eight to ten times as effective as $p$-aminobenzoic acid in preventing the toxicity of sulfanilamide for Streptobacterium plantarum $10 \mathrm{~S}$.

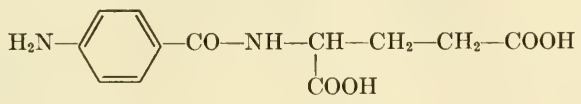

$N$-(p-aminobenzoyl $)-\mathrm{L}-$ glutamic acid

The corresponding $p$-aminobenzoyl derivatives of D-glutamic acid, L-aspartic acid, D- or L-leucine, glycine or glycylglycine were compara- 


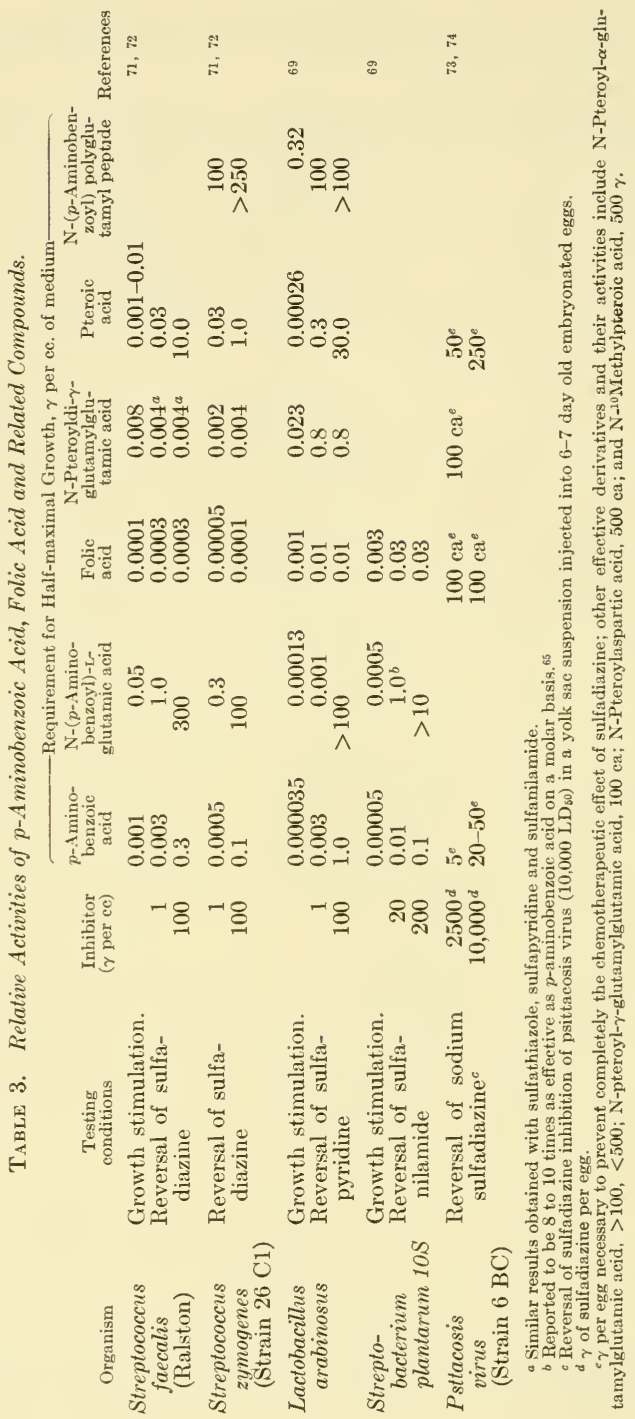


tively inactive in preventing the bacteriostasis induced by sulfanilamide for this organism. ${ }^{65}$

These tests have been repeated at various concentrations of sulfanilamide or sulfathiazole to obtain data which indicate that $\mathrm{N}$-( $p$-aminobenzoyl)-L-glutamic acid competitively prevents the toxicity of the sulfonamides and presumably is not the metabolic product of the enzyme system inhibited by sulfonamides. ${ }^{66}$

The pronounced activity of $p$-aminobenzoylglutamic acid over $p$-aminobenzoic acid has been observed only with two organisms under specific conditions. Even with Streptobacterium plantarum, differences in strains or testing conditions have resulted in failures $67,68,69$ to duplicate the results of Auhagen. ${ }^{65}$ One such result is indicated in Table 3. However, $p$-aminobenzoylglutamic acid is indicated in the same table to be considerably more effective than $p$-aminobenzoic acid for Lactobacillus arabinosus, but only in preventing the toxicity of low concentrations of sulfapyridine. ${ }^{69}$ However, adaptation to grow without $p$-aminobenzoic acid occurs under such conditions and may account for the unusual activity. At higher concentrations of inhibitor, $p$-aminobenzoylglutamic acid becomes relatively ineffective. ${ }^{69}$

In promoting growth of Lactobacillus arabinosus, $p$-aminobenzoylglutamic acid is only about 25 per cent as effective as $p$-aminobenzoic acid after 20-24 hours' incubation, but approaches the activity of $p$-aminobenzoic acid after 64 hours' incubation ( 77 per cent) ${ }^{69,} 70$

In preventing the bacteriostasis resulting from the action of sulfanilamide on Escherichia coli, Clostridium acetobutylicum, Streptococcus pyogenes, Diplococcus pneumoniae Type I, and Lactobacillus arabinosus, $\mathrm{N}$-( $p$-aminobenzoyl)-L-glutamic acid is only $0.017,0.012,0.25,0.1$ and 5.0 per cent, respectively, as effective as $p$-aminobenzoic acid. ${ }^{68}$ The glutamate is only 0.067 and 0.13 per cent as effective as $p$-aminobenzoic acid in promoting the growth of Clostridium acetobutylicum and Acetobacter suboxydans, respectively. ${ }^{68} \mathrm{~N}$ - $(p$-Aminobenzoyl $)$-L-glutamic acid is also less active than $p$-aminobenzoic acid in preventing the toxicity of sulfapyridine for Diplococcus pneumoniae Type III and Streptococcus hemolyticus Group A, ${ }^{16}$ and the toxicity of sulfadiazine for Streptococcus faecalis Ralston and Streptococcus zymogenes $26 \mathrm{C} 1$.

While the enhanced activity of $\mathrm{N}$-( $p$-aminobenzoyl)-L-glutamic acid over $p$-aminobenzoic acid is not widespread, the results with a single organism gave an early indication of the involvement of glutamic acid in the metabolism of $p$-aminobenzoic acid. With the discovery of $p$-aminobenzoic acid as a constituent of folic acid, the presence of glutamic acid in the folic acid molecule became an obvious possibility. 
p-Aminobenzoylpolyglutamyl Peptide from Yeast. A conjugate of $p$-aminobenzoic acid with 10 or $11 \mathrm{~L}$-glutamic acid resiclues and one unknown amino acid residue presumably acidic in nature has been isolated from yeast. ${ }^{75,76}$ This conjugate accounts for 20 to 30 per cent of the total amount of $p$-aminobenzoic acid occurring in yeast. Approximately 400 $\mathrm{mg}$ of the purified pepticle was obtained from $50 \mathrm{~kg}$ of dried yeast. As the amino group of $p$-aminobenzoyl peptide is diazotizable, it appears that the aromatic acid is conjugated by means of the carboxyl group to a peptide chain of 10 or $11 \mathrm{~L}$-glutamic acids with presumably a terminal unidentified amino acid. By analogy with the folic acid group, it seems probable that the conjugate may have $\gamma$-glutamyl units in the peptide chain. The ability of this conjugate to prevent the hydrolysis of a conjugate of folic acid (p. 571) further emphasizes this similarity.

As indicated in Table 3 , the conjugate of $p$-aminobenzoic acid is relatively inert in promoting growth of organisms requiring $p$-aminobenzoic acid or in preventing the toxicity of sulfonamides. ${ }^{75}$

Folic Acid and Related Compounds. The first conclusive indication of an interrelationship of $p$-aminobenzoic acid and folic acid was the report of Miller, ${ }^{77}$ who indicated that for both normal and resistant strains of Escherichia coli, sulfonamides at concentrations which do not affect growth decrease markedly the production of microbiologically active forms of folic acid. The biosynthesis of biotin is not affected under these conditions. Earlier work had indicated that $p$-aminobenzoic acid stimulated growth and increased approximately threefold the formation of microbiologically active forms of folic acid in mixed culture of bacteria from the fowl intestine. ${ }^{78} p$-Aminobenzoic acid was also reported to enhance the synthesis by Mycobacterium tuberculosis of factors with activities of vitamin $B_{10}$ and $B_{11}$, which are chick factors replaceable by folic acid..$^{79}$

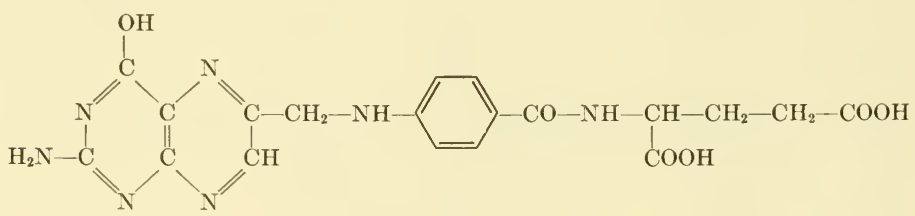

folic acid

The elucidation of the structure of folic acid (p. 565) made the relationship apparent, since folic acid contains $p$-aminobenzoic acid in combination with a pteridine group and glutamic acid. Initially the possibility that $p$-aminobenzoic acid functioned solely in the biosynthesis of folic acid presented itself. This possibility was also indicated by the resistance to sulfonamide-inhibition of organisms requiring folic acid for growth. ${ }^{80}$ 
However, it was observed even before the elucidation of the structure of folic acid that concentrations of this vitamin corresponding to those ordinarily required for organisms incapable of folic acid synthesis do not prevent the toxicity of sulfonamide for a number of organisms. ${ }^{66,80,81}$ Even high concentrations of folic acid do not affect the toxicity of sulfonamides for Escherichia coli ${ }^{66}$ and many other organisms. ${ }^{66,80,81,82}$ Furthermore, mutant strains of Escherichia coli ${ }^{83}$ and of Neurospora crassa, which require $p$-aminobenzoic acid for growth, do not respond to folic acid. For many organisms which require $p$-aminobenzoic acid, folic acid is either not utilized at any concentration or is utilized less effectively than $p$-aminobenzoic acid. ${ }^{6}$ However, Lampen and Jones ${ }^{71,72}$ have shown that folic acid is more active than $p$-aminobenzoic acid in supporting the growth of Streptococcus faecalis (Ralston), and that it prevents noncompetitively the toxicity of sulfonamides for this organism as indicated in Table 3 . The amount of folic acid necessary for reversal of sulfonamides approximates that necessary for growth of the organisms in the absence of sulfonamides. The toxicity of sulfonamides for Streptococcus faecalis G1Y2, Streptococcus zymogenes 26 C1, Streptococcus durans S10, or Streptococcus liquefaciens 815 is similarly reversed by low concentrations of folic acid. ${ }^{71,72}$ For a number of organisms, such as Lactobacillus arabinosus and Streptobacterium plantarum, the concentration of folic acid necessary to prevent the toxicity of sulfonamides is considerably higher than the concentration of $p$-aminobenzoic acid necessary to stimulate growth of the organisms. ${ }^{69}$ However, approximately the same amount of folic acid is required for reversal of any concentration of sulfonamides ${ }^{66,69}$ The noncompetitive reversal suggests that folic acid can supply the normal metabolic intermediate involved in the biosynthesis of the appropriate coenzyme, but is considerably less effectively utilized than $p$-aminobenzoic acid. The utilization of folic acid for these organisms cannot be ascribed to its conversion to $p$-aminobenzoic acid. $p$-Aminobenzoic acid, however, is converted by Lactobacillus arabinosus to microbiologically active forms of folic acid. ${ }^{70}$ The reversal by folic acid of the sulfadiazine inhibition of psittacosis virus (Strain $6 \mathrm{BC}$ ) in embryonated eggs (Table 3 ) is somewhat analogous to the results with Lactobacillus arabinosus and Streptobacterium plantarum. ${ }^{73,7+}$ Either $p$-aminobenzoic acid or folic acid prevents the chemotherapeutic effect of sulfathiazole in mice infected with Toxoplasma (RH strain) ${ }^{84}$

The activity of folic acid under certain conditions in preventing the toxicity of sulfonamides (p. 471) for Escherichia coli indicates that the organism has some slight ability to utilize folic acid in the biosynthesis of the coenzyme involved in production of thymine. However, folic acid cannot substitute for $p$-aminobenzoic acid in the biosynthesis of methionine, purines and serine in Escherichia coli (p. 470). 
Two different explanations for the various published data interrelating $p$-aminobenzoic acid and folic acid are possible. Folic acid can be considered as differing from the normal metabolic intermediate in the biosynthesis of the coenzyme derived from $p$-aminobenzoic acid; and organisms would be expected to have varying abilities to utilize folic acid in forming the coenzyme. In Escherichia coli, the addition of a number of products (methionine, purines and serine) might be expected to exert a sparing action such that the small amount of folic acid utilized by the organism would be sufficient for growth. This explanation would conform to the theory that a single coenzyme is derived from both $p$-aminobenzoic acid and folic acid.

The other explanation would require that more than one coenzyme is formed from folic acid and $p$-aminobenzoic acid. Thus, folic acid would perform only part of the biochemical functions of $p$-aminobenzoic acid. The data which are difficult to explain by such a theory include the ability of folic acid to prevent completely the toxicity of sulfonamides and to fulfill the growth requirements in place of $p$-aminobenzoic acid for certain organisms. If this second explanation is valid, such data would indicate that a rapid conversion of folic acid to the other coenzymes occurs by a process resistant to sulfonamides, or that the products of the functioning of the coenzyme derived from $p$-aminobenzoic acid but not folic acid are supplied in the medium of such organisms.

Although published reports do not indicate conclusively whether or not two or more coenzymes are derived from $p$-aminobenzoic acid and folic acid, the low activity of folic acid for many organisms suggests that other active forms of the vitamin will be found.

The exact biochemical step in the conversion of $p$-aminobenzoic acid to folic acid which is prevented by sulfonamides has not been elucidated. It is interesting that pteroic acid and $p$-aminobenzoylglutamic acid reverse the toxicity of sulfonamides competitively, so that neither represents the product of the inhibited enzyme system. Since both $p$-aminobenzoylglutamic acid and pteroic acid are less effective than $p$-aminobenzoic acid over a range of concentrations (Table 3), it appears that the biosynthesis of folic acid does not proceed through these compounds as intermediates. It has been suggested that sulfonamirles may prevent a combination of $p$-aminobenzoic acid with reductone- $\alpha, \beta$-dihydroxyacrolein. If so, this may represent a stage in the biosynthesis of folic acid, but convincing biochemical evidence has not been presented. ${ }^{85}$ 2-Amino4-hydroxypteridine-6-carboxaldehyde has been suggested as a possible intermediate which is prevented by sulfonamide from combining with $p$-aminobenzoic acid. ${ }^{86}$ Only glucose and $p$-aminobenzoic acid are essential, and glutamic acid is stimulatory in the biosynthesis of microbiologi- 
cally active forms of folic acid by Streptobacterium plantarum. ${ }^{87}$ Synthesis of this factor is inhibited by either sulfanilamide or sulfathiazole, and the inhibition is prevented competitively by $p$-aminobenzoic acid. ${ }^{87}$ The synthesis of the folic acid group by yeast is also inhibited by sulfonamides. ${ }^{s 8}$

The utilization of pteroic acid by Streptococcus faecalis R, which requires folic acid for growth, is not inhibited by sulfonamides. ${ }^{71}$

Pteroyl-di- $\gamma$-glutamylglutamic acid has an action similar to that of folic acid for organisms which utilize either $p$-aminobenzoic acid or folic acid (Table 3). The action of sulfonamides on such organisms is noncompetitively reversed by the triglutamate at a concentration somewhat higher than that of folic acid essential for a similar response.

\section{Inhibitory Analogues of $p$-Aminobenzoic Acid}

After the discovery of the therapeutic activity of sulfanilamide, ${ }^{8}$ a tremendous number of analogous compounds were prepared and tested as possible chemotherapeutic agents. Actually, many of the more efficient sulfonamides known today were in common use before the discovery by Woods ${ }^{1}$ of the interrelationship of these compounds with $p$-aminobenzoic acid. Although not among the first analogues synthesized and tested, sulfapyridine was the first of the $\mathrm{N}^{1}$-substituted sulfonamides found to

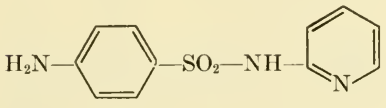

sulfapyridine

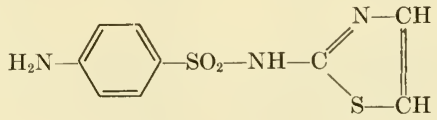

sulfathiazole

be superior to sulfanilamide. Sulfapyridine was particularly outstanding at the time for its curative effect in pneumonia, ${ }^{89}$ and was apparently first prepared by Ewins and Phillips ${ }^{90}$ but was prepared by several others. ${ }^{91}$ One of the most potent of the sulfonamides, sulfathiazole, was first reported by Fosbinder and Walter. ${ }^{92}$ The synthesis of two more very effective sulfonamides, sulfadiazine and sulfamethyldiazine (sulfamerazine), was first reported by Roblin, et al. ${ }^{93}$

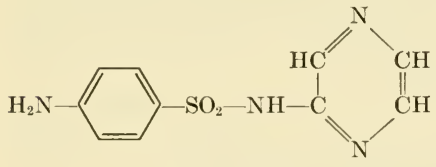

sulfadiazine

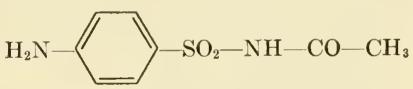

sulfacetamide 
TABlE 4. Sulfanilamide, Sulfathiazole, and Sulfapyridine as Inhibitory Analogues of p-Aminobenzoic Acid

Organism

Aerobacter aerogenes
Clostridium
acetobutylicum
Corynebacterium
diphtheriae
Diplococcus
pneumoniae

Escherichia coli

Lactobacillus acidophilus

Lactobacillus arabinosus 17-5

Lactobacillus pentosus 124-2

Mycobacterium tuberculosis

Neisseria gonorrhoeae

Neisseria meningococcus

Pasteurella pestis

Proteus vulgaris

Pseudomonas aeruginosa

Salmonella enteritidis

Salmonella typhimurium

Staphylococcus aureus

Streptobacterium plantarum

Streptococcus hemolyticus (Richards)

Aspergillus niger

Neurospora crassa ${ }^{\circ}$

Saccharomyces cerevisiae 139

Polytomella caeca

Strigomonas oncopelti

Trichophyton purpureum

Rice seedings

Tomato roots

Wheat seedings

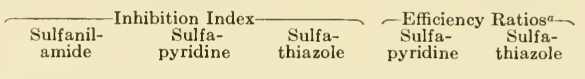

$45^{b} 100$

$72^{100}$

Supplementary References

$3220^{b} 100$

$23000^{c 13}$

200-1600101

$$
1600
$$

$3330^{b} 100$

$8000^{b 100}$

280

ca. 102

$450^{b 100}$

110

ca. ${ }^{102}$

$41^{b 100}$

$133^{b 100}$

$5.7^{102}$

$15^{102}$

$7.4^{100}$

$81^{100}$

$1,49,101$

$102,105-113$

$60^{100}$

$100^{d 80}$

$200^{d} 80$

$\begin{array}{crrrrr}16600^{b 114} & 2800^{b 114} & 106^{b 114} & 5.9^{114} & 157^{114} & 116-118 \\ 1000^{c 115} & & 20^{c 115} & & 50^{115} & \\ 100^{101} & & & & 119-121\end{array}$

$5-1000^{e} 101$

$4000^{b 100}$

$4.5^{c 113}$

$4.5^{c 113}$ $55^{b} 100$

$184^{b 100}$

$13330^{b} 100$

$100^{123}$

$6650^{b} 100$

$4660^{b 100}$

$416^{\text {b } 100}$

$92^{b 100}$

$53^{b 100}$

$11.2^{100}$

$72^{100}$

$88^{100} 36,109,11 y$. 124-127

$$
150^{b 26}
$$

$63^{b 26}$

$35^{b 26}$

$2.4^{26}$

$4.3^{26} \quad 128,129$

$5000^{c 1} 1000^{c 1}$

$10^{c} f 105$

$1500^{b 24}$

$1590^{b 133}$

$3.7^{105}$

$210,000^{c 137}$

$1270^{b} 133$

$540^{b 133}$

$1.3^{133}$

$2.9^{133} \quad 134-136$ 130,131 132

$$
400^{c 138}
$$

$4 \mathrm{ca}^{139}$

$\begin{array}{ccrrr}35-50^{b} & 27-36^{b} & 17^{b} & 1-2^{140} & 2-3^{140} \\ \text { ca. }{ }^{140} & \text { ca. }^{140} & \text { ca. }^{141} & & \end{array}$

Other organisms for which the toxicity of sulfonamides in vitro is prevented by $p$-aminobenzoic acid include: Bacillus subtilis; ${ }^{142}$ Brucella paramelitensis; ${ }^{109}$ Eberthella typhosa;111 Proteus friedlanderi; 110 Pseudomonas pyocyanea; ${ }^{142}$ Salmonella paratyph $; ;_{111}$ Salmonella schottmuelleri; $i 11$ Shigella dysenteria;111 Botrytis allii Munn;14 Fusarium caeruleum (Lib.) Sacc. ${ }^{141}$ Penicillium digitatum Sacc.; ${ }^{141}$ Chlorella (pigmented and nonpigmented) ${ }^{143}$. ${ }^{44} ;$ Nitzschia polea var. debilis (fresh water diatom)145, 146, 147; Flax seed;148 Onion rootlets; 149 Pea roots 150,151 and shoots. ${ }^{151}$

${ }^{a}$ Ratio of the activity of the sulfonamide to sulfanilamide. 100

$b$ For half-maximum growth.

c Molecular equivalents of sulfonamide neutralized by one molecular equivalent of $p$-aminobenzoic acid.

$d$ In the absence of purines.

- Varies with different strains.

At $\mathrm{pH} 7.1,2000$ at pH 3.7.

o Either p-aminobenzoicless strains 1663 or 5359 or parent strain.

$h$ Inhibition index depends on strain. Mitis, intermedius, and gravis strains are susceptible. 
Sulfacetamide (albucid) ${ }^{94}$ because of low toxicity, is useful in urinary infections. Sulfaguanidine, ${ }^{95,96}$ succinylsulfathiazole, ${ }^{97,98}$ and phthalylsulfathiazole ${ }^{97,98}$ have been synthesized and, being sparingly absorbed from the bowel, are useful in treatment of intestinal infections.

TABLE 5. Sulfonamides, Sulfones, Sulfoxides and Related Inhibitory Analogues of $p-$ Aminobenzoic Acid

\author{
Compound
}

Sulfadiazine

Sulfacetamide

Sulfaguanidine

Sulfanilic acid

bis(4-Aminophenyl)sulfone
Organism

Escherichia coli

Staphylococcus aureus

Mycobacterium tuberculosis

Pasteurella pestis

Proteus vulgaris

Escherichia coli

Staphylococcus aureus

Streptococcus hemolyticus B

Neisseria gonorrhoeae

Escherichia coli

Staphylococcus aureus

Streptococcus hemolyticus B

Saccharomyces cerevisiae 139

Streptococcus hemolyticus

Escherichia coli

Streptobacterium plantarum $10 \mathrm{~S}$

Staphylococcus aureus

\section{Escherichia coli \\ Mycobacterium tuberculosis \\ Streptobacterium plantarum $10 \mathrm{~S}$ \\ Streptococcus hemolyticus B}

$\begin{array}{ccc}\begin{array}{c}\text { Inhibition } \\ \text { Index }\end{array} & \begin{array}{c}\text { Efficiency } \\ \text { Ratio }^{a}\end{array} & \begin{array}{c}\text { Supplemen- } \\ \text { tary } \\ \text { References }\end{array} \\ 43^{b 100} & 78^{100} & 106,108,110 \\ 92^{b 100} & 51^{100} & 124,125 \\ 143^{b 114} & 116^{114} & \\ 4.5^{c} 113 & & \\ 534^{b 100} & 50^{110} & \\ 534^{b 100} & 6^{100} & \\ 800^{c} 130 & 2.9^{130} & \\ 3960^{b 100} & 0.84^{100} & 108 \\ 4570^{b 100} & 1.0^{100} & \\ 2500^{c 130} & 0.59^{130} & \\ 1280^{b 133} & 1.2^{133} & \\ 15,000^{c 1} & 0.33^{1} & 130 \\ 1,000^{c} 112 & 0.1^{112} & \\ 5,000^{b 26} & 0.03^{26} & 129 \\ 10^{c a} .^{152} & & 153 \\ 1390^{c 108} & 2.0^{108} & 49 \\ 332^{b 114} & 50^{114} & 154,155 \\ 28^{b} 26 & 5.3^{26} & 129 \\ 249^{c} 130 & 6.6^{130} & 49 \\ & & \end{array}$

Other sulfonamides, sulfones, sulfoxides and related compounds the toxicity of which is prevented by $p$-aminobenzoic acid include: $N^{1}$-arylsulfanilamides: $\mathrm{N}^{2}$ phenyl-, $130 \mathrm{~N} \mathrm{~N}^{1}-0$-tolyl-, 130 $\mathrm{N}^{1}-0$-chlorophenyl-, 130 $\mathrm{N}^{1}-o$-hydroxyphenyl-,130 $\mathrm{N}^{2}-p$-hydroxyphenyl-130 $\mathrm{N}^{1}-p$-aminophenyl-, $130 \quad \mathrm{~N}^{1}-p$-nitrophenyl-, 127 and $\mathrm{N}^{1}-m-$ earboxyphenylsulfanilamides, 130 and $\mathrm{N}^{1}, \mathrm{~N}^{1}$-dimethyl-4-sulfanilamidobenzenesulfonamide. ${ }^{27}, \quad 129,130 \quad N^{1}$ Heterocyclicsulfanilamides: 2-sulfanilamido derivatives of pyrimidine, ${ }^{151}$ 4-methylpyrimidine, 130 5-chloropyrimidine, ${ }^{d} 156$ 5-bromopyrimidine, $d 156$ 4-amino-5-bromopyrimidine, ${ }^{156}$ 5-bromo-4-methylpyrimidine, 156 5-bromo-4,6-dimethylpyrimidine, ${ }^{156}$ 5-(2,3-dibromopropyl)-4, 6-dimethylpyrimidine, ${ }^{155}$ 4-methylthia zole, 127, 130 4-phenyl-5-methylthiazole, 127, 130 5-bromothiazole, 156 5-chlorothiazole, 155 5-bromo-4-methylthiazole, ${ }^{156}$ 5-chloro-4-methylthiazole, ${ }^{156}$ 5-pyridinesulfonamide, 127, t30 4-methyldiazine, 110 4,6-dimethyldiazine, 110 5-chloropyridine, 156 thiazoline,127, 130 5-methylthiadiazole,121, 131 5-ethylthiadiazole,121, ${ }_{131}$ 5- $n$-propylthiadiazole, ${ }^{121}$ 5-isopropylthiadiazole, ${ }^{121}$ and 5-isobutylthiadiazole 121 and 2-(2-chlorosulfanilamido)pyrimidine ${ }^{156}$ and 3,4-dimethyl-5-sulfanilamidoiso-oxazole..$^{157} \mathrm{~N}^{1}$-Acylsulfanilamides and miscellaneous sulfonamides: $\mathrm{N}^{4}$-sulfoxymethylsulfanilamide (sodium salt), $130 \quad \mathrm{~N}^{1}-3,4$-dimethylbenzoylsulfanilamide, 158 sulfanilamidoacetic acid, 130 p-hydroxylaminobenzenesulfonamide. 101 Sulfones and sulfoxides: 2-aminophenyl 4-aminophenyl sulfone, ${ }^{154}$ bis(2-aminophenyl) sulfone, 154 bis (3-aminophenyl) sulfone, 154 4-acetylaminophenyl 4-nitrophenyl sulfone, ${ }^{154}$ 4-aminophenyl 4-hydroxyphenyl sulfone,49 4-aminophenyl 4-benzylidineaminophenyl sulfone, ${ }^{134}$ 4-aminophenyl 5-amino-2-pyridyl sulfone, 159 promin, 155 bis (4-aminophenyl) sulfoxide, 149,154 bis (4-acetamidophenyl) sulfoxide, ${ }^{154} 4$-aminophenyl phenyl sulfoxide, ${ }^{154} 4$-aminophenyl 4-nitrophenyl sulfoxide, ${ }^{49}$, 154 4-acetamidophenyl 4-nitrophenyl sulfone ${ }^{49}$, 154 4-aminophenyl 4-chlorophenyl sulfone,154 4-aminophenyl 4-iodophenyl sulfone, 154 4-iodophenyl 4-nitrophenyl sulfone.154 Miscellaneous Analogues: $p$-aminophenyldimethylsulfonium $\beta$-naphthalenesulfonate, ${ }^{160}$ bis(4-aminophenyl) disulfide, 109 bis (4-aminophenyl)diselenide, d109

a Ratio of the activity of the sulfonamide to that of sulfanilamide. ${ }^{100}$

$b$ For half-maximum growth.

- Molecular equivalents of sulfonamide neutralized by one molecular equivalent of $p$-aminobenzoic acid.

$d$ Toxicity of these compounds only partially reversed by $p$-aminobenzoic acid.

The remarkable chemotherapeutic activity of this group of compounds has stimulated the preparation of thousands of compounds somewhat related in structure to sulfanilamide. An excellent monograph by Northey ${ }^{99}$ on sulfonamides and related compounds includes comprehen- 
sive lists of these compounds together with their activities. Consequently, only these compounds which have been studied with respect to their relationship to $p$-aminobenzoic acid are included in this monograph.

\section{Sulfonamides, Sulfones and Related Analogues: Activity and Reversals with $p$-Aminobenzoic Acid in Vitro}

After the appearance of the report of Woods ${ }^{1}$ indicating the competitive interrelationship of $p$-aminobenzoic acid and the sulfonamide drugs, this effect was confirmed by many others, and the effect of $p$-aminobenzoic acid on the toxicity of related inhibitory analogues was determined for a wide variety of organisms. The organisms for which the toxicity of sulfanilamide, sulfathiazole or sulfapyridine in vitro is prevented by $p$-aminobenzoic acid are indicated in Table 4 . It is apparent that numerous species of bacteria, fungi, higher plants, diatoms, yeast and flagellates are inhibited by these sulfonamides, and the inhibition is prevented competitively by $p$-aminobenzoic acid. Similar results have been obtained with a large number of $\mathrm{N}^{1}$-substituted sulfanilamides, sulfones, sulfoxides and similar compounds structurally related to $p$-aminobenzoic acid (Table 5).

As indicated in these tables, the inhibition indices of an individual inhibitor vary considerably for different organisms. Also, organisms which require folic acid for growth are highly resistant to inhibition by any of the analogues of $p$-aminobenzoic acid. However, the relative efficiencies of the different sulfonamides in inhibiting the growth of various organisms are rather consistent, and it has been suggested that there is no specificity in the ability of sulfonamides to inhibit specific organisms. ${ }^{100}$ While this is true for most organisms, there are many exceptions. For example, sulfathiazole and sulfapyridine are only slightly more effective for Streptobacterium plantarum than sulfanilamide, and bis(4-aminophenyl)sulfone, which is only slightly more effective for Escherichia coli than sulfanilamide, is fifty times as efficient as sulfanilamide for Mycobacterium tuberculosis. The sulfone is more active than

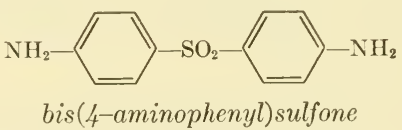

sulfathiazole for Streptobacterium plantarum, while sulfathiazole is considerably more effective than the sulfone against Escherichia coli. Even though the order of the relative activities of sulfanilamide, sulfapyridine and sulfathiazole is the same for essentially all organisms, there is little difference in their inhibitory ability against organisms such as Saccha- 
romyces cerevisiae, tomato roots and Streptobacterium plantarum. The order of effectiveness in some cases depends upon the experimental method, since the dose-response curves for the inhibitory action of the various sulfonamides are not identical under different testing conditions. For example, the growth of the fresh-water diatom, Nitzschia palea var. debilis, is inhibited by sulfanilamide, sulfapyridine and sulfathiazole, with decreasing effectiveness in the order named; however, $p$-aminobenzoic acid increases in effectiveness in preventing the toxicities of the sulfonamides in the reverse of the order named. ${ }^{145-147} \mathrm{~A}$ similar situation exists with Saccharomyces cerevisiae ${ }^{133}$ and with the nonpigmented alga, chlorella. ${ }^{143}$ At low concentrations of $p$-aminobenzoic acid, sulfapyridine is less effective for Saccharomyces cerevisiae than sulfanilamide, but at higher concentrations of $p$-aminobenzoic acid such that considerable growth occurs, sulfapyridine is more inhibitory to the organism than is sulfanilamide. ${ }^{133}$ Differences in the shape of dose-response curves appear to account for these unusual effects.

The sulfonamides, sulfones, sulfoxides, etc., which inhibit the utilization of $p$-aminobenzoic acid usually possess a free amino group in the position para to the sulfur-containing substituent. In a few cases, inhibitory activity has been reported for compounds in which the amino group is replaced by groups, such as nitro, acetylamino, alkylamino, glycosidoamino, etc., which presumably may be converted into a free amino group in the inhibited biological system. Usually these compounds are less active than the analogous compound with the free amino group. Also substitution of the aromatic ring of this series of sulfur analogues of $p$-aminobenzoic acid usually results in a decrease or complete loss of inhibitory activity.

The most effective modifications of sulfanilamicle involve $\mathrm{N}^{1}$-substituents. In general the inhibitory activity is decreased if the substituent is an alkyl or cycloalkyl group, but is usually increased if the substituent is an aromatic heterocyclic group. Similarly, the most effective sulfones and sulfoxides contain an aromatic group in conjunction with a $p$-aminophenyl substituent.

In contrast to the unusual activity of $\mathrm{N}$-( $p$-aminobenzoyl $)$-L-glutamic acid compared with p-aminobenzoic acid in preventing the toxicity of sulfonamides for certain organisms under specific conditions (p. 487), the corresponding sulfonamide, $\mathrm{N}$-sulfanilyl-L-glutamic acid not only does not have increased inhibitory power but is relatively inactive. ${ }^{67,161}$

\section{Reversals with p-Aminobenzoic Acid in Vivo}

Shortly after the preliminary report of Woods and Fildes ${ }^{1}$ concerning the ability of $p$-aminobenzoic acid to prevent the inhibitory action of sulfonamides, Selbie ${ }^{162}$ found that the therapeutic activity of sulfanila- 


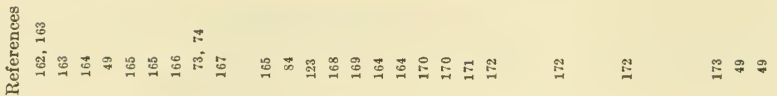

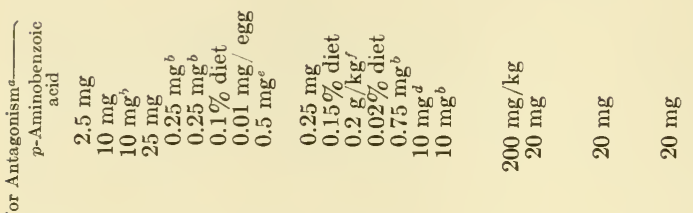

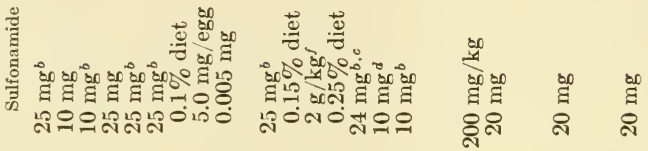

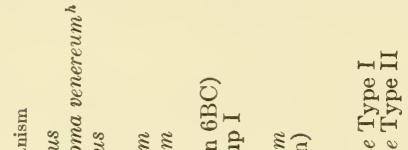

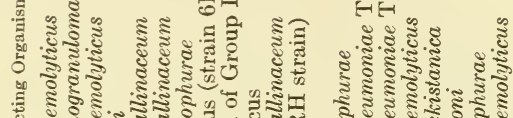

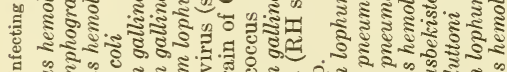
ป ई ర్. ठ․

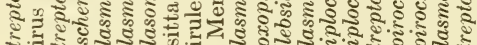

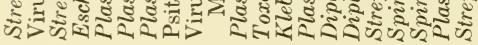
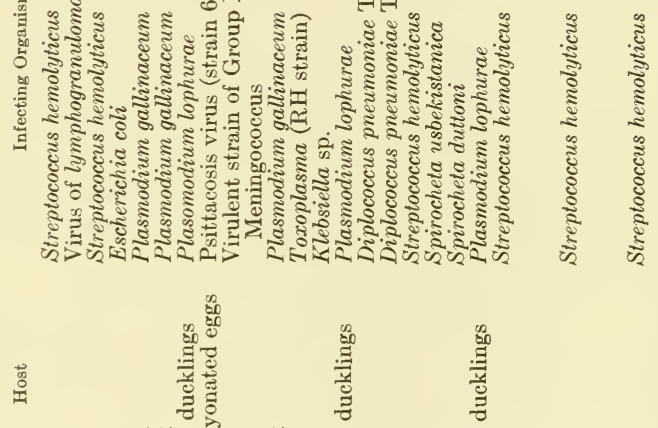

国

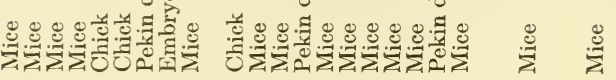

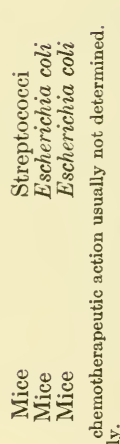

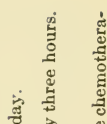

葋

更 
mide against infections of Streptococcus hemolyticus in mice was also antagonized by $p$-aminobenzoic acid. These findings were rapidly confirmed with this and other organisms for a wide variety of sulfonamides, sulfones and sulfoxides as indicated in Table 6 . This in vivo testing allowed extensions of the interrelationship of $p$-aminobenzoic acid and the sulfonamide drugs to pathogenic protozoa such as Toxoplasma, Plasmodium lophurae and Plasmodium gallinaceum, and to such viruses as' psittacosis and the virus of lymphogranuloma venereum.

Because $p$-aminobenzoic acid is rapidly converted into inactive forms, e.g., conjugates in animals, and is rapidly excreted in comparison to sulfonamides and related compounds, the amount of the factor necessary to prevent the therapeutic action of the sulfonamide drugs is often high in comparison with amounts required for in vitro testing. ${ }^{167,}{ }^{174}$ However, if the concentration of $p$-aminobenzoic acid at the site of the infection is compared with the corresponding concentration of sulfonamide, results comparable with those for in vitro testing are obtained. ${ }^{174}$ Failure to make such comparisons has resulted in conflicting reports in some cases.

The specificity of particular sulfonamide drugs for certain organisms appears to be greater in vivo than in vitro. Of a group of 33 sulfanilamide derivatives, including the $\mathrm{N}^{1}$-heterocyclic sulfanilamides, none was found to have significantly greater therapeutic activity than sulfanilamide against Streptococcus hemolyticus C-203 infections in mice. However, the $\mathrm{N}^{1}$-heterocyclic sulfanilamides were more effective than sulfanilamide against infections of Diplococcus pneumoniae. ${ }^{175}$ Of a group of $\mathrm{N}^{1}$-acylsulfanilamides, a few were found to be effective against Streptococcus hemolyticus, Diplococcus pneumoniae and Escherichia coli infections in mice; however, some of the compounds were effective only against Streptococcus hemolyticus and Escherichia coli, while another was effective only against Escherichia coli and not against the other two. ${ }^{176}$ Consequently, there appears to be a higher degree of specificity of chemotherapeutic activity in vivo than in vitro.

Sulfonamides, sulfones and related analogues of $p$-aminobenzoic acid are reported to prevent the effects of endotoxins of microorganisms. ${ }^{177}$ Although some attempts to verify the early work failed, ${ }^{178,} 179$ the effects of sulfonamides and related compounds on the action of endotoxins of certain bacteria have been verified. ${ }^{180-183}$ Sulfanilamide allows a significant increase in the number of mice surviving injection of the endotoxins of Salmonella typhimurium without affecting the process of immunization. ${ }^{182,183}$ The therapcutic effect of sulfanilamide was prevented by administration of p-aminobenzoic acid. ${ }^{183}$ The effect of 4 -aminophenyl 4 -nitrophenyl sulfoxide in protecting 40 to 80 per cent of the mice from a lethal dose of typhoid endotoxin is antagonized by injected $p$-amino- 
benzoic acid. ${ }^{184}$ The treatment with the sulfoxide does not affect the immunization of the animal against the endotoxin or the organism. ${ }^{184}$ Similar results were obtained with endotoxin from a particularly virulent strain of Escherichia coli. ${ }^{184}$

\section{Inhibitions by Sulfonamides Unaffected by $p$-Aminobenzoic Acid}

Sulfapyrazine, sulfadiazine and sulfathiazole inhibit completely the growth of Bacterium tularense, but $p$-aminobenzoic acid does not affect the inhibition. ${ }^{113}$ Although such cases are unusual for these analogues, there are a number of sulfonamide derivatives related structurally to $p$-aminobenzoic acid which are inhibitory, but the inhibition is not reversed by $p$-aminobenzoic acid. The toxicity of $2-, 3-, 5$ - and 7 -sulfanilamidoindazoles for Brucella melitensis is only slightly reversed by $p$-aminobenzoic acid. ${ }^{185} 3^{\prime}, 5^{\prime}$-Dibromosulfanilanilide is strongly inhibitory to numerous strains of pneumococci, hemolytic streptococci and staphylococci, but is only slightly inhibitory to Friedlanders' bacillus, Escherichia coli, Pseudomonas aeruginosa and various types of dysentery bacilli. However, the strong inhibition observed with the gram-positive cocei is not prevented by $p$-aminobenzoic acid, but this vitamin reverses the slight inhibition obtained with the gram-negative bacilli. ${ }^{186}$ Similarly, the toxicity of a series of $3^{\prime}, 4^{\prime}$ - and $3^{\prime}, 5^{\prime}$-halogenosulfanilanilides is also unaffected by $p$-aminobenzoic acid for certain organisms. ${ }^{187,}{ }^{188}$ The toxicity of $p$-aminomethylbenzenesulfonamide is not affected by $p$-aminobenzoic acid or by $p$-aminomethylbenzoic acid for a wide variety of organisms. ${ }^{189,} 190$

For some organisms the toxicity of sulfanilamide is only partially counteracted by $p$-aminobenzoic acid. This is true of onion rootlets, for which $p$-aminobenzoic acid is toxic at higher concentrations. ${ }^{149}$ Although very effective at low concentrations in preventing the toxicity of sulfanilamide, $p$-aminobenzoic acid is toxic at higher concentrations for pea roots. ${ }^{150} \mathrm{~A}$ similar situation exists with flax seed, which germinates slowly or not at all in the presence of relatively high concentrations of sulfanilamide, and the growth of the seedlings is retarded by lower concentrations of the drug. Although $p$-aminobenzoic acid counteracts the inhibitory action, root development is not quite restored to normal. Higher concentrations of $p$-aminobenzoic acid are toxic. ${ }^{148}$ For Lupinus albus seedlings ${ }^{191} p$-aminobenzoic acid enhances the toxicity of sulfanilamide.

In systems in which the toxicity of sulfonamides or related compounds is not affected by $p$-aminobenzoic acid, it appears that in most instances enzymatic reactions other than those concerned with the utilization of $p$-aminobenzoic acid are involved. This does not preclude the possibility that in some instances a sequence of two reactions is prevented by the 
analogue or that the analogue combines irreversibly with the enzyme, or that some other such phenomenon prevents a competitive reversal by $p$-aminobenzoic acid.

\section{Mechanism of Action of Sulfonamides and Correlation of Activities with Physical Properties and Structure}

Attempts to correlate the inhibitory activities of the $\mathrm{N}^{1}$-substituted sulfonamides and related compounds with structure or some physical property of the compounds have resulted in several different theories as to the mode of action of these substances. However, the theory of competitive inhibition of the utilization of $p$-aminobenzoic acid as an essential metabolite ${ }^{1}$ explains more effectively the data which have accumulated.

Application of the Michaelis-Menten equations adapted to the rate of an inhibited reaction was made by Wyss ${ }^{192}$ to demonstrate the competitive nature of the relationship between $p$-aminobenzoic acid and sulfanilamide for Escherichia coli (p. 455). A similar treatment was applied to the inhibition index by Wood, who suggested that the variations in the bacteriostatic activity of the different sulfonamides might be the result of differences in affinities for the enzyme involved in the functioning of $p$-aminobenzoic acid. ${ }^{108}$

Sulfanilamide does not displace any appreciable amount of $p$-aminobenzoic acid from cells of Streptococcus hemolyticus, ${ }^{193}$ and there is no appreciable binding of sulfanilamide labelled with radioactive sulfur in the cells of Escherichia coli. ${ }^{194}$ Thus, $p$-aminobenzoic acid appears to be converted into a coenzyme form, and very little of the total $p$-aminobenzoic acid of cells exists in a combination from which it is displaced by sulfonamide. This is further indicated by the observation that bacteria are capable of undergoing a definite, limited number (six or seven) of cell divisions in the presence of any effective drug concentration, regardless of the inoculum employed. ${ }^{195,196}$ After inhibition is obtained, $p$-aminobenzoic acid is reported to exert its effect immediately under certain conditions. ${ }^{158,}, 197$

Correlation of Activities with Ionization of Sulfonamides. The influence of $\mathrm{pH}$ on the inhibitory activity of sulfonamides and on the ability of $p$-aminobenzoic acid to prevent the toxicity of these drugs was first indicated by Lwoff and co-workers. ${ }^{198}$ The amount of sulfanilamide necessary to prevent the reproduction of the flagellate, Polytomella caeca, was five times greater at $\mathrm{pH}$ values below 3.1 than at values above 5.5. The change in inhibitory activity of sulfanilamide appeared to occur only between these two points. The ratio of sulfanilamide to $p$-aminobenzoic acid at which complete reversal of the inhibition is obtained increases 
from 1 at a $\mathrm{pH}$ range of $9.2-7.55$ to 1,200 at $\mathrm{pH} 3.65$, but then decreases to 380 at $\mathrm{pH} 2.25$. These values corrected for the relative activity of sulfanilamide give 1, 245 and 76 , respectively, for the relative activities of $p$-aminobenzoic acid. Since the point of maximum activity corresponds closely to the isoelectric point of $p$-aminobenzoic acid, it was proposed that the undissociated molecules penetrate into the cell at a greater rate than the ions.

For Escherichia coli and Aspergillus niger, sulfanilamide was less effective in acidic than in neutral media; however, the variation with Escherichia coli was very slight and only fivefold for Aspergillus niger. ${ }^{198}$ Consequently, any theory must account for such small variations.

Some degree of correlation of the inhibitory activity of sulfonamides with their ability to dissociate to sulfonamide ions was subsequently noted. ${ }^{199,200}$ The ratios of the concentration of sulfanilamide, sulfapyridine, sulfathiazole or sulfadiazine to that of $p$-aminobenzoic acid at which inhibition of growth of Escherichia coli becomes apparent are approximately $500,40,8$ and 8 , respectively, at $\mathrm{pH} 7$. However, these ratios calculated on the basis of the ratio of sulfonamide anion to $p$-aminobenzoate, are $1.4,1.4,4.9$ and 6.4 , respectively. ${ }^{199}$ Approximately eight times as much sulfanilamide is required to inhibit the growth of Escherichia coli at $\mathrm{pH} 6.8$ than at $\mathrm{pH} 7.8 .{ }^{199} \mathrm{At} \mathrm{pH} 9$, there is comparatively little difference in the activity of sulfathiazole and sulfanilamide. ${ }^{200}$ Since an increase in the $\mathrm{pH}$ of the culture medium favors ionization of the sulfonamides, the increased effectiveness of sulfonamides with increased $\mathrm{pH}$ of the medium and the similar activities of certain sulfonamide ions led to the consideration that the anion is the active form of the ionizable sulfonamides. ${ }^{199,} 200$

Subsequently, it was observed that the activity of the sulfonamides increased with $\mathrm{pH}$ of the culture medium only up to the point where ionization is approximately half complete. ${ }^{124,201}$ Actual decreases in bacteriostatic activity are observed with highly ionized sulfonamides such as sulfadiazine, when the $\mathrm{pH}$ is increased above the point of 50 per cent ionization. Thus, when the $\mathrm{pH}$ of the medium exceeds the $\mathrm{pK}_{\mathrm{a}}$ of the sulfonamide, the activity begins to decline. For sulfadiazine, an increase of approximately 8.8 fold in concentration is required for bacteriostasis when the $\mathrm{pH}$ is increased from 6.5 , where the sulfonamide is approximately 50 per cent ionized, to 8.9 where the sulfadiazine is approximately 99.6 per cent ionized.

These results led to the suggestion that only the molecular form penetrated the cell wall of bacteria while only the ionic form combines with the enzyme which is inhibited. ${ }^{201}$

The decreasing ability of $p$-aminobenzoic acid $\left(\mathrm{pK}_{\mathrm{a}} 4.68\right)$ to prevent 


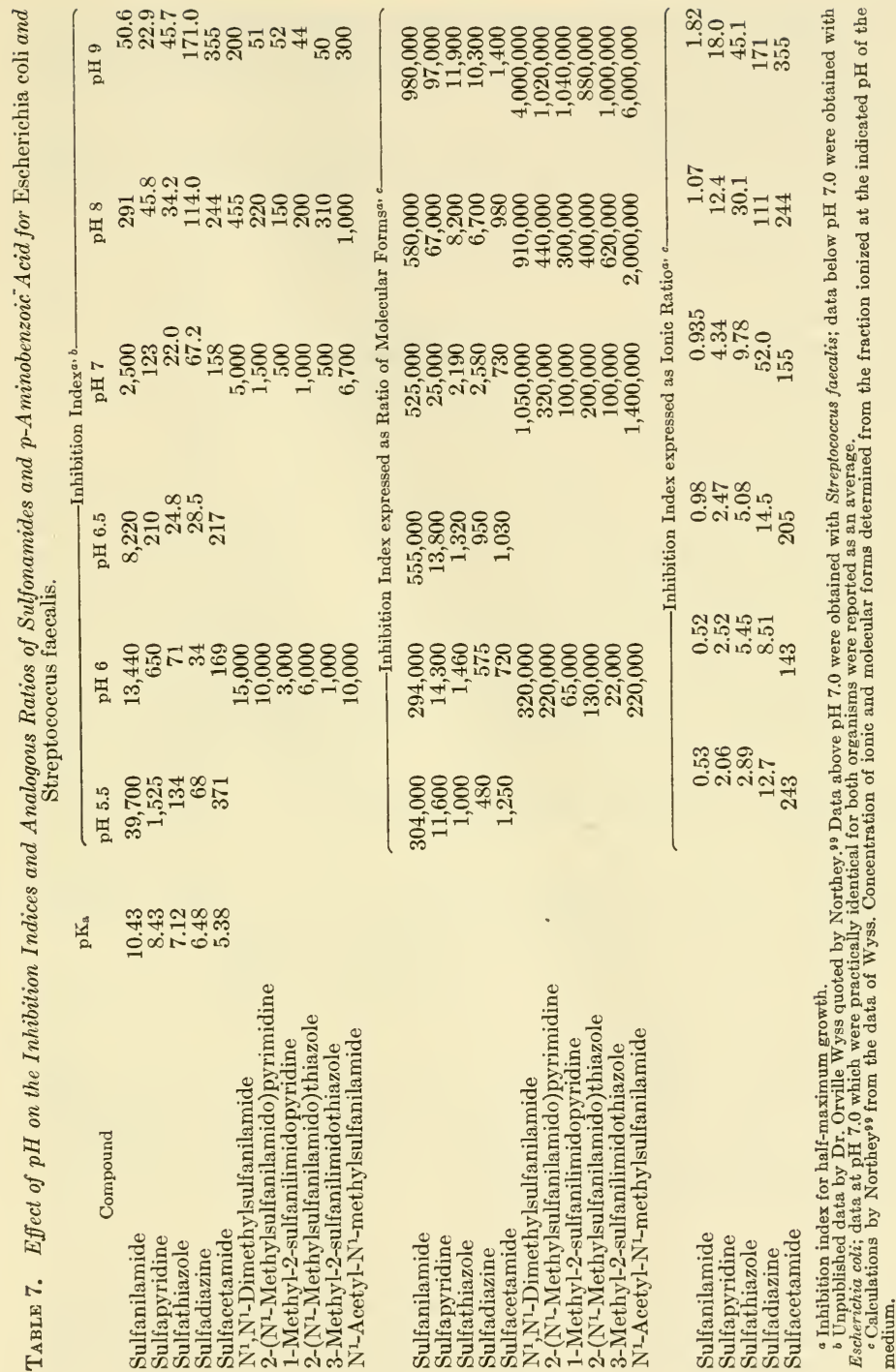


the toxicity of sulfonamide with increasing $\mathrm{pH}$ of the medium ${ }^{124,198}$ is parallelled by growth studies with a mutant strain of Neurospora crassa which requires considerably less $p$-aminobenzoic acid in media at $\mathrm{pH} 4$ than at higher $\mathrm{pH}$ levels. ${ }^{202}$

The effects of $\mathrm{pH}$ on the activities of several sulfonamides, including some which do not ionize, are indicated in Table 7. These data were derived by Northey ${ }^{99}$ from original data supplied by Wyss. Escherichia coli was used for the data below $\mathrm{pH} 7$ and Streptococcus faecalis in tests above $\mathrm{pH}$ 7. At $\mathrm{pH} 7$ the results with both organisms agreed so closely that only an average of the results was indicated. The inhibition indices are expressed in terms of the concentration of the sulfonamide to $p$-aminobenzoic acid, the ratio of the molecular forms of the substances in the medium, and the ratio of the ionic forms in the medium.

If only the molecular forms penetrate to the site of action, and if the $\mathrm{pH}$ within the cell is assumed to be constant over the $\mathrm{pH}$ range indicated, the ratio of molecular forms of sulfonamide to $p$-aminobenzoic acid in the medium would be proportional to the corresponding ratio of ionic forms within the cell. Since this ratio is not constant for the inhibition, it does not appear that the relative activity of sulfonamides can be explained solely on the basis of greater permeability of the molecular form and greater activity of the ionic form. However, the $\mathrm{pH}$ inside the cell may be affected more by the $\mathrm{pH}$ of the medium than is generally realized.

From the data presented in Table 7 , it is apparent that the sulfonamides are usually most effective at a $\mathrm{pH}$ almost equal to their $\mathrm{pK}_{\mathrm{a}}$, that at any given $\mathrm{pH}$ the most active sulfonamide is usually the one with a $\mathrm{pK}_{\mathrm{a}}$ approximating that $\mathrm{pH}$, and that non-ionic sulfonamides increase in activity with increases in $\mathrm{pH}$ over the range indicated. Similar results for changes in the activity of sulfonamides with changing $\mathrm{pH}$ of the medium have been reported for Mycobacterium tuberculosis. ${ }^{203}$

On the assumptions that (1) only the molecular form of sulfonamides penetrate the cell wall, (2) only the ionic form of sulfonamides acting within the cell inhibit the biological system, (3) that all sulfonamide ions have equal inhibitory activity within the cell, and (4) that the $\mathrm{pH}$ within the cells of Escherichia coli is 6, Northey ${ }^{99}$ derived an empirical equation relating activity of a sulfonamide to the fraction ionized in the medium and within the cell. Thus,

$$
\log 1 / C_{R}=\log X_{i}\left(1-X_{o}\right)+7.2573
$$

where $C_{R}$ is the minimum molar concentration producing the inhibition in medium at $\mathrm{pH} 7, X_{i}$ is the fraction of drug ionized within the cell, $X_{o}$ is the fraction ionized in the medium, and the last figure is $-\log k$, where 
$k$ is a proportionality constant derived empirically from the experimental maximum activity of sulfonamides at $\mathrm{pH} 7$, as indicated in Figure 7.

The curve for this expression when plotted as indicated in Figure 7 agrees closely with the experimental curve for Escherichia coli obtained from the data given in Table 8. Similar experimental data are obtained with other organisms, as indicated in Table 8. This theory, of course,

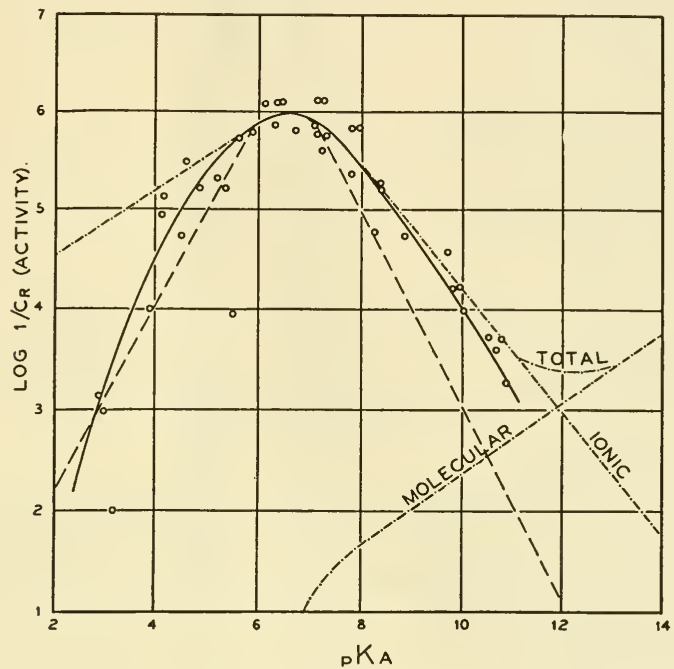

Figure 7. Experimental and theoretical relationships of activity of sulfonamides to acid dissociation constants.

Experimental curve. ${ }^{204}$

Theoretical (Negativity of $\mathrm{SO}_{2}$ group). ${ }^{204}$

- - - Theoretical (Negativity of

does not account for the high activity of certain sulfonamides which do not ionize.

Correlation with Negativity of the $\mathrm{SO}_{2}$ Group. Bell and Roblin ${ }^{204}$ have presented a theory correlating the negativity of the $\mathrm{SO}_{2}$ group of $\mathrm{N}^{1}$-substituted sulfonamides with their ability to prevent the growth of Escherichia coli. According to the theory, the more negative the $\mathrm{SO}_{2}$ group of an $\mathrm{N}^{1}$-substituted sulfanilamide derivative, the greater its bacteriostatic power. Since $p$-aminobenzoic acid is more than 99 per cent ionized in a medium buffered at $\mathrm{pH} 7$, the first formula of Figure 8 indicates the main form in which the vitamin exists in the medium and also within 
TABle 8. Dissociation Constants and Bacteriostatic Activity of N-Substituted Sulfonamides and Related Compounds

\section{Compound}

$p$-Aminobenzoic acid

$\mathrm{N}^{1}$-Sulfanilylsulfanilamide

Sulfanilylcyanamide

$\mathrm{N}^{1}$-Ethylsulfonylsulfanilamide

Sulfanilylglycine

$\mathrm{N}^{1}$-Chloroacetylsulfanilamide

3-Sulfanilamido-4-methylfurazan

5-Sulfanilamido-3-methylisoöxazole

3-Sulfanilamido-5-methyloxadiazole

$\mathrm{N}^{1}$-Benzoylsulfanilamide

4-Sulfanilamido-1,2,4-triazole

2-Sulfanilamido-1,3,4-thiadiazole

$\mathrm{N}^{1}-p$-Aminobenzoylsulfanilamide

$\mathrm{N}^{1}$-Acetylsulfanilamide

Sulfanilylurea

2-Sulfanilamido-5-methylthiadiazole

5-Sulfanilamido-2-chloropyrimidine

2-Sulfanilamidopyrazine

4-Sulfanilamidopyrimidine

Sulfadiazine

2-Sulfanilamidoöxazole

5-Sulfanilamidopyrimidine

3-Sulfanilamidopyridazine

2-Sulfanilamido-4-methylpyrimidine

5-Sulfanilamido-2-bromopyridine

Sulfathiazole

2-Sulfanilamido-5-bromopyridine

2-Sulfanilamido-4,6-dimethylpyrimidine

2-Sulfanilamido-4-methylthiazole

$\mathrm{N}^{4}$-Sulfanilylsulfanilamide

3-Sulfanilamidopyridine

4-Sulfanilamidopyridine

$\mathrm{N}^{3}$-Sulfanilylmetanilamide

Sulfapyridine

2-Sulfanilamido-5-aminopyridine

5-Sulfanilamido-2-aminopyridine

2-Sulfamilamido-4-aminopyrimidine

$\mathrm{N}^{1}$-Phenylsulfanilamide

2-Sulfanilamidoimidazole

$\mathrm{N}^{1}-m$-Tolylsulfanilamide

$\mathrm{N}^{1}-p$-Tolylsulfanilamide

$\mathrm{N}^{1}-o-$ Tolylsulfanilamide

$\mathrm{N}^{1}-p$-Aminophenylsulfanilamide

Sulfanilamide

$\mathrm{N}^{1}$-Methylsulfanilamide

$\mathrm{N}^{1}$-Furfurylsulfanilamide

$\mathrm{N}^{1}$-Hydroxyethylsulfanilamide

$\mathrm{N}^{1}, \mathrm{~N}^{2}-$ Dimethylsulfanilamide

Sulfanilylguanidine

Sulfanilylaminoguanidine

4,4'-Diaminodiphenylsulfone
Minimum Inhibitory Concentration $\times 10^{5}$ Mycobacterium

tuberculosis Streptococcus Escherichia var. hominis
coli 204

$\mathrm{pK}_{\mathrm{\Omega}}{ }^{204}$

4.68

2.89

2.92

3.10

3.52

3.79

4.10

60.0

100

1000

$>90.0$

10.0

1.0

$4.40 \quad 2.0$

$\begin{array}{ll}4.57 & 0.3\end{array}$

$4.66>80.0$

$4.77 \quad 0.6$

$5.20 \quad 0.5$

$\begin{array}{ll}5.38 & 0.7\end{array}$

$5.42 \quad 10.0$

$5.45 \quad 0.2$

$5.80 \quad 0.1$

6.04

6.17

0.08

46

10

6.0

0.6

6.48

6.5

6.62

7.06

7.06

7.12

7.12

7.15

7.37

7.79

7.85

7.89

8.00

8.23

8.43

8.47

8.82

9.44

9.60

9.72

9.74

9.82

9.96

10.22

10.43

10.77

10.88

10.92

0.1

0.08

0.08

0.2

0.08

0.2

0.2

0.08

0.5

0.3

0.2

0.5

0.2

2.0

0.6

0.6

2.0

20.0

3.0

40.0

5.0

5.0

10.0

5.0

20.0

30.0

20.0

50.0

30.0

10.0

0.9

2.0

0.62

0.15

1.03

1.2

4.7

10

13

13

26

3.57

1.72

14

13

4.54

0.69 
the cell of the organism which has a buffering capacity presumably in the same $\mathrm{pH}$ range. The molecular and ionized forms of $\mathrm{N}^{1}$-substituted sulfonamides are also indicated in Figure 8. From the standpoint of geometrical considerations, the $p$-aminobenzenesulfonyl group and the $p$-aminobenzoate ion are very similar, the bond distances differing only slightly, as indicated in Figure 8. Sulfones likewise are similar to the $p$-aminobenzoate ion.

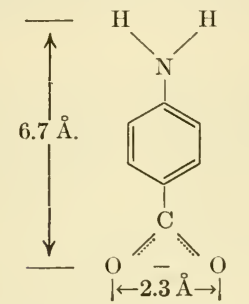

$p$-Aminobenzoate ion

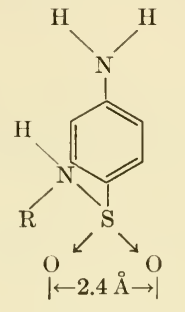

Sulfonamide

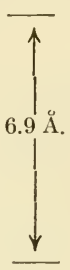

$|\leftarrow 2.4 \AA \rightarrow|$

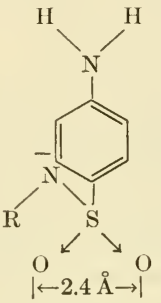

Sulfonamide ion

FIGURE 8. The Structures of the p-Aminobenzoate Ion and Molecular and Ionic Forms of Sulfonamides.

If the substituent group, $\mathrm{R}$, of the $\mathrm{N}^{1}$-substituted sulfonamide functions solely in affecting the combining power of the $p$-aminobenzenesulfonyl group with the enzyme and the substituent within itself does not possess groups which aid in this combining power, the activity of the sulfonamide will depend upon the effect of the substituent group on the combining power of the reactive groups, presumably the basic amino group and the $\mathrm{SO}_{2}$ group. Since only slight variations occur in the ionization constants for the basic amino group of the $\mathrm{N}^{1}$-substituted sulfonamides, differences in combining power of these sulfonamides with the inhibited enzyme cannot be attributed to the reactivity of the basic amino group, and no relationship between the bacteriostatic activity and these constants has been noted for these analogues. However, this may become an important consideration for other analogues.

The acid dissociation constants of $\mathrm{N}^{1}$-substituted sulfonamides vary over a wide range, indicating that the properties of the sulfonamide group are influenced greatly by the $\mathrm{N}^{1}$ substituent.

Since the $p$-aminobenzoate ion possesses an electronic charge which greatly increases the negative character of the $\mathrm{CO}_{2}^{-}$group, Bell and Roblin postulate that this negative character of the $\mathrm{CO}_{2}{ }^{-}$increases the affinity with which the molecule combines with the enzyme, and that the more negative the $\mathrm{SO}_{2}$ group in the sulfonamides, the greater the ability 
of the sulfonamide to compete with the $p$-aminobenzoate ion for the enzyme.

The ionic form of a sulfonamide has an electronic charge on the amide nitrogen. Since the $\mathrm{SO}_{2}$ group is electron-attracting, the charge on the adjacent atom is shared. This results in a more negative $\mathrm{SO}_{2}$ group in the ionized sulfonamide as compared with the corresponding group in the un-ionized form. Consequently, the ionized form of the sulfonamide would be expected by this theory to be considerably more active than the molecular form.

Sulfanilamide $\left(\mathrm{pK}_{\mathrm{a}} 3.7 \times 10^{-11}\right)$ at $\mathrm{pH} 7$ is only slightly ionized. Substitution of an electron-attracting group at the $\mathrm{N}^{1}$ position allows the hydrogen on this nitrogen to escape as a proton much more easily, since the electron density of the nitrogen atom is decreased by the electronattracting group. As the electron-attracting power of the $\mathrm{N}^{1}$ substituent increases, the degree of ionization of the sulfonamide increases.

However, as the electron-attracting power of the $\mathrm{N}^{1}$ substituent increases, the $\mathrm{SO}_{2}$ group becomes less negative, since the two groups compete for the electrons surrounding the nitrogen. Conversely, as the electron donating power of the $\mathrm{N}^{1}$ substituent increases, the $\mathrm{SO}_{2}$ group becomes more negative; however, simultaneously the degree of ionization decreases. These two opposing effects on activity of the sulfonamide would be expected to result in a maximum activity at a definite $\mathrm{pK}_{\mathrm{a}}$ value, according to the theory.

With the assumption that the bacteriostatic activity of $\mathrm{N}^{1}$-substituted sulfonamides is proportional to the potential of the $\mathrm{SO}_{2}$ group and that the potential of the $\mathrm{SO}_{2}$ group is influenced by the inductive effect of the $\mathrm{N}^{1}$ substituent, Bell and Roblin derived equations relating the biological activity of sulfonamide ion with the inductive constant of the $\mathrm{N}^{1}$ substituent. Thus,

$$
\log \left(k / x C_{R}\right)=\alpha\left(12.3-I_{R} \alpha\right)-I_{R} \alpha^{2}=4.39-0.255 I_{R}
$$

Similarly, the biological activity of the molecular form was related to the inductive constant. Thus,

$$
\log \left(k /(1-x) C_{R}\right)=\alpha\left(-1.3-I_{R} \alpha\right)-I_{R} \alpha^{2}=-0.464-0.255 I_{R}
$$

where $k$ is a proportionality constant, determined experimentally to be $0.001, x$ is the fraction of the compound in the ionized state, $C_{R}$ is the minimum molar concentration of the compound required for bacteriostasis, $I_{R}$ is the inductive constant of the $\mathrm{N}^{1}$ substituent, and $\alpha$ is the fraction of the inductive effect transmitted across each bond and is taken as $1 / 2.8$, the value of Branch and Calvin for a covalent bond.

From the above equations, the logarithm of the ratio of the activity of 
the molecular form to the ionized form is 4.85 , which means that the ion is approximately $10^{4.85}$ times as active as the corresponding molecular form. This seems to be somewhat high when the activities of nonionizing analogues are compared to similar ionizing forms.

The inductive constants of Branch and Calvin for various radicals are, according to Bell and Roblin, ${ }^{204}$ a linear function of the $\mathrm{pK}_{\mathrm{a}}$ values of the corresponding sulfanilamides with the radicals substituted in the $\mathrm{N}^{1}$ position.

$$
I_{R}=-1.33 p K_{\sigma}+13.88
$$

With these three equations, the activity of a sulfonamide could be determined from the ionization constant. In Figure 7, theoretical activities for the ionic and molecular forms at $\mathrm{pH} 7$ are plotted against $\mathrm{pK}_{\mathrm{a}}$ and compared with the experimental activities. The point of maximum activity occurs at a $\mathrm{pK}_{\mathrm{a}}$ value of 6.7 , which agrees very well with the experimentally determined maximum activity. The $\mathrm{pK}_{\mathrm{a}}$ of the most active sulfonamide varies with the $\mathrm{pH}$, since the fraction ionized changes for the various sulfonamides with changes in $\mathrm{pH}$.

The theoretical considerations applicable to the data with one organism, Escherichia coli in this instance, are not necessarily applicable to other organisms, since the combining power of the sulfonamide group may not in all cases be the limiting factor for interaction with the enzyme, and the enzyme may in itself differ to some extent from organism to organism.

Correlation With Ionization Constant. With the assumptions (1) that only the sulfonamide ion combines with the enzyme; (2) that a constant quantity of enzyme must be combined with the sulfonamide for inhibition of growth of Escherichia coli to occur; (3) that the dissociation constant for the enzyme-sulfonamide ion complex is a function of the ionization constant of the sulfonamide; and (4) that a maximum exists for the activity of sulfonamides correlated with ionization constant, an equation derived from the equilibrium constants by Klotz ${ }^{205}$ indicates that at this maximum, the logarithm of the dissociation constant of the enzyme-sulfonamide ion complex is a linear function of the logarithm of the ionization constant of the sulfonamide. Thus,

$$
\frac{d \ln K_{p}}{d \ln K_{a}}=\frac{\left[H^{+}\right]}{K_{a}^{0}+\left[H^{+}\right]}=J
$$

where $K_{p}$ represents the dissociation constant of the enzyme-sulfonamide complex, $K_{a}$ is the ionization constant of the sulfonamide, and $K_{a}^{0}$ is the ionization constant of the most effective sulfonamide at the hydrogen ion concentration, $\left[\mathrm{H}^{+}\right]$. Since the ratio of $d \ln K_{p}$ to $d \ln K_{a}$ is con- 
stant under the assumed conditions over a range of $K_{a}$ values at a con$\operatorname{stant}\left[\mathrm{H}^{+}\right], K_{p}=k K_{a}^{\dagger}$, where $k$ is an integration constant. Substitution of $k K_{a}^{f}$ for $K_{p}$ in an expression relating the ionization constants of sulfonamide and dissociation constants of enzyme-drug complexes resulted in an equation relating the activity of the sulfonamides to their ionization constants. However, the evaluation of $k$ and $f$ is dependent upon experimental data. Since the assumptions used in obtaining the equation are the theoretical considerations for which proof is desirable in advance of data, the equation has little to offer other than an empirical expression of the interrelationship of $\mathrm{pK}_{\mathrm{a}}$ and sulfonamide activity. The final expression is very similar to the one derived by Bell and Roblin. ${ }^{204}$

The assumption that a maximum exists in the activity for variable $\mathrm{pK}_{\mathrm{a}}$ values is based on the theory that the basic ionic form of the sulfonamide combines with the enzyme which acts as an acid. ${ }^{206}$ With increasing ionization constants, the sulfonamides would be expected to increase in activity; however, the increase in acidity of the sulfonamides would result in a decrease in basicity of the ion and decrease the combining power of the ion with the enzyme. Consequently, a maximum in activity may be expected from these theoretical considerations. These considerations do not, however, account for the relatively high activity of certain nonionizing analogues of $p$-aminobenzoic acid.

Effect of Resonance. It has been suggested that the activity of sulfanilamides and related compounds is associated with the contribution of the resonating form in which there is a separation of charge, such that a positively charged coplanar amino group is the fundamental factor and the negative character of the $\mathrm{SO}_{2}$ group is a concomitant factor associated with the resonating form. ${ }^{207,}{ }^{208}$ The interpretation of some experimental data on physical properties of sulfanilamide supporting this conclusion 209 has been questioned. ${ }^{210}$ Compounds such as a vinylog of sulfanilamide ${ }^{211}$ and the $p$-aminophenyldimethylsulfonium ion, ${ }^{160}$ which might be expected to have appreciable activity according to this theory, have been found to be relatively inactive. Other related theories have been proposed.212

\section{Miscellaneous Factors Influencing Sulfonamide Activity}

Inhibitory analogues of two metabolites of a biosynthetic sequence usually exert synergistic inhibitory effects. Either ethionine ${ }^{213}$ or methoxinine, ${ }^{214}$ both of which are inhibitory analogues of methionine for Escherichia coli, displays synergistic effects with sulfonamides. Similarly 5-amino-7-hydroxy-l- $v$-triazolo[d]pyrimidine, an inhibitory analogue of guanine, and sulfonamides are synergistic in inhibiting the growth of Staphylococcus aureus and Escherichia coli.

Many compounds have been reported to act synergistically with sul- 
fonamides. Some of these are: azochloramide, ${ }^{215-217}$ urea, ${ }^{218-225}$ guanidine, ${ }^{225,} 226$ thiourea, ${ }^{225,} 226$ urethane, ${ }^{215,216,} 223$ asparagine, ${ }^{217}$ hexyl carbamate, ${ }^{227}$ 6-benzylthiouracil, ${ }^{228} 5,6$-tetramethylene thiouracil, ${ }^{228}$ O-ethylisourea, ${ }^{224}$ dicyandiamide, $\mathrm{N}$-methyl thiourea, ${ }^{2 \cdot 24}$ penicillin, ${ }^{229}$ and $n$-propyl, isopropyl, $n$-butyl and isobutyl carbamates. ${ }^{230}$

Although the synergism of sulfonamides with urea has been confirmed, ${ }^{231}, 232$ failures to confirm the synergism have also been reported. ${ }^{233}$ Actually either additive or synergistic activity may be observed depending upon the experimental conditions employed.

Ethyl carbamate is reported to exert an antisulfonamide effect on luminous bacteria, ${ }^{234}$ and a slight effect on the toxicity of sulfanamide for Streptococcus hemolyticus ${ }^{235}$ and Escherichia coli. ${ }^{23.5}$

The bacteriostatic activity of sulfathiazole is reported to increase with temperature above $37^{\circ} \mathrm{C}$ for Escherichia coli and for Streptococcus pyogenes. ${ }^{236} p$-Aminobenzoic acid becomes less effective in preventing the toxicity of sulfathiazole and becomes a more potent inhibitor itself at higher concentrations under these temperature conditions. ${ }^{236}$

\section{Biological Effects of Sulfonamides and Related Compounds}

Effect of Biochemical Transformation. The effect of methionine, purines (or derivatives), serine or thymine (or derivatives, e.g., thymidine) in preventing the toxicity of sulfonamides are discussed separately (pp. 469 and 473). The involvement of $p$-aminobenzoic acid and related catalytic factors in the biosyntheses of these factors is indicated by the results of such inhibition studies.

The possibility that $p$-aminobenzoic acid has a role in the biosynthesis of other metabolites has been indicated by the ability of certain metabolites to exert an effect on the toxicity of sulfonamides. For example, arginine, histidine, lysine, methionine, glutamic acid and aspartic acid are reported to have some ability to prevent the toxicity of sulfonamides for Proteus vulgaris. ${ }^{237}$ Valine, and to a lesser extent lysine and isoleucine, prevent the toxicity of sulfanilamide for Escherichia coli in a medium containing methionine, purines, serine and either thymine or folic acid. ${ }^{238}$ Tryptophan has some ability to prevent the toxicity of sulfathiazole for Staphylococcus aureus. ${ }^{239,240}$

Various sulfonamides inhibit the growth of Eremothecium ashbyii, and the inhibition is paralleled by a decrease in formation of flavin, presumably riboflavin. ${ }^{241}$

The phosphorus content of yeast is increased by growth in the presence of sulfanilamide ( $200 \gamma$ per cc) from 1.9 to $2.6 \mathrm{mg}$ per $\mathrm{g}$ of dry cells. A slight increase in the nitrogen content is also noted under similar conditions; $p$-aminobenzoic acid $\left(1 \gamma\right.$ per cc) counteracts these effects. ${ }^{134,242}$ 
Effect on Respiration. Since the early indication ${ }^{243,244}$ that sulfonamides inhibit the respiration of certain bacteria and other microorganisms, numerous investigators have attempted to correlate inhibition of respiration with inhibition of growth by sulfonamides. While both inhibitions occur simultaneously in some organisms, ${ }^{2+4-246}$ the inhibition of growth of most organisms by sulfonamides appears to involve essential metabolic reactions not directly associated with respiration..$^{247}$ Respiration is inhibited by certain sulfonamides which are without chemotherapeutic activity. ${ }^{248}$

The inhibition of growth of Staphylococcus aureus by sulfapyridine is reported to be prevented partially by coenzymes I or II, but not by nicotinic acid. ${ }^{249}$ The ability of these coenzymes to prevent the toxicity of sulfapyridine has been questioned on the basis of failures to confirm this effect with both Staphylococcus aureus and a strain of Escherichia coli requiring nicotinic acid for growth. ${ }^{250}$ However, it has been shown that the ability of coenzyme I to exert such an effect on Staphylococcus aureus is dependent on the use of a small inoculum, and apparently is related to the growth-stimulating action of the coenzyme. ${ }^{251,252}$ High concentrations of nicotinic acid ( $100 \gamma$ per cc) are reported to prevent the toxicity of low concentrations of sulfapyridine for Lactobacillus arabinosus. ${ }^{253}$ Nicotinamide, cozymase, and nicotinamide-riboside exert a similar effect at somewhat lower concentrations ( 1 to $5 \gamma$ per cc) in preventing the toxicity of sulfapyridine $\left(2 \gamma\right.$ per cc).$^{253}$

Some attempts were unsuccessful in demonstrating interference of sulfanilamide, sulfapyridine or sulfathiazole in the functioning of cozymase in yeast fermentation and in several systems in rat liver. ${ }^{254}$

However, sulfapyridine appears to inhibit competitively the stimulation by nicotinamide of the respiration of nicotinamide-deficient cells of dysentery bacilli utilizing glucose. ${ }^{255-257}$ Greater inhibitory activity was observed if the sulfapyridine was added prior to the vitamin. Similar results were obtained with cozymase; but since $p$-aminobenzoic acid did not affect the inhibition, the inhibitory action does not appear to be related to growth inhibitions which are prevented by $p$-aminobenzoic acid. Other sulfonamides showed no definite inhibition of nicotinamidestimulated respiration. ${ }^{255-257}$

The sulfonamides, particularly sulfanilamide, prevent the combination of coenzyme II with the apoenzyme from yeast which oxidizes glucose6-phosphate to phosphohexonic acid. ${ }^{258}$ The sulfonamides react irreversibly with the apoenzyme and compete with the prosthetic group for the apoenzyme. Coenzyme II counteracts approximately fifty times its concentration of sulfanilamide. Glucose-6-phosphate also counteracts the inhibition to some extent, but $p$-aminobenzoic acid is ineffective in pre- 
venting the inhibition. The effect of the sulfonamides on cytochrome c, cytochrome $\mathrm{c}$ reductase, and lactic dehydrogenase is much less pronounced, and cytochrome oxidase is not affected. ${ }^{258} p$-Aminobenzoic acid and a number of other aromatic acids are reported to inhibit lactic acid dehydrogenase..$^{259}$

Sulfathiazole is reported to inhibit to a greater extent than other sulfonamides the anaerobic decarboxylation of pyruvic acid by Staphylococcus aureus, Escherichia coli and yeast. ${ }^{260}$ The partial inhibition of carboxylase of yeast or Staphylococcus aureus is prevented to some extent by cocarboxylase ${ }^{261}$ and to a greater extent by $p$-aminobenzoic acid. ${ }^{262} p$-Aminobenzoic acid at higher concentrations was inhibitory to carboxylase. ${ }^{261,262,263}$ It was considered that these results provided additional evidence for the hypothesis that the mode of action of sulfonamides involves the respiratory enzymes.

Although sulfonamides have been reported to inhibit the oxidation of glucose, ${ }^{246,255,256,263,264,265}$ glycerol, lactate and pyruvate, ${ }^{266-267}$ inhibitions of respiration by sulfonamides appear to be either unrelated or at most indirectly related to the inhibition of the catalytic role of $p$-aminobenzoic acid.

Effects on Nutrition of Animals. Certain sulfonamides fed to rats in a highly purified diet which alone supports normal growth and development cause the appearance of typical signs of dietary deficiencies. ${ }^{268-272}$ These symptoms often do not develop in animals receiving the sulfonamide in stock or natural diet. ${ }^{272,273}$ Although the mechanism by which sulfonamides exert such an effect is not completely understood, experimental evidence has usually been interpreted as indicating that the deficiencies develop as a result of the bacteriostatic action of the sulfonamides on the intestinal bacteria which synthesize certain factors required by the animals. The intestinal flora is usually markedly affected in animals receiving sulfonamide.

The symptoms which develop on administration of sulfaguanidine or succinylsulfathiazole in highly purified diets to rats include alopecia, achromotrichia, porphyrin-stained whiskers, anemia, leukopenia, agranulocytosis and hypocellularity of the bone marrow. These symptoms, as well as the retardation in growth and increase in prothrombin time which result from the toxic action of the sulfonamides, are overcome by supplements of folic acid and biotin. ${ }^{271},{ }^{273-279}$ Vitamin K counteracts only the prothrombin effect. 274,275 -Aminobenzoic acid prevents the effect of sulfaguanidine, ${ }^{268-274}$ but is reported not to counteract the effect of succinylsulfathiazole. ${ }^{272}$

The hepatic storage of folic acid and biotin decreases in rats on a highly purified diet as compared with stock or natural diets; however, 
inclusion of succinylsulfathiazole further decreases hepatic storage of these vitamins. ${ }^{280}$ Administration of folic acid and biotin returns the hepatic storage of these vitamins to normal. A marked decrease in hepatic storage of pantothenic acid which occurs on administration of succinylsulfathiazole is not corrected by administration of large amounts of pantothenic acid, whether administered orally or parenterally; however, supplements of folic acid and biotin allow normal storage of pantothenic acid in the liver, besides alleviating the symptoms normally attributed to pantothenic acid deficiency. ${ }^{280}$

Rats on stock diets containing succinylsulfathiazole are normal with respect to hepatic vitamin storage, with the exception of folic acid. The amount of folic acid in the liver is significantly decreased, but is still many times that found in deficient animals. ${ }^{281}$

Succinylsulfathiazole accentuates the folic acid deficiency induced by strain of lactation in rats. The leucopenia and granulocytopenia produced under these conditions are especially severe. ${ }^{282}$

The leukopenia, granulocytopenia and anemia produced in rats by sulfanilamide, sulfathiazole and sulfadiazine are prevented by either folic acid or $p$-aminobenzoic acid. ${ }^{283}$

Sulfapyridine fed at a level of 1 per cent of the diet produces symptoms of pantothenic acid deficieney, i.e., roughening of the fur, coproporphyrin deposits on the noses, wrists and whiskers, hemorrhagic necrosis of the adrenal glands, retardation of growth, and in black rats achromotrichia. All these symptoms except retardation of growth are relieved by relatively large supplements of pantothenic acid. ${ }^{284,285}$ The ability of pantothenic acid to counteract these effects is in contrast to the deficiency obtained with succinylsulfathiazole.

Xanthopterin has been reported to alleviate the leukopenia of rats fed a purified diet containing succinylsulfathiazole. ${ }^{285 a}$ However, several attempts to confirm this effect were not successful. ${ }^{278,279,286,287}$ More recently, xanthopterin has been reported to produce an immediate response in alleviating the anemia in rats fed a synthetic diet containing 1 per cent sulfathiazole. ${ }^{288}$ Folic acid was effective only after a delay of three to five days. ${ }^{2 s 8}$ Subsequent results indicated that the optimal dose of xanthopterin was $1 \mathrm{mg}$ per $\mathrm{kg}$ of body weight and that $10 \mathrm{mg}$ not only was ineffective but intensified the anemia. ${ }^{289}$ Similar results were obtained with xanthopterin and folic acid in stimulating cell proliferation of isolated bone marrow. ${ }^{290}$

Ascorbic acid is reported to be effective in treatment of the leucopenia occurring in rats fed a purified diet containing cither succinylsulfathiazole or phthalylsulfathiazole. ${ }^{291}$

Sulfonamides are reported to have a delayed carcinogenic action, though less than that of dibenzanthracene in albino rats and mice. ${ }^{292}$ 
Increased excretion of urobilins has been observed in rats treated with sulfonamides. ${ }^{293}$

The addition of succinylsulfathiazole to a highly purified diet deficient in inositol inhibited the growth of rats and caused alopecia, which was cured with inositol. ${ }^{294}$

Phthalylsulfathiazole administered to the pig on a diet deficient in inositol and biotin caused a syndrome which could be prevented by the addition of biotin, but was also largely alleviated by inositol. ${ }^{295}$ It has been suggested that the sulfa drugs inhibit intestinal microorganisms which synthesize inositol and thereby cause a deficiency. ${ }^{296-298}$ Sulfaguanidine in combination with inositol reduces the fertility of female albino rats, whereas either compound alone has no effect. ${ }^{299}$

Succinylsulfathiazole fed to pigs on a purified diet produces an anemia ${ }^{300,301}$ which responds to treatment with either folic acid, or less effectively with purified liver extracts. Earlier failures ${ }^{302,303}$ to accomplish this result have been explained on the basis of the relatively long period of treatment necessary to obtain this effect. Both folic acid and biotin are necessary to prevent the effects of the sulfonamide on the pig; however, folic acid administered without biotin accelerates the appearance of biotin deficiency symptoms. ${ }^{301}$ Thus, the interrelationships of these factors in the pig appear to parallel those in the rat.

The nutritional requirements of rabbits ${ }^{304}$ and chicks ${ }^{305,306}$ are reported to be affected by sulfaguanidine. On the other hand, succinylsulfathiazole does not appear to increase the nutritional requirements of chicks. ${ }^{307}$

Succinylsulfathiazole added to purified diets fed to mice retards the growth of the animals. ${ }^{308}$ Supplements of folic acid and biotin together do not counteract this effect; but these factors, together with liver extract, prevent the toxic action of the sulfonamide on growth of the animals. Concentrates of vitamin $\mathrm{B}_{12}$ almost completely replace the liver extract. ${ }^{308}$

Sulfanilamide, $125 \mathrm{mg}$ injected daily, prolonged nerve chronaxia and shortened muscle chronaxia in rats. ${ }^{308 a}$ These effects, which are similar to those in chronic alkalosis, are prevented by daily oral administration of ethyl $p$-aminobenzoate $(50 \gamma)$, nicotinic acid $(400 \gamma)$, riboflavin $(20 \gamma)$, or ascorbic acid $(5 \mathrm{mg})$. No effects were observed with thiamine, adenine, pantothenic acid or pyridoxine.

3-Hydroxysulfanilamide has been identified in human urine as a metabolic product of sulfanilamide. ${ }^{309}$

It has been reported that the toxicity for rats of bis $(4-\alpha$-aminovalerylphenyl)sulfone or related sulfones is diminished by administration of either $p$-aminobenzoic acid or ascorbic acid. ${ }^{310}$

Miscellaneous Effects. $p$-Aminobenzoic acid is reported to counteract the depressant effect of sulfanilamide on isolated frog hearts. ${ }^{311}$ While 
$p$-aminobenzoic acid alone at low concentrations exerts no effect on rhythm or amplitude, sulfanilamide at low concentrations increases amplitude, but does not accelerate rhythm, and at high concentrations stops the frog heart. ${ }^{311} p$-Aminobenzoic acid may under certain conditions inhibit the contractions of isolated frog heart. ${ }^{312}$ The incidence of convulsions after intramuscular injection of procaine in guinea pigs is reduced by previous administration of either diethylaminoethanol or $p$-aminobenzoic acid, or to a greater extent by both compounds. ${ }^{313}$ Other compounds structurally related to $p$-aminobenzoic acid and diethylaminoethanol exert a similar effect. ${ }^{313}$ No inhibition of the peripheral local anesthetic action of procaine occurs with these compounds. ${ }^{313}$ The effects are presumably unrelated to the catalytic roles of $p$-aminobenzoic acid.

The effect on intestinal contractions of procaine in physiologically active concentrations is suppressed by sulfanilamide in concentrations eight times that of procaine. ${ }^{314}$

Either $p$-aminobenzoic acid or diethylaminoethanol prevents competitively the typical convulsive action of procaine in guinea pigs. ${ }^{315}$

The formation of a yellow pigment in cultures of Mycobacterium tuberculosis in the presence of $p$-aminobenzoic acid or procaine is inhibited by sulfanilamide. ${ }^{316,317}$ The oxidation of $p$-aminobenzoic acid to a red-colored substance by peroxidase from horseradish is also prevented by sulfanilamide. ${ }^{317}$

Sulfanilamide, sulfathiazole or sulfapyridine inhibits the peroxidase reaction, but not phenol oxidase with $p$-aminobenzoic acid as a substrate. ${ }^{317}$

Carbonic anhydrase is inhibited by sulfanilamide, ${ }^{318}$ but the inhibition is only partially counteracted by $p$-aminobenzoic acid. ${ }^{319}$

An interesting adsorption effect has been reported in which sulfanilamide ( 0.1 per cent) decreases by 44 per cent the amount of methylene blue adsorbed by charcoal. $p$-Aminobenzoic acid (0.028 per cent) completely prevented the effect of sulfanilamide on the adsorption. ${ }^{320}$ The possibility that sulfonamides act bacteriostatically by reducing cellular and colloidal adsorption was suggested. ${ }^{320}$

\section{Resistance to Sulfonamides}

Natural Resistance. Many organisms, either isolated from patients or tested at random, have been found to possess a "natural" resistance to the sulfonamides. ${ }^{321-333}$ For example, two strains of Staphylococcus aureus isolated from patients with severe infections differed in their susceptibility to sodium sulfathiazole before treatment was administered;321 and from 168 patients with pneumonia, meningitis or endocarditis, moderately resistant organisms were isolated from six cases. ${ }^{322}$ 
Different types of pneumococei ${ }^{323,324,327,328,332}$ vary in their susceptibility to various sulfonamides. Similar results are obtained with different strains of clostridium, ${ }^{326}$ gonococcus ${ }^{329}$ and $\beta$-hemolytic streptococci. ${ }^{331,333}$ Marked disparity in the inhibitory ability of a specific sulfonamide for 10 strains of Shigella sonnei has been noted. ${ }^{325}$

Although clinical and in vitro observations usually correspond, ${ }^{329,} 334,335$ in vitro resistance does not always indicate in vivo resistance, and vice versa. $328,329,332,336-339$ Sulfathiazole resistance was induced in vitro in Shigella paradysenteriae Flexner, but this resistance was not exhibited in vivo in white mice. ${ }^{337}$ By isolating pneumococei from patients and following the changes in their resistance during the course of therapy, it was found that the in vitro resistance of an organism was decreased. ${ }^{332}$ Of organisms isolated from patients who have shown resistance in sulfonamide therapy, some are not resistant in vitro. Although variation in resistance of isolated gonococci corresponded to the clinical reaction of the patient in most cases, an in vitro-susceptible strain has been isolated from a patient resistant to sulfathiazole therapy, and an in vitro-resistant strain has been isolated from a patient who had responded to this treatment. ${ }^{329}$ These and similar observations in clinical studies with gonococci ${ }^{339}$ suggest that this particular type of resistance is dependent entirely upon the environment. It appears that in vivo resistance may sometimes be due to a host factor (and in vitro resistance, to a constituent of the culture medium), which counteracts the action of the sulfonamide.

Acquired Resistance. Organisms serially transferred in vitro in increasing concentrations of the sulfonamides may be made resistant to the action of these drugs. Resistance may also be developed in vivo during sulfonamide administration to the host. Many organisms have been shown capable of developing resistance to the sulfonamides: Escherichia coli, ${ }^{77}, 250,340-344$ hemolytic streptococci, ${ }^{331,345-349}$ Streptococcus pyogenes ${ }^{350}$ Neisseria gonorrheae, ${ }^{334,335,350 \mathrm{a}-357}$ Neisseria meningitidis, ${ }^{356}$ Neisseria catarrhalis, ${ }^{356}$ and Neisseria sicca, ${ }^{356}$ Brucella abortus, ${ }^{12}$ Brucella paramelitensis, ${ }^{342}$ pneumococci, ${ }^{18,}$, 266, 322, 324, 330, 332, 336, 338, 358-369 Staphylococcus aureus, ${ }^{1,18,321,342,370-376}$ Staphylococcus pyogenes, ${ }^{350}$ Shigella paradysenteriae, ${ }^{337,}$, 377-379 Shigella sonnei, ${ }^{18,325,337,377,378}$ Mycobacterium ranae, ${ }^{380}$ Friedlander's bacillus, ${ }^{343}$ Acetobacter suboxydans, ${ }^{381}$ Polytomella caeca, ${ }^{104}$ and Endamoeba histolytica. ${ }^{382}$ Between strains of one species there is often a wide variation in the ease with which resistance may be developed, ${ }^{351}, 359,362$ but the number of resistant organisms developing from a single strain often does not vary significantly. ${ }^{351}$

Development of resistance in vivo may be rather difficult or may occur readily, depending upon the organism. In a study of pneumococei from 72 infected patients, a strain with striking resistance developed in only 
one case $;{ }^{364}$ however, resistance of organisms isolated from patients after prolonged treatment is usually increased. Gonococci isolated from infected patients not cured after sulfathiazole had been administered for 6 days (6 $\mathrm{gm} /$ day) were resistant to $0.5 \mathrm{mg}$ per cent of sulfathiazole in vitro, and some grew in 25-50 mg per cent, while strains from cured patients were susceptible to this concentration. ${ }^{355}$

A wide variation in the extent to which resistance may be developed in different strains has also been observed. ${ }^{321,359,377}$

Although resistance to a certain bacteriostatic concentration of one sulfonamide is usually accompanied by an equal resistance to similar bacteriostatic concentrations of the other sulfonamides, $327,341,351,353,356$, $357,362,363,365$ this is not a rule without exceptions. ${ }^{345,350,377}$ For example, strains of Shigella paradysenteriae Flexner, Shigella sonnei $(\mathrm{Ch})$ and Shigella sonnei (Ma), made resistant to sulfathiazole by transfer in increasing concentrations, were resistant also to sulfapyridine, sulfadiazine and sulfanilamide, but not to sulfapyrazine. Their resistance to sulfacetamide varied. ${ }^{377,}, 378$

Cross-resistance between sulfonamides and penicillin does not appear to take place, ${ }^{330,344,350,359}$ and it has been shown that development of resistance to sulfanilamide does not influence susceptibility to streptomycin, atebrin, sodium salicylate, 3,5-dibromosalicylic acid and synthelin (decamethylenediguanidine).$^{344}$

A high degree of resistance acquired after a great many transfers on

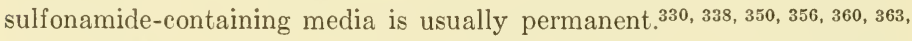
383, 384 However, partial resistance acquired through short contact with the drug is usually temporary, and is lost after repeated transfer on sulfonamide-free media. ${ }^{338,}, 360,367,383$

Several factors affecting the degree of resistance aequired have been observed. It has been indicated that, unless the drug is present in suffcient quantity to inhibit growth of Escherichia coli, very little resistance is developed, and that the degree of resistance developed varies with the concentration used. ${ }^{341}$ However, it is also reported that by repeated transfer of cultures of Escherichia coli on low concentrations of the drug, resistant strains can be developed. ${ }^{250}$ With Brucella abortus an increase in the time of incubation increases the degree of resistance developed. ${ }^{12}$ In the presence of $p$-aminobenzoic acid, no sulfonamide resistance could be produced with Escherichia coli, and the presence of methionine delayed its acquisition..$^{250}$ Organisms made resistant in a plain infusion broth containing peptone were not resistant when tested in a synthetic medium. ${ }^{250}$

Because pneumococei surviving the first exposure to sulfapyridine were significantly more resistant than any organisms of the original parent 
strain, ${ }^{385}$ it was suggested that the acquisition of sulfonamide-resistance is a sudden, spontaneous "mutation" occurring continuously, but becoming evident only when conditions are suitable for selective propagation of resistant cells. ${ }^{351}$ The gradual increase in resistance of Staphylococcus aureus to sulfonamides occurring at random time during serial transfer in sodium sulfathiazole ${ }^{376}$ was interpreted to indicate mutation and selection. The slow and apparently multiple process in obtaining resistant strains of Bacterium lactis aerogenes and early stage reversal of resistance in the absence of the inhibitor, indicating a slower growth rate for the resistant strain, have been presented as evidence in favor of an adaptive mechanism. ${ }^{383}$

From the standpoint of the permanence of resistance in organisms as related to the clinical use of sulfonamides, it is of interest that the cultivation of a mixed population of sulfanilamide-resistant and susceptible cells of Escherichia coli for 10 passages in synthetic media free of sulfanilamide resulted in a "weeding out" of the resistant strain, as shown by subsequent exposure of the culture to the drug. ${ }^{344}$

The converse of resistance is obtained by culturing in hemolyzed horse blood staphylococci and some streptococci which are relatively insensitive to sulfonamide. The organisms develop marked sensitivity to the sulfonamides. ${ }^{386}$

Mechanism of Resistance. A significant increase in production of $p$-aminobenzoic acid or a related anti-sulfonamide compound over that in parent or nonresistant strains has been observed in sulfonamide-resistant strains of Staphylococcus aureus, ${ }^{18,353,371,373,387,389}$ gonococci, ${ }^{334}$ strains of elostridium, ${ }^{326}$ Brucella paramelitensis, ${ }^{342}$ Escherichia coli, ${ }^{340}$ streptococci, ${ }^{340}$ Polytomella caeca, ${ }^{104}$ Diplococcus pneumoniae, ${ }^{340,388,390}$ and

TABLE 9. The Inhibition Index and Minimum Inhibitory Concentration of 2-Sulfanilamido-4-methylthiazole for Resistant and Original Strains of

Staphylococcus aureus ${ }^{127}$

$\begin{array}{cc} & \begin{array}{c}\text { Minimum } \\ \text { Effective } \\ \text { Inhibitor } \\ \text { Concentration } \\ \times 10^{5}, a\end{array} \\ \text { Strain } & \end{array}$

$\mathrm{B}$ (original)

B (resistant)

Nr. VI (original)

Nr. VI (resistant)

Nr. IX (original)

Nr. IX (resistant)

Nr. X (original)

Nr. X (resistant)

Nr. VII (original)
0.31

10.0

0.18

6.67

0.21

4.67

1.67

12.0

1.25
Inhibition Index ${ }^{b}$

Relative
Antisulfonamide
Produced $^{c}$

$$
<20
$$

14

220

7

133

31

66

21

35

30

a Concentration necessary to reduce growth to one-third of controls.

$b$ Ratio of concentration of sulfamethylthiazole $(0.001 \mathrm{M})$ to $p$-aminobenzoic acid.

- Relative antisulfonamide activity against strain B of extracts prepared from the various strains.

d Arbitrary value. 
Shigella sonnei. ${ }^{388}$ The synthesis of anti-sulfonamides occurs in both the presence and the absence of sulfonamides. ${ }^{371,373}$ The sulfonamide inhibitors may be restricted to the cells or released into the medium. ${ }^{340}$ Although the inhibitor in most cases noted is $p$-aminobenzoic acid, some inhibitors exhibit different properties and presumably are not $p$-aminobenzoic acid. ${ }^{340}$

Conversely, in experiments with certain resistant strains of Shigella paradysenteriae Sonne, ${ }^{18}$ Diplococcus pneumoniae Type $I^{18}$ and Staphylococcus aureus, ${ }^{391}$ there was no demonstrable inerease in synthesis of $p$-aminobenzoic acid, ${ }^{18}$ and for certain resistant strains of Neisseria gonorrheae increased synthesis of $p$-aminobenzoic acid was insufficient to account entirely for such resistance. ${ }^{355}$ A sulfathiazole-resistant strain of staphylococci with no increased production of an anti-sulfonamide was found to require smaller amounts of $p$-aminobenzoic acid for reversal of sulfonamides than the parent strain. ${ }^{389}$

Ivanovics ${ }^{127}$ compared the minimum inhibitory concentrations of sulfamethylthiazole and the inhibition indices obtained with resistant and parent strains of Staphylococcus aureus. The data listed in Table 9 indicate that the resistance of this organism can be accounted for by increased production of $p$-aminobenzoic acid or a related sulfonamide antagonist, or by more efficient utilization of $p$-aminobenzoic acid, or by a combination of the two mechanisms. The former mechanism is characterized by an increased minimum inhibitory concentration for the resistant organisms, but does not involve a change in the inhibition index. More efficient utilization of $p$-aminobenzoic acid results in an increased inhibition index.

Differences Between Resistant and Parent Strains. Although the virulence of an organism is usually unchanged in the acquisition of sulfonamide resistance, ${ }^{266}, 331,358,359,392$ in some cases contrary observations have been made. ${ }^{336,337,348,393}$ Certain strains of Shigella sonne $i^{337}$ and $\beta$-hemolytic streptococci ${ }^{348}$ became nonvirulent on becoming resistant to sulfonamides. Meningococci which became resistant to sulfanilamide lost their virulence, while those resistant to sulfapyridine remained virulent. ${ }^{393} \mathrm{~A}$ sulfonamideresistant strain of gonococcus lost its pathogenicity, and it could not be restored by treatment with $p$-aminobenzoic acid. ${ }^{120}$

More often than not, the morphology of the resistant strain remains the same as that of the parent strain ; $266,338,3.6,363,367$ however, changes have been noticed in some cases. The more resistant strains of staphylococci studied produced a nonfat-soluble yellow pigment in the presence of sulfonamides. ${ }^{372}$ However, strains which were not as highly resistant did not produce this pigment. It is suggested that this pigment may be derived from $p$-aminobenzoic acid. 
Resistant strains of pneumococci did not lose type-specific characteristics, and were still susceptible to anti-serum. ${ }^{363}$

Sulfonamide-resistant strains of pneumococci ${ }^{266}$ produce less hydrogen peroxide than the parent strain, and the ability to form hydrogen peroxide is lost by strains of $\alpha$-streptococci which become resistant to sulfathiazole $;^{349}$ but these strains no longer require riboflavin for growth and are more effective than the parent strain in oxidizing a number of substrates. $^{349}$ This contrasts to resistant strains of pneumococci, which are less effective in oxidizing glycerol, lactate and pyruvate but not glucose. ${ }^{266}$ Strains of Shigella with the greatest fermentation activity are more resistant to sulfonamides. ${ }^{325}$ Resistant strains of Mycobacterium ranae ${ }^{380}$ produce diazotizable arylamines. Development of resistance and increased arylamine formation in Staphylococcus aureus is reported not to be associated, but the arylamine in this case is apparently derived from tryptophan, and is not $p$-aminobenzoic acid. ${ }^{375}$

\section{Other Inhibitory Analogues of $p$-Aminobenzoic Acid}

Shortly after the discovery of the competitive relationship of $p$-aminobenzoic acid and the sulfonamides, many compounds differing from the sulfonamides but related in structure to $p$-aminobenzoic acid were prepared and tested. The compounds of this group which inhibit the utilization of $p$-aminobenzoic acid by various organisms are indicated in Table 10. It is apparent that neither a free amino nor an acidic radical is essential for the inhibitory action of an analogue of $p$-aminobenzoic acid. Nitro and acetamido groups may replace the amino group for certain inhibitory analogues, while the modification of the carboxyl group can be extended to a variety of changes. Utilization of carboxamide, ketone and alcohol groups, as well as arsonic, phosphonic and phosphonous acid groups in place of the carboxyl group of $p$-aminobenzoic acid and related analogues results in some instances in inhibitory analogues. Some inhibitory analogues contain an isosteric, heterocyclic ring in place of the aromatic ring structure of $p$-aminobenzoic acid, while other inhibitory analogues are substituted $p$-aminobenzoic acids. Of a large number of analogues of $p$-aminobenzoic acid with substituents in the aromatic nucleus, only a few inhibit the utilization of this vitamin. ${ }^{16,21}$ Usually disubstituted $p$-aminobenzoic acids are inactive, indicating the possibility that one side of the ring structure of the vitamin must be intact for combination with the appropriate enzymes.

Halogeno-4-aminobenzoic Acid. Although 2-fluoro-4-aminobenzoic acid is approximately one-third as effective as $p$-aminobenzoic acid in promoting growth of certain organisms and in preventing the toxicity of sulfanilamide (p. 484), 3-fluoro-4-aminobenzoic acid is almost as effective as 


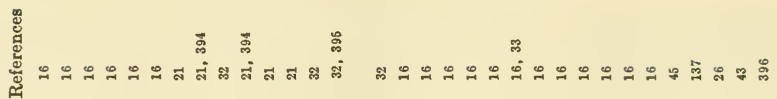

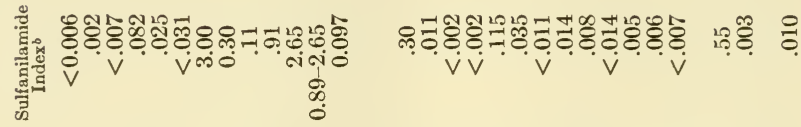

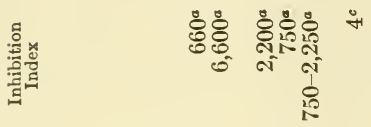

ชี่

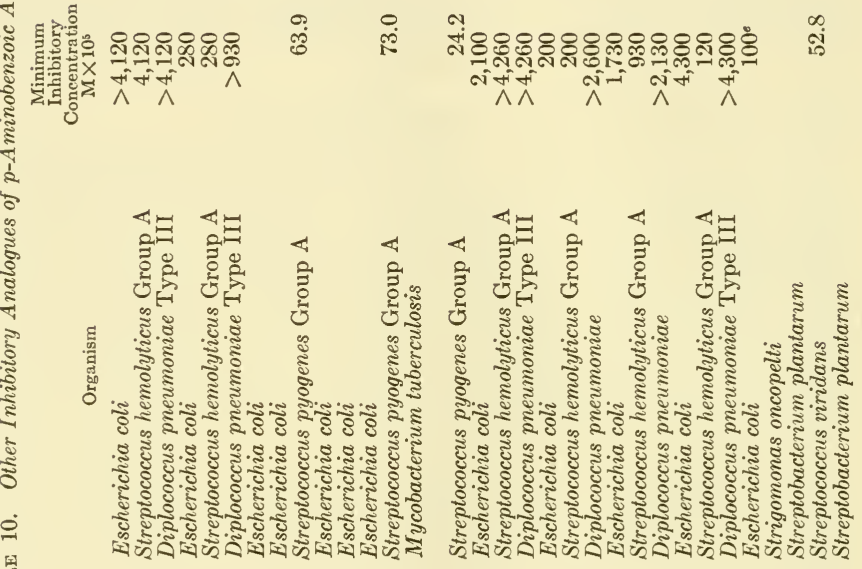

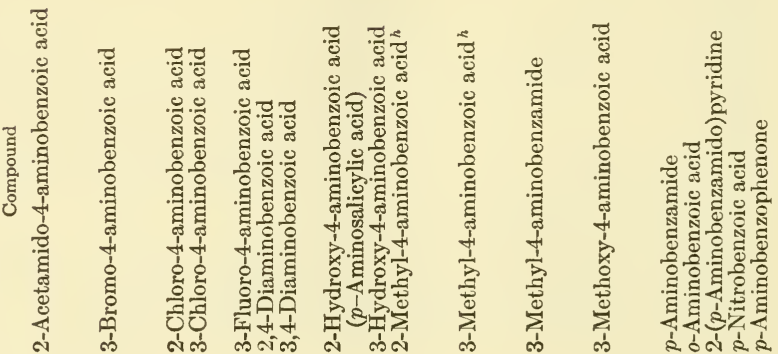




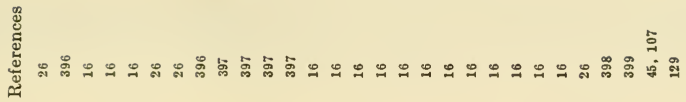

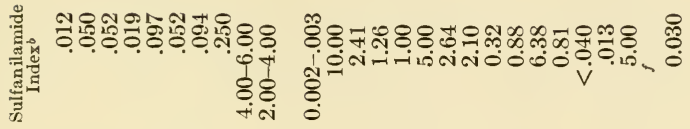

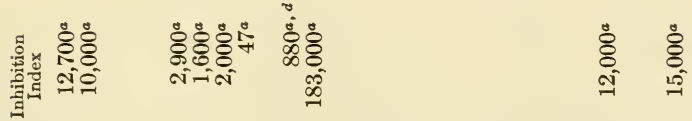

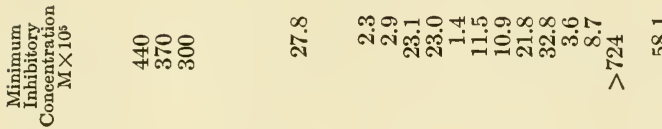

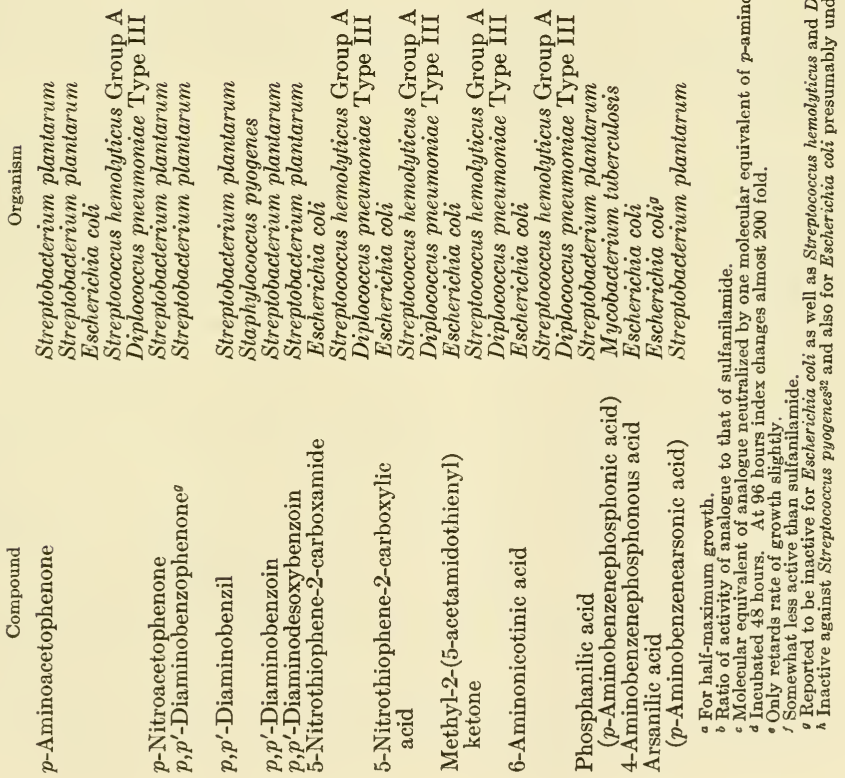


sulfanilamide in preventing the growth of Escherichia coli, and 2-chloro4-aminobenzoic acid is more active than sulfanilamide against Escherichia<smiles>Nc1ccc(C(=O)O)c(Cl)c1</smiles>

2-chloro-4-aminobenzoic acid<smiles>Nc1ccc(C(=O)O)cc1F</smiles>

3-fluoro-4-aminobenzoic acid

coli. ${ }^{21}$ However, in the presence of methionine, 2-chloro-4-aminobenzoic acid not only does not exert an inhibitory effect on Escherichia coli but has a slight ability to prevent the toxicity of sulfanilamide for that organism. The 2-chloro derivative also has some ability to prevent the toxicity of sulfonamides for Diplococcus pneumoniae, Streptococcus pyogenes and Streptococcus hemolyticus. ${ }^{16,32,33}$ Strains of Escherichia coli which are resistant to inhibition by 2 -chloro-4-aminobenzoic acid are obtained in a single transfer in the presence of the analogue; however, these strains resistant to 2-chloro-4-aminobenzoic acid are not resistant to sulfonamides, but sulfonamide-resistant strains are resistant to inhibition by the 2 -chloro derivative. ${ }^{400}$

Pantothenic acid is reported to be as effective as $p$-aminobenzoic acid in preventing the toxicity of 2-chloro-4-aminobenzoic acid; 401 however, since this effect is not observed after short incubation periods ${ }^{402}$ and since resistance ${ }^{400}$ to the inhibition occurs after a longer incubation period in the absence of pantothenic acid, it appears that this effect may be associated with the resistance phenomenon. Either thiamine or glutamic acid exerts effects analogous to pantothenic acid on this inhibition. ${ }^{402}$

In contrast to slight growth-promoting activity of 2-bromo-4-aminobenzoic acid, ${ }^{21,33}$ which may possibly be attributed to contamination with $p$-aminobenzoic acid, 3-bromo-4-aminobenzoic acid has some inhibitory action against Escherichia coli and Streptococcus hemolyticus Group A. ${ }^{16}$ 3-Chloro-4-aminobenzoic acid is somewhat more inhibitory than the corresponding 3-bromo derivative for Escherichia coli. The 3chloro derivative is also effective against Streptococcus pyogenes.

4-Amino-2-hydroxybenzoic Acid ( $p$-Aminosalicylic Acid) and Related Analogues. Since benzoic and salicylic acids were reported to stimulate the oxygen consumption and carbon dioxide production of tubercle bacilli, ${ }^{403}$ Lehmann, ${ }^{404,}{ }^{405}$ in attempting to find an inhibitor of the stimulatory action, tested 60 substances of similar structure and found that the 
most effective compound, $p$-aminosalicylic acid, inhibited growth of the tubercle bacillus at concentrations as low as $1.5 \gamma$ per cc. At this concen-

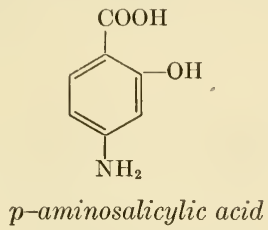

tration the inhibitor was bacteriostatic rather than bactericidal. The compound fed at a concentration of 5 per cent of the diet was not toxic for rats, mice or rabbits, but guinea pigs became emaciated, lost hair and died within two weeks on such a diet. In guinea pigs infected with Mycobacterium tuberculosis, the analogue tended to retard the disease, but only at concentrations at which some of the animals died. However, no toxic manifestations on administration of 10 to $15 \mathrm{~g}$ daily were observed in patients with tuberculosis. Patients responded with a prompt fall in temperature, improvement in general condition, gains in weight and appetite, and increases in red cells and hemoglobin.

$p$-Aminosalicylic acid in vitro is a very effective bacteriostatic agent at concentrations of 0.1-1.5 $\gamma$ per ce for tubercle bacillus, ${ }^{404-411}$ but does not have appreciable inhibitory activity against other microorganisms. ${ }^{408,} 412,413$

In the presence of $1 \gamma$ per cc of $p$-aminobenzoic acid, the amount of $p$-aminosalicylic acid necessary for inhibition of growth of Mycobacterium tuberculosis H37Rv is increased approximately 16 fold. ${ }^{42}$ Salicylic acid also prevents the toxicity of $p$-aminosalicylic acid in a somewhat competitive manner, but is only $2-6$ per cent as effective as $p$-aminobenzoic acid. ${ }^{395}$

Contrasting with the inhibitory action on Mycobacterium tuberculosis, $p$-aminosalicylic acid replaces $p$-aminobenzoic acid in stimulating the growth of two mutant strains of Escherichia coli which require $p$-aminobenzoic acid for growth. ${ }^{28}$ The analogue depending upon the testing conditions is from 4 to 16 per cent as effective as $p$-aminobenzoic acid. ${ }^{28}$ It appears that $p$-aminosalicylic acid inhibits the utilization of $p$-aminobenzoic acid for tubercle bacilli, but is utilized in place of the vitamin by Escherichia coli. Such activity, if general among microorganisms, would account for the unusual specificity of $p$-aminosalicylic acid as an inhibitory analogue.

Strains of tubercle bacilli which are resistant to streptomycin are sensitive to $p$-aminosalicylic acid, ${ }^{42,407}$ and the two compounds have been 
reported to act synergistically in inhibiting the growth of some strains of the organism.411 With other strains, only additive effects are observed. ${ }^{42,414}$ Nevertheless, the utilization of $p$-aminosalicylic acid concurrent with streptomycin to prevent the selection of resistant strains is indicated.

Although $p$-aminosalicylic acid is reported to have little therapeutic activity in rabbits or guinea pigs infected with certain strains of tubercle bacilli, ${ }^{405}, 408,414$ the analogue appears to suppress experimental tubereulosis in mice, ${ }^{42}$ as determined by histopathologic examination. ${ }^{415}$ The effect of streptomycin on the analogue appears to be additive in vivo.

$p$-Aminosalicylic acid is rapidly adsorbed and excreted by human subjects as well as by laboratory animals. ${ }^{414,416-419}$ Although blood levels of the compound can be maintained in the animals for several hours, approximately 85 per cent of the administered dose is recoverable from the urine within ten hours. ${ }^{416}$ Recovery as conjugated amines was highest (60 per cent of total dose) in man and apparently negligible in the dog. ${ }^{416}$ Both the free $p$-aminosalicylic acid and the $\mathrm{N}$-acetyl derivative can be isolated from the urine of rabbits after administration of $p$-aminosalicylic acid. ${ }^{417}$ In the urine of one human subject, three compounds containing a free amino group and two conjugated amines have been detected subsequent to the administration of $p$-aminosalicylic acid. ${ }^{416}$ Among these, unchanged $p$-aminosalicylic acid, $p$-aminosalicyluric acid and $\mathrm{N}$-acetyl$p$-aminobenzoic acid have been identified. ${ }^{416}$

4-Amino-3-hydroxybenzoic acid is about one-third as effective as sulfanilamide against Streptococcus pyogenes, and has a slight chemotherapeutic action in mice infected with that organism. ${ }^{32}$ The corresponding 3-methyoxy derivative is somewhat less inhibitory to Streptococcus hemolyticus Group A. ${ }^{16}$

Other Substituted p-Aminobenzoic Acids. Both 2,4-diamino- and 3,4-diaminobenzoic acids are antagonists of $p$-aminobenzoic acid for Diplococcus pneumoniae, ${ }^{33}$ and compare favorably with sulfanilamide in inhibiting the growth of Escherichia coli. ${ }^{21}$ Considerable loss in activity is observed ${ }^{16}$ with acetylation of the 2 -amino group of the former compound. ${ }^{16}$

3-Methyl-4-aminobenzoic acid has some inhibitory activity against Escherichia coli and Streptococcus hemolyticus. The corresponding 2-methyl derivative is less effective against Escherichia coli, and is inactive against Streptococcus hemolyticus. The amide of the 3-methyl derivative is less active than the parent compound.

Isosteres. A number of compounds which are isosteric with p-aminobenzoic acid are rather potent inhibitory analogues of the vitamin. 5-Nitrothiophene-2-carboxylic acid and the corresponding amide are more 
active than sulfanilamide for several organisms (Table 9). The latter compound is approximately ten times as active as sulfanilamide for<smiles>NC(=O)c1ccc([N+](=O)[O-])s1</smiles>

5-nitrothiophene-2-carboxamide<smiles>CC(=O)Nc1ccc(C(C)=O)s1</smiles>

methyl-2-(5-acetamidothienyl) ketone

Escherichia coli. While the heterocyclic amide is approximately ten times as active as the free acid for Escherichia coli, the acid is approximately twice as effective as the amide against both Streptococcus hemolyticus Group A and Diplococcus pneumoniae Type III. The corresponding amino compound, 5-aminothiophene-2-carboxamide, is inactive as an inhibitor for these organisms. ${ }^{16}$ Methyl 2-(5-acetamidothienyl) ketone is more active than sulfanilamide in inhibiting growth of Escherichia coli, but is somewhat less effective against Streptococcus hemolyticus and Diplococcus pneumoniae. A similar situation exists for 6-aminonicotinic acid, which is several times as active as sulfanilamide against Escherichia coli and is essentially as active against Streptococcus hemolyticus; but the analogue does not inhibit the growth of Diplococcus pneumoniae Type III. It is interesting that this analogue is reported to be an antagonist of nicotinic acid rather than of $p$-aminobenzoic acid in Staphylococ-<smiles>Nc1ccc(C(=O)O)cn1</smiles>

\section{6-aminonicotinic acid}

cus aureus. ${ }^{40}$ The pyrimidine derivative corresponding to this pyridine analogue, 2-amino-5-pyrimidinecarboxylic acid, is reported to have slight activity in preventing the toxicity of sulfonamides (p. 484).

Ketone Analogues. As indicated in Table 9, p-aminophenyl ketones are very effective inhibitory analogues of $p$-aminobenzoic acid. $p, p^{\prime}$ Diaminobenzil is several times as active as sulfanilamide in inhibiting the growth of either Streptobacterium plantarum or Staphylococcus pyogenes. This combination of two carbonyl groups results in a more effective inhibitor than a single carbonyl. Thus, the diketone is approximately 20 to 60 times as effective as $p, p^{\prime}$-diaminobenzophenone. Slight chemotherapeutic activity was observed with $p, p^{\prime}$-diaminobenzophenone

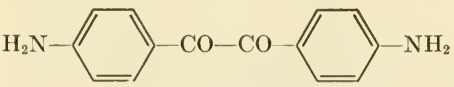

$p, p^{\prime}$-diaminobenzil

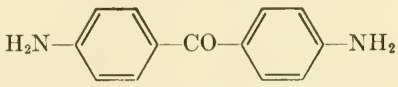

$p, p^{\prime}$-diaminobenzophenone 
in mice infected with streptococci, gonococci and meningococci. The compound had only a slight effect against staphylococci and pneumococci in vivo. ${ }^{396}$ Although the inhibitory activity of $p, p^{\prime}$-diaminobenzoin against Streptobacterium plantarum approached that of $p, p^{\prime}$-diaminobenzil for short periods of incubation, the benzoin derivative decreased markedly in activity with increased incubation periods to only a small fraction of the activity of the benzil derivative. ${ }^{26}$ It was suggested that the organism

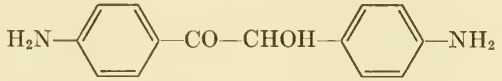

$p, p^{\prime}$-diaminobenzoin

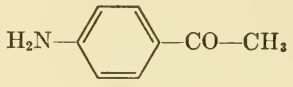

$p$-aminoacetophenone

oxidized the benzoin derivative to $p$-aminobenzoic acid, thereby effecting a reversal of the inhibition. $p$-Aminobenzophenone is about 10 to 25 per cent as effective as sulfanilamide in preventing growth of Streptobacterium plantarum, and $p, p^{\prime}$-diaminodesoxybenzoin and $p$-aminobenzophenone are only slightly inhibitory to that organism. $p$-Nitroacetophenone appears to be more effective than $p$-aminoacetophenone against Streptobacterium plantarum.

Miscellaneous Analogues. $p$-Nitrobenzoic acid at low concentrations inhibits the growth of Streptococcus viridans, and the growth inhibition is prevented by $p$-aminobenzoic acid; at higher concentrations the nitro compound does not inhibit growth of the organism. Under these conditions, the organism produces a diazotizable amine in the medium which has anti-sulfonamide properties. ${ }^{44}$ Consequently, it appears that reversals noted at high concentrations are the result of the action of $p$-aminobenzoic acid accumulating from reduction of the inhibitor by the organism.

$p$-Aminobenzamide is an inhibitory analogue of $p$-aminobenzoic acid for Escherichia coli, but is not particularly effective. Similar results have been obtained with 2-(4-aminobenzamido)pyridine against Streptobacterium plantarum.

Ethyl $p$-aminobenzoate is an inhibitory analogue of $p$-aminobenzoic acid for Streptobacterium plantarum. ${ }^{421}$

o-Aminobenzoic acid at a very high inhibition index is toxic for Strigomonas oncopelti, ${ }^{137}$ and $m$-aminobenzoic acid has some inhibitory activity against Streptobacterium plantarum. ${ }^{421}$ For the latter organism a high concentration of benzoic acid is toxic, and the inhibition is prevented by $p$-aminobenzoic acid. ${ }^{421}$

Even $p$-aminobenzyl alcohol has some ability to inhibit the utilization of $p$-aminobenzoic acid by some organisms. ${ }^{420 a}$ 6-Amino-2-naphthoic acid is slightly inhibitory to Streptobacterium plantarum. ${ }^{396}$ 
Analogues of $p$-Aminobenzoic Acid Containing Arsenic, Antimony or Phosphorus. The sodium salt of arsanilic acid ( $p$-aminobenzenearsonic acid), which has been termed atoxyl, was one of the first compounds found to have trypanosomacidal activity. The discovery of the activity of $p$-aminobenzoic acid in preventing the toxicity of the sulfonamides for bacteria prompted analogous research on arsanilic acid. The effect of arsanilic acid in retarding the growth of Escherichia coli as measured by oxygen consumption is counteracted by $p$-aminobenzoic acid. ${ }^{45}$ With some strains of the organism, complete inhibition of growth occurs, and in one case the inhibition index for half-maximum growth has been found to be approximately $15,000 .{ }^{107}$ The analogue is only about 3 per cent as effective as sulfanilamide. Methionine also prevents the toxicity of arsanilic acid, as well as sulfonamides, for Escherichia coli. ${ }^{107}$

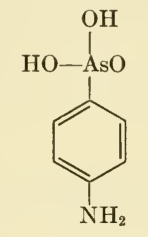

arsanilic acid<smiles>Nc1ccc([R](O)(O)O)cc1</smiles>

phosphanilic acid

The trypanosomacidal activity of arsanilic acid, which is attributed to the formation of the arsenoxide, is not affected by $p$-aminobenzoic acid in a test which renders Trypanosoma equiperdum noninfective for young rats. ${ }^{107}$ However, the chemotherapeutic action of arsanilic acid in Trypanosoma equiperdum infections in mice is reported to be counteracted by certain doses of $p$-aminobenzoic acid and sulfanilamide. ${ }^{421 a}$ These results suggest the possibility that substances of analogous structure prevent the conversion of arsanilic acid to the active form. Further evidence for such possibilities is indicated by reports that relatively large amounts of $p$-aminobenzoic acid prevent the toxicity for rats of otherwise lethal doses of arsanilic acid and related arsonic acids. ${ }^{422-425}$ However, $p$-aminobenzoic acid apparently does not prevent the therapeutic effects of these arsenicals against Trypanosoma equiperdum in rats. If $p$-aminobenzoic acid is not administered within a few hours after the arsenical, it is not effective in preventing the toxic manifestations of the drugs. ${ }^{425} p$-Aminobenzoic acid is not particularly effective in preventing the toxic effects of trivalent arsenicals, especially that of $m$-amino- $p$-hydroxyphenylarsenoxide. ${ }^{425}$ The effect of an otherwise lethal dose of neoarsphenamine is counteracted by $p$-aminobenzoic acid in dogs. ${ }^{426}$ The trypanocidal activity of $\gamma$-( $p$-arsenosophenyl) butyric acid both in vivo and in vitro is 
also counteracted by $p$-aminobenzoic acid. ${ }^{427}$ The question of specificity of $p$-aminobenzoic acid in eliciting some of these responses has not been adequately determined.

The toxicity of salvarsan, neosalvarsan, or neostibosan for Streptobacterium plantarum is prevented by $p$-aminobenzoic acid..$^{129}$

Similarly, the toxic manifestations caused by administration of sodium $m$-chloro- $p$-acetylaminobenzenestibonate to rats are counteracted by $p$-aminobenzoic acid, which does not appreciably affect the therapeutic activity of the drug against Trypanosoma equiperdum..$^{428}$

Phosphanilic acid inhibits the growth of Streptobacterium plantarum, and $p$-aminobenzoic acid prevents the inhibition. The inhibition index for half-maximum growth is approximately $12,000 .{ }^{26}$ Both phosphanilic acid and phosphanilamide are reported to inhibit with varying degrees of activity the growth of Escherichia coli, Staphylococcus aureus and Salmonella typhimurium. ${ }^{429}$ Phosphanilic acid also inhibits the growth of Mycobacterium tuberculosis in vitro. ${ }^{430,431}$ Administered three times daily in doses of $15 \mathrm{mg}$, it is not toxic to mice, but only low concentrations in the blood are attained. ${ }^{431}$

p-Aminobenzenephosphorous acid is slightly less inhibitory than sulfanilamide to the growth of Escherichia coli. $p$-Aminobenzoic acid prevents the inhibitory effect of the analogue. ${ }^{432}$

\section{Bibliography}

1. Woods, D. D., Brit. J. Exptl. Path., 21, 74 (1940); Woods, D. D., and Fildes, P., Chemistry and Industry, 59, 133 (1940).

2. Fischer, G., Ann., 127, 137 (1863).

3. Gelmo, P., J. prakt. Chem., 77, 369 (1908).

4. Heidelberger, M., and Jacobs, W. A., J. Am. Chem. Soc., 41, 2145 (1919).

5. Mietzsch, F., Ber., 71A, 15 (1938).

6. Mietzsch, F., and Klarer, J., Ger. 607, 537, Jan. 2, 1935; U. S. 2,085,037, June 29, 1937.

7. Domagk, G., Deut. med. Wochschr., 61, 250 (1935).

8. Tréfouël, J., Tréfouël, Mme. J., Nitti, F., and Bovet, D., Compt. rend. soc. biol., 120, 756 (1935).

9. Lockwood, J. S., J. Immunol., 35, 155 (1938).

10. McIntosch, J., and Whitby, L. E. H., Lancet, 1939, I, 431.

11. Stamp, T. C., Lancet, 1939, II, 10.

12. Green, H. N. G., Brit. J. Exptl. Path., 21, 38 (1940).

13. Rubbo, S. D., and Gillespie, J. M., Nature, 146, 838 (1940); Lancet, 1942, I, 36.

14. Blanchard, K. C., J. Biol. Chem., 140, 919 (1941).

15. Kuhn, R., and Schwarz, K., Ber., 74B, 1617 (1941).

16. Johnson, O. H., Green, D. E., and Pauli, R., J. Biol. Chem., 153, 37 (1944).

17. Rubbo, S. D., Maxwell, M., Fairbridge, R. A., and Gillespie, J. M., Australian J. Exptl. Biol. Med. Sci., 19, 185 (1941).

18. Housewright, R. D., and Koser, S. A., J. Infectious Diseases, 75, 113 (1944).

19. Lampen, J. O., and Peterson, W. H., Arch. Biochem., 2, 443 (1943).

20. Lampen, J. O., and Peterson, W. H., J. Biol. Chem., 153, 193 (1944). 
21. Wyss, O., Rubin, M., and Strandskov, F. B., Proc. Soc. Exptl. Biol. Med., 52, 155 (1943).

22. Landy, M., and Dicken, D. M., J. Biol. Chem., 146, 109 (1942).

23. Lampen, J. O., Underkofler, L. A., and Peterson, W. H., J. Biol. Chem., 146, 277 (1942) ; Underkofler, L. A., Bantz, A. C., and Peterson, WV. H., J. Bact. 45, 183 (1943).

24. Tatum, E. L., and Beadle, G. W., Proc. Natl. Acad. Sci. U.S., 28, 234 (1942); Beadle, G. W., and Tatum, E. L., Proc. Natl. Acad. Sci. U.S., 27, 499 (1941).

25. Thompson, R. C., Isbell, F. R., and Mitchell, H. K., J. Biol. Chem., 148, 281 (1943).

26. Kuhn, R., Möller, E. F., Wendt, G., and Beinert, H., Ber., 75B, 711 (1942).

27. Dann, O., and Möller, E. F., Chem. Ber., 80, 21 (1947).

28. 'Tobie, W. C., and Jones, M. J., J. Bact., 57, 573 (1949).

29. Euler, H. v., and Karrer, P., Helv. Chim. Acta, 27, 1697 (1944).

30. Goetchius, G. R., and Lawrence, C. A., J. Bact., 48, 683 (1944).

31. Martin, A. R., Rose, F. L., and Swain, G., Nature, 154, 639 (1944).

32. Martin, A. R., and Rose, F. L., Biochem. J., 39, 91 (1945).

33. Sirks, J. L., "Antonie von Leeuwenhoek," J. Microbiol. Serol., 11, 171 (1946).

34. Levaditi, C., Mentzer, C., and Pérault, R., Compt. rend. soc. biol., 136, 769 (1942).

35. Dansi, A., Farm. sci. e tec. (Pavia), 2, 195 (1947); Chem. Abstr., 42, 639 (1948).

36. Landy, M., and Wyeno, J., Proc. Soc. Exptl. Biol. Med., 46, 59 (1941).

37. Isbell, H., J. Biol. Chem., 144, 567 (1942).

38. Lewis, J. C., J. Biol. Chem., 146, 441 (1942).

39. Beyer, K. H., Mattis, P. A., Patch, E. A., and Russo, H. F., J. Pharmacol. Exptl. Therap., 84, 136 (1945).

40. Keltch, A. K., Baker, L. A., Krahl, M. E., and Clowes, G. H. A., Proc. Soc. Exptl. Biol. Med., 47, 533 (1941).

41. Lehmann, J., Lancet, 1946, I, 14, 15.

42. Youmans, G. P., Raleigh, G. W., and Youmans, A. S., J. Bact., 54, 409 (1947).

43. Miller, J. K., J. Pharmacol. Exptl. Therap., 71, 14 (1941).

44. Miller, J. K., J. Pharmacol. Exptl. Therap., 72, 354 (1941).

45. Hirsch, J., Science, 96, 139 (1942).

46. Hirsch, J., Compt. rend. annw. arch. soc. turque dcs sci. phys. nat., 10, 115 (1942); Chem. Abstr., 38, 3092 (1944).

47. Rubbo, S. D., and Gillispie, J. M., Lancet, 1942, I, 37.

48. Boroff, D. A., Cooper, A., and Bullowa, J. G. M., Proc. Soc. Exptl. Biol. Med., 47, 182 (1941).

49. Levaditi, C., and Pérault, R., Compt. rend. soc. biol., 135, 1043 (1941).

50. Legge, J. W., and Durie, E. B., Mcd. J. Australia, 29, 561 (1942) ; Chem. Abstr., 37, 2077 (1943).

51. Powell, H. M., Krahl, M. E., and Clowes, G. H. A., J. Indiana State Med. Assoc., 35, 62 (1942); Chem. Abstr., 36, 2017 (1942).

52. Allen, J. G., and Livingstone, H., Anesthesia and Analgesia, 21, 285 (1942); Chem. Abslr., 37, 465 (1943).

53. de Waal, H. L., Kanaar, A. C., and MeNaughtan, J., Lancet, 243, 724 (1942).

54. Benigno, P., Arch. expll. Path. Pharmakol., 199, 265 (1943); Chem. Abstr., 37, 5489 (1943).

55. Casten, D., Fried, J. J., and Hallman, F. A., Surgery, Gynecol. Obslet., 76, 726 (1943); Chem. Abstr., 37, 4757 (1943).

56. Surrey, A. R., and Hammer, H. F., J. Am. Chem. Soc., 66, 2127 (1944).

57. Lawrence, C. A., and Goetchius, G. R., Proc. Soc. Exptl. Biol. Med., 57, 180 (1944).

58. Peterson, O. L., Finland, M., Barnes, M. W., and Wilcox, C., Am. J. Med. Sci., 207, 166 (1944). 
59. Maevskii, V. E., and Fetisova, O. I., Farmakol. i. Toksikol., 7, No. 4, 39 (1944); Chem. Abstr., 39, 3592 (1945).

60. Pfeiffer, C. C., and Grant, C. W., Anesthesiology, 5, 605 (1944); Chem. Abstr., 39, 2331 (1945).

61. Hazard, R., and Vaille, C., Compt. rend. soc. biol., 139, 535 (1945).

62. Winkler, A., Zentr. Bakt. Parasitenk. I. (Abt., Orig.), 151, 106 (1944); Chem. Abstr., 39, 322 (1945).

63. Desbordes, J., Compt. rend. soc. biol., 137, 596 (1943); Chem. Abstr., 39, 3028 (1945).

64. Di Fonzo, M., and De Vanna, F., Farm. sci. e tec. (Pavia), 2, 493 (1947); Chem. Abstr., 42, 3021 (1948).

65. Auhagen, E., Z. physiol. Chem., 277, 197 (1942).

66. Nimmo-Smith, R. H., and Woods, D. D., unpublished observations referred to by Woods, D. D., Ann. Rev. Biochem., 16, 616 (1947); Abstrs. XVII Int. physiol. Congr. 328 (1947).

67. Wagner-Jauregg, T., and Wagner, W. H., Z. Naturforsch., 1, 229 (1946).

68. Williams, R. J., J. Biol. Chem., 156, 85 (1944).

69. Lampen, J. O., and Jones, M. J., J. Biol. Chem., 170, 133 (1947).

70. Sarett, H. P., J. Biol. Chem., 171, 265 (1947).

71. Lampen, J. O., and Jones, M. J., J. Biol. Chem., 166, 435 (1946).

72. Lampen, J. O., and Jones, M. J., J. Biol. Chem., 164, 485 (1946).

73. Morgan, H. R., J. Exptl. Med., 88, 285 (1948).

74. Morgan, H. R., Proc. Soc. Exptl. Biol. Med., 67, 29 (1948); Early, R. L., and Morgan, H. R., J. Immunol., 53, 151 (1946).

75. Ratner, S. Blanchard, M., Coburn, A. F., and Green, D. E., J. Biol. Chem., 155, 689 (1944).

76. Ratner, S., Blanchard, M., and Green, D. E., J. Biol. Chem., 164, 691 (1946).

77. Miller, A. K., Proc. Soc. Exptl. Biol. Med., 57, 151 (1944).

78. Briggs, G. M., Jr., Luckey, T. D., Mills, R. C., Elvehjem, C. A., and Hart, E. B., Proc. Soc. Exptl. Biol. Med., 52, 7 (1943).

79. Mills, R. C., Briggs, G. M., Jr., Luckey, T. D., and Elvehjem, C. A., Proc. Soc. Exptl. Biol. Med., 56, 240 (1944).

80. Snell, E. E., and Mitchell, H. K., Arch. Biochem., 1, 93 (1942).

81. Shive, W., and Roberts, E. C., J. Biol. Chem., 162, 463 (1946).

82. Tschesche, R., Soehring, K., and Harder, K., Z. Naturforsch., 2b, 244 (1947).

83. Lampen, J. O., Roepke, R. R., and Jones, M. J., J. Biol. Chem., 164, 789 (1946).

84. Summers, W. A., Proc. Soc. Exptl. Biol. Med, 66, 509 (1947).

85. O'Meara, R. A. Q., McNally, P. A., and Nelson, H. G., Nature, 154, 796 (1944); Lancet, 253, 747 (1947).

86. Tschesche, R., Z. Naturforsch., 2b, 10 (1947).

87. Nimmo-Smith, R. H., Lascelles, J., and Woods, D. D., Brit. J. Exptl. Path., 29, 264 (1948).

88. Eppright, M. A., and Williams, R. J., J. Gen. Physiol., 30, 61 (1946).

89. Whitby, L. E. H., Lancet, 1938, 1, 1210.

90. Ewins, A. J., and Phillips, M. A., U. S. 2,335,221, Nov. 23, 1943; Brit. 512,145, Aug. 30, 1939.

91. Goldyrev. L. N., and Postovski, I. Y., J. Applied Chem. (U.S.S.R.), 11, 316 (1938) ; Crossley, M. L., Northey, E. H., and Hultquist, M. E., J. Am. Chem. Soc., 62, 372 (1940).

92. Fosbinder, R. J., and Walter, L. A., J. Am. Chem. Soc., 61, 2032 (1939).

93. Roblin, R. O., Jr., Williams, J. H., Winnek, P. S., and English, J. P., J. Am. Chem. Soc., 62, 2002 (1940).

94. Dohrn, M., and Diedrich, P., Münch. med. Wochschr., 85, 2017 (1938); Crossley, M. L., Northey, E. H., and Hultquist, M. E., J. Am. Chem. Soc., 61, 2950 (1939). 
95. Winnek, P. S., U. S. 2,218,490, Oct. 15, 1940; U. S. 2,229,784, Jan. 28, 1941; U. S. 2,233,569, Mar. 4, 1941.

96. Marshall, E. K., Jr., Bratton, A. C., White, H. J., and Litchfield, J. T., Jr., Bull. Johns Hopkins Hosp., 57, 163 (1940).

97. Moore, M. L., U. S. 2,324,013-5, July 13, 1943.

98. Poth, E. J., Knotts, F. L., Lee, J. T., and Inui, F., Arch. Surg., 44, 187 (1942); Poth, E. J., and Ross, C. A., Texas Repts. Biol. and Med., 1, 345 (1943).

99. Northey, E. H., "The Sulfonamides and Allied Compounds," Am. Chem. Soc. Monograph, Reinhold Publishing Corp. New York, 1948, 660 pp.

100. Wyss, O., Grubaugh, K. K., and Schmelkes, F. C., Proc. Soc. Exptl. Med., 49, 618 (1942).

101. McLeod, J. W., Mayr-Harting, A., and Walker, N., Brit. J. Exptl. Path., 25, 27 (1944).

102. Strauss, E., Lowell, F. C., and Finland, M., J. Clin. Invest., 20, 189 (1941).

103. Strauss, E., Dingle, J. H., and Finland, M., Proc. Soc. Exptl. Biol. Med., 46, 131 (1941).

104. Gordon, I., Rept. N. Y. State Dept. Health, Div. Lab. Res., 1940, 10; Chem. Abstr., 35, 7442 (1941).

105. Lwoff, A., Nitti, F., Tréfouël, J., and Hamon, V., Ann. inst. Pasteur, 67, 9 (1941).

106. White, H. J., Litchfield, J. T., Jr., and Marshall, E. K., Jr., J. Pharmacol. Exptl. Therap., 73, 104 (1941).

107. Peters, L., J. Pharmacol. Exptl. Therap., 79, 32 (1943).

108. Wood, W. B., Jr., J. Exptl. Med., 75, 369 (1942).

109. Green, H. N., and Bielschowsky, F., Brit. J. Exptl. Path., 23, 13 (1942).

110. Nitti, F., Cosar, C., and Boyer, F., Ann. inst. Pasteur, 71, 142 (1945).

111. Gonzales, F., and Sotomayor, A., Rev. med. alimentacion (Santiago, Chile), 5, 53 (1942); Chem. Abstr., 39, 325 (1945).

112. Hirsch, J., Compt. rend. Ann. Arch. Soc. turque sci. phys. nat., 10, No. 10, 109 pp. (1941-1942); Chem. Abstr., 38, 3310 (1944).

113. Tamura, J. T., J. Bact., 47, 529 (1944).

114. Fitzgerald, F. J., and Feinstone, W. H., Proc. Soc. Exptl. Biol. Med., 52, 27 (1943).

115. Frisk, A. R., Acta Med. Scand., 125, 487 (1946); Chem. Abstr., 41, 1011 (1947).

116. Gromwall, A., and Zetterberg, B., Acta Med. Scand. Suppl. 170, 517 (1946); Chem. Abstr., 40, 5797 (1946).

117. Mayer, R. L., J. Bact., 48, 93, (1944); 48, 337 (1944).

118. Sjögren, B., "Thé Svedberg" (Mem. Vol.) 1944, 547; Chcm. Abstr., 39, 1460 (1945).

119. Ottolenghi-Lodigigiani, F., Boll. soc. ital. sper., 17, 697-698 (1942); Chem. Abstr., 41, 202 (1947).

120. Kimmig, J., Klin. Wochschr., 22, 31 (1943); Chem. Abstr., 38, 4002 (1944).

121. Kimmig, J., Klin. Wochschr., 20, 235 (1941); Chem. Abstr., 36, 7060 (1942).

122. Nitti, F., Tabone, J., and Mousset, H., Ann. inst. Pasteur, 68, 474 (1942).

123. Muir, R. D., Shamliffer, V. J., and Jones, L. R., J. Bact., 44, 95 (1942); 41, 84 (1941).

124. Brueckner, A. H., Yale J. Biol. Med., 15, 813 (1945).

125. Spink, W. W., and Jermsta, J., Proc. Soc. Exptl. Biol. Med., 47, 395 (1941).

126. Weis, O., and Jones, L. R., J. Bact. (Proceedings), 41, 82 (1941).

127. Ivánovies, G., Z. Immunitäts, 101, 58 (1942).

128. Hartelius, V., and Roholt, K., Compt. rend. trav. lab. Carlsberg, Sér. physiol., 24, 156 (1946).

129. Möller, E. F., and Swarz, K., Ber., 74B, 1612 (1941).

130. Ivánovies, G., Z. Immunitäts, 103, 469 (1943).

131. Seelemann, M., Deut. tierärztl. Wochschr., 50, 221 (1942); Chem. Abstr., 37, 4761 (1943). 
132. Hartelius, V., and Roholt, K., Compt. rend. trav. lab. Carlsberg, Sér. physiol., 24, 163 (1946).

133. Landy, M., and Dicken, D. M., Nature, 149, 244 (1942).

134. Nielsen, N., Compt. rend. trav. lab. Carlsberg, Sér. physiol., 24, 55 (1944).

135. Hartelius, V., and Roholt, K., Compt. rend. trav. lab. Carlsberg, Ser. physiol., 24, 141 (1946).

136. Hartelius, V., Compt. rend. trav. lab. Carlsberg, Sér. physiol., 24, 178 (1946).

137. Lwoff, M., and Lwoff, A., Ann. inst. Pasteur, 71, 206 (1945).

138. Dimond, N. S., Science, 94, 420 (1941).

139. Ribeiro, F., J. Biol. Chem., 152, 665 (1944).

140. Bonner, J., Proc. Nat. Acad. Sci., 28, 321 (1942).

141. Brian, P. W., Nature, 153, 83 (1944).

142. Ungar, J., Nature, 152, 22 (1943).

143. Ciferri, R., and Scaramuzzi, G., Farm sci. e. tec., 2, 9 (1947); Chem. Abstr., 41, 4196 (1947).

144. Chodat, F., and Soloweitchik, S., Compt. rend. soc. phys. hist. nat. Genève, 59, 167 (in Arch. sci. phys. nat., 24, 1942); Chem. Abstr., 38, 5530 (1944).

145. Wiedling, S., Botan. Notiser. (Sweden), 1941, 375; Chem. Abstr., 36, 4523 (1942).

146. Wiedling, S., Nature, 150, 290 (1942).

147. Wiedling, S., Science, 94, 389 (1941).

148. Hazard, R., Compt. rend. soc. biol., 138, 972 (1944).

149. Stoll, R., Compt. rend. soc. biol., 137, 170 (1943).

150. Mangenot, G., and Carpentier, S., Compt. rend. soc. biol., 135, 1053, 1152 (1941).

151. Wiedling, S., Naturwiss., 31, 114 (1945).

152. Euler, H. v., Högberg, B., Karrer, P., Salomon, H., Ruchstuhl, H., Helv. chim. Acta, 27, 382 (1944).

153. Euler, H. v., Ahlström, L., and Högberg, B., Arkiv Kemi., Mineral Gcol., 17A, No. 20, 11 pp. (1944); Chem. Abstr., 39, 3311 (1945).

154. Friedlander, B. L., and French, F., Proc. Soc. Exptl. Biol. Med., 63, 361 (1946)

155. Steenken, W., Jr., and Heise, F. H., Proc. Soc. Exptl. Biol. Med., 52, 180 (1943).

156. English, J. P., Clark, J. H., Clapp, J. W., Seeger, D., and Ebel, R. H., J. Am. Chem. Soc., 68, 453 (1946).

157. Schnitzer, R. J., Foster, R. H. K., Ercoli, N., Soo-Hoo, G., Mangieri, C. N., and Roe, M. D., J. Pharmacol. Exptl. Therap., 88, 47 (1946).

158. Hirsch, J., J. Immunol., 48, 199 (1944).

159. Nitti, F., and Matti, J., Compt. rend. soc. biol., 136, 401 (1942); Chem. Abstr., 37, 4089 (1943).

160. Wilcox, P. E., Owen, J. H., and Stahmann, M. A., J. Am. Chem. Soc., 70, 1931 (1948).

161. Dewing, T., Gray, W. H., Platt, B. C., and Stephenson, D., J. Chem. Soc., $1942,239$.

162. Selbie, F. R., Brit. J. Exptl. Path., 21, 356 (1940).

163. Findlay, G. M., Brit. J. Exptl. Path., 21, 356 (1940).

164. Raiziss, G. W., Severac, M., and Moetsch, J. C., J. Pharm. Assoc., 31, 198 (1942).

165. Maier, J., and Riley, E., Proc. Soc. Exptl. Biol. Med., 50, 152 (1942).

166. Marshall, E. K., Jr., Litchfield, J. T., and White, H. J., J. Pharmacol. Exptl. Therap., 86, 273 (1946).

167. Thomas, L., and Dingle, J. H., Proc. Soc. Exptl. Biol. Med., 51, 76 (1942).

168. Marshall, E. K., Jr., Litchfield, J. T., and White, H. J., J. Pharmacol. Exptl. Therap., 75, 89 (1942).

169. MeCarty, M., Proc. Soc. Exptl. Biol. Med., 46, 133 (1941).

170. Mudrow, L., and Bock, M., Z. Immunitäts, 104, 463 (1943); Chem. Abstr., 39, 4401 (1945). 
171. Seeler, A. O., Graessle, O., and Dusenberg, E. D., J. Bact., 45, 205 (1943).

172. Levaditi, C., Compt. rend. soc. biol., 135, 1109 (1941).

173. Auffarth, F., Süddent. Apoth.-Ztg., 88, 127 (1948); Chem. Abstr., 42, 7821 (1948).

174. McIlwain, H., Brit. J. Exptl. Path., 23, 265 (1942).

175. Marshall, E. K., Jr., Litchfield, J. T., Jr., White, H. J., Bratton, A. C., and Shepherd, R. G., J. Pharmacol., 76, 226 (1942).

176. Laüger, P., Suter, R., and Martin, H., Z. Immunitäts, 105, 78 (1944).

177. Levaditi, C., and Yaisman, A., Compt. rend. soc. biol., 120, 1077 (1935); 131, 33 (1939).

178. Gross, P., and Cooper, F. B., J. Infectious Diseases, 63, 245 (1938).

179. Gross, P., and Cooper, F. B., Proc. Soc. Exptl. Biol. Med., 38, 275 (1938).

180. Carpenter, C. M., and Barbour, G. M., Proc. Soc. Exptl. Biol. Med., 41, 255, 354 (1939).

181. Carpenter, C. M., Hawley, P. L., and Barbour, G. M., Science, 88, 530 (1938).

182. Zahl, P. A., Hutner, S. H., and Cooper, F. S., Proc. Soc. Exptl. Biol. Med., 55, 4 (1944).

183. Hunter, S. H., and Zahl, P. A., Science, 96, 563 (1942).

184. Levaditi, C., Compt. rend. soc. biol., 135, 619 (1941).

185. Lawrence, C. A., and Goetchius, G. R., Proc. Soc. Exptl. Biol. Med., 58, 356 (1945).

186. Schmidt, L. H., and Sesler, C. L., J. Pharmacol., 87, 313 (1946).

187. Goetchius, G. R., and Lawrence, C. A., J. Bact., 49, 575 (1945).

188. Kaplan, H., and Leubner, G. W., J. Am. Chem. Soc., 67, 1076 (1945).

189. Schreus, H. Th., Klin. Wochschr., 21, 671 (1942); Chem. Abstr., 38, 2061 (1944).

190. Lawrence, C. A., J. Bact., 49, 149 (1945).

191. Macht, D. I., Proc. Soc. Exptl. Biol. Med., 60, 217 (1945).

192. Wyss, O., Proc. Soc. Exptl. Biol. Med., 48, 122 (1941).

193. McIlwain, H., Biochem. J., 39, 329 (1945).

194. Klotz, I. M., and Melchoir, J. B., Arch. Biochem., 21, 35, (1949).

195. Rose, H. M., and Fox, C. L., Jr., Science, 95, 412 (1942).

196. Lwoff, A., Nitti, F., and Tréfouël, J., Ann. inst. Pasteur, 67, 173 (1941).

197. Hirsch, J., Bull. faculte med. Instanbul, 7, 3531 (1944); Chem. Abstr., 40, 7284 (1946).

198. Lwoff, A., Nitti, F., Tréfouël, Mme. J., and Hamon, V., Ann. inst. Pasteur, $67,9,19$ (1941).

199. Fox, C. L. Jr., and Rose, H. M., Proc. Soc. Exptl. Biol. Med., 50, 142 (1942).

200. Schmelkes, F. C., Wyss, O., Marks, H. C., Ludwig, B. J., and Strandskov, F. B., Proc. Soc. Exptl. Biol. Med., 50, 145 (1942); Schmelkes, F. C., J. Bact., 45, 67 (1943).

201. Cowles, P. B., Yale J. Biol. Med., 14, 599 (1942).

202. Wyss, O., Lilly, V. G., and Leonian, L. H., Science, 99, 18 (1944).

203. Middlebrook, G., and Lloyd, J. B., Am. Rev. Tuberc., 49, 535 (1944).

204. Bell, P. H., and Roblin, R. O., Jr., J. Am. Chem. Soc., 64, 2905 (1942).

205. Klotz, I. M., J. Am. Chem. Soc., 66, 459 (1944).

206. Klotz, I. M., Science, 98, 62 (1943).

207. Kumler, W. D., and Halverstadt, I. F., J. Am. Chem. Soc., 63, 2182 (1941).

208. Kumler, W. D., and Daniels, T. C., J. Am. Chem. Soc., 65, 2190 (1943).

209. Kumler, W. D., and Strait, L. A., J. Am. Chem. Soc., 65, 2349 (1943).

210. Bell, P. H., Bone, J. F., and Roblin, R. O., Jr., J. Am. Chem. Soc., 66, 847 (1944).

211. Bordwell, F. G., and Klotz, I. M., J. Am. Chem. Soc., 66, 660 (1944).

212. Jensen, K. A., and Schmith, K., Z Immunitäts, 102, 261 (1942).

213. Harris, J. S., and Kohn, H. I., J. Pharmacol., 73, 383 (1941).

214. Roblin, R. O., Jr., Lampen, J. O., English, J. P., Cole, Q. P., and Vaughan, J. R., Jr., J. Am. Chem. Soc., 67, 290 (1945).

215. Goldberger, H. A., Am. J. Surg., 56, 353 (1942). 
216. Neter, E. R., Proc. Soc. Exptl. Biol. Med., 47, 303 (1941).

217. Schmelkes, F. C., and Wyss, O., Proc. Soc. Exptl. Biol. Med., 49, 263 (1942).

218. Lee, S. W., Epstein, J. A., and Foley, E. J., Proc. Soc. Exptl. Biol. Med., 54, 107 (1943).

219. Tenenberg, D. J., Tsuchiya, H. M., Clark, W. G., and Strakosch, E. A., Proc. Soc. Exptl. Biol. Med., 51, 247 (1942).

220. Tsuchiya, H. M., Tenenberg, D. J., Clark, W. G., and Strakosch, E. A., Proc. Soc. Exptl. Biol. Med., 50, 262 (1942).

221. Tsuchiya, H. M., Tenenberg, D. J., Strakosch, E. A., and Clark, W. G., Proc. Soc. Exptl. Biol. Med., 51, 245 (1942).

222. Weinstein, L., and McDonald, A., Science, 101, 44 (1945).

223. Weinstein, L., and McDonald, A., J. Immunol., 54, 131 (1946).

224. Tenenberg, D. J., Tsuchiya, H. M., Clark, W. G., and Larson, N., J. Immunol., 58, 121-32 (1948); Chem. Abstr., 42, 2319 (1948).

225. McClintock, L. A., and Goodale, R. H., U. S. Naval Med. Bull., 41, 1057 (1943).

226. Lee, S. W., Epstein, J. A., and Foley, E. J., Proc. Soc. Exptl. Biol. Med., 54, 105 (1943).

227. Weinstein, L., J. Immunol., 59, 331-3 (1948); Chem. Abstr., 42, 8875 (1948).

228. Libermann, D., and Boyer, F., Compt. rend., 227, 377 (1948); Chem. Abstr., 43, 1106 (1949).

229. Ungar, Nature, 152, 245 (1943).

230. Weinstein, L., J. Immunol., 56, 195, 203 (1947).

231. Neter, E. R., and Clark, P., J. Urology, 51, 101 (1944).

232. Schmelkes, F. C., Surg. Gyn. Obst., 77, 69 (1943).

233. Kirby, W. M., Proc. Soc. Exptl. Biol. Med., 53, 109 (1913).

234. Johnson, F. H., Science, 95, 104 (1942).

235. MeIlwain, H., Science, 95, 509 (1942).

236. Lee, S. W., and Foley, E. J., Proc. Soc. Exptl. Biol. Med., 53, 243, 245 (1943).

237. Nitti, F., Tabone, J., and Mousset, H., Ann. inst. Pasteur, 70, 366, 379 (1944).

238. Winkler, K. C., and De Haan, P. G., Arch. Biochem., 18, 97 (1948).

239. Sevag, M. G., and Green, M. M., J. Bact., 48, 623 (1944).

240. Sevag, M. G., and Green, M. M., J. Bact., 48, 631 (1944).

241. Schopfer, W. H., and Guiloud, M., Experienta, 1, 333 (1945).

242. Nielsen, N., Naturwiss., 32, 293 (1944).

243. Barron, E. S. G., and Jacobs, H. R., Proc. Soc. Exptl. Biol. Med., 37, 10 (1937).

244. Foureau, E. S. G., Tréfouël, J., Nitti, F., and Bovet, D., Compt. rend. soc. biol., 122, 652 (1936).

245. Sevag, M. G., and Shelburne, M., J. Bact., 43, 447 (1942).

246. Clifton, C. E., and Loewinger, I. E., Proc. Soc. Exptl. Biol. Med., 52, 225 (1943).

247. Tatum, E. L., and Giese, A. C., Arch. Biochem., 9, 15 (1946).

248. Wyss, O., Strandskov, F. B., and Schmelkes, F. C., Science, 96, 236 (1942).

249. West, R., and Coburn, A. F., J. Exptl. Med., 72, 91 (1940).

250. Straus, E., Dingle, J. H., and Finland, M., J. Immunol., 42, 313, 331 (1941).

251. Spink, W. W., Vivino, J. J., and Mickelson, O., Proc. Soc. Exptl. Biol. Med., $50,32(1942)$.

252. Woods, W. B., Jr., and Austrian, R., J. Exptl. Med., 75, 383 (1942).

253. Tipley, L. J., Axelrod, A. E., and Elvehjem, C. A., J. Pharmacol., 77, 207 (1943).

254. Anderson, E. G., Pilgrim, F. J., and Elvehjem, C. A., Proc. Soc. Exptl. Biol. Med., 55, 39 (1944).

255. Dorfman, A., Rice, L., Koser, S. A., and Saunders, F., Proc. Soc. Exptl. Biol. Med., 45, 750 (1940).

256. Dorfman, A., and Koser, S. A., J. Infectious Diseases, 71, 241 (1942).

257. Berkman, S., and Koser, S. A., J. Bact., 41, 38 (1941).

258. Altman, K. I., J. Biol. Chem., 166, 149 (1946).

259. Euler, H. v., Ber., 75, 1876 (1942). 
260. Sevag, M. G., Shelburne, M., and Mudd, S., J. Gen. Physiol., 25, 805 (1942).

261. Sevag, M. G., Shelburne, M., and Mudd, S., J. Bact., 49, 65 (1945).

262. Sevag, M. G., Richardson, R. A., and Henry, J., J. Bact., 49, 79 (1945).

263. Sevag, M. G., Henry, J., and Richardson, R. A., J. Bact., 49, 71 (1945).

264. Frei, W., Schweiz. med. Wochschr., 72, 763 (1942).

265. Sevag, M. G., and Shelburne, M., J. Bact., 43, 147, 421 (1942).

266. MacLeod, C. M., Proc. Soc. Exptl. Biol. Med., 41, 215 (1939).

267. MacLeod, C. M., J. Am. Med. Assoc., 113, 1405 (1939).

268. Black, S., McKibbon, J. M., and Elvehjem, C. A., Proc. Soc. Exptl. Biol. Med., 47, 308 (1941).

269. MacKenzie, J. B., MacKenzie, C. G., and McCollum, E. V., Science, 94, 518 (1941).

270. Daft, F. S., Ashburn, L. L., Spicer, S. S., and Sebrell, W. H., Public Health Reports, 57, 217 (1942).

271. Daft, F. S., Ashburn, L. L., and Sebrell, W. H., Science, 96, 321 (1942).

272. Welch, A. D., Federation Proc., 1, 171 (1942).

273. Welch, A. D., and Wright, L. D., J. Nutrition, 25, 555 (1943).

274. Black, S., Overman, R. S., Elvehjem, C. A., and Link, K. P., J. Biol. Chem., 145, 137 (1942).

275. Nielsen, E., and Elvehjem, C. A., J. Biol. Chem., 145, 713 (1942).

276. Spicer, S. S., Daft, F. S., Sebrell, W. H., and Ashburn, L. L., U. S. Pub. Health Service, Pub. Health Reports, 57, 1559 (1942).

277. Kornberg, A., Daft, F. S., and Sebrell, W. H., Science, 98, 20 (1943).

278. Daft, F. S., and Sebrell, W. H., U. S. Pub. Health Service, Pub. Health Reports, 58,1542 (1943).

279. Axelrod, A. E., Bosse, M. O., Gross, P., and Gregg, H., Arch. Biochem., 7, 91 (1945).

280. Wright, L. D., and Welch, A. D., J. Nutrition, 27, 55 (1944).

281. Wright, L. D., and Skeggs, H. R., Am. J. Med. Sci., 212, 312 (1946).

282. Nelson, M. M., and Evans, H. M., Arch. Biochem., 18, 153 (1948).

283. Petering, H. G., Delor, R. A., and Murray, H. C., Blood, 2, 440 (1947); Chem. Abstr., 42, 678 (1948).

284. West, H. D., Jefferson, N. C., and Rivera, R. E., J. Nutrition, 25, 471 (1943).

285. West, H. D., and Elliott, R. R., Arch. Biochem., 16, 47 (1947).

285a. Totter, J. R., and Day, P. L., J. Biol. Chem., 147, 257 (1943).

286. Wright, L. D., and Welch, A. D., Science, 98, 179 (1943).

287. Ransome, B., and Elvehjem, C. A., J. Biol. Chem., 151, 109 (1943).

288. Norris, E. R., and Majnarich, J. J., Am. J. Physoiol., 152, 179 (1948).

289. Norris, E. R., and Majnarich, J. J., Am. J. Physiol., 153, 133 (1948).

290. Norris, E. R., and Majnarich, J. J., Am. J. Physiol., 152, 175, 652 (1948).

291. Johnson, B. C., and Dana, A. S., Science, 108, 210 (1948).

292. Haerem, A. T., Proc. Soc. Exptl. Biol. Med., 68, 330 (1948).

293. Legge, J. W., Biochem. J., 44, 105 (1949).

294. Nielsen, E., and Black, A., Proc. Soc. Exptl. Biol. Med., 55, 14 (1944).

295. Lindley, D. C., and Cunha, T. J., J. Nutrition, 32, 47 (1946).

296. Woolley, D. W., J. Exptl. Med., 75, 277 (1942).

297. Woolley, D. W., J. Nutrition, 28, 305 (1944).

298. Martin, G. J., Proc. Soc. Exptl. Biol. Med., 51, 56 (1942).

299. Ershoff, B. H., and McWilliams, H. B., Proc. Soc. Exptl. Biol. Med., 54, 227 (1943).

300. Cartwright, G. E., Wintrobe, M. M., and Humphreys, S., J. Lab. Clin. Med., 31, 423 (1946).

301. Cunha, T. J., Colby, R. N., Bustad, L. K., and Bone, J. F., J. Nutrition, 36, 215 (1948). 
302. Cunha, T. J., Bustad, L. K., Ham, W. E., Cordy, D. R., McCulloch, E. C., Woods, I. F., Conner, G. H., and McGregor, M. A., J. Nutrition, 34, 173 (1947).

303. Welch, A. D., Heinle, R. W., Sharpe, G., George, W. L., and Epstein, M., Proc. Soc. Exptl. Biol. Med., 65, 364 (1947).

304. Corwin, W. C., Bull. Johns Hopkins Hosp., 69, 39 (1941).

305. Farr, M. M., and Allen, R. W., J. Am. Vet. Med. Assn., 100, 47 (1942).

306. Lewis, K. H., Ham, W. E., and Jensen, W. I., Proc. Soc. Exptl. Biol. Med., 52, 53 (1913).

307. Moore, P. R., Evenson, A., Luckey, T. D., McCoy, E., Elvehjem, C. A., and Hart, E. B., J. Biol. Chem., 165, 437 (1946).

308. Bosshardt, D. K., Huff, J. W., Paul, W. J., and Barncs, R. H., Federation Proc., 8, 378 (1949).

308a. Lecoq, R., Chauchard, P., and Mazoue, H., Compt. rend. soc. biol., 140, 279 (1947).

309. Williams, R. T., Biochem. J., 41, 1 (1947).

310. Vacirca, F., Boll. ist. sieroterap. milanése, 22, 396 (1943).

311. Efimov, N. I., Farmakol. $i$ Toksikol., 8, No. 5, 28 (1945); Chem. Abstr., 40, 7409 (1945).

312. Danielopolu, D., and Rureanu, A., Bull. acad. méd. Roumanie, 13, No. 112, 185 (1943); Chem. Abstr., 41, 4570 (1947).

313. Richards, R. E., and Kueter, K. E., J. Pharmacol. Exptl. Therap., 87, 42 (1946).

314. Hazard, R., and Vaille, C., Compt. rend. soc. biol., 138, 114 (1943).

315. Richards, R. K., J. Biol. Chem., 159, 241 (1945).

316. Mayer, R. L., Science, 98, 203 (1943).

317. Lipmann, F., J. Biol. Chem., 139, 977 (1941).

318. Mann, T., and Kirlin, D., Nature, 146, 164 (1940).

319. H. van Goor, Enzymologia, 11, 174 (1944).

320. Eyster, H. C., J. Cellular Comp. Physiol., 21, 191 (1943).

321. Vivino, J. J., and Spink, W. W., Proc. Soc. Exptl. Biol. Med., 50, 336 (1942).

322. Schmidt, L. H., Sesler, C. L., and Hamburger, M., Jr., J. Bact., 45, 27 (1943).

323. Schmidt, L. H., Hilles, C., Dettwiler, H. A., and Starks, E., J. Infectious Diseases, 67, 232 (1940).

324. Schmidt, L. H., and Hilles, C., J. Infectious Discases, 65, 273 (1939).

325. Felsenfeld, O., J. Bact., 45, 25 (1943).

326. Reed, G. B., Orr, J. H., and Reed, R. W., J. Bact., 48, 233 (1944).

327. Lowell, F. C., Strauss, E., and Finland, M., Ann. Internal Med., 14, 1001 (1940).

328. Long, P. H., and Bliss, E. A., Ann. Internal. Med., 13, 232 (1939).

329. Cohn, A., Steer, A., and Seyo, I., Am. J. Med. Sci., 203, 276 (1942).

330. Schmidt, L. H., and Sesler, C. L., Proc. Soc. Exptl. Biol. Med., 52, 353 (1943).

331. Schmidt, L. H., and Sesler, C. L., J. Pharmacol. Exptl. Therap., 77, 277 (1943).

332. Grumbach, A., and Hegglin, R., Schweiz. med. Wochschr., 72, 1369 (1942); Chem. Zentr., 1943, I, 1077; Chem. Abstr., 38, 4006 (1944).

333. Swain, R. H. A., Brit. Med. J., 1940, I, 722; Chem. Abstr., 34, 5870 (1910).

334. Landy, M., and Gerstung, R. B., J. Bact., 47, 448 (1944).

335. Hagerman, G., Acta Path. Microbiol. Scand. Suppl., 46, 1 (1942); Chem. Abstr., 37,6693 (1943).

336. McKee, C. M., and Rake, G., Proc. Soc. Exptl. Biol. Med., 51, 275 (1942).

337. Cooper, M. L., and Keller, H. M., Proc. Soc. Exptl. Biol. Med., 51, 238 (1942).

338. Schmidt, L. H., Sesler, C. L., and Dettwiler, H. A., J. Pharmacol. Exptl. Therap., 74, 175 (1942).

339. Harkness, A. H., Lancet, 1943, 2, 116.

340. MacLeod, C. M., J. Exptl. Med., 72, 217 (1940).

341. Kirby, W. M. M., and Rantz, L. A., J. Exptl. Med., 77, 29 (1943).

342. Green, H. N., and Bielschowsky, F., Brit. J. Exptl. Path., 23, 1 (1942). 
343. Vacirca, F., Boll. ist. sierotcrap. milan, 22, 172 (1943); Chem. Abstr., 40, 4760 (1946).

344. Diczfalusy, E., and Euler, H. v., Artiv. Kemi, Mineral Geol., 25A, No. 12, 12 pp. (1947); Chem. Abstr., 42, 6412 (1948).

315. Colebrook, L., Lancet, 1943, 2, 207.

346. Cutts, M., and Troppoli, A. V., J. Lab. Clin. Med., 28, 14 (1942).

347. Selbie, F. R., Brit. J. Expll. Palh., 21, 90 (1940).

348. Hadley, P., and Hadley, F. P., J. Infectious Discases, 68, 246 (1941).

349. Clapper, W. E., and Heatherman, M. E., Proc. Soc. Exptl. Biol. Med., 68, 392 (1948).

350. McIntosh, J., and Selbie, F. R., Brit. J. Exptl. Path., 24, 246 (1943).

350a. Carpenter, C. M., Ackerman, H., Winchester, M. E., and Whittle, F., Proc. Soc. Exptl. Biol. Med., 46, 527 (1941).

351. Lankford, C. E., Scott, V., and Cooke, W. R., J. Bact., 45, 201 (1943).

352. Hüllstrung, H., Med. Klin. (Munich), 1946, 234; Chem. Zentr., 1947, I, 58; Chem. Abstr., 41, 6306 (1947).

353. Kirby, W. M. M., Proc. Soc. Exptl. Biol. Med., 52, 175 (1943).

354. Carpenter, C. M., Ackerman, H., Winchester, M. E., and Whittle, J., Am. J. Pub. Health, 34, 250 (1944).

355. Landy, M., and Gerstung, R. B., J. Immunol., 51, 269 (1945).

356. Westphal, L., Charles, R. L., and Carpenter, C. M., Venereal Disease Inform., 21, $183(1940)$.

357. Spitzer, R., Schweiz. Z. Path. Bakt., 5, 275 (1942); Chem. Abstr., 38, 5523 (1944).

358. MacLeod, C. M., and Daddi, G., Proc. Soc. Exptl. Biol. Med., 41, 69 (1939).

359. Powell, H. M., and Jamieson, W. H., Proc. Soc. Exptl. Biol. Med., 49, 387 (1942).

360. Sesler, C. L., Schmidt, L. H., and Belden, J., Proc. Soc. Exptl. Biol. Med., 56, 42 (1944).

361. Schmidt, L. H., and Dettwiler, H., J. Biol. Chem., 133, 85 (1940).

362. Sesler, C. L., and Schmidt, L. H., J. Bact., 43, 73 (1942); J. Pharmacol. Exptl. Therap., 75, 356 (1942).

363. MacLeod, C. M., and Mirick, G. S., Am. J. Pub. Health, 31, 34 (1941).

364. Sesler, C. L., Hamburger, M., Jr., and Schmidt, L. H., J. Bact., 45, 27 (1943).

365. Hamburger, M., Jr., Schmidt, L. H., and Sesler, C. L., J. Bact., 45, 28 (1943).

366. Hamburger, M., Jr., Schmidt, L. H., Sesler, C. L., Ruegsegger, J. M., and Grupen, E. S., J. Infectious Diseases, 73, 12 (1943).

367. Dettwiler, H. A., and Schmidt, L. H., J. Bact., 40, 160 (1940).

368. Schmith, K., Acta Path. Microbiol. Scand., 20, 563 (1943); Chem. Abstr., 38, 2685 (1944).

369. Jensen, K. A., Schmith, K., and Brandt, P., Klin. Wochschr., 21, 1042 (1942); Chem. Abstr., 38, 2681 (1944).

370. Spink, W. W., Ferris, V., and Vivino, J. J., Proc. Soc. Exptl. Biol. Med., 55, 207 (1944).

371. Landy, M., Larkum, N. W., Oswald, E. J., and Streightoff, F., Science, 97, 265 (1943).

372. Spink, W., and Vivino, J., Science, 98, 44 (1943).

373. Landy, M., Larkum, N. W., Oswald, E. J., and Streightoff, F., J. Bact., 45, 99 (1943).

374. Strauss, E., Dingle, J. H., and Finland, M., J. Immunol., 42, 331 (1941).

375. Sevag, M. G., and Green, M. M., J. Bact., 48, 615 (1944).

376. Oakberg, E. F., and Luria, S. E., Genetics, 32, 249 (1947); Chem. Abstr., 41, 5581 (1947).

377. Cooper, M. L., and Keller, H. M., Proc. Soc. Exptl. Biol. Med., 52, 92 (1943).

378. Cooper, M. L., and Keller, H. M., J. Bact., 45, 26 (1943).

379. Stewart, F. H., J. Hyg., 45, 28 (1947). 
380. Yegian, D., Budd, V., and Middlebrook, G., J. Bact., 51, 479 (1946).

381. Landy, M., Larkum, N. W., and Oswald, E. J., J. Bact., 45, 24 (1943).

382. Rodaniche, E. C., and Kirsner, J. B., J. Parasitol., 28, 441 (1942).

383. Davies, D. S., and Hinshelwood, C. N., Trans. Faraday Soc., 34, 431 (1943).

384. Davies, D. S., Hinshelwood, C. N., and James, A. M., Trans. Faraday Soc., 43, 138 (1947).

385. Schmidt, L. H., and Sesler, C. L., J. Pharmacol. Exptl. Therap., 77, 165 (1943).

386. Walker, N., Philip, R., Smyth, M. M., and McLeod, J. W., J. Path. Bact., 59, 631 (1947).

387. Spink, W. W., Wright, L. D., Vivino, J. J., and Skeggs, H. R., J. Exptl. Med., 79, 331 (1944).

388. Zimmerman, A., and Pike, R. M., J. Bact., 45, 522 (1943).

389. Link, Th., Z. Iminunitäts., 104, 441 (1943); Klin. Wochschr., 22, 744 (1943).

390. Mirick, G. S., J. Bact., 45, 66 (1943).

391. Liebermeister, K., Z. Immunitäts., 103, 439 (1943); Chem. Abstr., 40, 7287 (1946).

392. Gay, F. P., Clark, A. R., Street, J. A., and Miles, D. W., J. Exptl. Med., 69, 607 (1939).

393. Roux, E., and Chevé, J., Compt. rend. soc. biol., 136, 272 (1942); 135, 989 (1941); Chem. Abstr., 37, 3468 (1943).

394. Schmelkes, F. C., and Rubin, M., J. Am. Chem. Soc., 66, 1631 (1944).

395. Ivanovics, G., Proc. Soc. Exptl. Biol. Med., 70, 462 (1949).

396. Auhagen, E., Z. physiol. Chem., 274, 48 (1942).

397. Kuhn, R., Möller, E. F., and Wendt, G., Ber., 76B, 405 (1943).

398. Snith, M. I., Emmart, E. W., and Westfall, B. B., J. Pharmacol. Exptl. Therap., 74, 163 (1942).

399. Klotz, I. M., and Morrison, R. T., J. Am. Chem. Soc., 69, 473 (1947).

400. Strandskov, F. B., J. Bact., 53, 555 (1947).

401. King, T. E., Stearman, R. L., and Cheldelin, V. H., J. Am. Chem. Soc., 70, 3969 (1948).

402. Shive, W., unpublished work.

403. Bernhein, F., Science, 92, 204 (1940); J. Bact., 41, 38 (1941).

404. Lehmann, J., Lancet, 1, 14, 15 (1946).

405. Lehmann, J., Svenska Läkartidn., 43, 2029 (1946); Chem. Abstr., 41, 1334 (1947).

406. Lehmann, J., Nord. Med., 33, 140 (1947).

407. Youmans, G. P., Quart. Bull. Northwestern Univ. Med. School, 20, 420 (1946).

408. Sievers, O., Nord. Med., 33, 145 (1947).

409. Erlenmeyer, H., Prijs, B., Sorkin, E., and Suter, E., Helv. Chim. Acta, 31, 988 (1948).

410. Duca, C. J., Williams, R. D., and Scudi, J. V., Proc. Soc. Exptl. Biol. Med., 67, 149 (1948).

411. Vennesland, K., Ebert, R. H., and Bloch, R. G., Proc. Soc. Exptl. Biol. Med., 68, 250 (1948).

412. Lehmann, J., Rev. gén. sci., 54, 222 (1947); Chem. Abstr., 42, 4641 (1948).

413. Diczfalusy, E., Arkiv. Kemi, Mineral. Geol., 24B, No. 13, 1 (1947); Chem. Abstr., 42, 5506 (1948).

414. MeClosky, W. T., Smith, M. I., and Frias, J. E. G., J. Pharmacol. Exptl. Therap., 92, 447 (1948).

415. Youmans, G. P., and Raleigh, G. W., J. Infectious Diseases, 82, 221 (1948).

416. Way, E. L., Smith, P. K., Howie, D. L., Weiss, R., and Swanson, R., J. Pharmacol. Exptl. Therap., 93, 368 (1948).

417. Venkataraman, A., Venkataraman, P. R., and Lewis, H. B., J. Biol. Chem., 173, 641 (1948).

418. Paraf, J., Desbordes, J., Henry, J., and Paraf, M., Compt. rend. soc. biol., 142, 162 (1948). 
419. Desbordes, J., and Henry, J., Ann. pharm. franc., 6, 98 (1948); Chem. Abstr., 42, 7815 (1948).

420. Schmidt-Thomé, Z. Naturforsch., 3b, 136 (1948); Chem. Abstr., 43, 3884 (1949). 420a. Snell, E. E., and Shive, W., J. Biol. Chem., 158, 551 (1945).

421. Roholt, K., Compt. rend. trav. lab. Carlsberg, Ser. physiol., 24, 172 (1946).

421.a. Moshkovskiř, Sh. D., and Stoyanova, A. V., Byull. Eksptl. Biol. Med., 23, 202 (1947); Chem. Abstr., 42, 2672 (1948).

422. Sandground, J. H., Science, 97, 73 (1943).

423. Sandground, J. H., and Hamilton, C. R., J. Pharmacol. Exptl. Therap., 78, 109 (1943)

424. Sandground, J. H., and Hamilton, C. R., J. Pharmacol. Exptl. Therap., 78, 203 (1943).

425. Sandground, J. H., J. Pharmacol. Exptl. Therap., 78, 209 (1943).

426. Vidal, A. Z., Farmacopterap. actual (Madrid), 3, 847 (1946); Chem. Abstr., 41, 3215 (1947).

427. Williamson, J., and Lourie, E. M., Ann. Trop. Med. Parasitol., 40, 255 (1946); Nature, 161, 103 (1948).

428. Sandground, J. H., Proc. Soc. Exptl. Biol. Med., 52, 188 (1943).

429. Kanitkar, U. K., and Bhide, B. V., Current Sci. (India), 16, 223 (1947); Chem. Abstr., 41, 7447 (1947).

430. Smith, M. I., Emmart, E. W., and Westfall, B. B., J. Pharmacol. Exptl. Therap., 74, 162 (1942).

431. Pendse, G. S., and Bhide, B. V., Current Sci. (India), 17, 125 (1948); Chem. Abstr., 42, 5556 (1948).

432. Klotz, I. M., and Morrison, R. T., J. Am. Chem. Soc., 69, 473 (1947). 


\section{Chapter IVD}

\section{BIOTIN}

The stimulating effect of small amounts of natural extracts on the growth of yeast was first described in 1901 by Wildiers, ${ }^{1}$ who gave the name "bios" to this growth-promoting substance. Bios was subsequently shown to be a group of factors, one of which was isolated from dried Chinese chick egg yolk in 1935 by Kögl, ${ }^{2}$ who characterized the compound as the methyl ester, determined its empirical formula and named it "biotin." ${ }^{3}$ du Vigneaud and co-workers isolated biotin from liver ${ }^{4}$ and from a milk concentrate ${ }^{5}$ and completed the proof of structure. ${ }^{6}$ The biotin molecule is indicated by the following formula:

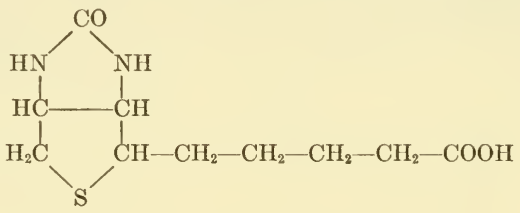

biotin

(cis-hexahydro-2-oxo-1H-thieno [3,4] imidazole-4-valeric acid)

The synthesis of the vitamin first reported by Harris et $a l^{7}{ }^{7}$ has since been accomplished in a number of laboratories by different methods.

\section{Specificity}

There are four diastereoisomers or eight optically active modifications corresponding to the structure of biotin. All the racemic diastereoisomers, DL-biotin, ${ }^{7,8}$ DL-epi-biotin, ${ }^{9}$ DL-allobiotin ${ }^{7,8}$ and DL-epi-allobiotin, ${ }^{8}$

* A naturally-occurring complex of biotin, biocytin, has recently been isolated in crystalline form from yeast extract (Wright, L. D., et al., J. Am. Chem. Soc., 72, 1048 (1950). Biocytin, m. p. $230-240^{\circ}$ (dec.) is on a molar basis just as active as biotin for Lactobacillus casei but is inactive for Lactobacillus arabinosus. On acid hydrolysis of biocytin, an equivalent of $40 \pm 4$ per cent of biotin determined microbiologically is produced. Biocytin also replaces biotin in the nutrition of Lactobacillus delbruckii $\mathrm{LD}_{5}$, Lactobacillus acidophilus, Streptococcus faecalis R, Neurospora crassa and Saccharomyces carlsbergensis, but the complex is inactive for Lactobacillus pentosus and Leuconostoc mesenteroides P-60 as well as Lactobacillus arabinosus. 
have been synthesized. Elucidation of the structure of the four racemic modifications has been accomplished to some extent by the conversion of the biotin stereoisomers to the corresponding desthio derivatives. This conversion is effected by the removal of the sulfur of biotin by reduction with Raney's nickel. ${ }^{10}$ The structure of the resulting product, desthiobiotin, allows only two racemic forms, since the asymmetry of carbon atom 2 in biotin is destroyed by the reduction. DL-Biotin and DL-epi-biotin are

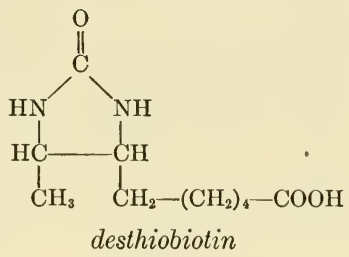

reduced to the same DL-desthiobiotin, ${ }^{9}$ while both DL-allobiotin and DL-epi-allobiotin give rise to the same DL-allodesthiobiotin. ${ }^{11}$ Thus, biotin and $e p i$-biotin differ by being epimeric at carbon 2 where the side chain is attached. Allobiotin is similarly epimeric with epi-allobiotin. Although the exact configuration of these compounds is unknown, the relationship between the epimeric biotins and epimeric allobiotins has been resolved by the relative ease of hydrolysis of the ureylene groups of allobiotin and epi-allobiotin as compared with biotin. The resulting diamino compounds derived from hydrolysis of the allobiotins are also less easily reconverted by the action of phosgene into the original compounds than are epimeric biotins. These results suggest that nitrogens of the ureylene group of the epimeric biotins have a cis-configuration, whereas the nitrogens of this group in the epimeric allobiotins have a trans configuration as illustrated below:

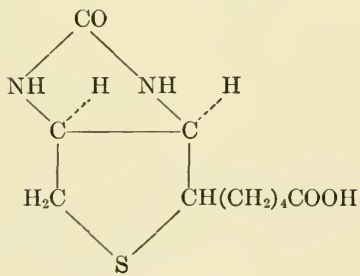

biotin, epi-biotin

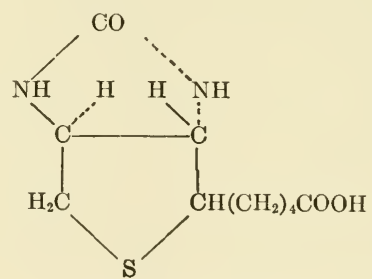

allobiotin, epi-allobiotin

Of the four racemic diastereoisomers, all except DL-biotin have been reported to be essentially inactive biologically as indicated in Table 11 . 
TABLE 11. Specificity of the Configuration of Biotin.

Compound

(+)-Biotin

$(-)$-Biotin

DL-Biotin

DL-epi-Biotin

DL-Allobiotin

DL-epi-Allobiotin
Organism

Rat

Chick

Lactobacillus casei

Lactobacillus arabinosus

Rat

Chick

Lactobacillus casei

Lactobacillus arabinosus

Saccharomyces cerevisiae

Saccharomyces cerevisiae

Rat

Chick

Lactobacillus casei

Lactobacillus arabinosus

Lactobacillus arabinosus
Activity

Reference 100

$\begin{array}{ll}0(<13) & 12 \\ 0 & 13 \\ 0.023 & 1 \\ 0.006-0.019 & 1\end{array}$

\section{0}

$50 \mathrm{ca}$

49

51,50

50

0

0

0

0.0029

0.002

0

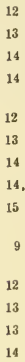

4, 15 3 4

8

However, the racemic biotin is only half as active as the natural biotin which is dextrorotatory. Synthetic $(+)$ biotin is just as active as natural biotin, whereas the synthetic (-) biotin is essentially inactive. The small amount of activity which has been found is usually attributed to contamination of the sample with traces of the dextrorotatory form.

Kögl and co-workers ${ }^{16-19}$ have concluded that two isomeric forms of biotin exist in nature, $\alpha$-biotin isolated from egg yolk and $\beta$-biotin isolated from liver by the procedure of du Vigneaud et $a l^{4}{ }^{4} \mathrm{~A}$ mixture of $\alpha$-biotin (m. p. $220^{\circ} \mathrm{C}$ ) and $\beta$-biotin (m. p. $232-3^{\circ} \mathrm{C}$ ) melted at 197 $202^{\circ} \mathrm{C}$; and a mixture of the methyl ester of $\alpha$-biotin (m. p. $161-2^{\circ} \mathrm{C}$ ) and the methyl ester of $\beta$-biotin (m. p. $163-4^{\circ} \mathrm{C}$ ) showed a melting point depression of $20-30^{\circ} \mathrm{C}$. Specific rotations of the two biotins and their methyl esters differed markedly. More significant was the isolation of a sulfocaproic acid by hydrolysis of $\alpha$-biotin to a diaminocarboxylic acid, oxidation of the latter with lead tetracetate to an aldehyde, and finally oxidation of the aldehyde with potassium permanganate in a series of steps. This sulfocaproic acid, which was converted by alkali fusion to a product identified as $\alpha, \beta$-dimethylbutyric acid, was found to be identical with the synthetic (-) form of $\alpha$-isopropyl- $\beta$-sulfopropionic acid when the melting points and mixed melting points of the anilides and $m$-toluidine salts of each were studied. Since there is no branching in the carbon skeleton of biotin, such a degradation product would indicate that another structural form exists.

Early work on the biological activity of the two biotins indicated that $\beta$-biotin was approximately twice as active as $\alpha$-biotin for yeast, Rhizo- 
bium trifolii and Clostridium butylicum. ${ }^{4,20}$ However, more recent experiments ${ }^{21}$ on a sample of $\alpha$-biotin isolated by Kögl indicate that with corrections for impurity of the natural biotin and corrections for the inactivity of $(-)$ biotin in DL- $\beta$-biotin, $\alpha$-biotin possesses $90-96$ per cent of the activity of synthetic DL- $\beta$-biotin for Lactobacillus casei 7469 , - Lactobacillus pentosus 124-2, Saccharomyces cerevisiae Y-30, Clostridium acetobutylicum S-9 and Neurospora crassa 1-A wild. If two such isomeric forms of biotin exist, it is indeed remarkable that for a wide variety of organisms the isomers have essentially identical biological activities.

Oxybiotin (O-Heterobiotin). The synthesis of DL-hexahydro-2-oxo-1Hfuro [3,4] imidazole-4-valeric acid, the biologically active oxygen analogue of biotin, was reported almost simultaneously by Hofmann ${ }^{22}$ and by Duschinsky et al..$^{23}$ Both O-heterobiotin ${ }^{23}$ and oxybiotin ${ }^{24}$ have been suggested as trivial names for the analogue which is indicated by the following formula:

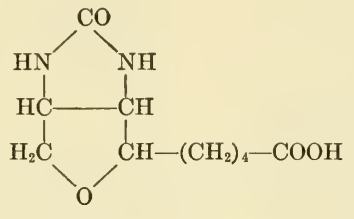

oxybiotin (O-heterobiotin)

Since this analogue might exist in stereoisomeric modifications similar to those of biotin, all four theoretically possible racemic forms of 3,4diamino-2-tetrahydrofuranvaleric acids were prepared ${ }^{25}$ Attempts to convert these to the corresponding hexahydro-2-oxo- $1 \mathrm{H}$-furo $[3,4]$ imidazole4 -valeric acids were successful with only two of the four diastereoisomers. Since only the two cis-3,4-diamino-2-tetrahydrofuranvaleric acids formed bicyclic ureylene derivatives, it appears that the tetrahydrofuran ring is more planar in its configuration than the tetrahydrothiophene ring, which apparently allows the formation of trans-ureylene derivatives. As in the case of biotin, the spatial arrangement of the side chain with respect to the ureylene group of the two diastereoisomers is still unknown.

Of the two racemic diastereoisomers of the oxygen analogue of biotin, only one, DL-oxybiotin (which is identical with DL-O-heterobiotin), ${ }^{26}$ possesses appreciable biological activity. The other, DL-epi-oxybiotin, ${ }^{25}$ is reported to have slight activity ( 0.1 per cent that of oxybiotin) for Lactobacillus arabinosus; however, it is suggested that it is likely that the activity is caused by DL-oxybiotin as a contaminant. The racemic modification of the biologically active form has not as yet been resolved; 
however, if it is analogous to biotin, only one of the optically active forms would be expected to be biologically active.

As indicated in Table 12, this oxygen analogue of biotin is capable of replacing the vitamin in the nutrition of a wide variety of organisms. For Lactobacillus arabinosus and Lactobacillus pentosus 124-2, DL-oxybiotin is as active as DL-biotin, giving growth responses at various concentrations identical with those obtained with biotin. However, the

TABLE 12. The Biological Activity of DL-Oxybiotin (O-Heterobiotin).

Organism

Rat

Chicks

Lactobacillus arabinosus

Lactobacillus pentosus 124-2

Lactobacillus casei

Streptococcus faecalis $\mathrm{R}$

Rhizobium trifolii

Saccharomyces cerevisiae

Saccharomyces carlsbergensis

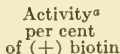

$6.0-2.9^{b, c}$
$17^{b}$
$20-2^{c, d}$
$50^{e}$
50
40
$22-25$
$0(\mathrm{pH} 6.6)^{f}$
$7.5(\mathrm{pH} 7.3)^{f}$
$12-1.3^{c}$
$25-10^{c}$
$20^{g}$

Refercnce

$27,28,31$

27,29

30

24, 41

33

24,34

23,31

32

32

34

$23,24,31,34$

31

a If the biological activity resides in only one of the optically active forms of the oxygen analogue, these values should be doubled.

$b$ Biotin deficiency induced by feeding raw egg white with biotin deficient diet; DL-oxybiotin injected intramuscularly or subcutaneously.

- The activity of the analogue relative to $(+)$ biotin varies at different concentrations of the analogue; i.e., the dose-response plots differ in shape for the two compounds. The relative activity of the analogue tends to decrease with increases in concentrations.

d Animals on biotin deficient diet only.

- Activities of 29-44 per cent dependent upon $\mathrm{pH}$ of the medium have been reported. ${ }^{2}$

$f$ Activity reported to be dependent upon pH of medium.

- Slight variations at different concentrations.

activity of this analogue is not so pronounced for other organisms, and relative to biotin, this analogue is often less effective in obtaining the maximum response of the organism than in eliciting a suboptimal response. This is particularly true of Rhizobium trifolii and Saccharomyces cerevisiae. For Saccharomyces cerevisiae, oxybiotin is approximately 25 per cent as effective as $(+)$ biotin in stimulating the fermentation rate of biotin-deficient cells. ${ }^{40}$

It is interesting to note that the activity of oxybiotin for Streptococcus faecalis $\mathrm{R}$ has been reported to be dependent upon the $\mathrm{pH}$ of the medium. The analogue is approximately 7.5 per cent as active as $(+)$ biotin at $\mathrm{pH} 7.3$, but it is essentially inactive at $\mathrm{pH}$ 6.6.

In the rat oxybiotin administered by daily subcutaneous injections completely cured the skin lesions resulting from biotin deficiency caused by feeding raw egg white, and spastic paralysis of the hind legs observed in a few animals was also completely cured with the oxygen analogue. ${ }^{28}$ In stimulating growth, DL-oxybiotin was from 2.9 to 6.0 per cent as 
effective as $(+)$ biotin. The lower figure was obtained for near maximal response of the organism, whereas the higher value represents the relative efficiency in eliciting a suboptimal response. Hence, in plots of doseresponse for the rat, the general shape of the curves for oxybiotin and biotin differ.

As little as $8 \gamma$ of DL-oxybiotin injected intramuscularly in chicks (White Leghorn cockerels) rendered deficient in biotin by supplementing a biotin-deficient diet with raw egg white caused the disappearance of the mandibular lesions within a week, and some healing of the feet. After three weeks, the hard, scaly, cracked skin of the bottom of the feet sloughed off, leaving normal tissue; but edema was still evident in some segments of the toes of the chicks. No deficiency symptoms of chicks on a biotin-deficient diet were obtained if a supplement of oxybiotin was provided; chicks becoming deficient on the diet were cured by oxybiotin. Injected intramuscularly, DL-oxybiotin was approximately 17 per cent as active as $(+)$ biotin in eliciting growth response of dayold chicks maintained for one week on a biotin-deficient diet containing raw egg white. The plots of dose-response for both compounds are similar in shape. ${ }^{29}$

These results contrast sharply with those of another group, ${ }^{30}$ who report that the oxygen analogue, when administered as a supplement in the diet instead of being injected intramuscularly, fails to replace biotin completely for growth of the chick (White Leghorn cockerels) on a biotin-deficient diet. It is reported that the racemic analogue at $20 \gamma$ per $100 \mathrm{~g}$ of diet is approximately 20 per cent as active as $(+)$ biotin in promoting growth, but at high concentrations of the analogue in the diet it is only $0.5-3$ per cent as effective as biotin. The relative activities are reported to vary inversely with the amount of analogue fed. Since optimal growth as obtained with biotin was not attained with any concentration of the analogue up to $1000 \gamma$ per $100 \mathrm{~g}$ of diet, it was suggested that the analogue fulfilled only a part of the function of biotin in the chick. The oxygen analogue is reported to have about one-third the activity of biotin in curing the dermatitis which develops in the deficient animals. It will be interesting indeed if the activity of oxybiotin (O-heterobiotin) can be demonstrated to be dependent upon the method of administration to the chick.

Although several analogues which were very active in replacing the corresponding vitamins had previously been prepared and tested, the oxygen analogue of biotin afforded the first opportunity for development of specific assays useful in determining whether the analogue was converted into the vitamin or was utilized as such by organisms in which it had vitamin activity. Two direct assays for oxybiotin have been 
developed; they depend upon the destruction of biotin by Raney's nickel ${ }^{35}$ and by oxidation with permanganate. ${ }^{36}$ The oxygen analogue is not appreciably affected by either of the reagents, but Raney's nickel quantitatively converts biotin to desthiobiotin and permanganate oxidizes biotin to the corresponding sulfone. Since neither desthiobiotin nor the sulfone of biotin possesses any appreciable activity for Lactobacillus arabinosus, as well as for several other organisms, such organisms which respond to oxybiotin may then be utilized for a direct assay for the oxygen analogue.

Indirect assays for oxybiotin by assaying directly for biotin in the presence of oxybiotin have been developed. The response of Lactobacillus arabinosus to moderate amounts of oxybiotin is prevented by either 800 $\mathrm{m} \gamma$ per $10 \mathrm{cc}$ of biotin sulfone or $70 \gamma$ per $10 \mathrm{ec}$ of $\gamma$ - (3,4-ureylenecyclohexyl)butyric acid. ${ }^{37}$ Under these conditions, neither of the inhibitors appreciably affects the utilization of biotin by the organism. The biotin is thus determined directly, and from the response of the organism to the assay sample in the absence of the inhibitors a differential assay for oxybiotin is obtained. Of course, large amounts of oxybiotin overcome the toxicity of the inhibitors and would prevent the determination of relatively small amounts of biotin.

Another indirect assay depends upon the inability of Streptococcus faecalis $\mathrm{R}$ to utilize the oxygen analogue effectively. This permits the estimation of biotin in an extract with only slight interference from the oxygen analogue. By simultaneous assays with Lactobacillus arabinosus and Streptococcus faecalis R, a direct assay for biotin and a differential assay for the oxygen analogue of biotin have been developed. ${ }^{22}$

With these assays, oxybiotin has been demonstrated not to occur naturally in any of the organisms tested, and it has been possible to show that the analogue is utilized as such without prior conversion to biotin. Thus, permanganate destroys the biotin activity of hydrolysates of cells of either Saccharomyces cerevisiae or Rhizobium trifolii grown in a biotincontaining medium, but does not destroy the biotin-like activity of such hydrolysates from cells of the organism grown in the presence of oxybiotin instead of biotin. ${ }^{36}$ In balance experiments ${ }^{38}$ with Saccharomyces cerevisiae 139 grown in a medium containing $10 \mathrm{~m} \gamma$ of DL-oxybiotin per $250 \mathrm{cc}$, essentially all (94-97 per cent of the compound) was recovered from the medium and cells. At higher concentrations (100 m $\gamma$ per $250 \mathrm{cc}$ ), the recovery was lower ( 84 per cent). By three different methods, the Raney's nickel method, the permanganate method, and the differential growth inhibitor method, it was demonstrated that oxybiotin alone (with the exception of the small amount of biotin added with the inoculum) accounted for all the biotin-like activity in the cells. ${ }^{38}$ 
With the permanganate method, oxybiotin has been demonstrated as the substance accounting for the biotin-like activity of hydrolysates of cells of Lactobacillus pentosus 124-2 grown in an oxybiotin medium. ${ }^{33}$ No biotin could be demonstrated in the hydrolysates of the cells by the differential growth inhibitor method. There was no significant difference in the growth rate of the organism when oxybiotin replaced biotin in stimulating growth. Either oxybiotin or biotin was absorbed from the medium into the cell in larger amounts than necessary for growth, and balance studies indicated that destruction of either compound became apparent only at relatively high concentrations, where recoveries were as low as 15 per cent.

After several weeks of intramuscular injections of oxybiotin, the liver, heart, spleen, lung and a sample of leg muscle were removed from chicks which had been maintained on a biotin-deficient diet containing dry raw egg white. ${ }^{39}$ All these tissues assayed for oxybiotin by both the permanganate method and the Raney's nickel method had a high content of oxybiotin. The actual biotin content of the tissues was essentially identical with that of tissues from biotin-deficient chicks. Injections of chicks with microbiologically equivalent amounts for Lactobacillus arabinosus of either oxybiotin or biotin result in storage of these compounds in similarly equivalent concentrations in the various tissues. Oxybiotin was found to be bound in the tissues in a manner similar to that of biotin. It was only partially liberated by hot water, but was readily freed by acid hydrolysis.

In contrast to this, it has been reported that administration of the oxygen analogue orally to biotin-deficient chicks does not result in appreciable accumulation of the analogue in the liver or leg muscle, even though the analogue gives a growth response and cures the dermatitis of the chicks. ${ }^{30}$

However, in total balance studies, ${ }^{39}$ analysis of acid hydrolysates of whole chicks grown on a biotin-deficient diet and of their combined excreta by both the permanganate method and the Raney's nickel method has demonstrated that the biotin content of chicks injected with oxybiotin was identical with that of control chicks and not significantly different from that of newly hatched chicks. The amount of biotin excreted by chicks injected with oxybiotin was identical with that excreted by control chicks on the same biotin-deficient diet. Also, of a total dosage of 32 or $64 \gamma$ of DL-oxybiotin injected into chicks over a period of two weeks, essentially all (75 per cent) of the oxybiotin was recovered and was distributed almost equally between the chick and excreta. Injected biotin was similarly recovered.

These results with the chick and other organisms demonstrate that the 
biological action of oxybiotin is a property of the molecule. Since it is not converted by the organisms into biotin, it must be utilized per se in the formation of the necessary coenzyme(s), which in turn can function

TABLE 13. Growth-promoting Activities of Analogues of Biotin and Oxybiotin.

Compound

(+)-Desthiobiotina,e

DL-Desthiobiotina,f

DL-Desthioallobiotin

Biotin methyl ester $f$

DL-Oxybiotin methyl ester ${ }^{f}$

Biotin sulfoxide methyl ester ${ }^{e}$

Biotin sulfone ${ }^{\circ}$

cis-3,4-Diamino-2-tetrahydrothiophenevaleric acid $f$

DL-cis-3,4-Diamino-2tetrahydrothiophenevaleric $\operatorname{acid}^{f}$

DL-cis-3,4-Diamino-2tetrahydrofuranvaleric acidf $^{\prime}$

DL-Hexahydro-2-oxo-1Hfuro[3,4]imidazole-4pentanolf

DL-Hexahydro-2-oxo-4methyl-1H-furo[3,4]imidazole

(+)- $\zeta, \eta$-Diaminopelargonic acide $^{\circ}$

DL-5-Methyl-2-thiono-4imidazolidinecaproic acid
Organism

Saccharomyces cerevisiae

Lactobacillus casei

Saccharomyces cerevisiae

Rats ${ }^{\circ}$

Saccharomyces cerevisiae

Saccharomyces cerevisiae

Lactobacillus casei

Saccharomyces cerevisiae

Saccharomyces cerevisiae

Lactobacillus casei

Saccharomyces cerevisiae

Rats $^{c}$

Lactobacillus casei

Saccharomyces cerevisiae

Saccharomyces cerevisiae F.B.

Lactobacillus casei

Rats

Lactobacillus arabinosus

Lactobacillus casei

Rats

Lactobacillus arabinosus

Lactobacillus casei

Lactobacillus arabinosus

Lactobacillus casei

Saccharomyces cerevisiae

Lactobacillus arabinosus

Lactobacillus casei

Saccharomyces cerevisiae

Lactobacillus casei

Saccharomyces cerevisiae

Lactobacillus casei

\section{Activity}

(t) biotin

References

100

$0^{b}$

60-50

0.1-0.01

0

100

0

16-10

$100 \mathrm{ca}$.

0

$0.1^{d}$

0.1

0

10

0

$0(<0.01)$

$0(<1)$

1.8

3.6

$0(0.4)$

0.01

0.35-0.5

0.13

0.07-0.03

$\begin{array}{ll}0.0001 & 34 \\ 0.0001 & 34 \\ 0.0001 & 34 \\ 10 & 10,48 \\ 0 & 10,48 \\ 0.06 & 52 \\ 0^{b} & 52\end{array}$

- Active for Saccharomyces cerevisiae (25 strains), Saccharomyces chodati, Saccharomyces macedoniensis, Endomycopsis fibuliger, Debaryomyces matruchotiv subolobosus, Mycoderma valida, Mycotorula lactis, Schizosaccharomyces pombe, Torula lactosa, Zygosaccharomyces lactis, Zyoosaccharomyces marxianus, Neurospora crassa, Neurospora sitophila, Ceratostomella ips 438, Ceratostomella montium, Leuconostoc mesenteroides, Penicillium notatum 21464, Escherichia coli 58, Ceratostomella reukanfi, Schwanniomyces occidentalis $116.14,51$ 54. 55 Inactive for Ceratostomella pini 416, Sordaria fimicola, Lactobacillus arabinosus, amd Rhizobium trifolii 205, 11 Penicillium chrysogenum 62078,14, 51

${ }^{b}$ Compound competitively inhibits utilization of biotin for this organism.

- Biotin deficiency induced with raw egg white.

d Only 35-50 per cent of optimal growth obtained with biotin is attained; inactive in the absence of aspartic acid.

- Configuration analogous to $(+)$ biotin.

$f$ Configuration analogous to DL-biotin.

rather efficiently in place of the natural coenzyme. It appears that the sulfur atom of biotin is not essential for its biological action.

Desthiobiotin. In the course of structural studies, ${ }^{10}$ natural biotin was treated with Raney's nickel to remove the sulfur atom of the tetrahydro- 
thiophene ring. The resulting product, $(+)$ desthiobiotin, gave an unusual result in the biotin assay with Saccharomyces cerevisiae. ${ }^{41}(+)$ Desthiobiotin was just as active as $(+)$ biotin, and the dose-response curves of the assays were essentially identical. However, the derivative not only was inactive for Lactobacillus casei but inhibited competitively the response of the organism to biotin. ${ }^{42,51}$

The activity of desthiobiotin is limited to only one of the four optically active forms of the compound. Synthetic DL-desthioallobiotin is inactive for Saccharomyces cerevisiae; ${ }^{8}$ synthetic DL-desthiobiotin is approximately half as active as $(+)$ desthiobiotin. ${ }^{43,44}$

As indicated in Table 13, the utilization of desthiobiotin in place of biotin is rather widespread. Organisms which utilize desthiobiotin have been found to convert the compound into substances which possess biotin-like activity for organisms which require biotin, but do not utilize desthiobiotin. ${ }^{14,42,63}$ The biotin content of cells of Saccharomyces cerevisiae grown on desthiobiotin was determined by differential assay with Lactobacillus casei and the yeast. When present in low concentration, the desthio compound was apparently converted quantitatively into biotin; however, at higher concentrations it was less effectively converted into biotin, and resting cells did not convert any measurable amounts of the compound to biotin or biotin-like substances. ${ }^{14,42}$ Further evidence for the conversion of desthiobiotin to biotin is afforded by the observation that for Lactobacillus casei, Lactobacillus arabinosus and Rhizobium trifolii, Raney's nickel destroys the biotin activity of cells of Saccharomyces cerevisiae grown on either biotin or desthiobiotin. ${ }^{14}$ Permanganate also destroys the activity. ${ }^{36}$ This contrasts with the results obtained with oxybiotin.

The natural occurrence of desthiobiotin is suggested by the accumulation in an x-ray induced biotinless mutant of Penicillium chrysogenum, strain 62078 , of a substance which has the biological properties of desthiobiotin. The substance is inactive for Lactobacillus casei, but active for Neurospora crassa and Escherichia coli 58, a mutant strain requiring biotin. ${ }^{54}$ The last two organisms utilize either desthiobiotin or biotin while the Penicillium mutant requires biotin and is unable to utilize desthiobiotin. Escherichia coli accumulates a biotin precursor which may be desthiobiotin. ${ }^{62}$

From these observations it appears that desthiobiotin is a normal precursor, or is converted to a normal precursor of biotin by a number of organisms. These results are further substantiated by results with a desthiobiotin inhibitor (p. 468).

Pimelic acid, which was shown to be an essential growth factor for certain strains of diphtheria bacillus, ${ }^{56}$ can be replaced by biotin in the 
nutrition of the Allen strain of the organism. ${ }^{57}$ Pimelic acid, however, does not replace biotin or desthiobiotin for a majority of organisms requiring biotin. ${ }^{54,} 58$ Pimelic acid, and also suberic and azelaic acids, enhanced effectively the biosynthesis of biotin in Aspergillus niger, an organism which requires neither biotin nor pimelic acid. ${ }^{59}$ The biosynthesis is further enhanced by certain sulfur compounds, such as cystine and cysteine. An increase in the accumulation of a substance similar to desthiobiotin in the biotinless mutant of Penicillium chrysogenum, strain 62078, is obtained on supplementing the medium with pimelic acid. ${ }^{54}$ Hence, the effects of pimelic acid and desthiobiotin appear to be those of precursors of biotin as indicated below:

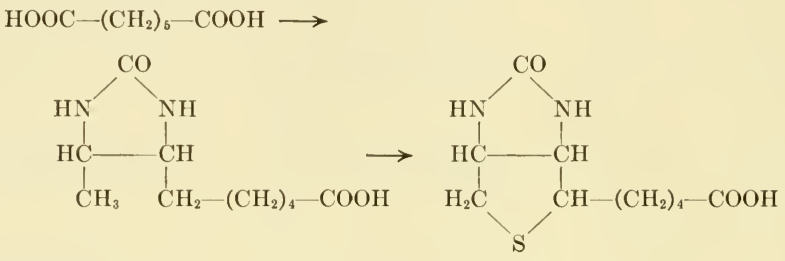

Other Stimulatory Biotin Analogues. As indicated above, the oxygen analogue of biotin and precursors of biotin replace the vitamin in the nutrition of a wide variety of organisms. A considerable number of analogues and derivatives of biotin and oxybiotin possess considerable activity. The activities of these compounds are indicated in Table 13 . In many instances, biotin is formed from the derivatives, but in several cases the activities appear to be inherent in the analogue.

It is interesting to note that the methyl ester of biotin is inactive for Lactobacillus casei but fully active for Saccharomyces cerevisiae. The methyl ester of oxybiotin is slightly less active than the free acid for the latter organism.

The sulfoxide of biotin is as active as biotin for Saccharomyces cerevisiae, but the sulfone which is an antagonist of biotin for Lactobacillus casei has only slight growth-promoting activity for the yeast. This slight activity is dependent upon the presence of aspartic acid in the medium. Hence, it appears that the sulfone cannot replace all the functions of biotin for this organism. Growth obtained with the sulfone at any concentration never attained more than 35 to 50 per cent of the maximum growth obtained with cultures grown on biotin. The results indicate that the sulfone may be utilized as such without prior conversion to biotin.

The diamino acids obtained on hydrolysis of biotin, desthiobiotin and 
oxybiotin have activities up to 10 per cent of that of biotin for a number of organisms.<smiles>NC1CSC(CC(=O)O)C1N</smiles>

cis-3,4-diamino-2-tetrahydrothiophenevaleric acid<smiles>CC(N)C(N)CCC(=O)O</smiles>

$\zeta, \eta$-diaminopelargonic acid<smiles>NC1COC(CC(=O)O)C1N</smiles>

cis-3,4-diamino-2-tetrahydrofuranvaleric acid

On the basis of the activity of the diaminocarboxylic acid derived from biotin, it has been proposed that biotin may function as a coenzyme involved in utilization of carbon dioxide. ${ }^{60}$

Slight activity has been noted for an oxybiotin analogue in which the valeric acid side chain has been replaced by a methyl group. The activity of the 4-pentanol derivative corresponding to oxybiotin may be the result of oxidation of the compound to oxybiotin. Analogous to this result, it has been reported that replacement of the alcohol group to form a number of sulfur analogues results in compounds possessing slight activity. Thus, DL-hexahydro-2-oxo-1H-furo[3,4] imidazole-4-(5-pentanesulfonic acid) has slight growth-promoting activity for both Lactobacillus arabinosus and Saccharomyces cerevisiae; and both DL-hexahydro-2-oxo-4-(5-benzylthiopentyl)-1H-furo[3,4] imidazole and DL-hexahydro-2-oxo-4-(5-mercaptopentyl)- $1 \mathrm{H}$-furo[3,4]imidazole are slightly active in replacing biotin for Lactobacillus arabinosus. ${ }^{61}$

\section{Inhibitory Analogues of Biotin}

Desthiobiotin and Related Compounds. Desthiobiotin, which was obtained by $d u$ Vigneaud and associates ${ }^{10}$ during structural studies on cleavage of biotin with Raney's nickel, was found to possess growthpromoting activity comparable to biotin for Saccharomyces cerevisiae. ${ }^{41}$ On the other hand, it was found to prevent competitively the utilization of biotin by Lactobacillus casei. ${ }^{42,51}$ The activities of this derivative stimulated the preparation and testing of numerous analogues. These compounds are listed in Table 14 . The inhibitory activities, unless indi- 


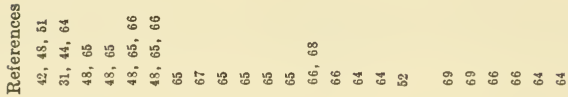

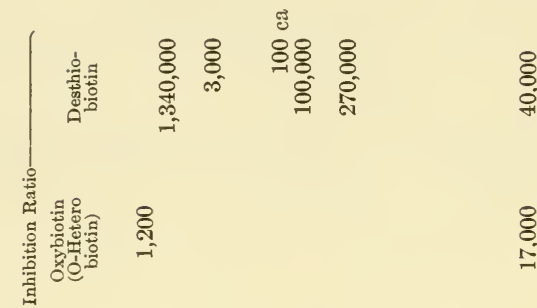

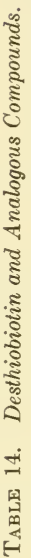

ఫ్ల

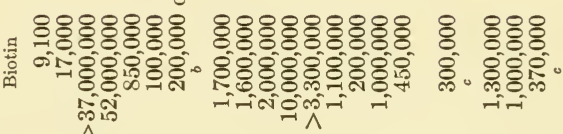
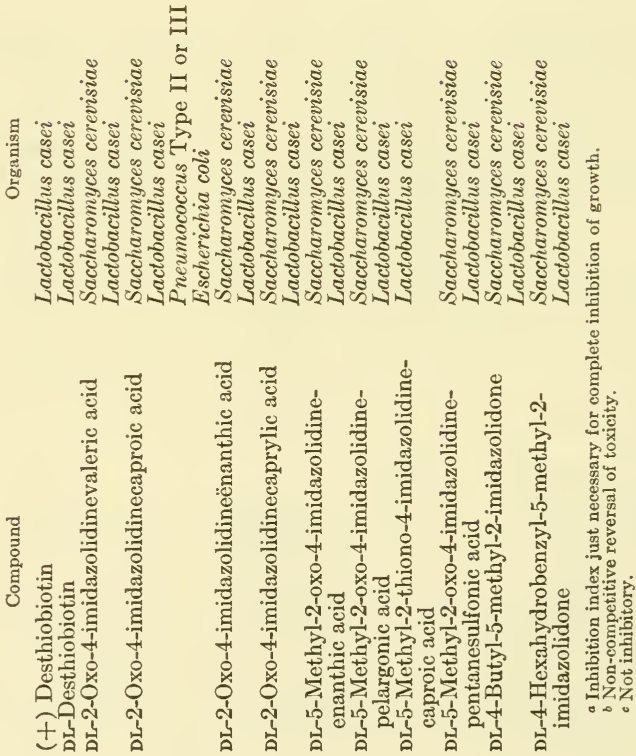
cated otherwise, are listed in terms of the molar inhibition ratio, ${ }^{48}$ which has been defined as the number of molecules of the inhibitor necessary to prevent the biological effect of one molecule of biotin. The molar inhibition ratio ${ }^{48}$ of inhibitor to biotin is obtained experimentally by determining the amount of the inhibitor required to reduce the growth obtained with $0.0002 \gamma$ of biotin to the level of growth obtained with $0.0001 \gamma$ of biotin. The molar inhibition ratio is approximately twice the inhibition index for half-maximum inhibition of growth, and is usually considerably less than the inhibition index just necessary for complete inhibition of growth. In replacing biotin with oxybiotin (O-heterobiotin) or desthiobiotin, amounts of the compounds biologically equivalent to the indicated amounts of biotin are used for determining the molar inhibition ratios.

Since ( + ) desthiobiotin is approximately twice as active as DLdesthiobiotin in preventing the utilization of biotin by Lactobacillus casei, the inhibitory action appears to be the result of the action of only the dextrorotatory form, which is also the only form which appears to exert a growth-promoting activity for Saccharomyces cerevisiae. The oxygen analogue of biotin is also capable of preventing the toxicity of desthiobiotin for Lactobacillus casei, but is much less effective. The results indicate that the affinity of oxybiotin for the enzyme involved is considerably less than that of biotin.

The more effective inhibitory analogues of desthiobiotin, which are indicated by the following formulas, represent modifications of desthiobiotin in which the length of the side chain containing the carboxyl group

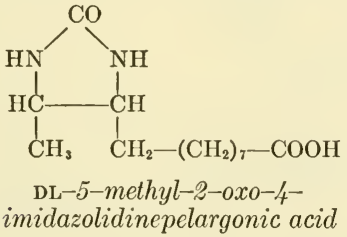

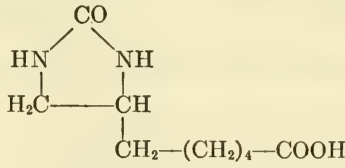

DL-2-oxo-4-imidazolidinecaproic acid

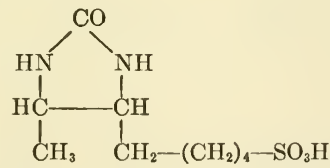

DL-5-methyl-2-oxo-4-imidazolidinepentanesulfonic acid

is varied, the 5-methyl group is omitted, or the carboxyl group is replaced by a sulfonic acid group. These compounds have not as yet been reported to be particularly effective antagonists of biotin. 
Nevertheless, a few of these analogues appear to be very potent in preventing the utilization of desthiobiotin for certain organisms (Table 14). This is especially true of DL-2-oxo-4-imidazolidinecaproic acid for Escherichia coli. Since biotin prevents the inhibition noncompetitively, the analogue appears to prevent the conversion of desthiobiotin to biotin (p. 468). Whether a similar situation exists with other analogues has not been determined. In some cases both the conversion of desthiobiotin to biotin and the utilization of biotin may be prevented by the inhibitor.

TABLE 15. Sulfide and Sulfone Analogues of Biotin.

\begin{tabular}{|c|c|c|c|c|}
\hline \multirow[b]{2}{*}{ Compound } & \multicolumn{4}{|c|}{ Molar Inhibition Ratio } \\
\hline & Organism & Biotin & $\begin{array}{l}\text { Desthio- } \\
\text { biotin }\end{array}$ & References \\
\hline L-Norbiotin & $\begin{array}{l}\text { Saccharomyces cerevisiae } \\
\text { Lactobacillus casei }\end{array}$ & $\begin{array}{r}1,000 \\
13,000\end{array}$ & & $\begin{array}{l}71 \\
71\end{array}$ \\
\hline L-Homobiotin & $\begin{array}{l}\text { Saccharomyces cerevisiae } \\
\text { Lactobacillus casei } \\
\text { Lactobacillus arabinosus }\end{array}$ & $\begin{array}{r}700 \\
130 \\
43,000\end{array}$ & & $\begin{array}{l}71 \\
71 \\
71\end{array}$ \\
\hline L-Bishomobiotin & $\begin{array}{l}\text { Saccharomyces cerevisiae } \\
\text { Lactobacillus casei }\end{array}$ & $\begin{array}{r}30,000 \\
7,000\end{array}$ & & $\begin{array}{l}71 \\
71\end{array}$ \\
\hline L-Trishomobiotin & $\begin{array}{l}\text { Saccharomyces cerevisiae } \\
\text { Lactobacillus casei }\end{array}$ & $\begin{array}{r}50,000 \\
3,000\end{array}$ & & $\begin{array}{l}71 \\
71\end{array}$ \\
\hline Biotin sulfone $e^{a}$ & $\begin{array}{l}\text { Lactobacillus casei } \\
\text { Neurospora crassa } \\
\text { Escherichia coli } 58^{b}\end{array}$ & $\begin{array}{c}280 \\
1,000 \mathrm{ca} \\
1,000 \mathrm{ca}\end{array}$ & $\begin{array}{l}1,000 \mathrm{ca} \\
1,000 \mathrm{ca}\end{array}$ & $\begin{array}{l}48 \\
54 \\
54\end{array}$ \\
\hline L-Homobiotin sulfone & $\begin{array}{l}\text { Saccharomyces cerevisiae } \\
\text { Lactobacillus casei }\end{array}$ & $\begin{array}{r}40,000 \\
400\end{array}$ & & $\begin{array}{l}71 \\
71\end{array}$ \\
\hline $\begin{array}{l}\text { L-Bishomobiotin } \\
\text { sulfone }\end{array}$ & $\begin{array}{l}\text { Saccharomyces cerevisiae } \\
\text { Lactobacillus casei }\end{array}$ & $\begin{array}{r}60,000 \\
8,000\end{array}$ & & $\begin{array}{l}71 \\
71\end{array}$ \\
\hline $\begin{array}{l}\text { L-Trishomobiotin } \\
\text { sulfone }\end{array}$ & $\begin{array}{l}\text { Saccharomyces cerevisiae } \\
\text { Lactobacillus casei }\end{array}$ & $\begin{array}{r}60,000 \\
6,000\end{array}$ & & $\begin{array}{l}71 \\
71\end{array}$ \\
\hline
\end{tabular}

a Also inhibitory to Lactobacillus arabinosus and Staphylococcus aureus. ${ }^{18}$

$b$ A mutant strain requiring biotin or desthiobiotin for growth.

Desthiobiotin is reported to inhibit the functioning of biotin in Sordari fimicola and Ceratostomella pini 416. Desthiobiotin is also reported to have an inhibitory effect on the growth of tumors. ${ }^{70}$ The response of Lactobacillus arabinosus to biotin is increased by the presence of desthiobiotin, even though the desthio compound is inactive alone. ${ }^{51}$

Neither 5-methyl-2-oxo-4-imidazolidinebutyric acid ${ }^{64}$ nor 5-methyl-4hexyl-2-imidazolidone ${ }^{66}$ has appreciable inhibitory activity for either Lactobacillus casei or Saccharomyces cerevisiae.

Sulfide and Sulfone Analogues of Biotin. The most potent inhibitors of the utilization of biotin which have been reported are the homologues of biotin, and the sulfone of biotin and its homologues. ${ }^{48,} 54,71$ Of these, biotin sulfone and DL-homobiotin are the most effective antagonists of biotin, as indicated in Table 15 . When comparing the potencies of vitamin 
analogues, the organism must be specified. While biotin sulfone can, in the presence of aspartic acid, replace biotin in stimulating the growth of<smiles>O=C(O)CC1COC(=O)C1CC(=O)O</smiles><smiles>O=C(O)CC1SCC2NC(=O)NC21</smiles>

homobiotin

Saccharomyces cerevisiae, it is an effective antagonist of biotin for Lactobacillus casei. Even homobiotin is not a particularly effective antagonist of biotin for Lactobacillus arabinosus.

Analogues of Oxybiotin (O-Heterobiotin). The activities of the homologues of oxybiotin and related compounds are indicated in Table 16. Oxybiotin analogues are either inactive or relatively ineffective in preventing the functioning of biotin, but they may prevent the utilization of oxybiotin. It appears that oxybiotin and its analogues do not combine

\section{TABLE 16. Analogues of Oxybiotin (O-Heterobiotin).}

TABLE 16.
Compound
DL-Bisnoroxybiotin
DL-Noroxybiotin
DL-Homoöxybiotin
DL-Bishomoöxybiotin
DL-Hexahydro-2-oxo-1H-
furo[3,4]imidazole-4-
(4-butanesulfonic acid)
(Oxybiotin sulfonic acid)
DL-Hexahydro-2-oxo-4-
(4-benzylthiobutyl)-1H-
furo[3,4]imidazole
a No significant inhibition.

DL-Hexahydro-2-oxo-4(4-benzylthiobutyl)-1HMolar Inhibition Ratio

\begin{tabular}{|c|c|c|c|}
\hline Organism & Biotin & $\begin{array}{l}\text { Oxybioten } \\
\text { (O-Hetero } \\
\text { biotin) }\end{array}$ & References \\
\hline $\begin{array}{l}\text { Saccharomyces cerevisiae } \\
\text { Lactobacillus arabinosus }\end{array}$ & $\begin{array}{l}>500,000 \\
>500,000\end{array}$ & $a$ & $\begin{array}{l}72 \\
72\end{array}$ \\
\hline $\begin{array}{l}\text { Saccharomyces cerevisiae } \\
\text { Lactobacillus arabinosus }\end{array}$ & $\begin{array}{l}>500,000 \\
>500,000\end{array}$ & 143,000 & $\begin{array}{l}72 \\
72\end{array}$ \\
\hline $\begin{array}{l}\text { Saccharomyces cerevisiae } \\
\text { Lactobacillus arabinosus }\end{array}$ & $\begin{array}{r}445,000 \\
>500,000\end{array}$ & $\begin{array}{r}7,400 \\
225,000\end{array}$ & $\begin{array}{l}40,72 \\
72\end{array}$ \\
\hline $\begin{array}{l}\text { Saccharomyces cerevisiae } \\
\text { Lactobacillus arabinosus }\end{array}$ & $\begin{array}{l}>500,000 \\
>500,000\end{array}$ & 30,000 & $\begin{array}{l}72 \\
72\end{array}$ \\
\hline Saccharomyces cerevisiae & $1,460,000$ & 16,600 & 40,61 \\
\hline Saccharomyces cerevisiae & 740,000 & 9,300 & 40,61 \\
\hline
\end{tabular}

as strongly with the enzyme involved as does biotin and its corresponding analogues. The preparation of the lower and higher homologues of oxybiotin has allowed some comparison of their inhibitory activities in the presence of oxybiotin with the activities of the corresponding analogues of biotin in the presence of biotin. For Saccharomyces cerevisiae, DL- 
bishomoöxybiotin prevents the utilization of oxybiotin at approximately the same molar ratio at which DL-bishomobiotin prevents the utilization of biotin. However, this does not hold true for inhibitions with homoöxybiotin and homobiotin. The molar ratios determined with the corresponding growth factors differ by a factor of ten, and the discrepancy increases to over 100 fold for the next lower homologues of oxybiotin and biotin.

In the presence of ammonium sulfate, biotin or oxybiotin stimulates the fermentation rate of biotin-deficient yeast. If either DL-homoöxybiotin or the sulfonic acid corresponding to oxybiotin is added before

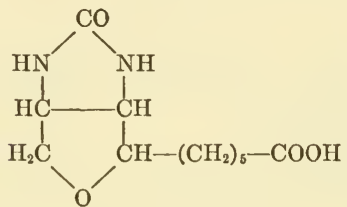

homoöxybiotin

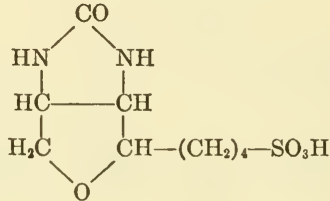

hexahydro-2-oxo-1H-furo[3,4]imidazole4-(4-butanesulfonic acid) (oxybiotin sulfonic acid)

oxybiotin or biotin, the analogues prevent the fermentation at inhibition ratios of 3,750 and 5,000 respectively, for oxybiotin, and 375,000 and 600,000 for biotin. If the inhibitors are added after the addition of biotin, no inhibitory effect is observed, indicating that the inhibitors prevent the conversion of biotin to a functional form which is not antagonized by the analogues. The effect of aspartic acid on fermentation is not altered by the inhibitors. ${ }^{40}$

DL-Hexahydro-2-oxo - 4 - (5 - benzylthiopentyl) - $1 \mathrm{H}$-furo [3,4] imidazole, DL-hexahydro-2-oxo-4-(4-mercaptobutyl)- $1 \mathrm{H}$-furo [3,4] imidazole, and DL-hexahydro-2-oxo-4-(5-mercaptopentyl) - $1 \mathrm{H}$-furo[3,4] imidazole are reported to have an inhibitory effect on Saccharomyces cerevisiae.

Ureylenephenyl and Ureylenecyclohexylbutyric and Valeric Acids. A group of 2,3- and 3,4-ureylenephenyl and ureylenecyclohexylbutyric and valeric acids have been synthesized and found to inhibit the utilization of biotin in a number of organisms. The activities of these compounds against Lactobacillus casei and Saccharomyces cerevisiae are indicated in Table 17. $\gamma$-(2,3-Ureylenecyclohexyl) butyric acid and $\delta$-(2,3-ureylenecyclohexyl)valeric acid are the most effective compounds against yeast, whereas $\gamma$-(3,4-ureylenecyclohexyl) butyric acid was the most effective against Lactobacillus casei. Two diastereoisomers distinguished by different melting points were obtained in the case of each of the 2,3ureylenecyclohexyl-derivatives; however, in contrast to other biotin 
<smiles>O=C(O)CCC1CCCC2NC(=O)NC12</smiles>

$\gamma$-(2,3-ureylenecyclohexyl)butyric acid<smiles>O=C(O)CCC1CCCC2NC(=O)NC12</smiles>

$\delta$-(2,3-ureylenecyclohexyl) valeric acid<smiles>O=C(O)CC1CCC2NC(=O)NC12</smiles>

$\boldsymbol{\gamma}$-(3,4-ureylenecyclohexyl)butyric acid

analogues and stereoisomers, the biological activities of the diastereoisomers were almost identical.

TABLE 17. Ureylenebenzene and Cyclohexane Derivatives as Inhibitory Biotin Analogues

\section{Analogue}

$\gamma$-(2,3-Ureylenephenyl)butyric acid

$\gamma$-(2,3-Ureylenecyclohexyl) butyric acid, ${ }^{a}$

m. p. $218-220^{\circ}$

$\gamma-\left(2,3-\right.$ Ureylenecyclohexyl)butyric acid, ${ }^{a}$

m. p. $192-194^{\circ}$

$\delta$-(2,3-Ureylenephenyl)valeric acid

$\delta$-(2,3-Ureylenecyclohexyl)valeric acid, ${ }^{a}$

m. p. $222-226^{\circ}$

$\delta-\left(2,3,-\right.$ Ureylenecyclohexyl)valeric acid, ${ }^{\circ}$

m. p. $183-184^{\circ}$

$\gamma$-(3,4-Ureylenephenyl)butyric acid ${ }^{b}$

$\gamma$-(3,4-Ureylenecyclohexyl) butyric acid ${ }^{b}$

$\delta$-(3,4-Ureylenephenyl)valeric acid

$\delta$-(3,4-Ureylenecyclohexyl)valeric acid

$\begin{array}{cr}\begin{array}{c}\text { Lactobacillus } \\ \text { casei }\end{array} & \begin{array}{r}\text { Raccharomyces } \\ \text { Sarevisiae }\end{array} \\ 25,000,000 & 310,000 \\ 12,500,000 & 1,500 \\ 6,250,000 & 1,500 \\ 6,250,000 & 2,500,000 \\ 31,000 & 3,000 \\ & \\ 31,000 & 3,000 \\ & \\ 1,500,000 & 6,250,000 \\ 4,000 & 156,000 \\ 750,000 & 1,560,000 \\ 31,000 & 156,000\end{array}$

a Stereoisomeric modifications distinguished only by melting point.

$b$ Inhibits growth of Lactobacillus arabinosus.

\section{Avidin}

On a well balanced diet to which relatively large quantities of dried egg white have been added, rats lose their hair and develop a severe dermatitis and skin hemorrhages; these symptoms are accompanied by 
nervous disorders and loss of weight. A spasticity develops which in the later stages of the deficiency causes the rats to assume a typical kangaroolike posture, and unless the condition is remedied, death ensues. ${ }^{74}$

The principle in egg white which is responsible for the detrimental effect has the properties characteristic of a protein; that is, it is destroyed by heat or mild hydrolysis with dilute acid and is precipitated by such agents as ammonium sulfate. A naturally occurring substance which exerts a protective action against this egg white injury has been termed vitamin $\mathrm{H}$ by György. ${ }^{75}$ The identity of vitamin $\mathrm{H}$ with biotin was finally established ${ }^{76,77,78}$ by testing a sample of biotin methyl ester isolated by Kögl. ${ }^{2}$

The protein in raw egg white which renders biotin unavailable to animals was found also to prevent the utilization of biotin by Saccharomyces cerevisiae ${ }^{79}$ and other microorganisms requiring biotin for growth. Thus, a protein constituent of the egg white forms a stable, nondialyzable complex with biotin. The combination between the protein and biotin was found to occur in stoichiometric amounts. With the microbiological test for the egg white factor, the protein which combines with biotin has been isolated by Eakin and associates ${ }^{79,80}$ in crystalline form and named avidin. Purified avidin produced effects in rats similar to those caused by dried egg white. ${ }^{81}$ With the assumption that one molecule of biotin combines with one molecule of protein, the molecular weight of avidin has been calculated to be 43,500 . The isoelectric point occurs at approximately $\mathrm{pH} 10 .{ }^{82}$ Some loss of activity was inherent in crystallization, since the potency of a crystalline sample of avidin was approximately 4,000 units per gram as compared with 7,000 units per gram for amorphous preparations. By definition one unit of avidin combines with $1 \gamma$ of biotin. ${ }^{80}$

It is interesting to note that avidin passes through the alimentary tract of animals unchanged and can be demonstrated in the feces. ${ }^{83}$ Even liver, kidney and proteolytic enzymes are inactive in liberating biotin combined with avidin. ${ }^{84}$ However, destruction of avidin by heat treatment liberated material with biotin-like activity.

The ability of various analogues of biotin to combine with avidin has been determined. For example, it has been reported that sufficient avidin completely inhibits the growth promoting activity of biotin sulfone for Saccharomyces cerevisiae. ${ }^{47}$ Since limited amounts of avidin added to a medium containing excess biotin sulfone produce responses in the yeast characteristic of biotin but not of biotin sulfone, it appears that biotin sulfone liberates biotin from the complex.

A method for determining the avidin-combinability of biotin analogues has been developed by Wright and Skeggs, ${ }^{85,86}$ in which the "relative 
affinity" for avidin is determined by varying the analogue concentration when biotin and avidin are present in stoichiometric amounts. The "relative affinity" is expressed as a ratio of concentration of analogue to biotin at which one-half of the biotin remains free and available for growth of a test organism. With this technique, $(-)$ biotin and DL-allobiotin were found to have no significant affinity for avidin, but DL-epi-allobiotin has an affinity ratio of approximately 6 . The affinity ratios for DL-desthiobiotin and $\delta$-(2,3-ureylenecyclohexyl) valeric acid are 10 and 14 , respectively. $\gamma$-(3,4-Ureylenecyclohexyl) butyric acid and $\delta$-(3,4-ureylenecyclohexyl)valeric acid have a definite ability to combine with avidin, but the ratios are too high to be determined.

Other than those mentioned above, the following have been found to combine with avidin: DL-oxybiotin, ${ }^{34}$ DL-oxybiotin methyl ester, ${ }^{34}$ DLhexahydro-2-oxo-1H-furo[3,4] imidazole-4-pentanol ${ }^{34}$ and 2-oxo-4-imidazolidinevaleric, caproic, enanthic and caprylic acids. ${ }^{48}$

The inability of cis-3,4-diamino-2-tetrahydrothiophenevaleric acid (the diaminocarboxylic acid corresponding to biotin) to combine with avidin was the basis for the first suggestion that avidin combinability was a function of the imidazolidone ring. ${ }^{48,}{ }^{49}$ This is further substantiated by the inability of $\zeta, \eta$-diaminopelargonic acid, ${ }^{48}$ an analogous derivative of desthiobiotin, and DL-cis-3,4-diamino-2-tetrahydrofuranvaleric acid, ${ }^{34}$ corresponding to oxybiotin, to combine with avidin.

Since desthiobiotin ${ }^{48}$ and other derivatives not containing the sulfur atom combine with avidin, the sulfur atom does not appear essential for the bonding. Also, the methyl ester of oxybiotin ${ }^{34}$ and the alcohol analogue of oxybiotin ${ }^{34}$ in which the carbinol group replaces the carboxyl group combine with avidin, indicating that the carboxyl group is not essential for this activity. However, there appears to be structural specificity with regard to the configuration of the molecule necessary for avidin combinability.

\section{Bibliography}

1. Wildiers, E., Cellule, 18, 313 (1901).

2. Kögl, F., Ber., 68, 16 (1935); Kögl, F., and Tönnis, B., Z. physiol. Chem., 242, 43 (1936).

3. Kögl, F., Proc. Roy. Soc. (London), B124, 1 (1937) ; Kögl, F., Chem. and Ind., 57, 49 (1938).

4. du Vigneaud, V., Hofmann, K., Melville, D. B., and György, P., J. Biol. Chem., 140, 643 (1941); György, P., Rose, C. S., Hofmann, K., Melville, D. B., and du Vigneaud, V., Science, 92, 609 (1940).

5. Melville, D. B., Hofmann, K., Hague, E., and du Vigneaud, V., J. Biol. Chem., 142, 615 (1942). 
6. du Vigneaud, V., Science, 96, 455 (1942).

7. Harris, S. A., Wolf, D. E., Mozingo, R., and Folkers, K., Science, 97, 447 (1943); Harris, S. A., Wolf, D. E., Mozingo, R., Anderson, R. C., Arth, G. E., Easton, N. R., Heyl, D., Wilson, A. N., and Folkers, K., J. Am. Chem. Soc., 66, 1756 (1944).

8. Harris, S. A., Wolf, D. E., Mozingo, R., Arch, G. E., Anderson, R. C., Easton, N. R., and Folkers, K., J. Am. Chem. Soc., 67, 2096 (1945); Harris, S. A., Mozingo, R., Wolf, D. E., Wilson, A. N., Arth, G. E., Folkers, K., J. Am. Chem. Soc., 66, 1800 (1944).

9. Baker, B. R., MeEwen, W. L., and Kinley, W. N., J. Org. Chem., 12, 322 (1947).

10. du Vigneaud, V., Melville, D. B., Folkers, K., Wolf, D. E., Mozingo, R., Keresztesy, J. C., and Harris, S. A., J. Biol. Chem., 146, 475 (1942).

11. Harris, S. A., Mozingo, R., Wolf, D. E., Wilson, A. N., and Folkers, K., J. Am. Chem. Soc., 67, 2102 (1915).

12. Emerson, G. H., J. Biol. Chem., 157, 127 (1945).

13. Ott, W. H., J. Biol. Chem., 157, 131 (1945).

14. Stokes, J. L., and Gunness, M., J. Biol. Chem., 157, 121 (1945).

15. Baker, B. R., Querry, M. V., McEwen, W. L., Bernstein, S., Safir, S. R., Dorfman, L., and SubbaRow, Y., J. Org. Chem., 12, 186 (1947).

16. Kögl, F., and ten Ham, E. J., Naturwiss., 31, 208 (1943).

17. Kögl, F., and ten Ham, E. J., Z. physiol. Chem., 279, 140 (1943).

18. Kögl, F., Verbeek, J. H., Erxleben, H., and Borg, W. A. J., Z. physiol. Chem., 279, 121 (1943).

19. Kögl, F., and Borg, W. A. J., Z. physiol. Chem., 281, 65 (1944).

20. György, P., Rose, C. S., Hofmann, K., Melville, D. B., and du Vigneaud, V., Science, 92, 609 (1940).

21. Krueger, K. K., and Peterson, W. H., J. Biol. Chem., 173, 497 (1948).

22. Hofmann, K., J. Am. Chem. Soc., 67, 694 (1945); 67, 1459 (1945).

23. Duschinsky, R., Dolan, L. A., Flower, D., and Rubin, S. H., Arch. Biochem., 6, 480 (1945).

24. Pilgrim, F. J., Axelrod, A. E., Winnick, T., and Hofmann, K., Science, 102, 35 (1945).

25. Hofmann, K., J. Am. Chem. Soc., 71, 164 (1949).

26. Hofmann, K., and Axelrod, A. E., Arch. Biochem., 11, 375 (1946).

27. Hofmann, K., McCoy, R. H., Felton, J. R., Axelrod, A. E., and Pilgrim, F. J., Arch. Biochem., 7, 393 (1945).

28. Axelrod, A. E., Pilgrim, F. J., and Hofmann, K., J. Biol. Chem., 163, 191 (1946).

29. McCoy, R. H., Felton, J. R., and Hofmann, K., Arch. Biochem., 9, 141 (1946).

30. Moore, P. R., Luckey, T. D., Elvehjem, C. A., and Hart, E. B., Proc. Soc. Exptl. Biol. Med., 61, 185 (1946).

31. Rubin, S. H., Flower, D., Rosen, F., and Drekter, L., Arch. Biochem., 8, 79 (1945).

32. Luckey, T. D., Moore, P. R., and Elvehjem, C. A., Proc. Soc. Exptl. Biol. Med., 61, 97 (1946).

33. Krueger, K. K., and Peterson, W. H., J. Bact., 55, 693 (1948).

34. Winnick, T., Hofmann, K., Pilgrim, F. J., and Axelrod, A. E., J. Biol. Chem., 161, 405 (1945).

35. Hofmann, K., Winnick, T., and Axelrod, A. E., J. Biol. Chem., 169, 191 (1947).

36. Hofmann, K., and Winnick, T., J. Biol. Chem., 160, 449 (1945).

37. Axelrod, A. E., DelWoody, J., and Hofmann, K., J. Biol. Chem., 163, 771 (1946).

38. Axelrod, A. E., Flinn, B. C., and Hofmann, K., J. Biol. Chem., 169, 195 (1947).

39. McCoy, R. H., McKibben, J. N., Axelrod, A. E., and Hofmann, K., J. Biol. Chem., 176, 1319, 1327 (1948).

40. Axelrod, A. E., Purris, S. E., and Hofmann, K., J. Biol. Chem., 176, 695 (1948).

41. Melville, D. B., Dittmer, K., Brown, G. B., and du Vigneaud, V., Science, 98, 497 (1943). 
42. Dittmer, K., Melville, D. B., and du Vigneaud, V., Science, 99, 203 (1944).

43. Wood, J. L., and du Vigneaud, V., J. Am. Chem. Soc., 67, 210 (1945).

44. Rubin, S. H., Drekter, L., and Moyer, E. H., Proc. Soc. Exptl. Biol. Med., 58, 352 (1945).

45. Shull, G. M., Hutchings, B. L., and Peterson, W. H., J. Biol. Chem., 142, 913 (1942).

46. du Vigneaud, V., Chem. Eng. News, 23, 620 (1945).

47. Dittmer, K., du Vigneaud, V., György, P., and Rose, C. S., Arch. Biochem., 4, 229 (1944).

48. Dittmer, K., and du Vigneaud, V., Science, 100, 129 (1944).

49. du Vigneaud, V., Dittmer, K., Hofmann, K., and Melville, D. B., Proc. Soc. Exptl. Biol. Med., 50, 374 (1942).

50. Chu, E. J-H., and Williams, R. J., J. Am. Chem. Soc., 66, 1678 (1944).

51. Lilly, V. G., and Leonian, L. H., Science, 99, 205 (1944).

52. Brown, G. B., and du Vigneaud, V., J. Biol. Chem., 163, 761 (1946).

53. Hofmann, K., Melville, D. B., and du Vigneaud, V., J. Biol. Chem., 141, 207 (1941).

54. Tatum, E. L., J. Biol. Chem., 160, 455 (1945).

55. Hijner, J. A., Experientia, 2, 312 (1946).

56. Mueller, J. H., J. Biol. Chem., 119, 121 (1937); J. Bact., 34, 163 (1937).

57. du Vigneaud, V., Dittmer, K., Hague, E., and Long, B., Science, 96, 186 (1942).

58. Wright, L. D., Proc. Soc. Exptl. Biol. Med., 51, 27 (1942).

59. Eakin, R. E., and Eakin, E. A., Science, 96, 187 (1942).

60. Burk, D., and Winzler, R. J., Science, 97, 57 (1943).

61. Hofmann, K., Bridgwater, A., and Axelrod, A. E., J. Am. Chem. Soc., 69, 1550 (1947); 71, 1253 (1949).

62. Auhagen, E., Naturwiss., 33, 221 (1946).

63. Leonian, L. H., and Lilly, V. G., J. Bact., 49, 291 (1945).

64. Duschinsky, R., and Dolan, L. A., J. Am. Chem. Soc., 67, 2079 (1945).

65. Dittmer, K., and du Vigneaud, V., J. Biol. Chem., 169, 63 (1947).

66. Duschinsky, R., and Dolan, L. A., J. Am. Chem. Soc., 68, 2350 (1946).

67. Rogers, L. L., and Shive, W., J. Biol. Chem., 169, 57 (1947).

68. McKennis, H., Jr., and du Vigneaud, V., J. Am. Chem. Soc., 68, 832 (1946).

69. Duschinsky, R., and Rubin, S. H., J. Am. Chem. Soc., 70, 2546 (1948).

70. Keresztesy, J. C., Laszlo, D., and Leuchtenberger, C., Cancer Research, 6, 128 (1946).

71. Goldberg, M. W., Sternbach, L. H., Kaiser, S., Heineman, S. D., Scheiner, J., and Rubin, S. H., Arch. Biochem., 14, 480 (1947).

72. Hofmann, K., Chen, C., Bridgwater, A., and Axelrod, A. E., J. Am. Chem. Soc., 69, 191 (1947).

73. English, J. P., Clapp, R. C., Cole, Q. P., Halverstadt, I. F., Lampen, J. O., and Roblin, R. O., Jr., J. Am. Chem. Soc., 67, 295 (1945).

74. Boas, M. A., Biochem. J., 21, 712 (1927).

75. György, P., J. Biol. Chem., 131, 733 (1939).

76. György, P., Melville, D. B., Burk, D., and du Vigneaud, V., Science, 91, 243 (1940).

77. du Vigneaud, V., Melville, D. B., György, P., and Rose, C. S., Science, 92, 62 (1940).

78. György, P., Rose, C. S., Hofmann, K., Melville, D. B., and du Vigneaud, V., Science, 92, 609 (1940).

79. Eakin, R. E., Snell, E. E., and Williams, R. J., J. Biol. Chem., 140, 535 (1941).

80. Pennington, D., Snell, E. E., and Eakin, R. E., J. Am. Chem. Soc., 64, 469 (1942).

81. György, P., Rose, C. S., Eakin, R. E., Snell, E. E., and Williams, R. J., Science, 93, 477 (1941).

82. Woolley, D. W., and Longsworth, L. G., J. Biol. Chem., 142, 285 (1942). 
83. György, P., and Rose, C. S., Science, 94, 261 (1941).

84. György, P., and Rose, C. S., Proc. Soc. Exptl. Biol. Med., 53, 55 (1943).

85. Wright, L. D., and Skeggs, H. R., Arch. Biochem., 12, 27 (1947).

86. Wright, L. D., Skeggs, H. R., and Cresson, E. L., Proc. Soc. Exptl. Biol. Med.,
64,150 (1947). 


\section{Chapter VD}

\section{THE FOLIC ACID GROUP}

The isolation of members of the folic acid group brought together a number of divergent studies. The earliest report of a biological activity which can now be attributed to the folic acid group is the striking effect of yeast extract in relieving a nutritional anemia occurring particularly in pregnant women. ${ }^{1}$ The nutritional deficiency was reproduced in monkeys, ${ }^{2,3}$ and the factor necessary to prevent the deficiency was later termed vitamin M.45 The folic acid group also accounts for the active principles which were essential for growth (Factor $\mathrm{U})^{6}$ and prevention of nutritional anemia (vitamin $\mathrm{B}_{\mathrm{c}}$ ) in chicks, ${ }^{7,8}$ and essential for the growth of Lactobacillus casei (Norit Eluate Factor) ${ }^{9,10}$ and of Streptococcus faecalis R. ${ }^{11}$

The isolation of the first crystalline member of the folic acid group was reported by Pfiffner et $a l .,^{12}$ who obtained folic acid from liver. Shortly afterward, Stokstad ${ }^{13}$ also reported the isolation of the same compound from liver. An enzymatic digest of a yeast concentrate was used as another source material for the isolation of the crystalline material. ${ }^{14}$ Folic acid has also been isolated in essentially pure form from spinach. ${ }^{15}$ Other members of this group of factors were obtained in crystalline form within a short period of time. ${ }^{16,17,18}$ Structural studies on folic acid indicated a xanthopterin-like unit, ${ }^{19-23} p$-aminobenzoic acid, ${ }^{21-23}$ and glutamic acid, ${ }^{21-23}$ combined as indicated by the following formula:<smiles>Nc1nc(O)c2nc(CNc3ccc(C(=O)NC(CCC(=O)O)C(=O)O)cc3)cnc2n1</smiles>

folic acid (N-pteroyl-L-glutamic acid)

The complete structure and synthesis ${ }^{24,25}$ were announced simultaneously with the structure of another member of this complex, a factor derived from the cultural broth of an unidentified organism belonging to the genus Corynebacterium. ${ }^{17}$ This factor was identified as a pteroyldi- 
glutamylglutamic acid ${ }^{21-23}$ and later synthesized. ${ }^{26}$ By synthesis, the structure was established as $\mathrm{N}$-pteroyldi- $\gamma$-glutamylglutamic acid.<smiles>Nc1nc(O)c2nc(CNc3ccc(C(=O)NC(CCC(=O)NC(CCC(=O)O)C(=O)O)C(=O)O)cc3)cnc2n1</smiles>

\section{$N$-pteroyldi- $-\boldsymbol{\gamma}$-glutamylglutamic acid}

Another crystalline compound which prevents anemia in chicks, but is not appreciably active for either Lactobacillus casei or Streptococcus faecalis $R$, was isolated from yeast. ${ }^{18}$ This compound was termed vitamin $\mathrm{B}_{\mathrm{c}}$ conjugate, and later was demonstrated to be a pteroylhexaglutamylglutamic acid. ${ }^{27}$ An enzyme widely distributed in animal tissues ${ }^{28-32}$ hydrolyzes the conjugate to a form which is active for Streptococcus faecalis $\mathrm{R}$ and Lactobacillus casei. Folic acid was isolated after enzymatic digestion of a concentrate of the conjugate from yeast. ${ }^{14}$

A fourth crystalline member ${ }^{16}$ of the folic complex was isolated from the fermentation liquors of Rhizopus nigricans and given the trivial name rhizopterin. ${ }^{33}$ This compound was subsequently shown to be $\mathrm{N}^{10}$-formylpteroic acid and synthesized ${ }^{34,35}$ from pteroic acid which was previously prepared synthetically in the course of studies on folic acid. ${ }^{24}$

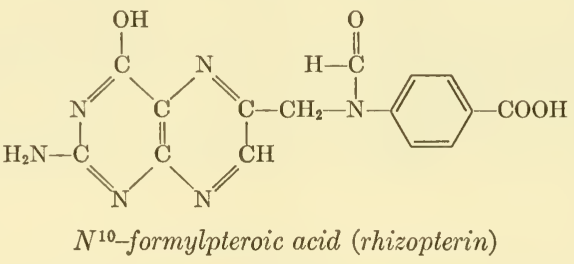

The relative biological activities of these naturally occurring members of the folic acid complex as well as a number of synthetic, related compounds are indicated in Table 18. All activities, except those for rat and man, represent the ability of derivatives to replace folic acid as an essential nutritional factor under controlled experimental conditions.

Supplementary folic acid is not ordinarily required by rats on a purified diet, but rats fed sulfaguanidine or succinylsulfathiazole in such a purified diet grow slowly ${ }^{66}$ and develop an anemia, an agranulocytosis, a leucopenia, and a hypocellularity of bone marrow, ${ }^{67,68}$ which are corrected by administration of folic acid..$^{5,69}$ The effect of sulfonamides, 


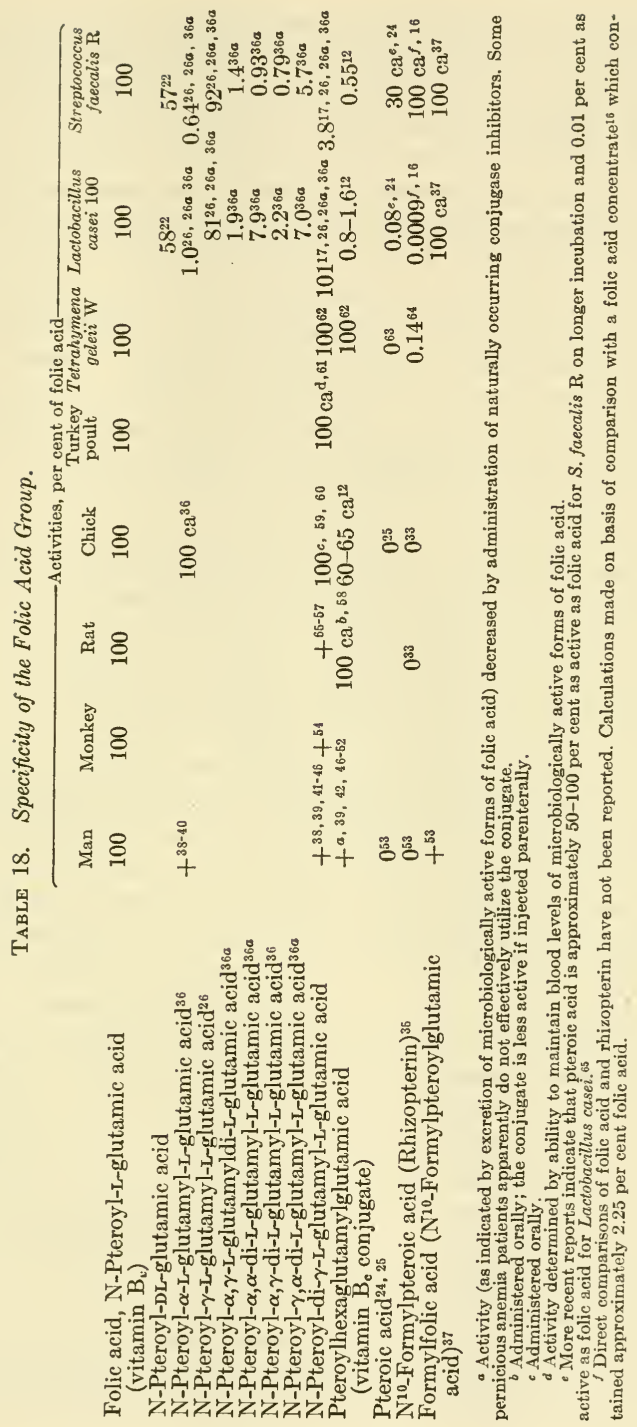


which has been attributed to decreased synthesis by intestinal bacteria, affords a deficiency with which the efficacy of folic acid and its derivatives can be evaluated for the rat. The effectiveness of folic acid in treatment of pernicious anemia, ${ }^{70-72}$ sprue,${ }^{73}$ and nutritional macrocytic anemia ${ }^{70-72}$ has made available a means of demonstrating in human subjects the activities of compounds related to folic acid. Increased excretion in the urine of microbiologically active forms of folic acid on administration of microbiologically inactive forms of the vitamin has also been used as a criterion for the utilization of the compounds in human subjects. Although only small amounts, less than 1 per cent of the normal total intake of folic acid in human subjects, are regularly excreted in the urine in microbiologically active forms, ${ }^{74,75}$ the administration of relatively large amounts of folic acid $(1-15 \mathrm{mg})$ often results in urinary excretion of 15 to 75 per cent of the administered dose within 24 hours $;^{42,76}$ however, the amount excreted depends somewhat on the individual.

Of the two optically active stercoisomers with the structure of folic acid, only the naturally occurring L-modification appears to be biologically active, since the racemic modification is approximately half as effective as the naturally occurring folic acid for Lactobacillus casei and Streptococcus faecalis $\mathrm{R}^{22}$ Also, D-folic acid (N-pteroyl-D-glutamic acid) does not replace L-folic acid in stimulating the production of the Rous sarcoma (p. 595) in folic acid-deficient chicks. ${ }^{77}$

Although N-pteroyl- $\alpha$-glutamylglutamic acid is only about 1 per cest as effective as folic acid in the nutrition of Lactobacillus casei and Streptococcus faecalis $\mathrm{R},{ }^{26}, 36$ this folic acid derivative is completely effective for the chick, ${ }^{36}$ and also replaces folic acid in stimulation of the Rous sarcoma virus in folic acid-deficient chicks. ${ }^{77}$ Administration of $\mathrm{N}$-pteroyl$\alpha$-glutamylglutamic acid $(6.5 \mathrm{mg})$ either orally or intravenously to normal male human subjects results in an increased excretion of folic acid amounting to 50 to 75 per cent of that excreted when an equivalent amount of folic acid $(5 \mathrm{mg})$ is administered. ${ }^{38}$ Although there is a lag in the initial rate of excretion, particularly following intravenous administration, a major portion of the excretion takes place within the first six hours. ${ }^{38}$ Pteroyl- $\alpha$-glutamylglutamic acid has also been reported to be effective in treatment of pernicious anemia and macrocytic nutritional anemia, but does not appear to be as active as folic acid.39,40

Contrasting markedly to the corresponding $\alpha$-glutamyl derivative, $\mathrm{N}$-pteroyl- $\gamma$-glutamylglutamic acid is almost as active as folic acid for Lactobacillus casei and Streptococcus faecalis R. ${ }^{26}$

With the exception of Streptococcus faecalis R, N-pteroyldi- $\gamma$-glutamylglutamic acid is essentially as effective as folic acid for all the organisms listed in Table 18. With Lactobacillus casei and particularly with Strepto- 
coccus faecalis $\mathrm{R}$, a sigmoid curve is obtained on plotting the growth response against concentration of the triglutamate. ${ }^{78,79}$

Pteroyldi- $\gamma$-glutamylglutamic acid, administered either orally or intravenously, is just as effective as folic acid in effecting an increase in the urinary excretion of forms of folic acid active for Streptococcus faecalis $\mathrm{R}$; however, a very slight lag in the rate of excretion is noted. ${ }^{38}$ Even so, most of the active substances derived from the triglutamate are excreted during the first six hours. ${ }^{38}$

The earliest indication of a beneficial effect of the triglutamate in human subjects was a report of slight activity in a patient with nutritional macrocytic anemia. ${ }^{41}$ Administration of $5 \mathrm{mg}$ of the triglutamate per day gave a reticulocyte response of 12 per cent on the ninth day, with a subsequent increase in red cell count. ${ }^{41}$ Subsequently, parenteral administration of the triglutamate (daily dose equivalent to $3.1 \mathrm{mg}$ of folic acid) was shown to increase markedly the excretion of folic acid, and to have pronounced beneficial effects in relieving the clinical and hematologic manifestations of sprue in a patient previously treated with liver extracts. ${ }^{42}$ Treatment of a sprue patient with intramuscular injections of a crystalline sample of the pteroyldi- $\gamma$-glutamylglutamic acid ( $5 \mathrm{mg}$ twice daily) alone resulted in a rise in erythrocyte count and hemoglobin, clinical improvement, and maximum reticulocytosis of 38 per cent on the fourth day of therapy. ${ }^{43}$ Administration of $3 \mathrm{mg}$ daily of the triglutamate by intramuscular injection to a patient with pernicious anemia in relapse resulted in a submaximal hemopoietic response, accompanied by subjective improvement. ${ }^{44}$ This is in contrast to an earlier report indicating that a concentrate of the triglutamate was inactive when administered to two patients in doses equivalent to 3.6 and $2.3 \mathrm{mg}$ of folic acid daily. ${ }^{45}$ However, from more recent work, it appears that the triglutamate is utilized by patients with pernicious anemia. ${ }^{39}$

Monkeys rendered anemic and leucopenic by vitamin M-deficient diets respond to an intramuscular injection of $3 \mathrm{mg}$ of pteroyldi- $\gamma$-glutamylglutamic acid. Administered in divided doses over a period of three days, it produces prompt and complete remission lasting for ten to thirty days. ${ }^{54}$

The deficiency of folic acid resulting from administration of succinylsulfathiazole or sulfaguanidine in the diet of rats is corrected by pteroyldi$\gamma$-glutamylglutamic acid as well as by folic acid. ${ }^{55}$ The triglutamyl derivative also has a preventive and corrective effect on anemia induced by bleeding rats fed a purified diet containing succinylsulfathiazole. ${ }^{56}$ The leucopenia which develops in rats given thyroid powder orally or thyroxine injections and fed thiourea in a purified diet is relieved by the triglutamate. ${ }^{57}$ Granulocytopenia, which develops in rats on a purified 
diet, deficient in riboflavin, is also corrected by pteroyldi- $\gamma$-glutamylglutamic acid. ${ }^{57 a}$

Pteroyldi- $\gamma$-glutamylglutamic acid is just as effective on a molar basis as folic acid in promoting growth and hemoglobin formation in chicks. ${ }^{17,59,60}$ Early work indicated that, while folic acid was effective alone, chicks required a supplementary factor for the utilization of the pteroyltriglutamic acid. This factor could be replaced by 5 - or 4-pyridoxic acid (p. 657) or their lactones ( $\alpha$ - or $\beta$-pyracin) ${ }^{80-82} 4$-Pyridoxic acid was the more effective of the two isomers. Attempts ${ }^{59,60}$ to confirm this supplementary effect have not been successful, with the exception of a single experiment involving a comparison between two groups of six chicks each. ${ }^{83}$ In hemorrhagic anemia in hens, either pteroyltriglutamic acid or 4-pyridoxic acid, or more effectively, a combination of the two compounds, was reported to exert a beneficial action in hastening the regeneration of hemoglobin. ${ }^{84}$ The yield of folic acid, determined with Streptococcus faecalis $\mathrm{R}$ from an incubation mixture of pteroyltriglutamic acid and chicken liver, increased twofold on supplementing the mixture with 4 - or 5 -pyridoxic acid. ${ }^{85}$

$\mathrm{N}$-Pteroyldi- $\gamma$-glutamylglutamic acid is also effective in replacing folic acid in stimulating the Rous sarcoma virus in folic acid-deficient chicks. ${ }^{77}$

This pteroyltriglutamic acid on a molar basis is just as effective as folic acid in maintaining the concentration of microbiologically active forms of folic acid in the blood of turkey poults on a folic acid-deficient diet. ${ }^{61}$ The triglutamate, however, is more effective than folic acid in increasing the blood concentrations of conjugates of folic acid which are hydrolyzed by chicken pancreas. ${ }^{61}$

A conjugase preparation from chicken pancreas ${ }^{86}$ hydrolyzes N-pteroyldi- $\gamma$-glutamylglutamic acid as well as $\mathrm{N}$-( $p$-aminobenzoyl) di- $\gamma$-glutamylglutamic acid and produces one molecule of glutamic acid for each carboxyl group liberated. ${ }^{87}$ Assay with $S$. faecalis $\mathrm{R}$ of the reaction mixture resulting from the action of the enzyme preparation on the pteroyltriglutamic acid indicated the liberation of activity equivalent to one folic acid for each glutamic acid. These results suggest that the triglutamate is hydrolyzed only to the diglutamate, which is approximately as active as folic acid for $S$. faecalis $R$. Since one equivalent of glutamic acid shows an inhibitory effect on the enzymatic reaction, it is suggested that the glutamic acid formed may prevent the reaction from going to completion. ${ }^{87}$

The pteroylhexaglutamylglutamic acid, vitamin $\mathrm{B}_{\mathrm{c}}$ conjugate, possesses on a molar basis only about one per cent of the activity of folic acid for the two bacteria, Lactobacillus casei and Streptococcus faecalis R, which are commonly used for the assay of folic acid. ${ }^{12}$ On the other hand, both 
this conjugate and the triglutamate, on a molar basis, are as active as folic acid for Tetrahymena gelii W. ${ }^{62}$ Earlier work on a deficient medium indicated the possibility that these conjugates may be more effective than folic acid. ${ }^{63}$

Oral or parenteral administration of pteroylhexaglutamylglutamic acid to normal human subjects produces a prompt excretion of microbiologically active forms of folic acid. On daily administration of equivalent amounts of the conjugate or folic acid $(4 \mathrm{mg})$, comparable amounts of microbiologically active forms of folic acid (approximately $30-35$ per cent of the administered dose) are excreted daily. ${ }^{46} \mathrm{~A}$ normal subject given $2.8 \mathrm{mg}$ of the conjugate daily by intramuscular injection excreted 8.3 per cent of microbiologically active forms equivalent to folic acid, whereas administration of an equivalent amount of folic acid resulted in the urinary excretion of 16 per cent of microbiologically active forms. ${ }^{47}$ Oral administration of pteroylhexaglutamylglutamic acid (equivalent to 8.4 $\mathrm{mg}$ of folic acid daily) caused a rapid clinical improvement in a patient with sprue; however, only small amounts of microbiologically active forms of the vitamin were excreted in the urine. ${ }^{42}$ The conjugate appears to be similarly active in nutritional macrocytic anemia. ${ }^{39,51}$

However, the ability of pernicious anemia patients to utilize the conjugate appears to vary. ${ }^{48}$ Many patients in relapse respond hematologically and excrete increased amounts of folic acid following the administration of pteroylhexaglutamylglutamic acid.39,49-51 Yet there are reports of failure by pernicious anemia patients in relapse to respond either hematologically or with increased excretion of microbiologically active forms of the vitamin. ${ }^{47-50,52}$ Quantities as high as $54 \mathrm{mg}$ of the conjugate administered daily in exceptional cases of pernicious anemia have failed to increase the urinary excretion of microbiologically active forms of folic acid. ${ }^{50}$ It has been demonstrated that even in normal individuals the ability of the conjugate to cause increased urinary excretion of microbiologically active forms of folic acid can be almost completely inhibited by administration of a conjugate preparation containing conjugase inhibitor or by administration of yeast extract ${ }^{46}$ which contains considerable amounts of conjugase inhibitor. ${ }^{88}$ No evidence for the excretion of the conjugate as such has been found. ${ }^{46}$

Nucleic acid, ${ }^{89}$ proteins, ${ }^{90}$ and a $p$-aminobenzoylpolyglutamyl derivative of an unidentified amino acid, ${ }^{91}$ which has been isolated from yeast, ${ }^{92}$ inhibit the conjugase enzyme. The inhibition with the polypeptide is competitive. ${ }^{91}$ Conjugase inhibitors appear to be widely distributed in nature and are known to occur in liver and spinach as well as in yeast. ${ }^{46}$ It has been suggested that the conjugase inhibitors may play an important role in the utilization of the conjugate and may account for at least some of 
the variations in the response of pernicious anemia patients to the conjugate. ${ }^{46,} 49$

Pteroylhexaglutamylglutamic acid is just as active as folic acid in correcting the succinylsulfathiazole-induced leucopenia in rats if the factors are administered orally. ${ }^{58}$ Injected parenterally, the conjugate is not quite so effective as folic acid. Simultaneous oral administration of a conjugase inhibitor and the heptaglutamate cause a 50 per cent decrease in the urinary excretion of microbiologically active forms of folic acid as compared with controls on the conjugate alone; however, the hematologic response is not decreased by the conjugase inhibitor. ${ }^{58}$

From these results, it appears that the hematopoietic activity of folic acid derivatives may not necessarily be reflected in urinary excretion of microbiologically available forms of folic acid on administration of the derivatives, and may not be dependent upon preliminary formation of folic acid before conversion to the active coenzyme.

On the basis of growth and production of hemoglobin, vitamin $\mathrm{B}_{\mathrm{c}}$ conjugate (pteroylheptaglutamic acid) administered in the diet is approximately 60-65 per cent as active on a molar basis as folic acid for chicks. ${ }^{12}$ It has been reported that livers of day-old chicks from eggs of hens maintained on a diet containing no animal protein are almost devoid of pteroylheptaglutamic acid conjugase; however, vitamin $\mathrm{B}_{12}$ and 4-pyridoxic acid are reported to be synergistic in producing a marked increase in the conjugase activity of preparations from such livers. ${ }^{93}$

Also of interest is the demonstration of pteroylheptaglutamic acid conjugase in the blood of turkey poults as well as other animals, including human beings. ${ }^{94}$

No hematologic responses have been noted after administration of either pteroic acid or $\mathrm{N}^{10}$-formylpteroic acid (rhizopterin) to patients with pernicious anemia or nutritional macrocytic anemia. ${ }^{53,95}$ These compounds are also inactive in replacing folic acid for other animals as indicated in Table 18. A slight response has been reported for $\mathrm{N}^{10}$-formylpteroic acid in replacing folic acid for Tetrahymena gelii $\mathrm{W}{ }^{64}$ Both the formyl derivative and pteroic acid have only very slight growthpromoting effect on Lactobacillus casei; ${ }^{16,24}$ however, the formyl derivative is just as effective as folic acid in the nutrition of Streptococcus faecalis R. ${ }^{16}$ Depending upon the time of incubation, the activity of pteroic acid approaches that of folic acid for this organism. ${ }^{65}$ Streptococcus faecalis 732, Streptococcus faecalis F24, Streptococcus zymogenes $5 \mathrm{C} 1$ and Streptococcus durans $98 \mathrm{~A}$ are also able to utilize either folic acid or formylpteroic acid (rhizopterin) ${ }^{96}$ Suspensions of resting cells of these organisms as well as Streptococcus faecalis $\mathrm{R}$ convert formylpteroic acid to folic acid or an analogous substance. ${ }^{96}$ Streptococcus faecalis S108 A, 
Lactobacillus bulgaricus 05 , and Lactobacillus delbruckii LD50 require folic acid for growth, but cannot utilize formylpteroic acid. ${ }^{96}$

Formylfolic acid, which is utilized as effectively as folic acid by Streptococcus faecalis $\mathrm{R}$ and Lactobacillus case $i,{ }^{37}$ produces reticulocytosis and increases the hemoglobin and the number of red and white blood cells and platelets in pernicious anemia patients, but is reported to be less active than folic acid when administered orally. ${ }^{53}$

$\mathrm{N}$-[p-(4-Quinazolyl) benzoyl] glutamic acid is reported to be approximately 1 to 10 per cent as active as folic acid in stimulating the growth of Streptococcus faecalis R. However, the results indicate that considerable growth was obtained in the absence of exogenous folic acid, at least one-half that which was obtained by addition of either folic acid or the analogue. ${ }^{97}$ The possibility of a sparing action of the analogue on folic acid cannot be excluded on the basis of the data presented; however, analogues of vitamins can, in many instances, carry out the metabolic functions of the vitamins. This quinazolyl analogue does not produce a hematologic response in patients with pernicious anemia. ${ }^{53}$

$\mathrm{N}^{10}$-Methylpteroylglutamic acid, which is inhibitory to some organisms (p. 580), replaces folic acid in stimulating the virus causing the Rous sarcoma in folic acid-deficient chicks. ${ }^{77}$

An x-methylfolic acid (p. 575), 2-desamino-2-hydroxypteroic acid, 2-desamino-2-hydroxypteroylglutamic acid, and pteroylaspartic acid are all inactive clinically in treatment of the anemias responding to folic acid. ${ }^{53}$

The diamide of folic acid neither increases the urinary folic acid concentrations in human subjects nor appreciably stimulates the growth of Lactobacillus casei or Streptococcus faecalis R.38 However, the methyl ester of folic acid is approximately 10 per cent as active as the free acid for Lactobacillus casei, ${ }^{13}$ but probably somewhat less active for Streptococcus faecalis R. ${ }^{19}$

Xanthopterin and Related Pterins. The hemopoietic effect of xanthopterin in alleviating the anemia resulting from the maintenance of rats on a diet of goat's milk was reported almost a decade before the structure of folic acid was known. ${ }^{98}$ Xanthopterin has also been reported to relieve the anemia produced by feeding a high-protein diet to fingerling Chinook salmon. ${ }^{99}$ Treatment of cytopenic monkeys maintained on a vitamin M-deficient diet with synthetic xanthopterin gave a reticulocyte response and increased the number of red and white blood cells. ${ }^{100}$ The growth inhibition and leucopenia in rats maintained on a purified diet deficient in folic acid and containing succinylsulfathiazole has been reported to respond partially to xanthopterin. ${ }^{101}$ Attempts to reproduce these results have been only partially successful; however, incubation of rat livers 
with xanthopterin increases the production of microbiologically active forms of folic acid. ${ }^{75}$

The leucopenia and anemia in rats resulting from the action of sulfathiazole is reported to be alleviated by xanthopterin, folic acid, or a substance termed vitamin $\mathrm{B}_{14}$, which increases cell proliferation in a beef bone marrow suspension but inhibits proliferation of a suspension of Brown Pearce rabbit tumor cells. ${ }^{102}$ Vitamin $\mathrm{B}_{14}$, isolated from human urine, is much more effective than xanthopterin; yet the activity of both xanthopterin and folic acid is reportedly increased by incubation with xanthine oxidase from milk, or with gastric mucosa of rats. 2-Amino-4hydroxy-7-methylpteridine counteracts the effects of both xanthopterin and vitamin $\mathrm{B}_{14} \cdot{ }^{102,103}$

Since xanthopterin is inactive in treatment of pernicious anemia ${ }^{95}$ and does not replace folic acid for chicks, ${ }^{104,105}$ the relationship between xanthopterin and folic acid in the rat is somewhat obscure. The possibility that this and related pterins may prevent the metabolism and loss of folic acid in enzymatic reactions not involved in hemapoiesis and growth must also be considered. This is further emphasized by the ability of a number of pterins and related substances to exert a beneficial action in chicks on a folic acid-deficient diet. Thus, fed at $20 \mathrm{mg}$ per $100 \mathrm{~g}$ diet over a four week period the following compounds gave on occasion what appeared to be significant growth stimulation of chicks: ${ }^{105}$ 2,4-dihydroxy-6,7-dicarboxypteridine, 2-mercapto-4-hydroxy-7-carboxypteridine, 2-amino-4hydroxy-7-carboxypteridine, 2-amino-4,6-dihydroxy-7-carboxypteridine, 2,4-dihydroxy-6-(or 7) -hydroxy-7 (or 6)-carboxymethylpteridine, 2-amino-4-hydroxy-6 (or 7) -hydroxy-7 (or 6) -methylpteridine, 2,4-dihydroxy-6 (or 7)-hydroxy-7 (or 6)-methylpteridine, bisalloxazine, 6-amino-2,4,8-trihydroxypyrimido (4,5-e) pteridine, 2,6-dihydroxy-4,5-diaminopyrimidine, and alloxan under conditions of suboptimal concentrations of folic acid. Of these compounds, only 2-amino-4-hydroxy-7-carboxypteridine and 2-mercapto-4-hydroxy-6,7-dicarboxypteridine under similar conditions stimulated hemoglobin formation. 2,4-Diamino-6,7-dicarboxypteridine, 2,4-diamino-7-carboxypteridine, 2,4-dihydroxy-7-carboxypteridine, and 2-mercapto-4-hydroxy-7-carboxypteridine were also found to stimulate, under certain conditions, hemoglobin formation in chicks on suboptimal concentrations of folic acid. ${ }^{105}$ The presence of folic acid in suboptimal amounts is essential for the response of all these factors. ${ }^{105}$ With 2-amino4-hydroxypteridine, 2,4-diaminopteridine, 2-amino-4-hydroxy-6,7-dicarboxypteridine, 2,4-diamino-6,7-dimethylpteridine, 2-amino-4-hydroxy-7methylpteridine, 2,4-diamino-7-methylpteridine, or 2,4-diamino-6,7diphenylpteridine, no stimulatory effects were noted on either growth or hemoglobin formation. ${ }^{105}$ 


\section{Inhibitory Analogues of Folic Acid}

The first synthetic inhibitory analogue of folic acid was reported by Martin, Tolman and Moss, ${ }^{106}$ who found that a D-methylfolic acid, prepared from 2,4,5-triamino-6-hydroxypyrimidine, $\alpha, \beta$-dibromobutyraldehyde and $\mathrm{N}$-( $p$-aminobenzoyl)-D-glutamic acid, competitively prevented the utilization of folic acid by Streptococcus faecalis R. Subsequently, numerous synthetic analogues of folic acid, which prevent the biological functions of the vitamin, have been reported. These inhibitory analogues represent a variety of modifications of the vitamin; some of the more active inhibitors are modifications in which a methyl group has been placed in the pteroyl radical, an amino group has replaced the 4-hydroxyl group of the pteridine ring, a substituent group has been placed at the $\mathrm{N}^{10}$-position, or the glutamic acid moiety has been replaced or, in case of modified pteroic acids, omitted. A group of pterins-particularly 2,4diaminopteridines, with only slight structural resemblance to folic acid in contrast to other folic acid analogues-have been reported to be effective antagonists of folic acid for a number of organisms, including some which synthesize folic acid.

\section{Substituted Folic, Pteroic and Pteroylaspartic Acids}

Inhibitory analogues of folic acid which can be classed as substituted folic, pteroic, or pteroylaspartic acids are listed with inhibition indices in Table 19.

$x$-Methylfolic Acids. The condensation product from $\alpha, \beta$-dibromobutyraldehyde, 2,4,5-triamino-6-hydroxypyrimidine, and $\mathrm{N}$-( $p$-aminobenzoyl)-D-glutamic acid inhibits the growth of Streptococcus faecalis $\mathrm{R}$, and the toxicity is competitively prevented by folic acid. The inhibition index is approximately $150 .{ }^{106}$ Although no evidence has been presented on the exact structure of this antagonist, it has been designated as 7-methylfolic acid. ${ }^{106}$

A similar product was reported which was derived from $\mathrm{N}$ - $(p$-aminobenzoyl)-L-glutamic acid and is considerably more inhibitory for Streptococcus faecalis R. ${ }^{107,108}$ The inhibition index for this $\mathrm{L}-x$-methylfolic acid is approximately 20 to $30 .{ }^{107}$ The product also inhibits the utilization of folic acid by Lactobacillus casei at an inhibition index of 100 to $1000 . .^{107,109}$

Either L- $x$-methylfolic acid or sulfathiazole $(1$ to $10 \mathrm{mg}$ ) prevents the growth of Staphylococcus aureus 209 in a bouillon medium. The toxicity of the methylfolic acid is reported to be prevented by relatively high concentrations (1-10 mg) of $p$-aminobenzoic acid, folic acid, pteroic acid or sulfathiazole, whereas the toxicity of sulfathiazole is prevented only by $p$-aminobenzoic acid and pteroic acid. ${ }^{109 b}$

Administration of this analogue to a wide variety of organisms results 


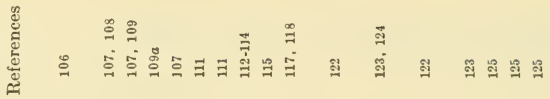

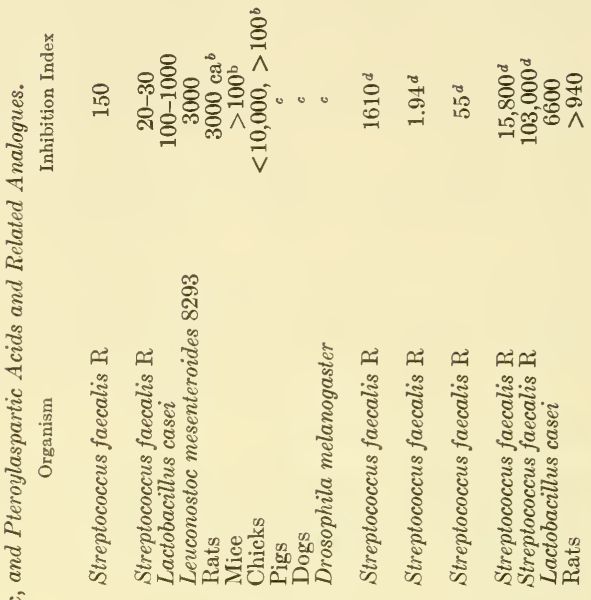

$\frac{0}{3}$

:

चु

i

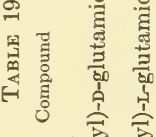

응

ज़

익

운융

록

屯ै है워

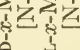

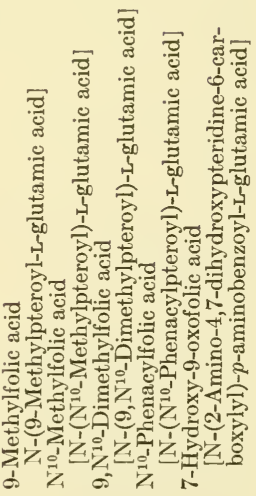




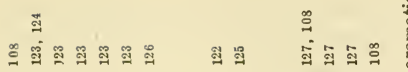

हू.

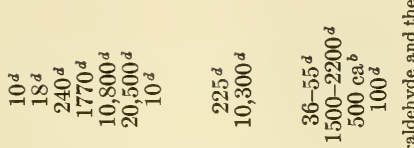

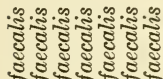

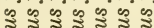

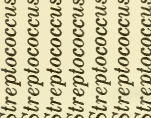

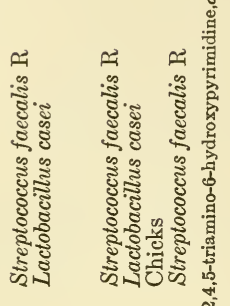

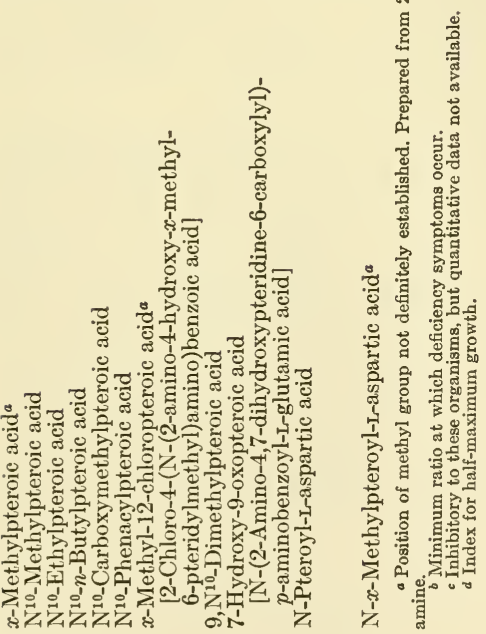

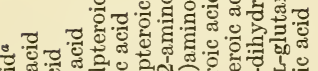

๘.0

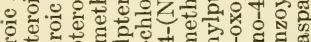

a

$$
\text { ํㅠㄴ }
$$

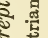

ส 
in the appearance of symptoms characteristic of folic acid deficiency ( $p$. 413). Thus, L- $x$-methylfolic acid inhibits competitively the utilization of folic acid in rats fed a purified diet supplemented with succinylsulfathiazole. ${ }^{107}$ The analogue-folic acid ratio at which the deficiency symptoms appear is approximately 3000 . The deficiency symptoms produced at this ratio and more acutely at higher ratios of analogue to vitamin include reduced rate of growth, lowered hemoglobin values, and pronounced reduction of the white cell count, with a greater reduction in granulocytes than in lymphocytes. Inanition and severe diarrhea develop, the fur becoming rough and unkempt in appearance. A red pigment accumulates about the vibrissae, and necrotic and ulcerative changes are produced in the oral cavity, particularly in animals which die. The oral lesions produced with the antagonist are usually not characteristic of a folic acid deficiency in rats, but are produced by folic acid deficiency in the monkey. ${ }^{110}$ In such animals, a general necrotic condition within the mouth and inflamed lungs with considerable congestion are noted. The gastrointestinal tracts of most animals are empty and atonic; the livers, spleens, hearts and other organs except the genital system are normal in size and appearance, but uteri are always small and atrophic. In the bone marrow, the maturation of cells of the erythroid series and of granulocytes are seriously impaired. If animals are treated with sufficient folic acid just prior to the expected appearance of the terminal moribund state, which usually occurs within one to two weeks after the onset of the syndrome, the rats recover rapidly and appear normal within four weeks. After recovery, the only abnormal effect is an enlarged spleen, which in some cases is four times the normal size. ${ }^{107}$

Although female mice on a purified basal diet containing succinylsulfathiazole do not develop deficiency symptoms within six weeks, supplementation of the diet with crude L- $x$-methylfolic acid (10 g per $\mathrm{kg}$ ) results in death of a majority of the animals within six weeks, and the surviving animals are left in a moribund condition. ${ }^{111}$ These surviving animals are emaciated but do not have the chromodacryorrhea, ruffled fur, and other characteristics of rats similarly treated. The livers are yellow, though normal in size and texture, but the uteri are atrophic. In contrast to the rat, in which the reduction of the granulocyte count is more pronounced, the cells of both the myeloid and lymphoid series are inhibited to almost the same extent by methylfolic acid in the mouse. Since complete protection against the analogue is afforded by $0.1 \mathrm{~g}$ of folic acid for each $10 \mathrm{~g}$ of crude inhibitor, the inhibition index is greater than $100 . .^{111}$

Chicks on a folic acid-deficient diet develop symptoms which include slow growth, poor feathering and low hemoglobin content of the blood. 
These symptoms are prevented by folic acid, but are aggravated by L- $x$ methylfolic acid, which prevents the utilization of folic acid. ${ }^{111}$ The inhibition index is somewhat lower than 10,000 . The symptoms of deficiency resulting from $1 \mathrm{~g}$ of the inhibitor per $\mathrm{kg}$ of diet are completely prevented by $10 \mathrm{mg}$ of folic acid per $\mathrm{kg}$ of diet. ${ }^{111}$

$\mathrm{L}$ - $x$-Methylfolic acid ( $1 \mathrm{~g}$ per $\mathrm{kg}$ of diet) administered to pigs on a purified diet deficient in folic acid and containing succinylsulfathiazole causes the development of deficiency symptoms characterized by severe anemia, profuse diarrhea, diminished appetite, decreased growth rate, some loss of hair and unkempt appearance. ${ }^{112}$ The formation of erythrocytes and granulocytes is inhibited. Adequate amounts of folic acid prevent toxicity of the analogue. Administration daily of the extrinsic factor for pernicious anemia (p. 415) derived from $100 \mathrm{~g}$ of crude casein together with 80 to $150 \mathrm{cc}$ of fresh neutralized human gastric juice resulted in improved appetite in one pig, and both growth and hematopoiesis were initiated and continued for many weeks after cessation of therapy.112 Liver extracts corresponding to those used in treatment of pernicious anemia also allow remissions, ${ }^{113}$ but the pig apparently cannot be maintained indefinitely without supplementation of folic acid. ${ }^{113}, 114$ While the inhibitory effect of the antagonist can be modified to some extent by these extracts, pigs receiving $2 \mathrm{~g}$ of the analogue per $\mathrm{kg}$ of a diet which contained adequate quantities of extrinsic factor respond only partially, if at all, to liver extract; however, administration of folic acid to such animals allows rapid relief of the anemia.114 The antipernicious anemia principle of liver does not appear to be present in normal amounts in the liver of pigs with remissions induced by folic acid, even with simultaneous feeding of extrinsic factor. ${ }^{113}$

Although dogs appear normal on a purified diet with folic acid omitted, the animals on the diet supplemented with L- $x$-methylfolic acid develop deficiency symptoms characterized by slow growth, or loss in weight, emaciation, alopecia, anemia and ulceration of the skin. Liver extract gives only a slight hemopoietic response, but administration of sufficient folic acid prevents the toxic effects of the analogue and causes marked responses in the deficient animals. ${ }^{115}$ Rhesus monkeys appear to be resistant to the $\mathrm{L}$ - $x$-methylfolic acid. ${ }^{116}$

The development of the larvae of Drosophila melanogaster in a synthetic medium containing growing yeast is arrested by supplementing the medium with $\mathrm{L}$ - $x$-methylfolic acid. ${ }^{117,118}$ In medium containing 0.25 per cent of the analogue, only 0.53 per cent of the larvae survived, and at a concentration of 1 per cent, all the larvae died. Supplementation of the medium containing 1 per cent of antagonist with an adequate quantity 
of folic acid increases the adult emergence to 64 per cent. Controls in the absence of the analogue showed a survival value of 74 per cent.117, 118

Estrogen-induced growth responses of the genital tract of the chick is prevented by L- $x$-methylfolic acid at a concentration of 1 per cent in the diet. ${ }^{119}$ The inhibitory effect is completely prevented by injection of $4 \mathrm{mg}$ of folic acid four times daily. ${ }^{119}$ It is interesting that such estrogen-induced growth is not obtained in folic acid-deficient chicks and monkeys. ${ }^{120}$

Addition of L- $x$-methylfolic acid (50 $\gamma$ per ce) to human blood cell cultures in a medium containing 30 to 35 per cent human umbilical cord serum in a balanced salt solution results in marked erythrophagocytosis by granulocytes, as compared with blood cell cultures in the absence of the analogue. ${ }^{121}$ Addition of folic acid ( $5 \gamma$ per cc) prevents the effect of the analogue. A maturation arrest in the erythroid series does not develop under these conditions. ${ }^{121}$

Other Methylfolic Acids. Effects similar to those of $x$-methylfolic acid have been noted recently with 9 -methylfolic acid, which competitively inhibits the utilization of folic acid for rats, mice and chicks. ${ }^{116}$<smiles>CC(Nc1ccc(C(=O)NC(CCC(=O)O)C(=O)O)cc1)c1cnc2nc(N)nc(O)c2n1</smiles>

9-methylfolic acid [N-(9-methylpteroyl)-L-glutamic acid]

However, $\mathrm{N}^{10}$-methylfolic acid is almost a thousand times as effective as 9 -methylfolic acid in competitively preventing the functions of folic acid in Streptococcus faecalis $\mathrm{R}$ (Table 19). The $\mathrm{N}^{10}$-methylfolic acid, in contrast to other analogues of folic acid, is capable of stimulating the growth of the Rous sarcoma in folic acid-deficient chicks. ${ }^{77}$ The $\mathrm{N}^{10}$ methyl analogue also has an effect analogous to L- $x$-methylfolic acid, causing marked erythrophagocytosis by granulocytes in human blood cell cultures. ${ }^{121}$<smiles>CN(Cc1cnc2nc(N)nc(O)c2n1)c1ccc(C(=O)NC(CCC(=O)O)C(=O)O)cc1</smiles>

$N^{10}$-methylfolic acid $\left[N-\left(N^{10}-\right.\right.$ methylpteroyl $)-\mathrm{L}-$ glutamic acid $]$ 
The analogue with a combination of two methyl groups at the 9-and $\mathrm{N}^{10}$-positions $\left(9, \mathrm{~N}^{10}\right.$-dimethylfolic acid) is considerably more effective than 9-methylfolic acid in inhibiting the growth of Streptococcus faecalis $\mathrm{R}$, but is less effective than $\mathrm{N}^{10}$-methylfolic acid.

Substituted Pteroic Acids and Related Compounds. A larger group, such as phenacyl, in the $\mathrm{N}^{10}$-position appears to be detrimental to the inhibitory activity of an analogue. This is indicated more clearly in the pteroic acid series of $\mathrm{N}^{10}$-derivatives, which become progressively less effective as antagonists of folic acid for Streptococcus faecalis $\mathrm{R}$ as the size of the substituent group increases from the $\mathrm{N}^{10}$-methyl (Table 19).

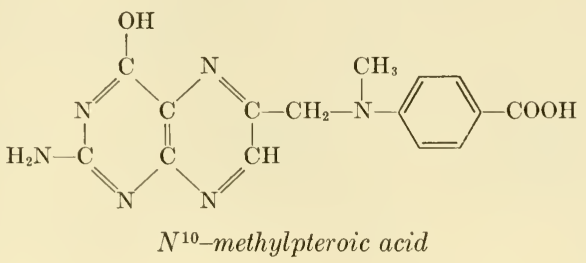

The pteroic acid analogues are as a general rule less effective than the corresponding folic acid analogues. Although the data in Table 19 indicate that $x$-methylpteroic acid approaches the activity of $x$-methylfolic acid, it is only 10 to 20 per cent as effective as $x$-methylfolic acid in preventing the utilization of folic acid by Streptococcus faecalis $\mathrm{R}$ when the two analogues are tested under identical conditions. ${ }^{108,126}$ The chloromethylpteroic acid prepared from 4-amino-2-chlorobenzoic acid, $\alpha, \beta$ dibromobutyraldehyde and 2,4,5-triamino-6-hydroxypyrimidine is approximately as inhibitory as $x$-methylfolic acid. It is interesting that 4-amino-2-chlorobenzoic acid can replace $p$-aminobenzoic acid in this folic acid analogue without appreciably altering its inhibitory action, and can also inhibit the utilization of $p$-aminobenzoic acid by Escherichia coli (p. 524).<smiles>Nc1nc(O)c2nc(C(=O)Nc3ccc(C(=O)NC(CCC(=O)O)C(=O)O)cc3)c(O)nc2n1</smiles>

7-hydroxy-9-oxofolic acid $[N-(N-(2-$ amino-4,7-dihydroxypteridine-6-carboxylyl)-p-aminobenzoyl)-L-glutamic acid] 
7-Hydroxy-9-oxofolic acid (2-amino-4,7-dihydroxypteridine-6-carboxylyl- $p$-aminobenzoylglutamic acid), which is more effective as an inhibitor of growth of Lactobacillus casei than of Streptococcus faecalis R, contrasts with a number of the other analogues listed in Table 19 which are more effective in preventing growth of Streptococcus faecalis $\mathrm{R}$. Whereas $1 \mathrm{mg}$ per day of this compound injected intraperitoneally is tolerated by rats weighing approximately $125 \mathrm{~g}$, a single dose of $10 \mathrm{mg}$ is fatal within 24 hours. Simultaneous administration of $10 \gamma$ of folic acid prevents the toxicity of the analogue.

An isomer of the 7-hydroxy-9-oxofolic acid with the 6- and 7-substituents exchanged (2-amino-4,6-dihydroxypteridine-7-carboxylyl- $p$-aminobenzoylglutamic acid) is less than one-third as active as the isomer with the general structure corresponding to folic acid.

Pteroylaspartic Acids. N-Pteroyl-L-aspartic acid inhibits the growth of both Lactobacillus casei and Streptococcus faecalis R, as indicated in Table 19. If pteroic acid, pteroyl- $\gamma$-glutamylglutamic acid, or pteroyldi- $\gamma$-glutamylglutamic acid are employed in place of folic acid for Strep-<smiles>Nc1nc(O)c2nc(CNc3ccc(C(=O)NC(CC(=O)O)C(=O)O)cc3)cnc2n1</smiles>

$N$-pteroyl-L-aspartic acid

tococcus faecalis $\mathrm{R}$, the inhibition indices for half maximum growth are 2-3, 4.2-17.2 and 0.21-0.32, respectively. Folic acid with an index of 37-55 prevents the toxicity of the inhibitor most effectively; the triglutamate is least effective.

A marked decrease in growth rate and decreased hemoglobin levels are observed in chicks receiving the inhibitor, which causes these deficiency symptoms to appear when administered at 500 times the concentration of folic acid.

Administration of the inhibitor to rats at concentrations up to.1.5 mg per day did not cause any significant decrease in growth rate or the appearance of deficiency symptoms even with animals receiving carboxysulfathiazole.

$\mathrm{N}$-( $x$-Methylpteroyl)-L-aspartic acid is somewhat less effective than pteroylaspartic acid in preventing the utilization of folic acid by Streptococcus faecalis $\mathrm{R}$. 


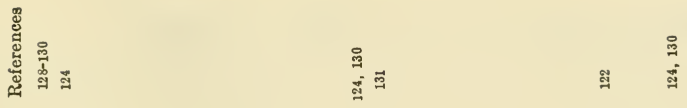

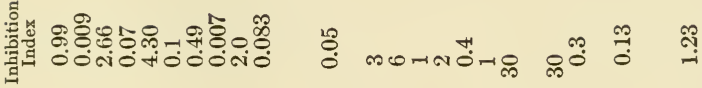

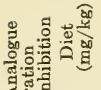
잇ำ

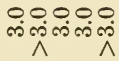
$<$

1.

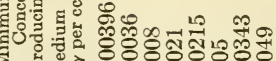

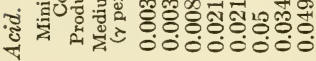

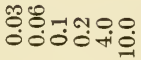

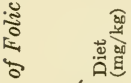

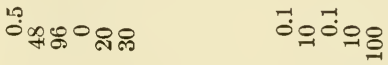

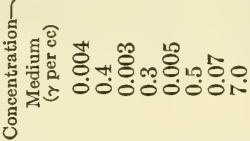

$\stackrel{\overrightarrow{0}}{\overrightarrow{0}} \overrightarrow{0} \stackrel{0}{0}$

$\ddot{\circ} \quad \overrightarrow{0}$

寻

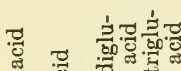

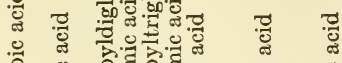

:

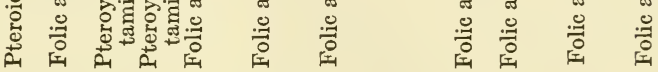

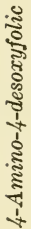

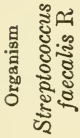

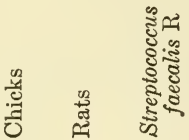

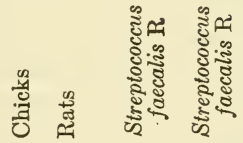

ลิ่
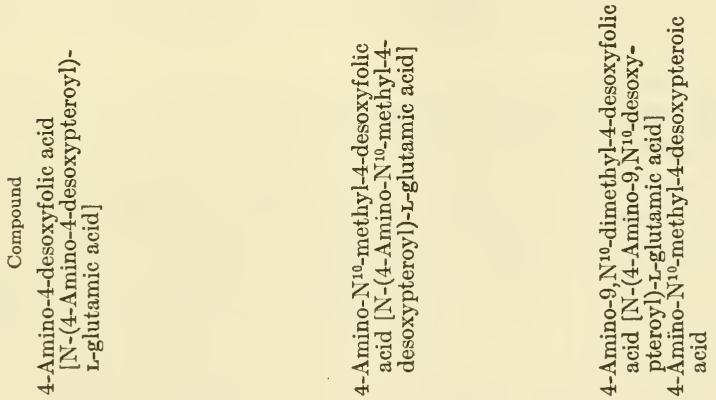


\section{4-Amino-4-desoxyfolic Acid and Related Analogues}

A series of synthetic analogues of folic acid which have an amino group in place of the 4-hydroxyl of the pteridyl moiety of folic acid are characterized by very potent inhibitory activities; with few exceptions these either are not prevented or are prevented only to a limited extent by folic acid. The analogues are extremely toxic for a majority of the organisms which require folic acid, and cause symptoms which are characteristic of folic acid deficiency even though folic acid does not in many instances show appreciable ability to prevent the toxic manifestations. The biological activities of analogues of this type are indicated in Table 20.

4-Amino-4-desoxyfolic Acid [N-(4-Amino-4-desoxypteroyl-L-glutamic Acid]. From Table 20, it is apparent that 2-amino-4-desoxyfolic acid inhibits markedly the growth of Streptococcus faecalis R; however, the various members of the folic acid group have very little effect on the amount of inhibitor necessary to prevent growth of the organism. ${ }^{129}$ Only

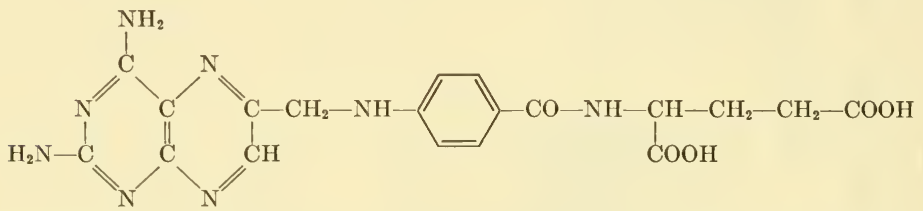

4-amino-4-desoxyfolic acid [ $\mathrm{N}$-(4-amino-4-desoxypteroyl)-L-glutamic acid]

about a three-fold increase in the concentration of the analogue is necessary to obtain the same degree of growth inhibition when the folic acid concentration is increased one hundred-fold. Growth stimulated by pteroic acid is most susceptible to the inhibitor, and no effect on the toxicity is noted by increasing one hundred-fold the concentration of ptéroic acid. ${ }^{129}$

The toxicity of the analogue becomes apparent in chicks at a concentration of $1 \mathrm{mg}$ per $\mathrm{kg}$ of diet containing $0.5 \mathrm{mg}$ of folic acid. At $5 \mathrm{mg}$ per $\mathrm{kg}$ of the diet, the 4-amino analogue is lethal for all the chicks. ${ }^{129}$ The onset of the symptoms is rapid in comparison with dietary depletions. To prevent the lethal effects of $4 \mathrm{mg}$ of analogue per $\mathrm{kg}$ of diet, approximately $96 \mathrm{mg}$ of folic acid per $\mathrm{kg}$ of diet are required. Intermediate concentrations of folic acid permit partial survivals, but there is little inhibition of growth of chicks surviving sub-lethal concentrations of the analogue. ${ }^{129}$

A similar situation exists with rats. As little as $10 \gamma$ per day or $1 \mathrm{mg}$ 
per $\mathrm{kg}$ of diet is fatal to all the animals. The symptoms of the toxicity include severe watery diarrhea, resulting in extreme dehydration, loss of weight, porphyrin-stained whiskers and hemoconcentration. Signs of nervous involvement and anoxia develop prior to death, and extensive tissue changes, including intestinal lesions and a very hypoplastic bone marrow, are observed. ${ }^{129}$ In the peripheral blood, marked granulocytopenia and reticulocytopenia and a moderate lymphopenia develop simultaneously. ${ }^{132}$ Very high concentrations of pteroylglutamic acid $(20-30$ $\mathrm{mg}$ per $\mathrm{kg}$ of diet) are required to prevent the effects of even the minimum lethal concentration. The folic acid content of the liver appears to be decreased as indicated by microbiological assay. ${ }^{133}$ The $\mathrm{LD}_{50}$ of the analogue for rats receiving single doses is $4.5 \pm 1.4 \mathrm{mg}$ per $\mathrm{kg}{ }^{132}$ Regardless of the size of the dose above the minimum lethal dose, the course of the fatal intoxication is not altered. The animals are unaffected for approximately one day, but all fatalities usually occur on the third or fourth day. Oral administration is as effective as parenteral injection, and fractional doses are cumulative and are possibly more effective than a single dose. Animals surviving the minimum lethal dose have a transient retardation in growth with subsequent rapid recovery.

At a concentration of $0.3 \mathrm{mg}$ per $\mathrm{kg}$ of diet, 4-amino-4-desoxyfolic acid tends to lower slightly the hemoglobin and white cell count of mice. ${ }^{134}$ The effect is prevented by high concentrations of folic acid, but the high mortality of mice fed $1 \mathrm{mg}$ of the analogue per $\mathrm{kg}$ of diet is not affected by folic acid even at high concentrations (100 mg per $\mathrm{kg}$ of diet). Gross examination at autopsy revealed no lesions. ${ }^{138}$ The $\mathrm{LD}_{50}$ for mice receiving a single dose of the analogue is $1.9 \pm 0.3 \mathrm{mg}$. per $\mathrm{kg} .{ }^{132}$ Fractional doses were cumulative and almost as toxic as the single dose. The $\mathrm{LD}_{50}$ can be increased several fold by repeated administration of folic acid (47 mg per $\mathrm{kg}$ daily) or pteroyltriglutamic acid (500 mg per $\mathrm{kg}$ daily) before and after administration of the 4-amino analogue. Neither thymine nor refined liver extract has such an effect on the toxicity of the analogue. ${ }^{132}$

Guinea pigs given daily subcutaneous injections of 0.5 to $5.0 \mathrm{mg}$ of 4-amino-4-desoxy folic acid lose weight and with few exceptions die within 11 to 28 days. ${ }^{135}$ The symptoms of the toxicity noted include normocytic anemia, leucopenia, agranulocytosis, thrombocytopenia and hypoplasia of the bone marrow. Although refined liver extract does not prevent the anemia or leucopenia, folic acid in concentrations 25 to 100 times that of the analogue appears to prevent the development of leucopenia or thrombocytopenia, but not the anemia. ${ }^{135}$

The 4-amino analogue administered to dogs in daily doses of 0.05 to $0.1 \mathrm{mg}$ per $\mathrm{kg}$ of body weight is fatal to approximately half the animals 
within ten days. ${ }^{136}$ The effects of the analogues are similar to those in other animals and include: hemorrhagic diarrhea, weight loss, leucopenia, hemoconcentration and degeneration of the bone marrow. Erythropoiesis and myelopoiesis are inhibited. Lymphopoiesis is less affected. ${ }^{136}$

Monkeys are susceptible to the inhibitory action of 4-amino-4-desoxyfolic acid, in contrast to the effect of L- $x$-methylfolic acid. ${ }^{116}$

The characteristic growth response to estrogens of the genital tracts of female chicks ${ }^{137}$ and rats ${ }^{137}$ and newly metamorphosed frogs ${ }^{138}$ (Rana clamitans) is inhibited by 4-amino-4-desoxyfolic acid. The decrease in the response of the female frog oviducts to estradiol resulting from administration of the amino analogue is not affected by supplemental folic acid even at 100 times $(5 \mathrm{mg}$ ) the concentration of the analogue $(0.05 \mathrm{mg}) .{ }^{138}$ High concentrations of folic acid in both the chick and rat tend to prevent the effect of the analogue. ${ }^{137}$

4-Amino-4-desoxyfolic acid also prevents the development of larvae of Drosophila melanogaster in a synthetic medium containing growing yeast. Folic acid does not appear to prevent this inhibition. ${ }^{117}, 118$

The analogue also causes a marked erythrophagocytosis by granulocytes in human blood cell cultures. ${ }^{121}$

Marked effects of the 4-amino analogue on the blood islets in 6- to 8 -day old chick embryos have been reported to be altered by folic acid, but not by either refined liver extract or vitamin $\mathrm{B}_{12} \cdot{ }^{139}$

Liver tissue from rats depleted of folic acid on a succinylsulfathiazole diet has a decreased ability to oxidize tyrosine as compared with liver tissue from normal animals. ${ }^{139 a}$ The addition of folic acid in vitro partially restores the ability to oxidize tyrosine, but neither liver extract nor pteroylheptaglutamate has this effect. Liver tissue from rats fed 4-amino-4-desoxyfolic acid is similarly deficient in its ability to oxidize tyrosine, but no effect on the system is obtained in vitro with folic acid, vitamin $\mathrm{B}_{12}$, or liver extract. The administration of folic acid or refined liver extract to the rat prevents the effects of the analogue on the ability of the liver to oxidize tyrosine. The analogue does not inhibit the oxidation of tyrosine by liver slices in vitro. ${ }^{139 a}$

4-Amino-4-desoxy- $\mathrm{N}^{10}$-methylfolic Acid [N-(4-Amino-4-desoxy- $\mathrm{N}^{10}$ - metl1ylpteroyl)-L-glutamic Acid]. In contrast to the effects of 4-amino-4desoxyfolic acid, the toxicity of 4-amino-4-desoxy- $\mathrm{N}^{10}$-methylfolic acid for Streptococcus faecalis $\mathrm{R}$ is prevented competitively by folic acid, as indicated in Table $20 .{ }^{131}$ However, folic acid has little ability to prevent the toxicity of the analogue for rats. ${ }^{131}$ Animals receiving sub-lethal amounts of 4-amino-4-desoxy- $\mathrm{N}^{10}$-methylfolic acid have normal growth; and except for a little alopecia and occasional but never severe chromodacryorrhea, anemia and leucopenia, the animals do not show symptoms 
characteristically caused by other analogues. At $3 \mathrm{mg}$ per $\mathrm{kg}$ of diet, the analogue is fatally toxic to all the rats, moderate anemia, leucopenia and granulocytopenia developing shortly before death. This fatal effect of the minimum lethal concentration is prevented only by high concentrations of folic acid (100 mg per $\mathrm{kg}$ of diet). Gross examination at autopsy does not reveal the usual lesions and pathology observed with other folic acid analogues. The viscera are normal in appearance, with no lesions or

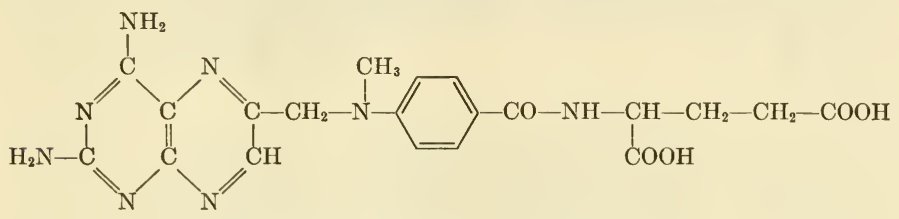

\section{4-amino-4-desoxy-N $\mathrm{N}^{10}$-methylfolic acid}

pathological changes other than a slight enteritis. Animals administered lethal amounts of the analogue seldom died before the fifth or sixth day, but all the animals susceptible to that concentration died within the second week. Animals surviving beyond the second week after administration of the analogue were not subsequently affected. ${ }^{131}$

It is interesting that different patterns of symptoms are obtained with various analogues of folic acid.

A slight growth-retarding effect of 4-amino-4-desoxy- $\mathrm{N}^{10}$-methylfolic acid (3 $\mathrm{mg}$ per $\mathrm{kg}$ of diet containing $0.1 \mathrm{mg}$ of folic acid) on chicks is prevented by folic acid (10 $\mathrm{mg}$ per $\mathrm{kg}$ of diet). ${ }^{131}$

The analogue also causes marked cytological changes in the blood islets of 6- to 8-day old chick embryos. Folic acid, but neither liver extracts nor vitamin $B_{12}$, alters this effect. ${ }^{139}$

Other 2,4-Diaminopteridyl Analogues of Folic Acid. Both 4-amino-4desoxypteroic acid and 4-amino-9, $\mathrm{N}^{10}$-dimethyl-4-desoxyfolic acid are effective inhibitory analogues of folic acid for Streptococcus faecalis R, as indicated in Table 20.122, 124, 130 4-Amino-4-desoxypteroyl-L-aspartic acid has some activity in inhibiting neoplastic growth (p. 593). A preliminary report indicates that the aspartic analogue is toxic for mice, rats and dogs, and produces pathologic changes similar to those caused by the other 4 -amino-4-desoxypteroyl derivatives. ${ }^{140 a}$

\section{Substituted Pteridines and Pyrimidines}

2,4-Diamino- and Related Pteridines. The first indication of the great affinity of certain 4-aminopteridines for enzymes related to the utilization of folic acid in biological systems was reported by Daniel, et al. ${ }^{140}$ They found that a group of 2,4-diaminopteridines synthesized by 
递

$\Xi \Xi \Xi \quad \Xi$

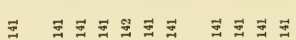

3

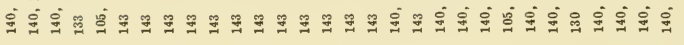

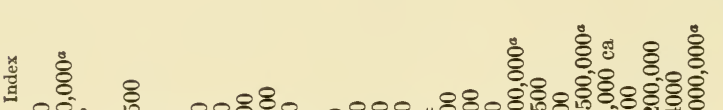

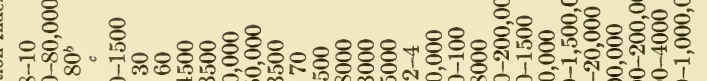

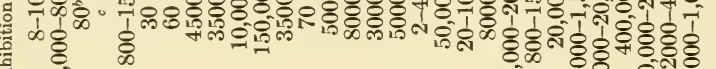

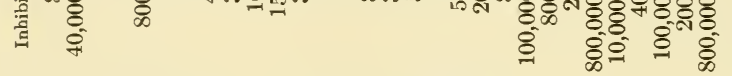

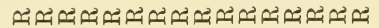

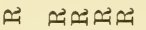

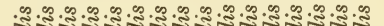

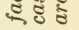

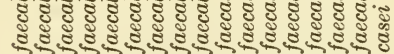

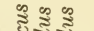
क क क क क क क क क क क क क क क क क क क क

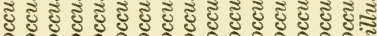

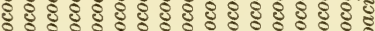
S:

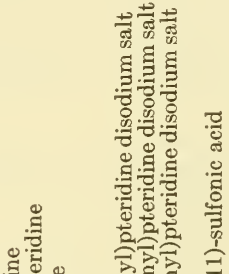

-

它 引 ๖.

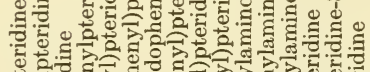

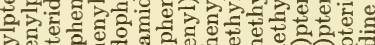

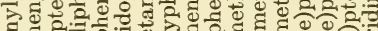

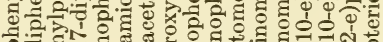

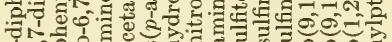

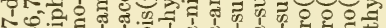
०० 1.0.

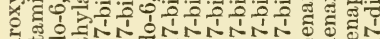

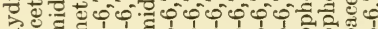
건 गे ङ

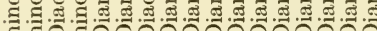

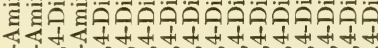

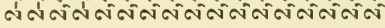


Mallette, Taylor and Cain ${ }^{141}$ was extremely effective in inhibiting the growth of Streptococcus faecalis $\mathrm{R}$. The effects of these pteridines and related compounds on a number of organisms are indicated in Table 21. The inhibitory effects of these pteridines are prevented in a competitive manner by folic acid, particularly for Streptococcus faecalis $\mathrm{R}$. The most effective inhibitors of these pteridines are those with aromatic substituents in the 6,7-positions, e.g., 2,4-diamino-6,7-diphenylpteridine, 2,4diaminophenanthro $(9,10$-e) pteridine and 2,4-diaminoacenaphtho(1,2-e)pteridine.

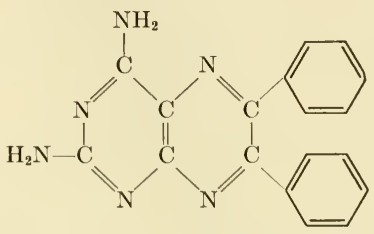

2,4-diamino-6,7-diphenylpteridine

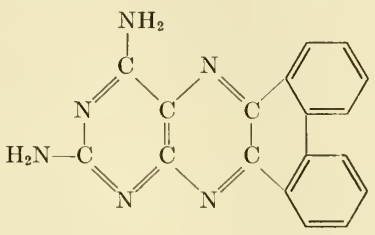

\section{2,4,-diamino-6,7-phenanthro $(9,10-e)$ pteridine}

A large number of the pteridines indicated in Table 21 were prepared in an effort to find more soluble derivatives with the same potent inhibitory properties as the 2,4-diaminopteridines with 6,7-aromatic substituents. However, substitution of the aromatic groups lowered the activity, regardless of the nature of the substituent group. Acetylation of the 2- or 4-amino groups did not appreciably alter the activity, but methylation of these amino groups resulted in considerable loss of inhibitory power. ${ }^{143}$

The 2,4-diaminopteridines contrast markedly with the 2-amino-4hydroxypteridines which, though more closely related structurally to folic acid, do not possess antibacterial activity; on the contrary, some have very slight growth-stimulating effects. ${ }^{140}$

2,4-Diamino-6,7-diphenylpteridine inhibits the growth of Lactobacillus arabinosus, which does not require exogenous folic acid. ${ }^{140}$ Both $p$-amino- 
benzoic acid and folic acid prevent the toxicity to a certain extent, but do not prevent the toxicity of concentrations of $3 \gamma$ per ce or greater. This type of phenomenon is characteristic of the corresponding 4-amino analogues of folic acid for a number of organisms. However, it is interesting that the substituted pteridine inhibits the system of enzymes related to folic acid in an organism which synthesizes the vitamin. Most folic acid analogues are not able to prevent effectively the utilization of this vitamin group in organisms which synthesize the vitamin.

Some of the 2,4-diaminopteridines act synergistically with sulfonamides in preventing growth of Escherichia coli, Staphylococcus aureus and Lactobacillus casei. ${ }^{144}$ For example, a minimum of either $1500 \gamma$ per $10 \mathrm{cc}$ of 2,4-diamino-6,7-dimethylpteridine or $1000 \gamma$ per $10 \mathrm{cc}$ of sulfathiazole is necessary to prevent the growth of Staphylococcus aureus, but a combination of $20 \gamma$ per $10 \mathrm{cc}$ of each of the two compounds allows approximately the same degree of inhibition. Similarly, a minimum of either $2000 \gamma$ per $10 \mathrm{ec}$ of the same pteridine or $50 \gamma$ per $10 \mathrm{cc}$ of sulfathiazole prevents the growth of Escherichia coli, but a mixture of $100 \gamma$ per 10 ce of the pteridine and $20 \gamma$ per 10 cc of sulfathiazole prevents growth of the organism. 2,4-Diamino-6,7-diphenylpteridine, at a concentration which is ineffective alone, will reduce more than ten-fold the amount of sulfathiazole necessary for inhibition of growth of Lactobacillus arabinosus. These results indicate that two biological processes in sequence are inhibited. ${ }^{144}$

Although 2,4-diamino-6,7-diphenylpteridine does not affect growth or hemoglobin formation in chicks, ${ }^{105}$ it is reported to affect blood formation, particularly leucocytes in rats. ${ }^{133}$ The pteridine appears to cause a decrease of folic acid in the liver of chicks, ${ }^{105}$ but no decrease in the folic acid content of the liver is noted for rats. ${ }^{133}$ Other pterins tested with rats included 2,4-diamino-6,7-dimethylpteridine, 2,4-diamino-6,7-di (4-aminophenyl)pteridine and 2-amino-4-hydroxy-6,7-diphenylpteridine. ${ }^{133}$

In chicks, both 2-amino-4-hydroxy-6,7-dimethylpteridine and 2-amino4-hydroxy-6,7-diphenylpteridine (20 mg per $100 \mathrm{~g}$ of diet containing $15 \gamma$ of folic acid) inhibit growth of chicks and decrease hemoglobin formation. The inhibitory effect is prevented by folic acid at $60 \gamma$ per $100 \mathrm{~g}$ of diet. ${ }^{105}$ 2-Amino-4-hydroxy-6 (or 7)-hydroxy-7 (or 6) -methylpteridine appears to inhibit hemoglobin formation, but stimulates growth of chicks on a diet containing suboptimal concentrations of folic acid. 2,4-Dihydroxybenzo (e) pteridine was slightly inhibitory to both growth and hemoglobin formation, whereas 2,4,6-trihydroxy-7-carboxypteridine was inhibitory only to hemoglobin formation. ${ }^{105}$ It is interesting that 2-amino-4hydroxypteridine-7-carboxylic acid and related pteridines prevent the oxidation of either xanthopterin or xanthine by a xanthine oxidase prepa- 
ration from whey. ${ }^{145}$ 2-Amino-4-hydroxy-6-formylpteridine is also an effective inhibitor of xanthine oxidase and related enzymes. ${ }^{146}$

Pyrimidine Derivatives. The toxicity of either 2,4-diamino-6,7-dimethylpteridine or 2,6-diaminopurine for Lactobacillus casei is reported to be prevented in a competitive manner by either folic acid or purines under highly specific conditions, particularly with concentrations of folic acid which limit growth. ${ }^{147}$ The reversal of the toxicity of the pteridine occurs over a relatively restricted range with purines, whereas the reversal by folic acid can be accomplished over a wide range of concentration of inhibitor. ${ }^{147}$ This indicates that the effect of the purines on the inhibited system is probably indirect. The reversal of the toxicity of 2,6-diaminopurine differs from the usual purine effect in that adenine prevents the toxicity over a much greater range of concentrations than does folic acid. ${ }^{147}$ This suggests the possibility that the folic acid effect is indirect, presumably in eliciting synthesis of adenine, which in turn competes with the diaminopurine. The possibility exists, however, that a single compound, such as 2,6-diaminopurine, may effectively inhibit the utilization of two different metabolites. 2-Amino-4-hydroxypteridine derivatives, 2-aminopurine, 2-amino- and 2,4-diamino-6,7-dihydroxypteridines similarly produce inhibitions of Lactobacillus casei stimulated by folic acid; these inhibitions are prevented by purines. These compounds, however, do not inhibit growth of Lactobacillus casei in the presence of thymine and purines. This contrasts to 2,4-diamino-6,7-dimethylpteridine, which inhibits growth of the organism under either condition. ${ }^{147}$ 2,4-Diamino5 -methyl- and 5,6-dimethylpyrimidines are intermediate in their behavior toward reversal with purines and folic acid. ${ }^{\mathbf{1 4 7}}$ Growth of Lactobacillus case $i$ stimulated by either thymine or folic acid is inhibited by 5 -nitrouracil ; ${ }^{148,149}$ however, the toxicity of 5 -nitrouracil is prevented by uracil under conditions in which folic acid exerts no effect. ${ }^{150}$

\section{Miscellaneous}

Miscellaneous analogues of folic acid which have been found to exert inhibitory effects on the utilization of folic acid by some organisms are indicated in Table 22.

4-Desoxyfolic Acid is reported to decrease the apparent folic acid content of the liver and produce both anemia and leucopenia in rats on a diet supplemented with succinylsulfathiazole. ${ }^{133}$

$N$-[N-(2-Amino-4,6-dihydroxypteridine-7-carboxylyl) - p-aminoben$z o y l]$-L-glutamic acid is isomeric with 7-hydroxy-9-oxofolic acid (Table 19) and is less than half as effective in inhibiting the growth of Lactobacillus casei as is the isomer resembling folic acid. ${ }^{125}$

Quinoxaline has a slight inhibitory effect on the growth of Streptococcus 


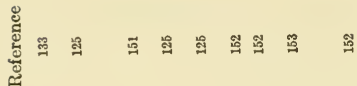

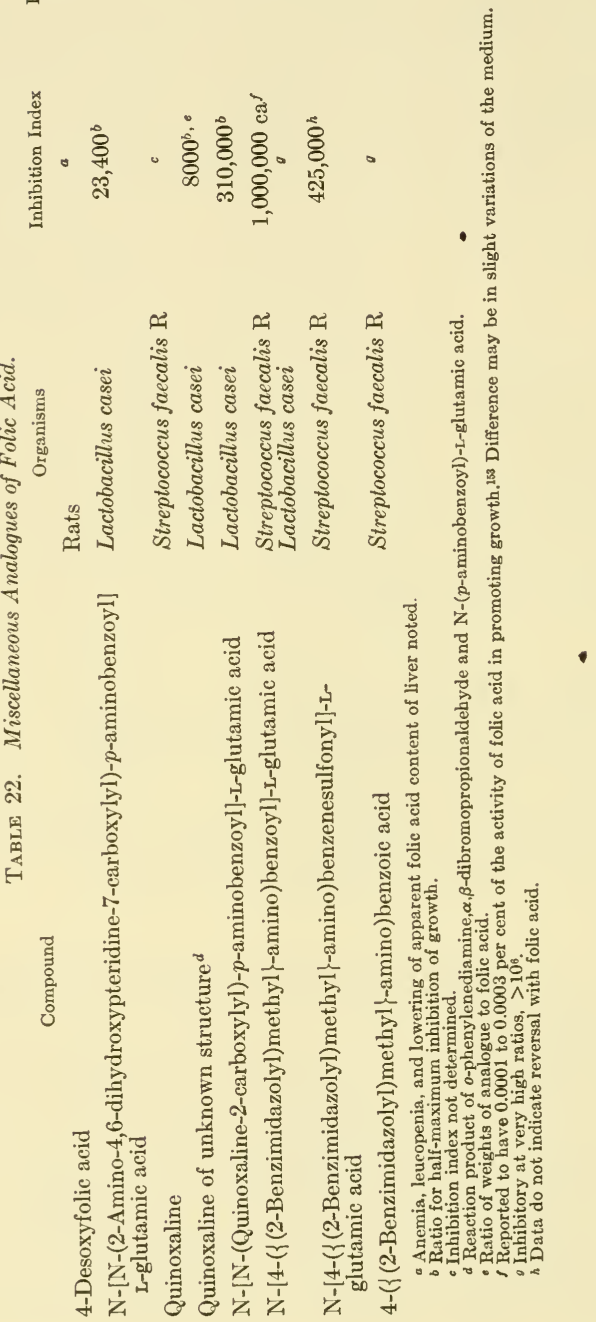


faecalis $\mathrm{R}$, which is prevented by folic acid. ${ }^{\mathbf{1 5 1}}$ Quinoxaline derivatives more closely related to folic acid are considerably more effective than the parent compound; ${ }^{125}$ however, the compounds with modified ring structures in place of the pteridine ring of folic acid have not as yet been found to be particularly effective as inhibitory analogues of folic acid (Table 22).

$N[N$-(2-Amino-4-hydroxy-6-pteridylmethyl) - p-aminobenzenesulfo$n y l]$-L-glutamic acid has recently been synthesized, but no biological data were reported. ${ }^{154}$

A number of analogues of folic acid including $\mathrm{N}$-(2-amino-4-hydroxy-6-pteridylmethyl)-p-aminophenol, N-(2-amino-4-hydroxy-6-pteridylmethyl)-p-aminohippuric acid, N-(2-amino-4-hydroxy-6-pteridylmethyl)-4-amino-2-chlorobenzoic acid, 2-hydroxy-2-desaminopteroic acid, 2-hydroxy-2-desaminofolic acid, N-(2-amino-4-hydroxy-6-pteridylmethyl)- $p$-aminobenzenesulfonic acid, $\mathrm{N}$-[N-(4-(6-aminoquinazoline)) $p$-aminobenzoyl] glutamic acid, $\mathrm{N}$-[N-(4-quinazoline)- $p$-aminobenzoyl]glutamic acid, $\mathrm{N}$-(4-aminoquinazoline)- $p$-aminobenzenesulfonamide, $\mathrm{N}$-[N-(4-(6-nitroquinazoline)) - $p$-aminobenzoyl] glutamic acid, $\mathrm{N}[\mathrm{N}-(4-$ (6-chloroquinazoline)) - $p$-aminobenzoyl] glutamic acid, N-[4-(6-chloroquinazoline) ]- $p$-aminobenzoic acid, $\mathrm{N}$-(2,4-dihydroxy-6-pteridylmethyl)$p$-aminobenzenesulfonic acid, and $\mathrm{N}$-[N-(2,4-dihydroxy- $x$-methyl-6-pteridylmethyl)-p-aminobenzoyl]glutamic acid have been prepared, and tested for their effects on the blood pressure of the dog, inhibitory effects on 3,4-dihydroxyphenylalanine decarboxylase, and their inhibitory action on Streptococcus faecalis R. ${ }^{126}$

Previous observations indicated that $L^{-} x$-methylfolic acid inhibited to some extent the action of the decarboxylase of 3,4-dihydroxyphenylalanine ${ }^{155}$ and lowered the blood pressure of the dog. ${ }^{156}$ Many of the above compounds, as well as other related inhibitory analogues at concentrations of 30 to $300 \gamma$ per cc, show some inhibitory action on the decarboxylase enzyme. ${ }^{136,157}$ The inhibition is reported to be prevented by folic acid in extremely high concentrations, ten to a hundred times the concentration of the inhibitors. Also, some of these analogues affect the blood pressure of the dog. These effects are reported to be nullified by the injection of $250 \mathrm{mg}$ of folic acid per $\mathrm{kg}$ of body weight. The pteroic acid analogues are more effective than the folic acid analogues for the hypotensive effect. ${ }^{126}$

\section{The Effect of Compounds Related to Folic Acid on Cancer}

An extensive study of $\mathrm{B}$ vitamins in normal and cancer tissue has indicated that folic acid is relatively the most abundant of the vitamins in cancer tissue. Since there is similarity in the content of the B vitamins 
in cancer tissues regardless of the host organism or site of appearance or means of induction, the general patterns of the metabolism of cancer tissues appear to be related. ${ }^{158}$

The folic acid from livers of rats bearing Walker tumor transplants is liberated by autolysis either in phosphate-sodium chloride or acetate buffer, whereas maximal release of folic acid from livers of normal rats requires both Clarase and phosphate-sodium chloride buffer. The acetate buffer does not replace the phosphate buffer.

Pteroyldi- $\gamma$-glutamylglutamic Acid. The first direct effect on growth of cancer by a member of the folic acid group was reported by Leuchtenberger, et al., ${ }^{160}$ who found that the growth of Sarcoma 180 in female Rockland mice was reduced to approximately 35 per cent of the controls by intravenous injection of $5 \gamma$ of a concentrate containing members of the folic acid group, or by the same number of injections of $0.4 \gamma$ of fermentation Lactobacillus case $i$ factor (pteroyldi- $\gamma$-glutamylglutamic acid). Complete regressions of spontaneous breast cancers in mice were observed in 38 among 89 (43 per cent) animals treated with daily injections of $5 \gamma$ of the pteroyltriglutamate. The incidence of the development of new tumors was decreased from 14 out of 60 mice in the controls to 1 out of 89 mice treated with the triglutamate. ${ }^{161}$ The liver Lactobacillus casei factor (pteroylglutamic acid) is inactive in effecting this inhibitory action on the tumors. ${ }^{161}$ Additional data on the inhibition of transplanted tumors and of spontaneous tumors have been reported by this group. ${ }^{162}$ However, failures in attempts to confirm these results using sarcoma 180 or spontaneous breast cancer of mice have been reported. ${ }^{163-166}$

Preliminary clinical reports of the use of pteroyldi- $\gamma$-glutamylglutamic acid (teropterin) in the treatment of cancer have indicated that it is nontoxic and in 500-mg doses relieves pain in most cases, if not all, thereby allowing a reduction in amount of sedation or analgesia required and in some cases obviating the necessity for use of opiates. The patients, after treatment with the compound, are cheerful, exhibit a sense of wellbeing and are more mentally alert. Although some patients with advanced malignancy have shown considerable improvement on treatment with the compound, the results in most instances cannot be evaluated objectively because other treatments known to have desirable effects preceded or were concurrent with the administration of the triglutamate. ${ }^{167-169}$

Effect of Folic Acid and Related Compounds on Rous Chicken Sarcoma. Folic acid and related compounds have a profound effect on the avian neoplasm first described by Rous. ${ }^{170}$ The Rous chicken sarcoma, which is transmitted by a filtrable agent, can be controlled either by regulation of the amount of folic acid in the diet of the chicks or by administration of certain folic acid antagonists. ${ }^{77}{ }^{170-172}$ Thus, injection of a sterile, 
aqueous suspension of infected tissue into the breast of baby chicks on an ordinary diet resulted in development of tumors in approximately 95 per cent of the chicks; but out of 40 similarly injected chicks on a folic acid-deficient diet, no tumors developed. However, the frequency of tumor development with chicks on the same deficient diet but injected daily with folic acid $(100 \gamma)$, pteroyl- $\alpha$-glutamylglutamic acid $(500 \gamma)$, pteroyltriglutamic acid $(500 \gamma)$, or $\mathrm{N}^{10}$-methylfolic acid $(100 \gamma)$ was $90,90,100$, and 80 per cent, respectively. No such action was noted with similar injections of pteroyl-D-glutamic acid, pteroic acid, pteroylaspartic acid, $\mathrm{N}^{10}$-methylpteroic acid or $p$-aminobenzoyltriglutamic acid.

On a normal diet on which 95 per cent of the chicks developed tumors by the twentieth day, folic acid antagonists tended to prolong or completely inhibit the development of the tumor, but were in many instances rather toxic to the host. Thus, daily intraperitoneal injections, beginning at the time of inoculation with tumor of 5-6 day old chicks, of 4-amino-4-desoxypteroyl-L-aspartic acid (400 $\gamma)$, 4-amino-4-desoxypteroyl-D-glutamic acid $(400 \gamma)$, 4-amino- $\mathrm{N}^{10}$-methyl-4-desoxypteroylglutamic acid $(100 \gamma)$, and 4-amino- $\mathrm{N}^{10}$-methyl-4-desoxypteroic acid $(100 \gamma)$ decreased the percentage of chicks developing tumors by the twentieth day to $40,0,70$, and 60 per cent, respectively. Pteroylaspartic acid and pteroyl-D-glutamic acid did not exhibit any appreciable inhibitory activity on the tumor. Concentrations of the compounds which were inhibitory to tumor growth resulted in impairment of health and eventual loss of life, particularly when the compounds were injected. The therapeutic index was slightly more favorable in older animals and least favorable with one-day old chicks. The method of administration was also important, and the best results were obtained on administering the antagonists in the diet. Thus, $80 \mathrm{mg}$ per $\mathrm{kg}$ of diet of 4 -amino-4-desoxypteroylaspartic acid or a similar amount of 4-amino-4-desoxypteroyl-Dglutamic acid was relatively nontoxic to one-day old chicks, and prevented for 17 days the development of tumors in 75 and 55 per cent of the chicks, respectively. All the untreated controls developed tumors.

4-Amino-4-desoxyfolic acid is suitable only for treatment of adult birds since it is extremely toxic for growing chicks. Any concentration affecting tumor growth was lethal to the chick. However, $1 \mathrm{mg}$ of 4-amino-4desoxyfolic acid, administered daily by intraperitoneal injection, prevented the development of the tumor in approximately 60 per cent of adult birds as compared with 20 per cent obtained with 4 -amino-4-desoxypteroylaspartic acid administered similarly. Even at this dosage for adult birds, 4-amino-4-desoxyfolic acid produces noticeable emaciation in adult birds, and a few injections of $10 \gamma$ are lethal to baby chicks. Doses of $0.25 \mathrm{mg}$ of folic acid, pteroyltriglutamic acid or pteroyldiglutamic acid 
protect approximately 60,50 and 10 per cent of baby chicks against the toxic effect of $10 \gamma$ doses of 4-amino-4-desoxyfolic acid which are otherwise fatal. The antagonist under these conditions did not prevent tumor growth in baby chicks.

Leukemia. The greater sensitivity to folic acid deficiency of leucopoiesis in the myeloid series ${ }^{107}$ led to the suggestion that folic acid analogues might be used as chemotherapeutic agents in myelogenous leukemia.

In a preliminary report on the use of folic acid derivatives in the treatment of human leukemia ${ }^{173}$ it is indicated that pteroyldi- $\gamma$-glutamylglutamic acid, pteroyldiglutamic acid, pteroylaspartic acid and N-methylpteroic acid do not alter the blood picture or bone marrow in cases of chronic leukemia and multiple myeloma; however, in cases of acute leukemia, administration of the folic acid antagonists resulted in a temporary reduction of the total white cells. ${ }^{173}$

A preliminary report of the effect of inhibitory folic acid analogues on acute leukemia in children indicated that temporary remissions can be induced with 4-amino-4-desoxyfolic acid. ${ }^{174}$ The effect of $\mathrm{N}^{10}$-methylpteroic acid and pteroylaspartic acid is questionable. Upon treatment with 4-amino-4-desoxyfolic acid, the white cell count tended to return to a normal level both in patients with an initially high count and in those with marked leukopenia at the outset of the therapy. A decline was observed in the percentage of immature cells with a marked decrease in blast forms, which disappeared in some cases from the peripheral blood. The relative percentages of mature leucocytes approached normal values in the peripheral blood. In the bone marrow a decrease or disappearance of leukemic cells and variation from hypoplasia to almost normal pattern was observed. However, the analogue is toxic, giving effects which include stomatitis with early ulceration. Indications are that the remissions are only temporary. Similar temporary favorable results have been reported by others. ${ }^{175}$

Only slight and irregular effects in increasing the survival time of mice injected with transmitted leukemia Ak 1394 and Akm 9417 are obtained on administration of 4-amino-4-desoxyfolic acid which was, however, somewhat more effective against the $\mathrm{Ak} 4$ and C 1498 strains of leukemia. ${ }^{176} 4$-Amino- $\mathrm{N}^{10}$-methyl-4-desoxyfolic acid allowed a longer survival time than did nitrogen mustards in mice injected with Akm 9417, $\mathrm{Ak} 4$ or C 1498 strains of leukemia, but was not effective with the Ak 1394 strain. Nitrogen mustards allow a slight increase in survival time of mice with the C 1498 strain, but 4 -amino- $\mathrm{N}^{10}$-methyl-4-desoxyfolic acid increases the survival time slightly more than 200 per cent. By the cytocidal method, no activity could be shown against the cells of leukemia Akm 
9417, which are completely inactivated by methylbis (chloroethyl) amine at four times the $\mathrm{LD}_{50} \cdot{ }^{176}$

The average survival time of mice with $\mathrm{Ak} 4$ strain of leukemia is prolonged significantly by 2,6 -diaminopurine as well as 4 -amino-4-desoxyfolic acid. ${ }^{177}$

Sarcoma 180. 4-Amino-4-desoxyfolic acid at 0.19 to $0.42 \mathrm{mg}$ per $\mathrm{kg}$ retards the rate of growth of sarcoma 180 in mice. Inhibitions of tumor growth to the extent of more than 50 per cent and on occasions as high as 94 per cent of the controls are obtained, but the mice lose weight up to 20 per cent under these conditions. ${ }^{178}$ Pteroyl- $\alpha$-glutamylglutamic acid administered intraperitoneally or intravenously does not affect growth of the tumor. ${ }^{176}$

4-Amino-4-desoxyfolic acid is fatal to many of the animals under conditions giving marked inhibition of tumor growth. ${ }^{179}$

A partial reversal of the inhibition of tumor growth is obtained by administration of folic acid with the 4-amino-4-desoxyfolic acid. Folic acid alone exerts some inhibition of tumor growth when administered at high concentration. ${ }^{180}$

4-Amino-4-desoxy- $\mathrm{N}^{10}$-methylfolic acid has a more favorable therapeutic index than 4-amino-4-desoxyfolic acid. ${ }^{181}$ At $1.5 \mathrm{mg}$ per $\mathrm{kg}$ per day, the amino methyl analogue shows marked inhibition of growth of sarcoma 180 , the tumors being only 3-10 per cent of the size of controls after one week, and causes very little loss in weight of the animals, with only 7 per cent fatalities during the injection period. Slightly higher concentrations (2.5 $\mathrm{mg}$ per $\mathrm{kg}$ per day) of the analogue are lethal to almost half the animals. Male mice appear to tolerate the analogue better than female mice. ${ }^{181}$

4-Amino-4-desoxypteroylaspartic acid also has a more favorable therapeutic index than 4-amino-4-desoxyfolic acid in inhibition of sarcoma 180 in mice. ${ }^{177}$

\section{Bibliography}

1. Wills, L., Brit. Med. J., 1, 1059 (1931).

2. Wills, L., and Bilimoria, H. S., Indian J. Med. Research, 20, 391 (1932).

3. Wills, L., and Stewart, A., Brit. J. Exper. Path., 16, 444 (1935).

4. Day, P. L., Langston, W. C., and Shukers, C. F., J. Nutrition, 9, 637 (1935).

5. Day, P. L., Langston, W. C., and Darby, W. J., Proc. Soc. Exptl. Biol. Med., 38, 860 (1938).

6. Stokstad, E. L. R., and Manning, P. D. V., J. Biol. Chem., 125, 687 (1938).

7. Hogan, A. G., and Parrott, E. M., J. Biol. Chem., 132, 507 (1940); Proc., 128, xlvi (1939).

8. O'Dell, B. L., and Hogan, A. G., J. Biol. Chem., 149, 323 (1943).

9. Snell, E. E., and Peterson, W. H., Proc. Am. Soc. Biol. Chem., J. Biol. Chem.. Proc., 128, xciv (1939). 
10. Snell, E. E., and Peterson, W. H., J. Bact., 39, 273 (1940).

11. Mitchell, H. K., Snell, E. E., and Williams, R. J., J. Am. Chem. Soc., 63, 2284 (1941).

12. Pfiffner, J. J., Calkins, D. G., O'Dell, B. L., Bloom, E. S., Brown, R. A., Campbell, C. J., and Bird, O. D., Science, 102, 228 (1945); Pfiffner, J. J., Binkley, S. B., Bloom, E. S., and O'Dell, B. L., J. Am. Chem. Soc., 69, 1476 (1947).

13. Stokstad, E. L. R., J. Biol. Chem., 149, 573 (1943) ; Stokstad, E. L. R., Hutchings, B. L., and SubbaRow, Y., Ann. New York Acad. Sci., 48, 261 (1946); J. Am. Chem. Soc., 70, 3 (1948).

14. Binkley, S. B., Bird, O. D., Bloom, E. S., Brown, R. A., Calkins, D. G., Campbell, C. J., Emmett, A. D., and Pfiffner, J. J., Science, 100, 36 (1944).

15. Mitchell, H. K., Snell, E. E., and Williams, R. J., J. Am. Chem. Soc., 66, 267 (1944).

16. Keresztesy, J. C., Rickes, E. L., and Stokes, J. L., Science, 97, 465 (1943).

17. Hutchings, B. L., Stokstad, E. L. R., Bohonos, N., and Slobodkin, N. H., Science, 99, 371 (1944) ; Hutchings, B. L., Stokstad, E. L. R., Bohonos, N., Sloane, N. H., and SubbaRow, Y., Ann. New York Acad. Sci., 48, 265 (1946); J. Am. Chem. Soc., ,70, 1 (1948).

18. Pfiffner, J. J., Calkins, D. G., O’Dell, B. L., Bloom, E. S., Brown, R. A., Campbell, C. J., and Bird, O. D., Science, 102, 228 (1945).

19. Mitchell, H. K., and Williams, R. J., J. Am. Chem. Soc., 66, 271 (1944).

20. Mitchell, H. K., J. Am. Chem. Soc., 66, 274 (1944).

21. Mowat, J. H., Boothe, J. H., Hutchings, B. L., Stokstad, E. L. R., Waller, C. W., Angier, R. B., Semb, J., Cosulich, D. B., and SubbaRow, Y., Ann. New York Acad. Sci., 48, 279 (1946); J. Am. Chem. Soc., 70, 14 (1948).

22. Stokstad, E. L. R., Hutchings, B. L., Mowat, J. H., Boothe, J. H., Waller, C. W., Angier, R. B., Semb, J., and SubbaRow, Y., Ann. New York Acad. Sci., 48, 269 (1946); J. Am. Chem. Soc., 70, 5 (1948).

23. Hutchings, B. L., Stokstad, E. L. R., Mowat, J. H., Boothe, J. H., Waller, C. W., Angier, R. B., Semb, J. and SubbaRow, Y., Ann. New York Acad. Sci., 48, 273 (1946); J. Am. Chem. Soc., 70, 10 (1948).

24. Waller, C. W., Hutchings, B. L., Mowat, J. H., Stokstad, E. L. R., Boothe, J. H., Angier, R. B., Semb, J., SubbaRow, Y., Cosulich, D. B., Fahrenbach, M. J., Hultquist, M. E., Kuh, E., Northey, E. H., Seeger, D. R., Sickels, J. P., and Smith, J. M., Jr., Ann. New York Acad. Sci., 48, 283 (1946); J. Am. Chem. Soc., 70, 19 (1948).

25. Angier, R. B., Boothe, J. H., Hutchings, B. L., Mowat, J. H., Semb, J., Stokstad, E. L. R., SubbaRow, Y., Waller, C. W., Cosulich, D. B., Fahrenbach, M. J., Hultquist, M. E., Kuh, E., Northey, E. H., Seeger, D. R., Sickels, J. P., and Smith, J. M., Jr., Science, 103, 667 (1946).

26. Boothe, J. H., Mowat, J. H., Hutchings, B. L., Angier, R. B., Waller, C. W., Stokstad, E. L. R., Semb, J., Gazzola, A. L., and SubbaRow, Y., Trans. New York Acad. Sci., 10, II, 70 (1948); J. Am. Chem. Soc., 70, 1099 (1948).

27. Pfiffner, J. J., Calkins, D. G., Bloom, E. S., and O'Dell, B. L., J. Am. Chem. Soc., 68, 1392 (1946).

28. Day, P. L., Langston, W. C., Darby, W. J., Wahlin, J. G., and Mims, V., J. Exptl. Med., 72, 463 (1940).

29. Mims, V., Totter, J. R., and Day, P. L., J. Biol. Chem., 155, 401 (1944).

30. Bird, O. D., Binkley, S. B., Bloom, E. S., Emmett, A. D., and Pfiffner, J J., J. Biol. Chem., 157, 413 (1945).

31. Bird, O. D., Bressler, B., Brown, R. A., Campbell, C. J., and Emmett, A. D., J. Biol. Chem., 159, 631 (1945).

32. Laskowski, M., Mims, V., and Day, P. L., J. Biol. Chem., 157, 731 (1945).

33. Rickes, E. L., Chaiet, L., and Keresztesy, J. C., J. Am. Chem. Soc., 69, 2749 (1947). 
34. Rickes, E. L., Trenner, N. R., Conn, J. B., and Keresztesy, J. C., J. Am. Chem. Soc., 69, 2751 (1947).

35. Wolf, D. F., Anderson, R. C., Kaczka, E. A., Harris, S. A., Arth, G. E., Southwick, P. L., Mozingo, R., and Folkers, K., J. Am. Chem. Soc., 69, 2753 (1947).

36. Mowat, J. H., Hutchings, B. L., Angier, R. B., Stokstad, E. L. R., Boothe, J. H., Waller, C. W., Semb, J., and SubbaRow, Y., J. Am. Chem. Soc., 70, 1096 (1948).

36a. Boothe, J. H., Semb, J., Waller, C. W., Angier, R. B., Mowat, J. H., Hutchings, B. L., Stokstad, E. L. R., and SubbaRow, Y., J. Am. Chem. Soc., 71, 2304 (1949) ; Mowat, J. H., Gazzola, A. L., Hutchings, B. L., Boothe, J. H., Waller, C. W., Angier, R. B., Semb, J., and SubbaRow, Y., J. Am. Chem. Soc., 71, 2308 (1949) ; Semb, J., Boothe, J. H., Angier, R. B., Waller, C. W., Mowat, J. H., Hutchings, B. L., and SubbaRow, Y., J. Am. Chem. Soc., 71, 2310 (1949).

37. Gordon, M., Ravel, J. M., Eakin, R. E., and Shive, W., J. Am. Chem. Soc., 70, 878 (1948).

38. Jukes, T. H., Franklin, A. L., Stokstad, E. L. R., and Boehne, J. W., III, J. Lab. Clin. Med., 32, 1350 (1947).

39. Spies, T. D., Lopez, G. G., Stone, R. E., Milanes, F., Brandenberg, R. O., and Aramburn, T., Intern. Z. Vitaminforsch., 19, 1 (1947); Chem., Abstr. 42, 4651 (1948).

40. Spies, T. D., Stone, R. E., and Toca, R. L., Southern Med. J., 40, 175 (1947).

41. Goldsmith, G. A., Reported at A.A.A.S. Conference on Vitamins, Gibson Island, July 23-27 (1945); ef. Darby, IV. J., Vitamins and Hormones, Vol. V., p. 140.

42. Suárez, R. M., Welch, A. D., Heinle, R. W., Suárez, R. M., Jr., and Nelson, E. M., J. Lab. and Clin. Med., 31, 1294 (1946).

43. Jones, E., Warden, H. F., and Darby, W. J., J. Lab. Clin. Med., 32, 387 (1947).

44. Spies, T. D., South Med. J., 39, 634 (1946).

45. Castle, W. B., Ross, J. B., Davidson, C. S., Burchenal, J. H., Fox, H. J., and Ham, T. H., Science, 100, 81 (1944).

46. Swendseid, M. E., Bird, O. D., Brown, R. A., and Bethell, F. H., J. Lab. Clin. Med., 32, 23 (1947).

47. Welch, A. D., Heinle, R. W., Nelson, E. M., and Nelson, H. V., J. Biol. Chem., 164, 787 (1946).

48. Welch, A. D., Fed. Proc., 6, 471 (1947).

49. Bethell, F. H., Meyers, M. C., Andrews, G. A., Swendseid, M. E., Bird, O. D., and Brown, R. A., J. Lab. Clin. Med., 32, 3 (1947).

50. Sharp, E. A., and Vonderheide, E. C., Am. J. Clin. Path., 17, 761 (1947).

51. Spies, T. D., and Stone, R. E., cf. Spies, "Experiences with Folic Acid" p. 61, The Year Book Publishers, Inc., Chicago, Ill. (1947).

52. Bethell, F. H., Swendseid, M. E., Bird, O. D., Meyers, M. C., Andrews, G. A., and Brown, R. A., Univ. Hosp. Bull., Ann Arbor, 12, 42 (1946).

53. Spies, T. D., Lopez, G. G., Stone, R. E., Milanes, F., Brandenberg, R. O., and Aramburu, T., Blood, 3, 121 (1948).

54. Day, P. L., Mims, V., Totter, J. R., Stokstad, E. L. R., Hutchings, B. L., and Sloane, N. H., J. Biol. Chem., 157, 423 (1945). Day, P. L., Mims, V., and Totter, J. R., J. Biol. Chem., 161, 45 (1945).

55. Daft, F. S., and Sebrell, W. H., U. S. Pub. Health Repts., 58, 1542 (1943).

56. Kornberg, A., Tabor, H., and Sebrell, W. H., Am. J. Physiol., 142, 604 (1944).

57. Daft, F. S., Kornberg, A., Ashburn, L. L., and Sebrell, W. H., Proc. Soc. Exptl. Biol. Med., 61, 154 (1946).

57a. Kornberg, A., Daft, F. S., and Sebrell, W. H., Arch. Biochem., 8, 431 (1945).

58. Swendseid, M. E., Brown, R. A., Bird, O. D., and Heinrich, R. A., Arch. Biochem., 16, 367 (1948).

59. Hutchings, B. L., Oleson, J. J., and Stokstad, E. L. R., J. Biol. Chem., 163, 447 (1946).

60. Jukes, T. H., and Stokstad, E. L. R., J. Biol. Chem., 168, 563 (1947). 
61. Schweigert, B. S., Arch. Biochem., 20, 41 (1949).

62. Kidder, G. W., and Dewey, V. C., Arch. Biochem., 21, 70 (1949).

63. Kidder, G. W., and Dewey, V. C., Proc. Natl. Acad. Sci. U. S., 33, 95 (1947).

64. Kidder, G. W., and Fuller, R. C., III, Science, 104, 160 (1946).

65. Jukes, T. H., and Stokstad, E. L. R., Phys. Rev., 28, 68 (1948).

66. Black, S., McKibbin, J. M., and Elvehjem, C. A., Proc. Soc. Exptl. Biol. Med., 4.7, 308 (1941).

67. Spicer, S. S., Daft, F. S., Sebrell, W. H., and Ashburn, L. L., U. S. Pub. Health Repts., 57, 1559 (1942).

68. Kornberg, A., Daft, F. S., and Sebrell, W. H., Science, 98, 20 (1943).

69. Nielsen, E., and Elvehjem, C. A., J. Biol. Chem., 145, 713 (1942).

70. Moore, C. V., Bierbaum, O. S., Welch, A. D., and Wright, L. D., J. Lab. Clin. Med., 30, 1056 (1945).

71. Spies, T. D., Vilter, C. F., Koch, M. B., and Caldwell, M. H., South. Med. J., 38, 707 (1945).

72. Vilter, C. F., Spies, T. D., and Koch, M. B., South. Med. J., 38, 781 (1945).

73. Darby, W. J., and Jones, E., Proc. Soc. Exptl. Biol. Med., 60, 259 (1945).

74. Wright, L. D., McMahan, J. R., Cheldelin, V. H., Taylor, A., Snell, E. E., and Williams, R. J., Univ. of Texas Publication No. 413.7, p. 38 (1941).

75. Wright, L. D., and Welch, A. D., Science, 98, 179 (1943).

76. Steinkamp, R., Shukers, C. F., Totter, J. R., and Day, P. L., Proc. Soc. Exptl. Biol. Med., 63, 556 (1946).

77. Little, P. A., Sampath, A., Paganelli, V., Locke, E., and SubbaRow, Y., Trans. New York Acad. Sci., 10, II, 91 (1948).

78. Johnson, B. C., Hamilton, T. S., and Mitchell, H. H., J. Biol. Chem., 159, 425 (1945).

79. Johnson, B. C., J. Biol. Chem., 163, 255 (1946).

80. Scott, M. L., Norris, L. C., Heuser, G. F., Bruce, W. F., Coover, H. W., Jr., Bellamy, IV. D., and Gunsalus, I. C., J. Biol. Chem., 154, 713 (1944).

81. Scott, M. L., Norris, L. C., Heuser, G. F., and Bruce, W. F., J. Biol. Chem., 158, 291 (1945).

82. Scott, M. L., Norris, L. C., Charkey, L. W., Daniel, L. J., and Heuser, G. F., J. Biol. Chem., 164, 403 (1946).

83. Petering, H. G., Marvel, J. P., Glauser, C. E., Jr., and Waddell, J., J. Biol. Chem., 162, 477 (1946).

84. Scott, M. L., Norris, L. C., and Heuser, G. F., Science, 103, 303 (1946).

85. Daniel, L. J., Scott, M. L., Norris, L. C., and Heuser, G. F., J. Biol. Chem., 160,265 (1945).

86. Mims, V., and Laskowski, M., J. Biol. Chem., 160, 493 (1945).

87. Kazenko, A., and Laskowski, M., J. Biol. Chem., 173, 217 (1948).

88. Bird, O. D., Robbins, M., Vandenbelt, J. M., and Pfiffner, J. J., J. Biol. Chem., 163, 649 (1946).

89. Mims, V., Swendseid, M. E., and Bird, O. D., J. Biol. Chem., 170, 367 (1947).

90. Hodson, A. Z., Arch. Biochem., 16, 309 (1948).

91. Sims, E. S., and Totter, J. R., Fed. Proc., 6, 291 (1947).

92. Ratner, S., Blanchard, M., and Green, D. E., J. Biol. Chem., 164, 691 (1946).

93. Scott, M. L., Hill, C. H., and Norris, L. C., Fed. Proc., 8, 249 (1949).

94. Simpson, R. E., and Schweigert, B. S., Arch. Biochem., 20, 32 (1949).

95. Spies, T. D., and Stone, R. E., Southern Med. J., 40, 46 (1947).

96. Stokes, J. L., Keresztesy, J. C., and Foster, J. W., Science, 100, 522 (1944); Stokes, J. L., and Larsen, A., J. Bact., 50, 219 (1945).

97. Martin, G. J., Moss, J., and Avakian, S., J. Biol. Chem., 167, 737 (1947).

98. Tschesche, R., and Wolf, H. J., Z. physiol. Chem., 244, I (1936).

99. Simmons, R. IV., and Norris, E. R., J. Biol. Chem., 140, 679 (1941).

100. Totter, J. R., Shukers, C. F., Kolson, J., Mims, V., and Day, P. L., J. Biol. Chem., 152, 147 (1944). 
101. Totter, J. R., and Day, P. L., J. Biol. Chem., 147, 257 (1943).

102. Norris, E. R., and Majnarich, J. J., Science, 109, 32 (1949); Norris, E. R., and Majnarich, J. J., Science, 109, 33 (1949).

103. Norris, E. R., and Majnarich, J. J., Fed. Proc., 8, 233 (1949).

104. O'Dell, B. L., and Hogan, A. G., J. Biol. Chem., 149, 323 (1943).

105. Daniel, L. J., Scott, M. L., Norris, L. C., and Heuser, G. F., J. Biol. Chem., 173, 123 (1948).

106. Martin, G. J., Tolman, L., and Moss, J., Arch. Biochem., 12, 318 (1947).

107. Franklin, A. L., Stokstad, E. L. R., Belt, M., and Jukes, T. H., J. Biol. Chem., 169, 427 (1947).

108. Martin, G. J., Avakian, S., Tolman, L., Urist, H., and Moss, J., Abstracts of Papers, 112th Meeting, Am. Chem. Soc., p. 2c, Sept. 1947.

109. Rogers, L. L., and Shive, W., J. Biol. Chem., 172, 751 (1948).

109a. Shive, W., Eakin, R. E., Harding, W. M., Ravel, J. M., and Sutherland, J. E., J. Am. Chem. Soc., 70, 2299 (1946).

109b. Martin, G. J., Tolman, L., and Moss, J., Scicnce, 106, 168 (1947).

110. Day, P. L., Langston, W. C., and Shukers, C. F., J. Biol. Chem., 114, p.xxv (1936).

111. Franklin, A. L., Stokstad, E. L. R., and Jukes, T. H., Proc. Soc. Exptl. Biol. Med., 65, 368 (1947).

112. Welch, A. D., Heinle, R. W., Sharpe, G., George, W. L., and Epstein, M., Proc. Soc. Exptl. Biol. Med., 65, 364 (1947); Heinle, R. W., Welch, A. D., George, W. L., Epstein, M., and Pritchard, J. A., Proc. Central Soc. Clin. Research, 20, 7 (1947).

113. Welch, A. D., Heinle, R. W., Pritchard, J. A., and Salis, H., Fed. Proc., 7, 300 (1948).

114. Cartwright, G. E., Fay, J., Tatting, B., and Winthrobe, M. M., J. Lab. Clin. Med., 33, 397 (1948).

115. Franklin, A. L., Jukes, T. H., Stokstad, E. L. R., and Belt, M., Fed. Proc., 8, 199 (1949).

116. Jukes, T. H., Stokstad, E. L. R., and Franklin, A. L., Conference on Development and Uses of Antimetabolites, New York Academy of Sciences, February, 1949 (in press).

117. Goldsmith, E. D., Tobias, E. B., and Harnly, M. H., Anat. Rec., 101, 104 (1948).

118. Goldsmith, E. D., Harnly, M. H., Nigrelli, R. F., and Schreiber, S. S., Conference on Development and Uses of Antimetabolites, New York Academy of Sciences, February, 1949 (in press).

119. Hertz, R., Science, 107, 300 (1948).

120. Hertz, R., Endocrinology, 37, 1 (1945).

121. Salis, H., Proc. Soc. Exptl. Biol. Med., 68, 382 (1948).

122. Hultquist, M. E., Smith, J. M., Jr., Seeger, D. R., Cosulich, D. B. and Kuh, E., J. Am. Chem. Soc., 71, 619 (1949).

123. Cosulich, D. B., and Smith, J. M., Jr., J. Am. Chem. Soc., 70, 1922 (1948).

124. Smith, J. M., Jr., Cosulich, D. B, Hultquist, M. E., and Seeger, D. R., Trans. N. Y. Acad. Sci., II 10, 82 (1948).

125. Woolley, D. W., and Pringle, A., J. Biol. Chem., 174, 327 (1948).

126. Martin, G. J., Brendel, R., Beiler, J. M., Moss, J., Avakian, S., Urist, H., Tolman, L., and Alpert, S., Am. J. Pharm., 120, 189 (1948).

127. Hutchings, B. L., Mowat, J. H., Oleson, J. J., Stokstad, E. L. R., Boothe, J. H., Waller, C. W., Angier, R. B., Semb, J., and SubbaRow, Y., J. Biol. Chem., 170, 323 (1947).

128. Seeger, D. R., Smith, J. M., Jr., and Hultquist, M. E., J. Am. Chem. Soc., 69, 2567 (1947).

129. Oleson, J. J., Hutchings, B. L., and SubbaRow, Y., J. Biol. Chem., 175, 359 (1948). 
130. Seeger, D. R., Cosulich, D. B., Smith, J. M., Jr., and Hultquist, M. E., J. Am. Chem. Soc., 71, 1753 (1919).

131. Franklin, A. L., Belt, M., Stokstad, E. L. R., and Jukes, T. H., J. Biol. Chem., 177, 621 (1949).

132. Philips, F. S., and Thiersch, J. B., J. Pharmacol. Exptl. Therap., 95, 303 (1949).

133. Swendseid, M. E., Wittle, E. L., Moersch, G. W., Bird, O. D., and Brown, R. A., Fed. Proc., 7, 299 (1948).

134. Franklin, A. L., Stokstad, E. L. R., and Jukes, T. H., Proc. Soc. Exptl. Biol. Med., 67, 398 (1948).

135. Minnich, V., and Moore, C. V., Fed. Proc., 7, 276 (1948).

136. Thiersch, J. B., and Philips, F. S., Fed. Proc., 8, 372 (1949).

137. Hertz, R., Proc. 39th Annual Meeting Am. Assn. Cancer Research, March, 1948; Private communication to Goldsmith et al. ${ }^{138}$

138. Goldsmith, E. D., Schreiber, S. S., and Nigrelli, R. F., Proc. Soc. Exptl. Biol. Med., 69, 299 (1948).

139. Morgan, H. R., and Wagley, P. F., Bull. Johns Hopkins Hosp., 83, 275 (1948).

139a. Rodney, G., Swendseid, M. E., and Swanson, A. L., J. Biol. Chem., 179, 19 (1949).

140. Daniel, L. J., Norris, L. C., Scott, M. L., and Heuser, G. F., J. Biol. Chem., 169, 689 (1947).

140a. Philips, F. S., Thiersch, J. B., and Ferguson, F. C., Conference on Development and Uses of Antimetabolites, New York Acad. of Sciences, Feb. 1949 (in press).

141. Mallette, M. F., Taylor, E. C., Jr., and Cain, C. K., J. Am. Chem. Soc., 69, 1814 (1947); Cain, C. K., Mallette, M. F., and Taylor, E. C., Jr., J. Am. Chem. Soc., 70, 3026 (1948).

142. Cain, C. K., Mallette, M. F., and Taylor, E. C., Jr., J. Am. Chem. Soc., 68, 1996 (1946).

143. Cain, C. K., Taylor, E. C., Jr., and Daniel, L. J., J. Am. Chem. Soc., 71, 892 (1949).

144. Daniel, L. J., and Norris, L. C., J. Biol. Chem., 170, 747 (1947).

145. Krebs, E. G., and Norris, E. R., Fed. Proc., 8, 216 (1949).

146. Kalckar, H. M., Kjeldgaard, N. O., and Klenow, H., J. Biol. Chem., 174, 771 (1948).

147. Hitchings, G. H., Elion, G. B., VanderWerff, H., and Falco, E. A., J. Biol. Chem., 174, 765 (1948).

148. Hitchings, G. H., Falco, E. A., and Slerwood, M. B., Science, 102, 251 (1945).

149. Hitchings, G. H., Elion, G. B., and VanderWerff, H., J. Biol. Chem., 174, 1037 (1948).

150. Shive, IV., Conference on Development and Uses of Antimetabolites, New York Acad. Science, Feb., 1949 (in press).

151. Hall, D. A., Biochem. J., 41, 294 (1947).

152. King, F. E., Spensley, P. C., and Nimmo-Smith, R. H., Nature, 162, 153 (1948).

153. Edwards, P. C., Starling, D., Mattocks, A. M., and Skipper, H. E., Science, 107, 119 (1948).

154. Viscontini, M., and Meier, J., Helv. Chim. Acta, 32, 877 (1949).

155. Martin, G. J., and Beiler, J. M., Arch. Biochem., 15, 201 (1947).

156. Martin, G. J., Tolman, L., and Brendel, R., Arch. Biochem., 15, 323 (1917).

157. Martin, G. J., and Beiler, J. M., J. Am. Pharm. Assoc., Sci. Ed., 37, 32 (1948); Martin, G. J., Avakian, S., Tolman, L., Urist, H., and Moss, J., Am. J. Digestive Diseases, 15, 55 (1948).

158. Williams, R. J., et al., University of Texas Publication No. 4137 (1941), 4237 (1942).

159. Loo, Y. H., and Williams, R. J., University of Texas Publication No. 4507, p. $123(1945)$. 
160. Leuchtenberger, C., Lewisohn, R., Laszlo, D., and Leuchtenberger, R., Proc. Soc. Exptl. Biol. Med., 55, 204 (1944).

161. Lewisohn, R., Leuchtenberger, C., Leuchtenberger, R., and Kieresztesy, J. C., Science, 104, 436 (1946).

162. Lewisohn, R., Laszlo, D., Leuchtenberger, C., and Leuchtenberger, R., "Approaches to Tumor Chemotherapy," Amer. Assoc. Adv. Sci. Symposium, p. 139 (1947).

163. Sugiura, K., "Approaches to Tumor Chemotherapy," Amer. Assoc. Adv. Sci. Symposium, p. 208 (1947).

164. Zahl, P. A., and Hutner, S. H., "Approaches to Tumor Chemotherapy," Amer. Assoc. Adv. Sci. Symposium, p. 214 (1947).

165. Morris, H. P., (Conference Discussion) "Approaches to Tumor Chemotherapy," Amer. Assoc. Adv. Sci. Symposium, p. 195 (1947).

166. Hesselback, M. L., (Conference Discussion) "Approaches to Tumor Chemotherapy," Amer. Assoc. Adv. Sci. Symposium, p. 196 (1947).

167. Farber, S., Cutler, E. C., Hawkins, J. W., Harrison, J. H., Pierce, E. C., 2nd, and Lenz, G. G., Science, 106, 619 (1947).

168. Klainer, M. J., Trans. New York Acad. Sci., II, 10, 71 (1948).

169. Lehv, S. P., Wright, L. T., Weinstraub, S., and Arons, I., Trans. New York Acad. Sci., II, 10, 75 (1948).

170. Rous, P., J. Exptl. Med., 12, 698 (1910); Little, P. A., Oleson, J. J. and SubbaRow, Y., J. Lab. Clin. Med., 33, 1139 (1948).

171. Little, P. A., Sampath, A., and SubbaRow, Y., J. Lab. Clin. Med., 33, 1144 (1948).

172. Woll, E., Trans. New York Acad. Sci., II, 10, 83 (1948).

173. Meyer, L. M., Trans. New York Acad. Sci., II, 10, 99 (1948).

174. Farber, S., Diamond, L. K., Mercer, R. D., Sylvester, R. F., Jr., and Wolf, J. A., New Eng. J. Med., 238, 787 (1948).

175. Levin, W. C., Jacobson, W., and Holt, G., Proc. Centr. Soc. Clin. Res., 21, 88 (1948); Pierce, M., and Alt, H., Proc. Centr. Soc. Clin. Res., 21, 89 (1948); Berman, L., Axelrod, A. R., Vonderheide, E. C., and Sharp, E. A., Proc. Centr. Soc. Clin. Res., 21, 90 (1948).

176. Burchenal, J. H., Burchenal, J. R., Kíushida, M. N., Johnston, S. F., and Williams, B. S., Cancer, 2, 113 (1949).

177. Stock, C. C., Burchenal, J. H., Biesele, J. J., Karnofsky, D. A., Moore, A. E., and Sugiura, K., "Conference on Development and Uses of Antimetabolites," New York Acad. Sci., Feb., 1949 (in press).

178. Schoenbach, E. B., Goldin, A., Goldberg, B., and Ortega, L. G., Cancer, 2, 57 (1949).

179. Sugiura, K., Moore, A., and Stock, C. C., Cancer (in press) (referred to in Ref. 181).

180. Goldin, A., Goldberg, B., Ortega, L. G., and Schoenbach, E. B., Fed. Proc., 8, 57 (1949).

181. Moore, A. E., Stock, C. C., Sugiura, K., and Rhoads, C. P., Proc. Soc. Exptl. Biol. Med., 70, 396 (1949). 


\section{Chapter VID}

\section{THE NICOTINIC ACID GROUP}

\section{Specificity}

Although nicotinic acid was prepared synthetically in 1867 by the oxidation of nicotine, ${ }^{1,2,3}$ it was not isolated from natural products until $1912.4,5,6$ In 1934, nicotinamide was isolated from coenzyme II by Warburg and Christian, ${ }^{7}$ who thereby demonstrated the first biological role of the factor since coenzyme II was recognized as a hydrogen-transporting coenzyme. Euler, Albers, and Schlenk ${ }^{8}$ shortly afterward obtained nicotinamide from coenzyme I. The structure of coenzyme I originally proposed by Schlenk and Euler ${ }^{9}$ is indicated as follows:

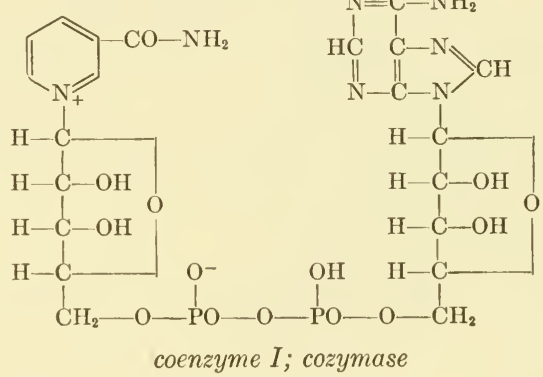

Coenzyme II contains an additional phosphate group and can be converted into coenzyme I.10 The exact location of this phosphate group is still questionable.

The first indication of the importance of this group of factors in nutrition was presented by A. Lwoff and M. Lwoff, ${ }^{11}$ who demonstrated that a factor essential for growth of certain bacteria of the Hemophilus group, ${ }^{12,13}$ the "V" factor, was replaced by either coenzyme I or II, both of which possess properties analogous to those of the "V" factor. Shortly thereafter, the role of nicotinic acid as an essential growth factor was demonstrated for Staphylococcus aureus by Knight ${ }^{14}$ and for Corynebacterium diphtheriae by Mueller. ${ }^{15}$ The activity of nicotinic acid and nicotinamide in preventing blacktongue in dogs was subsequently demon- 
strated by Elvehjem and co-workers. ${ }^{16}$ These reports, particularly with the extension of the biological role of nicotinic acid to treatment of human pellagra, ${ }^{17}, 18$ stimulated an intense search among analogues of nicotinic acid for those which possessed biological activity.

The specificity of the nicotinic acid group for various organisms is indicated in Table 23. It is interesting to note that nicotinic acid cannot replace nicotinamide in the nutrition of certain strains of Pasteurella, and that nicotinamide is essentially inactive in replacing nicotinic acid for Leuconostoc mesenteroides 9135 and 8293 and Leuconostoc dextranicum 8086. Quantitative variations in ability to utilize these compounds are common among various organisms. For example, nicotinamide is approximately ten times as active as nicotinic acid for certain dysentery bacilli, but nicotinic acid is ten times as effective as nicotinamide in stimulating the growth of Corynebacterium diphtheriae..$^{15}$ Most organisms, however, use nicotinic acid and its amide with about the same efficiency. The Hemophilus group which requires the "V" factor cannot utilize either nicotinic acid or its amide. For Hemophilus parainfuenzae and Hemophilus influenzae, the "V" factor requirement is satisfied most effectively by coenzyme I or by the equally active dihydrocoenzyme I. ${ }^{50,51}$ Desaminocoenzyme I is only 60 per cent as active as coenzyme I. As indicated in Table 23, nicotinamide riboside replaces the coenzyme, but is considerably less active on a molar basis. Growth response of the organism to increasing concentrations of the riboside is not proportional to that obtained with corresponding concentrations of coenzyme I. Coenzyme II is also less active than nicotinamide riboside, but the growth response closely parallels that of the riboside, indicating the possibility that it is utilized by prior conversion to the riboside before coenzyme I synthesis. ${ }^{50,} 51$ The amount of nicotinamide riboside just necessary for detectable growth of Hemophilus influenzae or Hemophilus parainfluenzae is less than that of coenzyme I for the same. response. However, for appreciable growth considerably more of the riboside than of coenzyme $\mathrm{I}$ is essential..$^{50,51}$

As indicated in Table 23, organisms which require nicotinic acid or nicotinamide for growth can usually utilize the coenzymes. However, Leuconostoc mesenteroides 8293 and Leuconostoc dextranicum 8086 cannot utilize effectively either coenzyme I or II. ${ }^{39 a}$ Furthermore, coenzyme I injected intravenously is reported to have no therapeutic effect on canine blacktongue, ${ }^{25}$ but administered orally in rats, it is reported to be more active than nicotinamide. ${ }^{54}$ In many instances, coenzymes I and II are less effective as microbial growth factors than either nicotinic acid or nicotinamide. ${ }^{38,46}$ Although neither Leuconostoc mesenteroides 8293 nor Leuconostoc dextranicum 8086 can utilize exogenous coenzyme I or II, both organisms synthesize from nicotinic acid a factor which replaces 


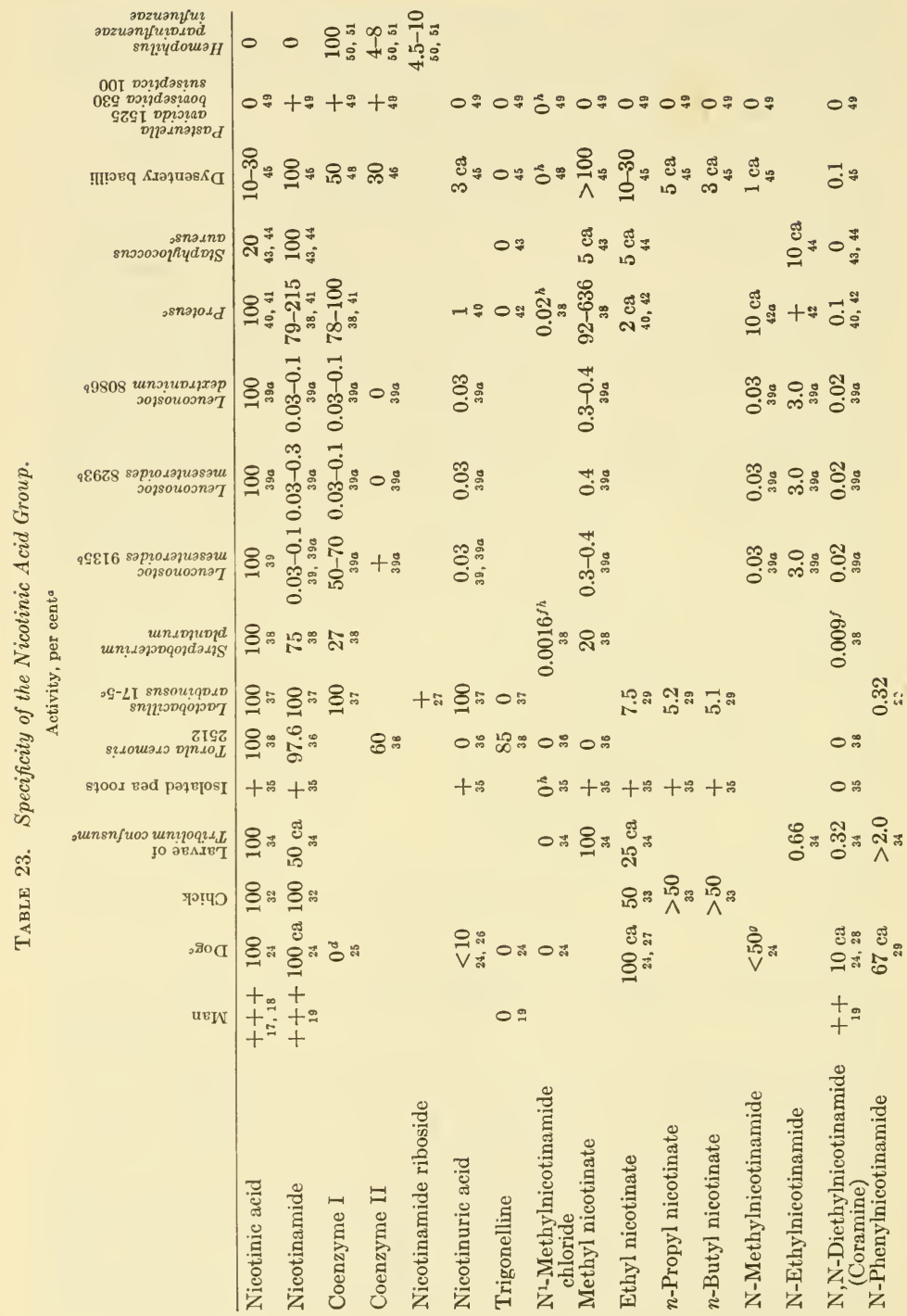




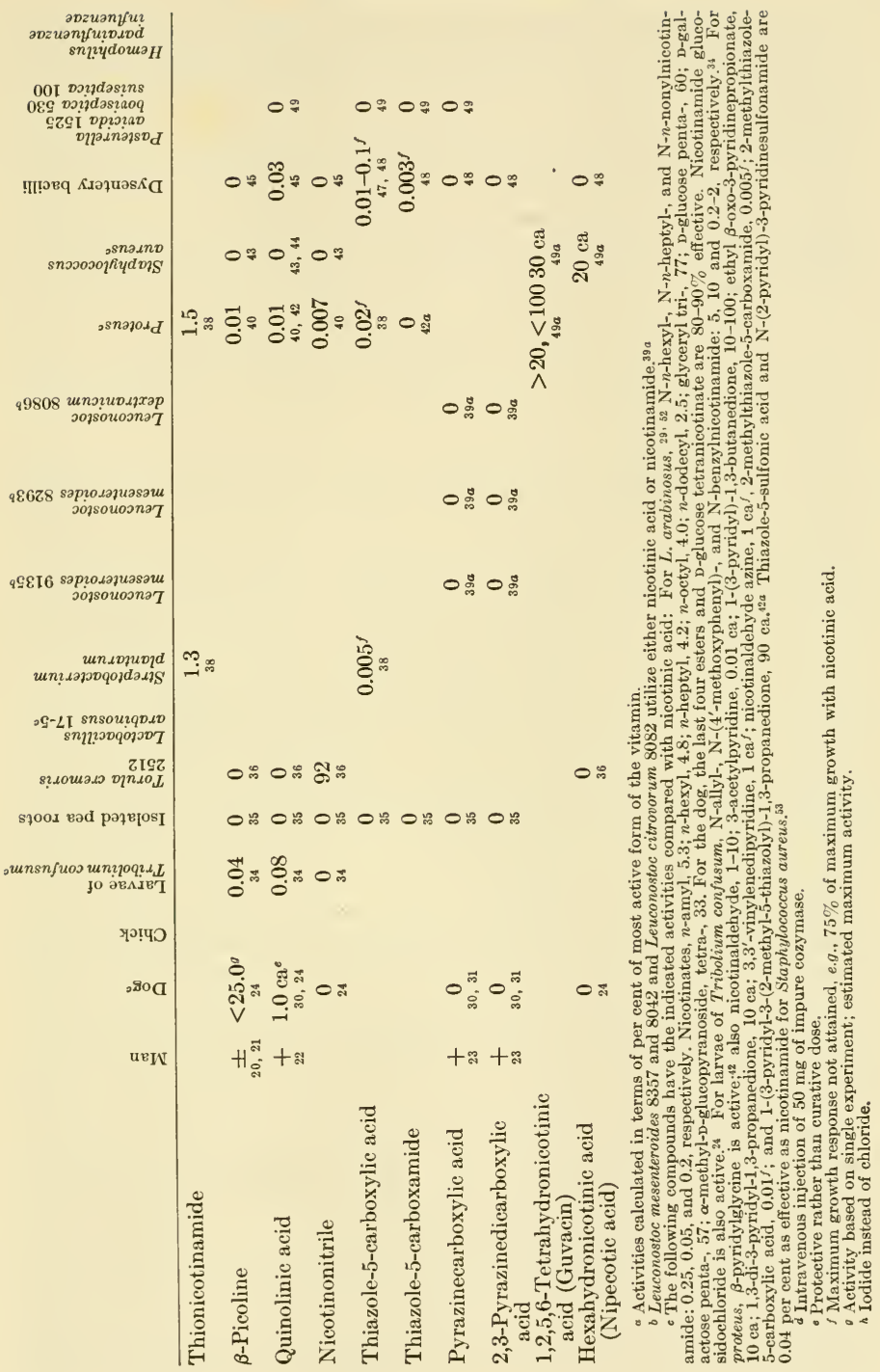


these coenzymes in the nutrition of Hemophilus parainfluenzae. ${ }^{39 a}$ Similar results are obtained with Leuconostoc mesenteroides $9135^{39 \mathrm{a}}$ and with Lactobacillus arabinosus $17-5$, in which nicotinic acid largely exists as coenzyme $I^{55}$

Most metabolites of nicotinic acid which are excreted in the urine of animals are not effectively utilized by many organisms which require nicotinic acid or nicotinamide for growth. Thus, trigonelline appears to be inactive for most organisms; however, for Torula cremoris 2512 it is almost as active as nicotinic acid. $\mathrm{N}^{1}$-Methyl nicotinamide chloride has only very slight ability to replace the nicotinic acid group for a few organisms. Nicotinuric acid is utilized only with difficulty by many organisms, but for some, e.g., Lactobacillus arabinosus, it is approximately as active as nicotinic acid.

Although nicotinuric acid was initially reported on the basis of a single test to have a relatively high curative action in eanine blacktongue, ${ }^{24}$ it has been proposed ${ }^{26}$ on the basis of additional tests that the compound probably should be grouped with pyrazinecarboxylic acid, ${ }^{30,31}$ pyrazine2,3-dicarboxylic acid, ${ }^{30,} 31$ quinolinic acid, ${ }^{30} 3$-aminopyridine, ${ }^{56} 2$-aminonicotinic acid, ${ }^{26}$ and pyrimidine-4-carboxylic acid. ${ }^{26}$ These compounds do not act regularly as blacktongue-preventives, but are sometimes able to replace nicotinic acid, at least partially, in the diet of dogs for extended periods. Nicotinuric acid administered to dogs maintained on a low nicotinic acid diet is excreted almost quantitatively without metabolic change. ${ }^{57}$ This offers further evidence of the inability of the dog to utilize this compound. It is also interesting that nicotinuric acid administered by intravenous injection to human subjects was almost quantitatively excreted unchanged in the urine. ${ }^{58}$

The majority of the biologically active analogues of nicotinic acid are compounds which can be converted to the vitamin by the organism. However, the activities, even though of a low order, of thiazole-5-carboxylic acid or its amide, thiazole-5-sulfonic acid, N-(2-pyridyl)-3-pyridinesulfonamide, pyrazinecarboxylic acid, and 2,3-pyrazinedicarboxylic acid cannot be explained on this basis. It has been suggested that some of these may actually be utilized as such without prior conversion to nicotinic acid.

Administration of pyrazinecarboxylic acid and quinolinic acid was reported to produce an increase in "V" factor activity in the blood and urine of human subjects;22, ${ }^{23}$ but this has not been verified by subsequent work, ${ }^{30}$ in which a slightly different assay method for the "V" factor was employed. Quinolinic acid, pyrazine monocarboxylic acid, or pyrazine-2,3-dicarboxylic acid administered orally to male subjects did not cause a rise in the "V" factor content of blood cells. Neither was synthesis of " $V$ " factor obtained on incubation of the three acids under 
sterile condition with defibrinated human blood. However, these results do not necessarily contradict the reported activity of these compounds in treatment of pellagra, since a change of the " $V$ " factor content of the blood is not a reliable method of determining utilization of analogues of nicotinic acid. This is particularly true in view of the fact that oral administration of nicotinamide to human subjects does not cause a change in the " $V$ " factor concentration of the blood; but oral administration of nicotinic acid is followed by a prompt elevation of the concentration of this factor, as determined by Hemophilus influenzae. ${ }^{59,60}$ The increase in concentration of the factor is paralleled by changes in the rate at which erythrocytes oxidize lactate and malate.

The activity of the esters of nicotinic acid for chicks increases with increasing chain length from ethyl to $n$-butyl. ${ }^{33} n$-Butyl nicotinate is almost as active as nicotinic acid. For bacteria, however, the activity appears to decrease with increasing chain length. Animals appear in most instances to utilize both the esters and $\mathrm{N}$-substituted amides more effectively than most bacteria. The search for utilizable but water-insoluble forms of nicotinic acid has largely been centered around the esters and $\mathrm{N}$-substituted amides. ${ }^{29,52,61,62}$ These forms of the vitamin are needed for enrichment of certain foods, such as corn grits and white rice, which are customarily subject to rinsing before cooking.

Methyl nicotinate has been reported to be the most active form of the vitamin for certain organisms. For example, with glucose as a substrate, the respiration of dysentery bacilli grown on a medium deficient in nicotinamide can be stimulated by coenzyme I, coenzyme II, nicotinamide, nicotinic acid, methyl nicotinate or other derivatives. ${ }^{63}$ Methyl nicotinate is reported to be more active than nicotinamide, which in turn is more active than coenzyme I or II or nicotinic acid. It was suggested that, since these variations cannot be explained by differences in rate of diffusion, the results are incompatible with the theory that nicotinamide serves simply as a precursor of coenzyme I or II or both. ${ }^{63}$

A similar high activity of methyl nicotinate has been reported ${ }^{38}$ for Proteus vulgaris 3056 . The activity of nicotinamide and methyl nicotinate varies with the $\mathrm{pH}$ of the medium. At $\mathrm{pH} 7.2$, these activities are 78 and 92 per cent, respectively, of nicotinic acid; however, at $\mathrm{pH}$ 7.8-8.1, the activities are 215 and 636 per cent, respectively. Thus, methyl nicotinate is significantly more active than either nicotinic acid or nicotinamide under these conditions. For other strains of Proteus, nicotinic acid, nicotinamide, and coenzyme I have been reported ${ }^{41}$ to be equally effective.

The growth requirements of 189 strains of Proteus were investigated, and strains of $P$. vulgaris, $P$. anindologenes, $P$. para-americanus, 
$P$. americanus, $P$. ammonia, $P$. mirabilis, $P$. asiaticus and $P$. nocutarnum were found to have essentially the specificity as indicated in Table 23 for Proteus, with the exception of quinolinic acid, which was inactive at the concentration employed. ${ }^{42}$

After injection into rats of compounds related to nicotinic acid, the increase in urinary excretion of nicotinamide methochloride has been reported in terms of per cent of theoretically possible increase ${ }^{34}$ as follows: nicotinic acid, 11.8; nicotinamide, $22.7 ; \mathrm{N}$-ethylnicotinamide, $17.2 ; \mathrm{N}, \mathrm{N}$ diethylnicotinamide, $16.8 ; \mathrm{N}$-phenylnicotinamide, $6.5 ; \mathrm{N}$-(4'-methyoxyphenyl) nicotinamide, $6.2 ; \mathrm{N}$-benzylnicotinamide, 5.8; nicotinamide methochloride, 62.7 ; quinolinic acid, 5.2; and 3-picoline, 41.3. No increase in urinary excretion of the metabolite was reported for nicotinonitrile, trigonelline, or $\mathrm{N}$-cyclohexylnicotinamide or related methylcyclohexyl derivative. The extent to which 3-picoline is metabolized is interesting.

Even compounds closely related to nicotinic acid are not capable of replacing the vitamin. Thus, isonicotinic acid, $24,35,36,39 \mathrm{a}, 42,43,44$ picolinic acid, ${ }^{20}, 24,35,36,38,39 a, 42,43,44$ dinicotinic acid, ${ }^{35}, 45$ cinchomeronic acid, ${ }^{45}$ 6-methylnicotinic acid, ${ }^{24,36,39 a, 45}$ 2,4-dimethylpyridine-3-carboxylic acid, ${ }^{43}$ 2,4,6-trimethylpyridine-3,5-dicarboxylic acid, ${ }^{40,43}$ 2-aminonicotinic acid, ${ }^{42,49}$ or pyridine betaine-3-carboxylic acid ${ }^{42,49}$ cannot replace nicotinic acid or its amide in the nutrition of a wide variety of organisms. With the exception of Staphylococcus aureus, the hexahydro derivative of nicotinic acid, nipecotic acid $\mathbf{2 4 , 3 6 , 4 5}$ is not dehydrogenated. Ethyl $\beta$-oxo-3-pyridinepropionate (ethyl nicotinoacetate) ${ }^{35,45}$ is not cleaved by some organisms, and nicotine ${ }^{36,43}$ is not oxidized by many organisms. $\mathrm{N}$-Cyclohexylnicotinamide as well as $\mathrm{N}$-(2-methyl-cyclohexyl) nicotinamide cannot replace nicotinic acid in the nutrition of larvae of Tribolium confusum, but the corresponding $\mathrm{N}$-phenyl derivative is active. ${ }^{34}$ Other compounds which are inactive in replacing the vitamin for some organisms include arecoline, ${ }^{35,45} \beta$-aminopyridine, ${ }^{20,35,45} \mathrm{~N}, \mathrm{~N}$-diethylthionicotinamide $^{38}$ thiopicolinamide, ${ }^{38}$ quinoline-2-carboxylic acid, ${ }^{38}$ quinoline-3carboxylic acid, ${ }^{38}$ pyridine-3-sulfonic acid, ${ }^{24}, 36,43,45$ pyridine- 3 -sulfonamide ${ }^{38} \beta$-acetylpyridine, ${ }^{24,}{ }^{45}$ benzoic acid, ${ }^{35}, 49$ pyridine, ${ }^{24,}{ }^{40,}{ }^{45} 2$-picoline, ${ }^{20,36,38,44}$ and 4 -picoline. ${ }^{44}$

Of the last group, 3-acetylpyridine and pyridine-3-sulfonic acid exerted a lethal toxic effect for dogs deficient in nicotinic acid, but did not exert such an action on normal animals. ${ }^{24}$ An occasional report has listed some members of this group as exerting a slight stimulating effect, ${ }^{38}$ or as being capable of replacing nicotinic acid: e.g., 2,6-dimethylpyridine-3,5dicarboxylic acid and dinicotinic acid were reported to give some improvement in pellagra $; 20$ pyridine-3-sulfonic acid and nicotine were reported to be active for Proteus ${ }^{38,40}$ and Streptobacterium plantarum. ${ }^{38}$ 
The order of activity reported is such that contamination with the vitamin cannot be ruled out, particularly since other reports indicate inactivity.

\section{Inhibitory Analogues of the Nicotinic Acid Group}

Although a large number of analogues of nicotinic acid and related compounds have been tested as inhibitors, only a few have been reported to inhibit competitively the functioning of nicotinic acid in biological systems.

3-Pyridinesulfonamide. The first specific reversal of the toxicity of an analogue by nicotinamide was reported by McIlwain, ${ }^{64}$ who found that the inhibition of growth of Staphylococcus aureus caused by 3-<smiles>NS(=O)(=O)c1cccnc1</smiles>

\section{3-pyridinesulfonamide}

pyridinesulfonamide was competitively prevented by the vitamin. The inhibition indices were $50,000,250,000$ and $1,250,000$ for incubation periods of 19, 26 and 43-96 hours, respectively. Growth promoted by nicotinic acid is affected much less by the analogue, and the inhibition under these conditions was essentially negligible, even though nicotinic acid is somewhat less effective than nicotinamide in promoting growth of the organism. Inhibition of growth promoted by a preparation of coenzyme I was not only more intense, but also was not prevented by additional amounts of coenzyme I. It was concluded that with Staphylococcus aureus, nicotinic acid is not used solely for synthesis of this coenzyme.

With Proteus vulgaris, ${ }^{64}$ growth promoted by nicotinamide was prevented by 3 -pyridinesulfonamide, but the analogue was less inhibitory to Proteus vulgaris than to Staphylococcus aureus. The inhibitory effect of the compound on growth changed with time and usually disappeared within a few days. The analogue was less effective in preventing growth stimulated by cozymase and was ineffective in preventing growth stimulated by nicotinic acid.

The toxicity of pyridine-3-sulfonamide for Streptobacterium plantarum is prevented over a narrow range of concentrations by nicotinic acid, nicotinamide, coenzyme I, 5-thiazolecarboxylic acid, and heavy metal salts, particularly iron salts. ${ }^{65}$

3-Pyridinesulfonamide does not inhibit the growth of Escherichia coli, ${ }^{64,66}$ some strains of Proteus vulgaris, or the flagellate, Polytomella caeca. ${ }^{66}$ 
3-Pyridinesulfonic Acid. The growth of Proteus vulgaris has been reported to be effectively inhibited by 3 -pyridinesulfonic acid $(\mathrm{M} / 100){ }^{64}$<smiles>O=S(=O)(O)c1cccnc1</smiles>

\section{3-pyridinesulfonic acid}

Although the toxicity of the inhibitor was not competitively prevented, increased concentrations of nicotinic acid reversed the inhibition after three to four days. When growth of the organism was promoted by an equivalent concentration of nicotinamide, 3-pyridinesulfonic acid did not inhibit it. Growth promoted by a preparation of coenzyme I was more strongly inhibited than that obtained with nicotinic acid as a growth stimulant. These indications that nicotinamide may not be used by Proteus vulgaris solely for the synthesis of coenzyme I have been pointed out by McIlwain. ${ }^{64}$

With Staphylococcus aureus, the inhibition of growth by 3-pyridinesulfonic acid was prevented to some extent by nicotinic acid in a somewhat competitive manner. ${ }^{64}$ The inhibition index was 10,000 and 250,000 for incubation periods of 23 hours and 5-6 days, respectively.

The toxicity of pyridine-3-sulfonic acid $\left(4 \times 10^{-3} \mathrm{M}\right)$ for Proteus vulgaris is reported ${ }^{67}$ to be prevented by either nicotinic acid $\left(1 \times 10^{-3} \mathrm{M}\right)$ or by thiazole-5-carboxamide $\left(1 \times 10^{-3} \mathrm{M}\right)$. The thiazole- 5 -carboxamide without the inhibitor shows slight inhibition of growth of the organism. The possibility that thiazole-5-carboxamide may actually function in the organism in place of nicotinic acid was suggested.

For Lactobacillus acidophilus (Hadley), 3-pyridinesulfonic acid caused half-maximum inhibition of growth at an index of $1700 .{ }^{68}$ However, no data indicating reversal of the inhibition were indicated.

Some inhibition of growth of rats was obtained by administration of 3 -pyridinesulfonic acid in a diet containing a low amount of protein. ${ }^{69}$ The inhibition of growth was prevented by either nicotinic acid or higher amounts of protein in the diet. However, 3-pyridinesulfonic acid did not appreciably affect the onset and reversibility of the effects of nicotinic acid deficiency in dogs. ${ }^{70}$ The sulfonic acid does not produce symptoms of nicotinic acid deficiency in mice. ${ }^{11}$

Another interesting effect is stimulation of growth of Staphylococcus aureus by low concentrations of 3-pyridinesulfonic acid (M/5000 to $\mathrm{M} / 1000$ ), which has been reported to occur in the presence of suboptimal concentrations of nicotinic acid; however, no stimulation was obtained even at high concentrations $(\mathrm{M} / 100)$ in the absence of nicotinic acid.53 
Methyl 3-Pyridyl Ketone (3-Acetylpyridine). For Streptobacterium plantarum, 3-acetylpyridine is toxic only at high concentrations, and the<smiles>CC(=O)c1cccnc1</smiles>

methyl 3-pyridyl ketone (3-acetylpyridine)

inhibition is not reversed by nicotinic acid..$^{72}$ However, the analogue is toxic for nicotinic acid-deficient dogs but not for normal dogs. ${ }^{24}$ This suggested the possibility that it might be used to produce symptoms of nicotinic acid deficiency in mice. ${ }^{73}$ With doses of 2 to $4 \mathrm{mg}$ per day, the animals began to breathe rapidly very soon after administration of the analogue. In a few hours, difficulties in control of the hind legs developed. Within two days, complete paralysis of the hind legs resulted. The mice appeared emaciated, extremely wet and unkempt. The skin became very red and inflamed, and, after four to seven days, fiery red tongues developed in about half the animals. Supplementing the ration with nicotinic acid for three or four days prior to administration of the analogue prevented the disease; however, only partial success was reported for attempts to cure animals ill with the deficiency disease.

Tryptophan also prevents the toxicity of 3-acetylpyridine, which causes the pellagra-like manifestations. ${ }^{74}$ The amino acid in amounts as little as 0.1 per cent of the diet was sufficient to protect the animals, and was as active as nicotinic acid in exerting the protective action.

3-Acetylpyridine injected into the yolk-sac is toxic for a 4-day old developing chick embryo. ${ }^{75}$ Sublethal concentrations cause certain maldevelopments of the chick, such as undersized, deformed legs and a general edema-like condition over the body. However, approximately $600 \gamma$ of 3 -acetylpyridine per egg was lethal within 24 hours. The toxicity of the analogue was prevented entirely when sufficient nicotinamide was injected simultaneously. The inhibition index required for the lethal effect in all the eggs was 15.4-16.7. The lowest ratio of analogue to metabolite just necessary not to exert any lethal effects was 13.6-14.5. However, in order to prevent maldevelopment of the chick, a still lower ratio of analogue to metabolite was essential. As compared with nicotinamide, nicotinic acid and tryptophan exerted much weaker effects in preventing the toxicity of the analogue for the chick embryo, but there appears to be some slight ability of the embryo to utilize nicotinic acid and tryptophan in place of nicotinamide at this stage of development.

Marked electrocardiographic abnormalities of an isolated rabbit's heart occurred on perfusion with 3 -acetylpyridine. ${ }^{76}$ Administration of nicotin- 
amide prevented these abnormalities. ${ }^{76} \mathrm{~A}$ beneficial effect of nicotinic acid on the isolated heart had been previously observed in the absence of an inhibitory analogue, ${ }^{77}$ and in clinical studies marked alterations in the electrocardiogram, which disappear promptly after nicotinic acid therapy, have been noted in patients with pellagra. ${ }^{78}$

Other Analogues of Nicotinic Acid and Nicotinamide. 6-Aminonicotinic acid which inhibits the utilization of $p$-aminobenzoic acid for a<smiles>Nc1ccc(C(=O)O)cn1</smiles>

6-aminonicotinic acid<smiles>O=C(CC(=O)c1ccccc1)c1ccccc1</smiles>

dibenzoylmethane (1,3-diphenyl-1,3-propanedione)

number of organisms (p. 527) prevents the growth of Staphylococcus aureus in a synthetic medium at a concentration of $1 \gamma$ per cc. ${ }^{79}$ The growth inhibition is reported to be prevented by either nicotinic acid or nicotinamide at concentration 0.1 to 0.01 that of the inhibitor. $p$-Aminobenzoic acid does not affect the inhibition. ${ }^{79}$

1,3-Diphenyl-1,3-propanedione (dibenzoylmethane) inhibits the growth of Proteus vulgaris. The inhibition of growth is prevented by sufficient nicotinamide. The inhibition index is reported to be approximately $100 .{ }^{42 a}$

Thiazole-5-carboxamide exerts a slight toxic effect for Staphylococcus aureus at relatively high concentrations. ${ }^{53}$ This effect contrasts with the ability of the compound to prevent the toxicity of 3-pyridinesulfonic acid for Proteus vulgaris.

2-(5'-Thiazolecarboxamido) pyridine neither inhibits nor promotes growth of Staphylococcus aureus. ${ }^{53}$

N-2-Pyridyl-3-pyridinesulfonamide is reported to be less effective as an inhibitor than the corresponding sulfonamide or sulfonic acid. ${ }^{80}$ It has been indicated (Table 23) that it replaces the requirement of Staphylococcus aureus for nicotinic acid. ${ }^{53}$

The toxicities of coramine, picolinic acid, pyridine-3-sulfonic acid, thionicotinamide, thiopicolinamide, quinoline-3-carboxylic acid, quinoline2-carboxylic acid (quinaldinic acid), and N,N-diethylpyridine-3-sulfonamide were all found to be of a low order for Proteus vulgaris and Streptobacterium plantarum, and were not prevented by nicotinic acid or nicotinamide. The latter two were toxic themselves at a concentration approaching that of some of these analogues ${ }^{65} 5$-Thiazolecarboxylic acid was toxic for Streptobacterium plantarum only at high concentrations at which its inhibitory effects were not prevented by nicotinic acid or amide. ${ }^{65}$ 
A number of analogues of nicotinic acid have been reported to be inactive as inhibitory analogues of the vitamin. These include; 1,2-dinicotinylhydrazine ${ }^{81}$ nicotinamide, ${ }^{82}$ nicotinhydrazide, 2 -fluoronicotinic acid, ${ }^{83}$ 6-fluoronicotinic acid, ${ }^{83}$ 5-bromonicotinic acid, and 5-bromonicotinamide.

The synthesis of 5 -fluoronicotinic acid and 5 -fluoronicotinamide has<smiles>O=C(O)c1cncc(F)c1</smiles>

5-fluoronicotinic acid<smiles>NC(=O)c1cncc(F)c1</smiles>

5-fluoronicotinamide

been recently reported, but the biological action of the analogues are not as yet available. ${ }^{84}$ Preliminary tests have indicated that these compounds are the most effective nicotinic acid antagonists as yet reported. The analogues, particularly the acid, inhibit a large number of bacteria at relatively low inhibition indices. ${ }^{85}$

Natural Antagonisms Related to Nicotinic Acid. Pellagra has long been associated with the eating of corn, and evidence has been presented which indicates that nicotinic acid is required in higher amounts if corn is included in the diet of animals. ${ }^{81,86,87}$ In fact, rats which ordinarily do not require nicotinic acid need either a supplement of nicotinic acid or additional tryptophan in the diet to overcome the retardations of growth resulting from the inclusion of corn grits in a low-protein diet. ${ }^{88}$ Since supplementary tryptophan administered either orally or subcutaneously to the rat causes increased excretion of metabolites of the nicotinic acid group, this animal appears to synthesize nicotinic acid from tryptophan. ${ }^{89}$ Administered to patients with pellagra, tryptophan (6-g oral doses) causes a remission of typical acute pellagrous lesions with increased urinary excretion of $\mathrm{N}^{1}$-methylnicotinamide. ${ }^{90}$ Tryptophan does not, however, affect the nicotinic acid requirement of many organisms, e.g., Tetrahymena geleii $\mathrm{W}^{91}$ and most lactobacilli. Evidence has been presented indicating that the conversion of tryptophan to nicotinic acid in the rat is impaired by certain amino acids, such as glycine and threonine, by tryptophan-free proteins, or by corn grits. ${ }^{92,93}$ However, the mode of action of the responsible substances is largely unknown. In mice fed a low-protein diet a weakly basic water-soluble substance which has been concentrated about 100,000 times from corn causes a disease similar to pellagra which is preventable or curable with nicotinamide. ${ }^{94}$ Indoleacetic acid has been reported to retard growth of rats in a manner similar to corn, and its effect on growth is prevented by either nicotinic acid or tryptophan. ${ }^{95}$ Although other reports fail to confirm this effect of indoleacetic acid ${ }^{69,96}$ there appear to be naturally occurring materials which 
exert an inhibition either directly or indirectly on nicotinic acid synthesis or functioning in animals. This type of action is probably more widespread than has been generally recognized.

The synthesis of nicotinamide from ornithine or ammonium lactate by Escherichia coli or by mixed cultures of rat caecum contents is inhibited by either 2-,4-,5-, or 7-methyltryptophan. ${ }^{97}$ The involvement of ornithine and $\delta$-amino- $n$-valeric acid in the biosynthesis of guvacin $(1,2,5,6$ tetrahydronicotinic acid) and nicotinic acid had been suggested before the interrelationship of tryptophan and nicotinic acid was discovered. ${ }^{98}$ The effect of these substances on nicotinic acid synthesis and its relationship to the tryptophan process is still obscure.

Analogues of 3-Hydroxyanthranilic Acid. A study of the requirements of a number of mutant strains of Neurospora revealed that one strain could utilize either tryptophan, kynurenine, 3-hydroxyanthranilic acid, or nicotinic acid for growth. ${ }^{99}$ Another strain could utilize only hydroxyanthranilic acid or nicotinic acid, ${ }^{100,101}$ and still another required nicotinic acid and could not utilize hydroxyanthranilic acid, which accumulated in the medium. ${ }^{100,} 101$ These results indicate that kynurenine and hydroxyanthranilic acid represent successive steps in the conversion of tryptophan to nicotinic acid or nicotinamide.

It has been reported that 3-methoxyanthranilic acid is significantly inhibitory to the conversion of tryptophan to nicotinic acid by Neurospora. ${ }^{102}$ Since methylation of 3-hydroxyanthranilic acid may occur in nature, and since methyl-2-methylamino-3-methoxybenzoate has been isolated from the seeds of two species of Nigella, these or similar naturally occurring substances as dietary constituents may affect the transformation of tryptophan to nicotinic acid.

Inhibitions Involving Coenzyme I. The functioning of coenzyme I in glucose dehydrogenase and lactic acid dehydrogenase is prevented competitively by 3 -pyridinesulfonic acid. ${ }^{103}$ The ratios of inhibitor to coenzyme for half-maximum inhibition are approximately 730 and 780 , respectively. Nicotinic acid and nicotinamide also effectively inhibit these enzymes, but nicotinamide methiodide is inactive. 3-Pyridinesulfonamide or its methiodide is somewhat less active than the corresponding sulfonic acid. Although a number of other compounds were inhibitory for these enzymes, the inhibitory effects of many of the substances, e.g., salicylic acid and adenosine, were not prevented by coenzyme I.

Salicylic acid prevents the function of coenzyme I in glucose fermentation by a zymase preparation (yeast). ${ }^{104}$ The inhibition is prevented in a competitive manner by coenzyme I over a range of concentrations. The ratio of concentration of salicylic acid to coenzyme I necessary for halfmaximum inhibition of the fermentation is 662-692. At high concentra- 
tions, nicotinic acid, nicotinamide, and trigonelline were found to inhibit glucose fermentation in this system.

\section{Bibliography}

1. Huber, C., Ann. Chem. Pharm., 141, 271 (1867); Ber., 3, 849 (1870).

2. Weidel, H., Ann. Chem. Pharm., 165, 328 (1873); Ber., 12, 1989 (1879).

3. Laiblin, R., Ber., 10, 2136 (1877).

4. Suzuki, U., Schimamura, T., and Odake, S., Biochem. Z., 43, 89 (1912).

5. Funk, C., J. Physiol., 46, 173 (1913); Brit. Med. J., 1, 814 (1913).

6. Vickery, H. B., J. Biol. Chem., 68, 585 (1926).

7. Warburg, O., and Christian, W., Biochem. Z., 274, 112 (1934); 275, 464 (1935).

8. Euler, H. v., Albers, H., and Schlenk, F., Z. physiol. Chem., 240, 113 (1936).

9. Schlenk, F., and Euler, H. v., Naturwiss., 24, 794 (1936).

10. Adler, E., Elliot, S., and Elliot, L., Enzymologia, 8, 80 (1940).

11. Lwoff, A., and Lwoff, M., Compt. rend., 203, 520, 846 (1936); Proc. Roy. Soc. (London), 122B, 352, 360 (1937).

12. Knight, B. C. J. G., Med. Research Council (Brit.) Special Rept. Series, No. 210 (1936).

13. Scott, W. M., System of Bacteriology, 2, Chap. 6, 326, H. M. Stat. Office, London (1929).

14. Knight, B. C. J. G., Nature, 139, 628 (1937); Biochem. J., 31, 731, 966 (1937).

15. Mueller, J. H., J. Biol. Chem., 120, 219 (1937); J. Bact., 34, 381, 429 (1937).

16. Elvehjem, C. A., Madden, R. J., Strong, F. M., and Woolley, D. W., J. Am. Chem. Soc., 59, 1767 (1937).

17. Fouts, P. J., Helmer, O. M., Lepkovsky, S., and Jukes, T. H., Proc. Soc. Exptl. Biol. Med., 37, 405 (1937).

18. Spies, T. D., Cooper, C., and Blankenhorn, M. A., J. Am. Med. Assoc., 110, 622 (1938).

19. Spies, T. D., Bean, W. B., and Stone, R. E., J. Am. Med. Assoc., 111, 584 (1938).

20. Spies, T. D., Grant, H. M., and Huff, N. E., Southern Med. J., 31, 901 (1938).

21. Vilter, S. P., Bean, W. B., and Spies, T. D., Southern Med. J., 31, 1163 (1938).

22. Vilter, R. W., and Spies, T. D., Lancet, 1939, II, 423.

23. Bills, C. E., McDonald, F. G., and Spies, T. D., Southern Med. J., 32, 793 (1939).

24. Woolley, D. W., Strong, F. M., Madden, R. J., and Elvehjem, C. A., J. Biol. Chem., 124, 715 (1938).

25. Daft, F. S., Fraser, H. F., Sebrell, W. H., and Pittman, M., Science, 88, 128 (1938).

26. Dann, W. J., and Handler, P., Proc. Soc. Exptl. Biol. Med., 48, 355 (1941).

27. Elvehjem, C. A., and Teply, L. J., Chem. Rev., 33, 185 (1943).

28. Smith, D. T., Margolis, G., and Margolis, L. H., J. Pharmacol. Exptl. Therap., 68, 458 (1940).

29. Badgett, C. O., Provost, R. C., Jr., Ogg, C. L., and Woodward, C. F., J. Am. Chem. Soc., 67, 1135 (1945).

30. Dann, W. J., Kohn, H. I., and Handler, P., J. Nutrition, 20, 477 (1940).

31. Waisman, H. A., Mickelson, O., McKibbin, J. M., and Elvehjem, C. A., J. Nutrition, 19, 483 (1940).

32. Briggs, G. M., Jr., Mills, R. C., Elvehjem, C. A., and Hart, E. B., Proc. Soc. Exptl. Biol. Med., 51, 59 (1942).

33. Briggs, G. M., Jr., Luckey, T. D., Teply, L. J., Elvehjem, C. A., and Hart, E. B., J. Biol. Chem., 148, 517 (1943).

34. Ellinger, P., Fraenkel, G., and Abdel Kader, M. M., Biochem. J., 41, 559 (1947).

35. Bonner, J., Plant Physiol., 15, 553 (1940).

36. Williams, W. L., J. Biol. Chem., 166, 397 (1946). 
37. Snell, E. E., and Wright, L. D., J. Biol. Chem., 139, 675 (1941).

38. Möller, E. F., and Birkofer, L., Ber., 75, 1108 (1912); Möller, E. F., Z. physiol. Chem., 260, 246 (1939).

39. Johnson, B. C., J. Biol. Chem., 159, 227 (1945); Gaines, S., and Stahly, G. L., J. Bact., 46, 441 (1943).

39a. Koser, S. A., and Kasai, G. J., J. Infectious Diseases, 83, 271 (1948).

40. Lwoff, A., and Querido, A., Compt. rend. soc. biol., 130, 1569 (1939).

41. Fildes, P., Brit. J. Exptl. Path., 19, 239 (1938).

42. Pelczar, M. J., Jr., and Porter, J. R., J. Bact.,. 39, 429 (1910).

42a. Raoul, Y., Bull. soc. chim. biol., 30, 896 (1948).

43. Knight, B. C. J. G., and McIlwain, H., Biochem. J., 32, 1241 (1938).

44. Landy, M., Proc. Soc. Exptl. Biol. Med., 38, 504 (1938).

45. Dorfman, A., Koser, S. A., Reames, H. R., Swingle, K. F., and Saunders, F., J. Infectious Diseases, 65, 163 (1939); Koser, S. A., Dorfman, A., and Saunders, F., Rept. Proc. Ird Intern. Congr. Microbiol., 1939, 496 (1940); Dorfman, A., Koser, S. A., and Saunders, F., J. Am. Chem. Soc., 60, 2004 (1938).

46. Dorfman, A., Koser, S. A., Horwitt, M. K., Berkman, S., and Saunders, F., Proc. Soc. Exptl. Biol. Med., 43, 434 (1940).

47. Schmelkes, F. C., Science, 90, 113 (1939).

48. Koser, S. A., Dorfman, A., and Saunders, F., Proc. Soc. Exptl. Biol. Med., 43, 391 (1940).

49. Berkman, S., J. Infectious Diseases, 71, 201 (1942).

49a. Euler, H. v., Högberg, B., Karrer, P., Salomon, H., and Ruckstuhl, H., Helv. Chim. Acta, 27, 382 (1944).

50. Schlenk, F., and Gingrich, W., J. Biol. Chem., 143, 295 (1942).

51. Gingrich, W., and Schlenk, F., J. Bact., 47, 535 (1944).

52. Strong, F. M., Lutwak, L., and Faroogue, M. A., Arch. Biochem., 18, 297 (1948).

53. Erlenmeyer, H., Bloch, H., and Kiefer, H., Helv. Chim. Acta, 25, 1066 (1942).

54. Euler, H. v., Malmberg, M., Robeznieks, I., and Schlenk, F., Naturwiss., 26, 45 (1938).

55. McIlwain, H., Stanley, D. A., and Hughes, D. E., Biochem. J., 44, 153 (1949).

56. SubbaRow, Y., Dann, W. J., and Meilman, E., J. Am. Chem. Soc., 60, 1510 (1938) ; Strong, F. M., Madden, R. J., and Elvehjem, C. A., J. Am. Chem. Soc., 60, 2564 (1938); SubbaRow, Y., and Dann, W. J., J. Am. Chem. Soc., 60, 2565 (1938).

57. Sarett, H. P., J. Nutrition, 23, 35 (1942).

58. Sarett, H. P., Huff, J. W., and Perlzweig, W. A., J. Nutrition, 23, 23 (1942).

59. Hoagland, C. L., Ward, S. M., and Shank, R. E., J. Biol. Chem., 151, 369 (1943).

60. Hoagland, C. L., and Ward, S. M., J. Biol. Chem., 146, 115 (1942).

61. Gunderson, F. L., Science, 98, 277 (1943).

62. Huber, W., Boehme, W., and Laskowski, S. C., J. Am. Chem. Soc., 68, 187 (1946).

63. Saunders, F., Dorfman, A., and Koser, S. A., J. Biol. Chem., 138, 69 (1941).

64. McIlwain, H., Brit. J. Exptl. Path., 21, 136 (1941).

65. Möller, E. F., and Birkofer, L., Ber., 75, 1118 (1942).

66. Matti, J., Nitti, F., Morel, M., and Lwoff, A., Ann. inst. Pasteur, 67, 240 (1941).

67. Erlenmeyer, H., and Würgler, W., Helv. Chim. Acta, 25, 249 (1942).

68. Dreizen, S., Scholz, E., and Spies, T. D., Proc. Soc. Exptl. Biol. Med., 68, 620 (1948).

69. Krehl, W. A., Henderson, L. M., Huerga, J. de la, and Elvehjem, C. A., J. Biol. Chem., 166, 531 (1946).

70. Gaebler, O. H., and Herman, E. V., Fed. Proc., 6, 254 (1947).

71. Woolley, D. W., and White, A. G. C., Proc. Soc. Exptl. Biol. Med., 52, 106 (1943).

72. Auhagen, E., Z. physiol. Chem., 274, 48 (1942). 
73. Woolley, D. W., J. Biol. Chem., 157, 455 (1945).

74. Woolley, D. W., J. Biol. Chem., 162, 179 (1946).

75. Ackermann, W. W., and Taylor, A., Proc. Soc. Exptl. Biol. Med., 67, 449 (1948).

76. Braun, K., J. Pharmacol. Exptl. Therap., 95, 58 (1949).

77. Calder, R. M., Proc. Soc. Exptl. Biol. Med., 65, 76 (1947).

78. Rachmilewitz, M., and Braun, K., Brit. Heart J., 7, 72 (1945); Am. Heart J., 36, 248 (1948); Braun, K., Roth, J., and Suesskind, S., J. Ped., 30, 177 (1947).

79. Schmidt-Thomé, Z. Naturforsch., 3b, 136 (1948); Chem. Abstr., 43, 3884 (1949).

80. McIlwain, H., Nature, 146, 653 (1940).

81. Gautier, J. A., Compt. rend., 222, 394 (1946).

82. Gautier, J. A., and Cordier, P., Bull. soc. chim. biol., 29, 514 (1947).

83. Minor, J. T., Hawkins, G. F., VanderWerf, C. A., and Roe, A., J. Am. Chem. Soc., 71, 1125 (1919).

84. Hawkins, G. F., and Roe, A., J. Org. Chem., 14, 328 (1949).

85. Unpublished work, Lilly Research Laboratories; Biochemical Institute, University of Texas.

86. Aykroyd, W. R., and Swaminathan, M., Indian J. Med. Research, 27, 667 (1940).

87. Krehl, W. A., Teply, L. J., and Elvehjem, C. A., Science, 101, 283 (1945).

88. Krehl, W. A., Teply, L. J., Sarma, P. S., and Elvehjem, C. A., Science, 101, 489 (1945).

89. Rosen, F., Huff, J. W., and Perlzweig, W. A., J. Biol. Chem., 163, 343 (1945).

90. Vilter, R. W., Mueller, J. F., and Bean, W. B., J. Lab. Clin. Med., 34, 409 (1949).

91. Kidder, G. W., Dewey, V. C., Andrews, M. B., and Kidder, R. R., J. Nutrition, 37, 521 (1949).

92. Hankes, L. V., Henderson, L. M., Brickson, W. L., and Elvehjem, C. A., J. Biol. Chem., 174, 873 (1948).

93. Rosen, F., and Perlzweig, W. A., J. Biol. Chem., 177, 163 (1949).

94. Woolley, D. W., J. Biol. Chem., 163, 773 (1946).

95. Kodicek, E., Carpenter, K. J., and Harris, L. J., Lancet, 251, II, 491 (1946).

96. Henderson, L. M., Deodhar, T., Krehl, W. A., and Elvehjem, C. A., J. Biol. Chem., 170, 261 (1947); Rosen, F., and Perlzweig, W. A., Arch. Biochem., 15, 111 (1947); Krehl, W. A., Carvalho, A., and Cowgill, G. R., Fed. Proc., 6, 413 (1947); Sarett, H. P., and Goldsmith, G. A., Fed. Proc., 6, 288 (1947).

97. Ellinger, P., and Abdel Kader, M. M., Nature, 163, 799 (1949) ; Biochem. J., 44, 506 (1919).

98. Huff, J. W., and Perlzweig, W. A., J. Biol. Chem., 142, 401 (1942); Guggenheim, M., Du biogene Amine, Basel and New York, 2nd Edition, p. 174, (1940).

99. Beadle, G. W., Mitchell, H. K., and Nyc, J. F., Proc. Natl. Acad. Sci., U. S., 33, 155 (1947).

100. Mitchell, H. K., and Nyc, J. F., Proc. Natl. Acad. Sci., U. S., 34, 1 (1948).

101. Bonner, D., Proc. Natl. Acad. Sci., U. S., 34, 5 (1948).

102. Mitchell, H. K., Nyc, J. F., and Owen, R. D., J. Biol. Chem., 175, 433 (1948).

103. Euler, H. v., and Skarzynski, B., Arkiv. Kemi Mineral. Geol., 16A, No. 9 (1943) ; Euler, H. v., Ber., 75B, 1876 (1942).

104. Euler, H. v., and Ahlström, L., Z. physiol. Chem., 279, 175 (1943). 


\section{Chapter VIID}

\section{PANTOTHENIC ACID}

\section{Specificity}

Even before the complete structure of pantothenic acid was determined to be $\mathrm{D}-\mathrm{N}$ - $(\alpha, \gamma$-dihydroxy- $\beta, \beta$-dimethylbutyryl $)-\beta$-alanine, preliminary data indicating that the factor was an hydroxy acid conjugated with $\beta$-alanine by an amide linkage ${ }^{1}$ led to the preparation of a number of analogous compounds. Some of these possessed partial activity. ${ }^{2-4}$ Subsequent to the announcement of the structure of pantothenic acid, a number of more closely related analogues of the vitamin were prepared and tested for activity in replacing pantothenic acid in the nutrition of organisms requiring the vitamin.

In Table 24, the specificity of pantothenic acid is indicated by comparison of its activity with that of its analogues. The activity of pantothenic acid resides only in the dextrorotatory form, which has been indicated to be the D-configuration by application of Hudson's amide rule. ${ }^{8,21,22} \mathrm{~L}-(-)$-Panthothenic acid appears to be inactive for organisms requiring the intact vitamin.

The methyl and ethyl esters of pantothenic acid are comparable to the vitamin in promoting the growth of rats, ${ }^{6-9}$ but ethyl pantothenate is only 6.8 per cent as effective as pantothenic acid for Lactobacillus casei ${ }^{6,}{ }^{6}$

Acetylation is reported to destroy the activity of pantothenic acid in natural extracts for chicks ${ }^{23}$ and bacteria. ${ }^{24}$ However, synthetic ethyl monoacetyl D-pantothenate (Table 24) is approximately as active as pantothenic acid for both rats and chicks, but is only 0.7 per cent as effective as the vitamin for Lactobacillus casei, ${ }^{6,7}$ It is necessary to hydrolyze methyl monoacetyl pantothenate to obtain maximal activity with Streptococcus faecalis $\mathrm{R}^{2}{ }^{2}$ Mono- $p$-nitrobenzoyl D-pantothenic acid $(\mathrm{N}-\alpha$ - $p$-nitrobenzoxy- $\gamma$-hydroxy- $\beta, \beta$-dimethylbutyryl- $\beta$-alanine) is inactive for Lactobacillus casei. ${ }^{7}$

The analogues which replace pantothenic acid are usually only partial substitutes for the vitamin in the nutrition of most organisms; however, some analogues appear to be capable of completely replacing the vitamin in the nutrition of some organisms. Of the analogues which appear to act without prior conversion to pantothenic acid, $\mathrm{N}-(\alpha$-hydroxy- $\beta, \beta-\mathrm{di}$ methylolbutyryl)- $\beta$-alanine ("hydroxypantothenic acid") and $\mathrm{N}-(\alpha-$ 
hydroxy - $\beta$-methyl- $\beta$-methylolvaleryl) - $\beta$-alanine ("methylpantothenic acid") are the most effective in replacing the nutritional requirement of pantothenic acid for a wide variety of organisms. The "methylpantothenic acid" appears to be somewhat mǫre active than "hydroxypantothenic acid" for several organisms, but neither of the substituted pantothenic acids exerts an action comparable with that of the vitamin on a wide variety of organisms. In many instances, the activity of the analogue compared with that of the vitamin is greater at low than at high dosage.

Preparation of two of the four diastereoisomers of "methylpantothenic acid" has recently been reported. $.^{25} \mathrm{DL}-\alpha$-Keto- $\beta$-methyl- $\beta$-methylolvalerolactone was resolved into the two optically active forms which were reduced to the corresponding $\alpha$-hydroxy lactones by yeast. Since yeast reduces $\alpha$-keto- $\beta, \beta$-dimethylbutyrolactone to $(-)$-pantolactone, ${ }^{26}$ the two optically active homologues of pantolactone presumably have the same D-configuration on the $\alpha$-carbon, and the two disastereoisomeric "methylpantothenic acids" prepared from these lactones differ only in configuration on the $\beta$-carbon and have a configuration analogous to pantothenic acid on the $\alpha$-carbon. The "methylpantothenic acid" A with a configuration on the $\beta$-carbon presumably similar to alloisoleucine determined by analogy in melting points is 27.8 per cent as active as pantothenic acid. The "methylpantothenic acid" B, presumably with configuration similar to isoleucine, is 62.5 per cent as active as pantothenic acid. Tested at a concentration of $14 \gamma$ per cc of cinchonidine salt, neither of these diastereoisomeric "methylpantothenic acids" allowed a maximum growth response of Streptobacterium plantarum $10 \mathrm{~S}$ in the absence of pantothenic acid..$^{25}$

Since $\mathrm{DL}-\mathrm{N}$ - $(\alpha$-hydroxy- $\beta$-ethyl- $\beta$-methylolvaleryl $)-\beta$-alanine is inactive, ${ }^{12}$ apparently only one of the $\beta$-methyl groups of the butyryl portion of pantothenic acid can be modified without complete loss of the biological activity of the vitamin.

All the analogues capable of replacing pantothenic acid to any extent retain at least one of the two hydroxyl groups. Modifications in vivo may account for the activity of a number of the less effective analogues.

Some analogues of pantothenic acid which are inhibitory have been found to replace pantothenic acid partially. These will be discussed separately.

The alcohol corresponding to pantothenic acid (pantothenyl alcohol) has been found to be as effective as pantothenic acid in preventing achromotrichia in rats. ${ }^{16}$ However, this activity is the result of conversion of the alcohol in vivo to pantothenic acid. ${ }^{17}$ Other warm-blooded animals have been found to carry out this oxidation. ${ }^{17}$ After administration of pantothenyl alcohol, pantothenic acid was excreted in the urine of male human subjects in amounts equal to or slightly greater than after administration of an equivalent amount of calcium pantothenate (100 


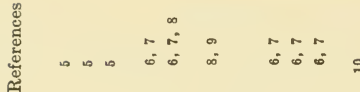

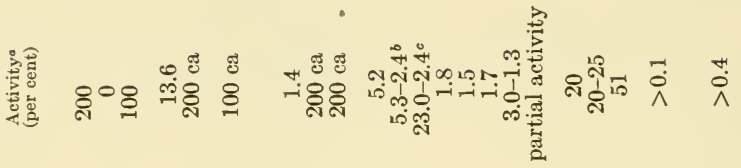
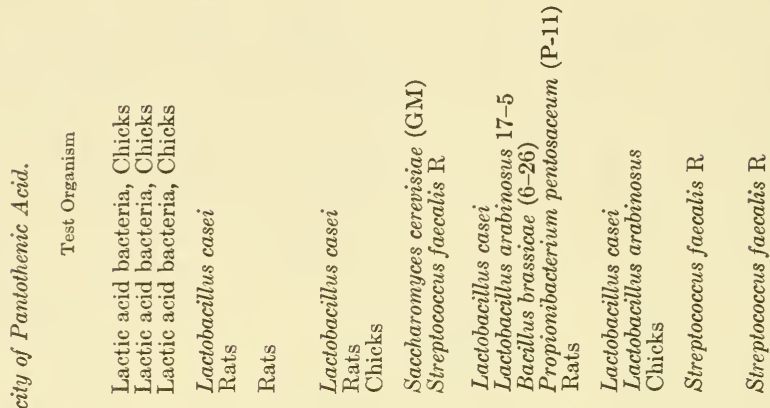

हूँ
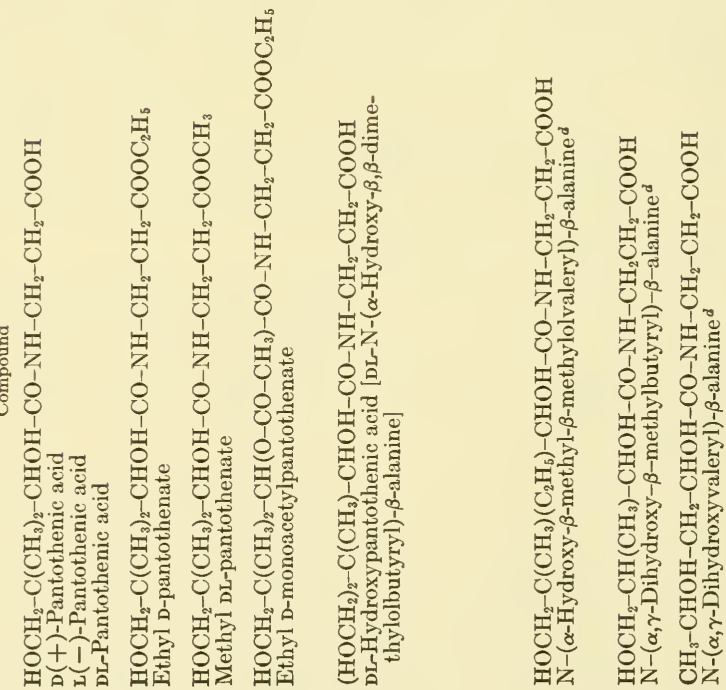
离州

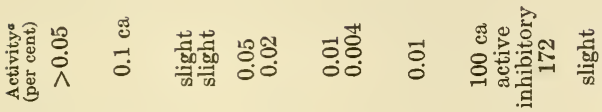

7

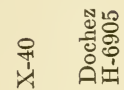

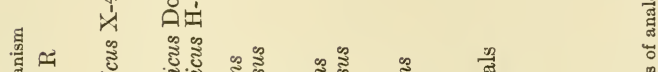

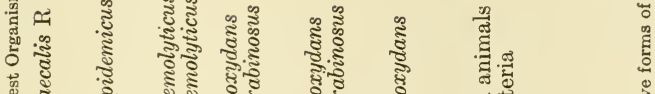

营

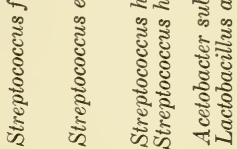

क्ष

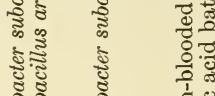

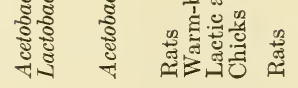

『ึ.

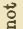



:

है

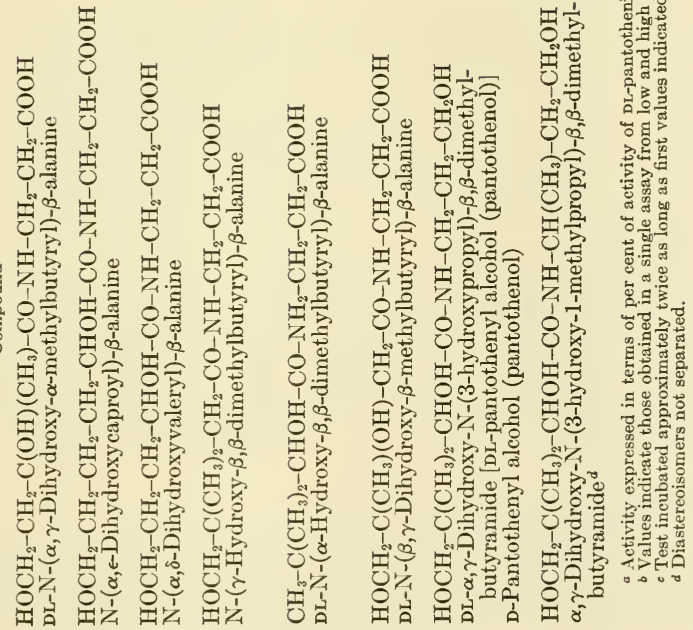


$\mathrm{mg}$ ). When larger amounts were administered, the urinary excretion of pantothenic acid was decidedly greater after administration of pantothenyl alcohol than after administration of an equivalent amount of either sodium or calcium pantothenate. ${ }^{17}, 27,28$ Pantothenyl alcohol cannot replace pantothenic acid in the nutrition of lactic acid bacteria. On the contrary, it inhibits the utilization of pantothenic acid by these organisms, ${ }^{19}$ as subsequently discussed.

While $\beta$-alanine but not pantoic acid can replace pantothenic acid in the nutrition of some organisms such as yeast ${ }^{29,30}$ and some strains of Corynebacterium diphtheriae, ${ }^{31-33}$ pantoic acid, but not $\beta$-alanine, is effective in replacing the vitamin for other organisms such as Acetobacter subdoxydans $621,{ }^{34}$ Streptococcus hemolyticus H 69 D, ${ }^{35}$ and one strain of Clostridium septicum..$^{36}$ Either pantoic acid or $\beta$-alanine alone accelerates the rate of growth of certain strains of Brucella suis; $;^{37}$ the two combined are more effective, but still are not as effective as the intact vitamin. When capable of replacing pantothenic acid, pantoic acid usually is considerably more active than pantolactone. ${ }^{38}$ The results suggest that pantolactone must be hydrolyzed before utilization in the synthesis of pantothenic acid.

Coenzyme $A,{ }^{39}$ the coenzyme which accounts for the major portion of the bound pantothenic acid, is inactive in replacing pantothenic acid in the nutrition of numerous organisms. These include Lactobacillus arabinosus 17-5, Saccharomyces cerevisiae, and Lactobacillus casei. However, coenzyme A administered intraperitoncally is fully active in the chick assay, but on oral administration only 61 per cent of the activity was observed. ${ }^{40}$ Both coenzyme $\mathrm{A}^{41}$ and a naturally occurring conjugate of pantothenic acid ${ }^{42}$ are appreciably more effective than pantothenic acid in promoting the growth of Acetobacter suboxydans 621. A product obtained from coenzyme A by enzymatic action of liver extracts has growthpromoting properties for Acetobacter suboxydans similar to that of the conjugate of pantothenic acid. ${ }^{41}$ Intestinal phosphatase acts on coenzyme A to form a still different product, which does not have enhanced activity for Acetobacter suboxydans ${ }^{41}$ and is not effective for other microorganisms. ${ }^{43}$ Both intestinal phosphatase and liver enzymes are required for the conversion of coenzyme A to a form which is utilized by Lactobacillus arabinosus and most other microorganisms, and is presumably free pantothenic acid. ${ }^{43}$

\section{Inhibitory Analogues of Pantothenic Acid}

Pantoyltaurine. The first growth inhibition specifically and competitively reversed by pantothenic acid was reported by Snell,44 who prepared and tested $\mathrm{N}$-( $\alpha, \gamma$-dihydroxy- $\beta, \beta$-dimethylbutyryl) taurine as an 
inhibitory analogue of pantothenic acid for lactic acid bacteria. Kuhn, Wieland, and Möller ${ }^{45}$ independently and almost simultaneously reported similar findings. Barnett and Robinson ${ }^{15}$ prepared, and McIlwain ${ }^{46}$ tested the same analogue independently, but published their results at a later date with the suggestion of the name "pantoyltaurine" for the analogue. The term "pantoyl" for the $\alpha, \gamma$-dihydroxy- $\beta, \beta$-dimethylbutyryl radical, as suggested by McIlwain, ${ }^{46}$ has since been widely used.

The two optically active forms of pantoyltaurine have been prepared from $\mathrm{D}-(-)$ - and $\mathrm{L}-(+)$-pantolactone by fusion with the sodium salt of taurine at $120^{\circ} \mathrm{C}$ for five hours. The resulting product from the $\mathrm{L}$ pantolactone was only about one-tenth as active as that from the lactone of D-configuration. ${ }^{44}$ If milder conditions were employed for the condensation and the products carefully purified by chromatography and conversion to the quinine salt, the differential in activity was even greater, about 32 -fold. ${ }^{45}$ Since the lactone intermediates were not optically pure and since the reactions are such that some racemization takes place, it seems probable that only the $\mathrm{D}-(+)$ form of pantoyltaurine corresponding to the configuration of the active form of pantothenic acid exerts a bacteriostatic activity. This specificity of configuration has subsequently been confirmed with other analogues of pantothenic acid.

\section{TABLE 25. Pantoyltaurine.}

Test Organism

Streptococcus hemolyticus

Corynebacterium diphtheriae (G1)

Lactobacillus arabinosus 17-5

Diplococcus pneumoniae

Streptobacterium plantarum

Propionibacterium pentosaceum P-11

Saccharomyces cerevisiae GM

Streptococcus faecalis $\mathrm{R}$

Lactobacillus pentosus 124-2

Leuconostoc mesenteroides $\mathrm{P}-60$

Other lactobacilli

$\begin{array}{cc}\text { Inhibition Index } & \text { Reference } \\ 500 & 47 \\ 500 & 47 \\ 1,000 & 44 \\ 1,000 & 47 \\ 2,000 & 45 \\ 5,000 & 44 \\ 8,000 & 44 \\ 10,000 & 44 \\ 150,000 & 44 \\ 150,000 & 44\end{array}$

Table 33

As indicated in Table 25, pantoyltaurine inhibits the growth of a wide variety of microorganisms which require pantothenic acid for growth. The growth inhibition is counteracted specifically by pantothenic acid, and becomes apparent only when the ratio of analogue to pantothenic acid surpasses a critical value. The minimum ratio necessary for maximum inhibition of growth, the inhibition index, is indicated in this table. This competitive relationship exists for each of the organisms over wide ranges in concentration. Inhibition indices vary with time of incubation, composition of the medium, size of inoculum, strain of the organism, etc. Consequently, the values indicated in Table 25 are only approximate, since the results from different laboratories do not agree exactly. 
Organisms which do not require pantothenic acid in their nutrition are usually not affected by pantoyltaurine. This phenomenon is widespread among the analogues of pantothenic acid and will be discussed separately. The toxicity of pantoyltaurine for Streptobacterium plantarum was prevented to some extent by large concentrations of $\beta$-alanine, and to a larger extent by mixtures of pantolactone and $\beta$-alanine. Since the mixtures of pantolactone and $\beta$-alanine do not promote growth in the absence of pantothenic acid, it appears that either a chemical or enzymatic conversion of the pantoyl radical of the analogue to pantothenic acid takes place during the testing.

Early reports ${ }^{48}$ indicated that pantothenic acid deficiency in mice could be produced by long-continued oral administration of pantoyltaurine, but this has not been substantiated. On the contrary, no toxic symptoms have been observed on the administration, either orally or subcutancously, of pantoyltaurine to mice ${ }^{49-51}$ or rats. ${ }^{51}$

Insulin-treated, depancreatized dogs on a diet deficient in pantothenic acid were fed $1 \mathrm{~g}$ of pantoyltaurine daily. After three days, complete refusal of food occurred, but during the first three days with constant food intake, the urinary nitrogen rose, whereas hemoglobin and cell volume fell sharply. ${ }^{52}$ It has not been indicated whether pantothenic acid has a beneficial effect in preventing these effects.

Pantoyltaurine, however, constitutes the first case of an effective chemotherapeutic agent being designed in accordance with the concept of competitive analogue-metabolite growth inhibition. McIlwain and Hawking ${ }^{51}$ reported that rats were protected from 10,000 lethal doses of a virulent strain of streptococcus and less completely from 1,000,000 lethal doses by frequent subcutaneous doses of pantoyltaurine. Although pantoyltaurine is rapidly excreted by rats, the ratio of pantoyltaurine to pantothenic acid in the blood could be maintained above the range necessary for in vitro inhibition. Administration of pantothenic acid with a subsequent increase in its concentration in the blood resulted in reversal of the therapeutic effect of pantoyltaurine, indicating that the mode of action in vivo was analogous to that in vitro. Because of a higher concentration of pantothenic acid in the blood of mice, pantoyltaurine did not exert such a protective action for these animals. Sulfonamide-resistant streptococci were just as sensitive to pantoyltaurine as the nonresistant strains.

In a series of studies on the mode of action of pantoyltaurine, McIlwain ${ }^{53}$ found that low concentrations of pantoyltaurine inhibited the initiation of growth of $\beta$-hemolytic streptococci, but, when the analogue was added to growing cultures, the inhibitory effect on growth was not apparent until after a latent period of an hour or more. The action of 
pantothenate in preventing growth inhibition was similarly delayed. The disappearance of pantothenic acid from the culture medium of either streptococci or Corynebacterium diphtheriae was quickly inhibited by pantoyltaurine and promptly recurred after removal of pantoyltaurine. By quantitative experiments, it was shown that the amount of pantothenic acid consumed in such a process by streptococci and other organisms during normal growth was in considerable excess of their ordinary needs. No correlation was apparent between the rate of destruction of pantothenic acid by an organism and the sensitivity of the organism to pantoyltaurine. The process of pantothenic acid destruction ${ }^{54}$ has been shown to be independent of growth and oxygen consumption, but was associated with the presence of glucose and a casein hydrolyzate (or related materials) and perhaps magnesium ions. Pantoyltaurine inhibited both growth and the destruction of pantothenic acid, but did not prevent glycolysis appreciably. However, inhibition of glycolysis prevented the inactivation of pantothenic acid. The concentrations of pantoyltaurine (or other analogues) necessary to prevent the disappearance of pantothenic acid from the medium varied over a 300 -fold range with strains of streptococci, of Corynebacterium diphtheriae, and of Proteus morganii, but were correlated with the concentrations required for inhibition of growth of the respective organisms.

Pantothenic acid was found to be present in a bound form in two strains of $\beta$-hemolytic streptococci. Autolysis or enzymatic digestion liberated free pantothenic acid, but even high concentrations of pantoyltaurine did not displace the pantothenic acid from the combined form. Pantothenic acid which was loosely bound was released into saline solutions, but pantoyltaurine did not decrease the quantity remaining with the organism. Thus, no gross displacement of pantothenic acid occurred. ${ }^{55}$

From these results, it was concluded that pantoyltaurine acts as a bacteriostatic agent by preventing the conversion of pantothenic acid to a functional derivative in susceptible bacteria.

In pantothenic acid-deficient yeast, pantoyltaurine does not inhibit fermentation processes stimulated by pantothenic acid. ${ }^{56}$ In Streptococcus hemolyticus,${ }^{57}$ pantoyltaurine inhibits the rate of glycolysis slightly (10-25 per cent), and the effect was prevented by pantothenic acid which alone accelerates glycolysis to a slight extent. ${ }^{57}$ The concentrations of pantoyltaurine affecting glycolysis had no effect on growth.

Pantoyltaurine depressed the growth of Streptococcus hemolyticus by increasing the lag period and decreasing the rate of growth. ${ }^{57}$ The logarithmic period of growth involved two phases - an initial phase during which the rate of growth was considerably decreased, as compared with normal growth, and a later phase characterized by an almost normal 
growth rate which was less susceptible to increasing concentrations of the inhibitor.

McIlwain ${ }^{33}$ obtained resistant strains of Streptococcus hemolyticus and Corynebacterium diphtheriae by serially subculturing the parent strains in increasing concentration of pantoyltaurine. The strains of streptococci which were resistant to pantoyltaurine were just as sensitive to sulfanilamide as the parent strain. Also, sulfanilamide-resistant strains were found to be as susceptible to pantoyltaurine as the parent strain. The resistant strains of streptococci retained their requirement for pantothenic acid and did not appear to destroy pantoyltaurine; but the resistant strains of diphtheriae bacilli were capable of utilizing $\beta$-alanine instead of pantothenic acid, whereas $\beta$-alanine did not replace pantothenic acid in the nutrition of the parent strain. Selection for strains of diphtheria bacilli which could utilize $\beta$-alanine was accomplished in the absence of pantoyltaurine. These strains were resistant to bacteriostasis by pantoyltaurine. Proteus morganii, which normally is not sensitive to inhibition by pantoyltaurine, was found to be inhibited by a mixture of salicylic acid and pantoyltaurine under conditions in which the single components of the mixture were inactive. A similar action was observed in both normal and resistant streptococci. Pantothenic acid was effective in preventing the toxic effects of the mixtures. Strains of Proteus morganii have since been found which are susceptible to inhibition by pantoyltaurine with very high inhibition indices, approximately $200,000 .^{33}$

Analogues Related to Pantoyltaurine. Pantoyltauramide and some of its substituted derivatives, as well as sulfones, sulfoxides, sulfides, a disulfide, and a mercaptan related to pantoyltaurine have been prepared. The formulas and names are listed in Table 26.

DL-N-Pantoyltauramide was found to be active against Streptococcus hemolyticus, Diplococcus pneumoniae, and Corynebacterium diphtheriae, the inhibition indices being $2000,10,000-50,000$, and $2000-10,000$, respectively. ${ }^{46}$ Although it was less active than pantoyltaurine in vitro, and against streptococcal infections in rats, D-N-pantoyltauramide administered to chicks intravenously in enormous doses $(2 \mathrm{~g}$ or more per $\mathrm{kg}$ per day) was found to exert a marked suppressive action upon the growth of Plasmodium gallinaceum in chicks, but not in ducks. The antimalarial activity in terms of quinine equivalents was 0.03 . The inactivity of pantothenic acid analogues in ducks is caused by a difference in the host rather than susceptibility of the organism, but this difference was not the result of variations in the pantothenic acid content of the blood of the hosts. The study of the antimalarial activity of analogues of pantothenic acid was begun subsequent to the discovery that the addition of pantothenic acid to an appropriate medium containing duck erythrocytes parasitized 
with Plasmodium lophurae lengthened the survival period of the parasites. $^{63}$

The condensation product of DL-pantolactone and DL- $\alpha$-phenyltaurine does not appreciably affect the growth of Lactobacillus arabinosus. ${ }^{60}$

DL-N-Pantoyl- $\beta$-mercaptoethylamine and the corresponding disulfide have been found to be approximately as active as pantoyltaurine in preventing the utilization of pantothenic acid by Lactobacillus arabinosus. $\mathrm{Di}(\mathrm{N}$-pantoyl- $\beta$-aminoethyl)sulfide and the corresponding sulfoxide

TABLE 26. Pantoyltaurine and Related Inhibitory Analogues of Pantothenic Acid.

Formula

$\mathrm{R}^{a}-\mathrm{NH}-\mathrm{CH}_{2}-\mathrm{CH}_{2}-\mathrm{SO}_{2}-\mathrm{OH}$

$\mathrm{R}-\mathrm{NH}-\mathrm{CH}_{2}-\mathrm{CH}_{2}-\mathrm{SO}_{2}-\mathrm{NH}_{2}$

$\mathrm{R}-\mathrm{NH}-\mathrm{CH}_{2}-\mathrm{CH}\left(\mathrm{C}_{6} \mathrm{H}_{6}\right)-\mathrm{SO}_{2}-\mathrm{OH}^{c}$

$\mathrm{R}-\mathrm{NH}-\mathrm{CH}_{2}-\mathrm{CH}_{2}-\mathrm{SO}_{2}-\mathrm{N}\left(\mathrm{R}_{1}\right)\left(\mathrm{R}_{2}\right)$

$\mathrm{R}-\mathrm{NH}-\mathrm{CH}_{2}-\mathrm{CH}_{2}-\mathrm{SH}$

$\left(\mathrm{R}-\mathrm{NH}-\mathrm{CH}_{2}-\mathrm{CH}_{2}\right)_{2}-\mathrm{S}_{2}$

$\left(\mathrm{R}-\mathrm{NH}-\mathrm{CH}_{2}-\mathrm{CH}_{2}\right)_{2} \mathrm{~S}$

$\left(\mathrm{R}-\mathrm{NH}-\mathrm{CH}_{2}-\mathrm{CH}_{2}\right)_{2} \mathrm{SO}$

$\left(\mathrm{R}-\mathrm{NH}-\mathrm{CH}_{2}-\mathrm{CH}_{2}\right)_{2} \mathrm{SO}_{2}$

$\mathrm{R}-\mathrm{NH}-\mathrm{CH}_{2}-\mathrm{CH}_{2}-\mathrm{S}-\mathrm{C}_{6} \mathrm{H}_{6}$

$\mathrm{R}-\mathrm{NH}-\mathrm{CH}_{2}-\mathrm{CH}_{2}-\mathrm{SO}-\mathrm{C}_{6} \mathrm{H}_{6}$

$\mathrm{R}-\mathrm{NH}-\mathrm{CH}_{2}-\mathrm{CH}_{2}-\mathrm{SO}_{2}-\mathrm{C}_{6} \mathrm{H}_{6}$

$\mathrm{R}-\mathrm{NH}-\mathrm{CH}_{2}-\mathrm{CH}_{2}-\mathrm{S}-\mathrm{C}_{6} \mathrm{H}_{4} \mathrm{Cl}$

$\mathrm{R}-\mathrm{NH}-\mathrm{CH}_{2}-\mathrm{CH}_{2}-\mathrm{SO}_{2}-\mathrm{C}_{6} \mathrm{H}_{4}-\mathrm{CH}_{3}$

$\mathrm{R}-\mathrm{NH}-\mathrm{CH}_{2}-\mathrm{CH}_{2}-\mathrm{SO}_{2}-\mathrm{C}_{6} \mathrm{H}_{4}-\mathrm{NH}_{2}$

$\mathrm{R}-\mathrm{NH}-\mathrm{CH}_{2}-\mathrm{CH}_{2}-\mathrm{SO}_{2}-\mathrm{C}_{6} \mathrm{H}_{4}-\mathrm{OCH}_{3}$
Name

DL-N-Pantoyltaurine

D-N-Pantoyltaurine

L-N-Pantoyltaurine

DL-N-Pantoyltauramide

D-N-Pantoyltauramide

N-Pantoyl- $\beta$-amino- $\alpha$-phenylethanesulfonic acid

Substituted Pantoyltauramides

DL-N-Pantoyl- $\beta$-mercaptoethylamine

Di(N-Pantoyl- $\beta$-aminoethyl) disulfide ${ }^{b}$

Di(N-Pantoyl- $\beta$-aminoethyl) sulfide ${ }^{c}$

Di(N-Pantoyl- $\beta$-aminoethyl) sulfoxide ${ }^{c} \quad 69$

Di(N-Pantoyl- $\beta$-aminoethyl) sulfone ${ }^{c} \quad 69$

D-(N-Pantoyl- $\beta$-aminoethyl) phenyl sulfide

D-(N-Pantoyl- $\beta$-aminoethyl) phenyl sulfoxide

D-(N-Pantoyl- $\beta$-aminoethyl) phenyl sulfone

D-(N-Pantoyl- $\beta$-aminoethyl) $p$-chlorophenyl sulfide

DL-(N-Pantoyl- $\beta$-aminoethyl) $p$-tolyl sulfone

DL-(N-Pantoyl- $\beta$-aminoethyl) $p$ aminophenyl sulfone

DL-(N-Pantoyl- $\beta$-aminoethyl) $p$ methoxyphenyl sulfone

61
References

$15,44,45,46$

44,45

44,45

16,46

58,69

60

Table 27

69

a $\mathrm{R}$ represents the pantoyl group, $\mathrm{HOCH}_{2}-\mathrm{C}\left(\mathrm{CH}_{3}\right)_{2}-\mathrm{CHOH}-\mathrm{CO}-$

b A crystalline compound, one of the two diastereoisomers.

c Presumably a mixture of diastereoisomers.

and sulfone were reported to be less effective than pantoyltaurine in inhibiting the growth of Lactobacillus arabinosus. In vivo tests indicated that these compounds were less effective than pantoyltaurine in preventing streptococcal infections in rats.

$\mathrm{D}$ (N-Pantoyl- $\beta$-aminoethyl) phenyl sulfide, the corresponding sulfoxide and sulfone, and the corresponding $p$-chlorophenyl sulfide have been prepared ${ }^{64}$ and tested for antimalarial activity ${ }^{61}$ in chicks infected with. Plasmodium gallinaceum. The quinine equivalents obtained for the suppressive action were $0.8,1.5,1.0$ and 1.0 , respectively.

The phenyl sulfide and sulfoxide have also been found to be effective. against Trichomonas vaginalis with inhibition indices of 3.1 and 140 , 
respectively, for 9 days' incubation in vitro. ${ }^{65}$ The phenyl sulfide is also effective in vitro against Trichomonas foetus and Trichomonas gallinae. However, even at high concentrations, it did not affect Trichomonas vaginalis infection in monkeys and human beings.

The phenyl sulfide has been reported to have an extremely low acute and chronic oral toxicity in several animal species, ${ }^{66}$ and local irritation effects are not obtained even upon repeated administration. An anemia developed in monkeys which gradually disappeared upon discontinuing the administration of the compound. Smooth muscle was generally stimulated by the analogue.

DL (N-Pantoyl- $\beta$-aminoethyl) $p$-tolyl sulfone and the corresponding $p$-aminophenyl and $p$-methoxyphenyl sulfones inhibited competitively the functioning of pantothenic acid in Lactobacillus casei with inhibition indices of approximately 6400,6400 and 1600, respectively. Streptococcus pyogenes was also inhibited by these compounds, but only a slight chemotherapeutic effect was obtained in experimental streptococcal infections in rats.

Substituted Pantoyltauramides. Since pantoyltaurine and its amide had been found to inhibit competitively the utilization of pantothenic acid in a relatively large number of bacteria, many substituted amides of pantoyltaurine were prepared as possible chemotherapeutic agents, particularly against the malarial parasites and streptococci. The activities of these compounds against Streptococcus hemolyticus C203 in vitro aild in infected mice and as suppressives against the malarial parasites in chicks are shown in Table 27. Streptococcus viridans, Streptococcus agalactiae, and pneumococci were also susceptible to this group of inhibitors. Sulfonamide-resistant streptococci were just as susceptible to the substituted pantoyltauramides as the normal strains.

It is interesting to note that the substituted amides are extremely effective inhibitors of the utilization of pantothenic acid in Streptococcus hemolyticus $\mathrm{C} 203 .{ }^{70}$ The more active of the compounds are approximately 10 to 20 times as effective as pantoyltaurine in vitro. Also, the protective activity of the substituted pantoyltauramides in experimental streptococcal infections in mice is in contrast to the inability of pantoyltaurine to exert any protective action for mice. Even the chemotherapeutic activity of pantoyltaurine which was observed in rats was obtained only with large doses administered frequently. ${ }^{51}$

All the substituted pantoyltauramides were relatively nontoxic to mice and rats, and with most of the analogues both the chemotherapeutic activity and in vitro inhibition of growth were prevented by higher concentrations of pantothenic acid. Of the compounds listed, the two most effective in maintaining blood levels and exerting a protective action on 


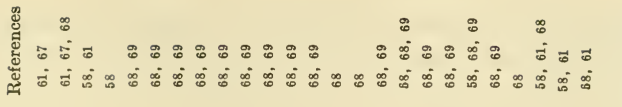

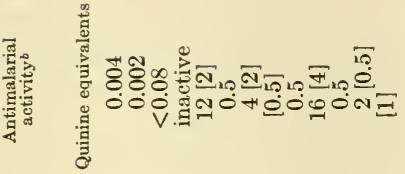

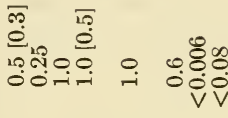

กิ

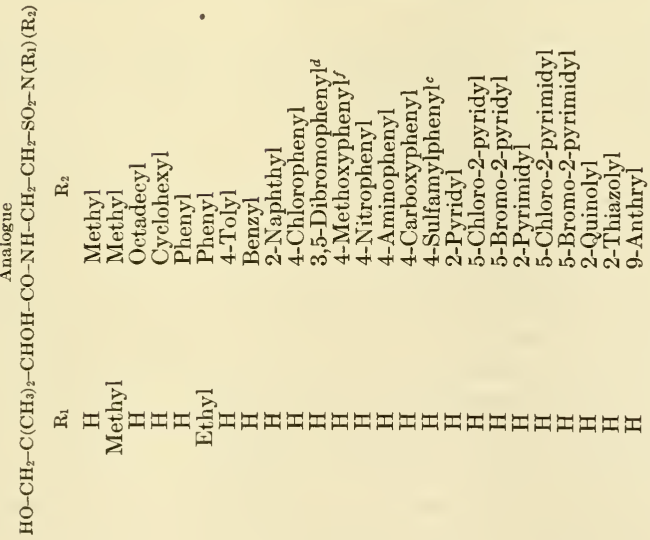


mice infected with Streptococcus hemolyticus C203 were D-pantoyltauramido-4-chlorobenzene and D-pantoyltauramido-3,5-dibromobenzene. However, only limited concentrations of these two compounds were found to be reversed by pantothenic acid. This afforded an interesting comparison of the relative activities of analogues of $\mathrm{D}$ - and L-configuration. The concentration of L-pantoyltauramido-3,5-dibromobenzene necessary for inhibition of growth was found to be essentially equal to the lowest concentration of the D-form which pantothenic acid did not competitively reverse. Also, L-2-(pantoyltauramido) pyridine was inactive at a ratio of inhibitor to pantothenic acid of 6400 , while the inhibition index of the compound of D-configuration was 100 . Hence, the analogues of L-configuration appear to be inactive as competitive antagonists of pantothenic acid.

These pantoyltauramide derivatives also have antiplasmodial activity in blood, but not in sporozoite-induced infections of Plasmodium gallinaceum in chickens. This suggests that the blood phases of this infection require pantothenic acid. The tissue phases either may not require the vitamin or may obtain sufficient supplies of pantothenic acid or other agents in the tissues to prevent the inhibitory action of the pantothenic acid analogues. The most active analogue is D-pantoyltauramido-4-chlorobenzene, which is four times as active as quinine in a standard four-day trophozoite-induced infection (in brackets in Table 27), or sixteen times as active in a more sensitive test where the peak parasitemias were not reached until seven days after infection. The suppressive action of the analogues was completely prevented by addition of adequate quantities of pantothenic acid to the diet.

The pantoyltauramides were found to be toxic for chicks, but only at concentrations well above those required for therapeutic doses. ${ }^{69}$ At a concentration of 0.5 per cent in the diet, D-pantoyltauramido-4-chlorobenzene reduced food intakes markedly and caused death of birds after the twelfth day. At concentrations of 0.1 per cent, the analogue caused only slightly reduced weight gains in a ten day period, and 0.025 per cent (40 mg/ $\mathrm{kg} /$ day) of the analogue did not affect growth of the chicks. The toxicity of even 0.5 per cent of the analogue in the diet was prevented by supplementation of 0.025 per cent of calcium D-pantothenate. An increase was obtained in immature erythrocytes which was directly related to the size of the dose of the analogue and length of treatment, although there was no effect on the total erythrocyte count. Concentrations in the diet as low as 0.0067 per cent gave significant increases in immature erythrocytes in ten days. However, rats receiving $0.25 \mathrm{~g} / \mathrm{kg} /$ day of the analogue maintained a normal blood picture even after 30 days of treatment. Pantoyltauramidobenzene, pantoyltauramido-5-bromobenzene, pantoyl- 
tauramido-4-methoxybenzene, pantoyltauramido-4-methylbenzene and pantoyltauramido-4-nitrobenzene caused similar alterations in the blood picture of chicks. The toxicities were roughly proportional to the antimalarial activities.

N-Pantoyl Amino Acids. Investigations concerning the specificity of pantothenic acid have resulted in the preparation of a number of $\mathrm{N}$-pantoyl derivatives of amino acids or their esters. DL-Pantolactone was condensed with the esters of DL-alanine, DL- $\beta$-aminobutyric acid, L-aspartic acid and DL-lysine, and the resulting products were found to be inactive in replacing pantothenic acid as a growth factor. ${ }^{71}$ In the isolation of pantothenic acid from livers of tunny fish, an impurity which was present in higher concentrations than pantothenic acid was found to be a homologue of pantothenic acid, composed of L-leucine and a pantoic acid homologue containing seven carbon atoms. This suggested the preparation of the pantoyl derivative of L-leucine, which was found to be inactive in replacing pantothenic acid for Streptobacterium plantarum. ${ }^{72}$ Pantoyl-y-aminobutyric acid has been found to be unable to replace pantothenic acid in preventing achromotrichia in rats. ${ }^{16}$ Also, the pantoyl derivatives of lysine, leucine, and valine have no marked activity in replacing the requirement of Proteus morganii for pantothenic acid. ${ }^{15}$

Condensation products of DL-pantolactone with glycine, DL- $\alpha$-aminobutyric acid, DL- $\alpha$-aminoisobutyric acid, DL- $\alpha$-amino- $\alpha$-ethylbutyric acid, and DL-norvaline are essentially inactive for Lactobacillus arabinosus $17-5 .{ }^{73}$ Corresponding condensation products of phenylalanine, 2-aminocyclohexanecarboxylic acid, and nipecotic acid are also essentially inactive for Lactobacillus casei ${ }^{62}$

Prepared as a possible substitute for pantothenic acid, the $\alpha$-methyl analogue of pantothenic acid was found to have slight growth-promoting activity, particularly for Saccharomyces cerevisiae G. M.; however, the compound was found to prevent the utilization of pantothenic acid by Lactobacillus casei. ${ }^{74}$ Several $\mathrm{N}$-pantoyl amino acids, as indicated in Table 28, have since been found to be competitive antagonists of pantothenic acid. With the possible exception of $\mathrm{N}$-pantoyl- $\epsilon$-aminocaproic acid, $N$-pantoyl- $\beta$-aminobutyric acid appears to be the most effective of this group as an antagonist of pantothenic acid. Since the product tested is a mixture of two racemic diastereoisomers, the inhibitory activity may result from the action of only one of the four forms, and certainly results from the action of no more than the two forms containing the pantoyl group of D-configuration. For comparison with the other analogues, the inhibition indices for $\mathrm{N}$-pantoyl- $\beta$-aminoisobutyric acid presumably should be doubled, since it alone was prepared from D-pantolactone.

In some cases with the $\alpha$-substituted pantothenic acids, $\mathrm{N}$-pantoyl- 


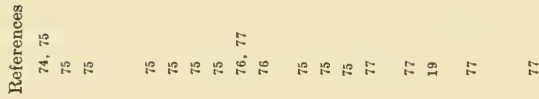

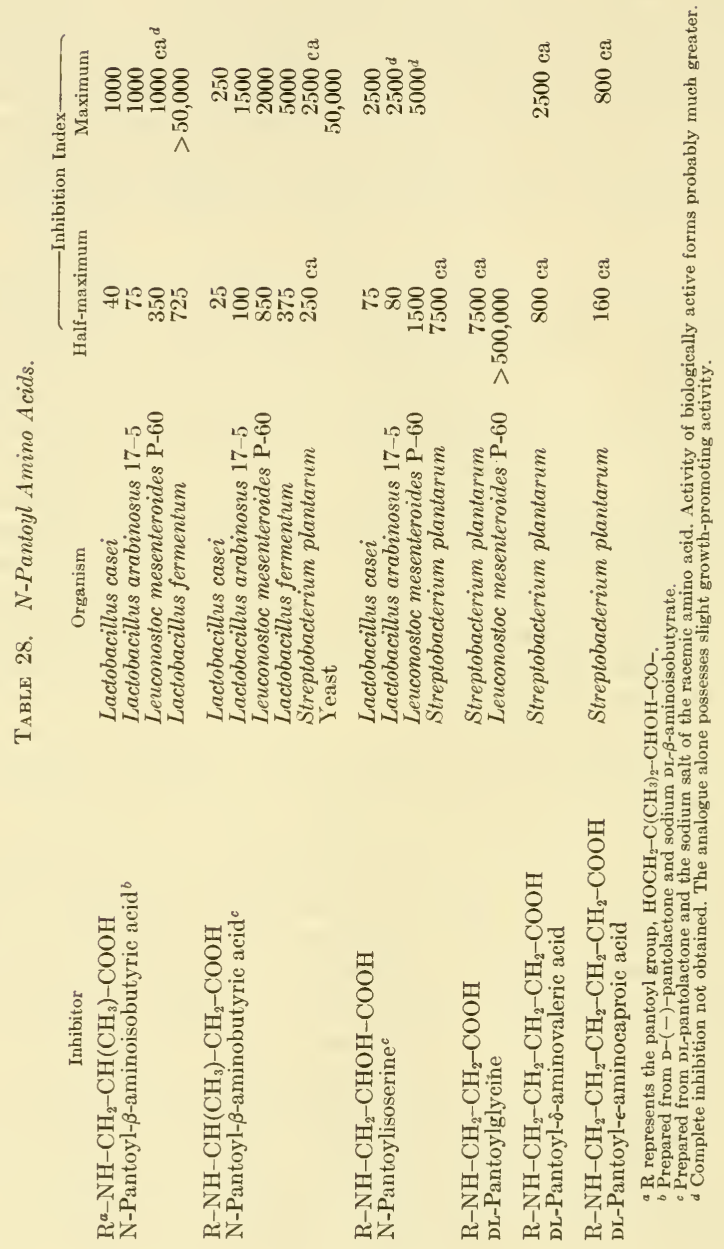


isoserine and $\mathrm{N}$-pantoyl- $\beta$-aminoisobutyric acid, complete inhibition of growth was not obtained regardless of the amount of inhibitor added. ${ }^{75}$ For such organisms not completely inhibited, these compounds alone partially and ineffectively replaced pantothenic acid in stimulating growth; however, growth induced by pantothenic acid was readily inlibited by these compounds to a level corresponding to their own stimulatory effect, but no further. In cases of this type, almost complete inhibition of growth was obtained before stimulation by the antimetabolite became apparent. The growth stimulated by any concentration of these analogues was only a fraction of that obtained with maximal concentrations of pantothenic acid. ${ }^{75}$

The relative concentrations of these antimetabolites required to produce half-maximum and maximum inhibition varied considerably with the various test organisms. The variation was from slightly more than twofold for Leuconostoc mesenteroides P-60 to more than tenfold for Lactobacillus arabinosus $17-5$ and Lactobacillus casei.75

The condensation products of DL-pantolactone and the sodium salts of DL-alanine, DL- $\alpha$-aminoisobutyric acid, DL-norvaline, DL-valine, DL-phenylalanine, DL-serine, L-asparagine, $p$-aminobenzoic acid and sulfanilamide were inactive as inhibitory analogues of pantothenic acid for Streptobacterium plantarum. However, the pantoyl derivatives of norvaline and alanine were somewhat inhibitory against yeast, whether the growth was stimulated by $\beta$-alanine or by pantothenic acid. ${ }^{77}$

Pantothenones. Replacement of the carboxyl group of $p$-aminobenzoic acid by various ketone groups resulted in compounds which competitively inhibited the corresponding metabolite. ${ }^{78}$ In determining whether or not this type of alteration could be used extensively in obtaining antimetabolites, Woolley and Collyer ${ }^{79}$ prepared phenyl-D-pantothenone and found it to be somewhat effective in preventing competitively the utilization of pantothenic acid for several organisms, as indicated in Table 29. It should be noted that the inhibition indices are for half-maximum inhibition. These values are usually only a fraction of those for maximum inhibition of growth. For Escherichia coli, Saccharomyces cerevisiae, and Endomyces vernalis, phenyl-D-pantothenone reduced the growth to halfmaximum at concentrations of 60,33 and $39 \gamma$ per cc, respectively, in the presence of $0.04 \gamma$ per ce of pantothenic acid. However, the inhibitions were not reversed by supplementary pantothenic acid for these organisms, which synthesize pantothenic acid. Saccharomyces cerevisiae requires, of course, the $\beta$-alanine portion in order to carry out this synthesis. It is interesting to note in Table 29 that the toxicity of phenyl pantothenone is reversed by pantothenic acid for a strain of Staphylococcus aureus, which was found to grow well without exogenous pantothenic acid but 
总을

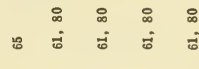

.

$\stackrel{0}{\stackrel{0}{2}} \stackrel{-}{0}$ m

|

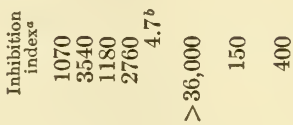

ิิ

을

8

:

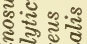

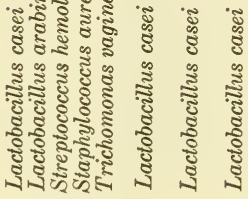

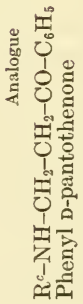

ज्ञ $\rightarrow$

I

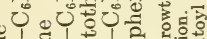

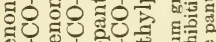

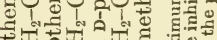

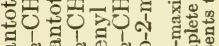

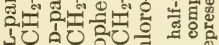
는 1 엘 हैंद 
was stimulated appreciably in its growth in the presence of the vitamin. Usually organisms which synthesize pantothenic acid are not affected by most of the analogues of that vitamin.

The activity of pantoyltauramide as an antimalarial led to the testing of other analogues of pantothenic acid including phenyl pantothenone, which was found to be effective as a suppressive agent in blood-induced infections of Plasmodium gallinaceum in chicks. ${ }^{61}$ As a result of this activity, other analogues related to phenyl pantothenone were prepared. ${ }^{80}$ The activities of these ketones are indicated in Table 29. Whereas $p$-tolyl D-pantothenone is the most effective inhibitor against Lactobacillus casei, $p$-chlorophenyl D-pantothenone is the most effective antimalarial agent.

An impure preparation of methyl pantothenone was found to inhibit the growth of Saccharomyces cerevisiae and Lactobacillus casei. ${ }^{79}$ This inhibitory effect was not reversed by pantothenic acid, and the preparation was at lower concentrations 1 per cent as active as the vitamin in stimulating growth of these two organisms. The possibility of contamination of the analogue with pantothenic acid appears possible.

The toxicity of phenyl pantothenone for Saccharomyces cerevisiae is not reversed by pantothenic acid, but certain amino acids at relatively high concentrations prevent the toxicity of the inhibitor. ${ }^{81}$ L-Histidine $(0.26 \mathrm{mg}$ per $\mathrm{cc}$ ) is the most effective amino acid in preventing the toxicity of $160 \gamma$ per ec of the inhibitor. L-Glutamic acid is somewhat less effective, and L-proline, L-aspartic acid, L-asparagine and glycine were about half as effective as L-glutamic acid, which also has a slight effect on the toxicity of phenyl pantothenone for Lactobacillus casei. Very slight effects were noted with serine, threonine, alanine and lysine, but other amino acids were inactive.

Pantothenyl Alcohol and Related Compounds. As previously indicated, pantothenyl alcohol is converted by warm-blooded animals into pantothenic acid and serves as an available source of that vitamin for these organisms; however, as indicated in Table 30, the alcohol analogue not only is not utilized in place of the vitamin by lactic acid bacteria, but on the contrary it prevents competitively the utilization of pantothenic acid by these organisms. ${ }^{19}$ Pantothenyl alcohol prevents the utilization of pantothenic acid in Leuconostoc mesenteroides P-60 at an inhibition index of 300 , whereas pantoyltaurine is inactive at a ratio of 200,000 . On the other hand, pantoyltaurine is approximately 5 times as active as pantothenyl alcohol in preventing the functioning of pantothenic acid in Lactobacillus arabinosus 17-5. Such variations in sensitivity to pantothenic acid analogues may result solely from differences in permeability of various microbial cells, but more likely the variations are the 


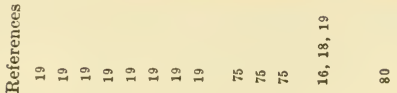

草

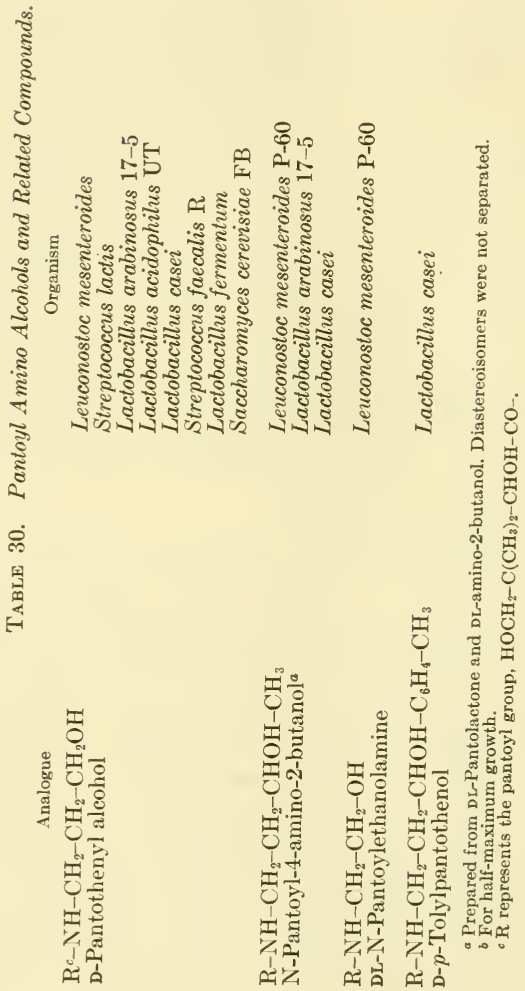




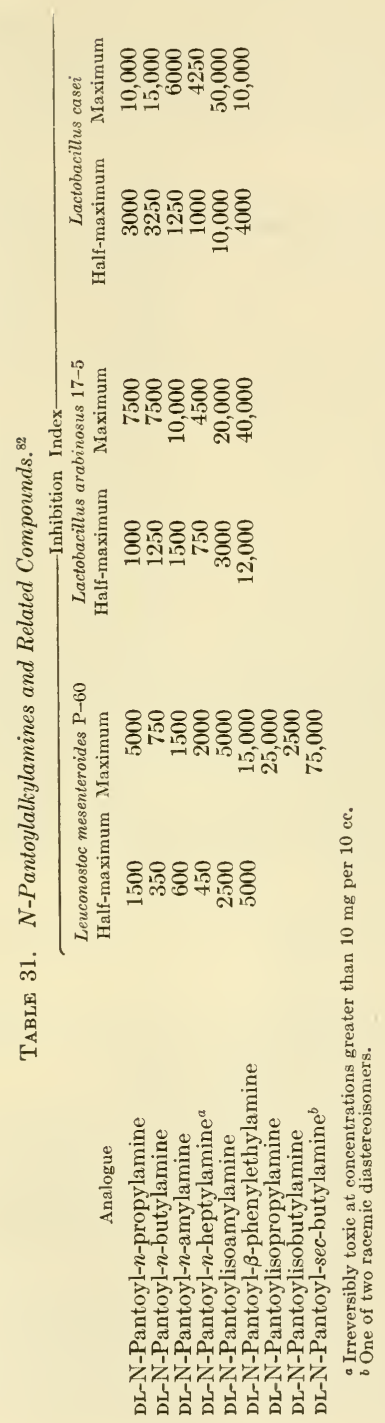


result of differences in cellular interactions. The structures of the enzymes utilizing pantothenic acid may be slightly different, the analogues may prevent different reactions in the utilization of pantothenic acid, or differences in the ability of the organisms to convert the analogues to other forms may exist.

As indicated in Table 30, alteration of the structure of pantothenyl alcohol tends to lower the inhibitory activity of the analogue. D- $p$-Tolylpantothenol is more active than the corresponding methylpantothenol, but is only 0.1 as active as the corresponding pantothenone for Lactobacillus casei. However, in chicks with blood-induced infections of Plasmodium gallinaceum, both $p$-tolylpantothenol and $p$-tolylpantothenone possess approximately the same activity as quinine. ${ }^{61}$

N-Pantoylalkylamines and Related Compounds. A series of N-pantoyl amines ${ }^{19,82}$ have been found to be relatively effective competitive antagonists of pantothenic acid for Leuconostoc mesenteroides P-60, but somewhat less effective as antimetabolites for Lactobacillus casei and Lactobacillus arabinosus 17-5, as indicated in Table 31 .

For Leuconostoc mesenteroides, the effectiveness of the different pantoyl alkyl amines as inhibitory analogues of pantothenic acid increases considerably as the length of the alkyl chain is increased to four carbons, and then slowly decreases with increasing chain length. The derivatives containing a branched alkyl are less active than the corresponding $n$-alkyl derivatives. For Lactobacillus arabinosus 17-5 and Lactobacillus casei, no definite trend in effectiveness with increasing chain length was apparent; both the $n$-propyl- and the $n$-heptylamides were more effective than the intervening members of the series.

D-N-Pantoyl- $\beta$-phenylethylamine ${ }^{80}$ also has a very slight activity (quinine equivalent, 0.03 ) against blood-induced Plasmodium gallinaceum infections in chicks. ${ }^{61}$

N-Pantoyl- $n$-butylamine and, less effectively, pantoyltaurine are reported to inhibit competitively the utilization of pantothenic acid as a carbon source for a strain of Pseudomonas. ${ }^{83}$

Miscellaneous Pantoyl Derivatives. DL-Pantamide and DL-panthydrazide have been synthesized and found to be antagonists of pantothenic acid functioning in Lactobacillus casei. ${ }^{62}$ At concentrations of 0.025 and $2.5 \gamma$ per cc of pantothenic acid, 1,250 and 10,000 $\gamma$ per ce, respectively, of pantamide, and 6 and $5,000 \gamma$ per cc, respectively, of panthydrazide inhibited growth of the organism. ${ }^{62}$

For Leuconostoc mesenteroides P-60, DL-pantothenonitrile, DL-pantothenyl amine, and DL-N-pantoyl- $\beta$-methoxyethylamine prevent competitively the functioning of pantothenic acid. ${ }^{82}$ The inhibition indices are $10,000,40,000$ and 15,000 , respectively. It is interesting to note 


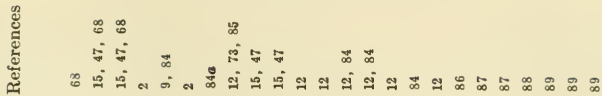

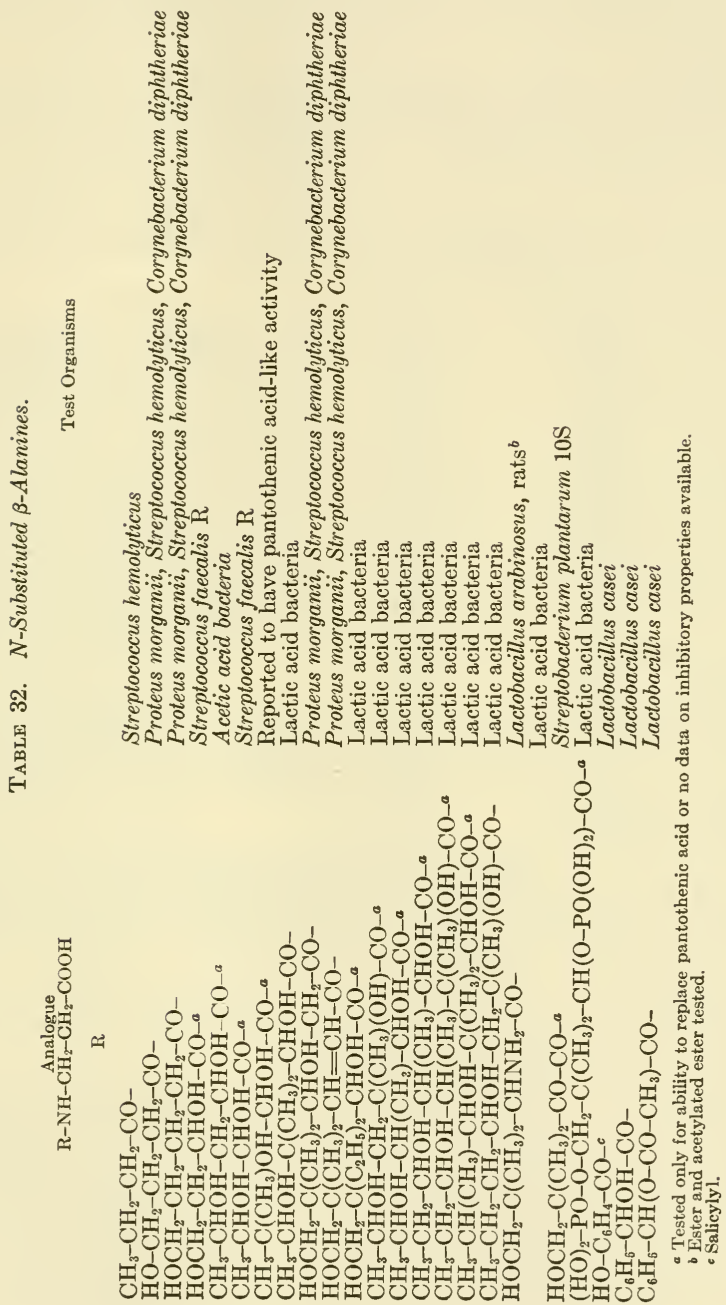


that the last compound is not so effective as either the $n$-butylamine or ethanolamine derivative. ${ }^{\$ 2}$ DL-N-Pantoyl- $\beta$-diethylaminoethylamine, DL-N-pantoyl-5-diethylamino-2-pentylamine, and DL-N-pantoyl- $p$-anisidine are essentially inactive as pantothenic acid antagonists for Lactobacillus casei. ${ }^{62}$

N-Substituted $\beta$-Alanines and Related Compounds. Modification of the pantoyl group of pantothenic acid has resulted with few exceptions in compounds possessing slight activity in replacing the vitamin or in compounds with neither stimulatory nor inhibitory properties. Almost onethird of the reported $\mathrm{N}$-substituted $\beta$-alanine analogues of pantothenic acid are active at least partially in replacing the vitamin in the nutrition of some organisms (cf. Table 24).

The $\mathrm{N}$-substituted derivatives of $\beta$-alanine which have been found to be inactive in replacing pantothenic acid are listed in Table 32 . The ester of $\alpha$-amino- $\gamma$-hydroxy- $\beta, \beta$-dimethylbutyryl- $\beta$-alanine, although inactive alone in replacing pantothenic acid, supplements suboptimal concentrations of pantothenic acid in stimulating growth of Lactobacillus arabinosus. As indicated in Table 32, nearly half of these compounds were not tested as possible antimetabolites of pantothenic acid. Although many of the compounds were found to be slightly inhibitory to the test organisms, the inhibitions were with few exceptions not prevented by pantothenic acid. Thus, $\gamma$-hydroxyvaleryl- and $\gamma$-hydroxybutyryl- $\beta$-alanine prevent the growth of several organisms, but the toxicity is not abolished by pantothenic acid.

Salicylyl- $\beta$-alanine, mandelyl- $\beta$-alanine, and acetyl mandelyl- $\beta$-alanine have been reported to be moderately active pantothenic acid antagonists; however, the data presented for mandelyl- $\beta$-alanine do not indicate a competitive inhibition.

Drell and Dunn ${ }^{73,84}$ have shown that $\mathrm{N}-\alpha, \gamma$-dihydroxy- $\beta, \beta$-dimethylvaleryl- $\beta$-alanine ( $\omega$-methylpantothenic acid) competitively prevents the utilization of pantothenic acid by a large number of lactic acid bacteria and by Streptococcus hemolyticus. This compound is the first N-substituted $\beta$-alanine to be reported as an effective antimetabolite of pantothenic acid. The analogue exists in two racemic diastereoisomeric forms, the relative activities of which are unknown.

As indicated in Table 33, $\omega$-methylpantothenic acid inhibits the growth of a wide variety of organisms for which pantothenic acid is essential; however, it does not affect the growth of organisms not requiring the vitamin for growth. As is common with a number of inhibitors, stimulation of growth was obtained in many instances at concentrations of the analogue just lower than that necessary for inhibition of growth. At relatively high concentrations of pantothenic acid, the inhibition index was 
significantly increased, particularly for Lactobacillus casei and Lactobacillus fermenti. After complete inhibition of growth of these organisms was attained, further increases in the concentration of the analogue stimulated growth of the organisms. Thus, at high concentrations of pantothenic acid, complete inhibition of growth is never attained, even though the analogue alone is incapable of stimulating growth of the organisms.

$\omega$-Methylpantothenic acid is effective against streptococei both in vitro and in vivo. Mice were protected from an 80 per cent fatal infection of

TABle 33. Comparison of Activity of $\omega$-Methylpantoyl Derivatives with Pantoyltaurine. ${ }^{73}$

\section{Organism}

Leuconostoc citrovorum $8082^{a}$

Lactobacillus fermentatus 4006

Lactobacillus pentoaceticus 367

Lactobacillus brevis 8257

Leuconostoc citrovorum 797

Leuconostoc citrovorum 7013

Streptococcus faecalis R 8043

Lactobacillus casei 7469

Lactobacillus helveticus 335

Lactobacillus helveticus 6345

Lactobacillus lycopersici 4005

Leuconostoc dextranicum 8358

Leuconostoc dextranicum 8086

Leuconostoc mesenteroides 9135

Leuconostoc mesenteroides 8293

Lactobacillus fermenti $36-9338$

Lactobacillus gayoni 8289

Leuconostoc dextranicum 8359

Leuconostoc mesenteroides P-60 (8042)

Lactobacillus pentosus 124-2

Lactobacillus arabinosus 17-5 (8014)

Lactobacillus brassicae 8041

Lactobacillus mannitopoeus

$\begin{array}{ccc}\begin{array}{c}\text { w-Methylpanto- } \\ \text { thenic acid }\end{array} & \begin{array}{c}\text { Inhibition Index-Methylpantoyl- } \\ \text { taurine }\end{array} & \begin{array}{c}\text { Pantoyl- } \\ \text { taurine }\end{array} \\ 80 & 2400 & 4200^{b} \\ 150 & 51,000^{b} & 113,000 \\ 270 & 175,000 & 85,000^{c} \\ 270 & 75,000 & 140,000^{c} \\ 330 & 7,300 & 8,500^{b} \\ 330 & 6,000^{b} & 5,100^{b} \\ 330 & 26,000 & 35,000 \\ 450^{d} & 16,500 & 15,000 \\ 500^{b} & 51,000^{b} & 42,500^{b} \\ 550^{b} & 44,000^{b} & 57,000^{b} \\ 800 & >333,000^{e} & 51,000 \\ 900 & 2,200 & 850 \\ 900 & 5,000 & 1,350 \\ 900 & >333,000^{e} & 1,350 \\ 1,100 & 4,400 & 7,000^{b} \\ 2,200^{b} & >1,000,000^{e} & 130,000 \\ 2,200^{b} & >333,000^{e} & 225,000^{b} \\ 2,700^{b} & 3,500 & 1,400 \\ 3,300 & >1,000,000^{b} & 250,000 \\ 4,000 & >333,000 & >333,000 \\ 5,200 & 22,000 & 4,700 \\ 7,500 & >333,000 & >333,000 \\ 13,000^{b} & >333,000^{e} & 225,000^{b}\end{array}$

a American Type Culture Collection number.

$b$ Stimulation of growth obtained at concentrations just below inhibitory levels.

- Half-maximum inhibition; complete inhibition of grow th not obtained at index of 280,000 .

d Stimulation of growth, no inhibition obtained.

- Stimulation of growth obtained at concentrations of analogue higher than that just necessary for maximum inhibition. Effect enhanced by higher concentrations of pantothenic acid.

a $\beta$-hemolytic streptococcus (Group A, type 23, No. 1072) when the analogue was administered in the diet for four days prior to infection at a concentration 200 times that of pantothenic acid.

Production of a pantothenic acid deficiency in weanling mice has been accomplished by supplementation of the diet with $\omega$-methylpantothenic acid. ${ }^{90}$ On a pantothenic acid-deficient diet, the survival time decreased from 8 to 9 weeks to $5,4,2.5$ and 1.5 weeks by supplementation with 0.06 , $0.2,0.5$ and 1.5 per cent, respectively, of the analogue in the diet. With diets containing 2 and $6 \mathrm{mg}$ per cent of pantothenic acid and a 100-fold excess of the analogue, the survival time was about the same as on the 
pantothenic acid-deficient diet. Administration of an adequate amount of pantothenic acid prevented or cured the deficiency disease caused by the analogue. Strain differences in mice were observed, and graying of fur was noted in only one of five experimental groups.

Analogues of Pantothenic Acid Modified in Both the Pantoyl and $\beta$-Alanyl Groups. A homologue of pantoyltaurine, DL- $N$ - $\beta, \delta$-dihydroxy$\gamma, \gamma$-dimethylvaleryltaurine (homopantoyltaurine) ${ }^{15}$ is an antagonist of pantothenic acid for Streptococcus hemolyticus with an inhibition index of $20,000 .{ }^{47}$ The inhibitory activity of this analogue is in contrast to that of the corresponding $\beta$-alanine derivative (homopantothenic acid). The $\beta$-alanine derivative exerts only a slight toxic effect, which is not reversed by pantothenic acid. On the other hand, homopantoyltaurine is much less effective than the corresponding pantoyl derivative (pantoyltaurine), which inhibits growth at an index of 500 .

Another homologue of pantoyltaurine, $\mathrm{N}$ - $\alpha, \gamma$-dihydroxy- $\beta, \beta$-dimethylvaleryltaurine ( $\omega$-methylpantoyltaurine), also has properties intermediate between the corresponding $\beta$-alanine and pantoyl derivatives. ${ }^{73} \omega$-Methylpantoyltaurine inhibits competitively the utilization of pantothenic acid in a large number of lactic acid bacteria as indicated in Table 33; however, it is much less effective than $\omega$-methylpantothenic acid, but is more effective in some cases than pantoyltaurine.

An unusual result was obtained with $\mathrm{N}$ - $\alpha, \gamma$-dihydroxy- $\beta, \beta$-dimethylvaleryl- $\beta$-aminobutyric acid, which prevents the growth of Escherichia coli at a concentration of $100 \gamma$ per cc. ${ }^{91}$ The toxicity is prevented by pantothenic acid. Usually analogues of pantothenic acid either do not prevent the growth of organisms which synthesize the vitamin, or, if growth is prevented at high concentrations, the toxicity is not prevented by supplements of the vitamin.

The activity of DL-N- $\alpha$-hydroxy- $\beta, \beta$-dimethylbutyryltaurine, ${ }^{14}$ which prevents the utilization of pantothenic acid (inhibition index, 2000 for half-maximum) or pantoic acid (inhibition index, 800 for half-maximum) by Acetobacter suboxydans, is in contrast with the corresponding $\beta$-alanine derivative, which possesses activity in replacing pantothenic acid as a growth factor for this organism (cf. Table 24).

$\mathrm{N}$ - $\left(\gamma\right.$-Hydroxybutyryl)taurine and $\mathrm{N}^{4}$-(diacetylpantoyl)sulfanilamide have been reported to be antagonists of pantothenic acid for Lactobacillus casei; ${ }^{89}$ however, the former was found to have little growth-inhibitory action with other organisms. ${ }^{68} \mathrm{~N}-\alpha, \gamma$-Dihydroxy- $\beta, \beta$ dimethylvaleryl-L-leucine, ${ }^{73} \mathrm{~N}$ - $\left(\beta\right.$-benzoylethyl) - $\alpha$-hydroxycaproamide, ${ }^{80}$ $\mathrm{N}$-( $\beta$-benzoylethyl) caproamide, ${ }^{80} \quad$ DL- $\mathrm{N}-\alpha, \gamma$-dihydroxy- $\beta, \beta$-diphenylbutyryltaurine, ${ }^{60}$ DL- $\mathrm{N}$ - $\alpha$-tosyl- $\gamma$-hydroxy- $\beta, \beta$-dimethylbutyryltaurine, ${ }^{60}$ 
$\mathrm{N}$-butyryltaurine ${ }^{68}$ and DL-N- $\alpha, \gamma$-dihydroxybutyryltaurine ${ }^{68}$ are also relatively inactive as inhibitors of pantothenic acid functioning.

The two optically active forms of $\mathrm{N}$ - $\alpha$-keto- $\beta$-methyl- $\beta$-methylolvaleryltaurine are reported to have slight activity in inhibiting the growth of Streptobacterium plantarum $105 .{ }^{25}$ However, $\mathrm{N}$ - $\alpha$-keto- $\beta, \beta$-dimethyl- $\gamma$ hydroxybutyryltaurine does not show such an inhibitory effect. ${ }^{87}$

Analogues of $\beta$-Alanine. The unintentional use of an inhibitor of the utilization of $\beta$-alanine led to the discovery of pantothenic acid as a growth factor for yeast by Williams and co-workers. ${ }^{29,92}$ Asparagine added to the medium prevented the response of yeast to $\beta$-alanine and allowed a very specific microbiological assay for the vitamin.

Not only asparagine, which resembles $\beta$-alanine structurally, but also other $\alpha$-amino acids in general prevent the utilization of $\beta$-alanine by yeast; ${ }^{93,94}$ thus L-glutamic acid, L-aspartic acid, L-glutamine, glycine, DL-alanine, DL-serine, DL- $\alpha$-aminobutyric acid, DL-threonine, DL-norvaline, DL-norleucine, DL-valine, DL-leucine, DL-methionine, and DL-cysteine show an inhibitory effect on yeast growth stimulated by $\beta$-alanine. However, no such effect is obtained when growth is stimulated by pantothenic acid, except for somewhat less specific inhibitions obtained only with methionine and cysteine. ${ }^{76}$ Proline, D-leucine, arginine, glycylglycine, and $\alpha$-aminoisobutyric acid do not inhibit growth stimulated by $\beta$ alanine.

The more effective inhibitors of the utilization of $\beta$-alanine have been $\beta$-amino acids. These include $\beta$-aminobutyric acid, ${ }^{95}$ isoserine, ${ }^{96}$ and phenyl- $\beta$-alanine. ${ }^{76} \beta$-Aminobutyric acid competitively inhibits the increase in respiration of yeast brought about by $\beta$-alanine when present in the ratio 1000:1, but does not have any effect on the increase brought about by the minimum effective concentration of pantothenic acid. ${ }^{97}$ The inhibitory effect on respiration which is paralleled by a similar effect on growth is less if the analogue is added several hours after the $\beta$-alanine. Taurine, ${ }^{95} \beta$-alanylglycine, ${ }^{95}$ and $\mathrm{N}$-methyl- $\beta$-alanine ${ }^{96}$ are ineffective for this strain of yeast. Both taurine and pantoyltaurine have been found to inhibit only one strain of yeast out of seventeen when growth was stimulated by $\beta$-alanine. ${ }^{30}$ Taurine had no effect on growth stimulated by pantothenic acid with this strain of yeast. However, pantoyltaurine inhibited the utilization of pantothenic acid in this and all other strains of yeast.

$\alpha, \beta$-Diaminopropionic acid is reported to have a marked growth-inhibiting effect on Corynebacterium diphtheriae, both mitis and gravis strains, presumably by competing with the essential growth factor, $\beta$-alanine. ${ }^{98}$

$\alpha$-Methyl- $\beta$-alanine has been found to be slightly active in replacing $\beta$-alanine for the growth of Saccharomyces cerevisiae G. M. ${ }^{74}$ 
L-Carnosine is approximately one-fourth as active as $\beta$-alanine for a strain of diphtheria bacillus ${ }^{99}$ and for Saccharomyces cerevisiae, Fleischmann's strain $139,{ }^{100}$ but is not active for Saccharomyces cerevisiae G. M. ${ }^{30}$ D-Carnosine was inactive for the diphtheria bacillus.

The inhibitory effect of propionic acid for Escherichia coli, ${ }^{101}$ for Saccharomyces cerevisiae, ${ }^{102}$ and to some extent for Acetobacter suboxydans, ${ }^{102}$ is prevented by $\beta$-alanine. For Saccharomyces cerevisiae the inhibition index is approximately 10,000. Pantothenic acid completely reverses the toxicity at its minimum effective concentration so that it appears that propionic acid also prevents the conversion of $\beta$-alanine to pantothenic acid in these organisms. Acetic acid acts in an analogous manner with this strain of yeast, but is much less effective.

Analogues of Pantoic Acid. Compounds structurally similar to pantoic acid and possessing growth inhibiting properties are listed in Table 34 . However, these compounds are not necessarily competitive antagonists of pantoic acid. On the contrary, most of them cannot be considered solely as competitive inhibitors of the functioning of pantoic acid.

Thus, a group of pantoic acid analogues reported by Cheldelin and Schink ${ }^{1+}$ prevent the growth of Acetobacter suboxydans either in the presence of pantothenic acid or pantoic acid as indicated in Table 34 . However, growth of Saccharomyces cerevisiae G. M. stimulated by pantothenic acid was not prevented by either $\alpha$-hydroxy- $\beta, \beta$-dimethylbutyric acid or $\beta, \gamma$-dihydroxy- $\beta$-methylbutyric acid, but the growth stimulated by $\beta$-alanine was prevented by the analogues. This suggests that the two analogues prevent the biosynthesis of pantothenic acid in the yeast under these conditions, presumably by competition with pantoic acid or a related metabolite. With Acetobacter suboxydans the analogues appear to prevent the utilization of pantothenic acid rather than its synthesis.

Ivanovics ${ }^{103}$ has reported that salicylic acid at low concentrations appears to prevent the synthesis of pantothenic acid in Escherichia coli. This effect is exerted to a lesser extent by acetylsalicylic acid or phenylsalicylate but not by thiosalicylic acid or salicylamide. The inhibitory action of low concentrations of salicylic acid was prevented in a somewhat noncompetitive manner by pantothenic acid. Also, larger amounts of pantoic acid as well as some amino acids were effective in preventing the toxicity. Although valine alone was singly the most active of the amino acids, mixtures of methionine with valine, leucine, isoleucine, or lysine were more effective. These mixtures were almost equivalent to a casein hydrolysate which caused an eight fold increase in pantothenic acid synthesis. Thiamine and vitamin $\mathrm{K}$ have also been reported to prevent partially the inhibitory effect of salicylic acid..$^{104}$

Pantoic acid is approximately nine times more active than pantolactone 


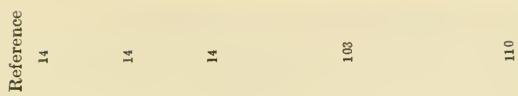

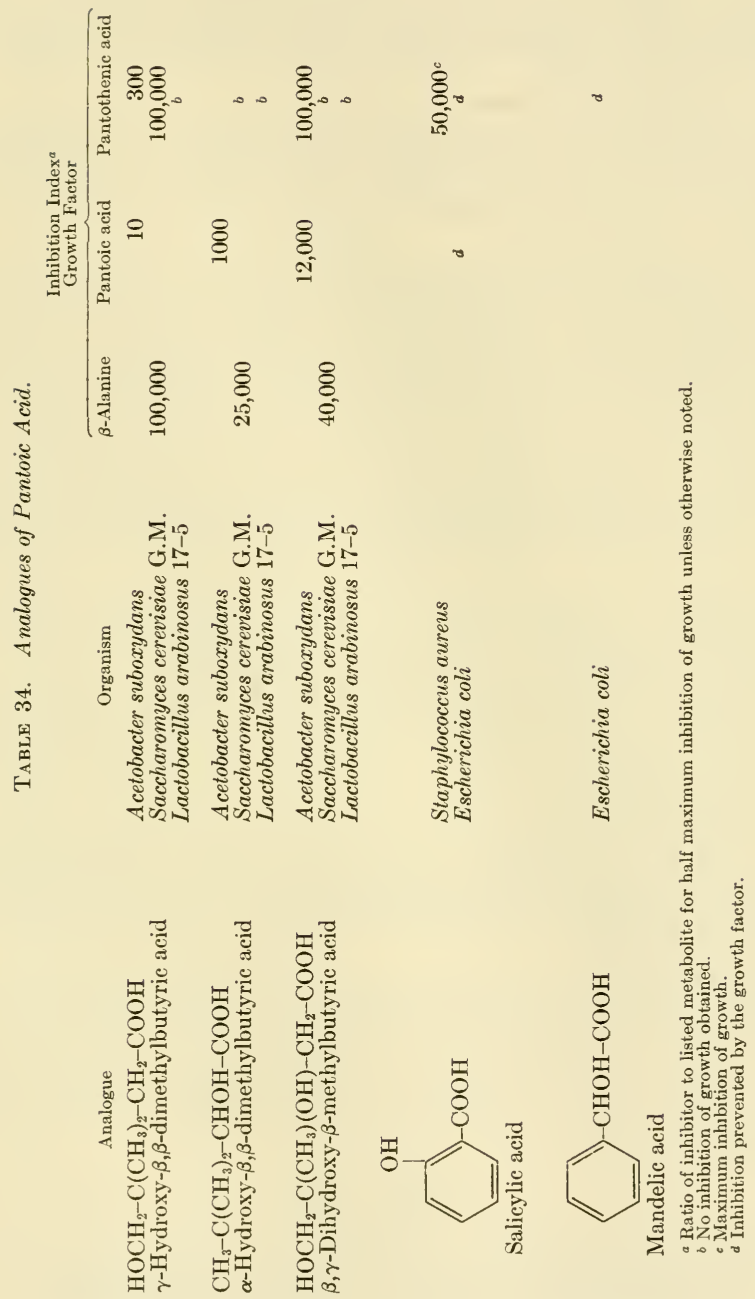


in antagonizing the inhibitory action of salicylic acid on Escherichia coli. ${ }^{105}$ Pantoyltaurine and certain pantoyltauramides have been found to prevent the toxicity of salicylate but are only about 3 per cent as active as pantoic acid. ${ }^{106}$ It is suggested that the organism effects a partial hydrolysis of the analogues forming pantoic acid which prevents the toxicity of salicylic acid.

DL- $\alpha$-Amino- $\beta, \beta$-dimethyl- $\gamma$-hydroxybutyric acid, which has been termed pantonine, has been found to be as effective as DL-pantoic acid in preventing the toxicity of salicylic acid for Escherichia coli, but was inactive in replacing the pantoic acid or pantothenic acid requirement of Acetobacter suboxydans. ${ }^{107}$

With Staphylococcus aureus, pantothenic acid prevented the toxicity of low concentrations of salicylic acid in almost a competitive manner; the anti-bacterial index varied only from 50,000 to 26,000 over a 16 -fold range of concentrations of pantothenic acid. ${ }^{103}$ The vitamin was approximately 20,000 times as active as pantolactone. Ascorbic acid, pimelic acid, and purine bases are reported to enhance the anti-salicylate action of pantothenic acid and to permit $\beta$-alanine, which is not otherwise effective, to prevent the toxicity of the inhibitor. ${ }^{108}$ The purine bases alone, particularly guanine, prevent to some extent the bacteriostatic action of salicylic acid.

The toxicity of salicylic acid for a strain of Clostridium septicum is reported to be related to the utilization of pantoic acid. ${ }^{36}$

A strain of Proteus morganii which required pantothenic acid or pantoic acid for growth was unaffected by salicylic acid except at high concentrations. ${ }^{103}$ The toxicity of such high concentrations of the inhibitor was not affected by pantothenic acid.

The reproductive phase of Trypanosoma lewisi infection is prolonged and the number of organisms is increased in rats by restricting pantothenic acid in the diet. Repeated administration of salicylic acid results similarly in prolonging the reproduction phase and in an exalted parasitemia in the rats. ${ }^{109}$

Mandelic acid has an inhibitory action which is largely prevented by pantothenic acid in some strains of Escherichia coli; ${ }^{110}$ however, inhibition of growth of many strains is not obtained with mandelic acid except at high concentrations, the effects of which are not prevented by pantothenic acid. ${ }^{111}$

\section{Bibliography}

1. Williams, R. J., Truesdail, J. H., Weinstock, H. H., Jr., Rohrmann, E., Lyman, C. M., and McBurney, C. H., J. Am. Chem. Soc., 60, 2719 (1938).

2. Mitchell, H. K., Weinstock, H. H., Jr., Snell, E. E., Stanberry, S. R., and Williams, R. J., J. Am. Chem. Soc., 62, 1776 (1940). 
3. Woolley, D. W., and Hutchings, B. L., J. Bact., 38, 285 (1939).

4. SubbaRow, Y., and Rane, L., J. Am. Chem. Soc., 61, 1616 (1939).

5. Stiller, E. T., Harris, S. A., Finkelstein, J., Keresztesy, J. C., and Folkers, K., J. Am. Chem. Soc., 62, 1785 (1940).

6. Unna, K., and Muschett, C. W., Am. J. Physiol., 135, 267 (1942).

7. Harris, S. A., Boyack, G. A., and Folkers, K., J. Am. Chem. Soc., 63, 2662 (1941).

8. Grüssner, A., Gätzi-Fichter, M., and Reichstein, T., Helv. Chim. Acta, 23, 1276 (1940).

9. Reichstein, T., and Grüssner, A., Helv. Chim. Acta, 23, 650 (1940).

10. Mitchell, H. K., Snell, E. E., and Williams, R. J., J. Am. Chem. Soc., 62, 1791 (1940).

11. Zschiesche, E., and Mitchell, H. K., Proc. Soc. Exptl. Biol. Med., 45, 565 (1940).

12. Henze, H. R., and Nease, A. H., (unpublished work); Nease, A. H., Dissertation, University of Texas (1943).

13. Woolley, D. W., and Hutchings, B. L., J. Bact., 39, 287 (1940).

14. Cheldelin, V. H., and Schink, C. A., J. Am. Chem. Soc., 69, 2625 (1947).

15. Barnett, J. W., and Robinson, F. A., Biochem. J., 36, 357, 364 (1942).

16. Pfaltz, Z., Z. Vitaminforsch., 13, 236 (1943); Chem. Abstr., 38, 6339 (1944).

17. Burlet, E., Jubilee Volume, Fmil Barell, Reinhardt, Ltd. Co., Basle, Switzerland, 1946, p. 92 ; Burlet, E., Z. Vitaminforsch., 14, 318 (1944); Chem. Abstr., 41, 2782 (1947).

18. Schnider, O., Jubilee Volume, Emil Barell, Reinhardt, Ltd. Co., Basle, Switzerland, 1946, p. 85.

19. Snell, E. E., and Shive, W., J. Biol. Chem., 158, 551 (1945).

20. Hegsted, D. M., Proc. Soc. Exptl. Biol. Med., 69, 571 (1948).

21. Parke, H. C., and Lawson, E. J., J. Am. Chem. Soc., 63, 2869 (1941).

22. Hudson, C. S., J. Am. Chem. Soc., 39, 462 (1917).

23. Woolley, D. W., Waisman, H. A., Michelsen, O., and Elvehjem, C. A., J. Biol. Chem., 125, 715 (1938).

24. Snell, E. E., Strong, F. M., and Peterson, N. H., Biochem. J., 31, 1789 (1937).

25. Wieland, T., and Möller, E. F., Chem. Abstr., 43, 4225 (1949); Ber., 81, 316 (1948).

26. Kuhn, R., and Wieland, T., Ber., 75, 121 (1942).

27. Schmidt, V., Acta Pharmacol. Toxicol. (Copenhagen), 1, 120 (1945); Chem. Abstr., 40, 6127 (1946).

28. Rubin, S. H., Cooperman, J. M., Moore, M. E., and Scheiner, J., J. Nutrition, 35, 499 (1948).

29. Williams, R. J., and Rohrmann, E., J. Am. Chem. Soc., 58, 695 (1936).

30. Sarett, H. P., and Cheldelin, V. H., J. Bact., 49, 31 (1945).

31. Mueller, J. H., Proc. Soc. Exptl. Biol. Med., 36, 706 (1937); Mueller, J. H., and Cohen, S., J. Bact., 34, 381 (1937); Mueller, J. H., and Klotz, A. W., J. Am. Chem. Soc., 60, 3086 (1938).

32. Evans, W. C., Handley, W. R. C., and Happold, F. C., Brit. J. Exptl. Path., 20, 823 (1939) ; Evans, W. C., Happold, F. C., and Handley, W. R. C., Brit. J. Exptl. Path., 20, 41 (1939).

33. MeIlwain, H., Brit. J. Exptl. Path., 24, 203, 212 (1943).

34. Underkofler, L. A., Bantz, A. C., and Peterson, W. H., J. Bact., 45, 113 (1943).

35. Woolley, D. W., J. Biol. Chem., 130, 417 (1939).

36. Ryan, F. J., Schneider, L. K., and Ballentine, R., J. Bact., 53, 417 (1947).

37. Koser, S. A., Breslove, B. B., and Dorfman, A., J. Infectious Diseases, 69, 114 (1941); J. Bact., 41, 37 (1941).

38. Sarett, H. P., and Cheldelin, V. H., J. Biol. Chem., 159, 311 (1945).

39. Lipmann, F., Kaplan, N. O., Novelli, G. D., Little L. C., and Guirard, B. M., J. Biol. Chem., 167, 869 (1947).

40. Hegsted, D. M., and Lipmann, F., J. Biol. Chem., 174, 89 (1948).

41. Novelli, G. D., Flynn, R. M., and Lipmann, F., J. Biol. Chem.. 177. 493 (1949) 
42. King, T. E., Locher, L. M., and Cheldelin, V. H., Arch. Biochem., 17, 483 (1948); Abstracts, Am. Chem. Soc., 114th Meeting 59e (1948); King, T. E., Fels, I. G., and Cheldelin, V. H., J. Am. Chem. Soc., 71, 131 (1919).

43. Novelli, G. D., Kaplan, N. O., and Lipmann, F., J. Biol. Chem., 177, 97 (1949); Lipmann, F., Kaplan, N. O., and Novelli, G. D., Fed. Proc., 6, 272 (1947).

44. Snell, E. E., J. Biol. Chem., 139, 975 (1941); 141, 121 (1941).

45. Kuhn, R., Wieland, W., and Möller, E. F., Ber., 74, 1605 (1941).

46. MeIlwain, H., Brit. J. Exptl. Path., 23, 95 (1942).

47. MeIlwain, H., Biochem. J., 36, 417 (1912); McIlwain, H., Barrett, J., and Robinson, F. A., Chem. and Ind., 61, 96 (1942).

48. Snell, E. E., Chan, L., Spiridanoff, S., Way, E. L., and Leake, C. D., Science, 97, 168 (1943).

49. Woolley, D. W., and White, A. G. C., Proc. Soc. Exptl. Biol. Med., 52, 106 (1943).

50. Unna, K., Proc. Soc. Exptl. Biol. Med., 54, 55 (1943).

51. MeIlwain, H., and Hawking, F., Lancet, 1943, I, 449.

52. Gaebler, O. H., and Herman, E. V., Fed. Proc., 6, 254 (1947).

53. McIlwain, H., Biochem. J., 39, 279 (1945); Proceeding, 38, XXVIII (1944).

54. McIlwain, H., and Hughes, D. E., Biochem. J., 38, 187 (1944); 39, 133 (1945).

55. Mellwain, H., Biochem. J., 39, 329 (1945).

56. Teague, P. C., and Williams, R. J., J. Gen. Physiol., 25, 777 (1942).

57. MeIlwain, H., Biochem. J., 38, 97 (1944).

58. Mead, J. F., Rapport, M. M., Senear, A. E., Maynard, J. T., and Koepfli, J. B., J. Biol. Chein., 163, 465 (1946).

59. Barnett, J., J. Chem. Soc., 1944, 5.

60. Barnett, J., Dupré, D. J., Holloway, B. J., and Robinson, F. A., J. Am. Chem. Soc., 1944, 94.

61. Wiselogle, F. Y., "A Survey of Antimalarial Drugs 1941-1945," Vol. I, p. 138, 174, J. WV. Edwards, Aun Arbor, Michigan, 1946.

62. Madinaveitia, J., Martin, A. R., Rose, F. L., and Swain, G., Biochem. J., 39, 85 (1945).

63. Trager, W., J. Exptl. Med., 77, 411 (1943).

64. Koepfli, J. B., et al. (unpublished work) California Institute of Technology.

65. Johnson, G., and Kupferberg, A. B., Proc. Soc. Exptl. Biol. Med., 67, 390 (1948).

66. Singher, H. O., Millman, N., and Bosworth, M. R., Proc. Soc. Exptl. Biol. Med., 67,388 (1948).

67. Mead, J. F., and Koepfli, J. B., J. Org. Chem., 12, 295 (1947).

68. Winterbottom, R., Clapp, J. W., Miller, W. H., English, J. P., and Roblin, R. O., Jr., J. Am. Chem. Soc., 69, 1393 (1947).

69. Brackett, S., Waletzky, E., and Baker, M., J. Parasitol., 32, 453 (1946).

70. White, H. J., Lee, M. E., Jackson, E. R., Himes, A. T., and Alverson, C., Fed. Proc., Part II, 5, 214 (1946).

71. Weinstock, H. H., Jr., May, E. L., Arnold, A., and Price, D., J. Biol. Chem., $135,343(1940)$.

72. Kuhn, R., and Wieland, T., Ber., 73, 962 (1940).

73. Drell, W., and Dunn, M. S., J. Am. Chem. Soc., 70, 2057 (1948).

74. Pollack, M. A., J. Am. Chem. Soc., 65, 1335 (1943).

75. Shive, W., and Snell, E. E., Science, 102, 401 (1945).

76. Nielsen, N., Hartelium, V., and Johansen, G., Naturwiss., 32, 294 (1944); Chem. Abstr., 40, 3154 (1946); Compt. rend. trav. lab. Carlsberg, Sér. physiol., 24, 39 (1944); Chem. Abstr., 40, 5796 (1946).

77. Nielsen, N., and Roholt, K., Acta Pharmacol. Toxicol. (Cophenhagen), 1, 207 (1945).

78. Auhagen, E., Z. physiol. Chem., 274, 48 (1942).

79. Woolley, D. W., and Collyer, M. L., J. Biol. Chem., 159, 263 (1945). 
80. Lutz, R. E., IVilson, J. W., III, Deinet, A. J., Harnest, G. H., Martin, T. A., and Freek, J. A., J. Org. Chem., 12, 96 (1947).

81. Woolley, D. WV., J. Biol. Chem., 163, 481 (1946).

82. Shive, W., and Snell, E. E., J. Biol. Chem., 160, 287 (1945).

83. Metzger, W. I., J. Bact., 54, 135 (1947).

84. Drell, W., and Dunn, M. S., Abstracts of Papers, 112th Meeting, Am. Chem. Soc., 5c (1947).

84a. F. Hoffman-La Roche and Co. A. G. Swiss 216,824 Cl. 116h, Sept. 15, 1941; Chem. Abstr., 42, 6377 (1948).

85. Drell, WV., and Dunn, M. S., J. Am. Chem. Soc., 68, 1868 (1946).

86. Holly, F. IV., Barnes, R. A., Koniuszy, F. R., and Folkers, K., J. Am. Chem. Soc., 70, 3088 (1948).

87. Wieland, T., Chem. Ber., 81, 323 (1948); Chem. Abstr., 43, 4223 (1949).

88. Woolley, D. W., J. Biol. Chem., 134, 461 (1940).

89. Moss, J. N., Urist, H., and Martin, G. J., Arch. Biochem., 19, 213 (1948).

90. Drell, W., and Dunn, M. S., Abstracts of Papers, 115th Meeting, Am. Chem. Soc., 13c (1919).

91. Shive, W., Ackermann, W. W., Ravel, J. M., and Sutherland, J. E., J. Am. Chem. Soc., 69, 2567 (1947).

92. Weinstock, H. H., Jr., Mitchell, H. K., Pratt, E. F., and Williams, R. J., J. Am. Chem. Soc., 61, 1421 (1939).

93. Nielsen, N., Naturwiss., 32, 80 (1944).

94. Hartelius, V., Compt. rend. trav. lab. Carlsberg, Sér. physiol., 24, 185 (1916); Chem. Abstr., 41, 2773 (1947).

95. Nielsen, N., Naturwiss., 31, 146 (1943).

96. Nielsen, N., and Johansen, G., Naturwiss., 31, 235 (1943).

97. Hartelius, V., Naturwiss., 31, 440 (1943).

98. Kjerulf-Jensen, K., and Schmidt, V., Acta. Pharmacol. Toxicol. (Copenhagen), 1, 346 (1945); Chem. Abstr., 40, 6541 (1946).

99. Mueller, J. H., J. Biol. Chem., 123, 421 (1938).

100. Schenck, J. R., and duVigneaud, V., J. Biol. Chem., 153, 501 (1944).

101. Wright, L. D., and Skeggs, H. R., Arch. Biochem., 10, 383 (1946).

102. King, T. E., and Cheldelin, V. H., J. Biol. Chem., 174, 273 (1948).

103. Ivanovics, G., Naturwiss., 30, 104 (1942); Z. physiol. Chem., 276, 33 (1942).

104. Vinet, A., Meunier, P., and Monfrais, J., Bull. soc. chim. biol., 28, 300 (1946).

105. Stansly, P. G., and Schlosser, M. E., J. Biol. Chem., 161, 513 (1945).

106. Stansly, P. G., and Alverson, C. M., Science, 103, 398 (1946).

107. Ackermann, W. W., and Shive, W., J. Biol. Chem., 175, 867 (1948).

108. Markees, S., Schweiz Z. Path. u. Bakt., 9, 88 (1946); Chem. Abstr., 40, 7278 (1946) ; Markees, S., Jubilee Volume, Emil Barell, Reinhardt, Ltd. Co., Basle, Switzerland, 1946, p. 405.

109. Becker, E. R., and Gallagher, P. L., Iowa State Coll. J. Sci., 21, 237, 351 (1947); Chem. Abstr., 41, 7437 (1947).

110. Pérault, R., and Greib, E, Compt. rend. soc. biol., 138, 506 (1944).

111. Roblin, R. O., Jr., Chem. Rev., 38, 255 (1946). 


\section{Chapter VIIID}

\section{THE VITAMIN $B_{6}$ GROUP}

Although a dermatitis, termed acrodynia, which is characteristic of vitamin $\mathrm{B}_{6}$ deficiency, was observed as early as 1926 in rats fed a deficient diet, ${ }^{1}$ it was not until 1934 that György ${ }^{2}$ established that the "rat pellagra preventitive" factor was a new B vitamin (vitamin $\mathrm{B}_{6}$ ) ; it was subsequently isolated in crystalline form independently in five different laboratories. ${ }^{3-\tau}$ However, in 1932 Ohdake $^{8}$ isolated from rice polishings a compound apparently identical with vitamin $\mathrm{B}_{6}$, but did not recognize it as a vitamin.

The structure of vitamin $\mathrm{B}_{6}$ indicated below was first elucidated by Kuhn and co-workers ${ }^{9,10,11}$ and subsequently was confirmed by independent work of other laboratories. ${ }^{12-15}$<smiles>Cc1ncc(CO)c(CO)c1O</smiles>

vitamin $B_{6}$ (pyridoxine, adermin)

The synthesis of vitamin $\mathrm{B}_{6}$ was accomplished shortly afterward by Harris and Folkers, ${ }^{16}$ by Kuhn and co-workers, ${ }^{17}$ and by Morii and Makino. ${ }^{18}$

\section{Specificity}

During the proof of structure and synthesis of vitamin $\mathrm{B}_{6}$, a number of structurally related compounds were prepared and tested for vitamin $\mathrm{B}_{6}$ activity. The activities of these compounds in replacing vitamin $B_{6}$ for various organisms are indicated in Table 35 .

Although many of the organisms respond similarly to the various analogues of pyridoxine, there are a few instances which contrast markedly. Thus, 2-ethyl-3-hydroxy-4,5-bis(hydroxymethyl)pyridine, which is as active as pyridoxine for stimulation of growth of excised tomato roots, inhibits the utilization of pyridoxine by Ceratostomella ulmi; but 2-methyl3-hydroxy-4-ethoxymethyl-5-hydroxymethylpyridine, which is 1 to 5 per cent as active as pyridoxine for Ceratostomella ulmi, is quite injurious 


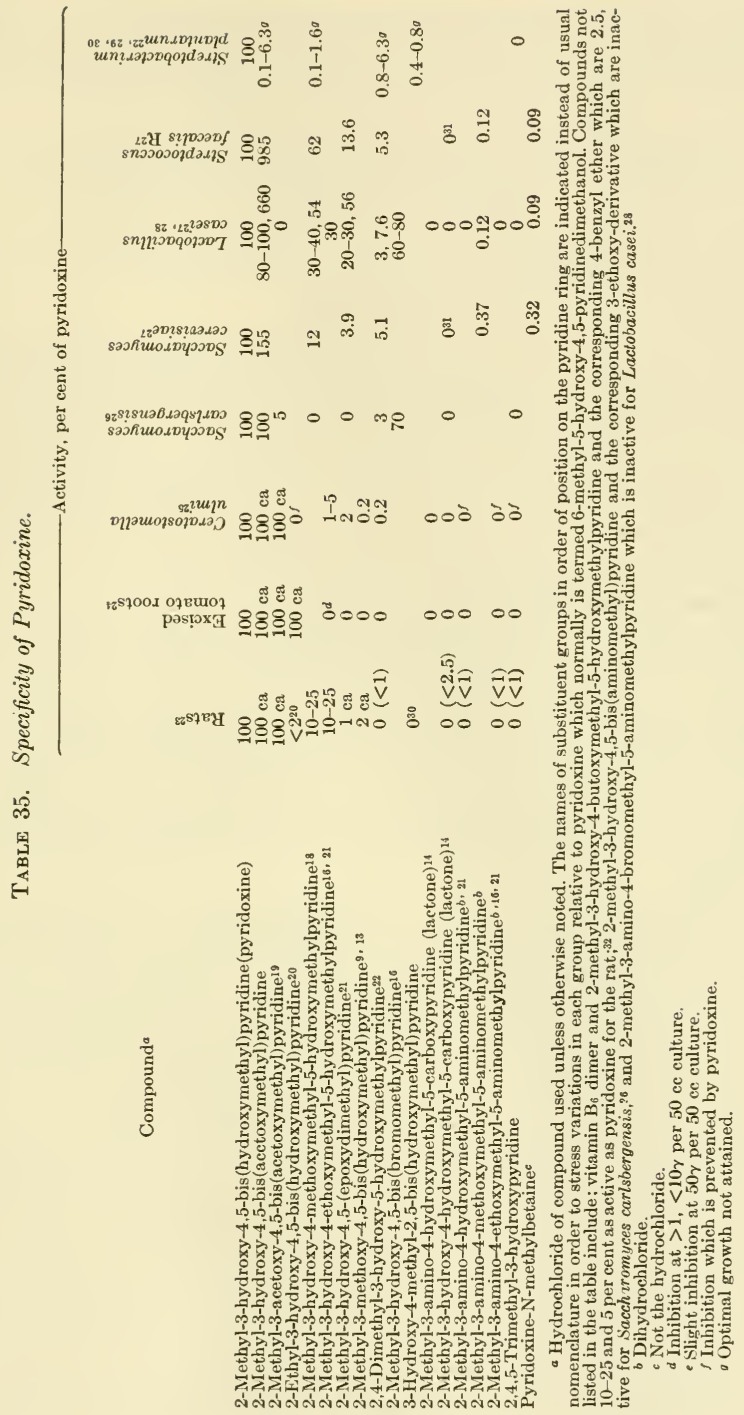


to tomato roots. Desoxypyridoxine (2,4-dimethyl-3-hydroxy-5-hydroxymethylpyridine) possesses growth-promoting activity for several microorganisms, but is an antagonist of pyridoxine for the rat and certain other animals (p. 661). 2-Methyl-3-hydroxy-4-methoxymethyl-5-hydroxymethylpyridine and 2-methyl-3-hydroxy-4,5-epoxydimethylpyridine are utilized effectively by Lactobacillus casei, but are inactive for Saccharomyces carlsbergensis. The relatively high activity of 2-methyl-3hydroxy-4,5-bis(bromomethyl)pyridine for both Lactobacillus casei and Saccharomyces carlsbergensis is interesting.

For many organisms, the triacetate of pyridoxine is as active as pyridoxine, but this compound is not utilized by Lactobacillus casei. On the other hand, both pyridoxine triacetate and tribenzoate are more effective for Streptococcus faecalis $\mathrm{R}$ than is pyridoxine. Pyridoxine diacetate is almost ten times as effective as pyridoxine for Streptococcus faecalis $\mathrm{R}$. These as well as some prior results indicated that compounds exist which are considerably more active than pyridoxine for Streptococcus faecalis $\mathrm{R}$.

Pyridoxamine and Pyridoxal. A naturally occurring form of vitamin $\mathrm{B}_{6}$ which was more active than pyridoxine was discovered by Snell, Guirard and Williams, ${ }^{33}$ who found that assays for the pyridoxine content of natural extracts with Streptococcus faecalis $\mathrm{R}$ gave values for the pyridoxine content several hundred to several thousand times as great as could be accounted for on the basis of pyridoxine actually present. This "pseudopyridoxine" was found to have properties similar to pyridoxine, and tissues of animals deficient in vitamin $\mathrm{B}_{6}$ also contained less "pseudopyridoxine."

It was demonstrated by Snell ${ }^{34}$ that pyridoxine was almost inactive unless autoclaved with the medium or with certain amino acids. Carpenter and Strong ${ }^{26}$ independently found that mild oxidation of pyridoxine yielded a substance with increased activity for Lactobacillus casei. Snell 27,35 had indicated that mixtures resulting from the reaction of pyridoxine with aminating agents or mild oxidizing agents were more active for Streptococcus faecalis $\mathrm{R}$ than the original vitamin. The properties of the active substance indicated that both an amine and an aldehyde derived chemically from pyridoxine were the active principles. With this and additional evidence, the structures of the two substances were limited essentially to four possibilities, which were synthesized in pure form by Harris, Heyl and Folkers ${ }^{36}$ by methods analogous to those used by Snell. Biological tests of the synthetic compounds revealed that 2-methyl-3hydroxy-4-aminomethyl-5-hydroxymethylpyridine and 2-methyl-3-hydroxy-4-formyl-5-hydroxymethylpyridine were the active amine and 
aldehyde which have been given the trivial names, pyridoxamine and pyridoxal, respectively.

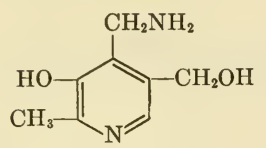

pyridoxamine<smiles>Cc1ncc(CO)c(C=O)c1O</smiles>

pyridoxal

On the basis of their structures, Snell ${ }^{35}$ proposed that pyridoxal and pyridoxamine might have a role in biological transamination, and showed that reversible interconversion of pyridoxal and pyridoxamine occurred by transamination reactions with amino acids. ${ }^{37}$ Vitamin $\mathrm{B}_{6}$-deficient rats were found to have a marked decrease in transaminase activity compared to normal rats. ${ }^{38}$

Pyridoxal, but not pyridoxamine, added to cells of Streptococcus faecalis $\mathrm{R}$ stimulated tyrosine decarboxylase, ${ }^{39}$ which was previously known to be influenced by the concentration of pyridoxine added to the growth medium. ${ }^{40}$ Gunsalus, Bellamy and Umbreit ${ }^{41}$ subsequently found that adenosine triphosphate was essential for maximum activity of pyridoxal in the system, and prepared a phosphorylated derivative of pyridoxal which was active in the absence of the phosphorylating agent. The enzyme was later isolated in a cell-free state and resolved into an apoenzyme and a coenzyme. The coenzyme was replaced by the phosphorylated pyridoxal. ${ }^{42}$ One of the methods of preparation ${ }^{41}$ indicates that the 5-hydroxymethyl is the point of phosphorylation, but definite proof of the structure is still lacking. ${ }^{41 a}$ This pyridoxal phosphate has been found to be a prosthetic group of transaminase and other enzymes (p. 177), and can be converted by heating with glutamic acid to pyridoxamine phosphate, which functions as a cotransaminase but not as a codecarboxylase. ${ }^{43}$ The discovery of this group of factors, which have been termed the vitamin $B_{6}$ group, stimulated studies of comparative activities which are indicated in Table 36 .

Injected pyridoxine, pyridoxal and pyridoxamine are equally active for dogs, rats and chicks; however, either pyridoxal or pyridoxamine is less active than pyridoxine for rats, mice and chicks when mixed with the diet. This loss of activity is prevented in the case of rats and chicks when these substances are fed by dropper. A possible explanation has been suggested ${ }^{45}$ that pyridoxal and pyridoxamine are more susceptible to destruction or utilization by intestinal bacteria than is pyridoxine. This conclusion is supported by the fact that Streptococcus faecalis $\mathrm{R}$ and presumably many other bacteria do not remove appreciable amounts of 
pyridoxine from the medium. ${ }^{32}$ Pyridoxal phosphate fed with the diet is similarly less active than pyridoxine, but when injected the two substances are equally active for rats. ${ }^{45}$

TABLE 36. Biological Activities of the Vitamin $B_{6}$ Group.

-Activity, per cent of most active form-

$\operatorname{Dogs}^{a}$

Rats

Mice

Chicks

Tetrahymena geleii

Lactobacillus helveticus

Lactobacillus acidophilus

Lactobacillus case $i$

Streptococcus faecalis $\mathrm{R}$

Streptococcus lactis L 101

Lactobacillus arabinosus

Leuconostoc mesenteroides

Streptococcus lactis UT

Streptococcus lactis 374

Streptococcus mastitidis G-2

Streptococcus mastititis 97-B

Streptococcus zymogenes H69D5

Bacillus lactis acidi B1-1

Clostridium perfringens

Clostridium welchi (B P6K)

Saccharomyces carlsbergensis

Saccharomyces cerevisiae GM

Saccharomyces oviformis

Ceratostomella ulmi

Neurospora sitophila 299

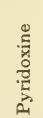

$100^{a}$

$100^{b}$

$100^{c}$

$100^{c}$

$100^{b}$

$100^{c}$

0.16

0

0

0.085

0.015

0.102

$0.001^{f}$

$0.013^{\circ}$

0.13

0.16

0.19

0.16

0.31

0.26

$0.01-0.03$

0.08

86.3

100

100

51.1

87.5

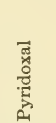

हี

$100^{a}$

$100^{b}$

$100^{a}$

$50 \mathrm{ca}^{c} 65 \mathrm{ca}^{\circ}$

$75 \mathrm{ca}^{c d} 55 \mathrm{ca}^{c d}$

$100^{b} \quad 100^{b}$

$50 \mathrm{ca}^{c} 80^{c}$

100

0

0

100

100.8

$100 \mathrm{ca}$

0

0

0
0.70

100

$5^{f} \quad 100^{f}$

$31.8^{\circ} \quad 100^{\circ}$

100

62.3

100

100

39.5

100

100

100

100

00

$30 \mathrm{ca}$

4.76

25

87.5

3.96

100

35 ca 100

100

93.6

33-98 $13-52$

$51-98 \quad 18-61$

$50 \quad 100$

$100 \quad 100$

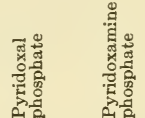

References

44

31,45

45,48

45

45

47

48

$20-33 \quad 100$

16 ca 100

$0.731,50,51$

$\begin{array}{lcc}6 \text { ca } & 0.7 & 31,50,51\end{array}$

7 ca $66-200^{e}$

31

51

52

$10 \mathrm{ca}$

31

31

31

31

31

31

53

54

$31,50,51$

$3 \quad 12 \mathrm{ca}$

31

31

31

31

a Injected in radial vein. Activities indicated are also the same for ability to promote blood regimentation in anemic dogs.

5 Injected intraperitoneally or fed by dropper; pyridoxal, pyridoxal phosphate or pyridoxamine are less active than pyridoxine if fed in the diet.

$c$ Fed in diet.
$d$ Ability to utilize pyridoxal increased after first week. Xanthurenic acid excretion data support the growth data.

- Dose-response curves for pyridoxamine phosphate and pyridoxamine differ. Figures express extreme variation in relative activity which increases as pyridoxamine is increased.

$f$ Activity in stimulating growth, vitamin $B_{6}$ not essential for growth.

- Activity in allowing growth to occur on indole in place of tryptophan.

It is interesting to note that two organisms-a strain of Lactobacillus helveticus and a strain of Lactobacillus acidophilus-require the phosphorylated forms of pyridoxamine or pyridoxal and cannot utilize pyridoxal, pyridoxamine or pyridoxine. Pyridoxamine phosphate is from 3 to 6 times as active as pyridoxal phosphate for these organisms.

Most bacteria utilize either pyridoxal or pyridoxamine more effectively than pyridoxine. The normal habitat of most of these organisms is of 
animal origin where pyridoxal and pyridoxamine predominate. ${ }^{55}$ Pyridoxine occurs in as large or larger amounts than pyridoxal and pyridoxamine in plants. $^{55}$

Yeast and molds utilized pyridoxine very effectively and in some instances more effectively than either pyridoxal or pyridoxamine.

For the few organisms tested, with the exception of the two requiring the phosphorylated form of the vitamin, pyridoxal phosphate is only 3 to 10 per cent as effective as pyridoxal, but pyridoxamine phosphate under certain conditions is more active than any other member of the vitamin $\mathrm{B}_{6}$ group for Streptococcus faecalis $\mathrm{R}$.

Pyridoxic Acid. A metabolite of pyridoxine occurring in human urine ${ }^{56}$ has been isolated, identified as 2-methyl-3-hydroxy-4-carboxy-5-hydroxymethylpyridine, synthesized and given the trivial name pyridoxic acid by Huff and Perlzweig. ${ }^{57,} 58$<smiles>Cc1ncc(CO)c(C(=O)O)c1O</smiles>

pyridoxic acid

Pyridoxic acid is the chief metabolic product of either pyridoxine, pyridoxal or pyridoxamine. ${ }^{59}$ Oral administration of pyridoxal to human subjects results in excretion of significantly higher amounts of pyridoxic acid than does administration of pyridoxine or pyridoxamine. After administration of pyridoxamine, almost equivalent amounts of pyridoxal and pyridoxamine are excreted; but when pyridoxal or pyridoxine is ingested, the form fed is the chief form of the vitamin in the urine. Although injection of pyridoxine increased the pyridoxamine and pyridoxal content of the urine, no evidence could be obtained for the conversion of either pyridoxal or pyridoxamine to pyridoxine. The recoveries in these four forms from ingested pyridoxal, pyridoxine or pyridoxamine were 70,45 and 31 per cent, respectively.

It is interesting to note that pyridoxic acid is inactive in replacing the vitamin $\mathrm{B}_{6}$ group in the nutrition of dogs, ${ }^{44}$ Streptococcus faecalis $\mathrm{R},{ }^{31}$ Lactobacillus case $i^{31}$ and Saccharomyces carlsbergensis. ${ }^{31}$

Both the lactone of pyridoxic acid and the lactone of 2-methyl-3hydroxy-4-hydroxymethyl-5-carboxypyridine have been reported to enhance the effect of pteroyldi- $\gamma$-glutamylglutamic acid in promoting growth and preventing anemia in chicks on a purified diet containing adequate amounts of pyridoxine (p. 570). ${ }^{60,61}$ Attempts to confirm these effects have been unsuccessful. ${ }^{61 a}$ The substances, designated as $\beta$ - and $\alpha$-pyracin, respectively, have been reported not to have any appreciable vitamin $B_{6}$ 
activity for certain microorganisms. ${ }^{31} \alpha$-Pyracin, as judged by survival of the animals on a vitamin $\mathrm{B}_{6}$-deficient diet, appears to have slight pyridoxine activity in mice. ${ }^{46}$ The possibility of contamination with pyridoxine was suggested, since $\alpha$-pyracin is inactive for chicks. ${ }^{47}$

Analogues of Pyridoxal and Pyridoxamine. 2-Methyl-3-hydroxy-4hydroxymethyl-5-aminomethylpyridine, an isomer of pyridoxamine, is only $0.002,1.4,0.22$ and 0.5 per cent as active as pyridoxamine for Streptococcus faecalis R, Lactobacillus casei, Saccharomyces carlsbergensis, and rats, respectively. The activity for Lactobacillus casei is slightly exaggerated, since it is compared with pyridoxamine, which is relatively inactive as compared with pyridoxal for this organism. ${ }^{31}$ 2-Methyl-3-hydroxy-4-hydroxymethyl-5-formylpyridine, the corresponding isomer of pyridoxal, is $0.005-0.01,0.03$, and $29-73$ per cent as effective as pyridoxal for Streptococcus faecalis R, Lactobacillus casei and Saccharomyces carlsbergensis, respectively. The 5-formyl derivative apparently can be utilized effectively by yeast, which presumably reduces the formyl group. Rats cannot effectively convert this compound to the vitamin. $^{31}$

The ethyl acetal derived from the hemiacetal of pyridoxal is 50 to 75 per cent as active as pyridoxal for Streptococcus faecalis $\mathrm{R}$ and Lactobacillus casei and just as effective as the vitamin for Saccharomyces carlsbergensis. This activity, however, is attributed to the hydrolysis of the acetal. ${ }^{31}$

Since vitamin $B_{6}$ functions in transamination reactions, the biological activity of Schiff bases and analogous compounds of the amino acids and pyridoxal are of interest. Eighteen pyridoxylamino acids corresponding to the formula indicated below have been prepared ${ }^{62}$ and tested ${ }^{63}$ for

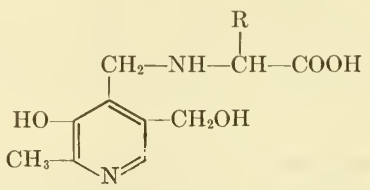

their ability to replace the vitamin $B_{6}$ group for a variety of organisms. These compounds were prepared by reductive condensation of pyridoxal with the following amino acids: DL-alanine, DL-aspartic acid, L-asparagine, DL-glutamic acid, L-glutamic acid, glycine, DL-isoleucine, DLleucine, L-leucine, L-lysine, DL-methionine, DL-norleucine, DL-phenylalanine, DL-serine, DL-threonine, DL-tryptophan, L-tyrosine and DLvaline. None of these compounds is more than 0.5 per cent as active as pyridoxal hydrochloride on a weight basis for Saccharomyces carlsber- 
gensis, Streptococcus faecalis and Lactobacillus casei. For a strain of Neurospora sitophila, the activities were for several of the compounds between 0.1 and 1 per cent that of pyridoxal hydrochloride. For the rat, definite but limited activity has been noted for some of the pyridoxalamino acids.

After pyridoxylamino acids are autoclaved in dilute aqueous solution, the resulting solution possesses high activity. ${ }^{63}$ Since the liberation of active substances could be prevented by antioxidants, such as ascorbic acid, cysteine or the complete basal medium, it was proposed that an oxidative and hydrolytic cleavage-presumably by intermediate formation of Schiff bases-occurred, with the formation of pyridoxal and pyridoxamine. This is substantiated by the fact that pyridoxylideneaniline and other Schiff bases tested were found to be as active as pyridoxal. The thiazolidinecarboxylic acid formed from L-cysteine and pyridoxal [2-(2-methyl-3-hydroxy-5-hydroxymethyl-4-pyridyl) - 4-thiazolidinecar boxylic acid] was also as active as pyridoxal for all organisms. The activity is attributed to pyridoxal formed by cleavage of the product in aqueous solutions, since the products of cysteine with other aldehydes act similarly. ${ }^{63}$

Pyridoxyl- $\beta$-alanine and the condensation product formed between histidine and pyridoxal[4-(2-methyl-3-hydroxyl-5-hydroxymethyl-4pyridyl)-1-imidazo(c)tetrahydropyridine-6-carboxylic acid] were inactive. $^{63}$

No significant antivitamin effect was noted for any of the pyridoxyl or pyridoxylidene compounds tested. ${ }^{63}$

By condensation of a number of amines, including some pressor amines, with pyridoxal and reduction of the pyridoxylidene derivative, a number of pyridoxyl amines have been prepared. These include pyridoxyltryptamine, pyridoxyl- $\beta$-phenylethylamine, pyridoxyltyramine and pyridoxylbenzylamine, which have activities between 50 and 100 per cent that of pyridoxine for rats. These compounds are considerably more effective than the corresponding pyridoxylamino acid in replacing the vitamin $B_{6}$ group. ${ }^{64}$

\section{Inhibitory Analogues of the Vitamin $B_{6}$ Group}

Demonstration of a growth inhibition prevented by pyridoxine was first reported by Robbins and $\mathrm{Ma}^{25}$ who showed that the toxicity of certain pyridoxine analogues for Ceratostomella ulmi was prevented by sufficient pyridoxine. These analogues were 2 -ethyl-3-hydroxy-4,5-bis(hydroxymethyl) pyridine, 2-methyl-3-amino-4-hydroxymethyl-5-aminomethylpyridine, 2-methyl-3-amino-4-ethoxymethyl-5-aminomethylpyridine, and 2,4,5-trimethyl-3-hydroxypyridine. Robbins ${ }^{24}$ previously 


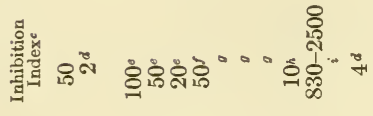
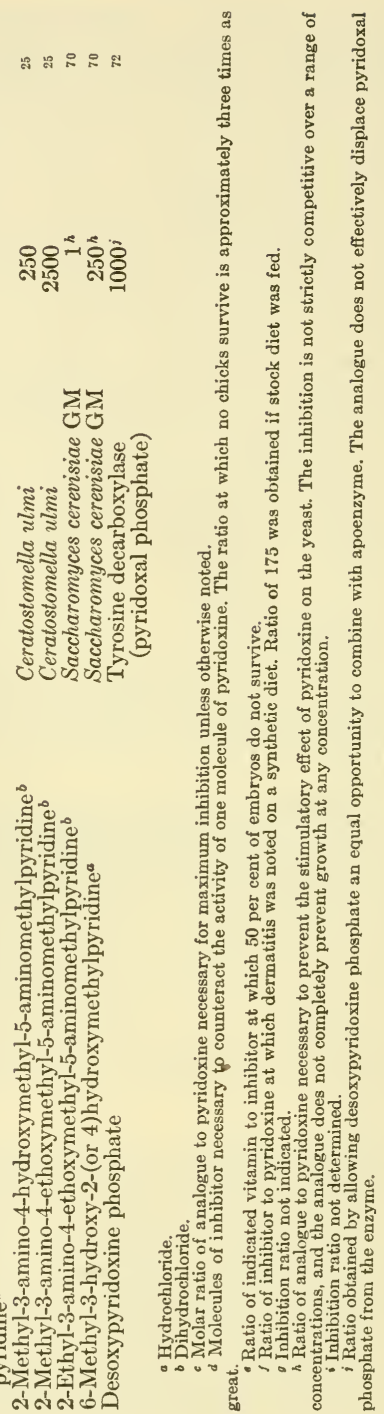
reported that 2,4,5-trimethyl-3-hydroxypyridine and 2-methyl-3-hydroxy-4-ethoxymethyl-5-hydroxymethylpyridine inhibited the growth of excised tomato roots, but did not show that the toxicity was prevented by pyridoxine. These and other inhibitory analogues of the vitamin $B_{6}$ group are listed in Table 37. The analogues, with one exception, contain one or more of the following variations from the structure of pyridoxine: 2-ethyl group replacing the 2-methyl group, a methyl group replacing an hydroxymethyl group, or either an amino or alkyoxyl group replacing an hydroxyl group. These types are illustrated by the following inhibitory analogues:<smiles>CCc1ncc(CO)c(CO)c1O</smiles>

2-ethyl-3-hydroxy-4,5-bis (hydroxymethyl) pyridine<smiles>COCc1cnc(C)c(O)c1CO</smiles>

2-methyl-3-hydroxy-4-methoxymethyl5-hydroxymethylpyridine<smiles>Cc1ncc(CO)c(C)c1O</smiles>

desoxypyridoxine<smiles>Cc1ncc(CN)c(CO)c1N</smiles>

2-methyl-3-amino-4-hydroxymethyl-5-aminomethylpyridine

2-Ethyl-3-hydroxy-4,5-bis(hydroxymethyl)pyridine. The index at which the ethyl homologue of pyridoxine inhibits the growth of Ceratostomella ulmi is approximately $50 .{ }^{25}$ At that ratio of analogue to vitamin, no growth occurs, but at a ratio of 10 , approximately half-maximal growth is obtained. This analogue is as effective as pyridoxine in stimulating the growth of excised tomato roots (Table 35 ).${ }^{24}$ This high activity in replacing the vitamin for one organism and the very potent inhibiting action on another organism illustrate that a line of demarkation cannot be drawn between stimulatory and inhibitory properties of analogues of metabolites.

Desoxypyridoxines. Investigation of the vitamin activity of analogues of pyridoxine led to the discovery that 2,4-dimethyl-3-hydroxy-5-hydroxymethylpyridine (a desoxypyridoxine) was very toxic for pyridoxinedeficient chicks. ${ }^{65}$ Day-old female single comb White Leghorn chicks, after being maintained on an adequate diet for three days, were placed on a purified diet deficient in pyridoxine for a period of six days before administration of the desoxypyridoxine. Administration of as little as 
two doses of desoxypyridoxine (100 $\gamma$ on alternate days) at approximately six times the concentration of pyridoxine intake $(16 \gamma)$ resulted in the death of all the chicks. Without simultaneous administration of pyridoxine, as little as $16 \gamma$ doses of desoxypyricloxine gave similar results. From the growth response of the chick to pyridoxine, the apparent pyridoxine activity resulting from administration of a mixture of the vitamin and analogue was determined. The difference between the amount of the vitamin administered and the apparent activity of the mixture was considered the amount of vitamin activity counteracted by the analogue. On this basis, two molecules of the inhibitor were necessary to counteract the vitamin activity of one molecule of pyridoxine at both suboptimal and optimal concentrations of the vitamin for the deficient chicks. ${ }^{65}$

Normal chicks on a diet containing adequate amounts of pyridoxine were able to tolerate a total dosage of at least $600 \gamma$ of the analogue, a level six times the lethal concentration for more than half the pyridoxinedeficient chicks on an analogous diet. ${ }^{65}$

Almost 100 per cent mortality of the chick embryos resulted from the injection of $1 \mathrm{mg}$ of desoxypyridoxine into eggs at the outset of incubation. ${ }^{66}$ The inhibitory effects were prevented by simultaneous injection of one of the vitamin $B_{6}$ group. The ratios of analogue to the vitamin at which only 50 per cent of the embryos survived were: 20 for pyridoxal hydrochloride, 50 for pyridoxamine dihydrochloride, and 100 for pyridoxine hydrochloride. However, desoxypyridoxine was not toxic to the embryo when injected after six days of incubation, except at high concentrations (2.5-5 mg per egg) at which the toxicity was not prevented by any of the three forms of vitamin $\mathrm{B}_{6}{ }^{66}$

If desoxypyridoxine is administered to weanling rats in a purified diet deficient in pyridoxine, the rate of production of acrodynia was increased and the symptoms of vitamin $B_{6}$ depletion were aggravated. ${ }^{67}$ Pyridoxine, as well as pyridoxal and pyridoxamine, prevents the toxicity of the analogue. The ratio of desoxypyridoxine to pyridoxine at which the dermatitis appears is approximately 50 . With stock rations, the ratio was 175 . Since the other members of the vitamin $B_{6}$ group act in a manner analogous to pyridoxine, the increased ratio with the stock ratios cannot be accounted for on the basis of their presence in the stock diet. Adult rats previously maintained on a stock diet were not affected appreciably during a test period on a purified diet deficient in pyridoxine; however, on the deficient diet supplemented with $0.5 \mathrm{mg}$ per cent of desoxypyridoxine, rats showed deficiency symptoms, acrodynia and loss of weight, at an average of 55 days. ${ }^{67}$

Marked reproductive upsets occur in normal female rats on a pyri- 
doxine-deficient diet containing $0.5 \mathrm{mg}$ per cent of desoxypyridoxine. ${ }^{73}$ If a change to the deficient diet containing the analogue is made on the day of breeding, the effects noted, such as 10 per cent resorption, are not as drastic as those resulting from placing the animals on the dict prior to breeding. If the animals are placed on the dict 22 days before breeding, resorption occurs in all cases and failure of implantation occurred to a significant extent (29 per cent). The percentage of resorptions and number of young born dead increased, whereas the average number of young per litter and average weight of the young decreased with the increase in number of days the animals were maintained on the deficient diet before breeding. Supplementation with pyridoxine on the day of breeding counteracted the adverse effects of the desoxypyridoxine. ${ }^{73}$

Administration of desoxypyridoxine to rats receiving tryptophan causes small increases in the excretion of xanthurenic acid and kynurenine, products which are known to be excreted as a result of vitamin $\mathrm{B}_{6}$ deficiency (p. 428). The increase produced by the analogue in excretion of xanthurenic acid and kynurenine was significantly greater in rats partially depleted of vitamin $\mathrm{B}_{6}$. Desoxypyridoxine produced this metabolic dysfunction almost immediately, in contrast with the period of time necessary for occurrence of deficiency symptoms. Supplements of pyridoxine prevented these effects of desoxypyridoxine.

With mice on a pyridoxine-deficient diet, desoxypyridoxine produces dermatitis, "ring tail" condition, unstable gait and other symptoms comparable to those produced in the rat. ${ }^{68}$

Desoxypyridoxine fed to an insulin-treated, depancreatized dog on a diet deficient in pyridoxine increased the fasting blood sugar but did not cause glycosuria or affect the hemoglobin, cell volume or serum chlorides. ${ }^{75}$

Administration of desoxypyridoxine to mice and rats causes atrophy of both normal and neoplastic lymphoid tissue. ${ }^{76}$ Lymphosarcoma transplants showed marked regression following administration of desoxypyridoxine. The regression was associated with extensive pyknosis and caryorrhexis of tumor lymphocytes and transformation of tumor cells into apparent multinucleated giant cells. ${ }^{76}$ When pyridoxine is given simultaneously with the analogue, the latter has no effect. Similarly, atrophy of the spleen, thymus and lymph nodes has been reported for puppies, chicks and monkeys following the administration of desoxypyridoxine. ${ }^{69}$ Impairment of the immune response in rats has also been noted, and the anamnestic reaction is abolished in acute pyridoxine deficiency. ${ }^{76}$ There is not, however, an increased rate of antibody destruction, as the disappearance of antibodies following passive immunization is not accelerated by a pyridoxine deficiency. ${ }^{76} \mathrm{~A}$ progressive decrease 
in erythrocyte count, hemoglobin, and hematocrit with a microcytic and hypochromic anemia was observed in puppies receiving desoxypyridoxine. ${ }^{69}$ The animals lost weight and died within two months. Monkeys receiving desoxypyridoxine similarly developed microcytic anemia, leucopenia and lymphopenia. ${ }^{69}$

Desoxypyridoxine inhibits the multiplication of $\mathrm{T}^{2} \mathrm{r}^{+}$Escherichia coli bacteriophage without affecting growth of the bacteria. The inhibition of virus production is prevented by sufficient pyridoxine, as well as by formic, acetic, butyric, valeric and pyruvic acids, glucose-6-phosphate, and less effectively by lactic, malic, fumaric and succinic acids. ${ }^{76 a}$

Desoxypyridoxine is reported to inhibit the stimulatory action of pyridoxine or pyridoxal for Saccharomyces cerevisiae. Pyridoxal appears to be more effective in preventing the inhibitory action of low concentrations of the analogue. ${ }^{70}$ At low concentrations the analogue alone is reported to stimulate the growth of a strain of Saccharomyces cerevisiae. ${ }^{27}$ It is interesting to note that desoxypyridoxine has slight growth-promoting activities for a number of microorganisms (Table 35).

Since the effect of desoxypyridoxine is more pronounced in animals with restricted vitamin $B_{6}$ intake, the mechanism of action of the inhibitor did not appear to be solely that of strict competition with the vitamin. Desoxypyridoxine, even at concentration of $1 \mathrm{mg}$ per cc, does not affect tyrosine decarboxylase from Streptococcus faecalis $\mathrm{R},{ }^{72,77}$ aspartic-glutamic transaminase from heart muscle of the horse, ${ }^{72}$ or the tryptophanase system of Escherichia coli. ${ }^{72}$ Once pyridoxal phosphate is associated with these enzymes, desoxypyridoxine does not displace it. Furthermore, desoxypyridoxine does not prevent the combination of pyridoxal phosphate with apoenzymes of the decarboxylase or the transaminase. ${ }^{72}$ Desoxypyridoxine has no effect on the formation of pyridoxal phosphate from pyridoxal and suboptimal amounts of adenosine triphosphate in Streptococcus faecalis $\mathrm{R}^{72}$ However, when adenosine triphosphate is present in excess and pyridoxal is limiting, desoxypyridoxine exerts some effect when the mixture is assayed with the apoenzyme of tyrosine decarboxylase from Streptococcus faecalis R. ${ }^{72}$ This inhibition is attributed to the formation of desoxypyridoxine phosphate.

Desoxypyridoxine phosphate exerts no effect on 3,4-dihydroxyphenylalanine decarboxylase ${ }^{77 a}$ and only a slight effect on tyrosine decarboxylase..$^{72,77}$ However, if it is allowed to compete with pyridoxal phosphate for the apoenzyme, either by addition prior to or simultaneous with the natural coenzyme, complete inhibition of the tyrosine decarboxylase occurs at a ratio of inhibitor to coenzyme of $1000 .^{72}$ If the apoenzyme is allowed to combine with the coenzyme first, desoxypyridoxine phosphate at the same relative concentration after a short incuba- 
tion inhibits only to the extent of 12 per cent. ${ }^{72}$ This indicates a very slow rate of dissociation of the enzyme and that considerable time would be required for attainment of equilibrium. This slow rate of attainment of equilibrium has been advanced as the explanation for the more pronounced effect of desoxypyridoxine in animals on a vitamin $\mathrm{B}_{6}$-deficient diet. $^{72}$

2,4,5-Trimethyl-3-hydroxypyridine, a bisdesoxy pyridoxine, is toxic for Ceratostomella ulmi with an inhibition index of $830-250,{ }^{25}$ and is also toxic for excised tomato roots. ${ }^{24}$

2-Methyl-3-hydroxy-4-alkoxy-5-hydroxymethylpyridines. Tests with chicks by methods analogous to those used for desoxypyridoxine indicate that 2-methyl-3-hydroxy-4-methoxymethyl-5-hydroxymethylpyridine is almost as effective as desoxypyridoxine in preventing the utilization of pyridoxine. Approximately 4 molecules of the methoxy analogue counteracts the response of 1 molecule of pyridoxine. Although both desoxypyridoxine and the methoxy analogue act similarly in many respects, the effects of otherwise lethal doses of desoxypyridoxine are easily counteracted, even after a considerable period of time, by administration of pyridoxine; however, pyridoxine administered subsequent to the methoxy analogue was generally ineffective in preventing death of chicks. Thus, if the modes of action of the two compounds are similar to the extent that the phosphorylated derivatives are the active inhibitory forms, the rate of dissociation of the complex of the phosphate of the methoxy analogue with appropriate apoenzymes would be expected to be similar to the slow rate observed with pyridoxal phosphate.

Hypoplasia, or failure of development of lymphoid elements, was the outstanding feature in the spleens of chicks receiving the methoxy analogue. ${ }^{69}$ Daily feeding of $1 \mathrm{mg}$ per $\mathrm{kg}$ of the methoxy compound to puppies on a vitamin $B_{6}$-deficient diet resulted in death after 1 to 4 weeks. One pup, however, on this dosage remained alive for a month and maintained blood values only slightly lower than those of the animals receiving pyridoxine, indicating some activity of the analogue in replacing pyridoxine for this animal. ${ }^{69}$

When the 4-methoxy analogue of pyridoxine and tryptophan was administered to rats deficient in vitamin $B_{6}$, the animals excreted less xanthurenic acid and kynurenine than the animals receiving tryptophan alone. ${ }^{74}$ However, in normal animals, the analogue tended to increase the amounts of these products excreted. This inclicates that the analogue may inhibit the action of pyridoxine to some extent; however, the increased excretion of pyridoxic acid on administration of the analogue indicated that it is cleaved and presumably utilized to some extent. ${ }^{74}$ 
This is also indicated by the activity of the analogue in replacing the vitamin in the nutrition of rats (Table 35).

2-Methyl-3-hydroxy-4-ethoxymethyl-5-hydroxymethylpyridine is reported to inhibit the growth of excised tomato roots. ${ }^{24}$

Other Analogues of Pyridoxine. Both 2-methyl-3-amino-4-hydroxymethyl-5-aminomethylpyridine and the corresponding 4-ethoxymethyl analogue are inhibitory for Ceratostomella ulmi, and the toxicity is prevented by sufficient pyridoxine. The inhibition indices are 250 and 2500 , respectively. ${ }^{25} \mathrm{~A}$ homologue of the last compound, 2-ethyl-3-amino-4ethoxymethyl-5-aminomethylpyridine, prevents the stimulatory action of pyridoxine for Saccharomyces cerevisiae. ${ }^{70}$ For relatively high concentrations the inhibitor appears to prevent the stimulatory action of an equivalent amount of pyridoxine. Growth, however, is never completely prevented by the analogue. ${ }^{70}$

Another inhibitory analogue of pyridoxine has been reported ${ }^{70}$ to be 2-methyl-3-hydroxy-4-hydroxymethylpyridine; however, this compound is actually derived from 2-picoline by sulfonation, fusion of the sulfonate with alkali, conversion to a dialkylaminomethyl derivative by the Mannich reaction and hydrolysis to a (hydroxymethyl) picolinol. The intermediate hydroxypicoline has been shown to be 6-methyl-3-hydroxypyridine. $^{78,79}$ Consequently, this analogue probably is 6-methyl-3hydroxy-2(or 4)-hydroxymethylpyridine. The analogue prevents the stimulatory action of pyridoxine on Saccharomyces cerevisiae at a molar ratio of $250 .^{70} \mathrm{~A}$ similar revision of structure is necessary for a group of (dialkylaminomethyl) picolinols prepared even earlier by analogous reactions. ${ }^{80}$

2-Methyl-3-hydroxy-5-hydroxymethylpyridine has been reported to be slightly inhibitory. ${ }^{81,82}$ Numerous other analogues have been prepared $^{81,82}$ including some pyrimidine analogues ${ }^{83,84}$ of pyridoxine, but neither growth-promoting nor growth-inhibiting properties have been reported for these compounds.

Irradiation of pyridoxamine under aerobic or anaerobic conditions produces a mixture with antibacterial properties. ${ }^{85}$ The active principle inhibits the growth of a wide variety of gram-negative organisms. The effect of the vitamin $\mathrm{B}_{6}$ group on the inhibition has not been determined. ${ }^{85}$

\section{Bibliography}

1. Goldberger, J., and Lillie, R. D., U. S. Pub. Health Service Pub. Health Rept., 41, 1025 (1926)

2. György, P., Nature, 133, 498 (1934); Biochem. J., 29, 741, 760, 767 (1935).

3. Kuhn, R., and Wendt, G., Ber., 71, 780, 1118 (1938).

4. Lepkovsky, S., Science, 87, 169 (1938). 
5. Keresztesy, J. C., and Stevens, J. R., Proc. Soc. Exptl. Biol. Med., 38, 64 (1938).

6. György, P., J. Am. Chem. Soc., 60, 983 (1938).

7. Ichiba, A., and Michi, K., Sci. Papers Inst. Phys. Chem. Research (Tokyo), 34, 623 (1938).

8. Ohdaki, S., Bull. Agr. Chem. Soc. Japan, 8, 111 (1932); Wiardi, P. W., Nature, 142, 1158 (1938).

9. Kuhn, R., and Wendt, G., Ber., 71, 1534 (1938).

10. Kuhn, R., Andersag, H., Westphal, K., and Wendt, G., Ber., 72, 309 (1939).

11. Kuhn, R., and Wendt, G., Ber., 72, 305, 311 (1939).

12. Keresztesy, J. C., and Stevens, J. R., J. Am. Chem. Soc., 60, 1267 (1938).

13. Stiller, E. T., Keresztesy, J. C., and Stevens, J. R., J. Am. Chem. Soc., 61, 1237 (1939).

14. Harris, S. A., Stiller, E. T., and Folkers, K., J. Am. Chem. Soc., 61, 1242 (1939).

15. Ichiba, A., and Michi, K., Sci. Papers Inst. Phys. Chem. Research (Tokyo), 34, 1014 (1938) ; 36, 1, 173 (1939).

16. Harris, S. A., and Folkers, K.., Science, 89, 347 (1939); J. Am. Chem. Soc., 61, 1245 (1939).

17. Kuhn, R., Westphal, K., Wendt, G., and Westphal, O., Naturwiss., 27, 469 (1939).

18. Morii, S., and Makino, K., Enzymologia, 7, 385 (1939).

19. Kuhn, R., and Wendt, G., Ber, 71, 780 (1938).

20. Harris, S. A., and Wilson, A. N., J. Am. Chem. Soc., 63, 2526 (1941).

21. Harris, S. A., and Folkers, K., J. Am. Chem. Soc., 61, 3307 (1939).

22. Möeller, E. F., Zima, O., Jung, F., and Moll, Th., Naturwiss., 27, 228 (1939).

23. Unna, K., Proc. Soc. Exptl. Biol. Med., 43, 122 (1940).

24. Robbins, W. J., Am J. Botany, 29, 241 (1942).

25. Robbins, W. J., and Ma, R., Bull. Torrey Botan. Club, 69, 342 (1942).

26. Carpenter, L. E., and Strong, F. M., Arch. Biochcm., 3, 375 (1944).

27. Snell, E. E., J. Am. Chem. Soc., 66, 2082 (1944).

28. Bohonos, N., Hutchings, B. L., and Peterson, W. H., J. Bact., 44, 479 (1942).

29. Möeller, E. F., Z. physiol. Chem., 260, 246 (1939).

30. Möeller, E. F., Angew. Chem., 53, 204 (1940).

31. Snell, E. E., and Rannefeld, A. N., J. Biol. Chem., 157, 475 (1945) .

32. Harris, S. A., J. Am. Chem. Soc., 63, 3363 (1941).

33. Snell, E. E., Guirard, B. M., and Williams, Re. J., J. Biol. Chem., 143, 519 (1942).

34. Snell, E. E., Proc. Soc. Exptl. Biol. Med., 51, 356 (1942).

35. Snell, E. E., J. Biol. Chem., 154, 313 (1944).

36. Harris, S. A., Heyl, D., and Folkers, K., J. Am. Chem. Soc., 66, 2088 (1944); J. Biol. Chem., 154, 315 (1944).

37. Snell, E. E., J. Am. Chem. Soc., 67, 194 (1945).

38. Schlenk, F., and Snell, E. E., J. Biol. Chem., 157, 425 (1945).

39. Gunsalus, I. C., and Bellamy, W. D., J. Biol. Chem., 155, 357 (1944).

40. Bellamy, W. D., and Gunsalus, I. C., J. Bact., 46, 573 (1943).

41. Gunsalus, I. C., Bellamy, IV. D., and Umbreit, WV. W., J. Biol. Chem., 155, 685 (1944).

41a. Umbreit, W. W., and Gunsalus, I. C., J. Biol. Chem., 179, 279 (1949).

42. Umbreit, W. W., Bellamy, W. D., and Gunsalus, I. C., Arch. Biochem., 7, 185 (1945).

43. Umbreit, W. W., O'Kane, D. J., and Gunsalus, I. C., J. Biol. Chem., 176, 629 (1948).

44. Sarma, P. S., Snell, E. E., and Elvehjem, C. A., Proc. Soc. Exptl. Biol. Med., 63, 284 (1946).

45. Sarma, P. S., Snell, E. E., and Elvehjem, C. A., J. Biol. Chem., 165, 55 (1946).

46. Miller, E. C., and Baumann, C. A., J. Biol. Chem., 159, 173 (1945).

47. Luckey, T. D., Briggs, G. M., Jr., Elvehjem, C. A., and Hart, E. B., Proc. Soc. Exptl. Biol. Med., 58, 340 (1945).

48. Kidder, G. W., and Dewey, V. C., Arch. Biochem., 21, 58 (1949). 
49. MeNutt, W. S., and Snell, E. E., J. Biol. Chem., 173, 801 (1948).

50. Rabinowitz, J. C., and Snell, E. E., Anal. Chem., 19, 277 (1947).

51. Rabinowitz, J. C., and Snell, E. E., J. Biol. Chem., 169, 643 (1947).

52. Schweigert, B. S., J. Biol. Chem., 168, 283 (1947).

53. Boyd, M. J., Logan, M. A., and Tytell, A. A., J. Biol. Chem., 174, 1013 (1948).

54. Boyd, M. J., Logan, M. A., and Tytell, A. A., J. Biol. Chem., 167, 879 (1947).

55. Rabinowitz, J. C., and Snell, E. E., J. Biol. Chem., 176, 1157 (1948).

56. Singal, S. A., and Sydenstricker, V. P., Science, 94, 545 (1941).

57. Huff, J. W., and Perlzweig, W. A., Science, 100, 15 (1944).

58. Huff, J. W., and Perlzweig, W. A., J. Biol. Chem., 155, 345 (1944).

59. Rabinowitz, J. C., and Snell, E. E., Proc. Soc. Exptl. Biol. Med., 70, 235 (1949).

60. Scott, M. L., Norris, L. C., Heuser, G. F., Bruce, W. F., Coover, H. W., Jr., Bellamy, W. D., and Gunsalus, I. C., J. Biol. Chem., 154, 713 (1944).

61. Scott, M. L., Norris, L. C., Heuser, G. F., and Bruce, W. F., J. Biol. Chem., 158, 291 (1945).

61a. Hutchings, B. L., Oleson, J. J., and Stokstad, E. L. R., J. Biol. Chem., 163, 447 (1946).

62. Heyl, D., Harris, S. A., and Folkers, K., J. Am. Chem. Soc., 70, 3429 (1948).

63. Snell, E. E., and Rabinowitz, J. C., J. Am. Chem. Soc, 70, 3432 (1948).

64. Heyl, D., Luz, E., Harris, S. A., and Folkers, K., J. Am. Chem. Soc., 70, 1670, 3669 (1948).

65. Ott, W. H., Proc. Soc. Exptl. Biol. Med., 61, 125 (1946).

66. Cravens, W. W., and Snell, E. E., Fed. Proc., 8, 380 (1949).

67. Emerson, G. A., Fed. Proc., 6, 406 (1947).

68. Cerecedo, L. R., and De Rinzo, E., Abstr. of Papers, 112th Meeting American Chemical Society, Sept. 1947, p. 25C

69. Mushett, C. W., Stebbins, R. B., and Barton, M. N., Trans. N. Y. Acad. Sci., 9, 291 (1947).

70. Martin, G. J., Avakian, S., and Moss, J., J. Biol. Chem., 174, 495 (1948).

71. Ott, W. H., Proc. Soc. Exptl. Biol. Med., 66, 215 (1947).

72. Umbreit, W. WV., and Waddell, J. G., Proc. Soc. Exptl. Biol. Med., 70, 293 (1949).

73. Nelson, M. M., and Erans, H. M., Proc. Soc. Extpl. Biol. Med., 68, 274 (1948).

74. Porter, C. C., Clark, I., and Silber, R. H., J. Biol. Chem., 167, 573 (1947).

75. Gaebler, O. H., and Herman, E. V., Fed. Proc., 6, 254 (1947).

76. Stoerck, H. C., Fed. Proc. 7, 281 (1948); J. Biol. Chem. 171, 437 (1948).

76a. Wooley, J. G., and Murphy, M. K., J. Biol. Chem., 178, 869 (1949).

77. Beiler, J. M., and Martin, G. J., J. Biol. Chem., 169, 345 (1947).

77a. Martin, G. J., and Beiler, J. M., Arch. Biochem., 15, 201 (1947).

78. Parker, E. D., and Shive, W., J. Am. Chem. Soc., 69, 63 (1947).

79. MeElvain, S. M., and Goese, M. A., J. Am. Chem. Soc., 65, 2233 (1943).

80. Brown, R. F., and Miller, S. J., J. Org. Chem. 11, 388 (1946).

81. Perez-Medina, L. A., Mariella, R. P., and MeElvain, S. M., J. Am. Chem. Soc., 69, 2574 (1947).

82. Mariella, R. P., and Leech, J. L., J. Am. Chem. Soc., 71, 331 (1949).

83. MeCasland, G. E., Tarbell, D. S., Carlin, R. B., and Shakespeare, N., J. Am. Chem. Soc., 68, 2390 (1946).

84. MeCasland, G. E., and Tarbell, D. S., J. Am. Chem. Soc., 68, 2393 (1946).

85. Shwartzman, G., and Fisher, H., J. Biol. Chem., 167, 345 (1947). 


\section{Chapter IXD}

\section{RIBOFLAVIN}

In 1933 , one of the members of the vitamin $\mathrm{B}_{2}$ complex was identified as lactochrome, a naturally occurring yellow pigment, ${ }^{1}$ first concentrated from milk in $1879,{ }^{2}$ and obtained in crude form from the same source in $1925 .^{3}$ This yellow pigment, which has a characteristic green fluorescence, was obtained in crystalline form from both egg white (ovoflavin) ${ }^{1}$ and milk (lactoflavin).1, 4 Subsequent chemical studies by Karrer and Kuhn and their co-workers limited the structural possibilities for the vitamin to the isomeric 6,7-dimethyl-9-(tetrahydroxyamyl)-isoalloxazines. By preparation of the possible isomers, the structure was finally established by Karrer and co-workers ${ }^{5,6}$ and Kuhn and co-workers, ${ }^{7,8} 8$ who independently synthesized the vitamin in 1935 . The vitamin which has since been termed riboflavin has the following structure:

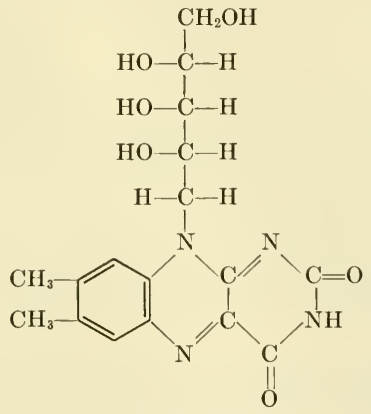

riboflavin

[6,7-dimethyl-9-(D, $1^{\prime}$-ribityl)-isoalloxazine $]$

While the purification and proof of structure of riboflavin were being accomplished, simultaneous investigations showed the vitamin to be associated with certain enzymes. In 1932, Warburg and Christian ${ }^{9}$ isolated a coenzyme essential for the functioning of the yellow enzyme. This coenzyme was found to be related to vitamin $\mathrm{B}_{2},{ }^{10}, 11$ and in 1936 its identity with synthetic riboflavin-5'-phosphoric acid was demonstrated. ${ }^{12}$ In vitro, riboflavin can replace the coenzyme, but only at 
relatively high concentrations, which suggests that the phosphate group facilitates combination of the coenzyme with the apoenzyme.

Since a large number of compounds analogous to riboflavin were prepared in the final synthetic approach to the structure of the vitamin and were tested for activity in replacing the vitamin in the nutrition of

TABLE 38. Specificity of Riboflavin.

\section{Compound}

6,7-Dimethyl-9-(D,1'-ribityl)isoalloxazine (riboflavin)

6-Ethyl-7-methyl-9-(D, 1'-ribityl)isoalloxazine

6-Methyl-9-(D, 1'-ribityl)isoalloxazine

7-Methyl-9-(D, 1'-ribityl)isoalloxazine

6,7-Dimethyl-9-(D, 1'-arabityl)isoalloxazine

6,7-Dimethyl-9-(L, 1'-arabityl)isoalloxazine

6-Methyl-9-(L, 1'-arabityl)isoalloxazine

6,7-Trimethylene-9-( $\mathrm{L}, 1^{\prime}$-arabityl)isoalloxazine

6,7-Tetramethylene-9-(L,1'arabityl)isoalloxazine

Riboflavin tetraacetate

Riboflavin-5'-phosphate

Flavin-adenine-dinucleotide

\begin{tabular}{|c|c|c|c|}
\hline Rat & $\begin{array}{l}\text { Lactobacillus } \\
\text { casei }\end{array}$ & $\begin{array}{l}\text { Bacillus } \\
\text { lactis } \\
\text { acidi }\end{array}$ & $\begin{array}{l}\text { Old Yellow } \\
\text { Enzyme }\end{array}$ \\
\hline 100 & 100 & 100 & 100 \\
\hline$>50^{13}$ & $\begin{array}{c}100 \mathrm{ca} \\
(90-100)^{e, 30}\end{array}$ & $\begin{array}{c}75-100 \\
(70-100)^{e, 30}\end{array}$ & \\
\hline$>25,<50$ & $\begin{array}{l}4-10 \\
(45-65)^{e}, 30\end{array}$ & $\begin{array}{l}6-15 \\
(45-65)^{e, 30}\end{array}$ & $87-92^{15}$ \\
\hline $\begin{array}{r}50 \mathrm{ca} \\
13,15,17\end{array}$ & $\begin{array}{c}12-20 \\
(75-100)^{e}, 30\end{array}$ & $\begin{array}{l}11-16 \\
(70-90)^{e}, 30\end{array}$ & \\
\hline $0^{b}$ & $0^{f, 30}$ & $0^{f, 30}$ & $0^{h, 32}$ \\
\hline $\begin{array}{r}30 \mathrm{ca}^{c} \\
6,18,23,26\end{array}$ & $0^{f, 30}$ & $0^{f, 30}$ & $76-81^{32}$ \\
\hline$<10^{d, 15}$ & & & $35 \mathrm{ca}^{15}$ \\
\hline$<10^{d, 15}$ & & & $46 \mathrm{ca}^{15}$ \\
\hline$<10^{d, 15}$ & & & $30-33^{15}$ \\
\hline $\begin{array}{l}100 \mathrm{ca}^{27} \\
100^{28,29}\end{array}$ & $\begin{array}{r}0^{30} \\
100^{31} \\
100^{31}\end{array}$ & $0^{30}$ & $\begin{array}{c}0^{h, 32} \\
150^{i, 32} \\
105^{j, 33}\end{array}$ \\
\hline
\end{tabular}

a The activities relative to that of riboflavin are calculated from data of each reference. Inactivity under the testing conditions is indicated by 0 . Upper limits and in one instance the lower limit of activity are indicated where data are not available for accurate estimate.

$b$ lnhibitory to growth.22 This compound was reported in earlier work to have slight activity ${ }^{13},{ }^{17-21}$ but an Amadori rearrangement appears to have occurred during synthesis resulting in contamination of the sample with riboflavin.

$c$ Maximum growth not obtained at any concentration.

$d$ Slightly active, $20 \gamma$ per day kept the animals alive without much growth, but animals receiving $10 \gamma$ per day died in 2 to 5 weeks.

- Maximum growth obtained with the analogue at any concentration reaches only the per cent indicated in brackets of the maximum response to riboflavin.

$f$ Analogue inactive alone but enhances the response of the organism to riboflavin.

o Data from separate experiments not strictly comparable on a quantitative basis. Per cent indicated represents the relative oxygen consumption in the presence of comparable quantities of analogue unless otherwise indicated.

$h$ Considered inactive; however, slight activities of enzyme preparations in absence of added analogue do not permit accurate estimates of low activities.

$i$ Relative oxygen consumption obtained with $30 \gamma$ riboflavin-5'-phosphate compared with $150 \gamma$ riboflavin. As little as $2.5 \gamma$ ribofla vin-5'-phosphate is essentially as active as $30 \gamma$ in this system.

$i$ Calculated on basis of activity $(70 \%)$ in comparison to riboflavin-5'-phosphate..$^{33}$

rats, the specificity of the structure for vitamin $B_{2}$ activity has been extensively studied by the time proof of structure of the vitamin was achieved. The activities of certain analogues in replacing riboflavin in the nutrition of rats and certain microörganisms are indicated in Table 38.

Many compounds analogous to riboflavin were also tested for activity as a coenzyme of the yellow coenzyme. Oxygen uptake and methylene blue decolorization were determined for the test substances when glucose- 
6-phosphate was oxidized to phosphogluconic acid by yeast hexosemonophosphate dehydrogenase. The coenzyme II required for the oxidation was supplied from horse blood, and the yellow enzyme necessary to complete the system was provided as the apoenzyme. The activities of the analogues in replacing riboflavin as a coenzyme for the yellow enzyme are shown in Table 38.

Another coenzyme containing riboflavin was discovered in 1936 by Das $^{34}$ as a dialyzable coenzyme of an amino acid oxidase, and was later isolated by Straub ${ }^{35}$ and by Warburg and Christian. ${ }^{36,37}$ The latter investigators demonstrated that the coenzyme contained a flavin and adenine in the form of a dinucleotide. This coenzyme, which is considered a combination of adenylic acid and riboflavin- 5 -phosphate by a pyrophosphate bond, is more versatile in its action than riboflavin-5'-phosphate, which cannot replace the dinucleotide for many apoenzymes. ${ }^{38}$ The dinucleotide can replace riboflavin phosphate in the yellow enzyme, and Lactobacillus casei utilizes either of these coenzymes as efficiently as riboflavin.

Of the eight stereoisomers corresponding to the structure of riboflavin, seven of these have been synthesized. These include the stereoisomers containing in place of the 9-D, $1^{\prime}$-ribityl group of riboflavin the following groups: L, $1^{\prime}$-ribityl, ${ }^{17} \quad \mathrm{D}, 1^{\prime}$-arabityl, ${ }^{12,}{ }^{17-22} \quad \mathrm{~L}, 1^{\prime}$-arabityl, ${ }^{6,18,23-26} \mathrm{D}, 1^{\prime}-$ lyxityl, $17 \quad \mathrm{D}, 1^{\prime}$-xylityl, ${ }^{5,6}, 23,27$ or 9 -L, $1^{\prime}$-lyxityl. The 9-L,1'-lyxityl stereoisomer ( $\mathrm{L}$-lyxoflavin) has recently been isolated from human heart muscle and synthesized, ${ }^{39}$ but no biological tests were reported. Of the other stereoisomers, only the $\mathrm{D}, \mathbf{1}^{\prime}$-xylityl and $\mathbf{L}, \mathbf{1}^{\prime}$-arabityl derivatives are reported to have activity in replacing the vitamin in the nutrition of rats. These two compounds are effective sometimes for only a few weeks with an average growth of the animals of about $30 \mathrm{gg}{ }^{18,27}$ The standard weight gain for rats of $40 \mathrm{~g}$ in 30 days with $8 \gamma$ per day of riboflavin was never obtained, even with as large amounts as $150 \gamma$ per day of either of the two stereoisomers. ${ }^{27}$

Only slight alterations of the riboflavin structure can be made if the biological activity is retained. The analogues most effective in replacing the vitamin are those containing modifications of the benzene ring of riboflavin (Table 38). The analogue with an ethyl group in place of the 6-methyl group appears to be almost as active as riboflavin for rats, Lactobacillus casei and Bacillus lactis acidi. Modifications involving the elimination of either the 6- or 7-methyl group possess appreciable biological activity of the vitamin; however, at least one of the methyl groups is essential for this activity, since, in contrast to these compounds and the corresponding 6,7-dimethyl derivatives, 9-(D,1-ribityl) isoalloxazine $^{40}$ and 9 -(D or $\mathbf{L}, 1^{\prime}$-arabityl) isoalloxazine ${ }^{6,26,41}$ are inactive in 
replacing riboflavin for rats and as a coenzyme in the yellow enzyme. ${ }^{32}$ Other known modifications of riboflavin involving the benzene ring have only slight stimulatory effects, or are inactive in replacing the vitamin. The 6,7-dimethyl groups of the L-arabityl stereoisomer of riboflavin can be replaced by either a 6,7-trimethylene or 6,7-tetramethylene group without complete loss of the biological activity for rats and the yellow enzyme (Table 38). 7-Ethyl-9(D,1'-ribityl)isoalloxazine has a growthpromoting effect for rats, but the response is not constant. ${ }^{13}$ Essentially inactive alone, 5,6-benzo-9-(D, $1^{\prime}$-ribityl) isoalloxazine ${ }^{13,30}$ and 6-ethyl7-methyl-9-(L, $1^{\prime}$-arabityl) isoalloxazine ${ }^{13,30}$ enhance the response of Lactobacillus casei and Bacillus lactis acidi to riboflavin. ${ }^{30}$ No growthpromoting activity for rats has been reported for either 5,6-dimethyl-9(L, $1^{\prime}$-arabityl) - or 6,8-dimethyl-9-(D,1'-ribityl) isoalloxazine. ${ }^{42} \quad 6,8, \mathrm{Di}$ methyl-9-(D or L, $1^{\prime}$-arabityl(isoalloxazine ${ }^{43}$ and 5,7-dimethyl-9-(D or $\mathrm{L}, 1^{\prime}$-arabityl)isoalloxazine $\mathrm{e}^{43}$ are inactive for rats and as a coenzyme for the yellow enzyme. 5,6-Benzo-9-( $\mathrm{L}, \mathrm{l}^{\prime}$-arabityl) isoalloxazine is inactive for rats. ${ }^{13}$

Replacement of the D-ribityl-group in riboflavin by glycosido-groupings results in total loss of biological activity. Thus, 6,7-dimethyl-9-(Dor L,1'-arabinosido) and 6,7-dimethyl-9-(D-ribosido)isoalloxazine are inactive in replacing riboflavin in the nutrition of the rat ${ }^{44}$ or in functioning as a coenzyme for the yellow enzyme. ${ }^{32}$

With exception of the D-xylityl and L-arabityl stereoisomers of riboflavin, substitution of similar groups for the 9-D, 1'-ribityl group in riboflavin produces inactive substances. These are exemplified by compounds containing the following substituents in the 9-position: D-1'-desoxyribityl, ${ }^{34}$ L, $1^{\prime}$-rhamnityl, ${ }^{6}$ or $n$-amyl. ${ }^{24,} 27,45$ Similarly, a complete loss of activity is obtained with analogues of 7-methyl-9-(D,1'-ribityl)isoalloxazine in which the ribityl group is replaced by the $\mathbf{L}, 1^{\prime}$-arabityl, ${ }^{6,26}$ D, 1'-xylityl, ${ }^{6,26} \mathrm{D}, 1^{\prime}$-sorbityl, ${ }^{6,26}$ D-1'-dulcityl, ${ }^{5,6}$ or $\mathrm{D}, 1^{\prime}$-mannityl ${ }^{5,6}$ group.

Substitution of a methyl group in the 3 position of riboflavin results in complete loss of vitamin activity for the rat. ${ }^{8}$ The 3,6,7-trimethyl9 -(D, $1^{\prime}$-ribityl) isoalloxazine is also inactive in the yellow enzyme test. Since this analogue does not combine with the protein, and since neither it nor the yellow enzyme shows the fluorescence characteristic of riboflavin and the free coenzyme, it has been proposed that the 3-position is one point of attachment of coenzyme to the apoenzyme. ${ }^{46}$ Because the riboflavin-5'-phosphate combines more readily than the free vitamin with the protein, the phosphate group has been considered as another point of combination with the apoenzyme. ${ }^{46}$

When riboflavin is esterified, the resulting derivatives vary considerably in their ability to replace riboflavin for biological systems. Activity 
appears to depend on whether or not the test organism is able to effect hydrolysis of a given ester. The tetra-acetyl derivative is almost as active as riboflavin for rats, ${ }^{27}$ but is inactive in the nutrition of Lactobacillus case $i$ and Bacillus lactis acidi ${ }^{30}$ and as a coenzyme for the yellow enzyme. ${ }^{32}$ The triacetate derivative of riboflavin- -5 -phosphate is also inactive in the yellow enzyme test. ${ }^{32}$ Riboflavin-5'-phosphate administered either orally or parenterally in the rat is fully as active as riboflavin. ${ }^{29}$ The sulfate of riboflavin shows some activity as a coenzyme for the yellow enzyme. ${ }^{32}$ Riboflavin mono-, di-, tri- and tetrasuccinates have been prepared in the search for more soluble forms of the vitamin. ${ }^{47}$ For Lactobacillus casei, the latter two are essentially inert, whereas the first two are respectively 60 and 18 per cent as active as riboflavin. For the rat, the activities on a molar basis are 100, 65, 21 and 0 per cent, respectively, of riboflavin. The inactivity of the tetrasuccinate contrasts with the high activity reported for the tetracetate in replacing riboflavin for the rat. ${ }^{47}$

Both the mono- and diacetone derivatives of riboflavin are active in the nutrition of rats. ${ }^{25,}{ }^{2 \tau}$ However, the condensation of riboflavin with chloral and with levulinic acid produced acetals which are inactive for both Lactobacillus casei and the rat. ${ }^{47}$

The reaction of riboflavin with formaldehyde produces methylol derivatives which are more soluble in water than the vitamin. The monomethylol derivative retains approximately 55 per cent of the activity of the vitamin but polymethylol derivatives are much less active. ${ }^{48}$

2-Amino-4,5-dimethyl-1'-D-ribitylaminobenzene increases the response of Lactobacillus casei to suboptimal concentrations of riboflavin, but alone at concentrations of 20 to $40 \gamma$ per ce, it is 0.003 per cent as effective as riboflavin. ${ }^{31}$ In the presence of alloxan, the activity is increased to as high as 0.35 per cent that of riboflavin. Alloxan alone is inactive, and neither riboflavin nor flavin-adenine-dinucleotide affects this transformation. It is suggested that the organism has some slight ability to combine these two components to form riboflavin. ${ }^{31}$

It has been reported that isoxanthopterincarboxylic acid, 2-thio-6hydroxypteridine, or lumazine can replace riboflavin or thiamine, or both, in preventing changes in chronaxia in rats. ${ }^{49}$ In similar experiments with pigeons, isoxanthopterin, 6-hydroxypteridine, 2,6-diaminopteridine, leucopterin, or lumazine are reportedly active in replacing riboflavin or thiamine. ${ }^{49}$

\section{Inhibitory Analogues of Riboflavin}

Although a number of analogues of riboflavin have been prepared, only a few appear to inhibit specifically the functioning of riboflavin in biological systems. These and related inhibitors are indicated in Table 
$\frac{3}{3}$

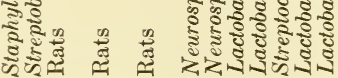
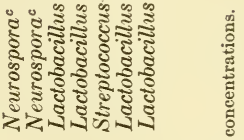

(1)

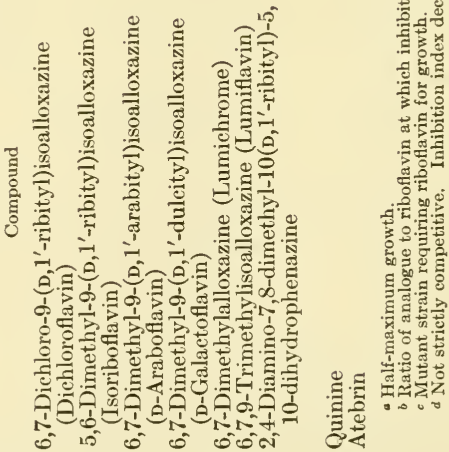


39. Of the substances listed, quinine and atebrin do not appear to be specific in their action since riboflavin, though affecting the inhibition, apparently does not act in a competitive manner. However, quinine, atebrin, and other antimalarials do affect certain isolated enzyme systems in which the riboflavin coenzymes function.

6,7-Dichloro-9(D,l'-ribityl)isoalloxazine [Dichloroflavin]. Noting that replacement of methyl by chloro substituents on the benzene nucleus of aromatic compounds changes the crystallographic properties so little that uninterrupted series of mixed crystals often result, Kuhn, Weygand, and Möller ${ }^{50}$ prepared 6,7-dichloro-9(D,1'-ribityl)isoalloxazine as a possible competitive inhibitor of riboflavin in biological systems. The analogue, which was the first antagonist of riboflavin to be reported, inhibits the growth of Staphylococcus aureus and Streptobacterium plantarum, but does not restrict the growth of yeast or Bacillus lactis acidi. Riboflavin competitively prevents the inhibitory action of the analogue, and the inhibition indices for half-maximum growth are 50, 70, and 280 for

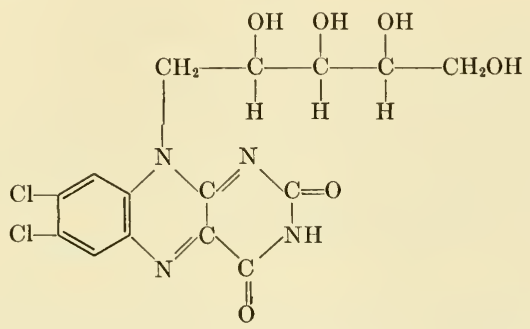

dichloroflavin[6,7-dichloro-9(D,1'-ribityl)isoalloxazine $]$

tests incubated 3, 4, and 6 days, respectively, with Staphylococcus aureus. With Streptobacterium plantarum, similar inhibition indices are 25, 60, 130, and 165 for incubation periods of 2, 3, 4, and 6 days, respectively. The inhibitory behavior of dichloroflavin has been explained on the basis of the difference in the redox potentials of dichloroflavin, $E_{0}=-0.095 \mathrm{~V}$ $(\mathrm{pH} 7)$, and riboflavin, $E_{o}=-0.185 \mathrm{~V}(\mathrm{pH}$ 7). The vitamin analogue probably cannot participate in the oxidation-reduction reactions mediated by the riboflavin coenzymes. ${ }^{50}$

Neither dichloroflavin nor its $5^{\prime}$-phosphate affect the activity in vitro of D-amino acid oxidase from liver or xanthine oxidase from milk even at concentrations 1000 -fold greater than that of the riboflavin coenzyme. ${ }^{56}$

Isoriboflavin [5,6-Dimethyl-9(b, 1'-ribityl)isoalloxazine]. An isomer of riboflavin, 5,6-dimethyl-9-(D,1'-ribityl)isoalloxazine, at levels of $2 \mathrm{mg}$ 
per day, restricts the growth of riboflavin-deficient rats to a much greater extent than does the deficiency of the vitamin alone.51 This intake of isoriboflavin almost completely inhibits the growth-promoting effect of $10 \gamma$ per day of riboflavin, but the inhibitory effect can be prevented entirely by the daily administration of $40 \gamma$ of the vitamin. ${ }^{51}$

Isoriboflavin even at concentrations 100,000 times that of riboflavin does not inhibit the growth of Lactobacillus casci; and negligible activity

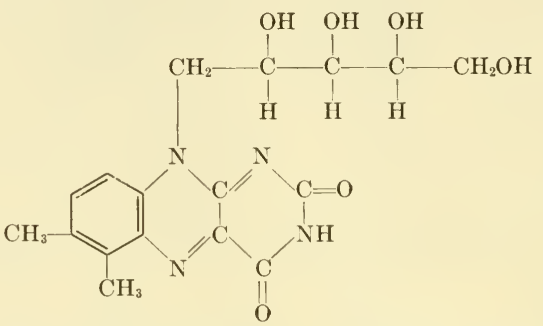

isoriboflavin [5,6-dimethyl-9-(D,1'-ribityl)isoalloxazine]

(less than 0.5 per cent) is obtained in attempts to replace the requirement for riboflavin, with either isoriboflavin or its tetra-acetyl derivative. ${ }^{57}$ However, isoriboflavin markedly stimulates the acid production and growth of Lactobacillus casei in the presence of suboptimal levels of riboflavin or flavin-adenine-dinucleotide. ${ }^{31}$

D-Araboflavin [6,7-Dimethyl-9-(D,1'-arabityl)isoalloxazine]. Administration of D-araboflavin (200 $\gamma$ per day) to rats on a riboflavin-deficient diet retards growth and increases the mortality rate beyond that which<smiles></smiles>

D-araboflavin [6,7-dimethyl-9-(D,1'-arabityl) isoalloxazine $]$

could be attributed to deficiency of the vitamin alone. ${ }^{22}$ Only one out of ten rats survives by the third week. The analogue $(200 \gamma$ per day) also decreases the rate of growth of rats receiving low amounts of riboflavin (10 $\gamma$ per day) to such an extent that no growth takes place by the third 
week. L-Araboflavin (200 $\gamma$ per day), which possesses some growthpromoting properties itself, appears to reduce very slightly the growth of rats receiving low amounts of riboflavin $\left(10 \gamma\right.$ per day). ${ }^{22}$ D-Araboflavin, at a concentration of $25 \gamma$ per ce, also inhibits the growth of an unidentified strain of lactic acid bacteria.

Galactoflavin [6,7-Dimethyl-9-(b,l'-dulcityl)isoalloxazine]. The administration of galactoflavin $(1.0$ to $2.16 \mathrm{mg})$ daily by stomach tube increases the mortality rate and decreases the rate of growth of rats on a riboflavin-free diet. The analogue $(2.16 \mathrm{mg}$ per day) completely inhibits the

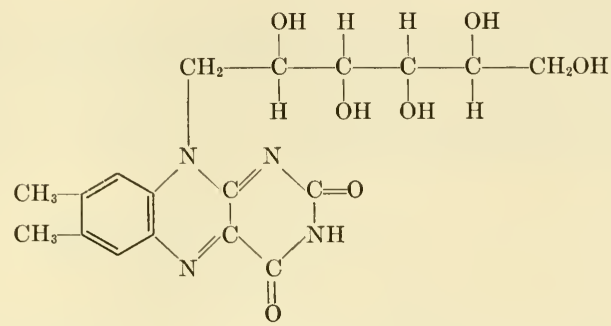

galactoflavin [6,7-dimethyl-9-(D,1'-dulcityl)isoalloxazine $]$

response of the animals to $10 \gamma$ daily of riboflavin and markedly inhibits the response to $40 \gamma$ daily of the vitamin. The inhibitory effect of $2.16 \mathrm{mg}$ of the analogue is almost, but not completely, prevented by $200 \gamma$ per day of riboflavin. The index at which growth inhibition is noted is approximately 10 .

Lumichrome and Lumiflavin. The growth of a mutant strain (51602) of Neurospora which requires riboflavin when incubated at $31-34^{\circ} \mathrm{C}$, but not when incubated at $25^{\circ} \mathrm{C}$, is inhibited by both lumichrome and lumiflavin. ${ }^{53}$ The inhibition resulting from either compound is competitively prevented by riboflavin. In tests incubated for 84 hours at $31^{\circ} \mathrm{C}$, the inhibition index for lumichrome was 2.2-2.5 for half-maximum and 6-8 for complete inhibition of growth. Lumiflavin was only about onetwentieth as effective as lumichrome. ${ }^{53}$<smiles></smiles>

lumichrome (6,7-dimethylalloxazine)<smiles></smiles>

lumiflavin (6,7,9-trimethylisoalloxazine) 
Natural extracts also contain a substance(s) which inhibits growth of the mutant. The toxicity is competitively prevented by riboflavin, but the properties of the toxic material suggest that neither lumichrome nor lumiflavin is responsible for the inhibitory activity of natural extracts. ${ }^{53}$

The growth of Lactobacillus casei stimulated by suboptimal amounts of riboflavin is inhibited by high concentrations of lumiflavin; however, in the presence of increased amounts of riboflavin, a stimulatory action is exerted by the analogue. ${ }^{31}$ Lumiflavin inhibits the utilization of flavinadenine-dinucleotide more effectively than the utilization of riboflavin by the organism. ${ }^{31}$

2,4-Diamino-7,8-dimethyl-10(D,1'-ribityl)5,10-dihydrophenazine. The growth of Lactobacillus case $i$ in the presence of $0.03 \gamma$ per ce of riboflavin is completely inhibited by $200 \gamma$ per ec of 2,4-diamino-7,8-dimethyl10 (D, $1^{\prime}$-ribityl) 5,10 -dihydrophenazine. ${ }^{54}$ The toxicity is prevented by

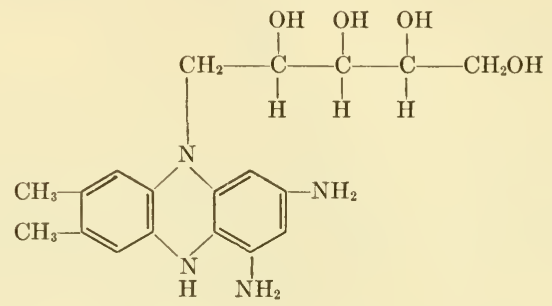

2,4-diamino-7,8-dimethyl-10-(D,1'-ribityl)-5,10-dihydrophenazine

increasing the concentration of riboflavin to $100 \gamma$ per cc. The inhibition index appears to be approximately 6600. Half-maximum inhibition of growth of Hemolytic streptococcus $\mathrm{H} 69 \mathrm{D}$ and Lactobacillus arabinosus resulted from addition of $330 \gamma$ per cc of the diaminophenazine to a medium containing $0.03 \gamma$ per cc of riboflavin. Higher concentrations of riboflavin prevent this inhibition of growth. Staphylococcus aureus, Streptococcus faecalis R, and Escherichia coli are not affected in their growth by the analogue. Because of the instability of the diaminophenazine, reduction of the corresponding 2,4-dinitrophenazine with finely divided iron in the culture medium is a desirable procedure. However, corresponding results are obtained with purified diaminophenazine previously prepared by reduction of the dinitrophenazine with tin. ${ }^{54}$

The dinitrophenazine produces in mice a very mild riboflavin deficiency characterized by greasy, unkempt fur, by hyperirritability, and by a slightly reduced rate of growth. Sufficient amounts of riboflavin prevent the appearance of these changes. 
Flavin-adenine-dinucleotide prevents the toxicity of the diaminophenazine for Lactobacillus casei in a manner analogous to riboflavin, and is equally effective. ${ }^{31}$

Atebrin, Quinine and Related Antimalarials. The discovery that atebrin inhibits the oxygen consumption of various organisms resulted in investigations of possible relationships to the riboflavin coenzymes. ${ }^{58,59,60}$ It was demonstrated that atebrin inhibits $\mathrm{D}$-amino acid oxidase ${ }^{60}$ and prevents the combination of the apoenzyme of cytochrome reductase with riboflavin- 5 '-phosphate. ${ }^{61}$ Although riboflavin-5'-phosphate at a

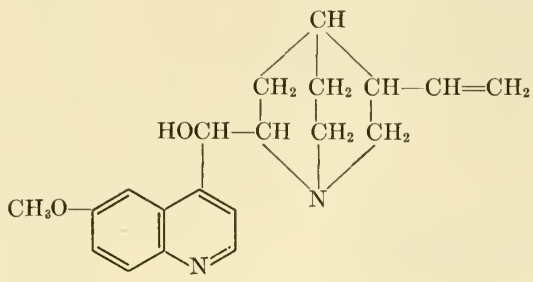

quinine<smiles>CCN(CC)CCCC(C)Nc1c2ccc(Cl)cc2nc2ccc(OC)cc12</smiles>

atebrin

ratio of 1 to 500 prevents the inhibitory action of atebrin, the inhibition resulting when atebrin is added to the apoenzyme of cytochrome reductase prior to the coenzyme is not affected by the coenzyme. ${ }^{61}$

A study of the effect of a number of compounds on the D-amino acid oxidase system has revealed that a large number of compounds related to quinine and atebrin inhibit the enzyme. ${ }^{62}$ Since increased concentrations of flavin-adenine-dinucleotide prevent the inhibition resulting from a number of these compounds, competitive inhibition is indicated. The relative activities of these compounds as compared with quinine at two different temperatures are indicated in Table 40. Atebrin, auramine, and novalauramine are somewhat more effective than quinine, while plasmochin and a number of quinoline derivatives are approximately as 
TABLE 40. Competitive Inhibitors of Flavin-Adenine-Dinucleotide for $D$-Amino Acid Oxidase ${ }^{62}$

Auramine

Atebrin

Novalauramine

Quinine

Quinine methochloride

6-Methoxyquinoline

Plasmochin

7-Chloro-4-(4-diethylamino-1-methylbutylamino)-2-methylquinoline

7-Chloro-4-(4-diethylamino-1-methylbutylamino)-3-methylquinoline

7-Chloro-4-(4-diethylamino-1-methylbutylamino)quinoline

$\alpha$-(Diamylaminomethyl)-1,2,3,4-tetrahydro9-phenanthrenemethanol

Sulfathiazole

Sulfapyridine

Sulfadiazine

Sulfanilamide

Benzenesulfonamide

$N$-(4-Diethylamino-1-methylbutyl)- $\beta$ -

( $p$-dimethylaminophenyl)alanine

Aniline

Pyridine

DL- $\alpha$ - $(p$-Dimethylaminophenyl)glycine
At $37^{\circ} \mathrm{C}$ Quinine Equivalent* ${ }^{\circ} \mathrm{At}$

7

2.5

2

1

1

1

1

1

0.4

0.5

0.5

0.5

0.2

0.07

0.04

0.04

0.07

0.015

0.04

0.015

0.04

0.04

0.02

$<0.03$

0.01

0.007

0.007

* The ratio of concentrations of quinine and inhibitor required to give the same amount of inhibition at any concentration of flavin-adenine-dinucleotide.

active as quinine. The sulfonamides are considerably less effective. The dissociation of the coenzyme from D-amino acid oxidase does not occur readily at $30^{\circ} \mathrm{C}$, but dissociation is readily detectable at $37^{\circ} \mathrm{C}$. At the latter temperature, the inhibitions resulting from quinine appear to be reversible and competitive with the coenzyme. The dissociation constants for two different enzyme preparations are $4.6 \times 10^{-7}$ and $6.9 \times 10^{-7}$ for the enzyme complex and $4.6 \times 10^{-4}$ and $8.9 \times 10^{-4}$ for the quinine-apoenzyme complex. With atebrin, two types of inhibition appear to exist. One is<smiles>CN(C)c1ccc(C(N)=C2C=CC(=[N+](C)C)C=C2)cc1</smiles>

auramine<smiles>CCN(CC)CCCC(C)Nc1cc(OC)cc2cccnc12</smiles>

plasmochin 
rapid, reversible, and competitive, but the other is slower and apparently irreversible. ${ }^{62}$

Quinine, atebrin, and a number of antimalarials prevent the growth of Lactobacillus case $i^{55}$ The inhibition is overcome to some extent, but apparently not strictly competitively in most instances, by increasing the riboflavin content of the medium. Thus, in media containing $0.25 \gamma$ and $2.5 \gamma$ per ce of riboflavin, the maximum concentrations, respectively, in $\mathrm{mg}$ per ce at which visible growth of Lactobacillus casei is observed are 0.6 and $1.75 \mathrm{mg}$ of quinine, 0.06 and $0.25 \mathrm{mg}$ of atebrin, 0.02 and 0.1 $\mathrm{mg}$ of propamidine, 0.0034 and $0.01 \mathrm{mg}$ of methylene blue, 0.29 and 1.22 $\mathrm{mg}$ of 2 - $p$-chloroanilino-4- $\beta$-diethylaminoethylamino-6-methylpyrimidine, ${ }^{63} 0.42$ and $2.5 \mathrm{mg}$ of $2-p$-chlorophenylguanidino-4- $\beta$-diethylaminoethylamino-6-methylpyrimidine, ${ }^{64}$ and 0.02 and $0.06 \mathrm{mg}$ of 2 - $\left(6^{\prime}\right.$-bromo$\beta$-naphthylamino) - 4-diethylaminoethylamino-6-methylpyrimidine. ${ }^{55}$, 65 Other inhibitory substances not related to these compounds structurally were not affected by additional riboflavin. ${ }^{55}$

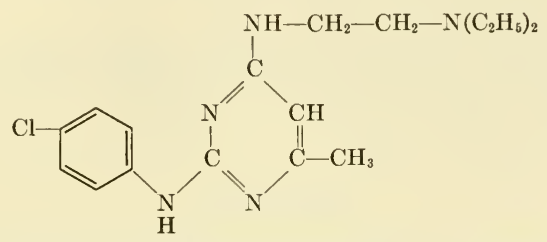

2-p-chloroanilino-4- $\beta$-diethylaminoethylamino-6-methylpyrimidine

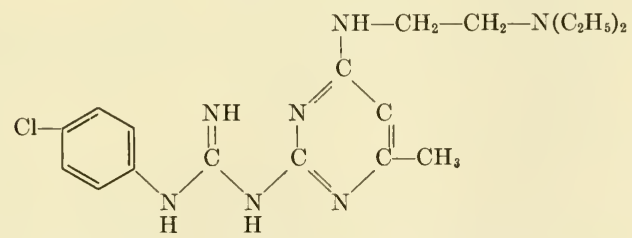

2-p-chlorophenylguanidino-4- $\beta$-diethylaminoethylamino-6-methylpyrimidine

Atebrin has an inhibitory action on the tryptophanase activity of viable cells of Escherichia coli but has little effect on the cell-free enzyme. The inhibitory action of atebrin on the cells is reduced by addition of supplementary riboflavin, and the effect has been attributed to the influence of accumulated pyruvate on the system.

Miscellaneous Analogues of Riboflavin. At concentrations of $25 \gamma$ per cc, 9-(D,1'-sorbityl)isoalloxazine inhibits the growth of Bacillus lactis 
acidi, and either 6,7-dimethyl-9-(D,1'-xylityl)isoalloxazine or 9-(D,1' arabityl)isoalloxazine inhibits the growth of an unidentified strain of lactic acid bacteria. ${ }^{50}$

The following isoalloxazines have been reported to be inactive as inhibitory analogues of riboflavin for Bacillus lactis acidi and the unidentified strain of lactic acid bacteria: 9-(L, $1^{\prime}$-arabityl)-, 9-hydroxyethyl-, 3-methyl-9-(D,1'-sorbityl) -, 5,6-dimethyl-9-(L,1'-arabityl)-, 6,7-dimethyl-9-(D,1'-sorbityl) -, 3,6,7-trimethyl-9-(D,1'-sorbityl)-, 6,7-tetramethylene-9-(L,1'-arabityl)-, 5,6-benzo-9-methyl-, 9-phenyl-, 6-methyl9-(D,1'-ribityl) isoalloxazine, 6,7-dimethyl-9-isoalloxazineacetic acid, and 9-isoalloxazineacetic acid. 1,2-Dimethyl-4-amino-5-D,1'-ribitylaminobenzene and the corresponding 4 -nitro derivative are also inactive. ${ }^{50}$

Although 2-amino-4,5-dimethyl-1,D-ribitylaminobenzene inhibits the oxidation of riboflavin by Pseudomonas riboflavina, the inhibition does not appear to be competitive..$^{57}$ No growth-inhibiting effect of 2-amino4,5-dimethyl-1-ribitylaminobenzene, even at relatively high concentrations, is obtained with Lactobacillus casei. ${ }^{57}$

$p$-Monomethylaminoazobenzene and p-dimethylaminoazobenzene, which are hepatic carcinogens, inhibit the growth of both Lactobacillus casei and Saccharomyces cerevisiae. ${ }^{67}$ Riboflavin tends to prevent the toxicity of these compounds for each organism. ${ }^{67}$

\section{Bibliography}

1. Kuhn, R., György, P., and Wagner-Jauregg, T., Ber., 66, 317, 576, 1034 (1933).

2. Blyth, A. W., J. Chem. Soc., 35, 530 (1879).

3. Bleyer, B., and Kallmann, O., Biochem. Z., 155, 54 (1925).

4. Ellinger, P., and Koschara, W., Ber., 66, 315, 808, 1411 (1933).

5. Karrer, P., Schöpp, K., and Benz, F., Helv. Chim. Acta, 18, 426 (1935).

6. Euler, H. v., Karrer, P., Malmberg, M., Schöpp, K., Benz, F., Becker, B., and Frei, P., Helv. Chim. Acta, 18, 522 (1935).

7. Kuhn, R., Reinemund, K., Káaltschmitt, H., Ströbele, K., and Trischmann, H., Naturwiss., 23, 260 (1935).

8. Kuhn, R., Reinemund, K., Weygand, F., and Ströbele, K., Ber., 68, 1765 (1935).

9. Warburg, O., and Christian, W., Naturwiss., 20, 688, 980 (1932); Biochem. Z., 258, $496(1933) ; 263,228(1933) ; 242,206(1931) ; 254,438(1932) ; 257,492$ (1933); 266, 377 (1933).

10. Banga, I., and Szent-Györgyi, A., Biochem. Z., 246, 203 (1932); Banga, I., SzentGyörgyi, A., and Vargha, L., Z. physiol. Chem., 210, 228 (1932).

11. Theorell, H., Biochem. Z., 275, 37 (1934); Kuhn, R., and Rudy, H., Ber., 68, 383 (1935).

12. Kuhn, R., Rudy, H., and Weygand, F., Ber., 69, 1543 (1936); Kuhn, R., and Rudy, H., Ber., 69, 1974 (1936).

13. Karrer, P., and Quibell, T. H., Helv. Chim. Acta, 19, 1034 (1936).

14. Karrer, P., and Strong, F. M., Helv. Chim. Acta, 18, 1343 (1935).

15. Kuhn, R., Vetter, H., and Rzeppa, H. W., Ber., 70, 1302 (1937).

16. Karrer, P., Euler, H. v., Malmberg, M., and Schöpp, K., Svensk. Kem. Tid., 47, 153 (1935).

17. Karrer, P., Salomon, H., Schöpp, K., Benz, F., and Becker, B., Helv. Chim. Acta, 18, 908 (1935). 
18. Euler, H. v., Karrer, P., and Malmberg, M., Helv. Chim. Acta, 18, 1336 (1935).

19. Kuhn, R., and Weygand, F., Ber., 68, 1282 (1935).

20. Kuhn, R., and Weygand, F., Ber., 70, 769 (1937); Karrer, P., Ber., 70, 2565 (1937).

21. Weygand, F., Ber., 73, 1259 (1940).

22. Euler, H. v., and Karrer, P., Helv. Chim. Acta, 29, 353 (1946).

23. Kuhn, R., and Weygand, F., Ber., 67, 1939, 2084 (1934).

24. Kuhn, R., and Weygand, F., Ber., 68, 166 (1935).

25. Kuhn, R., Rudy, H., and Weygand, F., Ber., 68, 625 (1935).

26. Karrer, P., Schöpp, K., Benz, F., and Pfachler, K., Helv. Chim. Acta, 18, 69 (1935).

27. Kuhn, R., Angew. Chem., 49, 6 (1936).

28. Kuhn, R., Rudy, H., and Weygand, F., Ber., 69, 1543 (1936); Kuhn, R., and Rudy, H., Ber., 68, 383 (1935); Z. physiol. Chem., 239, 47 (1936).

29. György, P., Proc. Soc. Exptl. Biol. Med., 35, 207 (1936).

30. Snell, E. E., and Strong, F. M., Enzymologia, 6, 186 (1939).

31. Sarett, H. P., J. Biol. Chem., 162, 87 (1946).

32. Kuhn, R., and Rudy, H., Ber., 69, 2557 (1936).

33. Warburg, O., and Christian, W., Biochem. Z., 298, 368 (1938).

34. Das, N. B., Biochem. J., 30, 1080, 1617 (1936).

35. Straub, F. B., Nature, 141, 603 (1938).

36. Warburg, O., and Christian, W., Naturwiss., 26, 201, 235 (1938).

37. Warburg, O., and Christian, W., Biochem. Z., 296, 294 (1938); 298, 150, 368 (1938).

38. Haas, E., Biochem. Z., 298, 378 (1938).

39. Pallares, E. S., and Garza, H. M., Arch. Biochem., 22, 63 (1949).

40. Karrer, P., Salomon, H., Schöpp, K., and Benz, F., Helv. Chim. Acta, 18, 1143 (1935).

41. Kuhn, R., and Weygand, F., Ber., 68, 1001 (1935).

42. Karrer, P., and Strong, F. M., Helv. Chim. Acta, 19, 483 (1936).

43. Kuhn, R., Desnuelle, P., and Weygand, F., Ber., 70, 1293 (1937).

44. Kuhn, R., and Ströbele, K., Ber., 70, 747 (1937).

45. Kuhn, R., and Weygand, F., Ber., 67, 1941 (1934).

46. Kuhn, R., and Boulanger, P., Ber., 69, 1557 (1936).

47. Furter, M. F., Haas, G. J., and Rubin, S. H., J. Biol. Chem., 160, 293 (1945).

48. Schoen, K., and Gordon, S. M., Arch. Biochem., 22, 149 (1949).

49. Busnel, R. G., Chauchard, P., Mazoué, H., and Polonovski, M., Compt. rend. soc. biol., 140, 50 (1946).

50. Kuhn, R., Weygand, F., and Möller, E. F., Ber., 76, 1044 (1943).

51. Emerson, G. A., and Tishler, M., Proc. Soc. Exptl. Biol. Med., 55, 184 (1944).

52. Emerson, G. A., Wurtz, E., and Johnson, O. H., J. Biol. Chem., 160, 165 (1945).

53. Mitchell, H. K., and Houlahan, M. B., Am. J. Botany, 33, 31 (1946).

54. Woolley, D. W., J. Biol. Chem., 154, 31 (1944).

55. Madinaveitia, J., Biochcm. J., 40, 373 (1946); Biochem. J., 38, xxvii (1944).

56. Karrer, P., and Ruckstuhl, H., Bl. Schweiz. Akad. Med. Wiss., 1, 236 (1945).

57. Foster, J. W., J. Bact., 48, 97 (1944).

58. Fulton, J. D., and Christophers, S. R., Ann. Trop. Med., 32, 77 (1938).

59. Martin, S. J., Cominole, B., and Clark, B. B., J. Pharmacol., 65, 156 (1939).

60. Wright, C. I., and Sabine, J. C., J. Biol. Chem., 155, 315 (1944).

61. Haas, E., J. Biol. Chem., 155, 321 (1944).

62. Hellerman, L., Lindsay, A., and Bovarnick, M. R., J. Biol. Chem., 163, 553 (1946).

63. Curd, F. H. S., and Rose, F. L., J. Chem. Soc., 1946, 343.

64. Curd, F. H. S., and Rose, F. L., J. Chem. Soc., 1946, 362.

65. Curd, F. H. S., Raison, C. G., and Rose, F. L., J. Chem. Soc., 1946, 366.

66. Dawson, J., Biochem. J., 40, xli (1946); Dawes, E. A., and Happold, F. C., Biochem. J., 44, 349 (1949).

67. Miller, E. C., Kingsley, H. N., and Miller, J. A., Cancer Research, 7, 730 (1947). 


\section{Chapter XD}

\section{THIAMINE*}

\section{Introduction}

Between 1884 and 1912, it was established that beriberi in man 1,2,3 and polyneuritis (beriberi) in fowls ${ }^{4}$ and in rats ${ }^{5}$ are deficiency diseases caused by the lack of some substance which is present only in certain foods, and it was shown that rice bran is a relatively rich source of this necessary substance. The isolation of this substance (which in the European literature is known as aneurine and in the American literature is called thiamine) presented many difficulties; it was not until 1926 to 1934 that crystalline preparations approaching purity were obtained ${ }^{6-10}$ and the empirical formula was established beyond reasonable doubt. In 1936, Williams ${ }^{11}$ and, independently, Grewe ${ }^{12}$ showed that thiamine has the structure represented by the following formula:

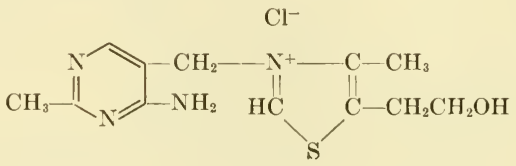

thiamine

Synthesis of this compound was achieved in the same year by Williams and Cline ${ }^{13}$ and by Andersag and Westphal. ${ }^{14}$

\section{Specificity}

The results of tests on pigeons and on rats indicate that on a molar basis, the hydrochloride, hydrobromide ${ }^{13}$ hydroiodide, ${ }^{15}$ the sulfate and nitrate salts as well as the acetate, benzoate, chaulmoograte and phosphate esters ${ }^{17}$ of thiamine possess substantially the same antineuritic activity.

Thiamine in the form of the pyrophosphate ester (cocarboxylase) is involved in tissue oxidation of carbohydrates, particularly in reactions involving decarboxylation of pyruvic acid $;^{18}$ consequently, it might be expected that thiamine would be capable of reversible oxidation and

* By A. D. Barton and Lorene L. Rogers. 
reduction. Early investigation of this possibility showed that thiamine, under suitable conditions, took up one mole of hydrogen when reduced catalytically or by means of sodium hydrosulfite, ${ }^{19}$ but the reduction product was biologically inactive. On the other hand dihydrothiamine pyrophosphate, prepared by catalytic hydrogenation, was found to be as active as thiamine pyrophosphate. ${ }^{19}$ However, none of these reduction products was autoxidizable.

Later investigations showed that under conditions as mild as those prevailing in growing tissues, thiamine ${ }^{20}$ and thiamine pyrophosphate ${ }^{21}$ (cocarboxylase) can be oxidized either by dilute hydrogen peroxide at $\mathrm{pH} 7.5$ or by iodine in alkaline solution to form the corresponding disulfide derivative without loss of vitamin $\mathrm{B}_{1}$ activity; this conversion involves the opening of the thiazole ring, and the disulfide may be represented by the following formula:

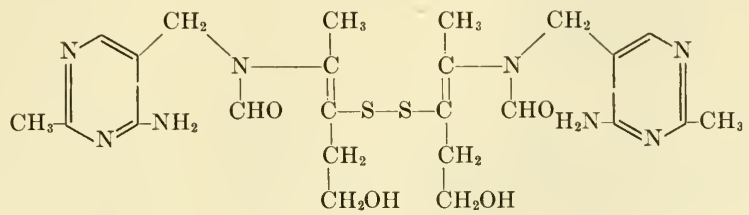

The disulfide can be reduced by cysteine or glutathione. ${ }^{22}$ More recently it has been shown that thiamine pyrophosphate disulfide is inactive in the yeast carboxylase enzyme system, ${ }^{23}$ and although fermenting yeast is able to reduce the cocarboxylase disulfide, it appears that the disulfide form may not be involved in the biological functioning of thiamine.

More vigorous oxidation of thiamine yields thiochrome, ${ }^{24}$ a yellow compound with intense blue fluorescence; this compound is biologically inactive, except for a few microorganisms. ${ }^{25}$<smiles>CC1=C(CO)SC2=Nc3nc(C)ncc3CN21</smiles>

thiochrome

In general, it appears that animals require the complete thiamine molecule, but plants and many microorganisms can utilize a mixture of the pyrimidine and thiazole components for the synthesis of vitamin $B_{1}$. Some organisms require only one of the components, and others are cap- 


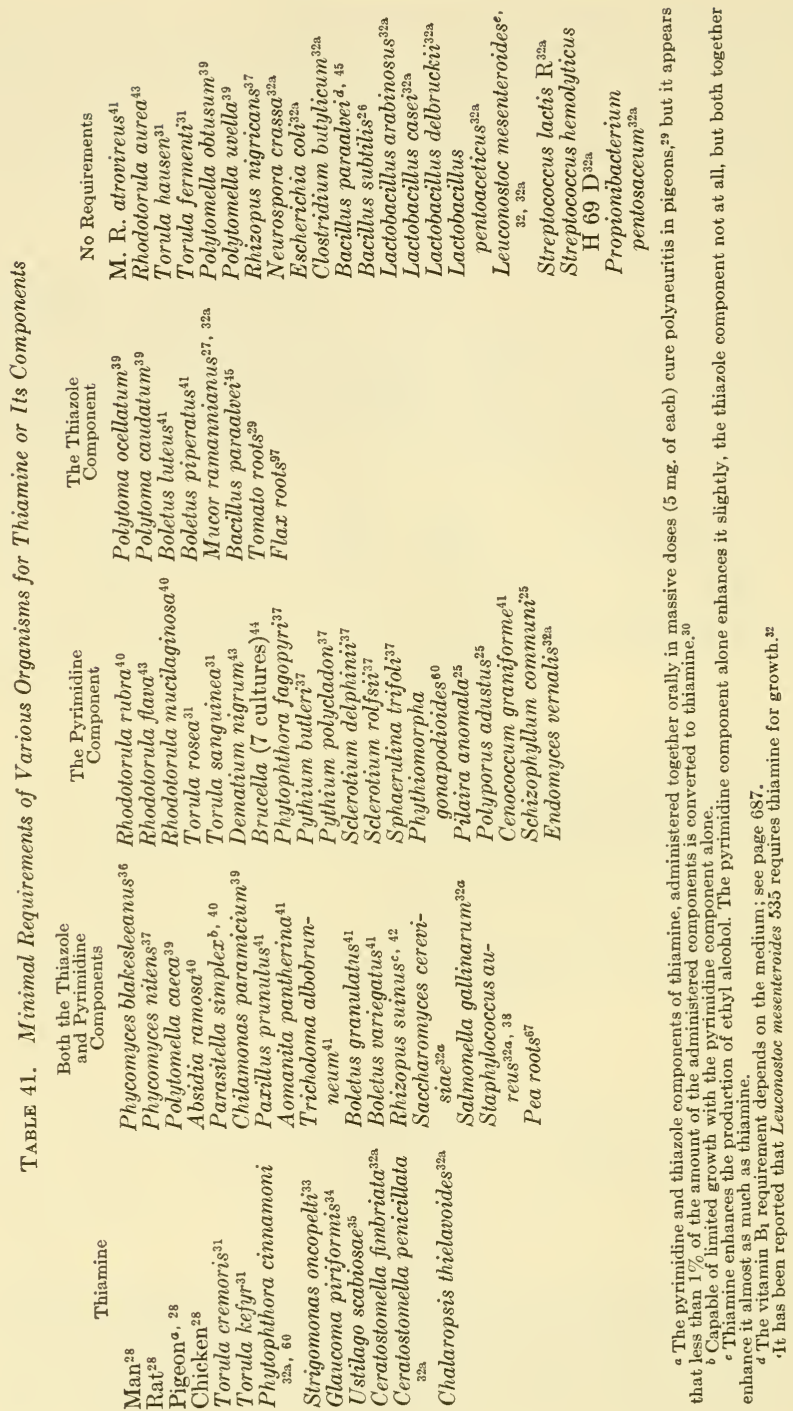


able of growth without an external supply of either of these components or thiamine. These requirements are summarized in Table 41. Some strains of Neisseria gonorrhoeae, when first isolated from a human host, cannot utilize thiamine but require cocarboxylase. ${ }^{25 a}$

Organisms which require only one or both of the components of thiamine rather than the intact molecule or do not require an external supply of thiamine or its components apparently achieve partial or in the latter case complete synthesis of thiamine. For example, Bacillus subtilis can be grown in a thiamine-free medium, and the resulting broth will support the growth of Staphylococcus aureus, which requires thiamine or its two components. ${ }^{26}$ Moreover, although Mucor ramannianus requires the thiazole component and Rhodotorula rubra requires the pyrimidine component of thiamine, these two organisms can grow together in thiaminefree media. ${ }^{27}$ Katznelson ${ }^{27 a}$ reported that Bacillus paraalvei requires thiamine or a mixture of its components, or at least the thiazole component, for growth in a medium devoid of cystine, phenylalanine, valine and leucine. Given the three last-named amino acids with either cysteine, glutathione, or cystine (in a reducing medium) or even sodium thioglycolate or sodium thiosulfate or ascorbic acid, this organism can grow without added thiamine or its components. These results suggest that this organism requires thiamine in its metabolism but in adequate media is able to synthesize the thiamine it requires.

The biological activity of a large number of analogues of thiamine and its pyrimidine and thiazole components has been determined, as indicated in Tables 42,43 , and 44 . As a result of these tests, it is evident that the thiamine molecule can undergo very little modification without extensive loss of vitamin $\mathrm{B}_{1}$ activity. Substitution of an ethyl, propyl or isopropyl group for the 2 -methyl group or an ethyl group for the 4 -methyl group are the only modifications which did not produce drastic reduction in the vitamin $B_{1}$ activity. In addition to the skeletal structure of thiamine, the 4 -amino group, the 5 - $\beta$-hydroxyethyl group and the absence of substitution in the 2-position appear to be essential for significant vitamin $B_{1}$ activity.

A number of thiamine analogues containing a pyridine ring instead of the thiazole ring ${ }^{58-63}$ have been found to be inactive in growth tests on the pigeon, the rat, and several microorganisms. The fact that two of these analogues are active in stimulating the production of carbon dioxide by yeast ${ }^{63}$ is due to the ability of the yeast to cleave these analogues and use them as a source of the pyrimidine component of the thiamine molecule.

Although many microorganisms can synthesize thiamine when supplied with suitable intermediates and thus do not require an external supply 
过

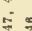

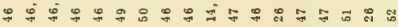

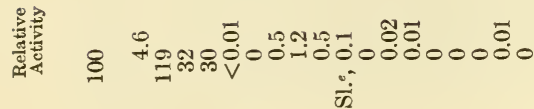

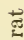

รี

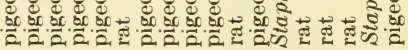

$\stackrel{\infty}{\circ}$

웅

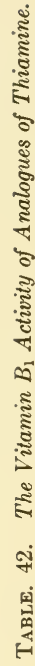

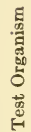

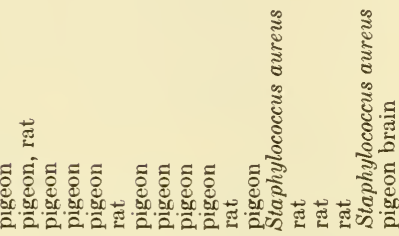

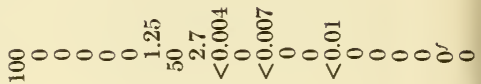

- ํㅗㄹ

仓ै दू

:

(ิ)

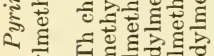

०

- इ है

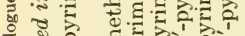

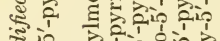

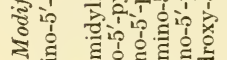

ม ह ह

ซึ क्ष

क्षँ

¿

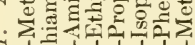

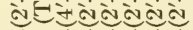

ले ले ले लें ले कैं
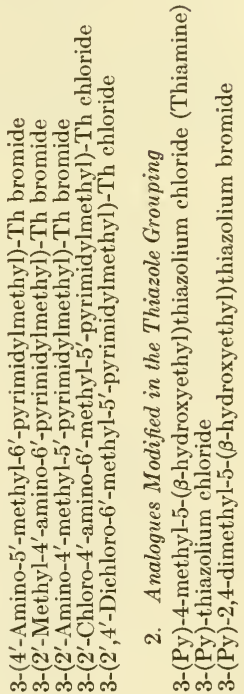

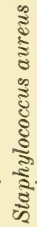

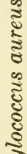

:

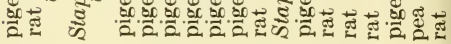




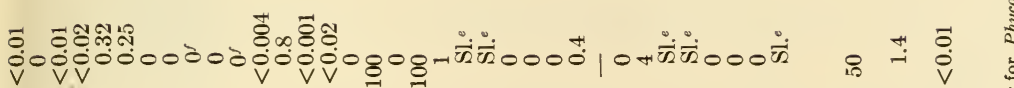

눈

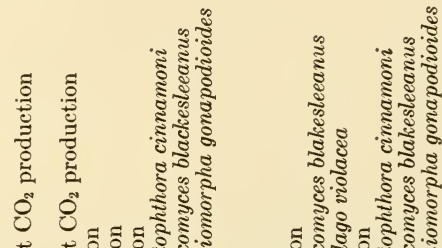

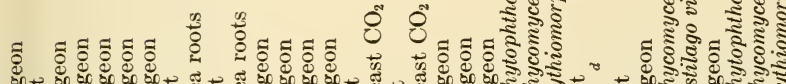

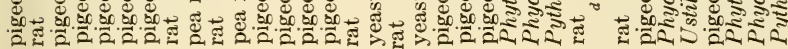

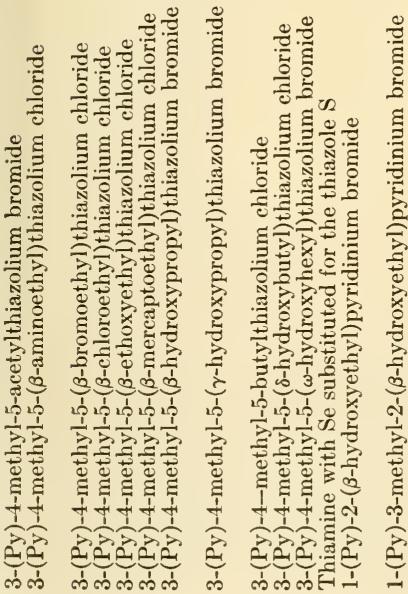

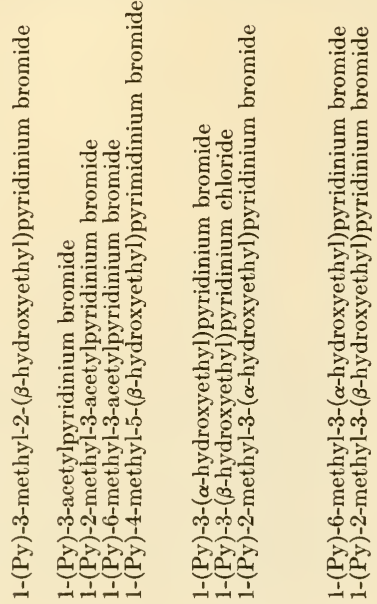




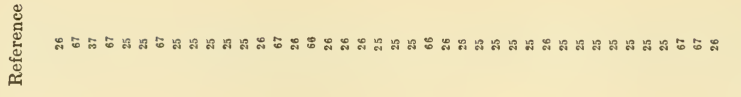

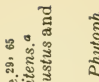


荧

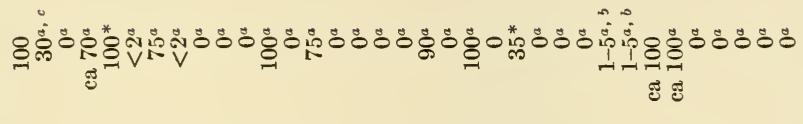

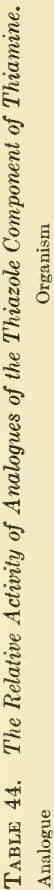

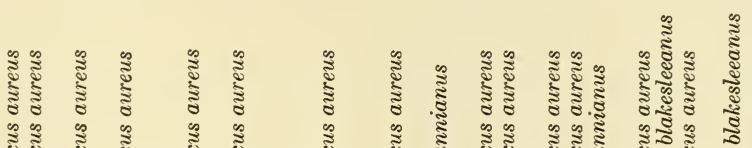
.

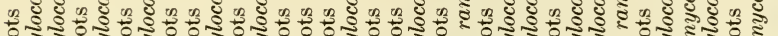

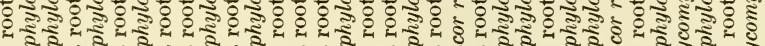

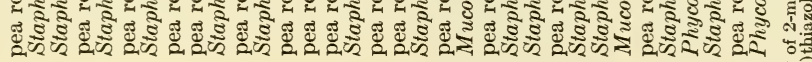

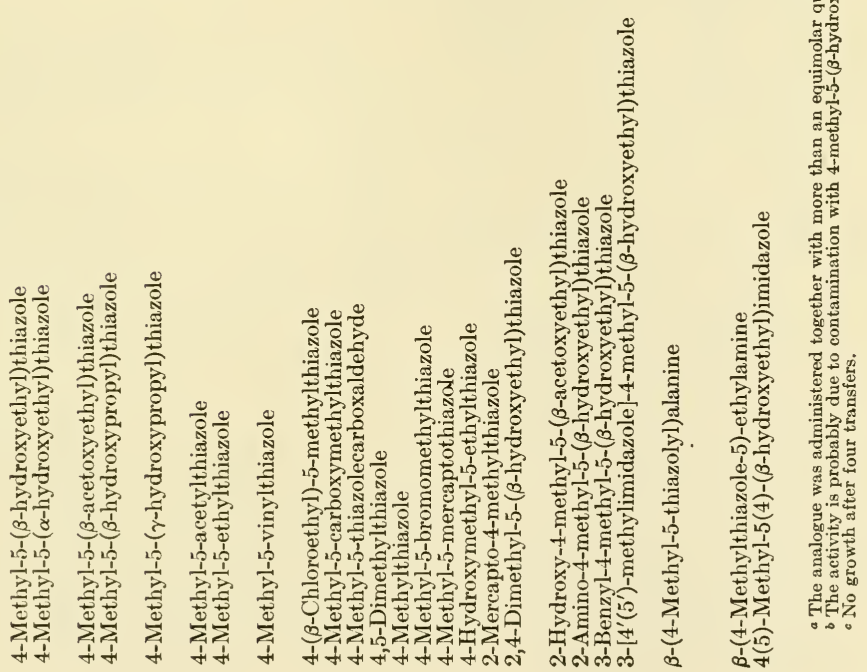


of thiamine, nevertheless it appears that vitamin $B_{1}$ is required in their metabolism, and the results of the experiments with analogues of the pyrimidine and thiazole components of thiamine (summarized in Tables 43 and 44) indicate that for many microorganisms the structural specificity of vitamin $B_{1}$ is substantially the same as for animals.

Recently it was reported that the administration of lumazine, xanthopterin, isoxanthopterin or folic acid to rats and pigeons on a low thiamine diet not only restores chronaxia to normal, but also cures polyneuritis and permits normal growth. This effect is observed when these compounds are injected into normal rats and when they are administered to caecumectomized rats, which indicates that their activity is not due to the synthesis of extra thiamine by the intestinal flora. However, these compounds do not replace thiamine as growth factors for Glaucoma or for Polytomella caeca. These results suggest that these compounds may exert a sparing action on thianine but are not able to replace it entirely.

Bonner ${ }^{67}$ has shown that pea roots require both the pyrimidine and thiazole components of thiamine for growth. Growth tests with analogues (Tables 43 and 44 ) indicate that their structural requirements for vitamin $B_{1}$ activity are not quite so stringent as those of many other organisms. Recently it was reported that 4-methyl-5-thiazoleacetamide stimulates plant germination. ${ }^{69 \mathrm{~b}}$

The possibility that the thiazole portion of thiamine may be deriver from an amino acid precursor, $\beta$-(4-methylthiazolyl-5)-alanine, was early considered. $^{68,69}$ While neither Staphylococcus aureus nor Phycomyces blakesleeanus utilized the amino acid derivative, the compound replaced the thiazole moiety in stimulating pea roots.

By assay with Phycomyces blakesleeanus, the formation by pea roots of the thiazole portion of thiamine was demonstrated with this amino acid as well as with 4-methylthiazole derivatives with the following substituents in the 5-position: $-\mathrm{CH}_{2}-\mathrm{CH}_{2} \mathrm{Cl},-\mathrm{CH}=\mathrm{CH}_{2},-\mathrm{CH}_{2}-\mathrm{CH}_{2} \mathrm{NH}_{2}$, and $-\mathrm{CH}_{2}-\mathrm{CH}_{2} \mathrm{OH}$. However, with 4-methylthiazole derivatives containing either $-\mathrm{CHOH}-\mathrm{CH}_{3}$ or $-\mathrm{CH}_{2}-\mathrm{CHOH}-\mathrm{CH}_{3}$ at position 5 , and with $-\mathrm{CH}_{2}-\mathrm{CH}_{2} \mathrm{OH}$ and $-\mathrm{CH}_{3}$ at positions 5 and 2 , respectively, synthesis of the thiazole moiety by pea roots could not be demonstrated. ${ }^{67 a}$ Consequently, since the last compounds are also effective in promoting growth of pea roots, it appears that these compounds possess activity without prior conversion to the normal thiazole moiety, while the activity of the other compounds may be ascribed at least in part to their conversion to the normal metabolite. The mode of action of these compounds which are not converted to the thiazole moiety is still obscure since analogues of thiamine possessing some of these modifications are inactive for pea roots (Table 42). 
TABLE 45. The Activity of Antagonists Related to Thiamine.

Aualogue

1-(2'-Methyl-4'-amino-5'-pyrimidylmethyl)2-methyl-3-( $\beta$-hydroxyethyl)pyridinium chloride (pyrithiamine)
3-(2'-Methyl-4'-hydroxy-5'-pyrimidylmethyl)-4-methyl-5-( $\beta$-hydroxyethyl) thiazolium chloride (oxythiamine)

3-(2'-Butyl-4'-amino-5'-pyrimidylmethyl)4-methyl-5-( $\beta$-hydroxyethyl) thiazolium chloride

2-Methyl-4-amino-5-bromoethylpyrimidine 2-Methyl-4-amino-5-bromomethylpyrimidine

2-Methyl-4-amino-5-chloromethylpyrimidine 2-Methyl-4-amino-5-hydroxymethylpyrimidine 2-Methyl-4-amino-5-aminomethylpyrimidine 2-Methyl-4-aminopyrimidine

\section{2-Methyl-4-amino-5-ethoxymethylpyrimidine}

4-Methyl-5-( $\beta$-hydroxyethyl)thiazole pyrophosphate

Sulfathiazole

\begin{tabular}{|c|c|c|}
\hline Organism & $\begin{array}{l}\text { Inhibition } \\
\text { Index }\end{array}$ & Refere \\
\hline Mouse & ca $20^{b}$ & 80 \\
\hline Rat & ca 2 & 81 \\
\hline $\begin{array}{l}\text { Ceratostomella } \\
\text { fimbriata }\end{array}$ & 7,19 & $32 \mathrm{a}$ \\
\hline $\begin{array}{l}\text { Ceratostomella } \\
\text { pennicillata }\end{array}$ & 10 & $32 \mathrm{a}$ \\
\hline $\begin{array}{l}\text { Phytophthora } \\
\text { cinnamoni }\end{array}$ & 12 & $32 \mathrm{a}$ \\
\hline $\begin{array}{l}\text { Chalaropsis } \\
\text { thielavioides }\end{array}$ & 11 & $32 \mathrm{a}$ \\
\hline $\begin{array}{l}\text { Lactobacillus } \\
\text { fermenti }\end{array}$ & $\begin{array}{l}50 \\
10^{c}\end{array}$ & 79 \\
\hline Endomyces vernalis & 130 & $32 \mathrm{a}$ \\
\hline $\begin{array}{l}\text { Penicillium } \\
\text { digitatum }\end{array}$ & $\begin{array}{l}160 \\
80^{c}\end{array}$ & 79 \\
\hline Mucor ramannianus & 800 & $32 \mathrm{a}$ \\
\hline $\begin{array}{l}\text { Saccharomyces } \\
\text { cerevisiae }\end{array}$ & 800 & $32 \mathrm{a}$ \\
\hline $\begin{array}{l}\text { Staphylococcus } \\
\text { aureus }\end{array}$ & 2000 & $32 \mathrm{a}$ \\
\hline $\begin{array}{l}\text { Staphylococcus } \\
\text { aureus } \\
\text { Salmonella }\end{array}$ & 700 & 78 \\
\hline $\begin{array}{l}\text { Salmonella } \\
\text { gallinarum } \\
\text { Lactobacillus }\end{array}$ & 1000 & $32 \mathrm{a}$ \\
\hline $\begin{array}{l}\text { Lactobacillus } \\
\text { acidophilus }\end{array}$ & 1900 & 80 \\
\hline $\begin{array}{l}\text { Neurospora crassa } \\
\text { Lactobacillus }\end{array}$ & 400,000 & $32 \mathrm{a}$ \\
\hline arabinosus & 40,000 & $32 \mathrm{a}$ \\
\hline Escherichia coli & $2,000,000^{d}$ & $32 \mathrm{a}$ \\
\hline Escherichia coli & 20,000 & 78 \\
\hline Mouse & $\begin{array}{c}\text { Not } \\
\text { greater } \\
\text { than } 25\end{array}$ & 74 \\
\hline Rat & ca $20^{b}$ & 82 \\
\hline Rat & $e$ & 70 \\
\hline Rat & - & 70 \\
\hline Mouse & $f$ & 30 \\
\hline Rat, mouse & $f$ & $\begin{array}{l}30 \\
30\end{array}$ \\
\hline $\begin{array}{l}\text { Rat, mouse } \\
\text { Rat, mouse }\end{array}$ & $\begin{array}{l}f \\
f\end{array}$ & $\begin{array}{c}30 \\
30\end{array}$ \\
\hline $\begin{array}{l}\text { Rat, mouse } \\
\text { Lactobacillus } \\
\text { fermentum }\end{array}$ & $\begin{array}{r}>20,000 \\
3000^{c}\end{array}$ & 79 \\
\hline $\begin{array}{l}\text { Lactobacillus } \\
\text { fermentum }\end{array}$ & $20,000^{g}$ & \\
\hline $\begin{array}{l}\text { Yeast } \\
\text { carboxylase }\end{array}$ & & 71 \\
\hline Yeast carboxylase & ca $160^{c, h}$ & 72 \\
\hline
\end{tabular}

a For half-maximum growth.

$b$ Approximately 40 moles of the inhibitor nullified the effect of one mole of thiamine.

c Using thiamine pyrophosphate as the growth factor.

d For Clostridium butylicum, Lactobacillus casei, Lactobacillus delbruckii, Lactobacillus pentoaceticus, Streptococcus lactis R, Propionibacterium pentosaceum and hemolytic streptococeus H69D, the pyrithiamine inhibition index is greater than $2 \times 10^{6}$.

- Quantitative data not available.

$f$ Subcutaneous administration of the inhibitor (7-9 mg to rats or 1-1.5 $\mathrm{mg}$ to mice) to animals maintained on a diet which appeared adequate for growth eaused severe cramps and often death.

If thiamine pyrophosphate is used as the growth factor, $90 \%$ inhibition occurs when the inhibitor/ growth factor ratio is 5000 .

${ }^{h}$ One mole of cocarboxylase was reported to prevent the union of 322 moles of sulfathiazole with the carboxylase protein of yeast washed with alkaline phosphate buffer solution. 
The interaction of thioformamide with 3-chloro-5-hydroxy-2-pentanone, which forms the thiazole portion of the thiamine molecule in vitro, also occurs in pea root cells. 5-Hydroxy-2-pentanone $\left(\mathrm{CH}_{3}-\mathrm{CO}-\mathrm{CH}_{2}-\mathrm{CH}_{2}\right.$ $\left.-\mathrm{CH}_{2} \mathrm{OH}\right)$ is also utilized by the root cells for the thiazole synthesis, but 4-hydroxy-2-pentanone did not allow formation of 4-methyl-5-( $\alpha$-hydroxyethyl) thiazole which has appreciable activity for pea roots. ${ }^{67 a}$

\section{Inhibitory Analogues of Thiamine}

The first report of an analogue of thiamine which may interfere with the utilization of this vitamin is that of Abderhalden, ${ }^{70}$ who observed in 1939 that the administration of 2-methyl-4-amino-5-bromomethylpyrimidine (as well as the 5-bromoethyl homologue) to rats maintained on a diet which appeared to be adequate for growth led to acute cramps and death of the animals. It was found that this effect could be prevented by the addition of corn sprouts or more dried yeast to the diet. These results were confirmed by Morii, ${ }^{30}$ who showed that the 5 -hydroxy, 5-bromo, and 5-chloro analogues, but not the 5-amino analogue, also produced spasms in mice as well as in rats.

In 1940, Buchman, et al. ${ }^{71}$ showed that the thiazole pyrophosphate portion of cocarboxylase (thiamine pyrophosphate) inhibited the car-

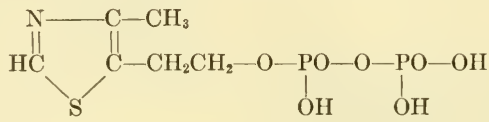

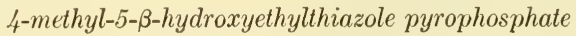

boxylase system of yeast, in which cocarboxylase is the coenzyme. Later, Sevag, et al..$^{72}$ reported that sulfathiazole specifically inhibits the cocarboxylase system of yeast. This effect can be overcome by the addition of cocarboxylase, one mole of cocarboxylase being able to prevent the union of 322 moles of sulfathiazole with the carboxylase protein. Subsequently, it was shown that $p$-aminobenzoic acid, although itself slightly inhibitory, largely overcame the inhibitory effect of sulfathiazole on the carboxylase system of Staphylococcus aureus and Escherichia coli. Moreover, these carboxylase systems are also inhibited (although to a lesser extent) by sulanilamide, sulfapyridine, sulfadiazine, 2-sulfanilamido5-ethyl-4-thiazolone, 2-aminopyridine, 2-aminothiazole and 2-aminopyrimidine, ${ }^{72}$ some of which possess very little structural similarity to thiamine. These results suggest that the relationship between thiamine and sulfathiazole may be more obscure than it appeared initially.

Robbins ${ }^{60}$ reported that pyrithiamine (heteroaneurine), 1- $\left(2^{\prime}\right.$-methyl$4^{\prime}$-amino - $5^{\prime}$-pyrimidylmethyl $)$ - 2 -methyl-3 - $(\beta$-hydroxyethyl $)$ pyridinium 
bromide, (the analogue of thiamine having a pyridine ring instead of the thiazole ring), shightly inhibited the growth of Phycomyces blakesleeanus, which requires thiamine or its pyrimidine and thiazole components for growth. However, the inhibition was reversed by the addition of the thiazole component, which indicated that this organism is able to cleave the pyrithiamine molecule and use the pyrimidine component. This interpretation was supported by the fact that in low concentration, this analogue stimulated the growth of Pythiomorpha gonapodioides, which requires only the pyrimidine component of thiamine. Large amounts of the analogue were toxic, but this effect could be overcome by the addition of the pyrimidine or the thiazole component of thiamine.

Woolley and White ${ }^{32 a}$ found that pyrithiamine competitively inhibited the growth of a number of organisms which require an external supply of thiamine or its components, whereas it was without effect on organisms which did not require thiamine. Those species which required intact thiamine were about ten times as sensitive as those which needed only the pyrimidine portion of thiamine, and about one hundred times as sensitive as those stimulated by the pyrimidine and thiazole components of thiamine. The pyrithiamine inhibition indices for Endomyces vernalis and Mucor ramannianus were 130 and 800, respectively, but these organisms were not inhibited by 2 -methyl-3-( $\beta$-hydroxyethyl)pyridine, even in concentrations as high as $100 \gamma$ per cc. The resistance of the organisms which do not require thiamine could not be attributed to their synthesis of abnormally large amounts of thiamine since there was no significant increase in the synthesis of this vitamin when the organisms were grown in the presence of pyrithiamine. Subsequently, Woolley ${ }^{76}$ reported that a new strain of Endomyces vernalis was obtained by growing a culture in media containing pyrithiamine; it was not inhibited by twenty-five times the concentration of pyrithiamine which produced 50 per cent inhibition in the parent strain. It still required thiamine or its pyrimidine portion as a growth factor, but it was able to utilize small amounts of the pyrithiamine also. It appears that resistance to inhibition by pyrithiamine may depend in part on the ability of the organism to cleave the molecule into its pyrimidine and pyridine components, since the latter does not interfere with the growth of organisms which are inhibited by pyrithiamine.

IVyss ${ }^{77}$ reported that the pyrithiamine inhibition indices for Staphylococcus aureus and Escherichia coli were 700 and 20,000, respectively. When injected into mice in concentrations which were not toxic to the animals, pyrithiamine was not anti-bacterial in the blood. Dreiser, Scholtz and Spies ${ }^{78}$ reported that pyrithiamine is inhibitory to the growth 
of Lactobacillus acidophilus; the inhibition index was found to be approximately 1900 .

Sarett and Cheldelin ${ }^{79}$ observed that pyrithiamine, 2-methyl-4-aminopyrimidine and 2-methyl-4-amino-5-ethoxymethylpyrimidine inhibit the utilization of either thiamine pyrophosphate or thiamine phosphate more effectively than the utilization of thiamine for growth of Lactobacillus fermentum and Penicillium digitatum. The possibility that dephosphorylation of the pyrophosphate was inhibited by the analogues and that some thiamine in the free form was essential for growth was considered, but small amounts of thiamine did not affect the inhibition by the analogues of the utilization of larger amounts of the pyrophosphate. On the basis of these results, it was suggested that thiamine is attached to the apoenzyme before phosphorylation.

In 1943, Woolley and White ${ }^{82}$ reported that the feeding of pyrithiamine to mice induced characteristic polyneuritic symptoms of thiamine deficiency, whereas the animals merely lost weight and died without polyneuritic symptoms on a low-thiamine diet. The effect could be prevented or reversed by the administration of thiamine; about forty moles of the pyrithiamine nullified one mole of thiamine. Emerson ${ }^{83}$ obtained similar results after administering pyrithiamine to rats. In this case also, the inhibition index was approximately 20 .

Wilson and Harris ${ }^{83 a}$ have recently pointed out that repeated analyses of pyrithiamine hydrobromide, which had been assigned the formula $\mathrm{C}_{14} \mathrm{H}_{20} \mathrm{~N}_{4} \mathrm{OBr}_{2}$, gave values which did not correspond closely to this formula. Furthermore, the nitrogen values on different samples were inconsistent. These authors report the preparation of a compound whose constants do correspond to this formula, and the new compound has been named neopyrithiamine. In rats its activity in inhibiting thiamine hydro-

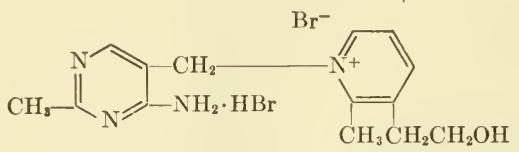

neopyrithiamine hydrobromide

chloride is four times as great as that of pyrithiamine. Rabinowitz and Snell ${ }^{83 b}$ have shown that neopyrithiamine alleviates the growth inhibition observed in yeast grown in the absence of vitamin $\mathrm{B}_{6}$, but in the presence of thiamine. It is concluded that neopyrithiamine acts as a competitive inhibitor to overcome the toxic effect of the added thiamine.

Although oxythiamine, the 4 -hydroxy analogue of thiamine, is reported to have 0.5 per cent of the antineuritic activity of thiamine for pigeons, ${ }^{50}$ 
it has no vitamin action on rats, ${ }^{49}$ and in doses of 25 to $50 \gamma$ per day it is fatally toxic to young mice maintained on a low vitamin $\mathrm{B}_{1}$ diet supple-

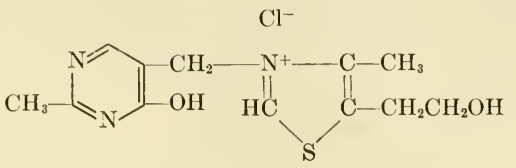

oxythiamine

mented with $1 \gamma$ of thiamine per day. ${ }^{74}$ The 5 - $\beta$-chloroethyl and 5 - $\beta$ bromoethyl analogues of oxythiamine were not toxic, even when administered in doses as high as $100 \gamma$ per day. More recently, it was reported that a low-thiamine diet provided some protection for mice against infection with the Lansing strain of poliomyelitis virus. Similar protection, though less marked, was afforded by the administration of oxythiamine. ${ }^{75}$

In 1945, Emerson and Southwick ${ }^{84}$ demonstrated that the administration of the 2'-butyl homologue of thiamine to rats maintained on a suboptimal intake of thiamine produced polyneuritis and subnormal growth, both characteristic of thiamine deficiency. This effect was counteracted

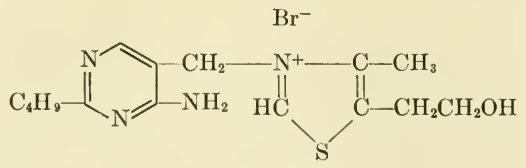

butylthiamine

by increasing the thiamine intake; the inhibition index was approximately 20 .

Ochoa and Peters ${ }^{80}$ reported that thiamine, as well as a number of pyrimidine analogues, stimulated the carboxylase system of yeast which had been washed with an alkaline phosphate buffer solution. Westenbrink, et al. ${ }^{81}$ showed that this apparent "stimulation" was actually due to the fact that these compounds inhibited the dephosphorylation of the cocarboxylase by a phosphatase present in the yeast. From the results which are summarized in Table 46 , it is evident that the inhibitory effect depends on the presence of the 4-aminopyrimidine group. Weil-Malherbe demonstrated that the presence of excess thiamine also slightly inhibits the synthesis of cocarboxylase by yeast. ${ }^{81 a}$

Weswig, Freed and Haag ${ }^{85}$ reported that rats placed on diets containing bracken fern which had been air-dried and ground developed symp- 
toms suggestive of thiamine deficiency after about ten days, and death ensued about twenty days later. If treated orally with $0.5 \mathrm{mg}$ of thiamine per day, animals showing severe anorexia, emaciation and polyneuritis promptly recovered. The toxicity was not decreased when the air-dried fern was heated at $105^{\circ} \mathrm{C}$ in air for 18 hours. The causative agent is essentially insoluble in ethyl ether and in acetone, but appears to be slightly soluble in 92 per cent ethyl alcohol.

TABLE 46. The Inhibition of the Dephosphorylation of Cocarboxylase in Yeast.

\section{Analogue}

Thiamine hydrochloride

3-(2'-Methyl-4'-amino-5'-pyrimidylmethyl)4-methyl-5-( $\beta$-hydroxypropyl)thiazolium chloride

2-Methyl-4-amino-5-aminomethylpyrimidine hydrochloride

2-Methyl-4-amino-5-thioformamidomethylpyrimidine hydrochloride

2-Methyl-4-hydroxy-5-thioformamidomethylpyrimidine hydrochloride
Inhibition Index

$15^{a}$

between 0.2 and 20

$75^{a}$

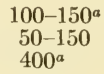

b
Reference

80

81

80

80

81

80

80

a Ochoa and Peters ${ }^{80}$ reported that these compounds stimulated the carboxylase system of yeast which had been washed with alkaline phosphate buffer solution. Westenbrink ${ }^{81}$, et al. showed that the apparent stimulation" was due to inhibition of the dephosphorylation of the cocarboxylase. These inhibition indices are calculated from the data of Ochoa and Peters on the assumption that 50 \% "stimulation" corresponded to $50 \%$ inhibition of dephosphorylation of the cocarboxylase present and they represent the ratio moles of inhibitor

moles of cocarboxylase which gives $50 \%$ "stimulation."

"Ochoa and Peters reported that this compound did not "stimulate" the carboxylase system.

The Chastek paralysis of foxes was shown by Green, Carlson and Evans ${ }^{86}$ to be caused by diets containing large amounts of raw fish, which apparently contained a substance capable of inactivating the thiamine in the feed and thus caused a thiamine deficiency. Later investigations demonstrated that a similar effect was produced in chicks ${ }^{87}$ and in cats. ${ }^{88}$ In each case, the toxicity could be overcome by the administration of large amounts of thiamine. The toxic substance was found to be an enzyme, ${ }^{89}$ and it was shown by Krampitz, Woolley and White ${ }^{90}$ to cleave the thiamine molecule at the methylene bridge, liberating ultimately 2-methyl-4-amino-5-hydroxymethylpyrimidine and 4-methyl-5( $\beta$-hydroxyethyl) thiazole. The thiazole component was liberated directly, but the pyrimidine portion apparently was first converted to an intermediate and liberated in a subsequent reaction. This was confirmed by Hennessy, Warner, Falk and Truhlar, ${ }^{91}$ who isolated a erystalline intermediate after the destruction of thiamine by clam tissue. Analysis indicated that the molecular formula was $\mathrm{C}_{8} \mathrm{H}_{16} \mathrm{~N}_{4} \mathrm{O}_{3} \mathrm{~S} .2 \mathrm{HCl}$ but the structure of the compound was not reported. Sealock and Davis ${ }^{92}$ found that $m$-nitroaniline and $m$-aminobenzoic acid stimulated the Chastek paralysis thiaminase in vitro, and they concluded that this effect was due to the 
formation of a secondary amine by combination of the amino group of the "activator" with the 5-methylene group of the pyrimidine component of the thiamine. They succeeded in isolating $\mathrm{N}$-(2-methyl-4-amino-5pyrimidylmethyl)-m-nitroaniline from the reaction mixture produced when thiamine was enzymatically destroyed in the presence of $m$-nitroaniline.

Sealock and Goodland ${ }^{93}$ found that the cleavage of thiamine by the Chastek paralysis enzyme is inhibited by a number of thiazole derivatives. 3-o-Aminobenzyl-4-methylthiazolium chloride at a concentration

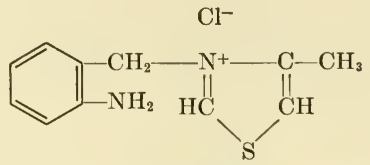

\section{3-o-aminobenzyl-4-methylthiazolium chloride}

of $5 \times 10^{-4}$ mole/liter was found to produce 100 per cent inhibition of the enzymatic destruction of thiamine at the same molar concentration, and the extent of the inhibition proved to be dependent upon the inhibitorthiamine ratio. A like concentration of 3 - $\beta$-aminoethyl-4-methylthiazolium chloride caused 56 per cent inhibition of the destruction of the vitamin. The other compounds tested (3-o-nitrobenzyl-, 3- $\beta$-phthalimidoethyl-, 3-ethyl-, 3-phenyl-, 3-ethyl-2-methyl-, and 3-methyl-5- $\beta$ hydroxyethyl-4-methylthiazolium chlorides as well as several 6-aminopyrimidine compounds) were either only slightly effective or completely without activity as inhibitors. The importance for inhibitory activity of the amino group in the position analogous to that of the $4^{\prime}$-amino group of thiamine is indicated by the high activity of the $o$-aminobenzyl derivative and the low activity of the corresponding compounds which do not possess this group. Further evidence for the importance of the position of the amino group in the benzyl portion of the thiazole derivative was obtained by Sealock and Livermore, ${ }^{94}$ who showed that whereas the $o$-aminobenzyl derivative was highly inhibitory, the corresponding $p$-aminobenzyl analogue was almost without inhibitory action and the $m$-aminobenzyl compound was markedly stimulatory. It was also demonstrated that the 4-methyl group is not an essential feature of the inhibitor molecule, for the corresponding 2-methyl derivative had equal or slightly greater inhibitory action. However, placing a methyl group in both the 2 and 4 positions of the thiazole ring resulted in a compound with less than 50 per cent of the inhibitory activity of the corresponding analogues in which only one of those positions was substituted. 
Soodak and Cerecedo ${ }^{74}$ reported that oxythiamine inhibits the Chastek paralysis thiaminase, but quantitative data are not available. Bhagvat and Devi ${ }^{95}$ found that certain cereals, legumes and oil seeds contain a factor capable of destroying thiamine. Apparently, the factor is not an enzyme, since it is extracted by chloroform-water mixtures and is stable to heat, including autoclaving. The end products are believed to be pyrimidine and thiazole derivatives, since mosquito larvae were able to utilize for growth the breakdown products produced when thiamine was destroyed by extracts from ragi or carp tissue, but were unable to utilize the breakdown products produced by autoclaving or treating thiamine with sulfite or sodium hydroxide. The extraction from the flesh of Corbicula strata of a thiaminase which deaminized the pyrimidine ring but did not open the thiazole ring was announced by Murata. ${ }^{96}$

\section{Bibliography}

1. Takaki, K., Lancet, (1906) I, 1369, 1451, 1520.

2. Fletcher, W., J. Trop. Med. Hyg., 12, 127 (1909).

3. Fraser, H., and Stanton, A. T., Lancet, (1909) II, 406.

4. Eijkman, C., Arch. Path. Anat. (Virchow's), 148, 523 (1897).

5. Funk, C., J. Physiol., 45, 75 (1912).

6. Jansen, B. C. P., and Donath, W. F., Chem. Welkblad., 23, 201 (1926).

7. Windaus, A., Tschesche, R., Ruhkopf, H., Laquer, F. and Schultz, F., Nach. v. der Ges. Wiss. Göttinger, III, 207 (1931).

8. Ohdake, S., Bull. Agric. Chem. Soc., Japan, 8, 179 (1932).

9. Kinnersley, N. W., O'Brien, J. R., and Peters, R. A., Biochem. J., 27, 232 (1933).

10. Wintersteiner, O., Williams, R. R., and Ruehle, A. E., J. Am. Chem. Soc., 57, 517 (1935)

11. Williams, R. R., J. Am. Chem. Soc., 58, 1063 (1936).

12. Grewe, R., Z. physiol. Chem., 242, 89 (1936).

13. Williams, R. R., and Cline, J. K., J. Am. Chem. Soc., 58, 1504 (1936).

14. Andersag, H., and Westphal, K., Ber., 70, 2035 (1937).

15. Tolpin, J. G., Foy, J. R., and Cerecedo, L. R., J. Am. Chem. Soc., 63, 2848 (1941).

16. Kinnersley, N. W., O'Brien, J. R., and Peters, R. A., Biochem. J., 29, 701 (1935).

17. Leporati, I. F., Boll. soc. ital. biol. sper., 15, 1073 (1940); C. A., 40, 6128 (1946); Schultz, F., Z. physiol. Chem., 265, 113 (1940).

18. Lohmann, K., and Schuster, P., Biochem. Z., 294, 188 (1937).

19. Lipmann, F., and Perlman, G., J. Am. Chem. Soc., 60, 2574 (1938); Stern, K. G., and Melnick, J. L., J. Biol. Chem., 131, 597 (1939).

20. Zima, O., and Williams, R. R., Ber., 73B, 941 (1940).

21. Myrbäck, K.. Vallin, I., and Magnell, I., Svensk. Kem. Tid., 57, 124 (1945); C. A., 40, 4094 (1946).

22. Zima, O., Ritsert, K. and Moll, T., Z. physiol. Chem., 267, 210 (1941).

23. Karrer, P., and Visconti, M., Helv. Chim. Acta, 29, 711 (1946); Wachtmeister, C. A., and Myrbäck, K., Arkiv. Kemi. Mineral. Geol., 24A, No. 8 (1946).

24. Peters, R. A., Nature, 135, 107 (1935).

25. Sehopfer, W. H., and Blumer, S., Enzymologia, 8, 261 (1940).

25a. Lankford, C. E., and Skaggs, P. K., Arch. Biochem., 9, 265 (1945).

26. Knight, B. C. J. G., and MeIlwain, H., Biochem. J., 32, 1241 (1938).

27. Müller, W., and Schopfer, W. H., Compt. rend., 205, 687 (1937). 
27a. Katznelson, H., J. Biol. Chem., 167, 615 (1947).

28. Williams, R. R., and Spies, T., "Vitamin $B_{1}$," The Macmillan Co., New York, 1938, p. 180.

29. Robbins, W. J., Bartley, M. A., Hogan, A. G., and Richardson, L. R., Proc. Natl. Acad. Sci., U. S., 23, 388 (1937); Robbins, W. J., and Bartley, M. A., ibid, 385 (1937).

30. Morii, S., Biochem. Z., 309, 354 (1941).

31. Robbins, W. J., and Kavanagh, F., Plant Physiol., 13, 611 (1938).

32. Gaines, S., and Stahley, G. L., J. Bact., 46, 441 (1943).

32a. Woolley, D. W., and White, A. G. C., J. Exptl. Med., 78, 489 (1943).

33. Lwoff, M., Compt. rend. soc. biol., 126, 771 (1937).

34. Lwoff, A., and Lwoff, M., Compt. rend soc. biol., 126, 644 (1937).

35. Schopfer, W. H., and Blumer, S., Compt. rend., 206, 1141 (1938).

36. Schopfer, W. H., and Jung, A., Compt. rend., 204, 1500 (1937).

37. Robbins, W. J., and Kavanagh, F., Am. J. Botany, 25, 229 (1938).

38. Knight, B. C. J. G., Biochem. J., 31, 966 (1937).

39. Lwoff, A., and Dusi, H., Compt. rend., 205, 882 (1937).

40. Schopfer, W. H., Compt. rend. soc. biol., 126, 842 (1937).

41. Melin, E., and Norkrans, B., Svensk. Botan. Tid., 36, 271 (1942).

42. Schopfer, W. H., Helv. Physiol. Pharmacol. Acta, 1, C83 (1943).

43. Schopfer, W. H., Protoplasma, 31, 105 (1938).

44. Koser, S. A., and Wright, M. H., J. Infectious Discases, 71, 86 (1942).

45. Lutz, A., Compt. rend. soc. biol., 142, 1229 (1948).

46. Schultz, F., Z. physiol. Chem., 265, 113 (1940).

47. Stein, G. A., Sampson, W. L., Cline, J. K., and Stevens, J. R., J. Am. Chem. Soc., 63, 2059 (1941).

48. Schultz, F., Z. physiol. Chem., 272, 29 (1941).

48a. Schopfer, W. H., Ann. inst. Pasteur, 61, 781 (1938).

49. Bergel, F., and Todd. A. R., J. Chem. Soc., 1504 (1937).

50. Slobodin, Y. M., and Zigel, M. S., J. Gon. Chem. U.S.S.R., 11, 1019 (1941); C. A., 36, 6542 (1942).

51. Price, C. C., Leonard, N. J., and Reitsema, R. H., J. Am. Chem. Soc., 68, 766 (1946).

52. Bowman, A., J. Chem. Soc., 494 (1937).

53. Pesina, A. G., and Zigel, M. S., Proc. Sci. Inst. Vitamin Research, U.S.S.R., 3, No. 1, 94 (1941); C. A., 36, 3008 (1942).

54. Buchman, E. R., and Richardson, E. M., J. Am. Chem. Soc., 67, 395 (1945).

55. Price, D., and Pickel, F. D., J. Am. Chem. Soc., 63, 1067 (1941).

56. Dornow, A., Ber., 73, 156 (1940).

57. Dornow, A., and Schacht, W., Chem. Ber., 80, 505 (1947).

58. Baumgarten, P., and Dornow, A., Ber., 73, 44 (1940).

59. Abderhalden, E., and Abderhalden, R., Arch. ges. Physiol. (Pflüger's), 244, 142 (1940).

60. Robbins, W. J., Proc. Natl. Acad. Sci. U. S., 27, 419 (1941).

61. Schopfer, W. H., Compt. rend. soc. phys. hist. nat. Genève, 58, 64 (1941); (in Arch. sci. phys. nat., 23, suppl. 64 (1941)).

62. Dornow, A., and Machens, H., Chem. Ber., 80, 502 (1947).

63. Finkelstein, J., and Elderfield, R. C., J. Org. Chem., 4, 365 (1939).

64. Robbins, W. J., Proc. Natl. Acad. Sci., U. S., 28, 352 (1942).

65. Abderhalden, E., and Abderhalden, R., Arch. ges. Physiol. (Pflüger's), 240, 746 (1938).

66. Robbins, W. J., and Kavanagh, F., Proc. Natl. Acad. Sci. U. S., 24, 229 (1938).

67. Bonner, J., Am. J. Botany, 25, 543 (1938).

67a. Bonner, J., and Buchman, E. R., Proc. Natl. Acad. Sci. U. S., 24, 431 (1938).

68. Buchman, E. R., and Richardson, E. M., J. Am. Chem. Soc., 61, 891 (1939).

69. Harington, C. R., and Moggridge, R. C. G., J. Chem. Soc., 443 (1939). 
69a. Fox, S. N., Sargent, H., and Buchman, E. R., J. Am. Chem. Soc., 67, 496 (1945).

69b. Sahashi, Y., Nakayama, A., Uchida, M., Ionoue, J., and Mizuno, H., J. Sci. Research Inst. Tokyo, 43, No. 1191, 56 (1948).

70. Abderhalden, R., Klin. Wochschr., 18, 171 (1939).

71. Buchman, E. R., Heegard, E., and Bonner, J., Proc. Natl. Acad. Sci. U. S., 26, 561 (1940).

72. Sevag, M. G., Shelburne, M., and Mudd, S., J. Bact., 49, 65 (1945); J. Gen. Physiol., 25, 805 (1942).

73. Sevag, M. G., Henry, J., and Richardson, R. A., J. Bact., 49, 71 (1945).

74. Soodak, M., and Cerecedo, L. R., J. Am. Chem. Soc., 66, 1988 (1944); Fed. Proc., 6, 293 (1947).

75. Jones, J. H., Foster, C., and Henle, W., Proc. Soc., Exptl. Biol. Med., 69, 454 (1948).

76. Woolley, D. W., Proc. Soc. Exptl. Biol. Med., 55, 179 (1944).

77. Wyss, O., J. Bact., 46, 483 (1943).

78. Dreizen, S., Scholz, E., and Spies, T. D., Proc. Soc. Exptl. Biol. Med., 68, 620 (1948).

79. Sarett, H. P., and Cheldelin, V. H., J. Biol. Chem., 156, 91 (1944).

80. Ochoa, S., and Peters, R. A., Biochem. J., 32, 1501 (1938).

81. Westenbrink, H. G. K., Van Dorp, D. A., Gruber, M, and Veldman, H, Enzymologia, 9, 73 (1940)

81a. Weil-Malherbe, H., Biochem. J., 33, 1997 (1939).

82. Woolley, D. W., and White, A. G. C., J. Biol. Chem., 149, 285 (1943).

83. Emerson, G. A., Abstract of Papers, ACS, April 14-18, 1947, p. 42 b.

83a. Wilson, A. N., and Harris, S. A., J. Am. Chem. Soc., 71, 2231 (1949).

83b. Rabinowitz, J. C., and Snell, E. E., Federation Proc., 8, 240 (1949).

84. Emerson, G. A., and Southwick, P. L., J. Biol. Chem., 160, 169 (1945).

85. Weswig, P. H., Freed, A. M., and Haag, J. R., J. Biol. Chem., 165, 737 (1946).

86. Green, R. G., Carlson, W. E., and Evans, C. A., J. Nutrition, 21, 243 (1941).

87. Spitzer, E. H., Coombes, A. I., Elvehjem, C. A., and Wisnicky, W., Proc. Soc. Exptl. Biol. Med., 48, 376 (1941).

88. Smith, D. C., and Proutt, L. M., Proc. Soc. Exptl. Biol. Med., 56, 1 (1944).

89. Sealock, R. R., Livermore, A. H., and Evans, C. A., J. Am. Chem. Soc., 65, 935 (1943).

90. Krampitz, L. O., and Woolley, D. W., J. Biol. Chem., 152, 9 (1944).

91. Hennessy, D. J., Warner, S., Falk, K. G., and Truhlar, J., Abstract of Papers, ACS, April 8-12, 1946 (p. 20B).

92. Sealock, R. R., and Davis, N. C., J. Biol. Chem., 177, 987 (1949).

93. Sealock, R. R., and Goodland, R. L., J. Am. Chem. Soc., 66, 507 (1944).

94. Sealock, R. R., and Livermore, A. H., J. Biol. Chem., 177, 553 (1949).

95. Bhagvat, K., and Devi, P., Indian J. Med. Research, 32, 131 (1944).

96. Murata, K., Rept. Osaka Municipal Research Inst. Domestic Sci., 17, No. 1, 137 (1946).

97. Bonner, J., and Bonner, H., Vitamins and Hormones, 6, 225 (1948). 


\title{
Chapter XID
}

\section{BIOLOGICAL ACTIVITIES OF OTHER NUTRITIONAL FACTORS OF DOUBTFUL STATUS*}

\author{
Choline
}

\section{Introduction}

The presence of choline as an integral structural unit as phospholipides was recognized soon after the middle of the last century; lecithins and sphingomyelins from both plant and animal sources were found to contain the substance. The nutritional importance of choline was not demonstrated until relatively recently.

In a series of investigations which followed the discovery of insulin by Banting and Best in 1922, it was found that the administration of lecithin prevents the abnormal accumulation of fat in the livers of depancreatized dogs injected with insulin. ${ }^{1,2,3}$ Subsequently it was shown that the development of fatty livers in rats maintained on a high-fat, low-protein ration can be prevented or cured by the inclusion of choline in the diet. ${ }^{4}$ The lipotropic action of lecithin was therefore attributed to the presence of choline in its structure.

It is now known that a dietary deficiency of choline may cause a variety of metabolic disturbances. These effects are discussed in detail in several excellent review papers. ${ }^{5,6,7}$ For rats, a lack of choline results in cessation of growth, infiltration of fat in the livers, and hemorrhagic degeneration of the kidneys. For chicks and turkey poults, a choline deficiency causes cessation of growth and perosis or "slipped tendon disease."

In addition to its involvement in animal and avian nutrition, choline is required as a growth factor by a number of microorganisms including certain strains of pneumococci (Types I, II, III, V, VIII) 8,9 and "cholineless" mutants of Neurospora crassa. ${ }^{10,11}$

Studies dealing with the choline molecule suggest that the metabolic effects of choline may be divided into two groups: (a) those which depend on the effectiveness of choline as a source of the "transferable methyl group," and (b) those which depend on the presence of the intact choline molecule.

* By Thomas J. Bardos, Lorene L. Rogers, and A. D. Barton. 


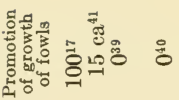

皱

$\stackrel{7}{+} \stackrel{9}{+}$

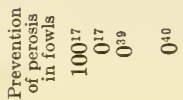

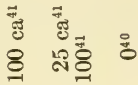

:

ฆ ํำ

윤: n

क.

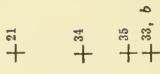

s

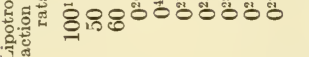

$800+$ 敬

$$
\stackrel{\infty}{+}+\stackrel{\infty}{+} \text { क क }
$$

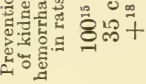

$\stackrel{\infty}{+} \stackrel{\infty}{+}+\frac{\infty}{+} \quad$ g

हैं क्षे

产

它

芯

赵

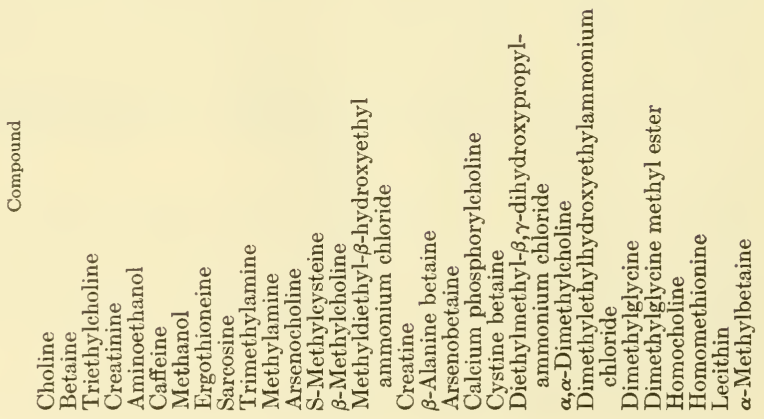


\#:

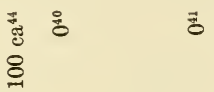

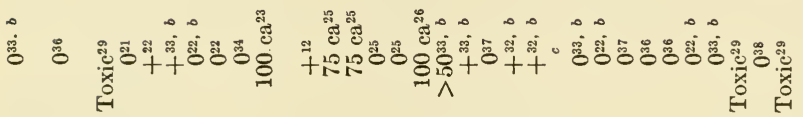

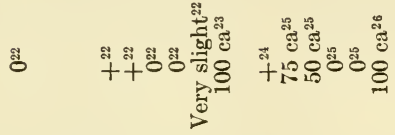

के 픙

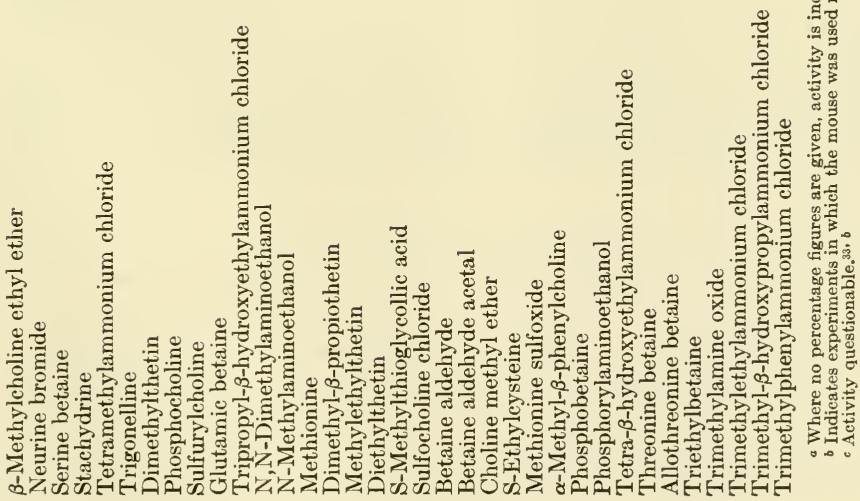


Efforts to determine what specific actions of choline are responsible for the gross physiological changes observed in choline deficiency led to the discovery of a functional relationship between choline and methionine. In 1938 Tucker and Eckstein ${ }^{12}$ reported that methionine exerts an effect on liver fat similar to that shown by choline. du Vigneaud and co-workers ${ }^{13}$ later observed that methionine can be replaced in the diet of rats by homocysteine, provided choline or betaine is simultaneously supplied. They suggested that this effect is due to the conversion of homocysteine to methionine by the transfer of methyl groups from choline. ${ }^{14}$ As a result of these and subsequent investigations it is now apparent that choline, betaine and methionine can serve as metabolic sources of the "transferable methyl group," which may be an essential dietary constituent.

Choline apparently functions as the intact molecule in the synthesis of some of the phospholipides which play an important role in the regulation of fat metabolism as well as in the synthesis of acetylcholine. The latter compound has attracted much attention as a "chemical transmitter" of the nerve impulse.

\section{Specificity}

A considerable number of compounds have been tested for their ability to alleviate the various symptoms produced by choline deficiencies in rats and in chicks and turkey poults. The results of these tests are summarized in Table 47.

The ability of various compounds to permit growth of the rat or the chick on a choline-methionine-free diet supplemented with homocysteine has been interpreted to mean that these compounds are able to transfer methyl groups to homocysteine to form methionine. This conversion has been demonstrated following the administration of deuteriocholine ${ }^{45}$ and deuteriobetaine. ${ }^{46}$ On this basis the following compounds have been found to be methyl donors: choline, choline derivatives such as lecithin and phosphorylcholine, monoethylcholine, betaine, dimethylthetin (sulfobetaine), methylethylthetin and dimethylpropiothetin. The minimal requirement for methyl donor activity appears to be the presence of at least one methyl group attached directly to an onium pole. That such activity may also be conditioned by enzyme specificity is suggested by the fact that transmethylations utilizing choline, betaine and dimethylthetin are catalyzed by three different enzymes. ${ }^{47}$ This view is further supported by the observation that sulfobetaine is an active methyl donor, while sulfocholine is inactive in this respect. Moreover, dimethylpropiothetin exhibits a marked growth-promoting activity, whereas its nitrogen analogue, 
$\beta$-alanine betaine, is toxic and apparently does not serve as a methyl donor.

The effects of choline in preventing fatty livers and hemorrhagic kidneys in rats and mice as well as the antiperosis effect in chicks and turkeys and the growth promoting effects on mutants No. 34486 and

TABLE 48. Activity of Choline Derivatives on Growth of a Strain of Pneumococcus (Type III). ${ }^{9}$

Compound

Structural Formula

1. Choline chloride

2. Dimethylethanolamine

3. Methyldiethanolamine

4. Triethylcholine chloride

5. Diethylethanolamine

6. Dimethylethylhydroxyethylammonium chloride

7. Ethanolamine

8. Diethanolamine

9. Triethanolamine

10. Tetraethanolammonium hydroxide

11. N-Acetylethanolamine

12. $\alpha$-Ethylethanolamine

13. $\alpha$-Ethyl- $\alpha$-hydroxymethylethanolamine

14. $\alpha$-Methyl- $\alpha$-hydroxymethylethanolamine

15. $\alpha, \alpha$-Dimethylethanolamine

16. $\alpha, \alpha$-Dimethylcholine chloride

17. $\alpha, \alpha$-Dihydroxymethylethanolamine

18. $\gamma$-Diethylaminopropanol

19. $\beta, \gamma$-Propanediol- $\alpha$-diethylamine

20 . Diethylmethyl- $\beta, \gamma$-dihydroxypropylammonium chloride

21. Acetylcholine chloride

22 . Acetyl- $\beta$-methylcholine chloride

\begin{tabular}{|c|c|}
\hline $\begin{array}{l}\text { Structural Formula } \\
\left(\mathrm{CH}_{3}\right)_{3} \mathrm{~N}(\mathrm{Cl}) \cdot \mathrm{CH}_{2} \cdot \mathrm{CH}_{2} \cdot \mathrm{OH} \\
\left(\mathrm{CH}_{3}\right)_{2} \mathrm{~N} \cdot \mathrm{CH}_{2} \cdot \mathrm{CH}_{2} \mathrm{OH} \\
\left(\mathrm{CH}_{3}\right) \cdot \mathrm{N}\left(\mathrm{CH}_{2} \cdot \mathrm{CH}_{2} \cdot \mathrm{OH}\right)_{2} \\
\left(\mathrm{C}_{2} \mathrm{H}_{6}\right)_{3} \cdot \mathrm{N}(\mathrm{Cl}) \cdot \mathrm{CH}_{2} \cdot \mathrm{CH}_{2} \cdot \mathrm{OH} \\
\left(\mathrm{C}_{2} \mathrm{H}_{6}\right)_{2} \cdot \mathrm{N} \cdot \mathrm{CH}_{2} \cdot \mathrm{CH}_{2} \cdot \mathrm{OH} \\
\left(\mathrm{CH}_{3}\right)_{2}\left(\mathrm{C}_{2} \mathrm{H}_{5}\right) \mathrm{N}(\mathrm{Cl}) \cdot \mathrm{CH}_{2} \cdot \mathrm{CH}_{2} \cdot \mathrm{OH}\end{array}$ & $\begin{array}{l}\text { Activity } \\
100 \\
100 \\
100 \\
100 \\
100 \\
100\end{array}$ \\
\hline $\begin{array}{l}\mathrm{H}_{2} \mathrm{~N} \cdot \mathrm{CH}_{2} \cdot \mathrm{CH}_{2} \cdot \mathrm{OH} \\
\mathrm{HN}\left(\mathrm{CH}_{2} \cdot \mathrm{CH}_{2} \cdot \mathrm{OH}\right)_{2} \\
\mathrm{~N}\left(\mathrm{CH}_{2} \cdot \mathrm{CH}_{2} \cdot \mathrm{OH}\right)_{3} \\
(\mathrm{HO}) \mathrm{N}\left(\mathrm{CH}_{2} \cdot \mathrm{CH}_{2} \cdot \mathrm{OH}\right)_{4}\end{array}$ & $\begin{array}{r}10(80 \\
50(95 \\
100(95 \\
25(85\end{array}$ \\
\hline $\begin{array}{l}\mathrm{CH}_{3} \cdot \mathrm{CO} \cdot \mathrm{NH} \cdot \mathrm{CH}_{2} \cdot \mathrm{CH}_{2} \cdot \mathrm{OH} \\
\mathrm{H}_{2} \mathrm{~N} \cdot \mathrm{CH}\left(\mathrm{C}_{2} \mathrm{H}_{5}\right) \cdot \mathrm{CH}_{2} \cdot \mathrm{OH} \\
\mathrm{H}_{2} \mathrm{~N} \cdot \mathrm{C}\left(\mathrm{C}_{2} \mathrm{H}_{5}\right)\left(\mathrm{CH}_{2} \cdot \mathrm{OH}\right) \cdot \mathrm{CH}_{2} \cdot \mathrm{OH}\end{array}$ & $\begin{array}{r}10(23 \\
67(80 \\
25(100\end{array}$ \\
\hline $\mathrm{H}_{2} \mathrm{~N} \cdot \mathrm{C}\left(\mathrm{CH}_{2} \cdot \mathrm{OH}\right)\left(\mathrm{CH}_{3}\right) \cdot \mathrm{CH}_{2} \cdot \mathrm{OH}$ & $12(90$ \\
\hline $\begin{array}{l}\mathrm{H}_{2} \mathrm{~N} \cdot \mathrm{C}\left(\mathrm{CH}_{3}\right)_{2} \cdot \mathrm{CH}_{2} \cdot \mathrm{OH} \\
\left(\mathrm{CH}_{3}\right)_{3} \mathrm{~N}(\mathrm{Cl}) \cdot \mathrm{C}\left(\mathrm{CH}_{3}\right)_{2} \cdot \mathrm{CH}_{2} \cdot \mathrm{OH} \\
\mathrm{H}_{2} \mathrm{~N} \cdot \mathrm{C}\left(\mathrm{CH}_{2} \mathrm{OH}\right)_{2} \cdot \mathrm{CH}_{2} \cdot \mathrm{OH}\end{array}$ & $\begin{array}{r}10(31 \\
100(20 \\
10(5\end{array}$ \\
\hline $\begin{array}{l}\left(\mathrm{C}_{2} \mathrm{H}_{5}\right)_{2} \mathrm{~N} \cdot \mathrm{CH}_{2} \cdot \mathrm{CH}_{2} \cdot \mathrm{CH}_{2} \cdot \mathrm{OH} \\
\left(\mathrm{C}_{2} \mathrm{H}_{5}\right)_{2} \mathrm{~N} \cdot \mathrm{CH}_{2} \cdot \mathrm{CH}(\mathrm{OH}) \cdot \mathrm{CH}_{2} \cdot \mathrm{OH} \\
\left(\mathrm{C}_{2} \mathrm{H}_{5}\right)_{2}\left(\mathrm{CH}_{3}\right) \mathrm{N}(\mathrm{Cl}) \cdot \mathrm{CH}_{2} \cdot \mathrm{CHOH} \cdot \mathrm{CH}_{2} \mathrm{OH}\end{array}$ & $\begin{array}{l}83(100 \\
50(100 \\
100(20)\end{array}$ \\
\hline $\begin{array}{l}\left(\mathrm{CH}_{3}\right)_{3} \mathrm{~N}(\mathrm{Cl}) \cdot \mathrm{CH}_{2} \cdot \mathrm{CH}_{2} \cdot \mathrm{O} \cdot \mathrm{CO} \cdot \mathrm{CH}_{3} \\
\left(\mathrm{CH}_{3}\right)_{3} \mathrm{~N}(\mathrm{Cl}) \cdot \mathrm{CH}_{2} \cdot \mathrm{CH}\left(\mathrm{CH}_{3}\right) \cdot \mathrm{O} \cdot \mathrm{CO} \cdot \mathrm{CH}_{3}\end{array}$ & $\begin{array}{l}100(7 \\
100(7\end{array}$ \\
\hline
\end{tabular}

Even when tested at concentrations up to $50 \mathrm{\gamma} / \mathrm{ml}$. of medium the following compounds were inactive: N-phenylethanolamine, 2-nitro-1-butanol; serine; ethylamine; ethylenediamine; glycine; sarcosine; betaine; $\beta$-methoxyethylamine; carnitine; calcium phosphorylcholine; urethane of $\beta$-methylcholine chloride; carbamylcholine chloride.

a Percent activity on molar basis relative to cholne chloride necessary for maximal response of the organism to the compound. Bracketed figures indicate percent of maximal growth attained with the most effective concentration of the compounds which do not give growth equivalent to that obtained with $5 \gamma$ per ec. of choline chloride.

No. 47904 of Neurospora crassa appear to depend on a function of choline which involves the utilization of the intact molecule. The ability of choline to form phospholipides may be a critical factor, since it has been shown that analogues possessing choline activity, e.g., triethylcholine ${ }^{48,49}$ and sulfocholine, ${ }^{26}$ can be incorporated into the body phospholipides of rats. Inasmuch as these two compounds are not methyl donors, it appears that the utilization of choline as an intact molecule is independent of its ability to furnish methyl groups. Additional support for this hypothesis 
is provided by the fact that compounds which fail to function as methyl donors such as arsenocholine, diethylmethylhydroxyethylammonium chloride and homocholine, are active in preventing fatty livers in the rat or perosis in the chick, whereas such methyl donors as betaine and methionine do not prevent perosis in the chick.

Choline has been found to be essential for the growth of certain strains of pneumococci (Types I, II, V, VIII) ${ }^{8}$ A number of choline analogues have been tested as growth factors for a Type III strain ; $^{9}$ the results of these tests are summarized in Table 48. Of the known functions of choline in animal nutrition, its utilization in phospholipide formation appears to be its most likely role in the metabolism of the pneumococcus, since ethanolamine, diethanolamine and triethanolamine are also effective. The activity of these compounds, together with the inactivity of methionine, betaine, phosphorylcholine, etc., suggest that for this organism choline does not have a significant role in transmethylation.

Two mutant strains, No. 34486 and No. 47904, which arose from the ultraviolet irradiation of a culture of wild type Neurospora crassa were found 10,11 to require choline for growth in a medium which supported the growth of the wild type organism. Both mutants also responded to acetylcholine, arsenocholine, phosphorylcholine, dimethylaminoethanol, dimethylethylhydroxyethylammonium chloride, diethylmethylhydroxyethylammonium chloride, triethylcholine and methionine. The following compounds were inactive for both mutants: betaine, creatine, sarcosine, ethanolamine, neurine, diethylaminoethanol, dimethylamine, trimethylamine and tetramethylammonium chloride. Of considerable interest is the fact that mutant No. 34486 can utilize monomethylaminoethanol but No. 47904 cannot; under suitable conditions, this compound accumulates in the latter organism. ${ }^{48}$ These results were considered to indicate that methylaminoethanol is an intermediate in the synthesis of choline by Neurospora and that the block in mutant No. 34486 precedes the formation of methylaminoethanol, whereas the block in mutant No. 47904 follows it. The structural specificity exhibited suggests that in Neurospora the predominant function of choline depends on its acting as an intact molecule rather than as a methyl donor.

\section{Inhibitors Related to Choline}

Triethylcholine. Keston and Wortis ${ }^{50}$ reported in 1946 that even though triethylcholine is lipotropic when fed in small quantities to rats, the compound is acutely toxic when injected into mice. The toxicity is completely prevented by the simultaneous injection of an equal weight of choline chloride. It was further observed that the action of choline in the contraction of isolated frog muscle is blocked by triethylcholine while 
<smiles>CCC(CC)(CCO)[N+](CC)(CC)CCO</smiles>

the action of acetylcholine is unaffected. These data indicate that the choline analogue may interfere with the synthesis of acetylcholine from choline.

L-Penicillamine ( $\beta, \beta$-Dimethylcysteine). Wilson and $d u$ Vigneaud ${ }^{51}$ found that the growth of young albino rats was inhibited when L-penicil-<smiles>CCC(C)(S)C(N)C(=O)O</smiles>

lamine hydrochloride hydrate was added to the diet, and that normal growth was restored by feeding choline chloride. However, this inhibitor appears not to be competitive with choline, for aminoethanol was found to be even more effective than choline in overcoming the growth inhibition. Dimethylaminoethanol and monomethylaminoethanol were also effective in restoring the normal rate of growth, but methionine, cysteine, cystine, homocysteine and homocystine were without activity. These investigators suggested that "penicillamine may exert its toxic action by blocking either the synthesis or the utilization of aminoethanol."

Coramine (N,N-Diethylnicotinamide). A type of inhibition involving choline was observed by Wilson and Leduc. ${ }^{52}$ Weanling mice were fed on a low protein, choline-deficient diet to which coramine was added in varying amounts. The compound at a concentration of 0.25 per cent per-<smiles>CCN(CC)C(=O)c1cccnc1</smiles>

coramine

mitted very slight growth; 0.5 per cent allowed maintenance without growth; and 1.0 per cent caused a loss in weight. The inhibitory effects of coramine are reversed by the addition of choline to the diet. Even though coramine is structurally unrelated to choline, its detoxication apparently takes place by transmethylation and its presence in the diet increases the requirement for a methyl donor. Choline must function here only as 
a methyl donor and not as a lipotropic agent, since fatty livers do not develop on a 1 per cent coramine diet.

Ethionine (S-Ethylhomocysteine). It was early shown ${ }^{53}$ that ethionine is toxic to rats on a low methionine diet and that the toxicity is prevented by the simultaneous administration of methionine. Methionine also re-

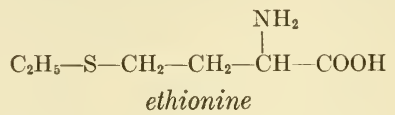

verses the growth inhibition produced in Escherichia coli by ethionine. ${ }^{54}$ The latter compound was used by Stekol and Weiss ${ }^{55}$ in an effort to determine whether the inhibition of growth in rats is the result of a block in the utilization of methionine per se, or whether there is interference with the utilization of some of the metabolites which normally originate from methionine. They found that choline alone can alleviate the growth inhibition caused by ethionine just as methionine can. The other substances tested, cystine, homocystine and cystathionine, were without effect on the inhibition. It is suggested that the increased need for choline which is created by the administration of ethionine may result in a diversion of the labile methyl group for greater synthesis of choline, thus decreasing the amount of methionine available for growth. The possibility of the incorporation of ethionine into the tissue protein of the rat was also considered, but it was stressed that further experimentation must be done before a rigorous interpretation of the data will be possible.

In this discussion no attempt has been made to treat the many inhibitors of enzyme systems in which choline is involved, but only those which may have a bearing on the status of choline as a doubtful member of the $\mathrm{B}$ vitamin complex.

\section{INOSITOL}

The role of inositol as a growth factor for yeast was discovered in 1928 by Eastcott, ${ }^{56}$ who isolated it from tea and recognized it as the active constituent of Lukas' "Bios I." Subsequent work has shown that it is a complementary growth factor for some strains of yeast; i.e., it is relatively ineffective alone, but in combination with other $B$ vitamins, it often causes a striking increase in growth. ${ }^{57,58}$ The amount of inositol necessary to produce these growth effects is from 100 to 1000 times larger than the effective concentrations of the other B vitamins, and the effects obtained are largely dependent on the particular strain of yeast under investigation. Inositol is also a complementary growth factor for various 

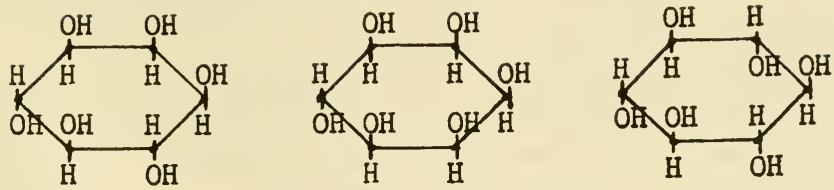

Meso-inositol

Epi-inositol

$\frac{1,2,3,5}{4,6}$

$\frac{1,2,3,4,5}{6}$

Scyllitol

$\frac{1,3,5}{2,4,6}$
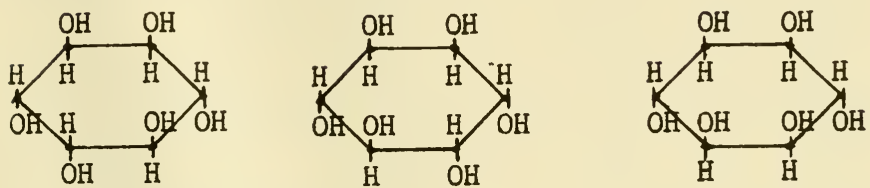

$\frac{1}{1}-$ inosit
$\frac{1,2,4}{3,5,6}$

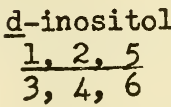

Yuco-1nositol
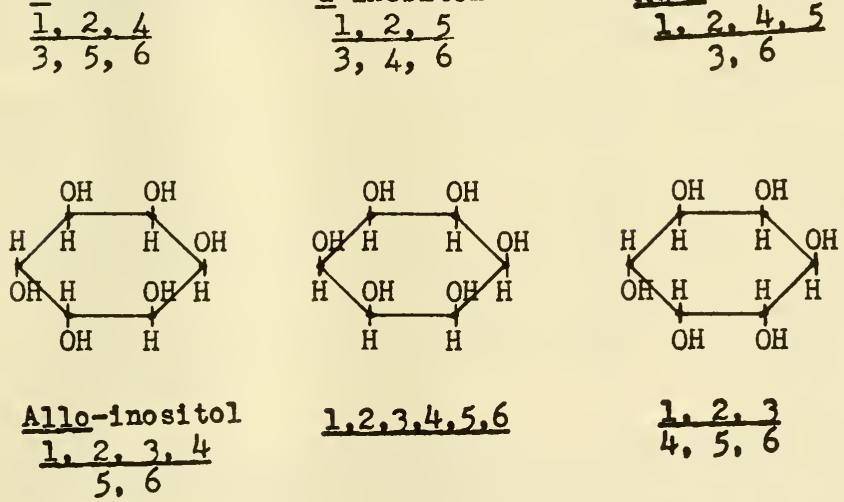

$1,2,3,4,5,6$

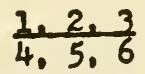

Figure 9. 
fungi, including Nematospora gossypii, ${ }^{59,60}$ Lophodermium pinastri ${ }^{60}$ and Eremothecium ashbyii. ${ }^{61}$ The compound has been shown to be an absolute essential for only a few organisms, such as Rhizopus suinus ${ }^{62}$ and "inositolless" mutant strains of Neurospora crassa. ${ }^{63}$

Woolley in 1940 isolated the "mouse anti-alopecia factor" from liver concentrate and identified it as inositol. ${ }^{64,65}$ He showed that a loss of hair and a severe dermatitis developed in mice on a purified diet and that the administration of inositol cured these symptoms.

Vitamin-like effects of inositol have also been reported for rats, ${ }^{66,67,68,69}$ guinea pigs, ${ }^{70}$ hamsters, ${ }^{71,72}$ chicks, ${ }^{73,74}$ and pigs. ${ }^{75}$

\section{Specificity}

Inositol, in contrast to other B vitamins, has a number of naturally occurring, closely related analogues. Several of its geometrical isomers and their derivatives are present in many natural products.

Nine geometrical isomers of inositol are theoretically possible if the six carbon atoms of the cyclohexane ring are considered to be coplanar. These nine forms ${ }^{76}$ are schematically represented below (Figure 9). Four of these isomers (meso-inositol, (+)- and (-)-inositol, and scyllitol) are known to occur naturally either in the free state or in the form of their esters or ethers. Their configurations as they are represented in Figure 9 have been established by the work of Posternak. ${ }^{79,} 80,81$ Three others isomers (epi-inositol, allo-inositol and muco-inositol) have been synthesized and characterized. ${ }^{79}, 82,83$

The possibility of additional isomers of compounds structurally related to inositol has been indicated by $\mathrm{x}$-ray studies on the $\beta$-isomer of $1,2,3,4,-$ 5,6-hexachlorocyclohexane and the corresponding bromo derivative. These studies 77,78 indicate that the six carbon atoms do not lie in one plane but in two parallel planes. This so-called "puckered ring" (chair form) would make 16 isomers theoretically possible including six optically active forms. While such structures may be stable in crystalline form, it is possible that in solution such isomers resulting solely because of the "puckered ring" or chair form of the cyclohexane ring may become interconvertible.

The biological activity of the four naturally occurring isomers and some of their most common derivatives ${ }^{76}$ are listed in Table 49. Of all these substances only meso-inositol was found to have vitamin activity for both yeast and mice. ${ }^{84}$ Indeed, in most instances only meso-inositol or compounds which can readily form meso-inositol appear to be effective. Esters of meso-inositol are active for mice but inactive for yeast. For yeast only mytilitol (the methyl homologue of scyllitol) and hydroxymytilitol have some activity in addition to meso-inositol itself. 
TABLE 49. Specificity of meso-Inositol.

\begin{tabular}{|c|c|c|c|c|c|c|}
\hline Compound & 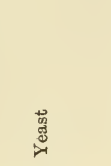 & 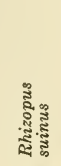 & 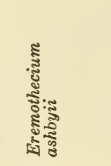 & 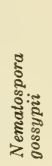 & 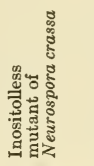 & 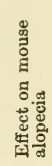 \\
\hline meso-Inositol & $\begin{array}{l}100 \\
107\end{array}$ & 100 & $\begin{array}{r}100 \\
61\end{array}$ & $\begin{array}{c}100 \\
108\end{array}$ & 100 & $\underset{106}{+}$ \\
\hline meso-Inositol monophosphate & ${ }_{100}^{5}$ & & $\underset{109}{50-100}$ & & $\begin{array}{c}0 \\
109\end{array}$ & \\
\hline meso-Inositol tetraphosphate & $\underset{106}{2}$ & & & & & \\
\hline $\begin{array}{l}\text { meso-Inositol hexaphosphate } \\
\text { (Phytin) }\end{array}$ & $<_{108}^{<1}$ & ${ }_{62}^{0}$ & & & & $+\underset{106}{+}$ \\
\hline meso-Inositol hexaacetate & $\underset{106}{<1}$ & & & & & $\underset{106}{+}$ \\
\hline epi-Inositol & & & $\underset{109}{2.9}$ & & & \\
\hline (-)-Inositol & $\underset{106}{<1}$ & ${ }_{62}^{0}$ & & $\begin{array}{r}0 \\
108\end{array}$ & $\begin{array}{l}4.5 \\
109\end{array}$ & $\overline{106}$ \\
\hline $\begin{array}{l}\text { (-)-Inositol monomethylether } \\
\text { (Quebrachitol) }\end{array}$ & $\begin{array}{l}0,<1 \\
106,107\end{array}$ & & $\begin{array}{l}\left(10.4^{109}\right) \\
22 \\
109\end{array}$ & $\begin{array}{r}0 \\
108\end{array}$ & $\begin{array}{c}20.7 \\
109\end{array}$ & $\overline{106}$ \\
\hline$(+)$-Inositol & $<_{106}^{<1}$ & & $\begin{array}{r}0 \\
109\end{array}$ & $\begin{array}{r}0 \\
108\end{array}$ & $\begin{array}{c}0 \\
109\end{array}$ & $\overline{106}$ \\
\hline $\begin{array}{l}\text { (+)-Inositol monomethylether } \\
\text { (Pinitol) }\end{array}$ & $\underset{106}{<1}$ & & $\begin{array}{r}0 \\
109\end{array}$ & & $\begin{array}{c}0 \\
109\end{array}$ & $\overline{106}$ \\
\hline Scyllitol & ${ }_{107}^{0}$ & ${ }_{62} 0$ & $\left\{\begin{array}{lc}0 & 61 \\
2.9^{109}\end{array}\right\}$ & & ${ }_{109}{ }^{0}$ & \\
\hline Mytilitol* & $\underset{106}{10}$ & $<10$ & $\underset{109}{8.1}$ & & & $\underset{106}{+}$ \\
\hline Isomytilitol* & & ${ }_{62}^{10}$ & $\begin{array}{l}19.7 \\
109\end{array}$ & & $\begin{array}{l}0.87 \\
109\end{array}$ & \\
\hline Hydroxymytilitol & & ${ }_{62}^{0}$ & $\underset{109}{7.5}$ & & & \\
\hline Hydroxyisomytilitol & & $<_{62} 10$ & $\underset{109}{2.9}$ & & $\underset{109}{0.15}$ & \\
\hline $\begin{array}{l}\text { Quercitol (pentahydroxy- } \\
\text { cyclohexane) }\end{array}$ & $\underset{108,107}{0,}<1$ & & & ${ }_{108}{ }^{0}$ & & $\overline{108}$ \\
\hline 5-Desoxy-(+)-inositol & ${ }_{109}^{1}$ & & $\underset{100}{2.9}$ & & & \\
\hline $\begin{array}{l}\text { Scyllo-ms-inosose } \\
\text { ("biochemical inosose") }\end{array}$ & $\underset{106}{<1}$ & ${ }_{62}^{0}$ & $\begin{array}{l}93.6 \\
109\end{array}$ & & $\begin{array}{c}0 \\
109\end{array}$ & \\
\hline $\begin{array}{l}\text { epi-ms-Inosose } \\
\text { ("chemical inosose") }\end{array}$ & & ${ }_{62}^{0}$ & $\begin{array}{ll}17.4 \\
109\end{array}$ & & $\begin{array}{l}13.9 \\
109\end{array}$ & \\
\hline Soybean cephalin & $<1$ & & & & & $\underset{108}{+}$ \\
\hline
\end{tabular}

Arabitol, sorbitol, dulcitol, mannitol and other sugar alcohols are inactive for yeast. ${ }^{107}$

*According to Posternak, ${ }^{81}$ mytilitol is methylscyllitol, and isomytilitol is methyl-meso-inositol.

Schopfer $62,85,86$ has tested the specificity of inositol for Rhizopus suinus and the "inositolless" mutant of Neurospora crassa. Meso-inositol is highly specific for both these organisms. (Table 49)

An interesting stereochemical specificity has been observed in the rate of the enzymatic oxidation of the inositols to ketones and diketones by 
Acetobacter suboxydans. ${ }^{80,87,88,89}$ According to Magasanik and Chargaff, ${ }^{89}$ "only those hydroxyls are oxidized that are situated in a polar plane." This would mean that in the case of meso-inositol only the cishydroxyl in the 2 position is attacked. Schopfer ${ }^{62}$ believed this hydroxyl to be necessary for vitamin activity. If this is true, scyllo-ms-inosose, which is active for Eremothecium ashbyii (Table 49), must be reduced to the corresponding meso-inositol before it is utilized by the organism.

\section{Inhibitory Analogues of Inositol}

The theory was advanced by Slade ${ }^{90,91}$ in 1945 that the powerful insecticidal action of $\gamma$-hexachlorocyclohexane ("Gammexane") could be explained on the basis of its structural similarity to meso-inositol. $\mathrm{He}$ assigned to the $\gamma$-isomer the configuration corresponding to that of mesoinositol and pointed out that this compound has a much higher toxicity for insects than do the $\alpha-, \beta$-, and $\delta$-isomers. It was proposed that the $\gamma$-isomer exerts its inhibitory action by blocking the functioning of inositol in some important enzyme system.

Recent $\mathrm{x}$-ray studies ${ }^{92}$ have indicated that the configuration of the $\gamma$-isomer does not correspond to that of meso-inositol; nevertheless, Slade's theory concerning the antagonism between "Gammexane" and meso-inositol stimulated several studies, and it was demonstrated that in some cases the toxic action of $\gamma$-hexachlorocyclohexane could be affected by meso-inositol.

Kirkwood and Philips ${ }^{93}$ found that the Gebruder Mayer strain of yeast, which normally requires $1 \gamma$ of inositol per $\mathrm{ml}$ of medium for maximal growth, was strongly inhibited by $60 \gamma$ per $\mathrm{ml}$ of $\gamma$-hexachlorocyclohexane. This inhibition was "progressively but not completely" reversed by the addition of 1 to $6 \gamma$ of meso-inositol per ml of medium. This would correspond to a molar inhibition ratio of about 30 . The $\alpha-$, $\beta$-, and $\delta$-isomers of hexachlorocyclohexane also had a slight inhibitory effect, but only the inhibition caused by the $\gamma$-isomer could be reversed by meso-inositol.

The $\gamma$-isomer of hexachlorocyclohexane completely inhibits the growth of Nematospora gossypii, whereas the $\beta$-isomer is inactive and the $\alpha$-isomer is only slightly inhibitory. ${ }^{94}$ The inhibition caused by $10-60 \gamma$ per $\mathrm{ml}$ of the $\gamma$-isomer was reduced to 50 per cent by the addition of $60 \gamma$ per $\mathrm{ml}$ of meso-inositol and to 6 per cent by $100 \gamma$ per $\mathrm{ml}$ of the vitamin.

Schopfer et $a l .^{95}$ found that flavinogenesis is inhibited in Eremothecium ashbyii var gossypii by $\gamma$-hexachlorocyclohexane and that the growth medium is completely decolorized. The effects of $800 \gamma$ of the inhibitor are prevented by the addition of $5 \gamma$ of meso-inositol. Scyllo-ms-inosose 
is also effective in preventing the inhibition, but $(+)$-inositol, scyllitol and other related compounds are inactive.

Chargaff et al..$^{96}$ found that meso-inositol is able to prevent the metaphase arrest and tumor formation induced in Allium Cepa by either colchicine or $\gamma$-hexachlorocyclohexane, first observed by Nybom and Knutsson. ${ }^{97}$ Meso-inositol appears to be specific in producing this effect; other related compounds were inactive.

It was recently reported ${ }^{98}$ that $\gamma$-hexachlorocyclohexane, after an incubation period of 16 hours, completely inhibited the enzymatic activity of a sample of purified pancreatic $\alpha$-amylase which had been shown to have a relatively high inositol content. The inhibition was competitively prevented by the addition of meso-inositol. Fischer and Bernfeld ${ }^{99}$ were not able to repeat this work, and suggested that the inositol-containing amylase was only partially purified.

Meillon ${ }^{100}$ observed that the blood of rabbits injected with $\gamma$-hexachlorocyclohexane was toxic for certain blood-sucking anthropods and that this toxicity was not reversed by injections of inositol. Schopfer ${ }^{95}$ found that either the toxicity of the inhibitor or the reversing action of meso-inositol was negligible or inconsistent in many fungi. He suggested that these inconsistencies may be due to the biosynthesis of inositol by these organisms.

Other studies have indicated that $\delta$-hexachlorocyclohexane is more toxic than the $\gamma$-isomer for the ciliate Glaucoma piriformis, ${ }^{101}$ for the eggs of sea urchins, ${ }^{102}$ and for many bacteria. ${ }^{103}$ In none of these cases was meso-inositol effective in preventing the toxicity.

In view of all the above experiments, it seems reasonable to conclude that the toxicity of $\gamma$-hexachlorocyclohexane for various organisms is not dependent on a single mechanism. Even though the $\gamma$-isomer may in some cases interfere with enzymatic reactions involving meso-inositol, there are apparently interrelationships other than these which are involved.

Carter and his co-workers ${ }^{104}$ found an interesting relationship between streptomycin and lipositol, a phospholipide containing 16 per cent inositol in a combined form. Soy bean lipositol, as well as preparations from brain infusion, prevented the antibacterial action of streptomycin on Eberthella typhosa and Staphylococcus aureus. Since there is present in lipositol an inositol-galactose structure which bears some resemblance to the streptomycin molecule, the authors suggested the possibility of a metabolite-antimetabolite relationship. It has also been observed ${ }^{105}$ that lipositol is slightly active in replacing streptomycin for streptomycinrequiring mutants of Escherichia coli. 


\section{Iron Porphyrins (Hemes) As Growth Factors and Inhibitors}

The same porphyrin nucleus which is present in heme, the nonprotein component of hemoglobin, occurs in the prosthetic group of various im-

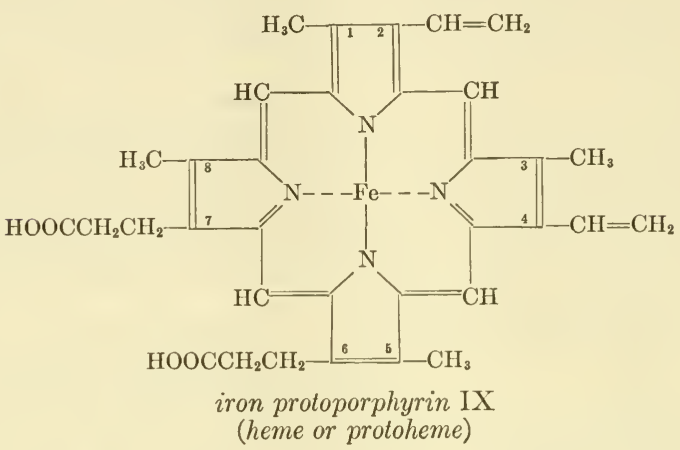

portant enzymes, including catalase, peroxidase and the cytochromes. Compounds whose structures are identical with or closely related to that of iron protoporphyrin IX have been found to be present in all animal and plant cells that have been examined with the exception of some anaerobic bacteria.

Among the organisms which have been found unable to synthesize protoporphyrin IX, the precursor of heme, are bacteria (Hemophilus influenzae), protozoa (certain trypanosomidae), and one insect species (Triatoma infestans). Consequently, protoporphyrin IX is an essential growth factor for these organisms. Although it has never been classified as a $\mathrm{B}$ vitamin, its mode of action, its role in respiratory enzyme systems, and its probable (but still unknown) relationships to some of the B vitamins may justify its inclusion here.

The role, mechanism of action and chemical structure of the iron porphyrins have been discussed in two excellent review papers by Granick and Gilder ${ }^{110}$ and by Lwoff. ${ }^{111}$

It was discovered as early as 1892 that Hemophilus influenzae did not grow unless a small amount of blood or hemoglobin was added to the culture medium. ${ }^{112}$ It was shown by Davis ${ }^{113}$ and by Thjötta and Avery ${ }^{114}$ that this organism required two growth factors, a heat-stable substance $X$ which is found in hemoglobin and a heat-labile factor $V$ which is present in yeast and in fresh animal and vegetable tissues.

Lwoff and Lwoff ${ }^{115}$ found that the $V$ factor could be replaced by coen- 
zymes I or II. Olsen ${ }^{116}$ showed that the growth-stimulating properties of the $X$ factor could be produced by heme (iron protoporphyrin), as well as by hemoglobin, but that hematoporphyrin, hemocyanin, bilirubin, chlorophyll, and pyrrole were inactive. He concluded that the growthpromoting activity of heme was connected with its function in the peroxidase enzyme system. This conclusion was later shown to be erroneous by Lwoff, who demonstrated that heme is also used for the synthesis of cytochrome $\mathrm{c}^{117}$ and that the growth-promoting activity and the peroxidase activity are not necessarily related.111

Granick and Gilder 118, 119, 120, 121 have investigated thoroughly the specificity of heme as the growth factor $X$ for Hemophilus influenzae. They found that protoporphyrin IX could replace heme in all cases; in fact, as is shown in Table 50, the iron-free compound has even higher activity in some instances than heme itself. Evidence was obtained that protoporphyrin IX was converted into heme by these organisms, showing that Hemophilus influenzae is capable of inserting iron into the protoporphyrin ring. It was also demonstrated that peroxidase and catalase enzyme systems were formed from the protoporphyrin added to the hemefree culture medium. The observation that the direct addition of heme to the medium was often less effective than the addition of protoporphyrin was attributed to the fact that heme is quite readily destroyed by even traces of hydrogen peroxide. This view was supported by the fact that substances which are able to destroy $\mathrm{H}_{2} \mathrm{O}_{2}$ enhance the growth of the organism in the presence of the various iron porphyrins whereas they do not affect the activity of the iron-free protoporphyrin. ${ }^{119}$ Neither cytochrome c nor crystalline beef catalase replaced factor $X$ in stimulating the growth of these organisms.

The iron-free porphyrins which do not contain vinyl groups, including deutero-, hemato-, meso-, and coproporphyrins, do not replace protoporphyrin in promoting growth of Hemophilus influenzae. Mesoporphyrin in small concentration did support growth of the "rough" Turner strain but larger concentrations of the compound were inhibitory. However, when these porphyrins lacking vinyl groups were converted into the corresponding iron porphyrins and then supplied to the organisms, they were found to support growth in seven of the ten strains tested (See Table 50). These data suggest that the vinyl group is essential for the insertion of iron into the porphyrin ring, but not for the growth-promoting activity of the iron porphyrins. It appears that the nonvinyl-containing iron porphyrins cannot carry out all the functions of iron protoporphyrin, since the cultures grown on the former compounds do not possess the ability to reduce nitrates to nitrites (Table 50). Also, maximum growth is not always obtained in the presence of such iron porphyrins. Theorell 


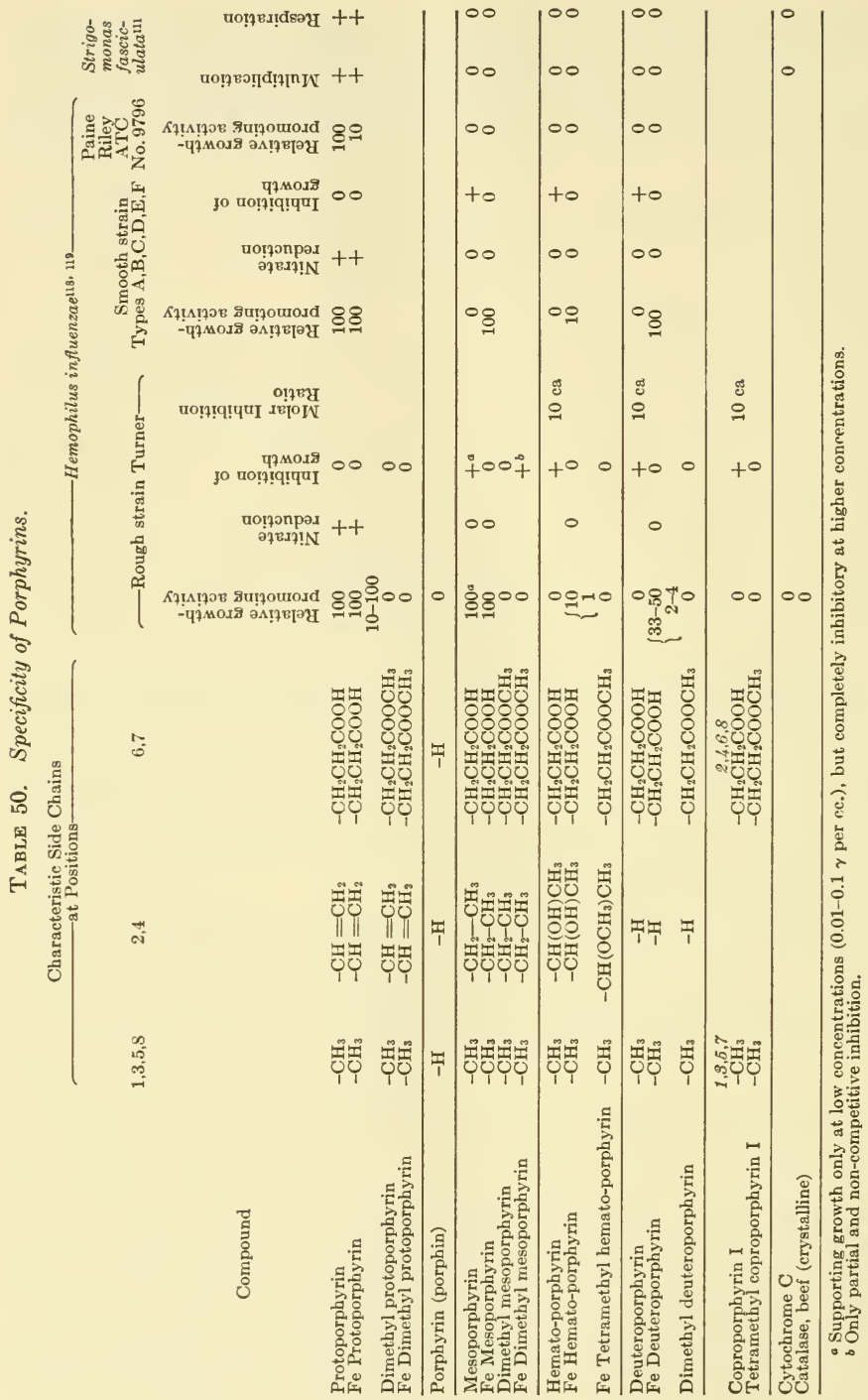


and his co-workers ${ }^{122}$ have reported that the protein moiety of horseradish peroxidase combines with protoheme, mesolieme, and deuteroheme to form substances with 100,53 , and 63 per cent, respectively, of the original enzyme activity.

Since heme is known to be a constituent of oxygen-activating enzyme systems, it has been generally accepted that it was indispensable only for aerobic life processes. It was thought that Hemophilus influenzae, a facultative anaerobe, would not require heme when grown under anaerobic conditions. This view was supported by experiments of Kopp ${ }^{123}$ and Eirund, ${ }^{124}$ but it was later shown by Gilder and Granick ${ }^{119}$ that a small amount of heme was required even for anaerobic growth. According to Lwoff, ${ }^{111}$ heme possibly functions also in enzyme systems other than those concerned with the activation of oxygen.

The nutritional requirements of some trypanosomidae were studied by Lwoff and Lwoff. ${ }^{111}, 115,117,125,126,127,128,129,130$ These parasites, which live in the digestive tubes of certain flies, could be grown on artificial medium only if it contained blood. Lwoff showed that blood can be replaced by either heme or the iron-free protoporphyrin in its role of stimulating the multiplication and respiration of Strigomonas fasciculata, but that all the nonvinyl-containing porphyrins and hemes, as well as cytochrome c, peroxidase and the "active iron" of Bandisch, were inactive. Hence, Strigomonas fasciculata like Hemophilus influenzae is able to insert iron into the protoporphyrin molecule.

The only insect which has been found to require heme for growth is the assassin bug, Triatoma infestans. ${ }^{131}, 132$ The artificially fed larvae of this insect require either blood or heme in their diet in order for normal growth to occur.

In the course of their investigations Granick and Gilder ${ }^{118}$ discovered that when iron-free porphyrins were added to a medium containing either protoporphyrin or iron protoporphyrin, the former substances inhibited the growth of Hemophilus influenzae. The inhibition was of the competitive type, the molecular ratio of the nonvinyl-containing porphyrin to protoporphyrin at almost complete inhibition being nearly constant. The molecular ratio of hemato-, deutero-, and coproporphyrin to protoporphyrin for almost complete inhibition was approximately 10 to 1 . A similar competition was also observed between iron protoporphyrin and other iron porphyrins. All these compounds support growth of the organism, but only iron protoporphyrin forms enzymes which reduce nitrate to nitrite. When iron mesoporphyrin was added to a medium containing a suboptimal concentration of heme, the growth of Hemophilus influenzae was enhanced but its ability to reduce nitrates was decreased. These observations led Granick and Gilder to conclude that a competition exists 
between the vinyl-containing and nonvinyl-containing porphyrins for certain apoenzymes.

The free ionizable propionic acid side chains seem to be essential for biological activity of the various porphyrins. When these propionic groups were esterified, none of the compounds tested either supported or inhibited the growth of Hemophilus influenzae. ${ }^{118}$ Apparently the free carboxyl groups are necessary for the attachment of the iron porphyrins to the basic groups of the apoenzyme.

High concentrations of heme inhibit the growth of some bacteria or even cause lysis. Cultures of Bacillus subtilis disappear 24 hours after the addition of blood to the medium and their growth is completely inhibited by heme in a concentration of $1: 125,000 .{ }^{133}$ Corynebacterium diphtheriae is inhibited by even low concentrations of heme, especially under aerobic conditions. ${ }^{134}$ Lwoff ${ }^{111}$ attributes these effects to the inhibition in vivo of the succinic acid dehydrogenase enzyme system by heme, a phenomenon observed in vitro by Keilen and Hartree. ${ }^{135}$ Since the biosynthesis of heme occurs in these same bacteria, this may be considered an antibiotic effect. Iron mesoporphyrin has also been reported to be an inhibitor for many bacteria. ${ }^{136}$

\section{Bibliography}

1. Allan, F. N., Bowie, D. J., Macleod, J. J. R., and Robinson, W. L., Brit. J. Exptl. Path., 5, 75 (1924).

2. Hershey, J. M., Am. J. Physiol., 93, 657 (1930).

3. Best, C. H., and Hershey, J. M., J. Physiol., 75, 49 (1932).

4. Best, C. H., and Huntsman, M. E., J. Physiol., 75, 405 (1932).

5. Best, C. H., and Lucas, C. C., Vitamins and Hormones, I, 1 (1943).

6. Jukes, T. H., Ann. Rev. Biochem., XVI, 193 (1947).

7. Griffith, W. H., "The Biological Action of the Vitamins," (E. A. Evans, Jr., ed.) University of Chicago Press; 1944, p. 169.

8. Rane, L., and SubbaRow, Y., J. Biol. Chem., 134, 455 (1940).

9. Badger, E., J. Biol. Chem., 153, 183 (1944).

10. Horowitz, N. H., and Beadle, G. W., J. Biol. Chem., 150, 325 (1943).

11. Horowitz, N. H., Bonner, D., and Houlahan, M. B., J. Biol. Chem., 159, 145 (1945).

12. Tucker, H. F., and Eckstein, H. C., J. Biol. Chem., 121, 479 (1937); 126, 117 (1938):

13. du Vigneaud, V., Chandler, J. P., Moyer, A. W., and Keppel, D. M., J. Biol. Chem., 131, 57 (1939).

14. Simmonds, S., Cohn, M., Chandler, J. P., and du Vigneaud, V., J. Biol. Chem., 149, 519 (1943).

15. Griffith, W. H., and Wade, N. J., Proc. Soc. Exptl. Biol. Med., 41, 188 (1939).

16. Best, C. H., Hershey, J. M., and Huntsman, M. E., Am. J. Physiol., 101, 7 (1932).

17. Jukes, T. H., J. Nutrition, 20, 445 (1940).

18. Welch, A. D., presented by Jukes, T. H., before the American Institute of Nutrition at Chicago, April 16, 1941. See Ref. 21.

19. Welch, A. D., J. Biol. Chem., 137, 173 (1941). 
20. du Vigneaud, V., Chandler, J. P., and Moyer, A. W., J. Biol. Chem., 139, 917 (1941).

21. Moyer, A. W., and du Vigneaud, V., J. Biol. Chem., 143, 373 (1942).

22. Welch, A. D., personal communication to Moyer, A. W., and du Vigneaud, V.; See Ref. 21.

23. du Vigneaud, V., Chandler, J. P., Simmonds, S., Moyer, A. W., and Cohn, M., J. Biol. Chem., 164, 603 (1946).

24. Griffith, W. H., and Wade, N. J., Proc. Soc. Exptl. Biol. Med., 41, 333 (1939).

25. Maw, G. A., and du Vigneaud, V., J. Biol. Chem., 176, 1037 (1948).

26. Maw, G. A., and du Vigneaud, V., J. Biol. Chem., 176, 1029 (1948).

27. Channon, H. J., and Smith, J. A. B., Biochem. J., 30, 115 (1936).

28. Best, C. H., and Ridout, J. H., Ann. Rev. Biochem., 8, 349 (1939).

29. Mawson, E. H., and Welch, A. D., Biochem. J., 30, 417 (1936).

30. Welch, A. D., Proc. Soc. Exptl. Biol. Med., 35, 107 (1936).

31. Channon, H. J., Manifold, M. C., and Platt, A. P., Biochem. J., 34, 866 (1940).

32. Singal, S. A., and Eckstein, H. C., J. Biol. Chem., 140, 27 (1941).

33. Welch, A. D., and Welch, M. S., Proc. Soc. Exptl. Biol. Med., 39, 7 (1938).

34. Channon, H. J., Platt, A. P., and Smith, J. A. B., Biochem. J., 31, 1736 (1937).

35. Best, C. H., Hershey, J. M., and Huntsman, M. E., J. Physiol., 75, 56 (1932).

36. Carter, H. E., and Melville, D. B., J. Biol. Chem., 133, 109 (1940).

37. Platt, A. P., Biochem. J., 33, 505 (1939).

38. Best, C. H., and Ridout, J. H., Can. Med. Assoc. J., 39, 188 (1938).

39. Jukes, T. H., Proc. Am. Inst. Nutrition, J. Nutrition, 21, suppl., 13 (1941).

40. Jukes, T. H., J. Nutrition, 22, 315 (1941).

41. Jukes, T. H., and Welch, A. D., J. Biol. Chem., 146, 19 (1942).

42. Jukes, T. H., personal communication to Moyer, A. W., and du Vigneaud, V., Ref. 21.

43. Jukes, T. H., Poultry Sci., 20, 251 (1941).

44. Jukes, T. H., Oleson, J. J., and Dornbush, A. C., J. Nutrition, 30, 219 (1945).

45. du Vigneaud, V., Simmonds, S., Chandler, J. P., and Cohn, M., J. Biol. Chem., 165, 639 (1946).

46. du Vigneaud, V., Chandler, J. P., Cohn, M., and Brown, G. B., J. Biol. Chem., 134, 787 (1940).

47. Dubnoff, J. W., and Borsook, H., Federation Proc., 7, 152 (1948).

48. McArthur, C. S., Science, 104, 222 (1946).

49. McArthur, C. S., Lucas, C. C., and Best, C. H., Biochem. J., 41, 612 (1948).

50. Keston, A. S., and Wortis, S. B., Proc. Soc. Exptl. Biol. Med., 61, 439 (1946).

51. Wilson, J. E., and du Vigneaud, V., Science, 107, 653 (1948).

52. Wilson, J. W., and Leduc, E. H., Federation Proc., 8, 169 (1949).

53. Dyer, H. M., J. Biol. Chem., 124, 519 (1938).

54. Harris, J. S., and Kohn, H. I., J. Pharmacol. Exptl. Therap., 73, 383 (1941).

55. Stekol, J. A., and Weiss, K., J. Biol. Chem., 179, 1049 (1949).

56. Eastcott, E. V., J. Phys. Chem., 32, 1094 (1928).

57. Williams, R. J., and Saunders, D. H., Biochem. J., 28, 1887 (1934).

58. Williams, R. J., Eakin, R. E., and Snell, E. E., J. Am. Chem. Soc., 62, 1204 (1940).

59. Buston, H. W., and Pramanik, B. N., Biochem. J., 25, 1656 (1931).

60. Kögl, F., and Fries, N., Z. Physiol. Chem., 249, 93 (1937).

61. Schopfer, W. H., Helv. Chim. Acta, 27, 1017 (1944).

62. Schopfer, W. H., Helv. Chim. Acta, 27, 468 (1944).

63. Beadle, G. W., J. Biol. Chem., 156, 683 (1944).

64. Woolley, D. W., Science, 92, 384 (1940).

65. Woolley, D. W., J. Biol. Chem., 139, 29 (1941).

66. Gavin, G., and McHenry, E. W., J. Biol. Chem., 139, 485 (1941).

67. Gavin, G., Patterson, J. M., and McHenry, E. W., J. Biol. Chem., 148, 275 (1943). 
68. Pavcek, P. L., and Baum, H. M., Science, 93, 502 (1941).

69. Martin, G. J., Am. J. Physiol., 136, 124 (1942).

70. Hogan, A. G., and Hamilton, J. W., J. Nutrition, 23, 533 (1942)

71. Hamilton, J. W., and Hogan, A. G., J. Nutrition, 27, 213 (1944).

72. Cooperman, J. M., Waisman, H. A., and Elvehjem, C. A., Proc. Soc. Exptl. Biol. Med., 52, 250 (1943).

73. Hegsted, D. M., Briggs, G. M., Mills, R. C., Elvehjem, C. A., and Hart, E. B., Proc. Soc. Exptl. Biol. Med., 47, 376 (1941).

74. Dam, H., J. Nutrition, 27, 193 (1944).

75. Lindley, D. C., and Cunha, T. J., J. Nutrition, 32, 47 (1946).

76. Ploetz, T., Chemie, Die, 56, 231 (1943).

77. Dickinson, R. G., and Bilicke, C., J. Am. Chem. Soc., 50, 764 (1928).

78. Kauer, K. C., DuVall, R. B., and Alquist, F. N., Ind. Eng. Chem., 39, 1335 (1947).

79. Posternak, S., and Posternak, T., Helv. Chim. Acta, 12, 1165 (1929) ; Posternak, T., Helv. Chim. Acta, 18, 1284 (1935); 19, 1333 (1936).

80. Posternak, T., Helv. Chim. Acta, 24, 1045 (1941) ; 29, 1991 (1946).

81. Posternak, T., Helv. Chim. Acta, 25, 746 (1942); 27, 457 (1944).

82. Dangschat, G., and Fischer, H. O. L., Naturwiss., 27, 756 (1939).

83. Dangschat, G., Naturwiss., 30, 146 (1942).

84. Woolley, D. W., J. Nutrition, 21, suppl., 17 (1941).

85. Schopfer, W. H., Posternak, T., and Guilloud, M., "Antonie von Leeuwenhoek," J. Microbiol. Serol., 12, 133 (1947).

86. Schopfer, W. H., Posternak, T., and Boss, M. L., Z. Vitaminforsch, 20, 121 (1948).

87. Kluyver, A. J., and Boezaardt, A. G., Rec. trav. chim., 58, 956 (1939).

88. Fulmer, E. I., and Underkofler, L. A., Iowa State Coll. J. Sci., 21, 251 (1947).

89. Magasanik, B., and Chargaff, E., J. Biol. Chem., 174, 173 (1948).

90. Slade, R. E., Chemistry and Industry, 64, 314 (1945).

91. Slade, R. E., Chem. Age, 52, 244 (1945).

92. van Vloten, G. W., Kruissink, C. A., Strijk, B., and Bijvoet, J. M., Nature, 162, 771 (1948).

93. Kirkwood, S., and Phillips, P. H., J. Biol. Chem., 163, 251 (1946).

94. Buston, H. W., Jacobs, S. E., and Goldstein, A., Nature, 158, 22 (1946).

95. Schopfer, WV. H., Posternak, T., and Boss, M. L., Schweiz. Z. Path. u. Bakt., 10, 443 (1947); through C. A., 42, 7821 (1948).

96. Chargaff, E., Stewart, R. N., and Magasanik, B., Science, 108, 556 (1948).

97. Nybom, N., and Knutsson, B., Hereditas, 33, 220 (1947).

98. Lane, R. L., and Williams, R. J., Arch. Biochem., 19, 329 (1948).

99. Fischer, E. H., and Bernfeld, P., Helv. Chim. Acta, 32, 1146 (1949).

100. de Meillon, B., Nature, 158, 839 (1946).

101. Chaix, P., Lacroix, L., and Fromageot, C., Biochim. et Biophys. Acta, 2, 57 (1948).

102. Chaix, P., and Lacroix, L., Biochim. et Biophys. Acta, 2, 86 (1948).

103. Fromageot, C., and Confino, M., Biochim. et Biophys. Acta, 2, 142 (1948).

104. Rhymer, I., Wallace, G. I., Byers, L. W., and Carter, H. E., J. Biol. Chem., 169, 457 (1947).

105. Rake, G., Proc. Soc. Exptl. Biol. Med., 67, 249 (1948).

106. Woolley, D. W., J. Biol. Chem., 140, 461 (1941).

107. Miller, W. L., J. Chem. Education, .7, 257 (1930).

108. Buston, H. W., and Kasinathan, S., Biochem. J., 27, 1859 (1933).

109. Chaix, P., Bull. soc. chim. biol., 30, 835 (1948).

110. Granick, S., and Gilder, H., Advances in Enzymol., 7, 305 (1947).

111. Lwoff, A., Bull. soc. chim. biol., 30, 817 (1948).

112. Pfeiffer, R., Deut. med. Wochschr., 18, 28 (1892).

113. Davis, D. J., J. Infectious Diseases, 21, 392 (1917). 
114. Thjötta, T., and Avery, O. T., J. Exptl. Med., 34, 97 (1921).

115. Lwoff, A., and Lwoff, M., Ann. inst. Pasteur, 59, 129 (1937).

116. Olsen, O., Centr. Bakt. Parasitenk. I Abt., 85, 12 (1920).

117. Lwoff, A., Compt. rend soc. biol., 122, 1041 (1936).

118. Granick, S., and Gilder, H., J. Gen. Physiol., 30, 1 (1916).

119. Gilder, H., and Granick, S., J. Gen. Physiol., 31, 103 (1947).

120. Granick, S., and Gilder, H., Science, 101, 540 (1945).

121. Granick, S., Ann. N.Y. Acad. Sci., 48, 657 (1947).

122. Theorell, H., Bergström, S., and Åkeson, A., Arkiv. Kemi., Mineral. Geol., Ser. A16, No. 13, 1 (1943).

123. Kopp, H., Centr. Bakt. Parasitenk., I Abt. 105, 54 (1927).

124. Eirund, A., Centr. Bakt. Parasitenk, I Abt., 111, 195 (1929).

125. Lwoff, M., Ann. inst. Pasteur, 51, 55 and 707 (1933).

126. Lwoff, A., Compt. rend. soc. biol., 113, 231 (1933).

127. Lwoff, A., Centr. Bakt. Parasitenk., I Abt., 130, 498 (1934).

128. Lwoff, A., Proc. Roy. Soc. (London), B124, 5 (1937).

129. Lwoff, M., Compt. rend. soc. biol., 121, 419 (1936).

130. Lwoff, M., "Recherches sur le pouvoir de synthese des Flagelles Trypanosomides," Monographies del' Institut Pasteur, Paris, 1940.

131. Lwoff, M., and Nicolle, P., Compt. rend. soc. biol., 138, 164, 205, 341, (1944).

132. Lwoff, M., and Nicolle, P., Compt. rend. soc. biol., 139, 879 (1945).

133. Van Heyningen, W. E., Nature, 162, 114 (1948).

134. Glass, V., J. Path. Bact., 49, 548 (1939).

135. Keilin, D., and Hartree, E. F., Biochem. J., 41, 503 (1947).

136. Kämmerer, H., Verhandl. deut. Ges. inn. Med., 31, 704 (1914).

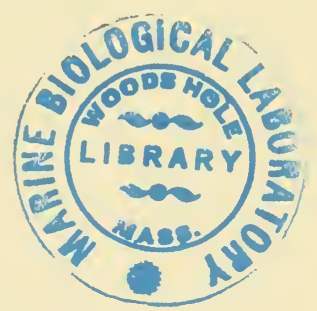





\section{INDEX}

Absorption of $\mathrm{B}$ vitamins, factors influencing, 344

in animals, $343-345$

in plants, $336-337$

Abundance of $B$ vitamins, in whole organisms, 24

Acetaldehyde, formation of, 221

Acetate factor, $16 \mathrm{ff}$

Acetic acid, aerobic production of, 164 as metabolic intermediate, 190

Acetic acid-lactic acid dismutation, 164 hydrogen carriers for, 164

Acetoin, from pyruvate, 161

Acetylcholine, 39

formation in nerve tissue, 106

role in production of electrical energy, 237

Acetylmethylcarbinol, formation of, 221

Acetylphosphorylase, 191

Achlorhydria, 266

Acid phosphates, formation of, 195

Acid labile forms of pyridoxine, 37

cis-Aconitate, formation of, 195

Activated acetyl molecule, 190

"Active acetate," 190

Active forms of B vitamins (see coenzymes of individual vitamins)

Addisonian pernicious anemia, 415 therapy, 415

Addison's disease, pigmentation in, 425

Adenine, toxicity of, 296

Adenosine diphosphate, as phosphorylating agent, 191 from DPN, 134

Adenosine triphosphate, in biosynthesis of thiamine, 157 in muscular contraction, 236

Adenylic acid, role in coenzyme formation, 117

Adermin, 652

Adrenal cortical relationships, 381

Adrenal cortex, effect of pantothenate deficiency on, 380,424

Adrenal hypertrophy, 382

Adrenalectomy, effects of, 425

Adrenaline, from DOPA, 181

Aerobic respiration, energy conservation in, 150

Age, effect on vitamin requirements, 265266

Aged, vitamin requirements of, 266

Agenizing of flour, 297
Alanine, relation to bacterial pyridoxine requirement, 184

$\beta$-Alanine, 35

from aspartic acid, 85,181

relation to pantothenic acid, 466

role in biosynthesis of pantothenic acid, 85

Alcohol, effect on $B$ vitamin requirements, 276

Alcoholism, chronic,

as genetotrophic disease, 433

with nutritional polyneuritis, 400

Aldehyde oxidases, 148

Aldol cleavage, in glycolysis, 219

Aldolase, 188

Alkaloids, in plants, 336

Alloxazine, 143

Amides, formation of, 195

Amines, detoxification of, 106

Amino acid decarboxylation, pyridoxal phosphate in, 181

a-Amino acids,

coenzyme activating, 174

effect on choline requirement, 278

effect on riboflavin requirement, 277

folic acid in synthesis of, 201

PABA in synthesis of, 201

relation to bacterial pyridoxine requirements, 184

requirements for plants of, 316-317

role of $\alpha$-ketoglutarate in synthesis of, 232

synthesis of aromatic, 195

vitamins required for degradation of, 233

vitamins required for synthesis of, 231

D-Amino acid oxidase, 147

L-Amino acid oxidase, 32, 147, 148

$p$-Aminobenzoic acid

analogues, 291, 484-486

anti-pernicious anemia vitamin in utilization of, 203,207

assay methods, 71-72

biochemical function, 11-12, 469, 488

biochemical interrelationships, 470

biosynthesis, 89

combined forms, 40-41

deficiency, 429-430

distribution, 18-19

effect of species size on requirement of, 323

excretion of, $365-370$ 
p-Aminobenzoic acid-Continued

glutamic acid in metabolism of, 487489

hydrolytic conditions, 41

inhibitory analogues, $493-500,521-530$ isosteres, $527-528$

metabolic relations to purines, pyrimidines, 198

metabolism of, 363-364

relation to folic acid, $202,472,490-493$

relation to nicotinic acid, 282

relation to pantothenic acid, 282

relation to thymidine, 474

requirements, 326,327

role in amino acid synthesis, 201

role in carbon to carbon bond formation, 189

role in purine and pyrimidine synthesis, 201

role in single carbon unit metabolism, 472

specificity, $482-483$

structure, 11

sulfonamide antagonism to, 199

sulfonamides in inhibition analysis of function, 470-471

toxicity, 391

p-Aminobenzoylglutamyl peptide, 490

$\gamma$-Aminobutyric acid, formation of, 181

Amino sugars, 223

$\alpha$-Amylase,

dissociation of, 125

inositol in, 10, 38, 106, 125

occurrence, 126

Analogues of B vitamins, $443,456-460$

(See also individual vitamins)

effect on coenzyme formation, 461

pharmacological properties, 391

Androgens,

inactivation of, 382

role in nitrogen, phosphorus and potassium retention, $381-382$

Anemia, pernicious, 204, 415-417

excretion of folic acid in, 571

thymine in therapy of, 414

Anemias,

associated with folic acid, vitamin $B_{12}$ deficiency, 413

due to cobalt deficiency, 206

"goat's milk," 419

megaloblastic macrocytic, 419

nutritional macrocytic, 419

pregnancy macrocytic, 419

tapeworm in macrocytic, 419-420

"temporary pernicious," 419

Aneurin, 6

Aneurin pyrophosphate, 164

Animal-protein factor, 14

Animals,

absorption of B vitamins in, 343-345

catabolism of $\mathrm{B}$ vitamins in, 351-370

digestion in, 338-343
Animals-Continued

germ-free, 300

synthesis of B vitamins in, 351-356

Annelida, B vitamin requirements of, 309

Anoxia,

coenzyme breakdown in, 272

effect on B vitamin requirements, 272

Antagonists, biochemical genetics in competitive, 477-478

Anthranilic acid, 287

Antibiotics, use in intestinal studies, 298

"Anti-dermatitis factor," 422

Anti-malarials, relation to riboflavin coenzymes, 679-681

Antipernicious anemia vitamin,

absorption spectra, 206

activity of nucleosides, 205

activity of thymidine, 205

as folic acid analogue, 290

characterization of active compounds, 205

coenzymatic role of, 112

coenzymes, 206

description, 14

dosage, 14

function, 14, 207, 474-475

inhibition studies, 207

interrelation to ascorbic acid, 204

interrelation to folic acid, 206-207

reactions catalyzed by, 206

role in carbon to carbon bond formation, 189

role in formation of serine and methionine, 207

role in utilization of PABA, 203, 207

Antipernicious anemia vitamin, deficiency of,

anemias associated with, 413

pernicious anemia, 415-416

sprue, $417-418$

Anti-thiamine effects, 295

"Anti-thiamine enzyme," in raw fish, 155

"Anti-vitamins," use of, 260-261

Apocarboxylase, 157

Apoenzymes, 31, 109

differential affinity for nicotinic acid coenzymes, 118

Apoerythein, 415

Apophosphorylases, 134

APP, 154

Araboflavin, 296

Arecoline, 336, 389

Arginine, formation of, 181

Arthropoda, B vitamin requirements of, 309

Ascorbic acid,

distribution, 21-22

effect on riboflavin storage, 258

from carbohydrates, 223

interrelation to carotenoids, 22

interrelation to vitamin $\mathrm{B}_{12}, 204$

oxidation-reduction potential effect, 21 
Aspartic acid, $\beta$-alanine from, 85, 181

function of biotin in synthesis of, 172 interrelation to pantothenic acid, 466

Aspartic-alanine system, 182

Assay methods, (See also individual vitamins)

biological, advantages of, 46

chemical and physical-chemical, advantages of, 45

microbiological, advantages of, 46 purposes, 45

Assays of urinary B vitamins, in humans, $252,254,283$

Atebrin, relation to riboflavin coenzymes, 679-681

Auramine, 680

Autoclaving, effect on pyridoxine, 176

Auximers, 316

Auxin activity of vitamins, 316

Availability of B vitamins, 46, 283

Avidin, 12, 37, 559-561

as egg white constituent, 294

production in oviduct, 383

properties of, 560

\section{Bacteria,}

$B$ vitamin requirements of, 307

intestinal, effect on $B$ vitamin requirements, 297

synthesis of B vitamins by, 299-300

Beriberi,

analysis of diets, in $250-251$

historical, $39 \mathrm{~S}$

incidence, 398, 399

infant, 399

symptomology, 400-401

Betaine, 10

Biochemical genetics, competitive antagonists and, 477-478

Biochemical reactions, 95 et seq. types of, 103 et seq.

Biocytin, $542 \mathrm{ff}$

Biological effects of $B$ vitamins, 377 et seq.

Biological materials, B vitamin levels in, 257-259

Biological oxidations and reductions (See dehydrogenations)

coenzymes mediating, 128 mechanisms, 128

Biological potency of $\mathrm{B}$ vitamins, 283

Biological systems, energy transformations in, 100

Bioluminescence, 237-238

"Bios I," 710

Biosynthesis of coenzymes, 112

Biotin

analogues, 289, 553-559

as essential cell constituent, 174

assay methods, 61-64

bacterial production of, $87-88$
Biotin-Continued

biochemical interrelationships, 467-468

biosynthesis, $87-88$

coenzymes, 173

combined forms, 37

distribution, $23-24$

effect of potassium on deficiency, 249

effect of species size on requirements of, 321

effect on pantothenate requirements, 278

effect on root production, 88

excretion, $87,365-370$

extraction, 37

functions, 170,172

hormone activity of, 337

in deaminations, 172-173

in synthesis of aspartic acid, 172

in synthesis of oleic acid, 173

inhibitory analogues, 553-559

requirements, $326,327,329$

role in $\beta$-decarboxylations, 154,171

sparing action of oleic acid on, 227

specificity, 542-545

stereoisomers, $542-544$

stimulatory analogues, 545-553

structure, 8

toxicity, 391

"uncombinable," 62

Biotin deficiency,

biochemical aspects of, 429

in dogs, 249

symptomology, 428

$\alpha$-Biotin, 173

$\beta$-Biotin, 173

Blacktongue, 412

Blood,

$B$ vitamin levels in, 345-347

nicotinic acid content of, 258

Bound forms of B vitamins, 284

Bradycardia tests, 384

2,3-Butylene glycol, 222

Cadaverine, formation of, 181

Caffeine, toxicity of, 297

Caloric intake, effect on $B$ vitamin requirements, 276

Cancer tissue, (See also tumors)

B vitamin content, 27

vitamin uniformity in, 27

water content, 27

Carbohydrates,

effect on $B$ vitamin requirements, 276

related compounds from, 223

synthesis of fatty acids from, 222

utilization of, 217 et seq.

Carbon to carbon bond formation, 106, 181 et seq.

role of PABA in, 189

role of thiamine in, 189

role of vitamin $B_{12}$ in, 189 
Carbonyl-phosphoric acid addition product, 219

Carboxylase, 30, 154

Carboxylation of keto acids, coenzymes for, 153

Carotenoids, 22

Carriers, 110

Catabolism, of $\mathrm{B}$ vitamins in animals, $351-370$

Catalase, 153

"Catalins," 5

Catatorulin effect, 403

Cecectomized rats, 285

Cecectomy, in study of intestinal vitamin synthesis, 299

Cell aggregates, 243

Chastek paralysis, 292, 402 of foxes, 292, 400

Chemical energy, conservation of, 101

Chemosynthesis, 238

Chicks,

B vitamin requirements of, 327

DPN content of embryos, 352 strain differences, 265

Children, B vitamin requirements of, 266

Chloroamino acids, 297

Cholamine (see ethanolamine)

Cholic acid, relation to thiamine, 282

Choline, 10

analogues, 290, 704-705

as source of "formate," 234

assay methods, 66 et seq.

biological activity, 706-708

biosynthesis, 88-89, 234-235, 353

combined forms, 39

deficiency, 430-431, 703

distribution, 18

effect of amino acids on requirements of, 278

effect of species size on requirements of, 322

essential, 278-279

ethanolamine as precursor, 89

excretion, $365-370$

extraction, 39

functions, 430

inhibitory analogues, 708-710

in phospholipids, 11,39

methyl groups from, 10-11

pharmacological action, 389-390

relation to ethanolamine, 89

requirements, $326,327,328,329$

specificity, 706-708

structure, 10

toxicity, 390

Choline acetylase, 237

Choline esterase, increase of associated with folic acid, 204

CI, 404

Citrovorum factor, $16 \mathrm{ff}$

Claisen-type condensation, 188
Climate, effect on B vitamin requirements, 267

Cobalt deficiency, anemia in cattle from, 206

Cocarboxylase, 154

Codecarboxylase, 177

"Codehydrogenase," 133

Codehydrogenase I, 133

Coefficient of uniformity, 27

Coelenterata, nutrition of, 307

Coenzyme A, assay methods, 192

biosynthesis, 194

in acetylation of choline, 237

occurrence, 193-194

reactions catalyzed by, 194 et seq.

structural studies on, 192

Coenzyme I, 33, 133, 286, 604

inhibitions involving, 616-617

Coenzyme II, 33, 132, 286

Coenzyme of Lipmann, 35

Coenzymes,

analytical methods for, 119 et seq.

biosynthesis of, 112

classification of, 109

from $B$ vitamins, $110-111$

meaning of, 108

"mobile," 137

number of, 114

occurrence of, 120 et seq.

role of adenylic acid in formation of, 114

separation of, 109

specificity of, 115,119

Coenzyme synthesis, $343-344,352-353$

extent of, 113

rate of, 113

Coenzymatic activity of simple vitamins, 111

Cofactors, 108

Coffee,

toxicity of, 297

trigonellin in, 288

"Coferment," 133

Coferment of alcoholic fermentation, 133

Cold, effect on $\mathrm{B}$ vitamin requirements, 268

Colostrum, B vitamins in, 347

Combined forms of B vitamins, (See also individual vitamins) 30 et seq.

Competitive inhibition, 295, 443

"Concentrates" of B vitamins, meaning, 13

Condensations, carbon to earbon bonds, 187 et seq. 195

Conservation of chemical energy, 101

Controlled diets, 247 et seq.

Cooking, effect on B vitamins, 339 effect on riboflavin, 33

"Cophosphorylase" activity, 135

Coramine, 287 
"Coreductase," 133

Corn, pellagragenic agent in, 280, 292

Cotransaminase, 177

"Cotryptophanase," 177

Cow-manure factor, 14, 421

"Cozymase," 132, 133

in sex hormone inactivation, 382

Crustacea, B vitamin requirements of, 309

Customs, effect on B vitamin requirements, 282

Cuttings, $B$ vitamin requirements of plant, 316

Cysteic acid, taurine from, 181

Cystic mastitis, B vitamin treatment of, 382

Cytochrome-C, 152

reduction mechanism, 152-153

reoxidation, 153

Cytochrome-C reductase, 32 oxidation of reduced TPN by, 150

Cytochromes (see also porphyrins), absorption spectra, 151

Daily dietary allowances of B vitamins for humans, table of, 324

"Dark reactions," 238

Deamination, biotin as catalyst for, 172173

Decarboxylation, of amino acids, 181

of keto acids, enzymes for, 153 et seq.

$\beta$-decarboxylation, biotin in, 154,171

Decarboxylative processes, 153 , et seq.

Deficiency states of B vitamins, 395 et seq. (See also individual vitamins) beriberi, 398-406

compound, 396

criteria, 248

in higher animals, 398 et seq.

in insects, $312-313$

in lower forms of life, 397-398

in primitive tribes, 296

in young ruminants, 298

muscular activity in, 399

pathology from, 395

pellagra, 398, 408-412

subclinical, 396-397

symptomology, 248 et seq., 396

Definition of $\mathrm{B}$ vitamins, 5

Dehydration-hydration, in glycolysis, 219

Dehydrogenation, enzymes in, 129

in glycolysis, 219

Deprivation of $B$ vitamins, effect on insects, 312

Dermal excretion of B vitamins, 368-369

Desthiobiotin, 468, 543, 550-552

Desoxycorticosterone, relation to pantothenic acid, 381

Desoxypentoses, from carbohydrates, 223
Desoxypentoses-Continued mechanism of formation, 224

Desoxyribosides, Vitamin $\mathrm{B}_{12}$ activity of, 205

Destruction of B vitamins in intestine, 337-338

Detoxification of amines, 106

Diacetyl, dismutation of, 166

Diaphorases, 149

Dietary surveys, 252-254

Diets,

controlled, 247 et seq.

special, use of in study of intestinal syntheses, 298

vitamin $\mathrm{B}$ content of mixed, 254

Digestion,

in animals, $338-343$

in plants, $336-338$

Dihydroluciferin, 238

3,4-dihydroxyphenylalanine, 181

3,4-dihydroxyphenylethylamine, formation of, 181

Dimethylethanolamine, 290

Dinicotinyl ornithine, 287, 357

Diphosphopyridine nucleotide, structure of, 132

as hydrogen acceptor in glycolysis, 219

Diphosphothiamine, 154

Disease, effect on B vitamin requirements, 273

Dismutation, acetic acid-lactic acid, 164

diacetyl, 166

Distribution of B vitamins, 18 et seq., 345 et seq. (See also individual vitamins)

in body fluids, 350-351

in body tissues, $350-351$

in circulating blood, 345-347

in human tissues, 26

in milks, $347-350$

in tumors, 27

quantitative relationships, 23

significance of, 24-25

uniformity of, 25

Dogfood, vitamin B content of, 254

Domestic birds, Vitamin B requirements of, 328

DOPA, 181

DOPA decarboxylase, 182

relation of coenzyme of, to folic acid, 204

DPN, 133

oxidation of reduced form by diaphorases, 149

ratio of reduced to oxidized form in malignancy, 138

DPT, 154

Dropsy, epidemic, 400

Dulcitoflavin, 296 
Echinodermata, B vitamin requirements of, 309

Effects of B vitamins, 377 et seq. (See also specific vitamins)

pharmacological, 385-391

toxicological, 385-391

Eggs,

experiments with, 246

riboflavin content of, 351

Egg white diets, effect on insects, 314

Embryonic development, B vitamins and, 383

Endocrine function, effect of $\mathrm{B}$ vitamins on, 380

Endocrine glands, metabolic activity of, $380-384$

Energy,

acetylcholine metabolism in production of, 237

chemical, 235

conservation of chemical, 101

conservation of, in aerobic respiration, 150

electrical, 236-237

from pyrophosphate bonds, 163

mechanical, 236

radiant, 237-238

thermal, 236

Energy transformations,

in biological systems, 100, 235 et seq. nicotinic acid coenzyme in, 140

Environment, effect on B vitamin requirement, 264

Enzymatic action, inhibition of, 445

Enzymatic reactions, 99 et seq.

general processes of, 100

inactivation of B vitamins, 292

outline of types, 104-105

requirements for characterization, 100

vitamins required, 104-105

Enzyme activators, 110

Enzyme systems, components of, 108

"Enzyme-substrate union," 116

Enzymes, 108

changes in total effect of concentration of, 462

coenzymes, 108

cofactors, 108

environmental conditions, 108

hydrolytic, 125

"poisons," 443

Ergot alkaloids, 336

Erythein, 14-15, 415

Erythropoiesis, stimulation of, 203

folic acid and, 383

Erythro-, 475

Erythrotide, 475

Erythrotin, 14-15, 415, 475

Essential choline, 278-279

Esters, formation of, 195

$\alpha$-Estradiol, detoxification of, 382
Estrogens, inactivation by liver, 382

effect of $B$ vitamins on inactivation, 382

Estrone, detoxification of, 382

Estrus cycle, B vitamins and, 383

Ethanol, formation of, 222

Ethanolamine, 10, 181, 290

Excretion of B vitamins

dermal, 368-369

fecal levels, 366-369

individual variations, $369-370$

in plants, $337-338$

studies on, 254-257

urinary levels, $364-368$

Excretion of pyramine, 255

Extraction of B vitamins, 30 et seq.

"Extrinsic" factors, 206

Exuviation, B vitamin requirements in, 311

Factors, unidentified, 244

"Factor V," 133

FAD, 143

Fasting urine specimens, 255

Fat metabolism, relation of vitamin $B_{8}$ to, $185-186$

vitamin requirements for, 229

Fats,

effect on B vitamin requirements, 276

formation of, 195,228

hydrolysis of, 228

Fat soluble compounds, 4

Fat soluble vitamins, relation to reproduction, 381

Fatty acid dehydrogenase, 152

Fatty acids, metabolism of, 225 et seq.

role of Coenzyme A in formation, 195 role of phosphoryl-acetyl compound in metabolism, 225

sparing effect on vitamins, 227

synthesis from carbohydrates, 222

unsaturated, formation of, 227

vitamins required in metabolism, 225

Fatty liver, prevention of, $\mathbf{1 2 7}$

Feces,

B vitamin excretion in, 256, 257, 300, 344, 366-367, 368-369

pantothenate content, 259

daily excretion of $\mathrm{B}$ vitamins in, 366367

Fermentation $L$, casei factor, 39

"Fern poisoning," 295

Fertilizers, effect of on B vitamins in crops, 80

Fever, effect on $\mathrm{B}$ vitamin requirements, 273

"Filtrate factor," 54

"Fitness," 245

Flavinadeninedinucleotide, 142, 143

Flavoproteins, 7, 32, 141 
Flavoproteins-Continued

L-amino acid oxidase, 32

comparison of reactions catalyzed by

to those of nicotinic acid system, 145

cytochrome-C reductase, 32

diaphorases, 149

in production of ethylenic bonds, 151

intracellular forms, 147

"old yellow enzyme," 32

properties of, 145

reaction types catalyzed by, 146

redox properties of, 145

types of, 147

union with apoenzyme, 145

Flour, agenizing of, 297

Fluids, distribution and storage of B vitamins in, $350-351$

Fluorescyanine, 285-286

Folic acid,

$p$-aminobenzoic acid in activity of xanthopterin, 421

analogues, 290

assay methods, 68-71

biological activity of, 568-574

biosynthesis, 88

choline esterase, increase associated with, 204

combined forms, 39-40

defective metabolism of tyrosine and, 204

distribution, 40

effect of related compounds on cancer, 593-597

effect of species size on requirements of, 322

excretion, 365

formyl derivative, 39

functions, 488

hematopoieses from, 202

inhibitory analogues, 575-593

in single carbon unit metabolism, 200

isolation, 565

liberation by enzymes, 40

metabolism, 363

oviduct response to stilbestrol and, 383

pyridoxal-like activity of, 204-205

relation to $\mathrm{PABA}, 202,472,490-493$

relation to pernicious anemia, 204

relation to vitamin $B_{12}, 206$

relation to thymidine, 424

requirements, $326,327,328,329$

role in amino acid synthesis, 201

role in erythropoiesis, 383

role in purine and nyrimidine synthesis, 202

specificity, 566-568

structure, 9, 493

therapeutic use in pernicious anemia, 416

toxicity, 390-391

Folic acid coenzymes, biosynthesis, 203

Folic acid deficiency, 413-422
Folic acid deficiency-Continued

anemias associated with, 413

in vertebrates, 420

in chicks, 248

pernicious anemia and, 415, 416

sprue, 417

Folinic acid, $16 \mathrm{ff}, 203 \mathrm{ff}$

Foods, processing of, effect on B vitamin content, 282

"Formate carrying," coenzyme, 197

Formic acid, formation of, 197

incorporation into purines, 196

production from pyruvate, 162

Formic acid dehydrogenase, 152

Formylfolic acid, 39 structure, 200

"Formyl group," 197

Formylpteroic acid, structure, 200, 422

Fowls, B vitamin requirements of, 259

Foxes, thiamin deficiency in, 292, 400

Chastek paralysis, 292, 400

Free energy, prediction of reactions from, 101

Fumaric acid, conversion to succinic acid, 151, 221 formation, 221

Fumaric dehydrogenase, 150

Fumaric reductase, 221

Function of B vitamins in nutrition, 5

Function of B vitamins in metabolism, 216 et seq.

Functional forms of B vitamins, 284

Galvanic cell, action of, 129

Gametogenesis, B vitamins in, 383

Genetic blocks, partial, 217

Genetotrophic diseases, 433

Geriatrics, 266

Germ-free animals, studies with, 300

Germination, B vitamin requirements for, 316

Glucose oxidase, 148

Glucose-6-phosphate, oxidation of, 136

Glutamic acid, as amino donor, 176

in metabolism of PABA, 487-489

Glutamic-alanine system, 182

Glutamic-aspartic system, 182

Glutamic-aspartic transaminase, 187

Glutamic-cysteic acid system, 183

Glutathione, in function of glyoxalase, 110

Glycerol, formation and utilization, 227228

D-Glycerophosphate dehydrogenase, 152

Glycine, conversion to serine, 233

Glycine oxidase, 148

Glycogen, phosphorolysis of, 218-219

Glycolytic process,

energy considerations, 218 
Glycolytic process-Continued

mechanism, 218

Grave's disease, thiamine administration in, 381

Gray hair syndrome, 381

Green plants,

as food source, 244

$\mathrm{B}$ vitamin deficiencies in, 397

$B$ vitamin requirements of, 316-318

Growth,

as criterion for vitamin sufficiency, 250

effect of B vitamins on, 379

"improvement" in, 250

"increase" in, 250

Guvacine, 336

Gynecomastia, in malnutrition, 382

Habits, effect on $\mathrm{B}$ vitamin requirements, 282

Harden's coferment, 133

Heart action, effect of thiamine on, 384

Heat lability as applied to vitamins, 37

Heavy metal poisons, 153

Hematopoiesis, 202

Hemes, 716-720 (See porphyrins)

Hepatic fatty infiltration, 248

Heteroauxin, 294

Hexonic acid, from carbohydrates, 223

Hexuronic acid, from carbohydrates, 223

High energy phosphate bonds, 101, 163

Higher animals, B vitamin deficiencies in, 398 et seq.

Histamine, formation of, 181

Histidine,

as single carbon unit donor, 198

histamine from, 181

Holoenzymes, 30, 109

formation of, 116

stability of, 117

Homobiotin, 468

Homocysteine, conversion to methionine, 233,234

Hormone-vitamin interrelationships, 380 384

Human experimentation,

bioassay methods for vitamins in, 252 , 254-257, 283

variables in, 249

Human tissue, distribution of $\mathrm{B}$ vitamins in, 26

Humans,

$B$ vitamin requirements of, 326

table of daily dietary allowances for, 324

Humidity, effect on B vitamin requirements, 267

Hungers, specific, 260

Hydrases, 127 et seq.

Hydrogen, production of from pyruvate, 163

Hydrogen acceptor, DPN as, 219
Hydrogen carriers, for acetate-lactate dismutation, 164

Hydrogenation-dehydrogenation, in glycolysis, 219

$\beta$-hydroxy acids, dehydration of, 128

L-Hydroxyacid oxidase, 148

3-Hydroxyanthranilic acid, 287

Hyperthyroidism,

symptomology of, 380

$B$ vitamins in treatment of, 381

Hypophysis, 380

Hypoxanthine, formation of, 199

Illness, effect on B vitamin requirements, 271

Inactivation of $\mathrm{B}$ vitamins, enzymatic, 292

in intestine, 337-338

Inactivators, natural, 292

Index of Carbohydrate Metabolism, 404

Indole, tryptophan from, 183

"Infantile pellagra," 407

Inhibition,

competitive, 443

determination of type, 452-453

index, 445-449

mass action effect in, 444

non-competitive, 443

of enzymatic action, 445

"quadratic," 454

reaction rates in, $450-451$

synergistic action of, 463

Inhibition analysis,

application of, 464

in assay development for unknown factors, 473

Inhibitors,

destruction of, 463

mechanism of resistance to competitive, $475-477$

natural, 292

Injury, effect on B vitamin requirements, 272

Inositol,

analogues, 291

assay methods, 64-66

biological activity, 712-714

biosynthesis, 89

combined forms, 38

deficiency, 430

distribution, 18

effect of species size on requirement of, 323

excretion of, 365

extraction, $38-39$

formation from carbohydrates, 223

in amylase, $10,38,106,125,218$

in phospholipides, $38,106,127$

inhibition studies on, 125

inhibitory analogues, 714-715

requirements, $326,327,328$

specificity, 712-714 
Inosital-Continued stereoisomers, 711-712

structure, 10, 711-712

therapeutic use, 430

toxicity, 391

Insects,

$B$ vitamin requirements of, 309-315

effect of egg-white diets on, 314

pyruvism in, 313

symbiosis in, 309

vitamin metabolism in, 314-315

Intake of B vitamins, recommended, 245

Intermediates, transport of labile, 121

"Intermediate carrier," 146

Interrelationships of B vitamins, 281-282, 379-380

thiamine-vitamin A, 282

Intestinal flora, 264 effect on B vitamin requirements, 297

Intestinal synthesis of B vitamins, 298

"Intrinsic factors," 206

Invertebrates, B vitamin requirements of, 306-315

Iodoacetate inhibition of thiamine phosphorylations, 155

Isoalloxazine, 143

Isoalloxazine adenine dinucleotide, 143

Isomerases, 127 et seq.

Isomerization, in glycolysis, 219

Isotels, 173

Isotopic labeling, 102

Isoxanthopterin, 285

Jackbean mean, crystalline urease from, 96

Ketenyl radical, reactions of, 160

$\alpha$-Ketoglutaric acid,

as amino acceptor, 176

as source of single carbon unit, 197

decarboxylation by thiamine coenzyme, 158

reactions catalyzed by TPP, 167

role in synthesis of amino acids, 232

role of coenzyme in synthesis, 195

Korsakoff's syndrome, 401

Kwashiorkor, 407

Kynurenin, 287

in synthesis of nicotinic acid, 83

Labile intermediates, transport of, 121

Labor, effect on $\mathrm{B}$ vitamin requirements, 267

Labor pains, thiamine in treatment of, 356

Lactation,

$B$ vitamins and, 383

effect on B vitamin requirements, 269

"Lactation factors," 383

Lactic acid dehydrogenase, 152

Lactobacillus bulgaricus factor, $16 \mathrm{ff}$

Lactochrome, 669
Lactoflavin, 669

Larvae, B vitamin requirements of, 311

Lecithins, 39

Leukemia, effect of folic acid analogues, 596

Liberation of B vitamins, in intestinal tract, 338

"Light reaction," 238

Linseed meal, anti-pyridoxine effect of, 295

Lipides, metabolism of, 225 , et seq.

"Lipocaic," 127

Lipositol, 38, 430

Liver, inactivation of sex hormones by, 382

Loading test, 255

Lower forms of life, B vitamin deficiencies in, 397

Luciferase, 238

Luciferin, 238

Lumazine, 285

Lumichrome, 363

Lycomarasmine, 260, 296

Lysine, relation to bacterial vitamin $B_{8}$ requirements, 184

Macrocytic anemias, 419-420

Magnesium ions, as cofactors for thiamine coenzyme, 158

Malic acid, formation of, 221

Malignant tissues, ratio of oxidized to reduced form of DPN in, 138

Mammals, B vitamin requirements of, 329

Manioc, 296

Manganese ions,

as cofactors for thiamine coenzyme, 158

as cofactors in biotin enzymes, 171

Mass action effect in inhibition, 444

Maternal instinct, B vitamins and, 383

Mating behavior, $\mathrm{B}$ vitamins and, 383

Melanin production, 381

Menorrhagia, B vitamin treatment of, 382

Mental activity,

effect of $\mathrm{B}$ vitamins on, 385

effect on B vitamin requirements, 267

Mental response, effect of thiamine on, 385

Metabolic interrelationships of B vitamins, $379-380$

Metabolic products,

of nicotinic acid, 356-361

of other B vitamins, 361-364

Metabolic rate, 264,379

effect of B vitamins on, 379

effect on $B$ vitamin requirements, 273

Metabolism, "total," 379

Metamorphosis, nutrition in, 309

Methionine, 10

essential level, 279 
Methionine-Continued

from homocysteine, 233, 234

methyl groups from, 10, 234

role of vitamin $B_{12}$ in formation, 207

Methyl groups,

from choline, 10-11

from methionine, 234

$\mathrm{N}^{\prime}$-methyl nicotinamide, 357

N'-methyl-6-pyridone-3-carboxylamide, 357

Metorrhagia, B vitamin treatment of, 382

Mice, $\mathrm{B}$ vitamin requirements of, 327

Microbiological tests in discovery of B vitamins, 15

Milks,

aldehyde oxidases in, 148

$B$ vitamins in, $20,258,347-350$

diaphorases from, 149

pantothenate in, 85

riboflavin in, 82,271

thiamine in, 271

xanthine oxidase in, 148

"Mobile coenzymes," 137

Modification of B vitamins in intestinal tract, $337-338$

Molds, riboflavin excretion in, 338

Monomethylethanolamine, 290

"Mouse anti-alopecia factor," 712

Muscle,

diaphorases from, 149

DPN from, 135

Muscular activity in B vitamin deficiency, 399

Mycorrhizial fungi, 80-81

Myelin sheath, 384

National Research Council, table of recommended dietary allowances for humans, 324

Natural selection studies, 259-260

Nerve function, effect of $\mathrm{B}$ vitamins on, 384

Nerve metabolism, role of vitamins in, 384

Neuron, 384

Neurospora, 10

Niacin (see nicotinic acid)

Nicotinamide (see nicotinic acid)

Nicotine, 336, 389

Nicotinic acid, (see also nicotinic acidtype compounds)

activity of glutamic acid and asparagine, 84

analogues, 286

assay methods, 54-56

biological activity of, 604-611

biosynthesis, $82-83$, 330, 353-356

combined forms, 33

content in blood, 259

distribution, 18
Nicotinic acid-Continued

effect of species size on requirement of, 320

effect of tryptophan on requirement of, 279

excretion, 365

extraction, 34

inhibitory analogues, 280, 611-617

interrelation to $\mathrm{PABA}, 282$

interrelation to pantothenic acid, 282

lethal dosage, 389

liberation by enzymes, 34

natural antagonisms related to, $615-616$

pharmacological action, 387-388

products of, $356-361$

replaceability by tryptophan, 279

requirements, $317,324,326,327,328$, 329

specificity, 604, 611

stimulatory action on plant roots, 8283

structure, 7

therapeutic use, 411

toxicity, 387-388

Nicotinic acid coenzymes, (see also DPN, TPN)

absorption spectra, 134

assay methods, 135

biosynthesis, 138

extraction, 137

inactivation of, 134

Nicotinic acid deficiency, 408-412

biochemical changes in, 412

blacktongue, 412

para-sprue, 410

pellagra, 408-412

symptomology, 409

Nicotinic acid type compounds, pharmacological action of, 388 occurrence, 137

oxidized and reduced forms, 133-134

reactions catalyzed by, 139 et seq.

redox systems coupled with, 140

role in energy transformations, 140

sources, 135

specificity, 139

Nicotinuric acid, 287, 357

Nitrogen compounds, metabolism of, 230 et seq.

Nitrogen retention, effect of androgens on, 381-382

Nitroid reaction, 387

"Norite-eluate factor," 69

Nucleosides, vitamin $B_{12}$ activity of, 205

Nucleotides, 7

Nutrition, effect of sulfonamides on, 512

Nutritional customs, effect on B vitamin requirements, 202

Nutritional function of $\mathrm{B}$ vitamins, 5

"Nutritional requirement," 244

Nutritional status, 249 
Nutritional viewpoint of meaning of $B$ vitamins, 13

Nutritional polyneuritis, 400

with chronic alcoholism, 400

Occupation, effect on $\mathrm{B}$ vitamin requirements, 267

Occurrence of $\mathrm{B}$ vitamins, reason for universal, 98

"Old yellow enzyme," 32, 144, 150

Oleic acid,

biotin in synthesis of, 173

sparing action on biotin, 227

Ornithine, putrescine from, 181

Oviduct hypertrophy, effect of stilbestrol on, 383

Ovoflavin, 669

Oxalacetic acid, formation of, 221

Oxidases, amino acid, 147-148

Oxidation-reduction potential, effect of ascorbic acid, 21

Oxybiotin, 8, 545-550

PAC, 192-193

Pancreatic amylase, 38, 218

Pantoic acid, 35

from pantonine, 85

"Pantonine," 85

Pantothen-, 20

Pantothenic acid, analogues, 289

assay methods, 56-59

biological activity, 620-621

biosynthesis, $84-86$

coenzyme (see coenzyme A)

combined forms, 34

conjugate, 192-193

distribution, $19-20$

effect of biotin on requirement of, 278

effect of species size on requirement of, 321

excretion, 365

extraction, 34

fecal content of, 259

functions, 465-466

inhibition of, 464

inhibitory analogues, 624-648

interrelation to $\beta$-alanine, 466

interrelation to aspartic acid, 466

interrelation to nicotinic acid, 282

interrelation to $\mathrm{PABA}, 282$

interrelation to desoxycorticosterone, melanin, 381

v liberation by enzymes, 34-35

metabolism, 362-363

oviduct response to stilbestrol and, 383

relation to plant growth, 86

role in sterol synthesis, 230

requirements, $326,327,328,329$

sparing effect of fatty acids on, 227

specificity, 620-624

structure, 7
Pantothenic acid-Continued toxicity, 391

$\checkmark$ universal occurrence, 19

urinary content of, 259

Pantothenic acid deficiency, adrenal hypertrophy in, 424

biochemical nature of, 424

symptomology, 423

Partial genetic block, 217

Pasteur reaction, 32

Pathological states,

effect on $B$ vitamin requirements, 271 relation of $\mathrm{B}$ vitamins to, 431-433

Pellagra, causation, 409

distribution, 408

effect of tryptophan in, 615

historical, 398

in pigs, 412

incidence, 398

mental symptoms, 410

pathology, 410-411

role of sunlight in, 409

symptomology, 409

Pellagragenic agent, in corn, 280, 294

"Pellagra-preventive factor," 407

Pentoses,

from carbohydrates, 223

mechanism of formation, 224

"Perleche," 406

Pernicious anemia,

Addisonian, 415

excretion of folic acid in, 571

hematological response in, 416

relation of folic acid to, 204

symptomology, 415

therapy, 415

thymine, folic acid, vitamin $B_{12}$ in therapy, 416

Perspiration, B vitamins in, 269, 368-369

PGA, 39

$\mathrm{pH}$, effect on sulfonamide activity, 502505

Pharmacological action of $B$ vitamins (see individual vitamins)

Pliarmacological level, 377

Phenylethylamine, formation of, 181

Plilorglucinol-like compounds, formation of, 195

Phosphatases, 157

Phosphate bonds, high energy, 101, 236

Phospholipides,

choline in, 11, 39

inositol in, 38

Phosphoroclastic reaction, 162,335

Phosphorus retention, effect of androgens on, 381-382

"Phosphoryl-acetyl intermediate," 162 end products of, associated with thiamine metabolism, 165 
"Phosphoryl-acetyl intermediate-Cont'd origin of, 191

role in fatty acid metabolism, 225

Phosphorylases, 157

Phosphorylation, in glycolysis, 219 of B vitamins, $343-344$

Photosynthesis, 237-238, 337

Phototrophism, 337

Phycomyces test, 80,384

Phyla, pantothenate in various, 19-20

"Physical fitness," 245

Physiological interrelationships of B vitamins, $378-379$

Physiological level, 377

Physiological requirements, 264

Phytic acid, 38

Phytin, 38

$\beta$-Picoline, 286-287

Pigs, pellagra in, 412

Pimelic acid, as biotin precursor, 88

effect on biotin production, 88

Pituitary gland, 380

Placebos, use of, 249

Plant cuttings, B vitamin requirements of, 316

Plant embryos, B vitamin requirements of, 307,316

Plant roots, B vitamin requirements of, 307,316

Plants, green,

absorption in, 336-337

$B$ vitamin deficiencies in, 397

digestion in, 336-337

distribution of $\mathrm{B}$ vitamins in, 337

excretion of $B$ vitamins in, 336-338

metabolism of $\mathrm{B}$ vitamins in, 336-338 origin of $B$ vitamins in, 337

Plant tissues, aldehyde-oxidases in, 148

amino acid requirements of, 316-317

$B$ vitamin requirements of, $316-318$

mitotic rates of, 379

Plasmochin, 680

Platyhelminthes, B vitamin requirements of, 308

Poising agents, enzyme activators as, 110

Poisons, heavy-metal, 153

Pollen grains, B vitamin requirements of, 316

Polyneuritis, nutritional, 400 time required for development, 264 with chronic alcoholism, 400

Polysaccharides, synthesis and cleavage of, 217 et seq.

Porifera, nutrition of, 308

Porphyrins,

as growth factors, 716

as inhibitors, 716

$B$ vitamin-like properties of, 151

biological activity, 716-720
Porphyrins,-Continued role of single carbon unit in biosynthesis of, 235

Potassium, effect of androgens on retention of, 381382

relation to biotin deficiency, 249

Potency of B vitamins, 283

Potentials, actual, 131

redox, 130

standard, 131

Precursors of B vitamins, 284

Pregnancy, anemia in, 419

effect on B vitamin requirements, 268

Premenstrual tension, $\mathrm{B}$ vitamin treatment of, 382

Primitive tribes, deficiencies in, 296

Prison camps, dietary surveys in, 252

Procaine, effect on sulfonamide therapy, 486

Processing of food, effect on B vitamin content of, 282

Products of B vitamins, 284

Prontosil, 481

Prosthetic groups, classification of, 109

Protein-complex, preparation of, 120-121

"Protein-complexes," 116

Proteins,

effect on B vitamin requirements, 276 effect on pyridoxine requirements, 278 effect on riboflavin requirements, 2.7 synthesis and hydrolysis of, 230 et seq.

Protogen, 16

Protozoa, B vitamin requirements of, 307

"Pseudopyridoxine," 654

Pterins, effects in anemia, 573-574

Pteroylglutamic acid, 9, 39-40

Pteroylheptaglutamate, 290

Pupation, $\mathrm{B}$ vitamin requirements for, 311

Purines, catabolism of, 234

folic acid in synthesis of, 201

incorporation of single carbon unit in, 196

PABA in synthesis of, 201

vitamins required in synthesis of, 233234

Putrescine, 181

$\alpha$-Pyracin, 421

$\beta$-Pyracin, 421

Pyramin, excretion of, 255, 369

Pyridine derivatives, excretion of, 389

Pyridoxal (see also pyridoxine, Vitamin $\mathrm{B}_{6}$ coenzyme)

analogues, 658-659

assay methods, 59-61

biological activity, 655, 656

dissociation constants of enzymes of, 180

isolation, 654

phosphate, $7,36,178$ 
Pyridoxal-Continued

structure, 8

sparing action on amino acids, 185

Pyridoxamine (see also pyridoxine, Vitamin $\mathrm{B}_{8}$ coenzyme)

analogues, 658-659

assay methods, $59-61$

biological activity, 655-656

formation from pyridoxal phosphate, 177

isolation, 654

phosphate, $7,36,178$

structure, 8

sparing action on amino acids, 184

4-Pyridoxic acid, 288, 290, 364, 657-658

Pyridoxine,

acid-labile forms, 37

analogues, 288

antagonistic effects, 295

as storage form, 86

assay methods, 59-61

biological activity, 652-654, 656

biosynthesis, $86-87$

coenzymes (see vitamin $\mathrm{B}_{6}$ coenzymes)

combined forms, 36

distribution, 36

effect of autoclaving on, 176

effect of protein on requirements of,

278

effect of species size on requirements of, 320

essentiality of, 187

excretion of, 365

extraction, 36

inhibitory analogues, 659-666

metabolism, 364

pharmacological action, 390

relation to fat metabolism, 185-186

relation to growth of plants, $86-87$

requirements, 317,318

specificity, 652-654

structure, 8

toxicity, 390

Pyridoxine deficiency,

biochemical aspects, 428

symptomology, 426-427

Pyrimidines,

folic acid in synthesis of, 202

PABA in synthesis of, 201

vitamins required in synthesis of, 233234

Pyrophosphate bond, energy from, 163

Pyruvate oxidation factor, $16 \mathrm{ff}$

Pyruvic acid,

acetate-lactate dismutation of, 164

acetoin from, 161

aerobic oxidation, without phosphate, 166

aerobic utilization of, 165,220

anaerobic utilization of, 220

as hydrogen acceptor, 220

$\beta$-decarboxylation of, 221
Pyruvic acid-Continued

decarboxylation of $158,160,221$

formic acid production from, 162

phosphoroclastic cleavage of, 163

reduction after carboxylation, 220-221

Pyruvism, in insects, 312

"Quadratic" inhibition, 454

Quinine, relation to riboflavin coenzymes, 679-681

Quinine oxidase, 296

Quinolinic acid, formation of, 287, 355, 357

Random urine specimens, 255

Rat carcass, vitamin B content of, 254

Rats, requirement for $\mathrm{B}$ vitamins, 326

requirement for vitamin $B_{6}, 299$

strain differences, 265

Reaction rate, effect of substrate in inhibition of, 450 in absence of inhibition, 451

in competitive inhibition, 451

in noncompetitive inhibition, 451

Reactions,

reversibility of, 101

types of biochemical, 103

Recommended daily intake of $B$ vitamins, 254

Redox dyes, 131

Redox potential, 130

Redox systems, coupled with nicotinic acid coenzyme, 141

"Refection," 298

Relaparotomy, B vitamin deficiency after, 272

Reproduction,

$\mathrm{B}$ vitamin deficiency and, 381-382

essentiality of $B$ vitamins in, 383

Requirements for B vitamins (see also individual vitamins)

bioassay methods, in humans, 252

comparative studies of, 246 et seq.

criteria in fixing of, 245

effect of age on, 265-266

effect of anoxia on, 272

effect of caloric intake on, 276

effect of carbohydrates on, 276

effect of climate on, 267

effect of customs on, 282

effect of disease on, 272

effect of fats on, 276

effect of fever on, 273

effect of illness on, 271

effect of injury on, 272

effect of intestinal flora on, 297

effect of lactation on, 269

affect of metabolic rate on, 273

affect of other nutritional components on, 275-276

effect of pregnancy on, 269

effect of proteins on, 276 
Requirements for B vitamins-Cont'd effect of sex on, 266-267

effect of shock on, 272

effect of size on, 264

effect of species size on, 319-323

effect of surgery on, 272

effect of thyroid on, 380-381

effect of weight on, 266

effect of work on, 267

environment as factor, 264

factors influencing, 264 , et seq.

growth as criterion for, 252

maximal, 245

methods of assessing, 243

of aged, 266

of arthropoda, 309-315

of bacteria, 307

of chicks, 327

of children, 266

of domestic birds, 328

of fetuses, 270

of fowls, 258

of humans, 326

of insects, 309-315

of invertebrates, 306-315

of mammals, 329

of mice, 327

of plant roots and embryos, 307

of protozoa, 307

of rats, 326

of tissues, 259

of vertebrates, $318-330$

of viruses, 307

of worms, 308

optimal, 245

qualitative requirements, 244

quantitative requirements, 245

recommended intake, 245

specie variability and, 264

strain variability and, 265

Resection, effect on $\mathrm{B}$ vitamin requirements, 272

Resistance, mechanism in competitive inhibition, 475-477

mechanism of sulfonamide, $519-520$

to sulfonamides, 516-521

Reversibility of a reaction, 101

Rhizopterin, 422, 566

in folic acid assay, 70

PABA in, 40

Riboflavin,

analogues, $285-286$

assay methods, $51-56$

biological activity of, 670-673

biosynthesis, $81-82$

combined forms, 32-33

cooking and storage effect on, 33

determination of, in serum, 259

distribution of, 19

effect of ascorbic acid on storage, 258

effect of protein on requirement, 277
Riboflavin-Continued

effect of species size on requirement of, 319

excretion, $271,338,365$

extraction, 33

flavoproteins, 32

function, in anaerobic systems, 150

inhibitory analogues, 673-682

in milk, 82

metabolism of, 362

"old yellow enzyme," 32

requirements, $317,324,326,327,328$, 329

sparing effect of fatty acids on, 227

specificity, $670-673$

stereoisomers, 671

structure, 6

toxicity, 390

Riboflavin coenzymes (see also flavoproteins)

as hydrogen carriers, 141

assay methods, 144

biosynthesis, 144

mechanism of oxidation and reduction, 146

occurrence, 144

Riboflavin deficiency,

biochemical changes in, 407-408

distribution, 406

symptomology, 406

Rice bran, crystalline thiamine from, 96

Rice moth larva, studies on, 311-312

Robinson ester, 136

Rous chicken sarcoma, 594-596

"Royal jelly," 22

Rumen,

synthesis of pantothenic acid in, 85

synthesis of riboflavin in, 82

synthesis of thiamine in, 78-79

Ruminants, deficiency in young of, 298

Sarcoma, 180, 597

effect of folic acid on, 594-596

Sarcosine dehydrogenases, 152

"Saturation" with B vitamins, 370

Schiff's base formation, 186

Secondary products, effect in inhibition, 461

Self-selection studies, $259-260$

Serine,

ethanolamine from, 181

from glycine, 233

role of vitamin $B_{12}$ in formation of, 207

trypotophan from, 183, 233

Sex, influence on $\mathrm{B}$ vitamin requirements, 266-267

Sexual function, B vitamin deficiency and, $381-382$

Shock,

coenzyme breakdown in, 272

effect on B vitamin requirements, 272 
Sickness, effect on B vitamin requirements, 271

Significance of distribution of B vitamins, $24-25$

Silverfish, 315

Single carbon unit,

blocking of reactions by sulfonamides, 200

coenzymes in utilization of, 196 et seq.

folic acid in, 200

in purines, 196

origin, 197

sources, 197

vitamins associated with metabolism of, 198 et seq.

Size, effect on B vitamin requirements of species, 319,323

SLR factor, 200,422

Sodium iodoacetate, inhibition of phosphorylation of thiamine, 156

Snake venom, 148

Solubility as criterion for classification of vitamins, 16

Solubility of B vitamins in water, 30

Sources of B vitamins, 30 et seq.

"Sparing effect" in inhibition studies, 461

Specificity of B vitamins (see specific B vitamins)

Specificity of coenzymes, 115

Species, variability of, 264

Species size, relation of B vitamin requirements to, $264,319-323$

Sphingomyelins, 39

Sprue, 417-418

symptomology, 417

therapy, 418

thymine in, 418

Standard potential, 131

Sterile animals,

studies with, 300

metabolism of, 228, 230

Sterility, B vitamin defieiency and, 381382

Sterols,

role of Coenzyme A in synthesis of, 195,230

vitamins required in synthesis of, 230

Stilbestrol, effect on oviduct, 383

Storage of B vitamins, 255, 258-259

in tissues and fluid, 350-351

Storage of foods, effect on riboflavin content, 33

Strain, variability of, 264

Strepogenin, 15, 260, 296, 397

Substrate, effect on rate of inhibited enzymatic reactions, 450

Succinic acid dehydrogenase, 152

Succinoxidase, 151

Sulfonamides,

activity in reversal with $\mathrm{PABA}, 496-499$
Sulfonamides-Continued

antagonism to chemotherapeutic action, 498

antagonism to PABA of, $199,494,500$

biological effects of, 511-516

blocking of single carbon unit metabolism by, 200

effect of ionization on activity, 501502

effect of mass action on activity, 501

effect of $\mathrm{pH}$ on activity, 502-505

effect of physical properties on activity, 501

effect of procaine on therapy with, 486

effect of resonance on activity, 510

effect of structure on activity, 501

effect of sulfonyl-negativity on activity, 505-509

effect on nutrition, 513

effect on respiration, 512

in inhibition analysis of PABA function, 470-471

inhibitions unaffected by PABA, 500

miscellaneous effects of, 515,516

resistance to, 516-521

Sulfones,

activity in reversal with PABA, 496499

antagonism to $\mathrm{PABA}, 495$

Sulfonyl group, effect of negativity on sulfonamides, 505-509

Symbiosis,

in green plants, 79

in insects, 309

Symbiotic microorganisms, 79

Synergism, in inhibition, 463

Synthesis of B vitamins in animals, 351 .

Synthesis of coenzymes,

extent of, 113

rate of, 113

Synthetic ability, impairment of, 217

Taboos, effect on B vitamin requirement, 282

Tapeworm, in anemia, 419-420

Temperature, effect on B vitamin requirements, 267-268

Testosterone, effect of on excretion of nitrogen, phosphorus, potassium, 381382

Thiaminase, 292-293

Thiamine,

activating effect on carboxylase, 155

analogues, 284-285, 688- 690

antagonistic effects, 295

as cofactor, 133

assay methods, 47-51

availability, from yeast, 291

biological activity of, 687-694

biosynthesis, $78-81$

cocarboxylase, 30

combined forms, $30-31$ 
Thiamine-Continued content of diets of, 253 curare-like action of, 386 decline in urinary and tissue levels, 405 distribution, 19

-disulfide, 32

effect of species size on requirement, 264,319

effect of yeast on dietary, 291

effect on heart action, 384

effect on mental activity, 385

effect on nervous impulse, 384

effect on plant and seedling growth, 80 excretion, 271, 365

extraction, 31

functions, 168,170

inhibition of phosphorylation of, 155

inhibitory analogues, 693-700

interrelation to cholic acid, 282

interrelation to vitamin A, 282

lethal dosage, 386

pharmacological action, 386

-pyrophosphate, 154

relation of structure to function, 167168

requirements, $264,317,324,326,327$, 328,329

sources, 30

sparing effect, 276

specificity, 681-694

structure, 4

therapeutic index, 386

therapeutic use, $356,381,386$

toxicity of, 386-387

variability in requirements of, 274

Thiamine coenzymes,

assay methods, 155

biosynthesis, 157

formation of holoenzyme from, 157

impermeability of cell membranes to, 158

inhibition by thiamine, 157

reactions catalyzed by, 158 et seq.

role in carbon to carbon bond metabolism, 189

Thiamine deficiency,

beriberi, 399-400

biochemical features of, 403

causes of, 399

in foxes, 292,402

mild, 404

nutritional polyneuritis, 400

requirements to prevent, 264

Thiamine disulfide, thiamine activity of, 168-169

Thiochrome, 685

Thiochrome method, 47-48

Thiochrome pyrophosphate, 155

Threonine, relation to pyridoxine requirement, 184

Thunberg technique, 149
Thymidine, 15

interrelation to antipernicious anemia vitamin, 205

interrelation to PABA and folic acid, 474

structure, 15

Thymine, 290, 414

in anemia therapy, 414, 416

in sprue, 418

structure, 414

Thyroid gland,

effect on B vitamin metabolism, 381

effect on $\mathrm{B}$ vitamin requirements, 380

Thysanura, 315

Ticks, 315

"Tissue hunger," 254

Tissues,

$B$ vitamins in, 20, 258, 350-351

$B$ vitamins in human tissue, 26

cancer, 27

requirements for B vitamins of, 259

"vitamin uniformity" in, 27

Tocopherols, distribution, 22

Toxicological level, 377

TPN, 133

oxidation of reduced forms by cytochrome-C-reductase, 150

TPP, synthetic preparation of, 154-155

Transaminases, 176

Transaminations, 176

Transesterifications, in glycolysis, 219

Transport of labile intermediates, 121

Tricarboxylic acid cycle, 223

Trigonellin, 83, 357

Triphosphopyridine nucleotide, 133

Tropical climate, effect on B vitamin requirements, 267

Tryptophan, 83

as substitute for nicotinic acid, 83, 279

effect on excretion of niacin products, 83

effect on niacin requirement, 279

non-oxidative degradation, 183

synthesis, 183,233

Tumors, 26-27

B vitamin content of, 28

inhibition of proliferation of cells of, 203

virus-induced, 28

Tyramine, formation of, 181

Tyrosine, defective metabolism of, 204

Tyrosine apodecarboxylase, 178

Tyrosine decarboxylase, 204

Uncombinable biotin, 62-63

Unidentified factors in animal nutrition, 244

Uniformity, coefficient of, 27

Uniformity of distribution of B vitamins, 25 
"Uniformity, vitamin," in tissues, 27

Universal distribution of B vitamins, 1920

reason for, 98

Unknown factors, inhibition analysis in assay development for, 473

Unsaturated fatty acids, formation of, 227

Urease, from jack-bean meal, 96

Urinary specimens,

$B$ vitamins in, 252, 254-257

pantothenate in, 259

Urine,

excretion of $\mathrm{B}$ vitamins in, 364-368

nicotinic acid products in, 356-361

other vitamin products in, 365

"V" factor activity, 608-609

Variability,

individual, 255, 369-370

species, 264

strain, 264

Variables in human experiments, 249

Variation, individual in thiamine requirements, 273-274

Venom, snake, 148

Verdoperoxidase, 184

Vertebrates, B vitamin requirements of, 318-330

Viruses, B vitamin requirements of, 307

Virus-induced tumors, 28

Vitamin A, distribution, 22

interrelation to thiamine, 282

Vitamin assays (see assay methods, individual vitamins)

Vitamin B

biosynthesis, 78 et seq. $234-235$

content of various materials, 254, 257259

end products of, 256

levels in feces, 256-257

studies in excretion of, 254-257

Vitamin $B_{1}$ (see thiamine)

Vitamin $B_{2}$ (see riboflavin)

Vitamin $B_{3}$ (see pantothenic acid)

Vitamin $B_{6}$ (see pyridoxine, pyridoxamine, and pyridoxal)

Vitamin $B_{12}$ (see antipernicious anemia vitamin)

Vitamin $B_{13}, 16$

Vitamin $\mathrm{B}_{14}, 16 \mathrm{ff}, 203$

Vitamin $\mathrm{B}_{\mathrm{c}}$ conjugase, 203

Vitamin $B_{c}$ conjugate, 40
Vitamin $\mathrm{B}_{8}$ coenzyme,

assay methods, 178

biosynthesis, 179-180

coenzyme for glutamic-aspartic system, 182

coenzyme for glutamic-alanine system, 182

condensations with, 189

in reactions of methylene groups, 183

in transaminations, 176

inhibition studies, 180

mechanism of action, 186

occurrence, 179

reactions catalyzed by, 180 et seq.

relation to bacterial amino acid requirements, 184

sources, 179

structure, 177

Vitamin C (see ascorbic acid)

Vitamin D, 22

Vitamin E (see tocopherols)

Vitamin G (see riboflavin)

Vitamin $\mathrm{H}$ (see biotin)

Vitamin K, 23

Vitamins required in enzymatic reactions, 104-105

"Vitamin-uniformity" in tissues, 27

Warburg's coferment, 133

Water content of cancer tissues, 27

Water solubility of $\mathrm{B}$ vitamins, 30

Weed killers, action of, 398

Weight, effect on B vitamin requirements, 266

Wernicke's disease, 400, 402

Work, effect on B vitamin requirements, 267

Worms, B vitamin requirements of, 308

Xanthine, uric acid from, 149

Xanthine oxidase, 148, 296

Xanthopterin, 285, 288

folic acid activity of, 421

effect in anemias, 573-574

"Xanthopterin oxidase," 203, 296

Xanthurenic acid excretion, 314

Yeast,

availability of thiamine in, 291

diaphorases from, 149

DPN from, 135

effect on dietary thiamine, 291

Yeast fermentation tests, 384

Yellow enzyme, 96

Zwischen ferment, 132

Zymase, 154 



